Gamma-Ray Library and Uncertainty Analysis: Passively Emitted Gamma Rays Used in Safeguards Technology

W. Parker

September 23, 2009 
This document was prepared as an account of work sponsored by an agency of the United States government. Neither the United States government nor Lawrence Livermore National Security, LLC, nor any of their employees makes any warranty, expressed or implied, or assumes any legal liability or responsibility for the accuracy, completeness, or usefulness of any information, apparatus, product, or process disclosed, or represents that its use would not infringe privately owned rights. Reference herein to any specific commercial product, process, or service by trade name, trademark, manufacturer, or otherwise does not necessarily constitute or imply its endorsement, recommendation, or favoring by the United States government or Lawrence Livermore National Security, LLC. The views and opinions of authors expressed herein do not necessarily state or reflect those of the United States government or Lawrence Livermore National Security, LLC, and shall not be used for advertising or product endorsement purposes.

This work performed under the auspices of the U.S. Department of Energy by Lawrence Livermore National Laboratory under Contract DE-AC52-07NA27344. 


\title{
Gamma-Ray Library and Uncertainty Analysis: Passively Emitted Gamma Rays Used in Safeguards Technology
}

\author{
Winifred Parker \\ Lawrence Livermore National Laboratory \\ P.O. Box 808, Livermore California 94550
}

\section{Introduction}

Non-destructive gamma-ray analysis is a fundamental part of nuclear safeguards, including nuclear energy safeguards technology. Developing safeguards capabilities for nuclear energy will certainly benefit from the advanced use of gamma-ray spectroscopy as well as the ability to model various reactor scenarios.

There is currently a wide variety of nuclear data that could be used in computer modeling and gamma-ray spectroscopy analysis. The data can be discrepant (with varying uncertainties), and it may difficult for a modeler or software developer to determine the best nuclear data set for a particular situation. To use gamma-ray spectroscopy to determine the relative isotopic composition of nuclear materials, the gamma-ray energies and the branching ratios or intensities of the gamma-rays emitted from the nuclides in the material must be well known. A variety of computer simulation codes will be used during the development of the nuclear energy safeguards, and, to compare the results of various codes, it will be essential to have all the $\gamma$-ray libraries agree. Assessing our nuclear data needs allows us to create a prioritized list of desired measurements, and provides uncertainties for energies and especially for branching intensities. Of interest are actinides, fission products, and activation products, and most particularly mixtures of all of these radioactive isotopes, including mixtures of actinides and other products.

Recent work includes ${ }^{1}$ the development of new detectors with increased energy resolution, and studies of gamma-rays and their lines used in simulation codes. Because new detectors are being developed, there is an increased need for well known nuclear data for radioactive isotopes of some elements. Safeguards technology should take advantage of all types of gamma-ray detectors, including new super cooled detectors, germanium detectors and cadmium zinc telluride detectors. Mixed isotopes, particularly mixed actinides found in nuclear reactor streams can be especially challenging to identify. The super cooled detectors have a marked improvement in energy resolution, allowing the possibility of deconvolution of mixtures of gamma rays that was unavailable with high purity germanium detectors.

Isotopic analysis codes require libraries of gamma rays. In certain situations, isotope identification can be made in the field, sometimes with a short turnaround time, depending on the choice of detector and software analysis package. Sodium iodide and 
high purity germanium detectors have been successfully used in field scenarios. The newer super cooled detectors offer dramatically increased resolution, but they have lower efficiency and so can require longer collection times. The different peak shapes require software development for the specific detector type and field application. Libraries can be tailored to specific scenarios; by eliminating isotopes that are certainly not present, the analysis time may be shortened and the accuracy may be increased.

The intent of this project was to create one accurate library of gamma rays emitted from isotopes of interest to be used as a reliable reference in safeguards work. All simulation and spectroscopy analysis codes can draw upon this best library to improve accuracy and cross-code consistency. Modeling codes may include MCNP and COG. Gamma-ray spectroscopy analysis codes may include MGA, MGAU, U235 and FRAM. The intent is to give developers and users the tools to use in nuclear energy safeguards work. In this project, the library created was limited to a selection of actinide isotopes of immediate interest to reactor technology. These isotopes included ${ }^{234-238} \mathrm{U},{ }^{237} \mathrm{~Np},{ }^{238-242} \mathrm{Pu},{ }^{241,243} \mathrm{Am}$ and ${ }^{244} \mathrm{Cm}$. These isotopes were examined, and the best of gamma-ray data, including line energies and relative strengths were selected.

There have been several isotopic analysis codes developed at Livermore, ${ }^{2}$ including GAMANAL, GRPANAL, MGA and U235/MGAU. GAMANAL (gamma-ray isotopic analysis) is an isotopic analysis code that analyzes gamma-ray spectra, collected with a germanium detector, and identifies isotopes that produced the gamma rays. GRPANAL is a group analysis program, which focuses on groups of isotopes, and was based on the GAMANAL code. MGA is the Multi Group Analysis code, which is a plutonium isotopic analysis code that uses an intrinsic calibration method to determine the efficiency and energy calibrations for the analysis. MGA uses data collected non-destructively with germanium detectors (small planar or two detectors) and determines isotopic ratios for $238,239,240,241 \mathrm{Pu}$, and also give a percent of ${ }^{241} \mathrm{Am},{ }^{235,238} \mathrm{U}$, determines the U/Pu ratio and the ${ }^{243} \mathrm{Am}^{239} \mathrm{~Np}$ and ${ }^{241} \mathrm{Am}$ inhomogeneity. MGA is part of the MGA++ code suite. The MGA++ code also includes U235 (MGAU) for determining the relative abundance of ${ }^{234} \mathrm{U},{ }^{235} \mathrm{U}$ and ${ }^{238} \mathrm{U}$. CZTU, a ${ }^{235} \mathrm{U}$ and ${ }^{238} \mathrm{U}$ isotopic analysis code for data collected with a cadmium zinc telluride detector, is also included in the MGA++ code suite. The MGAHI code determines relative isotopic ratios for $\mathrm{Pu}$ and ${ }^{241} \mathrm{Am}$ and sets a lower bound on the ${ }^{239} \mathrm{Pu}$ mass. The MGAHI code uses the $200 \mathrm{keV}-1 \mathrm{MeV}$ energy region, and is designed for situations in which the low energy gamma rays are not available.

New codes are being developed, including CZT-Pu, U-Pu HI, and U235HI. The CZTPu code will determine plutonium isotopic ratios using data collected with a cadmium zinc telluride detector. The U235HI code uses data collected with germanium detector to determine uranium isotopic ratios from the higher energy gamma-ray lines. The HI in these code names is for high energy and is for situations where low energy lines are unavailable, such as if the material is located in a thick walled container. U-Pu HI determines isotopic ratios for mixtures of uranium and plutonium, using higher energy gamma-ray data collected with Ge detectors. Table 1 gives a very general description of radiation detection history. ${ }^{3}$ The codes mentioned above all used subsets of the same library. Now that the super cooled detectors are becoming available, the exact energies of 
the gamma rays are more important, because gamma peaks that previously overlapped with each other will be distinct.

Table 1. A simple picture of radiation detection history. ${ }^{3}$
Decade Technology Volume
Operating
Energy Temperature
1930s
Gas
Large
$300 \mathrm{~K}$
Resolution detectors
1950s Scintillators
Large
$300 \mathrm{~K}$
$77 \mathrm{~K}$
Low
1970s Germanium
Medium
1990s Cryogenic:

$\begin{array}{cc}\text { Tunnel Jcts } & \text { Small } \\ \text { Calorimeters } & \text { Small }\end{array}$
$\sim 0.4 \mathrm{~K}$
$\sim 0.1 \mathrm{~K}$
Moderate
High
Very High
Extremely
High

In general, low temperature operation allows higher resolution and higher precision; however efficiency is often sacrificed as the detector size can decrease dramatically.

Our original proposal allowed for the investigation and creation of a gamma-ray library that would typically be found in a reactor environment, including actinides, fission products and activation products. Funding issues forced us to limit the project in scope. There are a variety of reactor fuels and daughter products, including uranium, plutonium americium and neptunium; for this project we focused on isotopes of these four elements.

\section{Data}

For selected isotopes of uranium, neptunium, plutonium, americium and curium, gammaray libraries were created using the RADSRC ${ }^{4}$ code (based on the GAMGEN program) and using the ENSDF library ${ }^{5}$ available from the National Nuclear Data Center. It was found that the gamma-ray energies and branching intensities are in good agreement, with a few exceptions in the lower intensity gamma rays.

Energies of gamma rays are well known, but there can be large uncertainties in gammaray branching intensities. ${ }^{6}$ Tables $2-4$ give isotopes of neptunium, americium, curium plutonium and uranium and the percent uncertainty in the gamma-ray branching ratio. Isotopes with large gamma-ray branching uncertainties are highlighted in red. Many plutonium isotopes $\left({ }^{237} \mathrm{Pu},{ }^{238} \mathrm{Pu},{ }^{239} \mathrm{Pu},{ }^{240} \mathrm{Pu}\right.$ and $\left.{ }^{241} \mathrm{Pu}\right)$ have small uncertainties in their gamma-ray branching intensities, while others $\left({ }^{235} \mathrm{Pu},{ }^{236} \mathrm{Pu},{ }^{242} \mathrm{Pu},{ }^{243} \mathrm{Pu},{ }^{244} \mathrm{Pu}\right)$ have higher uncertainties. There are high uncertainties in the gamma branching intensities for several uranium, protactinium, and thorium isotopes: ${ }^{233} \mathrm{U},{ }^{234} \mathrm{~Pa},{ }^{235} \mathrm{U},{ }^{236} \mathrm{U},{ }^{236} \mathrm{Th},{ }^{236} \mathrm{~Pa}$, ${ }^{237} \mathrm{U}$, and ${ }^{239} \mathrm{U}$

A selection of fission products (and daughters of fission products) are given in Table 5; some have large gamma-ray ranching ratio intensity uncertainties, including ${ }^{111} \mathrm{Ag},{ }^{143} \mathrm{Ce}$, 
${ }^{147} \mathrm{Nd}$, and ${ }^{161} \mathrm{~Tb}$. It should be noted that there is also uncertainty in the fission branch, and this uncertainty is not presented in this document. 
Table 2. Selected neptunium, americium and curium isotopes and the associated \% uncertainties in gamma-ray branching ratios.

\section{Isotope}

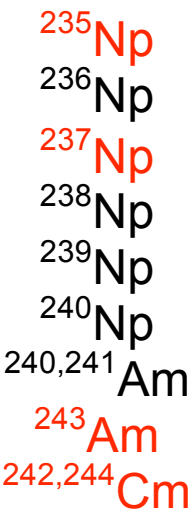

Half-life

1.085 years

$1.55 \mathrm{E} 5$ years

2.14E6 years

2.117 days

2.355 days

1.03 hours

2.12 days, 432.7 years

7.37E3 years

162.8 days, 18.1 years
$\%$ Uncertainty in $\gamma$-ray branching ratio $14 \%$

$6.0 \%$ for $\varepsilon, 2.1 \%$ for $\beta^{-}$

$11 \%$

$3.0 \%$

$2.8 \%$

$20 \%$

$5.0 \%, 1.4 \%$

$10 \%$

$8.8 \%, 10 \%$

Table 3. Selected plutonium isotopes and the associated \% uncertainties in gamma-ray branching ratios.

Isotope

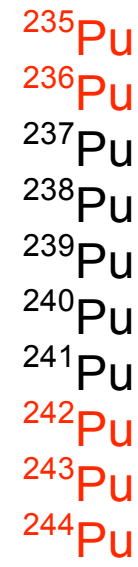

Half-life

25.3 minutes

2.87 years

45.2 days

87.7 years

$2.41 \mathrm{E} 4$ years

$6.56 \mathrm{E} 3$ years

14.4 years

$3.75 \mathrm{E} 5$ years

4.956 hours

8.0E7 years
$\%$ Uncertainty in $\gamma$-ray branching ratio

$17 \%$

$30 \%$

$3 \%$

$2 \%$

$3 \%$

$1 \%$

$1.7 \%$

$14 \%$

$8.7 \%$

$10 \%$ 
Table 4. Selected uranium, thorium, and protactinium isotopes and the associated $\%$ uncertainties in gamma-ray branching ratios.

$\begin{array}{ccr}\text { Isotope } & \text { Half-life } & \begin{array}{r}\% \text { uncertainty } \\ \text { ray branching }\end{array} \\ { }^{233} \mathrm{U} & 1.59 \mathrm{E} 5 \text { years } & 20 \% \\ { }^{234} \mathrm{U} & 2.46 \mathrm{E} 5 \text { years } & 8.5 \% \\ { }^{234} \mathrm{Th} & 24.1 \text { days } & 7.7 \% \\ { }^{234} \mathrm{~Pa} & 6.7 \text { hours } & 30 \% \\ { }^{235} \mathrm{U} & 7.04 \mathrm{E} 8 \text { years } & 10 \% \\ { }^{236} \mathrm{U} & 2.34 \mathrm{E} 7 \text { years } & 17 \% \\ { }^{236} \mathrm{Th} & 37.5 \text { minutes } & 34 \% \\ { }^{236} \mathrm{~Pa} & 9.1 \text { minutes } & 33 \% \\ { }^{237} \mathrm{U} & 6.75 \text { days } & 11 \% \\ { }^{239} \mathrm{U} & 23.5 \text { minutes } & 10 \%\end{array}$

Table 5. Selected fission products and the associated \% uncertainties in gamma-ray branching ratios.

$\begin{array}{ccc}\text { Isotope } & \text { Half-life } & \begin{array}{c}\% \text { Uncertainty in g-ray } \\ \text { branching ratio }\end{array} \\ { }^{89} \mathrm{Sr} & 50.5 \text { days } & 8.6 \% \text { (in equil. with }{ }^{89} \mathrm{Y} \text { ) } \\ { }^{95} \mathrm{Zr},{ }^{97} \mathrm{Zr} & 64 \text { days, } 18 \text { hours } & 0.37 \% 0.33 \% \\ { }^{99} \mathrm{Mo} & 2.75 \text { days } & 1.8 \% \\ { }^{111} \mathrm{Ag} & 7.47 \text { days } & 4.7 \% \text { for IT, 33\% for } \beta- \\ { }^{136} \mathrm{Cs},{ }^{137} \mathrm{Cs} & 13 \text { days, 30 years } & <0.1 \%, 0.08 \% \\ { }^{140} \mathrm{Ba} & 12.7 \text { days } & 0.9 \% \\ { }^{141} \mathrm{Ce},{ }^{143} \mathrm{Ce}, & 32 \text { days, } 1.4 \text { days, } 285 & 0.8 \%, 9.5 \%, 1.4 \% \\ { }^{144} \mathrm{Ce} & \text { days } & 6.4 \% \\ { }^{147} \mathrm{Nd} & 11 \text { days } & 4.9 \% \\ { }^{156} \mathrm{Eu} & 15 \text { days } & 10 \%\end{array}$


The RADSRC code allows the creation of libraries (generally intended for use in Monte Carlo simulations) of mixtures of isotopes at various ages. For example a radioactive source could be a mixture of uranium isotopes, five years old. In this report, only individual isotopes were run, at ages of zero years, five years and ten years. For example, at time zero, the gamma rays are associated with alpha decay from ${ }^{235} \mathrm{U} \rightarrow{ }^{231} \mathrm{Th}$. At an age of five years, the gamma rays are from an assortment of decay products, as seen in Figure 1 .

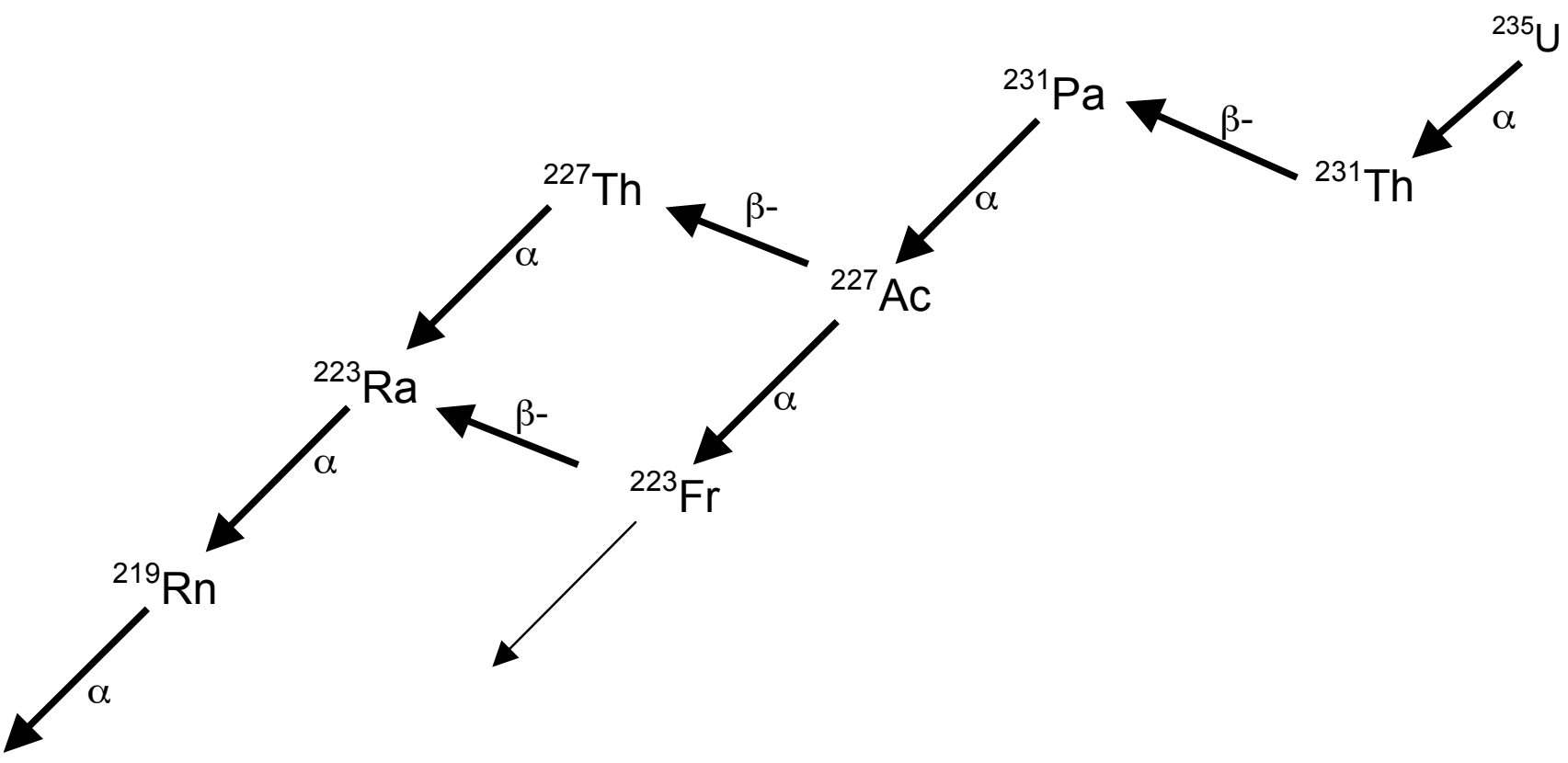

Figure 1. Naturally occurring decays from ${ }^{235} \mathrm{U}$. This is part of the actinium series. The dark arrows denote the major branch. The entire series, along with the thorium and uranium series may be found in Ref. [7].

The RADSRC gamma rays from the zero year-old isotopes are given in Appendix A, along with the energies found in the ENSDF library. The gamma rays from the five yearold isotopes are given in Appendix B, along with the radioactive decay associated with the gamma rays. The ten-year old gamma ray list was very similar to that generated from the five-year old material, so it is not included here.

\section{Suggestions for Future Work}

There are a variety of isotopic mixtures that are typically found in reactor materials that could be investigated using the RADSRC code. Particular mixtures of isotopes, such as aged uranium plus fission products, will produce unique spectra of gamma rays that could be used as a characteristic signature in safeguards work. In this report, we included the uncertainties for the gamma-ray branching intensities. There is also a need for the investigation of the impact of uncertainties on modeling radiation signatures. 
The activation products should also be investigated, both separately and within possible mixtures of radioactive isotopes. There are a variety of activation products which will appear due to the presence of structural materials (from a variety of neutron induced reactions). These materials include iron, cobalt, nickel, copper, zinc and molybdenum. Some activation products will have gamma rays suitable for fast characterization of waste streams or used materials. The ${ }^{55} \mathrm{Fe}$ and ${ }^{59} \mathrm{Fe}$ activation products have characteristic highenergy gamma-ray lines that could be used as a signature. Activation isotopes of cobalt include ${ }^{58,59.60} \mathrm{Co}$.

Other investigations could overlap with the creation of a gamma-ray library for safeguards. Measuring the cross sections for the creation of activation products could be important; the reactions $(\mathrm{n}, \mathrm{p}),(\mathrm{n}, \mathrm{d}),(\mathrm{n}, \mathrm{t}),(\mathrm{n}, \mathrm{alpha})$ and $(\mathrm{n}, \mathrm{gamma})$ will all create activation products. These cross sections are all neutron energy dependent. Some are well known, other important cross sections have only been modeled. The (n,gamma) cross sections can be difficult to model due to the exponential rise in cross section at the lower energy and a strong resonance region.

The important nuclear data needs highlighted in this study are the high overall uncertainty in branching intensity for some isotopes of uranium, protactinium, thorium, neptunium and plutonium americium and curium. The data presented here are only some of the possible nuclear data needs for safeguards technology.

\section{References}

1. W. E. Parker and S. Friedrich, "Nuclear Data Needs Project and Ultra High Resolution Gamma Detectors Project," Safeguards Working Group Meeting at Idaho State University, March 17-18, 2009, LLNL-PRES-411306.

2. W. E. Parker, W. Buckley, W. Ruhter and K. Raschke, "MGA Experts Class: Theory and Use of the MultiGroup Analysis Code," UCRL-MI-134577 (1999).

3. S. Friedrich, "Ultrahigh Resolution Gamma-Ray Spectrometers for Nuclear Safeguards," Lawrence Livermore National Laboratory. December 6. 2007.

4. T. B. Gosnell, "Automated Calculation of Photon Source Emission from Arbitrary Mixtures of Naturally Radioactive Heavy Nuclides," Nuclear Instruments and Methods A299, 682-686 (1990).

5. National Nuclear Data Center, Brookhaven National Laboratory, http://www.nndc.bnl.gov/

6. Edgardo Browne and Richard B. Firestone, Table of Radioactive Isotopes, Virginia S. Shirley, Editor, Lawrence Berkeley Laboratory, University of California, John Wiley and Sons, Inc. 1986. 
7. G. Friedlander, J. W. Kennedy, E. S. Macias and J. M. Miller, Nuclear and

Radiochemistry, $3^{\text {rd }}$ Edition, John Wiley and Sons, pp. 8-10 (1981).

This document was prepared as an account of work sponsored by an agency of the United States government. Neither the United States government nor Lawrence Livermore National Security, LLC, nor any of their employees makes any warranty, expressed or implied, or assumes any legal liability or responsibility for the accuracy, completeness, or usefulness of any information, apparatus, product, or process disclosed, or represents that its use would not infringe privately owned rights. Reference herein to any specific commercial product, process, or service by trade name, trademark, manufacturer, or otherwise does not necessarily constitute or imply its endorsement, recommendation, or favoring by the United States government or Lawrence Livermore National Security, LLC. The views and opinions of authors expressed herein do not necessarily state or reflect those of the United States government or Lawrence Livermore National Security, LLC, and shall not be used for advertising or product endorsement purposes. 
Appendix A. Gamma-ray energies from RADSRC and from ENSDF are compared for selected isotopes of uranium, neptunium, plutonium and americium. RADSRC was calculated for pure isotopes, that is age zero. Curium-244 is shown for RADSRC only.

$2340 \quad$ zero years

\begin{tabular}{|rccc|}
\hline \multicolumn{1}{c}{$\begin{array}{c}\text { RADSRC } \\
\text { energy (keV) }\end{array}$} & $\begin{array}{c}\text { RADSRC } \\
\text { intensity }\end{array}$ & $\begin{array}{c}\text { ENSDF } \\
\text { energy }(\mathrm{keV})\end{array}$ & \% diff \\
\hline & & 13 & \\
& & 53.2 & \\
& & 89.957 & \\
& & 93.35 & \\
& & 104.819 & \\
& & 105.604 & \\
120.912 & $9.44 \mathrm{E}+04$ & 120.9 & 0.010 \\
454.97 & $5.99 \mathrm{E}+01$ & 454.95 & 0.004 \\
503.53 & $2.30 \mathrm{E}+00$ & 503.5 & 0.006 \\
508.2 & $3.39 \mathrm{E}+01$ & 508.2 & 0.000 \\
581.78 & $2.76 \mathrm{E}+01$ & 581.7 & 0.014 \\
624.44 & $1.84 \mathrm{E}+00$ & 624.4 & 0.006 \\
677.67 & $2.30 \mathrm{E}+00$ & 677.6 & 0.010
\end{tabular}

$235 U$ zero years

\begin{tabular}{|cccc|}
\hline $\begin{array}{c}\text { RADSRC } \\
\text { energy (keV) }\end{array}$ & $\begin{array}{c}\text { RADSRC } \\
\text { intensity }\end{array}$ & $\begin{array}{c}\text { ENSDF } \\
\text { energy }(\mathrm{keV})\end{array}$ & \% diff \\
\hline 93.36 & $4.40 \mathrm{E}+03$ & 93.35 & 0.011 \\
96.154 & $6.88 \mathrm{E}+01$ & 96.09 & 0.067 \\
105.362 & $1.58 \mathrm{E}+03$ & 105.604 & 0.229 \\
108.99 & $5.28 \mathrm{E}+02$ & 108.582 & 0.376 \\
109.16 & $1.23 \mathrm{E}+03$ & 109.16 & 0.000 \\
116.11 & $5.60 \mathrm{E}+01$ & 115.45 & 0.572 \\
119.98 & $2.08 \mathrm{E}+01$ & 120.35 & 0.307 \\
136.72 & $9.60 \mathrm{E}+00$ & 136.55 & 0.124 \\
140.76 & $1.76 \mathrm{E}+02$ & 140.76 & 0.000 \\
& & 142.40 & \\
143.76 & $8.76 \mathrm{E}+03$ & 143.76 & 0.000 \\
150.93 & $6.40 \mathrm{E}+01$ & 150.93 & 0.000 \\
163.33 & $4.06 \mathrm{E}+03$ & 163.33 & 0.000 \\
172.27 & $8.00 \mathrm{E}+00$ & 173.3 & 0.594 \\
182.61 & $2.72 \mathrm{E}+02$ & 182.61 & 0.000 \\
185.72 & $4.57 \mathrm{E}+04$ & 185.715 & 0.003 \\
194.94 & $5.04 \mathrm{E}+02$ & 194.94 & 0.000 \\
198.90 & $3.36 \mathrm{E}+01$ & 198.90 & 0.000
\end{tabular}




\begin{tabular}{|c|c|c|c|}
\hline 202.11 & $8.64 \mathrm{E}+02$ & 202.11 & 0.000 \\
\hline 205.31 & $4.01 E+03$ & 205.311 & 0.000 \\
\hline 215.305 & $2.16 \mathrm{E}+01$ & 215.28 & 0.012 \\
\hline 221.38 & $9.60 \mathrm{E}+01$ & 221.38 & 0.000 \\
\hline 228.77 & $6.40 \mathrm{E}+00$ & 228.78 & 0.004 \\
\hline 233.5 & $2.32 \mathrm{E}+01$ & 233.5 & 0.000 \\
\hline 240.85 & $4.32 \mathrm{E}+01$ & 240.87 & 0.008 \\
\hline 246.84 & $4.24 \mathrm{E}+01$ & 246.84 & 0.000 \\
\hline \multirow[t]{2}{*}{266.483} & $4.80 \mathrm{E}+00$ & 266.45 & 0.012 \\
\hline & & 275.129 & \\
\hline 275.47 & $3.92 \mathrm{E}+00$ & 275.43 & 0.015 \\
\hline 281.45 & $4.80 E+00$ & 281.42 & 0.011 \\
\hline 282.98 & $4.00 \mathrm{E}+00$ & 282.92 & 0.021 \\
\hline 289.57 & $5.60 \mathrm{E}+00$ & 289.56 & 0.003 \\
\hline 291.72 & $1.12 \mathrm{E}+01$ & 291.65 & 0.024 \\
\hline 301.72 & $4.00 \mathrm{E}+00$ & 301.7 & 0.007 \\
\hline 310.71 & $3.20 \mathrm{E}+00$ & & \\
\hline 317.12 & $8.00 \mathrm{E}-01$ & 317.1 & 0.006 \\
\hline 343.78 & $2.40 \mathrm{E}+00$ & 343.5 & 0.082 \\
\hline 345.921 & $2.32 \mathrm{E}+01$ & 345.9 & 0.006 \\
\hline 356.068 & $4.00 \mathrm{E}+00$ & 356.03 & 0.011 \\
\hline 387.874 & $3.04 \mathrm{E}+00$ & 387.82 & 0.014 \\
\hline 390.32 & $8.00 \mathrm{E}-01$ & & \\
\hline \multirow[t]{2}{*}{410.269} & $2.40 \mathrm{E}+00$ & 410.29 & 0.005 \\
\hline & & 428.71 & \\
\hline 448.42 & $8.00 \mathrm{E}-01$ & 448.4 & 0.004 \\
\hline 455.12 & $6.40 \mathrm{E}-01$ & & \\
\hline 517.2 & $3.20 \mathrm{E}-01$ & & \\
\hline 742.52 & $3.20 \mathrm{E}-01$ & & \\
\hline 794.72 & $4.80 \mathrm{E}-01$ & & \\
\hline
\end{tabular}

\section{U zero years}

\begin{tabular}{|cccc|}
\hline $\begin{array}{c}\text { RADSRC } \\
\text { energy (keV) }\end{array}$ & $\begin{array}{c}\text { RADSRC } \\
\text { intensity }\end{array}$ & $\begin{array}{c}\text { ENSDF } \\
\text { energy }(\mathrm{keV})\end{array}$ & \% diff \\
\hline & & & \\
& & 13.0 & \\
& & 49.46 & \\
& 89.957 & \\
& 93.35 & \\
& 104.819 & \\
& & 105.604 & \\
& & 108.582 & \\
& & 112.79 & 0.035 \\
& & 171.15 & \\
& $4.55 \mathrm{E}+02$ & &
\end{tabular}




\begin{tabular}{|cccc|}
\hline $\begin{array}{c}\text { RADSRC } \\
\text { energy }(\mathrm{keV})\end{array}$ & $\begin{array}{c}\text { RADSRC } \\
\text { intensity }\end{array}$ & $\begin{array}{c}\text { ENSDF } \\
\text { energy }(\mathrm{keV})\end{array}$ & \% diff \\
\hline & & & \\
& & 13.0 & \\
& & 49.55 & \\
& 89.957 & \\
& 93.35 & \\
& & 104.819 & \\
& & 105.604 & \\
& & 108.582 & \\
& & 113.5 & 2.643 \\
\hline
\end{tabular}

\begin{tabular}{|c|c|c|c|c|}
\hline 237Np & ero years & & & \\
\hline $\begin{array}{c}\text { RADSRC } \\
\text { energy (keV) }\end{array}$ & $\begin{array}{l}\text { RADSRC } \\
\text { intensity }\end{array}$ & $\begin{array}{c}\text { ENSDF } \\
\text { energy (keV) }\end{array}$ & comment & $\%$ diff \\
\hline & & $\begin{array}{l}5.18 \\
8.22\end{array}$ & & \\
\hline 11.372 & $3.00 \mathrm{E}+05$ & & & \\
\hline 13.274 & $5.01 E+06$ & 13.3 & XR I & 0.195 \\
\hline 14.953 & $1.23 \mathrm{E}+05$ & & & \\
\hline 16.632 & $6.52 E+06$ & & & \\
\hline 19.718 & $1.51 \mathrm{E}+06$ & & & \\
\hline 29.378 & $3.36 \mathrm{E}+06$ & $\begin{array}{c}29.374 \\
36.32\end{array}$ & & 0.014 \\
\hline 46.57 & $3.47 E+04$ & 46.53 & & 0.086 \\
\hline 57.149 & $1.02 E+05$ & 57.104 & & 0.079 \\
\hline 62.66 & $3.13 E+03$ & 62.59 & & 0.112 \\
\hline 63.95 & $4.17 \mathrm{E}+03$ & 63.9 & & 0.078 \\
\hline 70.62 & $4.17 E+03$ & 70.49 & & 0.184 \\
\hline 74.46 & $2.89 E+03$ & 74.54 & & 0.107 \\
\hline 86.5 & $3.65 E+06$ & 86.477 & & 0.027 \\
\hline 88.05 & $4.69 E+04$ & 87.99 & & 0.068 \\
\hline 92.282 & $4.15 \mathrm{E}+05$ & 92.282 & XR ka2 & \\
\hline 94.723 & $1.98 \mathrm{E}+05$ & 94.64 & & 0.088 \\
\hline 95.858 & $7.56 \mathrm{E}+05$ & 95.863 & XR ka1 & 0.005 \\
\hline 106.13 & $1.46 \mathrm{E}+04$ & 106.15 & & 0.019 \\
\hline & & 107.595 & XR k $\beta 3$ & \\
\hline 108.166 & $2.45 E+05$ & 108.422 & $X R$ k $\beta 1$ & 0.236 \\
\hline 108.69 & $1.90 \mathrm{E}+04$ & 108.7 & & 0.009 \\
\hline
\end{tabular}




\begin{tabular}{|c|c|c|c|c|}
\hline 111.897 & $8.08 \mathrm{E}+04$ & 111.486 & $X R \mathrm{k} \beta 2$ & 0.369 \\
\hline 115.19 & $6.52 \mathrm{E}+02$ & 115.4 & & 0.182 \\
\hline 117.689 & $4.20 \mathrm{E}+04$ & 117.702 & & 0.011 \\
\hline 131.09 & $2.22 \mathrm{E}+04$ & 131.101 & & 0.008 \\
\hline \multirow{2}{*}{134.23} & $1.75 \mathrm{E}+04$ & 134.285 & & 0.041 \\
\hline & & $\begin{array}{c}139.9 ? \\
143.249\end{array}$ & & \\
\hline 151.423 & $6.26 \mathrm{E}+04$ & 151.414 & & 0.006 \\
\hline 153.72 & $1.80 \mathrm{E}+03$ & 153.37 & & 0.228 \\
\hline 155.263 & $2.40 \mathrm{E}+04$ & 155.239 & & 0.015 \\
\hline 162.51 & $9.65 E+03$ & 162.41 & & 0.062 \\
\hline 169.18 & $1.88 \mathrm{E}+04$ & 169.156 & & 0.014 \\
\hline 170.67 & $4.96 \mathrm{E}+03$ & 170.59 & & 0.047 \\
\hline 172.56 & $1.77 \mathrm{E}+03$ & & & \\
\hline 176.09 & $5.22 \mathrm{E}+03$ & 176.12 & & 0.017 \\
\hline 180.801 & $6.00 \mathrm{E}+03$ & 180.81 & & 0.005 \\
\hline 186.7 & $1.75 \mathrm{E}+03$ & 186.86 & & 0.086 \\
\hline 191.46 & 7.04E+03 & 191.46 & & \\
\hline 193.29 & $1.33 \mathrm{E}+04$ & 193.26 & & 0.016 \\
\hline 194.74 & $1.28 \mathrm{E}+04$ & 194.67 & & 0.036 \\
\hline 195.04 & $5.24 \mathrm{E}+04$ & 194.95 & & 0.046 \\
\hline 196.89 & $6.52 E+03$ & 196.86 & & 0.015 \\
\hline 200.17 & $9.13 \mathrm{E}+02$ & 199.95 & & 0.110 \\
\hline 201.72 & $1.10 \mathrm{E}+04$ & 201.62 & & 0.050 \\
\hline 202.85 & $9.39 \mathrm{E}+02$ & 202.9 & & 0.025 \\
\hline 209.19 & $4.04 \mathrm{E}+03$ & 209.19 & & \\
\hline 212.412 & $3.96 \mathrm{E}+04$ & 212.29 & & 0.057 \\
\hline \multirow[t]{2}{*}{214.08} & $1.10 \mathrm{E}+04$ & 214.01 & & 0.033 \\
\hline & & 222.6 & & \\
\hline 229.98 & $3.42 \mathrm{E}+03$ & 229.94 & & 0.017 \\
\hline 237.95 & $1.67 \mathrm{E}+04$ & 237.86 & & 0.038 \\
\hline 248.91 & $1.25 \mathrm{E}+03$ & 248.95 & & 0.016 \\
\hline 256.98 & $1.75 E+03$ & 257.09 & & 0.043 \\
\hline \multirow[t]{2}{*}{262.41} & $1.75 \mathrm{E}+03$ & 262.44 & & 0.011 \\
\hline & & 279.65 & & \\
\hline
\end{tabular}

238Pu zero years

\begin{tabular}{|ccccc|}
\hline $\begin{array}{c}\text { RADSRC } \\
\text { energy }(\mathrm{keV})\end{array}$ & $\begin{array}{c}\text { RADSRC } \\
\text { intensity }\end{array}$ & $\begin{array}{c}\text { ENSDF } \\
\text { energy }(\mathrm{keV})\end{array}$ & comment & \% diff \\
\hline 11.62 & $\begin{array}{c}\text { ph/s/gm } \\
1.58 \mathrm{E}+09\end{array}$ & & & \\
13.6 & $2.66 \mathrm{E}+10$ & 13.6 & & 0.000 \\
15.4 & $6.46 \mathrm{E}+08$ & & & \\
17.128 & $3.29 \mathrm{E}+10$ & & & \\
20.292 & $7.28 \mathrm{E}+09$ & & &
\end{tabular}




\begin{tabular}{|c|c|c|c|}
\hline 43.498 & $2.50 \mathrm{E}+08$ & 43.498 & 0.000 \\
\hline 62.78 & $2.53 E+02$ & 62.7 & 0.128 \\
\hline 94.66 & $7.28 \mathrm{E}+05$ & 94.654 & 0.006 \\
\hline 98.441 & $1.17 \mathrm{E}+06$ & 98.434 & 0.007 \\
\hline 99.864 & $4.50 \mathrm{E}+07$ & 99.853 & 0.011 \\
\hline 110.421 & $1.27 \mathrm{E}+05$ & 110.421 & 0.000 \\
\hline 111.3 & $2.47 \mathrm{E}+05$ & 111.298 & 0.002 \\
\hline 114.333 & $2.47 E+05$ & 114.445 & 0.098 \\
\hline \multirow[t]{2}{*}{114.561} & $1.00 \mathrm{E}+05$ & & \\
\hline & & 140.15 & \\
\hline 152.68 & $6.06 \mathrm{E}+06$ & 152.72 & 0.026 \\
\hline \multirow[t]{2}{*}{174.52} & $3.17 \mathrm{E}+01$ & & \\
\hline & & 192.91 & \\
\hline 200.98 & $2.60 E+04$ & 200.97 & 0.005 \\
\hline 203.2 & $6.33 E+01$ & 203.12 & 0.039 \\
\hline 236.03 & $6.33 \mathrm{E}+01$ & 235.9 & 0.055 \\
\hline 258.18 & $7.54 \mathrm{E}+02$ & 258.3 & 0.046 \\
\hline 299.08 & $8.23 E+02$ & 299.2 & 0.040 \\
\hline 706.02 & $9.56 \mathrm{E}+02$ & 705.9 & 0.017 \\
\hline 708.4 & $2.41 E+03$ & 708.42 & 0.003 \\
\hline 742.817 & $4.94 \mathrm{E}+04$ & 742.81 & 0.001 \\
\hline 766.412 & $2.09 E+05$ & 766.39 & 0.003 \\
\hline 783.38 & $2.91 \mathrm{E}+02$ & 783.4 & 0.003 \\
\hline \multirow[t]{2}{*}{786.287} & $2.98 \mathrm{E}+04$ & 786.3 & 0.002 \\
\hline & & 804.4 & \\
\hline 805.87 & $1.09 E+03$ & 805.6 & 0.034 \\
\hline 808.25 & $6.71 E+03$ & 808.25 & 0.000 \\
\hline 851.72 & $1.29 \mathrm{E}+04$ & 851.7 & 0.002 \\
\hline 860 & $0.00 \mathrm{E}+00$ & & \\
\hline 880.53 & $1.46 \mathrm{E}+03$ & 880.5 & 0.003 \\
\hline 883.24 & $7.03 E+03$ & 883.23 & 0.001 \\
\hline 904.38 & $6.33 E+02$ & 904.3 & 0.009 \\
\hline 926.71 & $5.19 \mathrm{E}+03$ & 926.72 & 0.001 \\
\hline 942.05 & $4.05 E+03$ & 941.9 & 0.016 \\
\hline 946.02 & $8.23 E+02$ & 946 & 0.002 \\
\hline 980.39 & $5.07 \mathrm{E}+02$ & & \\
\hline 1001 & $8.68 \mathrm{E}+03$ & 1001.03 & 0.003 \\
\hline 1041.9 & $1.83 E+03$ & 1041.8 & 0.010 \\
\hline 1085.37 & $6.65 E+02$ & 1085.4 & 0.003 \\
\hline
\end{tabular}

\section{Pu zero years}

\begin{tabular}{|ccccc|}
\hline $\begin{array}{c}\text { RADSRC } \\
\text { energy }(\mathrm{keV})\end{array}$ & $\begin{array}{c}\text { RADSRC } \\
\text { intensity }\end{array}$ & $\begin{array}{c}\text { ENSDF } \\
\text { energy }(\mathrm{keV})\end{array}$ & comment & \% diff \\
& ph/s/gm & & \\
& & 0.0765 & \\
& & 12.975
\end{tabular}




\begin{tabular}{|c|c|c|c|c|}
\hline \multicolumn{5}{|c|}{13.6} \\
\hline 30.08 & $2.41 \mathrm{E}+03$ & \multicolumn{2}{|l|}{30.04} & 0.133 \\
\hline 38.66 & $2.41 \mathrm{E}+05$ & \multicolumn{2}{|l|}{38.661} & 0.003 \\
\hline 40.41 & $3.72 E+03$ & \multicolumn{2}{|l|}{41.93} & 3.625 \\
\hline 42.06 & $3.79 E+03$ & & & \\
\hline 46.218 & $1.69 \mathrm{E}+04$ & \multicolumn{2}{|l|}{46.21} & 0.017 \\
\hline 46.69 & $1.33 \mathrm{E}+03$ & \multicolumn{2}{|l|}{46.68} & 0.021 \\
\hline 47.52 & $6.66 \mathrm{E}+02$ & \multicolumn{2}{|l|}{47.6} & 0.168 \\
\hline 51.624 & $6.22 \mathrm{E}+05$ & \multicolumn{2}{|l|}{51.624} & 0.000 \\
\hline 54.02 & $3.88 \mathrm{E}+03$ & \multicolumn{2}{|l|}{54.039} & 0.035 \\
\hline 56.825 & $2.59 E+04$ & \multicolumn{2}{|l|}{56.828} & 0.005 \\
\hline 65.7 & $7.58 \mathrm{E}+02$ & \multicolumn{2}{|l|}{65.708} & 0.012 \\
\hline 67.69 & $3.26 \mathrm{E}+03$ & \multicolumn{2}{|l|}{67.674} & 0.024 \\
\hline 68.73 & $2.76 \mathrm{E}+03$ & \multicolumn{2}{|l|}{68.696} & 0.049 \\
\hline 74.89 & $8.73 E+02$ & \multicolumn{2}{|l|}{68.74} & 8.947 \\
\hline 77.593 & $9.80 \mathrm{E}+03$ & \multicolumn{2}{|l|}{77.592} & 0.001 \\
\hline 78.37 & $3.88 \mathrm{E}+03$ & \multicolumn{2}{|l|}{78.43} & 0.077 \\
\hline & & \multicolumn{3}{|l|}{89.643} \\
\hline & & \multicolumn{3}{|l|}{$\approx 89.7$} \\
\hline 94.66 & $9.64 \mathrm{E}+04$ & 94.654 & XR ka2 & 0.006 \\
\hline \multirow[t]{2}{*}{96.16} & $8.45 E+02$ & 96.14 & & 0.021 \\
\hline & & \multicolumn{3}{|l|}{97.6} \\
\hline 98.441 & $1.57 \mathrm{E}+05$ & 98.434 & XR ka1 & 0.007 \\
\hline 98.79 & $2.80 E+04$ & \multicolumn{2}{|l|}{98.78} & 0.010 \\
\hline 103.035 & $4.87 E+03$ & \multicolumn{2}{|l|}{103.06} & 0.024 \\
\hline 110.421 & $1.83 \mathrm{E}+04$ & & XR k $\beta 3$ & 0.000 \\
\hline 111.3 & $3.56 \mathrm{E}+04$ & 111.298 & XR k $\beta 1$ & 0.002 \\
\hline 111.89 & $7.05 E+02$ & & & \\
\hline 114.333 & $1.44 \mathrm{E}+04$ & 114.445 & $X R \mathrm{k} \beta 2$ & 0.098 \\
\hline 115.38 & $1.49 \mathrm{E}+04$ & 115.38 & & 0.000 \\
\hline 116.266 & $1.37 \mathrm{E}+04$ & 116.26 & & 0.005 \\
\hline & & 119.7 & & \\
\hline & & $\approx 119.7 ?$ & & \\
\hline 122.34 & $6.89 \mathrm{E}+01$ & 122.35 & & 0.008 \\
\hline 123.55 & $0.00 \mathrm{E}+00$ & 123.228 & & 0.261 \\
\hline 123.62 & $4.52 E+02$ & 123.62 & & 0.000 \\
\hline 124.51 & $1.41 \mathrm{E}+03$ & 124.51 & & 0.000 \\
\hline 125.21 & $1.63 E+03$ & 125.21 & & 0.000 \\
\hline 129.296 & $1.44 \mathrm{E}+05$ & 129.296 & & 0.000 \\
\hline 141.657 & $7.35 E+02$ & 141.657 & & 0.000 \\
\hline 143.35 & $3.97 E+02$ & 143.35 & & 0.000 \\
\hline 144.201 & $6.97 \mathrm{E}+03$ & 144.201 & & 0.000 \\
\hline 146.094 & $2.73 E+03$ & 146.094 & & 0.000 \\
\hline & & 158.1 & & \\
\hline 160.19 & $1.42 \mathrm{E}+02$ & 160.19 & & 0.000 \\
\hline 161.482 & $2.82 E+03$ & 161.45 & & 0.020 \\
\hline 167.81 & $6.73 E+01$ & 167.81 & & 0.000 \\
\hline 171.393 & $2.53 E+03$ & 171.393 & & 0.000 \\
\hline & & 172.56 & & \\
\hline
\end{tabular}




\begin{tabular}{|c|c|c|c|}
\hline 173.7 & $9.18 \mathrm{E}+01$ & 173.7 & 0.000 \\
\hline 179.22 & $1.52 \mathrm{E}+03$ & 179.22 & 0.000 \\
\hline 184.55 & $4.87 \mathrm{E}+01$ & & \\
\hline 188.23 & $2.50 \mathrm{E}+02$ & 188.23 & 0.000 \\
\hline 189.36 & $1.91 \mathrm{E}+03$ & 189.36 & 0.000 \\
\hline 195.679 & $2.46 \mathrm{E}+03$ & 195.679 & 0.000 \\
\hline 196.87 & $8.50 E+01$ & & \\
\hline 203.54 & $1.29 \mathrm{E}+04$ & 203.55 & 0.005 \\
\hline 218.45 & $2.30 \mathrm{E}+01$ & 218.0 & 0.206 \\
\hline 225.39 & $3.74 \mathrm{E}+02$ & 225.42 & 0.013 \\
\hline 237.75 & $3.44 \mathrm{E}+02$ & 237.77 & 0.008 \\
\hline 242.12 & $1.91 \mathrm{E}+02$ & 242.08 & 0.017 \\
\hline 243.36 & $5.33 E+02$ & 243.38 & 0.008 \\
\hline 244.86 & $1.19 \mathrm{E}+02$ & 244.92 & 0.024 \\
\hline 248.88 & $1.72 \mathrm{E}+02$ & 248.95 & 0.028 \\
\hline 255.384 & $1.84 \mathrm{E}+03$ & 255.384 & 0.000 \\
\hline 263.9 & $5.86 \mathrm{E}+02$ & 263.95 & 0.019 \\
\hline 265.81 & $6.89 \mathrm{E}+01$ & 265.7 & 0.041 \\
\hline 281.12 & $4.82 \mathrm{E}+01$ & 281.2 & 0.028 \\
\hline 285.27 & $3.44 \mathrm{E}+01$ & 285.3 & 0.011 \\
\hline 297.46 & $1.14 \mathrm{E}+03$ & 297.46 & 0.000 \\
\hline 302.89 & $1.31 \mathrm{E}+02$ & 302.87 & 0.007 \\
\hline 307.82 & $1.42 \mathrm{E}+02$ & 307.85 & 0.010 \\
\hline 311.78 & $5.92 \mathrm{E}+02$ & 311.78 & 0.000 \\
\hline 313.39 & $4.59 \mathrm{E}+01$ & & \\
\hline \multirow[t]{3}{*}{316.4} & $3.24 \mathrm{E}+02$ & 316.41 & 0.003 \\
\hline & & 319.68 & \\
\hline & & 320.862 & \\
\hline 323.77 & $1.24 \mathrm{E}+03$ & 323.84 & 0.022 \\
\hline 332.853 & $1.16 \mathrm{E}+04$ & 332.845 & 0.002 \\
\hline 336.116 & $2.61 E+03$ & 336.113 & 0.001 \\
\hline \multirow[t]{2}{*}{341.526} & $1.52 \mathrm{E}+03$ & 341.506 & 0.006 \\
\hline & & 345.013 & \\
\hline 345.014 & $1.28 \mathrm{E}+04$ & 345.014 & 0.000 \\
\hline 354.02 & $1.84 \mathrm{E}+01$ & 354 & 0.006 \\
\hline 361.84 & $2.69 \mathrm{E}+02$ & 361.89 & 0.014 \\
\hline 367.048 & $1.99 \mathrm{E}+03$ & 367.073 & 0.007 \\
\hline 368.543 & $2.07 E+03$ & 368.554 & 0.003 \\
\hline 372.47 & $4.82 \mathrm{E}+01$ & & \\
\hline 375.039 & $3.60 \mathrm{E}+04$ & 375.054 & 0.004 \\
\hline 379.05 & $2.41 \mathrm{E}+01$ & & \\
\hline 380.16 & $7.00 \mathrm{E}+03$ & 380.191 & 0.008 \\
\hline 382.732 & $5.95 E+03$ & 382.75 & 0.005 \\
\hline \multirow[t]{2}{*}{392.914} & $1.27 \mathrm{E}+04$ & 392.53 & 0.098 \\
\hline & & 393.14 & \\
\hline \multirow[t]{3}{*}{399.46} & $1.40 \mathrm{E}+02$ & 399.53 & 0.018 \\
\hline & & 406.82 & \\
\hline & & 411.2 & \\
\hline 410.94 & $1.84 \mathrm{E}+02$ & & \\
\hline
\end{tabular}




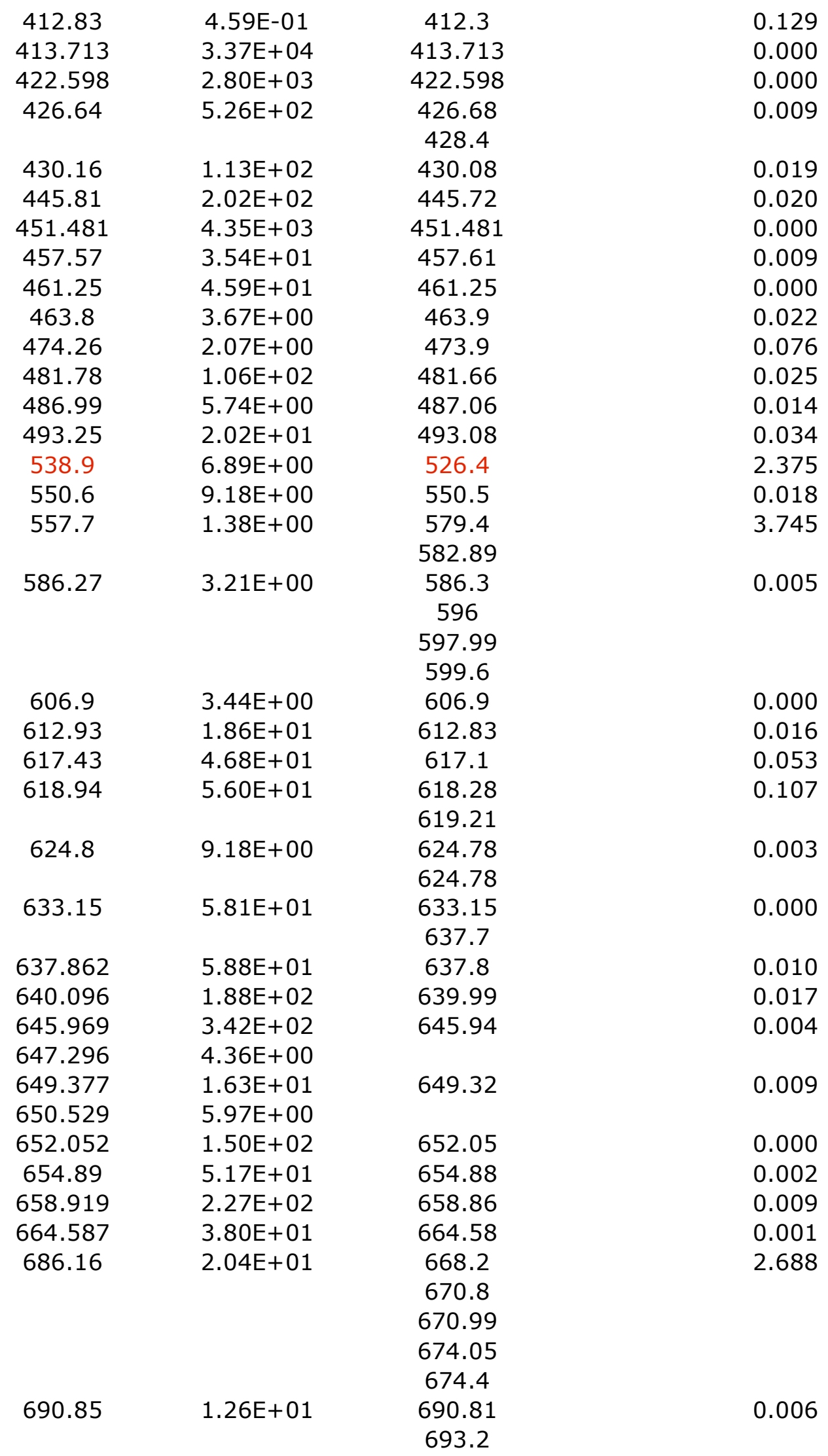




\begin{tabular}{|c|c|c|c|}
\hline & & $\begin{array}{l}697.8 \\
701.1\end{array}$ & \\
\hline 703.8 & $8.91 \mathrm{E}+01$ & 703.68 & 0.017 \\
\hline 714.58 & $2.07 E+00$ & 714.71 & 0.018 \\
\hline 718 & $6.43 \mathrm{E}+01$ & 718.0 & 0.000 \\
\hline & & 720.3 & \\
\hline 727.84 & $2.50 \mathrm{E}+01$ & 727.9 & 0.008 \\
\hline & & 736.5 & \\
\hline & & 747.4 & \\
\hline & & 756.4 & \\
\hline 756.42 & $7.74 \mathrm{E}+01$ & 756.4 & 0.003 \\
\hline 766.6 & $6.31 \mathrm{E}-02$ & 766.47 & 0.017 \\
\hline & & 767.29 & \\
\hline 769.19 & $2.73 \mathrm{E}+02$ & 769.15 & 0.005 \\
\hline & & 769.37 & \\
\hline 779.46 & $3.05 E+00$ & 779.4 & 0.008 \\
\hline 787.3 & $1.93 \mathrm{E}+00$ & & \\
\hline 793 & $5.74 \mathrm{E}-01$ & 792.9 & 0.013 \\
\hline 796.5 & $7.35 \mathrm{E}-01$ & & \\
\hline 803.3 & $1.01 \mathrm{E}+00$ & & \\
\hline & & 805.9 & \\
\hline 808.2 & $3.38 \mathrm{E}+00$ & 808.4 & 0.025 \\
\hline 813.9 & $1.42 \mathrm{E}+00$ & 813.7 & 0.025 \\
\hline & & 816 & \\
\hline 821.1 & $1.17 \mathrm{E}+00$ & 821.3 & 0.024 \\
\hline 828.8 & $3.17 \mathrm{E}+00$ & & \\
\hline 831.93 & $5.74 \mathrm{E}-01$ & 832.5 & 0.068 \\
\hline 839 & $9.18 \mathrm{E}-01$ & 840.4 & 0.167 \\
\hline 843.8 & $3.67 E+00$ & 843.78 & 0.002 \\
\hline 879 & $1.15 \mathrm{E}+00$ & 879.2 & 0.023 \\
\hline 891.1 & $1.77 \mathrm{E}+00$ & 891 & 0.011 \\
\hline & & 918.7 & \\
\hline 940.1 & $9.64 \mathrm{E}-01$ & 940.3 & 0.021 \\
\hline 956.4 & $1.29 \mathrm{E}+00$ & 955.6 & 0.084 \\
\hline & & 957.6 & \\
\hline 979.52 & $5.28 \mathrm{E}-01$ & 979.7 & 0.018 \\
\hline 986.7 & $2.76 \mathrm{E}-01$ & 986.9 & 0.020 \\
\hline 992.47 & $5.28 \mathrm{E}-01$ & 992.7 & 0.023 \\
\hline 1005.63 & $2.76 \mathrm{E}-01$ & 1005.7 & 0.007 \\
\hline 1057.32 & $1.03 \mathrm{E}+00$ & 1057.3 & 0.002 \\
\hline
\end{tabular}

\section{Pu zero years}

\begin{tabular}{|clcrl|}
\hline $\begin{array}{c}\text { RADSRC } \\
\text { energy (keV) }\end{array}$ & $\begin{array}{c}\text { RADSRC } \\
\text { intensity }\end{array}$ & $\begin{array}{c}\text { ENSDF } \\
\text { energy }(\mathrm{keV})\end{array}$ & comment & \% diff \\
\hline & $\mathrm{ph} / \mathrm{s} / \mathrm{gm}$ & & & \\
\hline
\end{tabular}




\begin{tabular}{|c|c|c|c|c|}
\hline & & $\begin{array}{c}13.6 \\
45.244\end{array}$ & XR I & \\
\hline 94.66 & $5.71 E+03$ & 94.654 & XR ka2 & 0.006 \\
\hline 98.441 & $8.40 E+03$ & 98.434 & XR ka1 & 0.007 \\
\hline 104.244 & $5.84 \mathrm{E}+05$ & 104.234 & & 0.010 \\
\hline 110.421 & $9.91 \mathrm{E}+02$ & 110.421 & XR k $\beta 3$ & 0.000 \\
\hline 111.3 & $3.02 E+03$ & 111.298 & XR k $\beta 1$ & 0.002 \\
\hline 114.866 & $8.40 E+02$ & 114.445 & $X R k \beta 2$ & 0.368 \\
\hline 160.28 & $3.53 E+04$ & 160.308 & & 0.017 \\
\hline 212.46 & $2.52 E+03$ & 212.46 & & 0.000 \\
\hline 538.11 & $1.01 \mathrm{E}+01$ & 538.09 & & 0.004 \\
\hline 642.5 & $1.06 \mathrm{E}+03$ & 642.35 & & 0.023 \\
\hline 687.59 & $2.27 E+02$ & $\begin{array}{c}687.57 \\
699\end{array}$ & & 0.003 \\
\hline 873.94 & $4.87 E+01$ & $\begin{array}{c}873.92 \\
958 \\
960 \\
967\end{array}$ & & 0.002 \\
\hline
\end{tabular}

\section{Pu zero years}

\begin{tabular}{|c|c|c|c|c|}
\hline \multirow[t]{4}{*}{$\begin{array}{c}\text { RADSRC } \\
\text { energy (keV) }\end{array}$} & $\begin{array}{l}\text { RADSRC } \\
\text { intensity }\end{array}$ & $\begin{array}{c}\text { ENSDF } \\
\text { energy (keV) }\end{array}$ & comment & $\%$ diff \\
\hline & $\mathrm{ph} / \mathrm{s} / \mathrm{gm}$ & & & \\
\hline & & 13.6 & XR I & \\
\hline & & 26.7 & & \\
\hline 44.2 & $3.84 \mathrm{E}+00$ & 44.2 & & 0.000 \\
\hline 44.86 & $7.68 \mathrm{E}-01$ & 44.86 & & 0.000 \\
\hline 56.32 & $2.30 \mathrm{E}+00$ & 56.32 & & 0.000 \\
\hline 56.76 & $8.96 \mathrm{E}-01$ & 56.76 & & 0.000 \\
\hline 71.6 & $2.57 \mathrm{E}+00$ & 71.6 & & 0.000 \\
\hline 77.1 & $1.99 \mathrm{E}+01$ & 77.1 & & 0.000 \\
\hline 94.66 & $2.74 \mathrm{E}+02$ & 94.654 & XR ka2 & 0.006 \\
\hline 98.441 & $4.45 E+02$ & 98.434 & XR ka1 & 0.007 \\
\hline & & 101 & & \\
\hline 103.68 & $9.05 E+01$ & 103.68 & & 0.000 \\
\hline 110.421 & $5.42 \mathrm{E}+01$ & 110.421 & XR kß3 & 0.000 \\
\hline 111.3 & $1.47 \mathrm{E}+02$ & 111.298 & XR k $\beta 1$ & 0.002 \\
\hline 111.89 & $3.04 \mathrm{E}+00$ & & & \\
\hline 114.333 & $1.47 \mathrm{E}+01$ & 114 & & 0.292 \\
\hline 114.561 & $3.34 \mathrm{E}+01$ & 114.445 & XR k $\beta 2$ & 0.101 \\
\hline 115.4 & $1.23 \mathrm{E}+01$ & & & \\
\hline 121.2 & $6.15 \mathrm{E}-01$ & 121.2 & & 0.000 \\
\hline 148.567 & $1.68 \mathrm{E}+02$ & 148.567 & & 0.000 \\
\hline & & 159.955 & & \\
\hline
\end{tabular}




\begin{tabular}{|c|c|c|c|c|}
\hline $\begin{array}{c}\text { RADSRC } \\
\text { energy (keV) }\end{array}$ & $\begin{array}{l}\text { RADSRC } \\
\text { intensity }\end{array}$ & $\begin{array}{c}\text { ENSDF } \\
\text { energy }(\mathrm{keV})\end{array}$ & comment & $\%$ diff \\
\hline & $\mathrm{ph} / \mathrm{s} / \mathrm{gm}$ & & & \\
\hline 11.62 & $2.90 \mathrm{E}+05$ & & & \\
\hline 13.6 & $4.79 E+06$ & 13.6 & XR I & 0.000 \\
\hline 15.4 & $1.18 \mathrm{E}+05$ & & & \\
\hline 17.128 & $5.95 E+06$ & & & \\
\hline 20.292 & $1.32 \mathrm{E}+06$ & & & \\
\hline 44.915 & $5.23 E+04$ & 44.915 & & 0.000 \\
\hline & & 94.654 & XR ka2 & \\
\hline & & 98.434 & XR ka1 & \\
\hline \multirow[t]{4}{*}{103.5} & $1.13 \mathrm{E}+04$ & 103.5 & & 0.000 \\
\hline & & 110.421 & $X R k \beta 3$ & \\
\hline & & 111.298 & XR k $\beta 1$ & \\
\hline & & 114.445 & $X R \mathrm{k} \beta 2$ & \\
\hline 158.8 & $6.54 \mathrm{E}+02$ & 158.8 & & 0.000 \\
\hline
\end{tabular}

241Am zero years

\begin{tabular}{|ccccc|}
\hline $\begin{array}{c}\text { RADSRC } \\
\text { energy (keV) }\end{array}$ & $\begin{array}{c}\text { RADSRC } \\
\text { intensity }\end{array}$ & $\begin{array}{c}\text { ENSDF } \\
\text { energy (keV) }\end{array}$ & comment & \% diff \\
\hline 11.871 & $1.03 \mathrm{E}+09$ & & & \\
13.927 & $1.65 \mathrm{E}+10$ & 13.9 & & 0.194 \\
15.861 & $4.18 \mathrm{E}+08$ & & & \\
17.611 & $2.56 \mathrm{E}+10$ & & & \\
20.997 & $6.59 \mathrm{E}+09$ & & & 0.000 \\
26.3445 & $3.04 \mathrm{E}+09$ & 26.3446 & & \\
32.2 & $2.21 \mathrm{E}+07$ & & & 0.012 \\
33.192 & $1.52 \mathrm{E}+08$ & 33.196 & & 0.012 \\
42.64 & $1.27 \mathrm{E}+06$ & 42.704 & & \\
43.415 & $9.26 \mathrm{E}+07$ & 43.42 & & \\
51.013 & $3.17 \mathrm{E}+04$ & 51.01 & & 0.058 \\
54.04 & $7.61 \mathrm{E}+05$ & & & 0.008 \\
55.528 & $2.54 \mathrm{E}+07$ & 55.56 & & 0.020 \\
59.536 & $4.55 \mathrm{E}+10$ & 59.5409 & & \\
64.817 & $1.77 \mathrm{E}+05$ & 64.83 & & \\
67.46 & $5.58 \mathrm{E}+05$ & 67.45 & & \\
69.76 & $3.04 \mathrm{E}+07$ & 69.76 & & \\
\end{tabular}




\begin{tabular}{|c|c|c|c|c|}
\hline 75.83 & $1.39 \mathrm{E}+05$ & 75.8 & & 0.040 \\
\hline 96.67 & $5.96 \mathrm{E}+04$ & & & \\
\hline 97.071 & $1.50 \mathrm{E}+06$ & 97.069 & XR ka2 & 0.002 \\
\hline 98.951 & $2.57 \mathrm{E}+07$ & 98.972 & & \\
\hline 101.066 & $2.50 E+06$ & 101.059 & XR ka1 & 0.007 \\
\hline 102.966 & $2.47 \mathrm{E}+07$ & $\begin{array}{l}102.982 \\
109.707\end{array}$ & & \\
\hline 113.3 & $3.00 \mathrm{E}+05$ & 113.303 & XR k $\beta 3$ & 0.003 \\
\hline 114.23 & $5.98 \mathrm{E}+05$ & 114.234 & XR k $\beta 1$ & 0.004 \\
\hline 117.58 & $2.92 \mathrm{E}+05$ & $\begin{array}{c}117.463 \\
120.36\end{array}$ & $X R \mathrm{k} \beta 2$ & 0.100 \\
\hline 122.994 & $1.27 \mathrm{E}+06$ & 123.052 & & 0.047 \\
\hline 125.292 & $5.17 \mathrm{E}+06$ & 125.3 & & 0.006 \\
\hline 139.53 & $6.77 \mathrm{E}+03$ & 139.44 & & 0.065 \\
\hline 146.557 & $5.83 \mathrm{E}+05$ & 146.55 & & 0.005 \\
\hline 150.11 & $1.01 \mathrm{E}+05$ & 150.04 & & 0.047 \\
\hline 154.4 & $1.01 \mathrm{E}+04$ & 154.27 & & 0.084 \\
\hline 156.4 & $1.52 \mathrm{E}+04$ & & & \\
\hline \multirow{2}{*}{158.48} & $1.77 \mathrm{E}+03$ & & & \\
\hline & & 159.26 & & \\
\hline 161.7 & $1.14 \mathrm{E}+04$ & 161.54 & & 0.099 \\
\hline 164.58 & $8.46 E+04$ & 164.69 & & 0.067 \\
\hline 165.92 & $3.04 \mathrm{E}+04$ & 165.81 & & 0.066 \\
\hline 169.56 & $2.13 \mathrm{E}+05$ & 169.56 & & \\
\hline 175.09 & $2.28 \mathrm{E}+04$ & 175.07 & & 0.011 \\
\hline 190.4 & $2.79 E+03$ & & & \\
\hline 191.9 & $2.74 \mathrm{E}+02$ & 191.96 & & 0.031 \\
\hline 197 & $1.65 E+04$ & 197 & & \\
\hline 203.9 & $3.68 \mathrm{E}+03$ & 204.06 & & 0.078 \\
\hline 208 & $1.00 \mathrm{E}+06$ & 208.01 & & 0.005 \\
\hline 221.45 & $5.32 E+04$ & 221.46 & & 0.005 \\
\hline 232.86 & $5.83 E+03$ & 232.81 & & 0.021 \\
\hline 234.352 & $8.87 E+02$ & 234.33 & & 0.009 \\
\hline 242.4 & $3.80 \mathrm{E}+04$ & & & \\
\hline 245 & $3.80 \mathrm{E}+04$ & & & \\
\hline 246.66 & $3.04 \mathrm{E}+03$ & 246.73 & & 0.028 \\
\hline 249.1 & $5.07 E+03$ & 249 & & 0.040 \\
\hline 260.9 & $1.27 \mathrm{E}+03$ & 260.8 & & 0.038 \\
\hline 264.87 & $1.14 \mathrm{E}+04$ & 264.89 & & 0.008 \\
\hline 267.54 & $3.33 E+04$ & 267.58 & & 0.015 \\
\hline 271.58 & $8.14 \mathrm{E}+02$ & & & \\
\hline \multirow[t]{2}{*}{275.68} & $8.37 E+03$ & 275.77 & & 0.033 \\
\hline & & 278.04 & & \\
\hline 291.21 & $3.93 E+03$ & 291.3 & & 0.031 \\
\hline 292.76 & $1.75 \mathrm{E}+04$ & 292.77 & & 0.003 \\
\hline 294.9 & $6.97 \mathrm{E}+04$ & & & \\
\hline 304.2 & $1.27 \mathrm{E}+03$ & $\begin{array}{c}304.21 \\
309.1\end{array}$ & & 0.003 \\
\hline 310.3 & $1.90 \mathrm{E}+04$ & & & \\
\hline
\end{tabular}




\begin{tabular}{|c|c|c|c|}
\hline & & 316.8 & \\
\hline 322.54 & $1.93 \mathrm{E}+05$ & 322.52 & 0.006 \\
\hline 332.39 & $1.88 \mathrm{E}+05$ & 332.35 & 0.012 \\
\hline 335.43 & $6.26 \mathrm{E}+05$ & 335.37 & 0.018 \\
\hline 337.727 & $5.32 E+03$ & 337.7 & 0.008 \\
\hline 349.46 & $1.52 \mathrm{E}+03$ & & \\
\hline 358.36 & $1.52 \mathrm{E}+03$ & 358.25 & 0.031 \\
\hline 368.61 & $2.75 E+05$ & 368.65 & 0.011 \\
\hline 370.933 & $6.66 \mathrm{E}+04$ & 370.94 & 0.002 \\
\hline 376.6 & $1.78 \mathrm{E}+05$ & 376.65 & 0.013 \\
\hline 383.744 & $3.58 \mathrm{E}+04$ & 383.81 & 0.017 \\
\hline 390.6 & $7.48 \mathrm{E}+03$ & & \\
\hline & & $\begin{array}{c}398.64 \\
401.3\end{array}$ & \\
\hline 406.4 & $1.77 \mathrm{E}+03$ & 406.35 & 0.012 \\
\hline 419.22 & $3.55 E+04$ & 419.33 & 0.026 \\
\hline 426.39 & $3.12 \mathrm{E}+04$ & 426.47 & 0.019 \\
\hline 429.8 & $1.27 \mathrm{E}+03$ & & \\
\hline 435.3 & $2.54 \mathrm{E}+03$ & & \\
\hline 442.75 & $4.44 \mathrm{E}+03$ & & \\
\hline & & 446.43 & \\
\hline 452.41 & $3.04 \mathrm{E}+03$ & 452.6 & 0.042 \\
\hline 454.67 & $1.23 \mathrm{E}+04$ & 454.66 & 0.002 \\
\hline 459.58 & $4.56 E+03$ & 459.68 & 0.022 \\
\hline & & 463.22 & \\
\hline 468 & $3.68 \mathrm{E}+03$ & 468.12 & 0.026 \\
\hline 486.3 & $1.27 \mathrm{E}+03$ & 487.3 & 0.205 \\
\hline 512 & $1.46 \mathrm{E}+03$ & 512.5 & 0.098 \\
\hline 514.2 & $3.30 E+03$ & 514 & 0.039 \\
\hline 522 & $1.14 \mathrm{E}+03$ & 522.06 & 0.011 \\
\hline & & 529.17 & \\
\hline & & 545.4 & \\
\hline & & 563.05 & \\
\hline 574.05 & $1.27 \mathrm{E}+03$ & 573.94 & 0.019 \\
\hline 582.6 & $2.54 \mathrm{E}+02$ & & \\
\hline 586.5 & $1.65 \mathrm{E}+03$ & 586.59 & 0.015 \\
\hline 590.3 & $3.55 E+03$ & 590.28 & 0.003 \\
\hline 597.41 & $1.19 \mathrm{E}+04$ & 597.48 & 0.012 \\
\hline 619.011 & $7.48 \mathrm{E}+04$ & 619.01 & 0.000 \\
\hline 620.87 & $0.00 \mathrm{E}+00$ & & \\
\hline 627.2 & $7.10 \mathrm{E}+02$ & 627.18 & 0.003 \\
\hline 633 & $1.60 \mathrm{E}+03$ & 632.93 & 0.011 \\
\hline 641.436 & $9.00 \mathrm{E}+03$ & 641.47 & 0.005 \\
\hline 652.99 & $4.78 \mathrm{E}+04$ & 653.02 & 0.005 \\
\hline 662.456 & $4.65 E+05$ & 662.4 & 0.008 \\
\hline 669.9 & $4.82 \mathrm{E}+02$ & 666.5 & 0.510 \\
\hline & & 669.83 & \\
\hline 675.8 & $8.11 \mathrm{E}+02$ & 676.03 & 0.034 \\
\hline 680.06 & $3.93 E+03$ & 680.1 & 0.006 \\
\hline
\end{tabular}




\begin{tabular}{|c|c|c|c|}
\hline 688.77 & $3.80 \mathrm{E}+04$ & 688.72 & 0.007 \\
\hline 693.5 & $4.69 E+03$ & & \\
\hline 696.36 & $6.34 \mathrm{E}+03$ & 696.6 & 0.034 \\
\hline 707.47 & $0.00 E+00$ & & \\
\hline 710.95 & $8.11 E+03$ & & \\
\hline 721.962 & $7.61 \mathrm{E}+04$ & 722.01 & 0.007 \\
\hline 722.7 & $1.65 \mathrm{E}+05$ & & \\
\hline 729.5 & $1.65 E+03$ & 729.72 & 0.030 \\
\hline 731.5 & $5.96 \mathrm{E}+02$ & & \\
\hline 737.3 & $1.01 \mathrm{E}+04$ & 737.34 & 0.005 \\
\hline 755.89 & $9.64 \mathrm{E}+03$ & 755.9 & 0.001 \\
\hline 759.5 & $2.16 \mathrm{E}+03$ & & \\
\hline 763.4 & $2.51 \mathrm{E}+02$ & & \\
\hline 766.79 & $6.34 \mathrm{E}+03$ & 767 & 0.027 \\
\hline 770.49 & $6.34 \mathrm{E}+03$ & 770.57 & 0.010 \\
\hline 772.1 & $3.37 E+03$ & & \\
\hline 777.2 & $7.61 \mathrm{E}+01$ & 772.4 & 0.621 \\
\hline 780.5 & $3.17 \mathrm{E}+02$ & & \\
\hline 788.8 & $4.94 \mathrm{E}+02$ & 786 & 0.356 \\
\hline 801.9 & $1.65 E+03$ & 801.94 & 0.005 \\
\hline 807.27 & $0.00 E+00$ & 806.26 & 0.125 \\
\hline 811.8 & $7.61 \mathrm{E}+02$ & & \\
\hline 819.3 & $5.07 E+02$ & & \\
\hline 822.6 & $2.79 E+02$ & & \\
\hline 828.5 & $3.04 \mathrm{E}+02$ & 828.5 & \\
\hline 851.5 & $4.82 E+02$ & & \\
\hline 854.7 & $2.54 \mathrm{E}+02$ & & \\
\hline 859.2 & $1.06 \mathrm{E}+02$ & 860.7 & 0.174 \\
\hline 862.6 & $6.72 \mathrm{E}+02$ & 862.7 & 0.012 \\
\hline 872.7 & $8.87 E+02$ & & \\
\hline 887.5 & $2.79 \mathrm{E}+02$ & 887.3 & 0.023 \\
\hline 898.4 & $8.87 E+01$ & & \\
\hline 902.5 & $3.80 \mathrm{E}+02$ & & \\
\hline 912.4 & $3.17 \mathrm{E}+02$ & & \\
\hline 922.2 & $2.41 \mathrm{E}+02$ & 921.5 & 0.076 \\
\hline 928.8 & $6.34 \mathrm{E}+01$ & & \\
\hline 945.7 & $6.34 \mathrm{E}+01$ & & \\
\hline 955.7 & $7.35 E+02$ & & \\
\hline
\end{tabular}

243Am zero years

\begin{tabular}{|c|c|c|c|}
\hline $\begin{array}{c}\text { RADSRC } \\
\text { energy (keV) }\end{array}$ & $\begin{array}{l}\text { RADSRC } \\
\text { intensity }\end{array}$ & $\begin{array}{c}\text { ENSDF } \\
\text { energy (keV) }\end{array}$ & $\%$ diff \\
\hline & & 13.9 & \\
\hline 31.136 & $4.87 E+06$ & 31.143 & \\
\hline 43.03 & $4.28 \mathrm{E}+06$ & 43.1 & \\
\hline 43.537 & $3.76 \mathrm{E}+08$ & 43.532 & \\
\hline
\end{tabular}




$\begin{array}{ccc}50.8 & 1.92 \mathrm{E}+05 & 50.6 \\ 55.4 & 6.78 \mathrm{E}+05 & 55.4 \\ 74.672 & 4.42 \mathrm{E}+09 & 74.662 \\ 86.57 & 2.21 \mathrm{E}+07 & 86.712 \\ & & 97.069 \\ 98.43 & 5.90 \mathrm{E}+05 & 98.5 \\ & & 101.059 \\ & & 113.303 \\ & & 114.234 \\ 117.7 & 4.06 \mathrm{E}+07 & 117.463 \\ & & 117.6015 \\ 141.97 & 8.41 \mathrm{E}+06 & 141.893 \\ 170.14 & 8.85 \mathrm{E}+04 & 169 \\ 195.3 & 6.19 \mathrm{E}+04 & 195 \\ 544.55 & 1.25 \mathrm{E}+03 & \\ 631.116 & 2.43 \mathrm{E}+04 & 631.09 \\ 662.251 & 7.01 \mathrm{E}+04 & 662.24\end{array}$

\section{Cm zero years}

\begin{tabular}{|rrrll|}
\hline \multicolumn{1}{l}{$\begin{array}{l}\text { Energy } \\
\text { keV }\end{array}$} & $\begin{array}{l}\text { Intensity } \\
\text { ph/s/gm }\end{array}$ & & \\
\hline 12.124 & $6.88 \mathrm{E}+09$ & $\mathrm{Cm}-244$ & $->$ & $\mathrm{Pu}-240$ \\
14.262 & $1.20 \mathrm{E}+11$ & $\mathrm{Cm}-244$ & $->$ & $\mathrm{Pu}-240$ \\
16.333 & $2.69 \mathrm{E}+09$ & $\mathrm{Cm}-244$ & $->$ & $\mathrm{Pu}-240$ \\
18.135 & $1.20 \mathrm{E}+11$ & $\mathrm{Cm}-244$ & $->$ & $\mathrm{Pu}-240$ \\
21.554 & $2.99 \mathrm{E}+10$ & $\mathrm{Cm}-244$ & $->$ & $\mathrm{Pu}-240$ \\
42.817 & $7.48 \mathrm{E}+08$ & $\mathrm{Cm}-244$ & $->$ & $\mathrm{Pu}-240$ \\
98.855 & $4.49 \mathrm{E}+07$ & $\mathrm{Cm}-244$ & $->$ & $\mathrm{Pu}-240$ \\
152.622 & $2.93 \mathrm{E}+07$ & $\mathrm{Cm}-244$ & $->$ & $\mathrm{Pu}-240$ \\
251.43 & $3.62 \mathrm{E}+05$ & $\mathrm{Cm}-244$ & $->$ & $\mathrm{Pu}-240$ \\
263.33 & $1.71 \mathrm{E}+06$ & $\mathrm{Cm}-244$ & $->$ & $\mathrm{Pu}-240$ \\
302.96 & $4.61 \mathrm{E}+05$ & $\mathrm{Cm}-244$ & $->$ & $\mathrm{Pu}-240$ \\
507.22 & $2.27 \mathrm{E}+05$ & $\mathrm{Cm}-244$ & $->$ & $\mathrm{Pu}-240$ \\
554.54 & $2.36 \mathrm{E}+06$ & $\mathrm{Cm}-244$ & $->$ & $\mathrm{Pu}-240$ \\
597.35 & $1.35 \mathrm{E}+06$ & $\mathrm{Cm}-244$ & $->$ & $\mathrm{Pu}-240$ \\
606.07 & $2.15 \mathrm{E}+05$ & $\mathrm{Cm}-244$ & $->$ & $\mathrm{Pu}-240$ \\
758.64 & $4.58 \mathrm{E}+05$ & $\mathrm{Cm}-244$ & $->$ & $\mathrm{Pu}-240$ \\
817.87 & $2.01 \mathrm{E}+06$ & $\mathrm{Cm}-244$ & $->$ & $\mathrm{Pu}-240$ \\
857.5 & $1.83 \mathrm{E}+05$ & $\mathrm{Cm}-244$ & $->$ & $\mathrm{Pu}-240$ \\
900.31 & $5.09 \mathrm{E}+04$ & $\mathrm{Cm}-244$ & $->$ & $\mathrm{Pu}-240$
\end{tabular}




\section{Appendix B.}

The gamma-ray lines calculated by the RADSRC code are given for selected isotopes of uranium, neptunium, plutonium, americium and curium. The materials here have been aged five years.

U-234 $\quad 100 \% \quad$ Aged 5 years

307 lines computed.

\begin{tabular}{|c|c|c|c|c|c|c|c|}
\hline \multirow{2}{*}{$\begin{array}{c}\text { Intensity } \\
\mathrm{keV}\end{array}$} & \multirow{2}{*}{$\begin{array}{c}\text { Energy } \\
\mathrm{ph} / \mathrm{s} / \mathrm{gm}\end{array}$} & \multicolumn{6}{|c|}{$\begin{array}{l}\text { Initial Isotopes and } \\
\text { Decays }\end{array}$} \\
\hline & & & & & & & \\
\hline 8.953 & $8.95 \mathrm{E}-10$ & $\mathrm{Hg}-206$ & $->$ & TI-206 & & & \\
\hline 9.185 & $9.79 E-06$ & TI-206 & $->$ & $\mathrm{Pb}-206$, & TI-210 & $->$ & $\mathrm{Pb}-210$ \\
\hline 9.419 & $3.56 \mathrm{E}-02$ & $\mathrm{~Pb}-210$ & $->$ & $\mathrm{Bi}-210$ & $\mathrm{~Pb}-214$ & $->$ & $\mathrm{Bi}-214$ \\
\hline 9.658 & $2.27 \mathrm{E}-03$ & $\mathrm{Bi}-214$ & $->$ & Рo-214 & & & \\
\hline 10.137 & $1.64 \mathrm{E}-03$ & $\mathrm{Ra}-226$ & $->$ & $\mathrm{Rn}-222$ & & & \\
\hline 10.259 & $1.87 \mathrm{E}-08$ & $\mathrm{Hg}-206$ & $->$ & TI-206 & & & \\
\hline 10.541 & $1.98 \mathrm{E}-04$ & Tl-206 & $->$ & $\mathrm{Pb}-206$ & TI-210 & $->$ & $\mathrm{Pb}-210$ \\
\hline 10.828 & $7.12 \mathrm{E}-01$ & $\mathrm{~Pb}-210$ & $->$ & $\mathrm{Bi}-210$ & $\mathrm{~Pb}-214$ & $->$ & $\mathrm{Bi}-214$ \\
\hline 10.994 & $2.36 \mathrm{E}-10$ & $\mathrm{Hg}-206$ & $->$ & TI-206 & & & \\
\hline 11.119 & $4.36 \mathrm{E}-02$ & $\mathrm{Bi}-214$ & $->$ & Рo-214 & & & \\
\hline 11.349 & $4.78 \mathrm{E}-06$ & TI-210 & $->$ & $\mathrm{Pb}-210$ & & & \\
\hline 11.439 & $2.77 \mathrm{E}-12$ & TI-206 & $->$ & $\mathrm{Pb}-206$ & & & \\
\hline 11.712 & $8.29 E-03$ & $\mathrm{~Pb}-210$ & $->$ & $\mathrm{Bi}-210$ & $\mathrm{~Pb}-214$ & $->$ & $\mathrm{Bi}-214$ \\
\hline 11.713 & $3.03 \mathrm{E}-02$ & Ra-226 & $->$ & $\mathrm{Rn}-222$ & & & \\
\hline 12.085 & $6.26 \mathrm{E}-04$ & $\mathrm{Bi}-214$ & $->$ & Рo-214 & & & \\
\hline 12.313 & $1.62 \mathrm{E}-08$ & $\mathrm{Hg}-206$ & $->$ & TI-206 & & & \\
\hline 12.678 & $2.63 \mathrm{E}-04$ & Tl-210 & $->$ & $\mathrm{Pb}-210$ & & & \\
\hline 12.71 & $1.74 \mathrm{E}-10$ & TI-206 & $->$ & $\mathrm{Pb}-206$ & & & \\
\hline 12.855 & $8.36 \mathrm{E}-04$ & $\mathrm{Ra}-226$ & $->$ & $\mathrm{Rn}-222$ & & & \\
\hline 13.066 & $5.88 \mathrm{E}-02$ & $\mathrm{~Pb}-210$ & $->$ & $\mathrm{Bi}-210$ & & & \\
\hline 13.088 & $6.48 \mathrm{E}-01$ & $\mathrm{~Pb}-214$ & $->$ & $\mathrm{Bi}-214$ & & & \\
\hline 13.502 & $4.21 \mathrm{E}-02$ & $\mathrm{Bi}-214$ & $->$ & Po-214 & & & \\
\hline 14.336 & $4.38 \mathrm{E}-02$ & $\mathrm{Ra}-226$ & $->$ & $\mathrm{Rn}-222$ & & & \\
\hline 14.407 & $2.98 \mathrm{E}-09$ & $\mathrm{Hg}-206$ & $->$ & TI-206 & & & \\
\hline 14.836 & $5.26 \mathrm{E}-05$ & TI-206 & $->$ & $\mathrm{Pb}-206$ & TI-210 & $->$ & $\mathrm{Pb}-210$ \\
\hline 15.439 & $1.32 \mathrm{E}-01$ & $\mathrm{~Pb}-214$ & $->$ & $\mathrm{Bi}-214$ & & & \\
\hline 15.537 & $1.41 \mathrm{E}-02$ & $\mathrm{~Pb}-210$ & $->$ & $\mathrm{Bi}-210$ & & & \\
\hline 15.874 & $8.08 \mathrm{E}-03$ & $\mathrm{Bi}-214$ & $->$ & Рo-214 & & & \\
\hline 16.874 & $9.61 \mathrm{E}-03$ & $\mathrm{Ra}-226$ & $->$ & $\mathrm{Rn}-222$ & & & \\
\hline 46.52 & $2.29 \mathrm{E}-02$ & $\mathrm{~Pb}-210$ & $->$ & $\mathrm{Bi}-210$ & & & \\
\hline 53.172 & $1.25 \mathrm{E}-01$ & $\mathrm{~Pb}-214$ & $->$ & $\mathrm{Bi}-214$ & & & \\
\hline 53.23 & $2.74 \mathrm{E}+05$ & U-234 & $->$ & Th-230 & & & \\
\hline
\end{tabular}




\begin{tabular}{|c|c|c|c|c|c|c|c|}
\hline 67.6758 & $3.98 \mathrm{E}+01$ & Th-230 & $->$ & $\mathrm{Ra}-226$ & & & \\
\hline 70.832 & $3.11 \mathrm{E}-08$ & $\mathrm{Hg}-206$ & $->$ & TI-206 & & & \\
\hline 72.803 & $6.69 \mathrm{E}-05$ & TI-206 & $->$ & $\mathrm{Pb}-206$ & TI-210 & $->$ & $\mathrm{Pb}-210$ \\
\hline 72.873 & $5.22 \mathrm{E}-08$ & $\mathrm{Hg}-206$ & $->$ & TI-206 & & & \\
\hline 74.814 & 7.42E-01 & $\mathrm{Pb}-214$ & $->$ & $\mathrm{Bi}-214$ & & & \\
\hline 74.969 & $1.10 \mathrm{E}-04$ & TI-206 & $->$ & $\mathrm{Pb}-206$, & TI-210 & $->$ & Pb-210 \\
\hline 76.838 & $6.60 \mathrm{E}-02$ & $\mathrm{Bi}-214$ & $->$ & Po-214 & & & \\
\hline 77.107 & $1.25 \mathrm{E}+00$ & $\mathrm{~Pb}-214$ & $->$ & $\mathrm{Bi}-214$ & & & \\
\hline 79.29 & $1.10 \mathrm{E}-01$ & $\mathrm{Bi}-214$ & $->$ & Po-214 & & & \\
\hline 81 & 4.78E-05 & TI-210 & $->$ & $\mathrm{Pb}-210$ & & & \\
\hline 81.067 & $2.01 \mathrm{E}-02$ & $\mathrm{Ra}-226$ & $->$ & $\mathrm{Rn}-222$ & & & \\
\hline 82.434 & $1.87 \mathrm{E}-08$ & $\mathrm{Hg}-206$ & $->$ & TI-206 & & & \\
\hline 83.787 & $3.34 \mathrm{E}-02$ & Ra-226 & $->$ & $\mathrm{Rn}-222$ & & & \\
\hline 84.789 & 3.92E-05 & TI-206 & $->$ & $\mathrm{Pb}-206$, & TI-210 & $->$ & $\mathrm{Pb}-210$ \\
\hline 85.185 & $5.22 \mathrm{E}-09$ & $\mathrm{Hg}-206$ & $->$ & TI-206 & & & \\
\hline 87.19 & $4.41 \mathrm{E}-01$ & $\mathrm{~Pb}-214$ & $->$ & $\mathrm{Bi}-214$ & & & \\
\hline 87.632 & $1.12 \mathrm{E}-05$ & TI-206 & $->$ & $\mathrm{Pb}-206$, & TI-210 & $->$ & $\mathrm{Pb}-210$ \\
\hline 89.639 & $3.92 \mathrm{E}-02$ & $\mathrm{Bi}-214$ & $->$ & Po-214 & & & \\
\hline 90.128 & $1.31 \mathrm{E}-01$ & $\mathrm{~Pb}-214$ & $->$ & $\mathrm{Bi}-214$ & & & \\
\hline 92.673 & $1.19 \mathrm{E}-02$ & $\mathrm{Bi}-214$ & $->$ & Po-214 & & & \\
\hline 94.677 & $1.18 \mathrm{E}-02$ & Ra-226 & $->$ & $\mathrm{Rn}-222$ & & & \\
\hline 95 & $9.56 \mathrm{E}-05$ & TI-210 & $->$ & $\mathrm{Pb}-210$ & & & \\
\hline 97.907 & 3.77E-03 & Ra-226 & $->$ & $\mathrm{Rn}-222$ & & & \\
\hline 109.97 & $6.24 \mathrm{E}-03$ & Th-230 & $->$ & Ra-226 & & & \\
\hline 120.912 & $9.44 \mathrm{E}+04$ & U-234 & $->$ & Th-230 & & & \\
\hline 137.4 & $6.71 E-03$ & $\mathrm{~Pb}-214$ & $->$ & $\mathrm{Bi}-214$ & & & \\
\hline 141.3 & $4.44 \mathrm{E}-03$ & $\mathrm{~Pb}-214$ & $->$ & $\mathrm{Bi}-214$ & & & \\
\hline 143.876 & $5.14 \mathrm{E}+00$ & Th-230 & $->$ & Ra-226 & & & \\
\hline 186.057 & $9.31 \mathrm{E}-01$ & Th-230 & $->$ & $\mathrm{Ra}-226$ & & & \\
\hline 186.11 & 3.75E-01 & Ra-226 & $->$ & $\mathrm{Rn}-222$ & & & \\
\hline 196.3 & $5.57 \mathrm{E}-03$ & $\mathrm{~Pb}-214$ & $->$ & $\mathrm{Bi}-214$ & & & \\
\hline 205.1 & $5.29 E-04$ & Th-230 & $->$ & Ra-226 & & & \\
\hline 235.01 & 8.57E-04 & Th-230 & $->$ & Ra-226 & & & \\
\hline 241.92 & $8.48 \mathrm{E}-01$ & $\mathrm{~Pb}-214$ & $->$ & $\mathrm{Bi}-214$ & & & \\
\hline 253.732 & $1.17 \mathrm{E}+00$ & Th-230 & $->$ & Ra-226 & & & \\
\hline 253.84 & 8.99E-02 & Th-230 & $->$ & Ra-226 & & & \\
\hline 258.94 & $6.26 \mathrm{E}-02$ & $\mathrm{~Pb}-214$ & $->$ & $\mathrm{Bi}-214$ & & & \\
\hline 262.41 & $6.18 \mathrm{E}-04$ & Ra-226 & $->$ & $\mathrm{Rn}-222$ & & & \\
\hline 266.15 & $2.95 \mathrm{E}-13$ & $\mathrm{Bi}-210$ & $->$ & TI-206 & & & \\
\hline 273.7 & $2.05 E-02$ & $\mathrm{Bi}-214$ & $->$ & Po-214 & & & \\
\hline 274.56 & 3.64E-02 & $\mathrm{Pb}-214$ & $->$ & $\mathrm{Bi}-214$ & & & \\
\hline 280.93 & $9.21 \mathrm{E}-03$ & $\mathrm{Bi}-214$ & $->$ & Po-214 & & & \\
\hline 286.9 & 3.64E-03 & $\mathrm{Bi}-214$ & $->$ & Рo-214 & & & \\
\hline 295.091 & $2.18 \mathrm{E}+00$ & $\mathrm{~Pb}-214$ & $->$ & $\mathrm{Bi}-214$ & & & \\
\hline 298.1 & $1.89 \mathrm{E}-03$ & TI-210 & $->$ & $\mathrm{Pb}-210$, & Рo-214 & $->$ & $\mathrm{Pb}-210$ \\
\hline 302.25 & $3.36 \mathrm{E}-07$ & $\mathrm{Hg}-206$ & $->$ & TI-206 & & & \\
\hline 304.42 & 3.87E-03 & $\mathrm{Bi}-214$ & $->$ & Po-214 & & & \\
\hline 305.5 & $2.62 \mathrm{E}-03$ & $\mathrm{~Pb}-214$ & $->$ & $\mathrm{Bi}-214$ & & & \\
\hline 314.2 & 8.99E-03 & $\mathrm{Pb}-214$ & $->$ & $\mathrm{Bi}-214$ & & & \\
\hline 324.3 & $2.27 \mathrm{E}-03$ & $\mathrm{~Pb}-214$ & $->$ & $\mathrm{Bi}-214$ & & & \\
\hline
\end{tabular}




\begin{tabular}{|c|c|c|c|c|}
\hline 333.6 & $1.08 \mathrm{E}-02$ & $\mathrm{Bi}-214$ & $->$ & Po-214 \\
\hline 334.9 & $6.48 \mathrm{E}-03$ & $\mathrm{Bi}-214$ & $->$ & Po-214 \\
\hline 338.5 & 4.44E-03 & $\mathrm{Bi}-214$ & $->$ & Po-214 \\
\hline 344.96 & 6.47E-09 & $\mathrm{Hg}-206$ & $->$ & TI-206 \\
\hline 347.1 & $6.82 \mathrm{E}-03$ & $\mathrm{Bi}-214$ & $->$ & Po-214 \\
\hline 351.87 & $4.22 E+00$ & $\mathrm{~Pb}-214$ & $->$ & $\mathrm{Bi}-214$ \\
\hline 354 & $9.56 \mathrm{E}-05$ & TI-210 & $->$ & $\mathrm{Pb}-210$ \\
\hline 364.2 & 7.17E-04 & $\mathrm{Bi}-214$ & $->$ & Po-214 \\
\hline 376.6 & 5.57E-04 & $\mathrm{Bi}-214$ & $->$ & Po-214 \\
\hline 380 & 7.17E-05 & TI-210 & $->$ & $\mathrm{Pb}-210$ \\
\hline 384.06 & $4.73 \mathrm{E}-11$ & $\mathrm{Hg}-206$ & $->$ & TI-206 \\
\hline 386.834 & 4.09E-02 & $\mathrm{Bi}-214$ & $->$ & Po-214 \\
\hline 388.95 & $4.66 \mathrm{E}-02$ & $\mathrm{Bi}-214$ & $->$ & Po-214 \\
\hline 394 & $1.02 \mathrm{E}-03$ & $\mathrm{Bi}-214$ & $->$ & Po-214 \\
\hline 396 & $3.41 \mathrm{E}-03$ & $\mathrm{Bi}-214$ & $->$ & Po-214 \\
\hline 405.73 & $1.90 \mathrm{E}-02$ & $\mathrm{Bi}-214$ & $->$ & Po-214 \\
\hline 414.72 & 4.46E-05 & Ra-226 & $->$ & $R n-222$ \\
\hline 426.5 & $1.25 \mathrm{E}-02$ & $\mathrm{Bi}-214$ & $->$ & Po-214 \\
\hline 440.4 & $3.30 \mathrm{E}-03$ & $\mathrm{Bi}-214$ & $->$ & Po-214 \\
\hline 449.5 & 3.09E-05 & $\mathrm{Ra}-226$ & $->$ & $\mathrm{Rn}-222$ \\
\hline 454.832 & $3.62 \mathrm{E}-02$ & $\mathrm{Bi}-214$ & $->$ & Po-214 \\
\hline 454.97 & $5.99 E+01$ & $U-234$ & $->$ & Th-230 \\
\hline 462.05 & $1.93 \mathrm{E}-02$ & $\mathrm{~Pb}-214$ & $->$ & $\mathrm{Bi}-214$ \\
\hline 469.76 & $1.51 \mathrm{E}-02$ & $\mathrm{Bi}-214$ & $->$ & Po-214 \\
\hline 470.8 & $1.14 \mathrm{E}-03$ & $\mathrm{~Pb}-214$ & $->$ & $\mathrm{Bi}-214$ \\
\hline 474.51 & $1.34 \mathrm{E}-02$ & $\mathrm{Bi}-214$ & $->$ & Po-214 \\
\hline 478 & $4.78 \mathrm{E}-05$ & TI-210 & $->$ & $\mathrm{Pb}-210$ \\
\hline 480.32 & 3.84E-02 & $\mathrm{Pb}-214$ & $->$ & $\mathrm{Bi}-214$ \\
\hline 487.13 & 4.99E-02 & $\mathrm{Pb}-214$ & $->$ & $\mathrm{Bi}-214$ \\
\hline 494.6 & $1.02 \mathrm{E}-03$ & $\mathrm{Bi}-214$ & $->$ & Po-214 \\
\hline 502.2 & $2.05 \mathrm{E}-03$ & $\mathrm{Bi}-214$ & $->$ & Po-214 \\
\hline 503.53 & $2.30 \mathrm{E}+00$ & $U-234$ & $->$ & Th-230 \\
\hline 508.2 & $3.39 E+01$ & $U-234$ & $->$ & Th-230 \\
\hline 510 & 7.96E-03 & $\mathrm{Rn}-222$ & $->$ & Po-218 \\
\hline 511 & 3.30E-03 & $\mathrm{Pb}-214$ & $->$ & $\mathrm{Bi}-214$ \\
\hline 520.4 & $6.48 \mathrm{E}-04$ & $\mathrm{Bi}-214$ & $->$ & Рo-214 \\
\hline 525 & $1.82 \mathrm{E}-03$ & $\mathrm{Bi}-214$ & $->$ & Po-214 \\
\hline 533.5 & $2.16 \mathrm{E}-02$ & $\mathrm{~Pb}-214$ & $->$ & $\mathrm{Bi}-214$ \\
\hline 536.93 & 8.08E-03 & $\mathrm{Bi}-214$ & $->$ & Po-214 \\
\hline 538.7 & 5.57E-04 & $\mathrm{Pb}-214$ & $->$ & $\mathrm{Bi}-214$ \\
\hline 542.84 & $9.67 \mathrm{E}-03$ & $\mathrm{Bi}-214$ & $->$ & Po-214 \\
\hline 543.91 & $2.62 \mathrm{E}-03$ & $\mathrm{~Pb}-214$ & $->$ & $\mathrm{Bi}-214$ \\
\hline 547.1 & $3.64 \mathrm{E}-03$ & $\mathrm{Bi}-214$ & $->$ & Po-214 \\
\hline 551.8 & $1.07 E-04$ & Th-230 & $->$ & Ra-226 \\
\hline 570.5 & $3.17 E-04$ & Th-230 & $->$ & Ra-226 \\
\hline 572.67 & $9.33 E-03$ & $\mathrm{Bi}-214$ & $->$ & Po-214 \\
\hline 580.06 & $4.14 \mathrm{E}-02$ & $\mathrm{~Pb}-214$ & $->$ & $\mathrm{Bi}-214$ \\
\hline 581.78 & $2.76 \mathrm{E}+01$ & $\mathrm{U}-234$ & $->$ & Th-230 \\
\hline 596 & $1.36 \mathrm{E}-03$ & $\mathrm{Bi}-214$ & $->$ & Рo-214 \\
\hline 600.83 & $6.98 \mathrm{E}-05$ & Ra-226 & $->$ & $\mathrm{Rn}-222$ \\
\hline
\end{tabular}




\begin{tabular}{|c|c|c|c|c|c|c|c|}
\hline 609.311 & $5.24 \mathrm{E}+00$ & $\mathrm{Bi}-214$ & $->$ & Po-214 & & & \\
\hline 615.77 & 7.96E-03 & $\mathrm{Bi}-214$ & $->$ & Рo-214 & & & \\
\hline 617.1 & 3.87E-03 & $\mathrm{Bi}-214$ & $->$ & Po- 214 & & & \\
\hline 620 & $1.07 \mathrm{E}-04$ & Th-230 & $->$ & $\mathrm{Ra}-226$ & & & \\
\hline 624.44 & $1.84 \mathrm{E}+00$ & $U-234$ & $->$ & Th-230 & & & \\
\hline 626.4 & $5.57 E-04$ & $\mathrm{Bi}-214$ & $->$ & Рo-214 & & & \\
\hline 631.2 & $1.93 \mathrm{E}-03$ & $\mathrm{Bi}-214$ & $->$ & Po-214 & & & \\
\hline 633.14 & $6.82 \mathrm{E}-03$ & $\mathrm{Bi}-214$ & $->$ & Рo-214 & & & \\
\hline 639.36 & $3.53 E-03$ & $\mathrm{Bi}-214$ & $->$ & Po-214 & & & \\
\hline 649.18 & $6.71 \mathrm{E}-03$ & $\mathrm{Bi}-214$ & $->$ & Рo-214 & & & \\
\hline 650.21 & $3.11 \mathrm{E}-08$ & $\mathrm{Hg}-206$ & $->$ & TI-206 & & & \\
\hline 660.75 & $4.89 E-03$ & $\mathrm{Bi}-214$ & $->$ & Po-214 & & & \\
\hline 665.442 & $1.77 \mathrm{E}-01$ & $\mathrm{Bi}-214$ & $->$ & Po-214 & & & \\
\hline 668 & $4.78 \mathrm{E}-05$ & TI-210 & $->$ & $\mathrm{Pb}-210$ & & & \\
\hline 677.67 & $2.30 E+00$ & $U-234$ & $->$ & Th-230 & & & \\
\hline 683.21 & 8.99E-03 & $\mathrm{Bi}-214$ & $->$ & Po-214 & & & \\
\hline 687.7 & $6.82 E-04$ & $\mathrm{Bi}-214$ & $->$ & Po-214 & & & \\
\hline 693.3 & $6.82 \mathrm{E}-04$ & $\mathrm{Bi}-214$ & $->$ & Po-214 & & & \\
\hline 697.89 & $4.21 \mathrm{E}-03$ & $\mathrm{Bi}-214$ & $->$ & Po-214 & & & \\
\hline 703.07 & $5.37 \mathrm{E}-02$ & $\mathrm{Bi}-214$ & $->$ & Po-214 & & & \\
\hline 710.84 & 8.50E-03 & $\mathrm{Bi}-214$ & $->$ & Рo-214 & & & \\
\hline 719.856 & $4.58 \mathrm{E}-02$ & $\mathrm{Bi}-214$ & $->$ & Po-214 & & & \\
\hline 723.32 & $5.12 \mathrm{E}-03$ & $\mathrm{Bi}-214$ & $->$ & Po-214 & & & \\
\hline 727.8 & $1.79 \mathrm{E}-03$ & $\mathrm{Bi}-214$ & $->$ & Po-214 & & & \\
\hline 733.64 & $5.35 \mathrm{E}-03$ & $\mathrm{Bi}-214$ & $->$ & Po-214 & & & \\
\hline 740.87 & 4.44E-03 & $\mathrm{Bi}-214$ & $->$ & Po-214 & & & \\
\hline 752.843 & $1.51 \mathrm{E}-02$ & $\mathrm{Bi}-214$ & $->$ & Po-214 & & & \\
\hline 765.9 & 8.99E-03 & $\mathrm{Pb}-214$ & $->$ & $\mathrm{Bi}-214$ & & & \\
\hline 768.35 & $5.55 \mathrm{E}-01$ & $\mathrm{Bi}-214$ & $->$ & Po-214 & & & \\
\hline 785.827 & $1.24 \mathrm{E}-01$ & $\mathrm{~Pb}-214$ & $->$ & $\mathrm{Bi}-214$ & & & \\
\hline 786.42 & $3.53 \mathrm{E}-02$ & $\mathrm{Bi}-214$ & $->$ & Po-214 & & & \\
\hline 797.88 & $3.55 E-03$ & Tl-210 & $->$ & $\mathrm{Pb}-210$, & Рo-214 & $->$ & $\mathrm{Pb}-210$ \\
\hline 799.75 & $4.66 \mathrm{E}-03$ & $\mathrm{Bi}-214$ & $->$ & Po-214 & & & \\
\hline 803.13 & $4.36 \mathrm{E}-06$ & TI-206 & $->$ & $\mathrm{Pb}-206$, & Po-210 & $->$ & $\mathrm{Pb}-206$ \\
\hline 806.155 & $1.40 \mathrm{E}-01$ & $\mathrm{Bi}-214$ & $->$ & Po-214 & & & \\
\hline 814.87 & $4.55 E-03$ & $\mathrm{Bi}-214$ & $->$ & Рo-214 & & & \\
\hline 821.166 & $1.71 \mathrm{E}-02$ & $\mathrm{Bi}-214$ & $->$ & Po-214 & & & \\
\hline 826.44 & $1.05 \mathrm{E}-02$ & $\mathrm{Bi}-214$ & $->$ & Po-214 & & & \\
\hline 832.34 & $2.62 E-03$ & $\mathrm{Bi}-214$ & $->$ & Po-214 & & & \\
\hline 838.999 & $6.68 \mathrm{E}-02$ & $\mathrm{~Pb}-214$ & $->$ & $\mathrm{Bi}-214$ & & & \\
\hline 847.2 & $1.93 \mathrm{E}-03$ & $\mathrm{Bi}-214$ & $->$ & Po-214 & & & \\
\hline 860 & $1.65 \mathrm{E}-04$ & TI-210 & $->$ & $\mathrm{Pb}-210$ & & & \\
\hline 904.33 & 1.19E-02 & $\mathrm{Bi}-214$ & $->$ & Po-214 & & & \\
\hline 908 & 7.17E-05 & Tl-210 & $->$ & $P b-210$ & & & \\
\hline 915.8 & $2.62 \mathrm{E}-03$ & $\mathrm{Bi}-214$ & $->$ & Рo-214 & & & \\
\hline 934.039 & $3.59 E-01$ & $\mathrm{Bi}-214$ & $->$ & Po-214 & & & \\
\hline 943.3 & $1.93 \mathrm{E}-03$ & $\mathrm{Bi}-214$ & $->$ & Po-214 & & & \\
\hline 964.07 & $4.36 \mathrm{E}-02$ & $\mathrm{Bi}-214$ & $->$ & Po-214 & & & \\
\hline 976.2 & $2.62 \mathrm{E}-03$ & $\mathrm{Bi}-214$ & $->$ & Po-214 & & & \\
\hline 989.2 & $1.36 \mathrm{E}-03$ & $\mathrm{Bi}-214$ & $->$ & Po-214 & & & \\
\hline
\end{tabular}




\begin{tabular}{|c|c|c|c|c|}
\hline 1013.4 & $1.14 \mathrm{E}-03$ & $\mathrm{Bi}-214$ & $->$ & Po-214 \\
\hline 1020.5 & $1.36 \mathrm{E}-03$ & $\mathrm{Bi}-214$ & $->$ & Po-214 \\
\hline 1032.22 & 1.09E-02 & $\mathrm{Bi}-214$ & $->$ & Po-214 \\
\hline 1038 & $1.93 \mathrm{E}-03$ & $\mathrm{Bi}-214$ & $->$ & Po-214 \\
\hline 1045.4 & $3.30 \mathrm{E}-03$ & $\mathrm{Bi}-214$ & $->$ & Po-214 \\
\hline 1051.95 & $3.58 \mathrm{E}-02$ & $\mathrm{Bi}-214$ & $->$ & Рo-214 \\
\hline 1067.3 & 3.30E-03 & $\mathrm{Bi}-214$ & $->$ & Po-214 \\
\hline 1068 & 2.87E-04 & TI-210 & $->$ & $\mathrm{Pb}-210$ \\
\hline 1070.02 & $3.24 \mathrm{E}-02$ & $\mathrm{Bi}-214$ & $->$ & Po-214 \\
\hline 1103.7 & $1.14 \mathrm{E}-02$ & $\mathrm{Bi}-214$ & $->$ & Po-214 \\
\hline 1104.766 & $9.10 \mathrm{E}-03$ & $\mathrm{Bi}-214$ & $->$ & Po-214 \\
\hline 1110 & $1.65 \mathrm{E}-04$ & TI-210 & $->$ & $\mathrm{Pb}-210$ \\
\hline 1120.273 & $1.71 \mathrm{E}+00$ & $\mathrm{Bi}-214$ & $->$ & Po-214 \\
\hline 1130.6 & $5.12 \mathrm{E}-03$ & $\mathrm{Bi}-214$ & $->$ & Po-214 \\
\hline 1133.65 & $2.90 \mathrm{E}-02$ & $\mathrm{Bi}-214$ & $->$ & Po-214 \\
\hline 1155.183 & $1.92 \mathrm{E}-01$ & $\mathrm{Bi}-214$ & $->$ & Po-214 \\
\hline 1172.93 & $6.60 \mathrm{E}-03$ & $\mathrm{Bi}-214$ & $->$ & Po-214 \\
\hline 1173.04 & $6.60 \mathrm{E}-03$ & $\mathrm{Bi}-214$ & $->$ & Po-214 \\
\hline 1207.764 & $5.23 \mathrm{E}-02$ & $\mathrm{Bi}-214$ & $->$ & Po-214 \\
\hline 1208 & $4.06 \mathrm{E}-04$ & TI-210 & $->$ & $P b-210$ \\
\hline 1226.8 & 3.07E-03 & $\mathrm{Bi}-214$ & $->$ & Po-214 \\
\hline 1230.84 & $2.50 \mathrm{E}-03$ & $\mathrm{Bi}-214$ & $->$ & Po-214 \\
\hline 1238.107 & $6.73 \mathrm{E}-01$ & $\mathrm{Bi}-214$ & $->$ & Po-214 \\
\hline 1280.952 & $1.67 \mathrm{E}-01$ & $\mathrm{Bi}-214$ & $->$ & Po-214 \\
\hline 1303.76 & $1.38 \mathrm{E}-02$ & $\mathrm{Bi}-214$ & $->$ & Po-214 \\
\hline 1314 & $5.02 \mathrm{E}-04$ & TI-210 & $->$ & $\mathrm{Pb}-210$ \\
\hline 1317.02 & $9.78 \mathrm{E}-03$ & $\mathrm{Bi}-214$ & $->$ & Po-214 \\
\hline 1330 & $1.25 \mathrm{E}-03$ & $\mathrm{Bi}-214$ & $->$ & Po-214 \\
\hline 1341.5 & $2.62 \mathrm{E}-03$ & $\mathrm{Bi}-214$ & $->$ & Po-214 \\
\hline 1353 & $5.12 \mathrm{E}-04$ & $\mathrm{Bi}-214$ & $->$ & Po-214 \\
\hline 1377.659 & 4.57E-01 & $\mathrm{Bi}-214$ & $->$ & Po-214 \\
\hline 1385.295 & 8.87E-02 & $\mathrm{Bi}-214$ & $->$ & Рo-214 \\
\hline 1392.5 & $2.16 \mathrm{E}-03$ & $\mathrm{Bi}-214$ & $->$ & Po-214 \\
\hline 1401.48 & $1.58 \mathrm{E}-01$ & $\mathrm{Bi}-214$ & $->$ & Po-214 \\
\hline 1407.97 & $2.82 \mathrm{E}-01$ & $\mathrm{Bi}-214$ & $->$ & Po-214 \\
\hline 1408 & $1.17 \mathrm{E}-04$ & TI-210 & $->$ & $\mathrm{Pb}-210$ \\
\hline 1419.7 & $5.80 \mathrm{E}-04$ & $\mathrm{Bi}-214$ & $->$ & Po-214 \\
\hline 1471.1 & $1.36 \mathrm{E}-03$ & $\mathrm{Bi}-214$ & $->$ & Po-214 \\
\hline 1479.19 & 7.85E-03 & $\mathrm{Bi}-214$ & $->$ & Рo-214 \\
\hline 1509.22 & $2.49 \mathrm{E}-01$ & $\mathrm{Bi}-214$ & $->$ & Рo-214 \\
\hline 1538 & 4.78E-05 & TI-210 & $->$ & $\mathrm{Pb}-210$ \\
\hline 1538.49 & $4.66 \mathrm{E}-02$ & $\mathrm{Bi}-214$ & $->$ & Po-214 \\
\hline 1543.347 & 3.98E-02 & $\mathrm{Bi}-214$ & $->$ & Рo-214 \\
\hline 1583.22 & 8.19E-02 & $\mathrm{Bi}-214$ & $->$ & Po-214 \\
\hline 1588 & 4.78E-05 & TI-210 & $->$ & $\mathrm{Pb}-210$ \\
\hline 1594.78 & 3.01E-02 & $\mathrm{Bi}-214$ & $->$ & Po-214 \\
\hline 1599.3 & $3.80 \mathrm{E}-02$ & $\mathrm{Bi}-214$ & $->$ & Po-214 \\
\hline 1636.6 & $2.16 \mathrm{E}-03$ & $\mathrm{Bi}-214$ & $->$ & Po-214 \\
\hline 1637.37 & 7.96E-03 & $\mathrm{Bi}-214$ & $->$ & Po-214 \\
\hline 1648 & 4.78E-05 & TI-210 & $->$ & $\mathrm{Pb}-210$ \\
\hline
\end{tabular}




\begin{tabular}{|c|c|c|c|c|}
\hline 1661.258 & $1.31 \mathrm{E}-01$ & $\mathrm{Bi}-214$ & $->$ & Po-214 \\
\hline 1683.99 & $2.68 \mathrm{E}-02$ & $\mathrm{Bi}-214$ & $->$ & Po-214 \\
\hline 1729.58 & $3.47 \mathrm{E}-01$ & $\mathrm{Bi}-214$ & $->$ & Po-214 \\
\hline 1764.49 & $1.81 \mathrm{E}+00$ & $\mathrm{Bi}-214$ & $->$ & Po-214 \\
\hline 1782.1 & $1.82 \mathrm{E}-03$ & $\mathrm{Bi}-214$ & $->$ & Po-214 \\
\hline 1814.01 & $1.36 \mathrm{E}-03$ & $\mathrm{Bi}-214$ & $->$ & Po-214 \\
\hline 1838.37 & 4.36E-02 & $\mathrm{Bi}-214$ & $->$ & Po-214 \\
\hline 1847.41 & $2.41 \mathrm{E}-01$ & $\mathrm{Bi}-214$ & $->$ & Po-214 \\
\hline 1873.112 & $2.57 \mathrm{E}-02$ & $\mathrm{Bi}-214$ & $->$ & Po-214 \\
\hline 1890.259 & $1.01 \mathrm{E}-02$ & $\mathrm{Bi}-214$ & $->$ & Po-214 \\
\hline 1896.28 & $2.01 \mathrm{E}-02$ & $\mathrm{Bi}-214$ & $->$ & Po-214 \\
\hline 1898.9 & 7.17E-03 & $\mathrm{Bi}-214$ & $->$ & Po-214 \\
\hline 1935.8 & $5.80 \mathrm{E}-03$ & $\mathrm{Bi}-214$ & $->$ & Po-214 \\
\hline 1994.7 & 5.69E-04 & $\mathrm{Bi}-214$ & $->$ & Po-214 \\
\hline 2004.5 & $3.30 \mathrm{E}-04$ & $\mathrm{Bi}-214$ & $->$ & Po-214 \\
\hline 2008 & $1.65 \mathrm{E}-04$ & TI-210 & $->$ & $\mathrm{Pb}-210$ \\
\hline 2010.79 & 5.57E-03 & $\mathrm{Bi}-214$ & $->$ & Po-214 \\
\hline 2021.7 & $2.16 \mathrm{E}-03$ & $\mathrm{Bi}-214$ & $->$ & Po-214 \\
\hline 2052.93 & 7.96E-03 & $\mathrm{Bi}-214$ & $->$ & Po-214 \\
\hline 2085 & $1.14 \mathrm{E}-03$ & $\mathrm{Bi}-214$ & $->$ & Po-214 \\
\hline 2088 & 1.17E-04 & TI-210 & $->$ & $\mathrm{Pb}-210$ \\
\hline 2089.55 & 6.37E-03 & $\mathrm{Bi}-214$ & $->$ & Po-214 \\
\hline 2109.91 & $9.89 E-03$ & $\mathrm{Bi}-214$ & $->$ & Po-214 \\
\hline 2118.53 & $1.38 \mathrm{E}-01$ & $\mathrm{Bi}-214$ & $->$ & Po-214 \\
\hline 2147.8 & $1.82 \mathrm{E}-03$ & $\mathrm{Bi}-214$ & $->$ & Po-214 \\
\hline 2192.52 & $6.94 \mathrm{E}-03$ & $\mathrm{Bi}-214$ & $->$ & Po-214 \\
\hline 2204.09 & $5.68 \mathrm{E}-01$ & $\mathrm{Bi}-214$ & $->$ & Po-214 \\
\hline 2251.2 & 7.96E-04 & $\mathrm{Bi}-214$ & $->$ & Po-214 \\
\hline 2259.7 & $1.02 \mathrm{E}-03$ & $\mathrm{Bi}-214$ & $->$ & Po-214 \\
\hline 2266.67 & $2.05 E-03$ & $\mathrm{Bi}-214$ & $->$ & Po-214 \\
\hline 2268 & 7.17E-05 & TI-210 & $->$ & $\mathrm{Pb}-210$ \\
\hline 2270 & $3.30 \mathrm{E}-04$ & $\mathrm{Bi}-214$ & $->$ & Po-214 \\
\hline 2284.4 & $5.80 \mathrm{E}-04$ & $\mathrm{Bi}-214$ & $->$ & Po-214 \\
\hline 2293.29 & 3.69E-02 & $\mathrm{Bi}-214$ & $->$ & Po-214 \\
\hline 2324.8 & $2.16 \mathrm{E}-04$ & $\mathrm{Bi}-214$ & $->$ & Po-214 \\
\hline 2331.2 & $2.50 \mathrm{E}-03$ & $\mathrm{Bi}-214$ & $->$ & Po-214 \\
\hline 2358 & $1.91 \mathrm{E}-04$ & TI-210 & $->$ & $\mathrm{Pb}-210$ \\
\hline 2360.9 & $2.16 \mathrm{E}-04$ & $\mathrm{Bi}-214$ & $->$ & Po-214 \\
\hline 2369.3 & $3.30 \mathrm{E}-04$ & $\mathrm{Bi}-214$ & $->$ & Po-214 \\
\hline 2376.99 & $1.34 \mathrm{E}-03$ & $\mathrm{Bi}-214$ & $->$ & Po-214 \\
\hline 2390.9 & 2.27E-04 & $\mathrm{Bi}-214$ & $->$ & Po-214 \\
\hline 2423.32 & $6.71 \mathrm{E}-04$ & $\mathrm{Bi}-214$ & $->$ & Po-214 \\
\hline 2428 & $2.15 \mathrm{E}-04$ & TI-210 & $->$ & $\mathrm{Pb}-210$ \\
\hline 2447.68 & $1.76 \mathrm{E}-01$ & $\mathrm{Bi}-214$ & $->$ & Po-214 \\
\hline 2482.417 & $2.39 E-04$ & $\mathrm{Bi}-214$ & $->$ & Po-214 \\
\hline 2505.58 & $6.71 \mathrm{E}-04$ & $\mathrm{Bi}-214$ & $->$ & Po-214 \\
\hline 2551 & 4.44E-05 & $\mathrm{Bi}-214$ & $->$ & Po-214 \\
\hline 2604.5 & $5.12 \mathrm{E}-05$ & $\mathrm{Bi}-214$ & $->$ & Po-214 \\
\hline 2631 & $1.02 \mathrm{E}-04$ & $\mathrm{Bi}-214$ & $->$ & Po-214 \\
\hline 2662.23 & $3.30 \mathrm{E}-05$ & $\mathrm{Bi}-214$ & $->$ & Po-214 \\
\hline
\end{tabular}




\begin{tabular}{|c|c|c|c|c|}
\hline 2694.67 & 3.69E-03 & $\mathrm{Bi}-214$ & $->$ & Рo-214 \\
\hline 2698.86 & $3.18 \mathrm{E}-04$ & $\mathrm{Bi}-214$ & $->$ & Po-214 \\
\hline 2719.21 & $2.05 E-04$ & $\mathrm{Bi}-214$ & $->$ & Po-214 \\
\hline 2769.99 & $2.91 \mathrm{E}-03$ & $\mathrm{Bi}-214$ & $->$ & Рo-214 \\
\hline 2786.09 & $6.71 \mathrm{E}-04$ & $\mathrm{Bi}-214$ & $->$ & Po-214 \\
\hline 2827 & $2.84 \mathrm{E}-04$ & $\mathrm{Bi}-214$ & $->$ & Рo-214 \\
\hline 2860.9 & 3.87E-05 & $\mathrm{Bi}-214$ & $->$ & Рo-214 \\
\hline 2880.4 & $1.05 E-03$ & $\mathrm{Bi}-214$ & $->$ & Po-214 \\
\hline 2893.59 & $7.28 \mathrm{E}-04$ & $\mathrm{Bi}-214$ & $->$ & Рo-214 \\
\hline 2922.09 & $1.79 \mathrm{E}-03$ & $\mathrm{Bi}-214$ & $->$ & Рo-214 \\
\hline 2928.7 & $1.36 \mathrm{E}-04$ & $\mathrm{Bi}-214$ & $->$ & Рo-214 \\
\hline 2934.9 & $6.48 \mathrm{E}-05$ & $\mathrm{Bi}-214$ & $->$ & Po-214 \\
\hline 2940.5 & $1.93 \mathrm{E}-04$ & $\mathrm{Bi}-214$ & $->$ & Рo-214 \\
\hline 2978.79 & $1.67 \mathrm{E}-03$ & $\mathrm{Bi}-214$ & $->$ & Po-214 \\
\hline 2988.7 & $1.25 \mathrm{E}-04$ & $\mathrm{Bi}-214$ & $->$ & Po-214 \\
\hline 2999.99 & $1.00 \mathrm{E}-03$ & $\mathrm{Bi}-214$ & $->$ & Рo-214 \\
\hline 3053.89 & $2.57 \mathrm{E}-03$ & $\mathrm{Bi}-214$ & $->$ & Рo-214 \\
\hline 3081.7 & 4.89E-04 & $\mathrm{Bi}-214$ & $->$ & Рo-214 \\
\hline 3093.9 & $5.80 \mathrm{E}-05$ & $\mathrm{Bi}-214$ & $->$ & Po-214 \\
\hline 3136.3 & 3.87E-05 & $\mathrm{Bi}-214$ & $->$ & Рo-214 \\
\hline 3142.6 & $1.82 \mathrm{E}-04$ & $\mathrm{Bi}-214$ & $->$ & Рo-214 \\
\hline 3160.5 & 5.80E-05 & $\mathrm{Bi}-214$ & $->$ & Рo-214 \\
\hline 3183.6 & $1.71 \mathrm{E}-04$ & $\mathrm{Bi}-214$ & $->$ & Po-214 \\
\hline 3233.3 & $2.27 \mathrm{E}-05$ & $\mathrm{Bi}-214$ & $->$ & Po-214 \\
\hline 3269.7 & $1.14 \mathrm{E}-05$ & $\mathrm{Bi}-214$ & $->$ & Po-214 \\
\hline
\end{tabular}

234U Aged 5 Sorted by Intensity

\begin{tabular}{|c|c|rrr|}
\hline Intensity & Energy & \multicolumn{2}{l}{$\begin{array}{l}\text { Initial Isotopes and } \\
\text { Decays }\end{array}$} \\
\hline $\mathrm{ph} / \mathrm{s} / \mathrm{gm}$ & $\mathrm{keV}$ & $\mathrm{U}-234$ & $->$ & $\mathrm{Th}-230$ \\
$2.74 \mathrm{E}+05$ & 53.23 & $\mathrm{U}-234$ & $->$ & $\mathrm{Th}-230$ \\
$9.44 \mathrm{E}+04$ & 120.912 & $\mathrm{U}-234$ & $->$ & $\mathrm{Th}-230$ \\
$5.99 \mathrm{E}+01$ & 454.97 & $\mathrm{Th}-230$ & $->$ & $\mathrm{Ra}-226$ \\
$3.98 \mathrm{E}+01$ & 67.6758 & $\mathrm{U}-234$ & $->$ & $\mathrm{Th}-230$ \\
$3.39 \mathrm{E}+01$ & 508.2 & $\mathrm{U}-234$ & $->$ & $\mathrm{Th}-230$ \\
$2.76 \mathrm{E}+01$ & 581.78 & $\mathrm{Bi}-214$ & $->$ & $\mathrm{Po}-214$ \\
$5.24 \mathrm{E}+00$ & 609.311 & $\mathrm{Th}-230$ & $->$ & $\mathrm{Ra}-226$ \\
$5.14 \mathrm{E}+00$ & 143.876 & $\mathrm{~Pb}-214$ & $->$ & $\mathrm{Bi}-214$ \\
$4.22 \mathrm{E}+00$ & 351.87 & $\mathrm{U}-234$ & $->$ & $\mathrm{Th}-230$ \\
$2.30 \mathrm{E}+00$ & 677.67 & $\mathrm{U}-234$ & $->$ & $\mathrm{Th}-230$ \\
$2.30 \mathrm{E}+00$ & 503.53 & $\mathrm{~Pb}-214$ & $->$ & $\mathrm{Bi}-214$ \\
$2.18 \mathrm{E}+00$ & 295.091 & $\mathrm{U}-234$ & $->$ & $\mathrm{Th}-230$ \\
$1.84 \mathrm{E}+00$ & 624.44 & $\mathrm{Bi}-214$ & $->$ & $\mathrm{Po}-214$ \\
$1.81 \mathrm{E}+00$ & 1764.49 & $\mathrm{Bi}-214$ & $->$ & $\mathrm{Po}-214$ \\
$1.71 \mathrm{E}+00$ & 1120.273 & $\mathrm{~Pb}-214$ & $->$ & $\mathrm{Bi}-214$ \\
$1.25 \mathrm{E}+00$ & 77.107 & &
\end{tabular}




\begin{tabular}{|c|c|c|c|c|c|c|c|}
\hline $1.17 \mathrm{E}+00$ & 253.732 & Th-230 & $->$ & Ra-226 & & & \\
\hline $9.31 \mathrm{E}-01$ & 186.057 & Th-230 & $->$ & Ra-226 & & & \\
\hline $8.48 \mathrm{E}-01$ & 241.92 & $\mathrm{~Pb}-214$ & $->$ & $\mathrm{Bi}-214$ & & & \\
\hline 7.42E-01 & 74.814 & $\mathrm{~Pb}-214$ & $->$ & $\mathrm{Bi}-214$ & & & \\
\hline 7.12E-01 & 10.828 & $\mathrm{~Pb}-210$ & $->$ & $\mathrm{Bi}-210$, & $\mathrm{Pb}-214$ & $->$ & $\mathrm{Bi}-214$ \\
\hline $6.73 E-01$ & 1238.107 & $\mathrm{Bi}-214$ & $->$ & Po-214 & & & \\
\hline $6.48 \mathrm{E}-01$ & 13.088 & $\mathrm{~Pb}-214$ & $->$ & $\mathrm{Bi}-214$ & & & \\
\hline $5.68 \mathrm{E}-01$ & 2204.09 & $\mathrm{Bi}-214$ & $->$ & Po-214 & & & \\
\hline $5.55 \mathrm{E}-01$ & 768.35 & $\mathrm{Bi}-214$ & $->$ & Po-214 & & & \\
\hline 4.57E-01 & 1377.659 & $\mathrm{Bi}-214$ & $->$ & Po-214 & & & \\
\hline $4.41 \mathrm{E}-01$ & 87.19 & $\mathrm{~Pb}-214$ & $->$ & $\mathrm{Bi}-214$ & & & \\
\hline 3.75E-01 & 186.11 & Ra-226 & $->$ & $\mathrm{Rn}-222$ & & & \\
\hline 3.59E-01 & 934.039 & $\mathrm{Bi}-214$ & $->$ & Po-214 & & & \\
\hline 3.47E-01 & 1729.58 & $\mathrm{Bi}-214$ & $->$ & Po-214 & & & \\
\hline $2.82 \mathrm{E}-01$ & 1407.97 & $\mathrm{Bi}-214$ & $->$ & Po-214 & & & \\
\hline $2.49 \mathrm{E}-01$ & 1509.22 & $\mathrm{Bi}-214$ & $->$ & Po-214 & & & \\
\hline $2.41 \mathrm{E}-01$ & 1847.41 & $\mathrm{Bi}-214$ & $->$ & Po-214 & & & \\
\hline $1.92 \mathrm{E}-01$ & 1155.183 & $\mathrm{Bi}-214$ & $->$ & Po-214 & & & \\
\hline $1.77 \mathrm{E}-01$ & 665.442 & $\mathrm{Bi}-214$ & $->$ & Po-214 & & & \\
\hline $1.76 \mathrm{E}-01$ & 2447.68 & $\mathrm{Bi}-214$ & $->$ & Po-214 & & & \\
\hline $1.67 \mathrm{E}-01$ & 1280.952 & $\mathrm{Bi}-214$ & $->$ & Po-214 & & & \\
\hline $1.58 \mathrm{E}-01$ & 1401.48 & $\mathrm{Bi}-214$ & $->$ & Po-214 & & & \\
\hline $1.40 \mathrm{E}-01$ & 806.155 & $\mathrm{Bi}-214$ & $->$ & Рo-214 & & & \\
\hline $1.38 \mathrm{E}-01$ & 2118.53 & $\mathrm{Bi}-214$ & $->$ & Po-214 & & & \\
\hline $1.32 \mathrm{E}-01$ & 15.439 & $\mathrm{~Pb}-214$ & $->$ & $\mathrm{Bi}-214$ & & & \\
\hline $1.31 \mathrm{E}-01$ & 90.128 & $\mathrm{~Pb}-214$ & $->$ & $\mathrm{Bi}-214$ & & & \\
\hline $1.31 \mathrm{E}-01$ & 1661.258 & $\mathrm{Bi}-214$ & $->$ & Po-214 & & & \\
\hline $1.25 \mathrm{E}-01$ & 53.172 & $\mathrm{~Pb}-214$ & $->$ & $\mathrm{Bi}-214$ & & & \\
\hline $1.24 \mathrm{E}-01$ & 785.827 & $\mathrm{~Pb}-214$ & $->$ & $\mathrm{Bi}-214$ & & & \\
\hline $1.10 \mathrm{E}-01$ & 79.29 & $\mathrm{Bi}-214$ & $->$ & Po-214 & & & \\
\hline 8.99E-02 & 253.84 & Th-230 & $->$ & Ra-226 & & & \\
\hline 8.87E-02 & 1385.295 & $\mathrm{Bi}-214$ & $->$ & Po-214 & & & \\
\hline 8.19E-02 & 1583.22 & $\mathrm{Bi}-214$ & $->$ & Po-214 & & & \\
\hline $6.68 \mathrm{E}-02$ & 838.999 & $\mathrm{~Pb}-214$ & $->$ & $\mathrm{Bi}-214$ & & & \\
\hline $6.60 \mathrm{E}-02$ & 76.838 & $\mathrm{Bi}-214$ & $->$ & Po-214 & & & \\
\hline $6.26 \mathrm{E}-02$ & 258.94 & $\mathrm{~Pb}-214$ & $->$ & $\mathrm{Bi}-214$ & & & \\
\hline $5.88 \mathrm{E}-02$ & 13.066 & $\mathrm{~Pb}-210$ & $->$ & $\mathrm{Bi}-210$ & & & \\
\hline $5.37 \mathrm{E}-02$ & 703.07 & $\mathrm{Bi}-214$ & $->$ & Po-214 & & & \\
\hline $5.23 \mathrm{E}-02$ & 1207.764 & $\mathrm{Bi}-214$ & $->$ & Po-214 & & & \\
\hline 4.99E-02 & 487.13 & $\mathrm{~Pb}-214$ & $->$ & $\mathrm{Bi}-214$ & & & \\
\hline $4.66 \mathrm{E}-02$ & 388.95 & $\mathrm{Bi}-214$ & $->$ & Po-214 & & & \\
\hline $4.66 \mathrm{E}-02$ & 1538.49 & $\mathrm{Bi}-214$ & $->$ & Po-214 & & & \\
\hline $4.58 \mathrm{E}-02$ & 719.856 & $\mathrm{Bi}-214$ & $->$ & Po-214 & & & \\
\hline $4.38 \mathrm{E}-02$ & 14.336 & $\mathrm{Ra}-226$ & $->$ & $\mathrm{Rn}-222$ & & & \\
\hline 4.36E-02 & 964.07 & $\mathrm{Bi}-214$ & $->$ & Рo-214 & & & \\
\hline $4.36 \mathrm{E}-02$ & 1838.37 & $\mathrm{Bi}-214$ & $->$ & Po-214 & & & \\
\hline 4.36E-02 & 11.119 & $\mathrm{Bi}-214$ & $->$ & Po-214 & & & \\
\hline $4.21 \mathrm{E}-02$ & 13.502 & $\mathrm{Bi}-214$ & $->$ & Po-214 & & & \\
\hline $4.14 \mathrm{E}-02$ & 580.06 & $\mathrm{~Pb}-214$ & $->$ & $\mathrm{Bi}-214$ & & & \\
\hline 4.09E-02 & 386.834 & $\mathrm{Bi}-214$ & $->$ & Po-214 & & & \\
\hline
\end{tabular}




\begin{tabular}{|c|c|c|c|c|}
\hline 3.98E-02 & 1543.347 & $\mathrm{Bi}-214$ & $->$ & Po-214 \\
\hline $3.92 \mathrm{E}-02$ & 89.639 & $\mathrm{Bi}-214$ & $->$ & Po-214 \\
\hline $3.84 \mathrm{E}-02$ & 480.32 & $\mathrm{~Pb}-214$ & $->$ & $\mathrm{Bi}-214$ \\
\hline $3.80 \mathrm{E}-02$ & 1599.3 & $\mathrm{Bi}-214$ & $->$ & Po-214 \\
\hline 3.69E-02 & 2293.29 & $\mathrm{Bi}-214$ & $->$ & Po-214 \\
\hline 3.64E-02 & 274.56 & $\mathrm{~Pb}-214$ & $->$ & $\mathrm{Bi}-214$ \\
\hline $3.62 \mathrm{E}-02$ & 454.832 & $\mathrm{Bi}-214$ & $->$ & Po-214 \\
\hline $3.58 \mathrm{E}-02$ & 1051.95 & $\mathrm{Bi}-214$ & $->$ & Po-214 \\
\hline $3.56 \mathrm{E}-02$ & 9.419 & $\mathrm{~Pb}-210$ & $->$ & $\mathrm{Bi}-210$, \\
\hline $3.53 E-02$ & 786.42 & $\mathrm{Bi}-214$ & $->$ & Po-214 \\
\hline $3.34 \mathrm{E}-02$ & 83.787 & $\mathrm{Ra}-226$ & $->$ & $\mathrm{Rn}-222$ \\
\hline $3.24 \mathrm{E}-02$ & 1070.02 & $\mathrm{Bi}-214$ & $->$ & Po-214 \\
\hline $3.03 \mathrm{E}-02$ & 11.713 & Ra-226 & $->$ & Rn-222 \\
\hline $3.01 \mathrm{E}-02$ & 1594.78 & $\mathrm{Bi}-214$ & $->$ & Po-214 \\
\hline $2.90 \mathrm{E}-02$ & 1133.65 & $\mathrm{Bi}-214$ & $->$ & Po-214 \\
\hline $2.68 \mathrm{E}-02$ & 1683.99 & $\mathrm{Bi}-214$ & $->$ & Po-214 \\
\hline $2.57 \mathrm{E}-02$ & 1873.112 & $\mathrm{Bi}-214$ & $->$ & Po-214 \\
\hline 2.29E-02 & 46.52 & $\mathrm{~Pb}-210$ & $->$ & $\mathrm{Bi}-210$ \\
\hline $2.16 \mathrm{E}-02$ & 533.5 & $\mathrm{~Pb}-214$ & $->$ & $\mathrm{Bi}-214$ \\
\hline $2.05 E-02$ & 273.7 & $\mathrm{Bi}-214$ & $->$ & Po-214 \\
\hline $2.01 \mathrm{E}-02$ & 81.067 & $\mathrm{Ra}-226$ & $->$ & $\mathrm{Rn}-222$ \\
\hline $2.01 \mathrm{E}-02$ & 1896.28 & $\mathrm{Bi}-214$ & $->$ & Po-214 \\
\hline $1.93 \mathrm{E}-02$ & 462.05 & $\mathrm{~Pb}-214$ & $->$ & $\mathrm{Bi}-214$ \\
\hline $1.90 \mathrm{E}-02$ & 405.73 & $\mathrm{Bi}-214$ & $->$ & Po-214 \\
\hline $1.71 \mathrm{E}-02$ & 821.166 & $\mathrm{Bi}-214$ & $->$ & Po-214 \\
\hline $1.51 \mathrm{E}-02$ & 469.76 & $\mathrm{Bi}-214$ & $->$ & Po-214 \\
\hline $1.51 \mathrm{E}-02$ & 752.843 & $\mathrm{Bi}-214$ & $->$ & Po-214 \\
\hline $1.41 \mathrm{E}-02$ & 15.537 & $\mathrm{~Pb}-210$ & $->$ & $\mathrm{Bi}-210$ \\
\hline $1.38 \mathrm{E}-02$ & 1303.76 & $\mathrm{Bi}-214$ & $->$ & Po-214 \\
\hline $1.34 \mathrm{E}-02$ & 474.51 & $\mathrm{Bi}-214$ & $->$ & Po-214 \\
\hline $1.25 \mathrm{E}-02$ & 426.5 & $\mathrm{Bi}-214$ & $->$ & Po-214 \\
\hline 1.19E-02 & 904.33 & $\mathrm{Bi}-214$ & $->$ & Po-214 \\
\hline $1.19 \mathrm{E}-02$ & 92.673 & $\mathrm{Bi}-214$ & $->$ & Po-214 \\
\hline $1.18 \mathrm{E}-02$ & 94.677 & $\mathrm{Ra}-226$ & $->$ & $\mathrm{Rn}-222$ \\
\hline $1.14 \mathrm{E}-02$ & 1103.7 & $\mathrm{Bi}-214$ & $->$ & Po-214 \\
\hline 1.09E-02 & 1032.22 & $\mathrm{Bi}-214$ & $->$ & Po-214 \\
\hline $1.08 \mathrm{E}-02$ & 333.6 & $\mathrm{Bi}-214$ & $->$ & Po-214 \\
\hline $1.05 \mathrm{E}-02$ & 826.44 & $\mathrm{Bi}-214$ & $->$ & Po-214 \\
\hline $1.01 \mathrm{E}-02$ & 1890.259 & $\mathrm{Bi}-214$ & $->$ & Po-214 \\
\hline 9.89E-03 & 2109.91 & $\mathrm{Bi}-214$ & $->$ & Po-214 \\
\hline $9.78 \mathrm{E}-03$ & 1317.02 & $\mathrm{Bi}-214$ & $->$ & Po-214 \\
\hline $9.67 \mathrm{E}-03$ & 542.84 & $\mathrm{Bi}-214$ & $->$ & Po-214 \\
\hline $9.61 \mathrm{E}-03$ & 16.874 & $\mathrm{Ra}-226$ & $->$ & $\mathrm{Rn}-222$ \\
\hline 9.33E-03 & 572.67 & $\mathrm{Bi}-214$ & $->$ & Po-214 \\
\hline $9.21 \mathrm{E}-03$ & 280.93 & $\mathrm{Bi}-214$ & $->$ & Po-214 \\
\hline $9.10 \mathrm{E}-03$ & 1104.766 & $\mathrm{Bi}-214$ & $->$ & Po-214 \\
\hline 8.99E-03 & 314.2 & $\mathrm{~Pb}-214$ & $->$ & $\mathrm{Bi}-214$ \\
\hline 8.99E-03 & 765.9 & $\mathrm{~Pb}-214$ & $->$ & $\mathrm{Bi}-214$ \\
\hline 8.99E-03 & 683.21 & $\mathrm{Bi}-214$ & $->$ & Po-214 \\
\hline 8.50E-03 & 710.84 & $\mathrm{Bi}-214$ & $->$ & Рo-214 \\
\hline
\end{tabular}




\begin{tabular}{|c|c|c|c|c|c|c|c|}
\hline 8.29E-03 & 11.712 & $\mathrm{~Pb}-210$ & $->$ & $\mathrm{Bi}-210$, & $\mathrm{Pb}-214$ & $->$ & $\mathrm{Bi}-214$ \\
\hline 8.08E-03 & 536.93 & $\mathrm{Bi}-214$ & $->$ & Po-214 & & & \\
\hline 8.08E-03 & 15.874 & $\mathrm{Bi}-214$ & $->$ & Po-214 & & & \\
\hline $7.96 \mathrm{E}-03$ & 510 & $\mathrm{Rn}-222$ & $->$ & Po-218 & & & \\
\hline 7.96E-03 & 615.77 & $\mathrm{Bi}-214$ & $->$ & Po-214 & & & \\
\hline 7.96E-03 & 1637.37 & $\mathrm{Bi}-214$ & $->$ & Po-214 & & & \\
\hline 7.96E-03 & 2052.93 & $\mathrm{Bi}-214$ & $->$ & Po-214 & & & \\
\hline 7.85E-03 & 1479.19 & $\mathrm{Bi}-214$ & $->$ & Po-214 & & & \\
\hline 7.17E-03 & 1898.9 & $\mathrm{Bi}-214$ & $->$ & Po-214 & & & \\
\hline 6.94E-03 & 2192.52 & $\mathrm{Bi}-214$ & $->$ & Po-214 & & & \\
\hline $6.82 \mathrm{E}-03$ & 347.1 & $\mathrm{Bi}-214$ & $->$ & Po-214 & & & \\
\hline $6.82 \mathrm{E}-03$ & 633.14 & $\mathrm{Bi}-214$ & $->$ & Po-214 & & & \\
\hline $6.71 \mathrm{E}-03$ & 137.4 & $\mathrm{~Pb}-214$ & $->$ & $\mathrm{Bi}-214$ & & & \\
\hline $6.71 \mathrm{E}-03$ & 649.18 & $\mathrm{Bi}-214$ & $->$ & Po-214 & & & \\
\hline $6.60 \mathrm{E}-03$ & 1172.93 & $\mathrm{Bi}-214$ & $->$ & Po-214 & & & \\
\hline 6.60E-03 & 1173.04 & $\mathrm{Bi}-214$ & $->$ & Po-214 & & & \\
\hline $6.48 \mathrm{E}-03$ & 334.9 & $\mathrm{Bi}-214$ & $->$ & Po-214 & & & \\
\hline $6.37 \mathrm{E}-03$ & 2089.55 & $\mathrm{Bi}-214$ & $->$ & Po-214 & & & \\
\hline $6.24 \mathrm{E}-03$ & 109.97 & Th-230 & $->$ & Ra-226 & & & \\
\hline 5.80E-03 & 1935.8 & $\mathrm{Bi}-214$ & $->$ & Po-214 & & & \\
\hline 5.57E-03 & 196.3 & $\mathrm{~Pb}-214$ & $->$ & $\mathrm{Bi}-214$ & & & \\
\hline 5.57E-03 & 2010.79 & $\mathrm{Bi}-214$ & $->$ & Po-214 & & & \\
\hline 5.35E-03 & 733.64 & $\mathrm{Bi}-214$ & $->$ & Po-214 & & & \\
\hline $5.12 \mathrm{E}-03$ & 723.32 & $\mathrm{Bi}-214$ & $->$ & Рo-214 & & & \\
\hline $5.12 \mathrm{E}-03$ & 1130.6 & $\mathrm{Bi}-214$ & $->$ & Po-214 & & & \\
\hline 4.89E-03 & 660.75 & $\mathrm{Bi}-214$ & $->$ & Po-214 & & & \\
\hline $4.66 \mathrm{E}-03$ & 799.75 & $\mathrm{Bi}-214$ & $->$ & Po-214 & & & \\
\hline $4.55 E-03$ & 814.87 & $\mathrm{Bi}-214$ & $->$ & Po-214 & & & \\
\hline 4.44E-03 & 141.3 & $\mathrm{~Pb}-214$ & $->$ & $\mathrm{Bi}-214$ & & & \\
\hline $4.44 \mathrm{E}-03$ & 338.5 & $\mathrm{Bi}-214$ & $->$ & Po-214 & & & \\
\hline 4.44E-03 & 740.87 & $\mathrm{Bi}-214$ & $->$ & Po-214 & & & \\
\hline $4.21 \mathrm{E}-03$ & 697.89 & $\mathrm{Bi}-214$ & $->$ & Po-214 & & & \\
\hline 3.87E-03 & 304.42 & $\mathrm{Bi}-214$ & $->$ & Po-214 & & & \\
\hline 3.87E-03 & 617.1 & $\mathrm{Bi}-214$ & $->$ & Po-214 & & & \\
\hline 3.77E-03 & 97.907 & $\mathrm{Ra}-226$ & $->$ & $\mathrm{Rn}-222$ & & & \\
\hline 3.69E-03 & 2694.67 & $\mathrm{Bi}-214$ & $->$ & Po-214 & & & \\
\hline 3.64E-03 & 286.9 & $\mathrm{Bi}-214$ & $->$ & Po-214 & & & \\
\hline 3.64E-03 & 547.1 & $\mathrm{Bi}-214$ & $->$ & Po-214 & & & \\
\hline $3.55 E-03$ & 797.88 & TI-210 & $->$ & $\mathrm{Pb}-210$, & Po-214 & $->$ & $\mathrm{Pb}-210$ \\
\hline 3.53E-03 & 639.36 & $\mathrm{Bi}-214$ & $->$ & Рo-214 & & & \\
\hline $3.41 \mathrm{E}-03$ & 396 & $\mathrm{Bi}-214$ & $->$ & Po-214 & & & \\
\hline $3.30 \mathrm{E}-03$ & 511 & $\mathrm{~Pb}-214$ & $->$ & $\mathrm{Bi}-214$ & & & \\
\hline 3.30E-03 & 440.4 & $\mathrm{Bi}-214$ & $->$ & Po-214 & & & \\
\hline $3.30 \mathrm{E}-03$ & 1045.4 & $\mathrm{Bi}-214$ & $->$ & Рo-214 & & & \\
\hline $3.30 \mathrm{E}-03$ & 1067.3 & $\mathrm{Bi}-214$ & $->$ & Po-214 & & & \\
\hline 3.07E-03 & 1226.8 & $\mathrm{Bi}-214$ & $->$ & Po-214 & & & \\
\hline $2.91 \mathrm{E}-03$ & 2769.99 & $\mathrm{Bi}-214$ & $->$ & Po-214 & & & \\
\hline 2.62E-03 & 305.5 & $\mathrm{~Pb}-214$ & $->$ & $\mathrm{Bi}-214$ & & & \\
\hline $2.62 \mathrm{E}-03$ & 543.91 & $\mathrm{~Pb}-214$ & $->$ & $\mathrm{Bi}-214$ & & & \\
\hline $2.62 \mathrm{E}-03$ & 1341.5 & $\mathrm{Bi}-214$ & $->$ & Po-214 & & & \\
\hline
\end{tabular}




\begin{tabular}{|c|c|c|c|c|c|c|c|}
\hline 2.62E-03 & 976.2 & $\mathrm{Bi}-214$ & $\rightarrow$ & Po-214 & & & \\
\hline $2.62 \mathrm{E}-03$ & 915.8 & $\mathrm{Bi}-214$ & $->$ & Po-214 & & & \\
\hline $2.62 \mathrm{E}-03$ & 832.34 & $\mathrm{Bi}-214$ & $->$ & Po- 214 & & & \\
\hline 2.57E-03 & 3053.89 & $\mathrm{Bi}-214$ & $->$ & Po-214 & & & \\
\hline $2.50 \mathrm{E}-03$ & 1230.84 & $\mathrm{Bi}-214$ & $->$ & Po-214 & & & \\
\hline $2.50 \mathrm{E}-03$ & 2331.2 & $\mathrm{Bi}-214$ & $->$ & Рo-214 & & & \\
\hline $2.27 \mathrm{E}-03$ & 324.3 & $\mathrm{~Pb}-214$ & $->$ & $\mathrm{Bi}-214$ & & & \\
\hline 2.27E-03 & 9.658 & $\mathrm{Bi}-214$ & $->$ & Рo-214 & & & \\
\hline $2.16 \mathrm{E}-03$ & 1636.6 & $\mathrm{Bi}-214$ & $->$ & Po-214 & & & \\
\hline $2.16 \mathrm{E}-03$ & 2021.7 & $\mathrm{Bi}-214$ & $->$ & Po-214 & & & \\
\hline $2.16 \mathrm{E}-03$ & 1392.5 & $\mathrm{Bi}-214$ & $->$ & Po-214 & & & \\
\hline $2.05 E-03$ & 2266.67 & $\mathrm{Bi}-214$ & $->$ & Рo-214 & & & \\
\hline $2.05 E-03$ & 502.2 & $\mathrm{Bi}-214$ & $->$ & Po-214 & & & \\
\hline 1.93E-03 & 943.3 & $\mathrm{Bi}-214$ & $->$ & Po-214 & & & \\
\hline 1.93E-03 & 847.2 & $\mathrm{Bi}-214$ & $->$ & Po-214 & & & \\
\hline 1.93E-03 & 631.2 & $\mathrm{Bi}-214$ & $->$ & Po-214 & & & \\
\hline 1.93E-03 & 1038 & $\mathrm{Bi}-214$ & $->$ & Po-214 & & & \\
\hline $1.89 \mathrm{E}-03$ & 298.1 & TI-210 & $->$ & $\mathrm{Pb}-210$ & Po-214 & $->$ & $\mathrm{Pb}-210$ \\
\hline $1.82 \mathrm{E}-03$ & 1782.1 & $\mathrm{Bi}-214$ & $->$ & Po-214 & & & \\
\hline $1.82 \mathrm{E}-03$ & 525 & $\mathrm{Bi}-214$ & $->$ & Po-214 & & & \\
\hline $1.82 \mathrm{E}-03$ & 2147.8 & $\mathrm{Bi}-214$ & $->$ & Po-214 & & & \\
\hline 1.79E-03 & 727.8 & $\mathrm{Bi}-214$ & $->$ & Po-214 & & & \\
\hline 1.79E-03 & 2922.09 & $\mathrm{Bi}-214$ & $->$ & Po-214 & & & \\
\hline 1.67E-03 & 2978.79 & $\mathrm{Bi}-214$ & $->$ & Po-214 & & & \\
\hline 1.64E-03 & 10.137 & Ra-226 & $->$ & $\mathrm{Rn}-222$ & & & \\
\hline $1.36 \mathrm{E}-03$ & 1020.5 & $\mathrm{Bi}-214$ & $->$ & Po-214 & & & \\
\hline $1.36 \mathrm{E}-03$ & 1814.01 & $\mathrm{Bi}-214$ & $->$ & Po-214 & & & \\
\hline $1.36 \mathrm{E}-03$ & 989.2 & $\mathrm{Bi}-214$ & $->$ & Po-214 & & & \\
\hline $1.36 \mathrm{E}-03$ & 1471.1 & $\mathrm{Bi}-214$ & $->$ & Po-214 & & & \\
\hline 1.36E-03 & 596 & $\mathrm{Bi}-214$ & $->$ & Po-214 & & & \\
\hline $1.34 \mathrm{E}-03$ & 2376.99 & $\mathrm{Bi}-214$ & $->$ & Po-214 & & & \\
\hline $1.25 \mathrm{E}-03$ & 1330 & $\mathrm{Bi}-214$ & $->$ & Po-214 & & & \\
\hline $1.14 \mathrm{E}-03$ & 470.8 & $\mathrm{~Pb}-214$ & $->$ & $\mathrm{Bi}-214$ & & & \\
\hline 1.14E-03 & 2085 & $\mathrm{Bi}-214$ & $->$ & Po-214 & & & \\
\hline $1.14 \mathrm{E}-03$ & 1013.4 & $\mathrm{Bi}-214$ & $->$ & Po-214 & & & \\
\hline $1.05 \mathrm{E}-03$ & 2880.4 & $\mathrm{Bi}-214$ & $->$ & Po-214 & & & \\
\hline $1.02 \mathrm{E}-03$ & 2259.7 & $\mathrm{Bi}-214$ & $->$ & Po-214 & & & \\
\hline $1.02 \mathrm{E}-03$ & 394 & $\mathrm{Bi}-214$ & $->$ & Po-214 & & & \\
\hline 1.02E-03 & 494.6 & $\mathrm{Bi}-214$ & $->$ & Po-214 & & & \\
\hline $1.00 \mathrm{E}-03$ & 2999.99 & $\mathrm{Bi}-214$ & $->$ & Po- 214 & & & \\
\hline 8.57E-04 & 235.01 & Th-230 & $->$ & Ra-226 & & & \\
\hline 8.36E-04 & 12.855 & $\mathrm{Ra}-226$ & $->$ & $\mathrm{Rn}-222$ & & & \\
\hline 7.96E-04 & 2251.2 & $\mathrm{Bi}-214$ & $->$ & Po-214 & & & \\
\hline $7.28 \mathrm{E}-04$ & 2893.59 & $\mathrm{Bi}-214$ & $->$ & Po-214 & & & \\
\hline 7.17E-04 & 364.2 & $\mathrm{Bi}-214$ & $->$ & Рo-214 & & & \\
\hline $6.82 \mathrm{E}-04$ & 693.3 & $\mathrm{Bi}-214$ & $->$ & Po-214 & & & \\
\hline $6.82 \mathrm{E}-04$ & 687.7 & $\mathrm{Bi}-214$ & $->$ & Po-214 & & & \\
\hline $6.71 \mathrm{E}-04$ & 2423.32 & $\mathrm{Bi}-214$ & $->$ & Po-214 & & & \\
\hline $6.71 \mathrm{E}-04$ & 2505.58 & $\mathrm{Bi}-214$ & $->$ & Po-214 & & & \\
\hline $6.71 \mathrm{E}-04$ & 2786.09 & $\mathrm{Bi}-214$ & $->$ & Po-214 & & & \\
\hline
\end{tabular}




\begin{tabular}{|c|c|c|c|c|c|c|c|}
\hline $6.48 \mathrm{E}-04$ & 520.4 & $\mathrm{Bi}-214$ & $->$ & Po-214 & & & \\
\hline $6.26 \mathrm{E}-04$ & 12.085 & $\mathrm{Bi}-214$ & $->$ & Po-214 & & & \\
\hline $6.18 \mathrm{E}-04$ & 262.41 & $\mathrm{Ra}-226$ & $->$ & $\mathrm{Rn}-222$ & & & \\
\hline 5.80E-04 & 1419.7 & $\mathrm{Bi}-214$ & $->$ & Po-214 & & & \\
\hline $5.80 \mathrm{E}-04$ & 2284.4 & $\mathrm{Bi}-214$ & $->$ & Po-214 & & & \\
\hline 5.69E-04 & 1994.7 & $\mathrm{Bi}-214$ & $->$ & Рo-214 & & & \\
\hline $5.57 \mathrm{E}-04$ & 538.7 & $\mathrm{~Pb}-214$ & $->$ & $\mathrm{Bi}-214$ & & & \\
\hline 5.57E-04 & 376.6 & $\mathrm{Bi}-214$ & $->$ & Рo-214 & & & \\
\hline 5.57E-04 & 626.4 & $\mathrm{Bi}-214$ & $->$ & Po-214 & & & \\
\hline $5.29 \mathrm{E}-04$ & 205.1 & Th-230 & $->$ & Ra-226 & & & \\
\hline $5.12 \mathrm{E}-04$ & 1353 & $\mathrm{Bi}-214$ & $->$ & Po-214 & & & \\
\hline 5.02E-04 & 1314 & TI-210 & $->$ & $\mathrm{Pb}-210$ & & & \\
\hline 4.89E-04 & 3081.7 & $\mathrm{Bi}-214$ & $->$ & Po-214 & & & \\
\hline $4.06 \mathrm{E}-04$ & 1208 & TI-210 & $->$ & $\mathrm{Pb}-210$ & & & \\
\hline $3.30 \mathrm{E}-04$ & 2369.3 & $\mathrm{Bi}-214$ & $->$ & Po-214 & & & \\
\hline $3.30 \mathrm{E}-04$ & 2270 & $\mathrm{Bi}-214$ & $->$ & Po- 214 & & & \\
\hline 3.30E-04 & 2004.5 & $\mathrm{Bi}-214$ & $->$ & Po-214 & & & \\
\hline $3.18 \mathrm{E}-04$ & 2698.86 & $\mathrm{Bi}-214$ & $->$ & Po-214 & & & \\
\hline 3.17E-04 & 570.5 & Th-230 & $->$ & Ra-226 & & & \\
\hline 2.87E-04 & 1068 & TI-210 & $->$ & $\mathrm{Pb}-210$ & & & \\
\hline $2.84 \mathrm{E}-04$ & 2827 & $\mathrm{Bi}-214$ & $->$ & Po-214 & & & \\
\hline $2.63 \mathrm{E}-04$ & 12.678 & TI-210 & $->$ & $\mathrm{Pb}-210$ & & & \\
\hline $2.39 \mathrm{E}-04$ & 2482.417 & $\mathrm{Bi}-214$ & $->$ & Po-214 & & & \\
\hline 2.27E-04 & 2390.9 & $\mathrm{Bi}-214$ & $->$ & Po-214 & & & \\
\hline $2.16 \mathrm{E}-04$ & 2360.9 & $\mathrm{Bi}-214$ & $->$ & Po-214 & & & \\
\hline $2.16 \mathrm{E}-04$ & 2324.8 & $\mathrm{Bi}-214$ & $->$ & Po-214 & & & \\
\hline $2.15 \mathrm{E}-04$ & 2428 & TI-210 & $->$ & $\mathrm{Pb}-210$ & & & \\
\hline $2.05 E-04$ & 2719.21 & $\mathrm{Bi}-214$ & $->$ & Po-214 & & & \\
\hline $1.98 \mathrm{E}-04$ & 10.541 & TI-206 & $->$ & $\mathrm{Pb}-206$ & TI-210 & $->$ & $\mathrm{Pb}-210$ \\
\hline 1.93E-04 & 2940.5 & $\mathrm{Bi}-214$ & $->$ & Po-214 & & & \\
\hline $1.91 \mathrm{E}-04$ & 2358 & TI-210 & $->$ & $\mathrm{Pb}-210$ & & & \\
\hline $1.82 \mathrm{E}-04$ & 3142.6 & $\mathrm{Bi}-214$ & $->$ & Po-214 & & & \\
\hline $1.71 \mathrm{E}-04$ & 3183.6 & $\mathrm{Bi}-214$ & $->$ & Po-214 & & & \\
\hline $1.65 \mathrm{E}-04$ & 1110 & TI-210 & $->$ & $\mathrm{Pb}-210$ & & & \\
\hline $1.65 \mathrm{E}-04$ & 2008 & TI-210 & $->$ & $\mathrm{Pb}-210$ & & & \\
\hline $1.65 \mathrm{E}-04$ & 860 & TI-210 & $->$ & $\mathrm{Pb}-210$ & & & \\
\hline $1.36 \mathrm{E}-04$ & 2928.7 & $\mathrm{Bi}-214$ & $->$ & Po-214 & & & \\
\hline $1.25 \mathrm{E}-04$ & 2988.7 & $\mathrm{Bi}-214$ & $->$ & Po-214 & & & \\
\hline 1.17E-04 & 1408 & TI-210 & $->$ & $\mathrm{Pb}-210$ & & & \\
\hline $1.17 \mathrm{E}-04$ & 2088 & TI-210 & $->$ & $\mathrm{Pb}-210$ & & & \\
\hline $1.10 \mathrm{E}-04$ & 74.969 & TI-206 & $->$ & $\mathrm{Pb}-206$, & TI-210 & $->$ & $\mathrm{Pb}-210$ \\
\hline 1.07E-04 & 620 & Th-230 & $->$ & Ra-226 & & & \\
\hline 1.07E-04 & 551.8 & Th-230 & $->$ & $\mathrm{Ra}-226$ & & & \\
\hline $1.02 \mathrm{E}-04$ & 2631 & $\mathrm{Bi}-214$ & $->$ & Po-214 & & & \\
\hline $9.56 \mathrm{E}-05$ & 95 & TI-210 & $->$ & $\mathrm{Pb}-210$ & & & \\
\hline $9.56 \mathrm{E}-05$ & 354 & TI-210 & $->$ & $\mathrm{Pb}-210$ & & & \\
\hline 7.17E-05 & 2268 & TI-210 & $->$ & $\mathrm{Pb}-210$ & & & \\
\hline 7.17E-05 & 908 & TI-210 & $->$ & $\mathrm{Pb}-210$ & & & \\
\hline 7.17E-05 & 380 & TI-210 & $->$ & $\mathrm{Pb}-210$ & & & \\
\hline $6.98 \mathrm{E}-05$ & 600.83 & Ra-226 & $->$ & $\mathrm{Rn}-222$ & & & \\
\hline
\end{tabular}




\begin{tabular}{|c|c|c|c|c|c|c|c|}
\hline 6.69E-05 & 72.803 & TI-206 & $->$ & $\mathrm{Pb}-206$ & TI-210 & $->$ & $\mathrm{Pb}-210$ \\
\hline $6.48 \mathrm{E}-05$ & 2934.9 & $\mathrm{Bi}-214$ & $->$ & Po-214 & & & \\
\hline 5.80E-05 & 3093.9 & $\mathrm{Bi}-214$ & $->$ & Po-214 & & & \\
\hline $5.80 \mathrm{E}-05$ & 3160.5 & $\mathrm{Bi}-214$ & $->$ & Po-214 & & & \\
\hline $5.26 \mathrm{E}-05$ & 14.836 & Tl-206 & $->$ & $\mathrm{Pb}-206$ & TI-210 & $->$ & $\mathrm{Pb}-210$ \\
\hline $5.12 \mathrm{E}-05$ & 2604.5 & $\mathrm{Bi}-214$ & $->$ & Po-214 & & & \\
\hline 4.78E-05 & 1538 & TI-210 & $->$ & $\mathrm{Pb}-210$ & & & \\
\hline 4.78E-05 & 1588 & $\mathrm{Tl}-210$ & $->$ & $\mathrm{Pb}-210$ & & & \\
\hline $4.78 \mathrm{E}-05$ & 668 & TI-210 & $->$ & $\mathrm{Pb}-210$ & & & \\
\hline 4.78E-05 & 1648 & TI-210 & $->$ & $\mathrm{Pb}-210$ & & & \\
\hline $4.78 \mathrm{E}-05$ & 478 & TI-210 & $->$ & $\mathrm{Pb}-210$ & & & \\
\hline $4.78 \mathrm{E}-05$ & 81 & $\mathrm{TI}-210$ & $->$ & $\mathrm{Pb}-210$ & & & \\
\hline $4.46 \mathrm{E}-05$ & 414.72 & $\mathrm{Ra}-226$ & $->$ & $\mathrm{Rn}-222$ & & & \\
\hline 4.44E-05 & 2551 & $\mathrm{Bi}-214$ & $->$ & Po-214 & & & \\
\hline $3.92 \mathrm{E}-05$ & 84.789 & TI-206 & $->$ & $\mathrm{Pb}-206$ & TI-210 & $->$ & $\mathrm{Pb}-210$ \\
\hline 3.87E-05 & 2860.9 & $\mathrm{Bi}-214$ & $->$ & Po-214 & & & \\
\hline 3.87E-05 & 3136.3 & $\mathrm{Bi}-214$ & $->$ & Po-214 & & & \\
\hline 3.30E-05 & 2662.23 & $\mathrm{Bi}-214$ & $->$ & Po-214 & & & \\
\hline 3.09E-05 & 449.5 & $\mathrm{Ra}-226$ & $->$ & $\mathrm{Rn}-222$ & & & \\
\hline 2.27E-05 & 3233.3 & $\mathrm{Bi}-214$ & $->$ & Po-214 & & & \\
\hline $1.14 \mathrm{E}-05$ & 3269.7 & $\mathrm{Bi}-214$ & $->$ & Po-214 & & & \\
\hline $1.12 \mathrm{E}-05$ & 87.632 & TI-206 & $->$ & $\mathrm{Pb}-206$, & TI-210 & $->$ & $\mathrm{Pb}-210$ \\
\hline $9.79 E-06$ & 9.185 & Tl-206 & $->$ & $\mathrm{Pb}-206$ & TI-210 & $->$ & $\mathrm{Pb}-210$ \\
\hline $4.78 \mathrm{E}-06$ & 11.349 & $\mathrm{Tl}-210$ & $->$ & $\mathrm{Pb}-210$ & & & \\
\hline $4.36 \mathrm{E}-06$ & 803.13 & TI-206 & $->$ & Pb-206, & Po-210 & $->$ & $\mathrm{Pb}-206$ \\
\hline $3.36 \mathrm{E}-07$ & 302.25 & $\mathrm{Hg}-206$ & $->$ & TI-206 & & & \\
\hline $5.22 \mathrm{E}-08$ & 72.873 & $\mathrm{Hg}-206$ & $->$ & TI-206 & & & \\
\hline $3.11 \mathrm{E}-08$ & 70.832 & $\mathrm{Hg}-206$ & $->$ & TI-206 & & & \\
\hline $3.11 \mathrm{E}-08$ & 650.21 & $\mathrm{Hg}-206$ & $->$ & TI-206 & & & \\
\hline $1.87 \mathrm{E}-08$ & 82.434 & $\mathrm{Hg}-206$ & $->$ & TI-206 & & & \\
\hline $1.87 \mathrm{E}-08$ & 10.259 & $\mathrm{Hg}-206$ & $->$ & Tl-206 & & & \\
\hline $1.62 \mathrm{E}-08$ & 12.313 & $\mathrm{Hg}-206$ & $->$ & TI-206 & & & \\
\hline 6.47E-09 & 344.96 & $\mathrm{Hg}-206$ & $->$ & TI-206 & & & \\
\hline 5.22E-09 & 85.185 & $\mathrm{Hg}-206$ & $->$ & TI-206 & & & \\
\hline 2.98E-09 & 14.407 & $\mathrm{Hg}-206$ & $->$ & TI-206 & & & \\
\hline 8.95E-10 & 8.953 & $\mathrm{Hg}-206$ & $->$ & TI-206 & & & \\
\hline $2.36 \mathrm{E}-10$ & 10.994 & $\mathrm{Hg}-206$ & $->$ & TI-206 & & & \\
\hline $1.74 \mathrm{E}-10$ & 12.71 & TI-206 & $->$ & $\mathrm{Pb}-206$ & & & \\
\hline $4.73 \mathrm{E}-11$ & 384.06 & $\mathrm{Hg}-206$ & $->$ & TI-206 & & & \\
\hline $2.77 \mathrm{E}-12$ & 11.439 & TI-206 & $->$ & $\mathrm{Pb}-206$ & & & \\
\hline $2.95 \mathrm{E}-13$ & 266.15 & $\mathrm{Bi}-210$ & $->$ & TI-206 & & & \\
\hline U-235 & $100 \%$ & & & ed 5 years & & & \\
\hline
\end{tabular}

604 lines computed. 
Sorted by Energy:

\begin{tabular}{|c|c|c|c|c|c|c|c|}
\hline \multirow{2}{*}{$\begin{array}{c}\text { Energy } \\
(\mathrm{keV})\end{array}$} & \multirow{2}{*}{$\begin{array}{l}\text { Intensity } \\
(\mathrm{ph} / \mathrm{s} / \mathrm{gm})\end{array}$} & \multicolumn{6}{|c|}{$\begin{array}{l}\text { Initial Isotopes and } \\
\text { Decays }\end{array}$} \\
\hline & & & & & & & \\
\hline 8.953 & $1.31 \mathrm{E}-04$ & $\mathrm{Bi}-211$ & $->$ & TI-207 & & & \\
\hline 9.185 & $2.73 E-07$ & TI-207 & $->$ & $\mathrm{Pb}-207$ & & & \\
\hline 9.3 & 8.17E-07 & Ac-227 & $->$ & Th-227 & & & \\
\hline 9.419 & $1.16 \mathrm{E}-04$ & $\mathrm{~Pb}-211$ & $->$ & $\mathrm{Bi}-211$ & & & \\
\hline 9.658 & $1.28 \mathrm{E}-04$ & $\mathrm{Rn}-219$ & $->$ & Po-215 & & & \\
\hline 10.137 & $3.35 E-03$ & Ra-223 & $->$ & $\mathrm{Rn}-219$ & & & \\
\hline 10.259 & $2.66 \mathrm{E}-03$ & $\mathrm{Bi}-211$ & $->$ & TI-207 & & & \\
\hline 10.541 & $5.46 \mathrm{E}-06$ & TI-207 & $->$ & $\mathrm{Pb}-207$ & & & \\
\hline 10.622 & $6.06 \mathrm{E}-03$ & Fr-223 & $->$ & Ra-223, & Th-227 & $->$ & Ra-223 \\
\hline 10.828 & $2.25 \mathrm{E}-03$ & $\mathrm{~Pb}-211$ & $->$ & $\mathrm{Bi}-211$ & & & \\
\hline 10.871 & $1.01 \mathrm{E}-01$ & $\mathrm{~Pa}-231$ & $->$ & $A c-227$ & & & \\
\hline 10.994 & $3.46 \mathrm{E}-05$ & $\mathrm{Bi}-211$ & $->$ & TI-207 & & & \\
\hline 11.118 & $8.00 E+02$ & $A c-227$ & $->$ & Th-227, & $U-235$ & $->$ & Th-231 \\
\hline 11.119 & 2.49E-03 & $\mathrm{Rn}-219$ & $->$ & Po-215 & & & \\
\hline 11.349 & $7.10 \mathrm{E}-08$ & TI-207 & $->$ & $\mathrm{Pb}-207$ & & & \\
\hline 11.372 & $2.40 \mathrm{E}+03$ & Th-231 & $->$ & Pa-231 & & & \\
\hline 11.712 & $3.65 \mathrm{E}-05$ & $\mathrm{~Pb}-211$ & $->$ & $\mathrm{Bi}-211$ & & & \\
\hline 11.713 & $6.20 \mathrm{E}-02$ & $\mathrm{Ra}-223$ & $->$ & $\mathrm{Rn}-219$ & & & \\
\hline 12.085 & 4.56E-05 & $\mathrm{Rn}-219$ & $->$ & Po-215 & & & \\
\hline 12.313 & $2.43 \mathrm{E}-03$ & $\mathrm{Bi}-211$ & $->$ & TI-207 & & & \\
\hline 12.325 & $1.05 \mathrm{E}-01$ & Fr-223 & $->$ & Ra-223, & Th-227 & $->$ & Ra-223 \\
\hline 12.636 & $1.86 \mathrm{E}+00$ & $\mathrm{~Pa}-231$ & $->$ & $A C-227$ & & & \\
\hline 12.703 & 4.97E-06 & TI-207 & $->$ & $\mathrm{Pb}-207$ & & & \\
\hline 12.855 & $8.82 \mathrm{E}-04$ & $\mathrm{Ra}-223$ & $->$ & $\mathrm{Rn}-219$ & & & \\
\hline 12.952 & $1.76 \mathrm{E}+04$ & Ac- 227 & $->$ & Th-227, & $U-235$ & $->$ & Th-231 \\
\hline 13.082 & $2.49 E-03$ & $\mathrm{~Pb}-211$ & $->$ & $\mathrm{Bi}-211$ & & & \\
\hline 13.495 & $2.74 \mathrm{E}-03$ & $\mathrm{Rn}-219$ & $->$ & Po-215 & & & \\
\hline 13.662 & $2.22 \mathrm{E}-03$ & Fr-223 & $->$ & Ra-223, & Th-227 & $->$ & Ra-223 \\
\hline 13.724 & $3.92 E+04$ & Th-231 & $->$ & $\mathrm{Pa}-231$ & & & \\
\hline 14.082 & $3.12 \mathrm{E}-02$ & Pa-231 & $->$ & $A c-227$ & & & \\
\hline 14.341 & $5.96 \mathrm{E}-02$ & Ra-223 & $->$ & $\mathrm{Rn}-219$ & & & \\
\hline 14.407 & $4.43 E-04$ & $\mathrm{Bi}-211$ & $->$ & TI-207 & & & \\
\hline 14.511 & $1.76 \mathrm{E}+02$ & $A c-227$ & $->$ & Th-227, & $U-235$ & $->$ & Th-231 \\
\hline 14.886 & $9.10 \mathrm{E}-07$ & TI-207 & $->$ & $\mathrm{Pb}-207$ & & & \\
\hline 14.953 & $3.20 \mathrm{E}+02$ & Th-231 & $->$ & $\mathrm{Pa}-231$ & & & \\
\hline 15.2 & $2.20 \mathrm{E}-04$ & $A c-227$ & $->$ & Th-227 & & & \\
\hline 15.213 & $1.67 \mathrm{E}-03$ & Fr-223 & $->$ & Ra-223 & & & \\
\hline 15.218 & $1.28 \mathrm{E}-01$ & Th-227 & $->$ & $\mathrm{Ra}-223$ & & & \\
\hline 15.397 & 5.47E-04 & $\mathrm{Pb}-211$ & $->$ & $\mathrm{Bi}-211$ & & & \\
\hline 15.69 & $2.11 \mathrm{E}+00$ & $\mathrm{~Pa}-231$ & $->$ & Ac- 227 & & & \\
\hline 15.856 & $5.53 E-04$ & $\mathrm{Rn}-219$ & $->$ & Po-215 & & & \\
\hline 16.074 & $9.42 \mathrm{E}-04$ & $A c-227$ & $->$ & Th-227 & & & \\
\hline 16.119 & $1.20 \mathrm{E}+04$ & U-235 & $->$ & Th-231 & & & \\
\hline 16.397 & $3.21 \mathrm{E}-02$ & Pa-231 & $->$ & $A c-227$ & & & \\
\hline 16.559 & $2.96 \mathrm{E}+04$ & Th-231 & $->$ & $\mathrm{Pa}-231$ & & & \\
\hline 16.918 & $1.18 \mathrm{E}-02$ & $\mathrm{Ra}-223$ & $->$ & $\mathrm{Rn}-219$ & & & \\
\hline
\end{tabular}




\begin{tabular}{|c|c|c|c|c|c|c|c|}
\hline 17.187 & $1.76 \mathrm{E}+02$ & Th-231 & $->$ & $\mathrm{Pa}-231$ & & & \\
\hline 17.997 & 2.93E-02 & Th-227 & $->$ & Ra-223 & & & \\
\hline 18.016 & $3.78 \mathrm{E}-04$ & Fr-223 & $->$ & Ra-223 & & & \\
\hline 18.607 & $4.98 \mathrm{E}-01$ & Pa-231 & $->$ & Ac- 227 & & & \\
\hline 18.998 & $2.79 \mathrm{E}-02$ & $\mathrm{~Pa}-231$ & $->$ & Ac- 227 & & & \\
\hline 19.118 & $2.08 E+03$ & U-235 & $->$ & Th-231 & & & \\
\hline 19.308 & $2.51 \mathrm{E}-04$ & Ac- 227 & $->$ & Th-227 & & & \\
\hline 19.811 & $5.92 E+03$ & Th-231 & $->$ & $\mathrm{Pa}-231$ & & & \\
\hline 20.27 & $1.29 \mathrm{E}-03$ & Fr-223 & $->$ & Ra-223, & Th-227 & $->$ & Ra-223 \\
\hline 23.55 & $1.44 \mathrm{E}-05$ & $\mathrm{~Pa}-231$ & $->$ & Ac- 227 & & & \\
\hline 24.5 & $2.20 \mathrm{E}-05$ & $A c-227$ & $->$ & Th-227 & & & \\
\hline 24.56 & $2.53 E-03$ & Pa-231 & $->$ & Ac-227 & & & \\
\hline 25.464 & 7.60E-03 & $\mathrm{Pa}-231$ & $->$ & Ac- 227 & & & \\
\hline 25.642 & $1.17 \mathrm{E}+04$ & Th-231 & $->$ & $\mathrm{Pa}-231$ & & & \\
\hline 27.396 & 7.85E-01 & $\mathrm{Pa}-231$ & $->$ & Ac- 227 & & & \\
\hline 29.58 & $3.88 \mathrm{E}-05$ & Fr-223 & $->$ & Ra-223, & Th-227 & $->$ & $\mathrm{Ra}-223$ \\
\hline 29.869 & $6.13 \mathrm{E}-04$ & $\mathrm{Fr}-223$ & $->$ & Ra-223, & Th-227 & $->$ & $\mathrm{Ra}-223$ \\
\hline 29.996 & 7.77E-03 & $\mathrm{Pa}-231$ & $->$ & $A c-227$ & & & \\
\hline 31.04 & 7.60E-04 & $\mathrm{Pa}-231$ & $->$ & Ac- 227 & & & \\
\hline 31.566 & $4.88 \mathrm{E}-04$ & Th-227 & $->$ & Ra-223 & & & \\
\hline 31.58 & 5.91E-04 & $\mathrm{Pa}-231$ & $->$ & Ac- 227 & & & \\
\hline 31.585 & $1.28 \mathrm{E}+01$ & U-235 & $->$ & Th-231 & & & \\
\hline 31.87 & $6.08 \mathrm{E}-07$ & Ra-223 & $->$ & $\mathrm{Rn}-219$ & & & \\
\hline 33.52 & $8.55 E-05$ & Th-227 & $->$ & Ra-223 & & & \\
\hline 35.884 & $1.35 \mathrm{E}-03$ & $\mathrm{~Pa}-231$ & $->$ & $A C-227$ & & & \\
\hline 38.235 & $1.26 \mathrm{E}-02$ & $\mathrm{~Pa}-231$ & $->$ & Ac- 227 & & & \\
\hline 39.61 & $1.18 \mathrm{E}-04$ & $\mathrm{~Pa}-231$ & $->$ & Ac- 227 & & & \\
\hline 40.006 & $1.01 \mathrm{E}-03$ & $\mathrm{~Pa}-231$ & $->$ & $A c-227$ & & & \\
\hline 40.16 & $1.22 \mathrm{E}-04$ & Th-227 & $->$ & Ra-223 & & & \\
\hline 41.13 & $2.40 E+01$ & U-235 & $->$ & Th-231 & & & \\
\hline 41.88 & 2.44E-04 & Th-227 & $->$ & Ra-223 & & & \\
\hline 41.96 & $4.80 E+01$ & U-235 & $->$ & Th-231 & & & \\
\hline 42.52 & $5.07 E-04$ & $\mathrm{~Pa}-231$ & $->$ & Ac- 227 & & & \\
\hline 42.828 & $4.64 \mathrm{E}+01$ & Th-231 & $->$ & $\mathrm{Pa}-231$ & & & \\
\hline 43.09 & 5.91E-04 & $\mathrm{Pa}-231$ & $->$ & Ac- 227 & & & \\
\hline 43.53 & $3.05 E-04$ & Th-227 & $->$ & Ra-223 & & & \\
\hline 43.73 & $1.40 \mathrm{E}-03$ & Fr-223 & $->$ & Ra-223, & Th-227 & $->$ & $\mathrm{Ra}-223$ \\
\hline 44.06 & $5.60 \mathrm{E}-01$ & Th-231 & $->$ & Pa-231 & & & \\
\hline 44.08 & 4.27E-05 & Th-227 & $->$ & Ra-223 & & & \\
\hline 44.213 & 5.07E-03 & $\mathrm{Pa}-231$ & $->$ & $A c-227$ & & & \\
\hline 44.37 & 7.94E-05 & Th-227 & $->$ & Ra-223 & & & \\
\hline 46.13 & $1.23 \mathrm{E}-07$ & Ac- 227 & $->$ & Fr-223 & & & \\
\hline 46.393 & $1.76 \mathrm{E}-02$ & $\mathrm{~Pa}-231$ & $->$ & $A c-227$ & & & \\
\hline 48.27 & $6.11 \mathrm{E}-05$ & $\mathrm{Fr}-223$ & $->$ & Ra-223, & Th-227 & $->$ & $\mathrm{Ra}-223$ \\
\hline 49.85 & $1.29 \mathrm{E}-03$ & Fr-223 & $->$ & Ra-223, & Th-227 & $->$ & $\mathrm{Ra}-223$ \\
\hline 50.14 & $5.48 \mathrm{E}-02$ & Fr-223 & $->$ & Ra-223, & Th-227 & $->$ & Ra-223 \\
\hline 50.82 & 9.77E-05 & Th-227 & $->$ & Ra-223 & & & \\
\hline 50.97 & $1.18 \mathrm{E}-04$ & Pa-231 & $->$ & $A c-227$ & & & \\
\hline 51.179 & $1.60 \mathrm{E}+01$ & U-235 & $->$ & Th-231 & & & \\
\hline 51.29 & $1.83 \mathrm{E}-05$ & Fr-223 & $->$ & Ra-223, & Th-227 & $->$ & Ra-223 \\
\hline
\end{tabular}




\begin{tabular}{|c|c|c|c|c|c|c|c|}
\hline 52.763 & 7.18E-03 & $\mathrm{Pa}-231$ & $->$ & Ac-227 & & & \\
\hline 54.18 & $4.88 \mathrm{E}-05$ & Fr-223 & $->$ & Ra-223, & Th-227 & $->$ & Ra-223 \\
\hline 54.632 & $6.84 \mathrm{E}-03$ & $\mathrm{~Pa}-231$ & $->$ & Ac- 227 & & & \\
\hline 56.03 & 3.05E-05 & Th-227 & $->$ & Ra-223 & & & \\
\hline 56.63 & 4.27E-05 & Th-227 & $->$ & Ra-223 & & & \\
\hline 56.8 & 5.07E-04 & $\mathrm{Pa}-231$ & $->$ & Ac- 227 & & & \\
\hline 56.9 & $9.29 E-04$ & $\mathrm{~Pa}-231$ & $->$ & Ac- 227 & & & \\
\hline 57.2 & 1.27E-03 & $\mathrm{Pa}-231$ & $->$ & Ac-227 & & & \\
\hline 57.233 & $2.11 \mathrm{E}-03$ & $\mathrm{~Pa}-231$ & $->$ & $A c-227$ & & & \\
\hline 58.562 & $3.84 \mathrm{E}+02$ & Th-231 & $->$ & $\mathrm{Pa}-231$ & & & \\
\hline 59.5 & $5.49 E-05$ & Th-227 & $->$ & $\mathrm{Ra}-223$ & & & \\
\hline 60.526 & $5.91 \mathrm{E}-04$ & $\mathrm{~Pa}-231$ & $->$ & Ac-227 & & & \\
\hline 61.435 & $4.88 \mathrm{E}-04$ & Th-227 & $->$ & Ra-223 & & & \\
\hline 62.05 & $1.22 \mathrm{E}-05$ & Th-227 & $->$ & Ra-223 & & & \\
\hline 62.36 & $1.46 \mathrm{E}-03$ & Th-227 & $->$ & Ra-223 & & & \\
\hline 62.54 & 5.49E-05 & Th-227 & $->$ & Ra-223 & & & \\
\hline 63.7 & $4.22 \mathrm{E}-03$ & $\mathrm{~Pa}-231$ & $->$ & $A c-227$ & & & \\
\hline 63.834 & $1.84 \mathrm{E}+01$ & Th-231 & $->$ & $\mathrm{Pa}-231$ & & & \\
\hline 64.348 & $1.60 \mathrm{E}+01$ & U-235 & $->$ & Th-231 & & & \\
\hline 64.37 & $1.71 \mathrm{E}-04$ & Th-227 & $->$ & Ra-223 & & & \\
\hline 65.516 & $4.68 \mathrm{E}-04$ & $\mathrm{~Pb}-211$ & $->$ & $\mathrm{Bi}-211$ & & & \\
\hline 66.1 & $3.66 \mathrm{E}-05$ & Th-227 & $->$ & Ra-223 & & & \\
\hline 66.3 & 4.27E-05 & Th-227 & $->$ & Ra-223 & & & \\
\hline 68.5 & $4.56 \mathrm{E}+00$ & Th-231 & $->$ & $\mathrm{Pa}-231$ & & & \\
\hline 68.7 & $3.48 \mathrm{E}-05$ & Th-227 & $->$ & Ra-223 & & & \\
\hline 68.75 & $2.44 \mathrm{E}-04$ & Th-227 & $->$ & Ra-223 & & & \\
\hline 69.13 & $4.84 \mathrm{E}-07$ & Ac-227 & $->$ & Fr-223 & & & \\
\hline 69.65 & 5.49E-05 & Th-227 & $->$ & Ra-223 & & & \\
\hline 69.83 & $1.49 \mathrm{E}-06$ & $A c-227$ & $->$ & Fr-223 & & & \\
\hline 70.54 & 5.91E-04 & $\mathrm{Pa}-231$ & $->$ & $A c-227$ & & & \\
\hline 70.832 & 4.55E-03 & $\mathrm{Bi}-211$ & $->$ & TI-207 & & & \\
\hline 71.92 & $1.69 \mathrm{E}-04$ & $\mathrm{~Pa}-231$ & $->$ & Ac- 227 & & & \\
\hline 72.57 & $3.38 \mathrm{E}-04$ & $\mathrm{~Pa}-231$ & $->$ & $A C-227$ & & & \\
\hline 72.71 & $8.80 E+01$ & $U-235$ & $->$ & Th-231 & & & \\
\hline 72.767 & $2.01 E+02$ & Th-231 & $->$ & $\mathrm{Pa}-231$ & & & \\
\hline 72.8 & $1.71 \mathrm{E}-04$ & Th-227 & $->$ & Ra-223 & & & \\
\hline 72.803 & $9.10 \mathrm{E}-06$ & TI-207 & $->$ & Pb-207 & & & \\
\hline 72.873 & 7.70E-03 & $\mathrm{Bi}-211$ & $->$ & TI-207 & & & \\
\hline 73.66 & $1.16 \mathrm{E}-04$ & Th-227 & $->$ & Ra-223 & & & \\
\hline 74.209 & $2.11 \mathrm{E}-03$ & $\mathrm{~Pa}-231$ & $->$ & $A c-227$ & & & \\
\hline 74.8 & $4.80 \mathrm{E}+01$ & U-235 & $->$ & Th-231 & & & \\
\hline 74.814 & $1.56 \mathrm{E}-03$ & $\mathrm{~Pb}-211$ & $->$ & $\mathrm{Bi}-211$ & & & \\
\hline 74.969 & $1.52 \mathrm{E}-05$ & TI-207 & $->$ & Pb-207 & & & \\
\hline 75.1 & 5.49E-05 & Th-227 & $->$ & $\mathrm{Ra}-223$ & & & \\
\hline 76.858 & $3.16 \mathrm{E}-03$ & $\mathrm{Rn}-219$ & $->$ & Po-215 & & & \\
\hline 77.107 & $2.62 \mathrm{E}-03$ & $\mathrm{~Pb}-211$ & $->$ & $\mathrm{Bi}-211$ & & & \\
\hline 77.405 & $5.74 \mathrm{E}-03$ & $\mathrm{~Pa}-231$ & $->$ & $A c-227$ & & & \\
\hline 79.29 & $5.29 \mathrm{E}-03$ & $\mathrm{Rn}-219$ & $->$ & Po-215 & & & \\
\hline 79.72 & $1.36 \mathrm{E}-02$ & Fr-223 & $->$ & Ra-223, & Th-227 & $->$ & Ra-223 \\
\hline 81 & $2.74 \mathrm{E}-04$ & $\mathrm{~Pb}-211$ & $->$ & $\mathrm{Bi}-211$ & & & \\
\hline
\end{tabular}




\begin{tabular}{|c|c|c|c|c|c|c|c|}
\hline 81.067 & $9.24 \mathrm{E}-02$ & Ra-223 & $->$ & $\mathrm{Rn}-219$ & & & \\
\hline 81.229 & $7.20 E+02$ & Th-231 & $->$ & $\mathrm{Pa}-231$ & & & \\
\hline 82.434 & 2.71E-03 & $\mathrm{Bi}-211$ & $->$ & TI-207 & & & \\
\hline 83.787 & $1.53 \mathrm{E}-01$ & Ra-223 & $->$ & $\mathrm{Rn}-219$ & & & \\
\hline 83.81 & $3.47 \mathrm{E}-04$ & $\mathrm{~Pb}-211$ & $->$ & $\mathrm{Bi}-211$ & & & \\
\hline 84.203 & $5.28 \mathrm{E}+03$ & Th-231 & $->$ & $\mathrm{Pa}-231$ & & & \\
\hline 84.979 & $5.40 \mathrm{E}-06$ & TI-207 & $->$ & Pb-207 & & & \\
\hline 85.185 & 7.64E-04 & $\mathrm{Bi}-211$ & $->$ & TI-207 & & & \\
\hline 85.429 & $1.16 \mathrm{E}-02$ & Fr-223 & $->$ & Ra-223, & Th-227 & $->$ & Ra-223 \\
\hline 87.19 & $9.24 \mathrm{E}-04$ & $\mathrm{~Pb}-211$ & $->$ & $\mathrm{Bi}-211$ & & & \\
\hline 87.632 & $1.58 \mathrm{E}-06$ & TI-207 & $->$ & Pb-207 & & & \\
\hline 87.673 & $4.02 E-02$ & $\mathrm{~Pa}-231$ & $->$ & Ac- 227 & & & \\
\hline 88.2 & $1.03 E-04$ & $\mathrm{~Pb}-211$ & $->$ & $\mathrm{Bi}-211$ & & & \\
\hline 88.471 & $1.92 \mathrm{E}-02$ & Fr-223 & $->$ & Ra-223, & Th-227 & $->$ & $\mathrm{Ra}-223$ \\
\hline 89.639 & $1.89 \mathrm{E}-03$ & $\mathrm{Rn}-219$ & $->$ & Po-215 & & & \\
\hline 89.85 & $2.08 \mathrm{E}-05$ & Th-227 & $->$ & Ra-223 & & & \\
\hline 89.954 & $7.52 E+02$ & Th-231 & $->$ & $\mathrm{Pa}-231$ & & & \\
\hline 89.955 & $2.69 E+03$ & U-235 & $->$ & Th-231 & & & \\
\hline 90.128 & $2.74 \mathrm{E}-04$ & $\mathrm{~Pb}-211$ & $->$ & $\mathrm{Bi}-211$ & & & \\
\hline 90.886 & 6.59E-02 & Pa-231 & $->$ & Ac- 227 & & & \\
\hline 92.279 & $3.12 E+02$ & Th-231 & $->$ & $\mathrm{Pa}-231$ & & & \\
\hline 92.673 & $5.72 \mathrm{E}-04$ & $\mathrm{Rn}-219$ & $->$ & Po-215 & & & \\
\hline 93.07 & $3.92 E+01$ & Th-231 & $->$ & $\mathrm{Pa}-231$ & & & \\
\hline 93.36 & $4.40 \mathrm{E}+03$ & U-235 & $->$ & Th-231 & & & \\
\hline 93.93 & $8.55 \mathrm{E}-03$ & Th-227 & $->$ & Ra-223 & & & \\
\hline 94.3 & 6.69E-05 & $\mathrm{Pb}-211$ & $->$ & $\mathrm{Bi}-211$ & & & \\
\hline 94.677 & 5.41E-02 & $\mathrm{Ra}-223$ & $->$ & $\mathrm{Rn}-219$ & & & \\
\hline 94.89 & $1.09 \mathrm{E}-04$ & $\mathrm{~Pb}-211$ & $->$ & $\mathrm{Bi}-211$ & & & \\
\hline 94.9 & 7.32E-05 & Th-227 & $->$ & Ra-223 & & & \\
\hline 95.863 & $5.04 \mathrm{E}+02$ & Th-231 & $->$ & Pa-231 & & & \\
\hline 96.06 & $3.66 \mathrm{E}-04$ & Th-227 & $->$ & $\mathrm{Ra}-223$ & & & \\
\hline 96.154 & $6.88 \mathrm{E}+01$ & U-235 & $->$ & Th-231 & & & \\
\hline 96.979 & $7.43 E-03$ & $\mathrm{~Pa}-231$ & $->$ & $A C-227$ & & & \\
\hline 97.3 & 6.99E-05 & $\mathrm{Pb}-211$ & $->$ & $\mathrm{Bi}-211$ & & & \\
\hline 97.907 & $1.73 \mathrm{E}-02$ & $\mathrm{Ra}-223$ & $->$ & $\mathrm{Rn}-219$ & & & \\
\hline 99.28 & $9.60 \mathrm{E}+01$ & Th-231 & $->$ & $\mathrm{Pa}-231$ & & & \\
\hline 99.56 & 7.94E-05 & Fr-223 & $->$ & Ra-223, & Th-227 & $->$ & $\mathrm{Ra}-223$ \\
\hline 99.64 & $1.22 \mathrm{E}-05$ & Th-227 & $->$ & $\mathrm{Ra}-223$ & & & \\
\hline 99.7 & $2.81 \mathrm{E}-06$ & Ac- 227 & $->$ & Fr-223 & & & \\
\hline 99.915 & $6.84 \mathrm{E}-03$ & Fr-223 & $->$ & Ra-223, & Th-227 & $->$ & $\mathrm{Ra}-223$ \\
\hline 100.31 & $6.13 E-04$ & Fr-223 & $->$ & Ra-223, & Th-227 & $->$ & Ra-223 \\
\hline 100.96 & $2.70 \mathrm{E}-03$ & $\mathrm{~Pa}-231$ & $->$ & $A c-227$ & & & \\
\hline 102.255 & $3.28 \mathrm{E}+02$ & Th-231 & $->$ & $\mathrm{Pa}-231$ & & & \\
\hline 102.45 & $7.32 \mathrm{E}-06$ & Th-227 & $->$ & $\mathrm{Ra}-223$ & & & \\
\hline 102.613 & $2.36 \mathrm{E}-02$ & $\mathrm{~Pa}-231$ & $->$ & $A C-227$ & & & \\
\hline 103.341 & $2.23 E-03$ & Fr-223 & $->$ & Ra-223, & Th-227 & $->$ & $\mathrm{Ra}-223$ \\
\hline 103.85 & $1.03 \mathrm{E}-04$ & $\mathrm{Ra}-223$ & $->$ & $\mathrm{Rn}-219$ & & & \\
\hline 104.9 & $2.44 \mathrm{E}-04$ & Th-227 & $->$ & Ra-223 & & & \\
\hline 105.362 & $1.58 \mathrm{E}+03$ & U-235 & $->$ & Th-231 & & & \\
\hline 105.803 & $5.68 \mathrm{E}+00$ & Th-231 & $->$ & $\mathrm{Pa}-231$ & & & \\
\hline
\end{tabular}




\begin{tabular}{|c|c|c|c|c|c|c|c|}
\hline 106.1 & $1.23 \mathrm{E}-07$ & $A c-227$ & $->$ & $\mathrm{Fr}-223$ & & & \\
\hline 106.137 & 7.77E-03 & Pa-231 & $->$ & Ac-227 & & & \\
\hline 106.581 & $1.36 \mathrm{E}+01$ & Th-231 & $->$ & $\mathrm{Pa}-231$ & & & \\
\hline 106.66 & $1.34 \mathrm{E}-04$ & $\mathrm{Ra}-223$ & $->$ & $\mathrm{Rn}-219$ & & & \\
\hline 107.92 & 4.27E-05 & Th-227 & $->$ & Ra-223 & & & \\
\hline 108.166 & $1.82 \mathrm{E}+02$ & Th-231 & $->$ & $\mathrm{Pa}-231$ & & & \\
\hline 108.99 & $5.28 \mathrm{E}+02$ & U-235 & $->$ & Th-231 & & & \\
\hline 109.1 & $3.66 \mathrm{E}-05$ & Th-227 & $->$ & Ra-223 & & & \\
\hline 109.16 & $1.23 E+03$ & U-235 & $->$ & Th-231 & & & \\
\hline 110.58 & $3.05 E-05$ & Th-227 & $->$ & Ra-223 & & & \\
\hline 110.8 & $2.92 \mathrm{E}-04$ & Ra-223 & $->$ & $\mathrm{Rn}-219$ & & & \\
\hline 111.897 & $6.08 E+01$ & Th-231 & $->$ & $\mathrm{Pa}-231$ & & & \\
\hline 112.54 & $4.88 \mathrm{E}-05$ & Th-227 & $->$ & Ra-223 & & & \\
\hline 113.12 & $9.16 \mathrm{E}-04$ & Th-227 & $->$ & Ra-223 & & & \\
\hline 113.19 & $3.42 \mathrm{E}-03$ & Th-227 & $->$ & Ra-223 & & & \\
\hline 114.5 & 5.47E-05 & $\mathrm{Ra}-223$ & $->$ & $\mathrm{Rn}-219$ & & & \\
\hline 115.3 & $2.01 \mathrm{E}-05$ & $\mathrm{Rn}-219$ & $->$ & Po-215 & & & \\
\hline 115.595 & 8.00E-01 & Th-231 & $->$ & $\mathrm{Pa}-231$ & & & \\
\hline 116.11 & $5.60 E+01$ & U-235 & $->$ & Th-231 & & & \\
\hline 116.827 & $1.66 \mathrm{E}+01$ & Th-231 & $->$ & $\mathrm{Pa}-231$ & & & \\
\hline 117.17 & $1.04 \mathrm{E}-03$ & Th-227 & $->$ & Ra-223 & & & \\
\hline 117.3 & $7.32 \mathrm{E}-05$ & Th-227 & $->$ & Ra-223 & & & \\
\hline 119.98 & $2.08 \mathrm{E}+01$ & U-235 & $->$ & Th-231 & & & \\
\hline 120.9 & $4.84 \mathrm{E}-07$ & $A c-227$ & $->$ & Fr-223 & & & \\
\hline 122.31 & $7.24 \mathrm{E}-03$ & Ra-223 & $->$ & Rn-219 & & & \\
\hline 123.55 & $4.88 \mathrm{E}-05$ & Th-227 & $->$ & Ra-223 & & & \\
\hline 124.48 & $3.05 E-05$ & Th-227 & $->$ & Ra-223 & & & \\
\hline 124.6 & $1.83 \mathrm{E}-05$ & Th-227 & $->$ & Ra-223 & & & \\
\hline 124.69 & $4.22 \mathrm{E}-04$ & $\mathrm{~Pa}-231$ & $->$ & $A c-227$ & & & \\
\hline 124.922 & $4.48 E+01$ & Th-231 & $->$ & $\mathrm{Pa}-231$ & & & \\
\hline 130.57 & $7.66 \mathrm{E}-04$ & $\mathrm{Rn}-219$ & $->$ & Po-215 & & & \\
\hline 131.1 & $3.04 \mathrm{E}-05$ & $\mathrm{Ra}-223$ & $->$ & $\mathrm{Rn}-219$ & & & \\
\hline 133.8 & $2.46 \mathrm{E}-07$ & $A c-227$ & $->$ & Fr-223 & & & \\
\hline 134.014 & $1.92 \mathrm{E}+01$ & Th-231 & $->$ & $\mathrm{Pa}-231$ & & & \\
\hline 134.5 & $2.03 E-04$ & Fr-223 & $->$ & Ra-223, & Th-227 & $\rightarrow$ & $\mathrm{Ra}-223$ \\
\hline 135.681 & $6.24 \mathrm{E}+01$ & Th-231 & $->$ & $\mathrm{Pa}-231$ & & & \\
\hline 136.705 & $3.36 \mathrm{E}+00$ & Th-231 & $->$ & $\mathrm{Pa}-231$ & & & \\
\hline 136.72 & $9.60 \mathrm{E}+00$ & U-235 & $->$ & Th-231 & & & \\
\hline 140.33 & $1.95 \mathrm{E}-04$ & Th-227 & $->$ & Ra-223 & & & \\
\hline 140.537 & $5.68 \mathrm{E}-01$ & Th-231 & $->$ & $\mathrm{Pa}-231$ & & & \\
\hline 140.76 & $1.76 \mathrm{E}+02$ & U-235 & $->$ & Th-231 & & & \\
\hline 141.44 & 7.94E-04 & Th-227 & $->$ & Ra-223 & & & \\
\hline 143.76 & $8.76 E+03$ & U-235 & $->$ & Th-231 & & & \\
\hline 144.18 & $1.98 \mathrm{E}-02$ & $\mathrm{Ra}-223$ & $->$ & $\mathrm{Rn}-219$ & & & \\
\hline 144.5 & $3.38 \mathrm{E}-04$ & Pa-231 & $->$ & Ac- 227 & & & \\
\hline 145.063 & $4.64 \mathrm{E}+00$ & Th-231 & $->$ & $\mathrm{Pa}-231$ & & & \\
\hline 145.927 & $2.56 \mathrm{E}+01$ & Th-231 & $->$ & $\mathrm{Pa}-231$ & & & \\
\hline 147.2 & $7.30 \mathrm{E}-07$ & $A c-227$ & $->$ & Fr-223 & & & \\
\hline 150.02 & $6.10 \mathrm{E}-05$ & Th-227 & $->$ & Ra-223 & & & \\
\hline 150.93 & $6.40 E+01$ & U-235 & $->$ & Th-231 & & & \\
\hline
\end{tabular}




\begin{tabular}{|c|c|c|c|c|c|c|c|}
\hline 154.18 & $3.40 \mathrm{E}-02$ & Ra-223 & $->$ & $\mathrm{Rn}-219$ & & & \\
\hline 158.59 & $4.18 \mathrm{E}-03$ & $\mathrm{Ra}-223$ & $->$ & $\mathrm{Rn}-219$ & & & \\
\hline 162.18 & 4.27E-05 & Th-227 & $->$ & Ra-223 & & & \\
\hline 163.114 & $1.24 \mathrm{E}+02$ & Th-231 & $->$ & $\mathrm{Pa}-231$ & & & \\
\hline 163.33 & $4.06 E+03$ & U-235 & $->$ & Th-231 & & & \\
\hline 164.97 & $3.12 E+00$ & Th-231 & $->$ & $\mathrm{Pa}-231$ & & & \\
\hline 165.5 & 3.04E-05 & Ra-223 & $->$ & $\mathrm{Rn}-219$ & & & \\
\hline 168.19 & $8.55 E-05$ & Th-227 & $->$ & Ra-223 & & & \\
\hline 169.637 & $9.60 \mathrm{E}-01$ & Th-231 & $->$ & $\mathrm{Pa}-231$ & & & \\
\hline 169.98 & $1.83 \mathrm{E}-05$ & Th-227 & $->$ & Ra-223 & & & \\
\hline 171.21 & $6.10 \mathrm{E}-06$ & Th-227 & $->$ & Ra-223 & & & \\
\hline 171.4 & $3.60 E-07$ & $A c-227$ & $->$ & Fr-223 & & & \\
\hline 172.27 & $8.00 E+00$ & U-235 & $->$ & Th-231 & & & \\
\hline 173.38 & $1.05 \mathrm{E}-04$ & Fr-223 & $->$ & Ra-223, & Th-227 & $\rightarrow$ & Ra-223 \\
\hline 174.157 & $1.46 \mathrm{E}+01$ & Th-231 & $->$ & $\mathrm{Pa}-231$ & & & \\
\hline 175.23 & $1.10 \mathrm{E}-04$ & Th-227 & $->$ & Ra-223 & & & \\
\hline 175.55 & $1.03 \mathrm{E}-04$ & Ra-223 & $->$ & $\mathrm{Rn}-219$ & & & \\
\hline 176.8 & $2.43 \mathrm{E}-05$ & Ra-223 & $->$ & $\mathrm{Rn}-219$ & & & \\
\hline 177.35 & $2.86 \mathrm{E}-04$ & Ra-223 & $->$ & $\mathrm{Rn}-219$ & & & \\
\hline 179.28 & $1.22 \mathrm{E}-05$ & Th-227 & $->$ & $\mathrm{Ra}-223$ & & & \\
\hline 179.69 & $9.31 \mathrm{E}-04$ & Ra-223 & $->$ & $\mathrm{Rn}-219$ & & & \\
\hline 182.61 & $2.72 E+02$ & U-235 & $->$ & Th-231 & & & \\
\hline 183.483 & $2.63 E+01$ & Th-231 & $->$ & $\mathrm{Pa}-231$ & & & \\
\hline 184.68 & $2.39 \mathrm{E}-04$ & $\mathrm{Fr}-223$ & $->$ & Ra-223, & Th-227 & $->$ & Ra-223 \\
\hline 185.72 & $4.57 E+04$ & $U-235$ & $->$ & Th-231 & & & \\
\hline 188.756 & $2.56 \mathrm{E}+00$ & Th-231 & $->$ & $\mathrm{Pa}-231$ & & & \\
\hline 194.94 & $5.04 \mathrm{E}+02$ & U-235 & $->$ & Th-231 & & & \\
\hline 197.61 & $7.32 \mathrm{E}-05$ & Th-227 & $->$ & Ra-223 & & & \\
\hline 198.9 & $3.36 \mathrm{E}+01$ & U-235 & $->$ & Th-231 & & & \\
\hline 198.96 & $4.73 E-04$ & Pa-231 & $->$ & Ac- 227 & & & \\
\hline 199.43 & $1.82 \mathrm{E}-05$ & Ra-223 & $->$ & $\mathrm{Rn}-219$ & & & \\
\hline 200.48 & $1.22 \mathrm{E}-04$ & Th-227 & $->$ & Ra-223 & & & \\
\hline 201.64 & $1.22 \mathrm{E}-04$ & Th-227 & $->$ & Ra-223 & & & \\
\hline 202.11 & $8.64 \mathrm{E}+02$ & U-235 & $->$ & Th-231 & & & \\
\hline 202.4 & $3.66 \mathrm{E}-05$ & Th-227 & $->$ & $\mathrm{Ra}-223$ & & & \\
\hline 204.2 & $1.40 \mathrm{E}-03$ & Fr-223 & $->$ & Ra-223, & Th-227 & $->$ & $\mathrm{Ra}-223$ \\
\hline 204.95 & $9.17 \mathrm{E}-04$ & Fr-223 & $->$ & $\mathrm{Ra}-223$, & Th-227 & $->$ & $\mathrm{Ra}-223$ \\
\hline 205.31 & $4.01 E+03$ & U-235 & $->$ & Th-231 & & & \\
\hline 206.04 & $1.40 \mathrm{E}-03$ & Fr-223 & $->$ & Ra-223, & Th-227 & $->$ & $\mathrm{Ra}-223$ \\
\hline 210.58 & $6.90 \mathrm{E}-03$ & Fr-223 & $->$ & Ra-223, & Th-227 & $->$ & $\mathrm{Ra}-223$ \\
\hline 212.56 & $4.27 E-04$ & Th-227 & $->$ & $\mathrm{Ra}-223$ & & & \\
\hline 212.69 & $1.04 \mathrm{E}-04$ & Th-227 & $->$ & Ra-223 & & & \\
\hline 215.305 & $2.16 \mathrm{E}+01$ & U-235 & $->$ & Th-231 & & & \\
\hline 217.934 & $3.20 E+01$ & Th-231 & $->$ & $\mathrm{Pa}-231$ & & & \\
\hline 218.77 & $3.66 \mathrm{E}-04$ & Th-227 & $->$ & Ra-223 & & & \\
\hline 218.94 & $2.44 \mathrm{E}-04$ & Th-227 & $->$ & Ra-223 & & & \\
\hline 220.2 & $2.03 E-05$ & $\mathrm{~Pa}-231$ & $->$ & $A c-227$ & & & \\
\hline 220.38 & $2.01 \mathrm{E}-04$ & Ra-223 & $->$ & Rn-219 & & & \\
\hline 221.38 & $9.60 E+01$ & U-235 & $->$ & Th-231 & & & \\
\hline 221.9 & $1.82 \mathrm{E}-04$ & $\mathrm{Rn}-219$ & $->$ & Po-215 & & & \\
\hline
\end{tabular}




\begin{tabular}{|c|c|c|c|c|c|c|c|}
\hline 224.68 & $9.16 \mathrm{E}-05$ & Fr-223 & $\rightarrow$ & Ra-223, & Th-227 & $\rightarrow$ & $\mathrm{Ra}-223$ \\
\hline 226.77 & $2.20 \mathrm{E}-04$ & $\mathrm{~Pa}-231$ & $->$ & Ac- 227 & & & \\
\hline 228.16 & $2.03 E-05$ & $\mathrm{~Pa}-231$ & $->$ & Ac- 227 & & & \\
\hline 228.77 & $6.40 E+00$ & U-235 & $->$ & Th-231 & & & \\
\hline 230.17 & 4.27E-06 & Th-227 & $->$ & Ra-223 & & & \\
\hline 233.5 & $2.32 \mathrm{E}+01$ & U-235 & $->$ & Th-231 & & & \\
\hline 234.82 & $3.01 \mathrm{E}-03$ & Fr-223 & $->$ & $\mathrm{Ra}-223$, & Th-227 & $->$ & $\mathrm{Ra}-223$ \\
\hline 235.97 & $6.84 \mathrm{E}-02$ & Fr-223 & $->$ & Ra-223, & Th-227 & $->$ & Ra-223 \\
\hline 235.985 & $7.36 \mathrm{E}+00$ & Th-231 & $->$ & $\mathrm{Pa}-231$ & & & \\
\hline 240.25 & $2.24 \mathrm{E}-01$ & Th-231 & $->$ & Pa-231 & & & \\
\hline 240.85 & $4.32 E+01$ & U-235 & $->$ & Th-231 & & & \\
\hline 242 & $6.76 \mathrm{E}-04$ & $\mathrm{~Pa}-231$ & $->$ & Ac- 227 & & & \\
\hline 242.508 & $6.72 \mathrm{E}-01$ & Th-231 & $->$ & Pa-231 & & & \\
\hline 243.17 & 3.97E-03 & $\mathrm{Pa}-231$ & $->$ & Ac- 227 & & & \\
\hline 245.19 & 5.47E-05 & Ra-223 & $->$ & $\mathrm{Rn}-219$ & & & \\
\hline 245.438 & 5.91E-04 & Pa-231 & $->$ & Ac- 227 & & & \\
\hline 245.77 & $5.83 \mathrm{E}-04$ & Pa-231 & $->$ & Ac- 227 & & & \\
\hline 246.07 & $6.71 \mathrm{E}-05$ & Th-227 & $->$ & Ra-223 & & & \\
\hline 246.84 & $4.24 E+01$ & U-235 & $->$ & Th-231 & & & \\
\hline 249.36 & $4.27 \mathrm{E}-05$ & Th-227 & $->$ & Ra-223 & & & \\
\hline 249.49 & $2.25 \mathrm{E}-04$ & Ra-223 & $->$ & $\mathrm{Rn}-219$ & & & \\
\hline 249.56 & $6.24 \mathrm{E}-01$ & Th-231 & $->$ & $\mathrm{Pa}-231$ & & & \\
\hline 250.12 & $2.26 \mathrm{E}-03$ & Fr-223 & $->$ & Ra-223, & Th-227 & $->$ & Ra-223 \\
\hline 250.33 & 7.94E-04 & Th-227 & $->$ & $\mathrm{Ra}-223$ & & & \\
\hline 250.43 & $5.20 \mathrm{E}-01$ & Th-231 & $->$ & $\mathrm{Pa}-231$ & & & \\
\hline 251.12 & $2.13 \mathrm{E}-04$ & Ra-223 & $->$ & $\mathrm{Rn}-219$ & & & \\
\hline 251.58 & $1.89 \mathrm{E}-04$ & Ra-223 & $->$ & $\mathrm{Rn}-219$ & & & \\
\hline 252.46 & $6.71 \mathrm{E}-04$ & Th-227 & $->$ & $\mathrm{Ra}-223$ & & & \\
\hline 254.66 & $4.88 \mathrm{E}-03$ & Fr-223 & $->$ & Ra-223, & Th-227 & $->$ & $\mathrm{Ra}-223$ \\
\hline 254.98 & $1.95 \mathrm{E}-04$ & Ra-223 & $->$ & $\mathrm{Rn}-219$ & & & \\
\hline 255.74 & $3.04 \mathrm{E}-05$ & Ra-223 & $->$ & $\mathrm{Rn}-219$ & & & \\
\hline 255.858 & $8.45 E-03$ & $\mathrm{~Pa}-231$ & $->$ & Ac- 227 & & & \\
\hline 256.24 & 4.09E-02 & Fr-223 & $->$ & Ra-223, & Th-227 & $->$ & $\mathrm{Ra}-223$ \\
\hline 258.43 & $1.94 \mathrm{E}-04$ & $\mathrm{~Pa}-231$ & $->$ & Ac- 227 & & & \\
\hline 260.29 & $1.54 \mathrm{E}-02$ & Pa-231 & $->$ & Ac- 227 & & & \\
\hline 260.4 & $3.65 E-05$ & Ra-223 & $->$ & Rn-219 & & & \\
\hline 262.72 & $6.11 \mathrm{E}-04$ & Fr-223 & $->$ & $\mathrm{Ra}-223$, & Th-227 & $->$ & $\mathrm{Ra}-223$ \\
\hline 266.3 & $1.53 \mathrm{E}-05$ & Th-227 & $->$ & $\mathrm{Ra}-223$ & & & \\
\hline 266.483 & $4.80 E+00$ & U-235 & $->$ & Th-231 & & & \\
\hline 267.62 & $9.28 \mathrm{E}-01$ & Th-231 & $->$ & $\mathrm{Pa}-231$ & & & \\
\hline 267.82 & $6.10 \mathrm{E}-05$ & Th-227 & $->$ & $\mathrm{Ra}-223$ & & & \\
\hline 269.39 & $8.27 \mathrm{E}-02$ & $\mathrm{Ra}-223$ & $->$ & Rn-219 & & & \\
\hline 270.34 & $4.88 \mathrm{E}-05$ & Th-227 & $->$ & Ra-223 & & & \\
\hline 270.76 & $1.95 \mathrm{E}-04$ & Th-227 & $->$ & Ra-223 & & & \\
\hline 271.13 & $6.02 \mathrm{E}-02$ & $R n-219$ & $->$ & Po-215 & & & \\
\hline 272.95 & $2.99 \mathrm{E}-03$ & Fr-223 & $->$ & $\mathrm{Ra}-223$, & Th-227 & $->$ & Ra-223 \\
\hline 273.17 & $4.81 \mathrm{E}-03$ & Pa-231 & $->$ & Ac- 227 & & & \\
\hline 274.14 & $2.40 \mathrm{E}-02$ & Th-231 & $->$ & $\mathrm{Pa}-231$ & & & \\
\hline 275.2 & $9.16 \mathrm{E}-06$ & Th-227 & $->$ & Ra-223 & & & \\
\hline 275.47 & $3.92 E+00$ & U-235 & $->$ & Th-231 & & & \\
\hline
\end{tabular}




\begin{tabular}{|c|c|c|c|c|c|c|c|}
\hline 277.17 & $5.24 \mathrm{E}-03$ & Pa-231 & $->$ & Ac- 227 & & & \\
\hline 277.43 & $2.79 E-04$ & $\mathrm{~Pa}-231$ & $->$ & Ac- 227 & & & \\
\hline 279.7 & $4.27 E-04$ & Fr-223 & $->$ & Ra-223, & Th-227 & $->$ & Ra-223 \\
\hline 281 & 4.27E-05 & Th-227 & $->$ & Ra-223 & & & \\
\hline 281.31 & $9.77 E-04$ & Th-227 & $->$ & Ra-223 & & & \\
\hline 281.45 & $4.80 E+00$ & $U-235$ & $->$ & Th-231 & & & \\
\hline 282.98 & $4.00 E+00$ & U-235 & $->$ & Th-231 & & & \\
\hline 283.673 & $1.34 \mathrm{E}-01$ & $\mathrm{~Pa}-231$ & $->$ & Ac-227 & & & \\
\hline 284.24 & $3.05 E-04$ & Fr-223 & $->$ & Ra-223, & Th-227 & $->$ & Ra-223 \\
\hline 285.48 & $3.36 \mathrm{E}-04$ & Th-227 & $->$ & Ra-223 & & & \\
\hline 286.11 & $9.71 \mathrm{E}-03$ & Fr-223 & $->$ & Ra-223, & Th-227 & $->$ & Ra-223 \\
\hline 288.15 & $9.37 \mathrm{E}-04$ & Ra-223 & $->$ & Rn-219 & & & \\
\hline 289.5 & 5.49E-05 & Th-227 & $->$ & Ra-223 & & & \\
\hline 289.57 & $5.60 E+00$ & U-235 & $->$ & Th-231 & & & \\
\hline 289.6 & 8.91E-05 & Fr-223 & $->$ & Ra-223, & Th-227 & $->$ & Ra-223 \\
\hline 291.72 & $1.12 \mathrm{E}+01$ & $U-235$ & $->$ & Th-231 & & & \\
\hline 292.3 & $3.67 E-04$ & $\mathrm{Fr}-223$ & $->$ & Ra-223, & Th-227 & $->$ & Ra-223 \\
\hline 293.8 & 3.95E-04 & $\mathrm{Rn}-219$ & $->$ & Po-215 & & & \\
\hline 296.54 & $2.62 E-03$ & Th-227 & $->$ & $\mathrm{Ra}-223$ & & & \\
\hline 299.97 & $1.28 \mathrm{E}-02$ & Fr-223 & $->$ & Ra-223, & Th-227 & $->$ & Ra-223 \\
\hline 300.07 & $2.01 \mathrm{E}-01$ & $\mathrm{~Pa}-231$ & $->$ & Ac- 227 & & & \\
\hline 300.34 & $1.22 \mathrm{E}-03$ & Th-227 & $->$ & Ra-223 & & & \\
\hline 301.72 & $4.00 \mathrm{E}+00$ & U-235 & $->$ & Th-231 & & & \\
\hline 302.638 & $5.41 \mathrm{E}-02$ & Pa-231 & $->$ & Ac- 227 & & & \\
\hline 302.671 & $1.44 \mathrm{E}-01$ & Pa-231 & $->$ & Ac- 227 & & & \\
\hline 304.51 & $6.65 E-03$ & $\mathrm{Fr}-223$ & $->$ & Ra-223, & Th-227 & $->$ & $\mathrm{Ra}-223$ \\
\hline 307.89 & $1.06 \mathrm{E}-05$ & Fr-223 & $->$ & Ra-223, & Th-227 & $->$ & Ra-223 \\
\hline 308.49 & $9.16 \mathrm{E}-05$ & Th-227 & $->$ & $\mathrm{Ra}-223$ & & & \\
\hline 308.75 & $3.12 \mathrm{E}-01$ & Th-231 & $->$ & Pa-231 & & & \\
\hline 310.19 & $1.18 \mathrm{E}-04$ & $\mathrm{~Pa}-231$ & $->$ & Ac- 227 & & & \\
\hline 310.71 & $3.20 \mathrm{E}+00$ & U-235 & $->$ & Th-231 & & & \\
\hline 311 & $2.32 E+00$ & Th-231 & $->$ & $\mathrm{Pa}-231$ & & & \\
\hline 312.57 & $2.87 \mathrm{E}-03$ & Fr-223 & $->$ & Ra-223, & Th-227 & $->$ & Ra-223 \\
\hline 313.058 & $9.54 \mathrm{E}-03$ & $\mathrm{~Pa}-231$ & $->$ & Ac- 227 & & & \\
\hline 313.85 & $1.89 E-04$ & $\mathrm{~Pb}-211$ & $->$ & $\mathrm{Bi}-211$ & & & \\
\hline 314.78 & $1.83 \mathrm{E}-04$ & Th-227 & $->$ & Ra-223 & & & \\
\hline 314.82 & $2.81 E-03$ & Th-227 & $->$ & $\mathrm{Ra}-223$ & & & \\
\hline 317.12 & $8.00 \mathrm{E}-01$ & $U-235$ & $->$ & Th-231 & & & \\
\hline 317.93 & $6.40 \mathrm{E}-02$ & Th-231 & $->$ & $\mathrm{Pa}-231$ & & & \\
\hline 318.1 & $1.69 \mathrm{E}-04$ & Pa-231 & $->$ & Ac- 227 & & & \\
\hline 318.58 & $3.66 \mathrm{E}-05$ & Th-227 & $->$ & Ra-223 & & & \\
\hline 319.18 & $1.94 \mathrm{E}-04$ & Fr-223 & $->$ & Ra-223, & Th-227 & $->$ & Ra-223 \\
\hline 320.188 & 8.80E-02 & Th-231 & $->$ & $\mathrm{Pa}-231$ & & & \\
\hline 323.88 & $2.37 \mathrm{E}-02$ & Ra-223 & $->$ & $\mathrm{Rn}-219$ & & & \\
\hline 325.08 & $3.66 \mathrm{E}-05$ & Th-227 & $->$ & $\mathrm{Ra}-223$ & & & \\
\hline 326.12 & 3.05E-05 & Th-227 & $->$ & Ra-223 & & & \\
\hline 327.23 & $2.54 \mathrm{E}-03$ & $\mathrm{~Pa}-231$ & $->$ & Ac-227 & & & \\
\hline 328.08 & $9.15 \mathrm{E}-06$ & TI-207 & $->$ & $\mathrm{Pb}-207$, & Po-211 & $->$ & $\mathrm{Pb}-207$ \\
\hline 328.49 & $1.20 \mathrm{E}-03$ & Ra-223 & $->$ & $\mathrm{Rn}-219$ & & & \\
\hline 329.84 & $1.67 \mathrm{E}-02$ & Fr-223 & $->$ & Ra-223, & Th-227 & $->$ & $\mathrm{Ra}-223$ \\
\hline
\end{tabular}




\begin{tabular}{|c|c|c|c|c|c|c|c|}
\hline 330.066 & $1.11 \mathrm{E}-01$ & Pa-231 & $->$ & Ac- 227 & & & \\
\hline 333.87 & $6.02 \mathrm{E}-04$ & Ra-223 & $->$ & $\mathrm{Rn}-219$ & & & \\
\hline 334.38 & $6.04 \mathrm{E}-03$ & Fr-223 & $->$ & Ra-223, & Th-227 & $->$ & Ra-223 \\
\hline 338.28 & 1.69E-02 & Ra-223 & $->$ & $\mathrm{Rn}-219$ & & & \\
\hline 339.1 & $8.55 E-06$ & Th-227 & $->$ & Ra-223 & & & \\
\hline 339.45 & $2.41 \mathrm{E}-05$ & Fr-223 & $->$ & $\mathrm{Ra}-223$, & Th-227 & $->$ & Ra-223 \\
\hline 340.873 & $1.47 \mathrm{E}-02$ & $\mathrm{~Pa}-231$ & $->$ & Ac- 227 & & & \\
\hline 341.17 & $1.86 \mathrm{E}-03$ & $\mathrm{~Pa}-231$ & $->$ & $A c-227$ & & & \\
\hline 342.44 & $2.32 \mathrm{E}-03$ & Fr-223 & $->$ & Ra-223, & Th-227 & $->$ & Ra-223 \\
\hline 342.9 & $1.22 \mathrm{E}-03$ & Ra-223 & $->$ & $\mathrm{Rn}-219$ & & & \\
\hline 343.02 & $2.07 E-04$ & $\mathrm{~Pb}-211$ & $->$ & $\mathrm{Bi}-211$ & & & \\
\hline 343.78 & $2.40 \mathrm{E}+00$ & U-235 & $->$ & Th-231 & & & \\
\hline 345.921 & $2.32 E+01$ & $U-235$ & $->$ & Th-231 & & & \\
\hline 346.39 & $4.58 \mathrm{E}-05$ & Th-227 & $->$ & Ra-223 & & & \\
\hline 348.4 & $3.66 \mathrm{E}-05$ & Th-227 & $->$ & Ra-223 & & & \\
\hline 350.1 & 7.76E-02 & $\mathrm{Bi}-211$ & $->$ & TI-207 & & & \\
\hline 350.48 & $6.71 \mathrm{E}-04$ & Th-227 & $->$ & Ra-223 & & & \\
\hline 351.82 & 5.60E-02 & Th-231 & $->$ & $\mathrm{Pa}-231$ & & & \\
\hline 352.57 & $6.10 \mathrm{E}-05$ & Th-227 & $->$ & Ra-223 & & & \\
\hline 354.63 & $6.76 \mathrm{E}-03$ & Pa-231 & $->$ & $A c-227$ & & & \\
\hline 356.068 & $4.00 E+00$ & $U-235$ & $->$ & Th-231 & & & \\
\hline 357.2 & $1.30 \mathrm{E}-02$ & Pa-231 & $->$ & Ac- 227 & & & \\
\hline 359.51 & 7.60E-04 & Pa-231 & $->$ & Ac- 227 & & & \\
\hline 361.8 & $2.62 \mathrm{E}-04$ & Ra-223 & $->$ & $\mathrm{Rn}-219$ & & & \\
\hline 362.55 & 3.05E-05 & Th-227 & $->$ & Ra-223 & & & \\
\hline 364.03 & $6.25 \mathrm{E}-04$ & $\mathrm{~Pa}-231$ & $->$ & $A C-227$ & & & \\
\hline 368.78 & 4.87E-05 & $\mathrm{Ra}-223$ & $->$ & $\mathrm{Rn}-219$ & & & \\
\hline 369.32 & 3.87E-05 & Fr-223 & $->$ & Ra-223, & Th-227 & $->$ & Ra-223 \\
\hline 369.48 & $1.22 \mathrm{E}-04$ & $\mathrm{Ra}-223$ & $->$ & Rn-219 & & & \\
\hline 370.85 & 4.27E-05 & Th-227 & $->$ & Ra-223 & & & \\
\hline 371.8 & $2.98 \mathrm{E}-03$ & $\mathrm{Ra}-223$ & $->$ & $\mathrm{Rn}-219$ & & & \\
\hline 374.93 & 8.55E-06 & Th-227 & $->$ & Ra-223 & & & \\
\hline 376.26 & $3.66 \mathrm{E}-05$ & Th-227 & $->$ & $\mathrm{Ra}-223$ & & & \\
\hline 376.9 & $4.26 \mathrm{E}-05$ & $\mathrm{Rn}-219$ & $->$ & Po-215 & & & \\
\hline 379.4 & $3.89 \mathrm{E}-03$ & $R n-219$ & $->$ & Po-215, & $\mathrm{Pa}-231$ & $->$ & Ac- 227 \\
\hline 382.15 & $3.66 \mathrm{E}-05$ & Th-227 & $->$ & $\mathrm{Ra}-223$ & & & \\
\hline 383.12 & $2.43 E-05$ & Ra-223 & $->$ & $\mathrm{Rn}-219$ & & & \\
\hline 383.47 & 2.93E-04 & Th-227 & $->$ & Ra-223 & & & \\
\hline 384.97 & $3.46 \mathrm{E}-04$ & $\mathrm{~Pa}-231$ & $->$ & $A c-227$ & & & \\
\hline 387.267 & $4.22 \mathrm{E}-05$ & $\mathrm{~Pa}-231$ & $->$ & $A c-227$ & & & \\
\hline 387.874 & $3.04 \mathrm{E}+00$ & U-235 & $->$ & Th-231 & & & \\
\hline 387.9 & $2.43 \mathrm{E}-06$ & $\mathrm{Rn}-219$ & $->$ & Po-215 & & & \\
\hline 388.3 & $8.51 \mathrm{E}-05$ & $\mathrm{Ra}-223$ & $->$ & $\mathrm{Rn}-219$ & & & \\
\hline 388.98 & $9.04 \mathrm{E}-05$ & $\mathrm{~Pa}-231$ & $->$ & $A c-227$ & & & \\
\hline 390.32 & 8.00E-01 & U-235 & $->$ & Th-231 & & & \\
\hline 391 & $1.82 \mathrm{E}-05$ & Ra-223 & $->$ & $\mathrm{Rn}-219$ & & & \\
\hline 391.85 & $5.74 \mathrm{E}-04$ & $\mathrm{~Pa}-231$ & $->$ & $A C-227$ & & & \\
\hline 392.24 & 5.49E-05 & Th-227 & $->$ & Ra-223 & & & \\
\hline 395.8 & $2.20 \mathrm{E}-04$ & Pa-231 & $->$ & Ac- 227 & & & \\
\hline 398.4 & 7.85E-04 & $\mathrm{Pa}-231$ & $->$ & Ac- 227 & & & \\
\hline
\end{tabular}




\begin{tabular}{|c|c|c|c|c|c|c|c|}
\hline 398.6 & 5.49E-05 & Th-227 & $->$ & Ra-223 & & & \\
\hline 401.7 & $4.04 \mathrm{E}-02$ & $\mathrm{Rn}-219$ & $->$ & Po-215 & & & \\
\hline 402.42 & 7.32E-05 & Th-227 & $->$ & Ra-223 & & & \\
\hline 404.86 & $2.33 \mathrm{E}-02$ & $\mathrm{~Pb}-211$ & $->$ & $\mathrm{Bi}-211$, & At-215 & $->$ & $\mathrm{Bi}-211$ \\
\hline 407.98 & $2.87 \mathrm{E}-03$ & $\mathrm{~Pa}-231$ & $->$ & Ac- 227 & & & \\
\hline 410.269 & $2.40 \mathrm{E}+00$ & U-235 & $->$ & Th-231 & & & \\
\hline 410.84 & $1.60 \mathrm{E}-04$ & $\mathrm{~Pa}-231$ & $->$ & Ac- 227 & & & \\
\hline 415.09 & $1.04 \mathrm{E}-05$ & Th-227 & $->$ & Ra-223 & & & \\
\hline 427 & $1.05 \mathrm{E}-02$ & $\mathrm{~Pb}-211$ & $->$ & $\mathrm{Bi}-211$ & & & \\
\hline 430.26 & 3.89E-05 & $\mathrm{Pb}-211$ & $->$ & $\mathrm{Bi}-211$ & & & \\
\hline 430.53 & $1.16 \mathrm{E}-04$ & Ra-223 & $->$ & $\mathrm{Rn}-219$ & & & \\
\hline 432.29 & $3.05 E-05$ & Th-227 & $->$ & Ra-223 & & & \\
\hline 432.34 & $2.01 \mathrm{E}-04$ & Ra-223 & $->$ & $\mathrm{Rn}-219$ & & & \\
\hline 435.38 & $2.79 E-04$ & $\mathrm{~Pa}-231$ & $->$ & Ac- 227 & & & \\
\hline 438.24 & $3.46 \mathrm{E}-04$ & $\mathrm{~Pa}-231$ & $->$ & Ac- 227 & & & \\
\hline 438.8 & $2.43 \mathrm{E}-04$ & Po-215 & $->$ & $\mathrm{Pb}-211$ & & & \\
\hline 444.94 & 7.72E-03 & Ra-223 & $->$ & Rn-219 & & & \\
\hline 448 & $9.95 \mathrm{E}-07$ & Fr-223 & $->$ & Ra-223, & Th-227 & $->$ & Ra-223 \\
\hline 448.42 & 8.00E-01 & U-235 & $->$ & Th-231 & & & \\
\hline 452.52 & 7.86E-07 & Fr-223 & $->$ & Ra-223, & Th-227 & $->$ & Ra-223 \\
\hline 455.12 & $6.40 \mathrm{E}-01$ & U-235 & $->$ & Th-231 & & & \\
\hline 457.07 & $5.50 E-07$ & Fr-223 & $->$ & Ra-223, & Th-227 & $->$ & Ra-223 \\
\hline 461.9 & $3.05 E-07$ & Th-227 & $->$ & Ra-223 & & & \\
\hline 466.2 & $3.05 E-07$ & Th-227 & $->$ & Ra-223 & & & \\
\hline 471.33 & $1.27 \mathrm{E}-05$ & $\mathrm{~Pa}-231$ & $->$ & Ac- 227 & & & \\
\hline 478 & 7.91E-05 & $\mathrm{Pb}-211$ & $->$ & $\mathrm{Bi}-211$ & & & \\
\hline 478.22 & $6.50 \mathrm{E}-06$ & $\mathrm{~Pa}-231$ & $->$ & $A c-227$ & & & \\
\hline 480.25 & $1.94 \mathrm{E}-06$ & Fr-223 & $->$ & Ra-223, & Th-227 & $->$ & $\mathrm{Ra}-223$ \\
\hline 481.1 & $1.58 \mathrm{E}-04$ & $\mathrm{~Pb}-211$ & $->$ & $\mathrm{Bi}-211$ & & & \\
\hline 482.14 & $1.01 \mathrm{E}-06$ & Fr-223 & $->$ & Ra-223, & Th-227 & $->$ & Ra-223 \\
\hline 487.59 & $6.08 \mathrm{E}-05$ & Ra-223 & $->$ & $\mathrm{Rn}-219$ & & & \\
\hline 492 & 8.51E-05 & $\mathrm{Pb}-211$ & $->$ & $\mathrm{Bi}-211$ & & & \\
\hline 493.15 & $3.55 \mathrm{E}-06$ & Fr-223 & $->$ & Ra-223, & Th-227 & $->$ & $\mathrm{Ra}-223$ \\
\hline 500.4 & 6.69E-05 & $\mathrm{Pb}-211$ & $->$ & $\mathrm{Bi}-211$ & & & \\
\hline 501.33 & 5.07E-05 & $\mathrm{Pa}-231$ & $->$ & $A c-227$ & & & \\
\hline 504.33 & $9.12 \mathrm{E}-05$ & $\mathrm{~Pb}-211$ & $->$ & $\mathrm{Bi}-211$ & & & \\
\hline 507.64 & $2.71 \mathrm{E}-06$ & Fr-223 & $->$ & Ra-223, & Th-227 & $->$ & Ra-223 \\
\hline 515.7 & $2.43 \mathrm{E}-04$ & $\mathrm{Rn}-219$ & $->$ & Po-215 & & & \\
\hline 515.96 & $1.30 \mathrm{E}-06$ & Fr-223 & $->$ & Ra-223, & Th-227 & $->$ & $\mathrm{Ra}-223$ \\
\hline 516.45 & $1.10 \mathrm{E}-04$ & $\mathrm{~Pa}-231$ & $->$ & AC- 227 & & & \\
\hline 517.2 & $3.20 \mathrm{E}-01$ & U-235 & $->$ & Th-231 & & & \\
\hline 524.02 & $1.08 \mathrm{E}-06$ & Fr-223 & $->$ & Ra-223, & Th-227 & $->$ & $\mathrm{Ra}-223$ \\
\hline 527.3 & $4.32 \mathrm{E}-04$ & Ra-223 & $->$ & $\mathrm{Rn}-219$ & & & \\
\hline 534.9 & $6.10 \mathrm{E}-07$ & Th-227 & $->$ & $\mathrm{Ra}-223$ & & & \\
\hline 535.45 & 4.73E-05 & $\mathrm{Pa}-231$ & $->$ & $A C-227$ & & & \\
\hline 536.88 & $7.10 \mathrm{E}-06$ & Fr-223 & $->$ & Ra-223, & Th-227 & $->$ & $\mathrm{Ra}-223$ \\
\hline 540.1 & $3.65 \mathrm{E}-05$ & $\mathrm{Rn}-219$ & $->$ & Po-215 & & & \\
\hline 546.9 & $4.22 \mathrm{E}-05$ & Pa-231 & $->$ & Ac-227 & & & \\
\hline 552.09 & $1.81 \mathrm{E}-06$ & Fr-223 & $->$ & Ra-223, & Th-227 & $->$ & Ra-223 \\
\hline 555.91 & $1.49 E-06$ & Fr-223 & $->$ & Ra-223, & Th-227 & $->$ & $\mathrm{Ra}-223$ \\
\hline
\end{tabular}




\begin{tabular}{|c|c|c|c|c|c|c|c|}
\hline 568.76 & $6.91 \mathrm{E}-06$ & Fr-223 & $->$ & Ra-223, & Th-227 & $->$ & Ra-223 \\
\hline 569.15 & 8.87E-06 & Po-211 & $->$ & $\mathrm{Pb}-207$ & & & \\
\hline 572.3 & $4.22 \mathrm{E}-05$ & $\mathrm{~Pa}-231$ & $->$ & Ac- 227 & & & \\
\hline 575.8 & $3.34 \mathrm{E}-06$ & Fr-223 & $->$ & Ra-223, & Th-227 & $->$ & Ra-223 \\
\hline 578.2 & $9.43 \mathrm{E}-07$ & Fr-223 & $->$ & Ra-223, & Th-227 & $->$ & Ra-223 \\
\hline 588.17 & $3.87 E-07$ & Fr-223 & $->$ & Ra-223, & Th-227 & $->$ & Ra-223 \\
\hline 596.44 & $2.54 \mathrm{E}-07$ & Fr-223 & $->$ & Ra-223, & Th-227 & $->$ & Ra-223 \\
\hline 598.69 & 5.47E-04 & Ra-223 & $->$ & $\mathrm{Rn}-219$ & & & \\
\hline 607.2 & $1.22 \mathrm{E}-06$ & Fr-223 & $->$ & Ra-223, & Th-227 & $->$ & Ra-223 \\
\hline 608.5 & $2.43 E-05$ & $\mathrm{Rn}-219$ & $->$ & Po-215 & & & \\
\hline 609.08 & $3.83 \mathrm{E}-04$ & Ra-223 & $->$ & $\mathrm{Rn}-219$ & & & \\
\hline 609.62 & $1.40 \mathrm{E}-04$ & $\mathrm{~Pb}-211$ & $->$ & $\mathrm{Bi}-211$ & & & \\
\hline 621.92 & 3.99E-07 & Fr-223 & $->$ & Ra-223, & Th-227 & $->$ & Ra-223 \\
\hline 623.38 & 4.87E-05 & Ra-223 & $->$ & Rn-219 & & & \\
\hline 623.52 & $9.77 \mathrm{E}-07$ & Th-227 & $->$ & Ra-223 & & & \\
\hline 633.2 & 7.94E-07 & Th-227 & $->$ & Ra-223 & & & \\
\hline 641.45 & $1.22 \mathrm{E}-07$ & Th-227 & $->$ & Ra-223 & & & \\
\hline 643.93 & $5.43 \mathrm{E}-07$ & Fr-223 & $->$ & Ra-223, & Th-227 & $->$ & Ra-223 \\
\hline 648.43 & $1.29 \mathrm{E}-07$ & Fr-223 & $->$ & Ra-223, & Th-227 & $->$ & Ra-223 \\
\hline 662.8 & $3.66 \mathrm{E}-07$ & Th-227 & $->$ & Ra-223 & & & \\
\hline 675.33 & 3.89E-05 & $\mathrm{Pb}-211$ & $->$ & $\mathrm{Bi}-211$ & & & \\
\hline 676.4 & $3.65 E-05$ & $\mathrm{Rn}-219$ & $->$ & Po-215 & & & \\
\hline 691.46 & $1.04 \mathrm{E}-06$ & Fr-223 & $->$ & Ra-223, & Th-227 & $->$ & Ra-223 \\
\hline 704.19 & 8.75E-07 & Fr-223 & $->$ & Ra-223, & Th-227 & $->$ & $\mathrm{Ra}-223$ \\
\hline 704.51 & 2.93E-03 & $\mathrm{Pb}-211$ & $->$ & $\mathrm{Bi}-211$ & & & \\
\hline 707.18 & $3.14 \mathrm{E}-07$ & Fr-223 & $->$ & Ra-223, & Th-227 & $->$ & Ra-223 \\
\hline 711.2 & $2.13 \mathrm{E}-05$ & Ra-223 & $->$ & Rn-219 & & & \\
\hline 718.22 & $2.04 \mathrm{E}-07$ & Fr-223 & $->$ & Ra-223, & Th-227 & $->$ & Ra-223 \\
\hline 722.6 & $4.52 E-06$ & Fr-223 & $->$ & Ra-223, & Th-227 & $->$ & Ra-223 \\
\hline 723.86 & $9.19 \mathrm{E}-06$ & Fr-223 & $->$ & Ra-223, & Th-227 & $->$ & Ra-223 \\
\hline 733.9 & $1.23 \mathrm{E}-06$ & Fr-223 & $->$ & Ra-223, & Th-227 & $->$ & Ra-223 \\
\hline 734.6 & $1.15 \mathrm{E}-06$ & Fr-223 & $->$ & Ra-223, & Th-227 & $->$ & Ra-223 \\
\hline 736.76 & $5.50 \mathrm{E}-07$ & Fr-223 & $->$ & Ra-223, & Th-227 & $->$ & Ra-223 \\
\hline 742.52 & $3.20 \mathrm{E}-01$ & $U-235$ & $->$ & Th-231 & & & \\
\hline 746.24 & $2.67 \mathrm{E}-06$ & Fr-223 & $->$ & Ra-223, & Th-227 & $->$ & Ra-223 \\
\hline 748.56 & $3.24 \mathrm{E}-06$ & Fr-223 & $->$ & Ra-223, & Th-227 & $->$ & Ra-223 \\
\hline 753.44 & $1.16 \mathrm{E}-06$ & Fr-223 & $->$ & Ra-223, & Th-227 & $->$ & Ra-223 \\
\hline 754.17 & $1.19 \mathrm{E}-06$ & Fr-223 & $->$ & Ra-223, & Th-227 & $->$ & Ra-223 \\
\hline 756.79 & $2.36 \mathrm{E}-06$ & Fr-223 & $->$ & Ra-223, & Th-227 & $->$ & Ra-223 \\
\hline 757.03 & $6.17 \mathrm{E}-06$ & Fr-223 & $->$ & Ra-223, & Th-227 & $->$ & Ra-223 \\
\hline 762.3 & $1.76 \mathrm{E}-06$ & Fr-223 & $->$ & Ra-223, & Th-227 & $->$ & Ra-223 \\
\hline 764.52 & $8.78 \mathrm{E}-06$ & Fr-223 & $->$ & Ra-223, & Th-227 & $->$ & Ra-223 \\
\hline 766.35 & $4.32 \mathrm{E}-03$ & $\mathrm{~Pb}-211$ & $->$ & $\mathrm{Bi}-211$ & & & \\
\hline 772.85 & $8.38 \mathrm{E}-07$ & Fr-223 & $->$ & Ra-223, & Th-227 & $->$ & Ra-223 \\
\hline 775.82 & $4.45 E-05$ & Fr-223 & $->$ & Ra-223, & Th-227 & $->$ & Ra-223 \\
\hline 780.59 & $2.17 \mathrm{E}-06$ & Fr-223 & $->$ & Ra-223, & Th-227 & $->$ & Ra-223 \\
\hline 784.04 & $1.19 \mathrm{E}-06$ & Fr-223 & $->$ & Ra-223, & Th-227 & $->$ & Ra-223 \\
\hline 786.9 & $8.26 \mathrm{E}-07$ & Fr-223 & $->$ & Ra-223, & Th-227 & $->$ & Ra-223 \\
\hline 788.36 & $2.44 \mathrm{E}-07$ & Th-227 & $->$ & $\mathrm{Ra}-223$ & & & \\
\hline 792.6 & $2.44 \mathrm{E}-07$ & Th-227 & $->$ & Ra-223 & & & \\
\hline
\end{tabular}




\begin{tabular}{|c|c|c|c|c|c|c|c|}
\hline 793.11 & $1.05 \mathrm{E}-08$ & Fr-223 & $->$ & Ra-223 & & & \\
\hline 793.12 & $1.83 \mathrm{E}-07$ & Th-227 & $->$ & Ra-223 & & & \\
\hline 794.72 & $4.80 \mathrm{E}-01$ & $U-235$ & $->$ & Th-231 & & & \\
\hline 796.96 & $5.71 \mathrm{E}-06$ & Fr-223 & $->$ & Ra-223, & Th-227 & $->$ & Ra-223 \\
\hline 803.58 & $1.09 \mathrm{E}-05$ & Fr-223 & $->$ & Ra-223, & Th-227 & $->$ & Ra-223 \\
\hline 808.26 & $3.58 \mathrm{E}-07$ & Fr-223 & $->$ & $\mathrm{Ra}-223$, & Th-227 & $->$ & Ra-223 \\
\hline 812.15 & $1.77 \mathrm{E}-05$ & Fr-223 & $->$ & Ra-223, & Th-227 & $->$ & Ra-223 \\
\hline 817.31 & $1.05 \mathrm{E}-06$ & Fr-223 & $->$ & Ra-223, & Th-227 & $->$ & Ra-223 \\
\hline 818.23 & $1.83 \mathrm{E}-07$ & Th-227 & $->$ & Ra-223 & & & \\
\hline 822.99 & $1.61 \mathrm{E}-05$ & Fr-223 & $->$ & Ra-223, & Th-227 & $->$ & Ra-223 \\
\hline 825.96 & $5.56 \mathrm{E}-06$ & Fr-223 & $->$ & Ra-223, & Th-227 & $->$ & Ra-223 \\
\hline 825.97 & $1.14 \mathrm{E}-07$ & Fr-223 & $->$ & Ra-223 & & & \\
\hline 828.31 & $1.33 \mathrm{E}-06$ & Fr-223 & $->$ & Ra-223, & Th-227 & $->$ & Ra-223 \\
\hline 828.53 & 7.25E-08 & Fr-223 & $->$ & Ra-223, & Th-227 & $->$ & Ra-223 \\
\hline 831.86 & $2.32 \mathrm{E}-02$ & $\mathrm{~Pb}-211$ & $->$ & $\mathrm{Bi}-211$ & & & \\
\hline 834.2 & $6.08 \mathrm{E}-06$ & Rn-219 & $->$ & Po-215 & & & \\
\hline 837.36 & $3.04 \mathrm{E}-06$ & Fr-223 & $->$ & Ra-223, & Th-227 & $->$ & Ra-223 \\
\hline 842.02 & $4.22 \mathrm{E}-06$ & Fr-223 & $->$ & Ra-223, & Th-227 & $->$ & Ra-223 \\
\hline 846.56 & $4.05 E-06$ & Fr-223 & $->$ & Ra-223, & Th-227 & $->$ & Ra-223 \\
\hline 848.88 & $6.56 \mathrm{E}-07$ & Fr-223 & $->$ & Ra-223, & Th-227 & $->$ & Ra-223 \\
\hline 854.2 & $4.27 E-07$ & Th-227 & $->$ & Ra-223 & & & \\
\hline 857.89 & 3.99E-07 & Fr-223 & $->$ & Ra-223, & Th-227 & $->$ & Ra-223 \\
\hline 858.4 & $1.74 \mathrm{E}-06$ & Fr-223 & $->$ & Ra-223, & Th-227 & $->$ & Ra-223 \\
\hline 863.2 & $2.98 \mathrm{E}-07$ & Fr-223 & $->$ & Ra-223, & Th-227 & $->$ & Ra-223 \\
\hline 865.81 & 3.89E-05 & $\mathrm{Pb}-211$ & $->$ & $\mathrm{Bi}-211$ & & & \\
\hline 867.23 & 5.39E-07 & Fr-223 & $->$ & Ra-223, & Th-227 & $->$ & Ra-223 \\
\hline 876.14 & $4.70 E-06$ & Fr-223 & $->$ & Ra-223, & Th-227 & $->$ & Ra-223 \\
\hline 878.16 & 7.37E-07 & Fr-223 & $->$ & Ra-223, & Th-227 & $->$ & Ra-223 \\
\hline 887.7 & $9.12 \mathrm{E}-06$ & $\mathrm{Rn}-219$ & $->$ & Po-215 & & & \\
\hline 891 & $2.98 \mathrm{E}-07$ & Fr-223 & $->$ & Ra-223, & Th-227 & $->$ & Ra-223 \\
\hline 892.8 & $1.94 \mathrm{E}-07$ & Fr-223 & $->$ & Ra-223, & Th-227 & $->$ & Ra-223 \\
\hline 896.41 & $2.18 \mathrm{E}-06$ & Fr-223 & $->$ & $\mathrm{Ra}-223$, & Th-227 & $->$ & Ra-223 \\
\hline 897.23 & $1.46 \mathrm{E}-03$ & TI-207 & $->$ & Pb-207, & Po-211 & $->$ & $\mathrm{Pb}-207$ \\
\hline 908.03 & $1.40 \mathrm{E}-05$ & Fr-223 & $->$ & Ra-223, & Th-227 & $->$ & Ra-223 \\
\hline 909.85 & $9.16 \mathrm{E}-08$ & Th-227 & $->$ & $\mathrm{Ra}-223$ & & & \\
\hline 920.06 & 7.32E-08 & Th-227 & $->$ & Ra-223 & & & \\
\hline 926.28 & $1.83 \mathrm{E}-07$ & Fr-223 & $->$ & Ra-223, & Th-227 & $->$ & Ra-223 \\
\hline 938.34 & $6.10 \mathrm{E}-08$ & Th-227 & $->$ & Ra-223 & & & \\
\hline 941.41 & 4.39E-07 & Th-227 & $->$ & Ra-223 & & & \\
\hline 951 & $1.34 \mathrm{E}-04$ & $\mathrm{~Pb}-211$ & $->$ & $\mathrm{Bi}-211$ & & & \\
\hline 958.6 & $3.78 \mathrm{E}-07$ & Th-227 & $->$ & Ra-223 & & & \\
\hline 969.91 & $1.83 \mathrm{E}-07$ & Th-227 & $->$ & Ra-223 & & & \\
\hline 971.28 & $6.10 \mathrm{E}-08$ & Th-227 & $->$ & Ra-223 & & & \\
\hline 990.2 & $2.14 \mathrm{E}-07$ & Th-227 & $->$ & Ra-223 & & & \\
\hline 994.9 & $4.27 E-08$ & Th-227 & $->$ & $\mathrm{Ra}-223$ & & & \\
\hline 999.78 & $1.83 \mathrm{E}-07$ & Th-227 & $->$ & Ra-223 & & & \\
\hline 1014.48 & $1.09 \mathrm{E}-04$ & $\mathrm{~Pb}-211$ & $->$ & $\mathrm{Bi}-211$ & & & \\
\hline 1015.1 & $9.16 \mathrm{E}-08$ & Th-227 & $->$ & Ra-223 & & & \\
\hline 1020 & $1.22 \mathrm{E}-07$ & Th-227 & $->$ & Ra-223 & & & \\
\hline 1024.8 & $9.16 \mathrm{E}-08$ & Th-227 & $->$ & Ra-223 & & & \\
\hline
\end{tabular}




$\begin{array}{ccccc}1055.8 & 3.65 \mathrm{E}-06 & \mathrm{Rn}-219 & \rightarrow & \mathrm{Po}-215 \\ 1080.19 & 9.31 \mathrm{E}-05 & \mathrm{~Pb}-211 & -> & \mathrm{Bi}-211 \\ 1090.5 & 1.58 \mathrm{E}-05 & \mathrm{~Pb}-211 & -> & \mathrm{Bi}-211 \\ 1103.4 & 3.10 \mathrm{E}-05 & \mathrm{~Pb}-211 & -> & \mathrm{Bi}-211 \\ 1109.37 & 8.94 \mathrm{E}-04 & \mathrm{~Pb}-211 & -> & \mathrm{Bi}-211 \\ 1196.61 & 7.78 \mathrm{E}-05 & \mathrm{~Pb}-211 & -> & \mathrm{Bi}-211 \\ 1234.3 & 7.91 \mathrm{E}-06 & \mathrm{~Pb}-211 & -> & \mathrm{Bi}-211 \\ 1270.67 & 5.41 \mathrm{E}-05 & \mathrm{~Pb}-211 & -> & \mathrm{Bi}-211\end{array}$

\begin{tabular}{|c|c|c|c|c|c|c|c|}
\hline $235 U$ & $\begin{array}{c}\text { Aged } 5 \\
\text { years }\end{array}$ & \multicolumn{6}{|c|}{ Sorted by Intensity } \\
\hline Intensity & Energy & \multicolumn{6}{|c|}{$\begin{array}{l}\text { Initial Isotopes and } \\
\text { Decays }\end{array}$} \\
\hline $\mathrm{ph} / \mathrm{s} / \mathrm{gm}$ & keV & & & & & & \\
\hline $4.57 E+04$ & 185.72 & $U-235$ & $->$ & Th-231 & & & \\
\hline $3.92 E+04$ & 13.724 & Th-231 & $->$ & $\mathrm{Pa}-231$ & & & \\
\hline $2.96 \mathrm{E}+04$ & 16.559 & Th-231 & $->$ & $\mathrm{Pa}-231$ & & & \\
\hline $1.76 \mathrm{E}+04$ & 12.952 & Ac-227 & $->$ & Th-227, & $U-235$ & $->$ & Th-231 \\
\hline $1.20 \mathrm{E}+04$ & 16.119 & $U-235$ & $->$ & Th-231 & & & \\
\hline $1.17 \mathrm{E}+04$ & 25.642 & Th-231 & $->$ & $\mathrm{Pa}-231$ & & & \\
\hline $8.76 E+03$ & 143.76 & $U-235$ & $->$ & Th-231 & & & \\
\hline $5.92 E+03$ & 19.811 & Th-231 & $->$ & $\mathrm{Pa}-231$ & & & \\
\hline $5.28 \mathrm{E}+03$ & 84.203 & Th-231 & $->$ & $\mathrm{Pa}-231$ & & & \\
\hline $4.40 E+03$ & 93.36 & U-235 & $->$ & Th-231 & & & \\
\hline $4.06 E+03$ & 163.33 & $U-235$ & $->$ & Th-231 & & & \\
\hline $4.01 E+03$ & 205.31 & $U-235$ & $->$ & Th-231 & & & \\
\hline $2.69 E+03$ & 89.955 & $U-235$ & $->$ & Th-231 & & & \\
\hline $2.40 E+03$ & 11.372 & Th-231 & $->$ & $\mathrm{Pa}-231$ & & & \\
\hline $2.08 \mathrm{E}+03$ & 19.118 & U-235 & $->$ & Th-231 & & & \\
\hline $1.58 \mathrm{E}+03$ & 105.362 & $U-235$ & $->$ & Th-231 & & & \\
\hline $1.23 E+03$ & 109.16 & U-235 & $->$ & Th-231 & & & \\
\hline $8.64 \mathrm{E}+02$ & 202.11 & U-235 & $->$ & Th-231 & & & \\
\hline $8.00 \mathrm{E}+02$ & 11.118 & Ac- 227 & $->$ & Th-227, & U-235 & $->$ & Th-231 \\
\hline $7.52 \mathrm{E}+02$ & 89.954 & Th-231 & $->$ & $\mathrm{Pa}-231$ & & & \\
\hline $7.20 \mathrm{E}+02$ & 81.229 & Th-231 & $->$ & $\mathrm{Pa}-231$ & & & \\
\hline $5.28 \mathrm{E}+02$ & 108.99 & U-235 & $->$ & Th-231 & & & \\
\hline $5.04 \mathrm{E}+02$ & 95.863 & Th-231 & $->$ & $\mathrm{Pa}-231$ & & & \\
\hline $5.04 \mathrm{E}+02$ & 194.94 & U-235 & $->$ & Th-231 & & & \\
\hline $3.84 \mathrm{E}+02$ & 58.562 & Th-231 & $->$ & $\mathrm{Pa}-231$ & & & \\
\hline $3.28 \mathrm{E}+02$ & 102.255 & Th-231 & $->$ & $\mathrm{Pa}-231$ & & & \\
\hline $3.20 \mathrm{E}+02$ & 14.953 & Th-231 & $->$ & $\mathrm{Pa}-231$ & & & \\
\hline $3.12 \mathrm{E}+02$ & 92.279 & Th-231 & $->$ & $\mathrm{Pa}-231$ & & & \\
\hline $2.72 E+02$ & 182.61 & U-235 & $->$ & Th-231 & & & \\
\hline $2.01 E+02$ & 72.767 & Th-231 & $->$ & $\mathrm{Pa}-231$ & & & \\
\hline $1.82 \mathrm{E}+02$ & 108.166 & Th-231 & $->$ & $\mathrm{Pa}-231$ & & & \\
\hline $1.76 \mathrm{E}+02$ & 14.511 & Ac- 227 & $->$ & Th-227, & U-235 & $->$ & Th-231 \\
\hline $1.76 \mathrm{E}+02$ & 17.187 & Th-231 & $->$ & $\mathrm{Pa}-231$ & & & \\
\hline $1.76 \mathrm{E}+02$ & 140.76 & U-235 & $->$ & Th-231 & & & \\
\hline
\end{tabular}




\begin{tabular}{|c|c|c|c|c|}
\hline $1.24 \mathrm{E}+02$ & 163.114 & Th-231 & $->$ & $\mathrm{Pa}-231$ \\
\hline $9.60 \mathrm{E}+01$ & 99.28 & Th-231 & $->$ & $\mathrm{Pa}-231$ \\
\hline $9.60 \mathrm{E}+01$ & 221.38 & $U-235$ & $->$ & Th-231 \\
\hline $8.80 E+01$ & 72.71 & U-235 & $->$ & Th-231 \\
\hline $6.88 \mathrm{E}+01$ & 96.154 & $U-235$ & $->$ & Th-23 \\
\hline $6.40 E+01$ & 150.93 & $U-235$ & $->$ & Th-23 \\
\hline $6.24 \mathrm{E}+01$ & 135.681 & Th-231 & $->$ & $\mathrm{Pa}-23$ \\
\hline $6.08 \mathrm{E}+01$ & 111.897 & Th-231 & $->$ & $\mathrm{Pa}-23$ \\
\hline $5.60 E+01$ & 116.11 & U-235 & $->$ & Th-23 \\
\hline $4.80 E+01$ & 74.8 & U-235 & $->$ & Th-23 \\
\hline $4.80 E+01$ & 41.96 & U-235 & $->$ & Th-23 \\
\hline $4.64 \mathrm{E}+01$ & 42.828 & Th-231 & $->$ & $\mathrm{Pa}-23$ \\
\hline $4.48 \mathrm{E}+01$ & 124.922 & Th-231 & $->$ & $\mathrm{Pa}-23$ \\
\hline $4.32 E+01$ & 240.85 & U-235 & $->$ & Th-23 \\
\hline $4.24 \mathrm{E}+01$ & 246.84 & U-235 & $->$ & Th-2: \\
\hline $3.92 E+01$ & 93.07 & Th-231 & $->$ & $\mathrm{Pa}-2$ \\
\hline $3.36 \mathrm{E}+01$ & 198.9 & $U-235$ & $->$ & Th-2: \\
\hline $3.20 E+01$ & 217.934 & Th-231 & $->$ & $\mathrm{Pa}-23$ \\
\hline $2.63 E+01$ & 183.483 & Th-231 & $->$ & $\mathrm{Pa}-2$ \\
\hline $2.56 \mathrm{E}+01$ & 145.927 & Th-231 & $->$ & $\mathrm{Pa}-23$ \\
\hline $2.40 \mathrm{E}+01$ & 41.13 & U-235 & $->$ & Th-23 \\
\hline $2.32 E+01$ & 233.5 & $U-235$ & $->$ & Th-23 \\
\hline $2.32 E+01$ & 345.921 & $U-235$ & $->$ & Th-23 \\
\hline $2.16 \mathrm{E}+01$ & 215.305 & U-235 & $->$ & Th-23 \\
\hline $2.08 \mathrm{E}+01$ & 119.98 & $U-235$ & $->$ & Th-23 \\
\hline $1.92 \mathrm{E}+01$ & 134.014 & Th-231 & $->$ & $\mathrm{Pa}-2$ \\
\hline $1.84 \mathrm{E}+01$ & 63.834 & Th-231 & $->$ & $\mathrm{Pa}-2$ \\
\hline $1.66 \mathrm{E}+01$ & 116.827 & Th-231 & $->$ & $\mathrm{Pa}-2$ \\
\hline $1.60 \mathrm{E}+01$ & 64.348 & U-235 & $->$ & Th-2: \\
\hline $1.60 \mathrm{E}+01$ & 51.179 & $U-235$ & $->$ & Th-2: \\
\hline $1.46 \mathrm{E}+01$ & 174.157 & Th-231 & $->$ & $\mathrm{Pa}-2$ \\
\hline $1.36 \mathrm{E}+01$ & 106.581 & Th-231 & $->$ & $\mathrm{Pa}-2$ \\
\hline $1.28 \mathrm{E}+01$ & 31.585 & U-235 & $->$ & Th-23 \\
\hline $1.12 \mathrm{E}+01$ & 291.72 & $U-235$ & $->$ & Th-23 \\
\hline $9.60 \mathrm{E}+00$ & 136.72 & U-235 & $->$ & Th-23 \\
\hline $8.00 E+00$ & 172.27 & $U-235$ & $->$ & Th-23 \\
\hline $7.36 \mathrm{E}+00$ & 235.985 & Th-231 & $->$ & $\mathrm{Pa}-23$ \\
\hline $6.40 E+00$ & 228.77 & U-235 & $->$ & Th-23 \\
\hline $5.68 \mathrm{E}+00$ & 105.803 & Th-231 & $->$ & $\mathrm{Pa}-2$ \\
\hline $5.60 E+00$ & 289.57 & U-235 & $->$ & Th-2: \\
\hline $4.80 E+00$ & 281.45 & U-235 & $->$ & Th-23 \\
\hline $4.80 E+00$ & 266.483 & U-235 & $->$ & Th-23 \\
\hline $4.64 \mathrm{E}+00$ & 145.063 & Th-231 & $->$ & $\mathrm{Pa}-23$ \\
\hline $4.56 \mathrm{E}+00$ & 68.5 & Th-231 & $->$ & $\mathrm{Pa}-23$ \\
\hline $4.00 E+00$ & 301.72 & U-235 & $->$ & Th-23 \\
\hline $4.00 E+00$ & 282.98 & U-235 & $->$ & Th-2: \\
\hline $4.00 \mathrm{E}+00$ & 356.068 & U-235 & $->$ & Th-23 \\
\hline $3.92 E+00$ & 275.47 & U-235 & $->$ & Th-23 \\
\hline $3.36 \mathrm{E}+00$ & 136.705 & Th-231 & $->$ & $\mathrm{Pa}-23$ \\
\hline $3.20 E+00$ & 310.71 & U-235 & $->$ & Th-23 \\
\hline
\end{tabular}




$\begin{array}{cc}3.12 \mathrm{E}+00 & 164.97 \\ 3.04 \mathrm{E}+00 & 387.874 \\ 2.56 \mathrm{E}+00 & 188.756 \\ 2.40 \mathrm{E}+00 & 410.269 \\ 2.40 \mathrm{E}+00 & 343.78 \\ 2.32 \mathrm{E}+00 & 311 \\ 2.11 \mathrm{E}+00 & 15.69 \\ 1.86 \mathrm{E}+00 & 12.636 \\ 9.60 \mathrm{E}-01 & 169.637 \\ 9.28 \mathrm{E}-01 & 267.62 \\ 8.00 \mathrm{E}-01 & 115.595 \\ 8.00 \mathrm{E}-01 & 317.12 \\ 8.00 \mathrm{E}-01 & 390.32 \\ 8.00 \mathrm{E}-01 & 448.42 \\ 7.85 \mathrm{E}-01 & 27.396 \\ 6.72 \mathrm{E}-01 & 242.508 \\ 6.40 \mathrm{E}-01 & 455.12 \\ 6.24 \mathrm{E}-01 & 249.56 \\ 5.68 \mathrm{E}-01 & 140.537 \\ 5.60 \mathrm{E}-01 & 44.06 \\ 5.20 \mathrm{E}-01 & 250.43 \\ 4.98 \mathrm{E}-01 & 18.607 \\ 4.80 \mathrm{E}-01 & 794.72 \\ 3.20 \mathrm{E}-01 & 517.2 \\ 3.20 \mathrm{E}-01 & 742.52 \\ 3.12 \mathrm{E}-01 & 308.75 \\ 2.24 \mathrm{E}-01 & 240.25 \\ 2.01 \mathrm{E}-01 & 300.07 \\ 1.53 \mathrm{E}-01 & 83.787 \\ 1.44 \mathrm{E}-01 & 302.671 \\ 1.34 \mathrm{E}-01 & 283.673 \\ 1.28 \mathrm{E}-01 & 15.218 \\ 1.11 \mathrm{E}-01 & 330.066 \\ 1.05 \mathrm{E}-01 & 12.325 \\ 1.01 \mathrm{E}-01 & 10.871 \\ 9.24 \mathrm{E}-02 & 81.067 \\ 8.80 \mathrm{E}-02 & 320.188 \\ 8.27 \mathrm{E}-02 & 269.39 \\ 7.76 \mathrm{E}-02 & 350.1 \\ 6.84 \mathrm{E}-02 & 235.97 \\ 6.59 \mathrm{E}-02 & 90.886 \\ 6.40 \mathrm{E}-02 & 317.93 \\ 6.20 \mathrm{E}-02 & 11.713 \\ 6.02 \mathrm{E}-02 & 271.13 \\ 5.96 \mathrm{E}-02 & 14.341 \\ 5.60 \mathrm{E}-02 & 351.82 \\ 5.48 \mathrm{E}-02 & 50.14 \\ 5.41 \mathrm{E}-02 & 94.677 \\ 5.41 \mathrm{E}-02 & 302.638 \\ 4.09 \mathrm{E}-02 & 256.24\end{array}$

\begin{tabular}{|c|c|c|c|c|c|}
\hline Th-231 & $->$ & $\mathrm{Pa}-231$ & & & \\
\hline U-235 & $->$ & Th-231 & & & \\
\hline Th-231 & $->$ & $\mathrm{Pa}-231$ & & & \\
\hline U-235 & $->$ & Th-231 & & & \\
\hline$U-235$ & $->$ & Th-231 & & & \\
\hline Th-231 & $->$ & $\mathrm{Pa}-231$ & & & \\
\hline $\mathrm{Pa}-231$ & $->$ & Ac- 227 & & & \\
\hline Pa-231 & $->$ & Ac- 227 & & & \\
\hline Th-231 & $->$ & $\mathrm{Pa}-231$ & & & \\
\hline Th-231 & $->$ & Pa-231 & & & \\
\hline Th-231 & $->$ & $\mathrm{Pa}-231$ & & & \\
\hline U-235 & $->$ & Th-231 & & & \\
\hline$U-235$ & $->$ & Th-231 & & & \\
\hline$U-235$ & $->$ & Th-231 & & & \\
\hline Pa-231 & $->$ & Ac- 227 & & & \\
\hline Th-231 & $->$ & Pa-231 & & & \\
\hline U-235 & $->$ & Th-231 & & & \\
\hline Th-231 & $->$ & $\mathrm{Pa}-231$ & & & \\
\hline Th-231 & $->$ & Pa-231 & & & \\
\hline Th-231 & $->$ & $\mathrm{Pa}-231$ & & & \\
\hline Th-231 & $->$ & Pa-231 & & & \\
\hline Pa-231 & $->$ & Ac- 227 & & & \\
\hline U-235 & $->$ & Th-231 & & & \\
\hline$U-235$ & $->$ & Th-231 & & & \\
\hline U-235 & $->$ & Th-231 & & & \\
\hline Th-231 & $->$ & $\mathrm{Pa}-231$ & & & \\
\hline Th-231 & $->$ & $\mathrm{Pa}-231$ & & & \\
\hline Pa-231 & $->$ & $A c-227$ & & & \\
\hline Ra-223 & $->$ & $\mathrm{Rn}-219$ & & & \\
\hline Pa-231 & $->$ & Ac- 227 & & & \\
\hline Pa-231 & $->$ & Ac- 227 & & & \\
\hline Th-227 & $->$ & Ra-223 & & & \\
\hline Pa-231 & $->$ & Ac- 227 & & & \\
\hline Fr-223 & $->$ & Ra-223, & Th-227 & $->$ & Ra-223 \\
\hline $\mathrm{Pa}-231$ & $->$ & $A c-227$ & & & \\
\hline Ra-223 & $->$ & $\mathrm{Rn}-219$ & & & \\
\hline Th-231 & $->$ & $\mathrm{Pa}-231$ & & & \\
\hline Ra-223 & $->$ & $\mathrm{Rn}-219$ & & & \\
\hline $\mathrm{Bi}-211$ & $->$ & TI-207 & & & \\
\hline Fr-223 & $->$ & Ra-223, & Th-227 & $->$ & $\mathrm{Ra}-223$ \\
\hline Pa-231 & $->$ & Ac-227 & & & \\
\hline Th-231 & $->$ & $\mathrm{Pa}-231$ & & & \\
\hline Ra-223 & $->$ & $R n-219$ & & & \\
\hline$R n-219$ & $->$ & Po-215 & & & \\
\hline Ra-223 & $->$ & $\mathrm{Rn}-219$ & & & \\
\hline Th-231 & $->$ & $\mathrm{Pa}-231$ & & & \\
\hline Fr-223 & $->$ & Ra-223, & Th-227 & $->$ & $\mathrm{Ra}-223$ \\
\hline Ra-223 & $->$ & $\mathrm{Rn}-219$ & & & \\
\hline Pa-231 & $->$ & Ac-227 & & & \\
\hline $\mathrm{Fr}-223$ & $->$ & Ra-223, & Th-227 & $->$ & $\mathrm{Ra}-223$ \\
\hline
\end{tabular}




\begin{tabular}{|c|c|c|c|c|c|c|c|}
\hline 4.04E-02 & 401.7 & Rn-219 & $->$ & Po-215 & & & \\
\hline $4.02 \mathrm{E}-02$ & 87.673 & $\mathrm{~Pa}-231$ & $->$ & Ac- 227 & & & \\
\hline $3.40 \mathrm{E}-02$ & 154.18 & Ra-223 & $->$ & $\mathrm{Rn}-219$ & & & \\
\hline $3.21 \mathrm{E}-02$ & 16.397 & $\mathrm{~Pa}-231$ & $->$ & $A c-227$ & & & \\
\hline $3.12 \mathrm{E}-02$ & 14.082 & $\mathrm{~Pa}-231$ & $->$ & Ac- 227 & & & \\
\hline $2.93 \mathrm{E}-02$ & 17.997 & Th-227 & $->$ & Ra-223 & & & \\
\hline 2.79E-02 & 18.998 & Pa-231 & $->$ & Ac- 227 & & & \\
\hline $2.40 \mathrm{E}-02$ & 274.14 & Th-231 & $->$ & $\mathrm{Pa}-231$ & & & \\
\hline $2.37 \mathrm{E}-02$ & 323.88 & $\mathrm{Ra}-223$ & $->$ & $\mathrm{Rn}-219$ & & & \\
\hline $2.36 \mathrm{E}-02$ & 102.613 & $\mathrm{~Pa}-231$ & $->$ & $A c-227$ & & & \\
\hline $2.33 \mathrm{E}-02$ & 404.86 & $\mathrm{~Pb}-211$ & $->$ & $\mathrm{Bi}-211$, & At-215 & $->$ & $\mathrm{Bi}-211$ \\
\hline $2.32 \mathrm{E}-02$ & 831.86 & $\mathrm{~Pb}-211$ & $->$ & $\mathrm{Bi}-211$ & & & \\
\hline $1.98 \mathrm{E}-02$ & 144.18 & $\mathrm{Ra}-223$ & $->$ & $\mathrm{Rn}-219$ & & & \\
\hline 1.92E-02 & 88.471 & Fr-223 & $->$ & Ra-223, & Th-227 & $->$ & Ra-223 \\
\hline $1.76 \mathrm{E}-02$ & 46.393 & $\mathrm{~Pa}-231$ & $->$ & Ac- 227 & & & \\
\hline $1.73 \mathrm{E}-02$ & 97.907 & $\mathrm{Ra}-223$ & $->$ & $\mathrm{Rn}-219$ & & & \\
\hline $1.69 \mathrm{E}-02$ & 338.28 & $\mathrm{Ra}-223$ & $->$ & $\mathrm{Rn}-219$ & & & \\
\hline $1.67 \mathrm{E}-02$ & 329.84 & Fr-223 & $->$ & Ra-223, & Th-227 & $->$ & Ra-223 \\
\hline $1.54 \mathrm{E}-02$ & 260.29 & $\mathrm{~Pa}-231$ & $->$ & Ac- 227 & & & \\
\hline $1.47 \mathrm{E}-02$ & 340.873 & $\mathrm{~Pa}-231$ & $->$ & Ac- 227 & & & \\
\hline $1.36 \mathrm{E}-02$ & 79.72 & Fr-223 & $->$ & Ra-223, & Th-227 & $->$ & $\mathrm{Ra}-223$ \\
\hline $1.30 \mathrm{E}-02$ & 357.2 & $\mathrm{~Pa}-231$ & $->$ & $A c-227$ & & & \\
\hline $1.28 \mathrm{E}-02$ & 299.97 & Fr-223 & $->$ & Ra-223, & Th-227 & $->$ & Ra-223 \\
\hline $1.26 \mathrm{E}-02$ & 38.235 & $\mathrm{~Pa}-231$ & $->$ & Ac- 227 & & & \\
\hline $1.18 \mathrm{E}-02$ & 16.918 & $\mathrm{Ra}-223$ & $->$ & $\mathrm{Rn}-219$ & & & \\
\hline $1.16 \mathrm{E}-02$ & 85.429 & Fr-223 & $->$ & Ra-223, & Th-227 & $->$ & $\mathrm{Ra}-223$ \\
\hline $1.05 \mathrm{E}-02$ & 427 & $\mathrm{~Pb}-211$ & $->$ & $\mathrm{Bi}-211$ & & & \\
\hline $9.71 \mathrm{E}-03$ & 286.11 & Fr-223 & $->$ & $\mathrm{Ra}-223$, & Th-227 & $->$ & Ra-223 \\
\hline $9.54 \mathrm{E}-03$ & 313.058 & $\mathrm{~Pa}-231$ & $->$ & $A c-227$ & & & \\
\hline $8.55 E-03$ & 93.93 & Th-227 & $->$ & Ra-223 & & & \\
\hline $8.45 E-03$ & 255.858 & $\mathrm{~Pa}-231$ & $->$ & Ac- 227 & & & \\
\hline 7.77E-03 & 29.996 & $\mathrm{~Pa}-231$ & $->$ & Ac- 227 & & & \\
\hline 7.77E-03 & 106.137 & $\mathrm{~Pa}-231$ & $->$ & Ac- 227 & & & \\
\hline 7.72E-03 & 444.94 & $\mathrm{Ra}-223$ & $->$ & $\mathrm{Rn}-219$ & & & \\
\hline 7.70E-03 & 72.873 & $\mathrm{Bi}-211$ & $->$ & TI-207 & & & \\
\hline 7.60E-03 & 25.464 & $\mathrm{~Pa}-231$ & $->$ & $A c-227$ & & & \\
\hline $7.43 E-03$ & 96.979 & $\mathrm{~Pa}-231$ & $->$ & Ac- 227 & & & \\
\hline 7.24E-03 & 122.31 & Ra-223 & $->$ & $\mathrm{Rn}-219$ & & & \\
\hline $7.18 \mathrm{E}-03$ & 52.763 & $\mathrm{~Pa}-231$ & $->$ & Ac- 227 & & & \\
\hline $6.90 \mathrm{E}-03$ & 210.58 & Fr-223 & $->$ & Ra-223, & Th-227 & $->$ & Ra-223 \\
\hline $6.84 \mathrm{E}-03$ & 54.632 & Pa-231 & $->$ & Ac- 227 & & & \\
\hline $6.84 \mathrm{E}-03$ & 99.915 & Fr-223 & $->$ & Ra-223, & Th-227 & $->$ & $\mathrm{Ra}-223$ \\
\hline $6.76 \mathrm{E}-03$ & 354.63 & $\mathrm{~Pa}-231$ & $->$ & $A c-227$ & & & \\
\hline $6.65 \mathrm{E}-03$ & 304.51 & Fr-223 & $->$ & Ra-223, & Th-227 & $->$ & Ra-223 \\
\hline $6.06 \mathrm{E}-03$ & 10.622 & Fr-223 & $->$ & Ra-223, & Th-227 & $->$ & Ra-223 \\
\hline $6.04 \mathrm{E}-03$ & 334.38 & Fr-223 & $->$ & Ra-223, & Th-227 & $->$ & Ra-223 \\
\hline $5.74 \mathrm{E}-03$ & 77.405 & $\mathrm{~Pa}-231$ & $->$ & Ac- 227 & & & \\
\hline $5.29 E-03$ & 79.29 & $\mathrm{Rn}-219$ & $->$ & Po-215 & & & \\
\hline $5.24 \mathrm{E}-03$ & 277.17 & $\mathrm{~Pa}-231$ & $->$ & $A c-227$ & & & \\
\hline 5.07E-03 & 44.213 & $\mathrm{~Pa}-231$ & $->$ & Ac- 227 & & & \\
\hline
\end{tabular}




$\begin{array}{lc}4.88 \mathrm{E}-03 & 254.66 \\ 4.81 \mathrm{E}-03 & 273.17 \\ 4.55 \mathrm{E}-03 & 70.832 \\ 4.32 \mathrm{E}-03 & 766.35 \\ 4.22 \mathrm{E}-03 & 63.7 \\ 4.18 \mathrm{E}-03 & 158.59 \\ 3.97 \mathrm{E}-03 & 243.17 \\ 3.89 \mathrm{E}-03 & 379.4 \\ 3.42 \mathrm{E}-03 & 113.19 \\ 3.35 \mathrm{E}-03 & 10.137 \\ 3.16 \mathrm{E}-03 & 76.858 \\ 3.01 \mathrm{E}-03 & 234.82 \\ 2.99 \mathrm{E}-03 & 272.95 \\ 2.98 \mathrm{E}-03 & 371.8 \\ 2.93 \mathrm{E}-03 & 704.51 \\ 2.87 \mathrm{E}-03 & 407.98 \\ 2.87 \mathrm{E}-03 & 312.57 \\ 2.81 \mathrm{E}-03 & 314.82 \\ 2.74 \mathrm{E}-03 & 13.495 \\ 2.71 \mathrm{E}-03 & 82.434 \\ 2.70 \mathrm{E}-03 & 100.96 \\ 2.66 \mathrm{E}-03 & 10.259 \\ 2.62 \mathrm{E}-03 & 296.54 \\ 2.62 \mathrm{E}-03 & 77.107 \\ 2.54 \mathrm{E}-03 & 327.23 \\ 2.53 \mathrm{E}-03 & 24.56 \\ 2.49 \mathrm{E}-03 & 11.119 \\ 2.49 \mathrm{E}-03 & 13.082 \\ 2.43 \mathrm{E}-03 & 12.313 \\ 2.32 \mathrm{E}-03 & 342.44 \\ 2.26 \mathrm{E}-03 & 250.12 \\ 2.25 \mathrm{E}-03 & 10.828 \\ 2.23 \mathrm{E}-03 & 103.341 \\ 2.22 \mathrm{E}-03 & 13.662 \\ 2.11 \mathrm{E}-03 & 74.209 \\ 2.11 \mathrm{E}-03 & 57.233 \\ 1.89 \mathrm{E}-03 & 89.639 \\ 1.86 \mathrm{E}-03 & 341.17 \\ 1.67 \mathrm{E}-03 & 15.213 \\ 1.56 \mathrm{E}-03 & 74.814 \\ 1.46 \mathrm{E}-03 & 62.36 \\ 1.46 \mathrm{E}-03 & 897.23 \\ 1.40 \mathrm{E}-03 & 204.2 \\ 1.40 \mathrm{E}-03 & 206.04 \\ 1.40 \mathrm{E}-03 & 43.73 \\ 1.35 \mathrm{E}-03 & 35.884 \\ 1.29 \mathrm{E}-03 & 20.27 \\ 1.29 \mathrm{E}-03 & 49.85 \\ 1.27 \mathrm{E}-03 & 57.2 \\ 1.22 \mathrm{E}-03 & 300.34\end{array}$

\begin{tabular}{|c|c|c|c|c|c|}
\hline Fr-223 & $->$ & $\mathrm{Ra}-223$, & Th-227 & $->$ & Ra-223 \\
\hline $\mathrm{Pa}-231$ & $->$ & $A c-227$ & & & \\
\hline $\mathrm{Bi}-211$ & $->$ & TI-207 & & & \\
\hline $\mathrm{Pb}-211$ & $->$ & $\mathrm{Bi}-211$ & & & \\
\hline Pa-231 & $->$ & $A c-227$ & & & \\
\hline Ra-223 & $->$ & $R n-219$ & & & \\
\hline Pa-231 & $->$ & $A c-227$ & & & \\
\hline $\mathrm{Rn}-219$ & $->$ & Po-215, & $\mathrm{Pa}-231$ & $->$ & $A c-227$ \\
\hline Th-227 & $->$ & $\mathrm{Ra}-223$ & & & \\
\hline Ra-223 & $->$ & $R n-219$ & & & \\
\hline $\mathrm{Rn}-219$ & $->$ & Po-215 & & & \\
\hline Fr-223 & $->$ & Ra-223, & Th-227 & $->$ & $\mathrm{Ra}-223$ \\
\hline Fr-223 & $->$ & Ra-223, & Th-227 & $->$ & Ra-223 \\
\hline Ra-223 & $->$ & Rn-219 & & & \\
\hline $\mathrm{Pb}-211$ & $->$ & $\mathrm{Bi}-211$ & & & \\
\hline Pa-231 & $->$ & Ac- 227 & & & \\
\hline $\mathrm{Fr}-223$ & $->$ & Ra-223, & Th-227 & $->$ & $\mathrm{Ra}-223$ \\
\hline Th-227 & $->$ & Ra-223 & & & \\
\hline $\mathrm{Rn}-219$ & $->$ & Po-215 & & & \\
\hline $\mathrm{Bi}-211$ & $->$ & TI-207 & & & \\
\hline $\mathrm{Pa}-231$ & $->$ & $A c-227$ & & & \\
\hline $\mathrm{Bi}-211$ & $->$ & TI-207 & & & \\
\hline Th-227 & $->$ & Ra-223 & & & \\
\hline $\mathrm{Pb}-211$ & $->$ & $\mathrm{Bi}-211$ & & & \\
\hline $\mathrm{Pa}-231$ & $->$ & $A c-227$ & & & \\
\hline $\mathrm{Pa}-231$ & $->$ & $A c-227$ & & & \\
\hline $\mathrm{Rn}-219$ & $->$ & Po-215 & & & \\
\hline $\mathrm{Pb}-211$ & $->$ & $\mathrm{Bi}-211$ & & & \\
\hline $\mathrm{Bi}-211$ & $->$ & TI-207 & & & \\
\hline Fr-223 & $->$ & Ra-223, & Th-227 & $->$ & $\mathrm{Ra}-223$ \\
\hline Fr-223 & $->$ & Ra-223, & Th-227 & $->$ & $\mathrm{Ra}-223$ \\
\hline $\mathrm{Pb}-211$ & $->$ & $\mathrm{Bi}-211$ & & & \\
\hline Fr-223 & $->$ & Ra-223, & Th-227 & $->$ & $\mathrm{Ra}-223$ \\
\hline $\mathrm{Fr}-223$ & $->$ & Ra-223, & Th-227 & $->$ & $\mathrm{Ra}-223$ \\
\hline $\mathrm{Pa}-231$ & $->$ & $A c-227$ & & & \\
\hline Pa-231 & $->$ & $A c-227$ & & & \\
\hline $\mathrm{Rn}-219$ & $->$ & Po-215 & & & \\
\hline $\mathrm{Pa}-231$ & $->$ & $A c-227$ & & & \\
\hline $\mathrm{Fr}-223$ & $->$ & Ra-223 & & & \\
\hline $\mathrm{Pb}-211$ & $->$ & $\mathrm{Bi}-211$ & & & \\
\hline Th-227 & $->$ & Ra-223 & & & \\
\hline TI-207 & $->$ & $\mathrm{Pb}-207$ & Po-211 & $->$ & $\mathrm{Pb}-207$ \\
\hline Fr-223 & $->$ & Ra-223, & Th-227 & $->$ & $\mathrm{Ra}-223$ \\
\hline Fr-223 & $->$ & Ra-223, & Th-227 & $->$ & Ra-223 \\
\hline Fr-223 & $->$ & Ra-223, & Th-227 & $->$ & $\mathrm{Ra}-223$ \\
\hline $\mathrm{Pa}-231$ & $->$ & $A c-227$ & & & \\
\hline Fr-223 & $->$ & Ra-223, & Th-227 & $->$ & Ra-223 \\
\hline Fr-223 & $->$ & Ra-223, & Th-227 & $->$ & $\mathrm{Ra}-223$ \\
\hline Pa-231 & $->$ & $A c-227$ & & & \\
\hline Th-227 & $->$ & Ra-223 & & & \\
\hline
\end{tabular}




\begin{tabular}{|c|c|c|c|c|c|c|c|}
\hline $1.22 \mathrm{E}-03$ & 342.9 & $\mathrm{Ra}-223$ & $->$ & $\mathrm{Rn}-219$ & & & \\
\hline $1.20 \mathrm{E}-03$ & 328.49 & $\mathrm{Ra}-223$ & $->$ & $\mathrm{Rn}-219$ & & & \\
\hline $1.04 \mathrm{E}-03$ & 117.17 & Th-227 & $->$ & Ra-223 & & & \\
\hline $1.01 \mathrm{E}-03$ & 40.006 & $\mathrm{~Pa}-231$ & $->$ & $A C-227$ & & & \\
\hline $9.77 \mathrm{E}-04$ & 281.31 & Th-227 & $->$ & Ra-223 & & & \\
\hline $9.42 \mathrm{E}-04$ & 16.074 & Ac- 227 & $->$ & Th-227 & & & \\
\hline $9.37 \mathrm{E}-04$ & 288.15 & Ra-223 & $->$ & $\mathrm{Rn}-219$ & & & \\
\hline $9.31 \mathrm{E}-04$ & 179.69 & Ra-223 & $->$ & $\mathrm{Rn}-219$ & & & \\
\hline $9.29 \mathrm{E}-04$ & 56.9 & $\mathrm{~Pa}-231$ & $->$ & $A c-227$ & & & \\
\hline $9.24 \mathrm{E}-04$ & 87.19 & $\mathrm{~Pb}-211$ & $->$ & $\mathrm{Bi}-211$ & & & \\
\hline $9.17 \mathrm{E}-04$ & 204.95 & Fr-223 & $->$ & Ra-223, & Th-227 & $->$ & Ra-223 \\
\hline $9.16 \mathrm{E}-04$ & 113.12 & Th-227 & $->$ & $\mathrm{Ra}-223$ & & & \\
\hline 8.94E-04 & 1109.37 & $\mathrm{~Pb}-211$ & $->$ & $\mathrm{Bi}-211$ & & & \\
\hline 8.82E-04 & 12.855 & Ra-223 & $->$ & Rn-219 & & & \\
\hline 7.94E-04 & 250.33 & Th-227 & $->$ & Ra-223 & & & \\
\hline 7.94E-04 & 141.44 & Th-227 & $->$ & Ra-223 & & & \\
\hline 7.85E-04 & 398.4 & $\mathrm{~Pa}-231$ & $->$ & Ac- 227 & & & \\
\hline $7.66 \mathrm{E}-04$ & 130.57 & $\mathrm{Rn}-219$ & $->$ & Po-215 & & & \\
\hline 7.64E-04 & 85.185 & $\mathrm{Bi}-211$ & $->$ & TI-207 & & & \\
\hline $7.60 \mathrm{E}-04$ & 31.04 & $\mathrm{~Pa}-231$ & $->$ & Ac- 227 & & & \\
\hline 7.60E-04 & 359.51 & Pa-231 & $->$ & Ac- 227 & & & \\
\hline $6.76 \mathrm{E}-04$ & 242 & $\mathrm{~Pa}-231$ & $->$ & Ac- 227 & & & \\
\hline $6.71 \mathrm{E}-04$ & 252.46 & Th-227 & $->$ & Ra-223 & & & \\
\hline $6.71 \mathrm{E}-04$ & 350.48 & Th-227 & $->$ & Ra-223 & & & \\
\hline $6.25 \mathrm{E}-04$ & 364.03 & $\mathrm{~Pa}-231$ & $->$ & $A c-227$ & & & \\
\hline $6.13 \mathrm{E}-04$ & 29.869 & Fr-223 & $->$ & Ra-223, & Th-227 & $->$ & Ra-223 \\
\hline $6.13 \mathrm{E}-04$ & 100.31 & Fr-223 & $->$ & Ra-223, & Th-227 & $->$ & Ra-223 \\
\hline $6.11 \mathrm{E}-04$ & 262.72 & Fr-223 & $->$ & Ra-223, & Th-227 & $->$ & Ra-223 \\
\hline $6.02 \mathrm{E}-04$ & 333.87 & Ra-223 & $->$ & $R n-219$ & & & \\
\hline $5.91 \mathrm{E}-04$ & 245.438 & Pa-231 & $->$ & $A c-227$ & & & \\
\hline 5.91E-04 & 60.526 & $\mathrm{~Pa}-231$ & $->$ & Ac- 227 & & & \\
\hline 5.91E-04 & 70.54 & $\mathrm{~Pa}-231$ & $->$ & $A c-227$ & & & \\
\hline $5.91 \mathrm{E}-04$ & 31.58 & $\mathrm{~Pa}-231$ & $->$ & Ac- 227 & & & \\
\hline $5.91 \mathrm{E}-04$ & 43.09 & Pa-231 & $->$ & Ac- 227 & & & \\
\hline $5.83 E-04$ & 245.77 & $\mathrm{~Pa}-231$ & $->$ & Ac- 227 & & & \\
\hline $5.74 \mathrm{E}-04$ & 391.85 & $\mathrm{~Pa}-231$ & $->$ & Ac- 227 & & & \\
\hline $5.72 \mathrm{E}-04$ & 92.673 & $\mathrm{Rn}-219$ & $->$ & Po-215 & & & \\
\hline $5.53 \mathrm{E}-04$ & 15.856 & $\mathrm{Rn}-219$ & $->$ & Po-215 & & & \\
\hline $5.47 E-04$ & 598.69 & $\mathrm{Ra}-223$ & $->$ & $\mathrm{Rn}-219$ & & & \\
\hline 5.47E-04 & 15.397 & $\mathrm{~Pb}-211$ & $->$ & $\mathrm{Bi}-211$ & & & \\
\hline 5.07E-04 & 56.8 & $\mathrm{~Pa}-231$ & $->$ & Ac- 227 & & & \\
\hline 5.07E-04 & 42.52 & $\mathrm{~Pa}-231$ & $->$ & Ac- 227 & & & \\
\hline 4.88E-04 & 61.435 & Th-227 & $->$ & Ra-223 & & & \\
\hline 4.88E-04 & 31.566 & Th-227 & $->$ & Ra-223 & & & \\
\hline 4.73E-04 & 198.96 & $\mathrm{~Pa}-231$ & $->$ & Ac- 227 & & & \\
\hline $4.68 \mathrm{E}-04$ & 65.516 & $\mathrm{~Pb}-211$ & $->$ & $\mathrm{Bi}-211$ & & & \\
\hline 4.43E-04 & 14.407 & $\mathrm{Bi}-211$ & $->$ & TI-207 & & & \\
\hline $4.32 \mathrm{E}-04$ & 527.3 & $\mathrm{Ra}-223$ & $->$ & $\mathrm{Rn}-219$ & & & \\
\hline $4.27 \mathrm{E}-04$ & 279.7 & Fr-223 & $->$ & Ra-223, & Th-227 & $->$ & Ra-223 \\
\hline 4.27E-04 & 212.56 & Th-227 & $->$ & $\mathrm{Ra}-223$ & & & \\
\hline
\end{tabular}




\begin{tabular}{|c|c|c|c|c|c|c|c|}
\hline $4.22 \mathrm{E}-04$ & 124.69 & $\mathrm{~Pa}-231$ & $->$ & $A c-227$ & & & \\
\hline $3.95 \mathrm{E}-04$ & 293.8 & $\mathrm{Rn}-219$ & $->$ & Po-215 & & & \\
\hline $3.83 \mathrm{E}-04$ & 609.08 & $\mathrm{Ra}-223$ & $->$ & $\mathrm{Rn}-219$ & & & \\
\hline $3.78 \mathrm{E}-04$ & 18.016 & Fr-223 & $->$ & Ra-223 & & & \\
\hline $3.67 \mathrm{E}-04$ & 292.3 & Fr-223 & $->$ & Ra-223, & Th-227 & $->$ & $\mathrm{Ra}-223$ \\
\hline $3.66 \mathrm{E}-04$ & 218.77 & Th-227 & $->$ & $\mathrm{Ra}-223$ & & & \\
\hline $3.66 \mathrm{E}-04$ & 96.06 & Th-227 & $->$ & Ra-223 & & & \\
\hline $3.47 E-04$ & 83.81 & $\mathrm{~Pb}-211$ & $->$ & $\mathrm{Bi}-211$ & & & \\
\hline $3.46 \mathrm{E}-04$ & 438.24 & $\mathrm{~Pa}-231$ & $->$ & Ac- 227 & & & \\
\hline $3.46 \mathrm{E}-04$ & 384.97 & $\mathrm{~Pa}-231$ & $->$ & Ac- 227 & & & \\
\hline $3.38 E-04$ & 72.57 & $\mathrm{~Pa}-231$ & $->$ & Ac- 227 & & & \\
\hline $3.38 E-04$ & 144.5 & $\mathrm{~Pa}-231$ & $->$ & Ac- 227 & & & \\
\hline $3.36 \mathrm{E}-04$ & 285.48 & Th-227 & $->$ & Ra-223 & & & \\
\hline $3.05 E-04$ & 284.24 & Fr-223 & $->$ & Ra-223, & Th-227 & $->$ & $\mathrm{Ra}-223$ \\
\hline $3.05 E-04$ & 43.53 & Th-227 & $->$ & $\mathrm{Ra}-223$ & & & \\
\hline $2.93 \mathrm{E}-04$ & 383.47 & Th-227 & $->$ & Ra-223 & & & \\
\hline $2.92 \mathrm{E}-04$ & 110.8 & $\mathrm{Ra}-223$ & $->$ & $\mathrm{Rn}-219$ & & & \\
\hline $2.86 \mathrm{E}-04$ & 177.35 & $\mathrm{Ra}-223$ & $->$ & $\mathrm{Rn}-219$ & & & \\
\hline $2.79 E-04$ & 435.38 & $\mathrm{~Pa}-231$ & $->$ & $A c-227$ & & & \\
\hline $2.79 E-04$ & 277.43 & $\mathrm{~Pa}-231$ & $->$ & Ac- 227 & & & \\
\hline $2.74 \mathrm{E}-04$ & 90.128 & $\mathrm{~Pb}-211$ & $->$ & $\mathrm{Bi}-211$ & & & \\
\hline $2.74 \mathrm{E}-04$ & 81 & $\mathrm{~Pb}-211$ & $->$ & $\mathrm{Bi}-211$ & & & \\
\hline $2.62 E-04$ & 361.8 & Ra-223 & $->$ & $\mathrm{Rn}-219$ & & & \\
\hline $2.51 \mathrm{E}-04$ & 19.308 & Ac- 227 & $->$ & Th-227 & & & \\
\hline $2.44 \mathrm{E}-04$ & 41.88 & Th-227 & $->$ & Ra-223 & & & \\
\hline $2.44 \mathrm{E}-04$ & 104.9 & Th-227 & $->$ & Ra-223 & & & \\
\hline $2.44 \mathrm{E}-04$ & 68.75 & Th-227 & $->$ & Ra-223 & & & \\
\hline $2.44 \mathrm{E}-04$ & 218.94 & Th-227 & $->$ & Ra-223 & & & \\
\hline $2.43 \mathrm{E}-04$ & 438.8 & Po-215 & $->$ & $\mathrm{Pb}-211$ & & & \\
\hline $2.43 \mathrm{E}-04$ & 515.7 & $R n-219$ & $->$ & Po-215 & & & \\
\hline $2.39 \mathrm{E}-04$ & 184.68 & Fr-223 & $->$ & Ra-223, & Th-227 & $->$ & $\mathrm{Ra}-223$ \\
\hline $2.25 \mathrm{E}-04$ & 249.49 & Ra-223 & $->$ & $\mathrm{Rn}-219$ & & & \\
\hline $2.20 \mathrm{E}-04$ & 15.2 & Ac- 227 & $->$ & Th-227 & & & \\
\hline $2.20 \mathrm{E}-04$ & 226.77 & $\mathrm{~Pa}-231$ & $->$ & $A c-227$ & & & \\
\hline $2.20 \mathrm{E}-04$ & 395.8 & $\mathrm{~Pa}-231$ & $->$ & $A c-227$ & & & \\
\hline $2.13 \mathrm{E}-04$ & 251.12 & Ra-223 & $->$ & $\mathrm{Rn}-219$ & & & \\
\hline 2.07E-04 & 343.02 & $\mathrm{~Pb}-211$ & $->$ & $\mathrm{Bi}-211$ & & & \\
\hline $2.03 E-04$ & 134.5 & Fr-223 & $->$ & Ra-223, & Th-227 & $->$ & Ra-223 \\
\hline $2.01 \mathrm{E}-04$ & 220.38 & $\mathrm{Ra}-223$ & $->$ & $\mathrm{Rn}-219$ & & & \\
\hline $2.01 \mathrm{E}-04$ & 432.34 & $\mathrm{Ra}-223$ & $->$ & $R n-219$ & & & \\
\hline $1.95 \mathrm{E}-04$ & 270.76 & Th-227 & $->$ & Ra-223 & & & \\
\hline $1.95 \mathrm{E}-04$ & 140.33 & Th-227 & $->$ & Ra-223 & & & \\
\hline $1.95 \mathrm{E}-04$ & 254.98 & $\mathrm{Ra}-223$ & $->$ & $\mathrm{Rn}-219$ & & & \\
\hline $1.94 \mathrm{E}-04$ & 258.43 & $\mathrm{~Pa}-231$ & $->$ & Ac-227 & & & \\
\hline $1.94 \mathrm{E}-04$ & 319.18 & Fr-223 & $->$ & Ra-223, & Th-227 & $->$ & Ra-223 \\
\hline $1.89 E-04$ & 251.58 & $\mathrm{Ra}-223$ & $->$ & Rn-219 & & & \\
\hline $1.89 \mathrm{E}-04$ & 313.85 & $\mathrm{~Pb}-211$ & $->$ & $\mathrm{Bi}-211$ & & & \\
\hline $1.83 \mathrm{E}-04$ & 314.78 & Th-227 & $->$ & Ra-223 & & & \\
\hline $1.82 \mathrm{E}-04$ & 221.9 & $R n-219$ & $->$ & Po-215 & & & \\
\hline $1.71 \mathrm{E}-04$ & 64.37 & Th-227 & $->$ & Ra-223 & & & \\
\hline
\end{tabular}




\begin{tabular}{|c|c|c|c|c|c|c|c|}
\hline $1.71 \mathrm{E}-04$ & 72.8 & Th-227 & $->$ & $\mathrm{Ra}-223$ & & & \\
\hline $1.69 \mathrm{E}-04$ & 318.1 & $\mathrm{~Pa}-231$ & $->$ & Ac- 227 & & & \\
\hline $1.69 \mathrm{E}-04$ & 71.92 & Pa-231 & $->$ & Ac- 227 & & & \\
\hline $1.60 \mathrm{E}-04$ & 410.84 & $\mathrm{~Pa}-231$ & $->$ & $A c-227$ & & & \\
\hline $1.58 \mathrm{E}-04$ & 481.1 & $\mathrm{~Pb}-211$ & $->$ & $\mathrm{Bi}-211$ & & & \\
\hline $1.40 \mathrm{E}-04$ & 609.62 & $\mathrm{~Pb}-211$ & $->$ & $\mathrm{Bi}-211$ & & & \\
\hline $1.34 \mathrm{E}-04$ & 106.66 & Ra-223 & $->$ & Rn-219 & & & \\
\hline $1.34 \mathrm{E}-04$ & 951 & $\mathrm{~Pb}-211$ & $->$ & $\mathrm{Bi}-211$ & & & \\
\hline $1.31 \mathrm{E}-04$ & 8.953 & $\mathrm{Bi}-211$ & $->$ & TI-207 & & & \\
\hline $1.28 \mathrm{E}-04$ & 9.658 & $\mathrm{Rn}-219$ & $->$ & Po-215 & & & \\
\hline $1.22 \mathrm{E}-04$ & 201.64 & Th-227 & $->$ & $\mathrm{Ra}-223$ & & & \\
\hline $1.22 \mathrm{E}-04$ & 200.48 & Th-227 & $->$ & Ra-223 & & & \\
\hline $1.22 \mathrm{E}-04$ & 40.16 & Th-227 & $->$ & $\mathrm{Ra}-223$ & & & \\
\hline $1.22 \mathrm{E}-04$ & 369.48 & Ra-223 & $->$ & $\mathrm{Rn}-219$ & & & \\
\hline $1.18 \mathrm{E}-04$ & 310.19 & $\mathrm{~Pa}-231$ & $->$ & $A c-227$ & & & \\
\hline $1.18 \mathrm{E}-04$ & 50.97 & $\mathrm{~Pa}-231$ & $->$ & Ac- 227 & & & \\
\hline $1.18 \mathrm{E}-04$ & 39.61 & $\mathrm{~Pa}-231$ & $->$ & Ac- 227 & & & \\
\hline $1.16 \mathrm{E}-04$ & 73.66 & Th-227 & $->$ & Ra-223 & & & \\
\hline $1.16 \mathrm{E}-04$ & 430.53 & Ra-223 & $->$ & $\mathrm{Rn}-219$ & & & \\
\hline $1.16 \mathrm{E}-04$ & 9.419 & $\mathrm{~Pb}-211$ & $->$ & $\mathrm{Bi}-211$ & & & \\
\hline 1.10E-04 & 175.23 & Th-227 & $->$ & Ra-223 & & & \\
\hline $1.10 \mathrm{E}-04$ & 516.45 & $\mathrm{~Pa}-231$ & $->$ & Ac-227 & & & \\
\hline 1.09E-04 & 94.89 & $\mathrm{~Pb}-211$ & $->$ & $\mathrm{Bi}-211$ & & & \\
\hline 1.09E-04 & 1014.48 & $\mathrm{~Pb}-211$ & $->$ & $\mathrm{Bi}-211$ & & & \\
\hline $1.05 \mathrm{E}-04$ & 173.38 & Fr-223 & $->$ & Ra-223, & Th-227 & $->$ & Ra-223 \\
\hline $1.04 \mathrm{E}-04$ & 212.69 & Th-227 & $->$ & $\mathrm{Ra}-223$ & & & \\
\hline 1.03E-04 & 175.55 & $\mathrm{Ra}-223$ & $->$ & $\mathrm{Rn}-219$ & & & \\
\hline $1.03 \mathrm{E}-04$ & 103.85 & Ra-223 & $->$ & $\mathrm{Rn}-219$ & & & \\
\hline 1.03E-04 & 88.2 & $\mathrm{~Pb}-211$ & $->$ & $\mathrm{Bi}-211$ & & & \\
\hline 9.77E-05 & 50.82 & Th-227 & $->$ & $\mathrm{Ra}-223$ & & & \\
\hline $9.31 \mathrm{E}-05$ & 1080.19 & $\mathrm{~Pb}-211$ & $->$ & $\mathrm{Bi}-211$ & & & \\
\hline $9.16 \mathrm{E}-05$ & 224.68 & Fr-223 & $->$ & Ra-223, & Th-227 & $->$ & Ra-223 \\
\hline $9.16 \mathrm{E}-05$ & 308.49 & Th-227 & $->$ & Ra-223 & & & \\
\hline $9.12 \mathrm{E}-05$ & 504.33 & $\mathrm{~Pb}-211$ & $->$ & $\mathrm{Bi}-211$ & & & \\
\hline $9.04 \mathrm{E}-05$ & 388.98 & Pa-231 & $->$ & Ac- 227 & & & \\
\hline 8.91E-05 & 289.6 & Fr-223 & $->$ & Ra-223, & Th-227 & $->$ & Ra-223 \\
\hline 8.55E-05 & 168.19 & Th-227 & $->$ & Ra-223 & & & \\
\hline 8.55E-05 & 33.52 & Th-227 & $->$ & Ra-223 & & & \\
\hline 8.51E-05 & 388.3 & $\mathrm{Ra}-223$ & $->$ & $\mathrm{Rn}-219$ & & & \\
\hline 8.51E-05 & 492 & $\mathrm{~Pb}-211$ & $->$ & $\mathrm{Bi}-211$ & & & \\
\hline 7.94E-05 & 99.56 & Fr-223 & $->$ & Ra-223, & Th-227 & $->$ & Ra-223 \\
\hline 7.94E-05 & 44.37 & Th-227 & $->$ & Ra-223 & & & \\
\hline 7.91E-05 & 478 & $\mathrm{~Pb}-211$ & $->$ & $\mathrm{Bi}-211$ & & & \\
\hline 7.78E-05 & 1196.61 & $\mathrm{~Pb}-211$ & $->$ & $\mathrm{Bi}-211$ & & & \\
\hline $7.32 \mathrm{E}-05$ & 197.61 & Th-227 & $->$ & $\mathrm{Ra}-223$ & & & \\
\hline 7.32E-05 & 402.42 & Th-227 & $->$ & Ra-223 & & & \\
\hline 7.32E-05 & 94.9 & Th-227 & $->$ & Ra-223 & & & \\
\hline 7.32E-05 & 117.3 & Th-227 & $->$ & Ra-223 & & & \\
\hline 6.99E-05 & 97.3 & $\mathrm{~Pb}-211$ & $->$ & $\mathrm{Bi}-211$ & & & \\
\hline $6.71 \mathrm{E}-05$ & 246.07 & Th-227 & $->$ & Ra-223 & & & \\
\hline
\end{tabular}




\begin{tabular}{|c|c|c|c|c|c|c|c|}
\hline 6.69E-05 & 94.3 & $\mathrm{~Pb}-211$ & $->$ & $\mathrm{Bi}-211$ & & & \\
\hline $6.69 \mathrm{E}-05$ & 500.4 & $\mathrm{~Pb}-211$ & $->$ & $\mathrm{Bi}-211$ & & & \\
\hline $6.11 \mathrm{E}-05$ & 48.27 & Fr-223 & $->$ & Ra-223, & Th-227 & $->$ & Ra-223 \\
\hline $6.10 \mathrm{E}-05$ & 267.82 & Th-227 & $->$ & $\mathrm{Ra}-223$ & & & \\
\hline $6.10 \mathrm{E}-05$ & 352.57 & Th-227 & $->$ & Ra-223 & & & \\
\hline $6.10 \mathrm{E}-05$ & 150.02 & Th-227 & $->$ & Ra-223 & & & \\
\hline $6.08 \mathrm{E}-05$ & 487.59 & Ra-223 & $->$ & Rn-219 & & & \\
\hline 5.49E-05 & 62.54 & Th-227 & $->$ & Ra-223 & & & \\
\hline 5.49E-05 & 392.24 & Th-227 & $->$ & $\mathrm{Ra}-223$ & & & \\
\hline 5.49E-05 & 289.5 & Th-227 & $->$ & Ra-223 & & & \\
\hline 5.49E-05 & 59.5 & Th-227 & $->$ & $\mathrm{Ra}-223$ & & & \\
\hline 5.49E-05 & 398.6 & Th-227 & $->$ & $\mathrm{Ra}-223$ & & & \\
\hline 5.49E-05 & 69.65 & Th-227 & $->$ & Ra-223 & & & \\
\hline $5.49 \mathrm{E}-05$ & 75.1 & Th-227 & $->$ & Ra-223 & & & \\
\hline 5.47E-05 & 245.19 & Ra-223 & $->$ & Rn-219 & & & \\
\hline $5.47 \mathrm{E}-05$ & 114.5 & Ra-223 & $->$ & $\mathrm{Rn}-219$ & & & \\
\hline 5.41E-05 & 1270.67 & $\mathrm{~Pb}-211$ & $->$ & $\mathrm{Bi}-211$ & & & \\
\hline 5.07E-05 & 501.33 & $\mathrm{~Pa}-231$ & $->$ & $A C-227$ & & & \\
\hline $4.88 \mathrm{E}-05$ & 54.18 & Fr-223 & $->$ & Ra-223, & Th-227 & $->$ & Ra-223 \\
\hline $4.88 \mathrm{E}-05$ & 270.34 & Th-227 & $->$ & $\mathrm{Ra}-223$ & & & \\
\hline $4.88 \mathrm{E}-05$ & 112.54 & Th-227 & $->$ & Ra-223 & & & \\
\hline $4.88 \mathrm{E}-05$ & 123.55 & Th-227 & $->$ & Ra-223 & & & \\
\hline $4.87 E-05$ & 368.78 & Ra-223 & $->$ & $\mathrm{Rn}-219$ & & & \\
\hline $4.87 \mathrm{E}-05$ & 623.38 & Ra-223 & $->$ & $\mathrm{Rn}-219$ & & & \\
\hline 4.73E-05 & 535.45 & $\mathrm{~Pa}-231$ & $->$ & $A c-227$ & & & \\
\hline $4.58 \mathrm{E}-05$ & 346.39 & Th-227 & $->$ & Ra-223 & & & \\
\hline $4.56 \mathrm{E}-05$ & 12.085 & $\mathrm{Rn}-219$ & $->$ & Po-215 & & & \\
\hline $4.45 E-05$ & 775.82 & $\mathrm{Fr}-223$ & $->$ & Ra-223, & Th-227 & $\rightarrow$ & Ra-223 \\
\hline $4.27 \mathrm{E}-05$ & 370.85 & Th-227 & $->$ & Ra-223 & & & \\
\hline $4.27 E-05$ & 44.08 & Th-227 & $->$ & Ra-223 & & & \\
\hline $4.27 E-05$ & 281 & Th-227 & $->$ & Ra-223 & & & \\
\hline 4.27E-05 & 249.36 & Th-227 & $->$ & $\mathrm{Ra}-223$ & & & \\
\hline $4.27 \mathrm{E}-05$ & 162.18 & Th-227 & $->$ & Ra-223 & & & \\
\hline 4.27E-05 & 66.3 & Th-227 & $->$ & Ra-223 & & & \\
\hline $4.27 E-05$ & 107.92 & Th-227 & $->$ & Ra-223 & & & \\
\hline $4.27 E-05$ & 56.63 & Th-227 & $->$ & Ra-223 & & & \\
\hline $4.26 \mathrm{E}-05$ & 376.9 & $\mathrm{Rn}-219$ & $->$ & Po-215 & & & \\
\hline $4.22 \mathrm{E}-05$ & 387.267 & $\mathrm{~Pa}-231$ & $->$ & Ac- 227 & & & \\
\hline $4.22 \mathrm{E}-05$ & 572.3 & $\mathrm{~Pa}-231$ & $->$ & Ac- 227 & & & \\
\hline $4.22 \mathrm{E}-05$ & 546.9 & $P a-231$ & $->$ & $A c-227$ & & & \\
\hline 3.89E-05 & 430.26 & $\mathrm{~Pb}-211$ & $->$ & $\mathrm{Bi}-211$ & & & \\
\hline $3.89 \mathrm{E}-05$ & 675.33 & $\mathrm{~Pb}-211$ & $->$ & $\mathrm{Bi}-211$ & & & \\
\hline 3.89E-05 & 865.81 & $\mathrm{~Pb}-211$ & $->$ & $\mathrm{Bi}-211$ & & & \\
\hline 3.88E-05 & 29.58 & Fr-223 & $->$ & Ra-223, & Th-227 & $->$ & Ra-223 \\
\hline $3.87 \mathrm{E}-05$ & 369.32 & Fr-223 & $->$ & Ra-223, & Th-227 & $->$ & Ra-223 \\
\hline $3.66 \mathrm{E}-05$ & 202.4 & Th-227 & $->$ & $\mathrm{Ra}-223$ & & & \\
\hline $3.66 \mathrm{E}-05$ & 348.4 & Th-227 & $->$ & Ra-223 & & & \\
\hline $3.66 \mathrm{E}-05$ & 318.58 & Th-227 & $->$ & Ra-223 & & & \\
\hline $3.66 \mathrm{E}-05$ & 109.1 & Th-227 & $->$ & Ra-223 & & & \\
\hline $3.66 \mathrm{E}-05$ & 325.08 & Th-227 & $->$ & Ra-223 & & & \\
\hline
\end{tabular}




\begin{tabular}{|c|c|c|c|c|c|c|c|}
\hline 3.66E-05 & 66.1 & Th-227 & $->$ & Ra-223 & & & \\
\hline 3.66E-05 & 382.15 & Th-227 & $->$ & Ra-223 & & & \\
\hline 3.66E-05 & 376.26 & Th-227 & $->$ & Ra-223 & & & \\
\hline 3.65E-05 & 260.4 & Ra-223 & $->$ & $\mathrm{Rn}-219$ & & & \\
\hline 3.65E-05 & 676.4 & $\mathrm{Rn}-219$ & $->$ & Po-215 & & & \\
\hline 3.65E-05 & 540.1 & $\mathrm{Rn}-219$ & $->$ & Po-215 & & & \\
\hline 3.65E-05 & 11.712 & $\mathrm{~Pb}-211$ & $->$ & $\mathrm{Bi}-211$ & & & \\
\hline 3.48E-05 & 68.7 & Th-227 & $->$ & Ra-223 & & & \\
\hline 3.46E-05 & 10.994 & $\mathrm{Bi}-211$ & $->$ & TI-207 & & & \\
\hline $3.10 \mathrm{E}-05$ & 1103.4 & $\mathrm{~Pb}-211$ & $->$ & $\mathrm{Bi}-211$ & & & \\
\hline 3.05E-05 & 326.12 & Th-227 & $->$ & $\mathrm{Ra}-223$ & & & \\
\hline $3.05 E-05$ & 56.03 & Th-227 & $->$ & Ra-223 & & & \\
\hline $3.05 \mathrm{E}-05$ & 124.48 & Th-227 & $->$ & Ra-223 & & & \\
\hline 3.05E-05 & 362.55 & Th-227 & $->$ & $\mathrm{Ra}-223$ & & & \\
\hline 3.05E-05 & 432.29 & Th-227 & $->$ & Ra-223 & & & \\
\hline 3.05E-05 & 110.58 & Th-227 & $->$ & Ra-223 & & & \\
\hline $3.04 \mathrm{E}-05$ & 165.5 & Ra-223 & $->$ & $\mathrm{Rn}-219$ & & & \\
\hline $3.04 \mathrm{E}-05$ & 255.74 & Ra-223 & $->$ & $\mathrm{Rn}-219$ & & & \\
\hline $3.04 \mathrm{E}-05$ & 131.1 & $\mathrm{Ra}-223$ & $->$ & $\mathrm{Rn}-219$ & & & \\
\hline 2.43E-05 & 383.12 & Ra-223 & $->$ & $\mathrm{Rn}-219$ & & & \\
\hline $2.43 E-05$ & 176.8 & Ra-223 & $->$ & $\mathrm{Rn}-219$ & & & \\
\hline 2.43E-05 & 608.5 & $\mathrm{Rn}-219$ & $->$ & Po-215 & & & \\
\hline $2.41 \mathrm{E}-05$ & 339.45 & Fr-223 & $->$ & Ra-223, & Th-227 & $->$ & Ra-223 \\
\hline $2.20 \mathrm{E}-05$ & 24.5 & Ac- 227 & $->$ & Th-227 & & & \\
\hline $2.13 \mathrm{E}-05$ & 711.2 & Ra-223 & $->$ & $\mathrm{Rn}-219$ & & & \\
\hline 2.08E-05 & 89.85 & Th-227 & $->$ & Ra-223 & & & \\
\hline 2.03E-05 & 228.16 & $\mathrm{~Pa}-231$ & $->$ & Ac- 227 & & & \\
\hline 2.03E-05 & 220.2 & Pa-231 & $->$ & Ac- 227 & & & \\
\hline 2.01E-05 & 115.3 & $\mathrm{Rn}-219$ & $->$ & Po-215 & & & \\
\hline 1.83E-05 & 51.29 & Fr-223 & $->$ & Ra-223, & Th-227 & $->$ & Ra-223 \\
\hline $1.83 \mathrm{E}-05$ & 169.98 & Th-227 & $->$ & Ra-223 & & & \\
\hline $1.83 \mathrm{E}-05$ & 124.6 & Th-227 & $->$ & $\mathrm{Ra}-223$ & & & \\
\hline $1.82 \mathrm{E}-05$ & 391 & Ra-223 & $->$ & $\mathrm{Rn}-219$ & & & \\
\hline 1.82E-05 & 199.43 & $\mathrm{Ra}-223$ & $->$ & $\mathrm{Rn}-219$ & & & \\
\hline 1.77E-05 & 812.15 & Fr-223 & $->$ & Ra-223, & Th-227 & $->$ & Ra-223 \\
\hline $1.61 \mathrm{E}-05$ & 822.99 & Fr-223 & $->$ & Ra-223, & Th-227 & $->$ & Ra-223 \\
\hline $1.58 \mathrm{E}-05$ & 1090.5 & $\mathrm{~Pb}-211$ & $->$ & $\mathrm{Bi}-211$ & & & \\
\hline 1.53E-05 & 266.3 & Th-227 & $->$ & $\mathrm{Ra}-223$ & & & \\
\hline $1.52 \mathrm{E}-05$ & 74.969 & TI-207 & $->$ & Pb-207 & & & \\
\hline $1.44 \mathrm{E}-05$ & 23.55 & $\mathrm{~Pa}-231$ & $->$ & $A c-227$ & & & \\
\hline $1.40 \mathrm{E}-05$ & 908.03 & Fr-223 & $->$ & Ra-223, & Th-227 & $->$ & Ra-223 \\
\hline 1.27E-05 & 471.33 & Pa-231 & $->$ & $A c-227$ & & & \\
\hline $1.22 \mathrm{E}-05$ & 99.64 & Th-227 & $->$ & $\mathrm{Ra}-223$ & & & \\
\hline $1.22 \mathrm{E}-05$ & 62.05 & Th-227 & $->$ & Ra-223 & & & \\
\hline $1.22 \mathrm{E}-05$ & 179.28 & Th-227 & $->$ & $\mathrm{Ra}-223$ & & & \\
\hline 1.09E-05 & 803.58 & Fr-223 & $->$ & Ra-223, & Th-227 & $->$ & Ra-223 \\
\hline $1.06 \mathrm{E}-05$ & 307.89 & Fr-223 & $->$ & Ra-223, & Th-227 & $->$ & Ra-223 \\
\hline $1.04 \mathrm{E}-05$ & 415.09 & Th-227 & $->$ & Ra-223 & & & \\
\hline 9.19E-06 & 723.86 & Fr-223 & $->$ & Ra-223, & Th-227 & $->$ & Ra-223 \\
\hline $9.16 \mathrm{E}-06$ & 275.2 & Th-227 & $->$ & Ra-223 & & & \\
\hline
\end{tabular}




$\begin{array}{cc}9.15 \mathrm{E}-06 & 328.08 \\ 9.12 \mathrm{E}-06 & 887.7 \\ 9.10 \mathrm{E}-06 & 72.803 \\ 8.87 \mathrm{E}-06 & 569.15 \\ 8.78 \mathrm{E}-06 & 764.52 \\ 8.55 \mathrm{E}-06 & 339.1 \\ 8.55 \mathrm{E}-06 & 374.93 \\ 7.91 \mathrm{E}-06 & 1234.3 \\ 7.32 \mathrm{E}-06 & 102.45 \\ 7.10 \mathrm{E}-06 & 536.88 \\ 6.91 \mathrm{E}-06 & 568.76 \\ 6.50 \mathrm{E}-06 & 478.22 \\ 6.17 \mathrm{E}-06 & 757.03 \\ 6.10 \mathrm{E}-06 & 171.21 \\ 6.08 \mathrm{E}-06 & 834.2 \\ 5.71 \mathrm{E}-06 & 796.96 \\ 5.56 \mathrm{E}-06 & 825.96 \\ 5.46 \mathrm{E}-06 & 10.541 \\ 5.40 \mathrm{E}-06 & 84.979 \\ 4.97 \mathrm{E}-06 & 12.703 \\ 4.70 \mathrm{E}-06 & 876.14 \\ 4.52 \mathrm{E}-06 & 722.6 \\ 4.27 \mathrm{E}-06 & 230.17 \\ 4.22 \mathrm{E}-06 & 842.02 \\ 4.05 \mathrm{E}-06 & 846.56 \\ 3.65 \mathrm{E}-06 & 1055.8 \\ 3.55 \mathrm{E}-06 & 493.15 \\ 3.34 \mathrm{E}-06 & 575.8 \\ 3.24 \mathrm{E}-06 & 748.56 \\ 3.04 \mathrm{E}-06 & 837.36 \\ 2.81 \mathrm{E}-06 & 99.7 \\ 2.71 \mathrm{E}-06 & 507.64 \\ 2.67 \mathrm{E}-06 & 746.24 \\ 2.43 \mathrm{E}-06 & 387.9 \\ 2.36 \mathrm{E}-06 & 756.79 \\ 2.18 \mathrm{E}-06 & 896.41 \\ 2.17 \mathrm{E}-06 & 780.59 \\ 1.94 \mathrm{E}-06 & 480.25 \\ 1.81 \mathrm{E}-06 & 552.09 \\ 1.76 \mathrm{E}-06 & 762.3 \\ 1.74 \mathrm{E}-06 & 858.4 \\ 1.58 \mathrm{E}-06 & 87.632 \\ 1.49 \mathrm{E}-06 & 69.83 \\ 1.49 \mathrm{E}-06 & 555.91 \\ 1.33 \mathrm{E}-06 & 828.31 \\ 1.30 \mathrm{E}-06 & 515.96 \\ 1.23 \mathrm{E}-06 & 733.9 \\ 1.22 \mathrm{E}-06 & 607.2 \\ 1.19 \mathrm{E}-06 & 784.04 \\ & 754.17\end{array}$

\begin{tabular}{|c|c|c|c|c|c|}
\hline TI-207 & $->$ & Pb-207, & Po-211 & $->$ & Pb-207 \\
\hline$R n-219$ & $->$ & Po-215 & & & \\
\hline TI-207 & $->$ & $\mathrm{Pb}-207$ & & & \\
\hline Po-211 & $->$ & Pb-207 & & & \\
\hline Fr-223 & $->$ & $\mathrm{Ra}-223$, & Th-227 & $->$ & $\mathrm{Ra}-223$ \\
\hline Th-227 & $->$ & $\mathrm{Ra}-223$ & & & \\
\hline Th-227 & $->$ & Ra-223 & & & \\
\hline $\mathrm{Pb}-211$ & $->$ & $\mathrm{Bi}-211$ & & & \\
\hline Th-227 & $->$ & Ra-223 & & & \\
\hline Fr-223 & $->$ & Ra-223, & Th-227 & $->$ & $\mathrm{Ra}-223$ \\
\hline Fr-223 & $->$ & Ra-223, & Th-227 & $->$ & $\mathrm{Ra}-223$ \\
\hline $\mathrm{Pa}-231$ & $->$ & Ac-227 & & & \\
\hline Fr-223 & $->$ & Ra-223, & Th-227 & $->$ & Ra-223 \\
\hline Th-227 & $->$ & $\mathrm{Ra}-223$ & & & \\
\hline $\mathrm{Rn}-219$ & $->$ & Po-215 & & & \\
\hline Fr-223 & $->$ & $\mathrm{Ra}-223$, & Th-227 & $->$ & $\mathrm{Ra}-223$ \\
\hline Fr-223 & $->$ & Ra-223, & Th-227 & $->$ & $\mathrm{Ra}-223$ \\
\hline TI-207 & $->$ & $\mathrm{Pb}-207$ & & & \\
\hline TI-207 & $->$ & $\mathrm{Pb}-207$ & & & \\
\hline TI-207 & $->$ & $\mathrm{Pb}-207$ & & & \\
\hline Fr-223 & $->$ & Ra-223, & Th-227 & $->$ & $\mathrm{Ra}-223$ \\
\hline Fr-223 & $->$ & Ra-223, & Th-227 & $->$ & Ra-223 \\
\hline Th-227 & $->$ & $\mathrm{Ra}-223$ & & & \\
\hline Fr-223 & $->$ & Ra-223, & Th-227 & $->$ & $\mathrm{Ra}-223$ \\
\hline Fr-223 & $->$ & Ra-223, & Th-227 & $->$ & $\mathrm{Ra}-223$ \\
\hline $\mathrm{Rn}-219$ & $->$ & Po-215 & & & \\
\hline Fr-223 & $->$ & Ra-223, & Th-227 & $->$ & $\mathrm{Ra}-223$ \\
\hline Fr-223 & $->$ & Ra-223, & Th-227 & $->$ & $\mathrm{Ra}-223$ \\
\hline Fr-223 & $->$ & Ra-223, & Th-227 & $->$ & $\mathrm{Ra}-223$ \\
\hline Fr-223 & $->$ & Ra-223, & Th-227 & $->$ & $\mathrm{Ra}-223$ \\
\hline Ac- 227 & $->$ & Fr-223 & & & \\
\hline Fr-223 & $->$ & Ra-223, & Th-227 & $->$ & $\mathrm{Ra}-223$ \\
\hline Fr-223 & $->$ & Ra-223, & Th-227 & $->$ & $\mathrm{Ra}-223$ \\
\hline $\mathrm{Rn}-219$ & $->$ & Po-215 & & & \\
\hline $\mathrm{Fr}-223$ & $->$ & Ra-223, & Th-227 & $->$ & $\mathrm{Ra}-223$ \\
\hline Fr-223 & $->$ & Ra-223, & Th-227 & $->$ & Ra-223 \\
\hline Fr-223 & $->$ & Ra-223, & Th-227 & $->$ & $\mathrm{Ra}-223$ \\
\hline Fr-223 & $->$ & Ra-223, & Th-227 & $->$ & $\mathrm{Ra}-223$ \\
\hline Fr-223 & $->$ & $\mathrm{Ra}-223$, & Th-227 & $->$ & $\mathrm{Ra}-223$ \\
\hline Fr-223 & $->$ & $\mathrm{Ra}-223$, & Th-227 & $->$ & $\mathrm{Ra}-223$ \\
\hline Fr-223 & $->$ & Ra-223, & Th-227 & $->$ & $\mathrm{Ra}-223$ \\
\hline TI-207 & $->$ & $\mathrm{Pb}-207$ & & & \\
\hline Ac- 227 & $->$ & Fr-223 & & & \\
\hline Fr-223 & $->$ & Ra-223, & Th-227 & $->$ & $\mathrm{Ra}-223$ \\
\hline Fr-223 & $->$ & Ra-223, & Th-227 & $->$ & $\mathrm{Ra}-223$ \\
\hline Fr-223 & $->$ & Ra-223, & Th-227 & $->$ & $\mathrm{Ra}-223$ \\
\hline Fr-223 & $->$ & Ra-223, & Th-227 & $->$ & Ra-223 \\
\hline Fr-223 & $->$ & Ra-223, & Th-227 & $->$ & $\mathrm{Ra}-223$ \\
\hline Fr-223 & $->$ & Ra-223, & Th-227 & $->$ & Ra-223 \\
\hline Fr-223 & $->$ & Ra-223, & Th-227 & $->$ & $\mathrm{Ra}-223$ \\
\hline
\end{tabular}




$\begin{array}{lc}1.16 \mathrm{E}-06 & 753.44 \\ 1.15 \mathrm{E}-06 & 734.6 \\ 1.08 \mathrm{E}-06 & 524.02 \\ 1.05 \mathrm{E}-06 & 817.31 \\ 1.04 \mathrm{E}-06 & 691.46 \\ 1.01 \mathrm{E}-06 & 482.14 \\ 9.95 \mathrm{E}-07 & 448 \\ 9.77 \mathrm{E}-07 & 623.52 \\ 9.43 \mathrm{E}-07 & 578.2 \\ 9.10 \mathrm{E}-07 & 14.886 \\ 8.75 \mathrm{E}-07 & 704.19 \\ 8.38 \mathrm{E}-07 & 772.85 \\ 8.26 \mathrm{E}-07 & 786.9 \\ 8.17 \mathrm{E}-07 & 9.3 \\ 7.94 \mathrm{E}-07 & 633.2 \\ 7.86 \mathrm{E}-07 & 452.52 \\ 7.37 \mathrm{E}-07 & 878.16 \\ 7.30 \mathrm{E}-07 & 147.2 \\ 6.56 \mathrm{E}-07 & 848.88 \\ 6.10 \mathrm{E}-07 & 534.9 \\ 6.08 \mathrm{E}-07 & 31.87 \\ 5.50 \mathrm{E}-07 & 457.07 \\ 5.50 \mathrm{E}-07 & 736.76 \\ 5.43 \mathrm{E}-07 & 643.93 \\ 5.39 \mathrm{E}-07 & 867.23 \\ 4.84 \mathrm{E}-07 & 69.13 \\ 4.84 \mathrm{E}-07 & 120.9 \\ 4.39 \mathrm{E}-07 & 941.41 \\ 4.27 \mathrm{E}-07 & 854.2 \\ 3.99 \mathrm{E}-07 & 621.92 \\ 3.99 \mathrm{E}-07 & 857.89 \\ 3.87 \mathrm{E}-07 & 588.17 \\ 3.78 \mathrm{E}-07 & 958.6 \\ 3.66 \mathrm{E}-07 & 662.8 \\ 3.60 \mathrm{E}-07 & 171.4 \\ 3.58 \mathrm{E}-07 & 808.26 \\ 3.14 \mathrm{E}-07 & 707.18 \\ 3.05 \mathrm{E}-07 & 461.9 \\ 3.05 \mathrm{E}-07 & 466.2 \\ 2.98 \mathrm{E}-07 & 863.2 \\ 2.98 \mathrm{E}-07 & 891 \\ 2.73 \mathrm{E}-07 & 9.185 \\ 2.54 \mathrm{E}-07 & 596.44 \\ 2.46 \mathrm{E}-07 & 133.8 \\ 2.44 \mathrm{E}-07 & 788.36 \\ 2.44 \mathrm{E}-07 & 792.6 \\ 2.14 \mathrm{E}-07 & 990.2 \\ 2.04 \mathrm{E}-07 & 718.22 \\ 1.94 \mathrm{E}-07 & 892.8 \\ 1.83 \mathrm{E}-07 & 926.28\end{array}$

\begin{tabular}{|c|c|c|c|c|c|}
\hline Fr-223 & $->$ & Ra-223, & Th-227 & $->$ & $\mathrm{Ra}-223$ \\
\hline Fr-223 & $->$ & Ra-223, & Th-227 & $->$ & $\mathrm{Ra}-223$ \\
\hline Fr-223 & $->$ & Ra-223, & Th-227 & $->$ & $\mathrm{Ra}-223$ \\
\hline Fr-223 & $->$ & $\mathrm{Ra}-223$, & Th-227 & $->$ & Ra-223 \\
\hline Fr-223 & $->$ & $\mathrm{Ra}-223$, & Th-227 & $->$ & $\mathrm{Ra}-223$ \\
\hline Fr-223 & $->$ & Ra-223, & Th-227 & $->$ & $\mathrm{Ra}-223$ \\
\hline Fr-223 & $->$ & Ra-223, & Th-227 & $->$ & Ra-223 \\
\hline Th-227 & $->$ & $\mathrm{Ra}-223$ & & & \\
\hline Fr-223 & $->$ & Ra-223, & Th-227 & $->$ & Ra-223 \\
\hline TI-207 & $->$ & $\mathrm{Pb}-207$ & & & \\
\hline Fr-223 & $->$ & Ra-223, & Th-227 & $->$ & $\mathrm{Ra}-223$ \\
\hline Fr-223 & $->$ & Ra-223, & Th-227 & $->$ & $\mathrm{Ra}-223$ \\
\hline Fr-223 & $->$ & Ra-223, & Th-227 & $->$ & $\mathrm{Ra}-223$ \\
\hline Ac- 227 & $->$ & Th-227 & & & \\
\hline Th-227 & $->$ & $\mathrm{Ra}-223$ & & & \\
\hline Fr-223 & $->$ & Ra-223, & Th-227 & $->$ & $\mathrm{Ra}-223$ \\
\hline Fr-223 & $->$ & Ra-223, & Th-227 & $->$ & Ra-223 \\
\hline Ac- 227 & $->$ & Fr-223 & & & \\
\hline Fr-223 & $->$ & Ra-223, & Th-227 & $->$ & $\mathrm{Ra}-223$ \\
\hline Th-227 & $->$ & Ra-223 & & & \\
\hline $\mathrm{Ra}-223$ & $->$ & $\mathrm{Rn}-219$ & & & \\
\hline Fr-223 & $->$ & Ra-223, & Th-227 & $->$ & Ra-223 \\
\hline Fr-223 & $->$ & Ra-223, & Th-227 & $->$ & Ra-223 \\
\hline Fr-223 & $->$ & Ra-223, & Th-227 & $->$ & $\mathrm{Ra}-223$ \\
\hline Fr-223 & $->$ & Ra-223, & Th-227 & $->$ & $\mathrm{Ra}-223$ \\
\hline$A c-227$ & $->$ & $\mathrm{Fr}-223$ & & & \\
\hline$A c-227$ & $->$ & Fr-223 & & & \\
\hline Th-227 & $->$ & Ra-223 & & & \\
\hline Th-227 & $->$ & Ra-223 & & & \\
\hline Fr-223 & $->$ & Ra-223, & Th-227 & $->$ & $\mathrm{Ra}-223$ \\
\hline Fr-223 & $->$ & Ra-223, & Th-227 & $->$ & $\mathrm{Ra}-223$ \\
\hline Fr-223 & $->$ & Ra-223, & Th-227 & $->$ & $\mathrm{Ra}-223$ \\
\hline Th-227 & $->$ & Ra-223 & & & \\
\hline Th-227 & $->$ & Ra-223 & & & \\
\hline Ac- 227 & $->$ & Fr-223 & & & \\
\hline Fr-223 & $->$ & Ra-223, & Th-227 & $->$ & Ra-223 \\
\hline Fr-223 & $->$ & Ra-223, & Th-227 & $->$ & $\mathrm{Ra}-223$ \\
\hline Th-227 & $->$ & $\mathrm{Ra}-223$ & & & \\
\hline Th-227 & $->$ & Ra-223 & & & \\
\hline Fr-223 & $->$ & Ra-223, & Th-227 & $->$ & $\mathrm{Ra}-223$ \\
\hline Fr-223 & $->$ & Ra-223, & Th-227 & $->$ & $\mathrm{Ra}-223$ \\
\hline TI-207 & $->$ & $\mathrm{Pb}-207$ & & & \\
\hline Fr-223 & $->$ & Ra-223, & Th-227 & $->$ & $\mathrm{Ra}-223$ \\
\hline Ac- 227 & $->$ & Fr-223 & & & \\
\hline Th-227 & $->$ & Ra-223 & & & \\
\hline Th-227 & $->$ & Ra-223 & & & \\
\hline Th-227 & $->$ & Ra-223 & & & \\
\hline Fr-223 & $->$ & Ra-223, & Th-227 & $->$ & $\mathrm{Ra}-223$ \\
\hline Fr-223 & $->$ & Ra-223, & Th-227 & $->$ & Ra-223 \\
\hline Fr-223 & $->$ & Ra-223, & Th-227 & $->$ & $\mathrm{Ra}-223$ \\
\hline
\end{tabular}




\begin{tabular}{|c|c|c|c|c|c|c|c|}
\hline $1.83 \mathrm{E}-07$ & 793.12 & Th-227 & $->$ & Ra-223 & & & \\
\hline $1.83 \mathrm{E}-07$ & 969.91 & Th-227 & $->$ & Ra-223 & & & \\
\hline $1.83 \mathrm{E}-07$ & 999.78 & Th-227 & $->$ & Ra-223 & & & \\
\hline $1.83 \mathrm{E}-07$ & 818.23 & Th-227 & $->$ & Ra-223 & & & \\
\hline $1.29 \mathrm{E}-07$ & 648.43 & Fr-223 & $->$ & Ra-223, & Th-227 & $->$ & $\mathrm{Ra}-223$ \\
\hline $1.23 \mathrm{E}-07$ & 46.13 & $A c-227$ & $->$ & Fr-223 & & & \\
\hline $1.23 \mathrm{E}-07$ & 106.1 & $A c-227$ & $->$ & Fr-223 & & & \\
\hline $1.22 \mathrm{E}-07$ & 641.45 & Th-227 & $->$ & $\mathrm{Ra}-223$ & & & \\
\hline $1.22 \mathrm{E}-07$ & 1020 & Th-227 & $->$ & Ra-223 & & & \\
\hline $1.14 \mathrm{E}-07$ & 825.97 & Fr-223 & $->$ & Ra-223 & & & \\
\hline $9.16 \mathrm{E}-08$ & 1024.8 & Th-227 & $->$ & Ra-223 & & & \\
\hline $9.16 \mathrm{E}-08$ & 1015.1 & Th-227 & $->$ & $\mathrm{Ra}-223$ & & & \\
\hline $9.16 \mathrm{E}-08$ & 909.85 & Th-227 & $->$ & Ra-223 & & & \\
\hline $7.32 \mathrm{E}-08$ & 920.06 & Th-227 & $->$ & $\mathrm{Ra}-223$ & & & \\
\hline $7.25 \mathrm{E}-08$ & 828.53 & Fr-223 & $->$ & Ra-223, & Th-227 & $\rightarrow$ & Ra-223 \\
\hline 7.10E-08 & 11.349 & TI-207 & $->$ & $\mathrm{Pb}-207$ & & & \\
\hline $6.10 \mathrm{E}-08$ & 971.28 & Th-227 & $->$ & Ra-223 & & & \\
\hline $6.10 \mathrm{E}-08$ & 938.34 & Th-227 & $->$ & $\mathrm{Ra}-223$ & & & \\
\hline $4.27 E-08$ & 994.9 & Th-227 & $->$ & Ra-223 & & & \\
\hline $1.05 \mathrm{E}-08$ & 793.11 & Fr-223 & $->$ & Ra-223 & & & \\
\hline U-236 & $100 \%$ & Aged 5 & ars & & & & \\
\hline
\end{tabular}

312 lines computed.

Sorted by Energy

\begin{tabular}{|c|c|c|c|c|c|c|c|}
\hline Energy & Intensity & \multicolumn{6}{|c|}{ Initial Isotopes and Decays } \\
\hline keV & $\mathrm{ph} / \mathrm{s} / \mathrm{gm}$ & & & & & & \\
\hline 8.953 & $3.30 \mathrm{E}-08$ & $\mathrm{Bi}-212$ & $->$ & TI-208 & & & \\
\hline 9.185 & $1.43 \mathrm{E}-08$ & Tl-208 & $->$ & $\mathrm{Pb}-208$ & & & \\
\hline 9.419 & $2.08 \mathrm{E}-07$ & $\mathrm{~Pb}-212$ & $->$ & $\mathrm{Bi}-212$ & & & \\
\hline 9.658 & $8.23 E-10$ & $\mathrm{Bi}-212$ & $->$ & Po-212 & & & \\
\hline 10.137 & 4.47E-09 & $\mathrm{Ra}-224$ & $->$ & $\mathrm{Rn}-220$ & & & \\
\hline 10.259 & $6.68 \mathrm{E}-07$ & $\mathrm{Bi}-212$ & $->$ & TI-208 & & & \\
\hline 10.541 & $2.86 \mathrm{E}-07$ & TI-208 & $->$ & $\mathrm{Pb}-208$ & & & \\
\hline 10.622 & $1.11 \mathrm{E}-07$ & Th-228 & $->$ & $\mathrm{Ra}-224$ & & & \\
\hline 10.828 & $4.04 \mathrm{E}-06$ & $\mathrm{~Pb}-212$ & $->$ & $\mathrm{Bi}-212$ & & & \\
\hline 10.994 & $6.22 \mathrm{E}-09$ & $\mathrm{Bi}-212$ & $->$ & TI-208 & & & \\
\hline 11.118 & $4.79 E+03$ & $A c-228$ & $->$ & Th-228, & U-236 & $->$ & Th-232 \\
\hline 11.119 & $1.61 \mathrm{E}-08$ & $\mathrm{Bi}-212$ & $->$ & Po-212 & & & \\
\hline 11.349 & 3.83E-09 & TI-208 & $->$ & $\mathrm{Pb}-208$ & & & \\
\hline 11.712 & $5.32 \mathrm{E}-08$ & $\mathrm{~Pb}-212$ & $->$ & $\mathrm{Bi}-212$ & & & \\
\hline 11.713 & $7.96 \mathrm{E}-08$ & Ra-224 & $->$ & $\mathrm{Rn}-220$ & & & \\
\hline 12.085 & $2.43 \mathrm{E}-10$ & $\mathrm{Bi}-212$ & $->$ & Po-212 & & & \\
\hline 12.282 & 7.69E-07 & $\mathrm{Bi}-212$ & $->$ & TI-208 & & & \\
\hline 12.325 & $1.91 \mathrm{E}-06$ & Th-228 & $->$ & $\mathrm{Ra}-224$ & & & \\
\hline 12.701 & $2.68 \mathrm{E}-07$ & TI-208 & $->$ & $\mathrm{Pb}-208$ & & & \\
\hline
\end{tabular}




\begin{tabular}{|c|c|c|c|c|c|c|c|}
\hline 12.76 & 2.79E-05 & $\mathrm{Ra}-228$ & $->$ & Ac- 228 & & & \\
\hline 12.855 & $2.08 \mathrm{E}-09$ & Ra-224 & $->$ & $\mathrm{Rn}-220$ & & & \\
\hline 12.952 & $8.14 \mathrm{E}+04$ & Ac- 228 & $->$ & Th-228, & $U-236$ & $->$ & Th-232 \\
\hline 13.099 & $3.67 \mathrm{E}-06$ & $\mathrm{~Pb}-212$ & $->$ & $\mathrm{Bi}-212$ & & & \\
\hline 13.5 & $1.47 \mathrm{E}-04$ & Ra-228 & $->$ & Ac- 228 & & & \\
\hline 13.501 & $1.61 \mathrm{E}-08$ & $\mathrm{Bi}-212$ & $->$ & Po-212 & & & \\
\hline 13.662 & $5.80 \mathrm{E}-08$ & Th-228 & $->$ & Ra-224 & & & \\
\hline 14.336 & $1.10 \mathrm{E}-07$ & Ra-224 & $->$ & $R n-220$ & & & \\
\hline 14.511 & $2.27 E+03$ & Ac- 228 & $->$ & Th-228, & $U-236$ & $->$ & Th-232 \\
\hline 14.563 & $1.80 \mathrm{E}-07$ & $\mathrm{Bi}-212$ & $->$ & TI-208 & & & \\
\hline 14.884 & 4.92E-08 & TI-208 & $->$ & $\mathrm{Pb}-208$ & & & \\
\hline 15.15 & $1.47 \mathrm{E}-05$ & Ra-228 & $->$ & Ac- 228 & & & \\
\hline 15.23 & $2.90 \mathrm{E}-06$ & Th-228 & $->$ & Ra-224 & & & \\
\hline 15.376 & $6.91 \mathrm{E}-07$ & $\mathrm{~Pb}-212$ & $->$ & $\mathrm{Bi}-212$ & & & \\
\hline 15.869 & 3.13E-09 & $\mathrm{Bi}-212$ & $->$ & Po-212 & & & \\
\hline 16.154 & $2.93 \mathrm{E}-05$ & Ac- 228 & $->$ & Th-228 & & & \\
\hline 16.161 & $1.12 \mathrm{E}+05$ & U-236 & $->$ & Th-232 & & & \\
\hline 16.18 & $6.60 \mathrm{E}-05$ & Ra-228 & $->$ & Ac- 228 & & & \\
\hline 16.881 & $2.39 E-08$ & Ra-224 & $->$ & $\mathrm{Rn}-220$ & & & \\
\hline 17.952 & $6.54 \mathrm{E}-07$ & Th-228 & $->$ & Ra-224 & & & \\
\hline 18.8 & $1.91 \mathrm{E}-05$ & $\mathrm{Ra}-228$ & $->$ & Ac- 228 & & & \\
\hline 19.094 & $2.51 \mathrm{E}+04$ & U-236 & $->$ & Th-232 & & & \\
\hline 19.113 & $6.75 \mathrm{E}-06$ & Ac- 228 & $->$ & Th-228 & & & \\
\hline 19.4 & $1.32 \mathrm{E}-06$ & Ra-228 & $->$ & Ac-228 & & & \\
\hline 39.846 & $2.42 \mathrm{E}-07$ & $\mathrm{Bi}-212$ & $->$ & TI-208 & & & \\
\hline 49.369 & $1.87 \mathrm{E}+03$ & U-236 & $->$ & Th-232 & & & \\
\hline 57.81 & 7.70E-07 & Ac- 228 & $->$ & Th-228 & & & \\
\hline 59 & $1.12 \mathrm{E}-06$ & Th-232 & $->$ & Ra-228 & & & \\
\hline 70.832 & $1.65 \mathrm{E}-08$ & $\mathrm{Bi}-212$ & $->$ & TI-208 & & & \\
\hline 72.803 & 4.70E-07 & TI-208 & $->$ & $\mathrm{Pb}-208$ & & & \\
\hline 72.873 & $2.79 \mathrm{E}-08$ & $\mathrm{Bi}-212$ & $->$ & TI-208 & & & \\
\hline 74.814 & $6.42 \mathrm{E}-06$ & $\mathrm{~Pb}-212$ & $->$ & $\mathrm{Bi}-212$ & & & \\
\hline 74.969 & 7.91E-07 & TI-208 & $->$ & $\mathrm{Pb}-208$ & & & \\
\hline 76.858 & $2.35 E-08$ & $\mathrm{Bi}-212$ & $->$ & Po-212 & & & \\
\hline 77.107 & $1.08 \mathrm{E}-05$ & $\mathrm{~Pb}-212$ & $->$ & $\mathrm{Bi}-212$ & & & \\
\hline 79.29 & 3.92E-08 & Bi-212 & $->$ & Po-212 & & & \\
\hline 81.067 & 7.35E-08 & Ra-224 & $->$ & $R n-220$ & & & \\
\hline 82.434 & 9.89E-09 & $\mathrm{Bi}-212$ & $->$ & TI-208 & & & \\
\hline 83.787 & $1.22 \mathrm{E}-07$ & Ra-224 & $->$ & $R n-220$ & & & \\
\hline 84.26 & 7.46E-07 & Th-228 & $->$ & Ra-224 & & & \\
\hline 84.789 & 2.79E-07 & TI-208 & $->$ & $\mathrm{Pb}-208$ & & & \\
\hline 85.185 & 2.75E-09 & $\mathrm{Bi}-212$ & $->$ & TI-208 & & & \\
\hline 85.429 & $1.08 \mathrm{E}-08$ & Th-228 & $->$ & Ra-224 & & & \\
\hline 87.19 & $3.84 \mathrm{E}-06$ & $\mathrm{~Pb}-212$ & $->$ & $\mathrm{Bi}-212$ & & & \\
\hline 87.632 & 8.07E-08 & TI-208 & $->$ & $\mathrm{Pb}-208$ & & & \\
\hline 88.471 & $1.78 \mathrm{E}-08$ & Th-228 & $->$ & Ra-224 & & & \\
\hline 89.639 & $1.37 \mathrm{E}-08$ & $\mathrm{Bi}-212$ & $->$ & Po-212 & & & \\
\hline 89.955 & 4.99E-06 & Ac- 228 & $->$ & Th-228 & & & \\
\hline 90.128 & $1.14 \mathrm{E}-06$ & $\mathrm{~Pb}-212$ & $->$ & $\mathrm{Bi}-212$ & & & \\
\hline 92.673 & 4.31E-09 & $\mathrm{Bi}-212$ & $->$ & Po-212 & & & \\
\hline
\end{tabular}




\begin{tabular}{|c|c|c|c|c|}
\hline 93.35 & $8.21 \mathrm{E}-06$ & $A c-228$ & $->$ & Th-228 \\
\hline 94.677 & $4.41 \mathrm{E}-08$ & Ra-224 & $->$ & $\mathrm{Rn}-220$ \\
\hline 97.907 & $1.41 \mathrm{E}-08$ & Ra-224 & $->$ & $\mathrm{Rn}-220$ \\
\hline 99.55 & $1.91 \mathrm{E}-06$ & Ac- 228 & $->$ & Th-228 \\
\hline 99.915 & $6.35 \mathrm{E}-09$ & Th-228 & $->$ & Ra-224 \\
\hline 103.341 & 2.07E-09 & Th-228 & $->$ & Ra-224 \\
\hline 105.362 & $2.93 \mathrm{E}-06$ & $A c-228$ & $->$ & Th-228 \\
\hline 108.99 & $9.83 \mathrm{E}-07$ & Ac- 228 & $->$ & Th-228 \\
\hline 112.75 & $4.55 E+02$ & U-236 & $->$ & Th-232 \\
\hline 115.122 & $3.62 \mathrm{E}-07$ & $\mathrm{~Pb}-212$ & $->$ & $\mathrm{Bi}-212$ \\
\hline 124 & $2.54 \mathrm{E}-07$ & Th-232 & $->$ & $\mathrm{Ra}-228$ \\
\hline 129.03 & $4.25 E-06$ & Ac-228 & $->$ & Th-228 \\
\hline 131.5 & 7.89E-08 & Th-228 & $->$ & $\mathrm{Ra}-224$ \\
\hline 135.68 & $2.49 \mathrm{E}-08$ & $A c-228$ & $->$ & Th-228 \\
\hline 141.19 & 7.19E-08 & $A c-228$ & $->$ & Th-228 \\
\hline 143.99 & 2.20E-09 & $\mathrm{Bi}-212$ & $->$ & TI-208 \\
\hline 146.06 & $4.25 E-07$ & $A c-228$ & $->$ & Th-228 \\
\hline 153.89 & $1.23 \mathrm{E}-06$ & $A c-228$ & $->$ & Th-228 \\
\hline 164 & $1.10 \mathrm{E}-09$ & $\mathrm{Bi}-212$ & $->$ & TI-208 \\
\hline 164.15 & $3.06 \mathrm{E}-09$ & $\mathrm{~Pb}-212$ & $->$ & $\mathrm{Bi}-212$ \\
\hline 166.43 & $5.06 \mathrm{E}-08$ & Th-228 & $->$ & Ra-224 \\
\hline 174.18 & 4.69E-08 & Ac-228 & $->$ & Th-228 \\
\hline 176.577 & $3.12 \mathrm{E}-08$ & $\mathrm{~Pb}-212$ & $->$ & $\mathrm{Bi}-212$ \\
\hline 184.72 & $2.05 E-07$ & Ac- 228 & $->$ & Th-228 \\
\hline 191.29 & $1.76 \mathrm{E}-07$ & $A c-228$ & $->$ & Th-228 \\
\hline 199.54 & $4.11 \mathrm{E}-07$ & $A c-228$ & $->$ & Th-228 \\
\hline 204.37 & $2.30 \mathrm{E}-07$ & $A c-228$ & $->$ & Th-228 \\
\hline 205.75 & $1.73 \mathrm{E}-08$ & Th-228 & $->$ & $\mathrm{Ra}-224$ \\
\hline 209.39 & $6.01 \mathrm{E}-06$ & Ac- 228 & $->$ & Th-228 \\
\hline 210.76 & 3.37E-07 & $A c-228$ & $->$ & Th-228 \\
\hline 211.31 & $3.74 \mathrm{E}-08$ & TI-208 & $->$ & $\mathrm{Pb}-208$ \\
\hline 215.75 & $1.71 \mathrm{E}-07$ & Th-228 & $->$ & $\mathrm{Ra}-224$ \\
\hline 216.24 & 1.17E-06 & $A c-228$ & $->$ & Th-228 \\
\hline 220.49 & $2.05 E-08$ & $A c-228$ & $->$ & Th-228 \\
\hline 223.72 & $9.68 \mathrm{E}-08$ & $A c-228$ & $->$ & Th-228 \\
\hline 232.3 & 8.80E-08 & $A c-228$ & $->$ & Th-228 \\
\hline 233.32 & $6.81 \mathrm{E}-08$ & TI-208 & $->$ & $\mathrm{Pb}-208$ \\
\hline 238.578 & $2.67 \mathrm{E}-05$ & $\mathrm{~Pb}-212$ & $->$ & $\mathrm{Bi}-212$ \\
\hline 240.76 & $2.39 \mathrm{E}-06$ & Ra-224 & $->$ & $\mathrm{Rn}-220$ \\
\hline 252.45 & $1.76 \mathrm{E}-07$ & TI-208 & $->$ & $\mathrm{Pb}-208$ \\
\hline 257.29 & 4.69E-08 & $A c-228$ & $->$ & Th-228 \\
\hline 263.57 & $8.51 \mathrm{E}-08$ & Ac- 228 & $->$ & Th-228 \\
\hline 270.26 & 5.57E-06 & $A c-228$ & $->$ & Th-228 \\
\hline 277.28 & $1.49 E-06$ & TI-208 & $->$ & $\mathrm{Pb}-208$ \\
\hline 279.3 & $3.37 \mathrm{E}-07$ & Ac- 228 & $->$ & Th-228 \\
\hline 282.02 & $1.31 \mathrm{E}-07$ & Ac-228 & $->$ & Th-228 \\
\hline 288.08 & 7.50E-08 & $\mathrm{Bi}-212$ & $->$ & TI-208 \\
\hline 290 & $5.51 \mathrm{E}-09$ & $\mathrm{Ra}-224$ & $->$ & $\mathrm{Rn}-220$ \\
\hline 300.034 & $2.04 \mathrm{E}-06$ & $\mathrm{~Pb}-212$ & $->$ & $\mathrm{Bi}-212$ \\
\hline 321.9 & $3.67 E-07$ & $A c-228$ & $->$ & Th-228 \\
\hline
\end{tabular}




\begin{tabular}{|c|c|c|c|c|c|c|c|}
\hline 327.67 & $1.91 \mathrm{E}-07$ & Ac-228 & $->$ & Th-228 & & & \\
\hline 327.93 & $3.01 \mathrm{E}-08$ & $\mathrm{Bi}-212$ & $->$ & TI-208 & & & \\
\hline 328.07 & $5.13 \mathrm{E}-06$ & Ac- 228 & $->$ & Th-228 & & & \\
\hline 332.48 & 6.89E-07 & Ac- 228 & $->$ & Th-228 & & & \\
\hline 338.42 & $1.82 \mathrm{E}-05$ & Ac- 228 & $->$ & Th-228 & & & \\
\hline 340.94 & 7.63E-07 & Ac- 228 & $->$ & Th-228 & & & \\
\hline 356.83 & $2.93 \mathrm{E}-08$ & Ac- 228 & $->$ & Th-228 & & & \\
\hline 372.3 & $1.32 \mathrm{E}-08$ & Ac- 228 & $->$ & Th-228 & & & \\
\hline 388.9 & $2.20 \mathrm{E}-08$ & Ac- 228 & $->$ & Th-228 & & & \\
\hline 396.9 & $4.69 E-08$ & Ac- 228 & $->$ & Th-228 & & & \\
\hline 399.2 & $5.43 E-08$ & $A c-228$ & $->$ & Th-228 & & & \\
\hline 409.3 & $2.45 \mathrm{E}-09$ & $\mathrm{Ra}-224$ & $->$ & $\mathrm{Rn}-220$ & & & \\
\hline 409.62 & $3.23 E-06$ & $A c-228$ & $->$ & Th-228 & & & \\
\hline 415.156 & $1.71 \mathrm{E}-08$ & $\mathrm{~Pb}-212$ & $->$ & $\mathrm{Bi}-212$ & & & \\
\hline 416.2 & $2.49 \mathrm{E}-08$ & Ac- 228 & $->$ & Th-228 & & & \\
\hline 419.23 & $3.81 \mathrm{E}-08$ & Ac- 228 & $->$ & Th-228 & & & \\
\hline 433.51 & $3.08 \mathrm{E}-09$ & $\mathrm{Bi}-212$ & $->$ & TI-208 & & & \\
\hline 440.49 & $2.20 \mathrm{E}-07$ & Ac- 228 & $->$ & Th-228 & & & \\
\hline 449.57 & $9.53 E-08$ & Ac- 228 & $->$ & Th-228 & & & \\
\hline 452.77 & 7.98E-08 & $\mathrm{Bi}-212$ & $->$ & TI-208 & & & \\
\hline 460.84 & 7.33E-08 & Ac- 228 & $->$ & Th-228 & & & \\
\hline 463.1 & $6.75 \mathrm{E}-06$ & Ac- 228 & $->$ & Th-228 & & & \\
\hline 471.4 & $5.13 \mathrm{E}-08$ & Ac- 228 & $->$ & Th-228 & & & \\
\hline 473.36 & $1.10 \mathrm{E}-08$ & $\mathrm{Bi}-212$ & $->$ & TI-208 & & & \\
\hline 474.32 & $4.25 E-08$ & Ac- 228 & $->$ & Th-228 & & & \\
\hline 478.2 & $3.52 \mathrm{E}-07$ & Ac- 228 & $->$ & Th-228 & & & \\
\hline 481.8 & $1.32 \mathrm{E}-08$ & $A c-228$ & $->$ & Th-228 & & & \\
\hline 485.78 & $1.10 \mathrm{E}-08$ & TI-208 & $->$ & $\mathrm{Pb}-208$ & & & \\
\hline 492.36 & $2.93 \mathrm{E}-08$ & Ac- 228 & $->$ & Th-228 & & & \\
\hline 492.62 & $1.34 \mathrm{E}-09$ & $\mathrm{Bi}-212$ & $->$ & TI-208 & & & \\
\hline 498.26 & $6.31 \mathrm{E}-08$ & Ac- 228 & $->$ & Th-228 & & & \\
\hline 503.7 & $3.08 \mathrm{E}-07$ & Ac- 228 & $->$ & Th-228 & & & \\
\hline 509.17 & 7.19E-07 & Ac- 228 & $->$ & Th-228 & & & \\
\hline 510.606 & $4.75 \mathrm{E}-06$ & TI-208 & $->$ & $\mathrm{Pb}-208$ & & & \\
\hline 515.2 & $6.31 \mathrm{E}-08$ & $A c-228$ & $->$ & Th-228 & & & \\
\hline 519.97 & $1.14 \mathrm{E}-07$ & Ac- 228 & $->$ & Th-228 & & & \\
\hline 523.18 & $1.76 \mathrm{E}-07$ & $A c-228$ & $->$ & Th-228 & & & \\
\hline 540.5 & $4.25 E-08$ & Ac- 228 & $->$ & Th-228 & & & \\
\hline 546.36 & $3.23 E-07$ & Ac- 228 & $->$ & Th-228 & & & \\
\hline 549.7 & $4.29 E-08$ & $\mathrm{Rn}-220$ & $->$ & Po-216 & & & \\
\hline 555.3 & 7.19E-08 & Ac- 228 & $->$ & Th-228 & & & \\
\hline 562.65 & $1.48 \mathrm{E}-06$ & Ac- 228 & $->$ & Th-228 & & & \\
\hline 570.2 & 2.79E-07 & Ac- 228 & $->$ & Th-228 & & & \\
\hline 572.5 & $2.79 E-07$ & Ac- 228 & $->$ & Th-228 & & & \\
\hline 580.4 & $1.74 \mathrm{E}-10$ & $\mathrm{Bi}-212$ & $->$ & TI-208 & & & \\
\hline 583.022 & $1.97 \mathrm{E}-05$ & TI-208 & $->$ & $\mathrm{Pb}-208$ & Po-212 & $->$ & $\mathrm{Pb}-208$ \\
\hline 583.28 & $2.20 \mathrm{E}-07$ & $A c-228$ & $->$ & Th-228 & & & \\
\hline 587.82 & 8.79E-09 & TI-208 & $->$ & $\mathrm{Pb}-208$ & & & \\
\hline 615.9 & $1.32 \mathrm{E}-07$ & Ac- 228 & $->$ & Th-228 & & & \\
\hline 619.88 & $1.61 \mathrm{E}-07$ & $A c-228$ & $->$ & Th-228 & & & \\
\hline
\end{tabular}




\begin{tabular}{|c|c|c|c|c|}
\hline 620.3 & $7.91 \mathrm{E}-10$ & $\mathrm{Bi}-212$ & $->$ & TI-208 \\
\hline 629.93 & 7.33E-08 & $A c-228$ & $->$ & Th-228 \\
\hline 640.7 & $8.95 E-08$ & $A c-228$ & $->$ & Th-228 \\
\hline 649.19 & $6.31 \mathrm{E}-08$ & Ac- 228 & $->$ & Th-228 \\
\hline 650.1 & 4.29E-09 & Ra-224 & $->$ & $R n-220$ \\
\hline 650.14 & 7.91E-09 & TI-208 & $->$ & $\mathrm{Pb}-208$ \\
\hline 651.44 & $1.48 \mathrm{E}-07$ & $A c-228$ & $->$ & Th-228 \\
\hline 666.4 & $6.75 \mathrm{E}-08$ & Ac- 228 & $->$ & Th-228 \\
\hline 673.86 & $1.47 \mathrm{E}-07$ & Ac-228 & $->$ & Th-228 \\
\hline 677.07 & $1.32 \mathrm{E}-06$ & Ac- 228 & $->$ & Th-228 \\
\hline 692.5 & 4.99E-09 & $A c-228$ & $->$ & Th-228 \\
\hline 701.8 & 2.79E-07 & Ac-228 & $->$ & Th-228 \\
\hline 705.24 & 4.84E-09 & TI-208 & $->$ & $\mathrm{Pb}-208$ \\
\hline 707.49 & $2.20 \mathrm{E}-07$ & $A c-228$ & $->$ & Th-228 \\
\hline 721.91 & $4.46 \mathrm{E}-08$ & TI-208 & $->$ & $\mathrm{Pb}-208$ \\
\hline 726.63 & $1.28 \mathrm{E}-06$ & Ac- 228 & $->$ & Th-228 \\
\hline 727.25 & $2.61 \mathrm{E}-06$ & $\mathrm{Bi}-212$ & $->$ & Po-212 \\
\hline 737.7 & $6.01 \mathrm{E}-08$ & $A c-228$ & $->$ & Th-228 \\
\hline 748.58 & $9.45 \mathrm{E}-09$ & TI-208 & $->$ & $\mathrm{Pb}-208$ \\
\hline 755.28 & $1.94 \mathrm{E}-06$ & Ac- 228 & $->$ & Th-228 \\
\hline 763.06 & $3.61 \mathrm{E}-07$ & TI-208 & $->$ & $\mathrm{Pb}-208$ \\
\hline 772.28 & $1.60 \mathrm{E}-06$ & Ac-228 & $->$ & Th-228 \\
\hline 774 & $1.32 \mathrm{E}-07$ & $A c-228$ & $->$ & Th-228 \\
\hline 782.12 & $8.65 E-07$ & Ac- 228 & $->$ & Th-228 \\
\hline 785.51 & $4.34 \mathrm{E}-07$ & $\mathrm{Bi}-212$ & $->$ & Po-212 \\
\hline 791.05 & $3.08 \mathrm{E}-08$ & $A c-228$ & $->$ & Th-228 \\
\hline 791.2 & $4.25 E-08$ & $A c-228$ & $->$ & Th-228 \\
\hline 794.79 & $6.75 \mathrm{E}-06$ & Ac- 228 & $->$ & Th-228 \\
\hline 804.9 & $1.10 \mathrm{E}-09$ & Po-216 & $->$ & $\mathrm{Pb}-212$ \\
\hline 816.62 & $5.13 \mathrm{E}-08$ & $A c-228$ & $->$ & Th-228 \\
\hline 821.14 & 8.79E-09 & TI-208 & $->$ & $\mathrm{Pb}-208$ \\
\hline 825.3 & $8.51 \mathrm{E}-08$ & Ac- 228 & $->$ & Th-228 \\
\hline 830.59 & $9.24 \mathrm{E}-07$ & $A c-228$ & $->$ & Th-228 \\
\hline 835.6 & $2.51 \mathrm{E}-06$ & $A c-228$ & $->$ & Th-228 \\
\hline 840.44 & $1.38 \mathrm{E}-06$ & $A c-228$ & $->$ & Th-228 \\
\hline 853.6 & $2.05 E-08$ & $A c-228$ & $->$ & Th-228 \\
\hline 860.3 & $2.64 \mathrm{E}-06$ & TI-208 & $->$ & $\mathrm{Pb}-208$ \\
\hline 870.47 & $9.68 \mathrm{E}-08$ & $A c-228$ & $->$ & Th-228 \\
\hline 874.43 & $1.23 \mathrm{E}-07$ & $A c-228$ & $->$ & Th-228 \\
\hline 877.65 & $2.93 E-08$ & $A c-228$ & $->$ & Th-228 \\
\hline 883.27 & 6.81E-09 & TI-208 & $->$ & $\mathrm{Pb}-208$ \\
\hline 884 & $1.47 \mathrm{E}-07$ & $A c-228$ & $->$ & Th-228 \\
\hline 887.46 & $2.93 E-08$ & $A c-228$ & $->$ & Th-228 \\
\hline 893.42 & $1.44 \mathrm{E}-07$ & $\mathrm{Bi}-212$ & $->$ & Po-212 \\
\hline 904.29 & $1.31 \mathrm{E}-06$ & $A c-228$ & $->$ & Th-228 \\
\hline 911.16 & $4.25 E-05$ & Ac-228 & $->$ & Th-228 \\
\hline 919.19 & $4.11 \mathrm{E}-08$ & $A c-228$ & $->$ & Th-228 \\
\hline 922.28 & $3.08 \mathrm{E}-08$ & Ac- 228 & $->$ & Th-228 \\
\hline 927.42 & $2.75 \mathrm{E}-08$ & TI-208 & $->$ & $\mathrm{Pb}-208$ \\
\hline 931.1 & $2.20 \mathrm{E}-08$ & $A c-228$ & $->$ & Th-228 \\
\hline
\end{tabular}




\begin{tabular}{|c|c|c|c|c|}
\hline 943.9 & $1.57 \mathrm{E}-07$ & Ac- 228 & $->$ & Th-228 \\
\hline 948.4 & $1.79 E-07$ & Ac- 228 & $->$ & Th-228 \\
\hline 952.17 & $6.90 \mathrm{E}-08$ & $\mathrm{Bi}-212$ & $->$ & Po-212 \\
\hline 958.3 & 4.55E-07 & $A c-228$ & $->$ & Th-228 \\
\hline 964.64 & $8.51 \mathrm{E}-06$ & Ac- 228 & $->$ & Th-228 \\
\hline 968.97 & $2.55 \mathrm{E}-05$ & Ac- 228 & $->$ & Th-228 \\
\hline 979.7 & 3.37E-08 & $A c-228$ & $->$ & Th-228 \\
\hline 982.52 & $4.33 \mathrm{E}-08$ & TI-208 & $->$ & $\mathrm{Pb}-208$ \\
\hline 987.87 & 4.99E-07 & Ac- 228 & $->$ & Th-228 \\
\hline 987.94 & $2.79 E-07$ & Ac- 228 & $->$ & Th-228 \\
\hline 1016.12 & $3.67 \mathrm{E}-08$ & Ac- 228 & $->$ & Th-228 \\
\hline 1019.7 & $3.67 \mathrm{E}-08$ & Ac- 228 & $->$ & Th-228 \\
\hline 1033.32 & $2.27 \mathrm{E}-07$ & Ac- 228 & $->$ & Th-228 \\
\hline 1039.97 & $8.07 E-08$ & $A C-228$ & $->$ & Th-228 \\
\hline 1041.35 & $4.69 E-08$ & $A c-228$ & $->$ & Th-228 \\
\hline 1054.3 & $4.69 E-08$ & $A c-228$ & $->$ & Th-228 \\
\hline 1065.05 & $2.51 \mathrm{E}-07$ & Ac- 228 & $->$ & Th-228 \\
\hline 1074.1 & 6.27E-09 & $\mathrm{Bi}-212$ & $->$ & Po-212 \\
\hline 1078.69 & $2.10 \mathrm{E}-07$ & $\mathrm{Bi}-212$ & $->$ & Po-212 \\
\hline 1093.63 & $8.13 E-08$ & TI-208 & $->$ & $\mathrm{Pb}-208$ \\
\hline 1095.87 & $1.94 \mathrm{E}-07$ & Ac- 228 & $->$ & Th-228 \\
\hline 1104.01 & $2.49 E-08$ & $A c-228$ & $->$ & Th-228 \\
\hline 1110.7 & $5.68 \mathrm{E}-07$ & Ac- 228 & $->$ & Th-228 \\
\hline 1116.97 & $8.95 \mathrm{E}-08$ & Ac- 228 & $->$ & Th-228 \\
\hline 1125.6 & $1.10 \mathrm{E}-09$ & TI-208 & $->$ & $\mathrm{Pb}-208$ \\
\hline 1135.39 & $1.76 \mathrm{E}-08$ & $A c-228$ & $->$ & Th-228 \\
\hline 1142.7 & $1.47 \mathrm{E}-08$ & $A c-228$ & $->$ & Th-228 \\
\hline 1153.69 & $2.33 \mathrm{E}-07$ & $A c-228$ & $->$ & Th-228 \\
\hline 1160.55 & $2.42 \mathrm{E}-09$ & TI-208 & $->$ & $\mathrm{Pb}-208$ \\
\hline 1163.6 & $1.10 \mathrm{E}-07$ & $A c-228$ & $->$ & Th-228 \\
\hline 1174.79 & $4.25 \mathrm{E}-08$ & Ac- 228 & $->$ & Th-228 \\
\hline 1185.1 & $3.74 \mathrm{E}-09$ & TI-208 & $->$ & $\mathrm{Pb}-208$ \\
\hline 1216.4 & $3.81 \mathrm{E}-08$ & Ac- 228 & $->$ & Th-228 \\
\hline 1245.23 & $1.32 \mathrm{E}-07$ & Ac- 228 & $->$ & Th-228 \\
\hline 1246.6 & $8.36 \mathrm{E}-07$ & $A c-228$ & $->$ & Th-228 \\
\hline 1249.81 & 8.80E-08 & Ac- 228 & $->$ & Th-228 \\
\hline 1277.5 & $2.93 \mathrm{E}-08$ & Ac- 228 & $->$ & Th-228 \\
\hline 1282.7 & $1.14 \mathrm{E}-08$ & TI-208 & $->$ & $\mathrm{Pb}-208$ \\
\hline 1286.6 & $1.75 \mathrm{E}-07$ & $A c-228$ & $->$ & Th-228 \\
\hline 1309.6 & $3.08 \mathrm{E}-08$ & $A c-228$ & $->$ & Th-228 \\
\hline 1314.76 & $3.08 \mathrm{E}-08$ & Ac- 228 & $->$ & Th-228 \\
\hline 1347.6 & $1.91 \mathrm{E}-08$ & $A c-228$ & $->$ & Th-228 \\
\hline 1357.6 & $4.25 E-08$ & $A c-228$ & $->$ & Th-228 \\
\hline 1374.26 & $2.93 E-08$ & Ac- 228 & $->$ & Th-228 \\
\hline 1381 & $1.54 \mathrm{E}-09$ & TI-208 & $->$ & $\mathrm{Pb}-208$ \\
\hline 1415.7 & 3.81E-08 & $A c-228$ & $->$ & Th-228 \\
\hline 1431 & 4.69E-08 & Ac- 228 & $->$ & Th-228 \\
\hline 1451.2 & $2.64 \mathrm{E}-08$ & Ac- 228 & $->$ & Th-228 \\
\hline 1459.19 & $1.55 \mathrm{E}-06$ & Ac- 228 & $->$ & Th-228 \\
\hline 1468.8 & $2.93 E-08$ & AC-228 & $->$ & Th-228 \\
\hline
\end{tabular}




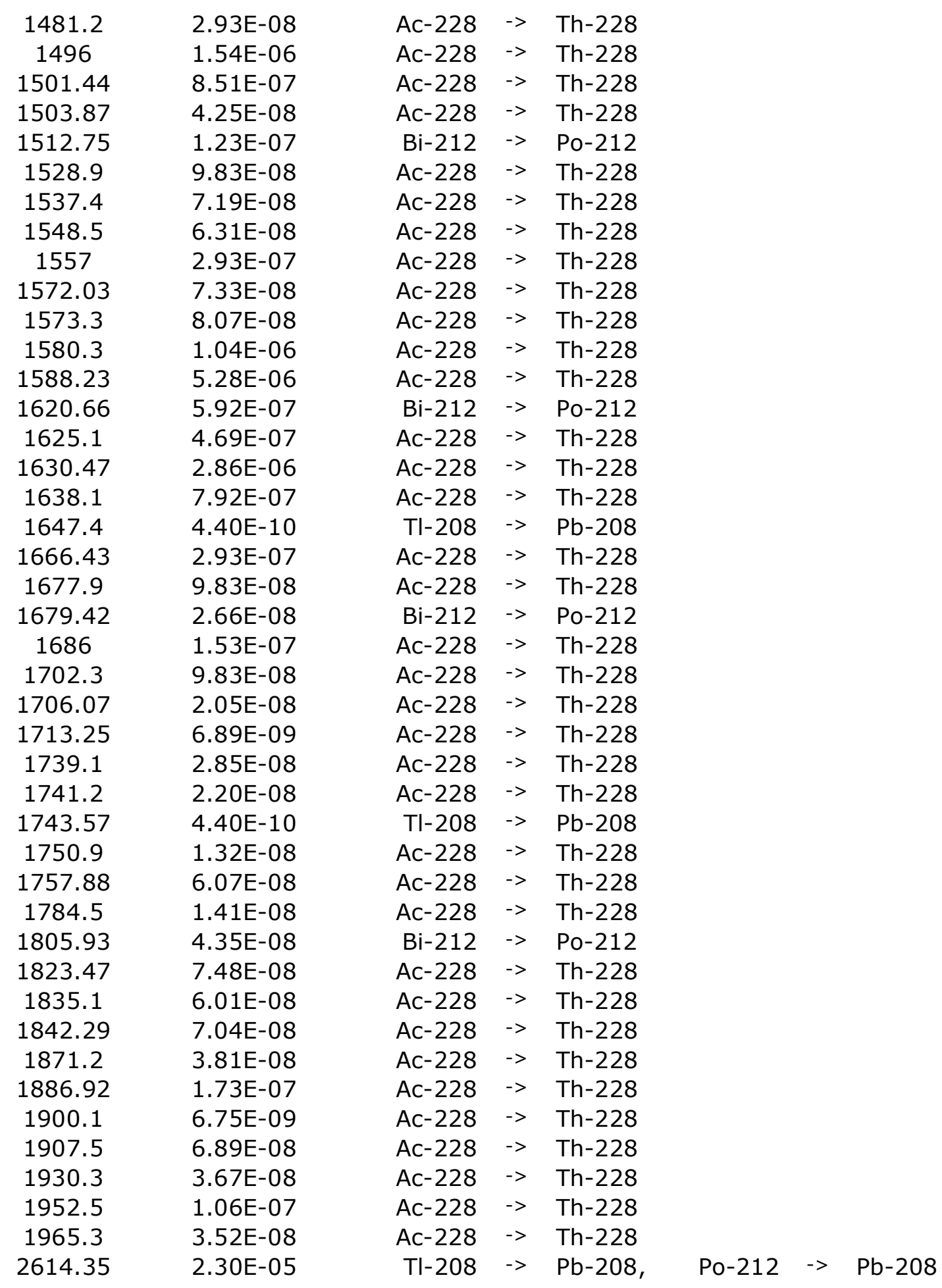

U236 Aged 5 years Sorted by Intensity

\begin{tabular}{|l|l|l|}
\hline Intensity & Energy & Initial Isotopes and Decays \\
\hline
\end{tabular}

$\mathrm{ph} / \mathrm{s} / \mathrm{gm} \quad \mathrm{keV}$ 


\begin{tabular}{|c|c|c|c|c|c|c|c|}
\hline $1.12 \mathrm{E}+05$ & 16.161 & $\mathrm{U}-236$ & $->$ & Th-232 & & & \\
\hline $8.14 \mathrm{E}+04$ & 12.952 & Ac- 228 & $->$ & Th-228, & $U-236$ & $->$ & Th-232 \\
\hline $2.51 \mathrm{E}+04$ & 19.094 & $U-236$ & $->$ & Th-232 & & & \\
\hline $4.79 E+03$ & 11.118 & Ac- 228 & $->$ & Th-228, & $U-236$ & $->$ & Th-232 \\
\hline $2.27 E+03$ & 14.511 & Ac- 228 & $->$ & Th-228, & $U-236$ & $->$ & Th-232 \\
\hline $1.87 E+03$ & 49.369 & $U-236$ & $->$ & Th-232 & & & \\
\hline $4.55 E+02$ & 112.75 & $U-236$ & $->$ & Th-232 & & & \\
\hline $1.47 \mathrm{E}-04$ & 13.5 & $\mathrm{Ra}-228$ & $->$ & Ac- 228 & & & \\
\hline 6.60E-05 & 16.18 & $\mathrm{Ra}-228$ & $->$ & Ac- 228 & & & \\
\hline $4.25 E-05$ & 911.16 & Ac- 228 & $->$ & Th-228 & & & \\
\hline 2.93E-05 & 16.154 & Ac- 228 & $->$ & Th-228 & & & \\
\hline 2.79E-05 & 12.76 & $\mathrm{Ra}-228$ & $->$ & Ac- 228 & & & \\
\hline 2.67E-05 & 238.578 & $\mathrm{~Pb}-212$ & $->$ & $\mathrm{Bi}-212$ & & & \\
\hline $2.55 \mathrm{E}-05$ & 968.97 & Ac- 228 & $->$ & Th-228 & & & \\
\hline $2.30 \mathrm{E}-05$ & 2614.35 & Tl-208 & $->$ & $\mathrm{Pb}-208$, & Po-212 & $->$ & $\mathrm{Pb}-208$ \\
\hline 1.97E-05 & 583.022 & Tl-208 & $->$ & $\mathrm{Pb}-208$, & Po-212 & $->$ & $\mathrm{Pb}-208$ \\
\hline $1.91 \mathrm{E}-05$ & 18.8 & $\mathrm{Ra}-228$ & $->$ & Ac- 228 & & & \\
\hline $1.82 \mathrm{E}-05$ & 338.42 & Ac- 228 & $->$ & Th-228 & & & \\
\hline $1.47 \mathrm{E}-05$ & 15.15 & Ra-228 & $->$ & Ac- 228 & & & \\
\hline $1.08 \mathrm{E}-05$ & 77.107 & $\mathrm{~Pb}-212$ & $->$ & $\mathrm{Bi}-212$ & & & \\
\hline $8.51 \mathrm{E}-06$ & 964.64 & Ac- 228 & $->$ & Th-228 & & & \\
\hline $8.21 \mathrm{E}-06$ & 93.35 & Ac- 228 & $->$ & Th-228 & & & \\
\hline $6.75 E-06$ & 463.1 & Ac- 228 & $->$ & Th-228 & & & \\
\hline $6.75 E-06$ & 19.113 & Ac- 228 & $->$ & Th-228 & & & \\
\hline $6.75 \mathrm{E}-06$ & 794.79 & Ac- 228 & $->$ & Th-228 & & & \\
\hline $6.42 \mathrm{E}-06$ & 74.814 & $\mathrm{~Pb}-212$ & $->$ & $\mathrm{Bi}-212$ & & & \\
\hline $6.01 \mathrm{E}-06$ & 209.39 & Ac- 228 & $->$ & Th-228 & & & \\
\hline $5.57 \mathrm{E}-06$ & 270.26 & Ac- 228 & $->$ & Th-228 & & & \\
\hline $5.28 \mathrm{E}-06$ & 1588.23 & Ac- 228 & $->$ & Th-228 & & & \\
\hline $5.13 \mathrm{E}-06$ & 328.07 & Ac- 228 & $->$ & Th-228 & & & \\
\hline 4.99E-06 & 89.955 & Ac- 228 & $->$ & Th-228 & & & \\
\hline $4.75 \mathrm{E}-06$ & 510.606 & TI-208 & $->$ & $\mathrm{Pb}-208$ & & & \\
\hline $4.25 \mathrm{E}-06$ & 129.03 & Ac- 228 & $->$ & Th-228 & & & \\
\hline $4.04 \mathrm{E}-06$ & 10.828 & $\mathrm{~Pb}-212$ & $->$ & $\mathrm{Bi}-212$ & & & \\
\hline 3.84E-06 & 87.19 & $\mathrm{~Pb}-212$ & $->$ & $\mathrm{Bi}-212$ & & & \\
\hline 3.67E-06 & 13.099 & $\mathrm{~Pb}-212$ & $->$ & $\mathrm{Bi}-212$ & & & \\
\hline $3.23 \mathrm{E}-06$ & 409.62 & Ac- 228 & $->$ & Th-228 & & & \\
\hline $2.93 \mathrm{E}-06$ & 105.362 & Ac- 228 & $->$ & Th-228 & & & \\
\hline 2.90E-06 & 15.23 & Th-228 & $->$ & Ra-224 & & & \\
\hline $2.86 \mathrm{E}-06$ & 1630.47 & Ac- 228 & $->$ & Th-228 & & & \\
\hline $2.64 \mathrm{E}-06$ & 860.3 & TI-208 & $->$ & $\mathrm{Pb}-208$ & & & \\
\hline $2.61 \mathrm{E}-06$ & 727.25 & $\mathrm{Bi}-212$ & $->$ & Po-212 & & & \\
\hline $2.51 \mathrm{E}-06$ & 835.6 & Ac- 228 & $->$ & Th-228 & & & \\
\hline 2.39E-06 & 240.76 & Ra-224 & $->$ & $\mathrm{Rn}-220$ & & & \\
\hline 2.04E-06 & 300.034 & $\mathrm{~Pb}-212$ & $->$ & $\mathrm{Bi}-212$ & & & \\
\hline 1.94E-06 & 755.28 & Ac- 228 & $->$ & Th-228 & & & \\
\hline $1.91 \mathrm{E}-06$ & 12.325 & Th-228 & $->$ & Ra-224 & & & \\
\hline 1.91E-06 & 99.55 & Ac- 228 & $->$ & Th-228 & & & \\
\hline $1.60 \mathrm{E}-06$ & 772.28 & Ac- 228 & $->$ & Th-228 & & & \\
\hline $1.55 \mathrm{E}-06$ & 1459.19 & Ac- 228 & $->$ & Th-228 & & & \\
\hline
\end{tabular}




\begin{tabular}{|c|c|c|c|c|}
\hline $1.54 \mathrm{E}-06$ & 1496 & $A C-228$ & $->$ & Th-228 \\
\hline $1.49 \mathrm{E}-06$ & 277.28 & TI-208 & $->$ & $\mathrm{Pb}-208$ \\
\hline $1.48 \mathrm{E}-06$ & 562.65 & Ac- 228 & $->$ & Th-228 \\
\hline $1.38 \mathrm{E}-06$ & 840.44 & Ac- 228 & $->$ & Th-228 \\
\hline $1.32 \mathrm{E}-06$ & 19.4 & Ra-228 & $->$ & Ac- 228 \\
\hline $1.32 \mathrm{E}-06$ & 677.07 & $A c-228$ & $->$ & Th-228 \\
\hline $1.31 \mathrm{E}-06$ & 904.29 & Ac- 228 & $->$ & Th-228 \\
\hline $1.28 \mathrm{E}-06$ & 726.63 & Ac- 228 & $->$ & Th-228 \\
\hline $1.23 \mathrm{E}-06$ & 153.89 & Ac- 228 & $->$ & Th-228 \\
\hline 1.17E-06 & 216.24 & Ac- 228 & $->$ & Th-228 \\
\hline $1.14 \mathrm{E}-06$ & 90.128 & $\mathrm{~Pb}-212$ & $->$ & $\mathrm{Bi}-212$ \\
\hline $1.12 \mathrm{E}-06$ & 59 & Th-232 & $->$ & Ra-228 \\
\hline 1.04E-06 & 1580.3 & Ac- 228 & $->$ & Th-228 \\
\hline $9.83 \mathrm{E}-07$ & 108.99 & Ac- 228 & $->$ & Th-228 \\
\hline $9.24 \mathrm{E}-07$ & 830.59 & Ac- 228 & $->$ & Th-228 \\
\hline 8.65E-07 & 782.12 & Ac- 228 & $->$ & Th-228 \\
\hline $8.51 \mathrm{E}-07$ & 1501.44 & Ac- 228 & $->$ & Th-228 \\
\hline $8.36 \mathrm{E}-07$ & 1246.6 & Ac- 228 & $->$ & Th-228 \\
\hline 7.92E-07 & 1638.1 & $A c-228$ & $->$ & Th-228 \\
\hline 7.91E-07 & 74.969 & TI-208 & $->$ & $\mathrm{Pb}-208$ \\
\hline 7.70E-07 & 57.81 & $A c-228$ & $->$ & Th-228 \\
\hline 7.69E-07 & 12.282 & $\mathrm{Bi}-212$ & $->$ & TI-208 \\
\hline 7.63E-07 & 340.94 & $A c-228$ & $->$ & Th-228 \\
\hline $7.46 \mathrm{E}-07$ & 84.26 & Th-228 & $->$ & Ra-224 \\
\hline 7.19E-07 & 509.17 & Ac- 228 & $->$ & Th-228 \\
\hline $6.91 \mathrm{E}-07$ & 15.376 & $\mathrm{~Pb}-212$ & $->$ & $\mathrm{Bi}-212$ \\
\hline $6.89 \mathrm{E}-07$ & 332.48 & $A c-228$ & $->$ & Th-228 \\
\hline $6.68 \mathrm{E}-07$ & 10.259 & $\mathrm{Bi}-212$ & $->$ & TI-208 \\
\hline $6.54 \mathrm{E}-07$ & 17.952 & Th-228 & $->$ & Ra-224 \\
\hline 5.92E-07 & 1620.66 & $\mathrm{Bi}-212$ & $->$ & Po-212 \\
\hline 5.68E-07 & 1110.7 & $A c-228$ & $->$ & Th-228 \\
\hline 4.99E-07 & 987.87 & Ac- 228 & $->$ & Th-228 \\
\hline 4.70E-07 & 72.803 & TI-208 & $->$ & $\mathrm{Pb}-208$ \\
\hline 4.69E-07 & 1625.1 & $A c-228$ & $->$ & Th-228 \\
\hline $4.55 E-07$ & 958.3 & $A c-228$ & $->$ & Th-228 \\
\hline 4.34E-07 & 785.51 & $\mathrm{Bi}-212$ & $->$ & Po-212 \\
\hline $4.25 \mathrm{E}-07$ & 146.06 & $A c-228$ & $->$ & Th-228 \\
\hline $4.11 \mathrm{E}-07$ & 199.54 & Ac- 228 & $->$ & Th-228 \\
\hline 3.67E-07 & 321.9 & Ac- 228 & $->$ & Th-228 \\
\hline $3.62 \mathrm{E}-07$ & 115.122 & $\mathrm{~Pb}-212$ & $->$ & $\mathrm{Bi}-212$ \\
\hline 3.61E-07 & 763.06 & TI-208 & $->$ & $\mathrm{Pb}-208$ \\
\hline 3.52E-07 & 478.2 & Ac- 228 & $->$ & Th-228 \\
\hline 3.37E-07 & 279.3 & $A c-228$ & $->$ & Th-228 \\
\hline 3.37E-07 & 210.76 & $A c-228$ & $->$ & Th-228 \\
\hline $3.23 E-07$ & 546.36 & $A c-228$ & $->$ & Th-228 \\
\hline $3.08 \mathrm{E}-07$ & 503.7 & $A c-228$ & $->$ & Th-228 \\
\hline 2.93E-07 & 1666.43 & Ac- 228 & $->$ & Th-228 \\
\hline 2.93E-07 & 1557 & $A c-228$ & $->$ & Th-228 \\
\hline $2.86 \mathrm{E}-07$ & 10.541 & TI-208 & $->$ & $\mathrm{Pb}-208$ \\
\hline $2.79 E-07$ & 84.789 & TI-208 & $->$ & $\mathrm{Pb}-208$ \\
\hline
\end{tabular}




\begin{tabular}{|c|c|c|c|c|}
\hline 2.79E-07 & 701.8 & AC- 228 & $->$ & Th-228 \\
\hline $2.79 \mathrm{E}-07$ & 572.5 & $A c-228$ & $->$ & Th-228 \\
\hline 2.79E-07 & 987.94 & $A c-228$ & $->$ & Th-228 \\
\hline 2.79E-07 & 570.2 & Ac- 228 & $->$ & Th-228 \\
\hline $2.68 \mathrm{E}-07$ & 12.701 & TI-208 & $->$ & $\mathrm{Pb}-208$ \\
\hline $2.54 \mathrm{E}-07$ & 124 & Th-232 & $->$ & $\mathrm{Ra}-228$ \\
\hline $2.51 \mathrm{E}-07$ & 1065.05 & $A c-228$ & $->$ & Th-228 \\
\hline $2.42 \mathrm{E}-07$ & 39.846 & $\mathrm{Bi}-212$ & $->$ & TI-208 \\
\hline $2.33 \mathrm{E}-07$ & 1153.69 & Ac-228 & $->$ & Th-228 \\
\hline $2.30 \mathrm{E}-07$ & 204.37 & $A c-228$ & $->$ & Th-228 \\
\hline $2.27 \mathrm{E}-07$ & 1033.32 & $A c-228$ & $->$ & Th-228 \\
\hline $2.20 \mathrm{E}-07$ & 440.49 & Ac- 228 & $->$ & Th-228 \\
\hline $2.20 \mathrm{E}-07$ & 707.49 & $A c-228$ & $->$ & Th-228 \\
\hline $2.20 \mathrm{E}-07$ & 583.28 & $A c-228$ & $->$ & Th-228 \\
\hline $2.10 \mathrm{E}-07$ & 1078.69 & $\mathrm{Bi}-212$ & $->$ & Po-212 \\
\hline $2.08 \mathrm{E}-07$ & 9.419 & $\mathrm{~Pb}-212$ & $->$ & $\mathrm{Bi}-212$ \\
\hline $2.05 E-07$ & 184.72 & Ac-228 & $->$ & Th-228 \\
\hline $1.94 \mathrm{E}-07$ & 1095.87 & $A c-228$ & $->$ & Th-228 \\
\hline $1.91 \mathrm{E}-07$ & 327.67 & Ac- 228 & $->$ & Th-228 \\
\hline $1.80 \mathrm{E}-07$ & 14.563 & $\mathrm{Bi}-212$ & $->$ & TI-208 \\
\hline 1.79E-07 & 948.4 & $A c-228$ & $->$ & Th-228 \\
\hline $1.76 \mathrm{E}-07$ & 191.29 & Ac-228 & $->$ & Th-228 \\
\hline $1.76 \mathrm{E}-07$ & 523.18 & Ac- 228 & $->$ & Th-228 \\
\hline $1.76 \mathrm{E}-07$ & 252.45 & TI-208 & $->$ & $\mathrm{Pb}-208$ \\
\hline $1.75 \mathrm{E}-07$ & 1286.6 & Ac-228 & $->$ & Th-228 \\
\hline $1.73 \mathrm{E}-07$ & 1886.92 & $A c-228$ & $->$ & Th-228 \\
\hline $1.71 \mathrm{E}-07$ & 215.75 & Th-228 & $->$ & Ra-224 \\
\hline $1.61 \mathrm{E}-07$ & 619.88 & $A C-228$ & $->$ & Th-228 \\
\hline $1.57 \mathrm{E}-07$ & 943.9 & Ac- 228 & $->$ & Th-228 \\
\hline 1.53E-07 & 1686 & $A c-228$ & $->$ & Th-228 \\
\hline $1.48 \mathrm{E}-07$ & 651.44 & $A c-228$ & $->$ & Th-228 \\
\hline $1.47 \mathrm{E}-07$ & 673.86 & Ac- 228 & $->$ & Th-228 \\
\hline $1.47 \mathrm{E}-07$ & 884 & $A C-228$ & $->$ & Th-228 \\
\hline $1.44 \mathrm{E}-07$ & 893.42 & $\mathrm{Bi}-212$ & $->$ & Po-212 \\
\hline $1.32 \mathrm{E}-07$ & 774 & Ac-228 & $->$ & Th-228 \\
\hline $1.32 \mathrm{E}-07$ & 1245.23 & Ac-228 & $->$ & Th-228 \\
\hline $1.32 \mathrm{E}-07$ & 615.9 & Ac- 228 & $->$ & Th-228 \\
\hline $1.31 \mathrm{E}-07$ & 282.02 & Ac- 228 & $->$ & Th-228 \\
\hline $1.23 \mathrm{E}-07$ & 874.43 & Ac-228 & $->$ & Th-228 \\
\hline $1.23 \mathrm{E}-07$ & 1512.75 & $\mathrm{Bi}-212$ & $->$ & Po-212 \\
\hline $1.22 \mathrm{E}-07$ & 83.787 & Ra-224 & $->$ & $\mathrm{Rn}-220$ \\
\hline $1.14 \mathrm{E}-07$ & 519.97 & Ac- 228 & $->$ & Th-228 \\
\hline $1.11 \mathrm{E}-07$ & 10.622 & Th-228 & $->$ & $\mathrm{Ra}-224$ \\
\hline $1.10 \mathrm{E}-07$ & 14.336 & Ra-224 & $->$ & $\mathrm{Rn}-220$ \\
\hline $1.10 \mathrm{E}-07$ & 1163.6 & Ac- 228 & $->$ & Th-228 \\
\hline $1.06 \mathrm{E}-07$ & 1952.5 & Ac-228 & $->$ & Th-228 \\
\hline $9.83 \mathrm{E}-08$ & 1528.9 & $A c-228$ & $->$ & Th-228 \\
\hline 9.83E-08 & 1677.9 & $A c-228$ & $->$ & Th-228 \\
\hline 9.83E-08 & 1702.3 & Ac-228 & $->$ & Th-228 \\
\hline 9.68E-08 & 870.47 & $A c-228$ & $->$ & Th-228 \\
\hline
\end{tabular}




\begin{tabular}{|c|c|c|c|c|}
\hline $9.68 \mathrm{E}-08$ & 223.72 & AC- 228 & $\rightarrow$ & Th-228 \\
\hline $9.53 E-08$ & 449.57 & $A c-228$ & $->$ & Th-228 \\
\hline 8.95E-08 & 640.7 & $A c-228$ & $->$ & Th-228 \\
\hline 8.95E-08 & 1116.97 & Ac- 228 & $->$ & Th-228 \\
\hline 8.80E-08 & 232.3 & $A c-228$ & $->$ & Th-228 \\
\hline 8.80E-08 & 1249.81 & $A c-228$ & $->$ & Th-228 \\
\hline $8.51 \mathrm{E}-08$ & 263.57 & $A c-228$ & $->$ & Th-228 \\
\hline 8.51E-08 & 825.3 & Ac- 228 & $->$ & Th-228 \\
\hline $8.13 \mathrm{E}-08$ & 1093.63 & TI-208 & $->$ & $\mathrm{Pb}-208$ \\
\hline 8.07E-08 & 87.632 & Tl-208 & $->$ & $\mathrm{Pb}-208$ \\
\hline 8.07E-08 & 1573.3 & $A c-228$ & $->$ & Th-228 \\
\hline 8.07E-08 & 1039.97 & Ac- 228 & $->$ & Th-228 \\
\hline 7.98E-08 & 452.77 & $\mathrm{Bi}-212$ & $->$ & TI-208 \\
\hline 7.96E-08 & 11.713 & Ra-224 & $->$ & $R n-220$ \\
\hline 7.89E-08 & 131.5 & Th-228 & $->$ & Ra-224 \\
\hline $7.50 \mathrm{E}-08$ & 288.08 & $\mathrm{Bi}-212$ & $->$ & TI-208 \\
\hline $7.48 \mathrm{E}-08$ & 1823.47 & Ac- 228 & $->$ & Th-228 \\
\hline 7.35E-08 & 81.067 & Ra-224 & $->$ & $R n-220$ \\
\hline 7.33E-08 & 460.84 & Ac- 228 & $->$ & Th-228 \\
\hline 7.33E-08 & 1572.03 & $A c-228$ & $->$ & Th-228 \\
\hline 7.33E-08 & 629.93 & $A c-228$ & $->$ & Th-228 \\
\hline 7.19E-08 & 555.3 & Ac-228 & $->$ & Th-228 \\
\hline 7.19E-08 & 141.19 & Ac- 228 & $->$ & Th-228 \\
\hline 7.19E-08 & 1537.4 & $A C-228$ & $->$ & Th-228 \\
\hline 7.04E-08 & 1842.29 & Ac-228 & $->$ & Th-228 \\
\hline 6.90E-08 & 952.17 & $\mathrm{Bi}-212$ & $->$ & Po-212 \\
\hline 6.89E-08 & 1907.5 & $A c-228$ & $->$ & Th-228 \\
\hline $6.81 \mathrm{E}-08$ & 233.32 & Tl-208 & $->$ & $\mathrm{Pb}-208$ \\
\hline $6.75 \mathrm{E}-08$ & 666.4 & $A C-228$ & $->$ & Th-228 \\
\hline $6.31 \mathrm{E}-08$ & 1548.5 & $A c-228$ & $->$ & Th-228 \\
\hline $6.31 \mathrm{E}-08$ & 498.26 & Ac- 228 & $->$ & Th-228 \\
\hline $6.31 \mathrm{E}-08$ & 515.2 & Ac- 228 & $->$ & Th-228 \\
\hline $6.31 \mathrm{E}-08$ & 649.19 & Ac- 228 & $->$ & Th-228 \\
\hline 6.07E-08 & 1757.88 & $A c-228$ & $->$ & Th-228 \\
\hline $6.01 \mathrm{E}-08$ & 1835.1 & Ac-228 & $->$ & Th-228 \\
\hline $6.01 \mathrm{E}-08$ & 737.7 & Ac-228 & $->$ & Th-228 \\
\hline $5.80 \mathrm{E}-08$ & 13.662 & Th-228 & $->$ & Ra-224 \\
\hline $5.43 \mathrm{E}-08$ & 399.2 & Ac- 228 & $->$ & Th-228 \\
\hline 5.32E-08 & 11.712 & $\mathrm{~Pb}-212$ & $->$ & $\mathrm{Bi}-212$ \\
\hline $5.13 \mathrm{E}-08$ & 471.4 & Ac- 228 & $->$ & Th-228 \\
\hline $5.13 \mathrm{E}-08$ & 816.62 & Ac- 228 & $->$ & Th-228 \\
\hline $5.06 \mathrm{E}-08$ & 166.43 & Th-228 & $->$ & Ra-224 \\
\hline 4.92E-08 & 14.884 & TI-208 & $->$ & $\mathrm{Pb}-208$ \\
\hline 4.69E-08 & 1431 & $A c-228$ & $->$ & Th-228 \\
\hline 4.69E-08 & 1041.35 & Ac- 228 & $->$ & Th-228 \\
\hline 4.69E-08 & 174.18 & Ac-228 & $->$ & Th-228 \\
\hline 4.69E-08 & 1054.3 & AC- 228 & $->$ & Th-228 \\
\hline 4.69E-08 & 396.9 & $A c-228$ & $->$ & Th-228 \\
\hline 4.69E-08 & 257.29 & Ac-228 & $->$ & Th-228 \\
\hline 4.46E-08 & 721.91 & TI-208 & $->$ & $\mathrm{Pb}-208$ \\
\hline
\end{tabular}




\begin{tabular}{|c|c|c|c|c|}
\hline $4.41 \mathrm{E}-08$ & 94.677 & Ra-224 & $->$ & Rn-220 \\
\hline 4.35E-08 & 1805.93 & $\mathrm{Bi}-212$ & $->$ & Po-212 \\
\hline $4.33 \mathrm{E}-08$ & 982.52 & TI-208 & $->$ & $\mathrm{Pb}-208$ \\
\hline 4.29E-08 & 549.7 & $\mathrm{Rn}-220$ & $->$ & Po-216 \\
\hline $4.25 \mathrm{E}-08$ & 1503.87 & $A c-228$ & $->$ & Th-228 \\
\hline $4.25 E-08$ & 1357.6 & $A c-228$ & $->$ & Th-228 \\
\hline $4.25 \mathrm{E}-08$ & 1174.79 & Ac- 228 & $->$ & Th-228 \\
\hline $4.25 \mathrm{E}-08$ & 540.5 & Ac- 228 & $->$ & Th-228 \\
\hline $4.25 \mathrm{E}-08$ & 791.2 & Ac- 228 & $->$ & Th-228 \\
\hline $4.25 \mathrm{E}-08$ & 474.32 & Ac- 228 & $->$ & Th-228 \\
\hline $4.11 \mathrm{E}-08$ & 919.19 & Ac-228 & $->$ & Th-228 \\
\hline 3.92E-08 & 79.29 & $\mathrm{Bi}-212$ & $->$ & Po-212 \\
\hline 3.81E-08 & 1871.2 & Ac- 228 & $->$ & Th-228 \\
\hline 3.81E-08 & 1415.7 & $A c-228$ & $->$ & Th-228 \\
\hline 3.81E-08 & 1216.4 & $A c-228$ & $->$ & Th-228 \\
\hline 3.81E-08 & 419.23 & Ac- 228 & $->$ & Th-228 \\
\hline 3.74E-08 & 211.31 & TI-208 & $->$ & $\mathrm{Pb}-208$ \\
\hline 3.67E-08 & 1930.3 & $A c-228$ & $->$ & Th-228 \\
\hline 3.67E-08 & 1016.12 & Ac- 228 & $->$ & Th-228 \\
\hline 3.67E-08 & 1019.7 & Ac- 228 & $->$ & Th-228 \\
\hline 3.52E-08 & 1965.3 & $A c-228$ & $->$ & Th-228 \\
\hline 3.37E-08 & 979.7 & Ac-228 & $->$ & Th-228 \\
\hline 3.30E-08 & 8.953 & $\mathrm{Bi}-212$ & $->$ & TI-208 \\
\hline $3.12 \mathrm{E}-08$ & 176.577 & $\mathrm{~Pb}-212$ & $->$ & $\mathrm{Bi}-212$ \\
\hline $3.08 \mathrm{E}-08$ & 1309.6 & $A c-228$ & $->$ & Th-228 \\
\hline $3.08 \mathrm{E}-08$ & 1314.76 & $A c-228$ & $->$ & Th-228 \\
\hline 3.08E-08 & 922.28 & $A c-228$ & $->$ & Th-228 \\
\hline $3.08 \mathrm{E}-08$ & 791.05 & $A c-228$ & $->$ & Th-228 \\
\hline 3.01E-08 & 327.93 & $\mathrm{Bi}-212$ & $->$ & TI-208 \\
\hline 2.93E-08 & 356.83 & $A c-228$ & $->$ & Th-228 \\
\hline 2.93E-08 & 1277.5 & Ac-228 & $->$ & Th-228 \\
\hline 2.93E-08 & 1374.26 & Ac- 228 & $->$ & Th-228 \\
\hline 2.93E-08 & 1468.8 & $A C-228$ & $->$ & Th-228 \\
\hline 2.93E-08 & 1481.2 & $A c-228$ & $->$ & Th-228 \\
\hline 2.93E-08 & 887.46 & Ac-228 & $->$ & Th-228 \\
\hline 2.93E-08 & 492.36 & Ac- 228 & $->$ & Th-228 \\
\hline 2.93E-08 & 877.65 & $A c-228$ & $->$ & Th-228 \\
\hline $2.85 \mathrm{E}-08$ & 1739.1 & $A c-228$ & $->$ & Th-228 \\
\hline 2.79E-08 & 72.873 & $\mathrm{Bi}-212$ & $->$ & TI-208 \\
\hline $2.75 \mathrm{E}-08$ & 927.42 & Tl-208 & $->$ & $\mathrm{Pb}-208$ \\
\hline $2.66 \mathrm{E}-08$ & 1679.42 & $\mathrm{Bi}-212$ & $->$ & Po-212 \\
\hline $2.64 \mathrm{E}-08$ & 1451.2 & Ac- 228 & $->$ & Th-228 \\
\hline $2.49 \mathrm{E}-08$ & 135.68 & Ac- 228 & $->$ & Th-228 \\
\hline 2.49E-08 & 1104.01 & Ac- 228 & $->$ & Th-228 \\
\hline $2.49 \mathrm{E}-08$ & 416.2 & AC- 228 & $->$ & Th-228 \\
\hline 2.39E-08 & 16.881 & $\mathrm{Ra}-224$ & $->$ & $\mathrm{Rn}-220$ \\
\hline $2.35 \mathrm{E}-08$ & 76.858 & $\mathrm{Bi}-212$ & $->$ & Po-212 \\
\hline $2.20 \mathrm{E}-08$ & 931.1 & Ac- 228 & $->$ & Th-228 \\
\hline $2.20 \mathrm{E}-08$ & 388.9 & Ac- 228 & $->$ & Th-228 \\
\hline $2.20 \mathrm{E}-08$ & 1741.2 & $A c-228$ & $->$ & Th-228 \\
\hline
\end{tabular}




\begin{tabular}{|c|c|c|c|c|}
\hline $2.05 E-08$ & 853.6 & $A c-228$ & $->$ & Th-228 \\
\hline $2.05 \mathrm{E}-08$ & 220.49 & $A c-228$ & $->$ & Th-228 \\
\hline $2.05 \mathrm{E}-08$ & 1706.07 & Ac- 228 & $->$ & Th-228 \\
\hline 1.91E-08 & 1347.6 & Ac- 228 & $->$ & Th-228 \\
\hline $1.78 \mathrm{E}-08$ & 88.471 & Th-228 & $->$ & Ra-224 \\
\hline $1.76 \mathrm{E}-08$ & 1135.39 & $A c-228$ & $->$ & Th-228 \\
\hline $1.73 \mathrm{E}-08$ & 205.75 & Th-228 & $->$ & Ra-224 \\
\hline $1.71 \mathrm{E}-08$ & 415.156 & $\mathrm{~Pb}-212$ & $->$ & $\mathrm{Bi}-212$ \\
\hline $1.65 \mathrm{E}-08$ & 70.832 & $\mathrm{Bi}-212$ & $->$ & TI-208 \\
\hline $1.61 \mathrm{E}-08$ & 13.501 & $\mathrm{Bi}-212$ & $->$ & Po-212 \\
\hline $1.61 \mathrm{E}-08$ & 11.119 & $\mathrm{Bi}-212$ & $->$ & Po-212 \\
\hline $1.47 \mathrm{E}-08$ & 1142.7 & Ac- 228 & $->$ & Th-228 \\
\hline $1.43 \mathrm{E}-08$ & 9.185 & TI-208 & $->$ & $\mathrm{Pb}-208$ \\
\hline $1.41 \mathrm{E}-08$ & 97.907 & Ra-224 & $->$ & $R n-220$ \\
\hline $1.41 \mathrm{E}-08$ & 1784.5 & $A c-228$ & $->$ & Th-228 \\
\hline $1.37 \mathrm{E}-08$ & 89.639 & $\mathrm{Bi}-212$ & $->$ & Po-212 \\
\hline 1.32E-08 & 372.3 & Ac-228 & $->$ & Th-228 \\
\hline $1.32 \mathrm{E}-08$ & 1750.9 & $A c-228$ & $->$ & Th-228 \\
\hline $1.32 \mathrm{E}-08$ & 481.8 & Ac- 228 & $->$ & Th-228 \\
\hline $1.14 \mathrm{E}-08$ & 1282.7 & TI-208 & $->$ & $\mathrm{Pb}-208$ \\
\hline 1.10E-08 & 473.36 & $\mathrm{Bi}-212$ & $->$ & TI-208 \\
\hline $1.10 \mathrm{E}-08$ & 485.78 & Tl-208 & $->$ & $\mathrm{Pb}-208$ \\
\hline $1.08 \mathrm{E}-08$ & 85.429 & Th-228 & $->$ & Ra-224 \\
\hline 9.89E-09 & 82.434 & $\mathrm{Bi}-212$ & $->$ & TI-208 \\
\hline 9.45E-09 & 748.58 & Tl-208 & $->$ & $\mathrm{Pb}-208$ \\
\hline 8.79E-09 & 587.82 & TI-208 & $->$ & $\mathrm{Pb}-208$ \\
\hline 8.79E-09 & 821.14 & TI-208 & $->$ & $\mathrm{Pb}-208$ \\
\hline 7.91E-09 & 650.14 & Tl-208 & $->$ & $\mathrm{Pb}-208$ \\
\hline $6.89 \mathrm{E}-09$ & 1713.25 & $A C-228$ & $->$ & Th-228 \\
\hline 6.81E-09 & 883.27 & TI-208 & $->$ & $\mathrm{Pb}-208$ \\
\hline $6.75 \mathrm{E}-09$ & 1900.1 & $A c-228$ & $->$ & Th-228 \\
\hline $6.35 \mathrm{E}-09$ & 99.915 & Th-228 & $->$ & Ra-224 \\
\hline $6.27 \mathrm{E}-09$ & 1074.1 & $\mathrm{Bi}-212$ & $->$ & Po-212 \\
\hline $6.22 \mathrm{E}-09$ & 10.994 & $\mathrm{Bi}-212$ & $->$ & TI-208 \\
\hline 5.51E-09 & 290 & Ra-224 & $->$ & $R n-220$ \\
\hline 4.99E-09 & 692.5 & Ac- 228 & $->$ & Th-228 \\
\hline 4.84E-09 & 705.24 & TI-208 & $->$ & $\mathrm{Pb}-208$ \\
\hline 4.47E-09 & 10.137 & Ra-224 & $->$ & $R n-220$ \\
\hline 4.31E-09 & 92.673 & $\mathrm{Bi}-212$ & $->$ & Po-212 \\
\hline 4.29E-09 & 650.1 & Ra-224 & $->$ & $R n-220$ \\
\hline 3.83E-09 & 11.349 & TI-208 & $->$ & $\mathrm{Pb}-208$ \\
\hline $3.74 \mathrm{E}-09$ & 1185.1 & TI-208 & $->$ & $\mathrm{Pb}-208$ \\
\hline 3.13E-09 & 15.869 & $\mathrm{Bi}-212$ & $->$ & Po-212 \\
\hline 3.08E-09 & 433.51 & $\mathrm{Bi}-212$ & $->$ & TI-208 \\
\hline 3.06E-09 & 164.15 & $\mathrm{~Pb}-212$ & $->$ & $\mathrm{Bi}-212$ \\
\hline 2.75E-09 & 85.185 & $\mathrm{Bi}-212$ & $->$ & TI-208 \\
\hline 2.45E-09 & 409.3 & Ra-224 & $->$ & $R n-220$ \\
\hline 2.42E-09 & 1160.55 & TI-208 & $->$ & $\mathrm{Pb}-208$ \\
\hline 2.20E-09 & 143.99 & $\mathrm{Bi}-212$ & $->$ & TI-208 \\
\hline 2.08E-09 & 12.855 & Ra-224 & $->$ & $\mathrm{Rn}-220$ \\
\hline
\end{tabular}




$\begin{array}{cc}2.07 \mathrm{E}-09 & 103.341 \\ 1.54 \mathrm{E}-09 & 1381 \\ 1.34 \mathrm{E}-09 & 492.62 \\ 1.10 \mathrm{E}-09 & 804.9 \\ 1.10 \mathrm{E}-09 & 164 \\ 1.10 \mathrm{E}-09 & 1125.6 \\ 8.23 \mathrm{E}-10 & 9.658 \\ 7.91 \mathrm{E}-10 & 620.3 \\ 4.40 \mathrm{E}-10 & 1743.57 \\ 4.40 \mathrm{E}-10 & 1647.4 \\ 2.43 \mathrm{E}-10 & 12.085 \\ 1.74 \mathrm{E}-10 & 580.4 \\ & \\ \mathbf{U}-\mathbf{2 3 8} & 100 \%\end{array}$

$\begin{array}{rll}\text { Th-228 } & -> & \mathrm{Ra}-224 \\ \mathrm{Tl}-208 & -> & \mathrm{Pb}-208 \\ \mathrm{Bi}-212 & -> & \mathrm{Tl}-208 \\ \mathrm{Po}-216 & -> & \mathrm{Pb}-212 \\ \mathrm{Bi}-212 & -> & \mathrm{Tl}-208 \\ \mathrm{Tl}-208 & -> & \mathrm{Pb}-208 \\ \mathrm{Bi}-212 & -> & \mathrm{Po}-212 \\ \mathrm{Bi}-212 & -> & \mathrm{Tl}-208 \\ \mathrm{Tl}-208 & -> & \mathrm{Pb}-208 \\ \mathrm{Tl}-208 & -> & \mathrm{Pb}-208 \\ \mathrm{Bi}-212 & -> & \mathrm{Po}-212 \\ \mathrm{Bi}-212 & -> & \mathrm{Tl}-208\end{array}$

Aged 5 years

651 lines computed.

Sorted by Energy

\begin{tabular}{|c|c|c|c|c|c|c|c|}
\hline Energy & Intensity & \multicolumn{6}{|c|}{ Initial Isotopes and Decays } \\
\hline keV & $\mathrm{ph} / \mathrm{s} / \mathrm{gm}$ & & & & & & \\
\hline 8.953 & $1.60 \mathrm{E}-19$ & $\mathrm{Hg}-206$ & $->$ & TI-206 & & & \\
\hline 9.185 & $2.34 \mathrm{E}-15$ & TI-206 & $->$ & $\mathrm{Pb}-206$ & TI-210 & $->$ & $\mathrm{Pb}-210$ \\
\hline 9.419 & $8.35 \mathrm{E}-12$ & $\mathrm{~Pb}-210$ & $->$ & $\mathrm{Bi}-210$ & $\mathrm{~Pb}-214$ & $->$ & $\mathrm{Bi}-214$ \\
\hline 9.658 & $5.43 E-13$ & $\mathrm{Bi}-214$ & $->$ & Po-214 & & & \\
\hline 10.137 & $3.93 E-13$ & $\mathrm{Ra}-226$ & $->$ & $\mathrm{Rn}-222$ & & & \\
\hline 10.259 & 3.33E-18 & $\mathrm{Hg}-206$ & $->$ & TI-206 & & & \\
\hline 10.541 & $4.73 E-14$ & Tl-206 & $->$ & $\mathrm{Pb}-206$, & $\mathrm{TI}-210$ & $->$ & $\mathrm{Pb}-210$ \\
\hline 10.828 & $1.67 \mathrm{E}-10$ & $\mathrm{~Pb}-210$ & $->$ & $\mathrm{Bi}-210$ & $\mathrm{~Pb}-214$ & $->$ & $\mathrm{Bi}-214$ \\
\hline 10.994 & $4.21 \mathrm{E}-20$ & $\mathrm{Hg}-206$ & $->$ & TI-206 & & & \\
\hline 11.118 & $2.24 \mathrm{E}+01$ & U-238 & $->$ & Th-234 & & & \\
\hline 11.119 & $1.04 \mathrm{E}-11$ & $\mathrm{Bi}-214$ & $->$ & Po-214 & & & \\
\hline 11.349 & $1.14 \mathrm{E}-15$ & $\mathrm{TI}-210$ & $->$ & $\mathrm{Pb}-210$ & & & \\
\hline 11.372 & $2.88 \mathrm{E}+01$ & Th-234 & $->$ & $\mathrm{Pa}-234 \mathrm{~m}$, & $\begin{array}{r}\text { Pa- } \\
234 \mathrm{~m}\end{array}$ & $->$ & $U-234$ \\
\hline 11.439 & $4.69 E-22$ & TI-206 & $->$ & $\mathrm{Pb}-206$ & & & \\
\hline 11.62 & $1.39 \mathrm{E}+00$ & $\mathrm{~Pa}-234$ & $->$ & U-234, & $\begin{array}{r}\text { Pa- } \\
234 m\end{array}$ & $->$ & $U-234$ \\
\hline 11.712 & $1.95 \mathrm{E}-12$ & $\mathrm{~Pb}-210$ & $->$ & $\mathrm{Bi}-210$ & $\mathrm{~Pb}-214$ & $->$ & $\mathrm{Bi}-214$ \\
\hline 11.713 & $7.28 \mathrm{E}-12$ & Ra-226 & $->$ & $\mathrm{Rn}-222$ & & & \\
\hline 12.085 & $1.49 \mathrm{E}-13$ & $\mathrm{Bi}-214$ & $->$ & Po-214 & & & \\
\hline 12.313 & $2.88 \mathrm{E}-18$ & $\mathrm{Hg}-206$ & $->$ & TI-206 & & & \\
\hline 12.678 & $6.27 \mathrm{E}-14$ & TI-210 & $->$ & $\mathrm{Pb}-210$ & & & \\
\hline 12.71 & $2.95 \mathrm{E}-20$ & TI-206 & $->$ & $\mathrm{Pb}-206$ & & & \\
\hline 12.855 & $2.01 \mathrm{E}-13$ & Ra-226 & $->$ & $\mathrm{Rn}-222$ & & & \\
\hline 12.952 & $3.73 E+02$ & $U-238$ & $->$ & Th-234 & & & \\
\hline 13.066 & $1.04 \mathrm{E}-11$ & $\mathrm{~Pb}-210$ & $->$ & $\mathrm{Bi}-210$ & & & \\
\hline 13.088 & $1.55 \mathrm{E}-10$ & $\mathrm{~Pb}-214$ & $->$ & $\mathrm{Bi}-214$ & & & \\
\hline
\end{tabular}




\begin{tabular}{|c|c|c|c|c|c|c|c|}
\hline 13.274 & $4.85 E+02$ & Th-234 & $->$ & $\mathrm{Pa}-234 \mathrm{~m}$ & & & \\
\hline 13.275 & $2.88 \mathrm{E}+00$ & $\mathrm{~Pa}-234 \mathrm{~m}$ & $->$ & U-234 & & & \\
\hline 13.502 & $1.00 \mathrm{E}-11$ & $\mathrm{Bi}-214$ & $->$ & Po-214 & & & \\
\hline 13.6 & $2.27 E+01$ & $\mathrm{~Pa}-234$ & $->$ & U-234, & $\begin{array}{c}\mathrm{Pa}- \\
234 \mathrm{~m}\end{array}$ & $->$ & U-234 \\
\hline 14.336 & $1.05 \mathrm{E}-11$ & Ra-226 & $->$ & $\mathrm{Rn}-222$ & & & \\
\hline 14.407 & $5.32 \mathrm{E}-19$ & $\mathrm{Hg}-206$ & $->$ & TI-206 & & & \\
\hline 14.511 & $1.03 \mathrm{E}+01$ & U-238 & $->$ & Th-234 & & & \\
\hline 14.836 & $1.25 \mathrm{E}-14$ & TI-206 & $->$ & $\mathrm{Pb}-206$ & TI-210 & $->$ & $\mathrm{Pb}-210$ \\
\hline 14.953 & $6.12 \mathrm{E}+00$ & Th-234 & $->$ & $\mathrm{Pa}-234 \mathrm{~m}$, & $\begin{array}{r}\text { Pa- } \\
234 \mathrm{~m}\end{array}$ & $->$ & U-234 \\
\hline 15.4 & $3.67 \mathrm{E}-01$ & $\mathrm{~Pa}-234$ & $->$ & U-234, & $\begin{array}{l}\text { Pa- } \\
234 \mathrm{~m}\end{array}$ & $->$ & U-234 \\
\hline 15.439 & $3.15 \mathrm{E}-11$ & $\mathrm{~Pb}-214$ & $->$ & Bi-214 & & & \\
\hline 15.537 & $2.50 \mathrm{E}-12$ & $\mathrm{~Pb}-210$ & $->$ & $\mathrm{Bi}-210$ & & & \\
\hline 15.874 & $1.93 \mathrm{E}-12$ & $\mathrm{Bi}-214$ & $->$ & Po-214 & & & \\
\hline 16.161 & $5.10 \mathrm{E}+02$ & $\mathrm{U}-238$ & $->$ & Th-234 & & & \\
\hline 16.547 & $3.34 \mathrm{E}+00$ & $\mathrm{~Pa}-234 \mathrm{~m}$ & $->$ & $U-234$ & & & \\
\hline 16.572 & $5.47 E+02$ & Th-234 & $->$ & $\mathrm{Pa}-234 \mathrm{~m}$ & & & \\
\hline 16.874 & $2.31 \mathrm{E}-12$ & $\mathrm{Ra}-226$ & $->$ & $\mathrm{Rn}-222$ & & & \\
\hline 17.069 & $1.46 \mathrm{E}+01$ & $\mathrm{~Pa}-234 \mathrm{~m}$ & $->$ & $U-234$ & & & \\
\hline 17.12 & $8.41 E+00$ & Рa-234 & $->$ & $U-234$ & & & \\
\hline 19.094 & $1.16 \mathrm{E}+02$ & U-238 & $->$ & Th-234 & & & \\
\hline 19.839 & $1.34 \mathrm{E}+02$ & Th-234 & $->$ & $\mathrm{Pa}-234 \mathrm{~m}$ & & & \\
\hline 19.891 & $3.97 \mathrm{E}-01$ & $P a-234 m$ & $->$ & $U-234$ & & & \\
\hline 20.019 & $1.24 \mathrm{E}+00$ & Th-234 & $->$ & $\mathrm{Pa}-234 \mathrm{~m}$ & & & \\
\hline 20.309 & $1.91 \mathrm{E}+00$ & Pa-234 & $->$ & $U-234$ & & & \\
\hline 20.366 & $3.18 \mathrm{E}+00$ & $\mathrm{~Pa}-234 \mathrm{~m}$ & $->$ & $U-234$ & & & \\
\hline 29.49 & $1.82 \mathrm{E}-01$ & Th-234 & $->$ & $\mathrm{Pa}-234 \mathrm{~m}$ & & & \\
\hline 34.37 & $5.82 \mathrm{E}-04$ & $\mathrm{~Pa}-234$ & $->$ & $U-234$ & & & \\
\hline 43.47 & $1.94 \mathrm{E}-02$ & $\mathrm{~Pa}-234$ & $->$ & $U-234$ & & & \\
\hline 45.28 & $1.78 \mathrm{E}-03$ & $\mathrm{~Pa}-234$ & $->$ & $U-234$ & & & \\
\hline 46.52 & $4.06 \mathrm{E}-12$ & $\mathrm{~Pb}-210$ & $->$ & $\mathrm{Bi}-210$ & & & \\
\hline 49.55 & $8.71 E+00$ & $U-238$ & $->$ & Th-234 & & & \\
\hline 53.172 & $2.99 \mathrm{E}-11$ & $\mathrm{~Pb}-214$ & $->$ & $\mathrm{Bi}-214$ & & & \\
\hline 53.23 & $2.05 E-04$ & U-234 & $->$ & Th-230 & & & \\
\hline 57.75 & $6.22 \mathrm{E}-01$ & Th-234 & $->$ & $\mathrm{Pa}-234 \mathrm{~m}$ & & & \\
\hline 58.31 & $1.46 \mathrm{E}-03$ & $\mathrm{~Pa}-234$ & $->$ & $U-234$ & & & \\
\hline 62.78 & $9.62 \mathrm{E}-01$ & $\mathrm{~Pa}-234$ & $->$ & U-234, & $\begin{array}{r}\mathrm{Pa}- \\
234 \mathrm{~m}\end{array}$ & $->$ & U-234 \\
\hline 62.862 & $2.36 \mathrm{E}+00$ & Th-234 & $->$ & $\mathrm{Pa}-234 \mathrm{~m}$ & & & \\
\hline 63.288 & $4.48 E+02$ & Th-234 & $->$ & $\mathrm{Pa}-234 \mathrm{~m}$ & & & \\
\hline 67.22 & $9.38 \mathrm{E}-03$ & $\mathrm{~Pa}-234$ & $->$ & U-234 & & & \\
\hline 67.6758 & $1.46 \mathrm{E}-08$ & Th-230 & $->$ & Ra-226 & & & \\
\hline 69.9 & $3.72 \mathrm{E}-02$ & $\mathrm{~Pa}-234$ & $->$ & U-234 & & & \\
\hline 70.832 & $5.54 \mathrm{E}-18$ & $\mathrm{Hg}-206$ & $->$ & TI-206 & & & \\
\hline 72.803 & $1.60 \mathrm{E}-14$ & TI-206 & $->$ & $\mathrm{Pb}-206$ & TI-210 & $->$ & $\mathrm{Pb}-21 \mathrm{C}$ \\
\hline 72.873 & $9.31 \mathrm{E}-18$ & $\mathrm{Hg}-206$ & $->$ & TI-206 & & & \\
\hline 73.9 & $1.76 \mathrm{E}-03$ & $\mathrm{~Pa}-234 \mathrm{~m}$ & $->$ & $\mathrm{Pa}-234$ & & & \\
\hline 74 & $5.30 \mathrm{E}+00$ & Th-234 & $->$ & $\mathrm{Pa}-234 \mathrm{~m}$ & & & \\
\hline
\end{tabular}




\begin{tabular}{|c|c|c|c|c|c|c|c|}
\hline 74.814 & $1.77 \mathrm{E}-10$ & $\mathrm{~Pb}-214$ & $->$ & $\mathrm{Bi}-214$ & & & \\
\hline 74.969 & $2.62 \mathrm{E}-14$ & TI-206 & $->$ & Pb-206, & TI-210 & $->$ & $\mathrm{Pb}-210$ \\
\hline 76.838 & $1.57 \mathrm{E}-11$ & $\mathrm{Bi}-214$ & $->$ & Po-214 & & & \\
\hline 77.107 & $2.99 \mathrm{E}-10$ & $\mathrm{~Pb}-214$ & $->$ & $\mathrm{Bi}-214$ & & & \\
\hline 79.29 & $2.63 \mathrm{E}-11$ & $\mathrm{Bi}-214$ & $->$ & Po-214 & & & \\
\hline 79.65 & 4.37E-02 & $\mathrm{Pa}-234$ & $->$ & U-234 & & & \\
\hline 81 & $1.14 \mathrm{E}-14$ & $\mathrm{Tl}-210$ & $->$ & $\mathrm{Pb}-210$ & & & \\
\hline 81.067 & $4.83 \mathrm{E}-12$ & Ra-226 & $->$ & Rn-222 & & & \\
\hline 82.434 & $3.33 \mathrm{E}-18$ & $\mathrm{Hg}-206$ & $->$ & TI-206 & & & \\
\hline 83.31 & $8.71 E+00$ & Th-234 & $->$ & $\mathrm{Pa}-234 \mathrm{~m}$ & & & \\
\hline 83.787 & $8.02 \mathrm{E}-12$ & $\mathrm{Ra}-226$ & $->$ & $\mathrm{Rn}-222$ & & & \\
\hline 84.789 & $9.35 E-15$ & TI-206 & $->$ & $\mathrm{Pb}-206$ & TI-210 & $->$ & $\mathrm{Pb}-210$ \\
\hline 85.185 & $9.31 \mathrm{E}-19$ & $\mathrm{Hg}-206$ & $->$ & TI-206 & & & \\
\hline 87.02 & $9.08 \mathrm{E}-01$ & Th-234 & $->$ & $\mathrm{Pa}-234 \mathrm{~m}$ & & & \\
\hline 87.19 & $1.05 \mathrm{E}-10$ & $\mathrm{~Pb}-214$ & $->$ & $\mathrm{Bi}-214$ & & & \\
\hline 87.632 & $2.68 \mathrm{E}-15$ & TI-206 & $->$ & $\mathrm{Pb}-206$ & TI-210 & $->$ & $\mathrm{Pb}-210$ \\
\hline 89.639 & $9.37 \mathrm{E}-12$ & $\mathrm{Bi}-214$ & $->$ & Po-214 & & & \\
\hline 90.128 & $3.12 \mathrm{E}-11$ & $\mathrm{~Pb}-214$ & $->$ & $\mathrm{Bi}-214$ & & & \\
\hline 92.279 & $9.95 \mathrm{E}-01$ & Th-234 & $->$ & $\mathrm{Pa}-234 \mathrm{~m}$ & & & \\
\hline 92.35 & $3.38 \mathrm{E}+02$ & Th-234 & $->$ & $\mathrm{Pa}-234 \mathrm{~m}$ & & & \\
\hline 92.673 & $2.85 \mathrm{E}-12$ & $\mathrm{Bi}-214$ & $->$ & Po-214 & & & \\
\hline 92.78 & $3.35 E+02$ & Th-234 & $->$ & $\mathrm{Pa}-234 \mathrm{~m}$ & & & \\
\hline 94.651 & $2.16 \mathrm{E}+01$ & $\mathrm{~Pa}-234$ & $->$ & U-234, & $\begin{array}{r}\mathrm{Pa}- \\
234 \mathrm{~m}\end{array}$ & $->$ & $U-234$ \\
\hline 94.677 & $2.83 \mathrm{E}-12$ & Ra-226 & $->$ & $\mathrm{Rn}-222$ & & & \\
\hline 95 & $2.28 \mathrm{E}-14$ & TI-210 & $->$ & $\mathrm{Pb}-210$ & & & \\
\hline 95.863 & $1.62 \mathrm{E}+00$ & Th-234 & $->$ & $\mathrm{Pa}-234 \mathrm{~m}$ & & & \\
\hline 97.907 & $9.04 \mathrm{E}-13$ & $\mathrm{Ra}-226$ & $->$ & $\mathrm{Rn}-222$ & & & \\
\hline 98.434 & $3.43 E+01$ & $\mathrm{~Pa}-234$ & $->$ & U-234, & $\begin{array}{r}\mathrm{Pa}- \\
234 \mathrm{~m}\end{array}$ & $->$ & $U-234$ \\
\hline 99.852 & 8.35E-01 & $\mathrm{Pa}-234$ & $->$ & U-234, & $\begin{array}{r}\mathrm{Pa}- \\
234 \mathrm{~m}\end{array}$ & $->$ & $U-234$ \\
\hline 103.35 & 4.35E-01 & Th-234 & $->$ & $\mathrm{Pa}-234 \mathrm{~m}$ & & & \\
\hline 103.7 & $1.94 \mathrm{E}-02$ & $\mathrm{~Pa}-234$ & $->$ & $U-234$ & & & \\
\hline 103.71 & 7.46E-01 & Th-234 & $->$ & $\mathrm{Pa}-234 \mathrm{~m}$ & & & \\
\hline 108 & 7.34E-01 & Th-234 & $->$ & $\mathrm{Pa}-234 \mathrm{~m}$ & & & \\
\hline 108.166 & $6.22 \mathrm{E}-01$ & Th-234 & $->$ & $\mathrm{Pa}-234 \mathrm{~m}$ & & & \\
\hline 109.97 & $2.29 \mathrm{E}-12$ & Th-230 & $->$ & $\mathrm{Ra}-226$ & & & \\
\hline 110.5 & $2.99 E+00$ & $\mathrm{U}-238$ & $->$ & Th-234 & & & \\
\hline 111.025 & $1.26 \mathrm{E}+01$ & $\mathrm{~Pa}-234$ & $->$ & U-234, & $\begin{array}{r}\text { Pa- } \\
234 m\end{array}$ & $->$ & U-234 \\
\hline 111.897 & $1.99 \mathrm{E}-01$ & Th-234 & $->$ & $\mathrm{Pa}-234 \mathrm{~m}$ & & & \\
\hline 112.8 & $3.01 \mathrm{E}+01$ & Th-234 & $->$ & $\mathrm{Pa}-234 \mathrm{~m}$ & & & \\
\hline 114.866 & $4.32 E+00$ & $\mathrm{~Pa}-234$ & $->$ & U-234, & $\begin{array}{r}\text { Pa- } \\
234 m\end{array}$ & $->$ & U-234 \\
\hline 120.912 & $7.06 \mathrm{E}-05$ & $U-234$ & $->$ & Th-230 & & & \\
\hline 125.53 & $1.62 \mathrm{E}-01$ & $\mathrm{~Pa}-234$ & $->$ & $U-234$ & & & \\
\hline 131.31 & $3.56 \mathrm{E}+00$ & Pa-234 & $->$ & $U-234$ & & & \\
\hline 134.3 & $3.40 \mathrm{E}-02$ & $\mathrm{~Pa}-234$ & $->$ & $U-234$ & & & \\
\hline 137.4 & $1.60 \mathrm{E}-12$ & $\mathrm{~Pb}-214$ & $->$ & $\mathrm{Bi}-214$ & & & \\
\hline
\end{tabular}




\begin{tabular}{|c|c|c|c|c|c|c|c|}
\hline 137.7 & $2.43 \mathrm{E}-02$ & Pa-234 & $->$ & U-234 & & & \\
\hline 140.15 & $2.89 \mathrm{E}-01$ & $\mathrm{~Pa}-234$ & $->$ & U-234, & $\begin{array}{r}\mathrm{Pa}- \\
234 \mathrm{~m}\end{array}$ & $->$ & $U-234$ \\
\hline 141.3 & $1.06 \mathrm{E}-12$ & $\mathrm{~Pb}-214$ & $->$ & $\mathrm{Bi}-214$ & & & \\
\hline 143.876 & $1.89 \mathrm{E}-09$ & Th-230 & $->$ & $\mathrm{Ra}-226$ & & & \\
\hline 143.9 & $5.66 \mathrm{E}-02$ & $\mathrm{~Pa}-234$ & $->$ & $U-234$ & & & \\
\hline 150 & $3.23 E-02$ & $\mathrm{~Pa}-234$ & $->$ & $U-234$ & & & \\
\hline 152.76 & $1.03 E+00$ & $\mathrm{~Pa}-234$ & $->$ & $U-234$ & & & \\
\hline 157.96 & $1.13 \mathrm{E}-01$ & $\mathrm{~Pa}-234$ & $->$ & $U-234$ & & & \\
\hline 165.8 & $1.46 \mathrm{E}-02$ & $\mathrm{~Pa}-234$ & $->$ & $U-234$ & & & \\
\hline 170.75 & $8.08 \mathrm{E}-02$ & $\mathrm{~Pa}-234$ & $->$ & $U-234$ & & & \\
\hline 174.52 & $3.23 E-02$ & $\mathrm{~Pa}-234$ & $->$ & $U-234$ & & & \\
\hline 184.6 & $1.91 \mathrm{E}-01$ & $\mathrm{~Pa}-234 \mathrm{~m}$ & $->$ & $U-234$ & & & \\
\hline 184.8 & $1.49 \mathrm{E}+00$ & Th-234 & $->$ & $\mathrm{Pa}-234 \mathrm{~m}$ & & & \\
\hline 185.92 & $3.23 E-01$ & $\mathrm{~Pa}-234$ & $->$ & U-234 & & & \\
\hline 186.057 & $3.42 \mathrm{E}-10$ & Th-230 & $->$ & Ra-226 & & & \\
\hline 186.11 & $9.01 \mathrm{E}-11$ & $\mathrm{Ra}-226$ & $->$ & $\mathrm{Rn}-222$ & & & \\
\hline 192.74 & 8.57E-03 & Pa-234m & $->$ & $U-234$ & & & \\
\hline 193.4 & 6.99E-02 & $\mathrm{Pa}-234 \mathrm{~m}$ & $->$ & $U-234$ & & & \\
\hline 193.55 & $9.70 \mathrm{E}-02$ & Pa-234 & $->$ & $U-234$ & & & \\
\hline 196.3 & $1.33 \mathrm{E}-12$ & $\mathrm{~Pb}-214$ & $->$ & $\mathrm{Bi}-214$ & & & \\
\hline 196.43 & $1.13 \mathrm{E}-02$ & $\mathrm{~Pa}-234$ & $->$ & $U-234$ & & & \\
\hline 199.46 & $1.41 \mathrm{E}-01$ & Pa-234 & $->$ & U-234, & $\begin{array}{r}\mathrm{Pa}- \\
234 \mathrm{~m}\end{array}$ & $->$ & U-234 \\
\hline 200.96 & $1.78 \mathrm{E}-01$ & $\mathrm{~Pa}-234$ & $->$ & $U-234$ & & & \\
\hline 203.12 & $3.36 \mathrm{E}-01$ & $\mathrm{~Pa}-234$ & $->$ & U-234, & $\begin{array}{r}\text { Pa- } \\
234 m\end{array}$ & $->$ & $U-234$ \\
\hline 205.1 & $1.94 \mathrm{E}-13$ & Th-230 & $->$ & $\mathrm{Ra}-226$ & & & \\
\hline 210.6 & $1.46 \mathrm{E}-01$ & $\mathrm{~Pa}-234 \mathrm{~m}$ & $->$ & $U-234$ & & & \\
\hline 210.7 & $8.08 \mathrm{E}-03$ & Pa-234 & $->$ & $U-234$ & & & \\
\hline 219.8 & $3.23 E-02$ & $\mathrm{~Pa}-234$ & $->$ & $U-234$ & & & \\
\hline 226.95 & $2.01 \mathrm{E}+00$ & Pa-234 & $->$ & $U-234$ & & & \\
\hline 235.01 & $3.14 \mathrm{E}-13$ & Th-230 & $->$ & $\mathrm{Ra}-226$ & & & \\
\hline 236.03 & $4.72 \mathrm{E}-03$ & $\mathrm{~Pa}-234 \mathrm{~m}$ & $->$ & $U-234$ & & & \\
\hline 241.92 & $2.03 \mathrm{E}-10$ & $\mathrm{~Pb}-214$ & $->$ & $\mathrm{Bi}-214$ & & & \\
\hline 243.5 & $5.56 \mathrm{E}-01$ & $\mathrm{~Pa}-234 \mathrm{~m}$ & $->$ & $U-234$ & & & \\
\hline 245.16 & $1.46 \mathrm{E}-01$ & Pa-234 & $->$ & $U-234$ & & & \\
\hline 247.7 & $8.29 E-02$ & $\mathrm{~Pa}-234 \mathrm{~m}$ & $->$ & $U-234$ & & & \\
\hline 248.94 & $3.52 \mathrm{E}-01$ & Pa-234 & $->$ & U-234 & & & \\
\hline 253.732 & $4.31 \mathrm{E}-10$ & Th-230 & $->$ & $\mathrm{Ra}-226$ & & & \\
\hline 253.84 & $3.30 \mathrm{E}-11$ & Th-230 & $->$ & Ra-226 & & & \\
\hline 258.26 & $9.07 \mathrm{E}+00$ & $\mathrm{~Pa}-234 \mathrm{~m}$ & $->$ & U-234 & & & \\
\hline 258.94 & $1.49 \mathrm{E}-11$ & $\mathrm{~Pb}-214$ & $->$ & $\mathrm{Bi}-214$ & & & \\
\hline 262.41 & $1.48 \mathrm{E}-13$ & $\mathrm{Ra}-226$ & $->$ & $\mathrm{Rn}-222$ & & & \\
\hline 266.15 & $5.21 \mathrm{E}-23$ & $\mathrm{Bi}-210$ & $->$ & TI-206 & & & \\
\hline 267.1 & $2.75 \mathrm{E}-02$ & $\mathrm{~Pa}-234$ & $->$ & $U-234$ & & & \\
\hline 272.2 & $2.24 \mathrm{E}-01$ & $\mathrm{~Pa}-234$ & $->$ & $U-234$ & & & \\
\hline 273.7 & $4.89 E-12$ & $\mathrm{Bi}-214$ & $->$ & Рo-214 & & & \\
\hline 274.45 & 8.83E-02 & $\mathrm{Pa}-234$ & $->$ & U-234, & $\begin{array}{r}\text { Pa- } \\
234 \mathrm{~m}\end{array}$ & $->$ & $U-234$ \\
\hline
\end{tabular}




\begin{tabular}{|c|c|c|c|c|c|c|c|}
\hline 274.56 & $8.69 \mathrm{E}-12$ & $\mathrm{~Pb}-214$ & $->$ & $\mathrm{Bi}-214$ & & & \\
\hline 276.83 & 7.69E-02 & $\mathrm{Pa}-234$ & $->$ & U-234, & $\begin{array}{r}\mathrm{Pa}- \\
234 \mathrm{~m}\end{array}$ & $->$ & $U-234$ \\
\hline 278.1 & $1.13 \mathrm{E}-02$ & Pa-234 & $->$ & $U-234$ & & & \\
\hline 280.93 & $2.20 \mathrm{E}-12$ & $\mathrm{Bi}-214$ & $->$ & Po-214 & & & \\
\hline 286.4 & $2.26 \mathrm{E}-02$ & $\mathrm{~Pa}-234$ & $->$ & $U-234$ & & & \\
\hline 286.9 & $8.69 \mathrm{E}-13$ & $\mathrm{Bi}-214$ & $->$ & Рo-214 & & & \\
\hline 289.25 & $1.78 \mathrm{E}-02$ & $\mathrm{~Pa}-234$ & $->$ & $U-234$ & & & \\
\hline 293.874 & $6.09 E-01$ & $\mathrm{~Pa}-234$ & $->$ & $U-234$ & & & \\
\hline 295.091 & $5.22 \mathrm{E}-10$ & $\mathrm{~Pb}-214$ & $->$ & $\mathrm{Bi}-214$ & & & \\
\hline 298.1 & $4.52 E-13$ & TI-210 & $->$ & $\mathrm{Pb}-210$, & Po-214 & $->$ & $\mathrm{Pb}-210$ \\
\hline 299.08 & 6.99E-02 & $\mathrm{Pa}-234 \mathrm{~m}$ & $->$ & $U-234$ & & & \\
\hline 302.25 & $5.99 \mathrm{E}-17$ & Hg-206 & $->$ & TI-206 & & & \\
\hline 304.42 & $9.23 E-13$ & $\mathrm{Bi}-214$ & $->$ & Po-214 & & & \\
\hline 305.5 & $6.25 \mathrm{E}-13$ & $\mathrm{~Pb}-214$ & $->$ & $\mathrm{Bi}-214$ & & & \\
\hline 309.6 & $1.62 \mathrm{E}-02$ & Pa-234 & $->$ & $U-234$ & & & \\
\hline 311 & $5.73 \mathrm{E}-02$ & $\mathrm{~Pa}-234 \mathrm{~m}$ & $->$ & U-234 & & & \\
\hline 313.83 & $4.85 \mathrm{E}-02$ & Pa-234 & $->$ & $U-234$ & & & \\
\hline 314.2 & $2.15 \mathrm{E}-12$ & $\mathrm{~Pb}-214$ & $->$ & $\mathrm{Bi}-214$ & & & \\
\hline 316.29 & $3.53 \mathrm{E}-02$ & $\mathrm{~Pa}-234$ & $->$ & U-234, & $\begin{array}{r}\mathrm{Pa}- \\
234 \mathrm{~m}\end{array}$ & $->$ & $U-234$ \\
\hline 320.7 & $1.94 \mathrm{E}-02$ & $\mathrm{~Pa}-234$ & $->$ & $U-234$ & & & \\
\hline 324.3 & $5.43 E-13$ & $\mathrm{~Pb}-214$ & $->$ & $\mathrm{Bi}-214$ & & & \\
\hline 330 & $4.85 E-02$ & $\mathrm{~Pa}-234$ & $->$ & $U-234$ & & & \\
\hline 330.62 & $9.70 \mathrm{E}-02$ & $\mathrm{~Pa}-234$ & $->$ & $U-234$ & & & \\
\hline 333.6 & $2.58 \mathrm{E}-12$ & $\mathrm{Bi}-214$ & $->$ & Po-214 & & & \\
\hline 334.9 & $1.55 \mathrm{E}-12$ & $\mathrm{Bi}-214$ & $->$ & Po-214 & & & \\
\hline 338.4 & $1.26 \mathrm{E}-01$ & $\mathrm{~Pa}-234 \mathrm{~m}$ & $->$ & $U-234$ & & & \\
\hline 338.5 & $1.06 \mathrm{E}-12$ & $\mathrm{Bi}-214$ & $->$ & Po-214 & & & \\
\hline 344.96 & $1.15 \mathrm{E}-18$ & $\mathrm{Hg}-206$ & $->$ & TI-206 & & & \\
\hline 347.1 & $1.63 \mathrm{E}-12$ & $\mathrm{Bi}-214$ & $->$ & Рo-214 & & & \\
\hline 351.87 & $1.01 \mathrm{E}-09$ & $\mathrm{~Pb}-214$ & $->$ & $\mathrm{Bi}-214$ & & & \\
\hline 352.16 & $9.70 \mathrm{E}-02$ & $\mathrm{~Pa}-234$ & $->$ & $U-234$ & & & \\
\hline 354 & $2.28 \mathrm{E}-14$ & TI-210 & $->$ & $\mathrm{Pb}-210$ & & & \\
\hline 357.9 & 8.89E-02 & $\mathrm{Pa}-234 \mathrm{~m}$ & $->$ & U-234 & & & \\
\hline 360.8 & 7.63E-02 & $\mathrm{Pa}-234 \mathrm{~m}$ & $->$ & $U-234$ & & & \\
\hline 364.2 & $1.71 \mathrm{E}-13$ & $\mathrm{Bi}-214$ & $->$ & Po-214 & & & \\
\hline 369.52 & 5.47E-01 & $\mathrm{Pa}-234$ & $->$ & $U-234$ & & & \\
\hline 372.02 & $2.86 \mathrm{E}-01$ & $\mathrm{~Pa}-234$ & $->$ & $\mathrm{U}-234$ & & & \\
\hline 376.6 & $1.33 \mathrm{E}-13$ & $\mathrm{Bi}-214$ & $->$ & Рo-214 & & & \\
\hline 380 & $1.71 \mathrm{E}-14$ & TI-210 & $->$ & $\mathrm{Pb}-210$ & & & \\
\hline 384.06 & $8.43 E-21$ & $\mathrm{Hg}-206$ & $->$ & TI-206 & & & \\
\hline 386.834 & $9.77 \mathrm{E}-12$ & $\mathrm{Bi}-214$ & $->$ & Po-214 & & & \\
\hline 387.8 & $1.11 \mathrm{E}-01$ & $\mathrm{~Pa}-234 \mathrm{~m}$ & $->$ & U-234 & & & \\
\hline 387.87 & 4.77E-02 & $\mathrm{Pa}-234 \mathrm{~m}$ & $->$ & $\mathrm{U}-234$ & & & \\
\hline 388.95 & $1.11 \mathrm{E}-11$ & $\mathrm{Bi}-214$ & $->$ & Po-214 & & & \\
\hline 394 & $2.44 \mathrm{E}-13$ & $\mathrm{Bi}-214$ & $->$ & Рo-214 & & & \\
\hline 396 & $8.15 \mathrm{E}-13$ & $\mathrm{Bi}-214$ & $->$ & Po-214 & & & \\
\hline 405.73 & $4.53 \mathrm{E}-12$ & $\mathrm{Bi}-214$ & $->$ & Po-214 & & & \\
\hline 409.8 & $6.47 \mathrm{E}-02$ & $\mathrm{~Pa}-234$ & $->$ & $U-234$ & & & \\
\hline
\end{tabular}




\begin{tabular}{|c|c|c|c|c|}
\hline 414.72 & $1.07 \mathrm{E}-14$ & $\mathrm{Ra}-226$ & $->$ & $\mathrm{Rn}-222$ \\
\hline 416 & $1.62 \mathrm{E}-02$ & $\mathrm{~Pa}-234$ & $->$ & $U-234$ \\
\hline 426.5 & 2.99E-12 & $\mathrm{Bi}-214$ & $->$ & Рo-214 \\
\hline 426.8 & $9.70 \mathrm{E}-02$ & $\mathrm{~Pa}-234$ & $->$ & U-234 \\
\hline 432.8 & $1.78 \mathrm{E}-03$ & Pa-234 & $->$ & $U-234$ \\
\hline 440.4 & 7.87E-13 & $\mathrm{Bi}-214$ & $->$ & Po-214 \\
\hline 446.1 & $1.94 \mathrm{E}-02$ & $\mathrm{~Pa}-234$ & $->$ & U-234 \\
\hline 449.5 & $7.42 \mathrm{E}-15$ & Ra-226 & $->$ & $\mathrm{Rn}-222$ \\
\hline 450.96 & 3.35E-01 & $\mathrm{Pa}-234 \mathrm{~m}$ & $->$ & U-234 \\
\hline 453.58 & $2.36 \mathrm{E}-01$ & $\mathrm{~Pa}-234 \mathrm{~m}$ & $->$ & $U-234$ \\
\hline 454.832 & $8.63 E-12$ & $\mathrm{Bi}-214$ & $->$ & Рo-214 \\
\hline 454.97 & $4.48 \mathrm{E}-08$ & $U-234$ & $->$ & Th-230 \\
\hline 456.5 & 7.95E-02 & $\mathrm{Pa}-234 \mathrm{~m}$ & $->$ & U-234 \\
\hline 458.63 & $2.49 \mathrm{E}-01$ & Pa-234 & $->$ & $U-234$ \\
\hline 461.3 & 2.59E-02 & $\mathrm{Pa}-234$ & $->$ & U-234 \\
\hline 462.05 & $4.62 \mathrm{E}-12$ & $\mathrm{~Pb}-214$ & $->$ & $\mathrm{Bi}-214$ \\
\hline 467.5 & $5.66 \mathrm{E}-02$ & $\mathrm{~Pa}-234$ & $->$ & $U-234$ \\
\hline 468.21 & $2.29 \mathrm{E}-01$ & $\mathrm{~Pa}-234 \mathrm{~m}$ & $->$ & $U-234$ \\
\hline 469.76 & $3.61 \mathrm{E}-12$ & $\mathrm{Bi}-214$ & $->$ & Po-214 \\
\hline 470.8 & $2.72 \mathrm{E}-13$ & $\mathrm{~Pb}-214$ & $->$ & Bi-214 \\
\hline 472.32 & $3.88 \mathrm{E}-02$ & $\mathrm{~Pa}-234$ & $->$ & $U-234$ \\
\hline 473.5 & $2.91 \mathrm{E}-02$ & $\mathrm{~Pa}-234$ & $->$ & $U-234$ \\
\hline 474.51 & $3.20 \mathrm{E}-12$ & $\mathrm{Bi}-214$ & $->$ & Po-214 \\
\hline 475.75 & $2.86 \mathrm{E}-01$ & $\mathrm{~Pa}-234 \mathrm{~m}$ & $->$ & U-234 \\
\hline 478 & $1.14 \mathrm{E}-14$ & TI-210 & $->$ & $\mathrm{Pb}-210$ \\
\hline 478.7 & $4.85 E-02$ & $\mathrm{~Pa}-234$ & $->$ & U-234 \\
\hline 480.32 & $9.18 \mathrm{E}-12$ & $\mathrm{~Pb}-214$ & $->$ & $\mathrm{Bi}-214$ \\
\hline 480.4 & 6.47E-02 & $\mathrm{Pa}-234$ & $->$ & $U-234$ \\
\hline 482.5 & $4.85 \mathrm{E}-02$ & $\mathrm{~Pa}-234$ & $->$ & $U-234$ \\
\hline 487.13 & $1.19 \mathrm{E}-11$ & $\mathrm{~Pb}-214$ & $->$ & $\mathrm{Bi}-214$ \\
\hline 494.6 & $2.44 \mathrm{E}-13$ & $\mathrm{Bi}-214$ & $->$ & Рo-214 \\
\hline 498.8 & $1.62 \mathrm{E}-02$ & $\mathrm{~Pa}-234$ & $->$ & U-234 \\
\hline 502.2 & $4.89 E-13$ & $\mathrm{Bi}-214$ & $->$ & Рo-214 \\
\hline 503.53 & $1.72 \mathrm{E}-09$ & U-234 & $->$ & Th-230 \\
\hline 506.69 & $2.60 \mathrm{E}-01$ & $\mathrm{~Pa}-234$ & $->$ & U-234 \\
\hline 507.3 & $1.75 \mathrm{E}-01$ & $\mathrm{~Pa}-234 \mathrm{~m}$ & $->$ & U-234 \\
\hline 508.2 & $2.53 E-08$ & U-234 & $->$ & Th-230 \\
\hline 509.11 & $2.38 \mathrm{E}-01$ & $\mathrm{~Pa}-234 \mathrm{~m}$ & $->$ & $U-234$ \\
\hline 510 & $1.90 \mathrm{E}-12$ & Rn-222 & $->$ & Po-218 \\
\hline 511 & $7.88 \mathrm{E}-13$ & $\mathrm{~Pb}-214$ & $->$ & $\mathrm{Bi}-214$ \\
\hline 513.6 & $2.10 \mathrm{E}-01$ & $\mathrm{~Pa}-234$ & $->$ & $U-234$ \\
\hline 516.8 & $1.86 \mathrm{E}-03$ & $\mathrm{~Pa}-234 \mathrm{~m}$ & $->$ & $U-234$ \\
\hline 520.2 & $9.70 \mathrm{E}-02$ & $\mathrm{~Pa}-234$ & $->$ & U-234 \\
\hline 520.4 & $1.55 \mathrm{E}-13$ & $\mathrm{Bi}-214$ & $->$ & Рo-214 \\
\hline 521.09 & $1.46 \mathrm{E}-01$ & $\mathrm{~Pa}-234$ & $->$ & U-234 \\
\hline 525 & $4.34 \mathrm{E}-13$ & $\mathrm{Bi}-214$ & $->$ & Po-214 \\
\hline 527.04 & $9.70 \mathrm{E}-02$ & $\mathrm{~Pa}-234$ & $->$ & U-234 \\
\hline 533.2 & $3.23 E-02$ & Pa-234 & $\rightarrow$ & $U-234$ \\
\hline 533.5 & $5.16 \mathrm{E}-12$ & $\mathrm{~Pb}-214$ & $->$ & $\mathrm{Bi}-214$ \\
\hline 536.93 & $1.93 \mathrm{E}-12$ & $\mathrm{Bi}-214$ & $->$ & Po-214 \\
\hline
\end{tabular}




\begin{tabular}{|c|c|c|c|c|}
\hline 537.1 & 2.59E-02 & Pa-234 & $->$ & $U-234$ \\
\hline 538.7 & $1.33 \mathrm{E}-13$ & $\mathrm{~Pb}-214$ & $->$ & $\mathrm{Bi}-214$ \\
\hline 542.84 & $2.31 \mathrm{E}-12$ & $\mathrm{Bi}-214$ & $->$ & Po-214 \\
\hline 543.91 & $6.25 \mathrm{E}-13$ & $\mathrm{~Pb}-214$ & $->$ & $\mathrm{Bi}-214$ \\
\hline 543.98 & 4.47E-01 & $\mathrm{Pa}-234 \mathrm{~m}$ & $->$ & U-234 \\
\hline 547.1 & 8.69E-13 & $\mathrm{Bi}-214$ & $->$ & Po-214 \\
\hline 551.8 & $3.92 \mathrm{E}-14$ & Th-230 & $->$ & $\mathrm{Ra}-226$ \\
\hline 557.3 & 7.95E-02 & Pa-234m & $->$ & U-234 \\
\hline 557.7 & 4.47E-03 & $\mathrm{Pa}-234 \mathrm{~m}$ & $->$ & $U-234$ \\
\hline 557.78 & $4.20 \mathrm{E}-02$ & Pa-234 & $->$ & U-234 \\
\hline 565.2 & $2.26 \mathrm{E}-01$ & $\mathrm{~Pa}-234$ & $->$ & $U-234$ \\
\hline 569.3 & $2.52 E+00$ & $\mathrm{~Pa}-234$ & $->$ & $U-234$ \\
\hline 570.5 & $1.16 \mathrm{E}-13$ & Th-230 & $->$ & Ra-226 \\
\hline 572 & 9.69E-02 & $\mathrm{Pa}-234 \mathrm{~m}$ & $->$ & U-234 \\
\hline 572.67 & $2.23 \mathrm{E}-12$ & $\mathrm{Bi}-214$ & $->$ & Po-214 \\
\hline 574.8 & 3.23E-01 & $\mathrm{Pa}-234$ & $->$ & U-234 \\
\hline 580.06 & $9.89 \mathrm{E}-12$ & $\mathrm{~Pb}-214$ & $->$ & $\mathrm{Bi}-214$ \\
\hline 581.78 & 2.07E-08 & U-234 & $->$ & Th-230 \\
\hline 585.8 & $2.43 \mathrm{E}-02$ & Pa-234 & $->$ & U-234 \\
\hline 596 & $3.26 \mathrm{E}-13$ & $\mathrm{Bi}-214$ & $->$ & Рo-214 \\
\hline 600.83 & $1.68 \mathrm{E}-14$ & Ra-226 & $->$ & $\mathrm{Rn}-222$ \\
\hline 602.1 & $1.46 \mathrm{E}-01$ & Pa-234 & $->$ & U-234 \\
\hline 609.311 & $1.25 \mathrm{E}-09$ & $\mathrm{Bi}-214$ & $->$ & Po-214 \\
\hline 611.5 & $1.29 \mathrm{E}-01$ & $\mathrm{~Pa}-234$ & $->$ & U-234 \\
\hline 615.77 & $1.90 \mathrm{E}-12$ & $\mathrm{Bi}-214$ & $->$ & Po-214 \\
\hline 617 & $3.23 \mathrm{E}-02$ & $\mathrm{~Pa}-234$ & $->$ & U-234 \\
\hline 617.1 & $9.23 \mathrm{E}-13$ & $\mathrm{Bi}-214$ & $->$ & Рo-214 \\
\hline 620 & $3.92 \mathrm{E}-14$ & Th-230 & $->$ & $\mathrm{Ra}-226$ \\
\hline 624.31 & $1.29 \mathrm{E}-01$ & $\mathrm{~Pa}-234$ & $->$ & U-234 \\
\hline 624.44 & $1.38 \mathrm{E}-09$ & U-234 & $->$ & Th-230 \\
\hline 625.5 & $1.59 \mathrm{E}-01$ & Pa-234m & $->$ & U-234 \\
\hline 626.4 & $1.33 \mathrm{E}-13$ & $\mathrm{Bi}-214$ & $->$ & Po-214 \\
\hline 627.5 & $1.29 \mathrm{E}-01$ & $\mathrm{~Pa}-234$ & $->$ & U-234 \\
\hline 631.2 & $4.62 E-13$ & $\mathrm{Bi}-214$ & $->$ & Po-214 \\
\hline 632 & 6.47E-02 & Pa-234 & $->$ & U-234 \\
\hline 633.14 & $1.63 \mathrm{E}-12$ & $\mathrm{Bi}-214$ & $->$ & Po-214 \\
\hline 634.4 & $4.85 E-02$ & Pa-234 & $->$ & U-234 \\
\hline 639.36 & $8.42 E-13$ & $\mathrm{Bi}-214$ & $->$ & Po-214 \\
\hline 639.7 & $3.23 E-02$ & Pa-234 & $->$ & $U-234$ \\
\hline 643.2 & $3.23 E-02$ & Pa-234 & $->$ & $U-234$ \\
\hline 646.84 & 4.85E-02 & Рa-234 & $->$ & $U-234$ \\
\hline 647.7 & $1.75 \mathrm{E}-01$ & $\mathrm{~Pa}-234 \mathrm{~m}$ & $->$ & U-234 \\
\hline 649.1 & $1.19 \mathrm{E}-01$ & Pa-234m & $->$ & U-234 \\
\hline 649.18 & $1.60 \mathrm{E}-12$ & $\mathrm{Bi}-214$ & $->$ & Po-214 \\
\hline 650.21 & $5.54 \mathrm{E}-18$ & $\mathrm{Hg}-206$ & $->$ & TI-206 \\
\hline 653.57 & $1.00 \mathrm{E}-01$ & $\mathrm{~Pa}-234$ & $->$ & $U-234$ \\
\hline 655 & 6.69E-02 & $\mathrm{Pa}-234$ & $->$ & U-234 \\
\hline 655.1 & $1.06 \mathrm{E}-01$ & $\mathrm{~Pa}-234 \mathrm{~m}$ & $->$ & U-234 \\
\hline 658 & $1.46 \mathrm{E}-01$ & Pa-234 & $->$ & U-234 \\
\hline 660.6 & $4.85 E-02$ & Pa-234 & $->$ & $U-234$ \\
\hline
\end{tabular}




\begin{tabular}{|c|c|c|c|c|c|c|c|}
\hline 660.75 & $1.17 \mathrm{E}-12$ & $\mathrm{Bi}-214$ & $->$ & Po-214 & & & \\
\hline 664.8 & $2.10 \mathrm{E}-01$ & $\mathrm{~Pa}-234$ & $->$ & U-234 & & & \\
\hline 665.442 & $4.24 \mathrm{E}-11$ & $\mathrm{Bi}-214$ & $->$ & Po-214 & & & \\
\hline 666.42 & $1.87 \mathrm{E}-01$ & $\mathrm{~Pa}-234$ & $->$ & U-234 & & & \\
\hline 668 & $1.14 \mathrm{E}-14$ & TI-210 & $->$ & $\mathrm{Pb}-210$ & & & \\
\hline 669.64 & $2.11 \mathrm{E}-01$ & $\mathrm{~Pa}-234$ & $->$ & $U-234$ & & & \\
\hline 674.24 & 7.15E-02 & $\mathrm{Pa}-234 \mathrm{~m}$ & $->$ & $U-234$ & & & \\
\hline 677.67 & $1.72 \mathrm{E}-09$ & U-234 & $->$ & Th-230 & & & \\
\hline 683 & $6.36 \mathrm{E}-02$ & $\mathrm{~Pa}-234 \mathrm{~m}$ & $->$ & U-234 & & & \\
\hline 683.21 & $2.14 \mathrm{E}-12$ & $\mathrm{Bi}-214$ & $->$ & Po-214 & & & \\
\hline 683.3 & $3.88 \mathrm{E}-02$ & $\mathrm{~Pa}-234$ & $->$ & U-234 & & & \\
\hline 687.7 & $1.63 \mathrm{E}-13$ & $\mathrm{Bi}-214$ & $->$ & Po-214 & & & \\
\hline 688.08 & $4.53 E-02$ & Pa-234 & $->$ & U-234 & & & \\
\hline 691.08 & $1.12 \mathrm{E}+00$ & $\mathrm{~Pa}-234 \mathrm{~m}$ & $->$ & $U-234$ & & & \\
\hline 692.7 & $2.43 \mathrm{E}-01$ & $\mathrm{~Pa}-234$ & $->$ & U-234 & & & \\
\hline 693.3 & $1.63 \mathrm{E}-13$ & $\mathrm{Bi}-214$ & $->$ & Po-214 & & & \\
\hline 695.9 & $1.75 \mathrm{E}-01$ & $\mathrm{~Pa}-234 \mathrm{~m}$ & $->$ & U-234 & & & \\
\hline 697.89 & $1.00 \mathrm{E}-12$ & $\mathrm{Bi}-214$ & $->$ & Po-214 & & & \\
\hline 698.85 & $6.55 \mathrm{E}-01$ & Pa-234 & $->$ & U-234 & & & \\
\hline 700.4 & 7.84E-02 & $\mathrm{Pa}-234 \mathrm{~m}$ & $->$ & $U-234$ & & & \\
\hline 702.05 & 8.82E-01 & $\mathrm{Pa}-234 \mathrm{~m}$ & $->$ & $U-234$ & & & \\
\hline 703.07 & $1.28 \mathrm{E}-11$ & $\mathrm{Bi}-214$ & $->$ & Po-214 & & & \\
\hline 705.9 & $8.08 \mathrm{E}-01$ & $\mathrm{~Pa}-234$ & $->$ & U-234, & $\begin{array}{r}\mathrm{Pa}- \\
234 \mathrm{~m}\end{array}$ & $->$ & $U-234$ \\
\hline 708.4 & $1.28 \mathrm{E}-01$ & $\mathrm{~Pa}-234$ & $->$ & U-234, & $\begin{array}{l}\mathrm{Pa}- \\
234 \mathrm{~m}\end{array}$ & $->$ & $U-234$ \\
\hline 710.84 & $2.03 \mathrm{E}-12$ & $\mathrm{Bi}-214$ & $->$ & Po-214 & & & \\
\hline 711.2 & $3.23 E-02$ & Pa-234 & $->$ & $U-234$ & & & \\
\hline 713.8 & $2.59 \mathrm{E}-02$ & Pa-234 & $->$ & $U-234$ & & & \\
\hline 718.94 & $3.85 E-03$ & $\mathrm{~Pa}-234 \mathrm{~m}$ & $->$ & $U-234$ & & & \\
\hline 719.856 & $1.09 \mathrm{E}-11$ & $\mathrm{Bi}-214$ & $->$ & Po-214 & & & \\
\hline 721.33 & $3.60 \mathrm{E}-03$ & $\mathrm{~Pa}-234 \mathrm{~m}$ & $->$ & U-234 & & & \\
\hline 723.32 & $1.22 \mathrm{E}-12$ & $\mathrm{Bi}-214$ & $->$ & Po-214 & & & \\
\hline 727.8 & $4.26 \mathrm{E}-13$ & $\mathrm{Bi}-214$ & $->$ & Po-214 & & & \\
\hline 732.7 & $1.45 \mathrm{E}-01$ & $\mathrm{~Pa}-234 \mathrm{~m}$ & $->$ & $U-234$ & & & \\
\hline 733.38 & $1.43 E+00$ & $\mathrm{~Pa}-234$ & $->$ & U-234 & & & \\
\hline 733.64 & $1.28 \mathrm{E}-12$ & $\mathrm{Bi}-214$ & $->$ & Po-214 & & & \\
\hline 738.88 & $2.61 \mathrm{E}-01$ & Pa-234 & $->$ & U-234 & & & \\
\hline 739.95 & $1.47 \mathrm{E}+00$ & $\mathrm{~Pa}-234 \mathrm{~m}$ & $->$ & $\mathrm{U}-234$ & & & \\
\hline 740.87 & $1.06 \mathrm{E}-12$ & $\mathrm{Bi}-214$ & $->$ & Po-214 & & & \\
\hline 742.817 & $1.18 \mathrm{E}+01$ & $\mathrm{~Pa}-234$ & $->$ & U-234, & $\begin{array}{r}\text { Pa- } \\
234 m\end{array}$ & $->$ & $U-234$ \\
\hline 746.5 & $2.10 \mathrm{E}-02$ & Pa-234 & $->$ & $U-234$ & & & \\
\hline 750.5 & $2.24 \mathrm{E}-03$ & $\mathrm{~Pa}-234 \mathrm{~m}$ & $->$ & $U-234$ & & & \\
\hline 752.843 & $3.61 \mathrm{E}-12$ & $\mathrm{Bi}-214$ & $->$ & Po-214 & & & \\
\hline 755 & $2.61 \mathrm{E}-01$ & $\mathrm{~Pa}-234$ & $->$ & $U-234$ & & & \\
\hline 760.1 & $2.59 \mathrm{E}-02$ & $\mathrm{~Pa}-234$ & $->$ & $U-234$ & & & \\
\hline 760.3 & $1.75 \mathrm{E}-01$ & $\mathrm{~Pa}-234 \mathrm{~m}$ & $->$ & $U-234$ & & & \\
\hline 765.9 & $2.15 \mathrm{E}-12$ & $\mathrm{~Pb}-214$ & $->$ & $\mathrm{Bi}-214$ & & & \\
\hline 766.369 & $4.85 \mathrm{E}-02$ & $\mathrm{~Pa}-234$ & $->$ & $\mathrm{U}-234$ & & & \\
\hline
\end{tabular}




\begin{tabular}{|c|c|c|c|c|c|c|c|}
\hline 766.412 & $4.00 \mathrm{E}+01$ & $\mathrm{~Pa}-234$ & $->$ & U-234, & $\begin{array}{r}\text { Pa- } \\
234 m\end{array}$ & $->$ & $U-234$ \\
\hline 768.35 & $1.32 \mathrm{E}-10$ & $\mathrm{Bi}-214$ & $->$ & Рo-214 & & & \\
\hline 768.5 & $9.05 \mathrm{E}-02$ & $\mathrm{~Pa}-234$ & $->$ & $U-234$ & & & \\
\hline 777.9 & $3.23 E-02$ & $\mathrm{~Pa}-234$ & $->$ & $U-234$ & & & \\
\hline 780.4 & $1.78 \mathrm{E}-01$ & Pa-234 & $->$ & $U-234$ & & & \\
\hline 782.8 & $8.42 \mathrm{E}-01$ & $\mathrm{~Pa}-234 \mathrm{~m}$ & $->$ & U-234 & & & \\
\hline 783.38 & 8.87E-02 & Pa-234 & $->$ & U-234, & $\begin{array}{r}\text { Pa- } \\
234 m\end{array}$ & $->$ & $U-234$ \\
\hline 785.827 & $2.96 \mathrm{E}-11$ & $\mathrm{~Pb}-214$ & $->$ & $\mathrm{Bi}-214$ & & & \\
\hline 786.287 & $6.88 \mathrm{E}+00$ & $\mathrm{~Pa}-234$ & $->$ & U-234, & $\begin{array}{r}\mathrm{Pa}- \\
234 \mathrm{~m}\end{array}$ & $->$ & $U-234$ \\
\hline 786.42 & $8.42 \mathrm{E}-12$ & $\mathrm{Bi}-214$ & $->$ & Po-214 & & & \\
\hline 793.6 & $2.43 E-01$ & $\mathrm{~Pa}-234$ & $->$ & $U-234$ & & & \\
\hline 796.42 & $6.72 \mathrm{E}-01$ & $\mathrm{~Pa}-234$ & $->$ & U-234 & & & \\
\hline 797.88 & $8.46 \mathrm{E}-13$ & Tl-210 & $->$ & $\mathrm{Pb}-210$, & Po-214 & $->$ & $\mathrm{Pb}-210$ \\
\hline 799.75 & $1.11 \mathrm{E}-12$ & $\mathrm{Bi}-214$ & $->$ & Po-214 & & & \\
\hline 803.13 & $6.76 \mathrm{E}-16$ & TI-206 & $->$ & $\mathrm{Pb}-206$, & Po-210 & $->$ & $\mathrm{Pb}-206$ \\
\hline 804.52 & $6.47 \mathrm{E}-02$ & $\mathrm{~Pa}-234$ & $->$ & $U-234$ & & & \\
\hline 805.74 & $1.09 \mathrm{E}+00$ & $\mathrm{~Pa}-234$ & $->$ & U-234, & $\begin{array}{r}\mathrm{Pa}- \\
234 \mathrm{~m}\end{array}$ & $->$ & $U-234$ \\
\hline 806.155 & $3.34 \mathrm{E}-11$ & $\mathrm{Bi}-214$ & $->$ & Рo-214 & & & \\
\hline 808.2 & $3.23 E-01$ & $\mathrm{~Pa}-234$ & $->$ & U-234, & $\begin{array}{r}\mathrm{Pa}- \\
234 \mathrm{~m}\end{array}$ & $->$ & $U-234$ \\
\hline 812.5 & $8.08 \mathrm{E}-02$ & $\mathrm{~Pa}-234$ & $->$ & $U-234$ & & & \\
\hline 814.87 & $1.09 \mathrm{E}-12$ & $\mathrm{Bi}-214$ & $->$ & Po-214 & & & \\
\hline 818 & $1.11 \mathrm{E}-01$ & $\mathrm{~Pa}-234 \mathrm{~m}$ & $->$ & $U-234$ & & & \\
\hline 819.21 & $4.60 \mathrm{E}-01$ & Pa-234 & $->$ & $U-234$ & & & \\
\hline 821.166 & $4.07 \mathrm{E}-12$ & $\mathrm{Bi}-214$ & $->$ & Po-214 & & & \\
\hline 824.94 & $8.46 \mathrm{E}-01$ & Pa-234 & $->$ & $U-234$ & & & \\
\hline 826.08 & $1.57 \mathrm{E}-01$ & $\mathrm{~Pa}-234 \mathrm{~m}$ & $->$ & $U-234$ & & & \\
\hline 826.44 & $2.50 \mathrm{E}-12$ & $\mathrm{Bi}-214$ & $->$ & Рo-214 & & & \\
\hline 831.39 & $9.70 \mathrm{E}-01$ & $\mathrm{~Pa}-234$ & $->$ & $U-234$ & & & \\
\hline 832.34 & $6.24 \mathrm{E}-13$ & $\mathrm{Bi}-214$ & $->$ & Рo-214 & & & \\
\hline 838.999 & $1.59 \mathrm{E}-11$ & $\mathrm{~Pb}-214$ & $->$ & $\mathrm{Bi}-214$ & & & \\
\hline 842.4 & $2.26 \mathrm{E}-02$ & $\mathrm{~Pa}-234$ & $->$ & U-234 & & & \\
\hline 844.4 & $2.02 \mathrm{E}-01$ & $\mathrm{~Pa}-234$ & $->$ & U-234, & $\begin{array}{r}\mathrm{Pa}- \\
234 \mathrm{~m}\end{array}$ & $->$ & $U-234$ \\
\hline 847.2 & $4.62 \mathrm{E}-13$ & $\mathrm{Bi}-214$ & $->$ & Po-214 & & & \\
\hline 851.57 & 8.70E-01 & Pa-234 & $->$ & U-234, & $\begin{array}{r}\mathrm{Pa}- \\
234 \mathrm{~m}\end{array}$ & $->$ & $U-234$ \\
\hline 860 & $3.93 E-14$ & TI-210 & $->$ & $\mathrm{Pb}-210$ & & & \\
\hline 866.98 & $1.19 \mathrm{E}-01$ & $\mathrm{~Pa}-234 \mathrm{~m}$ & $->$ & U-234 & & & \\
\hline 873.4 & $1.94 \mathrm{E}-02$ & Pa-234 & $->$ & $U-234$ & & & \\
\hline 875.94 & $5.22 \mathrm{E}-01$ & $\mathrm{~Pa}-234$ & $->$ & $U-234$ & & & \\
\hline 880.45 & $2.64 \mathrm{E}+00$ & Pa-234 & $->$ & $U-234$ & & & \\
\hline 881.1 & 4.29E-01 & $\mathrm{Pa}-234 \mathrm{~m}$ & $->$ & $U-234$ & & & \\
\hline 882.5 & 2.07E-01 & $\mathrm{Pa}-234 \mathrm{~m}$ & $->$ & U-234 & & & \\
\hline 883.22 & $2.62 \mathrm{E}+00$ & $\mathrm{~Pa}-234$ & $->$ & U-234, & $\begin{array}{r}\text { Pa- } \\
234 m\end{array}$ & $->$ & $U-234$ \\
\hline 887.28 & $8.82 \mathrm{E}-01$ & $\mathrm{~Pa}-234 \mathrm{~m}$ & $->$ & $U-234$ & & & \\
\hline
\end{tabular}




\begin{tabular}{|c|c|c|c|c|c|c|c|}
\hline 898.52 & 7.34E-01 & $\mathrm{Pa}-234$ & $->$ & $U-234$ & & & \\
\hline 904.33 & $2.85 \mathrm{E}-12$ & $\mathrm{Bi}-214$ & $->$ & Po-214 & & & \\
\hline 904.38 & 8.08E-02 & Pa-234 & $->$ & U-234 & & & \\
\hline 908 & $1.71 \mathrm{E}-14$ & TI-210 & $->$ & $\mathrm{Pb}-210$ & & & \\
\hline 915.8 & $6.24 \mathrm{E}-13$ & $\mathrm{Bi}-214$ & $->$ & Po-214 & & & \\
\hline 920 & $6.47 E-02$ & Pa-234 & $->$ & U-234 & & & \\
\hline 921.7 & $1.58 \mathrm{E}+00$ & $\mathrm{~Pa}-234 \mathrm{~m}$ & $->$ & $U-234$ & & & \\
\hline 924.98 & $1.77 \mathrm{E}+00$ & Рa-234 & $->$ & U-234 & & & \\
\hline 926.61 & $2.39 \mathrm{E}+00$ & $\mathrm{~Pa}-234$ & $->$ & U-234, & $\begin{array}{c}\text { Pa- } \\
234 \mathrm{~m}\end{array}$ & $->$ & $U-234$ \\
\hline 934.039 & $8.58 \mathrm{E}-11$ & $\mathrm{Bi}-214$ & $->$ & Po-214 & & & \\
\hline 936.8 & $2.07 E-01$ & $\mathrm{~Pa}-234 \mathrm{~m}$ & $->$ & U-234 & & & \\
\hline 941.94 & $3.11 \mathrm{E}-01$ & $\mathrm{~Pa}-234 \mathrm{~m}$ & $->$ & $U-234$ & & & \\
\hline 943.3 & $4.62 \mathrm{E}-13$ & $\mathrm{Bi}-214$ & $->$ & Po-214 & & & \\
\hline 945.9 & $4.16 \mathrm{E}+00$ & $\mathrm{~Pa}-234$ & $->$ & U-234, & $\begin{array}{c}\text { Pa- } \\
234 \mathrm{~m}\end{array}$ & $->$ & $U-234$ \\
\hline 948 & $1.29 \mathrm{E}+00$ & $\mathrm{~Pa}-234$ & $->$ & $U-234$ & & & \\
\hline 959.8 & $9.54 \mathrm{E}-02$ & $\mathrm{~Pa}-234 \mathrm{~m}$ & $->$ & U-234 & & & \\
\hline 960 & $1.62 \mathrm{E}-02$ & Pa-234 & $->$ & $U-234$ & & & \\
\hline 964.07 & $1.04 \mathrm{E}-11$ & $\mathrm{Bi}-214$ & $->$ & Po-214 & & & \\
\hline 967.33 & $9.70 \mathrm{E}-02$ & $\mathrm{~Pa}-234$ & $->$ & U-234 & & & \\
\hline 976.2 & $6.24 \mathrm{E}-13$ & $\mathrm{Bi}-214$ & $->$ & Po-214 & & & \\
\hline 978.8 & $1.62 \mathrm{E}-01$ & $\mathrm{~Pa}-234$ & $->$ & U-234 & & & \\
\hline 980.42 & $5.60 \mathrm{E}-01$ & $\mathrm{~Pa}-234$ & $->$ & $U-234$ & & & \\
\hline 984.12 & 3.07E-01 & $\mathrm{Pa}-234$ & $->$ & $U-234$ & & & \\
\hline 989.2 & $3.26 \mathrm{E}-13$ & $\mathrm{Bi}-214$ & $->$ & Po-214 & & & \\
\hline 994.93 & $7.08 \mathrm{E}-01$ & $\mathrm{~Pa}-234 \mathrm{~m}$ & $->$ & U-234 & & & \\
\hline 1000.99 & $1.04 \mathrm{E}+02$ & $\mathrm{~Pa}-234 \mathrm{~m}$ & $->$ & $U-234$ & & & \\
\hline 1013.4 & $2.72 \mathrm{E}-13$ & $\mathrm{Bi}-214$ & $->$ & Po-214 & & & \\
\hline 1020.5 & $3.26 \mathrm{E}-13$ & $\mathrm{Bi}-214$ & $->$ & Po-214 & & & \\
\hline 1022.23 & $9.70 \mathrm{E}-02$ & $\mathrm{~Pa}-234$ & $->$ & U-234 & & & \\
\hline 1029.03 & $1.29 \mathrm{E}-01$ & $\mathrm{~Pa}-234$ & $->$ & U-234 & & & \\
\hline 1032.22 & $2.61 \mathrm{E}-12$ & $\mathrm{Bi}-214$ & $->$ & Po-214 & & & \\
\hline 1038 & $4.62 \mathrm{E}-13$ & $\mathrm{Bi}-214$ & $->$ & Po-214 & & & \\
\hline 1041.7 & $1.49 \mathrm{E}-01$ & $\mathrm{~Pa}-234 \mathrm{~m}$ & $->$ & $U-234$ & & & \\
\hline 1044.9 & 8.08E-02 & $\mathrm{Pa}-234$ & $->$ & U-234 & & & \\
\hline 1045.4 & 7.87E-13 & $\mathrm{Bi}-214$ & $->$ & Po-214 & & & \\
\hline 1051.95 & $8.55 E-12$ & $\mathrm{Bi}-214$ & $->$ & Рo-214 & & & \\
\hline 1059.72 & $1.22 \mathrm{E}-01$ & $\mathrm{~Pa}-234 \mathrm{~m}$ & $->$ & $U-234$ & & & \\
\hline 1061.86 & $2.86 \mathrm{E}-01$ & $\mathrm{~Pa}-234 \mathrm{~m}$ & $->$ & U-234 & & & \\
\hline 1067.3 & $7.87 E-13$ & $\mathrm{Bi}-214$ & $->$ & Po-214 & & & \\
\hline 1068 & $6.84 \mathrm{E}-14$ & TI-210 & $->$ & $\mathrm{Pb}-210$ & & & \\
\hline 1070.02 & $7.74 \mathrm{E}-12$ & $\mathrm{Bi}-214$ & $->$ & Po-214 & & & \\
\hline 1074.6 & 4.04E-02 & $\mathrm{Pa}-234$ & $->$ & $U-234$ & & & \\
\hline 1084.25 & $1.49 \mathrm{E}-01$ & $\mathrm{~Pa}-234$ & $->$ & U-234, & $\begin{array}{r}\mathrm{Pa}- \\
234 \mathrm{~m}\end{array}$ & $->$ & $U-234$ \\
\hline 1085.37 & $5.56 \mathrm{E}-02$ & $\mathrm{~Pa}-234 \mathrm{~m}$ & $->$ & $U-234$ & & & \\
\hline 1103.7 & $2.72 \mathrm{E}-12$ & $\mathrm{Bi}-214$ & $->$ & Po-214 & & & \\
\hline 1104.766 & $2.17 \mathrm{E}-12$ & $\mathrm{Bi}-214$ & $->$ & Po-214 & & & \\
\hline 1108.5 & $4.85 \mathrm{E}-02$ & $\mathrm{~Pa}-234$ & $->$ & $U-234$ & & & \\
\hline
\end{tabular}




\begin{tabular}{|c|c|c|c|c|c|c|c|}
\hline 1110 & $3.93 \mathrm{E}-14$ & TI-210 & $->$ & $\mathrm{Pb}-210$ & & & \\
\hline 1120.273 & $4.07 E-10$ & $\mathrm{Bi}-214$ & $->$ & Po-214 & & & \\
\hline 1120.5 & $1.91 \mathrm{E}-01$ & $\mathrm{~Pa}-234 \mathrm{~m}$ & $->$ & $U-234$ & & & \\
\hline 1122.09 & 8.08E-02 & Pa-234 & $->$ & $U-234$ & & & \\
\hline 1124.93 & $5.22 \mathrm{E}-01$ & $\mathrm{~Pa}-234 \mathrm{~m}$ & $->$ & U-234 & & & \\
\hline 1126.17 & $2.36 \mathrm{E}-01$ & Pa-234 & $->$ & U-234, & $\begin{array}{r}\mathrm{Pa}- \\
234 \mathrm{~m}\end{array}$ & $->$ & $U-234$ \\
\hline 1130.6 & $1.22 \mathrm{E}-12$ & $\mathrm{Bi}-214$ & $->$ & Po-214 & & & \\
\hline 1133.65 & $6.92 \mathrm{E}-12$ & $\mathrm{Bi}-214$ & $->$ & Po-214 & & & \\
\hline 1153.6 & $4.85 E-02$ & $\mathrm{~Pa}-234$ & $->$ & $U-234$ & & & \\
\hline 1155.183 & $4.59 \mathrm{E}-11$ & $\mathrm{Bi}-214$ & $->$ & Рo-214 & & & \\
\hline 1171.3 & $3.88 \mathrm{E}-02$ & $\mathrm{~Pa}-234$ & $->$ & $U-234$ & & & \\
\hline 1172.93 & $1.57 \mathrm{E}-12$ & $\mathrm{Bi}-214$ & $->$ & Po-214 & & & \\
\hline 1173.04 & $1.57 \mathrm{E}-12$ & $\mathrm{Bi}-214$ & $->$ & Рo-214 & & & \\
\hline 1174.1 & $2.13 \mathrm{E}-01$ & $\mathrm{~Pa}-234 \mathrm{~m}$ & $->$ & $U-234$ & & & \\
\hline 1193.69 & $1.68 \mathrm{E}+00$ & $\mathrm{~Pa}-234 \mathrm{~m}$ & $->$ & $U-234$ & & & \\
\hline 1207.764 & $1.25 \mathrm{E}-11$ & $\mathrm{Bi}-214$ & $->$ & Po-214 & & & \\
\hline 1208 & $4.85 \mathrm{E}-02$ & TI-210 & $->$ & $\mathrm{Pb}-210$, & Pa-234 & $->$ & $U-234$ \\
\hline 1216.4 & 5.98E-02 & $\mathrm{Pa}-234$ & $->$ & U-234 & & & \\
\hline 1220.37 & $1.12 \mathrm{E}-01$ & $\mathrm{~Pa}-234 \mathrm{~m}$ & $->$ & $U-234$ & & & \\
\hline 1226.8 & $7.33 \mathrm{E}-13$ & $\mathrm{Bi}-214$ & $->$ & Po-214 & & & \\
\hline 1229 & $4.85 \mathrm{E}-02$ & Pa-234 & $->$ & $U-234$ & & & \\
\hline 1230.84 & $5.97 E-13$ & $\mathrm{Bi}-214$ & $->$ & Po-214 & & & \\
\hline 1237.24 & $6.58 \mathrm{E}-01$ & $\mathrm{~Pa}-234 \mathrm{~m}$ & $->$ & U-234 & & & \\
\hline 1238.107 & $1.61 \mathrm{E}-10$ & $\mathrm{Bi}-214$ & $->$ & Po-214 & & & \\
\hline 1241.4 & 8.08E-02 & $\mathrm{Pa}-234$ & $->$ & U-234 & & & \\
\hline 1251 & 4.85E-02 & $\mathrm{Pa}-234$ & $->$ & $U-234$ & & & \\
\hline 1277.4 & $3.23 E-02$ & $\mathrm{~Pa}-234$ & $->$ & U-234 & & & \\
\hline 1280.952 & $3.99 \mathrm{E}-11$ & $\mathrm{Bi}-214$ & $->$ & Po-214 & & & \\
\hline 1292.66 & $1.12 \mathrm{E}-01$ & $\mathrm{~Pa}-234$ & $->$ & U-234 & & & \\
\hline 1303.76 & $3.29 \mathrm{E}-12$ & $\mathrm{Bi}-214$ & $->$ & Po-214 & & & \\
\hline 1314 & $1.20 \mathrm{E}-13$ & TI-210 & $->$ & $\mathrm{Pb}-210$ & & & \\
\hline 1317.02 & $2.34 \mathrm{E}-12$ & $\mathrm{Bi}-214$ & $->$ & Po-214 & & & \\
\hline 1330 & $2.99 \mathrm{E}-13$ & $\mathrm{Bi}-214$ & $->$ & Po-214 & & & \\
\hline 1341.5 & $6.24 \mathrm{E}-13$ & $\mathrm{Bi}-214$ & $->$ & Po-214 & & & \\
\hline 1352.85 & $1.88 \mathrm{E}-01$ & $\mathrm{~Pa}-234$ & $->$ & $U-234$ & & & \\
\hline 1353 & 4.79E-02 & $\mathrm{Bi}-214$ & $->$ & Po-214, & $\begin{array}{r}\text { Pa- } \\
234 m\end{array}$ & $->$ & $U-234$ \\
\hline 1358.5 & $1.94 \mathrm{E}-02$ & $\mathrm{~Pa}-234$ & $->$ & $U-234$ & & & \\
\hline 1377.659 & $1.09 \mathrm{E}-10$ & $\mathrm{Bi}-214$ & $->$ & Po-214 & & & \\
\hline 1385.295 & $2.12 \mathrm{E}-11$ & $\mathrm{Bi}-214$ & $->$ & Po-214 & & & \\
\hline 1392 & $2.86 \mathrm{E}-01$ & $\mathrm{~Pa}-234 \mathrm{~m}$ & $->$ & U-234 & & & \\
\hline 1392.5 & $5.16 \mathrm{E}-13$ & $\mathrm{Bi}-214$ & $->$ & Po-214 & & & \\
\hline 1393.57 & 4.85E-01 & $\mathrm{Pa}-234$ & $->$ & U-234 & & & \\
\hline 1399.7 & $3.72 \mathrm{E}-02$ & $\mathrm{~Pa}-234$ & $->$ & $U-234$ & & & \\
\hline 1401.48 & $3.77 \mathrm{E}-11$ & $\mathrm{Bi}-214$ & $->$ & Po-214 & & & \\
\hline 1407.97 & $6.73 \mathrm{E}-11$ & $\mathrm{Bi}-214$ & $->$ & Po-214 & & & \\
\hline 1408 & $2.79 \mathrm{E}-14$ & TI-210 & $->$ & $\mathrm{Pb}-210$ & & & \\
\hline 1413.88 & $2.86 \mathrm{E}-01$ & $\mathrm{~Pa}-234 \mathrm{~m}$ & $->$ & $U-234$ & & & \\
\hline 1419.7 & $1.38 \mathrm{E}-13$ & $\mathrm{Bi}-214$ & $->$ & Po-214 & & & \\
\hline
\end{tabular}




\begin{tabular}{|c|c|c|c|c|}
\hline 1427.33 & $3.23 \mathrm{E}-02$ & Pa-234 & $->$ & $U-234$ \\
\hline 1434.13 & $1.20 \mathrm{E}+00$ & $\mathrm{~Pa}-234 \mathrm{~m}$ & $->$ & $U-234$ \\
\hline 1445.7 & $6.47 \mathrm{E}-02$ & Pa-234 & $->$ & $U-234$ \\
\hline 1452.63 & $1.49 \mathrm{E}-01$ & $\mathrm{~Pa}-234$ & $->$ & $U-234$ \\
\hline 1457.4 & $2.07 \mathrm{E}-01$ & $\mathrm{~Pa}-234 \mathrm{~m}$ & $->$ & $U-234$ \\
\hline 1460 & $4.85 \mathrm{E}-02$ & $\mathrm{~Pa}-234$ & $->$ & U-234 \\
\hline 1471.1 & $3.26 \mathrm{E}-13$ & $\mathrm{Bi}-214$ & $->$ & Po-214 \\
\hline 1479.19 & $1.87 \mathrm{E}-12$ & $\mathrm{Bi}-214$ & $->$ & Po-214 \\
\hline 1493.94 & $3.23 E-02$ & $\mathrm{~Pa}-234$ & $->$ & U-234 \\
\hline 1500.9 & $1.43 \mathrm{E}-01$ & $\mathrm{~Pa}-234 \mathrm{~m}$ & $->$ & $\mathrm{U}-234$ \\
\hline 1509.22 & $5.95 \mathrm{E}-11$ & $\mathrm{Bi}-214$ & $->$ & Po-214 \\
\hline 1510.2 & $1.60 \mathrm{E}+00$ & $\mathrm{~Pa}-234 \mathrm{~m}$ & $->$ & U-234 \\
\hline 1516 & $6.47 \mathrm{E}-02$ & Pa-234 & $->$ & $U-234$ \\
\hline 1527.27 & $2.98 \mathrm{E}-01$ & $\mathrm{~Pa}-234 \mathrm{~m}$ & $->$ & $U-234$ \\
\hline 1538 & $1.14 \mathrm{E}-14$ & TI-210 & $->$ & $\mathrm{Pb}-210$ \\
\hline 1538.49 & $1.11 \mathrm{E}-11$ & $\mathrm{Bi}-214$ & $->$ & Po-214 \\
\hline 1543.347 & $9.50 \mathrm{E}-12$ & $\mathrm{Bi}-214$ & $->$ & Po-214 \\
\hline 1548.12 & $1.74 \mathrm{E}-01$ & $\mathrm{~Pa}-234 \mathrm{~m}$ & $->$ & $U-234$ \\
\hline 1549.4 & $1.62 \mathrm{E}-02$ & $\mathrm{~Pa}-234$ & $->$ & $U-234$ \\
\hline 1553.74 & $1.01 E+00$ & Pa-234m & $->$ & $U-234$ \\
\hline 1558.7 & 8.58E-02 & $\mathrm{Pa}-234 \mathrm{~m}$ & $->$ & $U-234$ \\
\hline 1570.67 & $1.37 \mathrm{E}-01$ & $\mathrm{~Pa}-234 \mathrm{~m}$ & $->$ & $U-234$ \\
\hline 1580.02 & $2.75 \mathrm{E}-02$ & $\mathrm{~Pa}-234$ & $->$ & $\mathrm{U}-234$ \\
\hline 1583.22 & $1.95 \mathrm{E}-11$ & $\mathrm{Bi}-214$ & $->$ & Po-214 \\
\hline 1585.3 & $4.04 \mathrm{E}-02$ & $\mathrm{~Pa}-234$ & $->$ & U-234 \\
\hline 1588 & $1.14 \mathrm{E}-14$ & TI-210 & $->$ & $\mathrm{Pb}-210$ \\
\hline 1591.65 & $2.36 \mathrm{E}-01$ & $\mathrm{~Pa}-234 \mathrm{~m}$ & $->$ & $\mathrm{U}-234$ \\
\hline 1593.88 & $3.36 \mathrm{E}-01$ & Pa-234 & $->$ & U-234 \\
\hline 1594.78 & $7.20 \mathrm{E}-12$ & $\mathrm{Bi}-214$ & $->$ & Po-214 \\
\hline 1599.3 & $9.07 \mathrm{E}-12$ & $\mathrm{Bi}-214$ & $->$ & Po-214 \\
\hline 1602.2 & $4.61 \mathrm{E}-02$ & $\mathrm{~Pa}-234 \mathrm{~m}$ & $->$ & $U-234$ \\
\hline 1627.9 & $2.43 \mathrm{E}-02$ & $\mathrm{~Pa}-234$ & $->$ & $U-234$ \\
\hline 1636.6 & $5.16 \mathrm{E}-13$ & $\mathrm{Bi}-214$ & $->$ & Po-214 \\
\hline 1637.37 & $1.90 \mathrm{E}-12$ & $\mathrm{Bi}-214$ & $->$ & Po-214 \\
\hline 1638 & $6.47 \mathrm{E}-02$ & Pa-234 & $->$ & $U-234$ \\
\hline 1648 & $1.14 \mathrm{E}-14$ & TI-210 & $->$ & $\mathrm{Pb}-210$ \\
\hline 1656 & $2.43 \mathrm{E}-02$ & $\mathrm{~Pa}-234$ & $->$ & U-234 \\
\hline 1661.258 & $3.12 \mathrm{E}-11$ & $\mathrm{Bi}-214$ & $->$ & Po-214 \\
\hline 1667.3 & $9.22 \mathrm{E}-02$ & Pa-234m & $->$ & U-234 \\
\hline 1668.44 & $1.49 \mathrm{E}-01$ & Pa-234 & $->$ & $\mathrm{U}-234$ \\
\hline 1683.99 & $6.41 \mathrm{E}-12$ & $\mathrm{Bi}-214$ & $->$ & Po-214 \\
\hline 1686.2 & $8.08 \mathrm{E}-02$ & Pa-234 & $->$ & U-234 \\
\hline 1693.7 & $5.08 \mathrm{E}-02$ & $\mathrm{~Pa}-234 \mathrm{~m}$ & $->$ & $U-234$ \\
\hline 1694.08 & $1.62 \mathrm{E}-01$ & Pa-234 & $->$ & $U-234$ \\
\hline 1699.8 & $2.43 \mathrm{E}-02$ & $\mathrm{~Pa}-234$ & $->$ & $U-234$ \\
\hline 1719.5 & $3.23 E-03$ & Pa-234 & $->$ & $U-234$ \\
\hline 1720.5 & $3.65 \mathrm{E}-02$ & $\mathrm{~Pa}-234 \mathrm{~m}$ & $->$ & U-234 \\
\hline 1729.58 & 8.28E-11 & $\mathrm{Bi}-214$ & $->$ & Po-214 \\
\hline 1732.2 & $2.07 \mathrm{E}-01$ & $\mathrm{~Pa}-234 \mathrm{~m}$ & $->$ & U-234 \\
\hline 1737.73 & $2.63 E+00$ & $\mathrm{~Pa}-234 \mathrm{~m}$ & $->$ & $\mathrm{U}-234$ \\
\hline
\end{tabular}




\begin{tabular}{|c|c|c|c|c|}
\hline 1738 & $1.62 \mathrm{E}-02$ & $\mathrm{~Pa}-234$ & $->$ & $U-234$ \\
\hline 1741.7 & $1.62 \mathrm{E}-02$ & $\mathrm{~Pa}-234$ & $->$ & $U-234$ \\
\hline 1750.1 & 8.08E-03 & $\mathrm{Pa}-234$ & $->$ & $U-234$ \\
\hline 1756 & $4.04 \mathrm{E}-02$ & $\mathrm{~Pa}-234$ & $->$ & $U-234$ \\
\hline 1759.81 & $1.74 \mathrm{E}-01$ & Pa-234m & $->$ & $U-234$ \\
\hline 1764.49 & $4.32 \mathrm{E}-10$ & $\mathrm{Bi}-214$ & $->$ & Po-214 \\
\hline 1765.44 & $1.08 \mathrm{E}+00$ & $\mathrm{~Pa}-234 \mathrm{~m}$ & $->$ & $U-234$ \\
\hline 1768 & $9.70 \mathrm{E}-03$ & Pa-234 & $->$ & $U-234$ \\
\hline 1772.3 & $1.62 \mathrm{E}-02$ & $\mathrm{~Pa}-234$ & $->$ & $U-234$ \\
\hline 1782.1 & $4.34 \mathrm{E}-13$ & $\mathrm{Bi}-214$ & $->$ & Po-214 \\
\hline 1796.2 & $3.49 \mathrm{E}-02$ & $\mathrm{~Pa}-234 \mathrm{~m}$ & $->$ & U-234 \\
\hline 1797.3 & $4.85 \mathrm{E}-02$ & Pa-234 & $->$ & $U-234$ \\
\hline 1809.04 & $4.60 \mathrm{E}-01$ & $\mathrm{~Pa}-234 \mathrm{~m}$ & $->$ & $U-234$ \\
\hline 1814.01 & $3.26 \mathrm{E}-13$ & $\mathrm{Bi}-214$ & $->$ & Po-214 \\
\hline 1819.69 & $1.12 \mathrm{E}-01$ & $\mathrm{~Pa}-234 \mathrm{~m}$ & $->$ & $U-234$ \\
\hline 1828 & $4.85 E-03$ & $\mathrm{~Pa}-234$ & $->$ & $U-234$ \\
\hline 1831.36 & $2.14 \mathrm{E}+00$ & $\mathrm{~Pa}-234 \mathrm{~m}$ & $->$ & $U-234$ \\
\hline 1837.9 & $9.70 \mathrm{E}-03$ & $\mathrm{~Pa}-234$ & $->$ & $U-234$ \\
\hline 1838.37 & $1.04 \mathrm{E}-11$ & $\mathrm{Bi}-214$ & $->$ & Po-214 \\
\hline 1847.41 & $5.76 \mathrm{E}-11$ & $\mathrm{Bi}-214$ & $->$ & Po-214 \\
\hline 1850 & 8.08E-03 & $\mathrm{Pa}-234$ & $->$ & U-234 \\
\hline 1863.09 & $1.49 \mathrm{E}-01$ & $\mathrm{~Pa}-234 \mathrm{~m}$ & $->$ & $U-234$ \\
\hline 1867.68 & $1.14 \mathrm{E}+00$ & $\mathrm{~Pa}-234 \mathrm{~m}$ & $->$ & $U-234$ \\
\hline 1872.8 & $1.13 \mathrm{E}-02$ & Pa-234 & $->$ & $\mathrm{U}-234$ \\
\hline 1873.112 & $6.14 \mathrm{E}-12$ & $\mathrm{Bi}-214$ & $->$ & Po-214 \\
\hline 1874.85 & $1.02 \mathrm{E}+00$ & $\mathrm{~Pa}-234 \mathrm{~m}$ & $->$ & U-234 \\
\hline 1877.21 & $2.05 \mathrm{E}-01$ & $\mathrm{~Pa}-234 \mathrm{~m}$ & $->$ & $U-234$ \\
\hline 1890.1 & 3.07E-02 & $\mathrm{Pa}-234$ & $->$ & $U-234$ \\
\hline 1890.259 & $2.42 \mathrm{E}-12$ & $\mathrm{Bi}-214$ & $->$ & Po-214 \\
\hline 1893.5 & $2.73 \mathrm{E}-01$ & $\mathrm{~Pa}-234 \mathrm{~m}$ & $->$ & U-234 \\
\hline 1896.28 & $4.81 \mathrm{E}-12$ & $\mathrm{Bi}-214$ & $->$ & Po-214 \\
\hline 1897.1 & $2.43 \mathrm{E}-02$ & $\mathrm{~Pa}-234$ & $->$ & $U-234$ \\
\hline 1898.9 & $1.71 \mathrm{E}-12$ & $\mathrm{Bi}-214$ & $->$ & Po-214 \\
\hline 1905 & 4.53E-02 & $\mathrm{Pa}-234$ & $->$ & U-234 \\
\hline 1911.17 & 7.83E-01 & $\mathrm{Pa}-234 \mathrm{~m}$ & $->$ & $U-234$ \\
\hline 1926 & $3.86 \mathrm{E}-02$ & Pa-234 & $->$ & $U-234$ \\
\hline 1926.4 & $2.36 \mathrm{E}-02$ & $\mathrm{~Pa}-234 \mathrm{~m}$ & $->$ & U-234 \\
\hline 1935.8 & $1.38 \mathrm{E}-12$ & $\mathrm{Bi}-214$ & $->$ & Po-214 \\
\hline 1937.01 & $3.60 \mathrm{E}-01$ & $\mathrm{~Pa}-234 \mathrm{~m}$ & $->$ & U-234 \\
\hline 1937.7 & $8.08 \mathrm{E}-03$ & $\mathrm{~Pa}-234$ & $->$ & $U-234$ \\
\hline 1959 & $1.62 \mathrm{E}-04$ & $\mathrm{~Pa}-234$ & $->$ & $U-234$ \\
\hline 1969.9 & $6.20 \mathrm{E}-02$ & $\mathrm{~Pa}-234 \mathrm{~m}$ & $->$ & $U-234$ \\
\hline 1988 & $3.23 E-04$ & $\mathrm{~Pa}-234$ & $->$ & U-234 \\
\hline 1994.7 & $1.36 \mathrm{E}-13$ & $\mathrm{Bi}-214$ & $->$ & Po-214 \\
\hline 1998 & $1.62 \mathrm{E}-04$ & $\mathrm{~Pa}-234$ & $->$ & U-234 \\
\hline 2004.5 & 7.87E-14 & $\mathrm{Bi}-214$ & $->$ & Po-214 \\
\hline 2008 & $3.93 \mathrm{E}-14$ & $\mathrm{TI}-210$ & $->$ & $\mathrm{Pb}-210$ \\
\hline 2010.79 & $1.33 \mathrm{E}-12$ & $\mathrm{Bi}-214$ & $->$ & Po-214 \\
\hline 2021.7 & $5.16 \mathrm{E}-13$ & $\mathrm{Bi}-214$ & $->$ & Po-214 \\
\hline 2052.93 & $1.90 \mathrm{E}-12$ & $\mathrm{Bi}-214$ & $->$ & Po-214 \\
\hline
\end{tabular}




\begin{tabular}{|c|c|c|c|c|}
\hline 2085 & $2.72 \mathrm{E}-13$ & $\mathrm{Bi}-214$ & $->$ & Po-214 \\
\hline 2088 & $2.79 \mathrm{E}-14$ & TI-210 & $->$ & $\mathrm{Pb}-210$ \\
\hline 2089.55 & $1.52 \mathrm{E}-12$ & $\mathrm{Bi}-214$ & $->$ & Po-214 \\
\hline 2109.91 & $2.36 \mathrm{E}-12$ & $\mathrm{Bi}-214$ & $->$ & Po-214 \\
\hline 2118.53 & $3.29 \mathrm{E}-11$ & $\mathrm{Bi}-214$ & $->$ & Po-214 \\
\hline 2147.8 & 4.34E-13 & $\mathrm{Bi}-214$ & $->$ & Po-214 \\
\hline 2192.52 & $1.66 \mathrm{E}-12$ & $\mathrm{Bi}-214$ & $->$ & Po-214 \\
\hline 2204.09 & $1.35 \mathrm{E}-10$ & $\mathrm{Bi}-214$ & $->$ & Po-214 \\
\hline 2251.2 & $1.90 \mathrm{E}-13$ & $\mathrm{Bi}-214$ & $->$ & Po-214 \\
\hline 2259.7 & $2.44 \mathrm{E}-13$ & $\mathrm{Bi}-214$ & $->$ & Po-214 \\
\hline 2266.67 & $4.89 \mathrm{E}-13$ & $\mathrm{Bi}-214$ & $->$ & Po-214 \\
\hline 2268 & $1.71 \mathrm{E}-14$ & TI-210 & $->$ & $\mathrm{Pb}-210$ \\
\hline 2270 & 7.87E-14 & $\mathrm{Bi}-214$ & $->$ & Po-214 \\
\hline 2284.4 & $1.38 \mathrm{E}-13$ & $\mathrm{Bi}-214$ & $->$ & Po-214 \\
\hline 2293.29 & $8.80 \mathrm{E}-12$ & $\mathrm{Bi}-214$ & $->$ & Po-214 \\
\hline 2324.8 & $5.16 \mathrm{E}-14$ & $\mathrm{Bi}-214$ & $->$ & Po-214 \\
\hline 2331.2 & $5.97 \mathrm{E}-13$ & $\mathrm{Bi}-214$ & $->$ & Po-214 \\
\hline 2358 & $4.56 \mathrm{E}-14$ & TI-210 & $->$ & $\mathrm{Pb}-210$ \\
\hline 2360.9 & $5.16 \mathrm{E}-14$ & $\mathrm{Bi}-214$ & $->$ & Po-214 \\
\hline 2369.3 & 7.87E-14 & $\mathrm{Bi}-214$ & $->$ & Po-214 \\
\hline 2376.99 & $3.20 \mathrm{E}-13$ & $\mathrm{Bi}-214$ & $->$ & Po-214 \\
\hline 2390.9 & $5.43 E-14$ & $\mathrm{Bi}-214$ & $->$ & Po-214 \\
\hline 2423.32 & $1.60 \mathrm{E}-13$ & $\mathrm{Bi}-214$ & $->$ & Po-214 \\
\hline 2428 & $5.13 \mathrm{E}-14$ & TI-210 & $->$ & $\mathrm{Pb}-210$ \\
\hline 2447.68 & $4.21 \mathrm{E}-11$ & $\mathrm{Bi}-214$ & $->$ & Po-214 \\
\hline 2482.417 & $5.70 \mathrm{E}-14$ & $\mathrm{Bi}-214$ & $->$ & Po-214 \\
\hline 2505.58 & $1.60 \mathrm{E}-13$ & $\mathrm{Bi}-214$ & $->$ & Po-214 \\
\hline 2551 & $1.06 \mathrm{E}-14$ & $\mathrm{Bi}-214$ & $->$ & Po-214 \\
\hline 2604.5 & $1.22 \mathrm{E}-14$ & $\mathrm{Bi}-214$ & $->$ & Po-214 \\
\hline 2631 & $2.44 \mathrm{E}-14$ & $\mathrm{Bi}-214$ & $->$ & Po-214 \\
\hline 2662.23 & 7.87E-15 & $\mathrm{Bi}-214$ & $->$ & Po-214 \\
\hline 2694.67 & $8.80 \mathrm{E}-13$ & $\mathrm{Bi}-214$ & $->$ & Po-214 \\
\hline 2698.86 & $7.60 \mathrm{E}-14$ & $\mathrm{Bi}-214$ & $->$ & Po-214 \\
\hline 2719.21 & $4.89 E-14$ & $\mathrm{Bi}-214$ & $->$ & Po-214 \\
\hline 2769.99 & $6.95 E-13$ & $\mathrm{Bi}-214$ & $->$ & Po-214 \\
\hline 2786.09 & $1.60 \mathrm{E}-13$ & $\mathrm{Bi}-214$ & $->$ & Po-214 \\
\hline 2827 & $6.79 E-14$ & $\mathrm{Bi}-214$ & $->$ & Po-214 \\
\hline 2860.9 & $9.23 \mathrm{E}-15$ & $\mathrm{Bi}-214$ & $->$ & Po-214 \\
\hline 2880.4 & $2.50 \mathrm{E}-13$ & $\mathrm{Bi}-214$ & $->$ & Po-214 \\
\hline 2893.59 & $1.74 \mathrm{E}-13$ & $\mathrm{Bi}-214$ & $->$ & Po-214 \\
\hline 2922.09 & $4.26 \mathrm{E}-13$ & $\mathrm{Bi}-214$ & $->$ & Po-214 \\
\hline 2928.7 & $3.26 \mathrm{E}-14$ & $\mathrm{Bi}-214$ & $->$ & Po-214 \\
\hline 2934.9 & $1.55 \mathrm{E}-14$ & $\mathrm{Bi}-214$ & $->$ & Po-214 \\
\hline 2940.5 & $4.62 \mathrm{E}-14$ & $\mathrm{Bi}-214$ & $->$ & Po-214 \\
\hline 2978.79 & $3.99 E-13$ & $\mathrm{Bi}-214$ & $->$ & Po-214 \\
\hline 2988.7 & $2.99 \mathrm{E}-14$ & $\mathrm{Bi}-214$ & $->$ & Po-214 \\
\hline 2999.99 & $2.39 \mathrm{E}-13$ & $\mathrm{Bi}-214$ & $->$ & Po-214 \\
\hline 3053.89 & $6.14 \mathrm{E}-13$ & $\mathrm{Bi}-214$ & $->$ & Po-214 \\
\hline 3081.7 & $1.17 \mathrm{E}-13$ & $\mathrm{Bi}-214$ & $->$ & Po-214 \\
\hline 3093.9 & $1.38 \mathrm{E}-14$ & $\mathrm{Bi}-214$ & $->$ & Po-214 \\
\hline
\end{tabular}




$\begin{array}{lllll}3136.3 & 9.23 \mathrm{E}-15 & \mathrm{Bi}-214 & \rightarrow & \mathrm{Po}-214 \\ 3142.6 & 4.34 \mathrm{E}-14 & \mathrm{Bi}-214 & \rightarrow & \mathrm{Po}-214 \\ 3160.5 & 1.38 \mathrm{E}-14 & \mathrm{Bi}-214 & \rightarrow & \mathrm{Po}-214 \\ 3183.6 & 4.07 \mathrm{E}-14 & \mathrm{Bi}-214 & \rightarrow & \mathrm{Po}-214 \\ 3233.3 & 5.43 \mathrm{E}-15 & \mathrm{Bi}-214 & \rightarrow & \mathrm{Po}-214 \\ 3269.7 & 2.72 \mathrm{E}-15 & \mathrm{Bi}-214 & \rightarrow & \mathrm{Po}-214\end{array}$

\begin{tabular}{|c|c|c|c|c|c|c|c|}
\hline U238 & $\begin{array}{c}\text { Aged } 5 \\
\text { years }\end{array}$ & \multicolumn{6}{|c|}{ Sorted by Intensity } \\
\hline Intensity & Energy & Initial Isotop & es & nd Decays & & & \\
\hline pn/s/gm & kev & & & & & & \\
\hline $5.47 \mathrm{E}+02$ & 16.572 & Th-234 & $->$ & $\mathrm{Pa}-234 \mathrm{~m}$ & & & \\
\hline $5.10 \mathrm{E}+02$ & 16.161 & $U-238$ & $->$ & Th-234 & & & \\
\hline $4.85 E+02$ & 13.274 & Th-234 & $->$ & $\mathrm{Pa}-234 \mathrm{~m}$ & & & \\
\hline $4.48 \mathrm{E}+02$ & 63.288 & Th-234 & $->$ & $\mathrm{Pa}-234 \mathrm{~m}$ & & & \\
\hline $3.73 E+02$ & 12.952 & $U-238$ & $->$ & Th-234 & & & \\
\hline $3.38 \mathrm{E}+02$ & 92.35 & Th-234 & $->$ & $\mathrm{Pa}-234 \mathrm{~m}$ & & & \\
\hline $3.35 E+02$ & 92.78 & Th-234 & $->$ & $\mathrm{Pa}-234 \mathrm{~m}$ & & & \\
\hline $1.34 \mathrm{E}+02$ & 19.839 & Th-234 & $->$ & $\mathrm{Pa}-234 \mathrm{~m}$ & & & \\
\hline $1.16 \mathrm{E}+02$ & 19.094 & U-238 & $->$ & Th-234 & & & \\
\hline $1.04 \mathrm{E}+02$ & 1000.99 & $\mathrm{~Pa}-234 \mathrm{~m}$ & $->$ & $U-234$ & & & \\
\hline $4.00 \mathrm{E}+01$ & 766.412 & $\mathrm{~Pa}-234$ & $->$ & U-234, & $\begin{array}{r}\text { Pa- } \\
234 m\end{array}$ & $->$ & $U-234$ \\
\hline $3.43 E+01$ & 98.434 & $\mathrm{~Pa}-234$ & $->$ & U-234, & $\begin{array}{r}\text { Pa- } \\
234 m\end{array}$ & $->$ & $U-234$ \\
\hline $3.01 E+01$ & 112.8 & Th-234 & $->$ & $\mathrm{Pa}-234 \mathrm{~m}$ & & & \\
\hline $2.88 \mathrm{E}+01$ & 11.372 & Th-234 & $->$ & Pa-234m, & $\begin{array}{r}\mathrm{Pa}- \\
234 \mathrm{~m}\end{array}$ & $->$ & $U-234$ \\
\hline $2.27 E+01$ & 13.6 & $\mathrm{~Pa}-234$ & $->$ & U-234, & $\begin{array}{l}\text { Pa- } \\
234 m\end{array}$ & $->$ & $U-234$ \\
\hline $2.24 \mathrm{E}+01$ & 11.118 & U-238 & $->$ & Th-234 & & & \\
\hline $2.16 \mathrm{E}+01$ & 94.651 & $\mathrm{~Pa}-234$ & $->$ & U-234, & $\begin{array}{r}\text { Pa- } \\
234 m\end{array}$ & $->$ & $U-234$ \\
\hline $1.46 \mathrm{E}+01$ & 17.069 & $\mathrm{~Pa}-234 \mathrm{~m}$ & $->$ & U-234 & & & \\
\hline $1.26 \mathrm{E}+01$ & 111.025 & Pa-234 & $->$ & U-234, & $\begin{array}{r}\mathrm{Pa}- \\
234 \mathrm{~m}\end{array}$ & $->$ & $U-234$ \\
\hline $1.18 \mathrm{E}+01$ & 742.817 & $\mathrm{~Pa}-234$ & $->$ & U-234, & $\begin{array}{r}\mathrm{Pa}- \\
234 \mathrm{~m}\end{array}$ & $->$ & $U-234$ \\
\hline $1.03 \mathrm{E}+01$ & 14.511 & U-238 & $->$ & Th-234 & & & \\
\hline $9.07 E+00$ & 258.26 & $\mathrm{~Pa}-234 \mathrm{~m}$ & $->$ & $U-234$ & & & \\
\hline $8.71 E+00$ & 83.31 & Th-234 & $->$ & $\mathrm{Pa}-234 \mathrm{~m}$ & & & \\
\hline $8.71 \mathrm{E}+00$ & 49.55 & $U-238$ & $->$ & Th-234 & & & \\
\hline $8.41 E+00$ & 17.12 & $\mathrm{~Pa}-234$ & $->$ & $U-234$ & & & \\
\hline $6.88 \mathrm{E}+00$ & 786.287 & $\mathrm{~Pa}-234$ & $->$ & U-234, & $\begin{array}{r}\text { Pa- } \\
234 m\end{array}$ & $->$ & $U-234$ \\
\hline $6.12 E+00$ & 14.953 & Th-234 & $->$ & $\mathrm{Pa}-234 \mathrm{~m}$, & $\begin{array}{r}\mathrm{Pa}- \\
234 \mathrm{~m}\end{array}$ & $->$ & $U-234$ \\
\hline $5.30 E+00$ & 74 & Th-234 & $->$ & $\mathrm{Pa}-234 \mathrm{~m}$ & & & \\
\hline $4.32 \mathrm{E}+00$ & 114.866 & $\mathrm{~Pa}-234$ & $->$ & U-234, & $\mathrm{Pa}-$ & $->$ & $U-234$ \\
\hline
\end{tabular}




\begin{tabular}{|c|c|c|c|c|c|c|c|}
\hline $4.16 \mathrm{E}+00$ & 945.9 & Pa-234 & $->$ & U-234, & $\begin{array}{r}\mathrm{Pa}- \\
234 \mathrm{~m}\end{array}$ & $->$ & U-234 \\
\hline $3.56 \mathrm{E}+00$ & 131.31 & $\mathrm{~Pa}-234$ & $->$ & $U-234$ & & & \\
\hline $3.34 \mathrm{E}+00$ & 16.547 & $\mathrm{~Pa}-234 \mathrm{~m}$ & $->$ & U-234 & & & \\
\hline $3.18 \mathrm{E}+00$ & 20.366 & $\mathrm{~Pa}-234 \mathrm{~m}$ & $->$ & $U-234$ & & & \\
\hline $2.99 \mathrm{E}+00$ & 110.5 & U-238 & $->$ & Th-234 & & & \\
\hline $2.88 \mathrm{E}+00$ & 13.275 & $\mathrm{~Pa}-234 \mathrm{~m}$ & $->$ & $U-234$ & & & \\
\hline $2.64 \mathrm{E}+00$ & 880.45 & $\mathrm{~Pa}-234$ & $->$ & U-234 & & & \\
\hline $2.63 E+00$ & 1737.73 & $\mathrm{~Pa}-234 \mathrm{~m}$ & $->$ & U-234 & & & \\
\hline $2.62 \mathrm{E}+00$ & 883.22 & Pa-234 & $->$ & U-234, & $\begin{array}{l}\mathrm{Pa}- \\
234 \mathrm{~m}\end{array}$ & $->$ & U-234 \\
\hline $2.52 E+00$ & 569.3 & $\mathrm{~Pa}-234$ & $->$ & U-234 & & & \\
\hline $2.39 \mathrm{E}+00$ & 926.61 & $\mathrm{~Pa}-234$ & $->$ & U-234, & $\begin{array}{l}\text { Pa- } \\
234 \mathrm{~m}\end{array}$ & $->$ & $U-234$ \\
\hline $2.36 \mathrm{E}+00$ & 62.862 & Th-234 & $->$ & $\mathrm{Pa}-234 \mathrm{~m}$ & & & \\
\hline $2.14 \mathrm{E}+00$ & 1831.36 & $\mathrm{~Pa}-234 \mathrm{~m}$ & $->$ & $\mathrm{U}-234$ & & & \\
\hline $2.01 E+00$ & 226.95 & $\mathrm{~Pa}-234$ & $->$ & $U-234$ & & & \\
\hline $1.91 \mathrm{E}+00$ & 20.309 & Pa-234 & $->$ & $U-234$ & & & \\
\hline $1.77 \mathrm{E}+00$ & 924.98 & $\mathrm{~Pa}-234$ & $->$ & $U-234$ & & & \\
\hline $1.68 \mathrm{E}+00$ & 1193.69 & $\mathrm{~Pa}-234 \mathrm{~m}$ & $->$ & $\mathrm{U}-234$ & & & \\
\hline $1.62 \mathrm{E}+00$ & 95.863 & Th-234 & $->$ & $\mathrm{Pa}-234 \mathrm{~m}$ & & & \\
\hline $1.60 \mathrm{E}+00$ & 1510.2 & $\mathrm{~Pa}-234 \mathrm{~m}$ & $->$ & $U-234$ & & & \\
\hline $1.58 \mathrm{E}+00$ & 921.7 & $\mathrm{~Pa}-234 \mathrm{~m}$ & $->$ & $U-234$ & & & \\
\hline $1.49 \mathrm{E}+00$ & 184.8 & Th-234 & $->$ & $\mathrm{Pa}-234 \mathrm{~m}$ & & & \\
\hline $1.47 \mathrm{E}+00$ & 739.95 & $\mathrm{~Pa}-234 \mathrm{~m}$ & $->$ & $U-234$ & & & \\
\hline $1.43 \mathrm{E}+00$ & 733.38 & $\mathrm{~Pa}-234$ & $->$ & $U-234$ & & & \\
\hline $1.39 \mathrm{E}+00$ & 11.62 & Pa-234 & $->$ & U-234, & $\begin{array}{l}\text { Pa- } \\
234 \mathrm{~m}\end{array}$ & $->$ & U-234 \\
\hline $1.29 \mathrm{E}+00$ & 948 & $\mathrm{~Pa}-234$ & $->$ & $U-234$ & & & \\
\hline $1.24 \mathrm{E}+00$ & 20.019 & Th-234 & $->$ & $\mathrm{Pa}-234 \mathrm{~m}$ & & & \\
\hline $1.20 \mathrm{E}+00$ & 1434.13 & $\mathrm{~Pa}-234 \mathrm{~m}$ & $->$ & $U-234$ & & & \\
\hline $1.14 \mathrm{E}+00$ & 1867.68 & $\mathrm{~Pa}-234 \mathrm{~m}$ & $->$ & U-234 & & & \\
\hline $1.12 \mathrm{E}+00$ & 691.08 & $\mathrm{~Pa}-234 \mathrm{~m}$ & $->$ & U-234 & & & \\
\hline $1.09 \mathrm{E}+00$ & 805.74 & $\mathrm{~Pa}-234$ & $->$ & U-234, & $\begin{array}{r}\mathrm{Pa}- \\
234 \mathrm{~m}\end{array}$ & $->$ & $U-234$ \\
\hline $1.08 \mathrm{E}+00$ & 1765.44 & $\mathrm{~Pa}-234 \mathrm{~m}$ & $->$ & $U-234$ & & & \\
\hline $1.03 \mathrm{E}+00$ & 152.76 & Pa-234 & $->$ & U-234 & & & \\
\hline $1.02 \mathrm{E}+00$ & 1874.85 & $\mathrm{~Pa}-234 \mathrm{~m}$ & $->$ & $U-234$ & & & \\
\hline $1.01 \mathrm{E}+00$ & 1553.74 & $\mathrm{~Pa}-234 \mathrm{~m}$ & $->$ & U-234 & & & \\
\hline $9.95 \mathrm{E}-01$ & 92.279 & Th-234 & $->$ & $\mathrm{Pa}-234 \mathrm{~m}$ & & & \\
\hline $9.70 \mathrm{E}-01$ & 831.39 & Pa-234 & $->$ & $\mathrm{U}-234$ & & & \\
\hline $9.62 \mathrm{E}-01$ & 62.78 & $\mathrm{~Pa}-234$ & $->$ & U-234, & $\begin{array}{r}\mathrm{Pa}- \\
234 \mathrm{~m}\end{array}$ & $->$ & U-234 \\
\hline $9.08 \mathrm{E}-01$ & 87.02 & Th-234 & $->$ & Pa-234m & & & \\
\hline 8.82E-01 & 702.05 & $\mathrm{~Pa}-234 \mathrm{~m}$ & $->$ & U-234 & & & \\
\hline $8.82 \mathrm{E}-01$ & 887.28 & $\mathrm{~Pa}-234 \mathrm{~m}$ & $->$ & U-234 & & & \\
\hline $8.70 \mathrm{E}-01$ & 851.57 & Pa-234 & $->$ & U-234, & $\begin{array}{r}\mathrm{Pa}- \\
234 \mathrm{~m}\end{array}$ & $->$ & U-234 \\
\hline $8.46 \mathrm{E}-01$ & 824.94 & $\mathrm{~Pa}-234$ & $->$ & $U-234$ & & & \\
\hline
\end{tabular}




\begin{tabular}{|c|c|c|c|c|c|c|c|}
\hline $8.42 \mathrm{E}-01$ & 782.8 & $\mathrm{~Pa}-234 \mathrm{~m}$ & $->$ & U-234 & & & \\
\hline 8.35E-01 & 99.852 & $\mathrm{~Pa}-234$ & $->$ & U-234, & $\begin{array}{r}\mathrm{Pa}- \\
234 \mathrm{~m}\end{array}$ & $->$ & U-234 \\
\hline 8.08E-01 & 705.9 & Pa-234 & $->$ & U-234, & $\begin{array}{r}\mathrm{Pa}- \\
234 \mathrm{~m}\end{array}$ & $->$ & $U-234$ \\
\hline 7.83E-01 & 1911.17 & $\mathrm{~Pa}-234 \mathrm{~m}$ & $->$ & $U-234$ & & & \\
\hline 7.46E-01 & 103.71 & Th-234 & $->$ & $\mathrm{Pa}-234 \mathrm{~m}$ & & & \\
\hline 7.34E-01 & 108 & Th-234 & $->$ & $\mathrm{Pa}-234 \mathrm{~m}$ & & & \\
\hline 7.34E-01 & 898.52 & $\mathrm{~Pa}-234$ & $->$ & $U-234$ & & & \\
\hline 7.08E-01 & 994.93 & $\mathrm{~Pa}-234 \mathrm{~m}$ & $->$ & U-234 & & & \\
\hline $6.72 \mathrm{E}-01$ & 796.42 & Рa-234 & $->$ & U-234 & & & \\
\hline $6.58 \mathrm{E}-01$ & 1237.24 & $\mathrm{~Pa}-234 \mathrm{~m}$ & $->$ & U-234 & & & \\
\hline $6.55 \mathrm{E}-01$ & 698.85 & Pa-234 & $->$ & $U-234$ & & & \\
\hline $6.22 \mathrm{E}-01$ & 108.166 & Th-234 & $->$ & $\mathrm{Pa}-234 \mathrm{~m}$ & & & \\
\hline $6.22 \mathrm{E}-01$ & 57.75 & Th-234 & $->$ & Pa-234m & & & \\
\hline 6.09E-01 & 293.874 & $\mathrm{~Pa}-234$ & $->$ & $U-234$ & & & \\
\hline $5.60 \mathrm{E}-01$ & 980.42 & Рa-234 & $->$ & U-234 & & & \\
\hline $5.56 \mathrm{E}-01$ & 243.5 & $\mathrm{~Pa}-234 \mathrm{~m}$ & $->$ & U-234 & & & \\
\hline 5.47E-01 & 369.52 & Pa-234 & $->$ & U-234 & & & \\
\hline $5.22 \mathrm{E}-01$ & 875.94 & $\mathrm{~Pa}-234$ & $->$ & U-234 & & & \\
\hline $5.22 \mathrm{E}-01$ & 1124.93 & $\mathrm{~Pa}-234 \mathrm{~m}$ & $->$ & U-234 & & & \\
\hline $4.85 \mathrm{E}-01$ & 1393.57 & Pa-234 & $->$ & U-234 & & & \\
\hline 4.60E-01 & 819.21 & $\mathrm{~Pa}-234$ & $->$ & $U-234$ & & & \\
\hline $4.60 \mathrm{E}-01$ & 1809.04 & $\mathrm{~Pa}-234 \mathrm{~m}$ & $->$ & U-234 & & & \\
\hline 4.47E-01 & 543.98 & $\mathrm{~Pa}-234 \mathrm{~m}$ & $->$ & U-234 & & & \\
\hline 4.35E-01 & 103.35 & Th-234 & $->$ & $\mathrm{Pa}-234 \mathrm{~m}$ & & & \\
\hline 4.29E-01 & 881.1 & $\mathrm{~Pa}-234 \mathrm{~m}$ & $->$ & $U-234$ & & & \\
\hline 3.97E-01 & 19.891 & $\mathrm{~Pa}-234 \mathrm{~m}$ & $->$ & U-234 & & & \\
\hline 3.67E-01 & 15.4 & Pa-234 & $->$ & U-234, & $\begin{array}{r}\mathrm{Pa}- \\
234 \mathrm{~m}\end{array}$ & $->$ & $U-234$ \\
\hline 3.60E-01 & 1937.01 & $\mathrm{~Pa}-234 \mathrm{~m}$ & $->$ & U-234 & & & \\
\hline $3.52 \mathrm{E}-01$ & 248.94 & Pa-234 & $->$ & U-234 & & & \\
\hline 3.36E-01 & 1593.88 & $\mathrm{~Pa}-234$ & $->$ & U-234 & & & \\
\hline $3.36 \mathrm{E}-01$ & 203.12 & Pa-234 & $->$ & U-234, & $\begin{array}{r}\mathrm{Pa}- \\
234 \mathrm{~m}\end{array}$ & $->$ & $U-234$ \\
\hline 3.35E-01 & 450.96 & $\mathrm{~Pa}-234 \mathrm{~m}$ & $->$ & U-234 & & & \\
\hline $3.23 \mathrm{E}-01$ & 574.8 & Рa-234 & $->$ & U-234 & & & \\
\hline 3.23E-01 & 185.92 & Pa-234 & $->$ & U-234 & & & \\
\hline $3.23 \mathrm{E}-01$ & 808.2 & $\mathrm{~Pa}-234$ & $->$ & U-234, & $\begin{array}{r}\mathrm{Pa}- \\
234 \mathrm{~m}\end{array}$ & $->$ & $U-234$ \\
\hline $3.11 \mathrm{E}-01$ & 941.94 & Pa-234m & $->$ & U-234 & & & \\
\hline 3.07E-01 & 984.12 & Рa-234 & $->$ & U-234 & & & \\
\hline $2.98 \mathrm{E}-01$ & 1527.27 & $\mathrm{~Pa}-234 \mathrm{~m}$ & $->$ & U-234 & & & \\
\hline $2.89 \mathrm{E}-01$ & 140.15 & Pa-234 & $->$ & U-234, & $\begin{array}{r}\mathrm{Pa}- \\
234 \mathrm{~m}\end{array}$ & $->$ & $U-234$ \\
\hline $2.86 \mathrm{E}-01$ & 1392 & $\mathrm{~Pa}-234 \mathrm{~m}$ & $->$ & U-234 & & & \\
\hline $2.86 \mathrm{E}-01$ & 372.02 & Pa-234 & $->$ & $U-234$ & & & \\
\hline $2.86 \mathrm{E}-01$ & 475.75 & Pa-234m & $->$ & U-234 & & & \\
\hline $2.86 \mathrm{E}-01$ & 1061.86 & $\mathrm{~Pa}-234 \mathrm{~m}$ & $->$ & $U-234$ & & & \\
\hline $2.86 \mathrm{E}-01$ & 1413.88 & $\mathrm{~Pa}-234 \mathrm{~m}$ & $->$ & U-234 & & & \\
\hline
\end{tabular}




\begin{tabular}{|c|c|c|c|c|c|c|c|}
\hline $2.73 E-01$ & 1893.5 & $\mathrm{~Pa}-234 \mathrm{~m}$ & $\rightarrow$ & $U-234$ & & & \\
\hline $2.61 \mathrm{E}-01$ & 738.88 & $\mathrm{~Pa}-234$ & $->$ & $U-234$ & & & \\
\hline $2.61 \mathrm{E}-01$ & 755 & $\mathrm{~Pa}-234$ & $->$ & $U-234$ & & & \\
\hline $2.60 \mathrm{E}-01$ & 506.69 & $\mathrm{~Pa}-234$ & $->$ & $U-234$ & & & \\
\hline $2.49 \mathrm{E}-01$ & 458.63 & $\mathrm{~Pa}-234$ & $->$ & $U-234$ & & & \\
\hline $2.43 E-01$ & 692.7 & $\mathrm{~Pa}-234$ & $->$ & $U-234$ & & & \\
\hline $2.43 \mathrm{E}-01$ & 793.6 & Pa-234 & $->$ & $U-234$ & & & \\
\hline $2.38 \mathrm{E}-01$ & 509.11 & $\mathrm{~Pa}-234 \mathrm{~m}$ & $->$ & $U-234$ & & & \\
\hline $2.36 \mathrm{E}-01$ & 453.58 & $\mathrm{~Pa}-234 \mathrm{~m}$ & $->$ & $U-234$ & & & \\
\hline $2.36 \mathrm{E}-01$ & 1591.65 & $\mathrm{~Pa}-234 \mathrm{~m}$ & $->$ & $U-234$ & & & \\
\hline $2.36 \mathrm{E}-01$ & 1126.17 & Pa-234 & $->$ & U-234, & $\begin{array}{r}\mathrm{Pa}- \\
234 \mathrm{~m}\end{array}$ & $->$ & $U-234$ \\
\hline 2.29E-01 & 468.21 & $\mathrm{~Pa}-234 \mathrm{~m}$ & $->$ & $U-234$ & & & \\
\hline $2.26 \mathrm{E}-01$ & 565.2 & Pa-234 & $->$ & $U-234$ & & & \\
\hline $2.24 \mathrm{E}-01$ & 272.2 & $\mathrm{~Pa}-234$ & $->$ & $U-234$ & & & \\
\hline $2.13 \mathrm{E}-01$ & 1174.1 & $\mathrm{~Pa}-234 \mathrm{~m}$ & $->$ & $U-234$ & & & \\
\hline $2.11 \mathrm{E}-01$ & 669.64 & Pa-234 & $->$ & $U-234$ & & & \\
\hline $2.10 \mathrm{E}-01$ & 664.8 & $\mathrm{~Pa}-234$ & $->$ & $U-234$ & & & \\
\hline $2.10 \mathrm{E}-01$ & 513.6 & $\mathrm{~Pa}-234$ & $->$ & $U-234$ & & & \\
\hline 2.07E-01 & 882.5 & $\mathrm{~Pa}-234 \mathrm{~m}$ & $->$ & $U-234$ & & & \\
\hline $2.07 \mathrm{E}-01$ & 936.8 & $\mathrm{~Pa}-234 \mathrm{~m}$ & $->$ & $U-234$ & & & \\
\hline 2.07E-01 & 1457.4 & $\mathrm{~Pa}-234 \mathrm{~m}$ & $->$ & $U-234$ & & & \\
\hline 2.07E-01 & 1732.2 & $\mathrm{~Pa}-234 \mathrm{~m}$ & $->$ & $U-234$ & & & \\
\hline 2.05E-01 & 1877.21 & $\mathrm{~Pa}-234 \mathrm{~m}$ & $->$ & $U-234$ & & & \\
\hline $2.02 \mathrm{E}-01$ & 844.4 & $\mathrm{~Pa}-234$ & $->$ & U-234, & $\begin{array}{r}\mathrm{Pa}- \\
234 \mathrm{~m}\end{array}$ & $->$ & $U-234$ \\
\hline $1.99 \mathrm{E}-01$ & 111.897 & Th-234 & $->$ & $\mathrm{Pa}-234 \mathrm{~m}$ & & & \\
\hline $1.91 \mathrm{E}-01$ & 184.6 & $\mathrm{~Pa}-234 \mathrm{~m}$ & $->$ & $U-234$ & & & \\
\hline $1.91 \mathrm{E}-01$ & 1120.5 & $\mathrm{~Pa}-234 \mathrm{~m}$ & $->$ & $U-234$ & & & \\
\hline $1.88 \mathrm{E}-01$ & 1352.85 & Pa-234 & $->$ & $U-234$ & & & \\
\hline $1.87 \mathrm{E}-01$ & 666.42 & $\mathrm{~Pa}-234$ & $->$ & $U-234$ & & & \\
\hline $1.82 \mathrm{E}-01$ & 29.49 & Th-234 & $->$ & $\mathrm{Pa}-234 \mathrm{~m}$ & & & \\
\hline $1.78 \mathrm{E}-01$ & 200.96 & $\mathrm{~Pa}-234$ & $->$ & $U-234$ & & & \\
\hline $1.78 \mathrm{E}-01$ & 780.4 & $\mathrm{~Pa}-234$ & $->$ & $U-234$ & & & \\
\hline $1.75 \mathrm{E}-01$ & 507.3 & $\mathrm{~Pa}-234 \mathrm{~m}$ & $->$ & $U-234$ & & & \\
\hline $1.75 \mathrm{E}-01$ & 647.7 & $\mathrm{~Pa}-234 \mathrm{~m}$ & $->$ & $U-234$ & & & \\
\hline $1.75 \mathrm{E}-01$ & 695.9 & $\mathrm{~Pa}-234 \mathrm{~m}$ & $->$ & $U-234$ & & & \\
\hline $1.75 \mathrm{E}-01$ & 760.3 & $\mathrm{~Pa}-234 \mathrm{~m}$ & $->$ & $U-234$ & & & \\
\hline $1.74 \mathrm{E}-01$ & 1548.12 & $\mathrm{~Pa}-234 \mathrm{~m}$ & $->$ & $U-234$ & & & \\
\hline $1.74 \mathrm{E}-01$ & 1759.81 & $\mathrm{~Pa}-234 \mathrm{~m}$ & $->$ & $U-234$ & & & \\
\hline $1.62 \mathrm{E}-01$ & 978.8 & Pa-234 & $->$ & $U-234$ & & & \\
\hline $1.62 \mathrm{E}-01$ & 1694.08 & $\mathrm{~Pa}-234$ & $->$ & $U-234$ & & & \\
\hline $1.62 \mathrm{E}-01$ & 125.53 & $\mathrm{~Pa}-234$ & $->$ & $U-234$ & & & \\
\hline $1.59 \mathrm{E}-01$ & 625.5 & $\mathrm{~Pa}-234 \mathrm{~m}$ & $->$ & $U-234$ & & & \\
\hline $1.57 \mathrm{E}-01$ & 826.08 & $\mathrm{~Pa}-234 \mathrm{~m}$ & $->$ & $U-234$ & & & \\
\hline $1.49 \mathrm{E}-01$ & 1452.63 & $\mathrm{~Pa}-234$ & $->$ & $U-234$ & & & \\
\hline $1.49 \mathrm{E}-01$ & 1668.44 & $\mathrm{~Pa}-234$ & $->$ & $U-234$ & & & \\
\hline $1.49 \mathrm{E}-01$ & 1084.25 & $\mathrm{~Pa}-234$ & $->$ & U-234, & $\begin{array}{r}\mathrm{Pa}- \\
234 \mathrm{~m}\end{array}$ & $->$ & $U-234$ \\
\hline $1.49 \mathrm{E}-01$ & 1863.09 & $\mathrm{~Pa}-234 \mathrm{~m}$ & $->$ & $U-234$ & & & \\
\hline
\end{tabular}




\begin{tabular}{|c|c|c|c|c|c|c|c|}
\hline $1.49 \mathrm{E}-01$ & 1041.7 & $\mathrm{~Pa}-234 \mathrm{~m}$ & $\rightarrow$ & $U-234$ & & & \\
\hline $1.46 \mathrm{E}-01$ & 210.6 & $\mathrm{~Pa}-234 \mathrm{~m}$ & $->$ & $U-234$ & & & \\
\hline $1.46 \mathrm{E}-01$ & 602.1 & $\mathrm{~Pa}-234$ & $->$ & U-234 & & & \\
\hline $1.46 \mathrm{E}-01$ & 658 & $\mathrm{~Pa}-234$ & $->$ & $U-234$ & & & \\
\hline $1.46 \mathrm{E}-01$ & 245.16 & $\mathrm{~Pa}-234$ & $->$ & U-234 & & & \\
\hline $1.46 \mathrm{E}-01$ & 521.09 & $\mathrm{~Pa}-234$ & $->$ & U-234 & & & \\
\hline $1.45 \mathrm{E}-01$ & 732.7 & $\mathrm{~Pa}-234 \mathrm{~m}$ & $->$ & $U-234$ & & & \\
\hline $1.43 \mathrm{E}-01$ & 1500.9 & $\mathrm{~Pa}-234 \mathrm{~m}$ & $->$ & $U-234$ & & & \\
\hline $1.41 \mathrm{E}-01$ & 199.46 & $\mathrm{~Pa}-234$ & $->$ & U-234, & $\begin{array}{r}\mathrm{Pa}- \\
234 \mathrm{~m}\end{array}$ & $->$ & $U-234$ \\
\hline 1.37E-01 & 1570.67 & $\mathrm{~Pa}-234 \mathrm{~m}$ & $->$ & $U-234$ & & & \\
\hline $1.29 \mathrm{E}-01$ & 624.31 & $\mathrm{~Pa}-234$ & $->$ & U-234 & & & \\
\hline $1.29 \mathrm{E}-01$ & 627.5 & $\mathrm{~Pa}-234$ & $->$ & $U-234$ & & & \\
\hline $1.29 \mathrm{E}-01$ & 611.5 & $\mathrm{~Pa}-234$ & $->$ & $U-234$ & & & \\
\hline $1.29 \mathrm{E}-01$ & 1029.03 & $\mathrm{~Pa}-234$ & $->$ & U-234 & & & \\
\hline $1.28 \mathrm{E}-01$ & 708.4 & $\mathrm{~Pa}-234$ & $->$ & U-234, & $\begin{array}{r}\mathrm{Pa}- \\
234 \mathrm{~m}\end{array}$ & $->$ & $U-234$ \\
\hline $1.26 \mathrm{E}-01$ & 338.4 & $\mathrm{~Pa}-234 \mathrm{~m}$ & $->$ & U-234 & & & \\
\hline $1.22 \mathrm{E}-01$ & 1059.72 & $\mathrm{~Pa}-234 \mathrm{~m}$ & $->$ & $U-234$ & & & \\
\hline 1.19E-01 & 649.1 & $\mathrm{~Pa}-234 \mathrm{~m}$ & $->$ & $U-234$ & & & \\
\hline 1.19E-01 & 866.98 & $\mathrm{~Pa}-234 \mathrm{~m}$ & $->$ & U-234 & & & \\
\hline $1.13 \mathrm{E}-01$ & 157.96 & Pa-234 & $->$ & U-234 & & & \\
\hline $1.12 \mathrm{E}-01$ & 1292.66 & Pa-234 & $->$ & $U-234$ & & & \\
\hline $1.12 \mathrm{E}-01$ & 1819.69 & $\mathrm{~Pa}-234 \mathrm{~m}$ & $->$ & $U-234$ & & & \\
\hline $1.12 \mathrm{E}-01$ & 1220.37 & $\mathrm{~Pa}-234 \mathrm{~m}$ & $->$ & $U-234$ & & & \\
\hline $1.11 \mathrm{E}-01$ & 387.8 & $\mathrm{~Pa}-234 \mathrm{~m}$ & $->$ & U-234 & & & \\
\hline $1.11 \mathrm{E}-01$ & 818 & $\mathrm{~Pa}-234 \mathrm{~m}$ & $->$ & $U-234$ & & & \\
\hline $1.06 \mathrm{E}-01$ & 655.1 & $\mathrm{~Pa}-234 \mathrm{~m}$ & $->$ & U-234 & & & \\
\hline $1.00 \mathrm{E}-01$ & 653.57 & $\mathrm{~Pa}-234$ & $->$ & U-234 & & & \\
\hline $9.70 \mathrm{E}-02$ & 1022.23 & $\mathrm{~Pa}-234$ & $->$ & $U-234$ & & & \\
\hline $9.70 \mathrm{E}-02$ & 426.8 & $\mathrm{~Pa}-234$ & $->$ & U-234 & & & \\
\hline $9.70 \mathrm{E}-02$ & 527.04 & Pa-234 & $->$ & U-234 & & & \\
\hline $9.70 \mathrm{E}-02$ & 352.16 & $\mathrm{~Pa}-234$ & $->$ & $U-234$ & & & \\
\hline $9.70 \mathrm{E}-02$ & 520.2 & $\mathrm{~Pa}-234$ & $->$ & $U-234$ & & & \\
\hline $9.70 \mathrm{E}-02$ & 330.62 & $\mathrm{~Pa}-234$ & $->$ & $U-234$ & & & \\
\hline $9.70 \mathrm{E}-02$ & 967.33 & $\mathrm{~Pa}-234$ & $->$ & $U-234$ & & & \\
\hline $9.70 \mathrm{E}-02$ & 193.55 & $\mathrm{~Pa}-234$ & $->$ & U-234 & & & \\
\hline 9.69E-02 & 572 & $\mathrm{~Pa}-234 \mathrm{~m}$ & $->$ & $U-234$ & & & \\
\hline $9.54 \mathrm{E}-02$ & 959.8 & $\mathrm{~Pa}-234 \mathrm{~m}$ & $->$ & U-234 & & & \\
\hline $9.22 \mathrm{E}-02$ & 1667.3 & $\mathrm{~Pa}-234 \mathrm{~m}$ & $->$ & U-234 & & & \\
\hline $9.05 E-02$ & 768.5 & $\mathrm{~Pa}-234$ & $->$ & U-234 & & & \\
\hline 8.89E-02 & 357.9 & $\mathrm{~Pa}-234 \mathrm{~m}$ & $->$ & U-234 & & & \\
\hline 8.87E-02 & 783.38 & $\mathrm{~Pa}-234$ & $->$ & U-234, & $\begin{array}{r}\mathrm{Pa}- \\
234 \mathrm{~m}\end{array}$ & $->$ & $U-234$ \\
\hline 8.83E-02 & 274.45 & $\mathrm{~Pa}-234$ & $->$ & U-234, & $\begin{array}{r}\mathrm{Pa}- \\
234 \mathrm{~m}\end{array}$ & $->$ & $U-234$ \\
\hline $8.58 \mathrm{E}-02$ & 1558.7 & $\mathrm{~Pa}-234 \mathrm{~m}$ & $->$ & $U-234$ & & & \\
\hline 8.29E-02 & 247.7 & $\mathrm{~Pa}-234 \mathrm{~m}$ & $->$ & U-234 & & & \\
\hline 8.08E-02 & 812.5 & $\mathrm{~Pa}-234$ & $->$ & U-234 & & & \\
\hline 8.08E-02 & 904.38 & $\mathrm{~Pa}-234$ & $->$ & U-234 & & & \\
\hline
\end{tabular}




\begin{tabular}{|c|c|c|c|c|c|c|c|}
\hline 8.08E-02 & 1044.9 & $\mathrm{~Pa}-234$ & $\rightarrow$ & $U-234$ & & & \\
\hline 8.08E-02 & 1122.09 & $\mathrm{~Pa}-234$ & $->$ & $U-234$ & & & \\
\hline 8.08E-02 & 170.75 & $\mathrm{~Pa}-234$ & $->$ & U-234 & & & \\
\hline 8.08E-02 & 1241.4 & $\mathrm{~Pa}-234$ & $->$ & $U-234$ & & & \\
\hline 8.08E-02 & 1686.2 & $\mathrm{~Pa}-234$ & $->$ & U-234 & & & \\
\hline 7.95E-02 & 456.5 & $\mathrm{~Pa}-234 \mathrm{~m}$ & $->$ & U-234 & & & \\
\hline 7.95E-02 & 557.3 & $\mathrm{~Pa}-234 \mathrm{~m}$ & $->$ & U-234 & & & \\
\hline 7.84E-02 & 700.4 & $\mathrm{~Pa}-234 \mathrm{~m}$ & $->$ & $U-234$ & & & \\
\hline 7.69E-02 & 276.83 & $\mathrm{~Pa}-234$ & $->$ & U-234, & $\begin{array}{r}\text { Pa- } \\
234 m\end{array}$ & $->$ & $U-234$ \\
\hline 7.63E-02 & 360.8 & $\mathrm{~Pa}-234 \mathrm{~m}$ & $->$ & $U-234$ & & & \\
\hline $7.15 \mathrm{E}-02$ & 674.24 & $\mathrm{~Pa}-234 \mathrm{~m}$ & $->$ & $U-234$ & & & \\
\hline 6.99E-02 & 193.4 & $\mathrm{~Pa}-234 \mathrm{~m}$ & $->$ & $U-234$ & & & \\
\hline 6.99E-02 & 299.08 & $\mathrm{~Pa}-234 \mathrm{~m}$ & $->$ & $U-234$ & & & \\
\hline 6.69E-02 & 655 & $\mathrm{~Pa}-234$ & $->$ & U-234 & & & \\
\hline $6.47 \mathrm{E}-02$ & 632 & $\mathrm{~Pa}-234$ & $->$ & U-234 & & & \\
\hline $6.47 \mathrm{E}-02$ & 409.8 & $\mathrm{~Pa}-234$ & $->$ & U-234 & & & \\
\hline $6.47 \mathrm{E}-02$ & 920 & $\mathrm{~Pa}-234$ & $->$ & $U-234$ & & & \\
\hline 6.47E-02 & 480.4 & $\mathrm{~Pa}-234$ & $->$ & $U-234$ & & & \\
\hline 6.47E-02 & 1638 & Pa-234 & $->$ & $U-234$ & & & \\
\hline $6.47 \mathrm{E}-02$ & 804.52 & $\mathrm{~Pa}-234$ & $->$ & $U-234$ & & & \\
\hline $6.47 \mathrm{E}-02$ & 1516 & $\mathrm{~Pa}-234$ & $->$ & U-234 & & & \\
\hline $6.47 \mathrm{E}-02$ & 1445.7 & Рa-234 & $->$ & $U-234$ & & & \\
\hline $6.36 \mathrm{E}-02$ & 683 & $\mathrm{~Pa}-234 \mathrm{~m}$ & $->$ & U-234 & & & \\
\hline $6.20 \mathrm{E}-02$ & 1969.9 & $\mathrm{~Pa}-234 \mathrm{~m}$ & $->$ & U-234 & & & \\
\hline $5.98 \mathrm{E}-02$ & 1216.4 & $\mathrm{~Pa}-234$ & $->$ & U-234 & & & \\
\hline $5.73 \mathrm{E}-02$ & 311 & $\mathrm{~Pa}-234 \mathrm{~m}$ & $->$ & $U-234$ & & & \\
\hline $5.66 \mathrm{E}-02$ & 143.9 & $\mathrm{~Pa}-234$ & $->$ & U-234 & & & \\
\hline $5.66 \mathrm{E}-02$ & 467.5 & $\mathrm{~Pa}-234$ & $->$ & U-234 & & & \\
\hline $5.56 \mathrm{E}-02$ & 1085.37 & $\mathrm{~Pa}-234 \mathrm{~m}$ & $->$ & $U-234$ & & & \\
\hline $5.08 \mathrm{E}-02$ & 1693.7 & $\mathrm{~Pa}-234 \mathrm{~m}$ & $->$ & $U-234$ & & & \\
\hline $4.85 E-02$ & 1208 & Tl-210 & $->$ & $\mathrm{Pb}-210$ & $\mathrm{~Pa}-234$ & $->$ & $U-234$ \\
\hline 4.85E-02 & 1229 & $\mathrm{~Pa}-234$ & $->$ & $U-234$ & & & \\
\hline 4.85E-02 & 313.83 & $\mathrm{~Pa}-234$ & $->$ & $U-234$ & & & \\
\hline $4.85 E-02$ & 1251 & $\mathrm{~Pa}-234$ & $->$ & $U-234$ & & & \\
\hline 4.85E-02 & 766.369 & $\mathrm{~Pa}-234$ & $->$ & $U-234$ & & & \\
\hline $4.85 E-02$ & 330 & $\mathrm{~Pa}-234$ & $->$ & $U-234$ & & & \\
\hline $4.85 E-02$ & 1153.6 & $\mathrm{~Pa}-234$ & $->$ & U-234 & & & \\
\hline $4.85 E-02$ & 660.6 & $\mathrm{~Pa}-234$ & $->$ & U-234 & & & \\
\hline 4.85E-02 & 1460 & $\mathrm{~Pa}-234$ & $->$ & $U-234$ & & & \\
\hline $4.85 E-02$ & 1797.3 & $\mathrm{~Pa}-234$ & $->$ & U-234 & & & \\
\hline $4.85 E-02$ & 1108.5 & $\mathrm{~Pa}-234$ & $->$ & U-234 & & & \\
\hline $4.85 E-02$ & 634.4 & $\mathrm{~Pa}-234$ & $->$ & U-234 & & & \\
\hline $4.85 E-02$ & 482.5 & $\mathrm{~Pa}-234$ & $->$ & $U-234$ & & & \\
\hline $4.85 \mathrm{E}-02$ & 646.84 & $\mathrm{~Pa}-234$ & $->$ & $U-234$ & & & \\
\hline 4.85E-02 & 478.7 & $\mathrm{~Pa}-234$ & $->$ & $U-234$ & & & \\
\hline 4.79E-02 & 1353 & $\mathrm{Bi}-214$ & $->$ & Po-214, & $\begin{array}{r}\mathrm{Pa}- \\
234 \mathrm{~m}\end{array}$ & $->$ & $U-234$ \\
\hline 4.77E-02 & 387.87 & $\mathrm{~Pa}-234 \mathrm{~m}$ & $->$ & U-234 & & & \\
\hline $4.61 \mathrm{E}-02$ & 1602.2 & Pa-234m & $->$ & $U-234$ & & & \\
\hline
\end{tabular}




\begin{tabular}{|c|c|c|c|c|c|c|c|}
\hline 4.53E-02 & 1905 & Pa-234 & $\rightarrow>$ & $U-234$ & & & \\
\hline $4.53 \mathrm{E}-02$ & 688.08 & $\mathrm{~Pa}-234$ & $->$ & $U-234$ & & & \\
\hline 4.37E-02 & 79.65 & $\mathrm{~Pa}-234$ & $->$ & $U-234$ & & & \\
\hline $4.20 \mathrm{E}-02$ & 557.78 & $\mathrm{~Pa}-234$ & $->$ & $U-234$ & & & \\
\hline $4.04 \mathrm{E}-02$ & 1585.3 & $\mathrm{~Pa}-234$ & $->$ & $U-234$ & & & \\
\hline 4.04E-02 & 1756 & $\mathrm{~Pa}-234$ & $->$ & U-234 & & & \\
\hline $4.04 \mathrm{E}-02$ & 1074.6 & $\mathrm{~Pa}-234$ & $->$ & $U-234$ & & & \\
\hline 3.88E-02 & 472.32 & $\mathrm{~Pa}-234$ & $->$ & $U-234$ & & & \\
\hline 3.88E-02 & 1171.3 & $\mathrm{~Pa}-234$ & $->$ & $U-234$ & & & \\
\hline 3.88E-02 & 683.3 & $\mathrm{~Pa}-234$ & $->$ & $U-234$ & & & \\
\hline $3.86 \mathrm{E}-02$ & 1926 & $\mathrm{~Pa}-234$ & $->$ & $U-234$ & & & \\
\hline 3.72E-02 & 69.9 & $\mathrm{~Pa}-234$ & $->$ & $U-234$ & & & \\
\hline $3.72 \mathrm{E}-02$ & 1399.7 & $\mathrm{~Pa}-234$ & $->$ & $U-234$ & & & \\
\hline $3.65 \mathrm{E}-02$ & 1720.5 & $\mathrm{~Pa}-234 \mathrm{~m}$ & $->$ & $U-234$ & & & \\
\hline $3.53 \mathrm{E}-02$ & 316.29 & Pa-234 & $->$ & U-234, & $\begin{array}{r}\mathrm{Pa}- \\
234 \mathrm{~m}\end{array}$ & $->$ & $U-234$ \\
\hline 3.49E-02 & 1796.2 & $\mathrm{~Pa}-234 \mathrm{~m}$ & $->$ & $U-234$ & & & \\
\hline $3.40 \mathrm{E}-02$ & 134.3 & Pa-234 & $->$ & $U-234$ & & & \\
\hline $3.23 E-02$ & 643.2 & Pa-234 & $->$ & $U-234$ & & & \\
\hline $3.23 \mathrm{E}-02$ & 1493.94 & $\mathrm{~Pa}-234$ & $->$ & $U-234$ & & & \\
\hline $3.23 \mathrm{E}-02$ & 617 & $\mathrm{~Pa}-234$ & $->$ & $U-234$ & & & \\
\hline $3.23 \mathrm{E}-02$ & 219.8 & $\mathrm{~Pa}-234$ & $->$ & U-234 & & & \\
\hline $3.23 E-02$ & 777.9 & $\mathrm{~Pa}-234$ & $->$ & $U-234$ & & & \\
\hline $3.23 E-02$ & 1427.33 & $\mathrm{~Pa}-234$ & $->$ & $U-234$ & & & \\
\hline $3.23 \mathrm{E}-02$ & 639.7 & $\mathrm{~Pa}-234$ & $->$ & $U-234$ & & & \\
\hline $3.23 E-02$ & 533.2 & $\mathrm{~Pa}-234$ & $->$ & $U-234$ & & & \\
\hline $3.23 E-02$ & 711.2 & $\mathrm{~Pa}-234$ & $->$ & $U-234$ & & & \\
\hline $3.23 E-02$ & 174.52 & Pa-234 & $->$ & $U-234$ & & & \\
\hline $3.23 \mathrm{E}-02$ & 1277.4 & $\mathrm{~Pa}-234$ & $->$ & $U-234$ & & & \\
\hline $3.23 E-02$ & 150 & $\mathrm{~Pa}-234$ & $->$ & $U-234$ & & & \\
\hline 3.07E-02 & 1890.1 & $\mathrm{~Pa}-234$ & $->$ & $U-234$ & & & \\
\hline $2.91 \mathrm{E}-02$ & 473.5 & $\mathrm{~Pa}-234$ & $->$ & $U-234$ & & & \\
\hline $2.75 \mathrm{E}-02$ & 1580.02 & $\mathrm{~Pa}-234$ & $->$ & $U-234$ & & & \\
\hline $2.75 E-02$ & 267.1 & $\mathrm{~Pa}-234$ & $->$ & $U-234$ & & & \\
\hline $2.59 \mathrm{E}-02$ & 713.8 & $\mathrm{~Pa}-234$ & $->$ & $U-234$ & & & \\
\hline $2.59 \mathrm{E}-02$ & 760.1 & $\mathrm{~Pa}-234$ & $->$ & $U-234$ & & & \\
\hline $2.59 \mathrm{E}-02$ & 461.3 & $\mathrm{~Pa}-234$ & $->$ & $U-234$ & & & \\
\hline 2.59E-02 & 537.1 & $\mathrm{~Pa}-234$ & $->$ & $U-234$ & & & \\
\hline $2.43 E-02$ & 1656 & $\mathrm{~Pa}-234$ & $->$ & $U-234$ & & & \\
\hline $2.43 \mathrm{E}-02$ & 1897.1 & $\mathrm{~Pa}-234$ & $->$ & $U-234$ & & & \\
\hline $2.43 E-02$ & 585.8 & $\mathrm{~Pa}-234$ & $->$ & $U-234$ & & & \\
\hline $2.43 \mathrm{E}-02$ & 1699.8 & Pa-234 & $->$ & $U-234$ & & & \\
\hline $2.43 \mathrm{E}-02$ & 137.7 & $\mathrm{~Pa}-234$ & $->$ & $U-234$ & & & \\
\hline $2.43 \mathrm{E}-02$ & 1627.9 & $\mathrm{~Pa}-234$ & $->$ & $U-234$ & & & \\
\hline $2.36 \mathrm{E}-02$ & 1926.4 & $\mathrm{~Pa}-234 \mathrm{~m}$ & $->$ & $U-234$ & & & \\
\hline $2.26 \mathrm{E}-02$ & 842.4 & Pa-234 & $->$ & $U-234$ & & & \\
\hline $2.26 \mathrm{E}-02$ & 286.4 & Pa-234 & $->$ & $U-234$ & & & \\
\hline $2.10 \mathrm{E}-02$ & 746.5 & $\mathrm{~Pa}-234$ & $->$ & $U-234$ & & & \\
\hline $1.94 \mathrm{E}-02$ & 43.47 & $\mathrm{~Pa}-234$ & $->$ & $U-234$ & & & \\
\hline $1.94 \mathrm{E}-02$ & 1358.5 & $\mathrm{~Pa}-234$ & $->$ & $U-234$ & & & \\
\hline
\end{tabular}




\begin{tabular}{|c|c|c|c|c|}
\hline 1.94E-02 & 103.7 & $\mathrm{~Pa}-234$ & $->$ & $U-234$ \\
\hline 1.94E-02 & 446.1 & $\mathrm{~Pa}-234$ & $->$ & $U-234$ \\
\hline $1.94 \mathrm{E}-02$ & 873.4 & $\mathrm{~Pa}-234$ & $->$ & $U-234$ \\
\hline 1.94E-02 & 320.7 & $\mathrm{~Pa}-234$ & $->$ & $U-234$ \\
\hline $1.78 \mathrm{E}-02$ & 289.25 & $\mathrm{~Pa}-234$ & $->$ & $U-234$ \\
\hline $1.62 \mathrm{E}-02$ & 416 & $\mathrm{~Pa}-234$ & $->$ & $U-234$ \\
\hline $1.62 \mathrm{E}-02$ & 960 & $\mathrm{~Pa}-234$ & $->$ & $U-234$ \\
\hline 1.62E-02 & 1772.3 & $\mathrm{~Pa}-234$ & $->$ & $U-234$ \\
\hline $1.62 \mathrm{E}-02$ & 309.6 & $\mathrm{~Pa}-234$ & $->$ & $U-234$ \\
\hline $1.62 \mathrm{E}-02$ & 1741.7 & Pa-234 & $->$ & $U-234$ \\
\hline $1.62 \mathrm{E}-02$ & 1738 & $\mathrm{~Pa}-234$ & $->$ & $U-234$ \\
\hline $1.62 \mathrm{E}-02$ & 498.8 & $\mathrm{~Pa}-234$ & $->$ & $U-234$ \\
\hline $1.62 \mathrm{E}-02$ & 1549.4 & $\mathrm{~Pa}-234$ & $->$ & $U-234$ \\
\hline $1.46 \mathrm{E}-02$ & 165.8 & $\mathrm{~Pa}-234$ & $->$ & $U-234$ \\
\hline 1.13E-02 & 278.1 & $\mathrm{~Pa}-234$ & $->$ & $U-234$ \\
\hline $1.13 \mathrm{E}-02$ & 196.43 & $\mathrm{~Pa}-234$ & $->$ & $U-234$ \\
\hline $1.13 \mathrm{E}-02$ & 1872.8 & $\mathrm{~Pa}-234$ & $->$ & $U-234$ \\
\hline $9.70 \mathrm{E}-03$ & 1768 & $\mathrm{~Pa}-234$ & $->$ & $U-234$ \\
\hline $9.70 \mathrm{E}-03$ & 1837.9 & $\mathrm{~Pa}-234$ & $->$ & $U-234$ \\
\hline $9.38 \mathrm{E}-03$ & 67.22 & $\mathrm{~Pa}-234$ & $->$ & $U-234$ \\
\hline 8.57E-03 & 192.74 & $\mathrm{~Pa}-234 \mathrm{~m}$ & $->$ & U-234 \\
\hline 8.08E-03 & 1937.7 & Pa-234 & $->$ & $U-234$ \\
\hline 8.08E-03 & 1750.1 & $\mathrm{~Pa}-234$ & $->$ & $U-234$ \\
\hline 8.08E-03 & 210.7 & $\mathrm{~Pa}-234$ & $->$ & $U-234$ \\
\hline 8.08E-03 & 1850 & $\mathrm{~Pa}-234$ & $->$ & U-234 \\
\hline 4.85E-03 & 1828 & Pa-234 & $->$ & $U-234$ \\
\hline $4.72 \mathrm{E}-03$ & 236.03 & $\mathrm{~Pa}-234 \mathrm{~m}$ & $->$ & $U-234$ \\
\hline 4.47E-03 & 557.7 & $\mathrm{~Pa}-234 \mathrm{~m}$ & $->$ & $U-234$ \\
\hline $3.85 \mathrm{E}-03$ & 718.94 & $\mathrm{~Pa}-234 \mathrm{~m}$ & $->$ & $U-234$ \\
\hline 3.60E-03 & 721.33 & $\mathrm{~Pa}-234 \mathrm{~m}$ & $->$ & $U-234$ \\
\hline $3.23 E-03$ & 1719.5 & $\mathrm{~Pa}-234$ & $->$ & $U-234$ \\
\hline $2.24 \mathrm{E}-03$ & 750.5 & $\mathrm{~Pa}-234 \mathrm{~m}$ & $->$ & $U-234$ \\
\hline $1.86 \mathrm{E}-03$ & 516.8 & $\mathrm{~Pa}-234 \mathrm{~m}$ & $->$ & $U-234$ \\
\hline $1.78 \mathrm{E}-03$ & 432.8 & $\mathrm{~Pa}-234$ & $->$ & $U-234$ \\
\hline $1.78 \mathrm{E}-03$ & 45.28 & Pa-234 & $->$ & $U-234$ \\
\hline $1.76 \mathrm{E}-03$ & 73.9 & $\mathrm{~Pa}-234 \mathrm{~m}$ & $->$ & $\mathrm{Pa}-234$ \\
\hline $1.46 \mathrm{E}-03$ & 58.31 & $\mathrm{~Pa}-234$ & $->$ & $U-234$ \\
\hline $5.82 \mathrm{E}-04$ & 34.37 & $\mathrm{~Pa}-234$ & $->$ & $U-234$ \\
\hline $3.23 E-04$ & 1988 & $\mathrm{~Pa}-234$ & $->$ & $U-234$ \\
\hline 2.05E-04 & 53.23 & U-234 & $->$ & Th-230 \\
\hline $1.62 \mathrm{E}-04$ & 1959 & $\mathrm{~Pa}-234$ & $->$ & U-234 \\
\hline $1.62 \mathrm{E}-04$ & 1998 & $\mathrm{~Pa}-234$ & $->$ & $U-234$ \\
\hline 7.06E-05 & 120.912 & U-234 & $->$ & Th-230 \\
\hline 4.48E-08 & 454.97 & U-234 & $->$ & Th-230 \\
\hline $2.53 E-08$ & 508.2 & U-234 & $->$ & Th-230 \\
\hline 2.07E-08 & 581.78 & U-234 & $->$ & Th-230 \\
\hline $1.46 \mathrm{E}-08$ & 67.6758 & Th-230 & $->$ & Ra-226 \\
\hline 1.89E-09 & 143.876 & Th-230 & $->$ & $\mathrm{Ra}-226$ \\
\hline $1.72 \mathrm{E}-09$ & 503.53 & U-234 & $->$ & Th-230 \\
\hline $1.72 \mathrm{E}-09$ & 677.67 & $U-234$ & $->$ & Th-230 \\
\hline
\end{tabular}




\begin{tabular}{|c|c|c|c|c|c|c|c|}
\hline $1.38 \mathrm{E}-09$ & 624.44 & $U-234$ & $->$ & Th-230 & & & \\
\hline $1.25 \mathrm{E}-09$ & 609.311 & $\mathrm{Bi}-214$ & $->$ & Po-214 & & & \\
\hline $1.01 E-09$ & 351.87 & $\mathrm{~Pb}-214$ & $->$ & $\mathrm{Bi}-214$ & & & \\
\hline $5.22 \mathrm{E}-10$ & 295.091 & $\mathrm{~Pb}-214$ & $->$ & $\mathrm{Bi}-214$ & & & \\
\hline $4.32 \mathrm{E}-10$ & 1764.49 & $\mathrm{Bi}-214$ & $->$ & Po-214 & & & \\
\hline $4.31 \mathrm{E}-10$ & 253.732 & Th-230 & $->$ & Ra-226 & & & \\
\hline $4.07 E-10$ & 1120.273 & $\mathrm{Bi}-214$ & $->$ & Po-214 & & & \\
\hline $3.42 \mathrm{E}-10$ & 186.057 & Th-230 & $->$ & Ra-226 & & & \\
\hline $2.99 \mathrm{E}-10$ & 77.107 & $\mathrm{~Pb}-214$ & $->$ & $\mathrm{Bi}-214$ & & & \\
\hline $2.03 E-10$ & 241.92 & $\mathrm{~Pb}-214$ & $->$ & $\mathrm{Bi}-214$ & & & \\
\hline $1.77 \mathrm{E}-10$ & 74.814 & $\mathrm{~Pb}-214$ & $->$ & $\mathrm{Bi}-214$ & & & \\
\hline $1.67 \mathrm{E}-10$ & 10.828 & $\mathrm{~Pb}-210$ & $->$ & $\mathrm{Bi}-210$, & $\mathrm{Pb}-214$ & $->$ & $\mathrm{Bi}-214$ \\
\hline $1.61 \mathrm{E}-10$ & 1238.107 & $\mathrm{Bi}-214$ & $->$ & Po-214 & & & \\
\hline $1.55 \mathrm{E}-10$ & 13.088 & $\mathrm{~Pb}-214$ & $->$ & $\mathrm{Bi}-214$ & & & \\
\hline $1.35 \mathrm{E}-10$ & 2204.09 & $\mathrm{Bi}-214$ & $->$ & Po-214 & & & \\
\hline $1.32 \mathrm{E}-10$ & 768.35 & $\mathrm{Bi}-214$ & $->$ & Рo-214 & & & \\
\hline $1.09 \mathrm{E}-10$ & 1377.659 & $\mathrm{Bi}-214$ & $->$ & Po-214 & & & \\
\hline $1.05 \mathrm{E}-10$ & 87.19 & $\mathrm{~Pb}-214$ & $->$ & $\mathrm{Bi}-214$ & & & \\
\hline $9.01 E-11$ & 186.11 & $\mathrm{Ra}-226$ & $->$ & $\mathrm{Rn}-222$ & & & \\
\hline $8.58 \mathrm{E}-11$ & 934.039 & $\mathrm{Bi}-214$ & $->$ & Po-214 & & & \\
\hline $8.28 \mathrm{E}-11$ & 1729.58 & $\mathrm{Bi}-214$ & $->$ & Po-214 & & & \\
\hline $6.73 \mathrm{E}-11$ & 1407.97 & $\mathrm{Bi}-214$ & $->$ & Po-214 & & & \\
\hline $5.95 \mathrm{E}-11$ & 1509.22 & $\mathrm{Bi}-214$ & $->$ & Po-214 & & & \\
\hline $5.76 \mathrm{E}-11$ & 1847.41 & $\mathrm{Bi}-214$ & $->$ & Рo-214 & & & \\
\hline $4.59 E-11$ & 1155.183 & $\mathrm{Bi}-214$ & $->$ & Po-214 & & & \\
\hline $4.24 \mathrm{E}-11$ & 665.442 & $\mathrm{Bi}-214$ & $->$ & Po-214 & & & \\
\hline $4.21 \mathrm{E}-11$ & 2447.68 & $\mathrm{Bi}-214$ & $->$ & Рo-214 & & & \\
\hline $3.99 \mathrm{E}-11$ & 1280.952 & $\mathrm{Bi}-214$ & $->$ & Po-214 & & & \\
\hline 3.77E-11 & 1401.48 & $\mathrm{Bi}-214$ & $->$ & Рo-214 & & & \\
\hline $3.34 \mathrm{E}-11$ & 806.155 & $\mathrm{Bi}-214$ & $->$ & Po-214 & & & \\
\hline 3.30E-11 & 253.84 & Th-230 & $->$ & Ra-226 & & & \\
\hline $3.29 E-11$ & 2118.53 & $\mathrm{Bi}-214$ & $->$ & Po-214 & & & \\
\hline $3.15 \mathrm{E}-11$ & 15.439 & $\mathrm{~Pb}-214$ & $->$ & $\mathrm{Bi}-214$ & & & \\
\hline $3.12 \mathrm{E}-11$ & 90.128 & $\mathrm{~Pb}-214$ & $->$ & $\mathrm{Bi}-214$ & & & \\
\hline $3.12 \mathrm{E}-11$ & 1661.258 & $\mathrm{Bi}-214$ & $->$ & Po-214 & & & \\
\hline 2.99E-11 & 53.172 & $\mathrm{~Pb}-214$ & $->$ & $\mathrm{Bi}-214$ & & & \\
\hline $2.96 \mathrm{E}-11$ & 785.827 & $\mathrm{~Pb}-214$ & $->$ & $\mathrm{Bi}-214$ & & & \\
\hline $2.63 \mathrm{E}-11$ & 79.29 & $\mathrm{Bi}-214$ & $->$ & Рo-214 & & & \\
\hline $2.12 \mathrm{E}-11$ & 1385.295 & $\mathrm{Bi}-214$ & $->$ & Po-214 & & & \\
\hline $1.95 \mathrm{E}-11$ & 1583.22 & $\mathrm{Bi}-214$ & $->$ & Рo-214 & & & \\
\hline $1.59 \mathrm{E}-11$ & 838.999 & $\mathrm{~Pb}-214$ & $->$ & $\mathrm{Bi}-214$ & & & \\
\hline $1.57 \mathrm{E}-11$ & 76.838 & $\mathrm{Bi}-214$ & $->$ & Po-214 & & & \\
\hline $1.49 \mathrm{E}-11$ & 258.94 & $\mathrm{~Pb}-214$ & $->$ & $\mathrm{Bi}-214$ & & & \\
\hline $1.28 \mathrm{E}-11$ & 703.07 & $\mathrm{Bi}-214$ & $->$ & Po-214 & & & \\
\hline $1.25 \mathrm{E}-11$ & 1207.764 & $\mathrm{Bi}-214$ & $->$ & Po-214 & & & \\
\hline $1.19 \mathrm{E}-11$ & 487.13 & $\mathrm{~Pb}-214$ & $->$ & $\mathrm{Bi}-214$ & & & \\
\hline $1.11 \mathrm{E}-11$ & 388.95 & $\mathrm{Bi}-214$ & $->$ & Po-214 & & & \\
\hline $1.11 \mathrm{E}-11$ & 1538.49 & $\mathrm{Bi}-214$ & $->$ & Po-214 & & & \\
\hline $1.09 \mathrm{E}-11$ & 719.856 & $\mathrm{Bi}-214$ & $->$ & Po-214 & & & \\
\hline $1.05 \mathrm{E}-11$ & 14.336 & $\mathrm{Ra}-226$ & $->$ & $\mathrm{Rn}-222$ & & & \\
\hline
\end{tabular}




\begin{tabular}{|c|c|c|c|c|}
\hline $1.04 \mathrm{E}-11$ & 13.066 & $\mathrm{~Pb}-210$ & $->$ & $\mathrm{Bi}-210$ \\
\hline $1.04 \mathrm{E}-11$ & 964.07 & $\mathrm{Bi}-214$ & $->$ & Po-214 \\
\hline $1.04 \mathrm{E}-11$ & 1838.37 & $\mathrm{Bi}-214$ & $->$ & Po-214 \\
\hline $1.04 \mathrm{E}-11$ & 11.119 & $\mathrm{Bi}-214$ & $->$ & Po-214 \\
\hline $1.00 \mathrm{E}-11$ & 13.502 & $\mathrm{Bi}-214$ & $->$ & Po-214 \\
\hline $9.89 \mathrm{E}-12$ & 580.06 & $\mathrm{~Pb}-214$ & $->$ & $\mathrm{Bi}-214$ \\
\hline $9.77 \mathrm{E}-12$ & 386.834 & $\mathrm{Bi}-214$ & $->$ & Po-214 \\
\hline $9.50 \mathrm{E}-12$ & 1543.347 & $\mathrm{Bi}-214$ & $->$ & Po-214 \\
\hline $9.37 \mathrm{E}-12$ & 89.639 & $\mathrm{Bi}-214$ & $->$ & Po-214 \\
\hline $9.18 \mathrm{E}-12$ & 480.32 & $\mathrm{~Pb}-214$ & $->$ & $\mathrm{Bi}-214$ \\
\hline 9.07E-12 & 1599.3 & $\mathrm{Bi}-214$ & $->$ & Po-214 \\
\hline $8.80 \mathrm{E}-12$ & 2293.29 & $\mathrm{Bi}-214$ & $->$ & Po-214 \\
\hline 8.69E-12 & 274.56 & $\mathrm{~Pb}-214$ & $->$ & $\mathrm{Bi}-214$ \\
\hline 8.63E-12 & 454.832 & $\mathrm{Bi}-214$ & $->$ & Po-214 \\
\hline 8.55E-12 & 1051.95 & $\mathrm{Bi}-214$ & $->$ & Po-214 \\
\hline $8.42 \mathrm{E}-12$ & 786.42 & $\mathrm{Bi}-214$ & $->$ & Po-214 \\
\hline 8.35E-12 & 9.419 & $\mathrm{~Pb}-210$ & $->$ & $\mathrm{Bi}-210$, \\
\hline $8.02 \mathrm{E}-12$ & 83.787 & Ra-226 & $->$ & $\mathrm{Rn}-22$ \\
\hline 7.74E-12 & 1070.02 & $\mathrm{Bi}-214$ & $->$ & Po-21 \\
\hline $7.28 \mathrm{E}-12$ & 11.713 & Ra-226 & $->$ & $\mathrm{Rn}-22$ \\
\hline $7.20 \mathrm{E}-12$ & 1594.78 & $\mathrm{Bi}-214$ & $->$ & Po-21 \\
\hline $6.92 \mathrm{E}-12$ & 1133.65 & $\mathrm{Bi}-214$ & $->$ & Po-21 \\
\hline $6.41 \mathrm{E}-12$ & 1683.99 & $\mathrm{Bi}-214$ & $->$ & Po-21 \\
\hline $6.14 \mathrm{E}-12$ & 1873.112 & $\mathrm{Bi}-214$ & $->$ & Po-214 \\
\hline $5.16 \mathrm{E}-12$ & 533.5 & $\mathrm{~Pb}-214$ & $->$ & $\mathrm{Bi}-214$ \\
\hline 4.89E-12 & 273.7 & $\mathrm{Bi}-214$ & $->$ & Po-21 \\
\hline $4.83 \mathrm{E}-12$ & 81.067 & $\mathrm{Ra}-226$ & $->$ & $\mathrm{Rn}-22$ \\
\hline $4.81 \mathrm{E}-12$ & 1896.28 & $\mathrm{Bi}-214$ & $->$ & Po-21 \\
\hline $4.62 \mathrm{E}-12$ & 462.05 & $\mathrm{~Pb}-214$ & $->$ & $\mathrm{Bi}-214$ \\
\hline 4.53E-12 & 405.73 & $\mathrm{Bi}-214$ & $->$ & Po-21 \\
\hline 4.07E-12 & 821.166 & $\mathrm{Bi}-214$ & $->$ & Po-21 \\
\hline $4.06 \mathrm{E}-12$ & 46.52 & $\mathrm{~Pb}-210$ & $->$ & $\mathrm{Bi}-210$ \\
\hline $3.61 \mathrm{E}-12$ & 469.76 & $\mathrm{Bi}-214$ & $->$ & Po-21 \\
\hline $3.61 \mathrm{E}-12$ & 752.843 & $\mathrm{Bi}-214$ & $->$ & Po-21 \\
\hline $3.29 \mathrm{E}-12$ & 1303.76 & $\mathrm{Bi}-214$ & $->$ & Po-21 \\
\hline $3.20 \mathrm{E}-12$ & 474.51 & $\mathrm{Bi}-214$ & $->$ & Po-21 \\
\hline 2.99E-12 & 426.5 & $\mathrm{Bi}-214$ & $->$ & Po-21 \\
\hline $2.85 \mathrm{E}-12$ & 904.33 & $\mathrm{Bi}-214$ & $->$ & Po-21 \\
\hline $2.85 \mathrm{E}-12$ & 92.673 & $\mathrm{Bi}-214$ & $->$ & Po-21 \\
\hline $2.83 \mathrm{E}-12$ & 94.677 & $\mathrm{Ra}-226$ & $->$ & $\mathrm{Rn}-22$ \\
\hline $2.72 \mathrm{E}-12$ & 1103.7 & $\mathrm{Bi}-214$ & $->$ & Po-21 \\
\hline $2.61 \mathrm{E}-12$ & 1032.22 & $\mathrm{Bi}-214$ & $->$ & Po-21 \\
\hline $2.58 \mathrm{E}-12$ & 333.6 & $\mathrm{Bi}-214$ & $->$ & Po-214 \\
\hline $2.50 \mathrm{E}-12$ & 15.537 & $\mathrm{~Pb}-210$ & $->$ & $\mathrm{Bi}-210$ \\
\hline $2.50 \mathrm{E}-12$ & 826.44 & $\mathrm{Bi}-214$ & $->$ & Po-21 \\
\hline $2.42 \mathrm{E}-12$ & 1890.259 & $\mathrm{Bi}-214$ & $->$ & Po-21 \\
\hline $2.36 \mathrm{E}-12$ & 2109.91 & $\mathrm{Bi}-214$ & $->$ & Po-21 \\
\hline $2.34 \mathrm{E}-12$ & 1317.02 & $\mathrm{Bi}-214$ & $->$ & Po-21 \\
\hline $2.31 \mathrm{E}-12$ & 542.84 & $\mathrm{Bi}-214$ & $->$ & Po-21 \\
\hline $2.31 \mathrm{E}-12$ & 16.874 & $\mathrm{Ra}-226$ & $->$ & $\mathrm{Rn}-22$ \\
\hline
\end{tabular}




\begin{tabular}{|c|c|c|c|c|c|c|c|}
\hline $2.29 \mathrm{E}-12$ & 109.97 & Th-230 & $->$ & Ra-226 & & & \\
\hline $2.23 \mathrm{E}-12$ & 572.67 & $\mathrm{Bi}-214$ & $->$ & Po-214 & & & \\
\hline $2.20 \mathrm{E}-12$ & 280.93 & $\mathrm{Bi}-214$ & $->$ & Po-214 & & & \\
\hline $2.17 \mathrm{E}-12$ & 1104.766 & $\mathrm{Bi}-214$ & $->$ & Po-214 & & & \\
\hline $2.15 \mathrm{E}-12$ & 765.9 & $\mathrm{~Pb}-214$ & $->$ & $\mathrm{Bi}-214$ & & & \\
\hline $2.15 \mathrm{E}-12$ & 314.2 & $\mathrm{~Pb}-214$ & $->$ & $\mathrm{Bi}-214$ & & & \\
\hline $2.14 \mathrm{E}-12$ & 683.21 & $\mathrm{Bi}-214$ & $->$ & Po-214 & & & \\
\hline $2.03 E-12$ & 710.84 & $\mathrm{Bi}-214$ & $->$ & Po-214 & & & \\
\hline $1.95 \mathrm{E}-12$ & 11.712 & $\mathrm{~Pb}-210$ & $->$ & $\mathrm{Bi}-210$, & $\mathrm{Pb}-214$ & $->$ & $\mathrm{Bi}-214$ \\
\hline $1.93 \mathrm{E}-12$ & 15.874 & $\mathrm{Bi}-214$ & $->$ & Po-214 & & & \\
\hline $1.93 \mathrm{E}-12$ & 536.93 & $\mathrm{Bi}-214$ & $->$ & Po-214 & & & \\
\hline $1.90 \mathrm{E}-12$ & 510 & $\mathrm{Rn}-222$ & $->$ & Po-218 & & & \\
\hline $1.90 \mathrm{E}-12$ & 1637.37 & $\mathrm{Bi}-214$ & $->$ & Po-214 & & & \\
\hline $1.90 \mathrm{E}-12$ & 2052.93 & $\mathrm{Bi}-214$ & $->$ & Po-214 & & & \\
\hline $1.90 \mathrm{E}-12$ & 615.77 & $\mathrm{Bi}-214$ & $->$ & Po-214 & & & \\
\hline $1.87 \mathrm{E}-12$ & 1479.19 & $\mathrm{Bi}-214$ & $->$ & Po-214 & & & \\
\hline $1.71 \mathrm{E}-12$ & 1898.9 & $\mathrm{Bi}-214$ & $->$ & Po-214 & & & \\
\hline $1.66 \mathrm{E}-12$ & 2192.52 & $\mathrm{Bi}-214$ & $->$ & Po-214 & & & \\
\hline $1.63 \mathrm{E}-12$ & 633.14 & $\mathrm{Bi}-214$ & $->$ & Po-214 & & & \\
\hline $1.63 \mathrm{E}-12$ & 347.1 & $\mathrm{Bi}-214$ & $->$ & Po-214 & & & \\
\hline $1.60 \mathrm{E}-12$ & 137.4 & $\mathrm{~Pb}-214$ & $->$ & $\mathrm{Bi}-214$ & & & \\
\hline $1.60 \mathrm{E}-12$ & 649.18 & $\mathrm{Bi}-214$ & $->$ & Po-214 & & & \\
\hline $1.57 \mathrm{E}-12$ & 1173.04 & $\mathrm{Bi}-214$ & $->$ & Рo-214 & & & \\
\hline $1.57 \mathrm{E}-12$ & 1172.93 & $\mathrm{Bi}-214$ & $->$ & Po-214 & & & \\
\hline $1.55 \mathrm{E}-12$ & 334.9 & $\mathrm{Bi}-214$ & $->$ & Po-214 & & & \\
\hline $1.52 \mathrm{E}-12$ & 2089.55 & $\mathrm{Bi}-214$ & $->$ & Po-214 & & & \\
\hline $1.38 \mathrm{E}-12$ & 1935.8 & $\mathrm{Bi}-214$ & $->$ & Po-214 & & & \\
\hline $1.33 \mathrm{E}-12$ & 196.3 & $\mathrm{~Pb}-214$ & $->$ & $\mathrm{Bi}-214$ & & & \\
\hline $1.33 \mathrm{E}-12$ & 2010.79 & $\mathrm{Bi}-214$ & $->$ & Po-214 & & & \\
\hline $1.28 \mathrm{E}-12$ & 733.64 & $\mathrm{Bi}-214$ & $->$ & Po-214 & & & \\
\hline $1.22 \mathrm{E}-12$ & 1130.6 & $\mathrm{Bi}-214$ & $->$ & Po-214 & & & \\
\hline $1.22 \mathrm{E}-12$ & 723.32 & $\mathrm{Bi}-214$ & $->$ & Po-214 & & & \\
\hline $1.17 \mathrm{E}-12$ & 660.75 & $\mathrm{Bi}-214$ & $->$ & Po-214 & & & \\
\hline $1.11 \mathrm{E}-12$ & 799.75 & $\mathrm{Bi}-214$ & $->$ & Po-214 & & & \\
\hline $1.09 \mathrm{E}-12$ & 814.87 & $\mathrm{Bi}-214$ & $->$ & Po-214 & & & \\
\hline $1.06 \mathrm{E}-12$ & 141.3 & $\mathrm{~Pb}-214$ & $->$ & $\mathrm{Bi}-214$ & & & \\
\hline $1.06 \mathrm{E}-12$ & 740.87 & $\mathrm{Bi}-214$ & $->$ & Po-214 & & & \\
\hline $1.06 \mathrm{E}-12$ & 338.5 & $\mathrm{Bi}-214$ & $->$ & Po-214 & & & \\
\hline $1.00 \mathrm{E}-12$ & 697.89 & $\mathrm{Bi}-214$ & $->$ & Po-214 & & & \\
\hline $9.23 \mathrm{E}-13$ & 304.42 & $\mathrm{Bi}-214$ & $->$ & Рo-214 & & & \\
\hline $9.23 \mathrm{E}-13$ & 617.1 & $\mathrm{Bi}-214$ & $->$ & Po-214 & & & \\
\hline $9.04 \mathrm{E}-13$ & 97.907 & $\mathrm{Ra}-226$ & $->$ & $\mathrm{Rn}-222$ & & & \\
\hline $8.80 \mathrm{E}-13$ & 2694.67 & $\mathrm{Bi}-214$ & $->$ & Po-214 & & & \\
\hline 8.69E-13 & 286.9 & $\mathrm{Bi}-214$ & $->$ & Po-214 & & & \\
\hline 8.69E-13 & 547.1 & $\mathrm{Bi}-214$ & $->$ & Po-214 & & & \\
\hline $8.46 \mathrm{E}-13$ & 797.88 & $\mathrm{TI}-210$ & $->$ & $\mathrm{Pb}-210$, & Po-214 & $->$ & $\mathrm{Pb}-210$ \\
\hline $8.42 E-13$ & 639.36 & $\mathrm{Bi}-214$ & $->$ & Po-214 & & & \\
\hline $8.15 E-13$ & 396 & $\mathrm{Bi}-214$ & $->$ & Po-214 & & & \\
\hline $7.88 \mathrm{E}-13$ & 511 & $\mathrm{~Pb}-214$ & $->$ & $\mathrm{Bi}-214$ & & & \\
\hline 7.87E-13 & 440.4 & $\mathrm{Bi}-214$ & $->$ & Рo-214 & & & \\
\hline
\end{tabular}




\begin{tabular}{|c|c|c|c|c|c|c|c|}
\hline 7.87E-13 & 1045.4 & $\mathrm{Bi}-214$ & $\rightarrow$ & Po-214 & & & \\
\hline 7.87E-13 & 1067.3 & $\mathrm{Bi}-214$ & $->$ & Po-214 & & & \\
\hline 7.33E-13 & 1226.8 & $\mathrm{Bi}-214$ & $->$ & Po-214 & & & \\
\hline $6.95 \mathrm{E}-13$ & 2769.99 & $\mathrm{Bi}-214$ & $->$ & Po-214 & & & \\
\hline $6.25 \mathrm{E}-13$ & 543.91 & $\mathrm{~Pb}-214$ & $->$ & Bi-214 & & & \\
\hline $6.25 \mathrm{E}-13$ & 305.5 & $\mathrm{~Pb}-214$ & $->$ & $\mathrm{Bi}-214$ & & & \\
\hline $6.24 \mathrm{E}-13$ & 1341.5 & $\mathrm{Bi}-214$ & $->$ & Po-214 & & & \\
\hline $6.24 \mathrm{E}-13$ & 832.34 & $\mathrm{Bi}-214$ & $->$ & Po-214 & & & \\
\hline $6.24 \mathrm{E}-13$ & 976.2 & $\mathrm{Bi}-214$ & $->$ & Po-214 & & & \\
\hline $6.24 \mathrm{E}-13$ & 915.8 & $\mathrm{Bi}-214$ & $->$ & Po-214 & & & \\
\hline $6.14 \mathrm{E}-13$ & 3053.89 & $\mathrm{Bi}-214$ & $->$ & Po-214 & & & \\
\hline $5.97 \mathrm{E}-13$ & 1230.84 & $\mathrm{Bi}-214$ & $->$ & Po-214 & & & \\
\hline $5.97 \mathrm{E}-13$ & 2331.2 & $\mathrm{Bi}-214$ & $->$ & Po-214 & & & \\
\hline $5.43 \mathrm{E}-13$ & 324.3 & $\mathrm{~Pb}-214$ & $->$ & $\mathrm{Bi}-214$ & & & \\
\hline $5.43 \mathrm{E}-13$ & 9.658 & $\mathrm{Bi}-214$ & $->$ & Рo-214 & & & \\
\hline $5.16 \mathrm{E}-13$ & 1636.6 & $\mathrm{Bi}-214$ & $->$ & Po-214 & & & \\
\hline $5.16 \mathrm{E}-13$ & 2021.7 & $\mathrm{Bi}-214$ & $->$ & Po-214 & & & \\
\hline $5.16 \mathrm{E}-13$ & 1392.5 & $\mathrm{Bi}-214$ & $->$ & Po-214 & & & \\
\hline $4.89 \mathrm{E}-13$ & 2266.67 & $\mathrm{Bi}-214$ & $->$ & Рo-214 & & & \\
\hline $4.89 E-13$ & 502.2 & $\mathrm{Bi}-214$ & $->$ & Po-214 & & & \\
\hline $4.62 \mathrm{E}-13$ & 943.3 & $\mathrm{Bi}-214$ & $->$ & Po-214 & & & \\
\hline $4.62 \mathrm{E}-13$ & 847.2 & $\mathrm{Bi}-214$ & $->$ & Po-214 & & & \\
\hline $4.62 \mathrm{E}-13$ & 631.2 & $\mathrm{Bi}-214$ & $->$ & Po-214 & & & \\
\hline $4.62 \mathrm{E}-13$ & 1038 & $\mathrm{Bi}-214$ & $->$ & Po-214 & & & \\
\hline $4.52 \mathrm{E}-13$ & 298.1 & $\mathrm{Tl}-210$ & $->$ & $\mathrm{Pb}-210$, & Po-214 & $->$ & $\mathrm{Pb}-210$ \\
\hline $4.34 \mathrm{E}-13$ & 2147.8 & $\mathrm{Bi}-214$ & $->$ & Po-214 & & & \\
\hline $4.34 \mathrm{E}-13$ & 525 & $\mathrm{Bi}-214$ & $->$ & Po-214 & & & \\
\hline $4.34 \mathrm{E}-13$ & 1782.1 & $\mathrm{Bi}-214$ & $->$ & Po-214 & & & \\
\hline $4.26 \mathrm{E}-13$ & 2922.09 & $\mathrm{Bi}-214$ & $->$ & Po-214 & & & \\
\hline $4.26 \mathrm{E}-13$ & 727.8 & $\mathrm{Bi}-214$ & $->$ & Po-214 & & & \\
\hline $3.99 \mathrm{E}-13$ & 2978.79 & $\mathrm{Bi}-214$ & $->$ & Po-214 & & & \\
\hline $3.93 E-13$ & 10.137 & Ra-226 & $->$ & $\mathrm{Rn}-222$ & & & \\
\hline $3.26 \mathrm{E}-13$ & 1814.01 & $\mathrm{Bi}-214$ & $->$ & Po-214 & & & \\
\hline $3.26 \mathrm{E}-13$ & 989.2 & $\mathrm{Bi}-214$ & $->$ & Рo-214 & & & \\
\hline $3.26 \mathrm{E}-13$ & 1471.1 & $\mathrm{Bi}-214$ & $->$ & Po-214 & & & \\
\hline $3.26 \mathrm{E}-13$ & 1020.5 & $\mathrm{Bi}-214$ & $->$ & Po-214 & & & \\
\hline $3.26 \mathrm{E}-13$ & 596 & $\mathrm{Bi}-214$ & $->$ & Po-214 & & & \\
\hline $3.20 \mathrm{E}-13$ & 2376.99 & $\mathrm{Bi}-214$ & $->$ & Po-214 & & & \\
\hline $3.14 \mathrm{E}-13$ & 235.01 & Th-230 & $->$ & Ra-226 & & & \\
\hline $2.99 \mathrm{E}-13$ & 1330 & $\mathrm{Bi}-214$ & $->$ & Po-214 & & & \\
\hline $2.72 \mathrm{E}-13$ & 470.8 & $\mathrm{~Pb}-214$ & $->$ & $\mathrm{Bi}-214$ & & & \\
\hline $2.72 \mathrm{E}-13$ & 2085 & $\mathrm{Bi}-214$ & $->$ & Po-214 & & & \\
\hline $2.72 \mathrm{E}-13$ & 1013.4 & $\mathrm{Bi}-214$ & $->$ & Рo-214 & & & \\
\hline $2.50 \mathrm{E}-13$ & 2880.4 & $\mathrm{Bi}-214$ & $->$ & Po-214 & & & \\
\hline $2.44 \mathrm{E}-13$ & 494.6 & $\mathrm{Bi}-214$ & $->$ & Po-214 & & & \\
\hline $2.44 \mathrm{E}-13$ & 394 & $\mathrm{Bi}-214$ & $->$ & Po-214 & & & \\
\hline $2.44 \mathrm{E}-13$ & 2259.7 & $\mathrm{Bi}-214$ & $->$ & Рo-214 & & & \\
\hline $2.39 \mathrm{E}-13$ & 2999.99 & $\mathrm{Bi}-214$ & $->$ & Po-214 & & & \\
\hline $2.01 \mathrm{E}-13$ & 12.855 & $\mathrm{Ra}-226$ & $->$ & $\mathrm{Rn}-222$ & & & \\
\hline $1.94 \mathrm{E}-13$ & 205.1 & Th-230 & $->$ & Ra-226 & & & \\
\hline
\end{tabular}




\begin{tabular}{|c|c|c|c|c|c|c|c|}
\hline $1.90 \mathrm{E}-13$ & 2251.2 & $\mathrm{Bi}-214$ & $\rightarrow$ & Po-214 & & & \\
\hline $1.74 \mathrm{E}-13$ & 2893.59 & $\mathrm{Bi}-214$ & $->$ & Po-214 & & & \\
\hline $1.71 \mathrm{E}-13$ & 364.2 & $\mathrm{Bi}-214$ & $->$ & Po-214 & & & \\
\hline $1.63 \mathrm{E}-13$ & 693.3 & $\mathrm{Bi}-214$ & $->$ & Po-214 & & & \\
\hline $1.63 \mathrm{E}-13$ & 687.7 & $\mathrm{Bi}-214$ & $->$ & Po-214 & & & \\
\hline $1.60 \mathrm{E}-13$ & 2505.58 & $\mathrm{Bi}-214$ & $->$ & Рo-214 & & & \\
\hline $1.60 \mathrm{E}-13$ & 2423.32 & $\mathrm{Bi}-214$ & $->$ & Po-214 & & & \\
\hline $1.60 \mathrm{E}-13$ & 2786.09 & $\mathrm{Bi}-214$ & $->$ & Po-214 & & & \\
\hline $1.55 \mathrm{E}-13$ & 520.4 & $\mathrm{Bi}-214$ & $->$ & Po-214 & & & \\
\hline $1.49 \mathrm{E}-13$ & 12.085 & $\mathrm{Bi}-214$ & $->$ & Po-214 & & & \\
\hline $1.48 \mathrm{E}-13$ & 262.41 & $\mathrm{Ra}-226$ & $->$ & $\mathrm{Rn}-222$ & & & \\
\hline $1.38 \mathrm{E}-13$ & 2284.4 & $\mathrm{Bi}-214$ & $->$ & Po-214 & & & \\
\hline $1.38 \mathrm{E}-13$ & 1419.7 & $\mathrm{Bi}-214$ & $->$ & Po-214 & & & \\
\hline $1.36 \mathrm{E}-13$ & 1994.7 & $\mathrm{Bi}-214$ & $->$ & Po-214 & & & \\
\hline $1.33 \mathrm{E}-13$ & 538.7 & $\mathrm{~Pb}-214$ & $->$ & $\mathrm{Bi}-214$ & & & \\
\hline $1.33 \mathrm{E}-13$ & 626.4 & $\mathrm{Bi}-214$ & $->$ & Po-214 & & & \\
\hline $1.33 \mathrm{E}-13$ & 376.6 & $\mathrm{Bi}-214$ & $->$ & Po-214 & & & \\
\hline $1.20 \mathrm{E}-13$ & 1314 & TI-210 & $->$ & $\mathrm{Pb}-210$ & & & \\
\hline $1.17 \mathrm{E}-13$ & 3081.7 & $\mathrm{Bi}-214$ & $->$ & Рo-214 & & & \\
\hline $1.16 \mathrm{E}-13$ & 570.5 & Th-230 & $->$ & $\mathrm{Ra}-226$ & & & \\
\hline 7.87E-14 & 2004.5 & $\mathrm{Bi}-214$ & $->$ & Po-214 & & & \\
\hline 7.87E-14 & 2369.3 & $\mathrm{Bi}-214$ & $->$ & Po-214 & & & \\
\hline $7.87 E-14$ & 2270 & $\mathrm{Bi}-214$ & $->$ & Рo-214 & & & \\
\hline $7.60 \mathrm{E}-14$ & 2698.86 & $\mathrm{Bi}-214$ & $->$ & Po-214 & & & \\
\hline $6.84 \mathrm{E}-14$ & 1068 & TI-210 & $->$ & $\mathrm{Pb}-210$ & & & \\
\hline $6.79 \mathrm{E}-14$ & 2827 & $\mathrm{Bi}-214$ & $->$ & Po-214 & & & \\
\hline $6.27 \mathrm{E}-14$ & 12.678 & $\mathrm{Tl}-210$ & $->$ & $\mathrm{Pb}-210$ & & & \\
\hline $5.70 \mathrm{E}-14$ & 2482.417 & $\mathrm{Bi}-214$ & $->$ & Po-214 & & & \\
\hline $5.43 E-14$ & 2390.9 & $\mathrm{Bi}-214$ & $->$ & Po-214 & & & \\
\hline $5.16 \mathrm{E}-14$ & 2324.8 & $\mathrm{Bi}-214$ & $->$ & Po-214 & & & \\
\hline $5.16 \mathrm{E}-14$ & 2360.9 & $\mathrm{Bi}-214$ & $->$ & Po-214 & & & \\
\hline $5.13 \mathrm{E}-14$ & 2428 & $\mathrm{Tl}-210$ & $->$ & $\mathrm{Pb}-210$ & & & \\
\hline $4.89 \mathrm{E}-14$ & 2719.21 & $\mathrm{Bi}-214$ & $->$ & Po-214 & & & \\
\hline $4.73 E-14$ & 10.541 & TI-206 & $->$ & $\mathrm{Pb}-206$, & TI-210 & $->$ & $\mathrm{Pb}-210$ \\
\hline $4.62 \mathrm{E}-14$ & 2940.5 & $\mathrm{Bi}-214$ & $->$ & Po-214 & & & \\
\hline $4.56 \mathrm{E}-14$ & 2358 & $\mathrm{TI}-210$ & $->$ & $\mathrm{Pb}-210$ & & & \\
\hline $4.34 \mathrm{E}-14$ & 3142.6 & $\mathrm{Bi}-214$ & $->$ & Po-214 & & & \\
\hline $4.07 E-14$ & 3183.6 & $\mathrm{Bi}-214$ & $->$ & Po-214 & & & \\
\hline $3.93 E-14$ & 2008 & TI-210 & $->$ & $\mathrm{Pb}-210$ & & & \\
\hline $3.93 E-14$ & 1110 & Tl-210 & $->$ & $\mathrm{Pb}-210$ & & & \\
\hline $3.93 E-14$ & 860 & $\mathrm{TI}-210$ & $->$ & $\mathrm{Pb}-210$ & & & \\
\hline $3.92 \mathrm{E}-14$ & 551.8 & Th-230 & $->$ & $\mathrm{Ra}-226$ & & & \\
\hline $3.92 \mathrm{E}-14$ & 620 & Th-230 & $->$ & Ra-226 & & & \\
\hline $3.26 \mathrm{E}-14$ & 2928.7 & $\mathrm{Bi}-214$ & $->$ & Po-214 & & & \\
\hline $2.99 \mathrm{E}-14$ & 2988.7 & $\mathrm{Bi}-214$ & $->$ & Po-214 & & & \\
\hline $2.79 \mathrm{E}-14$ & 2088 & TI-210 & $->$ & $\mathrm{Pb}-210$ & & & \\
\hline $2.79 \mathrm{E}-14$ & 1408 & $\mathrm{Tl}-210$ & $->$ & $\mathrm{Pb}-210$ & & & \\
\hline $2.62 \mathrm{E}-14$ & 74.969 & Tl-206 & $->$ & $\mathrm{Pb}-206$ & TI-210 & $->$ & $\mathrm{Pb}-210$ \\
\hline $2.44 \mathrm{E}-14$ & 2631 & $\mathrm{Bi}-214$ & $->$ & Po-214 & & & \\
\hline $2.28 \mathrm{E}-14$ & 95 & TI-210 & $->$ & $\mathrm{Pb}-210$ & & & \\
\hline
\end{tabular}




\begin{tabular}{|c|c|}
\hline $2.28 \mathrm{E}-14$ & 354 \\
\hline $1.71 \mathrm{E}-14$ & 2268 \\
\hline $1.71 \mathrm{E}-14$ & 908 \\
\hline $1.71 \mathrm{E}-14$ & 380 \\
\hline $1.68 \mathrm{E}-14$ & 600.83 \\
\hline $1.60 \mathrm{E}-14$ & 72.803 \\
\hline $1.55 \mathrm{E}-14$ & 2934.9 \\
\hline $1.38 \mathrm{E}-14$ & 3093.9 \\
\hline $1.38 \mathrm{E}-14$ & 3160.5 \\
\hline $1.25 \mathrm{E}-14$ & 14.836 \\
\hline $1.22 \mathrm{E}-14$ & 2604.5 \\
\hline $1.14 \mathrm{E}-14$ & 81 \\
\hline $1.14 \mathrm{E}-14$ & 478 \\
\hline $1.14 \mathrm{E}-14$ & 668 \\
\hline $1.14 \mathrm{E}-14$ & 1538 \\
\hline $1.14 \mathrm{E}-14$ & 1588 \\
\hline $1.14 \mathrm{E}-14$ & 1648 \\
\hline $1.07 \mathrm{E}-14$ & 414.72 \\
\hline $1.06 \mathrm{E}-14$ & 2551 \\
\hline $9.35 \mathrm{E}-15$ & 84.789 \\
\hline $9.23 E-15$ & 3136.3 \\
\hline $9.23 \mathrm{E}-15$ & 2860.9 \\
\hline $7.87 E-15$ & 2662.23 \\
\hline $7.42 \mathrm{E}-15$ & 449.5 \\
\hline $5.43 \mathrm{E}-15$ & 3233.3 \\
\hline $2.72 \mathrm{E}-15$ & 3269.7 \\
\hline $2.68 \mathrm{E}-15$ & 87.632 \\
\hline $2.34 \mathrm{E}-15$ & 9.185 \\
\hline $1.14 \mathrm{E}-15$ & 11.349 \\
\hline $6.76 \mathrm{E}-16$ & 803.13 \\
\hline 5.99E-17 & 302.25 \\
\hline $9.31 \mathrm{E}-18$ & 72.873 \\
\hline $5.54 \mathrm{E}-18$ & 70.832 \\
\hline $5.54 \mathrm{E}-18$ & 650.21 \\
\hline $3.33 \mathrm{E}-18$ & 82.434 \\
\hline $3.33 \mathrm{E}-18$ & 10.259 \\
\hline $2.88 \mathrm{E}-18$ & 12.313 \\
\hline $1.15 \mathrm{E}-18$ & 344.96 \\
\hline $9.31 \mathrm{E}-19$ & 85.185 \\
\hline $5.32 \mathrm{E}-19$ & 14.407 \\
\hline $1.60 \mathrm{E}-19$ & 8.953 \\
\hline $4.21 \mathrm{E}-20$ & 10.994 \\
\hline $2.95 \mathrm{E}-20$ & 12.71 \\
\hline $8.43 E-21$ & 384.06 \\
\hline 4.69E-22 & 11.439 \\
\hline $5.21 \mathrm{E}-23$ & 266.15 \\
\hline Np-237 & $100 \%$ \\
\hline
\end{tabular}

\begin{tabular}{|c|c|c|c|c|c|}
\hline TI-210 & $->$ & $\mathrm{Pb}-210$ & & & \\
\hline TI-210 & $->$ & $\mathrm{Pb}-210$ & & & \\
\hline TI-210 & $->$ & $\mathrm{Pb}-210$ & & & \\
\hline TI-210 & $->$ & $\mathrm{Pb}-210$ & & & \\
\hline $\mathrm{Ra}-226$ & $->$ & $\mathrm{Rn}-222$ & & & \\
\hline TI-206 & $->$ & Pb-206, & TI-210 & $->$ & $\mathrm{Pb}-210$ \\
\hline $\mathrm{Bi}-214$ & $->$ & Po-214 & & & \\
\hline $\mathrm{Bi}-214$ & $->$ & Po-214 & & & \\
\hline $\mathrm{Bi}-214$ & $->$ & Po-214 & & & \\
\hline TI-206 & $->$ & $\mathrm{Pb}-206$, & TI-210 & $->$ & $\mathrm{Pb}-210$ \\
\hline $\mathrm{Bi}-214$ & $->$ & Po-214 & & & \\
\hline TI-210 & $->$ & $\mathrm{Pb}-210$ & & & \\
\hline TI-210 & $->$ & $\mathrm{Pb}-210$ & & & \\
\hline TI-210 & $->$ & $\mathrm{Pb}-210$ & & & \\
\hline TI-210 & $->$ & $\mathrm{Pb}-210$ & & & \\
\hline TI-210 & $->$ & $\mathrm{Pb}-210$ & & & \\
\hline TI-210 & $->$ & $\mathrm{Pb}-210$ & & & \\
\hline $\mathrm{Ra}-226$ & $->$ & $\mathrm{Rn}-222$ & & & \\
\hline $\mathrm{Bi}-214$ & $->$ & Po-214 & & & \\
\hline TI-206 & $->$ & $\mathrm{Pb}-206$, & TI-210 & $->$ & $\mathrm{Pb}-210$ \\
\hline $\mathrm{Bi}-214$ & $->$ & Po-214 & & & \\
\hline $\mathrm{Bi}-214$ & $->$ & Po-214 & & & \\
\hline $\mathrm{Bi}-214$ & $->$ & Po-214 & & & \\
\hline $\mathrm{Ra}-226$ & $->$ & $\mathrm{Rn}-222$ & & & \\
\hline $\mathrm{Bi}-214$ & $->$ & Po-214 & & & \\
\hline $\mathrm{Bi}-214$ & $->$ & Po-214 & & & \\
\hline TI-206 & $->$ & Pb-206, & TI-210 & $->$ & $\mathrm{Pb}-210$ \\
\hline TI-206 & $->$ & $\mathrm{Pb}-206$ & TI-210 & $->$ & $\mathrm{Pb}-210$ \\
\hline TI-210 & $->$ & $\mathrm{Pb}-210$ & & & \\
\hline TI-206 & $->$ & $\mathrm{Pb}-206$, & Po-210 & $->$ & $\mathrm{Pb}-206$ \\
\hline $\mathrm{Hg}-206$ & $->$ & TI-206 & & & \\
\hline $\mathrm{Hg}-206$ & $->$ & TI-206 & & & \\
\hline $\mathrm{Hg}-206$ & $->$ & TI-206 & & & \\
\hline $\mathrm{Hg}-206$ & $->$ & TI-206 & & & \\
\hline $\mathrm{Hg}-206$ & $->$ & TI-206 & & & \\
\hline $\mathrm{Hg}-206$ & $->$ & TI-206 & & & \\
\hline $\mathrm{Hg}-206$ & $->$ & TI-206 & & & \\
\hline $\mathrm{Hg}-206$ & $->$ & TI-206 & & & \\
\hline $\mathrm{Hg}-206$ & $->$ & TI-206 & & & \\
\hline $\mathrm{Hg}-206$ & $->$ & TI-206 & & & \\
\hline $\mathrm{Hg}-206$ & $->$ & TI-206 & & & \\
\hline $\mathrm{Hg}-206$ & $->$ & TI-206 & & & \\
\hline TI-206 & $->$ & $\mathrm{Pb}-206$ & & & \\
\hline $\mathrm{Hg}-206$ & $->$ & TI-206 & & & \\
\hline TI-206 & $->$ & Pb-206 & & & \\
\hline $\mathrm{Bi}-210$ & $->$ & TI-206 & & & \\
\hline
\end{tabular}


371 lines computed.

Sorted by Energy

\begin{tabular}{|c|c|c|c|c|}
\hline \multirow{2}{*}{$\begin{array}{c}\text { Energy } \\
\text { keV }\end{array}$} & \multirow{2}{*}{$\begin{array}{c}\text { Intensity } \\
\mathrm{ph} / \mathrm{s} / \mathrm{gm}\end{array}$} & \multicolumn{3}{|c|}{ Initial Isotopes and Decays } \\
\hline & & & & \\
\hline 8.953 & $6.12 \mathrm{E}-09$ & $\mathrm{Bi}-213$ & $\rightarrow$ & Tl-209 \\
\hline 9.185 & $5.06 \mathrm{E}-06$ & TI-209 & $->$ & $\mathrm{Pb}-209$ \\
\hline 9.658 & $3.11 \mathrm{E}-05$ & $\mathrm{Bi}-213$ & $->$ & Po-213 \\
\hline 9.897 & $5.17 \mathrm{E}-05$ & Fr-221 & $->$ & At-217 \\
\hline 10.259 & $1.33 \mathrm{E}-07$ & $\mathrm{Bi}-213$ & $->$ & TI-209 \\
\hline 10.381 & $5.30 \mathrm{E}-04$ & Ac- 225 & $->$ & Fr-221 \\
\hline 10.541 & $9.84 \mathrm{E}-05$ & TI-209 & $->$ & $\mathrm{Pb}-209$ \\
\hline 10.871 & $4.01 \mathrm{E}-04$ & $\mathrm{Ra}-225$ & $->$ & Ac- 225 \\
\hline 10.994 & $1.60 \mathrm{E}-09$ & $\mathrm{Bi}-213$ & $->$ & Tl-209 \\
\hline 11.118 & 8.33E-01 & $U-233$ & $->$ & Th-229 \\
\hline 11.119 & 5.97E-04 & $\mathrm{Bi}-213$ & $->$ & Po-213 \\
\hline 11.349 & $1.36 \mathrm{E}-06$ & TI-209 & $->$ & $\mathrm{Pb}-209$ \\
\hline 11.372 & $3.00 \mathrm{E}+05$ & $\mathrm{~Np}-237$ & $->$ & Pa-233 \\
\hline 11.414 & $9.73 E-04$ & Fr-221 & $->$ & At-217 \\
\hline 11.62 & $2.79 \mathrm{E}+05$ & Pa-233 & $->$ & U-233 \\
\hline 12.017 & $9.61 \mathrm{E}-03$ & $A c-225$ & $->$ & Fr-221 \\
\hline 12.085 & 8.07E-06 & $\mathrm{Bi}-213$ & $->$ & Po-213 \\
\hline 12.311 & $1.06 \mathrm{E}-07$ & $\mathrm{Bi}-213$ & $->$ & TI-209 \\
\hline 12.466 & $2.06 \mathrm{E}-05$ & Fr-221 & $->$ & At-217 \\
\hline 12.636 & $6.89 \mathrm{E}-03$ & $\mathrm{Ra}-225$ & $->$ & Ac- 225 \\
\hline 12.703 & $9.31 \mathrm{E}-05$ & TI-209 & $->$ & Pb-209 \\
\hline 12.952 & $1.44 \mathrm{E}+01$ & U-233 & $->$ & Th-229 \\
\hline 13.255 & $1.97 \mathrm{E}-04$ & Ac- 225 & $->$ & Fr-221 \\
\hline 13.274 & $5.01 E+06$ & $\mathrm{~Np}-237$ & $->$ & Pa-233 \\
\hline 13.504 & $5.54 \mathrm{E}-04$ & $\mathrm{Bi}-213$ & $->$ & Рo-213 \\
\hline 13.6 & $4.69 \mathrm{E}+06$ & $\mathrm{~Pa}-233$ & $->$ & U-233 \\
\hline 13.911 & $1.18 \mathrm{E}-03$ & Fr-221 & $->$ & At-217 \\
\hline 14.082 & $1.14 \mathrm{E}-04$ & $\mathrm{Ra}-225$ & $->$ & $A c-225$ \\
\hline 14.402 & $2.13 \mathrm{E}-08$ & $\mathrm{Bi}-213$ & $->$ & TI-209 \\
\hline 14.511 & $3.16 \mathrm{E}-01$ & U-233 & $->$ & Th-229 \\
\hline 14.772 & $1.18 \mathrm{E}-02$ & $A c-225$ & $->$ & Fr-221 \\
\hline 14.871 & $1.68 \mathrm{E}-05$ & TI-209 & $->$ & Pb-209 \\
\hline 14.953 & $1.23 \mathrm{E}+05$ & $\mathrm{~Np}-237$ & $->$ & $\mathrm{Pa}-233$ \\
\hline 15.4 & $6.00 \mathrm{E}+04$ & Pa-233 & $->$ & U-233 \\
\hline 15.662 & 7.64E-03 & Ra-225 & $->$ & Ac- 225 \\
\hline 15.878 & $1.06 \mathrm{E}-04$ & $\mathrm{Bi}-213$ & $->$ & Po-213 \\
\hline 16.141 & $1.83 \mathrm{E}+01$ & $U-233$ & $->$ & Th-229 \\
\hline 16.361 & $2.45 \mathrm{E}-04$ & Fr-221 & $->$ & At-217 \\
\hline 16.632 & $6.52 E+06$ & $\mathrm{~Np}-237$ & $->$ & Pa-233 \\
\hline 17.058 & $4.96 \mathrm{E}+06$ & $\mathrm{~Pa}-233$ & $->$ & U-233 \\
\hline 17.269 & $9.39 \mathrm{E}+02$ & $\mathrm{~Pa}-233$ & $->$ & U-233 \\
\hline
\end{tabular}




\begin{tabular}{|c|c|c|c|c|}
\hline 17.349 & $2.18 \mathrm{E}-04$ & Th-229 & $->$ & Ra-225 \\
\hline 17.449 & $2.59 \mathrm{E}-03$ & $A c-225$ & $->$ & Fr-221 \\
\hline 18.593 & $1.75 \mathrm{E}-03$ & $\mathrm{Ra}-225$ & $->$ & Ac- 225 \\
\hline 19.148 & $4.44 \mathrm{E}+00$ & U-233 & $->$ & Th-229 \\
\hline 19.718 & $1.51 \mathrm{E}+06$ & $\mathrm{~Np}-237$ & $->$ & $\mathrm{Pa}-233$ \\
\hline 20.42 & $1.17 \mathrm{E}+06$ & $\mathrm{~Pa}-233$ & $->$ & U-233 \\
\hline 25.304 & $6.72 \mathrm{E}-03$ & $U-233$ & $->$ & Th-229 \\
\hline 25.373 & 4.62E-05 & Th-229 & $->$ & Ra-225 \\
\hline 25.98 & $1.85 \mathrm{E}-06$ & Ac-225 & $->$ & Fr-221 \\
\hline 28.578 & $1.70 \mathrm{E}+04$ & Pa-233 & $->$ & $U-233$ \\
\hline 29.191 & $3.78 \mathrm{E}-02$ & U-233 & $->$ & Th-229 \\
\hline 29.378 & $3.36 \mathrm{E}+06$ & Np-237 & $->$ & $\mathrm{Pa}-233$ \\
\hline 31.24 & $1.50 \mathrm{E}-03$ & U-233 & $->$ & Th-229 \\
\hline 31.37 & $5.26 \mathrm{E}-03$ & Th-229 & $->$ & $\mathrm{Ra}-225$ \\
\hline 32.27 & $5.50 \mathrm{E}-03$ & U-233 & $->$ & Th-229 \\
\hline 36.65 & $1.85 \mathrm{E}-05$ & Ac- 225 & $->$ & Fr-221 \\
\hline 37.85 & 2.00E-03 & U-233 & $->$ & Th-229 \\
\hline 38.51 & $1.11 \mathrm{E}-05$ & Ac- 225 & $->$ & Fr-221 \\
\hline 40.34 & 3.63E-02 & $\mathrm{Ra}-225$ & $->$ & Ac- 225 \\
\hline 40.415 & $9.39 E+03$ & $\mathrm{~Pa}-233$ & $->$ & U-233 \\
\hline 41.718 & $3.39 E+03$ & $\mathrm{~Pa}-233$ & $->$ & $U-233$ \\
\hline 42.441 & 3.33E-01 & U-233 & $->$ & Th-229 \\
\hline 42.722 & $2.05 E-04$ & Th-229 & $->$ & Ra-225 \\
\hline 46.57 & $3.47 E+04$ & $\mathrm{~Np}-237$ & $->$ & Pa-233 \\
\hline 49.2 & $1.33 \mathrm{E}-05$ & Ac- 225 & $->$ & Fr-221 \\
\hline 52.613 & $1.39 \mathrm{E}-03$ & U-233 & $->$ & Th-229 \\
\hline 53.559 & $2.39 \mathrm{E}-02$ & $U-233$ & $->$ & Th-229 \\
\hline 53.82 & $2.34 \mathrm{E}-05$ & $A c-225$ & $->$ & Fr-221 \\
\hline 54.702 & 7.77E-02 & $U-233$ & $->$ & Th-229 \\
\hline 56.58 & $4.23 E-04$ & Th-229 & $->$ & Ra-225 \\
\hline 57.149 & $1.02 \mathrm{E}+05$ & $\mathrm{~Np}-237$ & $->$ & $\mathrm{Pa}-233$ \\
\hline 57.77 & 5.17E-06 & Ac- 225 & $->$ & Fr-221 \\
\hline 62.66 & $3.13 E+03$ & $\mathrm{~Np}-237$ & $->$ & $\mathrm{Pa}-233$ \\
\hline 62.93 & $6.78 \mathrm{E}-04$ & $A c-225$ & $->$ & Fr-221 \\
\hline 63.69 & $1.83 \mathrm{E}-04$ & U-233 & $->$ & Th-229 \\
\hline 63.95 & $4.17 E+03$ & $\mathrm{~Np}-237$ & $->$ & Pa-233 \\
\hline 64.23 & $6.90 \mathrm{E}-05$ & $A c-225$ & $->$ & Fr-221 \\
\hline 66.11 & $4.66 \mathrm{E}-03$ & $U-233$ & $->$ & Th-229 \\
\hline 67.96 & $1.78 \mathrm{E}-03$ & U-233 & $->$ & Th-229 \\
\hline 68.14 & $1.28 \mathrm{E}-04$ & Th-229 & $->$ & Ra-225 \\
\hline 68.88 & $1.41 \mathrm{E}-04$ & Th-229 & $->$ & Ra-225 \\
\hline 68.97 & $6.00 \mathrm{E}-04$ & U-233 & $->$ & Th-229 \\
\hline 69.85 & $6.28 \mathrm{E}-06$ & Ac- 225 & $->$ & Fr-221 \\
\hline 70.35 & $3.33 E-03$ & U-233 & $->$ & Th-229 \\
\hline 70.62 & $4.17 E+03$ & $\mathrm{~Np}-237$ & $->$ & $\mathrm{Pa}-233$ \\
\hline 70.832 & $2.13 \mathrm{E}-07$ & $\mathrm{Bi}-213$ & $->$ & TI-209 \\
\hline 70.92 & $1.23 \mathrm{E}-05$ & Ac-225 & $->$ & Fr-221 \\
\hline 71.71 & $1.81 \mathrm{E}-05$ & Ac-225 & $->$ & Fr-221 \\
\hline 71.847 & $1.50 \mathrm{E}-02$ & U-233 & $->$ & Th-229 \\
\hline 72.803 & $1.60 \mathrm{E}-04$ & TI-209 & $->$ & Pb-209 \\
\hline
\end{tabular}




\begin{tabular}{|c|c|c|c|c|}
\hline 72.821 & $3.28 \mathrm{E}-03$ & $U-233$ & $->$ & Th-229 \\
\hline 72.873 & $3.46 \mathrm{E}-07$ & $\mathrm{Bi}-213$ & $->$ & TI-209 \\
\hline 73.6 & $2.09 \mathrm{E}-05$ & $A c-225$ & $->$ & Fr-221 \\
\hline 73.84 & 3.99E-04 & $A c-225$ & $->$ & Fr-221 \\
\hline 74.46 & $2.89 E+03$ & $\mathrm{~Np}-237$ & $->$ & Pa-233 \\
\hline 74.5 & 8.33E-03 & U-233 & $->$ & Th-229 \\
\hline 74.9 & 4.44E-05 & $A c-225$ & $->$ & Fr-221 \\
\hline 74.969 & $2.69 \mathrm{E}-04$ & TI-209 & $->$ & Pb-209 \\
\hline 75.17 & $6.41 \mathrm{E}-04$ & Th-229 & $->$ & $\mathrm{Ra}-225$ \\
\hline 75.343 & $3.05 E+05$ & $\mathrm{~Pa}-233$ & $->$ & U-233 \\
\hline 76.32 & 2.17E-03 & U-233 & $->$ & Th-229 \\
\hline 76.858 & $9.40 \mathrm{E}-04$ & $\mathrm{Bi}-213$ & $->$ & Po-213 \\
\hline 77.1 & $4.00 E-03$ & $U-233$ & $->$ & Th-229 \\
\hline 78.41 & $3.33 \mathrm{E}-04$ & $U-233$ & $->$ & Th-229 \\
\hline 78.947 & $1.05 \mathrm{E}-03$ & Fr-221 & $->$ & At-217 \\
\hline 79.29 & $1.55 E-03$ & $\mathrm{Bi}-213$ & $->$ & Po-213 \\
\hline 81.517 & $1.74 \mathrm{E}-03$ & Fr-221 & $->$ & At-217 \\
\hline 82.434 & $1.33 \mathrm{E}-07$ & $\mathrm{Bi}-213$ & $->$ & TI-209 \\
\hline 82.9 & $1.85 \mathrm{E}-04$ & Ac- 225 & $->$ & Fr-221 \\
\hline 82.957 & 9.99E-04 & U-233 & $->$ & Th-229 \\
\hline 83.229 & $1.55 \mathrm{E}-03$ & Ac- -225 & $->$ & Fr-221 \\
\hline 84.27 & $4.16 \mathrm{E}-04$ & U-233 & $->$ & Th-229 \\
\hline 84.789 & $9.58 \mathrm{E}-05$ & TI-209 & $->$ & Pb-209 \\
\hline 85.185 & $3.46 \mathrm{E}-08$ & $\mathrm{Bi}-213$ & $->$ & TI-209 \\
\hline 85.402 & $1.05 \mathrm{E}-03$ & U-233 & $->$ & Th-229 \\
\hline 86.105 & $2.56 \mathrm{E}-03$ & $A c-225$ & $->$ & Fr-221 \\
\hline 86.23 & 4.87E-04 & Th-229 & $->$ & $\mathrm{Ra}-225$ \\
\hline 86.44 & $3.85 \mathrm{E}-03$ & Th-229 & $->$ & $\mathrm{Ra}-225$ \\
\hline 86.5 & $3.65 E+06$ & $\mathrm{~Np}-237$ & $->$ & $\mathrm{Pa}-233$ \\
\hline 86.652 & $4.59 E+05$ & $\mathrm{~Pa}-233$ & $->$ & $U-233$ \\
\hline 86.74 & $7.22 \mathrm{E}-04$ & U-233 & $->$ & Th-229 \\
\hline 87.24 & $1.05 \mathrm{E}-03$ & U-233 & $->$ & Th-229 \\
\hline 87.39 & 3.57E-04 & Ac- 225 & $->$ & Fr-221 \\
\hline 87.632 & $2.74 \mathrm{E}-05$ & TI-209 & $->$ & $\mathrm{Pb}-209$ \\
\hline 88.05 & $4.69 E+04$ & $\mathrm{~Np}-237$ & $->$ & $\mathrm{Pa}-233$ \\
\hline 88.43 & $2.44 \mathrm{E}-03$ & U-233 & $->$ & Th-229 \\
\hline 89.639 & $5.53 E-04$ & $\mathrm{Bi}-213$ & $->$ & Po-213 \\
\hline 89.957 & 5.77E-02 & $U-233$ & $->$ & Th-229 \\
\hline 90.994 & $1.83 \mathrm{E}-03$ & U-233 & $->$ & Th-229 \\
\hline 92.136 & $6.16 \mathrm{E}-04$ & Fr-221 & $->$ & At-217 \\
\hline 92.282 & $4.15 E+05$ & $\mathrm{~Np}-237$ & $->$ & $\mathrm{Pa}-233$ \\
\hline 92.673 & $1.69 \mathrm{E}-04$ & $\mathrm{Bi}-213$ & $->$ & Po-213 \\
\hline 93.36 & $9.38 \mathrm{E}-02$ & U-233 & $->$ & Th-229 \\
\hline 94.66 & $2.66 \mathrm{E}+06$ & $\mathrm{~Pa}-233$ & $->$ & $U-233$ \\
\hline 94.723 & $1.98 \mathrm{E}+05$ & $\mathrm{~Np}-237$ & $->$ & $\mathrm{Pa}-233$ \\
\hline 94.87 & 1.97E-04 & Ac- 225 & $->$ & Fr-221 \\
\hline 95.265 & $1.91 \mathrm{E}-04$ & Fr-221 & $->$ & At-217 \\
\hline 95.858 & $7.56 \mathrm{E}+05$ & $\mathrm{~Np}-237$ & $->$ & $\mathrm{Pa}-233$ \\
\hline 96.17 & 3.70E-05 & Ac- 225 & $->$ & Fr-221 \\
\hline 96.215 & 7.77E-03 & U-233 & $->$ & Th-22 \\
\hline
\end{tabular}




\begin{tabular}{|c|c|c|c|c|}
\hline 97.143 & $1.22 \mathrm{E}-01$ & $U-233$ & $->$ & Th-229 \\
\hline 97.2 & $2.34 \mathrm{E}-05$ & Fr-221 & $->$ & At-217 \\
\hline 97.272 & $9.24 \mathrm{E}-04$ & $A c-225$ & $->$ & Fr-221 \\
\hline 98.443 & $4.17 \mathrm{E}+06$ & $\mathrm{~Pa}-233$ & $->$ & $U-233$ \\
\hline 99.58 & $7.76 \mathrm{E}-04$ & Ac- 225 & $->$ & Fr-221 \\
\hline 99.82 & 2.07E-03 & Ac- 225 & $->$ & Fr-221 \\
\hline 100.008 & $3.05 \mathrm{E}-04$ & $U-233$ & $->$ & Th-229 \\
\hline 100.599 & $2.96 \mathrm{E}-04$ & Ac- 225 & $->$ & Fr-221 \\
\hline 100.88 & 7.39E-05 & Ac- 225 & $->$ & Fr-221 \\
\hline 101.8 & $5.00 \mathrm{E}-04$ & U-233 & $->$ & Th-229 \\
\hline 103.375 & 5.55E-04 & $U-233$ & $->$ & Th-229 \\
\hline 103.44 & $1.11 \mathrm{E}-05$ & Ac- 225 & $->$ & Fr-221 \\
\hline 103.921 & $1.80 E+05$ & $\mathrm{~Pa}-233$ & $->$ & $U-233$ \\
\hline 105.362 & 3.39E-02 & U-233 & $->$ & Th-229 \\
\hline 106.13 & $1.46 \mathrm{E}+04$ & $\mathrm{~Np}-237$ & $->$ & $\mathrm{Pa}-233$ \\
\hline 107.16 & $1.03 \mathrm{E}-03$ & Th-229 & $->$ & Ra-225 \\
\hline 108.166 & $2.45 \mathrm{E}+05$ & Np-237 & $->$ & $\mathrm{Pa}-233$ \\
\hline 108.36 & 3.33E-04 & Ac- 225 & $->$ & Fr-221 \\
\hline 108.69 & $1.90 \mathrm{E}+04$ & $\mathrm{~Np}-237$ & $->$ & $\mathrm{Pa}-233$ \\
\hline 108.99 & $1.13 \mathrm{E}-02$ & U-233 & $->$ & Th-229 \\
\hline 109.47 & $1.72 \mathrm{E}-03$ & U-233 & $->$ & Th-229 \\
\hline 111.298 & $1.56 \mathrm{E}+06$ & $\mathrm{~Pa}-233$ & $->$ & $U-233$ \\
\hline 111.56 & $4.03 E-04$ & Ac- 225 & $->$ & Fr-221 \\
\hline 111.897 & $8.08 \mathrm{E}+04$ & Np-237 & $->$ & $\mathrm{Pa}-233$ \\
\hline 111.935 & $2.61 \mathrm{E}-03$ & U-233 & $->$ & Th-229 \\
\hline 114.28 & $1.39 \mathrm{E}-03$ & U-233 & $->$ & Th-229 \\
\hline 114.445 & $5.22 \mathrm{E}+05$ & Pa-233 & $->$ & $U-233$ \\
\hline 115.19 & $6.52 E+02$ & $\mathrm{~Np}-237$ & $->$ & $\mathrm{Pa}-233$ \\
\hline 116.26 & $1.17 \mathrm{E}-03$ & $U-233$ & $->$ & Th-229 \\
\hline 117 & $2.16 \mathrm{E}-03$ & TI-209 & $->$ & $\mathrm{Pb}-209$ \\
\hline 117.155 & 1.55E-02 & $U-233$ & $->$ & Th-229 \\
\hline 117.689 & $4.20 \mathrm{E}+04$ & $\mathrm{~Np}-237$ & $->$ & $\mathrm{Pa}-233$ \\
\hline 118.47 & $4.31 \mathrm{E}-05$ & Fr-221 & $->$ & At-217 \\
\hline 118.97 & $1.78 \mathrm{E}-02$ & U-233 & $->$ & Th-229 \\
\hline 119.92 & 7.39E-05 & Ac- 225 & $->$ & Fr-221 \\
\hline 120.81 & 1.17E-02 & U-233 & $->$ & Th-229 \\
\hline 123.76 & $2.34 \mathrm{E}-04$ & Ac- 225 & $->$ & Fr-221 \\
\hline 123.91 & $3.61 \mathrm{E}-03$ & $U-233$ & $->$ & Th-229 \\
\hline 124.51 & $1.28 \mathrm{E}-03$ & Th-229 & $->$ & $\mathrm{Ra}-225$ \\
\hline 124.72 & 7.70E-04 & Th-229 & $->$ & $\mathrm{Ra}-225$ \\
\hline 124.81 & $6.16 \mathrm{E}-05$ & $A c-225$ & $->$ & Fr-221 \\
\hline 125.398 & $3.61 \mathrm{E}-04$ & $U-233$ & $->$ & Th-229 \\
\hline 126.23 & $1.72 \mathrm{E}-05$ & Ac- 225 & $->$ & Fr-221 \\
\hline 129.19 & $5.30 \mathrm{E}-06$ & Ac- 225 & $->$ & Fr-221 \\
\hline 129.2 & 3.89E-04 & U-233 & $->$ & Th-229 \\
\hline 131.09 & $2.22 \mathrm{E}+04$ & Np-237 & $->$ & Pa-233 \\
\hline 131.2 & $1.83 \mathrm{E}-04$ & U-233 & $->$ & Th-229 \\
\hline 131.92 & $4.23 E-04$ & Th-229 & $->$ & $\mathrm{Ra}-225$ \\
\hline 134.23 & $1.75 E+04$ & Np-237 & $->$ & $\mathrm{Pa}-233$ \\
\hline 135.01 & 4.93E-05 & Ac- 225 & $->$ & Fr-221 \\
\hline
\end{tabular}




\begin{tabular}{|c|c|c|c|c|}
\hline 135.328 & $1.22 \mathrm{E}-02$ & U-233 & $->$ & Th-229 \\
\hline 137.02 & $2.05 E-03$ & Th-229 & $->$ & Ra-225 \\
\hline 138.2 & $2.46 \mathrm{E}-05$ & Ac- 225 & $->$ & Fr-221 \\
\hline 138.5 & $1.11 \mathrm{E}-04$ & $U-233$ & $->$ & Th-229 \\
\hline 139.76 & $5.83 \mathrm{E}-04$ & U-233 & $->$ & Th-229 \\
\hline 140.61 & $4.69 E+03$ & $\mathrm{~Np}-237$ & $->$ & $\mathrm{Pa}-233$ \\
\hline 142.98 & $5.52 \mathrm{E}-04$ & Th-229 & $->$ & Ra-225 \\
\hline 143.227 & $1.02 E+05$ & $\mathrm{~Np}-237$ & $->$ & $\mathrm{Pa}-233$ \\
\hline 144.52 & $1.67 \mathrm{E}-03$ & U-233 & $->$ & Th-229 \\
\hline 145.15 & $1.55 \mathrm{E}-04$ & Ac- 225 & $->$ & Fr-221 \\
\hline 145.286 & 8.88E-03 & U-233 & $->$ & Th-22S \\
\hline 146.347 & $3.50 \mathrm{E}-02$ & $U-233$ & $->$ & Th-22S \\
\hline 148.15 & $2.00 \mathrm{E}-03$ & $U-233$ & $->$ & Th-22S \\
\hline 148.37 & $1.28 \mathrm{E}-03$ & Th-229 & $->$ & Ra-225 \\
\hline 149.8 & 7.05E-04 & U-233 & $->$ & Th-229 \\
\hline 149.99 & $8.62 E-05$ & Fr-221 & $->$ & At-217 \\
\hline 150.08 & $8.25 E-04$ & Ac- 225 & $->$ & Fr-221 \\
\hline 151.423 & $6.26 \mathrm{E}+04$ & Np-237 & $->$ & Pa-233 \\
\hline 152.64 & $4.56 \mathrm{E}-05$ & Ac- 225 & $->$ & Fr-221 \\
\hline 153.31 & $3.05 E-04$ & U-233 & $->$ & Th-229 \\
\hline 153.72 & $1.80 \mathrm{E}+03$ & $\mathrm{~Np}-237$ & $->$ & $\mathrm{Pa}-233$ \\
\hline 153.93 & $1.88 \mathrm{E}-04$ & Ac- 225 & $->$ & Fr-221 \\
\hline 154.37 & 8.98E-04 & Th-229 & $->$ & $\mathrm{Ra}-22$ \\
\hline 154.69 & 8.33E-04 & U-233 & $->$ & Th-229 \\
\hline 155.263 & $2.40 \mathrm{E}+04$ & $\mathrm{~Np}-237$ & $->$ & $\mathrm{Pa}-233$ \\
\hline 155.989 & $3.22 \mathrm{E}-04$ & U-233 & $->$ & Th-229 \\
\hline 156.46 & $1.28 \mathrm{E}-03$ & Th-229 & $->$ & $\mathrm{Ra}-225$ \\
\hline 157.24 & $3.82 \mathrm{E}-04$ & Ac- 225 & $->$ & Fr-221 \\
\hline 162.51 & $9.65 E+03$ & Np-237 & $->$ & $\mathrm{Pa}-233$ \\
\hline 162.52 & $4.16 \mathrm{E}-04$ & U-233 & $->$ & Th-229 \\
\hline 164.512 & $3.66 \mathrm{E}-02$ & $U-233$ & $->$ & Th-22S \\
\hline 165.493 & 2.17E-03 & $U-233$ & $->$ & Th-229 \\
\hline 168.98 & $3.72 E-04$ & U-233 & $->$ & Th-229 \\
\hline 169.18 & $1.88 \mathrm{E}+04$ & $\mathrm{~Np}-237$ & $->$ & $\mathrm{Pa}-233$ \\
\hline 170.67 & $4.96 \mathrm{E}+03$ & $\mathrm{~Np}-237$ & $->$ & $\mathrm{Pa}-233$ \\
\hline 170.794 & 7.77E-04 & U-233 & $->$ & Th-229 \\
\hline 170.91 & $1.11 \mathrm{E}-05$ & $A c-225$ & $->$ & Fr-221 \\
\hline 171.29 & $8.62 \mathrm{E}-05$ & $\mathrm{Fr}-221$ & $->$ & At-217 \\
\hline 172.35 & $1.94 \mathrm{E}-04$ & U-233 & $->$ & Th-229 \\
\hline 172.56 & $1.77 \mathrm{E}+03$ & $\mathrm{~Np}-237$ & $->$ & $\mathrm{Pa}-233$ \\
\hline 172.9 & $2.82 \mathrm{E}-04$ & Th-229 & $->$ & $\mathrm{Ra}-225$ \\
\hline 174.162 & $1.28 \mathrm{E}-03$ & U-233 & $->$ & Th-229 \\
\hline 176.09 & $5.22 \mathrm{E}+03$ & $\mathrm{~Np}-237$ & $->$ & $\mathrm{Pa}-233$ \\
\hline 176.1 & $2.39 \mathrm{E}-04$ & U-233 & $->$ & Th-22S \\
\hline 177.78 & $1.11 \mathrm{E}-04$ & U-233 & $->$ & Th-22 \\
\hline 178.39 & 1.97E-05 & Ac- 225 & $->$ & Fr-221 \\
\hline 179.74 & $6.54 \mathrm{E}-04$ & Th-229 & $->$ & $\mathrm{Ra}-225$ \\
\hline 180.801 & $6.00 E+03$ & $\mathrm{~Np}-237$ & $->$ & $\mathrm{Pa}-233$ \\
\hline 183.96 & $2.95 \mathrm{E}-04$ & Th-229 & $->$ & $\mathrm{Ra}-22$ \\
\hline 184.17 & $1.39 \mathrm{E}-04$ & U-233 & $->$ & Th-22 \\
\hline
\end{tabular}




\begin{tabular}{|c|c|c|c|c|}
\hline 185.782 & $2.22 \mathrm{E}-04$ & $U-233$ & $->$ & Th-229 \\
\hline 186.12 & $2.46 \mathrm{E}-05$ & $A c-225$ & $->$ & Fr-221 \\
\hline 186.7 & $1.75 E+03$ & $\mathrm{~Np}-237$ & $->$ & $\mathrm{Pa}-233$ \\
\hline 187.942 & $1.11 \mathrm{E}-02$ & U-233 & $->$ & Th-229 \\
\hline 187.99 & 5.67E-04 & Ac- 225 & $->$ & Fr-221 \\
\hline 191.46 & $7.04 E+03$ & $\mathrm{~Np}-237$ & $->$ & $\mathrm{Pa}-233$ \\
\hline 192.12 & $2.22 \mathrm{E}-04$ & U-233 & $->$ & Th-229 \\
\hline 193.29 & $1.33 E+04$ & Np-237 & $->$ & $\mathrm{Pa}-233$ \\
\hline 193.59 & 5.90E-03 & Th-229 & $->$ & Ra-225 \\
\hline 194.74 & $1.28 \mathrm{E}+04$ & $\mathrm{~Np}-237$ & $->$ & $\mathrm{Pa}-233$ \\
\hline 195.04 & $5.24 \mathrm{E}+04$ & $\mathrm{~Np}-237$ & $->$ & $\mathrm{Pa}-233$ \\
\hline 195.75 & $1.79 E-04$ & Ac- 225 & $->$ & Fr-221 \\
\hline 196.89 & $6.52 E+03$ & $\mathrm{~Np}-237$ & $->$ & $\mathrm{Pa}-233$ \\
\hline 198.72 & $2.46 \mathrm{E}-05$ & $A c-225$ & $->$ & Fr-221 \\
\hline 200.17 & $9.13 E+02$ & $\mathrm{~Np}-237$ & $->$ & $\mathrm{Pa}-233$ \\
\hline 200.67 & 5.55E-06 & U-233 & $->$ & Th-229 \\
\hline 201.72 & $1.10 \mathrm{E}+04$ & Np-237 & $->$ & $\mathrm{Pa}-233$ \\
\hline 202.85 & $9.39 E+02$ & $\mathrm{~Np}-237$ & $->$ & $\mathrm{Pa}-233$ \\
\hline 205.99 & $3.61 \mathrm{E}-04$ & U-233 & $->$ & Th-229 \\
\hline 208.149 & 1.39E-02 & $U-233$ & $->$ & Th-229 \\
\hline 209.19 & $4.04 \mathrm{E}+03$ & Np-237 & $->$ & $\mathrm{Pa}-233$ \\
\hline 210.94 & $4.23 E-03$ & Th-229 & $->$ & Ra-225 \\
\hline 212.29 & 7.77E-04 & U-233 & $->$ & Th-229 \\
\hline 212.412 & $3.96 \mathrm{E}+04$ & $\mathrm{~Np}-237$ & $->$ & $\mathrm{Pa}-233$ \\
\hline 214.08 & $1.10 \mathrm{E}+04$ & $\mathrm{~Np}-237$ & $->$ & $\mathrm{Pa}-233$ \\
\hline 216.08 & $3.66 \mathrm{E}-03$ & U-233 & $->$ & Th-229 \\
\hline 216.2 & 4.19E-04 & Ac-225 & $->$ & Fr-221 \\
\hline 216.86 & 5.17E-04 & Ac- 225 & $->$ & Fr-221 \\
\hline 217.133 & $1.94 \mathrm{E}-02$ & U-233 & $->$ & Th-229 \\
\hline 217.61 & $2.78 \mathrm{E}-04$ & $U-233$ & $->$ & Th-229 \\
\hline 217.98 & $1.34 \mathrm{E}-02$ & Fr-221 & $->$ & At-217 \\
\hline 218.15 & $1.80 \mathrm{E}-04$ & Th-229 & $->$ & $\mathrm{Ra}-225$ \\
\hline 219.424 & 8.33E-04 & U-233 & $->$ & Th-229 \\
\hline 223.357 & $1.83 \mathrm{E}-04$ & $U-233$ & $->$ & Th-229 \\
\hline 224.64 & $9.86 \mathrm{E}-05$ & $A c-225$ & $->$ & Fr-221 \\
\hline 225 & 5.55E-05 & U-233 & $->$ & Th-229 \\
\hline 226.74 & 5.55E-05 & $U-233$ & $->$ & Th-229 \\
\hline 228.07 & $1.11 \mathrm{E}-04$ & U-233 & $->$ & Th-229 \\
\hline 229.98 & $3.42 E+03$ & Np-237 & $->$ & $\mathrm{Pa}-233$ \\
\hline 230.086 & $3.72 E-04$ & U-233 & $->$ & Th-229 \\
\hline 236.32 & 4.62E-05 & Th-229 & $->$ & $\mathrm{Ra}-22$ \\
\hline 236.39 & $2.78 \mathrm{E}-04$ & $U-233$ & $->$ & Th-229 \\
\hline 237.95 & $1.67 \mathrm{E}+04$ & Np-237 & $->$ & $\mathrm{Pa}-233$ \\
\hline 240.364 & $2.11 \mathrm{E}-03$ & U-233 & $->$ & Th-229 \\
\hline 240.76 & $1.60 \mathrm{E}-05$ & Ac- 225 & $->$ & Fr-221 \\
\hline 245.294 & $1.94 \mathrm{E}-02$ & U-233 & $->$ & Th-229 \\
\hline 248.25 & $1.41 \mathrm{E}+04$ & $\mathrm{~Pa}-233$ & $->$ & U-233 \\
\hline 248.661 & $2.50 \mathrm{E}-02$ & U-233 & $->$ & Th-229 \\
\hline 248.7 & $2.46 \mathrm{E}-05$ & Ac- 225 & $->$ & Fr-221 \\
\hline 248.91 & $1.25 \mathrm{E}+03$ & $\mathrm{~Np}-237$ & $->$ & $\mathrm{Pa}-23$ \\
\hline
\end{tabular}




\begin{tabular}{|c|c|c|c|c|}
\hline 252.5 & $2.11 \mathrm{E}-04$ & U-233 & $->$ & Th-229 \\
\hline 253.51 & $1.23 \mathrm{E}-04$ & $A c-225$ & $->$ & Fr-221 \\
\hline 255.92 & $2.39 \mathrm{E}-04$ & U-233 & $->$ & Th-229 \\
\hline 256.98 & $1.75 \mathrm{E}+03$ & $\mathrm{~Np}-237$ & $->$ & $\mathrm{Pa}-233$ \\
\hline 258.46 & $1.41 \mathrm{E}+03$ & $\mathrm{~Pa}-233$ & $->$ & U-233 \\
\hline 259.3 & $9.99 E-04$ & U-233 & $->$ & Th-229 \\
\hline 260.4 & $6.00 \mathrm{E}-04$ & $U-233$ & $->$ & Th-229 \\
\hline 261.88 & $1.72 \mathrm{E}-03$ & U-233 & $->$ & Th-229 \\
\hline 262.41 & $1.75 \mathrm{E}+03$ & $\mathrm{~Np}-237$ & $->$ & $\mathrm{Pa}-233$ \\
\hline 268.619 & 1.39E-03 & U-233 & $->$ & Th-229 \\
\hline 271.597 & $7.30 \mathrm{E}+04$ & $\mathrm{~Pa}-233$ & $->$ & U-233 \\
\hline 272.27 & $3.50 \mathrm{E}-04$ & U-233 & $->$ & Th-229 \\
\hline 274.692 & $2.61 \mathrm{E}-03$ & $U-233$ & $->$ & Th-229 \\
\hline 278.059 & $6.77 E-03$ & U-233 & $->$ & Th-229 \\
\hline 279.27 & $4.31 \mathrm{E}-05$ & $A c-225$ & $->$ & Fr-221 \\
\hline 282.54 & $1.11 \mathrm{E}-05$ & Fr-221 & $->$ & At-217 \\
\hline 284.22 & 5.55E-05 & $U-233$ & $->$ & Th-229 \\
\hline 285.5 & $1.23 \mathrm{E}-05$ & Ac- 225 & $->$ & Fr-221 \\
\hline 287.949 & $6.00 \mathrm{E}-03$ & U-233 & $->$ & Th-229 \\
\hline 291.317 & $2.89 \mathrm{E}-02$ & $U-233$ & $->$ & Th-229 \\
\hline 292.79 & $5.30 \mathrm{E}-04$ & $\mathrm{Bi}-213$ & $->$ & Рo-213 \\
\hline 293.923 & 7.77E-04 & U-233 & $->$ & Th-229 \\
\hline 295.2 & $1.28 \mathrm{E}-04$ & $U-233$ & $->$ & Th-229 \\
\hline 298.87 & $6.60 \mathrm{E}+03$ & $\mathrm{~Pa}-233$ & $->$ & U-233 \\
\hline 300.175 & $1.62 E+06$ & $\mathrm{~Pa}-233$ & $->$ & $U-233$ \\
\hline 302.84 & $3.89 E-04$ & U-233 & $->$ & Th-229 \\
\hline 309.37 & $4.05 E-04$ & $U-233$ & $->$ & Th-229 \\
\hline 311.38 & $1.55 \mathrm{E}-04$ & $U-233$ & $->$ & Th-229 \\
\hline 311.9 & $9.91 E+06$ & Pa-233 & $->$ & U-233 \\
\hline 317.133 & $4.89 E-02$ & U-233 & $->$ & Th-229 \\
\hline 320.508 & $1.72 \mathrm{E}-02$ & $U-233$ & $->$ & Th-229 \\
\hline 323.321 & $4.66 \mathrm{E}-03$ & $U-233$ & $->$ & Th-229 \\
\hline 323.81 & $4.92 \mathrm{E}-06$ & $\mathrm{Bi}-213$ & $->$ & TI-209 \\
\hline 324.1 & 2.09E-05 & Fr-221 & $->$ & At-217 \\
\hline 328.69 & $3.61 \mathrm{E}-04$ & $U-233$ & $->$ & Th-229 \\
\hline 336.579 & $3.28 \mathrm{E}-03$ & $U-233$ & $->$ & Th-229 \\
\hline 339.64 & $4.16 \mathrm{E}-05$ & $U-233$ & $->$ & Th-229 \\
\hline 340.59 & $1.10 \mathrm{E}+06$ & Pa-233 & $->$ & U-233 \\
\hline 351.782 & $2.50 \mathrm{E}-04$ & U-233 & $->$ & Th-229 \\
\hline 354 & $3.22 \mathrm{E}-04$ & $U-233$ & $->$ & Th-229 \\
\hline 359.09 & $4.31 \mathrm{E}-05$ & Fr-221 & $->$ & At- 217 \\
\hline 365.762 & $4.55 E-03$ & U-233 & $->$ & Th-229 \\
\hline 375.518 & $1.51 \mathrm{E}+05$ & $\mathrm{~Pa}-233$ & $->$ & U-233 \\
\hline 382.05 & 4.31E-05 & Fr-221 & $->$ & At-217 \\
\hline 383.4 & 5.27E-04 & $U-233$ & $->$ & Th-229 \\
\hline 384 & $1.11 \mathrm{E}-04$ & $U-233$ & $->$ & Th-229 \\
\hline 394.34 & $4.16 \mathrm{E}-05$ & $U-233$ & $->$ & Th-229 \\
\hline 396.65 & $4.61 \mathrm{E}-05$ & U-233 & $->$ & Th-229 \\
\hline 398.663 & $3.10 \mathrm{E}+05$ & $\mathrm{~Pa}-233$ & $->$ & U-233 \\
\hline 402.37 & $4.61 \mathrm{E}-05$ & U-233 & $->$ & Th-229 \\
\hline
\end{tabular}




\begin{tabular}{|c|c|c|c|c|}
\hline 406.7 & $2.94 \mathrm{E}-05$ & $\mathrm{U}-233$ & $->$ & Th-229 \\
\hline 409.1 & $1.60 \mathrm{E}-04$ & Fr-221 & $->$ & At-217 \\
\hline 415.932 & $3.94 \mathrm{E}+05$ & Pa-233 & $->$ & $U-233$ \\
\hline 416.4 & 5.55E-05 & U-233 & $->$ & Th-229 \\
\hline 436.78 & $2.78 \mathrm{E}-05$ & $U-233$ & $->$ & Th-229 \\
\hline 440.34 & 1.99E-02 & $\mathrm{Bi}-213$ & $->$ & Po-213 \\
\hline 449.6 & 5.55E-05 & $U-233$ & $->$ & Th-229 \\
\hline 452.43 & $1.36 \mathrm{E}-04$ & Ac-225 & $->$ & Fr-221 \\
\hline 459.77 & $4.61 \mathrm{E}-05$ & U-233 & $->$ & Th-229 \\
\hline 467 & $2.16 \mathrm{E}-03$ & TI-209 & $->$ & $\mathrm{Pb}-209$ \\
\hline 471.3 & 8.33E-05 & $U-233$ & $->$ & Th-229 \\
\hline 479 & 8.33E-05 & $U-233$ & $->$ & Th-229 \\
\hline 480.5 & $3.70 E-05$ & $A c-225$ & $->$ & Fr-221 \\
\hline 484.05 & $2.39 \mathrm{E}-05$ & U-233 & $->$ & Th-229 \\
\hline 513.5 & $1.23 \mathrm{E}-05$ & Ac- 225 & $->$ & Fr-221 \\
\hline 526.03 & $1.23 E-05$ & $A c-225$ & $->$ & Fr-221 \\
\hline 540.27 & $2.94 \mathrm{E}-05$ & U-233 & $->$ & Th-229 \\
\hline 545.1 & $1.33 \mathrm{E}-05$ & $U-233$ & $->$ & Th-229 \\
\hline 569.37 & $2.11 \mathrm{E}-05$ & $U-233$ & $->$ & Th-229 \\
\hline 578.47 & $2.78 \mathrm{E}-05$ & $U-233$ & $->$ & Th-229 \\
\hline 620.87 & $1.28 \mathrm{E}-05$ & U-233 & $->$ & Th-229 \\
\hline 656.97 & $1.67 \mathrm{E}-05$ & $U-233$ & $->$ & Th-229 \\
\hline 659.72 & $1.05 \mathrm{E}-04$ & $\mathrm{Bi}-213$ & $->$ & Po-213 \\
\hline 707.47 & $1.50 \mathrm{E}-05$ & $U-233$ & $->$ & Th-229 \\
\hline 807.27 & $3.15 \mathrm{E}-04$ & $\mathrm{Bi}-213$ & $->$ & Po-213 \\
\hline 867.9 & 1.17E-05 & $U-233$ & $->$ & Th-229 \\
\hline 1003 & $4.61 \mathrm{E}-05$ & $U-233$ & $->$ & Th-229 \\
\hline 1100.06 & $3.40 \mathrm{E}-04$ & $\mathrm{Bi}-213$ & $->$ & Po-213 \\
\hline 1119 & $4.61 \mathrm{E}-05$ & $U-233$ & $->$ & Th-229 \\
\hline 1566 & $2.61 \mathrm{E}-03$ & Tl-209 & $->$ & Pb-209 \\
\hline
\end{tabular}

\begin{tabular}{|c|c|c|c|c|}
\hline Np237 & $\begin{array}{c}\text { Aged } 5 \\
\text { years }\end{array}$ & \multicolumn{3}{|c|}{ Sorted by Intensity } \\
\hline Intensity & Energy & \multicolumn{3}{|c|}{ Initial Isotopes and Decays } \\
\hline & & & & \\
\hline $9.91 E+06$ & 311.9 & $\mathrm{~Pa}-233$ & $->$ & $U-233$ \\
\hline $6.52 E+06$ & 16.632 & $\mathrm{~Np}-237$ & $->$ & $\mathrm{Pa}-233$ \\
\hline $5.01 E+06$ & 13.274 & $\mathrm{~Np}-237$ & $->$ & $\mathrm{Pa}-233$ \\
\hline $4.96 \mathrm{E}+06$ & 17.058 & $\mathrm{~Pa}-233$ & $->$ & $\mathrm{U}-233$ \\
\hline $4.69 E+06$ & 13.6 & $\mathrm{~Pa}-233$ & $->$ & $U-233$ \\
\hline 4.17E+06 & 98.443 & $\mathrm{~Pa}-233$ & $->$ & $U-233$ \\
\hline $3.65 E+06$ & 86.5 & $\mathrm{~Np}-237$ & $->$ & $\mathrm{Pa}-233$ \\
\hline $3.36 \mathrm{E}+06$ & 29.378 & $\mathrm{~Np}-237$ & $->$ & $\mathrm{Pa}-233$ \\
\hline $2.66 \mathrm{E}+06$ & 94.66 & $P a-233$ & $->$ & $U-233$ \\
\hline $1.62 E+06$ & 300.175 & $\mathrm{~Pa}-233$ & $->$ & $U-233$ \\
\hline $1.56 \mathrm{E}+06$ & 111.298 & $\mathrm{~Pa}-233$ & $->$ & $U-233$ \\
\hline $1.51 \mathrm{E}+06$ & 19.718 & $\mathrm{~Np}-237$ & $->$ & $\mathrm{Pa}-233$ \\
\hline
\end{tabular}




\begin{tabular}{|c|c|c|c|c|}
\hline $1.17 E+06$ & 20.42 & Pa-233 & $->$ & $U-233$ \\
\hline $1.10 \mathrm{E}+06$ & 340.59 & $\mathrm{~Pa}-233$ & $->$ & U-233 \\
\hline $7.56 \mathrm{E}+05$ & 95.858 & $\mathrm{~Np}-237$ & $->$ & $\mathrm{Pa}-233$ \\
\hline $5.22 E+05$ & 114.445 & $\mathrm{~Pa}-233$ & $->$ & U-233 \\
\hline $4.59 E+05$ & 86.652 & Pa-233 & $->$ & $U-233$ \\
\hline $4.15 E+05$ & 92.282 & $\mathrm{~Np}-237$ & $->$ & $\mathrm{Pa}-233$ \\
\hline $3.94 \mathrm{E}+05$ & 415.932 & $\mathrm{~Pa}-233$ & $->$ & U-233 \\
\hline $3.10 \mathrm{E}+05$ & 398.663 & $\mathrm{~Pa}-233$ & $->$ & U-233 \\
\hline $3.05 E+05$ & 75.343 & $\mathrm{~Pa}-233$ & $->$ & $U-233$ \\
\hline $3.00 E+05$ & 11.372 & $\mathrm{~Np}-237$ & $->$ & $\mathrm{Pa}-233$ \\
\hline $2.79 E+05$ & 11.62 & $\mathrm{~Pa}-233$ & $->$ & U-233 \\
\hline $2.45 E+05$ & 108.166 & Np-237 & $->$ & $\mathrm{Pa}-233$ \\
\hline $1.98 \mathrm{E}+05$ & 94.723 & $\mathrm{~Np}-237$ & $->$ & $\mathrm{Pa}-233$ \\
\hline $1.80 \mathrm{E}+05$ & 103.921 & $\mathrm{~Pa}-233$ & $->$ & U-233 \\
\hline $1.51 \mathrm{E}+05$ & 375.518 & $\mathrm{~Pa}-233$ & $->$ & U-233 \\
\hline $1.23 E+05$ & 14.953 & $\mathrm{~Np}-237$ & $->$ & Pa-233 \\
\hline $1.02 E+05$ & 143.227 & $\mathrm{~Np}-237$ & $->$ & $\mathrm{Pa}-233$ \\
\hline $1.02 E+05$ & 57.149 & $\mathrm{~Np}-237$ & $->$ & $\mathrm{Pa}-233$ \\
\hline $8.08 \mathrm{E}+04$ & 111.897 & $\mathrm{~Np}-237$ & $->$ & $\mathrm{Pa}-233$ \\
\hline $7.30 \mathrm{E}+04$ & 271.597 & $\mathrm{~Pa}-233$ & $->$ & U-233 \\
\hline $6.26 \mathrm{E}+04$ & 151.423 & $\mathrm{~Np}-237$ & $->$ & $\mathrm{Pa}-233$ \\
\hline $6.00 E+04$ & 15.4 & $\mathrm{~Pa}-233$ & $->$ & U-233 \\
\hline $5.24 \mathrm{E}+04$ & 195.04 & $\mathrm{~Np}-237$ & $->$ & $\mathrm{Pa}-233$ \\
\hline $4.69 E+04$ & 88.05 & $\mathrm{~Np}-237$ & $->$ & $\mathrm{Pa}-233$ \\
\hline $4.20 E+04$ & 117.689 & $\mathrm{~Np}-237$ & $->$ & $\mathrm{Pa}-233$ \\
\hline $3.96 \mathrm{E}+04$ & 212.412 & $\mathrm{~Np}-237$ & $->$ & $\mathrm{Pa}-233$ \\
\hline $3.47 E+04$ & 46.57 & Np-237 & $->$ & $\mathrm{Pa}-233$ \\
\hline $2.40 E+04$ & 155.263 & $\mathrm{~Np}-237$ & $->$ & $\mathrm{Pa}-233$ \\
\hline $2.22 \mathrm{E}+04$ & 131.09 & $\mathrm{~Np}-237$ & $->$ & $\mathrm{Pa}-233$ \\
\hline $1.90 \mathrm{E}+04$ & 108.69 & $\mathrm{~Np}-237$ & $->$ & $\mathrm{Pa}-233$ \\
\hline $1.88 \mathrm{E}+04$ & 169.18 & $\mathrm{~Np}-237$ & $->$ & $\mathrm{Pa}-233$ \\
\hline $1.75 \mathrm{E}+04$ & 134.23 & $N p-237$ & $->$ & $\mathrm{Pa}-233$ \\
\hline $1.70 \mathrm{E}+04$ & 28.578 & $\mathrm{~Pa}-233$ & $->$ & U-233 \\
\hline $1.67 \mathrm{E}+04$ & 237.95 & $\mathrm{~Np}-237$ & $->$ & $\mathrm{Pa}-233$ \\
\hline $1.46 \mathrm{E}+04$ & 106.13 & $\mathrm{~Np}-237$ & $->$ & $\mathrm{Pa}-233$ \\
\hline $1.41 \mathrm{E}+04$ & 248.25 & $\mathrm{~Pa}-233$ & $->$ & U-233 \\
\hline $1.33 \mathrm{E}+04$ & 193.29 & $\mathrm{~Np}-237$ & $->$ & $\mathrm{Pa}-233$ \\
\hline $1.28 \mathrm{E}+04$ & 194.74 & $\mathrm{~Np}-237$ & $->$ & Pa-233 \\
\hline $1.10 \mathrm{E}+04$ & 201.72 & $\mathrm{~Np}-237$ & $->$ & $\mathrm{Pa}-233$ \\
\hline $1.10 \mathrm{E}+04$ & 214.08 & $\mathrm{~Np}-237$ & $->$ & $\mathrm{Pa}-233$ \\
\hline $9.65 E+03$ & 162.51 & $\mathrm{~Np}-237$ & $->$ & $\mathrm{Pa}-233$ \\
\hline $9.39 E+03$ & 40.415 & $\mathrm{~Pa}-233$ & $->$ & U-233 \\
\hline $7.04 E+03$ & 191.46 & $\mathrm{~Np}-237$ & $->$ & $\mathrm{Pa}-233$ \\
\hline $6.60 \mathrm{E}+03$ & 298.87 & $\mathrm{~Pa}-233$ & $->$ & U-233 \\
\hline $6.52 E+03$ & 196.89 & $\mathrm{~Np}-237$ & $->$ & $\mathrm{Pa}-233$ \\
\hline $6.00 E+03$ & 180.801 & $\mathrm{~Np}-237$ & $->$ & $\mathrm{Pa}-233$ \\
\hline $5.22 E+03$ & 176.09 & $\mathrm{~Np}-237$ & $->$ & $\mathrm{Pa}-233$ \\
\hline $4.96 \mathrm{E}+03$ & 170.67 & $\mathrm{~Np}-237$ & $->$ & $\mathrm{Pa}-233$ \\
\hline $4.69 E+03$ & 140.61 & $\mathrm{~Np}-237$ & $->$ & $\mathrm{Pa}-233$ \\
\hline $4.17 E+03$ & 63.95 & Np-237 & $->$ & Pa-233 \\
\hline
\end{tabular}




\begin{tabular}{|c|c|c|c|c|}
\hline $4.17 E+03$ & 70.62 & $\mathrm{~Np}-237$ & $->$ & $\mathrm{Pa}-233$ \\
\hline $4.04 \mathrm{E}+03$ & 209.19 & $\mathrm{~Np}-237$ & $->$ & $\mathrm{Pa}-233$ \\
\hline $3.42 E+03$ & 229.98 & $N p-237$ & $->$ & Pa-233 \\
\hline $3.39 E+03$ & 41.718 & Pa-233 & $->$ & U-233 \\
\hline $3.13 E+03$ & 62.66 & $\mathrm{~Np}-237$ & $->$ & Pa-233 \\
\hline $2.89 E+03$ & 74.46 & $\mathrm{~Np}-237$ & $->$ & $\mathrm{Pa}-233$ \\
\hline $1.80 \mathrm{E}+03$ & 153.72 & $N p-237$ & $->$ & Pa-233 \\
\hline $1.77 \mathrm{E}+03$ & 172.56 & $\mathrm{~Np}-237$ & $->$ & $\mathrm{Pa}-233$ \\
\hline $1.75 E+03$ & 262.41 & $\mathrm{~Np}-237$ & $->$ & Pa-233 \\
\hline $1.75 E+03$ & 256.98 & $\mathrm{~Np}-237$ & $->$ & Pa-233 \\
\hline $1.75 E+03$ & 186.7 & $\mathrm{~Np}-237$ & $->$ & $\mathrm{Pa}-233$ \\
\hline $1.41 \mathrm{E}+03$ & 258.46 & $\mathrm{~Pa}-233$ & $->$ & U-233 \\
\hline $1.25 E+03$ & 248.91 & $N p-237$ & $->$ & Pa-233 \\
\hline $9.39 \mathrm{E}+02$ & 17.269 & $\mathrm{~Pa}-233$ & $->$ & U-233 \\
\hline $9.39 E+02$ & 202.85 & $N p-237$ & $->$ & Pa-233 \\
\hline $9.13 E+02$ & 200.17 & $\mathrm{~Np}-237$ & $->$ & Pa-233 \\
\hline $6.52 E+02$ & 115.19 & $N p-237$ & $->$ & Pa-233 \\
\hline $1.83 E+01$ & 16.141 & U-233 & $->$ & Th-229 \\
\hline $1.44 \mathrm{E}+01$ & 12.952 & $U-233$ & $->$ & Th-229 \\
\hline $4.44 E+00$ & 19.148 & $U-233$ & $->$ & Th-229 \\
\hline 8.33E-01 & 11.118 & $U-233$ & $->$ & Th-229 \\
\hline $3.33 \mathrm{E}-01$ & 42.441 & $U-233$ & $->$ & Th-229 \\
\hline $3.16 \mathrm{E}-01$ & 14.511 & $U-233$ & $->$ & Th-229 \\
\hline $1.22 \mathrm{E}-01$ & 97.143 & $U-233$ & $->$ & Th-229 \\
\hline $9.38 \mathrm{E}-02$ & 93.36 & $U-233$ & $->$ & Th-229 \\
\hline 7.77E-02 & 54.702 & $U-233$ & $->$ & Th-229 \\
\hline 5.77E-02 & 89.957 & $U-233$ & $->$ & Th-229 \\
\hline 4.89E-02 & 317.133 & $U-233$ & $->$ & Th-229 \\
\hline $3.78 \mathrm{E}-02$ & 29.191 & $U-233$ & $->$ & Th-229 \\
\hline $3.66 \mathrm{E}-02$ & 164.512 & $U-233$ & $->$ & Th-229 \\
\hline $3.63 E-02$ & 40.34 & Ra-225 & $->$ & Ac- 225 \\
\hline $3.50 \mathrm{E}-02$ & 146.347 & U-233 & $->$ & Th-229 \\
\hline 3.39E-02 & 105.362 & $U-233$ & $->$ & Th-229 \\
\hline 2.89E-02 & 291.317 & $U-233$ & $->$ & Th-229 \\
\hline $2.50 \mathrm{E}-02$ & 248.661 & $U-233$ & $->$ & Th-229 \\
\hline 2.39E-02 & 53.559 & U-233 & $->$ & Th-229 \\
\hline $1.99 \mathrm{E}-02$ & 440.34 & $\mathrm{Bi}-213$ & $->$ & Po-213 \\
\hline $1.94 \mathrm{E}-02$ & 245.294 & $U-233$ & $->$ & Th-229 \\
\hline $1.94 \mathrm{E}-02$ & 217.133 & $U-233$ & $->$ & Th-229 \\
\hline $1.78 \mathrm{E}-02$ & 118.97 & $U-233$ & $->$ & Th-229 \\
\hline $1.72 \mathrm{E}-02$ & 320.508 & $U-233$ & $->$ & Th-229 \\
\hline $1.55 \mathrm{E}-02$ & 117.155 & $U-233$ & $->$ & Th-229 \\
\hline $1.50 \mathrm{E}-02$ & 71.847 & $U-233$ & $->$ & Th-229 \\
\hline $1.39 \mathrm{E}-02$ & 208.149 & $U-233$ & $->$ & Th-229 \\
\hline $1.34 \mathrm{E}-02$ & 217.98 & Fr-221 & $->$ & At-217 \\
\hline $1.22 \mathrm{E}-02$ & 135.328 & U-233 & $->$ & Th-229 \\
\hline $1.18 \mathrm{E}-02$ & 14.772 & Ac-225 & $->$ & Fr-221 \\
\hline $1.17 \mathrm{E}-02$ & 120.81 & U-233 & $->$ & Th-229 \\
\hline $1.13 \mathrm{E}-02$ & 108.99 & U-233 & $->$ & Th-229 \\
\hline $1.11 \mathrm{E}-02$ & 187.942 & $U-233$ & $->$ & Th-229 \\
\hline
\end{tabular}




\begin{tabular}{|c|c|c|c|c|}
\hline $9.61 \mathrm{E}-03$ & 12.017 & $A c-225$ & $->$ & Fr-221 \\
\hline 8.88E-03 & 145.286 & $\mathrm{U}-233$ & $->$ & Th-229 \\
\hline 8.33E-03 & 74.5 & $U-233$ & $->$ & Th-229 \\
\hline 7.77E-03 & 96.215 & U-233 & $->$ & Th-229 \\
\hline 7.64E-03 & 15.662 & Ra-225 & $->$ & Ac- 225 \\
\hline 6.89E-03 & 12.636 & $\mathrm{Ra}-225$ & $->$ & Ac- 225 \\
\hline 6.77E-03 & 278.059 & U-233 & $->$ & Th-229 \\
\hline $6.72 \mathrm{E}-03$ & 25.304 & $U-233$ & $->$ & Th-229 \\
\hline $6.00 \mathrm{E}-03$ & 287.949 & $U-233$ & $->$ & Th-229 \\
\hline 5.90E-03 & 193.59 & Th-229 & $->$ & Ra-225 \\
\hline 5.50E-03 & 32.27 & U-233 & $->$ & Th-229 \\
\hline $5.26 \mathrm{E}-03$ & 31.37 & Th-229 & $->$ & Ra-225 \\
\hline $4.66 \mathrm{E}-03$ & 323.321 & U-233 & $->$ & Th-229 \\
\hline $4.66 \mathrm{E}-03$ & 66.11 & $U-233$ & $->$ & Th-229 \\
\hline $4.55 \mathrm{E}-03$ & 365.762 & $U-233$ & $->$ & Th-229 \\
\hline $4.23 E-03$ & 210.94 & Th-229 & $->$ & Ra-225 \\
\hline 4.00E-03 & 77.1 & U-233 & $->$ & Th-229 \\
\hline $3.85 E-03$ & 86.44 & Th-229 & $->$ & Ra-225 \\
\hline $3.66 \mathrm{E}-03$ & 216.08 & U-233 & $->$ & Th-229 \\
\hline $3.61 E-03$ & 123.91 & $U-233$ & $->$ & Th-229 \\
\hline $3.33 \mathrm{E}-03$ & 70.35 & $U-233$ & $->$ & Th-229 \\
\hline $3.28 \mathrm{E}-03$ & 336.579 & $U-233$ & $->$ & Th-229 \\
\hline $3.28 \mathrm{E}-03$ & 72.821 & $U-233$ & $->$ & Th-229 \\
\hline $2.61 \mathrm{E}-03$ & 111.935 & $U-233$ & $->$ & Th-229 \\
\hline $2.61 \mathrm{E}-03$ & 274.692 & U-233 & $->$ & Th-229 \\
\hline $2.61 \mathrm{E}-03$ & 1566 & TI-209 & $->$ & Pb-209 \\
\hline $2.59 E-03$ & 17.449 & $A c-225$ & $->$ & Fr-221 \\
\hline $2.56 \mathrm{E}-03$ & 86.105 & Ac-225 & $->$ & Fr-221 \\
\hline $2.44 \mathrm{E}-03$ & 88.43 & U-233 & $->$ & Th-229 \\
\hline $2.17 \mathrm{E}-03$ & 165.493 & $U-233$ & $->$ & Th-229 \\
\hline $2.17 \mathrm{E}-03$ & 76.32 & $U-233$ & $->$ & Th-229 \\
\hline $2.16 \mathrm{E}-03$ & 467 & Tl-209 & $->$ & Pb-209 \\
\hline $2.16 \mathrm{E}-03$ & 117 & TI-209 & $->$ & $P b-209$ \\
\hline $2.11 \mathrm{E}-03$ & 240.364 & U-233 & $->$ & Th-229 \\
\hline 2.07E-03 & 99.82 & $A c-225$ & $->$ & Fr-221 \\
\hline $2.05 E-03$ & 137.02 & Th-229 & $->$ & Ra-225 \\
\hline $2.00 \mathrm{E}-03$ & 148.15 & $\mathrm{U}-233$ & $->$ & Th-229 \\
\hline $2.00 \mathrm{E}-03$ & 37.85 & $U-233$ & $->$ & Th-229 \\
\hline $1.83 \mathrm{E}-03$ & 90.994 & $U-233$ & $->$ & Th-229 \\
\hline $1.78 \mathrm{E}-03$ & 67.96 & U-233 & $->$ & Th-229 \\
\hline $1.75 \mathrm{E}-03$ & 18.593 & $\mathrm{Ra}-225$ & $->$ & Ac- 225 \\
\hline $1.74 \mathrm{E}-03$ & 81.517 & Fr-221 & $->$ & At-217 \\
\hline $1.72 \mathrm{E}-03$ & 261.88 & U-233 & $->$ & Th-229 \\
\hline $1.72 \mathrm{E}-03$ & 109.47 & $U-233$ & $->$ & Th-229 \\
\hline $1.67 \mathrm{E}-03$ & 144.52 & U-233 & $->$ & Th-229 \\
\hline $1.55 E-03$ & 79.29 & $\mathrm{Bi}-213$ & $->$ & Po-213 \\
\hline $1.55 \mathrm{E}-03$ & 83.229 & Ac- 225 & $->$ & Fr-221 \\
\hline $1.50 \mathrm{E}-03$ & 31.24 & U-233 & $->$ & Th-229 \\
\hline $1.39 \mathrm{E}-03$ & 268.619 & $U-233$ & $->$ & Th-229 \\
\hline $1.39 \mathrm{E}-03$ & 52.613 & $U-233$ & $->$ & Th-229 \\
\hline
\end{tabular}




\begin{tabular}{|c|c|c|c|c|}
\hline $1.39 \mathrm{E}-03$ & 114.28 & $U-233$ & $->$ & Th-229 \\
\hline $1.28 \mathrm{E}-03$ & 148.37 & Th-229 & $->$ & Ra-225 \\
\hline $1.28 \mathrm{E}-03$ & 156.46 & Th-229 & $->$ & Ra-225 \\
\hline $1.28 \mathrm{E}-03$ & 124.51 & Th-229 & $->$ & Ra-225 \\
\hline $1.28 \mathrm{E}-03$ & 174.162 & U-233 & $->$ & Th-229 \\
\hline $1.18 \mathrm{E}-03$ & 13.911 & Fr-221 & $->$ & At-217 \\
\hline $1.17 \mathrm{E}-03$ & 116.26 & $U-233$ & $->$ & Th-229 \\
\hline $1.05 \mathrm{E}-03$ & 87.24 & U-233 & $->$ & Th-229 \\
\hline $1.05 E-03$ & 85.402 & $U-233$ & $->$ & Th-229 \\
\hline $1.05 \mathrm{E}-03$ & 78.947 & Fr-221 & $->$ & At-217 \\
\hline $1.03 E-03$ & 107.16 & Th-229 & $->$ & Ra-225 \\
\hline $9.99 E-04$ & 82.957 & $U-233$ & $->$ & Th-229 \\
\hline 9.99E-04 & 259.3 & $U-233$ & $->$ & Th-229 \\
\hline $9.73 E-04$ & 11.414 & Fr-221 & $->$ & At-217 \\
\hline $9.40 \mathrm{E}-04$ & 76.858 & $\mathrm{Bi}-213$ & $->$ & Po-213 \\
\hline $9.24 \mathrm{E}-04$ & 97.272 & Ac- 225 & $->$ & Fr-221 \\
\hline 8.98E-04 & 154.37 & Th-229 & $->$ & Ra-225 \\
\hline 8.33E-04 & 219.424 & U-233 & $->$ & Th-229 \\
\hline 8.33E-04 & 154.69 & U-233 & $->$ & Th-229 \\
\hline $8.25 E-04$ & 150.08 & Ac- 225 & $->$ & Fr-221 \\
\hline 7.77E-04 & 170.794 & U-233 & $->$ & Th-229 \\
\hline 7.77E-04 & 212.29 & $U-233$ & $->$ & Th-229 \\
\hline 7.77E-04 & 293.923 & U-233 & $->$ & Th-229 \\
\hline $7.76 \mathrm{E}-04$ & 99.58 & $A C-225$ & $->$ & Fr-221 \\
\hline 7.70E-04 & 124.72 & Th-229 & $->$ & Ra-225 \\
\hline 7.22E-04 & 86.74 & U-233 & $->$ & Th-229 \\
\hline 7.05E-04 & 149.8 & $U-233$ & $->$ & Th-229 \\
\hline $6.78 \mathrm{E}-04$ & 62.93 & Ac- 225 & $->$ & Fr-221 \\
\hline $6.54 \mathrm{E}-04$ & 179.74 & Th-229 & $->$ & Ra-225 \\
\hline $6.41 \mathrm{E}-04$ & 75.17 & Th-229 & $->$ & Ra-225 \\
\hline $6.16 \mathrm{E}-04$ & 92.136 & Fr-221 & $->$ & At-217 \\
\hline $6.00 \mathrm{E}-04$ & 68.97 & U-233 & $->$ & Th-229 \\
\hline $6.00 \mathrm{E}-04$ & 260.4 & $U-233$ & $->$ & Th-229 \\
\hline 5.97E-04 & 11.119 & $\mathrm{Bi}-213$ & $->$ & Po-213 \\
\hline 5.83E-04 & 139.76 & $U-233$ & $->$ & Th-229 \\
\hline 5.67E-04 & 187.99 & Ac- 225 & $->$ & Fr-221 \\
\hline $5.55 E-04$ & 103.375 & U-233 & $->$ & Th-229 \\
\hline $5.54 \mathrm{E}-04$ & 13.504 & $\mathrm{Bi}-213$ & $->$ & Po-213 \\
\hline 5.53E-04 & 89.639 & $\mathrm{Bi}-213$ & $->$ & Po-213 \\
\hline $5.52 \mathrm{E}-04$ & 142.98 & Th-229 & $->$ & Ra-225 \\
\hline $5.30 \mathrm{E}-04$ & 292.79 & $\mathrm{Bi}-213$ & $->$ & Po-213 \\
\hline $5.30 \mathrm{E}-04$ & 10.381 & Ac- 225 & $->$ & Fr-221 \\
\hline $5.27 E-04$ & 383.4 & U-233 & $->$ & Th-229 \\
\hline $5.17 \mathrm{E}-04$ & 216.86 & Ac- 225 & $->$ & Fr-221 \\
\hline $5.00 \mathrm{E}-04$ & 101.8 & U-233 & $->$ & Th-229 \\
\hline $4.87 E-04$ & 86.23 & Th-229 & $->$ & Ra-225 \\
\hline $4.23 E-04$ & 56.58 & Th-229 & $->$ & Ra-225 \\
\hline $4.23 E-04$ & 131.92 & Th-229 & $->$ & Ra-225 \\
\hline $4.19 E-04$ & 216.2 & $A c-225$ & $->$ & Fr-221 \\
\hline $4.16 \mathrm{E}-04$ & 84.27 & U-233 & $->$ & Th-229 \\
\hline
\end{tabular}




\begin{tabular}{|c|c|c|c|c|}
\hline $4.16 \mathrm{E}-04$ & 162.52 & $U-233$ & $->$ & Th-229 \\
\hline 4.05E-04 & 309.37 & $U-233$ & $->$ & Th-229 \\
\hline $4.03 E-04$ & 111.56 & Ac- 225 & $->$ & Fr-221 \\
\hline $4.01 \mathrm{E}-04$ & 10.871 & Ra-225 & $->$ & Ac- 225 \\
\hline $3.99 E-04$ & 73.84 & Ac- 225 & $->$ & Fr-221 \\
\hline 3.89E-04 & 302.84 & U-233 & $->$ & Th-229 \\
\hline 3.89E-04 & 129.2 & U-233 & $->$ & Th-229 \\
\hline $3.82 E-04$ & 157.24 & Ac- 225 & $->$ & Fr-221 \\
\hline $3.72 E-04$ & 230.086 & U-233 & $->$ & Th-229 \\
\hline $3.72 E-04$ & 168.98 & $U-233$ & $->$ & Th-229 \\
\hline $3.61 \mathrm{E}-04$ & 205.99 & $U-233$ & $->$ & Th-229 \\
\hline $3.61 \mathrm{E}-04$ & 125.398 & $U-233$ & $->$ & Th-229 \\
\hline $3.61 \mathrm{E}-04$ & 328.69 & $U-233$ & $->$ & Th-229 \\
\hline $3.57 E-04$ & 87.39 & $A c-225$ & $->$ & Fr-221 \\
\hline $3.50 \mathrm{E}-04$ & 272.27 & U-233 & $->$ & Th-229 \\
\hline $3.40 \mathrm{E}-04$ & 1100.06 & $\mathrm{Bi}-213$ & $->$ & Po-213 \\
\hline 3.33E-04 & 78.41 & $U-233$ & $->$ & Th-229 \\
\hline 3.33E-04 & 108.36 & Ac- 225 & $->$ & Fr-221 \\
\hline $3.22 \mathrm{E}-04$ & 155.989 & U-233 & $->$ & Th-229 \\
\hline $3.22 \mathrm{E}-04$ & 354 & $U-233$ & $->$ & Th-229 \\
\hline $3.15 \mathrm{E}-04$ & 807.27 & $\mathrm{Bi}-213$ & $->$ & Po-213 \\
\hline $3.05 E-04$ & 100.008 & U-233 & $->$ & Th-229 \\
\hline $3.05 E-04$ & 153.31 & U-233 & $->$ & Th-229 \\
\hline $2.96 \mathrm{E}-04$ & 100.599 & $A c-225$ & $->$ & Fr-221 \\
\hline 2.95E-04 & 183.96 & Th-229 & $->$ & Ra-225 \\
\hline $2.82 \mathrm{E}-04$ & 172.9 & Th-229 & $->$ & Ra-225 \\
\hline $2.78 \mathrm{E}-04$ & 217.61 & U-233 & $->$ & Th-229 \\
\hline $2.78 \mathrm{E}-04$ & 236.39 & $U-233$ & $->$ & Th-229 \\
\hline 2.69E-04 & 74.969 & TI-209 & $->$ & Pb-209 \\
\hline $2.50 \mathrm{E}-04$ & 351.782 & $U-233$ & $->$ & Th-229 \\
\hline $2.45 \mathrm{E}-04$ & 16.361 & Fr-221 & $->$ & At-217 \\
\hline 2.39E-04 & 255.92 & U-233 & $->$ & Th-229 \\
\hline 2.39E-04 & 176.1 & U-233 & $->$ & Th-229 \\
\hline $2.34 \mathrm{E}-04$ & 123.76 & $A c-225$ & $->$ & Fr-221 \\
\hline $2.22 \mathrm{E}-04$ & 185.782 & U-233 & $->$ & Th-229 \\
\hline $2.22 \mathrm{E}-04$ & 192.12 & U-233 & $->$ & Th-229 \\
\hline $2.18 \mathrm{E}-04$ & 17.349 & Th-229 & $->$ & Ra-225 \\
\hline $2.11 \mathrm{E}-04$ & 252.5 & U-233 & $->$ & Th-229 \\
\hline $2.05 E-04$ & 42.722 & Th-229 & $->$ & Ra-225 \\
\hline 1.97E-04 & 13.255 & $A c-225$ & $->$ & Fr-221 \\
\hline 1.97E-04 & 94.87 & AC-225 & $->$ & Fr-221 \\
\hline 1.94E-04 & 172.35 & U-233 & $->$ & Th-229 \\
\hline $1.91 \mathrm{E}-04$ & 95.265 & Fr-221 & $->$ & At-217 \\
\hline $1.88 \mathrm{E}-04$ & 153.93 & $A c-225$ & $->$ & Fr-221 \\
\hline $1.85 \mathrm{E}-04$ & 82.9 & AC-225 & $->$ & Fr-221 \\
\hline $1.83 \mathrm{E}-04$ & 223.357 & U-233 & $->$ & Th-229 \\
\hline $1.83 \mathrm{E}-04$ & 131.2 & $U-233$ & $->$ & Th-229 \\
\hline $1.83 \mathrm{E}-04$ & 63.69 & U-233 & $->$ & Th-229 \\
\hline $1.80 \mathrm{E}-04$ & 218.15 & Th-229 & $->$ & Ra-225 \\
\hline 1.79E-04 & 195.75 & Ac- 225 & $->$ & Fr-221 \\
\hline
\end{tabular}




\begin{tabular}{|c|c|c|c|c|}
\hline $1.69 \mathrm{E}-04$ & 92.673 & $\mathrm{Bi}-213$ & $->$ & Po-213 \\
\hline $1.60 \mathrm{E}-04$ & 409.1 & Fr-221 & $->$ & At-217 \\
\hline $1.60 \mathrm{E}-04$ & 72.803 & TI-209 & $->$ & $\mathrm{Pb}-209$ \\
\hline $1.55 \mathrm{E}-04$ & 311.38 & U-233 & $->$ & Th-229 \\
\hline $1.55 \mathrm{E}-04$ & 145.15 & Ac- 225 & $->$ & Fr-221 \\
\hline $1.41 \mathrm{E}-04$ & 68.88 & Th-229 & $->$ & $\mathrm{Ra}-225$ \\
\hline $1.39 \mathrm{E}-04$ & 184.17 & U-233 & $->$ & Th-229 \\
\hline $1.36 \mathrm{E}-04$ & 452.43 & Ac- 225 & $->$ & Fr-221 \\
\hline $1.28 \mathrm{E}-04$ & 68.14 & Th-229 & $->$ & $\mathrm{Ra}-225$ \\
\hline $1.28 \mathrm{E}-04$ & 295.2 & U-233 & $->$ & Th-229 \\
\hline $1.23 \mathrm{E}-04$ & 253.51 & $A c-225$ & $->$ & Fr-221 \\
\hline $1.14 \mathrm{E}-04$ & 14.082 & $\mathrm{Ra}-225$ & $->$ & Ac- 225 \\
\hline $1.11 \mathrm{E}-04$ & 228.07 & U-233 & $->$ & Th-229 \\
\hline $1.11 \mathrm{E}-04$ & 138.5 & $U-233$ & $->$ & Th-229 \\
\hline $1.11 \mathrm{E}-04$ & 177.78 & $U-233$ & $->$ & Th-229 \\
\hline $1.11 \mathrm{E}-04$ & 384 & $U-233$ & $->$ & Th-229 \\
\hline $1.06 \mathrm{E}-04$ & 15.878 & $\mathrm{Bi}-213$ & $->$ & Po-213 \\
\hline $1.05 \mathrm{E}-04$ & 659.72 & $\mathrm{Bi}-213$ & $->$ & Po-213 \\
\hline $9.86 \mathrm{E}-05$ & 224.64 & Ac- 225 & $->$ & Fr-221 \\
\hline $9.84 \mathrm{E}-05$ & 10.541 & TI-209 & $->$ & $\mathrm{Pb}-209$ \\
\hline $9.58 \mathrm{E}-05$ & 84.789 & Tl-209 & $->$ & Pb-209 \\
\hline $9.31 \mathrm{E}-05$ & 12.703 & TI-209 & $->$ & Pb-209 \\
\hline $8.62 \mathrm{E}-05$ & 171.29 & Fr-221 & $->$ & At-217 \\
\hline $8.62 \mathrm{E}-05$ & 149.99 & Fr-221 & $->$ & At-217 \\
\hline 8.33E-05 & 471.3 & $U-233$ & $->$ & Th-229 \\
\hline 8.33E-05 & 479 & $U-233$ & $->$ & Th-229 \\
\hline 7.39E-05 & 119.92 & $A c-225$ & $->$ & Fr-221 \\
\hline 7.39E-05 & 100.88 & Ac- 225 & $->$ & Fr-221 \\
\hline $6.90 \mathrm{E}-05$ & 64.23 & Ac- 225 & $->$ & Fr-221 \\
\hline $6.16 \mathrm{E}-05$ & 124.81 & Ac- 225 & $->$ & Fr-221 \\
\hline 5.55E-05 & 416.4 & U-233 & $->$ & Th-229 \\
\hline 5.55E-05 & 284.22 & $U-233$ & $->$ & Th-229 \\
\hline $5.55 E-05$ & 226.74 & $U-233$ & $->$ & Th-229 \\
\hline 5.55E-05 & 225 & U-233 & $->$ & Th-229 \\
\hline $5.55 E-05$ & 449.6 & $U-233$ & $->$ & Th-229 \\
\hline $5.17 \mathrm{E}-05$ & 9.897 & Fr-221 & $->$ & At-217 \\
\hline 4.93E-05 & 135.01 & $A c-225$ & $->$ & Fr-221 \\
\hline $4.62 E-05$ & 236.32 & Th-229 & $->$ & $\mathrm{Ra}-225$ \\
\hline $4.62 \mathrm{E}-05$ & 25.373 & Th-229 & $->$ & $\mathrm{Ra}-225$ \\
\hline 4.61E-05 & 396.65 & U-233 & $->$ & Th-229 \\
\hline 4.61E-05 & 1119 & $U-233$ & $->$ & Th-229 \\
\hline 4.61E-05 & 402.37 & $U-233$ & $->$ & Th-229 \\
\hline $4.61 \mathrm{E}-05$ & 1003 & $U-233$ & $->$ & Th-229 \\
\hline $4.61 \mathrm{E}-05$ & 459.77 & U-233 & $->$ & Th-229 \\
\hline $4.56 \mathrm{E}-05$ & 152.64 & Ac- 225 & $->$ & Fr-221 \\
\hline $4.44 \mathrm{E}-05$ & 74.9 & $A c-225$ & $->$ & Fr-221 \\
\hline $4.31 \mathrm{E}-05$ & 279.27 & Ac- 225 & $->$ & Fr-221 \\
\hline $4.31 \mathrm{E}-05$ & 382.05 & Fr-221 & $->$ & At-217 \\
\hline $4.31 \mathrm{E}-05$ & 359.09 & Fr-221 & $->$ & At-217 \\
\hline $4.31 \mathrm{E}-05$ & 118.47 & Fr-221 & $->$ & At-217 \\
\hline
\end{tabular}




\begin{tabular}{|c|c|c|c|c|}
\hline $4.16 \mathrm{E}-05$ & 394.34 & $U-233$ & $->$ & Th-229 \\
\hline $4.16 \mathrm{E}-05$ & 339.64 & U-233 & $->$ & Th-229 \\
\hline $3.70 \mathrm{E}-05$ & 480.5 & Ac- 225 & $->$ & Fr-221 \\
\hline $3.70 \mathrm{E}-05$ & 96.17 & Ac- 225 & $->$ & Fr-221 \\
\hline $3.11 \mathrm{E}-05$ & 9.658 & $\mathrm{Bi}-213$ & $->$ & Рo-213 \\
\hline $2.94 \mathrm{E}-05$ & 406.7 & $U-233$ & $->$ & Th-229 \\
\hline $2.94 \mathrm{E}-05$ & 540.27 & $U-233$ & $->$ & Th-229 \\
\hline $2.78 \mathrm{E}-05$ & 578.47 & U-233 & $->$ & Th-229 \\
\hline $2.78 \mathrm{E}-05$ & 436.78 & $U-233$ & $->$ & Th-229 \\
\hline $2.74 \mathrm{E}-05$ & 87.632 & TI-209 & $->$ & $\mathrm{Pb}-209$ \\
\hline $2.46 \mathrm{E}-05$ & 248.7 & $A c-225$ & $->$ & Fr-221 \\
\hline $2.46 \mathrm{E}-05$ & 186.12 & Ac- 225 & $->$ & Fr-221 \\
\hline $2.46 \mathrm{E}-05$ & 198.72 & $A c-225$ & $->$ & Fr-221 \\
\hline $2.46 \mathrm{E}-05$ & 138.2 & Ac- 225 & $->$ & Fr-221 \\
\hline $2.39 \mathrm{E}-05$ & 484.05 & $U-233$ & $->$ & Th-229 \\
\hline $2.34 \mathrm{E}-05$ & 53.82 & Ac- 225 & $->$ & Fr-221 \\
\hline $2.34 \mathrm{E}-05$ & 97.2 & Fr-221 & $->$ & At-217 \\
\hline $2.11 \mathrm{E}-05$ & 569.37 & U-233 & $->$ & Th-229 \\
\hline $2.09 E-05$ & 73.6 & Ac- 225 & $->$ & Fr-221 \\
\hline 2.09E-05 & 324.1 & Fr-221 & $->$ & At-217 \\
\hline $2.06 \mathrm{E}-05$ & 12.466 & Fr-221 & $->$ & At-217 \\
\hline $1.97 \mathrm{E}-05$ & 178.39 & $A c-225$ & $->$ & Fr-221 \\
\hline $1.85 \mathrm{E}-05$ & 36.65 & Ac- 225 & $->$ & Fr-221 \\
\hline $1.81 \mathrm{E}-05$ & 71.71 & $A C-225$ & $->$ & Fr-221 \\
\hline $1.72 \mathrm{E}-05$ & 126.23 & Ac- 225 & $->$ & Fr-221 \\
\hline $1.68 \mathrm{E}-05$ & 14.871 & TI-209 & $->$ & Pb-209 \\
\hline $1.67 \mathrm{E}-05$ & 656.97 & U-233 & $->$ & Th-229 \\
\hline $1.60 \mathrm{E}-05$ & 240.76 & Ac- 225 & $->$ & Fr-221 \\
\hline $1.50 \mathrm{E}-05$ & 707.47 & $U-233$ & $->$ & Th-229 \\
\hline $1.33 \mathrm{E}-05$ & 545.1 & U-233 & $->$ & Th-229 \\
\hline $1.33 \mathrm{E}-05$ & 49.2 & Ac- 225 & $->$ & Fr-221 \\
\hline $1.28 \mathrm{E}-05$ & 620.87 & U-233 & $->$ & Th-229 \\
\hline $1.23 E-05$ & 513.5 & Ac- 225 & $->$ & Fr-221 \\
\hline $1.23 \mathrm{E}-05$ & 70.92 & Ac- 225 & $->$ & Fr-221 \\
\hline $1.23 \mathrm{E}-05$ & 526.03 & $A C-225$ & $->$ & Fr-221 \\
\hline $1.23 \mathrm{E}-05$ & 285.5 & Ac- 225 & $->$ & Fr-221 \\
\hline $1.17 \mathrm{E}-05$ & 867.9 & $\mathrm{U}-233$ & $->$ & Th-229 \\
\hline $1.11 \mathrm{E}-05$ & 38.51 & Ac- 225 & $->$ & Fr-221 \\
\hline $1.11 \mathrm{E}-05$ & 103.44 & Ac- 225 & $->$ & Fr-221 \\
\hline $1.11 \mathrm{E}-05$ & 170.91 & Ac- 225 & $->$ & Fr-221 \\
\hline $1.11 \mathrm{E}-05$ & 282.54 & Fr-221 & $->$ & At-217 \\
\hline 8.07E-06 & 12.085 & $\mathrm{Bi}-213$ & $->$ & Po-213 \\
\hline $6.28 \mathrm{E}-06$ & 69.85 & Ac- 225 & $->$ & Fr-221 \\
\hline $5.55 E-06$ & 200.67 & U-233 & $->$ & Th-229 \\
\hline $5.30 \mathrm{E}-06$ & 129.19 & Ac- 225 & $->$ & Fr-221 \\
\hline $5.17 \mathrm{E}-06$ & 57.77 & Ac- 225 & $->$ & Fr-221 \\
\hline $5.06 \mathrm{E}-06$ & 9.185 & TI-209 & $->$ & Pb-209 \\
\hline 4.92E-06 & 323.81 & $\mathrm{Bi}-213$ & $->$ & TI-209 \\
\hline $1.85 \mathrm{E}-06$ & 25.98 & $A c-225$ & $->$ & Fr-221 \\
\hline $1.36 \mathrm{E}-06$ & 11.349 & TI-209 & $->$ & Pb-209 \\
\hline
\end{tabular}




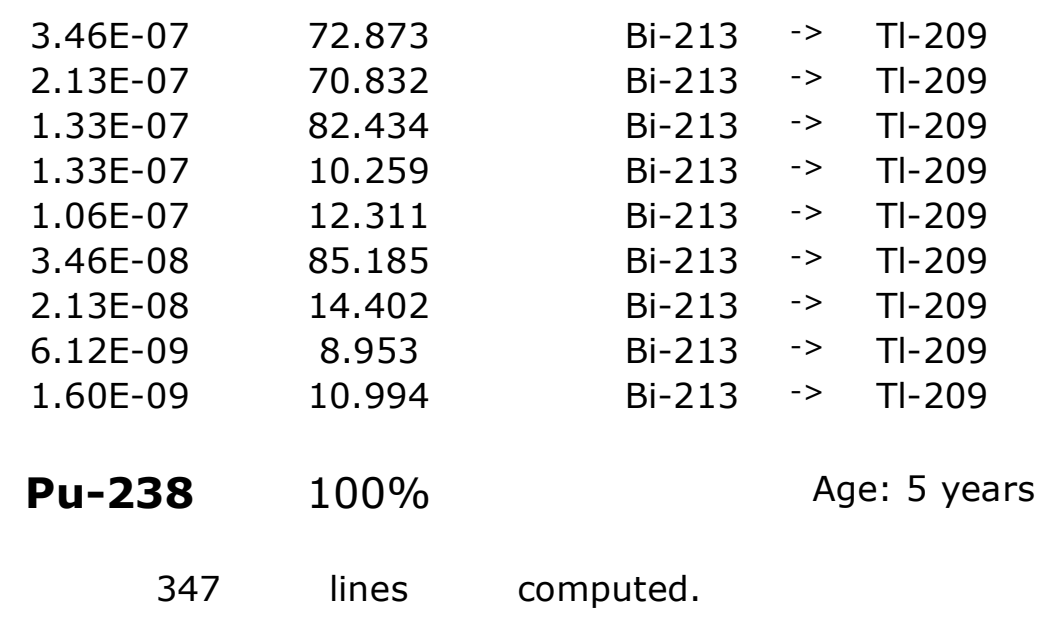

\begin{tabular}{|c|c|c|c|c|c|c|c|}
\hline \multirow{2}{*}{$\frac{\text { Energy }}{(\mathrm{keV})}$} & \multirow{2}{*}{$\begin{array}{l}\text { Intensity } \\
\text { (ph/s/gm) }\end{array}$} & \multicolumn{6}{|c|}{ Initial Isotopes and Decays } \\
\hline & & & & & & & \\
\hline 8.953 & $8.66 \mathrm{E}-12$ & $\mathrm{Hg}-206$ & $->$ & TI-206 & & & \\
\hline 9.185 & $1.25 \mathrm{E}-07$ & TI-206 & $->$ & $\mathrm{Pb}-206$, & $\mathrm{TI}-210$ & $->$ & $\mathrm{Pb}-210$ \\
\hline 9.419 & 4.47E-04 & $\mathrm{Pb}-210$ & $->$ & $\mathrm{Bi}-210$, & $\mathrm{Pb}-214$ & $->$ & $\mathrm{Bi}-214$ \\
\hline 9.658 & $2.91 \mathrm{E}-05$ & $\mathrm{Bi}-214$ & $->$ & Po-214 & & & \\
\hline 10.137 & $2.10 \mathrm{E}-05$ & $\mathrm{Ra}-226$ & $->$ & $\mathrm{Rn}-222$ & & & \\
\hline 10.259 & $1.80 \mathrm{E}-10$ & $\mathrm{Hg}-206$ & $->$ & TI-206 & & & \\
\hline 10.541 & $2.53 \mathrm{E}-06$ & TI-206 & $->$ & $\mathrm{Pb}-206$ & $\mathrm{TI}-210$ & $->$ & $\mathrm{Pb}-210$ \\
\hline 10.828 & 8.93E-03 & $\mathrm{Pb}-210$ & $->$ & $\mathrm{Bi}-210$, & $\mathrm{Pb}-214$ & $->$ & $\mathrm{Bi}-214$ \\
\hline 10.994 & $2.28 \mathrm{E}-12$ & $\mathrm{Hg}-206$ & $->$ & TI-206 & & & \\
\hline 11.119 & $5.56 \mathrm{E}-04$ & $\mathrm{Bi}-214$ & $->$ & Po-214 & & & \\
\hline 11.349 & $6.10 \mathrm{E}-08$ & TI-210 & $->$ & $\mathrm{Pb}-210$ & & & \\
\hline 11.439 & $2.68 \mathrm{E}-14$ & TI-206 & $->$ & $\mathrm{Pb}-206$ & & & \\
\hline 11.62 & $1.52 \mathrm{E}+09$ & $\mathrm{Pu}-238$ & $->$ & U-234 & & & \\
\hline 11.712 & $1.05 \mathrm{E}-04$ & $\mathrm{~Pb}-210$ & $->$ & $\mathrm{Bi}-210$ & $\mathrm{~Pb}-214$ & $->$ & $\mathrm{Bi}-214$ \\
\hline 11.713 & $3.89 \mathrm{E}-04$ & Ra-226 & $->$ & $\mathrm{Rn}-222$ & & & \\
\hline 12.085 & 7.99E-06 & Bi-214 & $->$ & Po-214 & & & \\
\hline 12.313 & $1.56 \mathrm{E}-10$ & $\mathrm{Hg}-206$ & $->$ & TI-206 & & & \\
\hline 12.678 & $3.36 \mathrm{E}-06$ & TI-210 & $->$ & $\mathrm{Pb}-210$ & & & \\
\hline 12.71 & $1.68 \mathrm{E}-12$ & TI-206 & $->$ & $\mathrm{Pb}-206$ & & & \\
\hline 12.855 & $1.07 \mathrm{E}-05$ & $\mathrm{Ra}-226$ & $->$ & $\mathrm{Rn}-222$ & & & \\
\hline 13.066 & 5.69E-04 & $\mathrm{Pb}-210$ & $->$ & $\mathrm{Bi}-210$ & & & \\
\hline 13.088 & $8.28 \mathrm{E}-03$ & $\mathrm{~Pb}-214$ & $->$ & $\mathrm{Bi}-214$ & & & \\
\hline 13.502 & $5.38 \mathrm{E}-04$ & $\mathrm{Bi}-214$ & $->$ & Рo-214 & & & \\
\hline 13.6 & $2.56 \mathrm{E}+10$ & Pu-238 & $->$ & U-234 & & & \\
\hline 14.336 & $5.62 \mathrm{E}-04$ & Ra-226 & $->$ & $\mathrm{Rn}-222$ & & & \\
\hline 14.407 & $2.89 \mathrm{E}-11$ & $\mathrm{Hg}-206$ & $->$ & TI-206 & & & \\
\hline 14.836 & $6.71 \mathrm{E}-07$ & TI-206 & $->$ & $\mathrm{Pb}-206$ & TI-210 & $->$ & $\mathrm{Pb}-210$ \\
\hline 15.4 & $6.21 \mathrm{E}+08$ & Pu-238 & $->$ & $U-234$ & & & \\
\hline 15.439 & $1.69 \mathrm{E}-03$ & $\mathrm{~Pb}-214$ & $->$ & $\mathrm{Bi}-214$ & & & \\
\hline 15.537 & $1.37 \mathrm{E}-04$ & $\mathrm{~Pb}-210$ & $->$ & $\mathrm{Bi}-210$ & & & \\
\hline
\end{tabular}




\begin{tabular}{|c|c|c|c|c|c|c|c|}
\hline 15.874 & $1.03 E-04$ & $\mathrm{Bi}-214$ & $->$ & Po-214 & & & \\
\hline 16.874 & $1.23 \mathrm{E}-04$ & $\mathrm{Ra}-226$ & $->$ & $\mathrm{Rn}-222$ & & & \\
\hline 17.128 & $3.17 E+10$ & Pu-238 & $->$ & U-234 & & & \\
\hline 20.292 & $7.00 E+09$ & Pu-238 & $->$ & $U-234$ & & & \\
\hline 43.498 & $2.41 E+08$ & Pu-238 & $->$ & $U-234$ & & & \\
\hline 46.52 & $2.21 \mathrm{E}-04$ & $\mathrm{~Pb}-210$ & $->$ & $\mathrm{Bi}-210$ & & & \\
\hline 53.172 & $1.60 \mathrm{E}-03$ & $\mathrm{~Pb}-214$ & $->$ & $\mathrm{Bi}-214$ & & & \\
\hline 53.23 & $1.04 \mathrm{E}+04$ & U-234 & $->$ & Th-230 & & & \\
\hline 62.78 & $2.44 \mathrm{E}+02$ & $\mathrm{Pu}-238$ & $->$ & $U-234$ & & & \\
\hline 67.6758 & $7.62 \mathrm{E}-01$ & Th-230 & $->$ & Ra-226 & & & \\
\hline 70.832 & $3.01 \mathrm{E}-10$ & $\mathrm{Hg}-206$ & $->$ & TI-206 & & & \\
\hline 72.803 & 8.54E-07 & TI-206 & $->$ & $\mathrm{Pb}-206$, & TI-210 & $->$ & $\mathrm{Pb}-210$ \\
\hline 72.873 & $5.05 E-10$ & $\mathrm{Hg}-206$ & $->$ & TI-206 & & & \\
\hline 74.814 & 9.47E-03 & $\mathrm{Pb}-214$ & $->$ & $\mathrm{Bi}-214$ & & & \\
\hline 74.969 & $1.40 \mathrm{E}-06$ & TI-206 & $->$ & $\mathrm{Pb}-206$, & TI-210 & $->$ & $\mathrm{Pb}-210$ \\
\hline 76.838 & $8.43 E-04$ & $\mathrm{Bi}-214$ & $->$ & Po-214 & & & \\
\hline 77.107 & $1.60 \mathrm{E}-02$ & $\mathrm{~Pb}-214$ & $->$ & $\mathrm{Bi}-214$ & & & \\
\hline 79.29 & $1.41 \mathrm{E}-03$ & $\mathrm{Bi}-214$ & $->$ & Po-214 & & & \\
\hline 81 & $6.10 \mathrm{E}-07$ & TI-210 & $->$ & $\mathrm{Pb}-210$ & & & \\
\hline 81.067 & $2.58 \mathrm{E}-04$ & Ra-226 & $->$ & $\mathrm{Rn}-222$ & & & \\
\hline 82.434 & $1.80 \mathrm{E}-10$ & $\mathrm{Hg}-206$ & $->$ & TI-206 & & & \\
\hline 83.787 & $4.28 \mathrm{E}-04$ & $\mathrm{Ra}-226$ & $->$ & $\mathrm{Rn}-222$ & & & \\
\hline 84.789 & $5.00 \mathrm{E}-07$ & TI-206 & $->$ & $\mathrm{Pb}-206$, & TI-210 & $->$ & $\mathrm{Pb}-210$ \\
\hline 85.185 & $5.05 \mathrm{E}-11$ & $\mathrm{Hg}-206$ & $->$ & Tl-206 & & & \\
\hline 87.19 & $5.64 \mathrm{E}-03$ & $\mathrm{~Pb}-214$ & $->$ & $\mathrm{Bi}-214$ & & & \\
\hline 87.632 & $1.43 \mathrm{E}-07$ & TI-206 & $->$ & $\mathrm{Pb}-206$, & TI-210 & $->$ & $\mathrm{Pb}-210$ \\
\hline 89.639 & $5.01 \mathrm{E}-04$ & $\mathrm{Bi}-214$ & $->$ & Po-214 & & & \\
\hline 90.128 & $1.67 \mathrm{E}-03$ & $\mathrm{~Pb}-214$ & $->$ & $\mathrm{Bi}-214$ & & & \\
\hline 92.673 & $1.53 \mathrm{E}-04$ & $\mathrm{Bi}-214$ & $->$ & Po-214 & & & \\
\hline 94.66 & $7.00 E+05$ & Pu-238 & $->$ & $U-234$ & & & \\
\hline 94.677 & $1.51 \mathrm{E}-04$ & Ra-226 & $->$ & $\mathrm{Rn}-222$ & & & \\
\hline 95 & $1.22 \mathrm{E}-06$ & TI-210 & $->$ & $\mathrm{Pb}-210$ & & & \\
\hline 97.907 & $4.82 \mathrm{E}-05$ & $\mathrm{Ra}-226$ & $->$ & $\mathrm{Rn}-222$ & & & \\
\hline 98.441 & $1.13 E+06$ & Pu-238 & $->$ & U-234 & & & \\
\hline 99.864 & $4.32 E+07$ & Pu-238 & $->$ & $U-234$ & & & \\
\hline 109.97 & $1.20 \mathrm{E}-04$ & Th-230 & $->$ & Ra-226 & & & \\
\hline 110.421 & $1.22 \mathrm{E}+05$ & Pu-238 & $->$ & U-234 & & & \\
\hline 111.3 & $2.37 E+05$ & Pu-238 & $->$ & $U-234$ & & & \\
\hline 114.333 & $2.37 E+05$ & Pu-238 & $->$ & $U-234$ & & & \\
\hline 114.561 & $9.62 E+04$ & Pu-238 & $->$ & $U-234$ & & & \\
\hline 120.912 & $3.59 E+03$ & U-234 & $->$ & Th-230 & & & \\
\hline 137.4 & 8.57E-05 & $\mathrm{Pb}-214$ & $->$ & $\mathrm{Bi}-214$ & & & \\
\hline 141.3 & 5.67E-05 & $\mathrm{Pb}-214$ & $->$ & $\mathrm{Bi}-214$ & & & \\
\hline 143.876 & $9.85 E-02$ & Th-230 & $->$ & $\mathrm{Ra}-226$ & & & \\
\hline 152.68 & $5.82 E+06$ & Pu-238 & $->$ & U-234 & & & \\
\hline 174.52 & $3.04 \mathrm{E}+01$ & Pu-238 & $->$ & $U-234$ & & & \\
\hline 186.057 & $1.78 \mathrm{E}-02$ & Th-230 & $->$ & Ra-226 & & & \\
\hline 186.11 & $4.81 \mathrm{E}-03$ & Ra-226 & $->$ & $\mathrm{Rn}-222$ & & & \\
\hline 196.3 & $7.12 \mathrm{E}-05$ & $\mathrm{~Pb}-214$ & $->$ & $\mathrm{Bi}-214$ & & & \\
\hline 200.98 & $2.50 E+04$ & Pu-238 & $->$ & $U-234$ & & & \\
\hline
\end{tabular}




\begin{tabular}{|c|c|c|c|c|c|c|c|}
\hline 203.2 & $6.09 E+01$ & Pu-238 & $->$ & $U-234$ & & & \\
\hline 205.1 & $1.01 \mathrm{E}-05$ & Th-230 & $->$ & $\mathrm{Ra}-226$ & & & \\
\hline 235.01 & $1.64 \mathrm{E}-05$ & Th-230 & $->$ & $\mathrm{Ra}-226$ & & & \\
\hline 236.03 & $6.09 \mathrm{E}+01$ & Pu-238 & $->$ & U-234 & & & \\
\hline 241.92 & $1.08 \mathrm{E}-02$ & $\mathrm{~Pb}-214$ & $->$ & $\mathrm{Bi}-214$ & & & \\
\hline 253.732 & $2.25 \mathrm{E}-02$ & Th-230 & $->$ & $\mathrm{Ra}-226$ & & & \\
\hline 253.84 & $1.72 \mathrm{E}-03$ & Th-230 & $->$ & Ra-226 & & & \\
\hline 258.18 & $7.25 E+02$ & Pu-238 & $->$ & U-234 & & & \\
\hline 258.94 & 7.99E-04 & $\mathrm{Pb}-214$ & $->$ & $\mathrm{Bi}-214$ & & & \\
\hline 262.41 & 7.92E-06 & Ra-226 & $->$ & $\mathrm{Rn}-222$ & & & \\
\hline 266.15 & $2.84 \mathrm{E}-15$ & $\mathrm{Bi}-210$ & $->$ & TI-206 & & & \\
\hline 273.7 & $2.61 \mathrm{E}-04$ & $\mathrm{Bi}-214$ & $->$ & Po-214 & & & \\
\hline 274.56 & 4.65E-04 & $\mathrm{Pb}-214$ & $->$ & $\mathrm{Bi}-214$ & & & \\
\hline 280.93 & $1.18 \mathrm{E}-04$ & $\mathrm{Bi}-214$ & $->$ & Po-214 & & & \\
\hline 286.9 & 4.65E-05 & $\mathrm{Bi}-214$ & $->$ & Po-214 & & & \\
\hline 295.091 & $2.79 \mathrm{E}-02$ & $\mathrm{~Pb}-214$ & $->$ & $\mathrm{Bi}-214$ & & & \\
\hline 298.1 & $2.42 \mathrm{E}-05$ & TI-210 & $->$ & $\mathrm{Pb}-210$ & Рo-214 & $->$ & $\mathrm{Pb}-210$ \\
\hline 299.08 & $7.92 E+02$ & Pu-238 & $->$ & $U-234$ & & & \\
\hline 302.25 & $3.25 \mathrm{E}-09$ & $\mathrm{Hg}-206$ & $->$ & TI-206 & & & \\
\hline 304.42 & $4.94 \mathrm{E}-05$ & $\mathrm{Bi}-214$ & $->$ & Рo-214 & & & \\
\hline 305.5 & 3.34E-05 & $\mathrm{Pb}-214$ & $->$ & $\mathrm{Bi}-214$ & & & \\
\hline 314.2 & $1.15 \mathrm{E}-04$ & $\mathrm{~Pb}-214$ & $->$ & $\mathrm{Bi}-214$ & & & \\
\hline 324.3 & $2.91 \mathrm{E}-05$ & $\mathrm{~Pb}-214$ & $->$ & $\mathrm{Bi}-214$ & & & \\
\hline 333.6 & $1.38 \mathrm{E}-04$ & $\mathrm{Bi}-214$ & $->$ & Po-214 & & & \\
\hline 334.9 & $8.28 \mathrm{E}-05$ & $\mathrm{Bi}-214$ & $->$ & Po-214 & & & \\
\hline 338.5 & $5.67 \mathrm{E}-05$ & $\mathrm{Bi}-214$ & $->$ & Po-214 & & & \\
\hline 344.96 & $6.25 \mathrm{E}-11$ & $\mathrm{Hg}-206$ & $->$ & TI-206 & & & \\
\hline 347.1 & $8.72 \mathrm{E}-05$ & $\mathrm{Bi}-214$ & $->$ & Po-214 & & & \\
\hline 351.87 & 5.39E-02 & $\mathrm{Pb}-214$ & $->$ & $\mathrm{Bi}-214$ & & & \\
\hline 354 & $1.22 \mathrm{E}-06$ & $\mathrm{TI}-210$ & $->$ & $\mathrm{Pb}-210$ & & & \\
\hline 364.2 & $9.15 \mathrm{E}-06$ & $\mathrm{Bi}-214$ & $->$ & Po-214 & & & \\
\hline 376.6 & $7.12 \mathrm{E}-06$ & $\mathrm{Bi}-214$ & $->$ & Po-214 & & & \\
\hline 380 & $9.15 \mathrm{E}-07$ & TI-210 & $->$ & $\mathrm{Pb}-210$ & & & \\
\hline 384.06 & $4.57 \mathrm{E}-13$ & $\mathrm{Hg}-206$ & $->$ & TI-206 & & & \\
\hline 386.834 & $5.23 E-04$ & $\mathrm{Bi}-214$ & $->$ & Po-214 & & & \\
\hline 388.95 & $5.96 \mathrm{E}-04$ & $\mathrm{Bi}-214$ & $->$ & Po-214 & & & \\
\hline 394 & $1.31 \mathrm{E}-05$ & $\mathrm{Bi}-214$ & $->$ & Po-214 & & & \\
\hline 396 & $4.36 \mathrm{E}-05$ & $\mathrm{Bi}-214$ & $->$ & Po-214 & & & \\
\hline 405.73 & $2.43 \mathrm{E}-04$ & $\mathrm{Bi}-214$ & $->$ & Po-214 & & & \\
\hline 414.72 & $5.72 \mathrm{E}-07$ & Ra-226 & $->$ & $\mathrm{Rn}-222$ & & & \\
\hline 426.5 & $1.60 \mathrm{E}-04$ & $\mathrm{Bi}-214$ & $->$ & Po-214 & & & \\
\hline 440.4 & $4.21 \mathrm{E}-05$ & $\mathrm{Bi}-214$ & $->$ & Po-214 & & & \\
\hline 449.5 & $3.96 \mathrm{E}-07$ & $\mathrm{Ra}-226$ & $->$ & $\mathrm{Rn}-222$ & & & \\
\hline 454.832 & $4.62 \mathrm{E}-04$ & $\mathrm{Bi}-214$ & $->$ & Po-214 & & & \\
\hline 454.97 & $2.28 \mathrm{E}+00$ & $U-234$ & $->$ & Th-230 & & & \\
\hline 462.05 & $2.47 \mathrm{E}-04$ & $\mathrm{~Pb}-214$ & $->$ & Bi-214 & & & \\
\hline 469.76 & $1.93 \mathrm{E}-04$ & $\mathrm{Bi}-214$ & $->$ & Po-214 & & & \\
\hline 470.8 & $1.45 \mathrm{E}-05$ & $\mathrm{~Pb}-214$ & $->$ & $\mathrm{Bi}-214$ & & & \\
\hline 474.51 & $1.71 \mathrm{E}-04$ & $\mathrm{Bi}-214$ & $->$ & Po-214 & & & \\
\hline 478 & $6.10 \mathrm{E}-07$ & TI- 210 & $->$ & $\mathrm{Pb}-210$ & & & \\
\hline
\end{tabular}




\begin{tabular}{|c|c|c|c|c|}
\hline 480.32 & $4.91 \mathrm{E}-04$ & $\mathrm{~Pb}-214$ & $\rightarrow$ & $\mathrm{Bi}-214$ \\
\hline 487.13 & $6.38 \mathrm{E}-04$ & $\mathrm{~Pb}-214$ & $->$ & $\mathrm{Bi}-214$ \\
\hline 494.6 & $1.31 \mathrm{E}-05$ & $\mathrm{Bi}-214$ & $->$ & Po-214 \\
\hline 502.2 & $2.61 \mathrm{E}-05$ & $\mathrm{Bi}-214$ & $->$ & Po-214 \\
\hline 503.53 & 8.77E-02 & $U-234$ & $->$ & Th-230 \\
\hline 508.2 & $1.29 \mathrm{E}+00$ & U-234 & $->$ & Th-230 \\
\hline 510 & $1.02 \mathrm{E}-04$ & $\mathrm{Rn}-222$ & $->$ & Po-218 \\
\hline 511 & $4.21 \mathrm{E}-05$ & $\mathrm{~Pb}-214$ & $->$ & $\mathrm{Bi}-214$ \\
\hline 520.4 & $8.28 \mathrm{E}-06$ & $\mathrm{Bi}-214$ & $->$ & Po-214 \\
\hline 525 & $2.32 \mathrm{E}-05$ & $\mathrm{Bi}-214$ & $->$ & Po-214 \\
\hline 533.5 & $2.76 \mathrm{E}-04$ & $\mathrm{~Pb}-214$ & $->$ & $\mathrm{Bi}-214$ \\
\hline 536.93 & $1.03 \mathrm{E}-04$ & $\mathrm{Bi}-214$ & $->$ & Po-214 \\
\hline 538.7 & $7.12 \mathrm{E}-06$ & $\mathrm{~Pb}-214$ & $->$ & $\mathrm{Bi}-214$ \\
\hline 542.84 & $1.23 \mathrm{E}-04$ & $\mathrm{Bi}-214$ & $->$ & Po-214 \\
\hline 543.91 & $3.34 \mathrm{E}-05$ & $\mathrm{~Pb}-214$ & $->$ & $\mathrm{Bi}-214$ \\
\hline 547.1 & $4.65 E-05$ & $\mathrm{Bi}-214$ & $->$ & Po-214 \\
\hline 551.8 & $2.05 E-06$ & Th-230 & $->$ & Ra-226 \\
\hline 570.5 & $6.08 \mathrm{E}-06$ & Th-230 & $->$ & Ra-226 \\
\hline 572.67 & $1.19 \mathrm{E}-04$ & $\mathrm{Bi}-214$ & $->$ & Po-214 \\
\hline 580.06 & $5.29 E-04$ & $\mathrm{~Pb}-214$ & $->$ & $\mathrm{Bi}-214$ \\
\hline 581.78 & $1.05 \mathrm{E}+00$ & U-234 & $->$ & Th-230 \\
\hline 596 & $1.74 \mathrm{E}-05$ & $\mathrm{Bi}-214$ & $->$ & Po-214 \\
\hline 600.83 & 8.94E-07 & $\mathrm{Ra}-226$ & $->$ & $\mathrm{Rn}-222$ \\
\hline 609.311 & $6.70 \mathrm{E}-02$ & $\mathrm{Bi}-214$ & $->$ & Po-214 \\
\hline 615.77 & $1.02 \mathrm{E}-04$ & $\mathrm{Bi}-214$ & $->$ & Po-214 \\
\hline 617.1 & 4.94E-05 & $\mathrm{Bi}-214$ & $->$ & Po-214 \\
\hline 620 & $2.05 \mathrm{E}-06$ & Th-230 & $->$ & Ra-226 \\
\hline 624.44 & 7.01E-02 & U-234 & $->$ & Th-230 \\
\hline 626.4 & $7.12 \mathrm{E}-06$ & $\mathrm{Bi}-214$ & $->$ & Po-214 \\
\hline 631.2 & 2.47E-05 & $\mathrm{Bi}-214$ & $->$ & Po-214 \\
\hline 633.14 & 8.72E-05 & $\mathrm{Bi}-214$ & $->$ & Po-214 \\
\hline 639.36 & 4.50E-05 & $\mathrm{Bi}-214$ & $->$ & Po-214 \\
\hline 649.18 & 8.57E-05 & $\mathrm{Bi}-214$ & $->$ & Po-214 \\
\hline 650.21 & $3.01 \mathrm{E}-10$ & $\mathrm{Hg}-206$ & $->$ & TI-206 \\
\hline 660.75 & $6.25 E-05$ & $\mathrm{Bi}-214$ & $->$ & Po-214 \\
\hline 665.442 & $2.27 \mathrm{E}-03$ & $\mathrm{Bi}-214$ & $->$ & Po-214 \\
\hline 668 & $6.10 \mathrm{E}-07$ & TI-210 & $->$ & $\mathrm{Pb}-210$ \\
\hline 677.67 & 8.77E-02 & $U-234$ & $->$ & Th-230 \\
\hline 683.21 & $1.15 \mathrm{E}-04$ & $\mathrm{Bi}-214$ & $->$ & Рo-214 \\
\hline 687.7 & $8.72 \mathrm{E}-06$ & $\mathrm{Bi}-214$ & $->$ & Po-214 \\
\hline 693.3 & $8.72 E-06$ & $\mathrm{Bi}-214$ & $->$ & Po-214 \\
\hline 697.89 & 5.38E-05 & $\mathrm{Bi}-214$ & $->$ & Po-214 \\
\hline 703.07 & $6.86 \mathrm{E}-04$ & $\mathrm{Bi}-214$ & $->$ & Po-214 \\
\hline 706.02 & $9.19 E+02$ & Pu-238 & $->$ & $U-234$ \\
\hline 708.4 & $2.31 E+03$ & Pu-238 & $->$ & $U-234$ \\
\hline 710.84 & $1.09 E-04$ & $\mathrm{Bi}-214$ & $->$ & Po-214 \\
\hline 719.856 & $5.85 E-04$ & $\mathrm{Bi}-214$ & $->$ & Po-214 \\
\hline 723.32 & $6.54 \mathrm{E}-05$ & $\mathrm{Bi}-214$ & $->$ & Po-214 \\
\hline 727.8 & $2.28 \mathrm{E}-05$ & $\mathrm{Bi}-214$ & $->$ & Po-214 \\
\hline 733.64 & $6.83 E-05$ & $\mathrm{Bi}-214$ & $->$ & Po-214 \\
\hline
\end{tabular}




\begin{tabular}{|c|c|c|c|c|c|c|c|}
\hline 740.87 & 5.67E-05 & $\mathrm{Bi}-214$ & $->$ & Po-214 & & & \\
\hline 742.817 & $4.75 E+04$ & Pu-238 & $->$ & $U-234$ & & & \\
\hline 752.843 & $1.93 \mathrm{E}-04$ & $\mathrm{Bi}-214$ & $->$ & Po-214 & & & \\
\hline 765.9 & $1.15 \mathrm{E}-04$ & $\mathrm{~Pb}-214$ & $->$ & $\mathrm{Bi}-214$ & & & \\
\hline 766.412 & $2.01 \mathrm{E}+05$ & Pu-238 & $->$ & $U-234$ & & & \\
\hline 768.35 & 7.09E-03 & $\mathrm{Bi}-214$ & $->$ & Рo-214 & & & \\
\hline 783.38 & $2.80 \mathrm{E}+02$ & Pu-238 & $->$ & U-234 & & & \\
\hline 785.827 & $1.58 \mathrm{E}-03$ & $\mathrm{~Pb}-214$ & $->$ & $\mathrm{Bi}-214$ & & & \\
\hline 786.287 & $2.86 \mathrm{E}+04$ & Pu-238 & $->$ & $U-234$ & & & \\
\hline 786.42 & $4.50 E-04$ & $\mathrm{Bi}-214$ & $->$ & Po-214 & & & \\
\hline 797.88 & 4.53E-05 & TI-210 & $->$ & $\mathrm{Pb}-210$, & Po-214 & $->$ & $\mathrm{Pb}-210$ \\
\hline 799.75 & $5.96 \mathrm{E}-05$ & $\mathrm{Bi}-214$ & $->$ & Po-214 & & & \\
\hline 803.13 & $3.87 E-08$ & TI-206 & $->$ & $\mathrm{Pb}-206$, & Po-210 & $->$ & Pb-206 \\
\hline 805.87 & $1.05 \mathrm{E}+03$ & Pu-238 & $->$ & $U-234$ & & & \\
\hline 806.155 & $1.79 \mathrm{E}-03$ & $\mathrm{Bi}-214$ & $->$ & Po-214 & & & \\
\hline 808.25 & $6.45 E+03$ & Pu-238 & $->$ & U-234 & & & \\
\hline 814.87 & $5.81 \mathrm{E}-05$ & $\mathrm{Bi}-214$ & $->$ & Po-214 & & & \\
\hline 821.166 & $2.18 \mathrm{E}-04$ & $\mathrm{Bi}-214$ & $->$ & Po-214 & & & \\
\hline 826.44 & $1.34 \mathrm{E}-04$ & $\mathrm{Bi}-214$ & $->$ & Po-214 & & & \\
\hline 832.34 & 3.34E-05 & $\mathrm{Bi}-214$ & $->$ & Po-214 & & & \\
\hline 838.999 & 8.53E-04 & $\mathrm{Pb}-214$ & $->$ & $\mathrm{Bi}-214$ & & & \\
\hline 847.2 & 2.47E-05 & $\mathrm{Bi}-214$ & $->$ & Po-214 & & & \\
\hline 851.72 & $1.24 \mathrm{E}+04$ & Pu-238 & $->$ & U-234 & & & \\
\hline 860 & $2.11 \mathrm{E}-06$ & TI-210 & $->$ & $\mathrm{Pb}-210$ & & & \\
\hline 880.53 & $1.40 \mathrm{E}+03$ & Pu-238 & $->$ & U-234 & & & \\
\hline 883.24 & $6.76 \mathrm{E}+03$ & Pu-238 & $->$ & $U-234$ & & & \\
\hline 904.33 & $1.53 \mathrm{E}-04$ & $\mathrm{Bi}-214$ & $->$ & Po-214 & & & \\
\hline 904.38 & $6.09 E+02$ & Pu-238 & $->$ & U-234 & & & \\
\hline 908 & $9.15 \mathrm{E}-07$ & TI-210 & $->$ & $\mathrm{Pb}-210$ & & & \\
\hline 915.8 & $3.34 \mathrm{E}-05$ & $\mathrm{Bi}-214$ & $->$ & Po-214 & & & \\
\hline 926.71 & $4.99 E+03$ & Pu-238 & $->$ & U-234 & & & \\
\hline 934.039 & 4.59E-03 & $\mathrm{Bi}-214$ & $->$ & Po-214 & & & \\
\hline 942.05 & $3.90 \mathrm{E}+03$ & Pu-238 & $->$ & U-234 & & & \\
\hline 943.3 & 2.47E-05 & $\mathrm{Bi}-214$ & $->$ & Po-214 & & & \\
\hline 946.02 & $7.92 \mathrm{E}+02$ & Pu-238 & $->$ & $U-234$ & & & \\
\hline 964.07 & $5.56 \mathrm{E}-04$ & $\mathrm{Bi}-214$ & $->$ & Рo-214 & & & \\
\hline 976.2 & 3.34E-05 & $B i-214$ & $->$ & Po-214 & & & \\
\hline 980.39 & $4.87 E+02$ & Pu-238 & $->$ & U-234 & & & \\
\hline 989.2 & $1.74 \mathrm{E}-05$ & $\mathrm{Bi}-214$ & $->$ & Po-214 & & & \\
\hline 1001 & $8.34 \mathrm{E}+03$ & Pu-238 & $->$ & U-234 & & & \\
\hline 1013.4 & $1.45 \mathrm{E}-05$ & $\mathrm{Bi}-214$ & $->$ & Po-214 & & & \\
\hline 1020.5 & $1.74 \mathrm{E}-05$ & $\mathrm{Bi}-214$ & $->$ & Po-214 & & & \\
\hline 1032.22 & $1.39 \mathrm{E}-04$ & $\mathrm{Bi}-214$ & $->$ & Po-214 & & & \\
\hline 1038 & $2.47 \mathrm{E}-05$ & $\mathrm{Bi}-214$ & $->$ & Po-214 & & & \\
\hline 1041.9 & $1.76 \mathrm{E}+03$ & Pu-238 & $->$ & U-234 & & & \\
\hline 1045.4 & $4.21 \mathrm{E}-05$ & $\mathrm{Bi}-214$ & $->$ & Po-214 & & & \\
\hline 1051.95 & $4.58 \mathrm{E}-04$ & $\mathrm{Bi}-214$ & $->$ & Po-214 & & & \\
\hline 1067.3 & $4.21 \mathrm{E}-05$ & $\mathrm{Bi}-214$ & $->$ & Po-214 & & & \\
\hline 1068 & $3.66 \mathrm{E}-06$ & TI-210 & $->$ & $\mathrm{Pb}-210$ & & & \\
\hline 1070.02 & $4.14 \mathrm{E}-04$ & $\mathrm{Bi}-214$ & $->$ & Po-214 & & & \\
\hline
\end{tabular}




\begin{tabular}{|c|c|c|c|c|}
\hline 1085.37 & $6.39 E+02$ & Pu-238 & $->$ & $U-234$ \\
\hline 1103.7 & $1.45 \mathrm{E}-04$ & $\mathrm{Bi}-214$ & $->$ & Po-214 \\
\hline 1104.766 & $1.16 \mathrm{E}-04$ & $\mathrm{Bi}-214$ & $->$ & Po-214 \\
\hline 1110 & $2.11 \mathrm{E}-06$ & TI-210 & $->$ & $\mathrm{Pb}-210$ \\
\hline 1120.273 & $2.18 \mathrm{E}-02$ & $\mathrm{Bi}-214$ & $->$ & Po-214 \\
\hline 1130.6 & $6.54 \mathrm{E}-05$ & $\mathrm{Bi}-214$ & $->$ & Po-214 \\
\hline 1133.65 & $3.70 \mathrm{E}-04$ & $\mathrm{Bi}-214$ & $->$ & Po-214 \\
\hline 1155.183 & $2.46 \mathrm{E}-03$ & $\mathrm{Bi}-214$ & $->$ & Po-214 \\
\hline 1172.93 & $8.43 E-05$ & $\mathrm{Bi}-214$ & $->$ & Po-214 \\
\hline 1173.04 & 8.43E-05 & $\mathrm{Bi}-214$ & $->$ & Po-214 \\
\hline 1207.764 & $6.68 \mathrm{E}-04$ & $\mathrm{Bi}-214$ & $->$ & Po-214 \\
\hline 1208 & 5.19E-06 & TI-210 & $->$ & $\mathrm{Pb}-210$ \\
\hline 1226.8 & 3.92E-05 & $\mathrm{Bi}-214$ & $->$ & Po-214 \\
\hline 1230.84 & $3.20 \mathrm{E}-05$ & $\mathrm{Bi}-214$ & $->$ & Po-214 \\
\hline 1238.107 & $8.60 \mathrm{E}-03$ & $\mathrm{Bi}-214$ & $->$ & Po-214 \\
\hline 1280.952 & $2.14 \mathrm{E}-03$ & $\mathrm{Bi}-214$ & $->$ & Po-214 \\
\hline 1303.76 & $1.76 \mathrm{E}-04$ & $\mathrm{Bi}-214$ & $->$ & Po-214 \\
\hline 1314 & $6.41 \mathrm{E}-06$ & TI-210 & $->$ & $\mathrm{Pb}-210$ \\
\hline 1317.02 & $1.25 \mathrm{E}-04$ & $\mathrm{Bi}-214$ & $->$ & Po-214 \\
\hline 1330 & $1.60 \mathrm{E}-05$ & $\mathrm{Bi}-214$ & $->$ & Po-214 \\
\hline 1341.5 & $3.34 \mathrm{E}-05$ & $\mathrm{Bi}-214$ & $->$ & Po-214 \\
\hline 1353 & $6.54 \mathrm{E}-06$ & $\mathrm{Bi}-214$ & $->$ & Po-214 \\
\hline 1377.659 & $5.84 \mathrm{E}-03$ & $\mathrm{Bi}-214$ & $->$ & Po-214 \\
\hline 1385.295 & $1.13 \mathrm{E}-03$ & $\mathrm{Bi}-214$ & $->$ & Po-214 \\
\hline 1392.5 & $2.76 \mathrm{E}-05$ & $\mathrm{Bi}-214$ & $->$ & Po-214 \\
\hline 1401.48 & $2.02 \mathrm{E}-03$ & $\mathrm{Bi}-214$ & $->$ & Po-214 \\
\hline 1407.97 & $3.60 \mathrm{E}-03$ & $\mathrm{Bi}-214$ & $->$ & Po-214 \\
\hline 1408 & $1.50 \mathrm{E}-06$ & TI-210 & $->$ & $\mathrm{Pb}-210$ \\
\hline 1419.7 & $7.41 \mathrm{E}-06$ & $\mathrm{Bi}-214$ & $->$ & Po-214 \\
\hline 1471.1 & $1.74 \mathrm{E}-05$ & $\mathrm{Bi}-214$ & $->$ & Po-214 \\
\hline 1479.19 & $1.00 \mathrm{E}-04$ & $\mathrm{Bi}-214$ & $->$ & Po-214 \\
\hline 1509.22 & $3.18 \mathrm{E}-03$ & $\mathrm{Bi}-214$ & $->$ & Po-214 \\
\hline 1538 & $6.10 \mathrm{E}-07$ & TI-210 & $->$ & $\mathrm{Pb}-210$ \\
\hline 1538.49 & $5.96 \mathrm{E}-04$ & $\mathrm{Bi}-214$ & $->$ & Po-214 \\
\hline 1543.347 & $5.08 \mathrm{E}-04$ & $\mathrm{Bi}-214$ & $->$ & Po-214 \\
\hline 1583.22 & $1.05 \mathrm{E}-03$ & $\mathrm{Bi}-214$ & $->$ & Po-214 \\
\hline 1588 & $6.10 \mathrm{E}-07$ & TI-210 & $->$ & $\mathrm{Pb}-210$ \\
\hline 1594.78 & $3.85 \mathrm{E}-04$ & $\mathrm{Bi}-214$ & $->$ & Po-214 \\
\hline 1599.3 & $4.85 E-04$ & $\mathrm{Bi}-214$ & $->$ & Po-214 \\
\hline 1636.6 & $2.76 \mathrm{E}-05$ & $\mathrm{Bi}-214$ & $->$ & Po-214 \\
\hline 1637.37 & $1.02 \mathrm{E}-04$ & $\mathrm{Bi}-214$ & $->$ & Po-214 \\
\hline 1648 & $6.10 \mathrm{E}-07$ & TI-210 & $->$ & $\mathrm{Pb}-210$ \\
\hline 1661.258 & $1.67 \mathrm{E}-03$ & $\mathrm{Bi}-214$ & $->$ & Po-214 \\
\hline 1683.99 & $3.43 \mathrm{E}-04$ & $\mathrm{Bi}-214$ & $->$ & Po-214 \\
\hline 1729.58 & $4.43 E-03$ & $\mathrm{Bi}-214$ & $->$ & Po-214 \\
\hline 1764.49 & $2.31 \mathrm{E}-02$ & $\mathrm{Bi}-214$ & $->$ & Po-214 \\
\hline 1782.1 & $2.32 \mathrm{E}-05$ & $\mathrm{Bi}-214$ & $->$ & Po-214 \\
\hline 1814.01 & $1.74 \mathrm{E}-05$ & $\mathrm{Bi}-214$ & $->$ & Po-214 \\
\hline 1838.37 & $5.56 \mathrm{E}-04$ & $\mathrm{Bi}-214$ & $->$ & Po-214 \\
\hline 1847.41 & $3.08 \mathrm{E}-03$ & $\mathrm{Bi}-214$ & $->$ & Po-214 \\
\hline
\end{tabular}




\begin{tabular}{|c|c|c|c|c|}
\hline 1873.112 & $3.28 \mathrm{E}-04$ & $\mathrm{Bi}-214$ & $\rightarrow$ & Po-214 \\
\hline 1890.259 & $1.29 \mathrm{E}-04$ & $\mathrm{Bi}-214$ & $->$ & Po-214 \\
\hline 1896.28 & 2.57E-04 & $\mathrm{Bi}-214$ & $->$ & Po-214 \\
\hline 1898.9 & $9.15 \mathrm{E}-05$ & $\mathrm{Bi}-214$ & $->$ & Po-214 \\
\hline 1935.8 & 7.41E-05 & $\mathrm{Bi}-214$ & $->$ & Po-214 \\
\hline 1994.7 & $7.26 \mathrm{E}-06$ & $\mathrm{Bi}-214$ & $->$ & Po-214 \\
\hline 2004.5 & $4.21 \mathrm{E}-06$ & $\mathrm{Bi}-214$ & $->$ & Po-214 \\
\hline 2008 & $2.11 \mathrm{E}-06$ & TI-210 & $->$ & $\mathrm{Pb}-210$ \\
\hline 2010.79 & $7.12 \mathrm{E}-05$ & $\mathrm{Bi}-214$ & $->$ & Po-214 \\
\hline 2021.7 & $2.76 \mathrm{E}-05$ & $\mathrm{Bi}-214$ & $->$ & Po-214 \\
\hline 2052.93 & $1.02 \mathrm{E}-04$ & $\mathrm{Bi}-214$ & $->$ & Po-214 \\
\hline 2085 & $1.45 \mathrm{E}-05$ & $\mathrm{Bi}-214$ & $->$ & Po-214 \\
\hline 2088 & $1.50 \mathrm{E}-06$ & TI-210 & $->$ & $\mathrm{Pb}-210$ \\
\hline 2089.55 & $8.14 \mathrm{E}-05$ & $\mathrm{Bi}-214$ & $->$ & Po-214 \\
\hline 2109.91 & $1.26 \mathrm{E}-04$ & $\mathrm{Bi}-214$ & $->$ & Po-214 \\
\hline 2118.53 & $1.76 \mathrm{E}-03$ & $\mathrm{Bi}-214$ & $->$ & Po-214 \\
\hline 2147.8 & $2.32 \mathrm{E}-05$ & $\mathrm{Bi}-214$ & $->$ & Po-214 \\
\hline 2192.52 & $8.86 E-05$ & $\mathrm{Bi}-214$ & $->$ & Po-214 \\
\hline 2204.09 & $7.25 \mathrm{E}-03$ & $\mathrm{Bi}-214$ & $->$ & Po-214 \\
\hline 2251.2 & $1.02 \mathrm{E}-05$ & $\mathrm{Bi}-214$ & $->$ & Po-214 \\
\hline 2259.7 & $1.31 \mathrm{E}-05$ & $\mathrm{Bi}-214$ & $->$ & Po-214 \\
\hline 2266.67 & $2.61 \mathrm{E}-05$ & $\mathrm{Bi}-214$ & $->$ & Po-214 \\
\hline 2268 & $9.15 \mathrm{E}-07$ & TI-210 & $->$ & $\mathrm{Pb}-210$ \\
\hline 2270 & $4.21 \mathrm{E}-06$ & $\mathrm{Bi}-214$ & $->$ & Po-214 \\
\hline 2284.4 & $7.41 \mathrm{E}-06$ & $\mathrm{Bi}-214$ & $->$ & Po-214 \\
\hline 2293.29 & $4.71 \mathrm{E}-04$ & $\mathrm{Bi}-214$ & $->$ & Po-214 \\
\hline 2324.8 & $2.76 \mathrm{E}-06$ & $\mathrm{Bi}-214$ & $->$ & Po-214 \\
\hline 2331.2 & $3.20 \mathrm{E}-05$ & $\mathrm{Bi}-214$ & $->$ & Po-214 \\
\hline 2358 & $2.44 \mathrm{E}-06$ & Tl-210 & $->$ & $\mathrm{Pb}-210$ \\
\hline 2360.9 & $2.76 \mathrm{E}-06$ & $\mathrm{Bi}-214$ & $->$ & Po-214 \\
\hline 2369.3 & $4.21 \mathrm{E}-06$ & $\mathrm{Bi}-214$ & $->$ & Po-214 \\
\hline 2376.99 & $1.71 \mathrm{E}-05$ & $\mathrm{Bi}-214$ & $->$ & Po-214 \\
\hline 2390.9 & $2.91 \mathrm{E}-06$ & $\mathrm{Bi}-214$ & $->$ & Po-214 \\
\hline 2423.32 & 8.57E-06 & $\mathrm{Bi}-214$ & $->$ & Po-214 \\
\hline 2428 & $2.75 \mathrm{E}-06$ & Tl-210 & $->$ & $\mathrm{Pb}-210$ \\
\hline 2447.68 & $2.25 \mathrm{E}-03$ & $\mathrm{Bi}-214$ & $->$ & Po-214 \\
\hline 2482.417 & $3.05 E-06$ & $\mathrm{Bi}-214$ & $->$ & Po-214 \\
\hline 2505.58 & 8.57E-06 & $\mathrm{Bi}-214$ & $->$ & Po-214 \\
\hline 2551 & 5.67E-07 & $\mathrm{Bi}-214$ & $->$ & Po-214 \\
\hline 2604.5 & $6.54 \mathrm{E}-07$ & $\mathrm{Bi}-214$ & $->$ & Po-214 \\
\hline 2631 & $1.31 \mathrm{E}-06$ & $\mathrm{Bi}-214$ & $->$ & Po-214 \\
\hline 2662.23 & $4.21 E-07$ & $\mathrm{Bi}-214$ & $->$ & Po-214 \\
\hline 2694.67 & 4.71E-05 & $\mathrm{Bi}-214$ & $->$ & Po-214 \\
\hline 2698.86 & 4.07E-06 & $\mathrm{Bi}-214$ & $->$ & Po-214 \\
\hline 2719.21 & $2.61 \mathrm{E}-06$ & $\mathrm{Bi}-214$ & $->$ & Po-214 \\
\hline 2769.99 & $3.72 \mathrm{E}-05$ & $\mathrm{Bi}-214$ & $->$ & Po-214 \\
\hline 2786.09 & 8.57E-06 & $\mathrm{Bi}-214$ & $->$ & Po-214 \\
\hline 2827 & $3.63 \mathrm{E}-06$ & $\mathrm{Bi}-214$ & $->$ & Po-214 \\
\hline 2860.9 & $4.94 \mathrm{E}-07$ & $\mathrm{Bi}-214$ & $->$ & Po-214 \\
\hline 2880.4 & $1.34 \mathrm{E}-05$ & $\mathrm{Bi}-214$ & $->$ & Po-214 \\
\hline
\end{tabular}




$\begin{array}{ccccc}2893.59 & 9.30 \mathrm{E}-06 & \mathrm{Bi}-214 & \rightarrow & \mathrm{Po}-214 \\ 2922.09 & 2.28 \mathrm{E}-05 & \mathrm{Bi}-214 & -> & \mathrm{Po}-214 \\ 2928.7 & 1.74 \mathrm{E}-06 & \mathrm{Bi}-214 & -> & \mathrm{Po}-214 \\ 2934.9 & 8.28 \mathrm{E}-07 & \mathrm{Bi}-214 & \rightarrow & \mathrm{Po}-214 \\ 2940.5 & 2.47 \mathrm{E}-06 & \mathrm{Bi}-214 & -> & \mathrm{Po}-214 \\ 2978.79 & 2.14 \mathrm{E}-05 & \mathrm{Bi}-214 & -> & \mathrm{Po}-214 \\ 2988.7 & 1.60 \mathrm{E}-06 & \mathrm{Bi}-214 & -> & \mathrm{Po}-214 \\ 2999.99 & 1.28 \mathrm{E}-05 & \mathrm{Bi}-214 & -> & \mathrm{Po}-214 \\ 3053.89 & 3.28 \mathrm{E}-05 & \mathrm{Bi}-214 & -> & \mathrm{Po}-214 \\ 3081.7 & 6.25 \mathrm{E}-06 & \mathrm{Bi}-214 & -> & \mathrm{Po}-214 \\ 3093.9 & 7.41 \mathrm{E}-07 & \mathrm{Bi}-214 & -> & \mathrm{Po}-214 \\ 3136.3 & 4.94 \mathrm{E}-07 & \mathrm{Bi}-214 & -> & \mathrm{Po}-214 \\ 3142.6 & 2.32 \mathrm{E}-06 & \mathrm{Bi}-214 & -> & \mathrm{Po}-214 \\ 3160.5 & 7.41 \mathrm{E}-07 & \mathrm{Bi}-214 & -> & \mathrm{Po}-214 \\ 3183.6 & 2.18 \mathrm{E}-06 & \mathrm{Bi}-214 & -> & \mathrm{Po}-214 \\ 3233.3 & 2.91 \mathrm{E}-07 & \mathrm{Bi}-214 & -> & \mathrm{Po}-214 \\ 3269.7 & 1.45 \mathrm{E}-07 & \mathrm{Bi}-214 & -> & \mathrm{Po}-214\end{array}$

\begin{tabular}{|c|c|c|c|c|}
\hline Pu238 & $\begin{array}{c}\text { Aged } 5 \\
\text { years }\end{array}$ & \multicolumn{3}{|c|}{ Sorted by Intensity } \\
\hline Intensity & Energy & \multicolumn{3}{|c|}{ Initial Isotopes and Decays } \\
\hline $3.17 E+10$ & 17.128 & Pu-238 & $->$ & $U-234$ \\
\hline $2.56 \mathrm{E}+10$ & 13.6 & Pu-238 & $->$ & $U-234$ \\
\hline $7.00 \mathrm{E}+09$ & 20.292 & Pu-238 & $->$ & $U-234$ \\
\hline $1.52 \mathrm{E}+09$ & 11.62 & Pu-238 & $->$ & $U-234$ \\
\hline $6.21 \mathrm{E}+08$ & 15.4 & Pu-238 & $->$ & $U-234$ \\
\hline $2.41 E+08$ & 43.498 & Pu-238 & $->$ & $U-234$ \\
\hline $4.32 E+07$ & 99.864 & Pu-238 & $->$ & $U-234$ \\
\hline $5.82 \mathrm{E}+06$ & 152.68 & Pu-238 & $->$ & $U-234$ \\
\hline $1.13 \mathrm{E}+06$ & 98.441 & Pu-238 & $->$ & $U-234$ \\
\hline $7.00 \mathrm{E}+05$ & 94.66 & Pu-238 & $->$ & $U-234$ \\
\hline $2.37 E+05$ & 111.3 & Pu-238 & $->$ & $U-234$ \\
\hline $2.37 E+05$ & 114.333 & Pu-238 & $->$ & $U-234$ \\
\hline $2.01 E+05$ & 766.412 & Pu-238 & $->$ & U-234 \\
\hline $1.22 \mathrm{E}+05$ & 110.421 & Pu-238 & $->$ & $U-234$ \\
\hline $9.62 \mathrm{E}+04$ & 114.561 & Pu-238 & $->$ & $U-234$ \\
\hline $4.75 \mathrm{E}+04$ & 742.817 & Pu-238 & $->$ & $U-234$ \\
\hline $2.86 \mathrm{E}+04$ & 786.287 & Pu-238 & $->$ & $U-234$ \\
\hline $2.50 E+04$ & 200.98 & Pu-238 & $->$ & $U-234$ \\
\hline $1.24 \mathrm{E}+04$ & 851.72 & Pu-238 & $->$ & $\mathrm{U}-234$ \\
\hline $1.04 \mathrm{E}+04$ & 53.23 & $U-234$ & $->$ & Th-230 \\
\hline $8.34 \mathrm{E}+03$ & 1001 & Pu-238 & $->$ & $U-234$ \\
\hline $6.76 \mathrm{E}+03$ & 883.24 & Pu-238 & $->$ & $U-234$ \\
\hline $6.45 \mathrm{E}+03$ & 808.25 & Pu-238 & $->$ & $U-234$ \\
\hline $4.99 E+03$ & 926.71 & Pu-238 & $->$ & $U-234$ \\
\hline $3.90 \mathrm{E}+03$ & 942.05 & Pu-238 & $->$ & $U-234$ \\
\hline
\end{tabular}




\begin{tabular}{|c|c|c|c|c|c|c|c|}
\hline $3.59 E+03$ & 120.912 & $U-234$ & $\rightarrow$ & Th-230 & & & \\
\hline $2.31 \mathrm{E}+03$ & 708.4 & Pu-238 & $->$ & $\mathrm{U}-234$ & & & \\
\hline $1.76 \mathrm{E}+03$ & 1041.9 & Pu-238 & $->$ & $U-234$ & & & \\
\hline $1.40 \mathrm{E}+03$ & 880.53 & Pu-238 & $->$ & $U-234$ & & & \\
\hline $1.05 \mathrm{E}+03$ & 805.87 & Pu-238 & $->$ & $U-234$ & & & \\
\hline $9.19 E+02$ & 706.02 & Pu-238 & $->$ & $U-234$ & & & \\
\hline $7.92 E+02$ & 946.02 & Pu-238 & $->$ & $U-234$ & & & \\
\hline $7.92 E+02$ & 299.08 & Pu-238 & $->$ & $U-234$ & & & \\
\hline $7.25 E+02$ & 258.18 & Pu-238 & $->$ & $U-234$ & & & \\
\hline $6.39 E+02$ & 1085.37 & Pu-238 & $->$ & $U-234$ & & & \\
\hline $6.09 E+02$ & 904.38 & Pu-238 & $->$ & $U-234$ & & & \\
\hline $4.87 E+02$ & 980.39 & Pu-238 & $->$ & $U-234$ & & & \\
\hline $2.80 E+02$ & 783.38 & Pu-238 & $->$ & $U-234$ & & & \\
\hline $2.44 \mathrm{E}+02$ & 62.78 & Pu-238 & $->$ & $U-234$ & & & \\
\hline $6.09 E+01$ & 236.03 & Pu-238 & $->$ & $U-234$ & & & \\
\hline $6.09 \mathrm{E}+01$ & 203.2 & Pu-238 & $->$ & $U-234$ & & & \\
\hline $3.04 \mathrm{E}+01$ & 174.52 & Pu-238 & $->$ & $U-234$ & & & \\
\hline $2.28 \mathrm{E}+00$ & 454.97 & U-234 & $->$ & Th-230 & & & \\
\hline $1.29 \mathrm{E}+00$ & 508.2 & $U-234$ & $->$ & Th-230 & & & \\
\hline $1.05 \mathrm{E}+00$ & 581.78 & U-234 & $->$ & Th-230 & & & \\
\hline 7.62E-01 & 67.6758 & Th-230 & $->$ & Ra-226 & & & \\
\hline $9.85 \mathrm{E}-02$ & 143.876 & Th-230 & $->$ & Ra-226 & & & \\
\hline 8.77E-02 & 677.67 & U-234 & $->$ & Th-230 & & & \\
\hline 8.77E-02 & 503.53 & $U-234$ & $->$ & Th-230 & & & \\
\hline 7.01E-02 & 624.44 & $U-234$ & $->$ & Th-230 & & & \\
\hline $6.70 \mathrm{E}-02$ & 609.311 & $\mathrm{Bi}-214$ & $->$ & Po-214 & & & \\
\hline 5.39E-02 & 351.87 & $\mathrm{~Pb}-214$ & $->$ & $\mathrm{Bi}-214$ & & & \\
\hline $2.79 \mathrm{E}-02$ & 295.091 & $\mathrm{~Pb}-214$ & $->$ & $\mathrm{Bi}-214$ & & & \\
\hline $2.31 \mathrm{E}-02$ & 1764.49 & $\mathrm{Bi}-214$ & $->$ & Po-214 & & & \\
\hline $2.25 \mathrm{E}-02$ & 253.732 & Th-230 & $->$ & $\mathrm{Ra}-226$ & & & \\
\hline $2.18 \mathrm{E}-02$ & 1120.273 & $\mathrm{Bi}-214$ & $->$ & Po-214 & & & \\
\hline $1.78 \mathrm{E}-02$ & 186.057 & Th-230 & $->$ & $\mathrm{Ra}-226$ & & & \\
\hline $1.60 \mathrm{E}-02$ & 77.107 & $\mathrm{~Pb}-214$ & $->$ & $\mathrm{Bi}-214$ & & & \\
\hline $1.08 \mathrm{E}-02$ & 241.92 & $\mathrm{~Pb}-214$ & $->$ & $\mathrm{Bi}-214$ & & & \\
\hline $9.47 \mathrm{E}-03$ & 74.814 & $\mathrm{~Pb}-214$ & $->$ & $\mathrm{Bi}-214$ & & & \\
\hline 8.93E-03 & 10.828 & $\mathrm{~Pb}-210$ & $->$ & $\mathrm{Bi}-210$, & $\mathrm{Pb}-214$ & $->$ & $\mathrm{Bi}-214$ \\
\hline 8.60E-03 & 1238.107 & $\mathrm{Bi}-214$ & $->$ & Po-214 & & & \\
\hline $8.28 \mathrm{E}-03$ & 13.088 & $\mathrm{~Pb}-214$ & $->$ & $\mathrm{Bi}-214$ & & & \\
\hline 7.25E-03 & 2204.09 & $\mathrm{Bi}-214$ & $->$ & Po-214 & & & \\
\hline 7.09E-03 & 768.35 & $\mathrm{Bi}-214$ & $->$ & Po-214 & & & \\
\hline $5.84 \mathrm{E}-03$ & 1377.659 & $\mathrm{Bi}-214$ & $->$ & Po-214 & & & \\
\hline $5.64 \mathrm{E}-03$ & 87.19 & $\mathrm{~Pb}-214$ & $->$ & $\mathrm{Bi}-214$ & & & \\
\hline $4.81 \mathrm{E}-03$ & 186.11 & $\mathrm{Ra}-226$ & $->$ & $\mathrm{Rn}-222$ & & & \\
\hline 4.59E-03 & 934.039 & $\mathrm{Bi}-214$ & $->$ & Po-214 & & & \\
\hline $4.43 E-03$ & 1729.58 & $\mathrm{Bi}-214$ & $->$ & Po-214 & & & \\
\hline $3.60 \mathrm{E}-03$ & 1407.97 & $\mathrm{Bi}-214$ & $->$ & Po-214 & & & \\
\hline $3.18 \mathrm{E}-03$ & 1509.22 & $\mathrm{Bi}-214$ & $->$ & Po-214 & & & \\
\hline $3.08 \mathrm{E}-03$ & 1847.41 & $\mathrm{Bi}-214$ & $->$ & Po-214 & & & \\
\hline $2.46 \mathrm{E}-03$ & 1155.183 & $\mathrm{Bi}-214$ & $->$ & Po-214 & & & \\
\hline $2.27 \mathrm{E}-03$ & 665.442 & $\mathrm{Bi}-214$ & $->$ & Po-214 & & & \\
\hline
\end{tabular}




\begin{tabular}{|c|c|c|c|c|}
\hline $2.25 \mathrm{E}-03$ & 2447.68 & $\mathrm{Bi}-214$ & $\rightarrow$ & Po-214 \\
\hline $2.14 \mathrm{E}-03$ & 1280.952 & $\mathrm{Bi}-214$ & $->$ & Po-214 \\
\hline 2.02E-03 & 1401.48 & $\mathrm{Bi}-214$ & $->$ & Po-214 \\
\hline 1.79E-03 & 806.155 & $\mathrm{Bi}-214$ & $->$ & Po-214 \\
\hline $1.76 \mathrm{E}-03$ & 2118.53 & $\mathrm{Bi}-214$ & $->$ & Po-214 \\
\hline $1.72 \mathrm{E}-03$ & 253.84 & Th-230 & $->$ & $\mathrm{Ra}-226$ \\
\hline 1.69E-03 & 15.439 & $\mathrm{~Pb}-214$ & $->$ & $\mathrm{Bi}-214$ \\
\hline 1.67E-03 & 90.128 & $\mathrm{~Pb}-214$ & $->$ & $\mathrm{Bi}-214$ \\
\hline $1.67 \mathrm{E}-03$ & 1661.258 & $\mathrm{Bi}-214$ & $->$ & Po-214 \\
\hline $1.60 \mathrm{E}-03$ & 53.172 & $\mathrm{~Pb}-214$ & $->$ & $\mathrm{Bi}-214$ \\
\hline $1.58 \mathrm{E}-03$ & 785.827 & $\mathrm{~Pb}-214$ & $->$ & $\mathrm{Bi}-214$ \\
\hline $1.41 \mathrm{E}-03$ & 79.29 & $\mathrm{Bi}-214$ & $->$ & Po-214 \\
\hline $1.13 \mathrm{E}-03$ & 1385.295 & $\mathrm{Bi}-214$ & $->$ & Po-214 \\
\hline $1.05 E-03$ & 1583.22 & $\mathrm{Bi}-214$ & $->$ & Po-214 \\
\hline 8.53E-04 & 838.999 & $\mathrm{~Pb}-214$ & $->$ & $\mathrm{Bi}-214$ \\
\hline 8.43E-04 & 76.838 & $\mathrm{Bi}-214$ & $->$ & Рo-214 \\
\hline 7.99E-04 & 258.94 & $\mathrm{~Pb}-214$ & $->$ & $\mathrm{Bi}-214$ \\
\hline $6.86 \mathrm{E}-04$ & 703.07 & $\mathrm{Bi}-214$ & $->$ & Po-214 \\
\hline $6.68 \mathrm{E}-04$ & 1207.764 & $\mathrm{Bi}-214$ & $->$ & Po-214 \\
\hline $6.38 \mathrm{E}-04$ & 487.13 & $\mathrm{~Pb}-214$ & $->$ & $\mathrm{Bi}-214$ \\
\hline $5.96 \mathrm{E}-04$ & 1538.49 & $\mathrm{Bi}-214$ & $->$ & Po-214 \\
\hline $5.96 \mathrm{E}-04$ & 388.95 & $\mathrm{Bi}-214$ & $->$ & Po-214 \\
\hline $5.85 \mathrm{E}-04$ & 719.856 & $\mathrm{Bi}-214$ & $->$ & Рo-214 \\
\hline $5.69 \mathrm{E}-04$ & 13.066 & $\mathrm{~Pb}-210$ & $->$ & $\mathrm{Bi}-210$ \\
\hline $5.62 \mathrm{E}-04$ & 14.336 & Ra-226 & $->$ & $\mathrm{Rn}-222$ \\
\hline $5.56 \mathrm{E}-04$ & 964.07 & $\mathrm{Bi}-214$ & $->$ & Po-214 \\
\hline $5.56 \mathrm{E}-04$ & 11.119 & $\mathrm{Bi}-214$ & $->$ & Po-214 \\
\hline $5.56 \mathrm{E}-04$ & 1838.37 & $\mathrm{Bi}-214$ & $->$ & Po-214 \\
\hline $5.38 \mathrm{E}-04$ & 13.502 & $\mathrm{Bi}-214$ & $->$ & Po-214 \\
\hline 5.29E-04 & 580.06 & $\mathrm{~Pb}-214$ & $->$ & $\mathrm{Bi}-214$ \\
\hline $5.23 E-04$ & 386.834 & $\mathrm{Bi}-214$ & $->$ & Po-214 \\
\hline $5.08 \mathrm{E}-04$ & 1543.347 & $\mathrm{Bi}-214$ & $->$ & Po-214 \\
\hline $5.01 \mathrm{E}-04$ & 89.639 & $\mathrm{Bi}-214$ & $->$ & Po-214 \\
\hline 4.91E-04 & 480.32 & $\mathrm{~Pb}-214$ & $->$ & $\mathrm{Bi}-214$ \\
\hline 4.85E-04 & 1599.3 & $\mathrm{Bi}-214$ & $->$ & Po-214 \\
\hline $4.71 \mathrm{E}-04$ & 2293.29 & $\mathrm{Bi}-214$ & $->$ & Po-214 \\
\hline $4.65 \mathrm{E}-04$ & 274.56 & $\mathrm{~Pb}-214$ & $->$ & $\mathrm{Bi}-214$ \\
\hline $4.62 E-04$ & 454.832 & $\mathrm{Bi}-214$ & $->$ & Po-214 \\
\hline $4.58 \mathrm{E}-04$ & 1051.95 & $\mathrm{Bi}-214$ & $->$ & Po-214 \\
\hline $4.50 \mathrm{E}-04$ & 786.42 & $\mathrm{Bi}-214$ & $->$ & Po-214 \\
\hline 4.47E-04 & 9.419 & $\mathrm{~Pb}-210$ & $->$ & $\mathrm{Bi}-210$, \\
\hline $4.28 \mathrm{E}-04$ & 83.787 & Ra-226 & $->$ & $R n-222$ \\
\hline $4.14 \mathrm{E}-04$ & 1070.02 & $\mathrm{Bi}-214$ & $->$ & Po-214 \\
\hline 3.89E-04 & 11.713 & Ra-226 & $->$ & $\mathrm{Rn}-222$ \\
\hline $3.85 E-04$ & 1594.78 & $\mathrm{Bi}-214$ & $->$ & Po-214 \\
\hline $3.70 \mathrm{E}-04$ & 1133.65 & $\mathrm{Bi}-214$ & $->$ & Po-214 \\
\hline $3.43 E-04$ & 1683.99 & $\mathrm{Bi}-214$ & $->$ & Po-214 \\
\hline $3.28 \mathrm{E}-04$ & 1873.112 & $\mathrm{Bi}-214$ & $->$ & Po-214 \\
\hline $2.76 \mathrm{E}-04$ & 533.5 & $\mathrm{~Pb}-214$ & $->$ & $\mathrm{Bi}-214$ \\
\hline $2.61 \mathrm{E}-04$ & 273.7 & $\mathrm{Bi}-214$ & $->$ & Рo-214 \\
\hline
\end{tabular}

$\mathrm{Pb}-214 \quad \rightarrow \quad \mathrm{Bi}-214$ 


$\begin{array}{lc}2.58 \mathrm{E}-04 & 81.067 \\ 2.57 \mathrm{E}-04 & 1896.28 \\ 2.47 \mathrm{E}-04 & 462.05 \\ 2.43 \mathrm{E}-04 & 405.73 \\ 2.21 \mathrm{E}-04 & 46.52 \\ 2.18 \mathrm{E}-04 & 821.166 \\ 1.93 \mathrm{E}-04 & 469.76 \\ 1.93 \mathrm{E}-04 & 752.843 \\ 1.76 \mathrm{E}-04 & 1303.76 \\ 1.71 \mathrm{E}-04 & 474.51 \\ 1.60 \mathrm{E}-04 & 426.5 \\ 1.53 \mathrm{E}-04 & 92.673 \\ 1.53 \mathrm{E}-04 & 904.33 \\ 1.51 \mathrm{E}-04 & 94.677 \\ 1.45 \mathrm{E}-04 & 1103.7 \\ 1.39 \mathrm{E}-04 & 1032.22 \\ 1.38 \mathrm{E}-04 & 333.6 \\ 1.37 \mathrm{E}-04 & 15.537 \\ 1.34 \mathrm{E}-04 & 826.44 \\ 1.29 \mathrm{E}-04 & 1890.259 \\ 1.26 \mathrm{E}-04 & 2109.91 \\ 1.25 \mathrm{E}-04 & 1317.02 \\ 1.23 \mathrm{E}-04 & 542.84 \\ 1.23 \mathrm{E}-04 & 16.874 \\ 1.20 \mathrm{E}-04 & 109.97 \\ 1.19 \mathrm{E}-04 & 572.67 \\ 1.18 \mathrm{E}-04 & 280.93 \\ 1.16 \mathrm{E}-04 & 1104.766 \\ 1.15 \mathrm{E}-04 & 765.9 \\ 1.15 \mathrm{E}-04 & 314.2 \\ 1.15 \mathrm{E}-04 & 683.21 \\ 1.09 \mathrm{E}-04 & 710.84 \\ 1.05 \mathrm{E}-04 & 11.712 \\ 1.03 \mathrm{E}-04 & 15.874 \\ 1.03 \mathrm{E}-04 & 536.93 \\ 1.02 \mathrm{E}-04 & 510 \\ 1.02 \mathrm{E}-04 & 1637.37 \\ 1.02 \mathrm{E}-04 & 2052.93 \\ 1.02 \mathrm{E}-04 & 615.77 \\ 1.00 \mathrm{E}-04 & 1479.19 \\ 9.15 \mathrm{E}-05 & 1898.9 \\ 8.86 \mathrm{E}-05 & 2192.52 \\ 8.72 \mathrm{E}-05 & 347.1 \\ 8.72 \mathrm{E}-05 & 633.14 \\ 8.57 \mathrm{E}-05 & 137.4 \\ 8.57 \mathrm{E}-05 & 649.18 \\ 8.43 \mathrm{E}-05 & 1173.04 \\ 8.43 \mathrm{E}-05 & 1172.93 \\ 8.28 \mathrm{E}-05 & 334.9 \\ 8.14 \mathrm{E}-05 & 2089.55\end{array}$

\begin{tabular}{|c|c|c|}
\hline Ra-226 & $->$ & $2 n-222$ \\
\hline $\mathrm{Bi}-214$ & $->$ & כo-214 \\
\hline $\mathrm{Pb}-214$ & -> & 214 \\
\hline $\mathrm{Bi}-214$ & $->$ & $0-214$ \\
\hline $\mathrm{Pb}-210$ & $\rightarrow$ & $3 i-210$ \\
\hline $\mathrm{Bi}-214$ & $->$ & م-214 \\
\hline $\mathrm{Bi}-214$ & -> & م-214 \\
\hline $\mathrm{Bi}-214$ & -> & م-214 \\
\hline $\mathrm{Bi}-214$ & -> & $0-21$ \\
\hline $\mathrm{Bi}-214$ & -> & $0-214$ \\
\hline $\mathrm{Bi}-214$ & -> & o-214 \\
\hline $\mathrm{Bi}-214$ & $->$ & $0-214$ \\
\hline $\mathrm{Bi}-214$ & $->$ & $0-21$ \\
\hline Ra-226 & -> & $n-222$ \\
\hline $\mathrm{Bi}-214$ & -> & $0-214$ \\
\hline $\mathrm{Bi}-214$ & -> & $0-214$ \\
\hline $\mathrm{Bi}-214$ & $->$ & $0-21$ \\
\hline $\mathrm{Pb}-210$ & -> & $i-210$ \\
\hline $\mathrm{Bi}-214$ & -> & Po-214 \\
\hline $\mathrm{Bi}-214$ & -> & Po-214 \\
\hline $\mathrm{Bi}-214$ & -> & $0-214$ \\
\hline $\mathrm{Bi}-214$ & -> & $0-21$ \\
\hline $\mathrm{Bi}-214$ & -> & $0-21$ \\
\hline Ra-226 & -> & $n-22$ \\
\hline Th-230 & -> & $a-226$ \\
\hline $\mathrm{Bi}-214$ & -> & $0-21$ \\
\hline $\mathrm{Bi}-214$ & -> & $0-21<$ \\
\hline $\mathrm{Bi}-214$ & -> & $0-214$ \\
\hline $\mathrm{Pb}-214$ & $->$ & $i-214$ \\
\hline $\mathrm{Pb}-214$ & $->$ & $3 i-214$ \\
\hline $\mathrm{Bi}-214$ & $->$ & $0-21$ \\
\hline $\mathrm{Bi}-214$ & $->$ & $0-214$ \\
\hline $\mathrm{Pb}-210$ & $->$ & $\mathrm{Bi}-210$ \\
\hline $\mathrm{Bi}-214$ & $->$ & Po-21< \\
\hline $\mathrm{Bi}-214$ & -> & Po-21 \\
\hline $\mathrm{Rn}-222$ & $->$ & Po-218 \\
\hline $\mathrm{Bi}-214$ & $->$ & Po-214 \\
\hline $\mathrm{Bi}-214$ & $->$ & Po-214 \\
\hline $\mathrm{Bi}-214$ & $->$ & Po-21 \\
\hline $\mathrm{Bi}-214$ & $->$ & Po-214 \\
\hline $\mathrm{Bi}-214$ & $->$ & Po-214 \\
\hline $\mathrm{Bi}-214$ & $->$ & Po-214 \\
\hline $\mathrm{Bi}-214$ & $->$ & Po-21 \\
\hline $\mathrm{Bi}-214$ & $->$ & Po-214 \\
\hline $\mathrm{Pb}-214$ & $->$ & $\mathrm{Bi}-214$ \\
\hline $\mathrm{Bi}-214$ & $->$ & Po-21 \\
\hline $\mathrm{Bi}-214$ & $->$ & Po-214 \\
\hline $\mathrm{Bi}-214$ & $->$ & Po-214 \\
\hline $\mathrm{Bi}-214$ & $->$ & Po-214 \\
\hline $\mathrm{Bi}-214$ & $->$ & Po-214 \\
\hline
\end{tabular}

$\mathrm{Pb}-214 \quad \rightarrow \quad \mathrm{Bi}-214$ 


\begin{tabular}{|c|c|c|c|c|c|c|c|}
\hline $7.41 \mathrm{E}-05$ & 1935.8 & $\mathrm{Bi}-214$ & $->$ & Рo-214 & & & \\
\hline $7.12 \mathrm{E}-05$ & 196.3 & $\mathrm{~Pb}-214$ & $->$ & $\mathrm{Bi}-214$ & & & \\
\hline $7.12 \mathrm{E}-05$ & 2010.79 & $\mathrm{Bi}-214$ & $->$ & Po-214 & & & \\
\hline $6.83 E-05$ & 733.64 & $\mathrm{Bi}-214$ & $->$ & Po-214 & & & \\
\hline $6.54 \mathrm{E}-05$ & 1130.6 & $\mathrm{Bi}-214$ & $->$ & Рo-214 & & & \\
\hline $6.54 \mathrm{E}-05$ & 723.32 & $\mathrm{Bi}-214$ & $->$ & Po-214 & & & \\
\hline $6.25 \mathrm{E}-05$ & 660.75 & $\mathrm{Bi}-214$ & $->$ & Po-214 & & & \\
\hline $5.96 \mathrm{E}-05$ & 799.75 & $\mathrm{Bi}-214$ & $->$ & Po-214 & & & \\
\hline 5.81E-05 & 814.87 & $\mathrm{Bi}-214$ & $->$ & Po-214 & & & \\
\hline $5.67 \mathrm{E}-05$ & 141.3 & $\mathrm{~Pb}-214$ & $->$ & $\mathrm{Bi}-214$ & & & \\
\hline 5.67E-05 & 740.87 & $\mathrm{Bi}-214$ & $->$ & Po-214 & & & \\
\hline 5.67E-05 & 338.5 & $\mathrm{Bi}-214$ & $->$ & Po-214 & & & \\
\hline $5.38 E-05$ & 697.89 & $\mathrm{Bi}-214$ & $->$ & Po-214 & & & \\
\hline 4.94E-05 & 304.42 & $\mathrm{Bi}-214$ & $->$ & Po-214 & & & \\
\hline 4.94E-05 & 617.1 & $\mathrm{Bi}-214$ & $->$ & Po-214 & & & \\
\hline $4.82 \mathrm{E}-05$ & 97.907 & Ra-226 & $->$ & $\mathrm{Rn}-222$ & & & \\
\hline $4.71 E-05$ & 2694.67 & $\mathrm{Bi}-214$ & $->$ & Po-214 & & & \\
\hline $4.65 E-05$ & 547.1 & $\mathrm{Bi}-214$ & $->$ & Рo-214 & & & \\
\hline $4.65 E-05$ & 286.9 & $\mathrm{Bi}-214$ & $->$ & Po-214 & & & \\
\hline 4.53E-05 & 797.88 & TI-210 & $->$ & $\mathrm{Pb}-210$, & Po-214 & $->$ & $\mathrm{Pb}-210$ \\
\hline $4.50 E-05$ & 639.36 & $\mathrm{Bi}-214$ & $->$ & Po-214 & & & \\
\hline $4.36 \mathrm{E}-05$ & 396 & $\mathrm{Bi}-214$ & $->$ & Po-214 & & & \\
\hline $4.21 \mathrm{E}-05$ & 511 & $\mathrm{~Pb}-214$ & $->$ & $\mathrm{Bi}-214$ & & & \\
\hline $4.21 E-05$ & 1045.4 & $\mathrm{Bi}-214$ & $->$ & Po-214 & & & \\
\hline $4.21 \mathrm{E}-05$ & 440.4 & $\mathrm{Bi}-214$ & $->$ & Po-214 & & & \\
\hline $4.21 \mathrm{E}-05$ & 1067.3 & $\mathrm{Bi}-214$ & $->$ & Po-214 & & & \\
\hline $3.92 \mathrm{E}-05$ & 1226.8 & $\mathrm{Bi}-214$ & $->$ & Рo-214 & & & \\
\hline $3.72 \mathrm{E}-05$ & 2769.99 & $\mathrm{Bi}-214$ & $->$ & Po-214 & & & \\
\hline $3.34 \mathrm{E}-05$ & 543.91 & $\mathrm{~Pb}-214$ & $->$ & $\mathrm{Bi}-214$ & & & \\
\hline $3.34 \mathrm{E}-05$ & 305.5 & $\mathrm{~Pb}-214$ & $->$ & $\mathrm{Bi}-214$ & & & \\
\hline $3.34 \mathrm{E}-05$ & 1341.5 & $\mathrm{Bi}-214$ & $->$ & Рo-214 & & & \\
\hline $3.34 \mathrm{E}-05$ & 832.34 & $\mathrm{Bi}-214$ & $->$ & Po-214 & & & \\
\hline $3.34 \mathrm{E}-05$ & 976.2 & $\mathrm{Bi}-214$ & $->$ & Po-214 & & & \\
\hline $3.34 \mathrm{E}-05$ & 915.8 & $\mathrm{Bi}-214$ & $->$ & Po-214 & & & \\
\hline $3.28 \mathrm{E}-05$ & 3053.89 & $\mathrm{Bi}-214$ & $->$ & Po-214 & & & \\
\hline $3.20 \mathrm{E}-05$ & 2331.2 & $\mathrm{Bi}-214$ & $->$ & Po-214 & & & \\
\hline $3.20 \mathrm{E}-05$ & 1230.84 & $\mathrm{Bi}-214$ & $->$ & Po-214 & & & \\
\hline $2.91 E-05$ & 324.3 & $\mathrm{~Pb}-214$ & $->$ & $\mathrm{Bi}-214$ & & & \\
\hline $2.91 E-05$ & 9.658 & $\mathrm{Bi}-214$ & $->$ & Po-214 & & & \\
\hline $2.76 \mathrm{E}-05$ & 2021.7 & $\mathrm{Bi}-214$ & $->$ & Po-214 & & & \\
\hline $2.76 \mathrm{E}-05$ & 1636.6 & $\mathrm{Bi}-214$ & $->$ & Po-214 & & & \\
\hline $2.76 \mathrm{E}-05$ & 1392.5 & $\mathrm{Bi}-214$ & $->$ & Po-214 & & & \\
\hline $2.61 \mathrm{E}-05$ & 2266.67 & $\mathrm{Bi}-214$ & $->$ & Po-214 & & & \\
\hline $2.61 \mathrm{E}-05$ & 502.2 & $\mathrm{Bi}-214$ & $->$ & Po-214 & & & \\
\hline $2.47 \mathrm{E}-05$ & 631.2 & $\mathrm{Bi}-214$ & $->$ & Po-214 & & & \\
\hline $2.47 E-05$ & 1038 & $\mathrm{Bi}-214$ & $->$ & Po-214 & & & \\
\hline $2.47 \mathrm{E}-05$ & 943.3 & $\mathrm{Bi}-214$ & $->$ & Po-214 & & & \\
\hline $2.47 \mathrm{E}-05$ & 847.2 & $\mathrm{Bi}-214$ & $->$ & Po-214 & & & \\
\hline $2.42 \mathrm{E}-05$ & 298.1 & TI-210 & $->$ & $\mathrm{Pb}-210$, & Рo-214 & $->$ & $\mathrm{Pb}-210$ \\
\hline $2.32 \mathrm{E}-05$ & 525 & $\mathrm{Bi}-214$ & $->$ & Po-214 & & & \\
\hline
\end{tabular}




\begin{tabular}{|c|c|c|c|c|}
\hline $2.32 \mathrm{E}-05$ & 1782.1 & $\mathrm{Bi}-214$ & $\rightarrow$ & Po-214 \\
\hline $2.32 \mathrm{E}-05$ & 2147.8 & $\mathrm{Bi}-214$ & $->$ & Po-214 \\
\hline $2.28 \mathrm{E}-05$ & 727.8 & $\mathrm{Bi}-214$ & $->$ & Po-214 \\
\hline $2.28 \mathrm{E}-05$ & 2922.09 & $\mathrm{Bi}-214$ & $->$ & Po-214 \\
\hline $2.14 \mathrm{E}-05$ & 2978.79 & $\mathrm{Bi}-214$ & $->$ & Po-214 \\
\hline $2.10 \mathrm{E}-05$ & 10.137 & $\mathrm{Ra}-226$ & $->$ & $\mathrm{Rn}-222$ \\
\hline $1.74 \mathrm{E}-05$ & 989.2 & $\mathrm{Bi}-214$ & $->$ & Po-214 \\
\hline $1.74 \mathrm{E}-05$ & 1814.01 & $\mathrm{Bi}-214$ & $->$ & Po-214 \\
\hline $1.74 \mathrm{E}-05$ & 1471.1 & $\mathrm{Bi}-214$ & $->$ & Po-214 \\
\hline $1.74 \mathrm{E}-05$ & 596 & $\mathrm{Bi}-214$ & $->$ & Po-214 \\
\hline $1.74 \mathrm{E}-05$ & 1020.5 & $\mathrm{Bi}-214$ & $->$ & Po-214 \\
\hline $1.71 \mathrm{E}-05$ & 2376.99 & $\mathrm{Bi}-214$ & $->$ & Po-214 \\
\hline 1.64E-05 & 235.01 & Th-230 & $->$ & Ra-226 \\
\hline $1.60 \mathrm{E}-05$ & 1330 & $\mathrm{Bi}-214$ & $->$ & Po-214 \\
\hline $1.45 \mathrm{E}-05$ & 470.8 & $\mathrm{~Pb}-214$ & $->$ & $\mathrm{Bi}-214$ \\
\hline $1.45 \mathrm{E}-05$ & 2085 & $\mathrm{Bi}-214$ & $->$ & Po-214 \\
\hline $1.45 \mathrm{E}-05$ & 1013.4 & $\mathrm{Bi}-214$ & $->$ & Po-214 \\
\hline $1.34 \mathrm{E}-05$ & 2880.4 & Bi-214 & $->$ & Po-214 \\
\hline $1.31 \mathrm{E}-05$ & 494.6 & $\mathrm{Bi}-214$ & $->$ & Po-214 \\
\hline $1.31 \mathrm{E}-05$ & 2259.7 & Bi-214 & $->$ & Po-214 \\
\hline $1.31 \mathrm{E}-05$ & 394 & $\mathrm{Bi}-214$ & $->$ & Po-214 \\
\hline $1.28 \mathrm{E}-05$ & 2999.99 & $\mathrm{Bi}-214$ & $->$ & Po-214 \\
\hline 1.07E-05 & 12.855 & $\mathrm{Ra}-226$ & $->$ & $\mathrm{Rn}-222$ \\
\hline $1.02 \mathrm{E}-05$ & 2251.2 & $\mathrm{Bi}-214$ & $->$ & Po-214 \\
\hline $1.01 \mathrm{E}-05$ & 205.1 & Th-230 & $->$ & Ra-226 \\
\hline $9.30 \mathrm{E}-06$ & 2893.59 & $\mathrm{Bi}-214$ & $->$ & Po-214 \\
\hline $9.15 \mathrm{E}-06$ & 364.2 & $\mathrm{Bi}-214$ & $->$ & Po-214 \\
\hline 8.72E-06 & 687.7 & $\mathrm{Bi}-214$ & $->$ & Po-214 \\
\hline $8.72 \mathrm{E}-06$ & 693.3 & $\mathrm{Bi}-214$ & $->$ & Po-214 \\
\hline 8.57E-06 & 2423.32 & $\mathrm{Bi}-214$ & $->$ & Po-214 \\
\hline $8.57 \mathrm{E}-06$ & 2505.58 & $\mathrm{Bi}-214$ & $->$ & Po-214 \\
\hline 8.57E-06 & 2786.09 & $\mathrm{Bi}-214$ & $->$ & Po-214 \\
\hline $8.28 \mathrm{E}-06$ & 520.4 & $\mathrm{Bi}-214$ & $->$ & Po-214 \\
\hline 7.99E-06 & 12.085 & $\mathrm{Bi}-214$ & $->$ & Po-214 \\
\hline $7.92 \mathrm{E}-06$ & 262.41 & $\mathrm{Ra}-226$ & $->$ & $\mathrm{Rn}-222$ \\
\hline 7.41E-06 & 1419.7 & $\mathrm{Bi}-214$ & $->$ & Po-214 \\
\hline $7.41 \mathrm{E}-06$ & 2284.4 & $\mathrm{Bi}-214$ & $->$ & Po-214 \\
\hline $7.26 \mathrm{E}-06$ & 1994.7 & $\mathrm{Bi}-214$ & $->$ & Po-214 \\
\hline 7.12E-06 & 538.7 & $\mathrm{~Pb}-214$ & $->$ & $\mathrm{Bi}-214$ \\
\hline $7.12 \mathrm{E}-06$ & 626.4 & $\mathrm{Bi}-214$ & $->$ & Po-214 \\
\hline 7.12E-06 & 376.6 & $\mathrm{Bi}-214$ & $->$ & Po-214 \\
\hline $6.54 \mathrm{E}-06$ & 1353 & $\mathrm{Bi}-214$ & $->$ & Po-214 \\
\hline $6.41 \mathrm{E}-06$ & 1314 & TI-210 & $->$ & $\mathrm{Pb}-210$ \\
\hline $6.25 \mathrm{E}-06$ & 3081.7 & $\mathrm{Bi}-214$ & $->$ & Po-214 \\
\hline $6.08 \mathrm{E}-06$ & 570.5 & Th-230 & $->$ & $\mathrm{Ra}-226$ \\
\hline 5.19E-06 & 1208 & TI-210 & $->$ & $\mathrm{Pb}-210$ \\
\hline $4.21 \mathrm{E}-06$ & 2004.5 & $\mathrm{Bi}-214$ & $->$ & Рo-214 \\
\hline $4.21 \mathrm{E}-06$ & 2270 & $\mathrm{Bi}-214$ & $->$ & Po-214 \\
\hline $4.21 \mathrm{E}-06$ & 2369.3 & $\mathrm{Bi}-214$ & $->$ & Po-214 \\
\hline 4.07E-06 & 2698.86 & $\mathrm{Bi}-214$ & $->$ & Po-214 \\
\hline
\end{tabular}




\begin{tabular}{|c|c|c|c|c|c|c|c|}
\hline $3.66 \mathrm{E}-06$ & 1068 & TI-210 & $->$ & $\mathrm{Pb}-210$ & & & \\
\hline 3.63E-06 & 2827 & $\mathrm{Bi}-214$ & $->$ & Po-214 & & & \\
\hline 3.36E-06 & 12.678 & TI-210 & $->$ & $\mathrm{Pb}-210$ & & & \\
\hline 3.05E-06 & 2482.417 & $\mathrm{Bi}-214$ & $->$ & Po-214 & & & \\
\hline $2.91 \mathrm{E}-06$ & 2390.9 & $\mathrm{Bi}-214$ & $->$ & Po-214 & & & \\
\hline $2.76 \mathrm{E}-06$ & 2360.9 & $\mathrm{Bi}-214$ & $->$ & Po-214 & & & \\
\hline $2.76 \mathrm{E}-06$ & 2324.8 & $\mathrm{Bi}-214$ & $->$ & Po-214 & & & \\
\hline $2.75 \mathrm{E}-06$ & 2428 & TI-210 & $->$ & $\mathrm{Pb}-210$ & & & \\
\hline 2.61E-06 & 2719.21 & $\mathrm{Bi}-214$ & $->$ & Po-214 & & & \\
\hline $2.53 E-06$ & 10.541 & TI-206 & $->$ & $\mathrm{Pb}-206$, & TI-210 & $->$ & $\mathrm{Pb}-210$ \\
\hline $2.47 \mathrm{E}-06$ & 2940.5 & $\mathrm{Bi}-214$ & $->$ & Po-214 & & & \\
\hline $2.44 \mathrm{E}-06$ & 2358 & TI-210 & $->$ & $\mathrm{Pb}-210$ & & & \\
\hline $2.32 \mathrm{E}-06$ & 3142.6 & $\mathrm{Bi}-214$ & $->$ & Po-214 & & & \\
\hline $2.18 \mathrm{E}-06$ & 3183.6 & $\mathrm{Bi}-214$ & $->$ & Po-214 & & & \\
\hline $2.11 \mathrm{E}-06$ & 1110 & $\mathrm{TI}-210$ & $->$ & $\mathrm{Pb}-210$ & & & \\
\hline $2.11 \mathrm{E}-06$ & 2008 & TI-210 & $->$ & $\mathrm{Pb}-210$ & & & \\
\hline $2.11 \mathrm{E}-06$ & 860 & TI-210 & $->$ & $\mathrm{Pb}-210$ & & & \\
\hline 2.05E-06 & 620 & Th-230 & $->$ & Ra-226 & & & \\
\hline $2.05 E-06$ & 551.8 & Th-230 & $->$ & $\mathrm{Ra}-226$ & & & \\
\hline $1.74 \mathrm{E}-06$ & 2928.7 & $\mathrm{Bi}-214$ & $->$ & Po-214 & & & \\
\hline $1.60 \mathrm{E}-06$ & 2988.7 & $\mathrm{Bi}-214$ & $->$ & Po-214 & & & \\
\hline $1.50 \mathrm{E}-06$ & 2088 & TI-210 & $->$ & $\mathrm{Pb}-210$ & & & \\
\hline $1.50 \mathrm{E}-06$ & 1408 & TI-210 & $->$ & $\mathrm{Pb}-210$ & & & \\
\hline $1.40 \mathrm{E}-06$ & 74.969 & TI-206 & $->$ & $\mathrm{Pb}-206$ & TI-210 & $->$ & $\mathrm{Pb}-210$ \\
\hline $1.31 \mathrm{E}-06$ & 2631 & $\mathrm{Bi}-214$ & $->$ & Po-214 & & & \\
\hline $1.22 \mathrm{E}-06$ & 95 & TI-210 & $->$ & $\mathrm{Pb}-210$ & & & \\
\hline $1.22 \mathrm{E}-06$ & 354 & TI-210 & $->$ & $\mathrm{Pb}-210$ & & & \\
\hline $9.15 \mathrm{E}-07$ & 2268 & TI-210 & $->$ & $\mathrm{Pb}-210$ & & & \\
\hline $9.15 \mathrm{E}-07$ & 380 & TI-210 & $->$ & $\mathrm{Pb}-210$ & & & \\
\hline $9.15 E-07$ & 908 & TI-210 & $->$ & $\mathrm{Pb}-210$ & & & \\
\hline 8.94E-07 & 600.83 & Ra-226 & $->$ & $\mathrm{Rn}-222$ & & & \\
\hline $8.54 \mathrm{E}-07$ & 72.803 & TI-206 & $->$ & $\mathrm{Pb}-206$ & TI-210 & $->$ & $\mathrm{Pb}-210$ \\
\hline $8.28 \mathrm{E}-07$ & 2934.9 & $\mathrm{Bi}-214$ & $->$ & Po-214 & & & \\
\hline $7.41 \mathrm{E}-07$ & 3093.9 & $\mathrm{Bi}-214$ & $->$ & Po-214 & & & \\
\hline $7.41 \mathrm{E}-07$ & 3160.5 & $\mathrm{Bi}-214$ & $->$ & Po-214 & & & \\
\hline $6.71 \mathrm{E}-07$ & 14.836 & TI-206 & $->$ & $\mathrm{Pb}-206$ & TI-210 & $->$ & $\mathrm{Pb}-210$ \\
\hline $6.54 \mathrm{E}-07$ & 2604.5 & $\mathrm{Bi}-214$ & $->$ & Po-214 & & & \\
\hline $6.10 \mathrm{E}-07$ & 1538 & TI-210 & $->$ & $\mathrm{Pb}-210$ & & & \\
\hline $6.10 \mathrm{E}-07$ & 1588 & TI-210 & $->$ & $\mathrm{Pb}-210$ & & & \\
\hline $6.10 \mathrm{E}-07$ & 668 & TI-210 & $->$ & $\mathrm{Pb}-210$ & & & \\
\hline $6.10 \mathrm{E}-07$ & 1648 & TI-210 & $->$ & $\mathrm{Pb}-210$ & & & \\
\hline $6.10 \mathrm{E}-07$ & 478 & $\mathrm{Tl}-210$ & $->$ & $\mathrm{Pb}-210$ & & & \\
\hline $6.10 \mathrm{E}-07$ & 81 & TI-210 & $->$ & $\mathrm{Pb}-210$ & & & \\
\hline $5.72 \mathrm{E}-07$ & 414.72 & $\mathrm{Ra}-226$ & $->$ & $\mathrm{Rn}-222$ & & & \\
\hline 5.67E-07 & 2551 & $\mathrm{Bi}-214$ & $->$ & Po-214 & & & \\
\hline $5.00 \mathrm{E}-07$ & 84.789 & TI-206 & $->$ & $\mathrm{Pb}-206$ & TI-210 & $->$ & $\mathrm{Pb}-210$ \\
\hline 4.94E-07 & 2860.9 & $\mathrm{Bi}-214$ & $->$ & Po-214 & & & \\
\hline 4.94E-07 & 3136.3 & $\mathrm{Bi}-214$ & $->$ & Po-214 & & & \\
\hline $4.21 \mathrm{E}-07$ & 2662.23 & $\mathrm{Bi}-214$ & $->$ & Po-214 & & & \\
\hline $3.96 \mathrm{E}-07$ & 449.5 & $\mathrm{Ra}-226$ & $->$ & $\mathrm{Rn}-222$ & & & \\
\hline
\end{tabular}




\begin{tabular}{|c|c|c|c|c|c|c|c|}
\hline $2.91 \mathrm{E}-07$ & 3233.3 & $\mathrm{Bi}-214$ & $\rightarrow$ & Po-214 & & & \\
\hline $1.45 \mathrm{E}-07$ & 3269.7 & $\mathrm{Bi}-214$ & $->$ & Po-214 & & & \\
\hline $1.43 \mathrm{E}-07$ & 87.632 & TI-206 & $->$ & $\mathrm{Pb}-206$, & TI-210 & $->$ & $\mathrm{Pb}-210$ \\
\hline $1.25 \mathrm{E}-07$ & 9.185 & TI-206 & $->$ & $\mathrm{Pb}-206$, & TI-210 & $->$ & $\mathrm{Pb}-210$ \\
\hline $6.10 \mathrm{E}-08$ & 11.349 & TI-210 & $->$ & $\mathrm{Pb}-210$ & & & \\
\hline 3.87E-08 & 803.13 & TI-206 & $->$ & $\mathrm{Pb}-206$, & Po-210 & $->$ & $\mathrm{Pb}-20 \epsilon$ \\
\hline $3.25 E-09$ & 302.25 & $\mathrm{Hg}-206$ & $->$ & TI-206 & & & \\
\hline $5.05 E-10$ & 72.873 & $\mathrm{Hg}-206$ & $->$ & TI-206 & & & \\
\hline $3.01 \mathrm{E}-10$ & 70.832 & $\mathrm{Hg}-206$ & $->$ & TI-206 & & & \\
\hline $3.01 \mathrm{E}-10$ & 650.21 & $\mathrm{Hg}-206$ & $->$ & TI-206 & & & \\
\hline $1.80 \mathrm{E}-10$ & 82.434 & $\mathrm{Hg}-206$ & $->$ & TI-206 & & & \\
\hline $1.80 \mathrm{E}-10$ & 10.259 & $\mathrm{Hg}-206$ & $->$ & TI-206 & & & \\
\hline $1.56 \mathrm{E}-10$ & 12.313 & $\mathrm{Hg}-206$ & $->$ & TI-206 & & & \\
\hline $6.25 \mathrm{E}-11$ & 344.96 & $\mathrm{Hg}-206$ & $->$ & TI-206 & & & \\
\hline $5.05 \mathrm{E}-11$ & 85.185 & $\mathrm{Hg}-206$ & $->$ & TI-206 & & & \\
\hline $2.89 \mathrm{E}-11$ & 14.407 & $\mathrm{Hg}-206$ & $->$ & TI-206 & & & \\
\hline $8.66 \mathrm{E}-12$ & 8.953 & $\mathrm{Hg}-206$ & $->$ & TI-206 & & & \\
\hline $2.28 \mathrm{E}-12$ & 10.994 & $\mathrm{Hg}-206$ & $->$ & TI-206 & & & \\
\hline $1.68 \mathrm{E}-12$ & 12.71 & TI-206 & $->$ & $\mathrm{Pb}-206$ & & & \\
\hline $4.57 \mathrm{E}-13$ & 384.06 & $\mathrm{Hg}-206$ & $->$ & TI-206 & & & \\
\hline $2.68 \mathrm{E}-14$ & 11.439 & TI-206 & $->$ & $P b-206$ & & & \\
\hline $2.84 \mathrm{E}-15$ & 266.15 & $\mathrm{Bi}-210$ & $->$ & TI-206 & & & \\
\hline Pu-239 & $100 \%$ & & $A c$ & 5 years & & & \\
\hline
\end{tabular}

751 lines computed.

Sorted by Energy

\begin{tabular}{|c|c|c|c|c|c|c|c|}
\hline \multirow{2}{*}{$\frac{\text { Energy }}{(\mathrm{keV})}$} & \multirow{2}{*}{$\begin{array}{l}\text { Intensity } \\
\text { (ph/s/gm) }\end{array}$} & \multicolumn{3}{|c|}{ Initial Isotopes and Decays } & & & \\
\hline & & & & & & & \\
\hline 8.953 & $6.10 \mathrm{E}-09$ & $\mathrm{Bi}-211$ & $->$ & TI-207 & & & \\
\hline 9.185 & $1.27 \mathrm{E}-11$ & TI-207 & $->$ & $\mathrm{Pb}-207$ & & & \\
\hline 9.3 & $3.89 \mathrm{E}-11$ & Ac-227 & $->$ & Th-227 & & & \\
\hline 9.419 & $5.38 \mathrm{E}-09$ & $\mathrm{~Pb}-211$ & $->$ & $\mathrm{Bi}-211$ & & & \\
\hline 9.658 & 5.97E-09 & $\mathrm{Rn}-219$ & $->$ & Po-215 & & & \\
\hline 10.137 & $1.56 \mathrm{E}-07$ & $\mathrm{Ra}-223$ & $->$ & $\mathrm{Rn}-219$ & & & \\
\hline 10.259 & $1.24 \mathrm{E}-07$ & $\mathrm{Bi}-211$ & $->$ & TI-207 & & & \\
\hline 10.541 & $2.54 \mathrm{E}-10$ & TI-207 & $->$ & $\mathrm{Pb}-207$ & & & \\
\hline 10.622 & $2.85 E-07$ & Fr-223 & $->$ & $\mathrm{Ra}-223$, & Th-227 & $->$ & $\mathrm{Ra}-223$ \\
\hline 10.828 & $1.05 \mathrm{E}-07$ & $\mathrm{~Pb}-211$ & $->$ & $\mathrm{Bi}-211$ & & & \\
\hline 10.871 & 7.15E-06 & $\mathrm{Pa}-231$ & $->$ & Ac- 227 & & & \\
\hline 10.994 & $1.61 \mathrm{E}-09$ & $\mathrm{Bi}-211$ & $->$ & TI-207 & & & \\
\hline 11.118 & $1.13 \mathrm{E}-01$ & Ac- 227 & $->$ & Th-227, & U-235 & $->$ & Th-231 \\
\hline 11.119 & $1.16 \mathrm{E}-07$ & $R n-219$ & $->$ & Po-215 & & & \\
\hline 11.349 & 3.30E-12 & TI-207 & $->$ & Pb-207 & & & \\
\hline 11.372 & 3.39E-01 & Th-231 & $->$ & $\mathrm{Pa}-231$ & & & \\
\hline 11.712 & $1.70 \mathrm{E}-09$ & $\mathrm{~Pb}-211$ & $->$ & $\mathrm{Bi}-211$ & & & \\
\hline
\end{tabular}




\begin{tabular}{|c|c|c|c|c|c|c|c|}
\hline 11.713 & 2.89E-06 & Ra-223 & $->$ & $\mathrm{Rn}-219$ & & & \\
\hline 12.085 & $2.12 \mathrm{E}-09$ & $\mathrm{Rn}-219$ & $->$ & Po-215 & & & \\
\hline 12.313 & $1.13 \mathrm{E}-07$ & $\mathrm{Bi}-211$ & $->$ & TI-207 & & & \\
\hline 12.325 & 4.94E-06 & Fr-223 & $->$ & Ra-223, & Th-227 & $->$ & $\mathrm{Ra}-223$ \\
\hline 12.636 & $1.31 \mathrm{E}-04$ & $\mathrm{~Pa}-231$ & $->$ & $A c-227$ & & & \\
\hline 12.703 & $2.32 \mathrm{E}-10$ & TI-207 & $->$ & $\mathrm{Pb}-207$ & & & \\
\hline 12.855 & $4.11 \mathrm{E}-08$ & $\mathrm{Ra}-223$ & $->$ & Rn-219 & & & \\
\hline 12.952 & $2.49 E+00$ & Ac- 227 & $->$ & Th-227, & U-235 & $->$ & Th-231 \\
\hline 13.082 & $1.16 \mathrm{E}-07$ & $\mathrm{~Pb}-211$ & $->$ & $\mathrm{Bi}-211$ & & & \\
\hline 13.495 & $1.27 \mathrm{E}-07$ & $\mathrm{Rn}-219$ & $->$ & Po-215 & & & \\
\hline 13.662 & $1.04 \mathrm{E}-07$ & Fr-223 & $->$ & $\mathrm{Ra}-223$, & Th-227 & $->$ & $\mathrm{Ra}-223$ \\
\hline 13.724 & $5.53 \mathrm{E}+00$ & Th-231 & $->$ & $\mathrm{Pa}-231$ & & & \\
\hline 14.082 & $2.21 \mathrm{E}-06$ & $\mathrm{~Pa}-231$ & $->$ & $A c-227$ & & & \\
\hline 14.341 & $2.78 \mathrm{E}-06$ & Ra-223 & $->$ & $\mathrm{Rn}-219$ & & & \\
\hline 14.407 & $2.06 \mathrm{E}-08$ & $\mathrm{Bi}-211$ & $->$ & TI-207 & & & \\
\hline 14.511 & $2.49 \mathrm{E}-02$ & Ac- 227 & $->$ & Th-227, & $U-235$ & $->$ & Th-231 \\
\hline 14.886 & $4.24 \mathrm{E}-11$ & TI-207 & $->$ & $\mathrm{Pb}-207$ & & & \\
\hline 14.953 & $4.52 \mathrm{E}-02$ & Th-231 & $->$ & $\mathrm{Pa}-231$ & & & \\
\hline 15.2 & $1.05 \mathrm{E}-08$ & $A c-227$ & $->$ & Th-227 & & & \\
\hline 15.213 & $7.96 \mathrm{E}-08$ & Fr-223 & $->$ & $\mathrm{Ra}-223$ & & & \\
\hline 15.218 & $6.02 E-06$ & Th-227 & $->$ & Ra-223 & & & \\
\hline 15.397 & $2.55 \mathrm{E}-08$ & $\mathrm{~Pb}-211$ & $->$ & $\mathrm{Bi}-211$ & & & \\
\hline 15.69 & $1.49 \mathrm{E}-04$ & $\mathrm{~Pa}-231$ & $->$ & Ac- 227 & & & \\
\hline 15.856 & $2.58 \mathrm{E}-08$ & $\mathrm{Rn}-219$ & $->$ & Po-215 & & & \\
\hline 16.074 & 4.49E-08 & Ac- 227 & $->$ & Th-227 & & & \\
\hline 16.119 & $1.69 \mathrm{E}+00$ & U-235 & $->$ & Th-231 & & & \\
\hline 16.397 & $2.27 \mathrm{E}-06$ & $\mathrm{~Pa}-231$ & $->$ & $A c-227$ & & & \\
\hline 16.559 & $4.18 \mathrm{E}+00$ & Th-231 & $->$ & $\mathrm{Pa}-231$ & & & \\
\hline 16.918 & $5.49 \mathrm{E}-07$ & $\mathrm{Ra}-223$ & $->$ & $\mathrm{Rn}-219$ & & & \\
\hline 17.187 & $2.48 \mathrm{E}-02$ & Th-231 & $->$ & $\mathrm{Pa}-231$ & & & \\
\hline 17.997 & $1.38 \mathrm{E}-06$ & Th-227 & $->$ & $\mathrm{Ra}-223$ & & & \\
\hline 18.016 & $1.80 \mathrm{E}-08$ & Fr-223 & $->$ & $\mathrm{Ra}-223$ & & & \\
\hline 18.607 & $3.52 \mathrm{E}-05$ & $\mathrm{~Pa}-231$ & $->$ & Ac- 227 & & & \\
\hline 18.998 & 1.97E-06 & $\mathrm{Pa}-231$ & $->$ & Ac- 227 & & & \\
\hline 19.118 & 2.94E-01 & U-235 & $->$ & Th-231 & & & \\
\hline 19.308 & $1.20 \mathrm{E}-08$ & Ac- 227 & $->$ & Th-227 & & & \\
\hline 19.811 & $8.35 \mathrm{E}-01$ & Th-231 & $->$ & $\mathrm{Pa}-231$ & & & \\
\hline 20.27 & 6.07E-08 & Fr-223 & $->$ & Ra-223, & Th-227 & $->$ & $\mathrm{Ra}-223$ \\
\hline 23.55 & 1.01E-09 & $\mathrm{Pa}-231$ & $->$ & $A c-227$ & & & \\
\hline 24.5 & $1.05 E-09$ & Ac- 227 & $->$ & Th-227 & & & \\
\hline 24.56 & $1.79 \mathrm{E}-07$ & $\mathrm{~Pa}-231$ & $->$ & Ac- 227 & & & \\
\hline 25.464 & 5.37E-07 & $\mathrm{Pa}-231$ & $->$ & Ac- 227 & & & \\
\hline 25.642 & $1.65 \mathrm{E}+00$ & Th-231 & $->$ & Pa-231 & & & \\
\hline 27.396 & 5.54E-05 & Pa-231 & $->$ & $A C-227$ & & & \\
\hline 29.58 & $1.83 \mathrm{E}-09$ & Fr-223 & $->$ & Ra-223, & Th-227 & $->$ & $\mathrm{Ra}-223$ \\
\hline 29.869 & $2.88 \mathrm{E}-08$ & Fr-223 & $->$ & Ra-223, & Th-227 & $->$ & $\mathrm{Ra}-223$ \\
\hline 29.996 & $5.48 \mathrm{E}-07$ & $\mathrm{~Pa}-231$ & $->$ & $A c-227$ & & & \\
\hline 30.08 & $2.41 E+03$ & Pu-239 & $->$ & U-235 & & & \\
\hline 31.04 & $5.37 \mathrm{E}-08$ & $\mathrm{~Pa}-231$ & $->$ & Ac- 227 & & & \\
\hline 31.566 & $2.29 \mathrm{E}-08$ & Th-227 & $->$ & Ra-223 & & & \\
\hline
\end{tabular}




\begin{tabular}{|c|c|c|c|c|c|c|c|}
\hline 31.58 & 4.17E-08 & $\mathrm{Pa}-231$ & $->$ & Ac-227 & & & \\
\hline 31.585 & $1.81 \mathrm{E}-03$ & U-235 & $->$ & Th-231 & & & \\
\hline 31.87 & $2.83 \mathrm{E}-11$ & $\mathrm{Ra}-223$ & $->$ & $\mathrm{Rn}-219$ & & & \\
\hline 33.52 & 4.01E-09 & Th-227 & $->$ & Ra-223 & & & \\
\hline 35.884 & $9.54 \mathrm{E}-08$ & Pa-231 & $->$ & Ac- 227 & & & \\
\hline 38.235 & 8.88E-07 & Pa-231 & $->$ & $A c-227$ & & & \\
\hline 38.66 & $2.41 \mathrm{E}+05$ & Pu-239 & $->$ & U-235 & & & \\
\hline 39.61 & 8.35E-09 & $\mathrm{Pa}-231$ & $->$ & $A c-227$ & & & \\
\hline 40.006 & 7.15E-08 & $\mathrm{Pa}-231$ & $->$ & Ac- 227 & & & \\
\hline 40.16 & $5.73 E-09$ & Th-227 & $->$ & Ra-223 & & & \\
\hline 40.41 & $3.72 E+03$ & Pu-239 & $->$ & U-235 & & & \\
\hline 41.13 & 3.39E-03 & U-235 & $->$ & Th-231 & & & \\
\hline 41.88 & $1.15 \mathrm{E}-08$ & Th-227 & $->$ & Ra-223 & & & \\
\hline 41.96 & $6.78 \mathrm{E}-03$ & U-235 & $->$ & Th-231 & & & \\
\hline 42.06 & $3.79 E+03$ & Pu-239 & $->$ & U-235 & & & \\
\hline 42.52 & $3.58 E-08$ & Pa-231 & $->$ & Ac- 227 & & & \\
\hline 42.828 & $6.55 \mathrm{E}-03$ & Th-231 & $->$ & $\mathrm{Pa}-231$ & & & \\
\hline 43.09 & $4.17 \mathrm{E}-08$ & $\mathrm{~Pa}-231$ & $->$ & Ac- 227 & & & \\
\hline 43.53 & $1.43 \mathrm{E}-08$ & Th-227 & $->$ & $\mathrm{Ra}-223$ & & & \\
\hline 43.73 & $6.60 \mathrm{E}-08$ & Fr-223 & $->$ & Ra-223, & Th-227 & $\rightarrow$ & Ra-223 \\
\hline 44.06 & 7.90E-05 & Th-231 & $->$ & $\mathrm{Pa}-231$ & & & \\
\hline 44.08 & 2.01E-09 & Th-227 & $->$ & Ra-223 & & & \\
\hline 44.213 & $3.58 \mathrm{E}-07$ & $\mathrm{~Pa}-231$ & $->$ & $A c-227$ & & & \\
\hline 44.37 & 3.73E-09 & Th-227 & $->$ & Ra-223 & & & \\
\hline 46.13 & $5.87 \mathrm{E}-12$ & Ac- -227 & $->$ & Fr-223 & & & \\
\hline 46.218 & $1.69 E+04$ & Pu-239 & $->$ & U-235 & & & \\
\hline 46.393 & $1.24 \mathrm{E}-06$ & $\mathrm{~Pa}-231$ & $->$ & Ac-227 & & & \\
\hline 46.69 & $1.33 E+03$ & Pu-239 & $->$ & $U-235$ & & & \\
\hline 47.52 & $6.66 \mathrm{E}+02$ & Pu-239 & $->$ & U-235 & & & \\
\hline 48.27 & 2.87E-09 & Fr-223 & $->$ & $\mathrm{Ra}-223$, & Th-227 & $->$ & Ra-223 \\
\hline 49.85 & $6.07 \mathrm{E}-08$ & Fr-223 & $->$ & Ra-223, & Th-227 & $->$ & $\mathrm{Ra}-223$ \\
\hline 50.14 & $2.58 \mathrm{E}-06$ & Fr-223 & $->$ & Ra-223, & Th-227 & $->$ & Ra-223 \\
\hline 50.82 & 4.59E-09 & Th-227 & $->$ & $\mathrm{Ra}-223$ & & & \\
\hline 50.97 & 8.35E-09 & $\mathrm{Pa}-231$ & $->$ & Ac- 227 & & & \\
\hline 51.179 & $2.26 \mathrm{E}-03$ & U-235 & $->$ & Th-231 & & & \\
\hline 51.29 & $8.60 \mathrm{E}-10$ & Fr-223 & $->$ & Ra-223, & Th-227 & $->$ & Ra-223 \\
\hline 51.624 & $6.22 \mathrm{E}+05$ & Pu-239 & $->$ & U-235 & & & \\
\hline 52.763 & 5.07E-07 & $\mathrm{Pa}-231$ & $->$ & $A c-227$ & & & \\
\hline 54.02 & $3.88 \mathrm{E}+03$ & Pu-239 & $->$ & $U-235$ & & & \\
\hline 54.18 & $2.29 \mathrm{E}-09$ & Fr-223 & $->$ & Ra-223, & Th-227 & $->$ & $\mathrm{Ra}-223$ \\
\hline 54.632 & $4.83 E-07$ & $\mathrm{~Pa}-231$ & $->$ & Ac- 227 & & & \\
\hline 56.03 & $1.43 \mathrm{E}-09$ & Th-227 & $->$ & $\mathrm{Ra}-223$ & & & \\
\hline 56.63 & $2.01 \mathrm{E}-09$ & Th-227 & $->$ & $\mathrm{Ra}-223$ & & & \\
\hline 56.8 & $3.58 \mathrm{E}-08$ & $\mathrm{~Pa}-231$ & $->$ & Ac- 227 & & & \\
\hline 56.825 & $2.59 \mathrm{E}+04$ & Pu-239 & $->$ & U-235 & & & \\
\hline 56.9 & $6.56 \mathrm{E}-08$ & $\mathrm{~Pa}-231$ & $->$ & Ac- 227 & & & \\
\hline 57.2 & $8.94 \mathrm{E}-08$ & $\mathrm{~Pa}-231$ & $->$ & Ac- 227 & & & \\
\hline 57.233 & $1.49 \mathrm{E}-07$ & $\mathrm{~Pa}-231$ & $->$ & Ac- 227 & & & \\
\hline 58.562 & $5.42 \mathrm{E}-02$ & Th-231 & $->$ & Pa-231 & & & \\
\hline 59.5 & $2.58 \mathrm{E}-09$ & Th-227 & $->$ & Ra-223 & & & \\
\hline
\end{tabular}




\begin{tabular}{|c|c|c|c|c|}
\hline 60.526 & $4.17 \mathrm{E}-08$ & Pa-231 & $->$ & Ac- 227 \\
\hline 61.435 & $2.29 E-08$ & Th-227 & $->$ & Ra-223 \\
\hline 62.05 & $5.73 \mathrm{E}-10$ & Th-227 & $->$ & Ra-223 \\
\hline 62.36 & $6.88 \mathrm{E}-08$ & Th-227 & $->$ & Ra-223 \\
\hline 62.54 & $2.58 \mathrm{E}-09$ & Th-227 & $->$ & Ra-223 \\
\hline 63.7 & $2.98 \mathrm{E}-07$ & $\mathrm{~Pa}-231$ & $->$ & Ac-227 \\
\hline 63.834 & $2.60 \mathrm{E}-03$ & Th-231 & $->$ & Pa-231 \\
\hline 64.348 & $2.26 \mathrm{E}-03$ & U-235 & $->$ & Th-231 \\
\hline 64.37 & 8.03E-09 & Th-227 & $->$ & Ra-223 \\
\hline 65.516 & $2.18 \mathrm{E}-08$ & $\mathrm{~Pb}-211$ & $->$ & $\mathrm{Bi}-211$ \\
\hline 65.7 & $7.58 \mathrm{E}+02$ & Pu-239 & $->$ & U-235 \\
\hline 66.1 & $1.72 \mathrm{E}-09$ & Th-227 & $->$ & $\mathrm{Ra}-223$ \\
\hline 66.3 & $2.01 \mathrm{E}-09$ & Th-227 & $->$ & Ra-223 \\
\hline 67.69 & $3.26 \mathrm{E}+03$ & Pu-239 & $->$ & U-235 \\
\hline 68.5 & $6.43 E-04$ & Th-231 & $->$ & $\mathrm{Pa}-231$ \\
\hline 68.7 & $1.63 \mathrm{E}-09$ & Th-227 & $->$ & $\mathrm{Ra}-223$ \\
\hline 68.73 & $2.75 E+03$ & Pu-239 & $->$ & U-235 \\
\hline 68.75 & $1.15 \mathrm{E}-08$ & Th-227 & $->$ & $\mathrm{Ra}-223$ \\
\hline 69.13 & $2.31 \mathrm{E}-11$ & $A c-227$ & $->$ & Fr-223 \\
\hline 69.65 & 2.58E-09 & Th-227 & $->$ & $\mathrm{Ra}-223$ \\
\hline 69.83 & $7.12 \mathrm{E}-11$ & $A c-227$ & $->$ & Fr-223 \\
\hline 70.54 & $4.17 \mathrm{E}-08$ & Pa-231 & $->$ & Ac- 227 \\
\hline 70.832 & $2.12 \mathrm{E}-07$ & $\mathrm{Bi}-211$ & $->$ & TI-207 \\
\hline 71.92 & $1.19 \mathrm{E}-08$ & Pa-231 & $->$ & $A c-227$ \\
\hline 72.57 & $2.38 \mathrm{E}-08$ & Pa-231 & $->$ & Ac- 227 \\
\hline 72.71 & $1.24 \mathrm{E}-02$ & U-235 & $->$ & Th-231 \\
\hline 72.767 & $2.83 \mathrm{E}-02$ & Th-231 & $->$ & Pa-231 \\
\hline 72.8 & 8.03E-09 & Th-227 & $->$ & Ra-223 \\
\hline 72.803 & $4.24 \mathrm{E}-10$ & TI-207 & $->$ & Pb-207 \\
\hline 72.873 & 3.59E-07 & $\mathrm{Bi}-211$ & $->$ & TI-207 \\
\hline 73.66 & 5.45E-09 & Th-227 & $->$ & $\mathrm{Ra}-223$ \\
\hline 74.209 & $1.49 \mathrm{E}-07$ & $\mathrm{~Pa}-231$ & $->$ & Ac- 227 \\
\hline 74.8 & $6.78 \mathrm{E}-03$ & U-235 & $->$ & Th-231 \\
\hline 74.814 & $7.25 \mathrm{E}-08$ & $\mathrm{~Pb}-211$ & $->$ & $\mathrm{Bi}-211$ \\
\hline 74.89 & $8.72 E+02$ & Pu-239 & $->$ & U-235 \\
\hline 74.969 & $7.06 \mathrm{E}-10$ & TI-207 & $->$ & $\mathrm{Pb}-207$ \\
\hline 75.1 & $2.58 \mathrm{E}-09$ & Th-227 & $->$ & Ra-223 \\
\hline 76.858 & $1.47 \mathrm{E}-07$ & Rn-219 & $->$ & Po-215 \\
\hline 77.107 & $1.22 \mathrm{E}-07$ & $\mathrm{~Pb}-211$ & $->$ & $\mathrm{Bi}-211$ \\
\hline 77.405 & $4.05 E-07$ & $\mathrm{~Pa}-231$ & $->$ & Ac- -227 \\
\hline 77.593 & $9.80 \mathrm{E}+03$ & Pu-239 & $->$ & U-235 \\
\hline 78.37 & $3.88 \mathrm{E}+03$ & Pu-239 & $->$ & $U-235$ \\
\hline 79.29 & $2.46 \mathrm{E}-07$ & $R n-219$ & $->$ & Po-215 \\
\hline 79.72 & 6.39E-07 & Fr-223 & $->$ & Ra-223 \\
\hline 81 & $1.27 \mathrm{E}-08$ & $\mathrm{~Pb}-211$ & $->$ & $\mathrm{Bi}-211$ \\
\hline 81.067 & 4.30E-06 & Ra-223 & $->$ & Rn-219 \\
\hline 81.229 & $1.02 \mathrm{E}-01$ & Th-231 & $->$ & $\mathrm{Pa}-231$ \\
\hline 82.434 & $1.26 \mathrm{E}-07$ & $\mathrm{Bi}-211$ & $->$ & TI-207 \\
\hline 83.787 & 7.14E-06 & Ra-223 & $->$ & $\mathrm{Rn}-219$ \\
\hline 83.81 & $1.61 \mathrm{E}-08$ & $\mathrm{~Pb}-211$ & $->$ & $\mathrm{Bi}-211$ \\
\hline
\end{tabular}




\begin{tabular}{|c|c|c|c|c|c|c|c|}
\hline 84.203 & 7.45E-01 & Th-231 & $->$ & $\mathrm{Pa}-231$ & & & \\
\hline 84.979 & $2.51 \mathrm{E}-10$ & TI-207 & $->$ & $\mathrm{Pb}-207$ & & & \\
\hline 85.185 & $3.56 \mathrm{E}-08$ & $\mathrm{Bi}-211$ & $->$ & TI-207 & & & \\
\hline 85.429 & $5.46 \mathrm{E}-07$ & Fr-223 & $->$ & Ra-223, & Th-227 & $->$ & $\mathrm{Ra}-223$ \\
\hline 87.19 & 4.30E-08 & $\mathrm{Pb}-211$ & $->$ & $\mathrm{Bi}-211$ & & & \\
\hline 87.632 & 7.34E-11 & TI-207 & $->$ & $\mathrm{Pb}-207$ & & & \\
\hline 87.673 & $2.84 \mathrm{E}-06$ & Pa-231 & $->$ & $A c-227$ & & & \\
\hline 88.2 & 4.81E-09 & $\mathrm{Pb}-211$ & $->$ & $\mathrm{Bi}-211$ & & & \\
\hline 88.471 & $9.00 \mathrm{E}-07$ & Fr-223 & $->$ & Ra-223, & Th-227 & $->$ & Ra-223 \\
\hline 89.639 & $8.78 \mathrm{E}-08$ & $\mathrm{Rn}-219$ & $->$ & Po-215 & & & \\
\hline 89.85 & $9.75 \mathrm{E}-10$ & Th-227 & $->$ & Ra-223 & & & \\
\hline 89.954 & $1.06 \mathrm{E}-01$ & Th-231 & $->$ & $\mathrm{Pa}-231$ & & & \\
\hline 89.955 & 3.80E-01 & U-235 & $->$ & Th-231 & & & \\
\hline 90.128 & $1.27 \mathrm{E}-08$ & $\mathrm{~Pb}-211$ & $->$ & $\mathrm{Bi}-211$ & & & \\
\hline 90.886 & $4.65 \mathrm{E}-06$ & $\mathrm{~Pa}-231$ & $->$ & $A c-227$ & & & \\
\hline 92.279 & $4.40 \mathrm{E}-02$ & Th-231 & $->$ & $\mathrm{Pa}-231$ & & & \\
\hline 92.673 & $2.66 \mathrm{E}-08$ & $R n-219$ & $->$ & Po-215 & & & \\
\hline 93.07 & $5.53 E-03$ & Th-231 & $->$ & $\mathrm{Pa}-231$ & & & \\
\hline 93.36 & $6.21 \mathrm{E}-01$ & U-235 & $->$ & Th-231 & & & \\
\hline 93.93 & $4.01 \mathrm{E}-07$ & Th-227 & $->$ & Ra-223 & & & \\
\hline 94.3 & $3.11 \mathrm{E}-09$ & $\mathrm{~Pb}-211$ & $->$ & $\mathrm{Bi}-211$ & & & \\
\hline 94.66 & $9.64 \mathrm{E}+04$ & Pu-239 & $->$ & U-235 & & & \\
\hline 94.677 & 2.52E-06 & Ra-223 & $->$ & $\mathrm{Rn}-219$ & & & \\
\hline 94.89 & 5.10E-09 & $\mathrm{Pb}-211$ & $->$ & $\mathrm{Bi}-211$ & & & \\
\hline 94.9 & $3.44 \mathrm{E}-09$ & Th-227 & $->$ & $\mathrm{Ra}-223$ & & & \\
\hline 95.863 & 7.11E-02 & Th-231 & $->$ & Pa-231 & & & \\
\hline 96.06 & $1.72 \mathrm{E}-08$ & Th-227 & $->$ & Ra-223 & & & \\
\hline 96.154 & $9.72 \mathrm{E}-03$ & U-235 & $->$ & Th-231 & & & \\
\hline 96.16 & $8.45 \mathrm{E}+02$ & Pu-239 & $->$ & U-235 & & & \\
\hline 96.979 & $5.25 \mathrm{E}-07$ & $\mathrm{~Pa}-231$ & $->$ & Ac- 227 & & & \\
\hline 97.3 & $3.26 \mathrm{E}-09$ & $\mathrm{~Pb}-211$ & $->$ & $\mathrm{Bi}-211$ & & & \\
\hline 97.907 & $8.04 \mathrm{E}-07$ & Ra-223 & $->$ & $\mathrm{Rn}-219$ & & & \\
\hline 98.441 & $1.57 \mathrm{E}+05$ & Pu-239 & $->$ & U-235 & & & \\
\hline 98.79 & $2.80 \mathrm{E}+04$ & Pu-239 & $->$ & $U-235$ & & & \\
\hline 99.28 & $1.35 \mathrm{E}-02$ & Th-231 & $->$ & $\mathrm{Pa}-231$ & & & \\
\hline 99.56 & 3.73E-09 & Fr-223 & $->$ & Ra-223, & Th-227 & $->$ & $\mathrm{Ra}-223$ \\
\hline 99.64 & $5.73 \mathrm{E}-10$ & Th-227 & $->$ & Ra-223 & & & \\
\hline 99.7 & $1.34 \mathrm{E}-10$ & Ac- 227 & $->$ & Fr-223 & & & \\
\hline 99.915 & $3.21 \mathrm{E}-07$ & Fr-223 & $->$ & Ra-223, & Th-227 & $->$ & $\mathrm{Ra}-223$ \\
\hline 100.31 & $2.89 E-08$ & Fr-223 & $->$ & Ra-223, & Th-227 & $->$ & $\mathrm{Ra}-223$ \\
\hline 100.96 & $1.91 \mathrm{E}-07$ & $\mathrm{~Pa}-231$ & $->$ & Ac- 227 & & & \\
\hline 102.255 & 4.63E-02 & Th-231 & $->$ & Pa-231 & & & \\
\hline 102.45 & $3.44 \mathrm{E}-10$ & Th-227 & $->$ & $\mathrm{Ra}-223$ & & & \\
\hline 102.613 & $1.67 \mathrm{E}-06$ & Pa-231 & $->$ & Ac- 227 & & & \\
\hline 103.035 & $4.87 E+03$ & Pu-239 & $->$ & U-235 & & & \\
\hline 103.341 & $1.05 \mathrm{E}-07$ & Fr-223 & $->$ & Ra-223, & Th-227 & $->$ & Ra-223 \\
\hline 103.85 & 4.81E-09 & $\mathrm{Ra}-223$ & $->$ & $R n-219$ & & & \\
\hline 104.9 & $1.15 \mathrm{E}-08$ & Th-227 & $->$ & $\mathrm{Ra}-223$ & & & \\
\hline 105.362 & 2.24E-01 & U-235 & $->$ & Th-231 & & & \\
\hline 105.803 & $8.02 \mathrm{E}-04$ & Th-231 & $->$ & $\mathrm{Pa}-231$ & & & \\
\hline
\end{tabular}




\begin{tabular}{|c|c|c|c|c|c|c|c|}
\hline 106.1 & $5.87 \mathrm{E}-12$ & $A c-227$ & $->$ & Fr-223 & & & \\
\hline 106.137 & $5.48 \mathrm{E}-07$ & $\mathrm{~Pa}-231$ & $->$ & $A c-227$ & & & \\
\hline 106.581 & $1.92 \mathrm{E}-03$ & Th-231 & $->$ & $\mathrm{Pa}-231$ & & & \\
\hline 106.66 & $6.23 E-09$ & $\mathrm{Ra}-223$ & $->$ & $R n-219$ & & & \\
\hline 107.92 & $2.01 \mathrm{E}-09$ & Th-227 & $->$ & $\mathrm{Ra}-223$ & & & \\
\hline 108.166 & $2.57 \mathrm{E}-02$ & Th-231 & $->$ & $\mathrm{Pa}-231$ & & & \\
\hline 108.99 & $7.46 \mathrm{E}-02$ & U-235 & $->$ & Th-231 & & & \\
\hline 109.1 & $1.72 \mathrm{E}-09$ & Th-227 & $->$ & Ra-223 & & & \\
\hline 109.16 & $1.74 \mathrm{E}-01$ & U-235 & $->$ & Th-231 & & & \\
\hline 110.421 & $1.83 \mathrm{E}+04$ & Pu-239 & $->$ & U-235 & & & \\
\hline 110.58 & $1.43 \mathrm{E}-09$ & Th-227 & $->$ & Ra-223 & & & \\
\hline 110.8 & $1.36 \mathrm{E}-08$ & Ra-223 & $->$ & $\mathrm{Rn}-219$ & & & \\
\hline 111.3 & $3.56 E+04$ & Pu-239 & $->$ & U-235 & & & \\
\hline 111.89 & $7.05 E+02$ & Pu-239 & $->$ & $U-235$ & & & \\
\hline 111.897 & 8.58E-03 & Th-231 & $->$ & $\mathrm{Pa}-231$ & & & \\
\hline 112.54 & 2.29E-09 & Th-227 & $->$ & Ra-223 & & & \\
\hline 113.12 & $4.30 \mathrm{E}-08$ & Th-227 & $->$ & $\mathrm{Ra}-223$ & & & \\
\hline 113.19 & $1.61 \mathrm{E}-07$ & Th-227 & $->$ & $\mathrm{Ra}-223$ & & & \\
\hline 114.333 & $1.44 \mathrm{E}+04$ & Pu-239 & $->$ & U-235 & & & \\
\hline 114.5 & $2.55 \mathrm{E}-09$ & $\mathrm{Ra}-223$ & $->$ & $\mathrm{Rn}-219$ & & & \\
\hline 115.3 & $9.34 \mathrm{E}-10$ & $\mathrm{Rn}-219$ & $->$ & Po-215 & & & \\
\hline 115.38 & $1.49 \mathrm{E}+04$ & Pu-239 & $->$ & U-235 & & & \\
\hline 115.595 & $1.13 \mathrm{E}-04$ & Th-231 & $->$ & Pa-231 & & & \\
\hline 116.11 & 7.91E-03 & U-235 & $->$ & Th-231 & & & \\
\hline 116.266 & $1.37 E+04$ & Pu-239 & $->$ & U-235 & & & \\
\hline 116.827 & $2.34 \mathrm{E}-03$ & Th-231 & $->$ & Pa-231 & & & \\
\hline 117.17 & 4.87E-08 & Th-227 & $->$ & $\mathrm{Ra}-223$ & & & \\
\hline 117.3 & $3.44 \mathrm{E}-09$ & Th-227 & $->$ & Ra-223 & & & \\
\hline 119.98 & $2.94 \mathrm{E}-03$ & U-235 & $->$ & Th-231 & & & \\
\hline 120.9 & $2.31 \mathrm{E}-11$ & $A c-227$ & $->$ & Fr-223 & & & \\
\hline 122.31 & 3.37E-07 & Ra-223 & $->$ & $\mathrm{Rn}-219$ & & & \\
\hline 122.34 & $6.89 E+01$ & Pu-239 & $->$ & U-235 & & & \\
\hline 123.55 & 2.29E-09 & Th-227 & $->$ & Ra-223 & & & \\
\hline 123.62 & $4.52 E+02$ & Pu-239 & $->$ & U-235 & & & \\
\hline 124.48 & $1.43 \mathrm{E}-09$ & Th-227 & $->$ & Ra-223 & & & \\
\hline 124.51 & $1.41 E+03$ & Pu-239 & $->$ & U-235 & & & \\
\hline 124.6 & $8.60 \mathrm{E}-10$ & Th-227 & $->$ & $\mathrm{Ra}-223$ & & & \\
\hline 124.69 & $2.98 \mathrm{E}-08$ & $\mathrm{~Pa}-231$ & $->$ & $A c-227$ & & & \\
\hline 124.922 & $6.32 \mathrm{E}-03$ & Th-231 & $->$ & Pa-231 & & & \\
\hline 125.21 & $1.63 E+03$ & Pu-239 & $->$ & U-235 & & & \\
\hline 129.296 & $1.44 \mathrm{E}+05$ & Pu-239 & $->$ & U-235 & & & \\
\hline 130.57 & $3.57 E-08$ & $R n-219$ & $->$ & Po-215 & & & \\
\hline 131.1 & $1.42 \mathrm{E}-09$ & Ra-223 & $->$ & Rn-219 & & & \\
\hline 133.8 & $1.17 \mathrm{E}-11$ & $A c-227$ & $->$ & Fr-223 & & & \\
\hline 134.014 & $2.71 \mathrm{E}-03$ & Th-231 & $->$ & $\mathrm{Pa}-231$ & & & \\
\hline 134.5 & $9.57 \mathrm{E}-09$ & Fr-223 & $->$ & Ra-223, & Th-227 & $->$ & $\mathrm{Ra}-223$ \\
\hline 135.681 & $8.81 \mathrm{E}-03$ & Th-231 & $->$ & $\mathrm{Pa}-231$ & & & \\
\hline 136.705 & $4.74 \mathrm{E}-04$ & Th-231 & $->$ & Pa-231 & & & \\
\hline 136.72 & $1.36 \mathrm{E}-03$ & U-235 & $->$ & Th-231 & & & \\
\hline 140.33 & $9.18 \mathrm{E}-09$ & Th-227 & $->$ & $\mathrm{Ra}-223$ & & & \\
\hline
\end{tabular}




\begin{tabular}{|c|c|c|c|c|c|c|c|}
\hline 140.537 & $8.02 \mathrm{E}-05$ & Th-231 & $->$ & Pa-231 & & & \\
\hline 140.76 & $2.49 \mathrm{E}-02$ & U-235 & $->$ & Th-231 & & & \\
\hline 141.44 & $3.73 E-08$ & Th-227 & $->$ & $\mathrm{Ra}-223$ & & & \\
\hline 141.657 & $7.35 E+02$ & Pu-239 & $->$ & U-235 & & & \\
\hline 143.35 & $3.97 E+02$ & Pu-239 & $->$ & U-235 & & & \\
\hline 143.76 & $1.24 \mathrm{E}+00$ & U-235 & $->$ & Th-231 & & & \\
\hline 144.18 & $9.23 \mathrm{E}-07$ & Ra-223 & $->$ & $\mathrm{Rn}-219$ & & & \\
\hline 144.201 & $6.97 E+03$ & Pu-239 & $->$ & U-235 & & & \\
\hline 144.5 & $2.38 \mathrm{E}-08$ & Pa-231 & $->$ & $A c-227$ & & & \\
\hline 145.063 & $6.55 \mathrm{E}-04$ & Th-231 & $->$ & $\mathrm{Pa}-231$ & & & \\
\hline 145.927 & $3.61 \mathrm{E}-03$ & Th-231 & $->$ & $\mathrm{Pa}-231$ & & & \\
\hline 146.094 & $2.73 E+03$ & Pu-239 & $->$ & U-235 & & & \\
\hline 147.2 & $3.48 \mathrm{E}-11$ & Ac- 227 & $->$ & Fr-223 & & & \\
\hline 150.02 & 2.87E-09 & Th-227 & $->$ & $\mathrm{Ra}-223$ & & & \\
\hline 150.93 & $9.04 \mathrm{E}-03$ & U-235 & $->$ & Th-231 & & & \\
\hline 154.18 & $1.58 \mathrm{E}-06$ & Ra-223 & $->$ & $\mathrm{Rn}-219$ & & & \\
\hline 158.59 & $1.95 \mathrm{E}-07$ & Ra-223 & $->$ & $\mathrm{Rn}-219$ & & & \\
\hline 160.19 & $1.42 \mathrm{E}+02$ & Pu-239 & $->$ & U-235 & & & \\
\hline 161.482 & $2.82 E+03$ & Pu-239 & $->$ & U-235 & & & \\
\hline 162.18 & 2.01E-09 & Th-227 & $->$ & Ra-223 & & & \\
\hline 163.114 & $1.75 \mathrm{E}-02$ & Th-231 & $->$ & $\mathrm{Pa}-231$ & & & \\
\hline 163.33 & $5.74 \mathrm{E}-01$ & U-235 & $->$ & Th-231 & & & \\
\hline 164.97 & $4.40 \mathrm{E}-04$ & Th-231 & $->$ & Pa-231 & & & \\
\hline 165.5 & $1.42 \mathrm{E}-09$ & Ra-223 & $->$ & $\mathrm{Rn}-219$ & & & \\
\hline 167.81 & $6.73 E+01$ & Pu-239 & $->$ & U-235 & & & \\
\hline 168.19 & $4.01 \mathrm{E}-09$ & Th-227 & $->$ & Ra-223 & & & \\
\hline 169.637 & $1.35 \mathrm{E}-04$ & Th-231 & $->$ & Pa-231 & & & \\
\hline 169.98 & $8.60 \mathrm{E}-10$ & Th-227 & $->$ & Ra-223 & & & \\
\hline 171.21 & $2.87 \mathrm{E}-10$ & Th-227 & $->$ & Ra-223 & & & \\
\hline 171.393 & $2.53 E+03$ & Pu-239 & $->$ & $U-235$ & & & \\
\hline 171.4 & $1.72 \mathrm{E}-11$ & $A c-227$ & $->$ & Fr-223 & & & \\
\hline 172.27 & $1.13 \mathrm{E}-03$ & U-235 & $->$ & Th-231 & & & \\
\hline 173.38 & 4.92E-09 & Fr-223 & $->$ & Ra-223, & Th-227 & $->$ & $\mathrm{Ra}-223$ \\
\hline 173.7 & $9.18 \mathrm{E}+01$ & Pu-239 & $->$ & $U-235$ & & & \\
\hline 174.157 & 2.07E-03 & Th-231 & $->$ & Pa-231 & & & \\
\hline 175.23 & $5.16 \mathrm{E}-09$ & Th-227 & $->$ & Ra-223 & & & \\
\hline 175.55 & 4.81E-09 & Ra-223 & $->$ & Rn-219 & & & \\
\hline 176.8 & $1.13 \mathrm{E}-09$ & Ra-223 & $->$ & $\mathrm{Rn}-219$ & & & \\
\hline 177.35 & $1.33 \mathrm{E}-08$ & Ra-223 & $->$ & $\mathrm{Rn}-219$ & & & \\
\hline 179.22 & $1.52 \mathrm{E}+03$ & Pu-239 & $->$ & U-235 & & & \\
\hline 179.28 & $5.73 E-10$ & Th-227 & $->$ & Ra-223 & & & \\
\hline 179.69 & $4.33 \mathrm{E}-08$ & Ra-223 & $->$ & $\mathrm{Rn}-219$ & & & \\
\hline 182.61 & $3.84 \mathrm{E}-02$ & U-235 & $->$ & Th-231 & & & \\
\hline 183.483 & 3.71E-03 & Th-231 & $->$ & $\mathrm{Pa}-231$ & & & \\
\hline 184.55 & $4.87 E+01$ & Pu-239 & $->$ & U-235 & & & \\
\hline 184.68 & $1.13 \mathrm{E}-08$ & Fr-223 & $->$ & Ra-223, & Th-227 & $->$ & Ra-223 \\
\hline 185.72 & $6.46 \mathrm{E}+00$ & U-235 & $->$ & Th-231 & & & \\
\hline 188.23 & $2.50 E+02$ & Pu-239 & $->$ & U-235 & & & \\
\hline 188.756 & $3.61 \mathrm{E}-04$ & Th-231 & $->$ & Pa-231 & & & \\
\hline 189.36 & $1.91 E+03$ & Pu-239 & $->$ & U-235 & & & \\
\hline
\end{tabular}




\begin{tabular}{|c|c|c|c|c|c|c|c|}
\hline 194.94 & $7.12 \mathrm{E}-02$ & U-235 & $->$ & Th-231 & & & \\
\hline 195.679 & $2.46 \mathrm{E}+03$ & Pu-239 & $->$ & U-235 & & & \\
\hline 196.87 & $8.49 \mathrm{E}+01$ & Pu-239 & $->$ & $U-235$ & & & \\
\hline 197.61 & $3.44 \mathrm{E}-09$ & Th-227 & $->$ & Ra-223 & & & \\
\hline 198.9 & $4.75 \mathrm{E}-03$ & U-235 & $->$ & Th-231 & & & \\
\hline 198.96 & $3.34 \mathrm{E}-08$ & $\mathrm{~Pa}-231$ & $->$ & $A c-227$ & & & \\
\hline 199.43 & $8.50 \mathrm{E}-10$ & Ra-223 & $->$ & $\mathrm{Rn}-219$ & & & \\
\hline 200.48 & 5.73E-09 & Th-227 & $->$ & Ra-223 & & & \\
\hline 201.64 & $5.73 E-09$ & Th-227 & $->$ & Ra-223 & & & \\
\hline 202.11 & $1.22 \mathrm{E}-01$ & U-235 & $->$ & Th-231 & & & \\
\hline 202.4 & $1.72 \mathrm{E}-09$ & Th-227 & $->$ & Ra-223 & & & \\
\hline 203.54 & $1.29 E+04$ & Pu-239 & $->$ & U-235 & & & \\
\hline 204.2 & $6.60 \mathrm{E}-08$ & Fr-223 & $->$ & Ra-223, & Th-227 & $->$ & $\mathrm{Ra}-223$ \\
\hline 204.95 & $4.32 \mathrm{E}-08$ & Fr-223 & $->$ & Ra-223, & Th-227 & $->$ & $\mathrm{Ra}-223$ \\
\hline 205.31 & $5.66 \mathrm{E}-01$ & U-235 & $->$ & Th-231 & & & \\
\hline 206.04 & $6.60 \mathrm{E}-08$ & Fr-223 & $->$ & Ra-223, & Th-227 & $->$ & $\mathrm{Ra}-223$ \\
\hline 210.58 & $3.24 \mathrm{E}-07$ & Fr-223 & $->$ & Ra-223, & Th-227 & $->$ & $\mathrm{Ra}-223$ \\
\hline 212.56 & $2.01 \mathrm{E}-08$ & Th-227 & $->$ & $\mathrm{Ra}-223$ & & & \\
\hline 212.69 & 4.87E-09 & Th-227 & $->$ & $\mathrm{Ra}-223$ & & & \\
\hline 215.305 & $3.05 E-03$ & U-235 & $->$ & Th-231 & & & \\
\hline 217.934 & 4.52E-03 & Th-231 & $->$ & $\mathrm{Pa}-231$ & & & \\
\hline 218.45 & $2.30 E+01$ & Pu-239 & $->$ & U-235 & & & \\
\hline 218.77 & $1.72 \mathrm{E}-08$ & Th-227 & $->$ & Ra-223 & & & \\
\hline 218.94 & $1.15 \mathrm{E}-08$ & Th-227 & $->$ & Ra-223 & & & \\
\hline 220.2 & $1.43 \mathrm{E}-09$ & $\mathrm{~Pa}-231$ & $->$ & AC- 227 & & & \\
\hline 220.38 & $9.34 \mathrm{E}-09$ & Ra-223 & $->$ & $\mathrm{Rn}-219$ & & & \\
\hline 221.38 & $1.36 \mathrm{E}-02$ & U-235 & $->$ & Th-231 & & & \\
\hline 221.9 & 8.50E-09 & $\mathrm{Rn}-219$ & $->$ & Po-215 & & & \\
\hline 224.68 & 4.30E-09 & Fr-223 & $->$ & Ra-223, & Th-227 & $->$ & $\mathrm{Ra}-223$ \\
\hline 225.39 & $3.74 \mathrm{E}+02$ & Pu-239 & $->$ & $U-235$ & & & \\
\hline 226.77 & $1.55 \mathrm{E}-08$ & Pa-231 & $->$ & $A c-227$ & & & \\
\hline 228.16 & $1.43 \mathrm{E}-09$ & $\mathrm{~Pa}-231$ & $->$ & $A c-227$ & & & \\
\hline 228.77 & $9.04 \mathrm{E}-04$ & $U-235$ & $->$ & Th-231 & & & \\
\hline 230.17 & $2.01 \mathrm{E}-10$ & Th-227 & $->$ & $\mathrm{Ra}-223$ & & & \\
\hline 233.5 & $3.28 \mathrm{E}-03$ & U-235 & $->$ & Th-231 & & & \\
\hline 234.82 & $1.42 \mathrm{E}-07$ & Fr-223 & $->$ & Ra-223, & Th-227 & $->$ & Ra-223 \\
\hline 235.97 & $3.21 \mathrm{E}-06$ & Fr-223 & $->$ & Ra-223, & Th-227 & $->$ & $\mathrm{Ra}-223$ \\
\hline 235.985 & $1.04 \mathrm{E}-03$ & Th-231 & $->$ & $\mathrm{Pa}-231$ & & & \\
\hline 237.75 & $3.44 \mathrm{E}+02$ & Pu-239 & $->$ & $U-235$ & & & \\
\hline 240.25 & $3.16 \mathrm{E}-05$ & Th-231 & $->$ & Pa-231 & & & \\
\hline 240.85 & $6.10 \mathrm{E}-03$ & U-235 & $->$ & Th-231 & & & \\
\hline 242 & 4.77E-08 & $\mathrm{Pa}-231$ & $->$ & $A c-227$ & & & \\
\hline 242.12 & $1.91 \mathrm{E}+02$ & Pu-239 & $->$ & $U-235$ & & & \\
\hline 242.508 & $9.48 \mathrm{E}-05$ & Th-231 & $->$ & $\mathrm{Pa}-231$ & & & \\
\hline 243.17 & $2.80 \mathrm{E}-07$ & $\mathrm{~Pa}-231$ & $->$ & $A c-227$ & & & \\
\hline 243.36 & $5.33 \mathrm{E}+02$ & Pu-239 & $->$ & U-235 & & & \\
\hline 244.86 & $1.19 \mathrm{E}+02$ & Pu-239 & $->$ & $U-235$ & & & \\
\hline 245.19 & $2.55 \mathrm{E}-09$ & $\mathrm{Ra}-223$ & $->$ & $\mathrm{Rn}-219$ & & & \\
\hline 245.438 & $4.17 \mathrm{E}-08$ & $\mathrm{~Pa}-231$ & $->$ & $A c-227$ & & & \\
\hline 245.77 & $4.11 \mathrm{E}-08$ & $\mathrm{~Pa}-231$ & $->$ & $A c-227$ & & & \\
\hline
\end{tabular}




\begin{tabular}{|c|c|}
\hline 246.07 & $3.15 \mathrm{E}-09$ \\
\hline 246.84 & 5.99E-03 \\
\hline 248.88 & $1.72 \mathrm{E}+02$ \\
\hline 249.36 & 2.01E-09 \\
\hline 249.49 & $1.05 \mathrm{E}-08$ \\
\hline 249.56 & 8.81E-05 \\
\hline 250.12 & $1.06 \mathrm{E}-07$ \\
\hline 250.33 & $3.73 E-08$ \\
\hline 250.43 & $7.34 \mathrm{E}-05$ \\
\hline 251.12 & $9.91 \mathrm{E}-0$ \\
\hline 251.58 & $8.78 \mathrm{E}-0$ \\
\hline 252.46 & $3.15 \mathrm{E}-08$ \\
\hline 254.66 & $2.29 \mathrm{E}-0$ \\
\hline 254.98 & $9.06 \mathrm{E}-0 \mathrm{~S}$ \\
\hline 255.384 & $1.84 \mathrm{E}+0$ \\
\hline 255.74 & $1.42 \mathrm{E}-0$ \\
\hline 255.858 & $5.96 \mathrm{E}-07$ \\
\hline 256.24 & $1.92 \mathrm{E}-0$ \\
\hline 258.43 & $1.37 \mathrm{E}-0$ \\
\hline 260.29 & $1.08 \mathrm{E}-0$ \\
\hline 260.4 & $1.70 \mathrm{E}-0$ \\
\hline 262.72 & $2.87 \mathrm{E}-0$ \\
\hline 263.9 & $5.85 E+0$ \\
\hline 265.81 & $6.89 E+0$ \\
\hline 266.3 & 7.17E-10 \\
\hline 266.483 & $6.78 \mathrm{E}-04$ \\
\hline 267.62 & $1.31 \mathrm{E}-0$ \\
\hline 267.82 & $2.87 \mathrm{E}-0$ \\
\hline 269.39 & $3.85 \mathrm{E}-06$ \\
\hline 270.34 & $2.29 \mathrm{E}-0$ \\
\hline 270.76 & $9.18 \mathrm{E}-0$ \\
\hline 271.13 & $2.80 \mathrm{E}-0$ \\
\hline 272.95 & $1.41 \mathrm{E}-0$ \\
\hline 273.17 & $3.40 \mathrm{E}-0$ \\
\hline 274.14 & 3.39E-0 \\
\hline 275.2 & 4.30E-1 \\
\hline 275.47 & $5.54 \mathrm{E}-0$ \\
\hline 277.17 & $3.70 \mathrm{E}-07$ \\
\hline 277.43 & $1.97 \mathrm{E}-0$ \\
\hline 279.7 & $2.01 \mathrm{E}-0$ \\
\hline 281 & $2.01 \mathrm{E}-0$ \\
\hline 281.12 & $4.82 E+0$ \\
\hline 281.31 & $4.59 E-0$ \\
\hline 281.45 & $6.78 \mathrm{E}-0$ \\
\hline 282.98 & $5.65 \mathrm{E}-0$ \\
\hline 283.673 & $9.48 \mathrm{E}-0$ \\
\hline 284.24 & $1.43 \mathrm{E}-0$ \\
\hline 285.27 & $3.44 \mathrm{E}+0$ \\
\hline 285.48 & $1.58 \mathrm{E}-0$ \\
\hline 286.11 & $4.56 \mathrm{E}-0$ \\
\hline
\end{tabular}

\begin{tabular}{|c|c|c|c|c|c|}
\hline Th-227 & $->$ & $\mathrm{Ra}-223$ & & & \\
\hline U-235 & $->$ & Th-231 & & & \\
\hline Pu-239 & $->$ & U-235 & & & \\
\hline Th-227 & $->$ & Ra-223 & & & \\
\hline Ra-223 & $->$ & $R n-219$ & & & \\
\hline Th-231 & $->$ & $\mathrm{Pa}-231$ & & & \\
\hline Fr-223 & $->$ & Ra-223, & Th-227 & $->$ & $\mathrm{Ra}-223$ \\
\hline Th-227 & $->$ & $\mathrm{Ra}-223$ & & & \\
\hline Th-231 & $->$ & Pa-231 & & & \\
\hline Ra-223 & $->$ & $\mathrm{Rn}-219$ & & & \\
\hline Ra-223 & $->$ & $\mathrm{Rn}-219$ & & & \\
\hline Th-227 & $->$ & $\mathrm{Ra}-223$ & & & \\
\hline Fr-223 & $->$ & Ra-223, & Th-227 & $->$ & Ra-223 \\
\hline Ra-223 & $->$ & $\mathrm{Rn}-219$ & & & \\
\hline Pu-239 & $->$ & U-235 & & & \\
\hline Ra-223 & $->$ & $\mathrm{Rn}-219$ & & & \\
\hline $\mathrm{Pa}-231$ & $->$ & Ac- 227 & & & \\
\hline $\mathrm{Fr}-223$ & $->$ & $\mathrm{Ra}-223$, & Th-227 & $->$ & $\mathrm{Ra}-223$ \\
\hline $\mathrm{Pa}-231$ & $->$ & Ac- 227 & & & \\
\hline $\mathrm{Pa}-231$ & $->$ & $A c-227$ & & & \\
\hline Ra-223 & $->$ & $\mathrm{Rn}-219$ & & & \\
\hline Fr-223 & $->$ & $\mathrm{Ra}-223$, & Th-227 & $->$ & $\mathrm{Ra}-223$ \\
\hline Pu-239 & $->$ & $U-235$ & & & \\
\hline Pu-239 & $->$ & U-235 & & & \\
\hline Th-227 & $->$ & Ra-223 & & & \\
\hline U-235 & $->$ & Th-231 & & & \\
\hline Th-231 & $->$ & $\mathrm{Pa}-231$ & & & \\
\hline Th-227 & $->$ & $\mathrm{Ra}-223$ & & & \\
\hline Ra-223 & $->$ & $R n-219$ & & & \\
\hline Th-227 & $->$ & Ra-223 & & & \\
\hline Th-227 & $->$ & Ra-223 & & & \\
\hline $\mathrm{Rn}-219$ & $->$ & Po-215 & & & \\
\hline Fr-223 & $->$ & $\mathrm{Ra}-223$, & Th-227 & $->$ & $\mathrm{Ra}-223$ \\
\hline $\mathrm{Pa}-231$ & $->$ & Ac- 227 & & & \\
\hline Th-231 & $->$ & $\mathrm{Pa}-231$ & & & \\
\hline Th-227 & $->$ & Ra-223 & & & \\
\hline$U-235$ & $->$ & Th-231 & & & \\
\hline $\mathrm{Pa}-231$ & $->$ & Ac- 227 & & & \\
\hline $\mathrm{Pa}-231$ & $->$ & $A c-227$ & & & \\
\hline Fr-223 & $->$ & Ra-223, & Th-227 & $->$ & $\mathrm{Ra}-223$ \\
\hline Th-227 & $->$ & $\mathrm{Ra}-223$ & & & \\
\hline Pu-239 & $->$ & U-235 & & & \\
\hline Th-227 & $->$ & $\mathrm{Ra}-223$ & & & \\
\hline$U-235$ & $->$ & Th-231 & & & \\
\hline U-235 & $->$ & Th-231 & & & \\
\hline Pa-231 & $->$ & Ac-227 & & & \\
\hline $\mathrm{Fr}-223$ & $->$ & Ra-223, & Th-227 & $->$ & $\mathrm{Ra}-223$ \\
\hline Pu-239 & $->$ & $U-235$ & & & \\
\hline Th-227 & $->$ & $\mathrm{Ra}-223$ & & & \\
\hline $\mathrm{Fr}-223$ & $->$ & Ra-223, & Th-227 & $->$ & $\mathrm{Ra}-223$ \\
\hline
\end{tabular}




\begin{tabular}{cc}
288.15 & $4.36 \mathrm{E}-08$ \\
289.5 & $2.58 \mathrm{E}-09$ \\
289.57 & $7.91 \mathrm{E}-04$ \\
289.6 & $4.20 \mathrm{E}-09$ \\
291.72 & $1.58 \mathrm{E}-03$ \\
292.3 & $1.72 \mathrm{E}-08$ \\
293.8 & $1.84 \mathrm{E}-08$ \\
296.54 & $1.23 \mathrm{E}-07$ \\
297.46 & $1.14 \mathrm{E}+03$ \\
299.97 & $6.02 \mathrm{E}-07$ \\
300.07 & $1.42 \mathrm{E}-05$ \\
300.34 & $5.73 \mathrm{E}-08$ \\
301.72 & $5.65 \mathrm{E}-04$ \\
302.638 & $3.82 \mathrm{E}-06$ \\
302.671 & $1.02 \mathrm{E}-05$ \\
302.89 & $1.31 \mathrm{E}+02$ \\
304.51 & $3.13 \mathrm{E}-07$ \\
307.82 & $1.42 \mathrm{E}+02$ \\
307.89 & $4.98 \mathrm{E}-10$ \\
308.49 & $4.30 \mathrm{E}-09$ \\
308.75 & $4.40 \mathrm{E}-05$ \\
310.19 & $8.35 \mathrm{E}-09$ \\
310.71 & $4.52 \mathrm{E}-04$ \\
311 & $3.27 \mathrm{E}-04$ \\
311.78 & $5.92 \mathrm{E}+02$ \\
312.57 & $1.35 \mathrm{E}-07$ \\
313.058 & $6.74 \mathrm{E}-07$ \\
313.39 & $4.59 \mathrm{E}+01$ \\
313.85 & $8.78 \mathrm{E}-09$ \\
314.78 & $8.60 \mathrm{E}-09$ \\
314.82 & $1.32 \mathrm{E}-07$ \\
316.4 & $3.24 \mathrm{E}+02$ \\
317.12 & $1.13 \mathrm{E}-04$ \\
317.93 & $9.03 \mathrm{E}-06$ \\
318.1 & $1.19 \mathrm{E}-08$ \\
318.58 & $1.72 \mathrm{E}-09$ \\
319.18 & $9.14 \mathrm{E}-09$ \\
320.188 & $1.24 \mathrm{E}-05$ \\
323.77 & $1.24 \mathrm{E}+03$ \\
323.88 & $1.10 \mathrm{E}-06$ \\
325.08 & $1.72 \mathrm{E}-09$ \\
326.12 & $1.43 \mathrm{E}-09$ \\
327.23 & $1.79 \mathrm{E}-07$ \\
328.08 & $4.26 \mathrm{E}-10$ \\
328.49 & $5.61 \mathrm{E}-08$ \\
330.84 & $7.83 \mathrm{E}-07$ \\
333.87 & $7.87 \mathrm{E}-06$ \\
334.38 & $2.16 \mathrm{E}+04$ \\
& $2.84 \mathrm{E}-07$ \\
\hline
\end{tabular}

\begin{tabular}{|c|c|c|c|c|c|}
\hline Ra-223 & $->$ & $\mathrm{Rn}-219$ & & & \\
\hline Th-227 & $->$ & Ra-223 & & & \\
\hline U-235 & $->$ & Th-231 & & & \\
\hline Fr-223 & $->$ & Ra-223, & Th-227 & $->$ & Ra-223 \\
\hline$U-235$ & $->$ & Th-231 & & & \\
\hline Fr-223 & $->$ & Ra-223, & Th-227 & $->$ & $\mathrm{Ra}-223$ \\
\hline$R n-219$ & $->$ & Po-215 & & & \\
\hline Th-227 & $->$ & Ra-223 & & & \\
\hline Pu-239 & $->$ & U-235 & & & \\
\hline Fr-223 & $->$ & Ra-223, & Th-227 & $->$ & Ra-223 \\
\hline $\mathrm{Pa}-231$ & $->$ & Ac- 227 & & & \\
\hline Th-227 & $->$ & Ra-223 & & & \\
\hline$U-235$ & $->$ & Th-231 & & & \\
\hline $\mathrm{Pa}-231$ & $->$ & Ac- 227 & & & \\
\hline $\mathrm{Pa}-231$ & $->$ & Ac- 227 & & & \\
\hline Pu-239 & $->$ & U-235 & & & \\
\hline Fr-223 & $->$ & Ra-223, & Th-227 & $->$ & Ra-223 \\
\hline Pu-239 & $->$ & $U-235$ & & & \\
\hline $\mathrm{Fr}-223$ & $->$ & Ra-223, & Th-227 & $->$ & Ra-223 \\
\hline Th-227 & $->$ & $\mathrm{Ra}-223$ & & & \\
\hline Th-231 & $->$ & $\mathrm{Pa}-231$ & & & \\
\hline $\mathrm{Pa}-231$ & $->$ & Ac- 227 & & & \\
\hline U-235 & $->$ & Th-231 & & & \\
\hline Th-231 & $->$ & $\mathrm{Pa}-231$ & & & \\
\hline Pu-239 & $->$ & U-235 & & & \\
\hline Fr-223 & $->$ & Ra-223, & Th-227 & $->$ & Ra-223 \\
\hline $\mathrm{Pa}-231$ & $->$ & Ac- 227 & & & \\
\hline Pu-239 & $->$ & U-235 & & & \\
\hline $\mathrm{Pb}-211$ & $->$ & $\mathrm{Bi}-211$ & & & \\
\hline Th-227 & $->$ & $\mathrm{Ra}-223$ & & & \\
\hline Th-227 & $->$ & $\mathrm{Ra}-223$ & & & \\
\hline Pu-239 & $->$ & $U-235$ & & & \\
\hline U-235 & $->$ & Th-231 & & & \\
\hline Th-231 & $->$ & $\mathrm{Pa}-231$ & & & \\
\hline $\mathrm{Pa}-231$ & $->$ & Ac- 227 & & & \\
\hline Th-227 & $->$ & Ra-223 & & & \\
\hline Fr-223 & $->$ & Ra-223, & Th-227 & $->$ & Ra-223 \\
\hline Th-231 & $->$ & $\mathrm{Pa}-231$ & & & \\
\hline Pu-239 & $->$ & U-235 & & & \\
\hline Ra-223 & $->$ & $\mathrm{Rn}-219$ & & & \\
\hline Th-227 & $->$ & Ra-223 & & & \\
\hline Th-227 & $->$ & Ra-223 & & & \\
\hline $\mathrm{Pa}-231$ & $->$ & Ac- 227 & & & \\
\hline TI-207 & $->$ & Pb-207, & Po-211 & $->$ & $\mathrm{Pb}-207$ \\
\hline Ra-223 & $->$ & $R n-219$ & & & \\
\hline Fr-223 & $->$ & Ra-223, & Th-227 & $->$ & $\mathrm{Ra}-223$ \\
\hline $\mathrm{Pa}-231$ & $->$ & $A c-227$ & & & \\
\hline Pu-239 & $->$ & U-235 & & & \\
\hline Ra-223 & $->$ & $\mathrm{Rn}-219$ & & & \\
\hline Fr-223 & $->$ & Ra-223, & Th-227 & $->$ & Ra-223 \\
\hline
\end{tabular}




\begin{tabular}{|c|c|c|c|c|c|c|c|}
\hline 336.116 & $2.61 E+03$ & Pu-239 & $->$ & $U-235$ & & & \\
\hline 338.28 & 7.87E-07 & $\mathrm{Ra}-223$ & $->$ & $\mathrm{Rn}-219$ & & & \\
\hline 339.1 & $4.01 \mathrm{E}-10$ & Th-227 & $->$ & Ra-223 & & & \\
\hline 339.45 & $1.14 \mathrm{E}-09$ & Fr-223 & $->$ & Ra-223, & Th-227 & $->$ & Ra-223 \\
\hline 340.873 & $1.04 \mathrm{E}-06$ & Pa-231 & $->$ & Ac- 227 & & & \\
\hline 341.17 & $1.31 \mathrm{E}-07$ & $\mathrm{~Pa}-231$ & $->$ & Ac- 227 & & & \\
\hline 341.526 & $1.52 \mathrm{E}+03$ & Pu-239 & $->$ & U-235 & & & \\
\hline 342.44 & $1.09 \mathrm{E}-07$ & Fr-223 & $->$ & Ra-223, & Th-227 & $->$ & $\mathrm{Ra}-223$ \\
\hline 342.9 & $5.66 \mathrm{E}-08$ & Ra-223 & $->$ & $\mathrm{Rn}-219$ & & & \\
\hline 343.02 & $9.63 \mathrm{E}-09$ & $\mathrm{~Pb}-211$ & $->$ & $\mathrm{Bi}-211$ & & & \\
\hline 343.78 & 3.39E-04 & U-235 & $->$ & Th-231 & & & \\
\hline 345.014 & $1.28 \mathrm{E}+04$ & Pu-239 & $->$ & U-235 & & & \\
\hline 345.921 & $3.28 \mathrm{E}-03$ & U-235 & $->$ & Th-231 & & & \\
\hline 346.39 & $2.15 \mathrm{E}-09$ & Th-227 & $->$ & Ra-223 & & & \\
\hline 348.4 & $1.72 \mathrm{E}-09$ & Th-227 & $->$ & Ra-223 & & & \\
\hline 350.1 & $3.61 \mathrm{E}-06$ & $\mathrm{Bi}-211$ & $->$ & TI-207 & & & \\
\hline 350.48 & $3.15 \mathrm{E}-08$ & Th-227 & $->$ & Ra-223 & & & \\
\hline 351.82 & 7.90E-06 & Th-231 & $->$ & $\mathrm{Pa}-231$ & & & \\
\hline 352.57 & 2.87E-09 & Th-227 & $->$ & $\mathrm{Ra}-223$ & & & \\
\hline 354.02 & $1.84 \mathrm{E}+01$ & Pu-239 & $->$ & U-235 & & & \\
\hline 354.63 & 4.77E-07 & $\mathrm{Pa}-231$ & $->$ & $A c-227$ & & & \\
\hline 356.068 & $5.65 E-04$ & U-235 & $->$ & Th-231 & & & \\
\hline 357.2 & $9.18 \mathrm{E}-07$ & Pa-231 & $->$ & Ac- 227 & & & \\
\hline 359.51 & $5.37 \mathrm{E}-08$ & Pa-231 & $->$ & Ac- 227 & & & \\
\hline 361.8 & $1.22 \mathrm{E}-08$ & Ra-223 & $->$ & $\mathrm{Rn}-219$ & & & \\
\hline 361.84 & $2.69 E+02$ & Pu-239 & $->$ & U-235 & & & \\
\hline 362.55 & $1.43 \mathrm{E}-09$ & Th-227 & $->$ & $\mathrm{Ra}-223$ & & & \\
\hline 364.03 & $4.41 \mathrm{E}-08$ & Pa-231 & $->$ & Ac- 227 & & & \\
\hline 367.048 & $1.99 \mathrm{E}+03$ & Pu-239 & $->$ & U-235 & & & \\
\hline 368.543 & $2.07 E+03$ & Pu-239 & $->$ & U-235 & & & \\
\hline 368.78 & 2.27E-09 & Ra-223 & $->$ & $\mathrm{Rn}-219$ & & & \\
\hline 369.32 & $1.82 \mathrm{E}-09$ & Fr-223 & $->$ & $\mathrm{Ra}-223$, & Th-227 & $->$ & Ra-223 \\
\hline 369.48 & $5.66 \mathrm{E}-09$ & Ra-223 & $->$ & Rn-219 & & & \\
\hline 370.85 & $2.01 E-09$ & Th-227 & $->$ & $\mathrm{Ra}-223$ & & & \\
\hline 371.8 & $1.39 \mathrm{E}-07$ & Ra-223 & $->$ & $\mathrm{Rn}-219$ & & & \\
\hline 372.47 & $4.82 E+01$ & Pu-239 & $->$ & U-235 & & & \\
\hline 374.93 & $4.01 \mathrm{E}-10$ & Th-227 & $->$ & Ra-223 & & & \\
\hline 375.039 & $3.60 E+04$ & Pu-239 & $->$ & U-235 & & & \\
\hline 376.26 & $1.72 \mathrm{E}-09$ & Th-227 & $->$ & $\mathrm{Ra}-223$ & & & \\
\hline 376.9 & $1.98 \mathrm{E}-09$ & $\mathrm{Rn}-219$ & $->$ & Po-215 & & & \\
\hline 379.05 & $2.41 E+01$ & Pu-239 & $->$ & U-235 & & & \\
\hline 379.4 & $2.74 \mathrm{E}-07$ & $\mathrm{Rn}-219$ & $->$ & Po-215, & Pa-231 & $->$ & Ac- 227 \\
\hline 380.16 & $7.00 E+03$ & Pu-239 & $->$ & U-235 & & & \\
\hline 382.15 & $1.72 \mathrm{E}-09$ & Th-227 & $->$ & $\mathrm{Ra}-223$ & & & \\
\hline 382.732 & $5.95 E+03$ & Pu-239 & $->$ & U-235 & & & \\
\hline 383.12 & $1.13 \mathrm{E}-09$ & Ra-223 & $->$ & $R n-219$ & & & \\
\hline 383.47 & $1.38 \mathrm{E}-08$ & Th-227 & $->$ & Ra-223 & & & \\
\hline 384.97 & $2.44 \mathrm{E}-08$ & Pa-231 & $->$ & $A c-227$ & & & \\
\hline 387.267 & $2.98 \mathrm{E}-09$ & Pa-231 & $->$ & $A c-227$ & & & \\
\hline 387.874 & $4.29 E-04$ & U-235 & $->$ & Th-231 & & & \\
\hline
\end{tabular}




\begin{tabular}{|c|c|c|c|c|c|c|c|}
\hline 387.9 & $1.13 \mathrm{E}-10$ & $\mathrm{Rn}-219$ & $->$ & Po-215 & & & \\
\hline 388.3 & $3.96 \mathrm{E}-09$ & $\mathrm{Ra}-223$ & $->$ & $\mathrm{Rn}-219$ & & & \\
\hline 388.98 & $6.38 \mathrm{E}-09$ & $\mathrm{~Pa}-231$ & $->$ & Ac- 227 & & & \\
\hline 390.32 & $1.13 \mathrm{E}-04$ & U-235 & $->$ & Th-231 & & & \\
\hline 391 & $8.50 \mathrm{E}-10$ & $\mathrm{Ra}-223$ & $->$ & $\mathrm{Rn}-219$ & & & \\
\hline 391.85 & $4.05 E-08$ & $\mathrm{~Pa}-231$ & $->$ & $A c-227$ & & & \\
\hline 392.24 & $2.58 \mathrm{E}-09$ & Th-227 & $->$ & Ra-223 & & & \\
\hline 392.914 & $1.27 \mathrm{E}+04$ & Pu-239 & $->$ & U-235 & & & \\
\hline 395.8 & $1.55 \mathrm{E}-08$ & $\mathrm{~Pa}-231$ & $->$ & Ac- 227 & & & \\
\hline 398.4 & $5.54 \mathrm{E}-08$ & $\mathrm{~Pa}-231$ & $->$ & Ac- 227 & & & \\
\hline 398.6 & $2.58 \mathrm{E}-09$ & Th-227 & $->$ & Ra-223 & & & \\
\hline 399.46 & $1.40 \mathrm{E}+02$ & Pu-239 & $->$ & U-235 & & & \\
\hline 401.7 & $1.88 \mathrm{E}-06$ & $\mathrm{Rn}-219$ & $->$ & Po-215 & & & \\
\hline 402.42 & 3.44E-09 & Th-227 & $->$ & Ra-223 & & & \\
\hline 404.86 & $1.08 \mathrm{E}-06$ & $\mathrm{~Pb}-211$ & $->$ & Bi-211, & At-215 & $->$ & $\mathrm{Bi}-211$ \\
\hline 407.98 & $2.03 \mathrm{E}-07$ & $\mathrm{~Pa}-231$ & $->$ & Ac- 227 & & & \\
\hline 410.269 & 3.39E-04 & U-235 & $->$ & Th-231 & & & \\
\hline 410.84 & $1.13 \mathrm{E}-08$ & $\mathrm{~Pa}-231$ & $->$ & Ac- 227 & & & \\
\hline 410.94 & $1.84 \mathrm{E}+02$ & Pu-239 & $->$ & U-235 & & & \\
\hline 412.83 & $4.59 \mathrm{E}-01$ & Pu-239 & $->$ & U-235 & & & \\
\hline 413.713 & $3.37 E+04$ & Pu-239 & $->$ & $U-235$ & & & \\
\hline 415.09 & $4.87 \mathrm{E}-10$ & Th-227 & $->$ & Ra-223 & & & \\
\hline 422.598 & $2.80 \mathrm{E}+03$ & Pu-239 & $->$ & U-235 & & & \\
\hline 426.64 & $5.26 \mathrm{E}+02$ & Pu-239 & $->$ & $U-235$ & & & \\
\hline 427 & 4.87E-07 & $\mathrm{Pb}-211$ & $->$ & $\mathrm{Bi}-211$ & & & \\
\hline 430.16 & $1.12 \mathrm{E}+02$ & Pu-239 & $->$ & $U-235$ & & & \\
\hline 430.26 & $1.81 \mathrm{E}-09$ & $\mathrm{~Pb}-211$ & $->$ & $\mathrm{Bi}-211$ & & & \\
\hline 430.53 & $5.38 \mathrm{E}-09$ & Ra-223 & $->$ & $\mathrm{Rn}-219$ & & & \\
\hline 432.29 & $1.43 \mathrm{E}-09$ & Th-227 & $->$ & Ra-223 & & & \\
\hline 432.34 & $9.34 \mathrm{E}-09$ & Ra-223 & $->$ & $\mathrm{Rn}-219$ & & & \\
\hline 435.38 & 1.97E-08 & $\mathrm{Pa}-231$ & $->$ & $A c-227$ & & & \\
\hline 438.24 & $2.44 \mathrm{E}-08$ & $\mathrm{~Pa}-231$ & $->$ & Ac- 227 & & & \\
\hline 438.8 & $1.13 \mathrm{E}-08$ & Po-215 & $->$ & $\mathrm{Pb}-211$ & & & \\
\hline 444.94 & $3.60 \mathrm{E}-07$ & Ra-223 & $->$ & $\mathrm{Rn}-219$ & & & \\
\hline 445.81 & $2.02 E+02$ & Pu-239 & $->$ & U-235 & & & \\
\hline 448 & $4.68 \mathrm{E}-11$ & Fr-223 & $->$ & Ra-223, & Th-227 & $->$ & Ra-223 \\
\hline 448.42 & $1.13 \mathrm{E}-04$ & U-235 & $->$ & Th-231 & & & \\
\hline 451.481 & $4.35 E+03$ & Pu-239 & $->$ & $U-235$ & & & \\
\hline 452.52 & $3.71 \mathrm{E}-11$ & Fr-223 & $->$ & Ra-223, & Th-227 & $->$ & $\mathrm{Ra}-223$ \\
\hline 455.12 & $9.04 \mathrm{E}-05$ & $U-235$ & $->$ & Th-231 & & & \\
\hline 457.07 & $2.59 \mathrm{E}-11$ & Fr-223 & $->$ & Ra-223, & Th-227 & $->$ & Ra-223 \\
\hline 457.57 & $3.54 \mathrm{E}+01$ & Pu-239 & $->$ & $U-235$ & & & \\
\hline 461.25 & $4.59 E+01$ & Pu-239 & $->$ & $U-235$ & & & \\
\hline 461.9 & $1.43 \mathrm{E}-11$ & Th-227 & $->$ & $\mathrm{Ra}-223$ & & & \\
\hline 463.8 & $3.67 E+00$ & Pu-239 & $->$ & U-235 & & & \\
\hline 466.2 & $1.43 \mathrm{E}-11$ & Th-227 & $->$ & Ra-223 & & & \\
\hline 471.33 & $8.94 \mathrm{E}-10$ & Pa-231 & $->$ & Ac- 227 & & & \\
\hline 474.26 & $2.07 E+00$ & Pu-239 & $->$ & $U-235$ & & & \\
\hline 478 & 3.68E-09 & $\mathrm{Pb}-211$ & $->$ & $\mathrm{Bi}-211$ & & & \\
\hline 478.22 & $4.59 \mathrm{E}-10$ & $\mathrm{~Pa}-231$ & $->$ & $A c-227$ & & & \\
\hline
\end{tabular}




$\begin{array}{cc}480.25 & 9.11 \mathrm{E}-11 \\ 481.1 & 7.36 \mathrm{E}-09 \\ 481.78 & 1.06 \mathrm{E}+02 \\ 482.14 & 4.77 \mathrm{E}-11 \\ 486.99 & 5.74 \mathrm{E}+00 \\ 487.59 & 2.83 \mathrm{E}-09 \\ 492 & 3.96 \mathrm{E}-09 \\ 493.15 & 1.67 \mathrm{E}-10 \\ 493.25 & 2.02 \mathrm{E}+01 \\ 500.4 & 3.11 \mathrm{E}-09 \\ 501.33 & 3.58 \mathrm{E}-09 \\ 504.33 & 4.25 \mathrm{E}-09 \\ 507.64 & 1.28 \mathrm{E}-10 \\ 515.7 & 1.13 \mathrm{E}-08 \\ 515.96 & 6.13 \mathrm{E}-11 \\ 516.45 & 7.75 \mathrm{E}-09 \\ 517.2 & 4.52 \mathrm{E}-05 \\ 524.02 & 5.10 \mathrm{E}-11 \\ 527.3 & 2.01 \mathrm{E}-08 \\ 534.9 & 2.87 \mathrm{E}-11 \\ 535.45 & 3.34 \mathrm{E}-09 \\ 536.88 & 3.34 \mathrm{E}-10 \\ 538.9 & 6.89 \mathrm{E}+00 \\ 540.1 & 1.70 \mathrm{E}-09 \\ 546.9 & 2.98 \mathrm{E}-09 \\ 550.6 & 9.18 \mathrm{E}+00 \\ 552.09 & 8.52 \mathrm{E}-11 \\ 555.91 & 7.02 \mathrm{E}-11 \\ 557.7 & 1.38 \mathrm{E}+00 \\ 568.76 & 3.27 \mathrm{E}-10 \\ 569.15 & 4.13 \mathrm{E}-10 \\ 572.3 & 2.98 \mathrm{E}-09 \\ 575.8 & 1.59 \mathrm{E}-10 \\ 578.2 & 4.44 \mathrm{E}-11 \\ 586.27 & 3.21 \mathrm{E}+00 \\ 588.17 & 1.82 \mathrm{E}-11 \\ 596.44 & 1.21 \mathrm{E}-11 \\ 598.69 & 2.55 \mathrm{E}-08 \\ 606.9 & 3.44 \mathrm{E}+00 \\ 607.2 & 5.75 \mathrm{E}-11 \\ 608.5 & 1.13 \mathrm{E}-09 \\ 609.08 & 1.78 \mathrm{E}-08 \\ 609.62 & 6.51 \mathrm{E}-09 \\ 612.93 & 1.86 \mathrm{E}+01 \\ 617.43 & 4.68 \mathrm{E}+01 \\ 618.94 & 5.60 \mathrm{E}+01 \\ 621.92 & 1.88 \mathrm{E}-11 \\ 623.38 & 2.27 \mathrm{E}-09 \\ 623.52 & 4.59 \mathrm{E}-11 \\ 624.8 & 9.18 \mathrm{E}+00\end{array}$

\begin{tabular}{|c|c|c|c|c|c|}
\hline Fr-223 & $->$ & Ra-223, & Th-227 & $->$ & Ra-223 \\
\hline $\mathrm{Pb}-211$ & $->$ & $\mathrm{Bi}-211$ & & & \\
\hline Pu-239 & $->$ & U-235 & & & \\
\hline Fr-223 & $->$ & Ra-223, & Th-227 & $->$ & $\mathrm{Ra}-223$ \\
\hline Pu-239 & $->$ & $U-235$ & & & \\
\hline Ra-223 & $->$ & $\mathrm{Rn}-219$ & & & \\
\hline $\mathrm{Pb}-211$ & $->$ & $\mathrm{Bi}-211$ & & & \\
\hline Fr-223 & $->$ & Ra-223, & Th-227 & $->$ & Ra-223 \\
\hline Pu-239 & $->$ & U-235 & & & \\
\hline $\mathrm{Pb}-211$ & $->$ & $\mathrm{Bi}-211$ & & & \\
\hline $\mathrm{Pa}-231$ & $->$ & $A c-227$ & & & \\
\hline$P b-211$ & $->$ & $\mathrm{Bi}-211$ & & & \\
\hline Fr-223 & $->$ & Ra-223, & Th-227 & $->$ & Ra-223 \\
\hline $\mathrm{Rn}-219$ & $->$ & Po-215 & & & \\
\hline $\mathrm{Fr}-223$ & $->$ & Ra-223, & Th-227 & $->$ & $\mathrm{Ra}-223$ \\
\hline $\mathrm{Pa}-231$ & $->$ & Ac- 227 & & & \\
\hline$U-235$ & $->$ & Th-231 & & & \\
\hline $\mathrm{Fr}-223$ & $->$ & Ra-223, & Th-227 & $->$ & $\mathrm{Ra}-223$ \\
\hline $\mathrm{Ra}-223$ & $->$ & $\mathrm{Rn}-219$ & & & \\
\hline Th-227 & $->$ & Ra-223 & & & \\
\hline $\mathrm{Pa}-231$ & $->$ & $A c-227$ & & & \\
\hline Fr-223 & $->$ & $\mathrm{Ra}-223$, & Th-227 & $->$ & Ra-223 \\
\hline Pu-239 & $->$ & U-235 & & & \\
\hline $\mathrm{Rn}-219$ & $->$ & Po-215 & & & \\
\hline Pa-231 & $->$ & Ac- 227 & & & \\
\hline Pu-239 & $->$ & $U-235$ & & & \\
\hline Fr-223 & $->$ & Ra-223, & Th-227 & $->$ & Ra-223 \\
\hline Fr-223 & $->$ & Ra-223, & Th-227 & $->$ & Ra-223 \\
\hline Pu-239 & $->$ & U-235 & & & \\
\hline Fr-223 & $->$ & Ra-223, & Th-227 & $->$ & $\mathrm{Ra}-223$ \\
\hline Po-211 & $->$ & $\mathrm{Pb}-207$ & & & \\
\hline $\mathrm{Pa}-231$ & $->$ & Ac- 227 & & & \\
\hline Fr-223 & $->$ & Ra-223, & Th-227 & $->$ & $\mathrm{Ra}-223$ \\
\hline $\mathrm{Fr}-223$ & $->$ & Ra-223, & Th-227 & $->$ & $\mathrm{Ra}-223$ \\
\hline Pu-239 & $->$ & $U-235$ & & & \\
\hline Fr-223 & $->$ & Ra-223, & Th-227 & $->$ & Ra-223 \\
\hline Fr-223 & $->$ & Ra-223, & Th-227 & $->$ & Ra-223 \\
\hline Ra-223 & $->$ & Rn-219 & & & \\
\hline Pu-239 & $->$ & $U-235$ & & & \\
\hline $\mathrm{Fr}-223$ & $->$ & $\mathrm{Ra}-223$, & Th-227 & $->$ & $\mathrm{Ra}-223$ \\
\hline $\mathrm{Rn}-219$ & $->$ & Po-215 & & & \\
\hline Ra-223 & $->$ & $\mathrm{Rn}-219$ & & & \\
\hline $\mathrm{Pb}-211$ & $->$ & $\mathrm{Bi}-211$ & & & \\
\hline Pu-239 & $->$ & $U-235$ & & & \\
\hline Pu-239 & $->$ & U-235 & & & \\
\hline Pu-239 & $->$ & U-235 & & & \\
\hline Fr-223 & $->$ & Ra-223, & Th-227 & $->$ & Ra-223 \\
\hline Ra-223 & $->$ & $\mathrm{Rn}-219$ & & & \\
\hline Th-227 & $->$ & Ra-223 & & & \\
\hline Pu-239 & $->$ & $U-235$ & & & \\
\hline
\end{tabular}




\begin{tabular}{|c|c|c|c|c|c|c|c|}
\hline 633.15 & $5.81 E+01$ & Pu-239 & $->$ & U-235 & & & \\
\hline 633.2 & $3.73 E-11$ & Th-227 & $->$ & $\mathrm{Ra}-223$ & & & \\
\hline 637.862 & $5.88 \mathrm{E}+01$ & Pu-239 & $->$ & $U-235$ & & & \\
\hline 640.096 & $1.88 \mathrm{E}+02$ & Pu-239 & $->$ & U-235 & & & \\
\hline 641.45 & $5.73 E-12$ & Th-227 & $->$ & $\mathrm{Ra}-223$ & & & \\
\hline 643.93 & $2.57 \mathrm{E}-11$ & Fr-223 & $->$ & Ra-223, & Th-227 & $->$ & Ra-223 \\
\hline 645.969 & $3.42 E+02$ & Pu-239 & $->$ & $U-235$ & & & \\
\hline 647.296 & $4.36 \mathrm{E}+00$ & Pu-239 & $->$ & U-235 & & & \\
\hline 648.43 & 6.07E-12 & Fr-223 & $->$ & Ra-223, & Th-227 & $->$ & Ra-223 \\
\hline 649.377 & $1.63 \mathrm{E}+01$ & Pu-239 & $->$ & $U-235$ & & & \\
\hline 650.529 & $5.97 E+00$ & Pu-239 & $->$ & U-235 & & & \\
\hline 652.052 & $1.50 \mathrm{E}+02$ & Pu-239 & $->$ & U-235 & & & \\
\hline 654.89 & $5.17 \mathrm{E}+01$ & Pu-239 & $->$ & U-235 & & & \\
\hline 658.919 & $2.27 E+02$ & Pu-239 & $->$ & U-235 & & & \\
\hline 662.8 & $1.72 \mathrm{E}-11$ & Th-227 & $->$ & Ra-223 & & & \\
\hline 664.587 & $3.80 E+01$ & Pu-239 & $->$ & U-235 & & & \\
\hline 675.33 & $1.81 \mathrm{E}-09$ & $\mathrm{~Pb}-211$ & $->$ & $\mathrm{Bi}-211$ & & & \\
\hline 676.4 & $1.70 \mathrm{E}-09$ & $\mathrm{Rn}-219$ & $->$ & Po-215 & & & \\
\hline 686.16 & $2.04 \mathrm{E}+01$ & Pu-239 & $->$ & U-235 & & & \\
\hline 690.85 & $1.26 \mathrm{E}+01$ & Pu-239 & $->$ & U-235 & & & \\
\hline 691.46 & $4.92 \mathrm{E}-11$ & Fr-223 & $->$ & Ra-223, & Th-227 & $->$ & Ra-223 \\
\hline 703.8 & $8.91 E+01$ & Pu-239 & $->$ & $U-235$ & & & \\
\hline 704.19 & $4.14 \mathrm{E}-11$ & Fr-223 & $->$ & Ra-223, & Th-227 & $->$ & Ra-223 \\
\hline 704.51 & $1.36 \mathrm{E}-07$ & $\mathrm{~Pb}-211$ & $->$ & $\mathrm{Bi}-211$ & & & \\
\hline 707.18 & $1.48 \mathrm{E}-11$ & Fr-223 & $->$ & Ra-223, & Th-227 & $->$ & Ra-223 \\
\hline 711.2 & $9.91 \mathrm{E}-10$ & Ra-223 & $->$ & $\mathrm{Rn}-219$ & & & \\
\hline 714.58 & $2.07 E+00$ & Pu-239 & $->$ & U-235 & & & \\
\hline 718 & $6.43 E+01$ & Pu-239 & $->$ & U-235 & & & \\
\hline 718.22 & $9.61 \mathrm{E}-12$ & Fr-223 & $->$ & Ra-223, & Th-227 & $->$ & $\mathrm{Ra}-223$ \\
\hline 722.6 & $2.14 \mathrm{E}-10$ & Fr-223 & $->$ & $\mathrm{Ra}-223$, & Th-227 & $->$ & $\mathrm{Ra}-223$ \\
\hline 723.86 & $4.35 \mathrm{E}-10$ & Fr-223 & $->$ & Ra-223, & Th-227 & $->$ & $\mathrm{Ra}-223$ \\
\hline 727.84 & $2.50 \mathrm{E}+01$ & Pu-239 & $->$ & $U-235$ & & & \\
\hline 733.9 & $5.80 \mathrm{E}-11$ & Fr-223 & $->$ & Ra-223, & Th-227 & $->$ & $\mathrm{Ra}-223$ \\
\hline 734.6 & $5.43 \mathrm{E}-11$ & Fr-223 & $->$ & Ra-223, & Th-227 & $->$ & Ra-223 \\
\hline 736.76 & $2.59 \mathrm{E}-11$ & Fr-223 & $->$ & Ra-223, & Th-227 & $->$ & $\mathrm{Ra}-223$ \\
\hline 742.52 & $4.52 \mathrm{E}-05$ & $\mathrm{U}-235$ & $->$ & Th-231 & & & \\
\hline 746.24 & $1.27 \mathrm{E}-10$ & Fr-223 & $->$ & Ra-223, & Th-227 & $->$ & $\mathrm{Ra}-223$ \\
\hline 748.56 & $1.53 \mathrm{E}-10$ & Fr-223 & $->$ & Ra-223, & Th-227 & $->$ & $\mathrm{Ra}-223$ \\
\hline 753.44 & $5.47 \mathrm{E}-11$ & Fr-223 & $->$ & Ra-223, & Th-227 & $->$ & $\mathrm{Ra}-223$ \\
\hline 754.17 & $5.63 \mathrm{E}-11$ & Fr-223 & $->$ & Ra-223, & Th-227 & $->$ & $\mathrm{Ra}-223$ \\
\hline 756.42 & $7.74 \mathrm{E}+01$ & Pu-239 & $->$ & $U-235$ & & & \\
\hline 756.79 & $1.12 \mathrm{E}-10$ & Fr-223 & $->$ & Ra-223, & Th-227 & $->$ & $\mathrm{Ra}-223$ \\
\hline 757.03 & $2.91 \mathrm{E}-10$ & Fr-223 & $->$ & Ra-223, & Th-227 & $->$ & Ra-223 \\
\hline 762.3 & $8.29 E-11$ & Fr-223 & $->$ & Ra-223, & Th-227 & $->$ & $\mathrm{Ra}-223$ \\
\hline 764.52 & $4.17 \mathrm{E}-10$ & Fr-223 & $->$ & Ra-223, & Th-227 & $->$ & Ra-223 \\
\hline 766.35 & $2.01 \mathrm{E}-07$ & $\mathrm{~Pb}-211$ & $->$ & $\mathrm{Bi}-211$ & & & \\
\hline 766.6 & $6.31 \mathrm{E}-02$ & Pu-239 & $->$ & U-235 & & & \\
\hline 769.19 & $2.73 E+02$ & Pu-239 & $->$ & $\mathrm{U}-235$ & & & \\
\hline 772.85 & $3.94 \mathrm{E}-11$ & Fr-223 & $->$ & Ra-223, & Th-227 & $->$ & $\mathrm{Ra}-223$ \\
\hline 775.82 & $2.11 \mathrm{E}-09$ & Fr-223 & $->$ & Ra-223, & Th-227 & $->$ & $\mathrm{Ra}-223$ \\
\hline
\end{tabular}




\begin{tabular}{|c|c|c|c|c|c|c|c|}
\hline 779.46 & $3.05 E+00$ & Pu-239 & $->$ & U-235 & & & \\
\hline 780.59 & $1.02 \mathrm{E}-10$ & Fr-223 & $->$ & Ra-223, & Th-227 & $->$ & Ra-223 \\
\hline 784.04 & $5.63 \mathrm{E}-11$ & Fr-223 & $->$ & Ra-223, & Th-227 & $->$ & Ra-223 \\
\hline 786.9 & $3.89 \mathrm{E}-11$ & Fr-223 & $->$ & Ra-223, & Th-227 & $->$ & Ra-223 \\
\hline 787.3 & $1.93 \mathrm{E}+00$ & Pu-239 & $->$ & U-235 & & & \\
\hline 788.36 & $1.15 \mathrm{E}-11$ & Th-227 & $->$ & $\mathrm{Ra}-223$ & & & \\
\hline 792.6 & $1.15 \mathrm{E}-11$ & Th-227 & $->$ & Ra-223 & & & \\
\hline 793 & $5.74 \mathrm{E}-01$ & Pu-239 & $->$ & U-235 & & & \\
\hline 793.11 & $5.03 E-13$ & Fr-223 & $->$ & Ra-223 & & & \\
\hline 793.12 & $8.60 \mathrm{E}-12$ & Th-227 & $->$ & Ra-223 & & & \\
\hline 794.72 & $6.78 \mathrm{E}-05$ & U-235 & $->$ & Th-231 & & & \\
\hline 796.5 & 7.35E-01 & Pu-239 & $->$ & U-235 & & & \\
\hline 796.96 & $2.69 \mathrm{E}-10$ & Fr-223 & $->$ & Ra-223, & Th-227 & $->$ & Ra-223 \\
\hline 803.3 & $1.01 \mathrm{E}+00$ & Pu-239 & $->$ & U-235 & & & \\
\hline 803.58 & $5.15 \mathrm{E}-10$ & $\mathrm{Fr}-223$ & $->$ & Ra-223, & Th-227 & $->$ & Ra-223 \\
\hline 808.2 & $3.37 \mathrm{E}+00$ & Pu-239 & $->$ & U-235 & & & \\
\hline 808.26 & $1.69 \mathrm{E}-11$ & Fr-223 & $->$ & Ra-223, & Th-227 & $->$ & Ra-223 \\
\hline 812.15 & $8.32 \mathrm{E}-10$ & Fr-223 & $->$ & Ra-223, & Th-227 & $->$ & $\mathrm{Ra}-223$ \\
\hline 813.9 & $1.42 \mathrm{E}+00$ & Pu-239 & $->$ & U-235 & & & \\
\hline 817.31 & $4.96 \mathrm{E}-11$ & Fr-223 & $->$ & Ra-223, & Th-227 & $->$ & Ra-223 \\
\hline 818.23 & $8.60 \mathrm{E}-12$ & Th-227 & $->$ & $\mathrm{Ra}-223$ & & & \\
\hline 821.1 & $1.17 \mathrm{E}+00$ & Pu-239 & $->$ & U-235 & & & \\
\hline 822.99 & $7.59 \mathrm{E}-10$ & Fr-223 & $->$ & Ra-223, & Th-227 & $->$ & Ra-223 \\
\hline 825.96 & $2.64 \mathrm{E}-10$ & Fr-223 & $->$ & Ra-223, & Th-227 & $->$ & Ra-223 \\
\hline 825.97 & $5.45 \mathrm{E}-12$ & Fr-223 & $->$ & $\mathrm{Ra}-223$ & & & \\
\hline 828.31 & $6.24 \mathrm{E}-11$ & Fr-223 & $->$ & Ra-223, & Th-227 & $->$ & Ra-223 \\
\hline 828.53 & $3.41 \mathrm{E}-12$ & Fr-223 & $->$ & Ra-223, & Th-227 & $->$ & Ra-223 \\
\hline 828.8 & $3.17 E+00$ & Pu-239 & $->$ & $U-235$ & & & \\
\hline 831.86 & $1.08 \mathrm{E}-06$ & $\mathrm{~Pb}-211$ & $->$ & $\mathrm{Bi}-211$ & & & \\
\hline 831.93 & $5.74 \mathrm{E}-01$ & Pu-239 & $->$ & U-235 & & & \\
\hline 834.2 & $2.83 \mathrm{E}-10$ & $\mathrm{Rn}-219$ & $->$ & Po-215 & & & \\
\hline 837.36 & $1.43 \mathrm{E}-10$ & Fr-223 & $->$ & Ra-223, & Th-227 & $->$ & $\mathrm{Ra}-223$ \\
\hline 839 & $9.18 \mathrm{E}-01$ & Pu-239 & $->$ & U-235 & & & \\
\hline 842.02 & $1.98 \mathrm{E}-10$ & Fr-223 & $->$ & Ra-223, & Th-227 & $->$ & $\mathrm{Ra}-223$ \\
\hline 843.8 & $3.67 E+00$ & Pu-239 & $->$ & U-235 & & & \\
\hline 846.56 & $1.93 \mathrm{E}-10$ & Fr-223 & $->$ & Ra-223, & Th-227 & $->$ & Ra-223 \\
\hline 848.88 & $3.10 \mathrm{E}-11$ & Fr-223 & $->$ & Ra-223, & Th-227 & $->$ & Ra-223 \\
\hline 854.2 & $2.01 \mathrm{E}-11$ & Th-227 & $->$ & Ra-223 & & & \\
\hline 857.89 & $1.88 \mathrm{E}-11$ & Fr-223 & $->$ & Ra-223, & Th-227 & $->$ & Ra-223 \\
\hline 858.4 & $8.18 \mathrm{E}-11$ & Fr-223 & $->$ & Ra-223, & Th-227 & $->$ & Ra-223 \\
\hline 863.2 & $1.41 \mathrm{E}-11$ & Fr-223 & $->$ & Ra-223, & Th-227 & $->$ & $\mathrm{Ra}-223$ \\
\hline 865.81 & $1.81 \mathrm{E}-09$ & $\mathrm{~Pb}-211$ & $->$ & $\mathrm{Bi}-211$ & & & \\
\hline 867.23 & $2.54 \mathrm{E}-11$ & Fr-223 & $->$ & Ra-223, & Th-227 & $->$ & $\mathrm{Ra}-223$ \\
\hline 876.14 & $2.23 \mathrm{E}-10$ & Fr-223 & $->$ & Ra-223, & Th-227 & $->$ & Ra-223 \\
\hline 878.16 & $3.47 \mathrm{E}-11$ & $\mathrm{Fr}-223$ & $->$ & Ra-223, & Th-227 & $->$ & $\mathrm{Ra}-223$ \\
\hline 879 & $1.15 \mathrm{E}+00$ & Pu-239 & $->$ & U-235 & & & \\
\hline 887.7 & $4.25 \mathrm{E}-10$ & $\mathrm{Rn}-219$ & $->$ & Po-215 & & & \\
\hline 891 & $1.41 \mathrm{E}-11$ & Fr-223 & $->$ & Ra-223, & Th-227 & $->$ & Ra-223 \\
\hline 891.1 & $1.77 \mathrm{E}+00$ & Pu-239 & $->$ & U-235 & & & \\
\hline 892.8 & $9.18 \mathrm{E}-12$ & Fr-223 & $->$ & Ra-223, & Th-227 & $->$ & Ra-223 \\
\hline
\end{tabular}




\begin{tabular}{|c|c|c|c|c|c|c|c|}
\hline 896.41 & $1.03 \mathrm{E}-10$ & Fr-223 & $->$ & Ra-223, & Th-227 & $->$ & $\mathrm{Ra}-223$ \\
\hline 897.23 & $6.82 \mathrm{E}-08$ & Tl-207 & $->$ & $\mathrm{Pb}-207$, & Po-211 & $->$ & Pb-207 \\
\hline 908.03 & $6.57 \mathrm{E}-10$ & Fr-223 & $->$ & $\mathrm{Ra}-223$, & Th-227 & $->$ & $\mathrm{Ra}-223$ \\
\hline 909.85 & $4.30 \mathrm{E}-12$ & Th-227 & $->$ & $\mathrm{Ra}-223$ & & & \\
\hline 920.06 & $3.44 \mathrm{E}-12$ & Th-227 & $->$ & Ra-223 & & & \\
\hline 926.28 & 8.71E-12 & Fr-223 & $->$ & Ra-223, & Th-227 & $->$ & Ra-223 \\
\hline 938.34 & $2.87 \mathrm{E}-12$ & Th-227 & $->$ & $\mathrm{Ra}-223$ & & & \\
\hline 940.1 & $9.64 \mathrm{E}-01$ & Pu-239 & $->$ & U-235 & & & \\
\hline 941.41 & $2.06 \mathrm{E}-11$ & Th-227 & $->$ & Ra-223 & & & \\
\hline 951 & $6.23 \mathrm{E}-09$ & $\mathrm{~Pb}-211$ & $->$ & $\mathrm{Bi}-211$ & & & \\
\hline 956.4 & $1.29 E+00$ & Pu-239 & $->$ & $U-235$ & & & \\
\hline 958.6 & $1.78 \mathrm{E}-11$ & Th-227 & $->$ & Ra-223 & & & \\
\hline 969.91 & $8.60 \mathrm{E}-12$ & Th-227 & $->$ & Ra-223 & & & \\
\hline 971.28 & $2.87 \mathrm{E}-12$ & Th-227 & $->$ & $\mathrm{Ra}-223$ & & & \\
\hline 979.52 & $5.28 \mathrm{E}-01$ & Pu-239 & $->$ & U-235 & & & \\
\hline 986.7 & $2.75 \mathrm{E}-01$ & Pu-239 & $->$ & $U-235$ & & & \\
\hline 990.2 & $1.00 \mathrm{E}-11$ & Th-227 & $->$ & Ra-223 & & & \\
\hline 992.47 & $5.28 \mathrm{E}-01$ & Pu-239 & $->$ & U-235 & & & \\
\hline 994.9 & $2.01 \mathrm{E}-12$ & Th-227 & $->$ & $\mathrm{Ra}-223$ & & & \\
\hline 999.78 & $8.60 \mathrm{E}-12$ & Th-227 & $->$ & Ra-223 & & & \\
\hline 1005.63 & $2.75 \mathrm{E}-01$ & Pu-239 & $->$ & U-235 & & & \\
\hline 1014.48 & 5.07E-09 & $\mathrm{Pb}-211$ & $->$ & $\mathrm{Bi}-211$ & & & \\
\hline 1015.1 & $4.30 \mathrm{E}-12$ & Th-227 & $->$ & Ra-223 & & & \\
\hline 1020 & $5.73 \mathrm{E}-12$ & Th-227 & $->$ & Ra-223 & & & \\
\hline 1024.8 & $4.30 \mathrm{E}-12$ & Th-227 & $->$ & Ra-223 & & & \\
\hline 1055.8 & $1.70 \mathrm{E}-10$ & $\mathrm{Rn}-219$ & $->$ & Po-215 & & & \\
\hline 1057.32 & $1.03 E+00$ & Pu-239 & $->$ & U-235 & & & \\
\hline 1080.19 & 4.33E-09 & $\mathrm{Pb}-211$ & $->$ & $\mathrm{Bi}-211$ & & & \\
\hline 1090.5 & $7.36 \mathrm{E}-10$ & $\mathrm{~Pb}-211$ & $->$ & $\mathrm{Bi}-211$ & & & \\
\hline 1103.4 & $1.44 \mathrm{E}-09$ & $\mathrm{~Pb}-211$ & $->$ & $\mathrm{Bi}-211$ & & & \\
\hline 1109.37 & $4.16 \mathrm{E}-08$ & $\mathrm{~Pb}-211$ & $->$ & $\mathrm{Bi}-211$ & & & \\
\hline 1196.61 & $3.62 E-09$ & $\mathrm{~Pb}-211$ & $->$ & $\mathrm{Bi}-211$ & & & \\
\hline 1234.3 & $3.68 \mathrm{E}-10$ & $\mathrm{~Pb}-211$ & $->$ & $\mathrm{Bi}-211$ & & & \\
\hline 1270.67 & $2.52 \mathrm{E}-09$ & $\mathrm{~Pb}-211$ & $->$ & $\mathrm{Bi}-211$ & & & \\
\hline
\end{tabular}

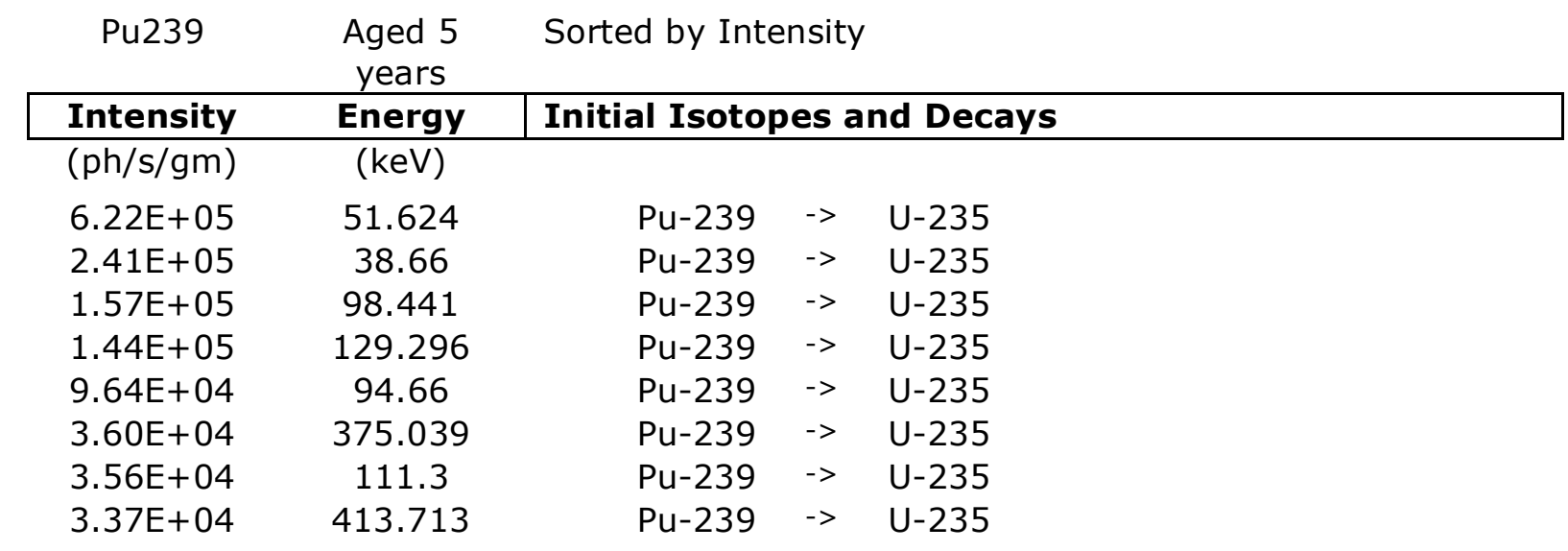




\begin{tabular}{|c|c|c|c|c|}
\hline $2.80 E+04$ & 98.79 & Pu-239 & $->$ & U-235 \\
\hline $2.59 \mathrm{E}+04$ & 56.825 & Pu-239 & $->$ & U-235 \\
\hline $1.83 E+04$ & 110.421 & Pu-239 & $->$ & U-235 \\
\hline $1.69 E+04$ & 46.218 & Pu-239 & $->$ & U-235 \\
\hline $1.49 E+04$ & 115.38 & Pu-239 & $->$ & $U-235$ \\
\hline $1.44 \mathrm{E}+04$ & 114.333 & Pu-239 & $->$ & $U-23$ \\
\hline $1.37 E+04$ & 116.266 & Pu-239 & $->$ & U-235 \\
\hline $1.29 E+04$ & 203.54 & Pu-239 & $->$ & $U-235$ \\
\hline $1.28 \mathrm{E}+04$ & 345.014 & Pu-239 & $->$ & U-23! \\
\hline $1.27 \mathrm{E}+04$ & 392.914 & Pu-239 & $->$ & $U-23$ \\
\hline $1.16 \mathrm{E}+04$ & 332.853 & Pu-239 & $->$ & $\mathrm{U}-23$ \\
\hline $9.80 E+03$ & 77.593 & Pu-239 & $->$ & $U-23$ \\
\hline $7.00 E+03$ & 380.16 & Pu-239 & $->$ & $U-23$ \\
\hline $6.97 E+03$ & 144.201 & Pu-239 & $->$ & $U-23$ \\
\hline $5.95 E+03$ & 382.732 & Pu-239 & $->$ & $U-23$ \\
\hline $4.87 E+03$ & 103.035 & Pu-239 & $->$ & U-235 \\
\hline $4.35 E+03$ & 451.481 & Pu-239 & $->$ & U-23: \\
\hline $3.88 E+03$ & 78.37 & Pu-239 & $->$ & $\mathrm{U}-23$ \\
\hline $3.88 E+03$ & 54.02 & Pu-239 & $->$ & $U-23$ \\
\hline $3.79 E+03$ & 42.06 & Pu-239 & $->$ & U-235 \\
\hline $3.72 E+03$ & 40.41 & Pu-239 & $->$ & $U-235$ \\
\hline $3.26 \mathrm{E}+03$ & 67.69 & Pu-239 & $->$ & $U-23$ \\
\hline $2.82 E+03$ & 161.482 & Pu-239 & $->$ & $U-23$ \\
\hline $2.80 E+03$ & 422.598 & Pu-239 & $->$ & $U-235$ \\
\hline $2.75 E+03$ & 68.73 & Pu-239 & $->$ & U-235 \\
\hline $2.73 E+03$ & 146.094 & Pu-239 & $->$ & $U-23$ \\
\hline $2.61 E+03$ & 336.116 & Pu-239 & $->$ & $U-23$ \\
\hline $2.53 E+03$ & 171.393 & Pu-239 & $->$ & U-23: \\
\hline $2.46 \mathrm{E}+03$ & 195.679 & Pu-239 & $->$ & $U-235$ \\
\hline $2.41 E+03$ & 30.08 & Pu-239 & $->$ & $U-23$ \\
\hline $2.07 E+03$ & 368.543 & Pu-239 & $->$ & $U-23$ \\
\hline $1.99 E+03$ & 367.048 & Pu-239 & $->$ & $U-23$ \\
\hline $1.91 E+03$ & 189.36 & Pu-239 & $->$ & $U-235$ \\
\hline $1.84 \mathrm{E}+03$ & 255.384 & Pu-239 & $->$ & $U-23$ \\
\hline $1.63 E+03$ & 125.21 & Pu-239 & $->$ & $U-23$ \\
\hline $1.52 E+03$ & 341.526 & Pu-239 & $->$ & $U-23$ \\
\hline $1.52 \mathrm{E}+03$ & 179.22 & Pu-239 & $->$ & $U-23$ \\
\hline $1.41 \mathrm{E}+03$ & 124.51 & Pu-239 & $->$ & $U-23$ \\
\hline $1.33 E+03$ & 46.69 & Pu-239 & $->$ & $U-23$ \\
\hline $1.24 \mathrm{E}+03$ & 323.77 & Pu-239 & $->$ & $U-23$ \\
\hline $1.14 \mathrm{E}+03$ & 297.46 & Pu-239 & $->$ & $U-23$ \\
\hline $8.72 E+02$ & 74.89 & Pu-239 & $->$ & $U-23$ \\
\hline $8.45 E+02$ & 96.16 & Pu-239 & $->$ & U-23 \\
\hline $7.58 \mathrm{E}+02$ & 65.7 & Pu-239 & $->$ & $\mathrm{U}-23$ \\
\hline $7.35 E+02$ & 141.657 & Pu-239 & $->$ & $U-23$ \\
\hline $7.05 E+02$ & 111.89 & Pu-239 & $->$ & U-23: \\
\hline $6.66 \mathrm{E}+02$ & 47.52 & Pu-239 & $->$ & $U-235$ \\
\hline $5.92 E+02$ & 311.78 & Pu-239 & $->$ & $U-23$ \\
\hline $5.85 E+02$ & 263.9 & Pu-239 & $->$ & $U-23$ \\
\hline $5.33 E+02$ & 243.36 & Pu-239 & $->$ & $U-23$ \\
\hline
\end{tabular}




\begin{tabular}{|c|c|c|c|c|}
\hline $5.26 E+02$ & 426.64 & Pu-239 & $->$ & U-235 \\
\hline $4.52 E+02$ & 123.62 & Pu-239 & $->$ & $U-235$ \\
\hline $3.97 \mathrm{E}+02$ & 143.35 & Pu-239 & $->$ & $U-235$ \\
\hline $3.74 \mathrm{E}+02$ & 225.39 & Pu-239 & $->$ & U-235 \\
\hline $3.44 \mathrm{E}+02$ & 237.75 & Pu-239 & $->$ & U-235 \\
\hline $3.42 E+02$ & 645.969 & Pu-239 & $->$ & $U-23$ \\
\hline $3.24 \mathrm{E}+02$ & 316.4 & Pu-239 & $->$ & U-23: \\
\hline $2.73 E+02$ & 769.19 & Pu-239 & $->$ & $U-235$ \\
\hline $2.69 E+02$ & 361.84 & Pu-239 & $->$ & U-235 \\
\hline $2.50 E+02$ & 188.23 & Pu-239 & $->$ & U-235 \\
\hline $2.27 E+02$ & 658.919 & Pu-239 & $->$ & U-235 \\
\hline $2.02 E+02$ & 445.81 & Pu-239 & $->$ & U-235 \\
\hline $1.91 \mathrm{E}+02$ & 242.12 & Pu-239 & $->$ & U-235 \\
\hline $1.88 \mathrm{E}+02$ & 640.096 & Pu-239 & $->$ & $U-235$ \\
\hline $1.84 \mathrm{E}+02$ & 410.94 & Pu-239 & $->$ & U-235 \\
\hline $1.72 \mathrm{E}+02$ & 248.88 & Pu-239 & $->$ & U-235 \\
\hline $1.50 \mathrm{E}+02$ & 652.052 & Pu-239 & $->$ & $U-235$ \\
\hline $1.42 \mathrm{E}+02$ & 160.19 & Pu-239 & $->$ & $U-235$ \\
\hline $1.42 E+02$ & 307.82 & Pu-239 & $->$ & $U-23$ \\
\hline $1.40 \mathrm{E}+02$ & 399.46 & Pu-239 & $->$ & U-23: \\
\hline $1.31 \mathrm{E}+02$ & 302.89 & Pu-239 & $->$ & U-235 \\
\hline $1.19 \mathrm{E}+02$ & 244.86 & Pu-239 & $->$ & U-235 \\
\hline $1.12 \mathrm{E}+02$ & 430.16 & Pu-239 & $->$ & U-235 \\
\hline $1.06 \mathrm{E}+02$ & 481.78 & Pu-239 & $->$ & U-235 \\
\hline $9.18 \mathrm{E}+01$ & 173.7 & Pu-239 & $->$ & U-235 \\
\hline $8.91 E+01$ & 703.8 & Pu-239 & $->$ & U-235 \\
\hline $8.49 E+01$ & 196.87 & Pu-239 & $->$ & U-235 \\
\hline $7.74 \mathrm{E}+01$ & 756.42 & Pu-239 & $->$ & $U-235$ \\
\hline $6.89 E+01$ & 265.81 & Pu-239 & $->$ & $U-235$ \\
\hline $6.89 E+01$ & 122.34 & Pu-239 & $->$ & U-235 \\
\hline $6.73 E+01$ & 167.81 & Pu-239 & $->$ & U-235 \\
\hline $6.43 E+01$ & 718 & Pu-239 & $->$ & $U-235$ \\
\hline $5.88 \mathrm{E}+01$ & 637.862 & Pu-239 & $->$ & $U-23$ \\
\hline $5.81 \mathrm{E}+01$ & 633.15 & Pu-239 & $->$ & U-235 \\
\hline $5.60 \mathrm{E}+01$ & 618.94 & Pu-239 & $->$ & U-235 \\
\hline $5.17 E+01$ & 654.89 & Pu-239 & $->$ & U-235 \\
\hline $4.87 E+01$ & 184.55 & Pu-239 & $->$ & $U-23$ \\
\hline $4.82 E+01$ & 281.12 & Pu-239 & $->$ & $U-23$ \\
\hline $4.82 E+01$ & 372.47 & Pu-239 & $->$ & U-235 \\
\hline $4.68 \mathrm{E}+01$ & 617.43 & Pu-239 & $->$ & $U-235$ \\
\hline $4.59 E+01$ & 461.25 & Pu-239 & $->$ & U-235 \\
\hline $4.59 E+01$ & 313.39 & Pu-239 & $->$ & U-23: \\
\hline $3.80 \mathrm{E}+01$ & 664.587 & Pu-239 & $->$ & $U-235$ \\
\hline $3.54 \mathrm{E}+01$ & 457.57 & Pu-239 & $->$ & $U-235$ \\
\hline $3.44 \mathrm{E}+01$ & 285.27 & Pu-239 & $->$ & U-235 \\
\hline $2.50 \mathrm{E}+01$ & 727.84 & Pu-239 & $->$ & U-23: \\
\hline $2.41 \mathrm{E}+01$ & 379.05 & Pu-239 & $->$ & $U-235$ \\
\hline $2.30 \mathrm{E}+01$ & 218.45 & Pu-239 & $->$ & U-235 \\
\hline $2.04 \mathrm{E}+01$ & 686.16 & Pu-239 & $->$ & U-235 \\
\hline $2.02 E+01$ & 493.25 & Pu-239 & $->$ & $U-23$ \\
\hline
\end{tabular}




\begin{tabular}{|c|c|c|c|c|c|c|c|}
\hline $1.86 \mathrm{E}+01$ & 612.93 & Pu-239 & $->$ & $U-235$ & & & \\
\hline $1.84 \mathrm{E}+01$ & 354.02 & Pu-239 & $->$ & U-235 & & & \\
\hline $1.63 E+01$ & 649.377 & Pu-239 & $->$ & U-235 & & & \\
\hline $1.26 \mathrm{E}+01$ & 690.85 & Pu-239 & $->$ & U-235 & & & \\
\hline $9.18 \mathrm{E}+00$ & 624.8 & Pu-239 & $->$ & U-235 & & & \\
\hline $9.18 \mathrm{E}+00$ & 550.6 & Pu-239 & $->$ & U-235 & & & \\
\hline $6.89 \mathrm{E}+00$ & 538.9 & Pu-239 & $->$ & U-235 & & & \\
\hline $6.46 \mathrm{E}+00$ & 185.72 & U-235 & $->$ & Th-231 & & & \\
\hline $5.97 E+00$ & 650.529 & Pu-239 & $->$ & U-235 & & & \\
\hline $5.74 \mathrm{E}+00$ & 486.99 & Pu-239 & $->$ & U-235 & & & \\
\hline $5.53 E+00$ & 13.724 & Th-231 & $->$ & $\mathrm{Pa}-231$ & & & \\
\hline $4.36 \mathrm{E}+00$ & 647.296 & Pu-239 & $->$ & U-235 & & & \\
\hline $4.18 \mathrm{E}+00$ & 16.559 & Th-231 & $->$ & Pa-231 & & & \\
\hline $3.67 E+00$ & 843.8 & Pu-239 & $->$ & U-235 & & & \\
\hline $3.67 E+00$ & 463.8 & Pu-239 & $->$ & U-235 & & & \\
\hline $3.44 \mathrm{E}+00$ & 606.9 & Pu-239 & $->$ & U-235 & & & \\
\hline $3.37 E+00$ & 808.2 & Pu-239 & $->$ & U-235 & & & \\
\hline $3.21 \mathrm{E}+00$ & 586.27 & Pu-239 & $->$ & $U-235$ & & & \\
\hline $3.17 E+00$ & 828.8 & Pu-239 & $->$ & U-235 & & & \\
\hline $3.05 E+00$ & 779.46 & Pu-239 & $->$ & U-235 & & & \\
\hline $2.49 E+00$ & 12.952 & Ac-227 & $->$ & Th-227, & $U-235$ & $->$ & Th-231 \\
\hline $2.07 E+00$ & 714.58 & Pu-239 & $->$ & $U-235$ & & & \\
\hline $2.07 E+00$ & 474.26 & Pu-239 & $->$ & U-235 & & & \\
\hline $1.93 E+00$ & 787.3 & Pu-239 & $->$ & $U-235$ & & & \\
\hline $1.77 E+00$ & 891.1 & Pu-239 & $->$ & U-235 & & & \\
\hline $1.69 \mathrm{E}+00$ & 16.119 & U-235 & $->$ & Th-231 & & & \\
\hline $1.65 \mathrm{E}+00$ & 25.642 & Th-231 & $->$ & Pa-231 & & & \\
\hline $1.42 \mathrm{E}+00$ & 813.9 & Pu-239 & $->$ & U-235 & & & \\
\hline $1.38 \mathrm{E}+00$ & 557.7 & Pu-239 & $->$ & U-235 & & & \\
\hline $1.29 E+00$ & 956.4 & Pu-239 & $->$ & $U-235$ & & & \\
\hline $1.24 \mathrm{E}+00$ & 143.76 & $U-235$ & $->$ & Th-231 & & & \\
\hline $1.17 \mathrm{E}+00$ & 821.1 & Pu-239 & $->$ & U-235 & & & \\
\hline $1.15 \mathrm{E}+00$ & 879 & Pu-239 & $->$ & U-235 & & & \\
\hline $1.03 E+00$ & 1057.32 & Pu-239 & $->$ & $U-235$ & & & \\
\hline $1.01 \mathrm{E}+00$ & 803.3 & Pu-239 & $->$ & U-235 & & & \\
\hline $9.64 \mathrm{E}-01$ & 940.1 & Pu-239 & $->$ & U-235 & & & \\
\hline $9.18 \mathrm{E}-01$ & 839 & Pu-239 & $->$ & U-235 & & & \\
\hline 8.35E-01 & 19.811 & Th-231 & $->$ & Pa-231 & & & \\
\hline 7.45E-01 & 84.203 & Th-231 & $->$ & $\mathrm{Pa}-231$ & & & \\
\hline 7.35E-01 & 796.5 & Pu-239 & $->$ & U-235 & & & \\
\hline $6.21 \mathrm{E}-01$ & 93.36 & U-235 & $->$ & Th-231 & & & \\
\hline $5.74 \mathrm{E}-01$ & 163.33 & U-235 & $->$ & Th-231 & & & \\
\hline $5.74 \mathrm{E}-01$ & 831.93 & Pu-239 & $->$ & U-235 & & & \\
\hline $5.74 \mathrm{E}-01$ & 793 & Pu-239 & $->$ & U-235 & & & \\
\hline $5.66 \mathrm{E}-01$ & 205.31 & U-235 & $->$ & Th-231 & & & \\
\hline $5.28 \mathrm{E}-01$ & 992.47 & Pu-239 & $->$ & $U-235$ & & & \\
\hline $5.28 \mathrm{E}-01$ & 979.52 & Pu-239 & $->$ & $U-235$ & & & \\
\hline $4.59 \mathrm{E}-01$ & 412.83 & Pu-239 & $->$ & U-235 & & & \\
\hline $3.80 \mathrm{E}-01$ & 89.955 & U-235 & $->$ & Th-231 & & & \\
\hline 3.39E-01 & 11.372 & Th-231 & $->$ & $\mathrm{Pa}-231$ & & & \\
\hline
\end{tabular}




\begin{tabular}{|c|c|c|c|c|c|c|c|}
\hline $2.94 \mathrm{E}-01$ & 19.118 & $U-235$ & $->$ & Th-231 & & & \\
\hline $2.75 \mathrm{E}-01$ & 986.7 & Pu-239 & $->$ & U-235 & & & \\
\hline $2.75 \mathrm{E}-01$ & 1005.63 & Pu-239 & $->$ & U-235 & & & \\
\hline $2.24 \mathrm{E}-01$ & 105.362 & U-235 & $->$ & Th-231 & & & \\
\hline $1.74 \mathrm{E}-01$ & 109.16 & U-235 & $->$ & Th-231 & & & \\
\hline $1.22 \mathrm{E}-01$ & 202.11 & U-235 & $->$ & Th-231 & & & \\
\hline $1.13 \mathrm{E}-01$ & 11.118 & Ac-227 & $->$ & Th-227, & U-235 & $->$ & Th-231 \\
\hline $1.06 \mathrm{E}-01$ & 89.954 & Th-231 & $->$ & $\mathrm{Pa}-231$ & & & \\
\hline $1.02 \mathrm{E}-01$ & 81.229 & Th-231 & $->$ & $\mathrm{Pa}-231$ & & & \\
\hline 7.46E-02 & 108.99 & U-235 & $->$ & Th-231 & & & \\
\hline $7.12 \mathrm{E}-02$ & 194.94 & $U-235$ & $->$ & Th-231 & & & \\
\hline $7.11 \mathrm{E}-02$ & 95.863 & Th-231 & $->$ & $\mathrm{Pa}-231$ & & & \\
\hline $6.31 \mathrm{E}-02$ & 766.6 & Pu-239 & $->$ & U-235 & & & \\
\hline $5.42 \mathrm{E}-02$ & 58.562 & Th-231 & $->$ & $\mathrm{Pa}-231$ & & & \\
\hline 4.63E-02 & 102.255 & Th-231 & $->$ & Pa-231 & & & \\
\hline $4.52 E-02$ & 14.953 & Th-231 & $->$ & $\mathrm{Pa}-231$ & & & \\
\hline 4.40E-02 & 92.279 & Th-231 & $->$ & $\mathrm{Pa}-231$ & & & \\
\hline $3.84 \mathrm{E}-02$ & 182.61 & $U-235$ & $->$ & Th-231 & & & \\
\hline $2.83 \mathrm{E}-02$ & 72.767 & Th-231 & $->$ & $\mathrm{Pa}-231$ & & & \\
\hline $2.57 \mathrm{E}-02$ & 108.166 & Th-231 & $->$ & $\mathrm{Pa}-231$ & & & \\
\hline $2.49 \mathrm{E}-02$ & 14.511 & Ac- 227 & $->$ & Th-227, & $U-235$ & $->$ & Th-231 \\
\hline $2.49 \mathrm{E}-02$ & 140.76 & U-235 & $->$ & Th-231 & & & \\
\hline $2.48 \mathrm{E}-02$ & 17.187 & Th-231 & $->$ & $\mathrm{Pa}-231$ & & & \\
\hline $1.75 \mathrm{E}-02$ & 163.114 & Th-231 & $->$ & $\mathrm{Pa}-231$ & & & \\
\hline $1.36 \mathrm{E}-02$ & 221.38 & U-235 & $->$ & Th-231 & & & \\
\hline $1.35 \mathrm{E}-02$ & 99.28 & Th-231 & $->$ & Pa-231 & & & \\
\hline $1.24 \mathrm{E}-02$ & 72.71 & U-235 & $->$ & Th-231 & & & \\
\hline $9.72 \mathrm{E}-03$ & 96.154 & $U-235$ & $->$ & Th-231 & & & \\
\hline $9.04 \mathrm{E}-03$ & 150.93 & U-235 & $->$ & Th-231 & & & \\
\hline 8.81E-03 & 135.681 & Th-231 & $->$ & $\mathrm{Pa}-231$ & & & \\
\hline $8.58 \mathrm{E}-03$ & 111.897 & Th-231 & $->$ & $\mathrm{Pa}-231$ & & & \\
\hline 7.91E-03 & 116.11 & U-235 & $->$ & Th-231 & & & \\
\hline $6.78 \mathrm{E}-03$ & 74.8 & $U-235$ & $->$ & Th-231 & & & \\
\hline $6.78 \mathrm{E}-03$ & 41.96 & U-235 & $->$ & Th-231 & & & \\
\hline $6.55 E-03$ & 42.828 & Th-231 & $->$ & $\mathrm{Pa}-231$ & & & \\
\hline $6.32 \mathrm{E}-03$ & 124.922 & Th-231 & $->$ & $\mathrm{Pa}-231$ & & & \\
\hline $6.10 \mathrm{E}-03$ & 240.85 & U-235 & $->$ & Th-231 & & & \\
\hline 5.99E-03 & 246.84 & U-235 & $->$ & Th-231 & & & \\
\hline $5.53 E-03$ & 93.07 & Th-231 & $->$ & $\mathrm{Pa}-231$ & & & \\
\hline 4.75E-03 & 198.9 & U-235 & $->$ & Th-231 & & & \\
\hline $4.52 E-03$ & 217.934 & Th-231 & $->$ & Pa-231 & & & \\
\hline $3.71 E-03$ & 183.483 & Th-231 & $->$ & $\mathrm{Pa}-231$ & & & \\
\hline $3.61 \mathrm{E}-03$ & 145.927 & Th-231 & $->$ & $\mathrm{Pa}-231$ & & & \\
\hline $3.39 \mathrm{E}-03$ & 41.13 & U-235 & $->$ & Th-231 & & & \\
\hline $3.28 \mathrm{E}-03$ & 345.921 & U-235 & $->$ & Th-231 & & & \\
\hline $3.28 \mathrm{E}-03$ & 233.5 & $U-235$ & $->$ & Th-231 & & & \\
\hline $3.05 E-03$ & 215.305 & U-235 & $->$ & Th-231 & & & \\
\hline $2.94 \mathrm{E}-03$ & 119.98 & U-235 & $->$ & Th-231 & & & \\
\hline $2.71 \mathrm{E}-03$ & 134.014 & Th-231 & $->$ & Pa-231 & & & \\
\hline $2.60 \mathrm{E}-03$ & 63.834 & Th-231 & $->$ & $\mathrm{Pa}-231$ & & & \\
\hline
\end{tabular}




\begin{tabular}{|c|c|c|c|c|}
\hline $2.34 \mathrm{E}-03$ & 116.827 & Th-231 & $->$ & $\mathrm{Pa}-231$ \\
\hline $2.26 \mathrm{E}-03$ & 51.179 & U-235 & $->$ & Th-231 \\
\hline $2.26 \mathrm{E}-03$ & 64.348 & $U-235$ & $->$ & Th-231 \\
\hline 2.07E-03 & 174.157 & Th-231 & $->$ & $\mathrm{Pa}-231$ \\
\hline $1.92 \mathrm{E}-03$ & 106.581 & Th-231 & $->$ & $\mathrm{Pa}-231$ \\
\hline $1.81 \mathrm{E}-03$ & 31.585 & U-235 & $->$ & Th-231 \\
\hline $1.58 \mathrm{E}-03$ & 291.72 & $U-235$ & $->$ & Th-231 \\
\hline $1.36 \mathrm{E}-03$ & 136.72 & U-235 & $->$ & Th-231 \\
\hline $1.13 \mathrm{E}-03$ & 172.27 & $U-235$ & $->$ & Th-231 \\
\hline $1.04 \mathrm{E}-03$ & 235.985 & Th-231 & $->$ & $\mathrm{Pa}-231$ \\
\hline $9.04 \mathrm{E}-04$ & 228.77 & U-235 & $->$ & Th-231 \\
\hline $8.02 E-04$ & 105.803 & Th-231 & $->$ & $\mathrm{Pa}-231$ \\
\hline 7.91E-04 & 289.57 & U-235 & $->$ & Th-231 \\
\hline $6.78 \mathrm{E}-04$ & 266.483 & $U-235$ & $->$ & Th-231 \\
\hline $6.78 \mathrm{E}-04$ & 281.45 & U-235 & $->$ & Th-231 \\
\hline $6.55 \mathrm{E}-04$ & 145.063 & Th-231 & $->$ & $\mathrm{Pa}-231$ \\
\hline $6.43 E-04$ & 68.5 & Th-231 & $->$ & $\mathrm{Pa}-231$ \\
\hline 5.65E-04 & 301.72 & U-235 & $->$ & Th-231 \\
\hline $5.65 E-04$ & 356.068 & U-235 & $->$ & Th-231 \\
\hline $5.65 \mathrm{E}-04$ & 282.98 & U-235 & $->$ & Th-231 \\
\hline $5.54 \mathrm{E}-04$ & 275.47 & U-235 & $->$ & Th-231 \\
\hline $4.74 \mathrm{E}-04$ & 136.705 & Th-231 & $->$ & $\mathrm{Pa}-231$ \\
\hline $4.52 E-04$ & 310.71 & U-235 & $->$ & Th-231 \\
\hline $4.40 E-04$ & 164.97 & Th-231 & $->$ & $\mathrm{Pa}-231$ \\
\hline 4.29E-04 & 387.874 & U-235 & $->$ & Th-231 \\
\hline $3.61 \mathrm{E}-04$ & 188.756 & Th-231 & $->$ & $\mathrm{Pa}-231$ \\
\hline $3.39 E-04$ & 410.269 & U-235 & $->$ & Th-231 \\
\hline $3.39 E-04$ & 343.78 & U-235 & $->$ & Th-231 \\
\hline $3.27 E-04$ & 311 & Th-231 & $->$ & $\mathrm{Pa}-231$ \\
\hline $1.49 E-04$ & 15.69 & $\mathrm{~Pa}-231$ & $->$ & Ac- 227 \\
\hline $1.35 \mathrm{E}-04$ & 169.637 & Th-231 & $->$ & $\mathrm{Pa}-231$ \\
\hline $1.31 \mathrm{E}-04$ & 12.636 & $\mathrm{~Pa}-231$ & $->$ & Ac- 227 \\
\hline $1.31 \mathrm{E}-04$ & 267.62 & Th-231 & $->$ & $\mathrm{Pa}-231$ \\
\hline $1.13 \mathrm{E}-04$ & 390.32 & U-235 & $->$ & Th-231 \\
\hline $1.13 \mathrm{E}-04$ & 448.42 & $U-235$ & $->$ & Th-231 \\
\hline $1.13 \mathrm{E}-04$ & 317.12 & U-235 & $->$ & Th-231 \\
\hline $1.13 \mathrm{E}-04$ & 115.595 & Th-231 & $->$ & $\mathrm{Pa}-231$ \\
\hline $9.48 \mathrm{E}-05$ & 242.508 & Th-231 & $->$ & $\mathrm{Pa}-231$ \\
\hline $9.04 \mathrm{E}-05$ & 455.12 & U-235 & $->$ & Th-231 \\
\hline 8.81E-05 & 249.56 & Th-231 & $->$ & $\mathrm{Pa}-231$ \\
\hline 8.02E-05 & 140.537 & Th-231 & $->$ & $\mathrm{Pa}-231$ \\
\hline 7.90E-05 & 44.06 & Th-231 & $->$ & $\mathrm{Pa}-231$ \\
\hline 7.34E-05 & 250.43 & Th-231 & $->$ & $\mathrm{Pa}-231$ \\
\hline $6.78 \mathrm{E}-05$ & 794.72 & U-235 & $->$ & Th-231 \\
\hline 5.54E-05 & 27.396 & $\mathrm{~Pa}-231$ & $->$ & Ac- 227 \\
\hline $4.52 E-05$ & 517.2 & U-235 & $->$ & Th-231 \\
\hline $4.52 E-05$ & 742.52 & U-235 & $->$ & Th-231 \\
\hline 4.40E-05 & 308.75 & Th-231 & $->$ & $\mathrm{Pa}-231$ \\
\hline $3.52 \mathrm{E}-05$ & 18.607 & $\mathrm{~Pa}-231$ & $->$ & Ac- 227 \\
\hline $3.16 \mathrm{E}-05$ & 240.25 & Th-231 & $->$ & $\mathrm{Pa}-231$ \\
\hline
\end{tabular}




$\begin{array}{lc}1.42 \mathrm{E}-05 & 300.07 \\ 1.24 \mathrm{E}-05 & 320.188 \\ 1.02 \mathrm{E}-05 & 302.671 \\ 9.48 \mathrm{E}-06 & 283.673 \\ 9.03 \mathrm{E}-06 & 317.93 \\ 7.90 \mathrm{E}-06 & 351.82 \\ 7.87 \mathrm{E}-06 & 330.066 \\ 7.15 \mathrm{E}-06 & 10.871 \\ 7.14 \mathrm{E}-06 & 83.787 \\ 6.02 \mathrm{E}-06 & 15.218 \\ 4.94 \mathrm{E}-06 & 12.325 \\ 4.65 \mathrm{E}-06 & 90.886 \\ 4.30 \mathrm{E}-06 & 81.067 \\ 3.85 \mathrm{E}-06 & 269.39 \\ 3.82 \mathrm{E}-06 & 302.638 \\ 3.61 \mathrm{E}-06 & 350.1 \\ 3.39 \mathrm{E}-06 & 274.14 \\ 3.21 \mathrm{E}-06 & 235.97 \\ 2.89 \mathrm{E}-06 & 11.713 \\ 2.84 \mathrm{E}-06 & 87.673 \\ 2.80 \mathrm{E}-06 & 271.13 \\ 2.78 \mathrm{E}-06 & 14.341 \\ 2.58 \mathrm{E}-06 & 50.14 \\ 2.52 \mathrm{E}-06 & 94.677 \\ 2.27 \mathrm{E}-06 & 16.397 \\ 2.21 \mathrm{E}-06 & 14.082 \\ 1.97 \mathrm{E}-06 & 18.998 \\ 1.92 \mathrm{E}-06 & 256.24 \\ 1.88 \mathrm{E}-06 & 401.7 \\ 1.67 \mathrm{E}-06 & 102.613 \\ 1.58 \mathrm{E}-06 & 154.18 \\ 1.38 \mathrm{E}-06 & 17.997 \\ 1.24 \mathrm{E}-06 & 46.393 \\ 1.10 \mathrm{E}-06 & 323.88 \\ 1.08 \mathrm{E}-06 & 260.29 \\ 1.08 \mathrm{E}-06 & 404.86 \\ 1.08 \mathrm{E}-06 & 831.86 \\ 1.04 \mathrm{E}-06 & 340.873 \\ 9.23 \mathrm{E}-07 & 144.18 \\ 9.18 \mathrm{E}-07 & 357.2 \\ 9.00 \mathrm{E}-07 & 88.471 \\ 8.88 \mathrm{E}-07 & 38.235 \\ 8.04 \mathrm{E}-07 & 97.907 \\ 7.87 \mathrm{E}-07 & 338.28 \\ 7.83 \mathrm{E}-07 & 329.84 \\ 6.74 \mathrm{E}-07 & 313.058 \\ 6.39 \mathrm{E}-07 & 79.72 \\ 6.02 \mathrm{E}-07 & 299.97 \\ 5.96 \mathrm{E}-07 & 255.858 \\ 5.49 \mathrm{E}-07 & 16.918\end{array}$

\begin{tabular}{|c|c|c|c|c|c|}
\hline Рa-231 & $->$ & $A c-227$ & & & \\
\hline Th-231 & $->$ & $\mathrm{Pa}-231$ & & & \\
\hline $\mathrm{Pa}-231$ & $->$ & $A c-227$ & & & \\
\hline $\mathrm{Pa}-231$ & $->$ & $A C-227$ & & & \\
\hline Th-231 & $->$ & $\mathrm{Pa}-231$ & & & \\
\hline Th-231 & $->$ & $\mathrm{Pa}-231$ & & & \\
\hline $\mathrm{Pa}-231$ & $->$ & Ac- 227 & & & \\
\hline $\mathrm{Pa}-231$ & $->$ & Ac-227 & & & \\
\hline Ra-223 & $->$ & Rn-219 & & & \\
\hline Th-227 & $->$ & Ra-223 & & & \\
\hline Fr-223 & $->$ & Ra-223, & Th-227 & $->$ & $\mathrm{Ra}-223$ \\
\hline $\mathrm{Pa}-231$ & $->$ & $A c-227$ & & & \\
\hline Ra-223 & $->$ & $\mathrm{Rn}-219$ & & & \\
\hline Ra-223 & $->$ & $\mathrm{Rn}-219$ & & & \\
\hline $\mathrm{Pa}-231$ & $->$ & Ac- 227 & & & \\
\hline $\mathrm{Bi}-211$ & $->$ & TI-207 & & & \\
\hline Th-231 & $->$ & $\mathrm{Pa}-231$ & & & \\
\hline Fr-223 & $->$ & Ra-223, & Th-227 & $->$ & $\mathrm{Ra}-223$ \\
\hline Ra-223 & $->$ & $\mathrm{Rn}-219$ & & & \\
\hline $\mathrm{Pa}-231$ & $->$ & Ac- 227 & & & \\
\hline $\mathrm{Rn}-219$ & $->$ & Po-215 & & & \\
\hline Ra-223 & $->$ & $\mathrm{Rn}-219$ & & & \\
\hline Fr-223 & $->$ & Ra-223, & Th-227 & $->$ & Ra-223 \\
\hline Ra-223 & $->$ & $\mathrm{Rn}-219$ & & & \\
\hline $\mathrm{Pa}-231$ & $->$ & $A c-227$ & & & \\
\hline $\mathrm{Pa}-231$ & $->$ & Ac- 227 & & & \\
\hline $\mathrm{Pa}-231$ & $->$ & $A c-227$ & & & \\
\hline Fr-223 & $->$ & Ra-223, & Th-227 & $->$ & Ra-223 \\
\hline$R n-219$ & $->$ & Po-215 & & & \\
\hline $\mathrm{Pa}-231$ & $->$ & $A c-227$ & & & \\
\hline $\mathrm{Ra}-223$ & $->$ & $\mathrm{Rn}-219$ & & & \\
\hline Th-227 & $->$ & Ra-223 & & & \\
\hline $\mathrm{Pa}-231$ & $->$ & Ac- 227 & & & \\
\hline $\mathrm{Ra}-223$ & $->$ & $\mathrm{Rn}-219$ & & & \\
\hline $\mathrm{Pa}-231$ & $->$ & $A c-227$ & & & \\
\hline $\mathrm{Pb}-211$ & $->$ & $\mathrm{Bi}-211$, & At-215 & $->$ & $\mathrm{Bi}-211$ \\
\hline $\mathrm{Pb}-211$ & $->$ & $\mathrm{Bi}-211$ & & & \\
\hline $\mathrm{Pa}-231$ & $->$ & Ac- 227 & & & \\
\hline $\mathrm{Ra}-223$ & $->$ & $\mathrm{Rn}-219$ & & & \\
\hline $\mathrm{Pa}-231$ & $->$ & Ac- 227 & & & \\
\hline Fr-223 & $->$ & Ra-223, & Th-227 & $->$ & $\mathrm{Ra}-223$ \\
\hline $\mathrm{Pa}-231$ & $->$ & Ac- 227 & & & \\
\hline $\mathrm{Ra}-223$ & $->$ & $\mathrm{Rn}-219$ & & & \\
\hline Ra-223 & $->$ & $\mathrm{Rn}-219$ & & & \\
\hline Fr-223 & $->$ & Ra-223, & Th-227 & $->$ & $\mathrm{Ra}-223$ \\
\hline $\mathrm{Pa}-231$ & $->$ & Ac- 227 & & & \\
\hline Fr-223 & $->$ & Ra-223, & Th-227 & $->$ & $\mathrm{Ra}-223$ \\
\hline $\mathrm{Fr}-223$ & $->$ & Ra-223, & Th-227 & $->$ & $\mathrm{Ra}-223$ \\
\hline $\mathrm{Pa}-231$ & $->$ & Ac- 227 & & & \\
\hline $\mathrm{Ra}-223$ & $->$ & $\mathrm{Rn}-219$ & & & \\
\hline
\end{tabular}




\begin{tabular}{lc}
$5.48 \mathrm{E}-07$ & 106.137 \\
$5.48 \mathrm{E}-07$ & 29.996 \\
$5.46 \mathrm{E}-07$ & 85.429 \\
$5.37 \mathrm{E}-07$ & 25.464 \\
$5.25 \mathrm{E}-07$ & 96.979 \\
$5.07 \mathrm{E}-07$ & 52.763 \\
$4.87 \mathrm{E}-07$ & 427 \\
$4.83 \mathrm{E}-07$ & 54.632 \\
$4.77 \mathrm{E}-07$ & 354.63 \\
$4.56 \mathrm{E}-07$ & 286.11 \\
$4.05 \mathrm{E}-07$ & 77.405 \\
$4.01 \mathrm{E}-07$ & 93.93 \\
$3.70 \mathrm{E}-07$ & 277.17 \\
$3.60 \mathrm{E}-07$ & 444.94 \\
$3.59 \mathrm{E}-07$ & 72.873 \\
$3.58 \mathrm{E}-07$ & 44.213 \\
$3.40 \mathrm{E}-07$ & 273.17 \\
$3.37 \mathrm{E}-07$ & 122.31 \\
$3.24 \mathrm{E}-07$ & 210.58 \\
$3.21 \mathrm{E}-07$ & 99.915 \\
$3.13 \mathrm{E}-07$ & 304.51 \\
$2.98 \mathrm{E}-07$ & 63.7 \\
$2.85 \mathrm{E}-07$ & 10.622 \\
$2.84 \mathrm{E}-07$ & 334.38 \\
$2.80 \mathrm{E}-07$ & 243.17 \\
$2.74 \mathrm{E}-07$ & 379.4 \\
$2.46 \mathrm{E}-07$ & 79.29 \\
$2.29 \mathrm{E}-07$ & 254.66 \\
$2.12 \mathrm{E}-07$ & 70.832 \\
$2.03 \mathrm{E}-07$ & 407.98 \\
$2.01 \mathrm{E}-07$ & 766.35 \\
$1.95 \mathrm{E}-07$ & 158.59 \\
$1.91 \mathrm{E}-07$ & 100.96 \\
$1.79 \mathrm{E}-07$ & 327.23 \\
$1.79 \mathrm{E}-07$ & 24.56 \\
$1.61 \mathrm{E}-07$ & 113.19 \\
$1.56 \mathrm{E}-07$ & 10.137 \\
$1.49 \mathrm{E}-07$ & 74.209 \\
$1.49 \mathrm{E}-07$ & 57.233 \\
$1.47 \mathrm{E}-07$ & 76.858 \\
$1.42 \mathrm{E}-07$ & 234.82 \\
$1.41 \mathrm{E}-07$ & 272.95 \\
$1.39 \mathrm{E}-07$ & 371.8 \\
$1.36 \mathrm{E}-07$ & 704.51 \\
$1.35 \mathrm{E}-07$ & 312.57 \\
$1.26 \mathrm{E}-07$ & 314.87 \\
$1.24 \mathrm{E}-07$ & 341.17 \\
& 13.495 \\
\hline & 10.259
\end{tabular}

\begin{tabular}{|c|c|c|c|c|c|}
\hline $\mathrm{Pa}-231$ & $\rightarrow$ & Ac-227 & & & \\
\hline $\mathrm{Pa}-231$ & $->$ & $A c-227$ & & & \\
\hline Fr-223 & $->$ & $\mathrm{Ra}-223$, & Th-227 & $->$ & $\mathrm{Ra}-223$ \\
\hline $\mathrm{Pa}-231$ & $->$ & Ac- 227 & & & \\
\hline $\mathrm{Pa}-231$ & $->$ & $A c-227$ & & & \\
\hline $\mathrm{Pa}-231$ & $->$ & $A c-227$ & & & \\
\hline $\mathrm{Pb}-211$ & $->$ & $\mathrm{Bi}-211$ & & & \\
\hline $\mathrm{Pa}-231$ & $->$ & $A c-227$ & & & \\
\hline $\mathrm{Pa}-231$ & $->$ & Ac- 227 & & & \\
\hline Fr-223 & $->$ & Ra-223, & Th-227 & $->$ & Ra-223 \\
\hline $\mathrm{Pa}-231$ & $->$ & $A c-227$ & & & \\
\hline Th-227 & $->$ & Ra-223 & & & \\
\hline $\mathrm{Pa}-231$ & $->$ & Ac- 227 & & & \\
\hline Ra-223 & $->$ & $R n-219$ & & & \\
\hline $\mathrm{Bi}-211$ & $->$ & TI-207 & & & \\
\hline $\mathrm{Pa}-231$ & $->$ & $A c-227$ & & & \\
\hline $\mathrm{Pa}-231$ & $->$ & Ac- 227 & & & \\
\hline Ra-223 & $->$ & $\mathrm{Rn}-219$ & & & \\
\hline Fr-223 & $->$ & Ra-223, & Th-227 & $->$ & $\mathrm{Ra}-223$ \\
\hline Fr-223 & $->$ & Ra-223, & Th-227 & $->$ & $\mathrm{Ra}-223$ \\
\hline Fr-223 & $->$ & Ra-223, & Th-227 & $->$ & $\mathrm{Ra}-223$ \\
\hline Pa-231 & $->$ & $A c-227$ & & & \\
\hline Fr-223 & $->$ & Ra-223, & Th-227 & $->$ & $\mathrm{Ra}-223$ \\
\hline Fr-223 & $->$ & Ra-223, & Th-227 & $->$ & $\mathrm{Ra}-223$ \\
\hline $\mathrm{Pa}-231$ & $->$ & $A c-227$ & & & \\
\hline $\mathrm{Rn}-219$ & $->$ & Po-215, & $\mathrm{Pa}-231$ & $->$ & $A c-227$ \\
\hline $\mathrm{Rn}-219$ & $->$ & Po-215 & & & \\
\hline Fr-223 & $->$ & Ra-223, & Th-227 & $->$ & $\mathrm{Ra}-223$ \\
\hline $\mathrm{Bi}-211$ & $->$ & TI-207 & & & \\
\hline $\mathrm{Pa}-231$ & $->$ & $A c-227$ & & & \\
\hline $\mathrm{Pb}-211$ & $->$ & $\mathrm{Bi}-211$ & & & \\
\hline $\mathrm{Ra}-223$ & $->$ & $\mathrm{Rn}-219$ & & & \\
\hline $\mathrm{Pa}-231$ & $->$ & $A c-227$ & & & \\
\hline $\mathrm{Pa}-231$ & $->$ & Ac- 227 & & & \\
\hline $\mathrm{Pa}-231$ & $->$ & $A c-227$ & & & \\
\hline Th-227 & $->$ & Ra-223 & & & \\
\hline Ra-223 & $->$ & $\mathrm{Rn}-219$ & & & \\
\hline $\mathrm{Pa}-231$ & $->$ & Ac- 227 & & & \\
\hline $\mathrm{Pa}-231$ & $->$ & $A c-227$ & & & \\
\hline $\mathrm{Rn}-219$ & $->$ & Po-215 & & & \\
\hline Fr-223 & $->$ & Ra-223, & Th-227 & $->$ & $\mathrm{Ra}-223$ \\
\hline Fr-223 & $->$ & Ra-223, & Th-227 & $->$ & Ra-223 \\
\hline Ra-223 & $->$ & $\mathrm{Rn}-219$ & & & \\
\hline $\mathrm{Pb}-211$ & $->$ & $\mathrm{Bi}-211$ & & & \\
\hline Fr-223 & $->$ & Ra-223, & Th-227 & $->$ & $\mathrm{Ra}-223$ \\
\hline Th-227 & $->$ & $\mathrm{Ra}-223$ & & & \\
\hline $\mathrm{Pa}-231$ & $\rightarrow$ & AC- 227 & & & \\
\hline $\mathrm{Rn}-219$ & $->$ & Po-215 & & & \\
\hline $\mathrm{Bi}-211$ & $->$ & TI-207 & & & \\
\hline $\mathrm{Bi}-211$ & $->$ & TI-207 & & & \\
\hline
\end{tabular}




\begin{tabular}{|c|c|c|c|c|c|c|c|}
\hline $1.23 \mathrm{E}-07$ & 296.54 & Th-227 & $->$ & Ra-223 & & & \\
\hline $1.22 \mathrm{E}-07$ & 77.107 & $\mathrm{~Pb}-211$ & $->$ & $\mathrm{Bi}-211$ & & & \\
\hline $1.16 \mathrm{E}-07$ & 11.119 & $\mathrm{Rn}-219$ & $->$ & Po-215 & & & \\
\hline $1.16 \mathrm{E}-07$ & 13.082 & $\mathrm{~Pb}-211$ & $->$ & $\mathrm{Bi}-211$ & & & \\
\hline $1.13 \mathrm{E}-07$ & 12.313 & $\mathrm{Bi}-211$ & $->$ & TI-207 & & & \\
\hline 1.09E-07 & 342.44 & Fr-223 & $->$ & Ra-223, & Th-227 & $->$ & Ra-223 \\
\hline $1.06 \mathrm{E}-07$ & 250.12 & Fr-223 & $->$ & Ra-223, & Th-227 & $->$ & Ra-223 \\
\hline $1.05 \mathrm{E}-07$ & 103.341 & Fr-223 & $->$ & Ra-223, & Th-227 & $->$ & Ra-223 \\
\hline $1.05 \mathrm{E}-07$ & 10.828 & $\mathrm{~Pb}-211$ & $->$ & $\mathrm{Bi}-211$ & & & \\
\hline $1.04 \mathrm{E}-07$ & 13.662 & Fr-223 & $->$ & Ra-223, & Th-227 & $->$ & Ra-223 \\
\hline 9.54E-08 & 35.884 & Pa-231 & $->$ & $A c-227$ & & & \\
\hline 8.94E-08 & 57.2 & $\mathrm{~Pa}-231$ & $->$ & Ac- 227 & & & \\
\hline 8.78E-08 & 89.639 & $\mathrm{Rn}-219$ & $->$ & Po-215 & & & \\
\hline 7.96E-08 & 15.213 & Fr-223 & $->$ & Ra-223 & & & \\
\hline $7.25 \mathrm{E}-08$ & 74.814 & $\mathrm{~Pb}-211$ & $->$ & $\mathrm{Bi}-211$ & & & \\
\hline $7.15 \mathrm{E}-08$ & 40.006 & $\mathrm{~Pa}-231$ & $->$ & Ac- 227 & & & \\
\hline $6.88 \mathrm{E}-08$ & 62.36 & Th-227 & $->$ & $\mathrm{Ra}-223$ & & & \\
\hline $6.82 \mathrm{E}-08$ & 897.23 & Tl-207 & $->$ & Pb-207, & Po-211 & $->$ & Pb-207 \\
\hline $6.60 \mathrm{E}-08$ & 204.2 & Fr-223 & $->$ & Ra-223, & Th-227 & $->$ & Ra-223 \\
\hline $6.60 \mathrm{E}-08$ & 43.73 & Fr-223 & $->$ & Ra-223, & Th-227 & $->$ & $\mathrm{Ra}-223$ \\
\hline 6.60E-08 & 206.04 & Fr-223 & $->$ & Ra-223, & Th-227 & $->$ & Ra-223 \\
\hline $6.56 \mathrm{E}-08$ & 56.9 & $\mathrm{~Pa}-231$ & $->$ & $A c-227$ & & & \\
\hline 6.07E-08 & 49.85 & Fr-223 & $->$ & Ra-223, & Th-227 & $->$ & $\mathrm{Ra}-223$ \\
\hline $6.07 \mathrm{E}-08$ & 20.27 & Fr-223 & $->$ & Ra-223, & Th-227 & $->$ & $\mathrm{Ra}-223$ \\
\hline $5.73 \mathrm{E}-08$ & 300.34 & Th-227 & $->$ & $\mathrm{Ra}-223$ & & & \\
\hline $5.66 \mathrm{E}-08$ & 342.9 & $\mathrm{Ra}-223$ & $->$ & $\mathrm{Rn}-219$ & & & \\
\hline 5.61E-08 & 328.49 & $\mathrm{Ra}-223$ & $->$ & $\mathrm{Rn}-219$ & & & \\
\hline 5.54E-08 & 398.4 & $\mathrm{~Pa}-231$ & $->$ & Ac- 227 & & & \\
\hline $5.37 \mathrm{E}-08$ & 359.51 & $\mathrm{~Pa}-231$ & $->$ & Ac- 227 & & & \\
\hline 5.37E-08 & 31.04 & $\mathrm{~Pa}-231$ & $->$ & Ac- 227 & & & \\
\hline $4.87 \mathrm{E}-08$ & 117.17 & Th-227 & $->$ & Ra-223 & & & \\
\hline 4.77E-08 & 242 & $\mathrm{~Pa}-231$ & $->$ & $A c-227$ & & & \\
\hline $4.59 E-08$ & 281.31 & Th-227 & $->$ & Ra-223 & & & \\
\hline 4.49E-08 & 16.074 & Ac- 227 & $->$ & Th-227 & & & \\
\hline $4.41 \mathrm{E}-08$ & 364.03 & $\mathrm{~Pa}-231$ & $->$ & Ac- 227 & & & \\
\hline $4.36 \mathrm{E}-08$ & 288.15 & $\mathrm{Ra}-223$ & $->$ & $\mathrm{Rn}-219$ & & & \\
\hline 4.33E-08 & 179.69 & $\mathrm{Ra}-223$ & $->$ & $\mathrm{Rn}-219$ & & & \\
\hline $4.32 \mathrm{E}-08$ & 204.95 & Fr-223 & $->$ & Ra-223, & Th-227 & $->$ & $\mathrm{Ra}-223$ \\
\hline 4.30E-08 & 87.19 & $\mathrm{~Pb}-211$ & $->$ & $\mathrm{Bi}-211$ & & & \\
\hline $4.30 \mathrm{E}-08$ & 113.12 & Th-227 & $->$ & Ra-223 & & & \\
\hline 4.17E-08 & 31.58 & $\mathrm{~Pa}-231$ & $->$ & Ac- 227 & & & \\
\hline 4.17E-08 & 43.09 & $\mathrm{~Pa}-231$ & $->$ & Ac- 227 & & & \\
\hline 4.17E-08 & 60.526 & $\mathrm{~Pa}-231$ & $->$ & Ac- 227 & & & \\
\hline $4.17 \mathrm{E}-08$ & 70.54 & $\mathrm{~Pa}-231$ & $->$ & Ac- 227 & & & \\
\hline 4.17E-08 & 245.438 & $\mathrm{~Pa}-231$ & $->$ & Ac- 227 & & & \\
\hline $4.16 \mathrm{E}-08$ & 1109.37 & $\mathrm{~Pb}-211$ & $->$ & $\mathrm{Bi}-211$ & & & \\
\hline $4.11 \mathrm{E}-08$ & 245.77 & $\mathrm{~Pa}-231$ & $->$ & Ac- 227 & & & \\
\hline $4.11 \mathrm{E}-08$ & 12.855 & $\mathrm{Ra}-223$ & $->$ & $\mathrm{Rn}-219$ & & & \\
\hline $4.05 E-08$ & 391.85 & Pa-231 & $->$ & Ac- 227 & & & \\
\hline $3.73 \mathrm{E}-08$ & 141.44 & Th-227 & $->$ & Ra-223 & & & \\
\hline
\end{tabular}




\begin{tabular}{|c|c|c|c|c|c|c|c|}
\hline $3.73 E-08$ & 250.33 & Th-227 & $\rightarrow$ & Ra-223 & & & \\
\hline $3.58 \mathrm{E}-08$ & 42.52 & $\mathrm{~Pa}-231$ & $->$ & Ac- 227 & & & \\
\hline $3.58 \mathrm{E}-08$ & 56.8 & $\mathrm{~Pa}-231$ & $->$ & Ac- 227 & & & \\
\hline $3.57 E-08$ & 130.57 & $\mathrm{Rn}-219$ & $->$ & Po-215 & & & \\
\hline $3.56 \mathrm{E}-08$ & 85.185 & $\mathrm{Bi}-211$ & $->$ & TI-207 & & & \\
\hline $3.34 \mathrm{E}-08$ & 198.96 & Pa-231 & $->$ & $A c-227$ & & & \\
\hline $3.15 \mathrm{E}-08$ & 350.48 & Th-227 & $->$ & Ra-223 & & & \\
\hline $3.15 \mathrm{E}-08$ & 252.46 & Th-227 & $->$ & Ra-223 & & & \\
\hline $2.98 \mathrm{E}-08$ & 124.69 & $\mathrm{~Pa}-231$ & $->$ & Ac- 227 & & & \\
\hline 2.89E-08 & 100.31 & Fr-223 & $->$ & Ra-223, & Th-227 & $->$ & $\mathrm{Ra}-223$ \\
\hline $2.88 \mathrm{E}-08$ & 29.869 & Fr-223 & $->$ & Ra-223, & Th-227 & $->$ & $\mathrm{Ra}-223$ \\
\hline $2.87 E-08$ & 262.72 & Fr-223 & $->$ & Ra-223, & Th-227 & $->$ & Ra-223 \\
\hline $2.80 \mathrm{E}-08$ & 333.87 & $\mathrm{Ra}-223$ & $->$ & $\mathrm{Rn}-219$ & & & \\
\hline $2.66 \mathrm{E}-08$ & 92.673 & $\mathrm{Rn}-219$ & $->$ & Po-215 & & & \\
\hline $2.58 \mathrm{E}-08$ & 15.856 & $\mathrm{Rn}-219$ & $->$ & Po-215 & & & \\
\hline $2.55 \mathrm{E}-08$ & 598.69 & $\mathrm{Ra}-223$ & $->$ & $\mathrm{Rn}-219$ & & & \\
\hline $2.55 E-08$ & 15.397 & $\mathrm{~Pb}-211$ & $->$ & $\mathrm{Bi}-211$ & & & \\
\hline $2.44 \mathrm{E}-08$ & 438.24 & $\mathrm{~Pa}-231$ & $->$ & Ac- 227 & & & \\
\hline $2.44 \mathrm{E}-08$ & 384.97 & $\mathrm{~Pa}-231$ & $->$ & Ac- 227 & & & \\
\hline $2.38 \mathrm{E}-08$ & 72.57 & $\mathrm{~Pa}-231$ & $->$ & Ac- 227 & & & \\
\hline $2.38 \mathrm{E}-08$ & 144.5 & $\mathrm{~Pa}-231$ & $->$ & Ac- 227 & & & \\
\hline $2.29 E-08$ & 61.435 & Th-227 & $->$ & Ra-223 & & & \\
\hline $2.29 E-08$ & 31.566 & Th-227 & $->$ & Ra-223 & & & \\
\hline $2.18 \mathrm{E}-08$ & 65.516 & $\mathrm{~Pb}-211$ & $->$ & $\mathrm{Bi}-211$ & & & \\
\hline $2.06 \mathrm{E}-08$ & 14.407 & $\mathrm{Bi}-211$ & $->$ & TI-207 & & & \\
\hline $2.01 \mathrm{E}-08$ & 527.3 & $\mathrm{Ra}-223$ & $->$ & $\mathrm{Rn}-219$ & & & \\
\hline $2.01 \mathrm{E}-08$ & 279.7 & Fr-223 & $->$ & Ra-223, & Th-227 & $->$ & $\mathrm{Ra}-223$ \\
\hline $2.01 \mathrm{E}-08$ & 212.56 & Th-227 & $->$ & $\mathrm{Ra}-223$ & & & \\
\hline 1.97E-08 & 435.38 & $\mathrm{~Pa}-231$ & $->$ & $A c-227$ & & & \\
\hline 1.97E-08 & 277.43 & $\mathrm{~Pa}-231$ & $->$ & Ac- 227 & & & \\
\hline $1.84 \mathrm{E}-08$ & 293.8 & $\mathrm{Rn}-219$ & $->$ & Po-215 & & & \\
\hline $1.80 \mathrm{E}-08$ & 18.016 & Fr-223 & $->$ & $\mathrm{Ra}-223$ & & & \\
\hline $1.78 \mathrm{E}-08$ & 609.08 & Ra-223 & $->$ & $R n-219$ & & & \\
\hline $1.72 \mathrm{E}-08$ & 292.3 & Fr-223 & $->$ & Ra-223, & Th-227 & $->$ & Ra-223 \\
\hline $1.72 \mathrm{E}-08$ & 96.06 & Th-227 & $->$ & $\mathrm{Ra}-223$ & & & \\
\hline $1.72 \mathrm{E}-08$ & 218.77 & Th-227 & $->$ & Ra-223 & & & \\
\hline $1.61 \mathrm{E}-08$ & 83.81 & $\mathrm{~Pb}-211$ & $->$ & $\mathrm{Bi}-211$ & & & \\
\hline $1.58 \mathrm{E}-08$ & 285.48 & Th-227 & $->$ & Ra-223 & & & \\
\hline $1.55 \mathrm{E}-08$ & 226.77 & $\mathrm{~Pa}-231$ & $->$ & $A c-227$ & & & \\
\hline $1.55 \mathrm{E}-08$ & 395.8 & $\mathrm{~Pa}-231$ & $->$ & $A c-227$ & & & \\
\hline $1.43 \mathrm{E}-08$ & 284.24 & Fr-223 & $->$ & Ra-223, & Th-227 & $->$ & $\mathrm{Ra}-223$ \\
\hline $1.43 \mathrm{E}-08$ & 43.53 & Th-227 & $->$ & $\mathrm{Ra}-223$ & & & \\
\hline $1.38 \mathrm{E}-08$ & 383.47 & Th-227 & $->$ & $\mathrm{Ra}-223$ & & & \\
\hline $1.37 \mathrm{E}-08$ & 258.43 & $\mathrm{~Pa}-231$ & $->$ & Ac- 227 & & & \\
\hline $1.36 \mathrm{E}-08$ & 110.8 & Ra-223 & $->$ & $R n-219$ & & & \\
\hline 1.33E-08 & 177.35 & $\mathrm{Ra}-223$ & $->$ & $R n-219$ & & & \\
\hline 1.27E-08 & 81 & $\mathrm{~Pb}-211$ & $->$ & $\mathrm{Bi}-211$ & & & \\
\hline $1.27 \mathrm{E}-08$ & 90.128 & $\mathrm{~Pb}-211$ & $->$ & $\mathrm{Bi}-211$ & & & \\
\hline $1.22 \mathrm{E}-08$ & 361.8 & $\mathrm{Ra}-223$ & $->$ & $\mathrm{Rn}-219$ & & & \\
\hline $1.20 \mathrm{E}-08$ & 19.308 & Ac- 227 & $->$ & Th-227 & & & \\
\hline
\end{tabular}




$\begin{array}{lc}1.19 E-08 & 318.1 \\ 1.19 E-08 & 71.92 \\ 1.15 E-08 & 104.9 \\ 1.15 E-08 & 218.94 \\ 1.15 E-08 & 41.88 \\ 1.15 E-08 & 68.75 \\ 1.13 E-08 & 515.7 \\ 1.13 E-08 & 438.8 \\ 1.13 E-08 & 410.84 \\ 1.13 E-08 & 184.68 \\ 1.05 E-08 & 15.2 \\ 1.05 E-08 & 249.49 \\ 9.91 E-09 & 251.12 \\ 9.63 E-09 & 343.02 \\ 9.57 E-09 & 134.5 \\ 9.34 E-09 & 432.34 \\ 9.34 E-09 & 220.38 \\ 9.18 E-09 & 270.76 \\ 9.18 E-09 & 140.33 \\ 9.14 E-09 & 319.18 \\ 9.06 E-09 & 254.98 \\ 8.78 E-09 & 251.58 \\ 8.78 E-09 & 313.85 \\ 8.60 E-09 & 314.78 \\ 8.50 E-09 & 221.9 \\ 8.35 E-09 & 39.61 \\ 8.35 E-09 & 310.19 \\ 8.35 E-09 & 50.97 \\ 8.03 E-09 & 64.37 \\ 8.03 E-09 & 72.8 \\ 7.75 E-09 & 516.45 \\ 7.36 E-09 & 481.1 \\ 6.51 E-09 & 609.62 \\ 6.38 E-09 & 388.98 \\ 6.23 E-09 & 106.66 \\ 6.23 E-09 & 951 \\ 6.10 E-09 & 8.953 \\ 5.97 E-09 & 9.658 \\ 5.73 E-09 & 200.48 \\ 5.73 E-09 & 201.64 \\ 5.73 E-09 & 40.16 \\ 5.66 E-09 & 369.48 \\ 5.45 E-09 & 73.66 \\ 5.38 E-09 & 430.53 \\ 5.38 E-09 & 9.419 \\ 5.16 E-09 & 175.23 \\ 5.10 E-09 & 94.89 \\ 5.07 E-09 & 1014.48 \\ 4.92 E-09 & 173.38 \\ 4.87 E-09 & 212.69\end{array}$

\begin{tabular}{|c|c|c|c|c|c|}
\hline $\mathrm{Pa}-231$ & $->$ & Ac- 227 & & & \\
\hline $\mathrm{Pa}-231$ & $->$ & $A c-227$ & & & \\
\hline Th-227 & $->$ & Ra-223 & & & \\
\hline Th-227 & $->$ & Ra-223 & & & \\
\hline Th-227 & $->$ & Ra-223 & & & \\
\hline Th-227 & $->$ & Ra-223 & & & \\
\hline $\mathrm{Rn}-219$ & $->$ & Po-215 & & & \\
\hline Po-215 & $->$ & $\mathrm{Pb}-211$ & & & \\
\hline $\mathrm{Pa}-231$ & $->$ & $A c-227$ & & & \\
\hline Fr-223 & $->$ & Ra-223, & Th-227 & $->$ & $\mathrm{Ra}-223$ \\
\hline Ac-227 & $->$ & Th-227 & & & \\
\hline Ra-223 & $->$ & $R n-219$ & & & \\
\hline Ra-223 & $->$ & $\mathrm{Rn}-219$ & & & \\
\hline $\mathrm{Pb}-211$ & $->$ & $\mathrm{Bi}-211$ & & & \\
\hline Fr-223 & $->$ & Ra-223, & Th-227 & $->$ & $\mathrm{Ra}-223$ \\
\hline Ra-223 & $->$ & $\mathrm{Rn}-219$ & & & \\
\hline Ra-223 & $->$ & $\mathrm{Rn}-219$ & & & \\
\hline Th-227 & $->$ & $\mathrm{Ra}-223$ & & & \\
\hline Th-227 & $->$ & $\mathrm{Ra}-223$ & & & \\
\hline Fr-223 & $->$ & Ra-223, & Th-227 & $->$ & $\mathrm{Ra}-223$ \\
\hline Ra-223 & $->$ & $R n-219$ & & & \\
\hline Ra-223 & $->$ & $\mathrm{Rn}-219$ & & & \\
\hline $\mathrm{Pb}-211$ & $->$ & $\mathrm{Bi}-211$ & & & \\
\hline Th-227 & $->$ & Ra-223 & & & \\
\hline $\mathrm{Rn}-219$ & $->$ & Po-215 & & & \\
\hline $\mathrm{Pa}-231$ & $->$ & Ac- 227 & & & \\
\hline Pa-231 & $->$ & Ac- 227 & & & \\
\hline $\mathrm{Pa}-231$ & $->$ & Ac- 227 & & & \\
\hline Th-227 & $->$ & Ra-223 & & & \\
\hline Th-227 & $->$ & Ra-223 & & & \\
\hline $\mathrm{Pa}-231$ & $->$ & $A c-227$ & & & \\
\hline $\mathrm{Pb}-211$ & $->$ & $\mathrm{Bi}-211$ & & & \\
\hline $\mathrm{Pb}-211$ & $->$ & $\mathrm{Bi}-211$ & & & \\
\hline $\mathrm{Pa}-231$ & $->$ & Ac- 227 & & & \\
\hline Ra-223 & $->$ & $\mathrm{Rn}-219$ & & & \\
\hline $\mathrm{Pb}-211$ & $->$ & $\mathrm{Bi}-211$ & & & \\
\hline $\mathrm{Bi}-211$ & $->$ & TI-207 & & & \\
\hline $\mathrm{Rn}-219$ & $->$ & Po-215 & & & \\
\hline Th-227 & $->$ & Ra-223 & & & \\
\hline Th-227 & $->$ & Ra-223 & & & \\
\hline Th-227 & $->$ & Ra-223 & & & \\
\hline Ra-223 & $->$ & $\mathrm{Rn}-219$ & & & \\
\hline Th-227 & $->$ & $\mathrm{Ra}-223$ & & & \\
\hline Ra-223 & $->$ & $\mathrm{Rn}-219$ & & & \\
\hline $\mathrm{Pb}-211$ & $->$ & $\mathrm{Bi}-211$ & & & \\
\hline Th-227 & $->$ & Ra-223 & & & \\
\hline $\mathrm{Pb}-211$ & $->$ & $\mathrm{Bi}-211$ & & & \\
\hline $\mathrm{Pb}-211$ & $->$ & $\mathrm{Bi}-211$ & & & \\
\hline Fr-223 & $->$ & Ra-223, & Th-227 & $->$ & Ra-223 \\
\hline Th-227 & $->$ & $\mathrm{Ra}-223$ & & & \\
\hline
\end{tabular}




\begin{tabular}{|c|c|c|c|c|c|c|c|}
\hline 4.81E-09 & 175.55 & $\mathrm{Ra}-223$ & $->$ & $\mathrm{Rn}-219$ & & & \\
\hline 4.81E-09 & 103.85 & $\mathrm{Ra}-223$ & $->$ & $\mathrm{Rn}-219$ & & & \\
\hline 4.81E-09 & 88.2 & $\mathrm{~Pb}-211$ & $->$ & $\mathrm{Bi}-211$ & & & \\
\hline 4.59E-09 & 50.82 & Th-227 & $->$ & Ra-223 & & & \\
\hline $4.33 \mathrm{E}-09$ & 1080.19 & $\mathrm{~Pb}-211$ & $->$ & $\mathrm{Bi}-211$ & & & \\
\hline 4.30E-09 & 224.68 & Fr-223 & $->$ & Ra-223, & Th-227 & $->$ & Ra-223 \\
\hline 4.30E-09 & 308.49 & Th-227 & $->$ & Ra-223 & & & \\
\hline 4.25E-09 & 504.33 & $\mathrm{~Pb}-211$ & $->$ & $\mathrm{Bi}-211$ & & & \\
\hline 4.20E-09 & 289.6 & Fr-223 & $->$ & Ra-223, & Th-227 & $->$ & Ra-223 \\
\hline $4.01 \mathrm{E}-09$ & 33.52 & Th-227 & $->$ & $\mathrm{Ra}-223$ & & & \\
\hline 4.01E-09 & 168.19 & Th-227 & $->$ & Ra-223 & & & \\
\hline 3.96E-09 & 388.3 & Ra-223 & $->$ & $\mathrm{Rn}-219$ & & & \\
\hline 3.96E-09 & 492 & $\mathrm{~Pb}-211$ & $->$ & $\mathrm{Bi}-211$ & & & \\
\hline 3.73E-09 & 99.56 & Fr-223 & $->$ & Ra-223, & Th-227 & $->$ & Ra-223 \\
\hline 3.73E-09 & 44.37 & Th-227 & $->$ & $\mathrm{Ra}-223$ & & & \\
\hline $3.68 \mathrm{E}-09$ & 478 & $\mathrm{~Pb}-211$ & $->$ & $\mathrm{Bi}-211$ & & & \\
\hline $3.62 E-09$ & 1196.61 & $\mathrm{~Pb}-211$ & $->$ & $\mathrm{Bi}-211$ & & & \\
\hline $3.58 E-09$ & 501.33 & $\mathrm{~Pa}-231$ & $->$ & Ac- 227 & & & \\
\hline $3.44 \mathrm{E}-09$ & 197.61 & Th-227 & $->$ & $\mathrm{Ra}-223$ & & & \\
\hline $3.44 \mathrm{E}-09$ & 402.42 & Th-227 & $->$ & Ra-223 & & & \\
\hline 3.44E-09 & 94.9 & Th-227 & $->$ & Ra-223 & & & \\
\hline $3.44 \mathrm{E}-09$ & 117.3 & Th-227 & $->$ & Ra-223 & & & \\
\hline 3.34E-09 & 535.45 & $\mathrm{~Pa}-231$ & $->$ & Ac- 227 & & & \\
\hline $3.26 \mathrm{E}-09$ & 97.3 & $\mathrm{~Pb}-211$ & $->$ & $\mathrm{Bi}-211$ & & & \\
\hline $3.15 \mathrm{E}-09$ & 246.07 & Th-227 & $->$ & Ra-223 & & & \\
\hline $3.11 \mathrm{E}-09$ & 500.4 & $\mathrm{~Pb}-211$ & $->$ & $\mathrm{Bi}-211$ & & & \\
\hline $3.11 \mathrm{E}-09$ & 94.3 & $\mathrm{~Pb}-211$ & $->$ & $\mathrm{Bi}-211$ & & & \\
\hline 2.98E-09 & 546.9 & Pa-231 & $->$ & Ac- 227 & & & \\
\hline $2.98 \mathrm{E}-09$ & 387.267 & $\mathrm{~Pa}-231$ & $->$ & Ac- 227 & & & \\
\hline 2.98E-09 & 572.3 & $\mathrm{~Pa}-231$ & $->$ & $A c-227$ & & & \\
\hline 2.87E-09 & 48.27 & $\mathrm{Fr}-223$ & $->$ & Ra-223, & Th-227 & $->$ & $\mathrm{Ra}-223$ \\
\hline 2.87E-09 & 150.02 & Th-227 & $->$ & $\mathrm{Ra}-223$ & & & \\
\hline 2.87E-09 & 352.57 & Th-227 & $->$ & Ra-223 & & & \\
\hline 2.87E-09 & 267.82 & Th-227 & $->$ & Ra-223 & & & \\
\hline 2.83E-09 & 487.59 & Ra-223 & $->$ & $\mathrm{Rn}-219$ & & & \\
\hline $2.58 \mathrm{E}-09$ & 59.5 & Th-227 & $->$ & Ra-223 & & & \\
\hline $2.58 \mathrm{E}-09$ & 62.54 & Th-227 & $->$ & $\mathrm{Ra}-223$ & & & \\
\hline $2.58 \mathrm{E}-09$ & 69.65 & Th-227 & $->$ & Ra-223 & & & \\
\hline $2.58 \mathrm{E}-09$ & 75.1 & Th-227 & $->$ & $\mathrm{Ra}-223$ & & & \\
\hline $2.58 \mathrm{E}-09$ & 398.6 & Th-227 & $->$ & $\mathrm{Ra}-223$ & & & \\
\hline $2.58 \mathrm{E}-09$ & 392.24 & Th-227 & $->$ & $\mathrm{Ra}-223$ & & & \\
\hline $2.58 \mathrm{E}-09$ & 289.5 & Th-227 & $->$ & Ra-223 & & & \\
\hline 2.55E-09 & 245.19 & $\mathrm{Ra}-223$ & $->$ & $\mathrm{Rn}-219$ & & & \\
\hline $2.55 \mathrm{E}-09$ & 114.5 & $\mathrm{Ra}-223$ & $->$ & $\mathrm{Rn}-219$ & & & \\
\hline $2.52 E-09$ & 1270.67 & $\mathrm{~Pb}-211$ & $->$ & $\mathrm{Bi}-211$ & & & \\
\hline 2.29E-09 & 54.18 & Fr-223 & $->$ & Ra-223, & Th-227 & $->$ & $\mathrm{Ra}-223$ \\
\hline 2.29E-09 & 112.54 & Th-227 & $->$ & $\mathrm{Ra}-223$ & & & \\
\hline 2.29E-09 & 123.55 & Th-227 & $->$ & $\mathrm{Ra}-223$ & & & \\
\hline 2.29E-09 & 270.34 & Th-227 & $->$ & Ra-223 & & & \\
\hline 2.27E-09 & 368.78 & Ra-223 & $->$ & $R n-219$ & & & \\
\hline
\end{tabular}




$\begin{array}{lc}2.27 \mathrm{E}-09 & 623.38 \\ 2.15 \mathrm{E}-09 & 346.39 \\ 2.12 \mathrm{E}-09 & 12.085 \\ 2.11 \mathrm{E}-09 & 775.82 \\ 2.01 \mathrm{E}-09 & 370.85 \\ 2.01 \mathrm{E}-09 & 56.63 \\ 2.01 \mathrm{E}-09 & 107.92 \\ 2.01 \mathrm{E}-09 & 66.3 \\ 2.01 \mathrm{E}-09 & 281 \\ 2.01 \mathrm{E}-09 & 44.08 \\ 2.01 \mathrm{E}-09 & 249.36 \\ 2.01 \mathrm{E}-09 & 162.18 \\ 1.98 \mathrm{E}-09 & 376.9 \\ 1.83 \mathrm{E}-09 & 29.58 \\ 1.82 \mathrm{E}-09 & 369.32 \\ 1.81 \mathrm{E}-09 & 675.33 \\ 1.81 \mathrm{E}-09 & 430.26 \\ 1.81 \mathrm{E}-09 & 865.81 \\ 1.72 \mathrm{E}-09 & 318.58 \\ 1.72 \mathrm{E}-09 & 382.15 \\ 1.72 \mathrm{E}-09 & 325.08 \\ 1.72 \mathrm{E}-09 & 348.4 \\ 1.72 \mathrm{E}-09 & 376.26 \\ 1.72 \mathrm{E}-09 & 202.4 \\ 1.72 \mathrm{E}-09 & 66.1 \\ 1.72 \mathrm{E}-09 & 109.1 \\ 1.70 \mathrm{E}-09 & 540.1 \\ 1.70 \mathrm{E}-09 & 676.4 \\ 1.70 \mathrm{E}-09 & 260.4 \\ 1.70 \mathrm{E}-09 & 11.712 \\ 1.63 \mathrm{E}-09 & 68.7 \\ 1.61 \mathrm{E}-09 & 10.994 \\ 1.44 \mathrm{E}-09 & 1103.4 \\ 1.43 \mathrm{E}-09 & 110.58 \\ 1.43 \mathrm{E}-09 & 56.03 \\ 1.43 \mathrm{E}-09 & 362.55 \\ 1.43 \mathrm{E}-09 & 432.29 \\ 1.43 \mathrm{E}-09 & 124.48 \\ 1.43 \mathrm{E}-09 & 326.12 \\ 1.43 \mathrm{E}-09 & 220.2 \\ 1.43 \mathrm{E}-09 & 228.16 \\ 1.42 \mathrm{E}-09 & 165.5 \\ 1.42 \mathrm{E}-09 & 131.1 \\ 1.42 \mathrm{E}-09 & 255.74 \\ 1.14 \mathrm{E}-09 & 339.45 \\ 1.13 \mathrm{E}-09 & 608.5 \\ 1.13 \mathrm{E}-09 & 176.8 \\ 1.13 \mathrm{E}-09 & 383.12 \\ 1.05 \mathrm{E}-09 & 24.5 \\ 1.01 \mathrm{E}-09 & 23.55\end{array}$

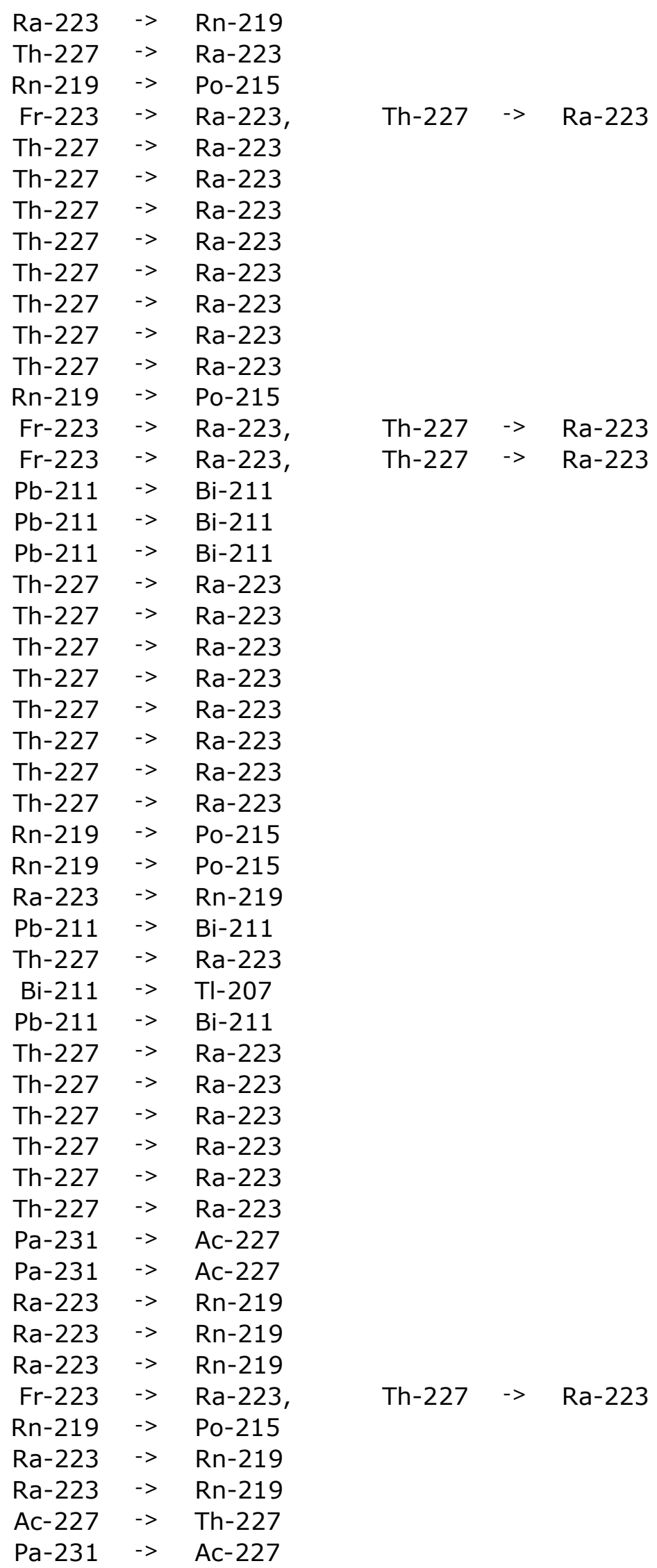




\begin{tabular}{|c|c|c|c|c|c|c|c|}
\hline $9.91 \mathrm{E}-10$ & 711.2 & $\mathrm{Ra}-223$ & $\rightarrow$ & $\mathrm{Rn}-219$ & & & \\
\hline $9.75 \mathrm{E}-10$ & 89.85 & Th-227 & $->$ & Ra-223 & & & \\
\hline $9.34 \mathrm{E}-10$ & 115.3 & $\mathrm{Rn}-219$ & $->$ & Po-215 & & & \\
\hline 8.94E-10 & 471.33 & $\mathrm{~Pa}-231$ & $->$ & $A c-227$ & & & \\
\hline $8.60 \mathrm{E}-10$ & 51.29 & Fr-223 & $->$ & Ra-223, & Th-227 & $->$ & Ra-223 \\
\hline $8.60 \mathrm{E}-10$ & 169.98 & Th-227 & $->$ & $\mathrm{Ra}-223$ & & & \\
\hline $8.60 \mathrm{E}-10$ & 124.6 & Th-227 & $->$ & Ra-223 & & & \\
\hline $8.50 \mathrm{E}-10$ & 199.43 & Ra-223 & $->$ & $\mathrm{Rn}-219$ & & & \\
\hline $8.50 \mathrm{E}-10$ & 391 & Ra-223 & $->$ & $R n-219$ & & & \\
\hline $8.32 \mathrm{E}-10$ & 812.15 & Fr-223 & $->$ & Ra-223, & Th-227 & $->$ & Ra-223 \\
\hline $7.59 \mathrm{E}-10$ & 822.99 & Fr-223 & $->$ & Ra-223, & Th-227 & $->$ & $\mathrm{Ra}-223$ \\
\hline $7.36 \mathrm{E}-10$ & 1090.5 & $\mathrm{~Pb}-211$ & $\rightarrow$ & $\mathrm{Bi}-211$ & & & \\
\hline 7.17E-10 & 266.3 & Th-227 & $->$ & Ra-223 & & & \\
\hline $7.06 \mathrm{E}-10$ & 74.969 & TI-207 & $->$ & $\mathrm{Pb}-207$ & & & \\
\hline $6.57 \mathrm{E}-10$ & 908.03 & Fr-223 & $->$ & Ra-223, & Th-227 & $->$ & Ra-223 \\
\hline $5.73 \mathrm{E}-10$ & 99.64 & Th-227 & $->$ & Ra-223 & & & \\
\hline $5.73 \mathrm{E}-10$ & 62.05 & Th-227 & $->$ & $\mathrm{Ra}-223$ & & & \\
\hline $5.73 E-10$ & 179.28 & Th-227 & $->$ & $\mathrm{Ra}-223$ & & & \\
\hline $5.15 \mathrm{E}-10$ & 803.58 & Fr-223 & $->$ & Ra-223, & Th-227 & $->$ & $\mathrm{Ra}-223$ \\
\hline $4.98 \mathrm{E}-10$ & 307.89 & Fr-223 & $->$ & Ra-223, & Th-227 & $->$ & Ra-223 \\
\hline $4.87 \mathrm{E}-10$ & 415.09 & Th-227 & $->$ & $\mathrm{Ra}-223$ & & & \\
\hline $4.59 \mathrm{E}-10$ & 478.22 & $\mathrm{~Pa}-231$ & $->$ & $A c-227$ & & & \\
\hline 4.35E-10 & 723.86 & Fr-223 & $->$ & Ra-223, & Th-227 & $->$ & $\mathrm{Ra}-223$ \\
\hline $4.30 \mathrm{E}-10$ & 275.2 & Th-227 & $->$ & $\mathrm{Ra}-223$ & & & \\
\hline $4.26 \mathrm{E}-10$ & 328.08 & TI-207 & $->$ & $\mathrm{Pb}-207$, & Po-211 & $->$ & $\mathrm{Pb}-207$ \\
\hline $4.25 \mathrm{E}-10$ & 887.7 & $\mathrm{Rn}-219$ & $->$ & Po-215 & & & \\
\hline $4.24 \mathrm{E}-10$ & 72.803 & TI-207 & $->$ & $\mathrm{Pb}-207$ & & & \\
\hline 4.17E-10 & 764.52 & Fr-223 & $->$ & Ra-223, & Th-227 & $->$ & $\mathrm{Ra}-223$ \\
\hline $4.13 \mathrm{E}-10$ & 569.15 & Po-211 & $->$ & $\mathrm{Pb}-207$ & & & \\
\hline $4.01 \mathrm{E}-10$ & 374.93 & Th-227 & $->$ & $\mathrm{Ra}-223$ & & & \\
\hline $4.01 \mathrm{E}-10$ & 339.1 & Th-227 & $->$ & Ra-223 & & & \\
\hline $3.68 \mathrm{E}-10$ & 1234.3 & $\mathrm{~Pb}-211$ & $->$ & $\mathrm{Bi}-211$ & & & \\
\hline $3.44 \mathrm{E}-10$ & 102.45 & Th-227 & $->$ & $\mathrm{Ra}-223$ & & & \\
\hline $3.34 \mathrm{E}-10$ & 536.88 & Fr-223 & $->$ & Ra-223, & Th-227 & $->$ & $\mathrm{Ra}-223$ \\
\hline $3.27 E-10$ & 568.76 & Fr-223 & $->$ & Ra-223, & Th-227 & $->$ & $\mathrm{Ra}-223$ \\
\hline $2.91 \mathrm{E}-10$ & 757.03 & Fr-223 & $->$ & Ra-223, & Th-227 & $->$ & $\mathrm{Ra}-223$ \\
\hline $2.87 \mathrm{E}-10$ & 171.21 & Th-227 & $->$ & $\mathrm{Ra}-223$ & & & \\
\hline $2.83 \mathrm{E}-10$ & 834.2 & $\mathrm{Rn}-219$ & $->$ & Po-215 & & & \\
\hline $2.69 \mathrm{E}-10$ & 796.96 & Fr-223 & $->$ & Ra-223, & Th-227 & $->$ & $\mathrm{Ra}-223$ \\
\hline $2.64 \mathrm{E}-10$ & 825.96 & Fr-223 & $->$ & Ra-223, & Th-227 & $->$ & $\mathrm{Ra}-223$ \\
\hline $2.54 \mathrm{E}-10$ & 10.541 & TI-207 & $->$ & $\mathrm{Pb}-207$ & & & \\
\hline $2.51 \mathrm{E}-10$ & 84.979 & TI-207 & $->$ & $\mathrm{Pb}-207$ & & & \\
\hline $2.32 \mathrm{E}-10$ & 12.703 & TI-207 & $->$ & Pb-207 & & & \\
\hline $2.23 \mathrm{E}-10$ & 876.14 & Fr-223 & $->$ & Ra-223, & Th-227 & $->$ & Ra-223 \\
\hline $2.14 \mathrm{E}-10$ & 722.6 & Fr-223 & $->$ & Ra-223, & Th-227 & $->$ & $\mathrm{Ra}-223$ \\
\hline $2.01 \mathrm{E}-10$ & 230.17 & Th-227 & $->$ & Ra-223 & & & \\
\hline $1.98 \mathrm{E}-10$ & 842.02 & Fr-223 & $->$ & Ra-223, & Th-227 & $->$ & Ra-223 \\
\hline $1.93 \mathrm{E}-10$ & 846.56 & Fr-223 & $->$ & Ra-223, & Th-227 & $->$ & $\mathrm{Ra}-223$ \\
\hline $1.70 \mathrm{E}-10$ & 1055.8 & $\mathrm{Rn}-219$ & $->$ & Po-215 & & & \\
\hline $1.67 \mathrm{E}-10$ & 493.15 & Fr-223 & $->$ & Ra-223, & Th-227 & $->$ & Ra-223 \\
\hline
\end{tabular}




\begin{tabular}{lc}
$1.59 \mathrm{E}-10$ & 575.8 \\
$1.53 \mathrm{E}-10$ & 748.56 \\
$1.43 \mathrm{E}-10$ & 837.36 \\
$1.34 \mathrm{E}-10$ & 99.7 \\
$1.28 \mathrm{E}-10$ & 507.64 \\
$1.27 \mathrm{E}-10$ & 746.24 \\
$1.13 \mathrm{E}-10$ & 387.9 \\
$1.12 \mathrm{E}-10$ & 756.79 \\
$1.03 \mathrm{E}-10$ & 896.41 \\
$1.02 \mathrm{E}-10$ & 780.59 \\
$9.11 \mathrm{E}-11$ & 480.25 \\
$8.52 \mathrm{E}-11$ & 552.09 \\
$8.29 \mathrm{E}-11$ & 762.3 \\
$8.18 \mathrm{E}-11$ & 858.4 \\
$7.34 \mathrm{E}-11$ & 87.632 \\
$7.12 \mathrm{E}-11$ & 69.83 \\
$7.02 \mathrm{E}-11$ & 555.91 \\
$6.24 \mathrm{E}-11$ & 828.31 \\
$6.13 \mathrm{E}-11$ & 515.96 \\
$5.80 \mathrm{E}-11$ & 733.9 \\
$5.75 \mathrm{E}-11$ & 607.2 \\
$5.63 \mathrm{E}-11$ & 754.17 \\
$5.63 \mathrm{E}-11$ & 784.04 \\
$5.47 \mathrm{E}-11$ & 753.44 \\
$5.43 \mathrm{E}-11$ & 734.6 \\
$5.10 \mathrm{E}-11$ & 524.02 \\
$4.96 \mathrm{E}-11$ & 817.31 \\
$4.92 \mathrm{E}-11$ & 691.46 \\
$4.77 \mathrm{E}-11$ & 482.14 \\
$4.68 \mathrm{E}-11$ & 448 \\
$4.59 \mathrm{E}-11$ & 623.52 \\
$4.44 \mathrm{E}-11$ & 578.2 \\
$4.24 \mathrm{E}-11$ & 14.886 \\
$4.14 \mathrm{E}-11$ & 704.19 \\
$3.94 \mathrm{E}-11$ & 772.85 \\
$3.89 \mathrm{E}-11$ & 9.3 \\
$3.89 \mathrm{E}-11$ & 786.9 \\
$3.73 \mathrm{E}-11$ & 633.2 \\
$3.71 \mathrm{E}-11$ & 452.52 \\
$3.48 \mathrm{E}-11$ & 147.2 \\
$3.47 \mathrm{E}-11$ & 878.16 \\
$3.10 \mathrm{E}-11$ & 848.88 \\
$2.87 \mathrm{E}-11$ & 534.9 \\
$2.83 \mathrm{E}-11$ & 31.87 \\
$2.59 \mathrm{E}-11$ & 457.07 \\
$2.59 \mathrm{E}-11$ & 736.76 \\
$2.31 \mathrm{E}-11$ & 643.93 \\
$2.31 \mathrm{E}-11$ & 867.23 \\
& 69.13 \\
\hline & 120.9
\end{tabular}

\begin{tabular}{|c|c|c|c|c|c|}
\hline Fr-223 & $\rightarrow$ & Ra-223, & Th-227 & $->$ & $\mathrm{Ra}-223$ \\
\hline Fr-223 & $->$ & Ra-223, & Th-227 & $->$ & Ra-223 \\
\hline Fr-223 & $->$ & Ra-223, & Th-227 & $->$ & $\mathrm{Ra}-223$ \\
\hline$A c-227$ & $->$ & Fr-223 & & & \\
\hline $\mathrm{Fr}-223$ & $->$ & Ra-223, & Th-227 & $->$ & $\mathrm{Ra}-223$ \\
\hline Fr-223 & $->$ & Ra-223, & Th-227 & $->$ & Ra-223 \\
\hline Rn-219 & $->$ & Po-215 & & & \\
\hline Fr-223 & $->$ & Ra-223, & Th-227 & $->$ & Ra-223 \\
\hline Fr-223 & $->$ & Ra-223, & Th-227 & $->$ & $\mathrm{Ra}-223$ \\
\hline Fr-223 & $->$ & Ra-223, & Th-227 & $->$ & $\mathrm{Ra}-223$ \\
\hline Fr-223 & $->$ & Ra-223, & Th-227 & $->$ & $\mathrm{Ra}-223$ \\
\hline Fr-223 & $->$ & Ra-223, & Th-227 & $->$ & Ra-223 \\
\hline Fr-223 & $->$ & Ra-223, & Th-227 & $->$ & $\mathrm{Ra}-223$ \\
\hline Fr-223 & $->$ & Ra-223, & Th-227 & $->$ & $\mathrm{Ra}-223$ \\
\hline TI-207 & $->$ & $\mathrm{Pb}-207$ & & & \\
\hline$A c-227$ & $->$ & Fr-223 & & & \\
\hline Fr-223 & $->$ & Ra-223, & Th-227 & $->$ & $\mathrm{Ra}-223$ \\
\hline Fr-223 & $->$ & Ra-223, & Th-227 & $->$ & $\mathrm{Ra}-223$ \\
\hline Fr-223 & $->$ & Ra-223, & Th-227 & $->$ & Ra-223 \\
\hline $\mathrm{Fr}-223$ & $->$ & Ra-223, & Th-227 & $->$ & $\mathrm{Ra}-223$ \\
\hline Fr-223 & $->$ & Ra-223, & Th-227 & $->$ & $\mathrm{Ra}-223$ \\
\hline Fr-223 & $->$ & Ra-223, & Th-227 & $->$ & $\mathrm{Ra}-223$ \\
\hline Fr-223 & $->$ & Ra-223, & Th-227 & $->$ & Ra-223 \\
\hline Fr-223 & $->$ & Ra-223, & Th-227 & $->$ & $\mathrm{Ra}-223$ \\
\hline Fr-223 & $->$ & Ra-223, & Th-227 & $->$ & Ra-223 \\
\hline Fr-223 & $->$ & Ra-223, & Th-227 & $->$ & $\mathrm{Ra}-223$ \\
\hline Fr-223 & $->$ & Ra-223, & Th-227 & $->$ & $\mathrm{Ra}-223$ \\
\hline Fr-223 & $->$ & Ra-223, & Th-227 & $->$ & $\mathrm{Ra}-223$ \\
\hline $\mathrm{Fr}-223$ & $->$ & $\mathrm{Ra}-223$, & Th-227 & $->$ & $\mathrm{Ra}-223$ \\
\hline Fr-223 & $->$ & $\mathrm{Ra}-223$, & Th-227 & $->$ & $\mathrm{Ra}-223$ \\
\hline Th-227 & $->$ & $\mathrm{Ra}-223$ & & & \\
\hline Fr-223 & $->$ & $\mathrm{Ra}-223$, & Th-227 & $->$ & $\mathrm{Ra}-223$ \\
\hline TI-207 & $->$ & $\mathrm{Pb}-207$ & & & \\
\hline Fr-223 & $->$ & $\mathrm{Ra}-223$, & Th-227 & $->$ & Ra-223 \\
\hline Fr-223 & $->$ & Ra-223, & Th-227 & $->$ & $\mathrm{Ra}-223$ \\
\hline Ac- 227 & $->$ & Th-227 & & & \\
\hline Fr-223 & $->$ & Ra-223, & Th-227 & $->$ & $\mathrm{Ra}-223$ \\
\hline Th-227 & $->$ & $\mathrm{Ra}-223$ & & & \\
\hline Fr-223 & $->$ & $\mathrm{Ra}-223$, & Th-227 & $->$ & $\mathrm{Ra}-223$ \\
\hline Ac- 227 & $->$ & Fr-223 & & & \\
\hline Fr-223 & $->$ & Ra-223, & Th-227 & $->$ & Ra-223 \\
\hline Fr-223 & $->$ & $\mathrm{Ra}-223$, & Th-227 & $->$ & Ra-223 \\
\hline Th-227 & $->$ & $\mathrm{Ra}-223$ & & & \\
\hline $\mathrm{Ra}-223$ & $->$ & $\mathrm{Rn}-219$ & & & \\
\hline Fr-223 & $->$ & Ra-223, & Th-227 & $->$ & Ra-223 \\
\hline Fr-223 & $->$ & Ra-223, & Th-227 & $->$ & Ra-223 \\
\hline Fr-223 & $->$ & Ra-223, & Th-227 & $->$ & Ra-223 \\
\hline Fr-223 & $->$ & Ra-223, & Th-227 & $->$ & $\mathrm{Ra}-223$ \\
\hline Ac- 227 & $->$ & Fr-223 & & & \\
\hline Ac- 227 & $->$ & Fr-223 & & & \\
\hline
\end{tabular}




\begin{tabular}{|c|c|c|c|c|c|c|c|}
\hline $2.06 \mathrm{E}-11$ & 941.41 & Th-227 & $->$ & Ra-223 & & & \\
\hline $2.01 \mathrm{E}-11$ & 854.2 & Th-227 & $->$ & $\mathrm{Ra}-223$ & & & \\
\hline $1.88 \mathrm{E}-11$ & 621.92 & Fr-223 & $->$ & Ra-223, & Th-227 & $->$ & Ra-223 \\
\hline $1.88 \mathrm{E}-11$ & 857.89 & Fr-223 & $->$ & Ra-223, & Th-227 & $->$ & $\mathrm{Ra}-223$ \\
\hline $1.82 \mathrm{E}-11$ & 588.17 & Fr-223 & $->$ & Ra-223, & Th-227 & $->$ & Ra-223 \\
\hline $1.78 \mathrm{E}-11$ & 958.6 & Th-227 & $->$ & $\mathrm{Ra}-223$ & & & \\
\hline $1.72 \mathrm{E}-11$ & 662.8 & Th-227 & $->$ & Ra-223 & & & \\
\hline $1.72 \mathrm{E}-11$ & 171.4 & Ac- 227 & $->$ & Fr-223 & & & \\
\hline $1.69 \mathrm{E}-11$ & 808.26 & Fr-223 & $->$ & Ra-223, & Th-227 & $->$ & Ra-223 \\
\hline $1.48 \mathrm{E}-11$ & 707.18 & Fr-223 & $->$ & Ra-223, & Th-227 & $->$ & $\mathrm{Ra}-223$ \\
\hline $1.43 \mathrm{E}-11$ & 466.2 & Th-227 & $->$ & $\mathrm{Ra}-223$ & & & \\
\hline $1.43 \mathrm{E}-11$ & 461.9 & Th-227 & $->$ & $\mathrm{Ra}-223$ & & & \\
\hline $1.41 \mathrm{E}-11$ & 863.2 & Fr-223 & $->$ & Ra-223, & Th-227 & $->$ & $\mathrm{Ra}-223$ \\
\hline $1.41 \mathrm{E}-11$ & 891 & Fr-223 & $->$ & Ra-223, & Th-227 & $->$ & Ra-223 \\
\hline $1.27 \mathrm{E}-11$ & 9.185 & Tl-207 & $->$ & $\mathrm{Pb}-207$ & & & \\
\hline $1.21 \mathrm{E}-11$ & 596.44 & Fr-223 & $->$ & Ra-223, & Th-227 & $->$ & Ra-223 \\
\hline $1.17 \mathrm{E}-11$ & 133.8 & $A c-227$ & $->$ & $\mathrm{Fr}-223$ & & & \\
\hline $1.15 \mathrm{E}-11$ & 788.36 & Th-227 & $->$ & $\mathrm{Ra}-223$ & & & \\
\hline $1.15 \mathrm{E}-11$ & 792.6 & Th-227 & $->$ & Ra-223 & & & \\
\hline $1.00 \mathrm{E}-11$ & 990.2 & Th-227 & $->$ & Ra-223 & & & \\
\hline $9.61 \mathrm{E}-12$ & 718.22 & Fr-223 & $->$ & Ra-223, & Th-227 & $->$ & Ra-223 \\
\hline $9.18 \mathrm{E}-12$ & 892.8 & Fr-223 & $->$ & $\mathrm{Ra}-223$, & Th-227 & $->$ & $\mathrm{Ra}-223$ \\
\hline $8.71 \mathrm{E}-12$ & 926.28 & Fr-223 & $->$ & Ra-223, & Th-227 & $->$ & Ra-223 \\
\hline 8.60E-12 & 999.78 & Th-227 & $->$ & $\mathrm{Ra}-223$ & & & \\
\hline 8.60E-12 & 969.91 & Th-227 & $->$ & Ra-223 & & & \\
\hline 8.60E-12 & 793.12 & Th-227 & $->$ & $\mathrm{Ra}-223$ & & & \\
\hline 8.60E-12 & 818.23 & Th-227 & $->$ & $\mathrm{Ra}-223$ & & & \\
\hline $6.07 \mathrm{E}-12$ & 648.43 & Fr-223 & $->$ & Ra-223, & Th-227 & $->$ & $\mathrm{Ra}-223$ \\
\hline $5.87 E-12$ & 46.13 & $A c-227$ & $->$ & Fr-223 & & & \\
\hline $5.87 \mathrm{E}-12$ & 106.1 & Ac-227 & $->$ & Fr-223 & & & \\
\hline $5.73 \mathrm{E}-12$ & 1020 & Th-227 & $->$ & Ra-223 & & & \\
\hline $5.73 E-12$ & 641.45 & Th-227 & $->$ & $\mathrm{Ra}-223$ & & & \\
\hline $5.45 \mathrm{E}-12$ & 825.97 & Fr-223 & $->$ & Ra-223 & & & \\
\hline $4.30 \mathrm{E}-12$ & 1015.1 & Th-227 & $->$ & Ra-223 & & & \\
\hline $4.30 \mathrm{E}-12$ & 1024.8 & Th-227 & $->$ & $\mathrm{Ra}-223$ & & & \\
\hline $4.30 \mathrm{E}-12$ & 909.85 & Th-227 & $->$ & $\mathrm{Ra}-223$ & & & \\
\hline $3.44 \mathrm{E}-12$ & 920.06 & Th-227 & $->$ & $\mathrm{Ra}-223$ & & & \\
\hline $3.41 \mathrm{E}-12$ & 828.53 & Fr-223 & $->$ & Ra-223, & Th-227 & $->$ & $\mathrm{Ra}-223$ \\
\hline $3.30 \mathrm{E}-12$ & 11.349 & TI-207 & $->$ & $\mathrm{Pb}-207$ & & & \\
\hline $2.87 \mathrm{E}-12$ & 971.28 & Th-227 & $->$ & $\mathrm{Ra}-223$ & & & \\
\hline $2.87 \mathrm{E}-12$ & 938.34 & Th-227 & $->$ & $\mathrm{Ra}-223$ & & & \\
\hline $2.01 \mathrm{E}-12$ & 994.9 & Th-227 & $->$ & Ra-223 & & & \\
\hline $5.03 E-13$ & 793.11 & Fr-223 & $->$ & Ra-223 & & & \\
\hline Pu-240 & $100 \%$ & & & tge: 5 years & & & \\
\hline 325 & lines & & & & & & \\
\hline
\end{tabular}


Sorted by Energy

\begin{tabular}{|c|c|c|c|c|c|c|c|}
\hline \multirow{2}{*}{$\frac{\text { Energy }}{8.953}$} & \multirow{2}{*}{$\begin{array}{c}\text { Intensity } \\
4.75 \mathrm{E}-12\end{array}$} & \multicolumn{6}{|c|}{ Initial Isotopes and Decays } \\
\hline & & $\mathrm{Bi}-212$ & $->$ & TI-208 & & & \\
\hline 9.185 & $2.06 \mathrm{E}-12$ & TI-208 & $->$ & $\mathrm{Pb}-208$ & & & \\
\hline 9.419 & $2.99 \mathrm{E}-11$ & $\mathrm{~Pb}-212$ & $->$ & $\mathrm{Bi}-212$ & & & \\
\hline 9.658 & $1.18 \mathrm{E}-13$ & $\mathrm{Bi}-212$ & $->$ & Po-212 & & & \\
\hline 10.137 & $6.44 \mathrm{E}-13$ & Ra-224 & $->$ & $\mathrm{Rn}-220$ & & & \\
\hline 10.259 & $9.62 \mathrm{E}-11$ & $\mathrm{Bi}-212$ & $->$ & TI-208 & & & \\
\hline 10.541 & $4.11 \mathrm{E}-11$ & TI-208 & $->$ & $\mathrm{Pb}-208$ & & & \\
\hline 10.622 & $1.60 \mathrm{E}-11$ & Th-228 & $->$ & $\mathrm{Ra}-224$ & & & \\
\hline 10.828 & $5.81 \mathrm{E}-10$ & $\mathrm{~Pb}-212$ & $->$ & $\mathrm{Bi}-212$ & & & \\
\hline 10.994 & $8.96 \mathrm{E}-13$ & $\mathrm{Bi}-212$ & $->$ & TI-208 & & & \\
\hline 11.118 & $2.48 \mathrm{E}+00$ & Ac- 228 & $->$ & Th-228, & $U-236$ & $->$ & Th-232 \\
\hline 11.119 & $2.31 \mathrm{E}-12$ & $\mathrm{Bi}-212$ & $->$ & Po-212 & & & \\
\hline 11.349 & $5.51 \mathrm{E}-13$ & TI-208 & $->$ & $\mathrm{Pb}-208$ & & & \\
\hline 11.712 & $7.66 \mathrm{E}-12$ & $\mathrm{~Pb}-212$ & $->$ & $\mathrm{Bi}-212$ & & & \\
\hline 11.713 & $1.15 \mathrm{E}-11$ & $\mathrm{Ra}-224$ & $->$ & $\mathrm{Rn}-220$ & & & \\
\hline 12.085 & $3.50 \mathrm{E}-14$ & $\mathrm{Bi}-212$ & $->$ & Po-212 & & & \\
\hline 12.282 & $1.11 \mathrm{E}-10$ & $\mathrm{Bi}-212$ & $->$ & TI-208 & & & \\
\hline 12.325 & $2.76 \mathrm{E}-10$ & Th-228 & $->$ & Ra-224 & & & \\
\hline 12.701 & $3.86 \mathrm{E}-11$ & TI-208 & $->$ & $\mathrm{Pb}-208$ & & & \\
\hline 12.76 & 5.05E-09 & $\mathrm{Ra}-228$ & $->$ & $A C-228$ & & & \\
\hline 12.855 & $3.00 \mathrm{E}-13$ & Ra-224 & $->$ & $\mathrm{Rn}-220$ & & & \\
\hline 12.952 & $4.22 \mathrm{E}+01$ & Ac- 228 & $->$ & Th-228, & $U-236$ & $->$ & Th-232 \\
\hline 13.099 & $5.28 \mathrm{E}-10$ & $\mathrm{~Pb}-212$ & $->$ & $\mathrm{Bi}-212$ & & & \\
\hline 13.5 & $2.66 \mathrm{E}-08$ & $\mathrm{Ra}-228$ & $->$ & $A C-228$ & & & \\
\hline 13.501 & $2.31 \mathrm{E}-12$ & $\mathrm{Bi}-212$ & $->$ & Po-212 & & & \\
\hline 13.662 & $8.37 \mathrm{E}-12$ & Th-228 & $->$ & $\mathrm{Ra}-224$ & & & \\
\hline 14.336 & $1.59 \mathrm{E}-11$ & Ra-224 & $->$ & $\mathrm{Rn}-220$ & & & \\
\hline 14.511 & $1.18 \mathrm{E}+00$ & Ac- 228 & $->$ & Th-228, & $U-236$ & $->$ & Th-232 \\
\hline 14.563 & $2.60 \mathrm{E}-11$ & $\mathrm{Bi}-212$ & $->$ & TI-208 & & & \\
\hline 14.884 & 7.09E-12 & TI-208 & $->$ & $\mathrm{Pb}-208$ & & & \\
\hline 15.15 & $2.66 \mathrm{E}-09$ & $\mathrm{Ra}-228$ & $->$ & Ac- 228 & & & \\
\hline 15.23 & $4.19 \mathrm{E}-10$ & Th-228 & $->$ & $\mathrm{Ra}-224$ & & & \\
\hline 15.376 & $9.95 \mathrm{E}-11$ & $\mathrm{~Pb}-212$ & $->$ & $\mathrm{Bi}-212$ & & & \\
\hline 15.869 & $4.51 \mathrm{E}-13$ & $\mathrm{Bi}-212$ & $->$ & Po-212 & & & \\
\hline 16.154 & $5.32 \mathrm{E}-09$ & $A c-228$ & $->$ & Th-228 & & & \\
\hline 16.161 & $5.83 \mathrm{E}+01$ & U-236 & $->$ & Th-232 & & & \\
\hline 16.18 & $1.20 \mathrm{E}-08$ & $\mathrm{Ra}-228$ & $->$ & $A C-228$ & & & \\
\hline 16.881 & $3.44 \mathrm{E}-12$ & $\mathrm{Ra}-224$ & $->$ & $\mathrm{Rn}-220$ & & & \\
\hline 17.952 & $9.44 \mathrm{E}-11$ & Th-228 & $->$ & $\mathrm{Ra}-224$ & & & \\
\hline 18.8 & $3.46 E-09$ & Ra-228 & $->$ & Ac- 228 & & & \\
\hline 19.094 & $1.30 \mathrm{E}+01$ & U-236 & $->$ & Th-232 & & & \\
\hline 19.113 & $1.22 \mathrm{E}-09$ & Ac- 228 & $->$ & Th-228 & & & \\
\hline 19.4 & $2.39 \mathrm{E}-10$ & $\mathrm{Ra}-228$ & $->$ & $A C-228$ & & & \\
\hline 39.846 & $3.48 \mathrm{E}-11$ & $\mathrm{Bi}-212$ & $->$ & Tl-208 & & & \\
\hline 45.244 & $3.78 \mathrm{E}+06$ & Pu-240 & $->$ & U-236 & & & \\
\hline
\end{tabular}




\begin{tabular}{|c|c|c|c|c|}
\hline 49.369 & $9.68 \mathrm{E}-01$ & U-236 & $->$ & Th-232 \\
\hline 57.81 & $1.40 \mathrm{E}-10$ & Ac- 228 & $->$ & Th-228 \\
\hline 59 & $2.91 \mathrm{E}-10$ & Th-232 & $->$ & $\mathrm{Ra}-228$ \\
\hline 70.832 & $2.37 \mathrm{E}-12$ & $\mathrm{Bi}-212$ & $->$ & TI-208 \\
\hline 72.803 & $6.77 \mathrm{E}-11$ & TI-208 & $->$ & $\mathrm{Pb}-208$ \\
\hline 72.873 & $4.02 \mathrm{E}-12$ & $\mathrm{Bi}-212$ & $->$ & TI-208 \\
\hline 74.814 & $9.25 \mathrm{E}-10$ & $\mathrm{~Pb}-212$ & $->$ & $\mathrm{Bi}-212$ \\
\hline 74.969 & $1.14 \mathrm{E}-10$ & TI-208 & $->$ & Pb-208 \\
\hline 76.858 & $3.38 \mathrm{E}-12$ & $\mathrm{Bi}-212$ & $->$ & Рo-212 \\
\hline 77.107 & $1.56 \mathrm{E}-09$ & $\mathrm{~Pb}-212$ & $->$ & $\mathrm{Bi}-212$ \\
\hline 79.29 & $5.64 \mathrm{E}-12$ & $\mathrm{Bi}-212$ & $->$ & Po-212 \\
\hline 81.067 & $1.06 \mathrm{E}-11$ & $\mathrm{Ra}-224$ & $->$ & $\mathrm{Rn}-220$ \\
\hline 82.434 & $1.42 \mathrm{E}-12$ & $\mathrm{Bi}-212$ & $->$ & TI-208 \\
\hline 83.787 & $1.76 \mathrm{E}-11$ & $\mathrm{Ra}-224$ & $->$ & $\mathrm{Rn}-220$ \\
\hline 84.26 & $1.08 \mathrm{E}-10$ & Th-228 & $->$ & $\mathrm{Ra}-224$ \\
\hline 84.789 & $4.02 \mathrm{E}-11$ & TI-208 & $->$ & $\mathrm{Pb}-208$ \\
\hline 85.185 & $3.96 \mathrm{E}-13$ & $\mathrm{Bi}-212$ & $->$ & TI-208 \\
\hline 85.429 & $1.56 \mathrm{E}-12$ & Th-228 & $->$ & $\mathrm{Ra}-224$ \\
\hline 87.19 & $5.52 \mathrm{E}-10$ & $\mathrm{~Pb}-212$ & $->$ & $\mathrm{Bi}-212$ \\
\hline 87.632 & $1.16 \mathrm{E}-11$ & TI-208 & $->$ & $\mathrm{Pb}-208$ \\
\hline 88.471 & $2.57 \mathrm{E}-12$ & Th-228 & $->$ & $\mathrm{Ra}-224$ \\
\hline 89.639 & $1.97 \mathrm{E}-12$ & $\mathrm{Bi}-212$ & $->$ & Po-212 \\
\hline 89.955 & $9.04 \mathrm{E}-10$ & Ac- 228 & $->$ & Th-228 \\
\hline 90.128 & $1.64 \mathrm{E}-10$ & $\mathrm{~Pb}-212$ & $->$ & $\mathrm{Bi}-212$ \\
\hline 92.673 & $6.21 \mathrm{E}-13$ & $\mathrm{Bi}-212$ & $->$ & Po-212 \\
\hline 93.35 & 1.49E-09 & Ac- 228 & $->$ & Th-228 \\
\hline 94.66 & $5.71 E+03$ & Pu-240 & $->$ & U-236 \\
\hline 94.677 & $6.35 \mathrm{E}-12$ & Ra-224 & $->$ & $\mathrm{Rn}-220$ \\
\hline 97.907 & $2.03 \mathrm{E}-12$ & $\mathrm{Ra}-224$ & $->$ & $\mathrm{Rn}-220$ \\
\hline 98.441 & $8.39 E+03$ & Pu-240 & $->$ & U-236 \\
\hline 99.55 & $3.46 \mathrm{E}-10$ & Ac- 228 & $->$ & Th-228 \\
\hline 99.915 & $9.18 \mathrm{E}-13$ & Th-228 & $->$ & $\mathrm{Ra}-224$ \\
\hline 103.341 & $2.99 \mathrm{E}-13$ & Th-228 & $->$ & $\mathrm{Ra}-224$ \\
\hline 104.244 & $5.84 \mathrm{E}+05$ & Pu-240 & $->$ & U-236 \\
\hline 105.362 & $5.32 \mathrm{E}-10$ & Ac- 228 & $->$ & Th-228 \\
\hline 108.99 & $1.78 \mathrm{E}-10$ & Ac- 228 & $->$ & Th-228 \\
\hline 110.421 & $9.90 \mathrm{E}+02$ & Pu-240 & $->$ & U-236 \\
\hline 111.3 & $3.02 E+03$ & Pu-240 & $->$ & $U-236$ \\
\hline 112.75 & $2.36 \mathrm{E}-01$ & U-236 & $->$ & Th-232 \\
\hline 114.866 & $8.39 E+02$ & Pu-240 & $->$ & $U-236$ \\
\hline 115.122 & $5.20 \mathrm{E}-11$ & $\mathrm{~Pb}-212$ & $->$ & $\mathrm{Bi}-212$ \\
\hline 124 & $6.58 \mathrm{E}-11$ & Th-232 & $->$ & $\mathrm{Ra}-228$ \\
\hline 129.03 & $7.71 \mathrm{E}-10$ & Ac- 228 & $->$ & Th-228 \\
\hline 131.5 & $1.14 \mathrm{E}-11$ & Th-228 & $->$ & Ra-224 \\
\hline 135.68 & $4.52 \mathrm{E}-12$ & Ac- 228 & $->$ & Th-228 \\
\hline 141.19 & $1.30 \mathrm{E}-11$ & Ac- 228 & $->$ & Th-228 \\
\hline 143.99 & $3.16 \mathrm{E}-13$ & $\mathrm{Bi}-212$ & $->$ & TI-208 \\
\hline 146.06 & 7.71E-11 & Ac- 228 & $->$ & Th-228 \\
\hline 153.89 & $2.23 \mathrm{E}-10$ & Ac- 228 & $->$ & Th-228 \\
\hline 160.28 & $3.52 E+04$ & Pu-240 & $->$ & $U-236$ \\
\hline
\end{tabular}




\begin{tabular}{|c|c|c|c|c|}
\hline 164 & $1.58 \mathrm{E}-13$ & $\mathrm{Bi}-212$ & $->$ & TI-208 \\
\hline 164.15 & $4.40 \mathrm{E}-13$ & $\mathrm{~Pb}-212$ & $->$ & $\mathrm{Bi}-212$ \\
\hline 166.43 & $7.31 \mathrm{E}-12$ & Th-228 & $->$ & $\mathrm{Ra}-224$ \\
\hline 174.18 & $8.51 \mathrm{E}-12$ & Ac- 228 & $->$ & Th-228 \\
\hline 176.577 & $4.49 \mathrm{E}-12$ & $\mathrm{~Pb}-212$ & $->$ & $\mathrm{Bi}-212$ \\
\hline 184.72 & $3.72 \mathrm{E}-11$ & Ac- 228 & $->$ & Th-228 \\
\hline 191.29 & $3.19 \mathrm{E}-11$ & Ac- 228 & $->$ & Th-228 \\
\hline 199.54 & 7.44E-11 & Ac- 228 & $->$ & Th-228 \\
\hline 204.37 & 4.17E-11 & Ac- 228 & $->$ & Th-228 \\
\hline 205.75 & $2.49 \mathrm{E}-12$ & Th-228 & $->$ & Ra-224 \\
\hline 209.39 & 1.09E-09 & Ac- 228 & $->$ & Th-228 \\
\hline 210.76 & $6.11 \mathrm{E}-11$ & Ac- 228 & $->$ & Th-228 \\
\hline 211.31 & $5.38 \mathrm{E}-12$ & TI-208 & $->$ & $\mathrm{Pb}-208$ \\
\hline 212.46 & $2.52 E+03$ & Pu-240 & $->$ & U-236 \\
\hline 215.75 & $2.47 \mathrm{E}-11$ & Th-228 & $->$ & $\mathrm{Ra}-224$ \\
\hline 216.24 & $2.13 \mathrm{E}-10$ & Ac- 228 & $->$ & Th-228 \\
\hline 220.49 & 3.72E-12 & Ac- -228 & $->$ & Th-228 \\
\hline 223.72 & $1.75 \mathrm{E}-11$ & Ac- 228 & $->$ & Th-228 \\
\hline 232.3 & $1.59 \mathrm{E}-11$ & Ac- 228 & $->$ & Th-228 \\
\hline 233.32 & $9.81 \mathrm{E}-12$ & TI-208 & $->$ & $\mathrm{Pb}-208$ \\
\hline 238.578 & 3.84E-09 & $\mathrm{Pb}-212$ & $->$ & $\mathrm{Bi}-212$ \\
\hline 240.76 & $3.44 \mathrm{E}-10$ & $\mathrm{Ra}-224$ & $->$ & $\mathrm{Rn}-220$ \\
\hline 252.45 & $2.53 \mathrm{E}-11$ & TI-208 & $->$ & $\mathrm{Pb}-208$ \\
\hline 257.29 & $8.51 \mathrm{E}-12$ & Ac- 228 & $->$ & Th-228 \\
\hline 263.57 & $1.54 \mathrm{E}-11$ & Ac- 228 & $->$ & Th-228 \\
\hline 270.26 & $1.01 \mathrm{E}-09$ & Ac- 228 & $->$ & Th-228 \\
\hline 277.28 & $2.15 \mathrm{E}-10$ & TI-208 & $->$ & Pb-208 \\
\hline 279.3 & $6.11 \mathrm{E}-11$ & Ac- 228 & $->$ & Th-228 \\
\hline 282.02 & $2.37 \mathrm{E}-11$ & Ac- 228 & $->$ & Th-228 \\
\hline 288.08 & $1.08 \mathrm{E}-11$ & $\mathrm{Bi}-212$ & $->$ & TI-208 \\
\hline 290 & 7.94E-13 & Ra-224 & $->$ & $\mathrm{Rn}-220$ \\
\hline 300.034 & $2.94 \mathrm{E}-10$ & $\mathrm{~Pb}-212$ & $->$ & $\mathrm{Bi}-212$ \\
\hline 321.9 & $6.65 E-11$ & Ac- 228 & $->$ & Th-228 \\
\hline 327.67 & $3.46 \mathrm{E}-11$ & Ac- 228 & $->$ & Th-228 \\
\hline 327.93 & $4.34 \mathrm{E}-12$ & $\mathrm{Bi}-212$ & $->$ & TI-208 \\
\hline 328.07 & $9.30 \mathrm{E}-10$ & Ac- 228 & $->$ & Th-228 \\
\hline 332.48 & $1.25 \mathrm{E}-10$ & Ac- 228 & $->$ & Th-228 \\
\hline 338.42 & 3.30E-09 & Ac- 228 & $->$ & Th-228 \\
\hline 340.94 & $1.38 \mathrm{E}-10$ & Ac- 228 & $->$ & Th-228 \\
\hline 356.83 & $5.32 \mathrm{E}-12$ & Ac- 228 & $->$ & Th-228 \\
\hline 372.3 & $2.39 \mathrm{E}-12$ & Ac- 228 & $->$ & Th-228 \\
\hline 388.9 & $3.99 \mathrm{E}-12$ & Ac- 228 & $->$ & Th-228 \\
\hline 396.9 & $8.51 \mathrm{E}-12$ & Ac- 228 & $->$ & Th-228 \\
\hline 399.2 & $9.84 \mathrm{E}-12$ & Ac- 228 & $\rightarrow$ & Th-228 \\
\hline 409.3 & $3.53 \mathrm{E}-13$ & $\mathrm{Ra}-224$ & $->$ & $\mathrm{Rn}-220$ \\
\hline 409.62 & $5.85 \mathrm{E}-10$ & Ac- 228 & $->$ & Th-228 \\
\hline 415.156 & $2.47 \mathrm{E}-12$ & $\mathrm{~Pb}-212$ & $->$ & $\mathrm{Bi}-212$ \\
\hline 416.2 & $4.52 \mathrm{E}-12$ & Ac- 228 & $->$ & Th-228 \\
\hline 419.23 & $6.91 \mathrm{E}-12$ & AC- 228 & $->$ & Th-228 \\
\hline 433.51 & $4.43 E-13$ & $\mathrm{Bi}-212$ & $->$ & TI-208 \\
\hline
\end{tabular}




\begin{tabular}{|c|c|c|c|c|c|c|c|}
\hline 440.49 & $3.99 \mathrm{E}-11$ & $A c-228$ & $->$ & Th-228 & & & \\
\hline 449.57 & $1.73 \mathrm{E}-11$ & Ac- 228 & $->$ & Th-228 & & & \\
\hline 452.77 & $1.15 \mathrm{E}-11$ & $\mathrm{Bi}-212$ & $->$ & TI-208 & & & \\
\hline 460.84 & $1.33 \mathrm{E}-11$ & $A c-228$ & $->$ & Th-228 & & & \\
\hline 463.1 & $1.22 \mathrm{E}-09$ & Ac- 228 & $->$ & Th-228 & & & \\
\hline 471.4 & $9.30 \mathrm{E}-12$ & Ac- 228 & $->$ & Th-228 & & & \\
\hline 473.36 & $1.58 \mathrm{E}-12$ & $\mathrm{Bi}-212$ & $->$ & TI-208 & & & \\
\hline 474.32 & 7.71E-12 & Ac- 228 & $->$ & Th-228 & & & \\
\hline 478.2 & $6.38 \mathrm{E}-11$ & $A c-228$ & $->$ & Th-228 & & & \\
\hline 481.8 & $2.39 \mathrm{E}-12$ & $A c-228$ & $->$ & Th-228 & & & \\
\hline 485.78 & $1.58 \mathrm{E}-12$ & TI-208 & $->$ & $\mathrm{Pb}-208$ & & & \\
\hline 492.36 & $5.32 \mathrm{E}-12$ & Ac- 228 & $->$ & Th-228 & & & \\
\hline 492.62 & $1.93 \mathrm{E}-13$ & $\mathrm{Bi}-212$ & $->$ & TI-208 & & & \\
\hline 498.26 & $1.14 \mathrm{E}-11$ & $A c-228$ & $->$ & Th-228 & & & \\
\hline 503.7 & $5.58 \mathrm{E}-11$ & $A c-228$ & $->$ & Th-228 & & & \\
\hline 509.17 & $1.30 \mathrm{E}-10$ & $A c-228$ & $->$ & Th-228 & & & \\
\hline 510.606 & $6.84 \mathrm{E}-10$ & TI-208 & $->$ & $\mathrm{Pb}-208$ & & & \\
\hline 515.2 & $1.14 \mathrm{E}-11$ & $A c-228$ & $->$ & Th-228 & & & \\
\hline 519.97 & $2.07 \mathrm{E}-11$ & Ac- 228 & $->$ & Th-228 & & & \\
\hline 523.18 & $3.19 \mathrm{E}-11$ & $A c-228$ & $->$ & Th-228 & & & \\
\hline 538.11 & $1.01 \mathrm{E}+01$ & Pu-240 & $->$ & $U-236$ & & & \\
\hline 540.5 & $7.71 \mathrm{E}-12$ & $A c-228$ & $->$ & Th-228 & & & \\
\hline 546.36 & $5.85 \mathrm{E}-11$ & Ac- 228 & $->$ & Th-228 & & & \\
\hline 549.7 & $6.17 \mathrm{E}-12$ & $\mathrm{Rn}-220$ & $->$ & Po-216 & & & \\
\hline 555.3 & $1.30 \mathrm{E}-11$ & Ac- 228 & $->$ & Th-228 & & & \\
\hline 562.65 & $2.68 \mathrm{E}-10$ & $A c-228$ & $->$ & Th-228 & & & \\
\hline 570.2 & $5.05 \mathrm{E}-11$ & $A c-228$ & $->$ & Th-228 & & & \\
\hline 572.5 & $5.05 E-11$ & $A c-228$ & $->$ & Th-228 & & & \\
\hline 580.4 & $2.50 \mathrm{E}-14$ & $\mathrm{Bi}-212$ & $->$ & TI-208 & & & \\
\hline 583.022 & 2.83E-09 & TI-208 & $->$ & $\mathrm{Pb}-208$, & Po-212 & $->$ & $\mathrm{Pb}-208$ \\
\hline 583.28 & $3.99 E-11$ & $A c-228$ & $->$ & Th-228 & & & \\
\hline 587.82 & $1.27 \mathrm{E}-12$ & TI-208 & $->$ & $\mathrm{Pb}-208$ & & & \\
\hline 615.9 & $2.39 \mathrm{E}-11$ & $A c-228$ & $->$ & Th-228 & & & \\
\hline 619.88 & $2.92 \mathrm{E}-11$ & Ac- 228 & $->$ & Th-228 & & & \\
\hline 620.3 & $1.14 \mathrm{E}-13$ & $\mathrm{Bi}-212$ & $->$ & TI-208 & & & \\
\hline 629.93 & $1.33 \mathrm{E}-11$ & Ac- 228 & $->$ & Th-228 & & & \\
\hline 640.7 & $1.62 \mathrm{E}-11$ & $A c-228$ & $->$ & Th-228 & & & \\
\hline 642.5 & $1.06 E+03$ & Pu-240 & $->$ & $U-236$ & & & \\
\hline 649.19 & $1.14 \mathrm{E}-11$ & $A c-228$ & $->$ & Th-228 & & & \\
\hline 650.1 & $6.17 \mathrm{E}-13$ & Ra-224 & $->$ & $\mathrm{Rn}-220$ & & & \\
\hline 650.14 & $1.14 \mathrm{E}-12$ & TI-208 & $->$ & $\mathrm{Pb}-208$ & & & \\
\hline 651.44 & $2.68 \mathrm{E}-11$ & Ac- 228 & $->$ & Th-228 & & & \\
\hline 666.4 & $1.22 \mathrm{E}-11$ & Ac- 228 & $->$ & Th-228 & & & \\
\hline 673.86 & $2.66 \mathrm{E}-11$ & $A c-228$ & $->$ & Th-228 & & & \\
\hline 677.07 & $2.39 \mathrm{E}-10$ & Ac- 228 & $->$ & Th-228 & & & \\
\hline 687.59 & $2.27 E+02$ & Pu-240 & $->$ & $U-236$ & & & \\
\hline 692.5 & $9.04 \mathrm{E}-13$ & Ac- 228 & $->$ & Th-228 & & & \\
\hline 701.8 & $5.05 \mathrm{E}-11$ & Ac- 228 & $->$ & Th-228 & & & \\
\hline 705.24 & $6.96 \mathrm{E}-13$ & TI-208 & $->$ & $\mathrm{Pb}-208$ & & & \\
\hline 707.49 & $3.99 E-11$ & $A c-228$ & $->$ & Th-228 & & & \\
\hline
\end{tabular}




\begin{tabular}{|c|c|c|c|c|}
\hline 721.91 & $6.42 \mathrm{E}-12$ & TI-208 & $->$ & $\mathrm{Pb}-208$ \\
\hline 726.63 & $2.31 \mathrm{E}-10$ & Ac- 228 & $->$ & Th-228 \\
\hline 727.25 & $3.75 \mathrm{E}-10$ & $\mathrm{Bi}-212$ & $->$ & Po-212 \\
\hline 737.7 & $1.09 \mathrm{E}-11$ & $A c-228$ & $->$ & Th-228 \\
\hline 748.58 & $1.36 \mathrm{E}-12$ & TI-208 & $->$ & $\mathrm{Pb}-208$ \\
\hline 755.28 & $3.51 \mathrm{E}-10$ & Ac- 228 & $->$ & Th-228 \\
\hline 763.06 & $5.19 \mathrm{E}-11$ & TI-208 & $->$ & $\mathrm{Pb}-208$ \\
\hline 772.28 & $2.90 \mathrm{E}-10$ & Ac- 228 & $->$ & Th-228 \\
\hline 774 & $2.39 \mathrm{E}-11$ & $A c-228$ & $->$ & Th-228 \\
\hline 782.12 & $1.57 \mathrm{E}-10$ & $A c-228$ & $->$ & Th-228 \\
\hline 785.51 & $6.24 \mathrm{E}-11$ & $\mathrm{Bi}-212$ & $->$ & Po-212 \\
\hline 791.05 & $5.58 \mathrm{E}-12$ & AC- 228 & $->$ & Th-228 \\
\hline 791.2 & $7.71 \mathrm{E}-12$ & $A c-228$ & $->$ & Th-228 \\
\hline 794.79 & $1.22 \mathrm{E}-09$ & $A c-228$ & $->$ & Th-228 \\
\hline 804.9 & $1.59 \mathrm{E}-13$ & Po-216 & $->$ & $\mathrm{Pb}-212$ \\
\hline 816.62 & $9.30 \mathrm{E}-12$ & Ac- 228 & $->$ & Th-228 \\
\hline 821.14 & $1.27 \mathrm{E}-12$ & TI-208 & $->$ & $\mathrm{Pb}-208$ \\
\hline 825.3 & $1.54 \mathrm{E}-11$ & $A c-228$ & $->$ & Th-228 \\
\hline 830.59 & $1.67 \mathrm{E}-10$ & Ac- 228 & $->$ & Th-228 \\
\hline 835.6 & $4.55 E-10$ & $A c-228$ & $->$ & Th-228 \\
\hline 840.44 & $2.50 \mathrm{E}-10$ & $A c-228$ & $->$ & Th-228 \\
\hline 853.6 & $3.72 \mathrm{E}-12$ & $A c-228$ & $->$ & Th-228 \\
\hline 860.3 & $3.80 \mathrm{E}-10$ & TI-208 & $->$ & $\mathrm{Pb}-208$ \\
\hline 870.47 & $1.75 \mathrm{E}-11$ & $A c-228$ & $->$ & Th-228 \\
\hline 873.94 & $4.87 E+01$ & Pu-240 & $->$ & $U-236$ \\
\hline 874.43 & $2.23 \mathrm{E}-11$ & $A c-228$ & $->$ & Th-228 \\
\hline 877.65 & $5.32 \mathrm{E}-12$ & $A c-228$ & $->$ & Th-228 \\
\hline 883.27 & $9.81 \mathrm{E}-13$ & TI-208 & $->$ & $\mathrm{Pb}-208$ \\
\hline 884 & $2.66 \mathrm{E}-11$ & Ac- 228 & $->$ & Th-228 \\
\hline 887.46 & $5.32 \mathrm{E}-12$ & $A c-228$ & $->$ & Th-228 \\
\hline 893.42 & 2.07E-11 & $\mathrm{Bi}-212$ & $->$ & Po-212 \\
\hline 904.29 & $2.37 \mathrm{E}-10$ & Ac- 228 & $->$ & Th-228 \\
\hline 911.16 & $7.71 \mathrm{E}-09$ & Ac- 228 & $->$ & Th-228 \\
\hline 919.19 & $7.44 \mathrm{E}-12$ & Ac- 228 & $->$ & Th-228 \\
\hline 922.28 & $5.58 \mathrm{E}-12$ & $A c-228$ & $->$ & Th-228 \\
\hline 927.42 & $3.96 \mathrm{E}-12$ & TI-208 & $->$ & $\mathrm{Pb}-208$ \\
\hline 931.1 & $3.99 \mathrm{E}-12$ & Ac- 228 & $->$ & Th-228 \\
\hline 943.9 & $2.84 \mathrm{E}-11$ & Ac- 228 & $->$ & Th-228 \\
\hline 948.4 & $3.24 \mathrm{E}-11$ & $A c-228$ & $->$ & Th-228 \\
\hline 952.17 & $9.93 \mathrm{E}-12$ & $\mathrm{Bi}-212$ & $->$ & Po-212 \\
\hline 958.3 & $8.24 \mathrm{E}-11$ & Ac- 228 & $->$ & Th-228 \\
\hline 964.64 & $1.54 \mathrm{E}-09$ & Ac- 228 & $->$ & Th-228 \\
\hline 968.97 & 4.63E-09 & Ac- 228 & $->$ & Th-228 \\
\hline 979.7 & $6.11 \mathrm{E}-12$ & Ac- 228 & $->$ & Th-228 \\
\hline 982.52 & $6.23 E-12$ & TI-208 & $->$ & $\mathrm{Pb}-208$ \\
\hline 987.87 & $9.04 \mathrm{E}-11$ & Ac- 228 & $->$ & Th-228 \\
\hline 987.94 & $5.05 \mathrm{E}-11$ & AC- 228 & $->$ & Th-228 \\
\hline 1016.12 & $6.65 \mathrm{E}-12$ & $A c-228$ & $->$ & Th-228 \\
\hline 1019.7 & $6.65 \mathrm{E}-12$ & Ac- 228 & $->$ & Th-228 \\
\hline 1033.32 & $4.12 \mathrm{E}-11$ & $A c-228$ & $->$ & Th-228 \\
\hline
\end{tabular}




\begin{tabular}{|c|c|c|c|c|}
\hline 1039.97 & $1.46 \mathrm{E}-11$ & $A c-228$ & $->$ & Th-228 \\
\hline 1041.35 & 8.51E-12 & $A c-228$ & $->$ & Th-228 \\
\hline 1054.3 & 8.51E-12 & Ac- 228 & $->$ & Th-228 \\
\hline 1065.05 & $4.55 \mathrm{E}-11$ & Ac- 228 & $->$ & Th-228 \\
\hline 1074.1 & $9.03 E-13$ & $\mathrm{Bi}-212$ & $->$ & Рo-212 \\
\hline 1078.69 & $3.02 \mathrm{E}-11$ & $\mathrm{Bi}-212$ & $->$ & Po-212 \\
\hline 1093.63 & $1.17 \mathrm{E}-11$ & TI-208 & $->$ & $\mathrm{Pb}-208$ \\
\hline 1095.87 & $3.51 \mathrm{E}-11$ & Ac- 228 & $->$ & Th-228 \\
\hline 1104.01 & $4.52 \mathrm{E}-12$ & $A c-228$ & $->$ & Th-228 \\
\hline 1110.7 & $1.03 \mathrm{E}-10$ & $A c-228$ & $->$ & Th-228 \\
\hline 1116.97 & $1.62 \mathrm{E}-11$ & Ac-228 & $->$ & Th-228 \\
\hline 1125.6 & $1.58 \mathrm{E}-13$ & TI-208 & $->$ & Pb-208 \\
\hline 1135.39 & $3.19 \mathrm{E}-12$ & $A c-228$ & $->$ & Th-228 \\
\hline 1142.7 & $2.66 \mathrm{E}-12$ & Ac- 228 & $->$ & Th-228 \\
\hline 1153.69 & $4.23 E-11$ & $A C-228$ & $->$ & Th-228 \\
\hline 1160.55 & $3.48 \mathrm{E}-13$ & Tl-208 & $->$ & $\mathrm{Pb}-208$ \\
\hline 1163.6 & $1.99 \mathrm{E}-11$ & $A c-228$ & $->$ & Th-228 \\
\hline 1174.79 & $7.71 \mathrm{E}-12$ & $A c-228$ & $->$ & Th-228 \\
\hline 1185.1 & $5.38 \mathrm{E}-13$ & TI-208 & $->$ & $\mathrm{Pb}-208$ \\
\hline 1216.4 & $6.91 \mathrm{E}-12$ & Ac- 228 & $->$ & Th-228 \\
\hline 1245.23 & $2.39 \mathrm{E}-11$ & $A c-228$ & $->$ & Th-228 \\
\hline 1246.6 & $1.52 \mathrm{E}-10$ & Ac-228 & $->$ & Th-228 \\
\hline 1249.81 & $1.59 \mathrm{E}-11$ & Ac- 228 & $->$ & Th-228 \\
\hline 1277.5 & $5.32 \mathrm{E}-12$ & $A c-228$ & $->$ & Th-228 \\
\hline 1282.7 & $1.65 \mathrm{E}-12$ & TI-208 & $->$ & Pb-208 \\
\hline 1286.6 & $3.16 \mathrm{E}-11$ & Ac-228 & $->$ & Th-228 \\
\hline 1309.6 & $5.58 \mathrm{E}-12$ & $A c-228$ & $->$ & Th-228 \\
\hline 1314.76 & $5.58 \mathrm{E}-12$ & $A c-228$ & $->$ & Th-228 \\
\hline 1347.6 & $3.46 \mathrm{E}-12$ & $A c-228$ & $->$ & Th-228 \\
\hline 1357.6 & 7.71E-12 & Ac- 228 & $->$ & Th-228 \\
\hline 1374.26 & $5.32 \mathrm{E}-12$ & Ac- 228 & $->$ & Th-228 \\
\hline 1381 & $2.22 \mathrm{E}-13$ & TI-208 & $->$ & $\mathrm{Pb}-208$ \\
\hline 1415.7 & $6.91 \mathrm{E}-12$ & Ac- 228 & $->$ & Th-228 \\
\hline 1431 & 8.51E-12 & $A c-228$ & $->$ & Th-228 \\
\hline 1451.2 & $4.78 \mathrm{E}-12$ & Ac-228 & $->$ & Th-228 \\
\hline 1459.19 & $2.82 \mathrm{E}-10$ & $A c-228$ & $->$ & Th-228 \\
\hline 1468.8 & $5.32 \mathrm{E}-12$ & $A c-228$ & $->$ & Th-228 \\
\hline 1481.2 & $5.32 \mathrm{E}-12$ & Ac- 228 & $->$ & Th-228 \\
\hline 1496 & $2.79 \mathrm{E}-10$ & Ac- 228 & $->$ & Th-228 \\
\hline 1501.44 & $1.54 \mathrm{E}-10$ & $A c-228$ & $->$ & Th-228 \\
\hline 1503.87 & 7.71E-12 & $A c-228$ & $->$ & Th-228 \\
\hline 1512.75 & $1.77 \mathrm{E}-11$ & Bi-212 & $->$ & Po-212 \\
\hline 1528.9 & $1.78 \mathrm{E}-11$ & Ac- 228 & $->$ & Th-228 \\
\hline 1537.4 & $1.30 \mathrm{E}-11$ & Ac- 228 & $->$ & Th-228 \\
\hline 1548.5 & $1.14 \mathrm{E}-11$ & Ac-228 & $->$ & Th-228 \\
\hline 1557 & $5.32 \mathrm{E}-11$ & $A c-228$ & $->$ & Th-228 \\
\hline 1572.03 & $1.33 \mathrm{E}-11$ & $A c-228$ & $->$ & Th-228 \\
\hline 1573.3 & $1.46 \mathrm{E}-11$ & Ac- 228 & $->$ & Th-228 \\
\hline 1580.3 & $1.89 \mathrm{E}-10$ & Ac- 228 & $->$ & Th-228 \\
\hline 1588.23 & $9.57 \mathrm{E}-10$ & Ac- 228 & $->$ & Th-228 \\
\hline
\end{tabular}




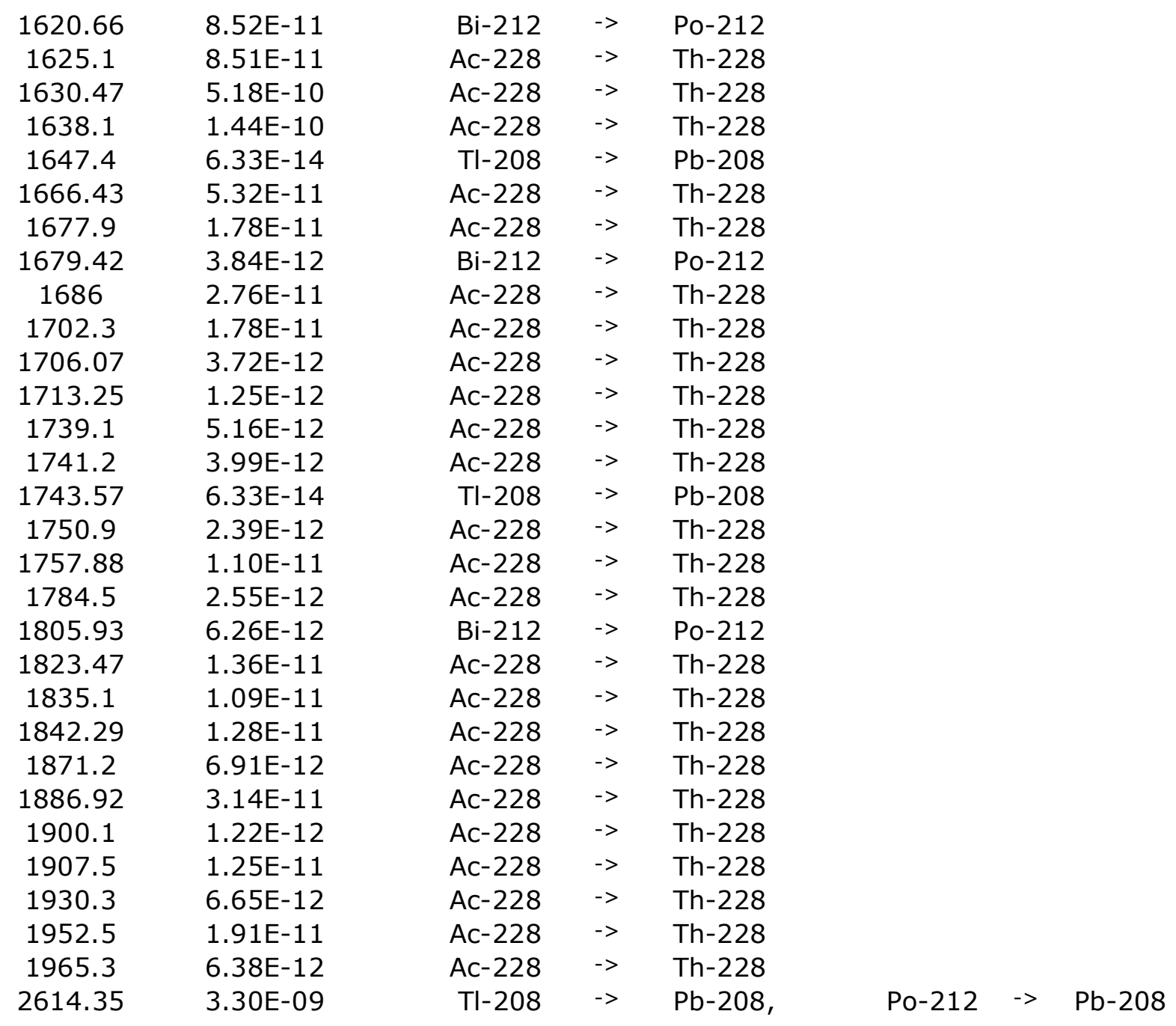

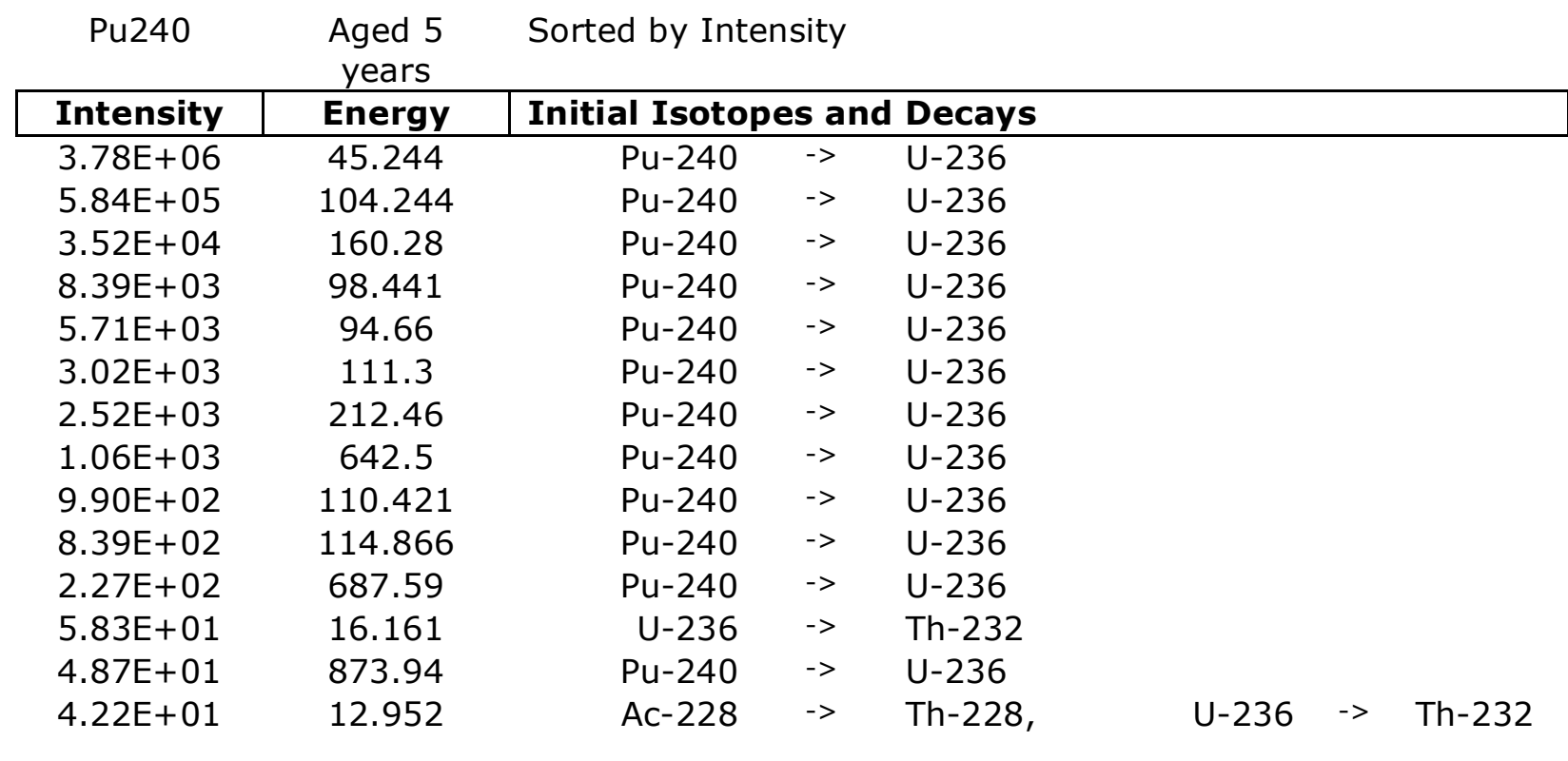




\begin{tabular}{|c|c|c|c|c|c|c|c|}
\hline $1.30 \mathrm{E}+01$ & 19.094 & $U-236$ & $->$ & Th-232 & & & \\
\hline $1.01 \mathrm{E}+01$ & 538.11 & Pu-240 & $->$ & $U-236$ & & & \\
\hline $2.48 \mathrm{E}+00$ & 11.118 & $A c-228$ & $->$ & Th-228, & $U-236$ & $->$ & Th-232 \\
\hline $1.18 \mathrm{E}+00$ & 14.511 & Ac- 228 & $->$ & Th-228, & $U-236$ & $->$ & Th-232 \\
\hline $9.68 \mathrm{E}-01$ & 49.369 & U-236 & $->$ & Th-232 & & & \\
\hline $2.36 \mathrm{E}-01$ & 112.75 & $U-236$ & $->$ & Th-232 & & & \\
\hline $2.66 \mathrm{E}-08$ & 13.5 & Ra-228 & $->$ & Ac- 228 & & & \\
\hline $1.20 \mathrm{E}-08$ & 16.18 & Ra-228 & $->$ & Ac- 228 & & & \\
\hline 7.71E-09 & 911.16 & Ac-228 & $->$ & Th-228 & & & \\
\hline 5.32E-09 & 16.154 & Ac- 228 & $->$ & Th-228 & & & \\
\hline 5.05E-09 & 12.76 & Ra-228 & $->$ & Ac- 228 & & & \\
\hline 4.63E-09 & 968.97 & Ac- 228 & $->$ & Th-228 & & & \\
\hline 3.84E-09 & 238.578 & $\mathrm{~Pb}-212$ & $->$ & $\mathrm{Bi}-212$ & & & \\
\hline $3.46 \mathrm{E}-09$ & 18.8 & Ra-228 & $->$ & $A c-228$ & & & \\
\hline $3.30 \mathrm{E}-09$ & 2614.35 & TI-208 & $->$ & $\mathrm{Pb}-208$, & Po-212 & $->$ & $\mathrm{Pb}-208$ \\
\hline 3.30E-09 & 338.42 & $A c-228$ & $->$ & Th-228 & & & \\
\hline 2.83E-09 & 583.022 & Tl-208 & $->$ & $\mathrm{Pb}-208$, & Po-212 & $->$ & $\mathrm{Pb}-208$ \\
\hline $2.66 \mathrm{E}-09$ & 15.15 & Ra-228 & $->$ & $A c-228$ & & & \\
\hline $1.56 \mathrm{E}-09$ & 77.107 & $\mathrm{~Pb}-212$ & $->$ & $\mathrm{Bi}-212$ & & & \\
\hline $1.54 \mathrm{E}-09$ & 964.64 & Ac- 228 & $->$ & Th-228 & & & \\
\hline 1.49E-09 & 93.35 & Ac- 228 & $->$ & Th-228 & & & \\
\hline $1.22 \mathrm{E}-09$ & 463.1 & $A c-228$ & $->$ & Th-228 & & & \\
\hline $1.22 \mathrm{E}-09$ & 794.79 & Ac- 228 & $->$ & Th-228 & & & \\
\hline $1.22 \mathrm{E}-09$ & 19.113 & Ac- 228 & $->$ & Th-228 & & & \\
\hline 1.09E-09 & 209.39 & $A c-228$ & $->$ & Th-228 & & & \\
\hline $1.01 \mathrm{E}-09$ & 270.26 & Ac- 228 & $->$ & Th-228 & & & \\
\hline $9.57 \mathrm{E}-10$ & 1588.23 & Ac- 228 & $->$ & Th-228 & & & \\
\hline $9.30 \mathrm{E}-10$ & 328.07 & Ac- 228 & $->$ & Th-228 & & & \\
\hline $9.25 \mathrm{E}-10$ & 74.814 & $\mathrm{~Pb}-212$ & $->$ & $\mathrm{Bi}-212$ & & & \\
\hline $9.04 \mathrm{E}-10$ & 89.955 & Ac- 228 & $->$ & Th-228 & & & \\
\hline $7.71 \mathrm{E}-10$ & 129.03 & Ac- 228 & $->$ & Th-228 & & & \\
\hline $6.84 \mathrm{E}-10$ & 510.606 & TI-208 & $->$ & $\mathrm{Pb}-208$ & & & \\
\hline $5.85 \mathrm{E}-10$ & 409.62 & Ac- 228 & $->$ & Th-228 & & & \\
\hline $5.81 \mathrm{E}-10$ & 10.828 & $\mathrm{~Pb}-212$ & $->$ & $\mathrm{Bi}-212$ & & & \\
\hline $5.52 \mathrm{E}-10$ & 87.19 & $\mathrm{~Pb}-212$ & $->$ & $\mathrm{Bi}-212$ & & & \\
\hline $5.32 \mathrm{E}-10$ & 105.362 & Ac- 228 & $->$ & Th-228 & & & \\
\hline $5.28 \mathrm{E}-10$ & 13.099 & $\mathrm{~Pb}-212$ & $->$ & $\mathrm{Bi}-212$ & & & \\
\hline $5.18 \mathrm{E}-10$ & 1630.47 & Ac- 228 & $->$ & Th-228 & & & \\
\hline $4.55 \mathrm{E}-10$ & 835.6 & Ac- 228 & $->$ & Th-228 & & & \\
\hline $4.19 \mathrm{E}-10$ & 15.23 & Th-228 & $->$ & Ra-224 & & & \\
\hline $3.80 \mathrm{E}-10$ & 860.3 & Tl-208 & $->$ & $\mathrm{Pb}-208$ & & & \\
\hline $3.75 \mathrm{E}-10$ & 727.25 & $\mathrm{Bi}-212$ & $->$ & Po-212 & & & \\
\hline $3.51 \mathrm{E}-10$ & 755.28 & Ac- 228 & $->$ & Th-228 & & & \\
\hline $3.46 \mathrm{E}-10$ & 99.55 & Ac- 228 & $->$ & Th-228 & & & \\
\hline $3.44 \mathrm{E}-10$ & 240.76 & Ra-224 & $->$ & $\mathrm{Rn}-220$ & & & \\
\hline $2.94 \mathrm{E}-10$ & 300.034 & $\mathrm{~Pb}-212$ & $->$ & $\mathrm{Bi}-212$ & & & \\
\hline $2.91 \mathrm{E}-10$ & 59 & Th-232 & $->$ & $\mathrm{Ra}-228$ & & & \\
\hline $2.90 \mathrm{E}-10$ & 772.28 & Ac- 228 & $->$ & Th-228 & & & \\
\hline $2.82 \mathrm{E}-10$ & 1459.19 & Ac- 228 & $->$ & Th-228 & & & \\
\hline $2.79 \mathrm{E}-10$ & 1496 & $A c-228$ & $->$ & Th-228 & & & \\
\hline
\end{tabular}




\begin{tabular}{|c|c|c|c|c|}
\hline $2.76 \mathrm{E}-10$ & 12.325 & Th-228 & $->$ & $\mathrm{Ra}-224$ \\
\hline $2.68 \mathrm{E}-10$ & 562.65 & $A c-228$ & $->$ & Th-228 \\
\hline $2.50 \mathrm{E}-10$ & 840.44 & Ac- 228 & $->$ & Th-228 \\
\hline $2.39 \mathrm{E}-10$ & 19.4 & $\mathrm{Ra}-228$ & $->$ & $A c-228$ \\
\hline $2.39 \mathrm{E}-10$ & 677.07 & $A c-228$ & $->$ & Th-228 \\
\hline $2.37 \mathrm{E}-10$ & 904.29 & Ac- 228 & $->$ & Th-228 \\
\hline $2.31 \mathrm{E}-10$ & 726.63 & Ac- 228 & $->$ & Th-228 \\
\hline $2.23 \mathrm{E}-10$ & 153.89 & $A c-228$ & $->$ & Th-228 \\
\hline $2.15 \mathrm{E}-10$ & 277.28 & TI-208 & $->$ & $\mathrm{Pb}-208$ \\
\hline $2.13 \mathrm{E}-10$ & 216.24 & Ac- 228 & $->$ & Th-228 \\
\hline $1.89 \mathrm{E}-10$ & 1580.3 & $A c-228$ & $->$ & Th-228 \\
\hline $1.78 \mathrm{E}-10$ & 108.99 & $A c-228$ & $->$ & Th-228 \\
\hline $1.67 \mathrm{E}-10$ & 830.59 & $A c-228$ & $->$ & Th-228 \\
\hline $1.64 \mathrm{E}-10$ & 90.128 & $\mathrm{~Pb}-212$ & $->$ & $\mathrm{Bi}-212$ \\
\hline $1.57 \mathrm{E}-10$ & 782.12 & Ac- 228 & $->$ & Th-228 \\
\hline $1.54 \mathrm{E}-10$ & 1501.44 & Ac- 228 & $->$ & Th-228 \\
\hline $1.52 \mathrm{E}-10$ & 1246.6 & Ac-228 & $->$ & Th-228 \\
\hline $1.44 \mathrm{E}-10$ & 1638.1 & $A c-228$ & $->$ & Th-228 \\
\hline $1.40 \mathrm{E}-10$ & 57.81 & Ac- 228 & $->$ & Th-228 \\
\hline $1.38 \mathrm{E}-10$ & 340.94 & $A c-228$ & $->$ & Th-228 \\
\hline $1.30 \mathrm{E}-10$ & 509.17 & $A c-228$ & $->$ & Th-228 \\
\hline $1.25 \mathrm{E}-10$ & 332.48 & Ac-228 & $->$ & Th-228 \\
\hline $1.14 \mathrm{E}-10$ & 74.969 & TI-208 & $->$ & $\mathrm{Pb}-208$ \\
\hline $1.11 \mathrm{E}-10$ & 12.282 & $\mathrm{Bi}-212$ & $->$ & TI-208 \\
\hline $1.08 \mathrm{E}-10$ & 84.26 & Th-228 & $->$ & Ra-224 \\
\hline $1.03 \mathrm{E}-10$ & 1110.7 & $A c-228$ & $->$ & Th-228 \\
\hline 9.95E-11 & 15.376 & $\mathrm{~Pb}-212$ & $->$ & $\mathrm{Bi}-212$ \\
\hline $9.62 \mathrm{E}-11$ & 10.259 & $\mathrm{Bi}-212$ & $->$ & TI-208 \\
\hline $9.44 \mathrm{E}-11$ & 17.952 & Th-228 & $->$ & $\mathrm{Ra}-224$ \\
\hline $9.04 \mathrm{E}-11$ & 987.87 & Ac-228 & $->$ & Th-228 \\
\hline $8.52 \mathrm{E}-11$ & 1620.66 & $\mathrm{Bi}-212$ & $->$ & Po-212 \\
\hline $8.51 \mathrm{E}-11$ & 1625.1 & Ac- 228 & $->$ & Th-228 \\
\hline $8.24 \mathrm{E}-11$ & 958.3 & Ac- 228 & $->$ & Th-228 \\
\hline 7.71E-11 & 146.06 & $A c-228$ & $->$ & Th-228 \\
\hline $7.44 \mathrm{E}-11$ & 199.54 & $A C-228$ & $->$ & Th-228 \\
\hline $6.77 \mathrm{E}-11$ & 72.803 & TI-208 & $->$ & $\mathrm{Pb}-208$ \\
\hline $6.65 \mathrm{E}-11$ & 321.9 & $A c-228$ & $->$ & Th-228 \\
\hline $6.58 \mathrm{E}-11$ & 124 & Th-232 & $->$ & Ra-228 \\
\hline $6.38 \mathrm{E}-11$ & 478.2 & $A c-228$ & $->$ & Th-228 \\
\hline $6.24 \mathrm{E}-11$ & 785.51 & $\mathrm{Bi}-212$ & $->$ & Po-212 \\
\hline $6.11 \mathrm{E}-11$ & 279.3 & Ac- 228 & $->$ & Th-228 \\
\hline $6.11 \mathrm{E}-11$ & 210.76 & Ac- 228 & $->$ & Th-228 \\
\hline $5.85 \mathrm{E}-11$ & 546.36 & $A c-228$ & $->$ & Th-228 \\
\hline $5.58 \mathrm{E}-11$ & 503.7 & Ac- 228 & $->$ & Th-228 \\
\hline $5.32 \mathrm{E}-11$ & 1557 & Ac- 228 & $->$ & Th-228 \\
\hline $5.32 \mathrm{E}-11$ & 1666.43 & Ac- 228 & $->$ & Th-228 \\
\hline $5.20 \mathrm{E}-11$ & 115.122 & $\mathrm{~Pb}-212$ & $->$ & $\mathrm{Bi}-212$ \\
\hline $5.19 \mathrm{E}-11$ & 763.06 & TI-208 & $->$ & $\mathrm{Pb}-208$ \\
\hline $5.05 \mathrm{E}-11$ & 701.8 & Ac- 228 & $->$ & Th-228 \\
\hline $5.05 \mathrm{E}-11$ & 570.2 & Ac- 228 & $->$ & Th-228 \\
\hline
\end{tabular}




\begin{tabular}{|c|c|c|c|c|}
\hline $5.05 \mathrm{E}-11$ & 572.5 & Ac- 228 & $->$ & Th-228 \\
\hline $5.05 \mathrm{E}-11$ & 987.94 & Ac- 228 & $->$ & Th-228 \\
\hline $4.55 E-11$ & 1065.05 & $A c-228$ & $->$ & Th-228 \\
\hline $4.23 E-11$ & 1153.69 & $A c-228$ & $->$ & Th-228 \\
\hline $4.17 \mathrm{E}-11$ & 204.37 & $A c-228$ & $->$ & Th-228 \\
\hline $4.12 \mathrm{E}-11$ & 1033.32 & Ac- 228 & $->$ & Th-228 \\
\hline $4.11 \mathrm{E}-11$ & 10.541 & TI-208 & $->$ & $\mathrm{Pb}-208$ \\
\hline $4.02 E-11$ & 84.789 & TI-208 & $->$ & $\mathrm{Pb}-208$ \\
\hline $3.99 E-11$ & 440.49 & Ac- 228 & $->$ & Th-228 \\
\hline 3.99E-11 & 707.49 & $A c-228$ & $->$ & Th-228 \\
\hline 3.99E-11 & 583.28 & $A c-228$ & $->$ & Th-228 \\
\hline $3.86 \mathrm{E}-11$ & 12.701 & TI-208 & $->$ & $\mathrm{Pb}-208$ \\
\hline $3.72 \mathrm{E}-11$ & 184.72 & Ac- 228 & $->$ & Th-228 \\
\hline $3.51 \mathrm{E}-11$ & 1095.87 & $A c-228$ & $->$ & Th-228 \\
\hline $3.48 \mathrm{E}-11$ & 39.846 & $\mathrm{Bi}-212$ & $->$ & TI-208 \\
\hline $3.46 \mathrm{E}-11$ & 327.67 & Ac -228 & $->$ & Th-228 \\
\hline $3.24 \mathrm{E}-11$ & 948.4 & $A c-228$ & $->$ & Th-228 \\
\hline $3.19 \mathrm{E}-11$ & 523.18 & $A c-228$ & $->$ & Th-228 \\
\hline $3.19 \mathrm{E}-11$ & 191.29 & Ac- 228 & $->$ & Th-228 \\
\hline $3.16 \mathrm{E}-11$ & 1286.6 & Ac- 228 & $->$ & Th-228 \\
\hline $3.14 \mathrm{E}-11$ & 1886.92 & Ac- 228 & $->$ & Th-228 \\
\hline $3.02 \mathrm{E}-11$ & 1078.69 & $\mathrm{Bi}-212$ & $->$ & Po-212 \\
\hline $2.99 \mathrm{E}-11$ & 9.419 & $\mathrm{~Pb}-212$ & $->$ & $\mathrm{Bi}-212$ \\
\hline $2.92 \mathrm{E}-11$ & 619.88 & Ac- 228 & $->$ & Th-228 \\
\hline $2.84 \mathrm{E}-11$ & 943.9 & Ac- 228 & $->$ & Th-228 \\
\hline $2.76 \mathrm{E}-11$ & 1686 & $A c-228$ & $->$ & Th-228 \\
\hline $2.68 \mathrm{E}-11$ & 651.44 & Ac- 228 & $->$ & Th-228 \\
\hline $2.66 \mathrm{E}-11$ & 673.86 & $A c-228$ & $->$ & Th-228 \\
\hline $2.66 \mathrm{E}-11$ & 884 & Ac- 228 & $->$ & Th-228 \\
\hline $2.60 \mathrm{E}-11$ & 14.563 & $\mathrm{Bi}-212$ & $->$ & TI-208 \\
\hline $2.53 \mathrm{E}-11$ & 252.45 & TI-208 & -> & Pb-208 \\
\hline $2.47 \mathrm{E}-11$ & 215.75 & Th-228 & $->$ & $\mathrm{Ra}-224$ \\
\hline $2.39 E-11$ & 774 & $A c-228$ & $->$ & Th-228 \\
\hline 2.39E-11 & 1245.23 & Ac- 228 & $->$ & Th-228 \\
\hline $2.39 \mathrm{E}-11$ & 615.9 & Ac- 228 & $->$ & Th-228 \\
\hline $2.37 \mathrm{E}-11$ & 282.02 & Ac- 228 & $->$ & Th-228 \\
\hline $2.23 \mathrm{E}-11$ & 874.43 & $A c-228$ & $->$ & Th-228 \\
\hline $2.07 E-11$ & 519.97 & Ac- 228 & $->$ & Th-228 \\
\hline $2.07 E-11$ & 893.42 & $\mathrm{Bi}-212$ & $->$ & Po-212 \\
\hline $1.99 \mathrm{E}-11$ & 1163.6 & Ac- 228 & $->$ & Th-228 \\
\hline $1.91 \mathrm{E}-11$ & 1952.5 & $A c-228$ & $->$ & Th-228 \\
\hline $1.78 \mathrm{E}-11$ & 1702.3 & Ac- 228 & $->$ & Th-228 \\
\hline $1.78 \mathrm{E}-11$ & 1677.9 & Ac- 228 & $->$ & Th-228 \\
\hline $1.78 \mathrm{E}-11$ & 1528.9 & Ac- 228 & $->$ & Th-228 \\
\hline $1.77 \mathrm{E}-11$ & 1512.75 & $\mathrm{Bi}-212$ & $->$ & Po-212 \\
\hline $1.76 \mathrm{E}-11$ & 83.787 & $\mathrm{Ra}-224$ & $->$ & $\mathrm{Rn}-220$ \\
\hline $1.75 \mathrm{E}-11$ & 870.47 & Ac- 228 & $->$ & Th-228 \\
\hline $1.75 \mathrm{E}-11$ & 223.72 & Ac- 228 & $->$ & Th-228 \\
\hline $1.73 \mathrm{E}-11$ & 449.57 & Ac- 228 & $->$ & Th-228 \\
\hline $1.62 \mathrm{E}-11$ & 1116.97 & Ac- 228 & $->$ & Th-228 \\
\hline
\end{tabular}




\begin{tabular}{|c|c|c|c|c|}
\hline $1.62 \mathrm{E}-11$ & 640.7 & Ac- 228 & $->$ & Th-228 \\
\hline $1.60 \mathrm{E}-11$ & 10.622 & Th-228 & $->$ & $\mathrm{Ra}-224$ \\
\hline $1.59 \mathrm{E}-11$ & 232.3 & Ac- 228 & $->$ & Th-228 \\
\hline $1.59 \mathrm{E}-11$ & 1249.81 & Ac- 228 & $->$ & Th-228 \\
\hline $1.59 \mathrm{E}-11$ & 14.336 & Ra-224 & $->$ & $\mathrm{Rn}-220$ \\
\hline $1.54 \mathrm{E}-11$ & 825.3 & $A c-228$ & $->$ & Th-228 \\
\hline $1.54 \mathrm{E}-11$ & 263.57 & Ac- 228 & $->$ & Th-228 \\
\hline $1.46 \mathrm{E}-11$ & 1573.3 & Ac- 228 & $->$ & Th-228 \\
\hline $1.46 \mathrm{E}-11$ & 1039.97 & Ac- 228 & $->$ & Th-228 \\
\hline $1.36 \mathrm{E}-11$ & 1823.47 & Ac- 228 & $->$ & Th-228 \\
\hline $1.33 \mathrm{E}-11$ & 460.84 & $A c-228$ & $->$ & Th-228 \\
\hline $1.33 \mathrm{E}-11$ & 1572.03 & $A c-228$ & $->$ & Th-228 \\
\hline $1.33 \mathrm{E}-11$ & 629.93 & Ac- 228 & $->$ & Th-228 \\
\hline $1.30 \mathrm{E}-11$ & 555.3 & Ac- 228 & $->$ & Th-228 \\
\hline $1.30 \mathrm{E}-11$ & 141.19 & Ac- 228 & $->$ & Th-228 \\
\hline $1.30 \mathrm{E}-11$ & 1537.4 & Ac- 228 & $->$ & Th-228 \\
\hline $1.28 \mathrm{E}-11$ & 1842.29 & Ac- 228 & $->$ & Th-228 \\
\hline $1.25 \mathrm{E}-11$ & 1907.5 & Ac- 228 & $->$ & Th-228 \\
\hline $1.22 \mathrm{E}-11$ & 666.4 & Ac- 228 & $->$ & Th-228 \\
\hline $1.17 \mathrm{E}-11$ & 1093.63 & TI-208 & $->$ & $\mathrm{Pb}-208$ \\
\hline $1.16 \mathrm{E}-11$ & 87.632 & TI-208 & $->$ & $P b-208$ \\
\hline $1.15 \mathrm{E}-11$ & 452.77 & $\mathrm{Bi}-212$ & $->$ & TI-208 \\
\hline $1.15 \mathrm{E}-11$ & 11.713 & Ra-224 & $->$ & $\mathrm{Rn}-220$ \\
\hline $1.14 \mathrm{E}-11$ & 1548.5 & Ac- 228 & $->$ & Th-228 \\
\hline $1.14 \mathrm{E}-11$ & 649.19 & Ac- 228 & $->$ & Th-228 \\
\hline $1.14 \mathrm{E}-11$ & 498.26 & Ac- 228 & $->$ & Th-228 \\
\hline $1.14 \mathrm{E}-11$ & 515.2 & Ac- 228 & $->$ & Th-228 \\
\hline $1.14 \mathrm{E}-11$ & 131.5 & Th-228 & $->$ & Ra-224 \\
\hline $1.10 \mathrm{E}-11$ & 1757.88 & Ac- 228 & $->$ & Th-228 \\
\hline $1.09 \mathrm{E}-11$ & 737.7 & Ac- 228 & $->$ & Th-228 \\
\hline $1.09 \mathrm{E}-11$ & 1835.1 & Ac- 228 & $->$ & Th-228 \\
\hline $1.08 \mathrm{E}-11$ & 288.08 & $\mathrm{Bi}-212$ & $->$ & TI-208 \\
\hline $1.06 \mathrm{E}-11$ & 81.067 & Ra-224 & $->$ & $R n-220$ \\
\hline $9.93 E-12$ & 952.17 & $\mathrm{Bi}-212$ & $->$ & Po-212 \\
\hline $9.84 \mathrm{E}-12$ & 399.2 & Ac- 228 & $->$ & Th-228 \\
\hline $9.81 E-12$ & 233.32 & TI-208 & $->$ & $P b-208$ \\
\hline $9.30 \mathrm{E}-12$ & 471.4 & Ac- 228 & $->$ & Th-228 \\
\hline $9.30 \mathrm{E}-12$ & 816.62 & Ac- 228 & $->$ & Th-228 \\
\hline $8.51 \mathrm{E}-12$ & 174.18 & Ac- 228 & $->$ & Th-228 \\
\hline $8.51 \mathrm{E}-12$ & 1041.35 & Ac- 228 & $->$ & Th-228 \\
\hline 8.51E-12 & 396.9 & Ac- 228 & $->$ & Th-228 \\
\hline $8.51 \mathrm{E}-12$ & 1431 & Ac- 228 & $->$ & Th-228 \\
\hline $8.51 \mathrm{E}-12$ & 1054.3 & Ac- 228 & $->$ & Th-228 \\
\hline $8.51 \mathrm{E}-12$ & 257.29 & $A C-228$ & $->$ & Th-228 \\
\hline 8.37E-12 & 13.662 & Th-228 & $->$ & Ra-224 \\
\hline $7.71 \mathrm{E}-12$ & 1174.79 & Ac- 228 & $->$ & Th-228 \\
\hline $7.71 \mathrm{E}-12$ & 1503.87 & Ac- 228 & $->$ & Th-228 \\
\hline 7.71E-12 & 1357.6 & Ac- 228 & $->$ & Th-228 \\
\hline 7.71E-12 & 540.5 & Ac- 228 & $->$ & Th-228 \\
\hline $7.71 \mathrm{E}-12$ & 791.2 & Ac- 228 & $->$ & Th-228 \\
\hline
\end{tabular}




\begin{tabular}{|c|c|c|c|c|}
\hline $7.71 \mathrm{E}-12$ & 474.32 & $A c-228$ & $->$ & Th-228 \\
\hline $7.66 \mathrm{E}-12$ & 11.712 & $\mathrm{~Pb}-212$ & $->$ & $\mathrm{Bi}-212$ \\
\hline $7.44 \mathrm{E}-12$ & 919.19 & Ac- 228 & $->$ & Th-228 \\
\hline $7.31 \mathrm{E}-12$ & 166.43 & Th-228 & $->$ & $\mathrm{Ra}-224$ \\
\hline 7.09E-12 & 14.884 & TI-208 & $->$ & $\mathrm{Pb}-208$ \\
\hline $6.91 \mathrm{E}-12$ & 1216.4 & Ac- 228 & $->$ & Th-228 \\
\hline $6.91 \mathrm{E}-12$ & 419.23 & $A c-228$ & $->$ & Th-228 \\
\hline $6.91 \mathrm{E}-12$ & 1415.7 & $A c-228$ & $->$ & Th-228 \\
\hline $6.91 \mathrm{E}-12$ & 1871.2 & Ac-228 & $->$ & Th-228 \\
\hline $6.65 E-12$ & 1019.7 & Ac- 228 & $->$ & Th-228 \\
\hline $6.65 \mathrm{E}-12$ & 1016.12 & $A c-228$ & $->$ & Th-228 \\
\hline $6.65 \mathrm{E}-12$ & 1930.3 & $A c-228$ & $->$ & Th-228 \\
\hline $6.42 \mathrm{E}-12$ & 721.91 & TI-208 & $->$ & $\mathrm{Pb}-208$ \\
\hline $6.38 \mathrm{E}-12$ & 1965.3 & Ac-228 & $->$ & Th-228 \\
\hline $6.35 \mathrm{E}-12$ & 94.677 & Ra-224 & $->$ & $\mathrm{Rn}-220$ \\
\hline $6.26 \mathrm{E}-12$ & 1805.93 & $\mathrm{Bi}-212$ & $->$ & Po-212 \\
\hline $6.23 E-12$ & 982.52 & TI-208 & $->$ & $\mathrm{Pb}-208$ \\
\hline $6.17 \mathrm{E}-12$ & 549.7 & $\mathrm{Rn}-220$ & $->$ & Po-216 \\
\hline $6.11 \mathrm{E}-12$ & 979.7 & Ac- 228 & $->$ & Th-228 \\
\hline $5.64 \mathrm{E}-12$ & 79.29 & $\mathrm{Bi}-212$ & $->$ & Po-212 \\
\hline $5.58 \mathrm{E}-12$ & 922.28 & $A c-228$ & $->$ & Th-228 \\
\hline $5.58 \mathrm{E}-12$ & 1309.6 & Ac-228 & $->$ & Th-228 \\
\hline $5.58 \mathrm{E}-12$ & 1314.76 & Ac- 228 & $->$ & Th-228 \\
\hline $5.58 \mathrm{E}-12$ & 791.05 & $A c-228$ & $->$ & Th-228 \\
\hline $5.38 \mathrm{E}-12$ & 211.31 & TI-208 & $->$ & $\mathrm{Pb}-208$ \\
\hline $5.32 \mathrm{E}-12$ & 356.83 & $A c-228$ & $->$ & Th-228 \\
\hline $5.32 \mathrm{E}-12$ & 887.46 & $A c-228$ & $->$ & Th-228 \\
\hline $5.32 \mathrm{E}-12$ & 877.65 & Ac-228 & $->$ & Th-228 \\
\hline $5.32 \mathrm{E}-12$ & 1277.5 & $A c-228$ & $->$ & Th-228 \\
\hline $5.32 \mathrm{E}-12$ & 1374.26 & $A c-228$ & $->$ & Th-228 \\
\hline $5.32 \mathrm{E}-12$ & 492.36 & Ac-228 & $->$ & Th-228 \\
\hline $5.32 \mathrm{E}-12$ & 1468.8 & Ac- 228 & $->$ & Th-228 \\
\hline $5.32 \mathrm{E}-12$ & 1481.2 & Ac- 228 & $->$ & Th-228 \\
\hline $5.16 \mathrm{E}-12$ & 1739.1 & $A c-228$ & $->$ & Th-228 \\
\hline $4.78 \mathrm{E}-12$ & 1451.2 & $A c-228$ & $->$ & Th-228 \\
\hline 4.75E-12 & 8.953 & $\mathrm{Bi}-212$ & $->$ & TI-208 \\
\hline $4.52 \mathrm{E}-12$ & 416.2 & $A c-228$ & $->$ & Th-228 \\
\hline $4.52 \mathrm{E}-12$ & 1104.01 & $A c-228$ & $->$ & Th-228 \\
\hline $4.52 \mathrm{E}-12$ & 135.68 & $A c-228$ & $->$ & Th-228 \\
\hline 4.49E-12 & 176.577 & $\mathrm{~Pb}-212$ & $->$ & $\mathrm{Bi}-212$ \\
\hline 4.34E-12 & 327.93 & $\mathrm{Bi}-212$ & $->$ & TI-208 \\
\hline $4.02 \mathrm{E}-12$ & 72.873 & $\mathrm{Bi}-212$ & $->$ & TI-208 \\
\hline 3.99E-12 & 388.9 & $A c-228$ & $->$ & Th-228 \\
\hline 3.99E-12 & 931.1 & Ac- 228 & $->$ & Th-228 \\
\hline 3.99E-12 & 1741.2 & Ac- 228 & $->$ & Th-228 \\
\hline $3.96 \mathrm{E}-12$ & 927.42 & TI-208 & $->$ & $\mathrm{Pb}-208$ \\
\hline $3.84 \mathrm{E}-12$ & 1679.42 & $\mathrm{Bi}-212$ & $->$ & Po-212 \\
\hline $3.72 \mathrm{E}-12$ & 1706.07 & Ac- 228 & $->$ & Th-228 \\
\hline $3.72 \mathrm{E}-12$ & 220.49 & Ac- 228 & $->$ & Th-228 \\
\hline $3.72 \mathrm{E}-12$ & 853.6 & Ac- 228 & $->$ & Th-228 \\
\hline
\end{tabular}




\begin{tabular}{|c|c|c|c|c|}
\hline $3.46 \mathrm{E}-12$ & 1347.6 & Ac-228 & $\rightarrow$ & Th-228 \\
\hline $3.44 \mathrm{E}-12$ & 16.881 & Ra-224 & $->$ & $\mathrm{Rn}-220$ \\
\hline $3.38 \mathrm{E}-12$ & 76.858 & $\mathrm{Bi}-212$ & $->$ & Po-212 \\
\hline $3.19 \mathrm{E}-12$ & 1135.39 & Ac- 228 & $->$ & Th-228 \\
\hline $2.66 \mathrm{E}-12$ & 1142.7 & $A c-228$ & $->$ & Th-228 \\
\hline $2.57 \mathrm{E}-12$ & 88.471 & Th-228 & $->$ & Ra-224 \\
\hline $2.55 \mathrm{E}-12$ & 1784.5 & Ac- 228 & $->$ & Th-228 \\
\hline $2.49 \mathrm{E}-12$ & 205.75 & Th-228 & $->$ & Ra-224 \\
\hline $2.47 \mathrm{E}-12$ & 415.156 & $\mathrm{~Pb}-212$ & $->$ & $\mathrm{Bi}-212$ \\
\hline $2.39 \mathrm{E}-12$ & 372.3 & $A c-228$ & $->$ & Th-228 \\
\hline $2.39 \mathrm{E}-12$ & 1750.9 & Ac-228 & $->$ & Th-228 \\
\hline 2.39E-12 & 481.8 & Ac- 228 & $->$ & Th-228 \\
\hline $2.37 \mathrm{E}-12$ & 70.832 & $\mathrm{Bi}-212$ & $->$ & TI-208 \\
\hline $2.31 \mathrm{E}-12$ & 13.501 & $\mathrm{Bi}-212$ & $->$ & Po-212 \\
\hline $2.31 \mathrm{E}-12$ & 11.119 & $\mathrm{Bi}-212$ & $->$ & Рo-212 \\
\hline $2.06 \mathrm{E}-12$ & 9.185 & TI-208 & $->$ & $\mathrm{Pb}-208$ \\
\hline $2.03 E-12$ & 97.907 & Ra-224 & $->$ & $\mathrm{Rn}-220$ \\
\hline $1.97 \mathrm{E}-12$ & 89.639 & $\mathrm{Bi}-212$ & $->$ & Po-212 \\
\hline $1.65 \mathrm{E}-12$ & 1282.7 & TI-208 & $->$ & $\mathrm{Pb}-208$ \\
\hline $1.58 \mathrm{E}-12$ & 473.36 & $\mathrm{Bi}-212$ & $->$ & TI-208 \\
\hline $1.58 \mathrm{E}-12$ & 485.78 & TI-208 & $->$ & $\mathrm{Pb}-208$ \\
\hline $1.56 \mathrm{E}-12$ & 85.429 & Th-228 & $->$ & $\mathrm{Ra}-224$ \\
\hline $1.42 \mathrm{E}-12$ & 82.434 & $\mathrm{Bi}-212$ & $->$ & TI-208 \\
\hline $1.36 \mathrm{E}-12$ & 748.58 & TI-208 & $->$ & $\mathrm{Pb}-208$ \\
\hline $1.27 \mathrm{E}-12$ & 587.82 & TI-208 & $->$ & $\mathrm{Pb}-208$ \\
\hline $1.27 \mathrm{E}-12$ & 821.14 & TI-208 & $->$ & $\mathrm{Pb}-208$ \\
\hline $1.25 \mathrm{E}-12$ & 1713.25 & $A c-228$ & $->$ & Th-228 \\
\hline $1.22 \mathrm{E}-12$ & 1900.1 & Ac- 228 & $->$ & Th-228 \\
\hline $1.14 \mathrm{E}-12$ & 650.14 & TI-208 & $->$ & Pb-208 \\
\hline $9.81 \mathrm{E}-13$ & 883.27 & TI-208 & $->$ & $\mathrm{Pb}-208$ \\
\hline $9.18 \mathrm{E}-13$ & 99.915 & Th-228 & $->$ & $\mathrm{Ra}-224$ \\
\hline $9.04 \mathrm{E}-13$ & 692.5 & Ac- 228 & $->$ & Th-228 \\
\hline $9.03 E-13$ & 1074.1 & $\mathrm{Bi}-212$ & $->$ & Po-212 \\
\hline 8.96E-13 & 10.994 & $\mathrm{Bi}-212$ & $->$ & TI-208 \\
\hline $7.94 \mathrm{E}-13$ & 290 & $\mathrm{Ra}-224$ & $->$ & $\mathrm{Rn}-220$ \\
\hline $6.96 \mathrm{E}-13$ & 705.24 & TI-208 & $->$ & $\mathrm{Pb}-208$ \\
\hline $6.44 \mathrm{E}-13$ & 10.137 & Ra-224 & $->$ & $\mathrm{Rn}-220$ \\
\hline $6.21 \mathrm{E}-13$ & 92.673 & $\mathrm{Bi}-212$ & $->$ & Po-212 \\
\hline $6.17 \mathrm{E}-13$ & 650.1 & Ra-224 & $->$ & Rn-220 \\
\hline $5.51 \mathrm{E}-13$ & 11.349 & TI-208 & $->$ & $\mathrm{Pb}-208$ \\
\hline $5.38 \mathrm{E}-13$ & 1185.1 & TI-208 & $->$ & $\mathrm{Pb}-208$ \\
\hline $4.51 \mathrm{E}-13$ & 15.869 & $\mathrm{Bi}-212$ & $->$ & Po-212 \\
\hline $4.43 E-13$ & 433.51 & $\mathrm{Bi}-212$ & $->$ & TI-208 \\
\hline $4.40 \mathrm{E}-13$ & 164.15 & $\mathrm{~Pb}-212$ & $->$ & $\mathrm{Bi}-212$ \\
\hline $3.96 \mathrm{E}-13$ & 85.185 & $\mathrm{Bi}-212$ & $->$ & TI-208 \\
\hline $3.53 E-13$ & 409.3 & Ra-224 & $->$ & $\mathrm{Rn}-220$ \\
\hline $3.48 \mathrm{E}-13$ & 1160.55 & TI-208 & $->$ & $\mathrm{Pb}-208$ \\
\hline $3.16 \mathrm{E}-13$ & 143.99 & $\mathrm{Bi}-212$ & $->$ & TI-208 \\
\hline $3.00 \mathrm{E}-13$ & 12.855 & Ra-224 & $->$ & $\mathrm{Rn}-220$ \\
\hline $2.99 \mathrm{E}-13$ & 103.341 & Th-228 & $->$ & Ra-224 \\
\hline
\end{tabular}




$\begin{array}{lccll}2.22 \mathrm{E}-13 & 1381 & \mathrm{Tl}-208 & \rightarrow & \mathrm{Pb}-208 \\ 1.93 \mathrm{E}-13 & 492.62 & \mathrm{Bi}-212 & \rightarrow & \mathrm{Tl}-208 \\ 1.59 \mathrm{E}-13 & 804.9 & \mathrm{Po}-216 & \rightarrow & \mathrm{Pb}-212 \\ 1.58 \mathrm{E}-13 & 164 & \mathrm{Bi}-212 & -> & \mathrm{Tl}-208 \\ 1.58 \mathrm{E}-13 & 1125.6 & \mathrm{Tl}-208 & \rightarrow & \mathrm{Pb}-208 \\ 1.18 \mathrm{E}-13 & 9.658 & \mathrm{Bi}-212 & -> & \mathrm{Po}-212 \\ 1.14 \mathrm{E}-13 & 620.3 & \mathrm{Bi}-212 & \rightarrow & \mathrm{Tl}-208 \\ 6.33 \mathrm{E}-14 & 1743.57 & \mathrm{Tl}-208 & -> & \mathrm{Pb}-208 \\ 6.33 \mathrm{E}-14 & 1647.4 & \mathrm{Tl}-208 & -> & \mathrm{Pb}-208 \\ 3.50 \mathrm{E}-14 & 12.085 & \mathrm{Bi}-212 & -> & \mathrm{Po}-212 \\ 2.50 \mathrm{E}-14 & 580.4 & \mathrm{Bi}-212 & -> & \mathrm{Tl}-208\end{array}$

Pu-241 100\%

Aged: 5 years

$538 \quad$ lines computed.

Sorted by energy

\begin{tabular}{|c|c|c|c|c|c|c|c|}
\hline \multirow{2}{*}{$\begin{array}{c}\text { Energy } \\
(\mathrm{keV})\end{array}$} & \multirow{2}{*}{$\begin{array}{l}\text { Intensity } \\
\text { (ph/s/gm) }\end{array}$} & \multicolumn{6}{|c|}{$\begin{array}{l}\text { Initial Isotopes and } \\
\text { Decays }\end{array}$} \\
\hline & & & & & & & \\
\hline 8.953 & $8.60 \mathrm{E}-13$ & $\mathrm{Bi}-213$ & $->$ & TI-209 & & & \\
\hline 9.185 & $7.11 \mathrm{E}-10$ & TI-209 & $->$ & Pb-209 & & & \\
\hline 9.658 & 4.37E-09 & $\mathrm{Bi}-213$ & $->$ & Po-213 & & & \\
\hline 9.897 & 7.27E-09 & Fr-221 & $->$ & At-217 & & & \\
\hline 10.259 & $1.87 \mathrm{E}-11$ & $\mathrm{Bi}-213$ & $->$ & TI-209 & & & \\
\hline 10.381 & 7.45E-08 & Ac- 225 & $->$ & Fr-221 & & & \\
\hline 10.541 & $1.38 \mathrm{E}-08$ & TI-209 & $->$ & $\mathrm{Pb}-209$ & & & \\
\hline 10.871 & $5.72 \mathrm{E}-08$ & $\mathrm{Ra}-225$ & $->$ & $A C-225$ & & & \\
\hline 10.994 & $2.24 \mathrm{E}-13$ & $\mathrm{Bi}-213$ & $->$ & TI-209 & & & \\
\hline 11.118 & $2.39 \mathrm{E}-04$ & U-233 & $->$ & Th-229 & & & \\
\hline 11.119 & $8.38 \mathrm{E}-08$ & $\mathrm{Bi}-213$ & $->$ & Po-213 & & & \\
\hline 11.349 & $1.91 \mathrm{E}-10$ & TI-209 & $->$ & Pb-209 & & & \\
\hline 11.372 & $2.63 E+02$ & Np-237 & $->$ & $\mathrm{Pa}-233$ & & & \\
\hline 11.414 & $1.37 \mathrm{E}-07$ & Fr-221 & $->$ & At-217 & & & \\
\hline 11.62 & $2.35 \mathrm{E}+02$ & $\mathrm{~Pa}-233$ & $->$ & U-233 & & & \\
\hline 11.871 & $2.20 E+08$ & U-237 & $->$ & Np-237, & Am-241 & $->$ & $\mathrm{Np}-237$ \\
\hline 12.017 & $1.35 \mathrm{E}-06$ & Ac- 225 & $->$ & Fr-221 & & & \\
\hline 12.085 & 1.13E-09 & $\mathrm{Bi}-213$ & $->$ & Po-213 & & & \\
\hline 12.311 & $1.50 \mathrm{E}-11$ & $\mathrm{Bi}-213$ & $->$ & Tl-209 & & & \\
\hline 12.466 & 2.89E-09 & Fr-221 & $->$ & At-217 & & & \\
\hline 12.636 & $9.83 \mathrm{E}-07$ & Ra-225 & $->$ & $A c-225$ & & & \\
\hline 12.703 & $1.31 \mathrm{E}-08$ & TI-209 & $->$ & Pb-209 & & & \\
\hline 12.952 & $4.15 \mathrm{E}-03$ & U-233 & $->$ & Th-229 & & & \\
\hline 13.255 & $2.77 \mathrm{E}-08$ & Ac- 225 & $->$ & Fr-221 & & & \\
\hline 13.274 & $4.39 E+03$ & Np-237 & $->$ & Pa-233 & & & \\
\hline 13.504 & $7.79 \mathrm{E}-08$ & $\mathrm{Bi}-213$ & $->$ & Po-213 & & & \\
\hline 13.6 & $3.95 E+03$ & $\mathrm{~Pa}-233$ & $->$ & U-233 & & & \\
\hline
\end{tabular}




\begin{tabular}{|c|c|c|c|c|c|c|c|}
\hline 13.804 & $7.30 E+04$ & $U-237$ & $\rightarrow$ & $\mathrm{Np}-237$ & & & \\
\hline 13.911 & $1.66 \mathrm{E}-07$ & Fr-221 & $->$ & At-217 & & & \\
\hline 13.927 & $3.53 E+09$ & $U-237$ & $->$ & Np-237, & Am-241 & $->$ & $\mathrm{Np}-237$ \\
\hline 14.082 & $1.63 \mathrm{E}-08$ & $\mathrm{Ra}-225$ & $->$ & $A c-225$ & & & \\
\hline 14.402 & $2.99 \mathrm{E}-12$ & $\mathrm{Bi}-213$ & $->$ & TI-209 & & & \\
\hline 14.511 & $9.10 \mathrm{E}-05$ & $U-233$ & $->$ & Th-229 & & & \\
\hline 14.772 & $1.66 \mathrm{E}-06$ & AC- 225 & $->$ & Fr-221 & & & \\
\hline 14.871 & $2.36 \mathrm{E}-09$ & TI-209 & $->$ & $\mathrm{Pb}-209$ & & & \\
\hline 14.953 & $1.08 \mathrm{E}+02$ & $\mathrm{~Np}-237$ & $->$ & Pa-233 & & & \\
\hline 15.4 & $5.05 E+01$ & $\mathrm{~Pa}-233$ & $->$ & U-233 & & & \\
\hline 15.662 & $1.09 \mathrm{E}-06$ & $\mathrm{Ra}-225$ & $->$ & Ac-225 & & & \\
\hline 15.861 & $8.94 \mathrm{E}+07$ & U-237 & $->$ & Np-237, & Am-241 & $\rightarrow$ & $\mathrm{Np}-237$ \\
\hline 15.878 & $1.49 \mathrm{E}-08$ & $\mathrm{Bi}-213$ & $->$ & Po-213 & & & \\
\hline 16.141 & $5.27 E-03$ & $U-233$ & $->$ & Th-229 & & & \\
\hline 16.361 & $3.45 \mathrm{E}-08$ & Fr-221 & $->$ & At-217 & & & \\
\hline 16.632 & $5.72 E+03$ & $\mathrm{~Np}-237$ & $->$ & $\mathrm{Pa}-233$ & & & \\
\hline 17.058 & $4.17 E+03$ & $\mathrm{~Pa}-233$ & $->$ & $U-233$ & & & \\
\hline 17.269 & $7.91 \mathrm{E}-01$ & $\mathrm{~Pa}-233$ & $->$ & $U-233$ & & & \\
\hline 17.349 & $3.18 \mathrm{E}-08$ & Th-229 & $->$ & $\mathrm{Ra}-225$ & & & \\
\hline 17.449 & $3.64 \mathrm{E}-07$ & $A c-225$ & $->$ & Fr-221 & & & \\
\hline 17.592 & $2.17 E+07$ & $U-237$ & $->$ & $\mathrm{Np}-237$ & & & \\
\hline 17.611 & $5.45 E+09$ & Am-241 & $->$ & $\mathrm{Np}-237$ & & & \\
\hline 18.593 & $2.50 \mathrm{E}-07$ & Ra-225 & $->$ & Ac- 225 & & & \\
\hline 19.148 & $1.28 \mathrm{E}-03$ & U-233 & $->$ & Th-229 & & & \\
\hline 19.718 & $1.33 E+03$ & $\mathrm{~Np}-237$ & $->$ & $\mathrm{Pa}-233$ & & & \\
\hline 20.42 & $9.88 \mathrm{E}+02$ & $\mathrm{~Pa}-233$ & $->$ & $U-233$ & & & \\
\hline 20.99 & $5.21 \mathrm{E}+06$ & U-237 & $->$ & $\mathrm{Np}-237$ & & & \\
\hline 20.997 & $1.40 \mathrm{E}+09$ & Am-241 & $->$ & $\mathrm{Np}-237$ & & & \\
\hline 25.304 & $1.93 \mathrm{E}-06$ & U-233 & $->$ & Th-229 & & & \\
\hline 25.373 & $6.74 \mathrm{E}-09$ & Th-229 & $->$ & Ra-225 & & & \\
\hline 25.98 & $2.60 \mathrm{E}-10$ & Ac- 225 & $->$ & Fr-221 & & & \\
\hline 26.3445 & $6.49 E+08$ & U-237 & $->$ & Np-237, & Am-241 & $->$ & $\mathrm{Np}-237$ \\
\hline 28.578 & $1.43 \mathrm{E}+01$ & $\mathrm{~Pa}-233$ & $->$ & $\mathrm{U}-233$ & & & \\
\hline 29.191 & $1.09 \mathrm{E}-05$ & U-233 & $->$ & Th-229 & & & \\
\hline 29.378 & $2.95 E+03$ & $\mathrm{~Np}-237$ & $->$ & Pa-233 & & & \\
\hline 31.24 & $4.31 \mathrm{E}-07$ & U-233 & $->$ & Th-229 & & & \\
\hline 31.37 & $7.68 \mathrm{E}-07$ & Th-229 & $->$ & $\mathrm{Ra}-225$ & & & \\
\hline 32.2 & $4.70 E+06$ & Am-241 & $->$ & $\mathrm{Np}-237$ & & & \\
\hline 32.27 & $1.58 \mathrm{E}-06$ & U-233 & $->$ & Th-229 & & & \\
\hline 33.192 & $3.25 E+07$ & $U-237$ & $->$ & Np-237, & Am-241 & $->$ & $\mathrm{Np}-237$ \\
\hline 36.65 & 2.60E-09 & Ac- 225 & $->$ & Fr-221 & & & \\
\hline 37.85 & $5.75 \mathrm{E}-07$ & U-233 & $->$ & Th-229 & & & \\
\hline 38.51 & $1.56 \mathrm{E}-09$ & Ac- 225 & $->$ & Fr-221 & & & \\
\hline 38.57 & $6.93 E+00$ & Pu-241 & $->$ & U-237 & & & \\
\hline 40.34 & 5.19E-06 & $\mathrm{Ra}-225$ & $->$ & Ac- 225 & & & \\
\hline 40.415 & $7.91 \mathrm{E}+00$ & $\mathrm{~Pa}-233$ & $->$ & $U-233$ & & & \\
\hline 41.718 & $2.86 \mathrm{E}+00$ & $\mathrm{~Pa}-233$ & $->$ & U-233 & & & \\
\hline 42.441 & $9.58 \mathrm{E}-05$ & U-233 & $->$ & Th-229 & & & \\
\hline 42.64 & $2.70 \mathrm{E}+05$ & $A m-241$ & $->$ & $\mathrm{Np}-237$ & & & \\
\hline 42.722 & $3.00 \mathrm{E}-08$ & Th-229 & $->$ & Ra-225 & & & \\
\hline
\end{tabular}




\begin{tabular}{|c|c|c|c|c|c|c|c|}
\hline 43.415 & $1.97 \mathrm{E}+07$ & $U-237$ & $->$ & $\mathrm{Np}-237$, & Am-241 & $->$ & Np-237 \\
\hline 44.2 & $3.02 E+00$ & Pu-241 & $->$ & $U-237$ & & & \\
\hline 44.86 & 6.04E-01 & Pu-241 & $->$ & $U-237$ & & & \\
\hline 46.57 & $3.04 \mathrm{E}+01$ & Np-237 & $->$ & $\mathrm{Pa}-233$ & & & \\
\hline 49.2 & $1.87 \mathrm{E}-09$ & Ac- 225 & $->$ & Fr-221 & & & \\
\hline 51.013 & $1.51 \mathrm{E}+05$ & U-237 & $->$ & Np-237, & Am-241 & $->$ & $N p-237$ \\
\hline 52.613 & $3.99 E-07$ & $U-233$ & $->$ & Th-229 & & & \\
\hline 53.559 & $6.86 \mathrm{E}-06$ & $\mathrm{U}-233$ & $->$ & Th-229 & & & \\
\hline 53.82 & 3.29E-09 & Ac-225 & $->$ & Fr-221 & & & \\
\hline 54.04 & $1.62 \mathrm{E}+05$ & Am-241 & $->$ & $\mathrm{Np}-237$ & & & \\
\hline 54.702 & $2.23 E-05$ & U-233 & $->$ & Th-229 & & & \\
\hline 55.528 & $5.40 \mathrm{E}+06$ & Am-241 & $->$ & $\mathrm{Np}-237$ & & & \\
\hline 56.32 & $1.81 \mathrm{E}+00$ & Pu-241 & $->$ & U-237 & & & \\
\hline 56.58 & $6.18 \mathrm{E}-08$ & Th-229 & $->$ & $\mathrm{Ra}-225$ & & & \\
\hline 56.76 & 7.04E-01 & Pu-241 & $->$ & U-237 & & & \\
\hline 57.149 & $8.92 \mathrm{E}+01$ & $\mathrm{~Np}-237$ & $->$ & $\mathrm{Pa}-233$ & & & \\
\hline 57.77 & $7.28 \mathrm{E}-10$ & $A c-225$ & $->$ & Fr-221 & & & \\
\hline 59.536 & $9.71 E+09$ & U-237 & $->$ & $\mathrm{Np}-237$, & Am-241 & $->$ & $\mathrm{Np}-237$ \\
\hline 62.66 & $2.75 E+00$ & $\mathrm{~Np}-237$ & $->$ & $\mathrm{Pa}-233$ & & & \\
\hline 62.93 & $9.53 E-08$ & $A c-225$ & $->$ & Fr-221 & & & \\
\hline 63.69 & $5.27 E-08$ & U-233 & $->$ & Th-229 & & & \\
\hline 63.95 & $3.66 \mathrm{E}+00$ & Np-237 & $->$ & $\mathrm{Pa}-233$ & & & \\
\hline 64.23 & $9.70 \mathrm{E}-09$ & Ac- 225 & $->$ & Fr-221 & & & \\
\hline 64.817 & 8.77E+05 & U-237 & $->$ & $\mathrm{Np}-237$ & Am-241 & $->$ & Np-237 \\
\hline 66.11 & $1.34 \mathrm{E}-06$ & U-233 & $->$ & Th-229 & & & \\
\hline 67.46 & $1.19 \mathrm{E}+05$ & Am-241 & $->$ & $\mathrm{Np}-237$ & & & \\
\hline 67.96 & 5.11E-07 & U-233 & $->$ & Th-229 & & & \\
\hline 68.14 & $1.87 \mathrm{E}-08$ & Th-229 & $->$ & Ra-225 & & & \\
\hline 68.88 & $2.06 \mathrm{E}-08$ & Th-229 & $->$ & $\mathrm{Ra}-225$ & & & \\
\hline 68.97 & $1.72 \mathrm{E}-07$ & U-233 & $->$ & Th-229 & & & \\
\hline 69.76 & $6.48 \mathrm{E}+06$ & Am-241 & $->$ & $\mathrm{Np}-237$ & & & \\
\hline 69.85 & 8.83E-10 & Ac-225 & $->$ & Fr-221 & & & \\
\hline 70.35 & $9.58 \mathrm{E}-07$ & U-233 & $->$ & Th-229 & & & \\
\hline 70.62 & $3.66 \mathrm{E}+00$ & $\mathrm{~Np}-237$ & $->$ & $\mathrm{Pa}-233$ & & & \\
\hline 70.832 & $2.99 \mathrm{E}-11$ & $\mathrm{Bi}-213$ & $->$ & TI-209 & & & \\
\hline 70.92 & $1.73 \mathrm{E}-09$ & $A c-225$ & $->$ & Fr-221 & & & \\
\hline 71.6 & $2.02 E+00$ & Pu-241 & $->$ & $U-237$ & & & \\
\hline 71.71 & $2.55 \mathrm{E}-09$ & Ac- 225 & $->$ & Fr-221 & & & \\
\hline 71.847 & $4.31 \mathrm{E}-06$ & U-233 & $->$ & Th-229 & & & \\
\hline 72.803 & $2.24 \mathrm{E}-08$ & TI-209 & $->$ & $\mathrm{Pb}-209$ & & & \\
\hline 72.821 & $9.42 \mathrm{E}-07$ & $U-233$ & $->$ & Th-229 & & & \\
\hline 72.873 & $4.86 \mathrm{E}-11$ & $\mathrm{Bi}-213$ & $->$ & TI-209 & & & \\
\hline 73.6 & 2.94E-09 & $A c-225$ & $->$ & Fr-221 & & & \\
\hline 73.84 & 5.61E-08 & Ac- 225 & $->$ & Fr-221 & & & \\
\hline 74.46 & $2.54 \mathrm{E}+00$ & $\mathrm{~Np}-237$ & $->$ & $\mathrm{Pa}-233$ & & & \\
\hline 74.5 & $2.39 \mathrm{E}-06$ & U-233 & $->$ & Th-229 & & & \\
\hline 74.9 & $6.24 \mathrm{E}-09$ & Ac- 225 & $->$ & Fr-221 & & & \\
\hline 74.969 & $3.78 \mathrm{E}-08$ & TI-209 & $->$ & $\mathrm{Pb}-209$ & & & \\
\hline 75.17 & $9.36 \mathrm{E}-08$ & Th-229 & $->$ & Ra-225 & & & \\
\hline 75.343 & $2.57 \mathrm{E}+02$ & Pa-233 & $->$ & U-233 & & & \\
\hline
\end{tabular}




\begin{tabular}{|c|c|c|c|c|c|c|c|}
\hline 75.83 & $2.97 E+04$ & Am-241 & $->$ & Np-237 & & & \\
\hline 76.32 & $6.23 E-07$ & $\mathrm{U}-233$ & $->$ & Th-229 & & & \\
\hline 76.858 & $1.32 \mathrm{E}-07$ & $\mathrm{Bi}-213$ & $->$ & Po-213 & & & \\
\hline 77.1 & $1.57 \mathrm{E}+01$ & $U-233$ & $->$ & Th-229, & Pu-241 & $->$ & $U-237$ \\
\hline 78.41 & $9.58 \mathrm{E}-08$ & $U-233$ & $->$ & Th-229 & & & \\
\hline 78.947 & $1.47 \mathrm{E}-07$ & Fr-221 & $->$ & At-217 & & & \\
\hline 79.29 & 2.19E-07 & $\mathrm{Bi}-213$ & $->$ & Po-213 & & & \\
\hline 81.517 & $2.44 \mathrm{E}-07$ & Fr-221 & $->$ & At-217 & & & \\
\hline 82.434 & $1.87 \mathrm{E}-11$ & $\mathrm{Bi}-213$ & $->$ & TI-209 & & & \\
\hline 82.9 & $2.60 \mathrm{E}-08$ & Ac- 225 & $->$ & Fr-221 & & & \\
\hline 82.957 & $2.87 \mathrm{E}-07$ & U-233 & $->$ & Th-229 & & & \\
\hline 83.229 & $2.18 \mathrm{E}-07$ & Ac- 225 & $->$ & Fr-221 & & & \\
\hline 84.27 & $1.20 \mathrm{E}-07$ & U-233 & $->$ & Th-229 & & & \\
\hline 84.789 & $1.35 \mathrm{E}-08$ & TI-209 & $->$ & Pb-209 & & & \\
\hline 85.185 & $4.86 \mathrm{E}-12$ & $\mathrm{Bi}-213$ & $->$ & TI-209 & & & \\
\hline 85.402 & $3.03 E-07$ & $U-233$ & $->$ & Th-229 & & & \\
\hline 86.105 & $3.60 \mathrm{E}-07$ & Ac- 225 & $->$ & Fr-221 & & & \\
\hline 86.23 & 7.12E-08 & Th-229 & $->$ & $\mathrm{Ra}-225$ & & & \\
\hline 86.44 & $5.62 \mathrm{E}-07$ & Th-229 & $->$ & $\mathrm{Ra}-225$ & & & \\
\hline 86.5 & $3.20 \mathrm{E}+03$ & Np-237 & $->$ & Pa-233 & & & \\
\hline 86.652 & $3.87 \mathrm{E}+02$ & Рa-233 & $->$ & U-233 & & & \\
\hline 86.74 & $2.08 \mathrm{E}-07$ & $\mathrm{U}-233$ & $->$ & Th-229 & & & \\
\hline 87.24 & 3.03E-07 & U-233 & $->$ & Th-229 & & & \\
\hline 87.39 & $5.02 \mathrm{E}-08$ & Ac- 225 & $->$ & Fr-221 & & & \\
\hline 87.632 & 3.85E-09 & TI-209 & $->$ & Pb-209 & & & \\
\hline 88.05 & $4.12 \mathrm{E}+01$ & Np-237 & $->$ & Pa-233 & & & \\
\hline 88.43 & $7.02 \mathrm{E}-07$ & U-233 & $->$ & Th-229 & & & \\
\hline 89.639 & 7.77E-08 & $\mathrm{Bi}-213$ & $->$ & Po-213 & & & \\
\hline 89.957 & $1.66 \mathrm{E}-05$ & $U-233$ & $->$ & Th-229 & & & \\
\hline 90.994 & 5.27E-07 & $U-233$ & $->$ & Th-229 & & & \\
\hline 92.136 & $8.66 \mathrm{E}-08$ & Fr-221 & $->$ & At-217 & & & \\
\hline 92.282 & $3.64 \mathrm{E}+02$ & $\mathrm{~Np}-237$ & $->$ & Pa-233 & & & \\
\hline 92.673 & $2.37 \mathrm{E}-08$ & $\mathrm{Bi}-213$ & $->$ & Po-213 & & & \\
\hline 93.36 & 2.70E-05 & $U-233$ & $->$ & Th-229 & & & \\
\hline 94.66 & $2.46 \mathrm{E}+03$ & Рa-233 & $->$ & U-233, & Pu-241 & $->$ & U-237 \\
\hline 94.723 & $1.74 \mathrm{E}+02$ & Np-237 & $->$ & Pa-233 & & & \\
\hline 94.87 & 2.77E-08 & Ac-225 & $->$ & Fr-221 & & & \\
\hline 95.265 & $2.68 \mathrm{E}-08$ & Fr-221 & $->$ & At-217 & & & \\
\hline 95.858 & $6.64 \mathrm{E}+02$ & $\mathrm{~Np}-237$ & $->$ & $\mathrm{Pa}-233$ & & & \\
\hline 96.17 & $5.20 \mathrm{E}-09$ & Ac- 225 & $->$ & Fr-221 & & & \\
\hline 96.215 & $2.23 \mathrm{E}-06$ & U-233 & $->$ & Th-229 & & & \\
\hline 96.67 & $1.27 \mathrm{E}+04$ & Am-241 & $->$ & Np-237 & & & \\
\hline 97.071 & $1.19 \mathrm{E}+07$ & U-237 & $->$ & Np-237, & Am-241 & $->$ & Np-237 \\
\hline 97.143 & 3.51E-05 & $U-233$ & $->$ & Th-229 & & & \\
\hline 97.2 & 3.29E-09 & Fr-221 & $->$ & At-217 & & & \\
\hline 97.272 & $1.30 \mathrm{E}-07$ & Ac-225 & $->$ & Fr-221 & & & \\
\hline 98.441 & $3.50 \mathrm{E}+02$ & Pu-241 & $->$ & U-237 & & & \\
\hline 98.443 & $3.51 E+03$ & Pa-233 & $->$ & U-233 & & & \\
\hline 98.951 & $5.48 \mathrm{E}+06$ & Am-241 & $->$ & $N p-237$ & & & \\
\hline 99.58 & $1.09 \mathrm{E}-07$ & AC-225 & $->$ & Fr-221 & & & \\
\hline
\end{tabular}




\begin{tabular}{|c|c|c|c|c|c|c|c|}
\hline 99.82 & $2.91 \mathrm{E}-07$ & $A c-225$ & $->$ & Fr-221 & & & \\
\hline 100.008 & $8.78 \mathrm{E}-08$ & U-233 & $->$ & Th-229 & & & \\
\hline 100.599 & $4.16 \mathrm{E}-08$ & Ac- 225 & $->$ & Fr-221 & & & \\
\hline 100.88 & $1.04 \mathrm{E}-08$ & Ac- 225 & $->$ & Fr-221 & & & \\
\hline 101.066 & $1.88 \mathrm{E}+07$ & $U-237$ & $->$ & Np-237, & Am-241 & $->$ & Np-237 \\
\hline 101.8 & $1.44 \mathrm{E}-07$ & $U-233$ & $->$ & Th-229 & & & \\
\hline 102.966 & $5.25 E+06$ & Am-241 & $->$ & $\mathrm{Np}-237$ & & & \\
\hline 103.375 & $1.60 \mathrm{E}-07$ & U-233 & $->$ & Th-229 & & & \\
\hline 103.44 & $1.56 \mathrm{E}-09$ & Ac- 225 & $->$ & Fr-221 & & & \\
\hline 103.68 & $7.11 \mathrm{E}+01$ & Pu-241 & $->$ & $U-237$ & & & \\
\hline 103.921 & $1.52 \mathrm{E}+02$ & $\mathrm{~Pa}-233$ & $->$ & $U-233$ & & & \\
\hline 105.362 & $9.74 \mathrm{E}-06$ & $U-233$ & $->$ & Th-229 & & & \\
\hline 106.13 & $1.28 \mathrm{E}+01$ & Np-237 & $->$ & $\mathrm{Pa}-233$ & & & \\
\hline 107.16 & $1.50 \mathrm{E}-07$ & Th-229 & $->$ & Ra-225 & & & \\
\hline 108.166 & $2.15 \mathrm{E}+02$ & $\mathrm{~Np}-237$ & $->$ & $\mathrm{Pa}-233$ & & & \\
\hline 108.36 & $4.68 \mathrm{E}-08$ & Ac- 225 & $->$ & Fr-221 & & & \\
\hline 108.69 & $1.67 \mathrm{E}+01$ & Np-237 & $->$ & $\mathrm{Pa}-233$ & & & \\
\hline 108.99 & $3.24 \mathrm{E}-06$ & U-233 & $->$ & Th-229 & & & \\
\hline 109.47 & 4.95E-07 & $U-233$ & $->$ & Th-229 & & & \\
\hline 110.421 & $4.26 \mathrm{E}+01$ & Pu-241 & $->$ & U-237 & & & \\
\hline 111.298 & $1.32 \mathrm{E}+03$ & Рa-233 & $->$ & $U-233$ & & & \\
\hline 111.3 & $1.16 \mathrm{E}+02$ & Pu-241 & $->$ & $U-237$ & & & \\
\hline 111.56 & $5.66 \mathrm{E}-08$ & Ac- 225 & $->$ & Fr-221 & & & \\
\hline 111.89 & $2.39 \mathrm{E}+00$ & Pu-241 & $->$ & $U-237$ & & & \\
\hline 111.897 & $7.09 E+01$ & Np-237 & $->$ & $\mathrm{Pa}-233$ & & & \\
\hline 111.935 & $7.50 E-07$ & U-233 & $->$ & Th-229 & & & \\
\hline 113.3 & $2.26 \mathrm{E}+06$ & $U-237$ & $->$ & Np-237, & Am-241 & $->$ & $\mathrm{Np}-237$ \\
\hline 114.23 & $4.36 \mathrm{E}+06$ & $U-237$ & $->$ & $\mathrm{Np}-237$ & Am-241 & $->$ & $\mathrm{Np}-237$ \\
\hline 114.28 & $3.99 \mathrm{E}-07$ & $U-233$ & $->$ & Th-229 & & & \\
\hline 114.333 & $1.16 \mathrm{E}+01$ & Pu-241 & $->$ & U-237 & & & \\
\hline 114.445 & $4.39 E+02$ & $\mathrm{~Pa}-233$ & $->$ & $U-233$ & & & \\
\hline 114.561 & $2.63 E+01$ & Pu-241 & $->$ & $U-237$ & & & \\
\hline 114.95 & $2.42 E+05$ & U-237 & $->$ & Np-237 & & & \\
\hline 115.19 & $5.72 E-01$ & Np-237 & $->$ & $\mathrm{Pa}-233$ & & & \\
\hline 115.4 & $9.68 \mathrm{E}+00$ & Pu-241 & $->$ & U-237 & & & \\
\hline 116.26 & $3.35 E-07$ & U-233 & $->$ & Th-229 & & & \\
\hline 117 & 3.03E-07 & TI-209 & $->$ & $\mathrm{Pb}-209$ & & & \\
\hline 117.155 & $4.47 \mathrm{E}-06$ & $U-233$ & $->$ & Th-229 & & & \\
\hline 117.34 & $5.71 E+05$ & $U-237$ & $->$ & Np-237 & & & \\
\hline 117.58 & $1.19 \mathrm{E}+06$ & U-237 & $->$ & Np-237, & $\mathrm{Am}-241$ & $->$ & $\mathrm{Np}-237$ \\
\hline 117.689 & $3.68 \mathrm{E}+01$ & Np-237 & $->$ & $\mathrm{Pa}-233$ & & & \\
\hline 118.43 & $5.95 \mathrm{E}+05$ & U-237 & $->$ & $\mathrm{Np}-237$ & & & \\
\hline 118.47 & $6.06 \mathrm{E}-09$ & Fr-221 & $->$ & At-217 & & & \\
\hline 118.97 & $5.11 \mathrm{E}-06$ & U-233 & $->$ & Th-229 & & & \\
\hline 119.92 & $1.04 \mathrm{E}-08$ & Ac- 225 & $->$ & Fr-221 & & & \\
\hline 120.81 & $3.35 \mathrm{E}-06$ & $U-233$ & $->$ & Th-229 & & & \\
\hline 121.2 & $4.84 \mathrm{E}-01$ & Pu-241 & $->$ & $U-237$ & & & \\
\hline 122.994 & $2.70 E+05$ & Am-241 & $->$ & Np-237 & & & \\
\hline 123.76 & $3.29 \mathrm{E}-08$ & Ac- 225 & $->$ & Fr-221 & & & \\
\hline 123.91 & $1.04 \mathrm{E}-06$ & U-233 & $->$ & Th-229 & & & \\
\hline
\end{tabular}




\begin{tabular}{|c|c|c|c|c|}
\hline 124.51 & $1.87 \mathrm{E}-07$ & Th-229 & $->$ & Ra-225 \\
\hline 124.72 & $1.12 \mathrm{E}-07$ & Th-229 & $->$ & Ra-225 \\
\hline 124.81 & 8.66E-09 & Ac- 225 & $->$ & Fr-221 \\
\hline 125.292 & $1.10 \mathrm{E}+06$ & Am-241 & $->$ & Np-237 \\
\hline 125.398 & $1.04 \mathrm{E}-07$ & U-233 & $->$ & Th-229 \\
\hline 126.23 & $2.43 E-09$ & Ac- 225 & $->$ & Fr-221 \\
\hline 129.19 & $7.45 \mathrm{E}-10$ & Ac- 225 & $->$ & Fr-221 \\
\hline 129.2 & $1.12 \mathrm{E}-07$ & U-233 & $->$ & Th-229 \\
\hline 131.09 & $1.94 \mathrm{E}+01$ & Np-237 & $->$ & $\mathrm{Pa}-233$ \\
\hline 131.2 & $5.27 E-08$ & U-233 & $->$ & Th-229 \\
\hline 131.92 & $6.18 \mathrm{E}-08$ & Th-229 & $->$ & $\mathrm{Ra}-225$ \\
\hline 134.23 & $1.53 \mathrm{E}+01$ & $\mathrm{~Np}-237$ & $->$ & $\mathrm{Pa}-233$ \\
\hline 135.01 & 6.93E-09 & Ac- 225 & $->$ & Fr-221 \\
\hline 135.328 & $3.51 \mathrm{E}-06$ & $U-233$ & $->$ & Th-229 \\
\hline 137.02 & $3.00 \mathrm{E}-07$ & Th-229 & $->$ & Ra-225 \\
\hline 138.2 & 3.46E-09 & Ac- 225 & $->$ & Fr-221 \\
\hline 138.5 & 3.19E-08 & $U-233$ & $->$ & Th-229 \\
\hline 139.53 & $1.44 \mathrm{E}+03$ & $\mathrm{Am}-241$ & $->$ & $\mathrm{Np}-237$ \\
\hline 139.76 & $1.68 \mathrm{E}-07$ & U-233 & $->$ & Th-229 \\
\hline 140.61 & $4.12 E+00$ & Np-237 & $->$ & Pa-233 \\
\hline 142.98 & 8.05E-08 & Th-229 & $->$ & $\mathrm{Ra}-225$ \\
\hline 143.227 & $8.92 E+01$ & Np-237 & $->$ & $\mathrm{Pa}-233$ \\
\hline 144.52 & 4.79E-07 & U-233 & $->$ & Th-229 \\
\hline 145.15 & $2.18 \mathrm{E}-08$ & Ac- 225 & $->$ & Fr-221 \\
\hline 145.286 & $2.55 \mathrm{E}-06$ & U-233 & $->$ & Th-229 \\
\hline 146.347 & $1.01 \mathrm{E}-05$ & $U-233$ & $->$ & Th-229 \\
\hline 146.557 & $1.24 \mathrm{E}+05$ & Am-241 & $->$ & $\mathrm{Np}-237$ \\
\hline 148.15 & $5.75 \mathrm{E}-07$ & $\mathrm{U}-233$ & $->$ & Th-229 \\
\hline 148.37 & $1.87 \mathrm{E}-07$ & Th-229 & $->$ & $\mathrm{Ra}-225$ \\
\hline 148.567 & $1.32 \mathrm{E}+02$ & Pu-241 & $->$ & $U-237$ \\
\hline 149.8 & $2.03 E-07$ & U-233 & $->$ & Th-229 \\
\hline 149.99 & $1.21 \mathrm{E}-08$ & Fr-221 & $->$ & At-217 \\
\hline 150.08 & $1.16 \mathrm{E}-07$ & Ac- 225 & $->$ & Fr-221 \\
\hline 150.11 & $2.16 \mathrm{E}+04$ & Am-241 & $->$ & $\mathrm{Np}-237$ \\
\hline 151.423 & $5.49 E+01$ & $N p-237$ & $->$ & $\mathrm{Pa}-233$ \\
\hline 152.64 & $6.41 \mathrm{E}-09$ & $A c-225$ & $->$ & Fr-221 \\
\hline 153.31 & $8.78 \mathrm{E}-08$ & U-233 & $->$ & Th-229 \\
\hline 153.72 & $1.58 \mathrm{E}+00$ & Np-237 & $->$ & $\mathrm{Pa}-233$ \\
\hline 153.93 & $2.65 E-08$ & Ac-225 & $->$ & Fr-221 \\
\hline 154.37 & $1.31 \mathrm{E}-07$ & Th-229 & $->$ & Ra-225 \\
\hline 154.4 & $2.16 \mathrm{E}+03$ & Am-241 & $->$ & $\mathrm{Np}-237$ \\
\hline 154.69 & $2.39 \mathrm{E}-07$ & $\mathrm{U}-233$ & $->$ & Th-229 \\
\hline 155.263 & $2.11 E+01$ & Np-237 & $->$ & $\mathrm{Pa}-233$ \\
\hline 155.989 & $9.26 \mathrm{E}-08$ & U-233 & $->$ & Th-229 \\
\hline 156.4 & $3.24 \mathrm{E}+03$ & Am-241 & $->$ & $\mathrm{Np}-237$ \\
\hline 156.46 & $1.87 \mathrm{E}-07$ & Th-229 & $->$ & $\mathrm{Ra}-225$ \\
\hline 157.24 & $5.37 E-08$ & Ac- 225 & $->$ & Fr-221 \\
\hline 158.48 & $3.78 \mathrm{E}+02$ & Am-241 & $->$ & $\mathrm{Np}-237$ \\
\hline 159.955 & $4.77 E+00$ & Pu-241 & $->$ & U-237 \\
\hline 161.7 & $2.43 E+03$ & Am-241 & $->$ & $\mathrm{Np}-237$ \\
\hline
\end{tabular}




\begin{tabular}{|c|c|c|c|c|c|c|c|}
\hline 162.51 & $8.47 E+00$ & Np-237 & $->$ & $\mathrm{Pa}-233$ & & & \\
\hline 162.52 & $1.20 \mathrm{E}-07$ & U-233 & $->$ & Th-229 & & & \\
\hline 164.512 & $1.05 E-05$ & U-233 & $->$ & Th-229 & & & \\
\hline 164.58 & $1.35 \mathrm{E}+06$ & $U-237$ & $->$ & Np-237, & Am-241 & $->$ & $\mathrm{Np}-237$ \\
\hline 165.493 & $6.23 \mathrm{E}-07$ & $U-233$ & $->$ & Th-229 & & & \\
\hline 165.92 & $6.48 \mathrm{E}+03$ & Am-241 & $->$ & $N p-237$ & & & \\
\hline 168.98 & $1.07 \mathrm{E}-07$ & U-233 & $->$ & Th-229 & & & \\
\hline 169.18 & $1.65 \mathrm{E}+01$ & $\mathrm{~Np}-237$ & $->$ & $\mathrm{Pa}-233$ & & & \\
\hline 169.56 & $4.53 E+04$ & Am-241 & $->$ & Np-237 & & & \\
\hline 170.67 & $4.35 E+00$ & $\mathrm{~Np}-237$ & $->$ & Pa-233 & & & \\
\hline 170.794 & $2.23 E-07$ & U-233 & $->$ & Th-229 & & & \\
\hline 170.91 & $1.56 \mathrm{E}-09$ & Ac- 225 & $->$ & Fr-221 & & & \\
\hline 171.29 & $1.21 \mathrm{E}-08$ & Fr-221 & $->$ & At-217 & & & \\
\hline 172.35 & 5.59E-08 & $U-233$ & $->$ & Th-229 & & & \\
\hline 172.56 & $1.56 \mathrm{E}+00$ & $\mathrm{~Np}-237$ & $->$ & $\mathrm{Pa}-233$ & & & \\
\hline 172.9 & $4.12 \mathrm{E}-08$ & Th-229 & $->$ & Ra-225 & & & \\
\hline 174.162 & 3.67E-07 & $U-233$ & $->$ & Th-229 & & & \\
\hline 175.09 & $4.86 \mathrm{E}+03$ & Am-241 & $->$ & $\mathrm{Np}-237$ & & & \\
\hline 176.09 & $4.58 \mathrm{E}+00$ & $\mathrm{~Np}-237$ & $->$ & $\mathrm{Pa}-233$ & & & \\
\hline 176.1 & $6.86 \mathrm{E}-08$ & U-233 & $->$ & Th-229 & & & \\
\hline 177.78 & 3.19E-08 & $U-233$ & $->$ & Th-229 & & & \\
\hline 178.39 & 2.77E-09 & Ac- 225 & $->$ & Fr-221 & & & \\
\hline 179.74 & $9.55 \mathrm{E}-08$ & Th-229 & $->$ & Ra-225 & & & \\
\hline 180.801 & $5.26 \mathrm{E}+00$ & Np-237 & $->$ & Pa-233 & & & \\
\hline 183.96 & 4.31E-08 & Th-229 & $->$ & Ra-225 & & & \\
\hline 184.17 & 3.99E-08 & U-233 & $->$ & Th-229 & & & \\
\hline 185.782 & $6.38 \mathrm{E}-08$ & $U-233$ & $->$ & Th-229 & & & \\
\hline 186.12 & $3.46 \mathrm{E}-09$ & Ac- 225 & $->$ & Fr-221 & & & \\
\hline 186.7 & $1.53 \mathrm{E}+00$ & $\mathrm{~Np}-237$ & $->$ & $\mathrm{Pa}-233$ & & & \\
\hline 187.942 & $3.19 \mathrm{E}-06$ & U-233 & $->$ & Th-229 & & & \\
\hline 187.99 & 7.97E-08 & $A c-225$ & $->$ & Fr-221 & & & \\
\hline 190.4 & $5.94 \mathrm{E}+02$ & Am-241 & $->$ & $N p-237$ & & & \\
\hline 191.46 & $6.18 \mathrm{E}+00$ & $\mathrm{~Np}-237$ & $->$ & $\mathrm{Pa}-233$ & & & \\
\hline 191.9 & $5.83 E+01$ & Am-241 & $->$ & $\mathrm{Np}-237$ & & & \\
\hline 192.12 & $6.38 \mathrm{E}-08$ & U-233 & $->$ & Th-229 & & & \\
\hline 193.29 & $1.17 \mathrm{E}+01$ & Np-237 & $->$ & $\mathrm{Pa}-233$ & & & \\
\hline 193.59 & $8.62 E-07$ & Th-229 & $->$ & Ra-225 & & & \\
\hline 194.74 & $1.12 \mathrm{E}+01$ & Np-237 & $->$ & $\mathrm{Pa}-233$ & & & \\
\hline 195.04 & $4.60 E+01$ & Np-237 & $->$ & $\mathrm{Pa}-233$ & & & \\
\hline 195.75 & $2.51 \mathrm{E}-08$ & Ac- 225 & $->$ & Fr-221 & & & \\
\hline 196.89 & $5.72 E+00$ & $\mathrm{~Np}-237$ & $->$ & $\mathrm{Pa}-233$ & & & \\
\hline 197 & $3.51 E+03$ & Am-241 & $->$ & $\mathrm{Np}-237$ & & & \\
\hline 198.72 & $3.46 \mathrm{E}-09$ & Ac- 225 & $->$ & Fr-221 & & & \\
\hline 200.17 & 8.01E-01 & $\mathrm{Np}-237$ & $->$ & $\mathrm{Pa}-233$ & & & \\
\hline 200.67 & $1.60 \mathrm{E}-09$ & U-233 & $->$ & Th-229 & & & \\
\hline 201.72 & $9.61 E+00$ & Np-237 & $->$ & $\mathrm{Pa}-233$ & & & \\
\hline 202.85 & 8.24E-01 & $\mathrm{Np}-237$ & $->$ & $\mathrm{Pa}-233$ & & & \\
\hline 203.9 & $7.83 E+02$ & Am-241 & $->$ & Np-237 & & & \\
\hline 205.99 & $1.04 \mathrm{E}-07$ & U-233 & $->$ & Th-229 & & & \\
\hline 208 & $1.59 E+07$ & $U-237$ & $->$ & $\mathrm{Np}-237$ & Am-241 & $->$ & $\mathrm{Np}-237$ \\
\hline
\end{tabular}




\begin{tabular}{|c|c|c|c|c|}
\hline 208.149 & $3.99 \mathrm{E}-06$ & U-233 & $->$ & Th-229 \\
\hline 209.19 & $3.55 \mathrm{E}+00$ & Np-237 & $->$ & $\mathrm{Pa}-233$ \\
\hline 210.94 & $6.18 \mathrm{E}-07$ & Th-229 & $->$ & $\mathrm{Ra}-225$ \\
\hline 212.29 & $2.23 \mathrm{E}-07$ & U-233 & $->$ & Th-229 \\
\hline 212.412 & $3.48 \mathrm{E}+01$ & $N p-237$ & $->$ & $\mathrm{Pa}-233$ \\
\hline 214.08 & $9.61 \mathrm{E}+00$ & Np-237 & $->$ & $\mathrm{Pa}-233$ \\
\hline 216.08 & $1.05 \mathrm{E}-06$ & U-233 & $->$ & Th-229 \\
\hline 216.2 & 5.89E-08 & $A c-225$ & $->$ & Fr-221 \\
\hline 216.86 & $7.28 \mathrm{E}-08$ & $A c-225$ & $->$ & Fr-221 \\
\hline 217.133 & 5.59E-06 & U-233 & $->$ & Th-229 \\
\hline 217.61 & 7.98E-08 & U-233 & $->$ & Th-229 \\
\hline 217.98 & $1.89 E-06$ & Fr-221 & $->$ & At-217 \\
\hline 218.15 & $2.62 \mathrm{E}-08$ & Th-229 & $->$ & $\mathrm{Ra}-225$ \\
\hline 219.424 & $2.39 \mathrm{E}-07$ & U-233 & $->$ & Th-229 \\
\hline 221.45 & $1.13 E+04$ & Am-241 & $->$ & $\mathrm{Np}-237$ \\
\hline 221.73 & $1.53 \mathrm{E}+04$ & U-237 & $->$ & $\mathrm{Np}-237$ \\
\hline 223.357 & $5.27 \mathrm{E}-08$ & U-233 & $->$ & Th-229 \\
\hline 224.64 & $1.39 \mathrm{E}-08$ & $A c-225$ & $->$ & Fr-221 \\
\hline 225 & $1.60 \mathrm{E}-08$ & U-233 & $->$ & Th-229 \\
\hline 226.74 & $1.60 \mathrm{E}-08$ & $U-233$ & $->$ & Th-229 \\
\hline 228.07 & 3.19E-08 & U-233 & $->$ & Th-229 \\
\hline 229.98 & $3.00 \mathrm{E}+00$ & $\mathrm{~Np}-237$ & $->$ & $\mathrm{Pa}-233$ \\
\hline 230.086 & $1.07 \mathrm{E}-07$ & U-233 & $->$ & Th-229 \\
\hline 232.86 & $1.24 \mathrm{E}+03$ & Am-241 & $->$ & $\mathrm{Np}-237$ \\
\hline 234.24 & $1.40 \mathrm{E}+04$ & U-237 & $->$ & $\mathrm{Np}-237$ \\
\hline 234.352 & $1.89 \mathrm{E}+02$ & Am-241 & $->$ & $\mathrm{Np}-237$ \\
\hline 236.32 & $6.74 \mathrm{E}-09$ & Th-229 & $->$ & $\mathrm{Ra}-225$ \\
\hline 236.39 & 7.98E-08 & U-233 & $->$ & Th-229 \\
\hline 237.95 & $1.46 \mathrm{E}+01$ & $\mathrm{~Np}-237$ & $->$ & $\mathrm{Pa}-233$ \\
\hline 240.364 & 6.07E-07 & U-233 & $->$ & Th-229 \\
\hline 240.76 & $2.25 \mathrm{E}-09$ & Ac-225 & $->$ & Fr-221 \\
\hline 242.4 & $8.10 E+03$ & Am-241 & $->$ & $\mathrm{Np}-237$ \\
\hline 245 & $8.10 \mathrm{E}+03$ & Am-241 & $->$ & $\mathrm{Np}-237$ \\
\hline 245.294 & 5.59E-06 & U-233 & $->$ & Th-229 \\
\hline 246.66 & $6.48 \mathrm{E}+02$ & Am-241 & $->$ & $\mathrm{Np}-237$ \\
\hline 248.25 & $1.19 \mathrm{E}+01$ & Pa-233 & $->$ & U-233 \\
\hline 248.661 & $7.18 \mathrm{E}-06$ & U-233 & $->$ & Th-229 \\
\hline 248.7 & $3.46 \mathrm{E}-09$ & $A c-225$ & $->$ & Fr-221 \\
\hline 248.91 & $1.10 \mathrm{E}+00$ & $\mathrm{~Np}-237$ & $->$ & $\mathrm{Pa}-233$ \\
\hline 249.1 & $1.08 \mathrm{E}+03$ & Am-241 & $->$ & $\mathrm{Np}-23$ \\
\hline 252.5 & 6.07E-08 & U-233 & $->$ & Th-229 \\
\hline 253.51 & $1.73 \mathrm{E}-08$ & Ac-225 & $->$ & Fr-221 \\
\hline 255.92 & $6.86 \mathrm{E}-08$ & U-233 & $->$ & Th-229 \\
\hline 256.98 & $1.53 \mathrm{E}+00$ & Np-237 & $->$ & $\mathrm{Pa}-23$ \\
\hline 258.46 & $1.19 \mathrm{E}+00$ & $\mathrm{~Pa}-233$ & $->$ & U-233 \\
\hline 259.3 & 2.87E-07 & U-233 & $->$ & Th-229 \\
\hline 260.4 & $1.72 \mathrm{E}-07$ & $\mathrm{U}-233$ & $->$ & Th-229 \\
\hline 260.9 & $2.70 E+02$ & Am-241 & $->$ & $\mathrm{Np}-237$ \\
\hline 261.88 & $4.95 \mathrm{E}-07$ & U-233 & $->$ & Th-22S \\
\hline 262.41 & $1.53 E+00$ & $\mathrm{~Np}-237$ & $->$ & $\mathrm{Pa}-23$ \\
\hline
\end{tabular}




\begin{tabular}{|c|c|c|c|c|c|c|c|}
\hline 264.87 & $2.43 E+03$ & Am-241 & $->$ & Np-237 & & & \\
\hline 267.54 & $5.22 \mathrm{E}+05$ & U-237 & $->$ & Np-237, & Am-241 & $->$ & $N p-237$ \\
\hline 268.619 & $3.99 E-07$ & $U-233$ & $->$ & Th-229 & & & \\
\hline 271.58 & $1.73 E+02$ & Am-241 & $->$ & $N p-237$ & & & \\
\hline 271.597 & $6.15 \mathrm{E}+01$ & $\mathrm{~Pa}-233$ & $->$ & $U-233$ & & & \\
\hline 272.27 & $1.01 \mathrm{E}-07$ & $U-233$ & $->$ & Th-229 & & & \\
\hline 274.692 & $7.50 E-07$ & $U-233$ & $->$ & Th-229 & & & \\
\hline 275.68 & $1.78 \mathrm{E}+03$ & Am-241 & $->$ & Np-237 & & & \\
\hline 278.059 & $1.95 \mathrm{E}-06$ & U-233 & $->$ & Th-229 & & & \\
\hline 279.27 & 6.06E-09 & Ac- 225 & $->$ & Fr-221 & & & \\
\hline 282.54 & $1.56 \mathrm{E}-09$ & Fr-221 & $->$ & At-217 & & & \\
\hline 284.22 & $1.60 \mathrm{E}-08$ & U-233 & $->$ & Th-229 & & & \\
\hline 285.5 & $1.73 \mathrm{E}-09$ & Ac- 225 & $->$ & Fr-221 & & & \\
\hline 287.949 & $1.72 \mathrm{E}-06$ & $U-233$ & $->$ & Th-229 & & & \\
\hline 291.21 & $8.37 E+02$ & Am-241 & $->$ & Np-237 & & & \\
\hline 291.317 & 8.30E-06 & U-233 & $->$ & Th-229 & & & \\
\hline 292.76 & $5.68 \mathrm{E}+03$ & $U-237$ & $->$ & Np-237, & Am-241 & $->$ & $\mathrm{Np}-237$ \\
\hline 292.79 & $7.45 \mathrm{E}-08$ & $\mathrm{Bi}-213$ & $->$ & Po-213 & & & \\
\hline 293.923 & $2.23 E-07$ & U-233 & $->$ & Th-229 & & & \\
\hline 294.9 & $1.48 \mathrm{E}+04$ & Am-241 & $->$ & $\mathrm{Np}-237$ & & & \\
\hline 295.2 & 3.67E-08 & U-233 & $->$ & Th-229 & & & \\
\hline 298.87 & $5.56 \mathrm{E}+00$ & Pa-233 & $->$ & U-233 & & & \\
\hline 300.175 & $1.36 \mathrm{E}+03$ & Pa-233 & $->$ & U-233 & & & \\
\hline 302.84 & $1.12 \mathrm{E}-07$ & U-233 & $->$ & Th-229 & & & \\
\hline 304.2 & $2.70 \mathrm{E}+02$ & Am-241 & $->$ & Np-237 & & & \\
\hline 309.37 & $1.17 \mathrm{E}-07$ & U-233 & $->$ & Th-229 & & & \\
\hline 310.3 & $4.05 E+03$ & Am-241 & $->$ & $N p-237$ & & & \\
\hline 311.38 & 4.47E-08 & U-233 & $->$ & Th-229 & & & \\
\hline 311.9 & $8.35 E+03$ & Pa-233 & $->$ & U-233 & & & \\
\hline 317.133 & $1.40 \mathrm{E}-05$ & U-233 & $->$ & Th-229 & & & \\
\hline 320.508 & 4.95E-06 & U-233 & $->$ & Th-229 & & & \\
\hline 322.54 & $4.10 \mathrm{E}+04$ & Am-241 & $->$ & $\mathrm{Np}-237$ & & & \\
\hline 323.321 & $1.34 \mathrm{E}-06$ & U-233 & $->$ & Th-229 & & & \\
\hline 323.81 & $6.92 \mathrm{E}-10$ & $\mathrm{Bi}-213$ & $->$ & TI-209 & & & \\
\hline 324.1 & 2.94E-09 & Fr-221 & $->$ & At-217 & & & \\
\hline 328.69 & $1.04 \mathrm{E}-07$ & U-233 & $->$ & Th-229 & & & \\
\hline 332.354 & $8.68 \mathrm{E}+05$ & $U-237$ & $->$ & $N p-237$ & & & \\
\hline 332.39 & $3.99 E+04$ & Am-241 & $->$ & $N p-237$ & & & \\
\hline 335.405 & $7.01 \mathrm{E}+04$ & U-237 & $->$ & Np-237 & & & \\
\hline 335.43 & $1.33 \mathrm{E}+05$ & Am-241 & $->$ & $\mathrm{Np}-237$ & & & \\
\hline 336.579 & $9.42 \mathrm{E}-07$ & U-233 & $->$ & Th-229 & & & \\
\hline 337.727 & $1.13 E+03$ & Am-241 & $->$ & $\mathrm{Np}-237$ & & & \\
\hline 337.75 & $6.22 \mathrm{E}+03$ & U-237 & $->$ & Np-237 & & & \\
\hline 339.64 & $1.20 \mathrm{E}-08$ & $U-233$ & $->$ & Th-229 & & & \\
\hline 340.45 & $1.19 \mathrm{E}+03$ & U-237 & $->$ & Np-237 & & & \\
\hline 340.59 & $9.23 \mathrm{E}+02$ & Pa-233 & $->$ & $U-233$ & & & \\
\hline 349.46 & $3.24 \mathrm{E}+02$ & Am-241 & $->$ & Np-237 & & & \\
\hline 351.782 & $7.18 \mathrm{E}-08$ & U-233 & $->$ & Th-229 & & & \\
\hline 354 & $9.26 \mathrm{E}-08$ & $U-233$ & $->$ & Th-229 & & & \\
\hline 358.36 & $3.24 \mathrm{E}+02$ & Am-241 & $->$ & Np-237 & & & \\
\hline
\end{tabular}




\begin{tabular}{|c|c|c|c|c|}
\hline 359.09 & $6.06 \mathrm{E}-09$ & Fr-221 & $->$ & At-217 \\
\hline 365.762 & $1.31 \mathrm{E}-06$ & U-233 & $->$ & Th-229 \\
\hline 368.605 & $3.10 \mathrm{E}+04$ & $U-237$ & $->$ & $\mathrm{Np}-237$ \\
\hline 368.61 & $5.85 E+04$ & Am-241 & $->$ & $\mathrm{Np}-237$ \\
\hline 370.933 & $1.42 E+04$ & Am-241 & $->$ & $\mathrm{Np}-237$ \\
\hline 370.934 & $7.98 \mathrm{E}+04$ & U-237 & $->$ & $\mathrm{Np}-237$ \\
\hline 375.518 & $1.27 E+02$ & Pa-233 & $->$ & U-233 \\
\hline 376.6 & $3.79 E+04$ & Am-241 & $->$ & $\mathrm{Np}-237$ \\
\hline 382.05 & $6.06 \mathrm{E}-09$ & Fr-221 & $->$ & At-217 \\
\hline 383.4 & $1.52 \mathrm{E}-07$ & U-233 & $->$ & Th-229 \\
\hline 383.744 & $7.61 E+03$ & Am-241 & $->$ & $\mathrm{Np}-237$ \\
\hline 384 & $3.19 \mathrm{E}-08$ & U-233 & $->$ & Th-229 \\
\hline 390.6 & $1.59 E+03$ & Am-241 & $->$ & $\mathrm{Np}-237$ \\
\hline 394.34 & $1.20 \mathrm{E}-08$ & U-233 & $->$ & Th-229 \\
\hline 396.65 & $1.32 \mathrm{E}-08$ & U-233 & $->$ & Th-229 \\
\hline 398.663 & $2.61 E+02$ & $\mathrm{~Pa}-233$ & $->$ & U-233 \\
\hline 402.37 & $1.32 \mathrm{E}-08$ & U-233 & $->$ & Th-229 \\
\hline 406.4 & $3.78 E+02$ & Am-241 & $->$ & $\mathrm{Np}-237$ \\
\hline 406.7 & 8.46E-09 & U-233 & $->$ & Th-229 \\
\hline 409.1 & $2.25 \mathrm{E}-08$ & Fr-221 & $->$ & At-217 \\
\hline 415.932 & $3.32 E+02$ & $\mathrm{~Pa}-233$ & $->$ & U-233 \\
\hline 416.4 & $1.60 \mathrm{E}-08$ & U-233 & $->$ & Th-229 \\
\hline 419.22 & $7.56 E+03$ & Am-241 & $->$ & $\mathrm{Np}-237$ \\
\hline 426.39 & $6.64 E+03$ & Am-241 & $->$ & $\mathrm{Np}-237$ \\
\hline 429.8 & $2.70 E+02$ & Am-241 & $->$ & $\mathrm{Np}-237$ \\
\hline 435.3 & $5.40 E+02$ & Am-241 & $->$ & $\mathrm{Np}-237$ \\
\hline 436.78 & 7.98E-09 & U-233 & $->$ & Th-229 \\
\hline 440.34 & $2.79 \mathrm{E}-06$ & $\mathrm{Bi}-213$ & $->$ & Po-213 \\
\hline 442.75 & $9.45 E+02$ & Am-241 & $->$ & $\mathrm{Np}-237$ \\
\hline 449.6 & $1.60 \mathrm{E}-08$ & U-233 & $->$ & Th-229 \\
\hline 452.41 & $6.48 E+02$ & Am-241 & $->$ & $\mathrm{Np}-237$ \\
\hline 452.43 & $1.91 \mathrm{E}-08$ & Ac- 225 & $->$ & Fr-221 \\
\hline 454.67 & $2.62 E+03$ & Am-241 & $->$ & $\mathrm{Np}-237$ \\
\hline 459.58 & $9.72 E+02$ & Am-241 & $->$ & $\mathrm{Np}-237$ \\
\hline 459.77 & $1.32 \mathrm{E}-08$ & U-233 & $->$ & Th-229 \\
\hline 467 & $3.03 E-07$ & TI-209 & $->$ & Pb-209 \\
\hline 468 & $7.83 E+02$ & Am-241 & $->$ & $\mathrm{Np}-237$ \\
\hline 471.3 & $2.39 \mathrm{E}-08$ & U-233 & $->$ & Th-229 \\
\hline 479 & $2.39 \mathrm{E}-08$ & U-233 & $->$ & Th-229 \\
\hline 480.5 & 5.20E-09 & $A c-225$ & $->$ & Fr-221 \\
\hline 484.05 & $6.86 \mathrm{E}-09$ & U-233 & $->$ & Th-229 \\
\hline 486.3 & $2.70 E+02$ & Am-241 & $->$ & $\mathrm{Np}-237$ \\
\hline 512 & $3.10 E+02$ & Am-241 & $->$ & $\mathrm{Np}-237$ \\
\hline 513.5 & $1.73 \mathrm{E}-09$ & Ac-225 & $->$ & Fr-221 \\
\hline 514.2 & $7.02 E+02$ & Am-241 & $->$ & $\mathrm{Np}-237$ \\
\hline 522 & $2.43 E+02$ & Am-241 & $->$ & $\mathrm{Np}-237$ \\
\hline 526.03 & $1.73 \mathrm{E}-09$ & Ac-225 & $->$ & Fr-221 \\
\hline 540.27 & 8.46E-09 & U-233 & $->$ & Th-229 \\
\hline 545.1 & 3.83E-09 & $U-233$ & $->$ & Th-229 \\
\hline 569.37 & 6.07E-09 & $U-233$ & $->$ & Th-229 \\
\hline
\end{tabular}




\begin{tabular}{|c|c|c|c|c|}
\hline 574.05 & $2.70 E+02$ & Am-241 & $->$ & $\mathrm{Np}-237$ \\
\hline 578.47 & 7.98E-09 & $\mathrm{U}-233$ & $->$ & Th-229 \\
\hline 582.6 & $5.40 E+01$ & Am-241 & $->$ & $\mathrm{Np}-237$ \\
\hline 586.5 & $3.51 \mathrm{E}+02$ & Am-241 & $->$ & $\mathrm{Np}-237$ \\
\hline 590.3 & $7.56 \mathrm{E}+02$ & Am-241 & $->$ & $\mathrm{Np}-237$ \\
\hline 597.41 & $2.54 E+03$ & Am-241 & $->$ & $\mathrm{Np}-237$ \\
\hline 619.011 & $1.59 E+04$ & Am-241 & $->$ & $\mathrm{Np}-237$ \\
\hline 620.87 & 3.67E-09 & U-233 & $->$ & Th-229 \\
\hline 627.2 & $1.51 \mathrm{E}+02$ & Am-241 & $->$ & $\mathrm{Np}-237$ \\
\hline 633 & $3.40 E+02$ & Am-241 & $->$ & $\mathrm{Np}-237$ \\
\hline 641.436 & $1.92 E+03$ & Am-241 & $->$ & $\mathrm{Np}-237$ \\
\hline 652.99 & $1.02 E+04$ & Am-241 & $->$ & $\mathrm{Np}-237$ \\
\hline 656.97 & 4.79E-09 & U-233 & $->$ & Th-229 \\
\hline 659.72 & $1.47 \mathrm{E}-08$ & $\mathrm{Bi}-213$ & $->$ & Po-213 \\
\hline 662.456 & $9.91 E+04$ & Am-241 & $->$ & $\mathrm{Np}-237$ \\
\hline 669.9 & $1.03 E+02$ & Am-241 & $->$ & $\mathrm{Np}-237$ \\
\hline 675.8 & $1.73 E+02$ & Am-241 & $->$ & $\mathrm{Np}-237$ \\
\hline 680.06 & $8.37 E+02$ & Am-241 & $->$ & $\mathrm{Np}-237$ \\
\hline 688.77 & $8.10 E+03$ & Am-241 & $->$ & $\mathrm{Np}-237$ \\
\hline 693.5 & $9.99 E+02$ & Am-241 & $->$ & $\mathrm{Np}-237$ \\
\hline 696.36 & $1.35 E+03$ & Am-241 & $->$ & $\mathrm{Np}-237$ \\
\hline 707.47 & $4.31 \mathrm{E}-09$ & U-233 & $->$ & Th-229 \\
\hline 710.95 & $1.73 E+03$ & Am-241 & $->$ & $\mathrm{Np}-237$ \\
\hline 721.962 & $1.62 E+04$ & Am-241 & $->$ & $\mathrm{Np}-237$ \\
\hline 722.7 & $3.51 E+04$ & Am-241 & $->$ & $\mathrm{Np}-237$ \\
\hline 729.5 & $3.51 E+02$ & Am-241 & $->$ & $\mathrm{Np}-237$ \\
\hline 731.5 & $1.27 E+02$ & Am-241 & $->$ & $\mathrm{Np}-237$ \\
\hline 737.3 & $2.16 E+03$ & Am-241 & $->$ & $\mathrm{Np}-237$ \\
\hline 755.89 & $2.05 E+03$ & Am-241 & $->$ & $\mathrm{Np}-237$ \\
\hline 759.5 & $4.59 E+02$ & Am-241 & $->$ & $\mathrm{Np}-237$ \\
\hline 763.4 & $5.34 \mathrm{E}+01$ & Am-241 & $->$ & $\mathrm{Np}-237$ \\
\hline 766.79 & $1.35 E+03$ & Am-241 & $->$ & $\mathrm{Np}-237$ \\
\hline 770.49 & $1.35 E+03$ & Am-241 & $->$ & $\mathrm{Np}-237$ \\
\hline 772.1 & $7.18 E+02$ & Am-241 & $->$ & $\mathrm{Np}-237$ \\
\hline 777.2 & $1.62 E+01$ & Am-241 & $->$ & $\mathrm{Np}-237$ \\
\hline 780.5 & $6.75 E+01$ & Am-241 & $->$ & $\mathrm{Np}-237$ \\
\hline 788.8 & $1.05 E+02$ & Am-241 & $->$ & $\mathrm{Np}-237$ \\
\hline 801.9 & $3.51 E+02$ & Am-241 & $->$ & $\mathrm{Np}-237$ \\
\hline 807.27 & $4.42 \mathrm{E}-08$ & $\mathrm{Bi}-213$ & $->$ & Po-213 \\
\hline 811.8 & $1.62 E+02$ & Am-241 & $->$ & $\mathrm{Np}-237$ \\
\hline 819.3 & $1.08 E+02$ & Am-241 & $->$ & $\mathrm{Np}-237$ \\
\hline 822.6 & $5.94 E+01$ & Am-241 & $->$ & $\mathrm{Np}-237$ \\
\hline 828.5 & $6.48 E+01$ & Am-241 & $->$ & $\mathrm{Np}-237$ \\
\hline 851.5 & $1.03 E+02$ & Am-241 & $->$ & $\mathrm{Np}-237$ \\
\hline 854.7 & $5.40 E+01$ & Am-241 & $->$ & $\mathrm{Np}-237$ \\
\hline 859.2 & $2.27 E+01$ & Am-241 & $->$ & $\mathrm{Np}-237$ \\
\hline 862.6 & $1.43 E+02$ & $A m-241$ & $->$ & $\mathrm{Np}-237$ \\
\hline 867.9 & $3.35 E-09$ & U-233 & $->$ & Th-229 \\
\hline 872.7 & $1.89 E+02$ & Am-241 & $->$ & $\mathrm{Np}-237$ \\
\hline 887.5 & $5.94 \mathrm{E}+01$ & Am-241 & $->$ & $\mathrm{Np}-237$ \\
\hline
\end{tabular}




$\begin{array}{ccccc}898.4 & 1.89 \mathrm{E}+01 & \mathrm{Am}-241 & -> & \mathrm{Np}-237 \\ 902.5 & 8.10 \mathrm{E}+01 & \mathrm{Am}-241 & -> & \mathrm{Np}-237 \\ 912.4 & 6.75 \mathrm{E}+01 & \mathrm{Am}-241 & -> & \mathrm{Np}-237 \\ 922.2 & 5.13 \mathrm{E}+01 & \mathrm{Am}-241 & -> & \mathrm{Np}-237 \\ 928.8 & 1.35 \mathrm{E}+01 & \mathrm{Am}-241 & -> & \mathrm{Np}-237 \\ 945.7 & 1.35 \mathrm{E}+01 & \mathrm{Am}-241 & -> & \mathrm{Np}-237 \\ 955.7 & 1.57 \mathrm{E}+02 & \mathrm{Am}-241 & -> & \mathrm{Np}-237 \\ 1003 & 1.32 \mathrm{E}-08 & \mathrm{U}-233 & -> & \mathrm{Th}-229 \\ 1100.06 & 4.78 \mathrm{E}-08 & \mathrm{Bi}-213 & -> & \mathrm{Po}-213 \\ 1119 & 1.32 \mathrm{E}-08 & \mathrm{U}-233 & -> & \mathrm{Th}-229 \\ 1566 & 3.67 \mathrm{E}-07 & \mathrm{Tl}-209 & -> & \mathrm{Pb}-209\end{array}$

\begin{tabular}{|c|c|c|c|c|c|c|c|}
\hline Pu241 & Aged 5 & \multicolumn{6}{|c|}{ Sorted by Intensity } \\
\hline Intensity & Energy & \multicolumn{6}{|c|}{$\begin{array}{l}\text { Initial Isotopes and } \\
\text { Decays }\end{array}$} \\
\hline$(\mathrm{ph} / \mathrm{s} / \mathrm{gm})$ & (keV) & & & & & & \\
\hline $9.71 E+09$ & 59.536 & U-237 & $->$ & Np-237, & Am-241 & $->$ & $\mathrm{Np}-237$ \\
\hline $5.45 E+09$ & 17.611 & Am-241 & $->$ & Np-237 & & & \\
\hline $3.53 E+09$ & 13.927 & U-237 & $->$ & Np-237, & Am-241 & $->$ & $\mathrm{Np}-237$ \\
\hline $1.40 \mathrm{E}+09$ & 20.997 & Am-241 & $->$ & $\mathrm{Np}-237$ & & & \\
\hline $6.49 \mathrm{E}+08$ & 26.3445 & $U-237$ & $->$ & $\mathrm{Np}-237$ & Am-241 & $->$ & $\mathrm{Np}-237$ \\
\hline $2.20 E+08$ & 11.871 & $U-237$ & $->$ & $\mathrm{Np}-237$ & Am-241 & $->$ & $\mathrm{Np}-237$ \\
\hline $8.94 \mathrm{E}+07$ & 15.861 & $U-237$ & $->$ & Np-237, & Am-241 & $->$ & $\mathrm{Np}-237$ \\
\hline $3.25 \mathrm{E}+07$ & 33.192 & $U-237$ & $->$ & $\mathrm{Np}-237$ & Am-241 & $->$ & $\mathrm{Np}-237$ \\
\hline $2.17 E+07$ & 17.592 & $U-237$ & $->$ & $\mathrm{Np}-237$ & & & \\
\hline $1.97 \mathrm{E}+07$ & 43.415 & $U-237$ & $->$ & Np-237, & Am-241 & $->$ & $\mathrm{Np}-237$ \\
\hline $1.88 \mathrm{E}+07$ & 101.066 & $U-237$ & $->$ & $\mathrm{Np}-237$ & Am-241 & $->$ & $\mathrm{Np}-237$ \\
\hline $1.59 \mathrm{E}+07$ & 208 & U-237 & $->$ & $\mathrm{Np}-237$, & Am-241 & $->$ & $\mathrm{Np}-237$ \\
\hline $1.19 \mathrm{E}+07$ & 97.071 & U-237 & $->$ & $\mathrm{Np}-237$ & Am-241 & $->$ & $\mathrm{Np}-237$ \\
\hline $6.48 \mathrm{E}+06$ & 69.76 & Am-241 & $->$ & $\mathrm{Np}-237$ & & & \\
\hline $5.48 \mathrm{E}+06$ & 98.951 & Am-241 & $->$ & $\mathrm{Np}-237$ & & & \\
\hline $5.40 \mathrm{E}+06$ & 55.528 & Am-241 & $->$ & $\mathrm{Np}-237$ & & & \\
\hline $5.25 E+06$ & 102.966 & Am-241 & $->$ & $\mathrm{Np}-237$ & & & \\
\hline $5.21 \mathrm{E}+06$ & 20.99 & U-237 & $->$ & $\mathrm{Np}-237$ & & & \\
\hline $4.70 E+06$ & 32.2 & Am-241 & $->$ & $\mathrm{Np}-237$ & & & \\
\hline $4.36 \mathrm{E}+06$ & 114.23 & U-237 & $->$ & $\mathrm{Np}-237$, & Am-241 & $->$ & $\mathrm{Np}-237$ \\
\hline $2.26 \mathrm{E}+06$ & 113.3 & U-237 & $->$ & $\mathrm{Np}-237$, & Am-241 & $->$ & $\mathrm{Np}-237$ \\
\hline $1.35 \mathrm{E}+06$ & 164.58 & U-237 & $->$ & Np-237, & Am-241 & $->$ & $\mathrm{Np}-237$ \\
\hline $1.19 \mathrm{E}+06$ & 117.58 & U-237 & $->$ & $\mathrm{Np}-237$ & Am-241 & $->$ & $\mathrm{Np}-237$ \\
\hline $1.10 \mathrm{E}+06$ & 125.292 & Am-241 & $->$ & $\mathrm{Np}-237$ & & & \\
\hline $8.77 E+05$ & 64.817 & U-237 & $->$ & Np-237, & Am-241 & $->$ & $\mathrm{Np}-237$ \\
\hline $8.68 \mathrm{E}+05$ & 332.354 & $U-237$ & $->$ & $\mathrm{Np}-237$ & & & \\
\hline $5.95 \mathrm{E}+05$ & 118.43 & U-237 & $->$ & $N p-237$ & & & \\
\hline $5.71 E+05$ & 117.34 & U-237 & $->$ & $\mathrm{Np}-237$ & & & \\
\hline $5.22 \mathrm{E}+05$ & 267.54 & U-237 & $->$ & $\mathrm{Np}-237$ & Am-241 & $->$ & $\mathrm{Np}-237$ \\
\hline $2.70 \mathrm{E}+05$ & 42.64 & Am-241 & $->$ & $\mathrm{Np}-237$ & & & \\
\hline
\end{tabular}




\begin{tabular}{|c|c|c|c|c|c|c|c|}
\hline $2.70 E+05$ & 122.994 & Am-241 & $->$ & $\mathrm{Np}-237$ & & & \\
\hline $2.42 E+05$ & 114.95 & U-237 & $->$ & Np-237 & & & \\
\hline $1.62 E+05$ & 54.04 & Am-241 & $->$ & Np-237 & & & \\
\hline $1.51 \mathrm{E}+05$ & 51.013 & U-237 & $->$ & $\mathrm{Np}-237$, & Am-241 & $->$ & $N p-237$ \\
\hline $1.33 \mathrm{E}+05$ & 335.43 & Am-241 & $->$ & $\mathrm{Np}-237$ & & & \\
\hline $1.24 \mathrm{E}+05$ & 146.557 & Am-241 & $->$ & $\mathrm{Np}-237$ & & & \\
\hline $1.19 E+05$ & 67.46 & Am-241 & $->$ & $\mathrm{Np}-237$ & & & \\
\hline $9.91 \mathrm{E}+04$ & 662.456 & Am-241 & $->$ & Np-237 & & & \\
\hline $7.98 E+04$ & 370.934 & U-237 & $->$ & $\mathrm{Np}-237$ & & & \\
\hline $7.30 E+04$ & 13.804 & U-237 & $->$ & $N p-237$ & & & \\
\hline $7.01 E+04$ & 335.405 & U-237 & $->$ & $\mathrm{Np}-237$ & & & \\
\hline $5.85 E+04$ & 368.61 & Am-241 & $->$ & Np-237 & & & \\
\hline $4.53 E+04$ & 169.56 & Am-241 & $->$ & $\mathrm{Np}-237$ & & & \\
\hline $4.10 \mathrm{E}+04$ & 322.54 & Am-241 & $->$ & $\mathrm{Np}-237$ & & & \\
\hline $3.99 E+04$ & 332.39 & Am-241 & $->$ & $\mathrm{Np}-237$ & & & \\
\hline $3.79 E+04$ & 376.6 & Am-241 & $->$ & $\mathrm{Np}-237$ & & & \\
\hline $3.51 E+04$ & 722.7 & Am-241 & $->$ & $\mathrm{Np}-237$ & & & \\
\hline $3.10 E+04$ & 368.605 & U-237 & $->$ & $\mathrm{Np}-237$ & & & \\
\hline $2.97 E+04$ & 75.83 & Am-241 & $->$ & Np-237 & & & \\
\hline $2.16 \mathrm{E}+04$ & 150.11 & Am-241 & $->$ & $\mathrm{Np}-237$ & & & \\
\hline $1.62 \mathrm{E}+04$ & 721.962 & Am-241 & $->$ & Np-237 & & & \\
\hline $1.59 E+04$ & 619.011 & Am-241 & $->$ & Np-237 & & & \\
\hline $1.53 \mathrm{E}+04$ & 221.73 & U-237 & $->$ & $\mathrm{Np}-237$ & & & \\
\hline $1.48 \mathrm{E}+04$ & 294.9 & Am-241 & $->$ & $\mathrm{Np}-237$ & & & \\
\hline $1.42 \mathrm{E}+04$ & 370.933 & Am-241 & $->$ & $\mathrm{Np}-237$ & & & \\
\hline $1.40 \mathrm{E}+04$ & 234.24 & U-237 & $->$ & $N p-237$ & & & \\
\hline $1.27 \mathrm{E}+04$ & 96.67 & Am-241 & $->$ & $\mathrm{Np}-237$ & & & \\
\hline $1.13 \mathrm{E}+04$ & 221.45 & Am-241 & $->$ & Np-237 & & & \\
\hline $1.02 \mathrm{E}+04$ & 652.99 & Am-241 & $->$ & $\mathrm{Np}-237$ & & & \\
\hline $8.35 E+03$ & 311.9 & $\mathrm{~Pa}-233$ & $->$ & U-233 & & & \\
\hline $8.10 E+03$ & 688.77 & Am-241 & $->$ & $\mathrm{Np}-237$ & & & \\
\hline $8.10 \mathrm{E}+03$ & 242.4 & Am-241 & $->$ & Np-237 & & & \\
\hline $8.10 E+03$ & 245 & Am-241 & $->$ & $\mathrm{Np}-237$ & & & \\
\hline $7.61 \mathrm{E}+03$ & 383.744 & Am-241 & $->$ & $\mathrm{Np}-237$ & & & \\
\hline $7.56 \mathrm{E}+03$ & 419.22 & Am-241 & $->$ & Np-237 & & & \\
\hline $6.64 E+03$ & 426.39 & Am-241 & $->$ & $\mathrm{Np}-237$ & & & \\
\hline $6.48 \mathrm{E}+03$ & 165.92 & Am-241 & $->$ & Np-237 & & & \\
\hline $6.22 E+03$ & 337.75 & U-237 & $->$ & $\mathrm{Np}-237$ & & & \\
\hline $5.72 E+03$ & 16.632 & $\mathrm{~Np}-237$ & $->$ & $\mathrm{Pa}-233$ & & & \\
\hline $5.68 \mathrm{E}+03$ & 292.76 & U-237 & $->$ & $\mathrm{Np}-237$ & Am-241 & $->$ & $\mathrm{Np}-237$ \\
\hline $4.86 \mathrm{E}+03$ & 175.09 & Am-241 & $->$ & $N p-237$ & & & \\
\hline $4.39 E+03$ & 13.274 & $\mathrm{~Np}-237$ & $->$ & $\mathrm{Pa}-233$ & & & \\
\hline $4.17 E+03$ & 17.058 & $\mathrm{~Pa}-233$ & $->$ & $U-233$ & & & \\
\hline $4.05 E+03$ & 310.3 & Am-241 & $->$ & $\mathrm{Np}-237$ & & & \\
\hline $3.95 E+03$ & 13.6 & $\mathrm{~Pa}-233$ & $->$ & U-233 & & & \\
\hline $3.51 E+03$ & 98.443 & $\mathrm{~Pa}-233$ & $->$ & $U-233$ & & & \\
\hline $3.51 E+03$ & 197 & Am-241 & $->$ & $\mathrm{Np}-237$ & & & \\
\hline $3.24 \mathrm{E}+03$ & 156.4 & Am-241 & $->$ & Np-237 & & & \\
\hline $3.20 \mathrm{E}+03$ & 86.5 & $\mathrm{~Np}-237$ & $->$ & $\mathrm{Pa}-233$ & & & \\
\hline $2.95 E+03$ & 29.378 & $\mathrm{~Np}-237$ & $->$ & Pa-233 & & & \\
\hline
\end{tabular}




\begin{tabular}{|c|c|c|c|c|c|c|c|}
\hline $2.62 E+03$ & 454.67 & Am-241 & $->$ & $\mathrm{Np}-237$ & & & \\
\hline $2.54 \mathrm{E}+03$ & 597.41 & Am-241 & $->$ & Np-237 & & & \\
\hline $2.46 \mathrm{E}+03$ & 94.66 & $\mathrm{~Pa}-233$ & $->$ & U-233, & Pu-241 & $->$ & U-237 \\
\hline $2.43 E+03$ & 264.87 & Am-241 & $->$ & $\mathrm{Np}-237$ & & & \\
\hline $2.43 E+03$ & 161.7 & Am-241 & $->$ & $\mathrm{Np}-237$ & & & \\
\hline $2.16 \mathrm{E}+03$ & 737.3 & Am-241 & $->$ & $\mathrm{Np}-237$ & & & \\
\hline $2.16 \mathrm{E}+03$ & 154.4 & Am-241 & $->$ & $\mathrm{Np}-237$ & & & \\
\hline $2.05 E+03$ & 755.89 & Am-241 & $->$ & $\mathrm{Np}-237$ & & & \\
\hline $1.92 E+03$ & 641.436 & Am-241 & $->$ & $\mathrm{Np}-237$ & & & \\
\hline $1.78 \mathrm{E}+03$ & 275.68 & Am-241 & $->$ & $\mathrm{Np}-237$ & & & \\
\hline $1.73 E+03$ & 710.95 & Am-241 & $->$ & Np-237 & & & \\
\hline $1.59 E+03$ & 390.6 & Am-241 & $->$ & $\mathrm{Np}-237$ & & & \\
\hline $1.44 \mathrm{E}+03$ & 139.53 & Am-241 & $->$ & $\mathrm{Np}-237$ & & & \\
\hline $1.36 \mathrm{E}+03$ & 300.175 & $\mathrm{~Pa}-233$ & $->$ & $U-233$ & & & \\
\hline $1.35 \mathrm{E}+03$ & 696.36 & Am-241 & $->$ & $\mathrm{Np}-237$ & & & \\
\hline $1.35 \mathrm{E}+03$ & 770.49 & Am-241 & $->$ & $\mathrm{Np}-237$ & & & \\
\hline $1.35 \mathrm{E}+03$ & 766.79 & Am-241 & $->$ & $\mathrm{Np}-237$ & & & \\
\hline $1.33 E+03$ & 19.718 & $\mathrm{~Np}-237$ & $->$ & $\mathrm{Pa}-233$ & & & \\
\hline $1.32 \mathrm{E}+03$ & 111.298 & $\mathrm{~Pa}-233$ & $->$ & $U-233$ & & & \\
\hline $1.24 \mathrm{E}+03$ & 232.86 & Am-241 & $->$ & $\mathrm{Np}-237$ & & & \\
\hline $1.19 \mathrm{E}+03$ & 340.45 & U-237 & $->$ & $\mathrm{Np}-237$ & & & \\
\hline $1.13 E+03$ & 337.727 & Am-241 & $->$ & $\mathrm{Np}-237$ & & & \\
\hline $1.08 \mathrm{E}+03$ & 249.1 & Am-241 & $->$ & Np-237 & & & \\
\hline $9.99 E+02$ & 693.5 & Am-241 & $->$ & Np-237 & & & \\
\hline $9.88 \mathrm{E}+02$ & 20.42 & $\mathrm{~Pa}-233$ & $->$ & U-233 & & & \\
\hline $9.72 E+02$ & 459.58 & Am-241 & $->$ & $\mathrm{Np}-237$ & & & \\
\hline $9.45 E+02$ & 442.75 & Am-241 & $->$ & $\mathrm{Np}-237$ & & & \\
\hline $9.23 E+02$ & 340.59 & $\mathrm{~Pa}-233$ & $->$ & $U-233$ & & & \\
\hline $8.37 E+02$ & 291.21 & Am-241 & $->$ & $\mathrm{Np}-237$ & & & \\
\hline $8.37 \mathrm{E}+02$ & 680.06 & Am-241 & $->$ & Np-237 & & & \\
\hline $7.83 E+02$ & 468 & Am-241 & $->$ & $N p-237$ & & & \\
\hline $7.83 E+02$ & 203.9 & Am-241 & $->$ & $\mathrm{Np}-237$ & & & \\
\hline $7.56 \mathrm{E}+02$ & 590.3 & Am-241 & $->$ & $\mathrm{Np}-237$ & & & \\
\hline $7.18 \mathrm{E}+02$ & 772.1 & Am-241 & $->$ & $\mathrm{Np}-237$ & & & \\
\hline $7.02 E+02$ & 514.2 & Am-241 & $->$ & $\mathrm{Np}-237$ & & & \\
\hline $6.64 \mathrm{E}+02$ & 95.858 & $\mathrm{~Np}-237$ & $->$ & $\mathrm{Pa}-233$ & & & \\
\hline $6.48 \mathrm{E}+02$ & 246.66 & Am-241 & $->$ & $\mathrm{Np}-237$ & & & \\
\hline $6.48 \mathrm{E}+02$ & 452.41 & Am-241 & $->$ & $\mathrm{Np}-237$ & & & \\
\hline $5.94 \mathrm{E}+02$ & 190.4 & Am-241 & $->$ & $\mathrm{Np}-237$ & & & \\
\hline $5.40 E+02$ & 435.3 & Am-241 & $->$ & $\mathrm{Np}-237$ & & & \\
\hline $4.59 \mathrm{E}+02$ & 759.5 & Am-241 & $->$ & $\mathrm{Np}-237$ & & & \\
\hline $4.39 E+02$ & 114.445 & $\mathrm{~Pa}-233$ & $->$ & $U-233$ & & & \\
\hline $3.87 E+02$ & 86.652 & $\mathrm{~Pa}-233$ & $->$ & $U-233$ & & & \\
\hline $3.78 \mathrm{E}+02$ & 406.4 & Am-241 & $->$ & $\mathrm{Np}-237$ & & & \\
\hline $3.78 \mathrm{E}+02$ & 158.48 & Am-241 & $->$ & $\mathrm{Np}-237$ & & & \\
\hline $3.64 \mathrm{E}+02$ & 92.282 & $\mathrm{~Np}-237$ & $->$ & $\mathrm{Pa}-233$ & & & \\
\hline $3.51 \mathrm{E}+02$ & 729.5 & Am-241 & $->$ & $\mathrm{Np}-237$ & & & \\
\hline $3.51 E+02$ & 586.5 & Am-241 & $->$ & Np-237 & & & \\
\hline $3.51 E+02$ & 801.9 & Am-241 & $->$ & $\mathrm{Np}-237$ & & & \\
\hline $3.50 \mathrm{E}+02$ & 98.441 & Pu-241 & $->$ & U-237 & & & \\
\hline
\end{tabular}




\begin{tabular}{|c|c|c|c|c|}
\hline $3.40 \mathrm{E}+02$ & 633 & Am-241 & $->$ & $\mathrm{Np}-237$ \\
\hline $3.32 E+02$ & 415.932 & $\mathrm{~Pa}-233$ & $->$ & U-233 \\
\hline $3.24 \mathrm{E}+02$ & 349.46 & Am-241 & $->$ & $\mathrm{Np}-237$ \\
\hline $3.24 \mathrm{E}+02$ & 358.36 & Am-241 & $->$ & Np-237 \\
\hline $3.10 \mathrm{E}+02$ & 512 & Am-241 & $->$ & $N p-237$ \\
\hline $2.70 E+02$ & 304.2 & Am-241 & $->$ & $N p-237$ \\
\hline $2.70 E+02$ & 260.9 & Am-241 & $->$ & $\mathrm{Np}-237$ \\
\hline $2.70 E+02$ & 429.8 & Am-241 & $->$ & $N p-237$ \\
\hline $2.70 E+02$ & 574.05 & Am-241 & $->$ & $N p-237$ \\
\hline $2.70 E+02$ & 486.3 & Am-241 & $->$ & $N p-237$ \\
\hline $2.63 E+02$ & 11.372 & Np-237 & $->$ & $\mathrm{Pa}-233$ \\
\hline $2.61 E+02$ & 398.663 & $\mathrm{~Pa}-233$ & $->$ & $U-233$ \\
\hline $2.57 E+02$ & 75.343 & $\mathrm{~Pa}-233$ & $->$ & U-233 \\
\hline $2.43 E+02$ & 522 & Am-241 & $->$ & $\mathrm{Np}-237$ \\
\hline $2.35 E+02$ & 11.62 & Pa-233 & $->$ & U-233 \\
\hline $2.15 E+02$ & 108.166 & $\mathrm{~Np}-237$ & $->$ & $\mathrm{Pa}-233$ \\
\hline $1.89 \mathrm{E}+02$ & 234.352 & Am-241 & $->$ & Np-237 \\
\hline $1.89 \mathrm{E}+02$ & 872.7 & Am-241 & $->$ & $N p-237$ \\
\hline $1.74 \mathrm{E}+02$ & 94.723 & Np-237 & $->$ & $\mathrm{Pa}-233$ \\
\hline $1.73 E+02$ & 271.58 & Am-241 & $->$ & $\mathrm{Np}-237$ \\
\hline $1.73 E+02$ & 675.8 & Am-241 & $->$ & $N p-237$ \\
\hline $1.62 \mathrm{E}+02$ & 811.8 & Am-241 & $->$ & $N p-237$ \\
\hline $1.57 \mathrm{E}+02$ & 955.7 & Am-241 & $->$ & $N p-237$ \\
\hline $1.52 \mathrm{E}+02$ & 103.921 & $\mathrm{~Pa}-233$ & $->$ & U-233 \\
\hline $1.51 \mathrm{E}+02$ & 627.2 & Am-241 & $->$ & $\mathrm{Np}-237$ \\
\hline $1.43 E+02$ & 862.6 & Am-241 & $->$ & Np-237 \\
\hline $1.32 \mathrm{E}+02$ & 148.567 & Pu-241 & $->$ & $U-237$ \\
\hline $1.27 \mathrm{E}+02$ & 375.518 & $\mathrm{~Pa}-233$ & $->$ & $U-233$ \\
\hline $1.27 \mathrm{E}+02$ & 731.5 & Am-241 & $->$ & $\mathrm{Np}-237$ \\
\hline $1.16 \mathrm{E}+02$ & 111.3 & Pu-241 & $->$ & U-237 \\
\hline $1.08 \mathrm{E}+02$ & 819.3 & Am-241 & $->$ & $\mathrm{Np}-237$ \\
\hline $1.08 \mathrm{E}+02$ & 14.953 & Np-237 & $->$ & $\mathrm{Pa}-233$ \\
\hline $1.05 E+02$ & 788.8 & Am-241 & $->$ & $\mathrm{Np}-237$ \\
\hline $1.03 \mathrm{E}+02$ & 851.5 & Am-241 & $->$ & $\mathrm{Np}-237$ \\
\hline $1.03 E+02$ & 669.9 & Am-241 & $->$ & $\mathrm{Np}-237$ \\
\hline $8.92 E+01$ & 57.149 & $\mathrm{~Np}-237$ & $->$ & $\mathrm{Pa}-233$ \\
\hline $8.92 E+01$ & 143.227 & Np-237 & $->$ & $\mathrm{Pa}-233$ \\
\hline $8.10 \mathrm{E}+01$ & 902.5 & Am-241 & $->$ & $\mathrm{Np}-237$ \\
\hline $7.11 \mathrm{E}+01$ & 103.68 & Pu-241 & $->$ & U-237 \\
\hline $7.09 E+01$ & 111.897 & $\mathrm{~Np}-237$ & $->$ & $\mathrm{Pa}-233$ \\
\hline $6.75 E+01$ & 912.4 & Am-241 & $->$ & $\mathrm{Np}-237$ \\
\hline $6.75 E+01$ & 780.5 & Am-241 & $->$ & $\mathrm{Np}-237$ \\
\hline $6.48 \mathrm{E}+01$ & 828.5 & Am-241 & $->$ & $\mathrm{Np}-237$ \\
\hline $6.15 E+01$ & 271.597 & Pa-233 & $->$ & U-233 \\
\hline $5.94 \mathrm{E}+01$ & 887.5 & Am-241 & $->$ & $\mathrm{Np}-237$ \\
\hline $5.94 \mathrm{E}+01$ & 822.6 & Am-241 & $->$ & $\mathrm{Np}-237$ \\
\hline $5.83 E+01$ & 191.9 & Am-241 & $->$ & $\mathrm{Np}-237$ \\
\hline $5.49 E+01$ & 151.423 & $\mathrm{~Np}-237$ & $->$ & $\mathrm{Pa}-233$ \\
\hline $5.40 \mathrm{E}+01$ & 582.6 & Am-241 & $->$ & $\mathrm{Np}-237$ \\
\hline $5.40 \mathrm{E}+01$ & 854.7 & Am-241 & $->$ & $\mathrm{Np}-237$ \\
\hline
\end{tabular}




\begin{tabular}{|c|c|c|c|c|c|c|c|}
\hline $5.34 \mathrm{E}+01$ & 763.4 & Am-241 & $->$ & $\mathrm{Np}-237$ & & & \\
\hline $5.13 E+01$ & 922.2 & Am-241 & $->$ & $\mathrm{Np}-237$ & & & \\
\hline $5.05 E+01$ & 15.4 & $\mathrm{~Pa}-233$ & $->$ & U-233 & & & \\
\hline $4.60 E+01$ & 195.04 & $\mathrm{~Np}-237$ & $->$ & $\mathrm{Pa}-233$ & & & \\
\hline $4.26 \mathrm{E}+01$ & 110.421 & Pu-241 & $->$ & U-237 & & & \\
\hline $4.12 \mathrm{E}+01$ & 88.05 & $\mathrm{~Np}-237$ & $->$ & $\mathrm{Pa}-233$ & & & \\
\hline $3.68 \mathrm{E}+01$ & 117.689 & $\mathrm{~Np}-237$ & $->$ & $\mathrm{Pa}-233$ & & & \\
\hline $3.48 \mathrm{E}+01$ & 212.412 & $\mathrm{~Np}-237$ & $->$ & $\mathrm{Pa}-233$ & & & \\
\hline $3.04 \mathrm{E}+01$ & 46.57 & $\mathrm{~Np}-237$ & $->$ & $\mathrm{Pa}-233$ & & & \\
\hline $2.63 E+01$ & 114.561 & Pu-241 & $->$ & U-237 & & & \\
\hline $2.27 E+01$ & 859.2 & Am-241 & $->$ & $\mathrm{Np}-237$ & & & \\
\hline $2.11 \mathrm{E}+01$ & 155.263 & $\mathrm{~Np}-237$ & $->$ & $\mathrm{Pa}-233$ & & & \\
\hline $1.94 \mathrm{E}+01$ & 131.09 & $\mathrm{~Np}-237$ & $->$ & Pa-233 & & & \\
\hline $1.89 \mathrm{E}+01$ & 898.4 & Am-241 & $->$ & $\mathrm{Np}-237$ & & & \\
\hline $1.67 E+01$ & 108.69 & $\mathrm{~Np}-237$ & $->$ & $\mathrm{Pa}-233$ & & & \\
\hline $1.65 \mathrm{E}+01$ & 169.18 & $\mathrm{~Np}-237$ & $->$ & $\mathrm{Pa}-233$ & & & \\
\hline $1.62 \mathrm{E}+01$ & 777.2 & Am-241 & $->$ & $\mathrm{Np}-237$ & & & \\
\hline $1.57 \mathrm{E}+01$ & 77.1 & $U-233$ & $->$ & Th-229, & Pu-241 & $->$ & U-237 \\
\hline $1.53 \mathrm{E}+01$ & 134.23 & $\mathrm{~Np}-237$ & $->$ & $\mathrm{Pa}-233$ & & & \\
\hline $1.46 \mathrm{E}+01$ & 237.95 & $\mathrm{~Np}-237$ & $->$ & $\mathrm{Pa}-233$ & & & \\
\hline $1.43 \mathrm{E}+01$ & 28.578 & $\mathrm{~Pa}-233$ & $->$ & U-233 & & & \\
\hline $1.35 \mathrm{E}+01$ & 945.7 & Am-241 & $->$ & $\mathrm{Np}-237$ & & & \\
\hline $1.35 \mathrm{E}+01$ & 928.8 & Am-241 & $->$ & $\mathrm{Np}-237$ & & & \\
\hline $1.28 \mathrm{E}+01$ & 106.13 & $\mathrm{~Np}-237$ & $->$ & $\mathrm{Pa}-233$ & & & \\
\hline $1.19 \mathrm{E}+01$ & 248.25 & $\mathrm{~Pa}-233$ & $->$ & U-233 & & & \\
\hline $1.17 \mathrm{E}+01$ & 193.29 & $\mathrm{~Np}-237$ & $->$ & Pa-233 & & & \\
\hline $1.16 \mathrm{E}+01$ & 114.333 & Pu-241 & $->$ & U-237 & & & \\
\hline $1.12 \mathrm{E}+01$ & 194.74 & $\mathrm{~Np}-237$ & $->$ & $\mathrm{Pa}-233$ & & & \\
\hline $9.68 \mathrm{E}+00$ & 115.4 & Pu-241 & $->$ & $U-237$ & & & \\
\hline $9.61 E+00$ & 201.72 & $\mathrm{~Np}-237$ & $->$ & $\mathrm{Pa}-233$ & & & \\
\hline $9.61 E+00$ & 214.08 & $\mathrm{~Np}-237$ & $->$ & Pa-233 & & & \\
\hline $8.47 E+00$ & 162.51 & $\mathrm{~Np}-237$ & $->$ & $\mathrm{Pa}-233$ & & & \\
\hline $7.91 \mathrm{E}+00$ & 40.415 & $\mathrm{~Pa}-233$ & $->$ & $U-233$ & & & \\
\hline $6.93 E+00$ & 38.57 & Pu-241 & $->$ & U-237 & & & \\
\hline $6.18 \mathrm{E}+00$ & 191.46 & $\mathrm{~Np}-237$ & $->$ & $\mathrm{Pa}-233$ & & & \\
\hline $5.72 E+00$ & 196.89 & $\mathrm{~Np}-237$ & $->$ & $\mathrm{Pa}-233$ & & & \\
\hline $5.56 \mathrm{E}+00$ & 298.87 & $\mathrm{~Pa}-233$ & $->$ & U-233 & & & \\
\hline $5.26 \mathrm{E}+00$ & 180.801 & $\mathrm{~Np}-237$ & $->$ & $\mathrm{Pa}-233$ & & & \\
\hline $4.77 E+00$ & 159.955 & Pu-241 & $->$ & U-237 & & & \\
\hline $4.58 \mathrm{E}+00$ & 176.09 & $\mathrm{~Np}-237$ & $->$ & $\mathrm{Pa}-233$ & & & \\
\hline $4.35 E+00$ & 170.67 & $\mathrm{~Np}-237$ & $->$ & $\mathrm{Pa}-233$ & & & \\
\hline $4.12 E+00$ & 140.61 & $\mathrm{~Np}-237$ & $->$ & $P a-233$ & & & \\
\hline $3.66 \mathrm{E}+00$ & 70.62 & $\mathrm{~Np}-237$ & $->$ & $\mathrm{Pa}-233$ & & & \\
\hline $3.66 \mathrm{E}+00$ & 63.95 & $\mathrm{~Np}-237$ & $->$ & $\mathrm{Pa}-233$ & & & \\
\hline $3.55 \mathrm{E}+00$ & 209.19 & $\mathrm{~Np}-237$ & $->$ & $\mathrm{Pa}-233$ & & & \\
\hline $3.02 E+00$ & 44.2 & Pu-241 & $->$ & U-237 & & & \\
\hline $3.00 E+00$ & 229.98 & $\mathrm{~Np}-237$ & $->$ & $\mathrm{Pa}-233$ & & & \\
\hline $2.86 \mathrm{E}+00$ & 41.718 & $\mathrm{~Pa}-233$ & $->$ & $U-233$ & & & \\
\hline $2.75 E+00$ & 62.66 & $\mathrm{~Np}-237$ & $->$ & Pa-233 & & & \\
\hline $2.54 \mathrm{E}+00$ & 74.46 & $\mathrm{~Np}-237$ & $->$ & $\mathrm{Pa}-233$ & & & \\
\hline
\end{tabular}




\begin{tabular}{|c|c|c|c|c|}
\hline $2.39 E+00$ & 111.89 & Pu-241 & $->$ & $U-237$ \\
\hline $2.02 E+00$ & 71.6 & Pu-241 & $->$ & U-237 \\
\hline $1.81 \mathrm{E}+00$ & 56.32 & Pu-241 & $->$ & $U-237$ \\
\hline $1.58 \mathrm{E}+00$ & 153.72 & $\mathrm{~Np}-237$ & $->$ & $\mathrm{Pa}-233$ \\
\hline $1.56 \mathrm{E}+00$ & 172.56 & $\mathrm{~Np}-237$ & $->$ & $\mathrm{Pa}-233$ \\
\hline $1.53 E+00$ & 186.7 & $\mathrm{~Np}-237$ & $->$ & $\mathrm{Pa}-233$ \\
\hline $1.53 E+00$ & 262.41 & $\mathrm{~Np}-237$ & $->$ & Pa-233 \\
\hline $1.53 E+00$ & 256.98 & $N p-237$ & $->$ & $\mathrm{Pa}-233$ \\
\hline $1.19 \mathrm{E}+00$ & 258.46 & $\mathrm{~Pa}-233$ & $->$ & $U-233$ \\
\hline $1.10 \mathrm{E}+00$ & 248.91 & $\mathrm{~Np}-237$ & $->$ & $\mathrm{Pa}-233$ \\
\hline $8.24 \mathrm{E}-01$ & 202.85 & $\mathrm{~Np}-237$ & $->$ & $\mathrm{Pa}-233$ \\
\hline $8.01 E-01$ & 200.17 & $\mathrm{~Np}-237$ & $->$ & $\mathrm{Pa}-233$ \\
\hline 7.91E-01 & 17.269 & $\mathrm{~Pa}-233$ & $->$ & $U-233$ \\
\hline 7.04E-01 & 56.76 & Pu-241 & $->$ & U-237 \\
\hline $6.04 \mathrm{E}-01$ & 44.86 & Pu-241 & $->$ & $U-237$ \\
\hline $5.72 \mathrm{E}-01$ & 115.19 & $N p-237$ & $->$ & $\mathrm{Pa}-233$ \\
\hline 4.84E-01 & 121.2 & Pu-241 & $->$ & $U-237$ \\
\hline $5.27 E-03$ & 16.141 & U-233 & $->$ & Th-229 \\
\hline $4.15 \mathrm{E}-03$ & 12.952 & $U-233$ & $->$ & Th-229 \\
\hline $1.28 \mathrm{E}-03$ & 19.148 & $U-233$ & $->$ & Th-229 \\
\hline $2.39 E-04$ & 11.118 & $U-233$ & $->$ & Th-229 \\
\hline $9.58 \mathrm{E}-05$ & 42.441 & U-233 & $->$ & Th-229 \\
\hline $9.10 \mathrm{E}-05$ & 14.511 & U-233 & $->$ & Th-229 \\
\hline $3.51 E-05$ & 97.143 & $U-233$ & $->$ & Th-229 \\
\hline $2.70 \mathrm{E}-05$ & 93.36 & U-233 & $->$ & Th-229 \\
\hline $2.23 \mathrm{E}-05$ & 54.702 & $U-233$ & $->$ & Th-229 \\
\hline $1.66 \mathrm{E}-05$ & 89.957 & $U-233$ & $->$ & Th-229 \\
\hline $1.40 \mathrm{E}-05$ & 317.133 & $U-233$ & $->$ & Th-229 \\
\hline $1.09 \mathrm{E}-05$ & 29.191 & $U-233$ & $->$ & Th-229 \\
\hline $1.05 \mathrm{E}-05$ & 164.512 & $U-233$ & $->$ & Th-229 \\
\hline $1.01 \mathrm{E}-05$ & 146.347 & $U-233$ & $->$ & Th-229 \\
\hline $9.74 \mathrm{E}-06$ & 105.362 & $U-233$ & $->$ & Th-229 \\
\hline 8.30E-06 & 291.317 & $U-233$ & $->$ & Th-229 \\
\hline $7.18 \mathrm{E}-06$ & 248.661 & $U-233$ & $->$ & Th-229 \\
\hline $6.86 \mathrm{E}-06$ & 53.559 & $U-233$ & $->$ & Th-229 \\
\hline 5.59E-06 & 217.133 & U-233 & $->$ & Th-229 \\
\hline 5.59E-06 & 245.294 & U-233 & $->$ & Th-229 \\
\hline 5.19E-06 & 40.34 & $\mathrm{Ra}-225$ & $->$ & Ac- 225 \\
\hline $5.11 \mathrm{E}-06$ & 118.97 & U-233 & $->$ & Th-229 \\
\hline 4.95E-06 & 320.508 & $U-233$ & $->$ & Th-229 \\
\hline 4.47E-06 & 117.155 & $U-233$ & $->$ & Th-229 \\
\hline $4.31 \mathrm{E}-06$ & 71.847 & $U-233$ & $->$ & Th-229 \\
\hline 3.99E-06 & 208.149 & $U-233$ & $->$ & Th-229 \\
\hline $3.51 \mathrm{E}-06$ & 135.328 & $U-233$ & $->$ & Th-229 \\
\hline $3.35 \mathrm{E}-06$ & 120.81 & U-233 & $->$ & Th-229 \\
\hline $3.24 \mathrm{E}-06$ & 108.99 & $U-233$ & $->$ & Th-229 \\
\hline 3.19E-06 & 187.942 & $U-233$ & $->$ & Th-229 \\
\hline $2.79 E-06$ & 440.34 & $\mathrm{Bi}-213$ & $->$ & Po-213 \\
\hline $2.55 \mathrm{E}-06$ & 145.286 & U-233 & $->$ & Th-229 \\
\hline $2.39 E-06$ & 74.5 & U-233 & $->$ & Th-229 \\
\hline
\end{tabular}




\begin{tabular}{|c|c|c|c|c|}
\hline $2.23 E-06$ & 96.215 & $U-233$ & $->$ & Th-229 \\
\hline $1.95 \mathrm{E}-06$ & 278.059 & $U-233$ & $->$ & Th-229 \\
\hline $1.93 \mathrm{E}-06$ & 25.304 & $U-233$ & $->$ & Th-229 \\
\hline $1.89 \mathrm{E}-06$ & 217.98 & Fr-221 & $->$ & At-217 \\
\hline $1.72 \mathrm{E}-06$ & 287.949 & $U-233$ & $->$ & Th-229 \\
\hline $1.66 \mathrm{E}-06$ & 14.772 & Ac- 225 & $->$ & Fr-221 \\
\hline $1.58 \mathrm{E}-06$ & 32.27 & U-233 & $->$ & Th-229 \\
\hline $1.35 \mathrm{E}-06$ & 12.017 & Ac- 225 & $->$ & Fr-221 \\
\hline $1.34 \mathrm{E}-06$ & 66.11 & U-233 & $->$ & Th-229 \\
\hline $1.34 \mathrm{E}-06$ & 323.321 & $U-233$ & $->$ & Th-229 \\
\hline $1.31 \mathrm{E}-06$ & 365.762 & $U-233$ & $->$ & Th-229 \\
\hline $1.09 \mathrm{E}-06$ & 15.662 & $\mathrm{Ra}-225$ & $->$ & Ac- 225 \\
\hline $1.05 \mathrm{E}-06$ & 216.08 & U-233 & $->$ & Th-229 \\
\hline $1.04 \mathrm{E}-06$ & 123.91 & $U-233$ & $->$ & Th-229 \\
\hline $9.83 E-07$ & 12.636 & $\mathrm{Ra}-225$ & $->$ & Ac- 225 \\
\hline $9.58 \mathrm{E}-07$ & 70.35 & U-233 & $->$ & Th-229 \\
\hline $9.42 \mathrm{E}-07$ & 336.579 & $U-233$ & $->$ & Th-229 \\
\hline $9.42 \mathrm{E}-07$ & 72.821 & $U-233$ & $->$ & Th-229 \\
\hline $8.62 E-07$ & 193.59 & Th-229 & $->$ & Ra-225 \\
\hline $7.68 \mathrm{E}-07$ & 31.37 & Th-229 & $->$ & Ra-225 \\
\hline 7.50E-07 & 111.935 & U-233 & $->$ & Th-229 \\
\hline $7.50 E-07$ & 274.692 & $U-233$ & $->$ & Th-229 \\
\hline $7.02 E-07$ & 88.43 & $U-233$ & $->$ & Th-229 \\
\hline $6.23 E-07$ & 165.493 & $U-233$ & $->$ & Th-229 \\
\hline $6.23 E-07$ & 76.32 & U-233 & $->$ & Th-229 \\
\hline $6.18 \mathrm{E}-07$ & 210.94 & Th-229 & $->$ & $\mathrm{Ra}-225$ \\
\hline 6.07E-07 & 240.364 & U-233 & $->$ & Th-229 \\
\hline $5.75 E-07$ & 37.85 & $U-233$ & $->$ & Th-229 \\
\hline $5.75 E-07$ & 148.15 & $U-233$ & $->$ & Th-229 \\
\hline $5.62 E-07$ & 86.44 & Th-229 & $->$ & $\mathrm{Ra}-225$ \\
\hline $5.27 \mathrm{E}-07$ & 90.994 & $\mathrm{U}-233$ & $->$ & Th-229 \\
\hline $5.11 \mathrm{E}-07$ & 67.96 & $U-233$ & $->$ & Th-229 \\
\hline $4.95 E-07$ & 109.47 & $U-233$ & $->$ & Th-229 \\
\hline 4.95E-07 & 261.88 & $U-233$ & $->$ & Th-229 \\
\hline 4.79E-07 & 144.52 & $U-233$ & $->$ & Th-229 \\
\hline $4.31 E-07$ & 31.24 & $U-233$ & $->$ & Th-229 \\
\hline 3.99E-07 & 52.613 & $U-233$ & $->$ & Th-229 \\
\hline 3.99E-07 & 114.28 & $U-233$ & $->$ & Th-229 \\
\hline 3.99E-07 & 268.619 & $U-233$ & $->$ & Th-229 \\
\hline $3.67 E-07$ & 174.162 & $U-233$ & $->$ & Th-229 \\
\hline $3.67 E-07$ & 1566 & TI-209 & $->$ & Pb-209 \\
\hline $3.64 \mathrm{E}-07$ & 17.449 & Ac- 225 & $->$ & Fr-221 \\
\hline $3.60 E-07$ & 86.105 & $A c-225$ & $->$ & Fr-221 \\
\hline $3.35 E-07$ & 116.26 & U-233 & $->$ & Th-229 \\
\hline $3.03 E-07$ & 87.24 & $U-233$ & $->$ & Th-229 \\
\hline $3.03 E-07$ & 85.402 & $U-233$ & $->$ & Th-229 \\
\hline $3.03 E-07$ & 467 & TI-209 & $->$ & Pb-209 \\
\hline $3.03 E-07$ & 117 & TI-209 & $->$ & $\mathrm{Pb}-209$ \\
\hline $3.00 \mathrm{E}-07$ & 137.02 & Th-229 & $->$ & $\mathrm{Ra}-225$ \\
\hline $2.91 \mathrm{E}-07$ & 99.82 & Ac- 225 & $->$ & Fr-221 \\
\hline
\end{tabular}




\begin{tabular}{|c|c|c|c|c|}
\hline 2.87E-07 & 82.957 & $U-233$ & $->$ & Th-229 \\
\hline $2.87 E-07$ & 259.3 & U-233 & $->$ & Th-229 \\
\hline $2.50 \mathrm{E}-07$ & 18.593 & $\mathrm{Ra}-225$ & $->$ & Ac- 225 \\
\hline $2.44 \mathrm{E}-07$ & 81.517 & Fr-221 & $->$ & At-217 \\
\hline $2.39 \mathrm{E}-07$ & 154.69 & U-233 & $->$ & Th-229 \\
\hline $2.39 E-07$ & 219.424 & U-233 & $->$ & Th-229 \\
\hline $2.23 E-07$ & 212.29 & $U-233$ & $->$ & Th-229 \\
\hline $2.23 E-07$ & 293.923 & U-233 & $->$ & Th-229 \\
\hline $2.23 E-07$ & 170.794 & $U-233$ & $->$ & Th-229 \\
\hline 2.19E-07 & 79.29 & $\mathrm{Bi}-213$ & $->$ & Po-213 \\
\hline $2.18 \mathrm{E}-07$ & 83.229 & $A c-225$ & $->$ & Fr-221 \\
\hline $2.08 \mathrm{E}-07$ & 86.74 & U-233 & $->$ & Th-229 \\
\hline $2.03 E-07$ & 149.8 & $U-233$ & $->$ & Th-229 \\
\hline 1.87E-07 & 156.46 & Th-229 & $->$ & $\mathrm{Ra}-225$ \\
\hline $1.87 \mathrm{E}-07$ & 148.37 & Th-229 & $->$ & Ra-225 \\
\hline $1.87 \mathrm{E}-07$ & 124.51 & Th-229 & $->$ & $\mathrm{Ra}-225$ \\
\hline $1.72 \mathrm{E}-07$ & 260.4 & U-233 & $->$ & Th-229 \\
\hline $1.72 \mathrm{E}-07$ & 68.97 & $U-233$ & $->$ & Th-229 \\
\hline $1.68 \mathrm{E}-07$ & 139.76 & $U-233$ & $->$ & Th-229 \\
\hline $1.66 \mathrm{E}-07$ & 13.911 & Fr-221 & $->$ & At-217 \\
\hline $1.60 \mathrm{E}-07$ & 103.375 & U-233 & $->$ & Th-229 \\
\hline $1.52 \mathrm{E}-07$ & 383.4 & $U-233$ & $->$ & Th-229 \\
\hline $1.50 \mathrm{E}-07$ & 107.16 & Th-229 & $->$ & Ra-225 \\
\hline $1.47 \mathrm{E}-07$ & 78.947 & Fr-221 & $->$ & At-217 \\
\hline $1.44 \mathrm{E}-07$ & 101.8 & U-233 & $->$ & Th-229 \\
\hline $1.37 \mathrm{E}-07$ & 11.414 & Fr-221 & $->$ & At-217 \\
\hline $1.32 \mathrm{E}-07$ & 76.858 & $\mathrm{Bi}-213$ & $->$ & Po-213 \\
\hline $1.31 \mathrm{E}-07$ & 154.37 & Th-229 & $->$ & $\mathrm{Ra}-225$ \\
\hline $1.30 \mathrm{E}-07$ & 97.272 & AC- 225 & $->$ & Fr-221 \\
\hline $1.20 \mathrm{E}-07$ & 162.52 & U-233 & $->$ & Th-229 \\
\hline $1.20 \mathrm{E}-07$ & 84.27 & $U-233$ & $->$ & Th-229 \\
\hline $1.17 \mathrm{E}-07$ & 309.37 & $U-233$ & $->$ & Th-229 \\
\hline $1.16 \mathrm{E}-07$ & 150.08 & Ac- 225 & $->$ & Fr-221 \\
\hline $1.12 \mathrm{E}-07$ & 124.72 & Th-229 & $->$ & $\mathrm{Ra}-225$ \\
\hline $1.12 \mathrm{E}-07$ & 129.2 & U-233 & $->$ & Th-229 \\
\hline $1.12 \mathrm{E}-07$ & 302.84 & U-233 & $->$ & Th-229 \\
\hline 1.09E-07 & 99.58 & Ac- 225 & $->$ & Fr-221 \\
\hline $1.07 E-07$ & 230.086 & U-233 & $->$ & Th-229 \\
\hline $1.07 \mathrm{E}-07$ & 168.98 & U-233 & $->$ & Th-229 \\
\hline $1.04 \mathrm{E}-07$ & 125.398 & $U-233$ & $->$ & Th-229 \\
\hline $1.04 \mathrm{E}-07$ & 328.69 & U-233 & $->$ & Th-229 \\
\hline $1.04 \mathrm{E}-07$ & 205.99 & U-233 & $->$ & Th-229 \\
\hline $1.01 \mathrm{E}-07$ & 272.27 & U-233 & $->$ & Th-229 \\
\hline $9.58 \mathrm{E}-08$ & 78.41 & U-233 & $->$ & Th-229 \\
\hline $9.55 E-08$ & 179.74 & Th-229 & $->$ & $\mathrm{Ra}-225$ \\
\hline $9.53 E-08$ & 62.93 & Ac-225 & $->$ & Fr-221 \\
\hline $9.36 \mathrm{E}-08$ & 75.17 & Th-229 & $->$ & $\mathrm{Ra}-225$ \\
\hline $9.26 \mathrm{E}-08$ & 354 & U-233 & $->$ & Th-229 \\
\hline $9.26 \mathrm{E}-08$ & 155.989 & U-233 & $->$ & Th-229 \\
\hline $8.78 \mathrm{E}-08$ & 153.31 & $U-233$ & $->$ & Th-229 \\
\hline
\end{tabular}




\begin{tabular}{|c|c|c|c|c|}
\hline $8.78 \mathrm{E}-08$ & 100.008 & $U-233$ & $->$ & Th-229 \\
\hline $8.66 \mathrm{E}-08$ & 92.136 & Fr-221 & $->$ & At-217 \\
\hline $8.38 \mathrm{E}-08$ & 11.119 & $\mathrm{Bi}-213$ & $->$ & Po-213 \\
\hline 8.05E-08 & 142.98 & Th-229 & $->$ & $\mathrm{Ra}-225$ \\
\hline $7.98 \mathrm{E}-08$ & 217.61 & U-233 & $->$ & Th-229 \\
\hline $7.98 \mathrm{E}-08$ & 236.39 & U-233 & $->$ & Th-229 \\
\hline 7.97E-08 & 187.99 & Ac- 225 & $->$ & Fr-221 \\
\hline 7.79E-08 & 13.504 & $\mathrm{Bi}-213$ & $->$ & Po-213 \\
\hline 7.77E-08 & 89.639 & $\mathrm{Bi}-213$ & $->$ & Po-213 \\
\hline $7.45 \mathrm{E}-08$ & 292.79 & $\mathrm{Bi}-213$ & $->$ & Po-213 \\
\hline $7.45 E-08$ & 10.381 & $A c-225$ & $->$ & Fr-221 \\
\hline $7.28 \mathrm{E}-08$ & 216.86 & Ac- 225 & $->$ & Fr-221 \\
\hline 7.18E-08 & 351.782 & U-233 & $->$ & Th-229 \\
\hline 7.12E-08 & 86.23 & Th-229 & $->$ & Ra-225 \\
\hline $6.86 \mathrm{E}-08$ & 255.92 & U-233 & $->$ & Th-229 \\
\hline $6.86 \mathrm{E}-08$ & 176.1 & $U-233$ & $->$ & Th-229 \\
\hline $6.38 \mathrm{E}-08$ & 185.782 & $U-233$ & $->$ & Th-229 \\
\hline $6.38 \mathrm{E}-08$ & 192.12 & $U-233$ & $->$ & Th-229 \\
\hline $6.18 \mathrm{E}-08$ & 131.92 & Th-229 & $->$ & Ra-225 \\
\hline $6.18 \mathrm{E}-08$ & 56.58 & Th-229 & $->$ & Ra-225 \\
\hline $6.07 E-08$ & 252.5 & U-233 & $->$ & Th-229 \\
\hline 5.89E-08 & 216.2 & $A c-225$ & $->$ & Fr-221 \\
\hline $5.72 \mathrm{E}-08$ & 10.871 & $\mathrm{Ra}-225$ & $->$ & Ac- 225 \\
\hline $5.66 \mathrm{E}-08$ & 111.56 & Ac- 225 & $->$ & Fr-221 \\
\hline $5.61 \mathrm{E}-08$ & 73.84 & Ac- 225 & $->$ & Fr-221 \\
\hline 5.59E-08 & 172.35 & U-233 & $->$ & Th-229 \\
\hline 5.37E-08 & 157.24 & $A c-225$ & $->$ & Fr-221 \\
\hline 5.27E-08 & 63.69 & U-233 & $->$ & Th-229 \\
\hline $5.27 E-08$ & 131.2 & $U-233$ & $->$ & Th-229 \\
\hline 5.27E-08 & 223.357 & U-233 & $->$ & Th-229 \\
\hline $5.02 \mathrm{E}-08$ & 87.39 & AC- 225 & $->$ & Fr-221 \\
\hline $4.78 \mathrm{E}-08$ & 1100.06 & $\mathrm{Bi}-213$ & $->$ & Po-213 \\
\hline $4.68 \mathrm{E}-08$ & 108.36 & Ac- 225 & $->$ & Fr-221 \\
\hline 4.47E-08 & 311.38 & U-233 & $->$ & Th-229 \\
\hline $4.42 E-08$ & 807.27 & $\mathrm{Bi}-213$ & $->$ & Po-213 \\
\hline $4.31 E-08$ & 183.96 & Th-229 & $->$ & Ra-225 \\
\hline $4.16 \mathrm{E}-08$ & 100.599 & $A c-225$ & $->$ & Fr-221 \\
\hline $4.12 \mathrm{E}-08$ & 172.9 & Th-229 & $->$ & $\mathrm{Ra}-225$ \\
\hline 3.99E-08 & 184.17 & U-233 & $->$ & Th-229 \\
\hline $3.78 \mathrm{E}-08$ & 74.969 & TI-209 & $->$ & Pb-209 \\
\hline $3.67 E-08$ & 295.2 & U-233 & $->$ & Th-229 \\
\hline $3.45 E-08$ & 16.361 & Fr-221 & $->$ & At-217 \\
\hline $3.29 E-08$ & 123.76 & Ac- 225 & $->$ & Fr-221 \\
\hline $3.19 E-08$ & 228.07 & U-233 & $->$ & Th-229 \\
\hline $3.19 \mathrm{E}-08$ & 384 & U-233 & $->$ & Th-229 \\
\hline $3.19 \mathrm{E}-08$ & 138.5 & $U-233$ & $->$ & Th-229 \\
\hline $3.19 \mathrm{E}-08$ & 177.78 & U-233 & $->$ & Th-229 \\
\hline $3.18 \mathrm{E}-08$ & 17.349 & Th-229 & $->$ & $\mathrm{Ra}-225$ \\
\hline $3.00 \mathrm{E}-08$ & 42.722 & Th-229 & $->$ & $\mathrm{Ra}-225$ \\
\hline $2.77 \mathrm{E}-08$ & 94.87 & $A c-225$ & $->$ & Fr-221 \\
\hline
\end{tabular}




\begin{tabular}{|c|c|c|c|c|}
\hline $2.77 \mathrm{E}-08$ & 13.255 & $A c-225$ & $->$ & Fr-221 \\
\hline $2.68 \mathrm{E}-08$ & 95.265 & Fr-221 & $->$ & At-217 \\
\hline $2.65 \mathrm{E}-08$ & 153.93 & Ac- 225 & $->$ & Fr-221 \\
\hline 2.62E-08 & 218.15 & Th-229 & $->$ & Ra-225 \\
\hline $2.60 \mathrm{E}-08$ & 82.9 & $A c-225$ & $->$ & Fr-221 \\
\hline $2.51 \mathrm{E}-08$ & 195.75 & Ac- 225 & $->$ & Fr-221 \\
\hline 2.39E-08 & 471.3 & U-233 & $->$ & Th-229 \\
\hline 2.39E-08 & 479 & U-233 & $->$ & Th-229 \\
\hline 2.37E-08 & 92.673 & $\mathrm{Bi}-213$ & $->$ & Po-213 \\
\hline $2.25 \mathrm{E}-08$ & 409.1 & Fr-221 & $->$ & At-217 \\
\hline $2.24 \mathrm{E}-08$ & 72.803 & TI-209 & $->$ & Pb-209 \\
\hline $2.18 \mathrm{E}-08$ & 145.15 & $A c-225$ & $->$ & Fr-221 \\
\hline $2.06 \mathrm{E}-08$ & 68.88 & Th-229 & $->$ & Ra-225 \\
\hline 1.91E-08 & 452.43 & Ac-225 & $->$ & Fr-221 \\
\hline 1.87E-08 & 68.14 & Th-229 & $->$ & Ra-225 \\
\hline $1.73 \mathrm{E}-08$ & 253.51 & $A c-225$ & $->$ & Fr-221 \\
\hline 1.63E-08 & 14.082 & Ra-225 & $->$ & Ac- 225 \\
\hline 1.60E-08 & 226.74 & U-233 & $->$ & Th-229 \\
\hline $1.60 \mathrm{E}-08$ & 225 & $U-233$ & $->$ & Th-229 \\
\hline $1.60 \mathrm{E}-08$ & 284.22 & $U-233$ & $->$ & Th-229 \\
\hline 1.60E-08 & 416.4 & $U-233$ & $->$ & Th-229 \\
\hline $1.60 \mathrm{E}-08$ & 449.6 & $U-233$ & $->$ & Th-229 \\
\hline $1.49 \mathrm{E}-08$ & 15.878 & $\mathrm{Bi}-213$ & $->$ & Po-213 \\
\hline $1.47 \mathrm{E}-08$ & 659.72 & $\mathrm{Bi}-213$ & $->$ & Po-213 \\
\hline $1.39 \mathrm{E}-08$ & 224.64 & $A c-225$ & $->$ & Fr-221 \\
\hline $1.38 \mathrm{E}-08$ & 10.541 & TI-209 & $->$ & Pb-209 \\
\hline $1.35 \mathrm{E}-08$ & 84.789 & TI-209 & $->$ & Pb-209 \\
\hline $1.32 \mathrm{E}-08$ & 459.77 & $U-233$ & $->$ & Th-229 \\
\hline $1.32 \mathrm{E}-08$ & 1119 & $U-233$ & $->$ & Th-229 \\
\hline $1.32 \mathrm{E}-08$ & 1003 & $U-233$ & $->$ & Th-229 \\
\hline $1.32 \mathrm{E}-08$ & 396.65 & $U-233$ & $->$ & Th-229 \\
\hline $1.32 \mathrm{E}-08$ & 402.37 & $U-233$ & $->$ & Th-229 \\
\hline $1.31 \mathrm{E}-08$ & 12.703 & TI-209 & $->$ & $\mathrm{Pb}-209$ \\
\hline $1.21 \mathrm{E}-08$ & 149.99 & Fr-221 & $->$ & At-217 \\
\hline $1.21 \mathrm{E}-08$ & 171.29 & Fr-221 & $->$ & At-217 \\
\hline $1.20 \mathrm{E}-08$ & 394.34 & $U-233$ & $->$ & Th-229 \\
\hline $1.20 \mathrm{E}-08$ & 339.64 & $U-233$ & $->$ & Th-229 \\
\hline $1.04 \mathrm{E}-08$ & 100.88 & $A c-225$ & $->$ & Fr-221 \\
\hline $1.04 \mathrm{E}-08$ & 119.92 & Ac-225 & $->$ & Fr-221 \\
\hline $9.70 \mathrm{E}-09$ & 64.23 & $A c-225$ & $->$ & Fr-221 \\
\hline 8.66E-09 & 124.81 & Ac-225 & $->$ & Fr-221 \\
\hline 8.46E-09 & 406.7 & U-233 & $->$ & Th-229 \\
\hline 8.46E-09 & 540.27 & $U-233$ & $->$ & Th-229 \\
\hline 7.98E-09 & 578.47 & $U-233$ & $->$ & Th-229 \\
\hline 7.98E-09 & 436.78 & $U-233$ & $->$ & Th-229 \\
\hline 7.27E-09 & 9.897 & Fr-221 & $->$ & At-217 \\
\hline 6.93E-09 & 135.01 & Ac- 225 & $->$ & Fr-221 \\
\hline $6.86 \mathrm{E}-09$ & 484.05 & U-233 & $->$ & Th-229 \\
\hline $6.74 \mathrm{E}-09$ & 25.373 & Th-229 & $->$ & $\mathrm{Ra}-225$ \\
\hline $6.74 \mathrm{E}-09$ & 236.32 & Th-229 & $->$ & $\mathrm{Ra}-225$ \\
\hline
\end{tabular}




\begin{tabular}{|c|c|c|c|c|}
\hline 6.41E-09 & 152.64 & $A c-225$ & $->$ & Fr-221 \\
\hline $6.24 \mathrm{E}-09$ & 74.9 & Ac-225 & $->$ & Fr-221 \\
\hline 6.07E-09 & 569.37 & U-233 & $->$ & Th-229 \\
\hline $6.06 \mathrm{E}-09$ & 279.27 & $A c-225$ & $->$ & Fr-221 \\
\hline $6.06 \mathrm{E}-09$ & 382.05 & Fr-221 & $->$ & At-217 \\
\hline $6.06 \mathrm{E}-09$ & 359.09 & Fr-221 & $->$ & At-217 \\
\hline $6.06 \mathrm{E}-09$ & 118.47 & Fr-221 & $->$ & At-217 \\
\hline 5.20E-09 & 480.5 & $A c-225$ & $->$ & Fr-221 \\
\hline 5.20E-09 & 96.17 & $A c-225$ & $->$ & Fr-221 \\
\hline 4.79E-09 & 656.97 & U-233 & $->$ & Th-229 \\
\hline 4.37E-09 & 9.658 & $\mathrm{Bi}-213$ & $->$ & Po-213 \\
\hline 4.31E-09 & 707.47 & U-233 & $->$ & Th-229 \\
\hline $3.85 E-09$ & 87.632 & TI-209 & $->$ & $\mathrm{Pb}-209$ \\
\hline 3.83E-09 & 545.1 & $U-233$ & $->$ & Th-229 \\
\hline 3.67E-09 & 620.87 & U-233 & $->$ & Th-229 \\
\hline $3.46 \mathrm{E}-09$ & 186.12 & Ac- 225 & $->$ & $\mathrm{Fr}-221$ \\
\hline $3.46 \mathrm{E}-09$ & 198.72 & $A c-225$ & $->$ & Fr-221 \\
\hline $3.46 E-09$ & 248.7 & Ac- 225 & $->$ & Fr-221 \\
\hline $3.46 \mathrm{E}-09$ & 138.2 & Ac- 225 & $->$ & Fr-221 \\
\hline $3.35 \mathrm{E}-09$ & 867.9 & U-233 & $->$ & Th-229 \\
\hline 3.29E-09 & 53.82 & Ac- 225 & $->$ & Fr-221 \\
\hline 3.29E-09 & 97.2 & Fr-221 & $->$ & At-217 \\
\hline $2.94 \mathrm{E}-09$ & 73.6 & Ac- 225 & $->$ & Fr-221 \\
\hline $2.94 \mathrm{E}-09$ & 324.1 & Fr-221 & $->$ & At-217 \\
\hline 2.89E-09 & 12.466 & Fr-221 & $->$ & At-217 \\
\hline 2.77E-09 & 178.39 & $A c-225$ & $->$ & Fr-221 \\
\hline $2.60 E-09$ & 36.65 & $A c-225$ & $->$ & Fr-221 \\
\hline $2.55 E-09$ & 71.71 & Ac- 225 & $->$ & Fr-221 \\
\hline 2.43E-09 & 126.23 & $A c-225$ & $->$ & Fr-221 \\
\hline $2.36 \mathrm{E}-09$ & 14.871 & TI-209 & $->$ & Pb-209 \\
\hline $2.25 \mathrm{E}-09$ & 240.76 & AC- 225 & $->$ & Fr-221 \\
\hline $1.87 \mathrm{E}-09$ & 49.2 & Ac- 225 & $->$ & Fr-221 \\
\hline $1.73 \mathrm{E}-09$ & 70.92 & Ac-225 & $->$ & Fr-221 \\
\hline $1.73 \mathrm{E}-09$ & 285.5 & Ac- 225 & $->$ & Fr-221 \\
\hline $1.73 \mathrm{E}-09$ & 526.03 & Ac-225 & $->$ & Fr-221 \\
\hline $1.73 \mathrm{E}-09$ & 513.5 & Ac- 225 & $->$ & Fr-221 \\
\hline $1.60 \mathrm{E}-09$ & 200.67 & U-233 & $->$ & Th-229 \\
\hline $1.56 \mathrm{E}-09$ & 38.51 & $A c-225$ & $->$ & Fr-221 \\
\hline $1.56 \mathrm{E}-09$ & 103.44 & $A c-225$ & $->$ & Fr-221 \\
\hline $1.56 \mathrm{E}-09$ & 170.91 & $A c-225$ & $->$ & Fr-221 \\
\hline $1.56 \mathrm{E}-09$ & 282.54 & Fr-221 & $->$ & At-217 \\
\hline 1.13E-09 & 12.085 & $\mathrm{Bi}-213$ & $->$ & Po-213 \\
\hline 8.83E-10 & 69.85 & Ac- 225 & $->$ & Fr-221 \\
\hline $7.45 \mathrm{E}-10$ & 129.19 & $A c-225$ & $->$ & Fr-221 \\
\hline $7.28 \mathrm{E}-10$ & 57.77 & $A c-225$ & $->$ & Fr-221 \\
\hline $7.11 \mathrm{E}-10$ & 9.185 & Tl-209 & $->$ & $\mathrm{Pb}-209$ \\
\hline $6.92 \mathrm{E}-10$ & 323.81 & $\mathrm{Bi}-213$ & $->$ & TI-209 \\
\hline $2.60 \mathrm{E}-10$ & 25.98 & Ac- 225 & $->$ & Fr-221 \\
\hline $1.91 \mathrm{E}-10$ & 11.349 & TI-209 & $->$ & $\mathrm{Pb}-209$ \\
\hline $4.86 \mathrm{E}-11$ & 72.873 & $\mathrm{Bi}-213$ & $->$ & TI-209 \\
\hline
\end{tabular}




$\begin{array}{lccll}2.99 \mathrm{E}-11 & 70.832 & \mathrm{Bi}-213 & -> & \mathrm{Tl}-209 \\ 1.87 \mathrm{E}-11 & 82.434 & \mathrm{Bi}-213 & -> & \text { Tl-209 } \\ 1.87 \mathrm{E}-11 & 10.259 & \mathrm{Bi}-213 & -> & \mathrm{Tl}-209 \\ 1.50 \mathrm{E}-11 & 12.311 & \mathrm{Bi}-213 & -> & \mathrm{Tl}-209 \\ 4.86 \mathrm{E}-12 & 85.185 & \mathrm{Bi}-213 & -> & \mathrm{Tl}-209 \\ 2.99 \mathrm{E}-12 & 14.402 & \mathrm{Bi}-213 & -> & \mathrm{Tl}-209 \\ 8.60 \mathrm{E}-13 & 8.953 & \mathrm{Bi}-213 & -> & \mathrm{Tl}-209 \\ 2.24 \mathrm{E}-13 & 10.994 & \mathrm{Bi}-213 & -> & \mathrm{Tl}-209\end{array}$

Pu-242 100\% $\quad$ Aged 5 years

656 lines computed.

Sorted by Energy:

\begin{tabular}{|c|c|c|c|c|c|c|c|c|c|c|}
\hline Energy & Intensity & Initial Is & to & es and & cays & & & & & \\
\hline (kev) & $\mathrm{ph} / \mathrm{s} / \mathrm{gm}$ & & & & & & & & & \\
\hline 8.953 & $1.50 \mathrm{E}-20$ & $\mathrm{Hg}-206$ & $->$ & TI-206 & & & & & & \\
\hline 9.185 & $4.32 \mathrm{E}-21$ & TI-206 & $->$ & $\mathrm{Pb}-206$, & TI-210 & $->$ & $\mathrm{Pb}-210$ & & & \\
\hline 9.419 & $1.88 \mathrm{E}-21$ & $\mathrm{~Pb}-210$ & $->$ & $\mathrm{Bi}-210$ & $\mathrm{~Pb}-214$ & -> & $\mathrm{Bi}-214$ & & & \\
\hline 9.658 & $3.57 E-19$ & $\mathrm{Bi}-214$ & -> & Po-214 & & & & & & \\
\hline 10.137 & $0.00 E+00$ & $\mathrm{Ra}-226$ & $->$ & $\mathrm{Rn}-222$ & & & & & & \\
\hline 10.259 & $3.11 \mathrm{E}-19$ & $\mathrm{Hg}-206$ & $->$ & TI-206 & & & & & & \\
\hline 10.541 & $8.73 E-20$ & TI-206 & $->$ & $\mathrm{Pb}-206$, & $\mathrm{TI}-210$ & $\rightarrow$ & $\mathrm{Pb}-210$ & & & \\
\hline 10.828 & $3.68 \mathrm{E}-20$ & $\mathrm{~Pb}-210$ & $->$ & $\mathrm{Bi}-210$ & $\mathrm{~Pb}-214$ & $->$ & $\mathrm{Bi}-214$ & & & \\
\hline 10.994 & $3.95 E-21$ & $\mathrm{Hg}-206$ & $->$ & TI-206 & & & & & & \\
\hline 11.118 & $2.03 E-04$ & U-238 & $->$ & Th-234 & & & & & & \\
\hline 11.119 & $6.83 \mathrm{E}-18$ & $\mathrm{Bi}-214$ & -> & Po-214 & & & & & & \\
\hline 11.349 & $2.07 E-21$ & TI-210 & -> & $\mathrm{Pb}-210$ & & & & & & \\
\hline 11.372 & $2.56 \mathrm{E}-04$ & Th-234 & -> & $\mathrm{Pa}-234 \mathrm{~m}$, & $\mathrm{Pa}-234 \mathrm{~m}$ & $->$ & $U-234$ & & & \\
\hline 11.439 & $2.30 \mathrm{E}-23$ & Tl-206 & -> & $\mathrm{Pb}-206$ & & & & & & \\
\hline 11.62 & $2.90 E+05$ & $\mathrm{~Pa}-234$ & $->$ & U-234, & Pa-234m & $->$ & U-234, & Pu-242 & - & $U-238$ \\
\hline 11.712 & $3.16 \mathrm{E}-22$ & $\mathrm{~Pb}-210$ & $->$ & $\mathrm{Bi}-210$, & $\mathrm{Pb}-214$ & $->$ & $\mathrm{Bi}-214$ & & & \\
\hline 11.713 & $0.00 E+00$ & $\mathrm{Ra}-226$ & $->$ & $\mathrm{Rn}-222$ & & & & & & \\
\hline 12.085 & $9.80 \mathrm{E}-20$ & $\mathrm{Bi}-214$ & -> & Po-214 & & & & & & \\
\hline 12.313 & $2.70 \mathrm{E}-19$ & $\mathrm{Hg}-206$ & $->$ & TI-206 & & & & & & \\
\hline 12.678 & $1.14 \mathrm{E}-19$ & $\mathrm{Tl}-210$ & $->$ & $\mathrm{Pb}-210$ & & & & & & \\
\hline 12.71 & $1.45 \mathrm{E}-21$ & TI-206 & $->$ & $\mathrm{Pb}-206$ & & & & & & \\
\hline 12.855 & $0.00 E+00$ & $\mathrm{Ra}-226$ & $->$ & $\mathrm{Rn}-222$ & & & & & & \\
\hline 12.952 & $3.38 \mathrm{E}-03$ & $\mathrm{U}-238$ & $->$ & Th-234 & & & & & & \\
\hline 13.066 & $4.16 \mathrm{E}-20$ & $\mathrm{~Pb}-210$ & $->$ & $\mathrm{Bi}-210$ & & & & & & \\
\hline 13.088 & $0.00 E+00$ & $\mathrm{~Pb}-214$ & $->$ & $\mathrm{Bi}-214$ & & & & & & \\
\hline 13.274 & $4.31 \mathrm{E}-03$ & Th-234 & $->$ & $\mathrm{Pa}-234 \mathrm{~m}$ & & & & & & \\
\hline 13.275 & $2.55 \mathrm{E}-05$ & $\mathrm{~Pa}-234 \mathrm{~m}$ & -> & $\mathrm{U}-234$ & & & & & & \\
\hline 13.502 & $6.60 \mathrm{E}-18$ & $\mathrm{Bi}-214$ & -> & Po-214 & & & & & & \\
\hline 13.6 & $4.79 E+06$ & $\mathrm{~Pa}-234$ & -> & U-234, & $\mathrm{Pa}-234 \mathrm{~m}$ & $->$ & U-234, & Pu-242 & $\begin{array}{l}- \\
>\end{array}$ & U-238 \\
\hline
\end{tabular}




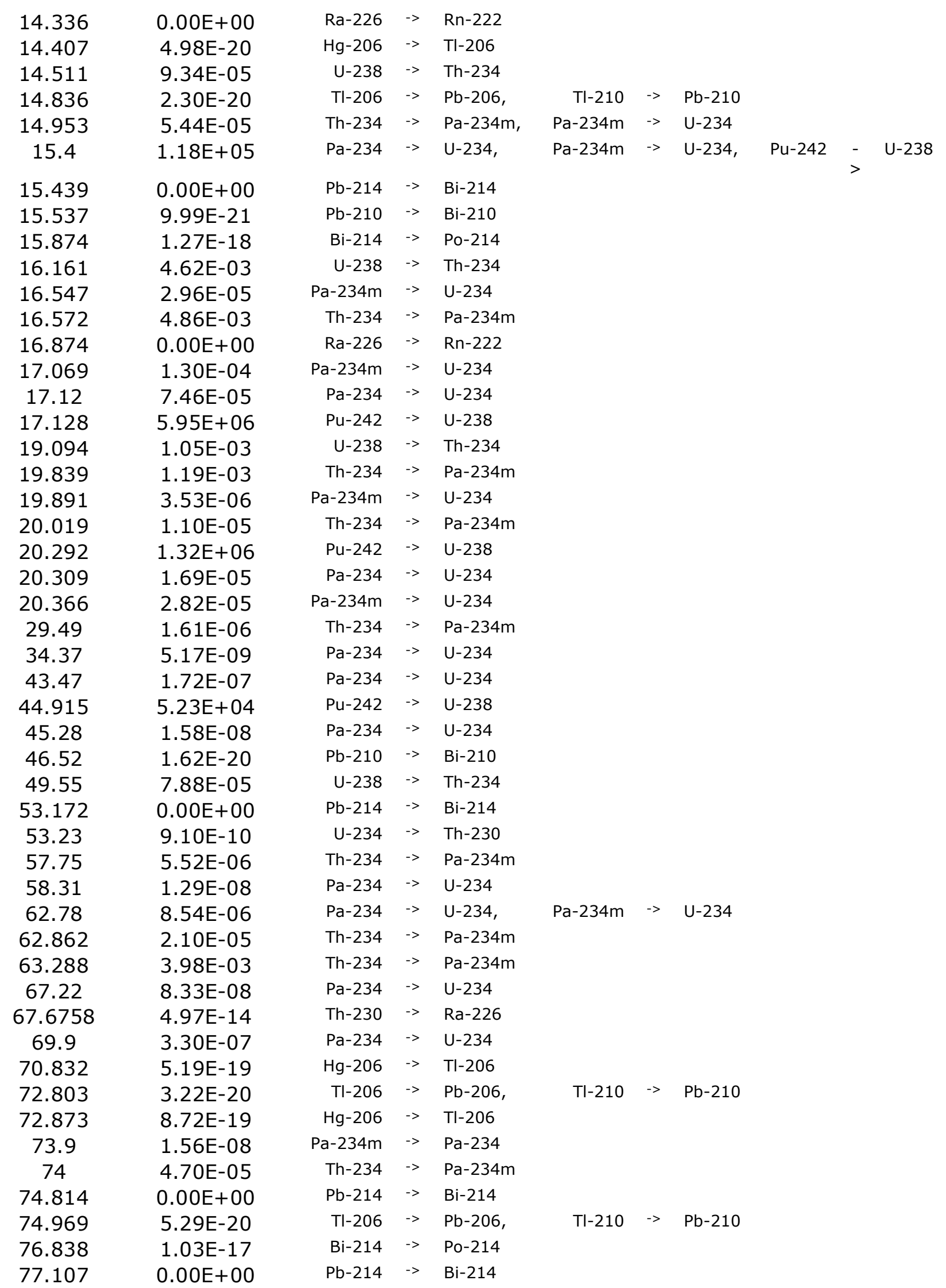




\begin{tabular}{|c|c|c|c|c|c|c|c|}
\hline 79.29 & $1.73 \mathrm{E}-17$ & $\mathrm{Bi}-214$ & $->$ & Po-214 & & & \\
\hline 79.65 & $3.88 E-07$ & $\mathrm{~Pa}-234$ & $->$ & $U-234$ & & & \\
\hline 81 & $2.07 E-20$ & TI-210 & $->$ & $\mathrm{Pb}-210$ & & & \\
\hline 81.067 & $0.00 E+00$ & $\mathrm{Ra}-226$ & $->$ & $\mathrm{Rn}-222$ & & & \\
\hline 82.434 & $3.11 \mathrm{E}-19$ & $\mathrm{Hg}-206$ & $->$ & TI-206 & & & \\
\hline 83.31 & $7.73 E-05$ & Th-234 & $->$ & $\mathrm{Pa}-234 \mathrm{~m}$ & & & \\
\hline 83.787 & $0.00 E+00$ & Ra-226 & $->$ & $\mathrm{Rn}-222$ & & & \\
\hline 84.789 & $1.89 \mathrm{E}-20$ & TI-206 & $->$ & $\mathrm{Pb}-206$ & $\mathrm{TI}-210$ & $->$ & $\mathrm{Pb}-210$ \\
\hline 85.185 & $8.72 E-20$ & $\mathrm{Hg}-206$ & $->$ & TI-206 & & & \\
\hline 87.02 & 8.06E-06 & Th-234 & $->$ & $\mathrm{Pa}-234 \mathrm{~m}$ & & & \\
\hline 87.19 & $0.00 E+00$ & $\mathrm{~Pb}-214$ & $->$ & $\mathrm{Bi}-214$ & & & \\
\hline 87.632 & $5.41 \mathrm{E}-21$ & TI-206 & $->$ & $\mathrm{Pb}-206$ & $\mathrm{TI}-210$ & $->$ & $\mathrm{Pb}-210$ \\
\hline 89.639 & $6.15 \mathrm{E}-18$ & $\mathrm{Bi}-214$ & $->$ & Po-214 & & & \\
\hline 90.128 & $0.00 E+00$ & $\mathrm{~Pb}-214$ & $->$ & $\mathrm{Bi}-214$ & & & \\
\hline 92.279 & 8.83E-06 & Th-234 & $->$ & $\mathrm{Pa}-234 \mathrm{~m}$ & & & \\
\hline 92.35 & 3.00E-03 & Th-234 & $->$ & $\mathrm{Pa}-234 \mathrm{~m}$ & & & \\
\hline 92.673 & $1.87 E-18$ & $\mathrm{Bi}-214$ & $->$ & Po-214 & & & \\
\hline 92.78 & 2.97E-03 & Th-234 & $->$ & $\mathrm{Pa}-234 \mathrm{~m}$ & & & \\
\hline 94.651 & $1.92 \mathrm{E}-04$ & $\mathrm{~Pa}-234$ & $->$ & U-234, & $\mathrm{Pa}-234 \mathrm{~m}$ & $->$ & $U-234$ \\
\hline 94.677 & $0.00 E+00$ & Ra-226 & $->$ & $\mathrm{Rn}-222$ & & & \\
\hline 95 & $4.13 E-20$ & TI-210 & $->$ & $\mathrm{Pb}-210$ & & & \\
\hline 95.863 & $1.44 \mathrm{E}-05$ & Th-234 & $->$ & $\mathrm{Pa}-234 \mathrm{~m}$ & & & \\
\hline 97.907 & $0.00 E+00$ & Ra-226 & $->$ & $\mathrm{Rn}-222$ & & & \\
\hline 98.434 & $3.04 \mathrm{E}-04$ & Pa-234 & $->$ & U-234, & $\mathrm{Pa}-234 \mathrm{~m}$ & $->$ & $U-234$ \\
\hline 99.852 & $7.41 \mathrm{E}-06$ & $\mathrm{~Pa}-234$ & $->$ & U-234, & $\mathrm{Pa}-234 \mathrm{~m}$ & -> & $U-234$ \\
\hline 103.35 & $3.87 E-06$ & Th-234 & $->$ & $\mathrm{Pa}-234 \mathrm{~m}$ & & & \\
\hline 103.5 & $1.13 E+04$ & Pu-242 & $->$ & $U-238$ & & & \\
\hline 103.7 & $1.72 \mathrm{E}-07$ & $\mathrm{~Pa}-234$ & $->$ & $U-234$ & & & \\
\hline 103.71 & $6.63 E-06$ & Th-234 & $->$ & $\mathrm{Pa}-234 \mathrm{~m}$ & & & \\
\hline 108 & $6.52 E-06$ & Th-234 & $->$ & $\mathrm{Pa}-234 \mathrm{~m}$ & & & \\
\hline 108.166 & $5.52 E-06$ & Th-234 & $->$ & $\mathrm{Pa}-234 \mathrm{~m}$ & & & \\
\hline 109.97 & $7.80 \mathrm{E}-18$ & Th-230 & $->$ & $\mathrm{Ra}-226$ & & & \\
\hline 110.5 & $2.70 E-05$ & $\mathrm{U}-238$ & $->$ & Th-234 & & & \\
\hline 111.025 & $1.12 \mathrm{E}-04$ & Pa-234 & $->$ & U-234, & $\mathrm{Pa}-234 \mathrm{~m}$ & $->$ & $U-234$ \\
\hline 111.897 & $1.77 \mathrm{E}-06$ & Th-234 & $->$ & $\mathrm{Pa}-234 \mathrm{~m}$ & & & \\
\hline 112.8 & $2.67 E-04$ & Th-234 & $->$ & $\mathrm{Pa}-234 \mathrm{~m}$ & & & \\
\hline 114.866 & $3.83 E-05$ & $\mathrm{~Pa}-234$ & $->$ & U-234, & $\mathrm{Pa}-234 \mathrm{~m}$ & $->$ & $U-234$ \\
\hline 120.912 & $3.14 \mathrm{E}-10$ & $\mathrm{U}-234$ & $->$ & Th-230 & & & \\
\hline 125.53 & $1.44 \mathrm{E}-06$ & $\mathrm{~Pa}-234$ & $->$ & $U-234$ & & & \\
\hline 131.31 & $3.16 \mathrm{E}-05$ & Pa-234 & $->$ & $U-234$ & & & \\
\hline 134.3 & $3.01 E-07$ & Pa-234 & $->$ & $U-234$ & & & \\
\hline 137.4 & $0.00 E+00$ & $\mathrm{~Pb}-214$ & $->$ & $\mathrm{Bi}-214$ & & & \\
\hline 137.7 & $2.15 \mathrm{E}-07$ & Pa-234 & $->$ & $U-234$ & & & \\
\hline 140.15 & $2.56 \mathrm{E}-06$ & $\mathrm{~Pa}-234$ & $->$ & U-234, & $\mathrm{Pa}-234 \mathrm{~m}$ & $->$ & $U-234$ \\
\hline 141.3 & $0.00 E+00$ & $\mathrm{~Pb}-214$ & $->$ & $\mathrm{Bi}-214$ & & & \\
\hline 143.876 & $6.42 E-15$ & Th-230 & $->$ & Ra-226 & & & \\
\hline 143.9 & 5.02E-07 & $\mathrm{Pa}-234$ & $->$ & $\mathrm{U}-234$ & & & \\
\hline 150 & $2.87 E-07$ & $\mathrm{~Pa}-234$ & $->$ & $U-234$ & & & \\
\hline 152.76 & $9.16 \mathrm{E}-06$ & Pa-234 & $->$ & $U-234$ & & & \\
\hline 157.96 & $1.00 \mathrm{E}-06$ & $\mathrm{~Pa}-234$ & $->$ & $U-234$ & & & \\
\hline
\end{tabular}




\begin{tabular}{|c|c|c|c|c|c|c|c|}
\hline 158.8 & $6.54 \mathrm{E}+02$ & Pu-242 & $->$ & $U-238$ & & & \\
\hline 165.8 & 1.29E-07 & $\mathrm{Pa}-234$ & $->$ & $U-234$ & & & \\
\hline 170.75 & $7.18 \mathrm{E}-07$ & $\mathrm{~Pa}-234$ & $->$ & $U-234$ & & & \\
\hline 174.52 & $2.87 E-07$ & Pa-234 & $->$ & $U-234$ & & & \\
\hline 184.6 & $1.69 E-06$ & $\mathrm{~Pa}-234 \mathrm{~m}$ & $->$ & $U-234$ & & & \\
\hline 184.8 & $1.33 E-05$ & Th-234 & $->$ & $\mathrm{Pa}-234 \mathrm{~m}$ & & & \\
\hline 185.92 & $2.87 E-06$ & Pa-234 & $->$ & U-234 & & & \\
\hline 186.057 & $1.16 \mathrm{E}-15$ & Th-230 & $->$ & Ra-226 & & & \\
\hline 186.11 & $0.00 E+00$ & Ra-226 & $->$ & $\mathrm{Rn}-222$ & & & \\
\hline 192.74 & $7.61 \mathrm{E}-08$ & $\mathrm{~Pa}-234 \mathrm{~m}$ & $->$ & U-234 & & & \\
\hline 193.4 & $6.21 E-07$ & Pa-234m & $->$ & $U-234$ & & & \\
\hline 193.55 & 8.61E-07 & Pa-234 & $->$ & $U-234$ & & & \\
\hline 196.3 & $0.00 E+00$ & $\mathrm{~Pb}-214$ & $->$ & $\mathrm{Bi}-214$ & & & \\
\hline 196.43 & $1.00 \mathrm{E}-07$ & Pa-234 & $->$ & U-234 & & & \\
\hline 199.46 & $1.25 \mathrm{E}-06$ & Pa-234 & $->$ & U-234, & $\mathrm{Pa}-234 \mathrm{~m}$ & $->$ & $U-234$ \\
\hline 200.96 & $1.58 \mathrm{E}-06$ & $\mathrm{~Pa}-234$ & $->$ & U-234 & & & \\
\hline 203.12 & $2.98 \mathrm{E}-06$ & Pa-234 & $->$ & U-234, & $\mathrm{Pa}-234 \mathrm{~m}$ & $->$ & $U-234$ \\
\hline 205.1 & $6.61 \mathrm{E}-19$ & Th-230 & $->$ & Ra-226 & & & \\
\hline 210.6 & $1.30 \mathrm{E}-06$ & $\mathrm{~Pa}-234 \mathrm{~m}$ & $->$ & $U-234$ & & & \\
\hline 210.7 & $7.18 \mathrm{E}-08$ & $\mathrm{~Pa}-234$ & $->$ & $U-234$ & & & \\
\hline 219.8 & 2.87E-07 & Pa-234 & $->$ & $U-234$ & & & \\
\hline 226.95 & $1.79 E-05$ & Pa-234 & $->$ & $U-234$ & & & \\
\hline 235.01 & $1.07 E-18$ & Th-230 & $->$ & $\mathrm{Ra}-226$ & & & \\
\hline 236.03 & 4.19E-08 & $\mathrm{Pa}-234 \mathrm{~m}$ & $->$ & U-234 & & & \\
\hline 241.92 & $0.00 E+00$ & $\mathrm{~Pb}-214$ & $->$ & $\mathrm{Bi}-214$ & & & \\
\hline 243.5 & $4.94 \mathrm{E}-06$ & $\mathrm{~Pa}-234 \mathrm{~m}$ & $->$ & $U-234$ & & & \\
\hline 245.16 & $1.29 \mathrm{E}-06$ & Pa-234 & $->$ & $U-234$ & & & \\
\hline 247.7 & $7.36 \mathrm{E}-07$ & $\mathrm{~Pa}-234 \mathrm{~m}$ & $->$ & $U-234$ & & & \\
\hline 248.94 & $3.13 E-06$ & Pa-234 & $->$ & U-234 & & & \\
\hline 253.732 & $1.47 \mathrm{E}-15$ & Th-230 & $->$ & Ra-226 & & & \\
\hline 253.84 & $1.12 \mathrm{E}-16$ & Th-230 & $->$ & Ra-226 & & & \\
\hline 258.26 & 8.05E-05 & $\mathrm{Pa}-234 \mathrm{~m}$ & $->$ & U-234 & & & \\
\hline 258.94 & $0.00 E+00$ & $\mathrm{~Pb}-214$ & $->$ & $\mathrm{Bi}-214$ & & & \\
\hline 262.41 & $0.00 E+00$ & Ra-226 & $->$ & $R n-222$ & & & \\
\hline 266.15 & $4.80 E-31$ & $\mathrm{Bi}-210$ & $->$ & TI-206 & & & \\
\hline 267.1 & $2.44 \mathrm{E}-07$ & Pa-234 & $->$ & $U-234$ & & & \\
\hline 272.2 & 1.99E-06 & $\mathrm{Pa}-234$ & $->$ & U-234 & & & \\
\hline 273.7 & $3.21 \mathrm{E}-18$ & $\mathrm{Bi}-214$ & $->$ & Po-214 & & & \\
\hline 274.45 & $7.84 \mathrm{E}-07$ & Pa-234 & $->$ & U-234, & Pa-234m & $->$ & U-234 \\
\hline 274.56 & $0.00 E+00$ & $\mathrm{~Pb}-214$ & $->$ & $\mathrm{Bi}-214$ & & & \\
\hline 276.83 & $6.83 E-07$ & Pa-234 & $->$ & U-234, & $\mathrm{Pa}-234 \mathrm{~m}$ & $->$ & $U-234$ \\
\hline 278.1 & $1.00 \mathrm{E}-07$ & $\mathrm{~Pa}-234$ & $->$ & U-234 & & & \\
\hline 280.93 & $1.44 \mathrm{E}-18$ & $\mathrm{Bi}-214$ & $->$ & Po-214 & & & \\
\hline 286.4 & 2.01E-07 & $\mathrm{Pa}-234$ & $->$ & U-234 & & & \\
\hline 286.9 & $5.70 E-19$ & $\mathrm{Bi}-214$ & $->$ & Po-214 & & & \\
\hline 289.25 & $1.58 \mathrm{E}-07$ & $\mathrm{~Pa}-234$ & $->$ & $U-234$ & & & \\
\hline 293.874 & $5.41 \mathrm{E}-06$ & $\mathrm{~Pa}-234$ & $->$ & $U-234$ & & & \\
\hline 295.091 & $0.00 E+00$ & $\mathrm{~Pb}-214$ & $->$ & $\mathrm{Bi}-214$ & & & \\
\hline 298.1 & $8.16 \mathrm{E}-19$ & TI-210 & $->$ & $\mathrm{Pb}-210$ & Po-214 & $->$ & $\mathrm{Pb}-210$ \\
\hline 299.08 & $6.21 \mathrm{E}-07$ & Pa-234m & $->$ & U-234 & & & \\
\hline
\end{tabular}




\begin{tabular}{|c|c|c|c|c|}
\hline 302.25 & $5.61 \mathrm{E}-18$ & $\mathrm{Hg}-206$ & $->$ & TI-206 \\
\hline 304.42 & $6.06 \mathrm{E}-19$ & $\mathrm{Bi}-214$ & $->$ & Po-214 \\
\hline 305.5 & $0.00 E+00$ & $\mathrm{~Pb}-214$ & $->$ & $\mathrm{Bi}-214$ \\
\hline 309.6 & $1.44 \mathrm{E}-07$ & $\mathrm{~Pa}-234$ & $->$ & $U-234$ \\
\hline 311 & $5.08 \mathrm{E}-07$ & $\mathrm{~Pa}-234 \mathrm{~m}$ & $->$ & U-234 \\
\hline 313.83 & $4.31 \mathrm{E}-07$ & $\mathrm{~Pa}-234$ & $->$ & $U-234$ \\
\hline 314.2 & $0.00 E+00$ & $\mathrm{~Pb}-214$ & $->$ & $\mathrm{Bi}-214$ \\
\hline 316.29 & $3.13 E-07$ & $\mathrm{~Pa}-234$ & $->$ & U-234, \\
\hline 320.7 & $1.72 \mathrm{E}-07$ & $\mathrm{~Pa}-234$ & $->$ & $U-234$ \\
\hline 324.3 & $0.00 E+00$ & $\mathrm{~Pb}-214$ & $->$ & $\mathrm{Bi}-214$ \\
\hline 330 & $4.31 \mathrm{E}-07$ & $\mathrm{~Pa}-234$ & $->$ & $U-234$ \\
\hline 330.62 & 8.61E-07 & $\mathrm{Pa}-234$ & $->$ & $U-234$ \\
\hline 333.6 & $1.69 \mathrm{E}-18$ & $\mathrm{Bi}-214$ & $->$ & Po-214 \\
\hline 334.9 & $1.02 \mathrm{E}-18$ & $\mathrm{Bi}-214$ & $->$ & Po-214 \\
\hline 338.4 & $1.12 \mathrm{E}-06$ & $\mathrm{~Pa}-234 \mathrm{~m}$ & $->$ & U-234 \\
\hline 338.5 & $6.95 \mathrm{E}-19$ & $\mathrm{Bi}-214$ & $->$ & Po-214 \\
\hline 344.96 & $1.08 \mathrm{E}-19$ & $\mathrm{Hg}-206$ & $->$ & TI-206 \\
\hline 347.1 & $1.07 \mathrm{E}-18$ & $\mathrm{Bi}-214$ & $->$ & Po-214 \\
\hline 351.87 & $0.00 E+00$ & $\mathrm{~Pb}-214$ & $->$ & $\mathrm{Bi}-214$ \\
\hline 352.16 & 8.61E-07 & $\mathrm{Pa}-234$ & $->$ & $U-234$ \\
\hline 354 & $4.13 E-20$ & TI-210 & $->$ & $\mathrm{Pb}-210$ \\
\hline 357.9 & 7.90E-07 & $\mathrm{Pa}-234 \mathrm{~m}$ & $->$ & $U-234$ \\
\hline 360.8 & $6.77 \mathrm{E}-07$ & $\mathrm{~Pa}-234 \mathrm{~m}$ & $->$ & U-234 \\
\hline 364.2 & $1.12 \mathrm{E}-19$ & $\mathrm{Bi}-214$ & $->$ & Po-214 \\
\hline 369.52 & $4.86 \mathrm{E}-06$ & $\mathrm{~Pa}-234$ & $->$ & $U-234$ \\
\hline 372.02 & $2.54 \mathrm{E}-06$ & $\mathrm{~Pa}-234$ & $->$ & $U-234$ \\
\hline 376.6 & $8.73 E-20$ & $\mathrm{Bi}-214$ & $->$ & Po-214 \\
\hline 380 & $3.10 \mathrm{E}-20$ & TI-210 & $->$ & $\mathrm{Pb}-210$ \\
\hline 384.06 & $7.89 E-22$ & $\mathrm{Hg}-206$ & $->$ & Tl-206 \\
\hline 386.834 & $6.42 \mathrm{E}-18$ & $\mathrm{Bi}-214$ & $->$ & Po-214 \\
\hline 387.8 & $9.88 \mathrm{E}-07$ & $\mathrm{~Pa}-234 \mathrm{~m}$ & $->$ & $U-234$ \\
\hline 387.87 & $4.24 \mathrm{E}-07$ & $\mathrm{~Pa}-234 \mathrm{~m}$ & $->$ & $\mathrm{U}-234$ \\
\hline 388.95 & $7.31 \mathrm{E}-18$ & $\mathrm{Bi}-214$ & $->$ & Po-214 \\
\hline 394 & $1.60 \mathrm{E}-19$ & $\mathrm{Bi}-214$ & -> & Po-214 \\
\hline 396 & $5.35 \mathrm{E}-19$ & $\mathrm{Bi}-214$ & $->$ & Po-214 \\
\hline 405.73 & $2.98 \mathrm{E}-18$ & $\mathrm{Bi}-214$ & $->$ & Po-214 \\
\hline 409.8 & $5.74 \mathrm{E}-07$ & $\mathrm{~Pa}-234$ & $->$ & $U-234$ \\
\hline 414.72 & $0.00 \mathrm{E}+00$ & Ra-226 & $->$ & $\mathrm{Rn}-222$ \\
\hline 416 & $1.44 \mathrm{E}-07$ & $\mathrm{~Pa}-234$ & $->$ & $U-234$ \\
\hline 426.5 & $1.96 \mathrm{E}-18$ & $\mathrm{Bi}-214$ & $->$ & Po-214 \\
\hline 426.8 & $8.61 \mathrm{E}-07$ & $\mathrm{~Pa}-234$ & $->$ & $U-234$ \\
\hline 432.8 & $1.58 \mathrm{E}-08$ & $\mathrm{~Pa}-234$ & -> & U-234 \\
\hline 440.4 & $5.17 \mathrm{E}-19$ & $\mathrm{Bi}-214$ & $->$ & Po-214 \\
\hline 446.1 & $1.72 \mathrm{E}-07$ & $\mathrm{~Pa}-234$ & $->$ & $U-234$ \\
\hline 449.5 & $0.00 \mathrm{E}+00$ & Ra-226 & $->$ & $\mathrm{Rn}-222$ \\
\hline 450.96 & $2.98 \mathrm{E}-06$ & $\mathrm{~Pa}-234 \mathrm{~m}$ & $->$ & $U-234$ \\
\hline 453.58 & $2.10 \mathrm{E}-06$ & $\mathrm{~Pa}-234 \mathrm{~m}$ & $->$ & $U-234$ \\
\hline 454.832 & $5.67 \mathrm{E}-18$ & $\mathrm{Bi}-214$ & $->$ & Po-214 \\
\hline 454.97 & $1.99 \mathrm{E}-13$ & U-234 & $->$ & Th-230 \\
\hline 456.5 & $7.06 E-07$ & $\mathrm{~Pa}-234 \mathrm{~m}$ & $->$ & $\mathrm{U}-234$ \\
\hline
\end{tabular}




\begin{tabular}{|c|c|c|c|c|}
\hline 458.63 & $2.21 \mathrm{E}-06$ & $\mathrm{~Pa}-234$ & -> & $U-234$ \\
\hline 461.3 & $2.30 \mathrm{E}-07$ & $\mathrm{~Pa}-234$ & $->$ & $U-234$ \\
\hline 462.05 & $0.00 \mathrm{E}+00$ & $\mathrm{~Pb}-214$ & $->$ & $\mathrm{Bi}-214$ \\
\hline 467.5 & $5.02 \mathrm{E}-07$ & Pa-234 & $->$ & U-234 \\
\hline 468.21 & $2.03 \mathrm{E}-06$ & $\mathrm{~Pa}-234 \mathrm{~m}$ & $->$ & U-234 \\
\hline 469.76 & $2.37 \mathrm{E}-18$ & $\mathrm{Bi}-214$ & $->$ & Po-214 \\
\hline 470.8 & $0.00 \mathrm{E}+00$ & $\mathrm{~Pb}-214$ & $->$ & $\mathrm{Bi}-214$ \\
\hline 472.32 & $3.44 \mathrm{E}-07$ & $\mathrm{~Pa}-234$ & -> & $U-234$ \\
\hline 473.5 & $2.58 \mathrm{E}-07$ & $\mathrm{~Pa}-234$ & $->$ & U-234 \\
\hline 474.51 & $2.10 \mathrm{E}-18$ & $\mathrm{Bi}-214$ & $->$ & Po-214 \\
\hline 475.75 & $2.54 \mathrm{E}-06$ & $\mathrm{~Pa}-234 \mathrm{~m}$ & $->$ & U-234 \\
\hline 478 & $2.07 E-20$ & TI-210 & $->$ & $\mathrm{Pb}-210$ \\
\hline 478.7 & $4.31 \mathrm{E}-07$ & $\mathrm{~Pa}-234$ & $->$ & U-234 \\
\hline 480.32 & $0.00 E+00$ & $\mathrm{~Pb}-214$ & $->$ & $\mathrm{Bi}-214$ \\
\hline 480.4 & $5.74 \mathrm{E}-07$ & $\mathrm{~Pa}-234$ & $->$ & $U-234$ \\
\hline 482.5 & $4.31 \mathrm{E}-07$ & $\mathrm{~Pa}-234$ & $->$ & $U-234$ \\
\hline 487.13 & $0.00 E+00$ & $\mathrm{~Pb}-214$ & $->$ & $\mathrm{Bi}-214$ \\
\hline 494.6 & $1.60 \mathrm{E}-19$ & $\mathrm{Bi}-214$ & $->$ & Po-214 \\
\hline 498.8 & $1.44 \mathrm{E}-07$ & $\mathrm{~Pa}-234$ & $->$ & $U-234$ \\
\hline 502.2 & $3.21 \mathrm{E}-19$ & $\mathrm{Bi}-214$ & $->$ & Po-214 \\
\hline 503.53 & $7.65 E-15$ & U-234 & -> & Th-230 \\
\hline 506.69 & $2.31 \mathrm{E}-06$ & Pa-234 & $->$ & $U-234$ \\
\hline 507.3 & $1.56 \mathrm{E}-06$ & $\mathrm{~Pa}-234 \mathrm{~m}$ & $->$ & $U-234$ \\
\hline 508.2 & $1.12 \mathrm{E}-13$ & U-234 & $->$ & Th-230 \\
\hline 509.11 & $2.12 \mathrm{E}-06$ & $\mathrm{~Pa}-234 \mathrm{~m}$ & $->$ & $U-234$ \\
\hline 510 & $0.00 \mathrm{E}+00$ & Rn-222 & $->$ & Po-218 \\
\hline 511 & $0.00 \mathrm{E}+00$ & $\mathrm{~Pb}-214$ & $->$ & $\mathrm{Bi}-214$ \\
\hline 513.6 & $1.87 \mathrm{E}-06$ & $\mathrm{~Pa}-234$ & $->$ & $\mathrm{U}-234$ \\
\hline 516.8 & $1.65 \mathrm{E}-08$ & $\mathrm{~Pa}-234 \mathrm{~m}$ & $->$ & $\mathrm{U}-234$ \\
\hline 520.2 & 8.61E-07 & Pa-234 & $->$ & $U-234$ \\
\hline 520.4 & $1.02 \mathrm{E}-19$ & $\mathrm{Bi}-214$ & $->$ & Po-214 \\
\hline 521.09 & $1.29 \mathrm{E}-06$ & $\mathrm{~Pa}-234$ & $->$ & $U-234$ \\
\hline 525 & $2.85 \mathrm{E}-19$ & $\mathrm{Bi}-214$ & $->$ & Po-214 \\
\hline 527.04 & $8.61 \mathrm{E}-07$ & Pa-234 & -> & $U-234$ \\
\hline 533.2 & 2.87E-07 & $\mathrm{Pa}-234$ & $->$ & $U-234$ \\
\hline 533.5 & $0.00 \mathrm{E}+00$ & $\mathrm{~Pb}-214$ & $->$ & $\mathrm{Bi}-214$ \\
\hline 536.93 & $1.27 \mathrm{E}-18$ & $\mathrm{Bi}-214$ & $->$ & Po-214 \\
\hline 537.1 & $2.30 \mathrm{E}-07$ & $\mathrm{~Pa}-234$ & $->$ & $\mathrm{U}-234$ \\
\hline 538.7 & $0.00 E+00$ & $\mathrm{~Pb}-214$ & $->$ & $\mathrm{Bi}-214$ \\
\hline 542.84 & $1.52 \mathrm{E}-18$ & $\mathrm{Bi}-214$ & $->$ & Po-214 \\
\hline 543.91 & $0.00 \mathrm{E}+00$ & $\mathrm{~Pb}-214$ & $->$ & $\mathrm{Bi}-214$ \\
\hline 543.98 & 3.97E-06 & $\mathrm{Pa}-234 \mathrm{~m}$ & $->$ & $U-234$ \\
\hline 547.1 & $5.70 \mathrm{E}-19$ & $\mathrm{Bi}-214$ & $->$ & Po-214 \\
\hline 551.8 & $1.33 \mathrm{E}-19$ & Th-230 & -> & Ra-226 \\
\hline 557.3 & $7.06 \mathrm{E}-07$ & $\mathrm{~Pa}-234 \mathrm{~m}$ & $->$ & $U-234$ \\
\hline 557.7 & 3.97E-08 & $\mathrm{Pa}-234 \mathrm{~m}$ & $->$ & $\mathrm{U}-234$ \\
\hline 557.78 & $3.73 \mathrm{E}-07$ & $\mathrm{~Pa}-234$ & -> & $U-234$ \\
\hline 565.2 & $2.01 \mathrm{E}-06$ & $\mathrm{~Pa}-234$ & $->$ & $U-234$ \\
\hline 569.3 & $2.24 \mathrm{E}-05$ & $\mathrm{~Pa}-234$ & $->$ & $U-234$ \\
\hline 570.5 & $3.96 \mathrm{E}-19$ & Th-230 & $->$ & Ra-226 \\
\hline
\end{tabular}




\begin{tabular}{|c|c|c|c|c|}
\hline 572 & $8.60 E-07$ & $\mathrm{~Pa}-234 \mathrm{~m}$ & $\rightarrow$ & $U-234$ \\
\hline 572.67 & $1.46 \mathrm{E}-18$ & $\mathrm{Bi}-214$ & -> & Po-214 \\
\hline 574.8 & $2.87 \mathrm{E}-06$ & $\mathrm{~Pa}-234$ & $->$ & U-234 \\
\hline 580.06 & $0.00 E+00$ & $\mathrm{~Pb}-214$ & -> & $\mathrm{Bi}-214$ \\
\hline 581.78 & $9.18 \mathrm{E}-14$ & U-234 & -> & Th-230 \\
\hline 585.8 & $2.15 \mathrm{E}-07$ & $\mathrm{~Pa}-234$ & $->$ & U-234 \\
\hline 596 & $2.14 \mathrm{E}-19$ & $\mathrm{Bi}-214$ & $->$ & Po-214 \\
\hline 600.83 & $0.00 E+00$ & Ra-226 & $->$ & $\mathrm{Rn}-222$ \\
\hline 602.1 & $1.29 \mathrm{E}-06$ & $\mathrm{~Pa}-234$ & -> & $U-234$ \\
\hline 609.311 & $8.22 \mathrm{E}-16$ & $\mathrm{Bi}-214$ & $->$ & Po-214 \\
\hline 611.5 & $1.15 \mathrm{E}-06$ & $\mathrm{~Pa}-234$ & -> & $U-234$ \\
\hline 615.77 & $1.25 \mathrm{E}-18$ & $\mathrm{Bi}-214$ & $->$ & Po-214 \\
\hline 617 & 2.87E-07 & $\mathrm{Pa}-234$ & $->$ & $U-234$ \\
\hline 617.1 & $6.06 \mathrm{E}-19$ & $\mathrm{Bi}-214$ & $->$ & Po-214 \\
\hline 620 & $1.33 \mathrm{E}-19$ & Th-230 & -> & $\mathrm{Ra}-226$ \\
\hline 624.31 & $1.15 \mathrm{E}-06$ & $\mathrm{~Pa}-234$ & $->$ & $U-234$ \\
\hline 624.44 & $6.12 \mathrm{E}-15$ & U-234 & $->$ & Th-230 \\
\hline 625.5 & $1.41 \mathrm{E}-06$ & $\mathrm{~Pa}-234 \mathrm{~m}$ & $->$ & $U-234$ \\
\hline 626.4 & $8.73 E-20$ & $\mathrm{Bi}-214$ & $->$ & Po-214 \\
\hline 627.5 & $1.15 \mathrm{E}-06$ & $\mathrm{~Pa}-234$ & -> & $U-234$ \\
\hline 631.2 & 3.03E-19 & $\mathrm{Bi}-214$ & $->$ & Po-214 \\
\hline 632 & $5.74 \mathrm{E}-07$ & $\mathrm{~Pa}-234$ & $->$ & $U-234$ \\
\hline 633.14 & $1.07 \mathrm{E}-18$ & $\mathrm{Bi}-214$ & $->$ & Po-214 \\
\hline 634.4 & $4.31 \mathrm{E}-07$ & $\mathrm{~Pa}-234$ & $->$ & $U-234$ \\
\hline 639.36 & $5.53 \mathrm{E}-19$ & $\mathrm{Bi}-214$ & -> & Po-214 \\
\hline 639.7 & $2.87 E-07$ & $\mathrm{~Pa}-234$ & $->$ & $U-234$ \\
\hline 643.2 & $2.87 E-07$ & $\mathrm{~Pa}-234$ & $->$ & U-234 \\
\hline 646.84 & $4.31 \mathrm{E}-07$ & $\mathrm{~Pa}-234$ & $->$ & U-234 \\
\hline 647.7 & $1.55 \mathrm{E}-06$ & $\mathrm{~Pa}-234 \mathrm{~m}$ & -> & U-234 \\
\hline 649.1 & $1.06 \mathrm{E}-06$ & $\mathrm{~Pa}-234 \mathrm{~m}$ & -> & $U-234$ \\
\hline 649.18 & $1.05 \mathrm{E}-18$ & $\mathrm{Bi}-214$ & $->$ & Po-214 \\
\hline 650.21 & 5.19E-19 & $\mathrm{Hg}-206$ & $->$ & TI-206 \\
\hline 653.57 & 8.90E-07 & $\mathrm{Pa}-234$ & -> & U-234 \\
\hline 655 & 5.94E-07 & Pa-234 & $->$ & U-234 \\
\hline 655.1 & $9.44 \mathrm{E}-07$ & Pa-234m & $->$ & $U-234$ \\
\hline 658 & $1.29 \mathrm{E}-06$ & Pa-234 & $->$ & U-234 \\
\hline 660.6 & $4.31 \mathrm{E}-07$ & $\mathrm{~Pa}-234$ & $->$ & $U-234$ \\
\hline 660.75 & 7.67E-19 & $\mathrm{Bi}-214$ & $->$ & Po-214 \\
\hline 664.8 & $1.87 \mathrm{E}-06$ & $\mathrm{~Pa}-234$ & $->$ & $U-234$ \\
\hline 665.442 & $2.78 \mathrm{E}-17$ & $\mathrm{Bi}-214$ & $->$ & Po-214 \\
\hline 666.42 & $1.66 \mathrm{E}-06$ & $\mathrm{~Pa}-234$ & $->$ & $U-234$ \\
\hline 668 & $2.07 E-20$ & Tl-210 & -> & $\mathrm{Pb}-210$ \\
\hline 669.64 & $1.88 \mathrm{E}-06$ & Pa-234 & -> & U-234 \\
\hline 674.24 & $6.35 \mathrm{E}-07$ & Pa-234m & -> & $U-234$ \\
\hline 677.67 & $7.65 \mathrm{E}-15$ & U-234 & $->$ & Th-230 \\
\hline 683 & $5.65 \mathrm{E}-07$ & $\mathrm{~Pa}-234 \mathrm{~m}$ & $->$ & $U-234$ \\
\hline 683.21 & $1.41 \mathrm{E}-18$ & $\mathrm{Bi}-214$ & -> & Po-214 \\
\hline 683.3 & $3.44 \mathrm{E}-07$ & $\mathrm{~Pa}-234$ & -> & $U-234$ \\
\hline 687.7 & $1.07 \mathrm{E}-19$ & $\mathrm{Bi}-214$ & $->$ & Po-214 \\
\hline 688.08 & $4.02 \mathrm{E}-07$ & $\mathrm{~Pa}-234$ & $->$ & U-234 \\
\hline
\end{tabular}




\begin{tabular}{|c|c|c|c|c|c|c|c|}
\hline 691.08 & 9.93E-06 & $\mathrm{Pa}-234 \mathrm{~m}$ & $->$ & $U-234$ & & & \\
\hline 692.7 & $2.15 \mathrm{E}-06$ & $\mathrm{~Pa}-234$ & $->$ & $\mathrm{U}-234$ & & & \\
\hline 693.3 & $1.07 E-19$ & $\mathrm{Bi}-214$ & $->$ & Po-214 & & & \\
\hline 695.9 & $1.55 \mathrm{E}-06$ & $\mathrm{~Pa}-234 \mathrm{~m}$ & $->$ & $U-234$ & & & \\
\hline 697.89 & $6.60 \mathrm{E}-19$ & $\mathrm{Bi}-214$ & $->$ & Po-214 & & & \\
\hline 698.85 & $5.82 \mathrm{E}-06$ & $\mathrm{~Pa}-234$ & $->$ & $U-234$ & & & \\
\hline 700.4 & $6.96 \mathrm{E}-07$ & $\mathrm{~Pa}-234 \mathrm{~m}$ & $->$ & $U-234$ & & & \\
\hline 702.05 & $7.83 E-06$ & $\mathrm{~Pa}-234 \mathrm{~m}$ & $->$ & $\mathrm{U}-234$ & & & \\
\hline 703.07 & $8.41 \mathrm{E}-18$ & $\mathrm{Bi}-214$ & $->$ & Po-214 & & & \\
\hline 705.9 & $7.17 \mathrm{E}-06$ & Pa-234 & $->$ & U-234, & $\mathrm{Pa}-234 \mathrm{~m}$ & $->$ & $U-234$ \\
\hline 708.4 & $1.14 \mathrm{E}-06$ & $\mathrm{~Pa}-234$ & $->$ & U-234, & $\mathrm{Pa}-234 \mathrm{~m}$ & $->$ & $U-234$ \\
\hline 710.84 & $1.33 E-18$ & $\mathrm{Bi}-214$ & $->$ & Po-214 & & & \\
\hline 711.2 & $2.87 E-07$ & Pa-234 & $->$ & $U-234$ & & & \\
\hline 713.8 & $2.30 \mathrm{E}-07$ & Pa-234 & $->$ & $U-234$ & & & \\
\hline 718.94 & $3.42 \mathrm{E}-08$ & $\mathrm{~Pa}-234 \mathrm{~m}$ & $->$ & $U-234$ & & & \\
\hline 719.856 & $7.18 \mathrm{E}-18$ & $\mathrm{Bi}-214$ & $->$ & Po-214 & & & \\
\hline 721.33 & $3.20 E-08$ & $\mathrm{~Pa}-234 \mathrm{~m}$ & $->$ & $U-234$ & & & \\
\hline 723.32 & $8.02 E-19$ & $\mathrm{Bi}-214$ & $->$ & Po-214 & & & \\
\hline 727.8 & $2.80 \mathrm{E}-19$ & $\mathrm{Bi}-214$ & $->$ & Po-214 & & & \\
\hline 732.7 & $1.28 \mathrm{E}-06$ & $\mathrm{~Pa}-234 \mathrm{~m}$ & $->$ & $U-234$ & & & \\
\hline 733.38 & $1.27 E-05$ & Pa-234 & $->$ & $\mathrm{U}-234$ & & & \\
\hline 733.64 & 8.38E-19 & $\mathrm{Bi}-214$ & $->$ & Po-214 & & & \\
\hline 738.88 & $2.32 E-06$ & Pa-234 & $->$ & $U-234$ & & & \\
\hline 739.95 & $1.30 \mathrm{E}-05$ & $\mathrm{~Pa}-234 \mathrm{~m}$ & $->$ & $U-234$ & & & \\
\hline 740.87 & $6.95 \mathrm{E}-19$ & $\mathrm{Bi}-214$ & $->$ & Po-214 & & & \\
\hline 742.817 & $1.04 \mathrm{E}-04$ & Pa-234 & $->$ & U-234, & $\mathrm{Pa}-234 \mathrm{~m}$ & $->$ & $U-234$ \\
\hline 746.5 & $1.87 E-07$ & Pa-234 & $->$ & $\mathrm{U}-234$ & & & \\
\hline 750.5 & $1.99 E-08$ & $\mathrm{~Pa}-234 \mathrm{~m}$ & $->$ & $U-234$ & & & \\
\hline 752.843 & $2.37 E-18$ & $\mathrm{Bi}-214$ & $->$ & Po-214 & & & \\
\hline 755 & $2.32 \mathrm{E}-06$ & $\mathrm{~Pa}-234$ & $->$ & $\mathrm{U}-234$ & & & \\
\hline 760.1 & $2.30 \mathrm{E}-07$ & Pa-234 & $->$ & $\mathrm{U}-234$ & & & \\
\hline 760.3 & $1.55 \mathrm{E}-06$ & $\mathrm{~Pa}-234 \mathrm{~m}$ & $->$ & U-234 & & & \\
\hline 765.9 & $0.00 E+00$ & $\mathrm{~Pb}-214$ & $->$ & $\mathrm{Bi}-214$ & & & \\
\hline 766.369 & $4.31 E-07$ & $\mathrm{~Pa}-234$ & $->$ & $U-234$ & & & \\
\hline 766.412 & $3.55 E-04$ & $\mathrm{~Pa}-234$ & $->$ & U-234, & $\mathrm{Pa}-234 \mathrm{~m}$ & $->$ & $U-234$ \\
\hline 768.35 & $8.70 \mathrm{E}-17$ & $\mathrm{Bi}-214$ & $->$ & Po-214 & & & \\
\hline 768.5 & 8.04E-07 & $\mathrm{Pa}-234$ & $->$ & $U-234$ & & & \\
\hline 777.9 & $2.87 E-07$ & $\mathrm{~Pa}-234$ & $->$ & $\mathrm{U}-234$ & & & \\
\hline 780.4 & $1.58 \mathrm{E}-06$ & Pa-234 & $->$ & $\mathrm{U}-234$ & & & \\
\hline 782.8 & $7.48 E-06$ & $\mathrm{~Pa}-234 \mathrm{~m}$ & $->$ & $U-234$ & & & \\
\hline 783.38 & 7.87E-07 & Pa-234 & $->$ & U-234, & $\mathrm{Pa}-234 \mathrm{~m}$ & $->$ & $U-234$ \\
\hline 785.827 & $0.00 E+00$ & $\mathrm{~Pb}-214$ & $->$ & $\mathrm{Bi}-214$ & & & \\
\hline 786.287 & $6.11 \mathrm{E}-05$ & $\mathrm{~Pa}-234$ & $->$ & U-234, & $\mathrm{Pa}-234 \mathrm{~m}$ & $->$ & $U-234$ \\
\hline 786.42 & $5.53 E-18$ & $\mathrm{Bi}-214$ & $->$ & Po-214 & & & \\
\hline 793.6 & $2.15 \mathrm{E}-06$ & $\mathrm{~Pa}-234$ & $->$ & $U-234$ & & & \\
\hline 796.42 & $5.96 \mathrm{E}-06$ & $\mathrm{~Pa}-234$ & $->$ & $U-234$ & & & \\
\hline 797.88 & $1.02 \mathrm{E}-18$ & TI-210 & $->$ & $\mathrm{Pb}-210$ & Po-214 & $->$ & $\mathrm{Pb}-210$ \\
\hline 799.75 & $7.31 \mathrm{E}-19$ & $\mathrm{Bi}-214$ & $->$ & Po-214 & & & \\
\hline 803.13 & $9.19 E-22$ & TI-206 & $->$ & $\mathrm{Pb}-206$ & Po-210 & $->$ & $\mathrm{Pb}-206$ \\
\hline 804.52 & $5.74 \mathrm{E}-07$ & $\mathrm{~Pa}-234$ & $->$ & $\mathrm{U}-234$ & & & \\
\hline
\end{tabular}




\begin{tabular}{|c|c|c|c|c|c|c|c|}
\hline 805.74 & $9.71 \mathrm{E}-06$ & $\mathrm{~Pa}-234$ & $->$ & U-234, & $\mathrm{Pa}-234 \mathrm{~m}$ & $->$ & $U-234$ \\
\hline 806.155 & $2.19 \mathrm{E}-17$ & $\mathrm{Bi}-214$ & $->$ & Po-214 & & & \\
\hline 808.2 & $2.87 E-06$ & $\mathrm{~Pa}-234$ & $->$ & U-234, & $\mathrm{Pa}-234 \mathrm{~m}$ & $->$ & $U-234$ \\
\hline 812.5 & $7.18 \mathrm{E}-07$ & $\mathrm{~Pa}-234$ & $->$ & $U-234$ & & & \\
\hline 814.87 & $7.13 \mathrm{E}-19$ & $\mathrm{Bi}-214$ & $->$ & Po-214 & & & \\
\hline 818 & $9.88 \mathrm{E}-07$ & $\mathrm{~Pa}-234 \mathrm{~m}$ & $->$ & $U-234$ & & & \\
\hline 819.21 & 4.09E-06 & $\mathrm{Pa}-234$ & $->$ & $U-234$ & & & \\
\hline 821.166 & $2.67 \mathrm{E}-18$ & $\mathrm{Bi}-214$ & $->$ & Po-214 & & & \\
\hline 824.94 & $7.51 \mathrm{E}-06$ & $\mathrm{~Pa}-234$ & $->$ & $U-234$ & & & \\
\hline 826.08 & $1.40 \mathrm{E}-06$ & $\mathrm{~Pa}-234 \mathrm{~m}$ & $->$ & $U-234$ & & & \\
\hline 826.44 & $1.64 \mathrm{E}-18$ & $\mathrm{Bi}-214$ & $->$ & Po-214 & & & \\
\hline 831.39 & 8.61E-06 & Pa-234 & $->$ & $U-234$ & & & \\
\hline 832.34 & $4.10 \mathrm{E}-19$ & $\mathrm{Bi}-214$ & $->$ & Po-214 & & & \\
\hline 838.999 & $0.00 E+00$ & $\mathrm{~Pb}-214$ & $->$ & $\mathrm{Bi}-214$ & & & \\
\hline 842.4 & $2.01 \mathrm{E}-07$ & $\mathrm{~Pa}-234$ & $->$ & $U-234$ & & & \\
\hline 844.4 & $1.79 \mathrm{E}-06$ & $\mathrm{~Pa}-234$ & $->$ & U-234, & $\mathrm{Pa}-234 \mathrm{~m}$ & $->$ & $U-234$ \\
\hline 847.2 & $3.03 E-19$ & $\mathrm{Bi}-214$ & $->$ & Po-214 & & & \\
\hline 851.57 & $7.72 \mathrm{E}-06$ & Pa-234 & $->$ & U-234, & $\mathrm{Pa}-234 \mathrm{~m}$ & $->$ & $U-234$ \\
\hline 860 & $7.12 \mathrm{E}-20$ & TI-210 & $->$ & $\mathrm{Pb}-210$ & & & \\
\hline 866.98 & $1.06 \mathrm{E}-06$ & $\mathrm{~Pa}-234 \mathrm{~m}$ & $->$ & $U-234$ & & & \\
\hline 873.4 & $1.72 \mathrm{E}-07$ & $\mathrm{~Pa}-234$ & $->$ & $U-234$ & & & \\
\hline 875.94 & $4.64 \mathrm{E}-06$ & $\mathrm{~Pa}-234$ & $->$ & $U-234$ & & & \\
\hline 880.45 & $2.34 \mathrm{E}-05$ & Pa-234 & $->$ & $U-234$ & & & \\
\hline 881.1 & $3.81 \mathrm{E}-06$ & $\mathrm{~Pa}-234 \mathrm{~m}$ & $->$ & $U-234$ & & & \\
\hline 882.5 & $1.83 \mathrm{E}-06$ & $\mathrm{~Pa}-234 \mathrm{~m}$ & $->$ & $U-234$ & & & \\
\hline 883.22 & $2.33 E-05$ & Pa-234 & $->$ & U-234, & $\mathrm{Pa}-234 \mathrm{~m}$ & $->$ & $U-234$ \\
\hline 887.28 & $7.83 E-06$ & $\mathrm{~Pa}-234 \mathrm{~m}$ & $->$ & U-234 & & & \\
\hline 898.52 & $6.51 \mathrm{E}-06$ & Pa-234 & $->$ & $\mathrm{U}-234$ & & & \\
\hline 904.33 & $1.87 \mathrm{E}-18$ & $\mathrm{Bi}-214$ & $->$ & Po-214 & & & \\
\hline 904.38 & $7.18 \mathrm{E}-07$ & $\mathrm{~Pa}-234$ & $->$ & $U-234$ & & & \\
\hline 908 & $3.10 \mathrm{E}-20$ & $\mathrm{TI}-210$ & $->$ & $\mathrm{Pb}-210$ & & & \\
\hline 915.8 & $4.10 \mathrm{E}-19$ & $\mathrm{Bi}-214$ & $->$ & Po-214 & & & \\
\hline 920 & $5.74 \mathrm{E}-07$ & $\mathrm{~Pa}-234$ & $->$ & $\mathrm{U}-234$ & & & \\
\hline 921.7 & $1.40 \mathrm{E}-05$ & $\mathrm{~Pa}-234 \mathrm{~m}$ & $->$ & $U-234$ & & & \\
\hline 924.98 & $1.57 \mathrm{E}-05$ & $\mathrm{~Pa}-234$ & $->$ & $U-234$ & & & \\
\hline 926.61 & $2.12 \mathrm{E}-05$ & $\mathrm{~Pa}-234$ & $->$ & U-234, & $\mathrm{Pa}-234 \mathrm{~m}$ & $->$ & $U-234$ \\
\hline 934.039 & $5.63 E-17$ & $\mathrm{Bi}-214$ & $->$ & Po-214 & & & \\
\hline 936.8 & $1.83 E-06$ & $\mathrm{~Pa}-234 \mathrm{~m}$ & $->$ & $U-234$ & & & \\
\hline 941.94 & $2.76 \mathrm{E}-06$ & $\mathrm{~Pa}-234 \mathrm{~m}$ & $->$ & $\mathrm{U}-234$ & & & \\
\hline 943.3 & $3.03 E-19$ & $\mathrm{Bi}-214$ & $->$ & Po-214 & & & \\
\hline 945.9 & $3.70 E-05$ & $\mathrm{~Pa}-234$ & $->$ & U-234, & $\mathrm{Pa}-234 \mathrm{~m}$ & $->$ & $U-234$ \\
\hline 948 & $1.15 \mathrm{E}-05$ & $\mathrm{~Pa}-234$ & $->$ & $\mathrm{U}-234$ & & & \\
\hline 959.8 & $8.47 E-07$ & $\mathrm{~Pa}-234 \mathrm{~m}$ & $->$ & $U-234$ & & & \\
\hline 960 & $1.44 \mathrm{E}-07$ & $\mathrm{~Pa}-234$ & $->$ & $U-234$ & & & \\
\hline 964.07 & $6.83 \mathrm{E}-18$ & $\mathrm{Bi}-214$ & $->$ & Po-214 & & & \\
\hline 967.33 & $8.61 \mathrm{E}-07$ & $\mathrm{~Pa}-234$ & $->$ & $\mathrm{U}-234$ & & & \\
\hline 976.2 & $4.10 \mathrm{E}-19$ & $\mathrm{Bi}-214$ & $->$ & Po-214 & & & \\
\hline 978.8 & $1.44 \mathrm{E}-06$ & $\mathrm{~Pa}-234$ & $->$ & $U-234$ & & & \\
\hline 980.42 & $4.97 E-06$ & $\mathrm{~Pa}-234$ & $->$ & $U-234$ & & & \\
\hline 984.12 & $2.73 \mathrm{E}-06$ & $\mathrm{~Pa}-234$ & $->$ & $U-234$ & & & \\
\hline
\end{tabular}




\begin{tabular}{|c|c|c|c|c|c|c|c|}
\hline 989.2 & $2.14 \mathrm{E}-19$ & $\mathrm{Bi}-214$ & $->$ & Po-214 & & & \\
\hline 994.93 & $6.29 E-06$ & $\mathrm{~Pa}-234 \mathrm{~m}$ & $->$ & $U-234$ & & & \\
\hline 1000.99 & $9.25 E-04$ & $\mathrm{~Pa}-234 \mathrm{~m}$ & $->$ & U-234 & & & \\
\hline 1013.4 & $1.78 \mathrm{E}-19$ & $\mathrm{Bi}-214$ & $->$ & Po-214 & & & \\
\hline 1020.5 & $2.14 \mathrm{E}-19$ & $\mathrm{Bi}-214$ & $->$ & Po-214 & & & \\
\hline 1022.23 & $8.61 \mathrm{E}-07$ & $\mathrm{~Pa}-234$ & $->$ & $U-234$ & & & \\
\hline 1029.03 & $1.15 \mathrm{E}-06$ & $\mathrm{~Pa}-234$ & $->$ & $U-234$ & & & \\
\hline 1032.22 & $1.71 \mathrm{E}-18$ & $\mathrm{Bi}-214$ & $->$ & Po-214 & & & \\
\hline 1038 & $3.03 E-19$ & $\mathrm{Bi}-214$ & $->$ & Po-214 & & & \\
\hline 1041.7 & $1.32 \mathrm{E}-06$ & $\mathrm{~Pa}-234 \mathrm{~m}$ & $->$ & $U-234$ & & & \\
\hline 1044.9 & $7.18 \mathrm{E}-07$ & $\mathrm{~Pa}-234$ & $->$ & $\mathrm{U}-234$ & & & \\
\hline 1045.4 & 5.17E-19 & $\mathrm{Bi}-214$ & $->$ & Po-214 & & & \\
\hline 1051.95 & $5.62 \mathrm{E}-18$ & $\mathrm{Bi}-214$ & $->$ & Po-214 & & & \\
\hline 1059.72 & 1.09E-06 & $\mathrm{Pa}-234 \mathrm{~m}$ & $->$ & $U-234$ & & & \\
\hline 1061.86 & $2.54 \mathrm{E}-06$ & $\mathrm{~Pa}-234 \mathrm{~m}$ & $->$ & $U-234$ & & & \\
\hline 1067.3 & $5.17 E-19$ & $\mathrm{Bi}-214$ & $->$ & Po-214 & & & \\
\hline 1068 & $1.24 \mathrm{E}-19$ & TI-210 & $->$ & $\mathrm{Pb}-210$ & & & \\
\hline 1070.02 & $5.08 \mathrm{E}-18$ & $\mathrm{Bi}-214$ & $->$ & Po-214 & & & \\
\hline 1074.6 & $3.59 E-07$ & Pa-234 & $->$ & $\mathrm{U}-234$ & & & \\
\hline 1084.25 & $1.32 E-06$ & Pa-234 & $->$ & U-234, & $\mathrm{Pa}-234 \mathrm{~m}$ & $->$ & $U-234$ \\
\hline 1085.37 & $4.94 E-07$ & $\mathrm{~Pa}-234 \mathrm{~m}$ & $->$ & $U-234$ & & & \\
\hline 1103.7 & $1.78 \mathrm{E}-18$ & $\mathrm{Bi}-214$ & $->$ & Po-214 & & & \\
\hline 1104.766 & $1.43 E-18$ & $\mathrm{Bi}-214$ & $->$ & Po-214 & & & \\
\hline 1108.5 & $4.31 \mathrm{E}-07$ & $\mathrm{~Pa}-234$ & $->$ & $U-234$ & & & \\
\hline 1110 & $7.12 \mathrm{E}-20$ & TI-210 & $->$ & $\mathrm{Pb}-210$ & & & \\
\hline 1120.273 & $2.67 E-16$ & $\mathrm{Bi}-214$ & $->$ & Po-214 & & & \\
\hline 1120.5 & $1.69 E-06$ & $\mathrm{~Pa}-234 \mathrm{~m}$ & $->$ & $\mathrm{U}-234$ & & & \\
\hline 1122.09 & $7.18 \mathrm{E}-07$ & Pa-234 & $->$ & $U-234$ & & & \\
\hline 1124.93 & $4.63 E-06$ & $\mathrm{~Pa}-234 \mathrm{~m}$ & $->$ & U-234 & & & \\
\hline 1126.17 & 2.09E-06 & Pa-234 & $->$ & U-234, & $\mathrm{Pa}-234 \mathrm{~m}$ & $->$ & $U-234$ \\
\hline 1130.6 & $8.02 E-19$ & $\mathrm{Bi}-214$ & $->$ & Po-214 & & & \\
\hline 1133.65 & $4.55 E-18$ & $\mathrm{Bi}-214$ & $->$ & Po-214 & & & \\
\hline 1153.6 & $4.31 E-07$ & $\mathrm{~Pa}-234$ & $->$ & $\mathrm{U}-234$ & & & \\
\hline 1155.183 & $3.01 \mathrm{E}-17$ & $\mathrm{Bi}-214$ & $->$ & Po-214 & & & \\
\hline 1171.3 & $3.44 \mathrm{E}-07$ & $\mathrm{~Pa}-234$ & $->$ & $\mathrm{U}-234$ & & & \\
\hline 1172.93 & $1.03 E-18$ & $\mathrm{Bi}-214$ & $->$ & Po-214 & & & \\
\hline 1173.04 & $1.03 E-18$ & $\mathrm{Bi}-214$ & $->$ & Po-214 & & & \\
\hline 1174.1 & $1.89 E-06$ & $\mathrm{~Pa}-234 \mathrm{~m}$ & $->$ & $U-234$ & & & \\
\hline 1193.69 & $1.49 E-05$ & $\mathrm{~Pa}-234 \mathrm{~m}$ & $->$ & $U-234$ & & & \\
\hline 1207.764 & $8.20 E-18$ & $\mathrm{Bi}-214$ & $->$ & Po-214 & & & \\
\hline 1208 & $4.31 \mathrm{E}-07$ & TI-210 & $->$ & $\mathrm{Pb}-210$, & $\mathrm{Pa}-234$ & $->$ & $U-234$ \\
\hline 1216.4 & $5.31 \mathrm{E}-07$ & Pa-234 & $->$ & $U-234$ & & & \\
\hline 1220.37 & $9.93 E-07$ & $\mathrm{~Pa}-234 \mathrm{~m}$ & $->$ & $U-234$ & & & \\
\hline 1226.8 & $4.81 E-19$ & $\mathrm{Bi}-214$ & $->$ & Po-214 & & & \\
\hline 1229 & $4.31 E-07$ & $\mathrm{~Pa}-234$ & $->$ & $U-234$ & & & \\
\hline 1230.84 & $3.92 E-19$ & $\mathrm{Bi}-214$ & $->$ & Po-214 & & & \\
\hline 1237.24 & $5.85 \mathrm{E}-06$ & $\mathrm{~Pa}-234 \mathrm{~m}$ & $->$ & $\mathrm{U}-234$ & & & \\
\hline 1238.107 & $1.06 \mathrm{E}-16$ & Bi-214 & $->$ & Po-214 & & & \\
\hline 1241.4 & $7.18 \mathrm{E}-07$ & $\mathrm{~Pa}-234$ & $->$ & $U-234$ & & & \\
\hline 1251 & $4.31 \mathrm{E}-07$ & $\mathrm{~Pa}-234$ & $->$ & $U-234$ & & & \\
\hline
\end{tabular}




\begin{tabular}{|c|c|c|c|c|}
\hline 1277.4 & 2.87E-07 & $\mathrm{Pa}-234$ & -> & U-234 \\
\hline 1280.952 & $2.62 \mathrm{E}-17$ & $\mathrm{Bi}-214$ & $->$ & Po-214 \\
\hline 1292.66 & $9.93 \mathrm{E}-07$ & $\mathrm{~Pa}-234$ & -> & U-234 \\
\hline 1303.76 & $2.16 \mathrm{E}-18$ & $\mathrm{Bi}-214$ & $->$ & Po-214 \\
\hline 1314 & $2.17 \mathrm{E}-19$ & TI-210 & $->$ & $\mathrm{Pb}-210$ \\
\hline 1317.02 & $1.53 \mathrm{E}-18$ & $\mathrm{Bi}-214$ & $->$ & Po-214 \\
\hline 1330 & $1.96 \mathrm{E}-19$ & $\mathrm{Bi}-214$ & $->$ & Po-214 \\
\hline 1341.5 & $4.10 \mathrm{E}-19$ & $\mathrm{Bi}-214$ & -> & Po-214 \\
\hline 1352.85 & $1.67 \mathrm{E}-06$ & $\mathrm{~Pa}-234$ & -> & U-234 \\
\hline 1353 & $4.26 \mathrm{E}-07$ & $\mathrm{Bi}-214$ & -> & Po-214, \\
\hline 1358.5 & $1.72 \mathrm{E}-07$ & $\mathrm{~Pa}-234$ & $->$ & $\mathrm{U}-234$ \\
\hline 1377.659 & 7.17E-17 & $\mathrm{Bi}-214$ & -> & Po-214 \\
\hline 1385.295 & $1.39 \mathrm{E}-17$ & $\mathrm{Bi}-214$ & $->$ & Po-214 \\
\hline 1392 & $2.54 \mathrm{E}-06$ & $\mathrm{~Pa}-234 \mathrm{~m}$ & $->$ & $\mathrm{U}-234$ \\
\hline 1392.5 & 3.39E-19 & $\mathrm{Bi}-214$ & $->$ & Po-214 \\
\hline 1393.57 & $4.31 \mathrm{E}-06$ & $\mathrm{~Pa}-234$ & -> & $\mathrm{U}-234$ \\
\hline 1399.7 & $3.30 \mathrm{E}-07$ & $\mathrm{~Pa}-234$ & $\rightarrow$ & U-234 \\
\hline 1401.48 & $2.48 \mathrm{E}-17$ & $\mathrm{Bi}-214$ & -> & Po-214 \\
\hline 1407.97 & $4.42 \mathrm{E}-17$ & $\mathrm{Bi}-214$ & -> & Po-214 \\
\hline 1408 & $5.06 \mathrm{E}-20$ & TI-210 & $->$ & $\mathrm{Pb}-210$ \\
\hline 1413.88 & $2.54 \mathrm{E}-06$ & $\mathrm{~Pa}-234 \mathrm{~m}$ & -> & U-234 \\
\hline 1419.7 & $9.09 E-20$ & $\mathrm{Bi}-214$ & $->$ & Po-214 \\
\hline 1427.33 & $2.87 \mathrm{E}-07$ & Pa-234 & $->$ & $U-234$ \\
\hline 1434.13 & $1.07 \mathrm{E}-05$ & $\mathrm{~Pa}-234 \mathrm{~m}$ & $->$ & $U-234$ \\
\hline 1445.7 & $5.74 \mathrm{E}-07$ & $\mathrm{~Pa}-234$ & $->$ & U-234 \\
\hline 1452.63 & $1.32 \mathrm{E}-06$ & $\mathrm{~Pa}-234$ & $->$ & $U-234$ \\
\hline 1457.4 & $1.83 \mathrm{E}-06$ & $\mathrm{~Pa}-234 \mathrm{~m}$ & $->$ & U-234 \\
\hline 1460 & $4.31 \mathrm{E}-07$ & Pa-234 & -> & $\mathrm{U}-234$ \\
\hline 1471.1 & $2.14 \mathrm{E}-19$ & $\mathrm{Bi}-214$ & -> & Po-214 \\
\hline 1479.19 & $1.23 \mathrm{E}-18$ & $\mathrm{Bi}-214$ & -> & Po-214 \\
\hline 1493.94 & $2.87 \mathrm{E}-07$ & $\mathrm{~Pa}-234$ & -> & U-234 \\
\hline 1500.9 & $1.27 \mathrm{E}-06$ & $\mathrm{~Pa}-234 \mathrm{~m}$ & -> & $\mathrm{U}-234$ \\
\hline 1509.22 & $3.90 \mathrm{E}-17$ & $\mathrm{Bi}-214$ & -> & Po-214 \\
\hline 1510.2 & $1.42 \mathrm{E}-05$ & $\mathrm{~Pa}-234 \mathrm{~m}$ & $->$ & $U-234$ \\
\hline 1516 & $5.74 \mathrm{E}-07$ & Pa-234 & $->$ & $U-234$ \\
\hline 1527.27 & $2.65 \mathrm{E}-06$ & $\mathrm{~Pa}-234 \mathrm{~m}$ & $->$ & U-234 \\
\hline 1538 & 2.07E-20 & TI-210 & -> & $\mathrm{Pb}-210$ \\
\hline 1538.49 & $7.31 \mathrm{E}-18$ & $\mathrm{Bi}-214$ & -> & Po-214 \\
\hline 1543.347 & $6.24 \mathrm{E}-18$ & $\mathrm{Bi}-214$ & -> & Po-214 \\
\hline 1548.12 & $1.54 \mathrm{E}-06$ & $\mathrm{~Pa}-234 \mathrm{~m}$ & $->$ & U-234 \\
\hline 1549.4 & $1.44 \mathrm{E}-07$ & Pa-234 & $->$ & $U-234$ \\
\hline 1553.74 & 8.93E-06 & $\mathrm{Pa}-234 \mathrm{~m}$ & -> & $U-234$ \\
\hline 1558.7 & $7.62 \mathrm{E}-07$ & $\mathrm{~Pa}-234 \mathrm{~m}$ & $->$ & $U-234$ \\
\hline 1570.67 & $1.21 \mathrm{E}-06$ & $\mathrm{~Pa}-234 \mathrm{~m}$ & $->$ & $U-234$ \\
\hline 1580.02 & $2.44 \mathrm{E}-07$ & Pa-234 & -> & $\mathrm{U}-234$ \\
\hline 1583.22 & $1.28 \mathrm{E}-17$ & $\mathrm{Bi}-214$ & $->$ & Po-214 \\
\hline 1585.3 & $3.59 \mathrm{E}-07$ & $\mathrm{~Pa}-234$ & $->$ & U-234 \\
\hline 1588 & $2.07 E-20$ & TI-210 & $->$ & $\mathrm{Pb}-210$ \\
\hline 1591.65 & $2.10 \mathrm{E}-06$ & $\mathrm{~Pa}-234 \mathrm{~m}$ & $->$ & $\mathrm{U}-234$ \\
\hline 1593.88 & $2.98 \mathrm{E}-06$ & Pa-234 & $->$ & U-234 \\
\hline
\end{tabular}




\begin{tabular}{|c|c|c|c|c|}
\hline 1594.78 & $4.72 \mathrm{E}-18$ & $\mathrm{Bi}-214$ & $\rightarrow$ & Po-214 \\
\hline 1599.3 & $5.95 \mathrm{E}-18$ & $\mathrm{Bi}-214$ & -> & Po-214 \\
\hline 1602.2 & 4.09E-07 & $\mathrm{Pa}-234 \mathrm{~m}$ & $->$ & $U-234$ \\
\hline 1627.9 & $2.15 \mathrm{E}-07$ & Pa-234 & $->$ & $U-234$ \\
\hline 1636.6 & $3.39 \mathrm{E}-19$ & $\mathrm{Bi}-214$ & -> & Po-214 \\
\hline 1637.37 & $1.25 \mathrm{E}-18$ & $\mathrm{Bi}-214$ & $->$ & Po-214 \\
\hline 1638 & $5.74 \mathrm{E}-07$ & $\mathrm{~Pa}-234$ & $->$ & U-234 \\
\hline 1648 & $2.07 E-20$ & Tl-210 & -> & $\mathrm{Pb}-210$ \\
\hline 1656 & $2.15 \mathrm{E}-07$ & $\mathrm{~Pa}-234$ & $->$ & $U-234$ \\
\hline 1661.258 & $2.05 \mathrm{E}-17$ & $\mathrm{Bi}-214$ & $->$ & Po-214 \\
\hline 1667.3 & $8.18 \mathrm{E}-07$ & $\mathrm{~Pa}-234 \mathrm{~m}$ & $->$ & U-234 \\
\hline 1668.44 & $1.32 \mathrm{E}-06$ & $\mathrm{~Pa}-234$ & $->$ & $U-234$ \\
\hline 1683.99 & $4.21 \mathrm{E}-18$ & $\mathrm{Bi}-214$ & $->$ & Po-214 \\
\hline 1686.2 & $7.18 \mathrm{E}-07$ & $\mathrm{~Pa}-234$ & $->$ & U-234 \\
\hline 1693.7 & $4.51 \mathrm{E}-07$ & $\mathrm{~Pa}-234 \mathrm{~m}$ & $->$ & $U-234$ \\
\hline 1694.08 & $1.44 \mathrm{E}-06$ & Pa-234 & $->$ & $U-234$ \\
\hline 1699.8 & $2.15 \mathrm{E}-07$ & $\mathrm{~Pa}-234$ & -> & $U-234$ \\
\hline 1719.5 & 2.87E-08 & Pa-234 & $->$ & U-234 \\
\hline 1720.5 & $3.24 \mathrm{E}-07$ & $\mathrm{~Pa}-234 \mathrm{~m}$ & -> & U-234 \\
\hline 1729.58 & $5.44 \mathrm{E}-17$ & $\mathrm{Bi}-214$ & -> & Po-214 \\
\hline 1732.2 & $1.83 \mathrm{E}-06$ & $\mathrm{~Pa}-234 \mathrm{~m}$ & -> & U-234 \\
\hline 1737.73 & $2.34 \mathrm{E}-05$ & $\mathrm{~Pa}-234 \mathrm{~m}$ & $->$ & $U-234$ \\
\hline 1738 & $1.44 \mathrm{E}-07$ & Pa-234 & -> & $U-234$ \\
\hline 1741.7 & $1.44 \mathrm{E}-07$ & $\mathrm{~Pa}-234$ & $->$ & $U-234$ \\
\hline 1750.1 & $7.18 \mathrm{E}-08$ & $\mathrm{~Pa}-234$ & $->$ & $U-234$ \\
\hline 1756 & 3.59E-07 & $\mathrm{Pa}-234$ & -> & U-234 \\
\hline 1759.81 & $1.54 \mathrm{E}-06$ & $\mathrm{~Pa}-234 \mathrm{~m}$ & $->$ & $U-234$ \\
\hline 1764.49 & $2.83 \mathrm{E}-16$ & Bi-214 & $->$ & Po-214 \\
\hline 1765.44 & $9.60 \mathrm{E}-06$ & $\mathrm{~Pa}-234 \mathrm{~m}$ & $->$ & U-234 \\
\hline 1768 & 8.61E-08 & Pa-234 & $->$ & $U-234$ \\
\hline 1772.3 & $1.44 \mathrm{E}-07$ & $\mathrm{~Pa}-234$ & $->$ & U-234 \\
\hline 1782.1 & $2.85 \mathrm{E}-19$ & $\mathrm{Bi}-214$ & $->$ & Po-214 \\
\hline 1796.2 & $3.10 \mathrm{E}-07$ & $\mathrm{~Pa}-234 \mathrm{~m}$ & -> & U-234 \\
\hline 1797.3 & $4.31 \mathrm{E}-07$ & Pa-234 & -> & $U-234$ \\
\hline 1809.04 & $4.08 \mathrm{E}-06$ & $\mathrm{~Pa}-234 \mathrm{~m}$ & -> & $U-234$ \\
\hline 1814.01 & $2.14 \mathrm{E}-19$ & $\mathrm{Bi}-214$ & -> & Po-214 \\
\hline 1819.69 & $9.93 \mathrm{E}-07$ & $\mathrm{~Pa}-234 \mathrm{~m}$ & $->$ & $U-234$ \\
\hline 1828 & $4.31 \mathrm{E}-08$ & Pa-234 & $->$ & $U-234$ \\
\hline 1831.36 & $1.90 \mathrm{E}-05$ & $\mathrm{~Pa}-234 \mathrm{~m}$ & -> & $U-234$ \\
\hline 1837.9 & $8.61 \mathrm{E}-08$ & $\mathrm{~Pa}-234$ & $->$ & $U-234$ \\
\hline 1838.37 & $6.83 \mathrm{E}-18$ & $\mathrm{Bi}-214$ & $->$ & Po-214 \\
\hline 1847.41 & $3.78 \mathrm{E}-17$ & $\mathrm{Bi}-214$ & -> & Po-214 \\
\hline 1850 & $7.18 \mathrm{E}-08$ & Pa-234 & $->$ & $\mathrm{U}-234$ \\
\hline 1863.09 & $1.32 \mathrm{E}-06$ & $\mathrm{~Pa}-234 \mathrm{~m}$ & $->$ & $U-234$ \\
\hline 1867.68 & $1.01 \mathrm{E}-05$ & $\mathrm{~Pa}-234 \mathrm{~m}$ & $->$ & U-234 \\
\hline 1872.8 & $1.00 \mathrm{E}-07$ & Pa-234 & -> & U-234 \\
\hline 1873.112 & $4.03 \mathrm{E}-18$ & $\mathrm{Bi}-214$ & -> & Po-214 \\
\hline 1874.85 & $9.04 \mathrm{E}-06$ & $\mathrm{~Pa}-234 \mathrm{~m}$ & $->$ & U-234 \\
\hline 1877.21 & $1.82 \mathrm{E}-06$ & $\mathrm{~Pa}-234 \mathrm{~m}$ & $->$ & $U-234$ \\
\hline 1890.1 & $2.73 \mathrm{E}-07$ & Pa-234 & $->$ & $\mathrm{U}-234$ \\
\hline
\end{tabular}




\begin{tabular}{|c|c|c|c|c|}
\hline 1890.259 & $1.59 \mathrm{E}-18$ & $\mathrm{Bi}-214$ & $->$ & Po-214 \\
\hline 1893.5 & $2.43 \mathrm{E}-06$ & $\mathrm{~Pa}-234 \mathrm{~m}$ & -> & $U-234$ \\
\hline 1896.28 & $3.16 \mathrm{E}-18$ & $\mathrm{Bi}-214$ & $->$ & Po-214 \\
\hline 1897.1 & $2.15 \mathrm{E}-07$ & Pa-234 & $->$ & $U-234$ \\
\hline 1898.9 & $1.12 \mathrm{E}-18$ & $\mathrm{Bi}-214$ & -> & Po-214 \\
\hline 1905 & $4.02 E-07$ & $\mathrm{~Pa}-234$ & $->$ & U-234 \\
\hline 1911.17 & 6.95E-06 & $\mathrm{Pa}-234 \mathrm{~m}$ & $->$ & $\mathrm{U}-234$ \\
\hline 1926 & $3.43 E-07$ & Pa-234 & -> & $U-234$ \\
\hline 1926.4 & $2.10 \mathrm{E}-07$ & $\mathrm{~Pa}-234 \mathrm{~m}$ & $->$ & $U-234$ \\
\hline 1935.8 & 9.09E-19 & $\mathrm{Bi}-214$ & $->$ & Po-214 \\
\hline 1937.01 & $3.20 \mathrm{E}-06$ & $\mathrm{~Pa}-234 \mathrm{~m}$ & $->$ & $\mathrm{U}-234$ \\
\hline 1937.7 & $7.18 \mathrm{E}-08$ & Pa-234 & $->$ & $U-234$ \\
\hline 1959 & $1.44 \mathrm{E}-09$ & $\mathrm{~Pa}-234$ & $->$ & $U-234$ \\
\hline 1969.9 & $5.50 E-07$ & $\mathrm{~Pa}-234 \mathrm{~m}$ & $->$ & U-234 \\
\hline 1988 & 2.87E-09 & Pa-234 & $->$ & U-234 \\
\hline 1994.7 & $8.91 \mathrm{E}-20$ & $\mathrm{Bi}-214$ & $->$ & Po-214 \\
\hline 1998 & $1.44 \mathrm{E}-09$ & $\mathrm{~Pa}-234$ & $->$ & U-234 \\
\hline 2004.5 & $5.17 \mathrm{E}-20$ & $\mathrm{Bi}-214$ & $->$ & Po-214 \\
\hline 2008 & $7.12 \mathrm{E}-20$ & TI-210 & $->$ & $\mathrm{Pb}-210$ \\
\hline 2010.79 & 8.73E-19 & $\mathrm{Bi}-214$ & $->$ & Po-214 \\
\hline 2021.7 & 3.39E-19 & $\mathrm{Bi}-214$ & $->$ & Po-214 \\
\hline 2052.93 & $1.25 \mathrm{E}-18$ & $\mathrm{Bi}-214$ & $->$ & Po-214 \\
\hline 2085 & $1.78 \mathrm{E}-19$ & $\mathrm{Bi}-214$ & -> & Po-214 \\
\hline 2088 & $5.06 \mathrm{E}-20$ & $\mathrm{TI}-210$ & $->$ & $\mathrm{Pb}-210$ \\
\hline 2089.55 & $9.98 \mathrm{E}-19$ & $\mathrm{Bi}-214$ & $->$ & Po-214 \\
\hline 2109.91 & $1.55 \mathrm{E}-18$ & $\mathrm{Bi}-214$ & -> & Po-214 \\
\hline 2118.53 & $2.16 \mathrm{E}-17$ & $\mathrm{Bi}-214$ & $->$ & Po-214 \\
\hline 2147.8 & $2.85 \mathrm{E}-19$ & $\mathrm{Bi}-214$ & $->$ & Po-214 \\
\hline 2192.52 & $1.09 \mathrm{E}-18$ & $\mathrm{Bi}-214$ & $->$ & Po-214 \\
\hline 2204.09 & $8.90 E-17$ & $\mathrm{Bi}-214$ & $->$ & Po-214 \\
\hline 2251.2 & $1.25 \mathrm{E}-19$ & $\mathrm{Bi}-214$ & $->$ & Po-214 \\
\hline 2259.7 & $1.60 \mathrm{E}-19$ & $\mathrm{Bi}-214$ & $->$ & Po-214 \\
\hline 2266.67 & $3.21 \mathrm{E}-19$ & $\mathrm{Bi}-214$ & $->$ & Po-214 \\
\hline 2268 & $3.10 \mathrm{E}-20$ & TI-210 & -> & $\mathrm{Pb}-210$ \\
\hline 2270 & $5.17 \mathrm{E}-20$ & $\mathrm{Bi}-214$ & $->$ & Po-214 \\
\hline 2284.4 & $9.09 \mathrm{E}-20$ & $\mathrm{Bi}-214$ & -> & Po-214 \\
\hline 2293.29 & $5.78 \mathrm{E}-18$ & $\mathrm{Bi}-214$ & $->$ & Po-214 \\
\hline 2324.8 & $3.39 \mathrm{E}-20$ & $\mathrm{Bi}-214$ & $->$ & Po-214 \\
\hline 2331.2 & $3.92 \mathrm{E}-19$ & $\mathrm{Bi}-214$ & $->$ & Po-214 \\
\hline 2358 & $8.26 \mathrm{E}-20$ & TI-210 & $->$ & $\mathrm{Pb}-210$ \\
\hline 2360.9 & $3.39 E-20$ & $\mathrm{Bi}-214$ & $->$ & Po-214 \\
\hline 2369.3 & $5.17 \mathrm{E}-20$ & $\mathrm{Bi}-214$ & $->$ & Po-214 \\
\hline 2376.99 & $2.10 \mathrm{E}-19$ & $\mathrm{Bi}-214$ & -> & Po-214 \\
\hline 2390.9 & $3.57 E-20$ & $\mathrm{Bi}-214$ & $->$ & Po-214 \\
\hline 2423.32 & $1.05 E-19$ & $\mathrm{Bi}-214$ & $->$ & Po-214 \\
\hline 2428 & $9.29 \mathrm{E}-20$ & TI-210 & $->$ & $\mathrm{Pb}-210$ \\
\hline 2447.68 & $2.76 \mathrm{E}-17$ & $\mathrm{Bi}-214$ & -> & Po-214 \\
\hline 2482.417 & $3.74 \mathrm{E}-20$ & $\mathrm{Bi}-214$ & $->$ & Po-214 \\
\hline 2505.58 & $1.05 \mathrm{E}-19$ & $\mathrm{Bi}-214$ & $->$ & Po-214 \\
\hline 2551 & $6.95 \mathrm{E}-21$ & $\mathrm{Bi}-214$ & $->$ & Po-214 \\
\hline
\end{tabular}




\begin{tabular}{|c|c|c|c|c|}
\hline 2604.5 & $8.02 \mathrm{E}-21$ & $\mathrm{Bi}-214$ & -> & Po-214 \\
\hline 2631 & $1.60 \mathrm{E}-20$ & $\mathrm{Bi}-214$ & $->$ & Po-214 \\
\hline 2662.23 & $5.17 \mathrm{E}-21$ & $\mathrm{Bi}-214$ & $->$ & Po-214 \\
\hline 2694.67 & $5.78 \mathrm{E}-19$ & $\mathrm{Bi}-214$ & $->$ & Po-214 \\
\hline 2698.86 & $4.99 \mathrm{E}-20$ & $\mathrm{Bi}-214$ & $->$ & Po-214 \\
\hline 2719.21 & $3.21 \mathrm{E}-20$ & $\mathrm{Bi}-214$ & $->$ & Po-214 \\
\hline 2769.99 & $4.56 \mathrm{E}-19$ & $\mathrm{Bi}-214$ & $->$ & Po-214 \\
\hline 2786.09 & $1.05 \mathrm{E}-19$ & $\mathrm{Bi}-214$ & -> & Po-214 \\
\hline 2827 & $4.46 \mathrm{E}-20$ & $\mathrm{Bi}-214$ & $->$ & Po-214 \\
\hline 2860.9 & $6.06 \mathrm{E}-21$ & $\mathrm{Bi}-214$ & $->$ & Po-214 \\
\hline 2880.4 & $1.64 \mathrm{E}-19$ & $\mathrm{Bi}-214$ & $->$ & Po-214 \\
\hline 2893.59 & $1.14 \mathrm{E}-19$ & $\mathrm{Bi}-214$ & -> & Po-214 \\
\hline 2922.09 & $2.80 \mathrm{E}-19$ & $\mathrm{Bi}-214$ & $->$ & Po-214 \\
\hline 2928.7 & $2.14 \mathrm{E}-20$ & $\mathrm{Bi}-214$ & $->$ & Po-214 \\
\hline 2934.9 & $1.02 \mathrm{E}-20$ & $\mathrm{Bi}-214$ & $->$ & Po-214 \\
\hline 2940.5 & $3.03 E-20$ & $\mathrm{Bi}-214$ & $->$ & Po-214 \\
\hline 2978.79 & $2.62 \mathrm{E}-19$ & $\mathrm{Bi}-214$ & $->$ & Po-214 \\
\hline 2988.7 & $1.96 \mathrm{E}-20$ & $\mathrm{Bi}-214$ & $->$ & Po-214 \\
\hline 2999.99 & $1.57 \mathrm{E}-19$ & $\mathrm{Bi}-214$ & $->$ & Po-214 \\
\hline 3053.89 & $4.03 E-19$ & $\mathrm{Bi}-214$ & $->$ & Po-214 \\
\hline 3081.7 & 7.67E-20 & $\mathrm{Bi}-214$ & -> & Po-214 \\
\hline 3093.9 & $9.09 \mathrm{E}-21$ & $\mathrm{Bi}-214$ & $->$ & Po-214 \\
\hline 3136.3 & $6.06 \mathrm{E}-21$ & $\mathrm{Bi}-214$ & $->$ & Po-214 \\
\hline 3142.6 & $2.85 \mathrm{E}-20$ & $\mathrm{Bi}-214$ & $->$ & Po-214 \\
\hline 3160.5 & $9.09 \mathrm{E}-21$ & $\mathrm{Bi}-214$ & $->$ & Po-214 \\
\hline 3183.6 & $2.67 \mathrm{E}-20$ & $\mathrm{Bi}-214$ & -> & Po-214 \\
\hline 3233.3 & $3.57 \mathrm{E}-21$ & $\mathrm{Bi}-214$ & $->$ & Po-214 \\
\hline 3269.7 & $1.78 \mathrm{E}-21$ & $\mathrm{Bi}-214$ & $->$ & Po-214 \\
\hline
\end{tabular}

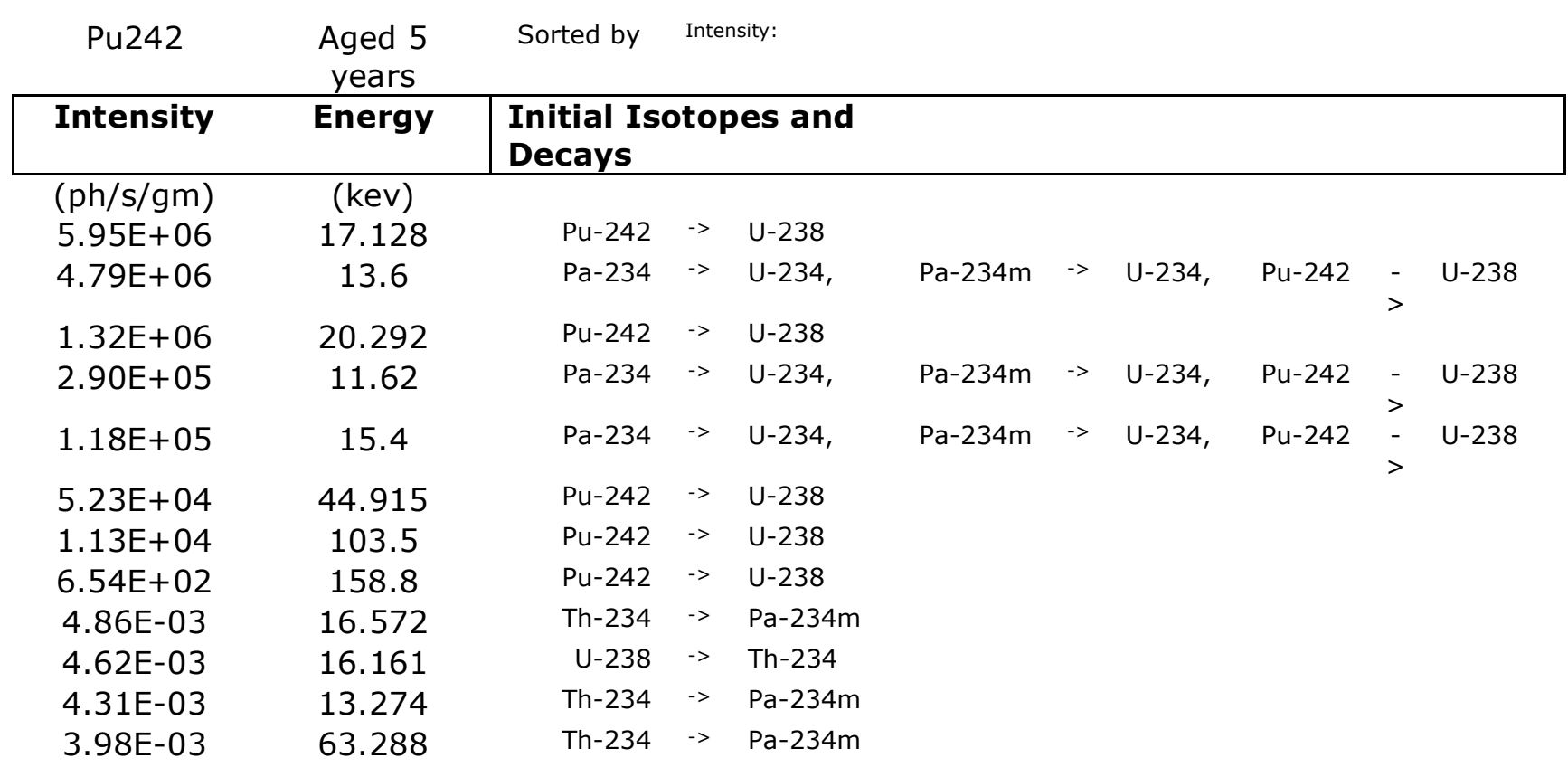




\begin{tabular}{|c|c|c|c|c|c|c|c|}
\hline $3.38 E-03$ & 12.952 & $\mathrm{U}-238$ & $\rightarrow$ & Th-234 & & & \\
\hline 3.00E-03 & 92.35 & Th-234 & $->$ & $\mathrm{Pa}-234 \mathrm{~m}$ & & & \\
\hline 2.97E-03 & 92.78 & Th-234 & $->$ & $\mathrm{Pa}-234 \mathrm{~m}$ & & & \\
\hline $1.19 \mathrm{E}-03$ & 19.839 & Th-234 & $->$ & $\mathrm{Pa}-234 \mathrm{~m}$ & & & \\
\hline $1.05 \mathrm{E}-03$ & 19.094 & $\mathrm{U}-238$ & $->$ & Th-234 & & & \\
\hline $9.25 \mathrm{E}-04$ & 1000.99 & $\mathrm{~Pa}-234 \mathrm{~m}$ & $->$ & $U-234$ & & & \\
\hline $3.55 \mathrm{E}-04$ & 766.412 & Pa-234 & $->$ & U-234, & $\mathrm{Pa}-234 \mathrm{~m}$ & $->$ & $U-234$ \\
\hline $3.04 \mathrm{E}-04$ & 98.434 & Pa-234 & $->$ & U-234, & $\mathrm{Pa}-234 \mathrm{~m}$ & $->$ & $U-234$ \\
\hline $2.67 E-04$ & 112.8 & Th-234 & $->$ & $\mathrm{Pa}-234 \mathrm{~m}$ & & & \\
\hline $2.56 \mathrm{E}-04$ & 11.372 & Th-234 & $->$ & $\mathrm{Pa}-234 \mathrm{~m}$, & $\mathrm{Pa}-234 \mathrm{~m}$ & $->$ & $U-234$ \\
\hline $2.03 E-04$ & 11.118 & $\mathrm{U}-238$ & $->$ & Th-234 & & & \\
\hline $1.92 \mathrm{E}-04$ & 94.651 & $\mathrm{~Pa}-234$ & $->$ & U-234, & $\mathrm{Pa}-234 \mathrm{~m}$ & $->$ & $U-234$ \\
\hline $1.30 \mathrm{E}-04$ & 17.069 & $\mathrm{~Pa}-234 \mathrm{~m}$ & $->$ & $\mathrm{U}-234$ & & & \\
\hline $1.12 \mathrm{E}-04$ & 111.025 & Pa-234 & $->$ & U-234, & $\mathrm{Pa}-234 \mathrm{~m}$ & $->$ & $U-234$ \\
\hline $1.04 \mathrm{E}-04$ & 742.817 & Pa-234 & $->$ & U-234, & $\mathrm{Pa}-234 \mathrm{~m}$ & $->$ & $U-234$ \\
\hline $9.34 \mathrm{E}-05$ & 14.511 & U-238 & $->$ & Th-234 & & & \\
\hline $8.05 E-05$ & 258.26 & $\mathrm{~Pa}-234 \mathrm{~m}$ & $->$ & $U-234$ & & & \\
\hline $7.88 \mathrm{E}-05$ & 49.55 & $\mathrm{U}-238$ & $->$ & Th-234 & & & \\
\hline $7.73 E-05$ & 83.31 & Th-234 & $->$ & $\mathrm{Pa}-234 \mathrm{~m}$ & & & \\
\hline $7.46 \mathrm{E}-05$ & 17.12 & Pa-234 & $->$ & $U-234$ & & & \\
\hline $6.11 E-05$ & 786.287 & Pa-234 & $->$ & U-234, & $\mathrm{Pa}-234 \mathrm{~m}$ & $->$ & $U-234$ \\
\hline $5.44 E-05$ & 14.953 & Th-234 & $->$ & $\mathrm{Pa}-234 \mathrm{~m}$, & $\mathrm{Pa}-234 \mathrm{~m}$ & $->$ & $U-234$ \\
\hline $4.70 E-05$ & 74 & Th-234 & $->$ & $\mathrm{Pa}-234 \mathrm{~m}$ & & & \\
\hline $3.83 E-05$ & 114.866 & Pa-234 & $->$ & U-234, & $\mathrm{Pa}-234 \mathrm{~m}$ & $->$ & $U-234$ \\
\hline $3.70 \mathrm{E}-05$ & 945.9 & Pa-234 & $->$ & U-234, & $\mathrm{Pa}-234 \mathrm{~m}$ & $->$ & $U-234$ \\
\hline $3.16 \mathrm{E}-05$ & 131.31 & Pa-234 & $->$ & $U-234$ & & & \\
\hline $2.96 \mathrm{E}-05$ & 16.547 & $\mathrm{~Pa}-234 \mathrm{~m}$ & $->$ & $U-234$ & & & \\
\hline $2.82 \mathrm{E}-05$ & 20.366 & $\mathrm{~Pa}-234 \mathrm{~m}$ & $->$ & $U-234$ & & & \\
\hline $2.70 \mathrm{E}-05$ & 110.5 & U-238 & $->$ & Th-234 & & & \\
\hline $2.55 E-05$ & 13.275 & $\mathrm{~Pa}-234 \mathrm{~m}$ & $->$ & $U-234$ & & & \\
\hline $2.34 E-05$ & 880.45 & Pa-234 & $->$ & $U-234$ & & & \\
\hline $2.34 \mathrm{E}-05$ & 1737.73 & $\mathrm{~Pa}-234 \mathrm{~m}$ & $->$ & $U-234$ & & & \\
\hline $2.33 E-05$ & 883.22 & Pa-234 & $->$ & U-234, & $\mathrm{Pa}-234 \mathrm{~m}$ & $->$ & $U-234$ \\
\hline $2.24 \mathrm{E}-05$ & 569.3 & $\mathrm{~Pa}-234$ & $->$ & $U-234$ & & & \\
\hline $2.12 E-05$ & 926.61 & $\mathrm{~Pa}-234$ & $->$ & U-234, & $\mathrm{Pa}-234 \mathrm{~m}$ & $->$ & $U-234$ \\
\hline $2.10 \mathrm{E}-05$ & 62.862 & Th-234 & $->$ & $\mathrm{Pa}-234 \mathrm{~m}$ & & & \\
\hline $1.90 \mathrm{E}-05$ & 1831.36 & $\mathrm{~Pa}-234 \mathrm{~m}$ & $->$ & $\mathrm{U}-234$ & & & \\
\hline $1.79 E-05$ & 226.95 & Pa-234 & $->$ & $U-234$ & & & \\
\hline $1.69 E-05$ & 20.309 & $\mathrm{~Pa}-234$ & $->$ & $U-234$ & & & \\
\hline $1.57 \mathrm{E}-05$ & 924.98 & Pa-234 & $->$ & $U-234$ & & & \\
\hline $1.49 E-05$ & 1193.69 & $\mathrm{~Pa}-234 \mathrm{~m}$ & $->$ & $\mathrm{U}-234$ & & & \\
\hline $1.44 \mathrm{E}-05$ & 95.863 & Th-234 & $->$ & $\mathrm{Pa}-234 \mathrm{~m}$ & & & \\
\hline $1.42 \mathrm{E}-05$ & 1510.2 & $\mathrm{~Pa}-234 \mathrm{~m}$ & $->$ & $\mathrm{U}-234$ & & & \\
\hline $1.40 \mathrm{E}-05$ & 921.7 & $\mathrm{~Pa}-234 \mathrm{~m}$ & $->$ & $\mathrm{U}-234$ & & & \\
\hline $1.33 E-05$ & 184.8 & Th-234 & $->$ & $\mathrm{Pa}-234 \mathrm{~m}$ & & & \\
\hline $1.30 \mathrm{E}-05$ & 739.95 & $\mathrm{~Pa}-234 \mathrm{~m}$ & $->$ & $\mathrm{U}-234$ & & & \\
\hline $1.27 E-05$ & 733.38 & Pa-234 & $->$ & $U-234$ & & & \\
\hline $1.15 \mathrm{E}-05$ & 948 & $\mathrm{~Pa}-234$ & $->$ & $\mathrm{U}-234$ & & & \\
\hline 1.10E-05 & 20.019 & Th-234 & $->$ & $\mathrm{Pa}-234 \mathrm{~m}$ & & & \\
\hline $1.07 \mathrm{E}-05$ & 1434.13 & $\mathrm{~Pa}-234 \mathrm{~m}$ & $->$ & $\mathrm{U}-234$ & & & \\
\hline
\end{tabular}




\begin{tabular}{|c|c|c|c|c|c|c|c|}
\hline $1.01 E-05$ & 1867.68 & $\mathrm{~Pa}-234 \mathrm{~m}$ & $->$ & $U-234$ & & & \\
\hline 9.93E-06 & 691.08 & $\mathrm{~Pa}-234 \mathrm{~m}$ & $->$ & $U-234$ & & & \\
\hline $9.71 \mathrm{E}-06$ & 805.74 & $\mathrm{~Pa}-234$ & $->$ & U-234, & $\mathrm{Pa}-234 \mathrm{~m}$ & $->$ & U-234 \\
\hline $9.60 \mathrm{E}-06$ & 1765.44 & $\mathrm{~Pa}-234 \mathrm{~m}$ & $->$ & $\mathrm{U}-234$ & & & \\
\hline $9.16 \mathrm{E}-06$ & 152.76 & $\mathrm{~Pa}-234$ & $->$ & $\mathrm{U}-234$ & & & \\
\hline $9.04 \mathrm{E}-06$ & 1874.85 & $\mathrm{~Pa}-234 \mathrm{~m}$ & $->$ & $\mathrm{U}-234$ & & & \\
\hline 8.93E-06 & 1553.74 & $\mathrm{~Pa}-234 \mathrm{~m}$ & $->$ & U-234 & & & \\
\hline 8.83E-06 & 92.279 & Th-234 & $->$ & $\mathrm{Pa}-234 \mathrm{~m}$ & & & \\
\hline 8.61E-06 & 831.39 & Pa-234 & $->$ & U-234 & & & \\
\hline $8.54 \mathrm{E}-06$ & 62.78 & Pa-234 & $->$ & U-234, & $\mathrm{Pa}-234 \mathrm{~m}$ & $->$ & $U-234$ \\
\hline 8.06E-06 & 87.02 & Th-234 & $->$ & Pa-234m & & & \\
\hline 7.83E-06 & 887.28 & $\mathrm{~Pa}-234 \mathrm{~m}$ & $->$ & $U-234$ & & & \\
\hline 7.83E-06 & 702.05 & $\mathrm{~Pa}-234 \mathrm{~m}$ & $->$ & $\mathrm{U}-234$ & & & \\
\hline $7.72 \mathrm{E}-06$ & 851.57 & Pa-234 & $->$ & U-234, & $\mathrm{Pa}-234 \mathrm{~m}$ & $->$ & U-234 \\
\hline $7.51 \mathrm{E}-06$ & 824.94 & Pa-234 & $->$ & U-234 & & & \\
\hline $7.48 E-06$ & 782.8 & $\mathrm{~Pa}-234 \mathrm{~m}$ & $->$ & $U-234$ & & & \\
\hline $7.41 \mathrm{E}-06$ & 99.852 & Pa-234 & $->$ & U-234, & $\mathrm{Pa}-234 \mathrm{~m}$ & $->$ & $U-234$ \\
\hline 7.17E-06 & 705.9 & Pa-234 & $->$ & U-234, & $\mathrm{Pa}-234 \mathrm{~m}$ & $->$ & $U-234$ \\
\hline $6.95 E-06$ & 1911.17 & $\mathrm{~Pa}-234 \mathrm{~m}$ & $->$ & $\mathrm{U}-234$ & & & \\
\hline 6.63E-06 & 103.71 & Th-234 & $->$ & $\mathrm{Pa}-234 \mathrm{~m}$ & & & \\
\hline $6.52 \mathrm{E}-06$ & 108 & Th-234 & $->$ & $\mathrm{Pa}-234 \mathrm{~m}$ & & & \\
\hline $6.51 \mathrm{E}-06$ & 898.52 & Pa-234 & $->$ & $U-234$ & & & \\
\hline $6.29 E-06$ & 994.93 & $\mathrm{~Pa}-234 \mathrm{~m}$ & $->$ & $U-234$ & & & \\
\hline $5.96 \mathrm{E}-06$ & 796.42 & $\mathrm{~Pa}-234$ & $->$ & $U-234$ & & & \\
\hline 5.85E-06 & 1237.24 & $\mathrm{~Pa}-234 \mathrm{~m}$ & $->$ & $U-234$ & & & \\
\hline $5.82 \mathrm{E}-06$ & 698.85 & Pa-234 & $->$ & $U-234$ & & & \\
\hline $5.52 E-06$ & 108.166 & Th-234 & $->$ & $\mathrm{Pa}-234 \mathrm{~m}$ & & & \\
\hline 5.52E-06 & 57.75 & Th-234 & $->$ & $\mathrm{Pa}-234 \mathrm{~m}$ & & & \\
\hline $5.41 E-06$ & 293.874 & Pa-234 & $->$ & $U-234$ & & & \\
\hline 4.97E-06 & 980.42 & $\mathrm{~Pa}-234$ & $->$ & $U-234$ & & & \\
\hline 4.94E-06 & 243.5 & $\mathrm{~Pa}-234 \mathrm{~m}$ & $->$ & U-234 & & & \\
\hline 4.86E-06 & 369.52 & $\mathrm{~Pa}-234$ & $->$ & $U-234$ & & & \\
\hline $4.64 \mathrm{E}-06$ & 875.94 & $\mathrm{~Pa}-234$ & $->$ & $U-234$ & & & \\
\hline $4.63 E-06$ & 1124.93 & $\mathrm{~Pa}-234 \mathrm{~m}$ & $->$ & $U-234$ & & & \\
\hline $4.31 \mathrm{E}-06$ & 1393.57 & Pa-234 & $->$ & $U-234$ & & & \\
\hline 4.09E-06 & 819.21 & Pa-234 & $->$ & $U-234$ & & & \\
\hline $4.08 E-06$ & 1809.04 & $\mathrm{~Pa}-234 \mathrm{~m}$ & $->$ & $U-234$ & & & \\
\hline 3.97E-06 & 543.98 & $\mathrm{~Pa}-234 \mathrm{~m}$ & $->$ & $U-234$ & & & \\
\hline 3.87E-06 & 103.35 & Th-234 & $->$ & $\mathrm{Pa}-234 \mathrm{~m}$ & & & \\
\hline $3.81 E-06$ & 881.1 & $\mathrm{~Pa}-234 \mathrm{~m}$ & $->$ & $U-234$ & & & \\
\hline 3.53E-06 & 19.891 & $\mathrm{~Pa}-234 \mathrm{~m}$ & $->$ & $U-234$ & & & \\
\hline $3.20 \mathrm{E}-06$ & 1937.01 & $\mathrm{~Pa}-234 \mathrm{~m}$ & $->$ & $U-234$ & & & \\
\hline $3.13 \mathrm{E}-06$ & 248.94 & Pa-234 & $->$ & U-234 & & & \\
\hline $2.98 \mathrm{E}-06$ & 1593.88 & Pa-234 & $->$ & U-234 & & & \\
\hline $2.98 \mathrm{E}-06$ & 203.12 & Pa-234 & $->$ & U-234, & $\mathrm{Pa}-234 \mathrm{~m}$ & $->$ & $U-234$ \\
\hline $2.98 \mathrm{E}-06$ & 450.96 & $\mathrm{~Pa}-234 \mathrm{~m}$ & $->$ & $\mathrm{U}-234$ & & & \\
\hline 2.87E-06 & 574.8 & Pa-234 & $->$ & $U-234$ & & & \\
\hline 2.87E-06 & 185.92 & Pa-234 & $->$ & $\mathrm{U}-234$ & & & \\
\hline 2.87E-06 & 808.2 & Pa-234 & $->$ & U-234, & $\mathrm{Pa}-234 \mathrm{~m}$ & $->$ & $U-234$ \\
\hline $2.76 \mathrm{E}-06$ & 941.94 & $\mathrm{~Pa}-234 \mathrm{~m}$ & $->$ & U-234 & & & \\
\hline
\end{tabular}




\begin{tabular}{|c|c|c|c|c|c|c|c|}
\hline $2.73 E-06$ & 984.12 & Pa-234 & $->$ & $\mathrm{U}-234$ & & & \\
\hline $2.65 \mathrm{E}-06$ & 1527.27 & $\mathrm{~Pa}-234 \mathrm{~m}$ & $->$ & $\mathrm{U}-234$ & & & \\
\hline $2.56 \mathrm{E}-06$ & 140.15 & Pa-234 & $->$ & U-234, & $\mathrm{Pa}-234 \mathrm{~m}$ & -> & $U-234$ \\
\hline $2.54 \mathrm{E}-06$ & 1392 & $\mathrm{~Pa}-234 \mathrm{~m}$ & $->$ & $U-234$ & & & \\
\hline $2.54 \mathrm{E}-06$ & 372.02 & Pa-234 & $->$ & $U-234$ & & & \\
\hline $2.54 \mathrm{E}-06$ & 1413.88 & $\mathrm{~Pa}-234 \mathrm{~m}$ & $->$ & $U-234$ & & & \\
\hline $2.54 \mathrm{E}-06$ & 1061.86 & $\mathrm{~Pa}-234 \mathrm{~m}$ & $->$ & $U-234$ & & & \\
\hline $2.54 \mathrm{E}-06$ & 475.75 & $\mathrm{~Pa}-234 \mathrm{~m}$ & $->$ & $U-234$ & & & \\
\hline $2.43 \mathrm{E}-06$ & 1893.5 & $\mathrm{~Pa}-234 \mathrm{~m}$ & $->$ & $U-234$ & & & \\
\hline $2.32 \mathrm{E}-06$ & 738.88 & Pa-234 & $->$ & $U-234$ & & & \\
\hline $2.32 \mathrm{E}-06$ & 755 & $\mathrm{~Pa}-234$ & $->$ & $U-234$ & & & \\
\hline $2.31 E-06$ & 506.69 & $\mathrm{~Pa}-234$ & $->$ & $U-234$ & & & \\
\hline $2.21 \mathrm{E}-06$ & 458.63 & Pa-234 & $->$ & $U-234$ & & & \\
\hline $2.15 \mathrm{E}-06$ & 793.6 & Pa-234 & $->$ & $U-234$ & & & \\
\hline $2.15 \mathrm{E}-06$ & 692.7 & Pa-234 & $->$ & $U-234$ & & & \\
\hline $2.12 \mathrm{E}-06$ & 509.11 & $\mathrm{~Pa}-234 \mathrm{~m}$ & $->$ & $U-234$ & & & \\
\hline $2.10 \mathrm{E}-06$ & 453.58 & $\mathrm{~Pa}-234 \mathrm{~m}$ & $->$ & $U-234$ & & & \\
\hline $2.10 \mathrm{E}-06$ & 1591.65 & $\mathrm{~Pa}-234 \mathrm{~m}$ & $->$ & U-234 & & & \\
\hline 2.09E-06 & 1126.17 & Pa-234 & $->$ & U-234, & $\mathrm{Pa}-234 \mathrm{~m}$ & $->$ & $U-234$ \\
\hline $2.03 E-06$ & 468.21 & $\mathrm{~Pa}-234 \mathrm{~m}$ & $->$ & $U-234$ & & & \\
\hline $2.01 \mathrm{E}-06$ & 565.2 & Pa-234 & $->$ & $\mathrm{U}-234$ & & & \\
\hline $1.99 E-06$ & 272.2 & Pa-234 & $->$ & $U-234$ & & & \\
\hline $1.89 E-06$ & 1174.1 & $\mathrm{~Pa}-234 \mathrm{~m}$ & $->$ & $\mathrm{U}-234$ & & & \\
\hline $1.88 \mathrm{E}-06$ & 669.64 & Pa-234 & $->$ & $U-234$ & & & \\
\hline $1.87 \mathrm{E}-06$ & 664.8 & Pa-234 & $->$ & $U-234$ & & & \\
\hline $1.87 \mathrm{E}-06$ & 513.6 & Pa-234 & $->$ & $U-234$ & & & \\
\hline $1.83 E-06$ & 1457.4 & $\mathrm{~Pa}-234 \mathrm{~m}$ & $->$ & $U-234$ & & & \\
\hline $1.83 E-06$ & 882.5 & $\mathrm{~Pa}-234 \mathrm{~m}$ & $->$ & $U-234$ & & & \\
\hline $1.83 E-06$ & 936.8 & $\mathrm{~Pa}-234 \mathrm{~m}$ & $->$ & $U-234$ & & & \\
\hline $1.83 E-06$ & 1732.2 & $\mathrm{~Pa}-234 \mathrm{~m}$ & $->$ & $U-234$ & & & \\
\hline $1.82 E-06$ & 1877.21 & $\mathrm{~Pa}-234 \mathrm{~m}$ & $->$ & U-234 & & & \\
\hline $1.79 \mathrm{E}-06$ & 844.4 & Pa-234 & $->$ & U-234, & $\mathrm{Pa}-234 \mathrm{~m}$ & $->$ & $U-234$ \\
\hline $1.77 E-06$ & 111.897 & Th-234 & $->$ & $\mathrm{Pa}-234 \mathrm{~m}$ & & & \\
\hline $1.69 E-06$ & 1120.5 & $\mathrm{~Pa}-234 \mathrm{~m}$ & $->$ & $\mathrm{U}-234$ & & & \\
\hline $1.69 \mathrm{E}-06$ & 184.6 & $\mathrm{~Pa}-234 \mathrm{~m}$ & $->$ & $\mathrm{U}-234$ & & & \\
\hline $1.67 E-06$ & 1352.85 & Pa-234 & $->$ & $U-234$ & & & \\
\hline $1.66 \mathrm{E}-06$ & 666.42 & $\mathrm{~Pa}-234$ & $->$ & $\mathrm{U}-234$ & & & \\
\hline $1.61 \mathrm{E}-06$ & 29.49 & Th-234 & $->$ & $\mathrm{Pa}-234 \mathrm{~m}$ & & & \\
\hline $1.58 \mathrm{E}-06$ & 780.4 & $\mathrm{~Pa}-234$ & $->$ & $U-234$ & & & \\
\hline $1.58 \mathrm{E}-06$ & 200.96 & Pa-234 & $->$ & $\mathrm{U}-234$ & & & \\
\hline $1.56 \mathrm{E}-06$ & 507.3 & $\mathrm{~Pa}-234 \mathrm{~m}$ & $->$ & $U-234$ & & & \\
\hline $1.55 \mathrm{E}-06$ & 647.7 & $\mathrm{~Pa}-234 \mathrm{~m}$ & $->$ & $\mathrm{U}-234$ & & & \\
\hline $1.55 \mathrm{E}-06$ & 695.9 & $\mathrm{~Pa}-234 \mathrm{~m}$ & $->$ & $\mathrm{U}-234$ & & & \\
\hline $1.55 \mathrm{E}-06$ & 760.3 & $\mathrm{~Pa}-234 \mathrm{~m}$ & $->$ & $\mathrm{U}-234$ & & & \\
\hline $1.54 \mathrm{E}-06$ & 1759.81 & $\mathrm{~Pa}-234 \mathrm{~m}$ & $->$ & $\mathrm{U}-234$ & & & \\
\hline $1.54 \mathrm{E}-06$ & 1548.12 & $\mathrm{~Pa}-234 \mathrm{~m}$ & $->$ & $\mathrm{U}-234$ & & & \\
\hline $1.44 \mathrm{E}-06$ & 1694.08 & Pa-234 & $->$ & $\mathrm{U}-234$ & & & \\
\hline $1.44 \mathrm{E}-06$ & 125.53 & $\mathrm{~Pa}-234$ & $->$ & $U-234$ & & & \\
\hline $1.44 \mathrm{E}-06$ & 978.8 & Pa-234 & $->$ & $U-234$ & & & \\
\hline $1.41 \mathrm{E}-06$ & 625.5 & $\mathrm{~Pa}-234 \mathrm{~m}$ & $->$ & $U-234$ & & & \\
\hline
\end{tabular}




\begin{tabular}{|c|c|c|c|c|c|c|c|}
\hline $1.40 \mathrm{E}-06$ & 826.08 & $\mathrm{~Pa}-234 \mathrm{~m}$ & $->$ & $U-234$ & & & \\
\hline $1.32 \mathrm{E}-06$ & 1452.63 & $\mathrm{~Pa}-234$ & $->$ & $U-234$ & & & \\
\hline $1.32 \mathrm{E}-06$ & 1668.44 & Pa-234 & $->$ & U-234 & & & \\
\hline $1.32 \mathrm{E}-06$ & 1084.25 & Pa-234 & $->$ & U-234, & $\mathrm{Pa}-234 \mathrm{~m}$ & $->$ & $U-234$ \\
\hline $1.32 \mathrm{E}-06$ & 1863.09 & $\mathrm{~Pa}-234 \mathrm{~m}$ & $->$ & U-234 & & & \\
\hline $1.32 \mathrm{E}-06$ & 1041.7 & Pa-234m & $->$ & U-234 & & & \\
\hline $1.30 \mathrm{E}-06$ & 210.6 & $\mathrm{~Pa}-234 \mathrm{~m}$ & $->$ & $U-234$ & & & \\
\hline $1.29 \mathrm{E}-06$ & 521.09 & Pa-234 & $->$ & U-234 & & & \\
\hline $1.29 \mathrm{E}-06$ & 658 & Pa-234 & $->$ & U-234 & & & \\
\hline $1.29 \mathrm{E}-06$ & 602.1 & Pa-234 & $->$ & $U-234$ & & & \\
\hline 1.29E-06 & 245.16 & Pa-234 & $->$ & $U-234$ & & & \\
\hline $1.28 \mathrm{E}-06$ & 732.7 & $\mathrm{~Pa}-234 \mathrm{~m}$ & $->$ & U-234 & & & \\
\hline 1.27E-06 & 1500.9 & Pa-234m & $->$ & U-234 & & & \\
\hline $1.25 \mathrm{E}-06$ & 199.46 & Pa-234 & $->$ & U-234, & $\mathrm{Pa}-234 \mathrm{~m}$ & $->$ & $U-234$ \\
\hline $1.21 \mathrm{E}-06$ & 1570.67 & $\mathrm{~Pa}-234 \mathrm{~m}$ & $->$ & $U-234$ & & & \\
\hline $1.15 \mathrm{E}-06$ & 1029.03 & Pa-234 & $->$ & $U-234$ & & & \\
\hline $1.15 \mathrm{E}-06$ & 627.5 & Pa-234 & $->$ & $U-234$ & & & \\
\hline $1.15 \mathrm{E}-06$ & 624.31 & Pa-234 & $->$ & $U-234$ & & & \\
\hline $1.15 \mathrm{E}-06$ & 611.5 & Pa-234 & $->$ & $U-234$ & & & \\
\hline $1.14 \mathrm{E}-06$ & 708.4 & Pa-234 & $->$ & U-234, & $\mathrm{Pa}-234 \mathrm{~m}$ & $->$ & $U-234$ \\
\hline $1.12 \mathrm{E}-06$ & 338.4 & $\mathrm{~Pa}-234 \mathrm{~m}$ & $->$ & $U-234$ & & & \\
\hline 1.09E-06 & 1059.72 & $\mathrm{~Pa}-234 \mathrm{~m}$ & $->$ & $U-234$ & & & \\
\hline $1.06 \mathrm{E}-06$ & 649.1 & $\mathrm{~Pa}-234 \mathrm{~m}$ & $->$ & $U-234$ & & & \\
\hline $1.06 \mathrm{E}-06$ & 866.98 & $\mathrm{~Pa}-234 \mathrm{~m}$ & $->$ & $U-234$ & & & \\
\hline $1.00 \mathrm{E}-06$ & 157.96 & Pa-234 & $->$ & $U-234$ & & & \\
\hline 9.93E-07 & 1292.66 & Pa-234 & $->$ & $U-234$ & & & \\
\hline 9.93E-07 & 1220.37 & $\mathrm{~Pa}-234 \mathrm{~m}$ & $->$ & $U-234$ & & & \\
\hline 9.93E-07 & 1819.69 & $\mathrm{~Pa}-234 \mathrm{~m}$ & $->$ & $U-234$ & & & \\
\hline $9.88 \mathrm{E}-07$ & 818 & $\mathrm{~Pa}-234 \mathrm{~m}$ & $->$ & $U-234$ & & & \\
\hline $9.88 \mathrm{E}-07$ & 387.8 & $\mathrm{~Pa}-234 \mathrm{~m}$ & $->$ & $U-234$ & & & \\
\hline $9.44 \mathrm{E}-07$ & 655.1 & $\mathrm{~Pa}-234 \mathrm{~m}$ & $->$ & $U-234$ & & & \\
\hline 8.90E-07 & 653.57 & Pa-234 & $->$ & $U-234$ & & & \\
\hline 8.61E-07 & 967.33 & Pa-234 & $->$ & $U-234$ & & & \\
\hline $8.61 \mathrm{E}-07$ & 1022.23 & Pa-234 & $->$ & U-234 & & & \\
\hline 8.61E-07 & 330.62 & $\mathrm{~Pa}-234$ & $->$ & $U-234$ & & & \\
\hline 8.61E-07 & 426.8 & Pa-234 & $->$ & $U-234$ & & & \\
\hline 8.61E-07 & 352.16 & Pa-234 & $->$ & $U-234$ & & & \\
\hline 8.61E-07 & 520.2 & Pa-234 & $->$ & $U-234$ & & & \\
\hline 8.61E-07 & 527.04 & Pa-234 & $->$ & $U-234$ & & & \\
\hline 8.61E-07 & 193.55 & Pa-234 & $->$ & $U-234$ & & & \\
\hline 8.60E-07 & 572 & $\mathrm{~Pa}-234 \mathrm{~m}$ & $->$ & $U-234$ & & & \\
\hline 8.47E-07 & 959.8 & $\mathrm{~Pa}-234 \mathrm{~m}$ & $->$ & U-234 & & & \\
\hline $8.18 \mathrm{E}-07$ & 1667.3 & $\mathrm{~Pa}-234 \mathrm{~m}$ & $->$ & $U-234$ & & & \\
\hline 8.04E-07 & 768.5 & Pa-234 & $->$ & $U-234$ & & & \\
\hline 7.90E-07 & 357.9 & $\mathrm{~Pa}-234 \mathrm{~m}$ & $->$ & $U-234$ & & & \\
\hline 7.87E-07 & 783.38 & Pa-234 & $->$ & U-234, & $\mathrm{Pa}-234 \mathrm{~m}$ & $->$ & $U-234$ \\
\hline 7.84E-07 & 274.45 & Pa-234 & $->$ & U-234, & $\mathrm{Pa}-234 \mathrm{~m}$ & $->$ & $U-234$ \\
\hline $7.62 \mathrm{E}-07$ & 1558.7 & $\mathrm{~Pa}-234 \mathrm{~m}$ & $->$ & $U-234$ & & & \\
\hline $7.36 \mathrm{E}-07$ & 247.7 & $\mathrm{~Pa}-234 \mathrm{~m}$ & $->$ & $U-234$ & & & \\
\hline $7.18 \mathrm{E}-07$ & 1044.9 & Pa-234 & $->$ & $U-234$ & & & \\
\hline
\end{tabular}




\begin{tabular}{|c|c|c|c|c|c|c|c|}
\hline $7.18 \mathrm{E}-07$ & 1241.4 & $\mathrm{~Pa}-234$ & $->$ & $U-234$ & & & \\
\hline $7.18 \mathrm{E}-07$ & 812.5 & $\mathrm{~Pa}-234$ & $->$ & $U-234$ & & & \\
\hline $7.18 \mathrm{E}-07$ & 1686.2 & $\mathrm{~Pa}-234$ & $->$ & $U-234$ & & & \\
\hline $7.18 \mathrm{E}-07$ & 1122.09 & $\mathrm{~Pa}-234$ & $->$ & $U-234$ & & & \\
\hline $7.18 \mathrm{E}-07$ & 170.75 & $\mathrm{~Pa}-234$ & $->$ & $U-234$ & & & \\
\hline $7.18 \mathrm{E}-07$ & 904.38 & $\mathrm{~Pa}-234$ & $->$ & $U-234$ & & & \\
\hline $7.06 \mathrm{E}-07$ & 557.3 & Pa-234m & $->$ & $U-234$ & & & \\
\hline $7.06 \mathrm{E}-07$ & 456.5 & Pa-234m & $->$ & $U-234$ & & & \\
\hline $6.96 \mathrm{E}-07$ & 700.4 & Pa-234m & $->$ & U-234 & & & \\
\hline $6.83 E-07$ & 276.83 & Pa-234 & $->$ & U-234, & $\mathrm{Pa}-234 \mathrm{~m}$ & $->$ & $U-234$ \\
\hline $6.77 E-07$ & 360.8 & $\mathrm{~Pa}-234 \mathrm{~m}$ & $->$ & $U-234$ & & & \\
\hline $6.35 E-07$ & 674.24 & $\mathrm{~Pa}-234 \mathrm{~m}$ & $->$ & $U-234$ & & & \\
\hline $6.21 \mathrm{E}-07$ & 193.4 & $\mathrm{~Pa}-234 \mathrm{~m}$ & $->$ & $\mathrm{U}-234$ & & & \\
\hline $6.21 \mathrm{E}-07$ & 299.08 & $\mathrm{~Pa}-234 \mathrm{~m}$ & $->$ & $U-234$ & & & \\
\hline 5.94E-07 & 655 & $\mathrm{~Pa}-234$ & $->$ & $U-234$ & & & \\
\hline $5.74 \mathrm{E}-07$ & 632 & $\mathrm{~Pa}-234$ & $->$ & $U-234$ & & & \\
\hline $5.74 \mathrm{E}-07$ & 409.8 & Pa-234 & $->$ & $U-234$ & & & \\
\hline $5.74 \mathrm{E}-07$ & 1638 & Pa-234 & $->$ & U-234 & & & \\
\hline $5.74 \mathrm{E}-07$ & 1516 & $\mathrm{~Pa}-234$ & $->$ & $U-234$ & & & \\
\hline $5.74 \mathrm{E}-07$ & 480.4 & Pa-234 & $->$ & $U-234$ & & & \\
\hline $5.74 \mathrm{E}-07$ & 1445.7 & $\mathrm{~Pa}-234$ & $->$ & $U-234$ & & & \\
\hline $5.74 \mathrm{E}-07$ & 804.52 & $\mathrm{~Pa}-234$ & $->$ & $U-234$ & & & \\
\hline $5.74 \mathrm{E}-07$ & 920 & $\mathrm{~Pa}-234$ & $->$ & $U-234$ & & & \\
\hline $5.65 E-07$ & 683 & $\mathrm{~Pa}-234 \mathrm{~m}$ & $->$ & $U-234$ & & & \\
\hline 5.50E-07 & 1969.9 & $\mathrm{~Pa}-234 \mathrm{~m}$ & $->$ & $U-234$ & & & \\
\hline $5.31 E-07$ & 1216.4 & Pa-234 & $->$ & $U-234$ & & & \\
\hline $5.08 E-07$ & 311 & $\mathrm{~Pa}-234 \mathrm{~m}$ & $->$ & $U-234$ & & & \\
\hline 5.02E-07 & 467.5 & Pa-234 & $->$ & $U-234$ & & & \\
\hline 5.02E-07 & 143.9 & $\mathrm{~Pa}-234$ & $->$ & $U-234$ & & & \\
\hline 4.94E-07 & 1085.37 & $\mathrm{~Pa}-234 \mathrm{~m}$ & $->$ & $U-234$ & & & \\
\hline $4.51 \mathrm{E}-07$ & 1693.7 & $\mathrm{~Pa}-234 \mathrm{~m}$ & $->$ & $\mathrm{U}-234$ & & & \\
\hline 4.31E-07 & 1208 & TI-210 & $->$ & $\mathrm{Pb}-210$ & $\mathrm{~Pa}-234$ & $->$ & $U-234$ \\
\hline 4.31E-07 & 1797.3 & Pa-234 & $->$ & U-234 & & & \\
\hline $4.31 \mathrm{E}-07$ & 660.6 & Pa-234 & $->$ & $U-234$ & & & \\
\hline $4.31 \mathrm{E}-07$ & 313.83 & Pa-234 & $->$ & $U-234$ & & & \\
\hline 4.31E-07 & 766.369 & Pa-234 & $->$ & $U-234$ & & & \\
\hline $4.31 \mathrm{E}-07$ & 1108.5 & Pa-234 & $->$ & $U-234$ & & & \\
\hline 4.31E-07 & 1251 & Pa-234 & $->$ & $U-234$ & & & \\
\hline $4.31 \mathrm{E}-07$ & 1460 & Pa-234 & $->$ & $U-234$ & & & \\
\hline 4.31E-07 & 1229 & Pa-234 & $->$ & $U-234$ & & & \\
\hline $4.31 \mathrm{E}-07$ & 646.84 & Pa-234 & $->$ & $U-234$ & & & \\
\hline $4.31 \mathrm{E}-07$ & 1153.6 & Pa-234 & $->$ & $U-234$ & & & \\
\hline $4.31 \mathrm{E}-07$ & 634.4 & Pa-234 & $->$ & $U-234$ & & & \\
\hline $4.31 \mathrm{E}-07$ & 330 & Pa-234 & $->$ & $U-234$ & & & \\
\hline $4.31 \mathrm{E}-07$ & 478.7 & Pa-234 & $->$ & $U-234$ & & & \\
\hline $4.31 \mathrm{E}-07$ & 482.5 & Рa-234 & $->$ & $\mathrm{U}-234$ & & & \\
\hline $4.26 \mathrm{E}-07$ & 1353 & $\mathrm{Bi}-214$ & $->$ & Po-214, & $\mathrm{Pa}-234 \mathrm{~m}$ & $->$ & $U-234$ \\
\hline 4.24E-07 & 387.87 & Pa-234m & $->$ & $U-234$ & & & \\
\hline 4.09E-07 & 1602.2 & $\mathrm{~Pa}-234 \mathrm{~m}$ & $->$ & $U-234$ & & & \\
\hline $4.02 E-07$ & 1905 & $\mathrm{~Pa}-234$ & $->$ & $\mathrm{U}-234$ & & & \\
\hline
\end{tabular}




\begin{tabular}{|c|c|c|c|c|}
\hline $4.02 \mathrm{E}-07$ & 688.08 & $\mathrm{~Pa}-234$ & $->$ & $U-234$ \\
\hline $3.88 \mathrm{E}-07$ & 79.65 & $\mathrm{~Pa}-234$ & $->$ & U-234 \\
\hline $3.73 E-07$ & 557.78 & $\mathrm{~Pa}-234$ & $->$ & $U-234$ \\
\hline 3.59E-07 & 1756 & $\mathrm{~Pa}-234$ & $->$ & $U-234$ \\
\hline 3.59E-07 & 1585.3 & $\mathrm{~Pa}-234$ & $->$ & U-234 \\
\hline 3.59E-07 & 1074.6 & $\mathrm{~Pa}-234$ & $->$ & U-234 \\
\hline $3.44 \mathrm{E}-07$ & 683.3 & $\mathrm{~Pa}-234$ & $->$ & $U-234$ \\
\hline $3.44 \mathrm{E}-07$ & 1171.3 & $\mathrm{~Pa}-234$ & $->$ & $U-234$ \\
\hline $3.44 \mathrm{E}-07$ & 472.32 & $\mathrm{~Pa}-234$ & $->$ & U-234 \\
\hline $3.43 E-07$ & 1926 & $\mathrm{~Pa}-234$ & $->$ & U-234 \\
\hline $3.30 \mathrm{E}-07$ & 1399.7 & $\mathrm{~Pa}-234$ & $->$ & U-234 \\
\hline $3.30 \mathrm{E}-07$ & 69.9 & $\mathrm{~Pa}-234$ & $->$ & $U-234$ \\
\hline $3.24 \mathrm{E}-07$ & 1720.5 & $\mathrm{~Pa}-234 \mathrm{~m}$ & $->$ & $U-234$ \\
\hline $3.13 \mathrm{E}-07$ & 316.29 & $\mathrm{~Pa}-234$ & $->$ & U-234, \\
\hline $3.10 \mathrm{E}-07$ & 1796.2 & $\mathrm{~Pa}-234 \mathrm{~m}$ & $->$ & U-234 \\
\hline $3.01 \mathrm{E}-07$ & 134.3 & $\mathrm{~Pa}-234$ & $->$ & U-234 \\
\hline $2.87 \mathrm{E}-07$ & 777.9 & $\mathrm{~Pa}-234$ & $->$ & $U-234$ \\
\hline 2.87E-07 & 617 & $\mathrm{~Pa}-234$ & $->$ & U-234 \\
\hline 2.87E-07 & 711.2 & $\mathrm{~Pa}-234$ & $->$ & U-234 \\
\hline $2.87 \mathrm{E}-07$ & 639.7 & $\mathrm{~Pa}-234$ & $->$ & U-234 \\
\hline $2.87 \mathrm{E}-07$ & 643.2 & Pa-234 & $->$ & U-234 \\
\hline $2.87 E-07$ & 1277.4 & $\mathrm{~Pa}-234$ & $->$ & U-234 \\
\hline $2.87 E-07$ & 174.52 & $\mathrm{~Pa}-234$ & $->$ & U-234 \\
\hline $2.87 E-07$ & 219.8 & $\mathrm{~Pa}-234$ & -> & U-234 \\
\hline $2.87 E-07$ & 150 & $\mathrm{~Pa}-234$ & $->$ & U-234 \\
\hline $2.87 E-07$ & 1427.33 & $\mathrm{~Pa}-234$ & $->$ & $U-234$ \\
\hline $2.87 E-07$ & 533.2 & $\mathrm{~Pa}-234$ & $->$ & $U-234$ \\
\hline $2.87 \mathrm{E}-07$ & 1493.94 & $\mathrm{~Pa}-234$ & $->$ & U-234 \\
\hline $2.73 E-07$ & 1890.1 & $\mathrm{~Pa}-234$ & $->$ & $U-234$ \\
\hline $2.58 \mathrm{E}-07$ & 473.5 & $\mathrm{~Pa}-234$ & $->$ & $U-234$ \\
\hline $2.44 \mathrm{E}-07$ & 267.1 & $\mathrm{~Pa}-234$ & $->$ & $U-234$ \\
\hline $2.44 \mathrm{E}-07$ & 1580.02 & $\mathrm{~Pa}-234$ & $->$ & U-234 \\
\hline $2.30 \mathrm{E}-07$ & 760.1 & Pa-234 & $->$ & U-234 \\
\hline $2.30 \mathrm{E}-07$ & 537.1 & Pa-234 & $->$ & $U-234$ \\
\hline $2.30 \mathrm{E}-07$ & 461.3 & $\mathrm{~Pa}-234$ & $->$ & U-234 \\
\hline $2.30 \mathrm{E}-07$ & 713.8 & $\mathrm{~Pa}-234$ & -> & U-234 \\
\hline $2.15 \mathrm{E}-07$ & 1699.8 & $\mathrm{~Pa}-234$ & $->$ & $U-234$ \\
\hline $2.15 E-07$ & 1627.9 & $\mathrm{~Pa}-234$ & $->$ & $U-234$ \\
\hline $2.15 \mathrm{E}-07$ & 137.7 & $\mathrm{~Pa}-234$ & $->$ & U-234 \\
\hline $2.15 \mathrm{E}-07$ & 1656 & $\mathrm{~Pa}-234$ & $->$ & $U-234$ \\
\hline $2.15 \mathrm{E}-07$ & 585.8 & Pa-234 & $->$ & U-234 \\
\hline $2.15 E-07$ & 1897.1 & $\mathrm{~Pa}-234$ & $->$ & U-234 \\
\hline $2.10 \mathrm{E}-07$ & 1926.4 & $\mathrm{~Pa}-234 \mathrm{~m}$ & $->$ & $U-234$ \\
\hline $2.01 \mathrm{E}-07$ & 842.4 & $\mathrm{~Pa}-234$ & $->$ & $U-234$ \\
\hline $2.01 \mathrm{E}-07$ & 286.4 & Pa-234 & -> & U-234 \\
\hline $1.87 \mathrm{E}-07$ & 746.5 & $\mathrm{~Pa}-234$ & $->$ & U-234 \\
\hline $1.72 \mathrm{E}-07$ & 873.4 & $\mathrm{~Pa}-234$ & -> & $U-234$ \\
\hline $1.72 \mathrm{E}-07$ & 1358.5 & $\mathrm{~Pa}-234$ & $->$ & $U-234$ \\
\hline $1.72 \mathrm{E}-07$ & 320.7 & Pa-234 & $->$ & U-234 \\
\hline $1.72 \mathrm{E}-07$ & 446.1 & $\mathrm{~Pa}-234$ & $->$ & $\mathrm{U}-234$ \\
\hline
\end{tabular}




\begin{tabular}{|c|c|c|c|c|}
\hline $1.72 \mathrm{E}-07$ & 103.7 & $\mathrm{~Pa}-234$ & $->$ & U-234 \\
\hline $1.72 \mathrm{E}-07$ & 43.47 & $\mathrm{~Pa}-234$ & $->$ & U-234 \\
\hline $1.58 \mathrm{E}-07$ & 289.25 & $\mathrm{~Pa}-234$ & $->$ & U-234 \\
\hline $1.44 \mathrm{E}-07$ & 498.8 & $\mathrm{~Pa}-234$ & -> & $U-234$ \\
\hline $1.44 \mathrm{E}-07$ & 1741.7 & $\mathrm{~Pa}-234$ & $->$ & $U-234$ \\
\hline $1.44 \mathrm{E}-07$ & 1772.3 & $\mathrm{~Pa}-234$ & $->$ & $U-234$ \\
\hline $1.44 \mathrm{E}-07$ & 960 & $\mathrm{~Pa}-234$ & -> & U-234 \\
\hline $1.44 \mathrm{E}-07$ & 1738 & $\mathrm{~Pa}-234$ & -> & $U-234$ \\
\hline $1.44 \mathrm{E}-07$ & 309.6 & $\mathrm{~Pa}-234$ & $->$ & U-234 \\
\hline $1.44 \mathrm{E}-07$ & 1549.4 & $\mathrm{~Pa}-234$ & $->$ & $U-234$ \\
\hline $1.44 \mathrm{E}-07$ & 416 & $\mathrm{~Pa}-234$ & -> & U-234 \\
\hline $1.29 \mathrm{E}-07$ & 165.8 & $\mathrm{~Pa}-234$ & -> & $U-234$ \\
\hline $1.00 \mathrm{E}-07$ & 1872.8 & $\mathrm{~Pa}-234$ & -> & U-234 \\
\hline $1.00 \mathrm{E}-07$ & 196.43 & $\mathrm{~Pa}-234$ & $->$ & U-234 \\
\hline $1.00 \mathrm{E}-07$ & 278.1 & $\mathrm{~Pa}-234$ & $->$ & $U-234$ \\
\hline $8.61 \mathrm{E}-08$ & 1837.9 & $\mathrm{~Pa}-234$ & -> & U-234 \\
\hline $8.61 \mathrm{E}-08$ & 1768 & $\mathrm{~Pa}-234$ & -> & $U-234$ \\
\hline 8.33E-08 & 67.22 & Pa-234 & $->$ & $U-234$ \\
\hline $7.61 \mathrm{E}-08$ & 192.74 & $\mathrm{~Pa}-234 \mathrm{~m}$ & -> & U-234 \\
\hline $7.18 \mathrm{E}-08$ & 210.7 & $\mathrm{~Pa}-234$ & -> & U-234 \\
\hline $7.18 \mathrm{E}-08$ & 1850 & $\mathrm{~Pa}-234$ & -> & $U-234$ \\
\hline $7.18 \mathrm{E}-08$ & 1937.7 & $\mathrm{~Pa}-234$ & $->$ & U-234 \\
\hline $7.18 \mathrm{E}-08$ & 1750.1 & $\mathrm{~Pa}-234$ & $->$ & U-234 \\
\hline $4.31 \mathrm{E}-08$ & 1828 & $\mathrm{~Pa}-234$ & -> & U-234 \\
\hline $4.19 \mathrm{E}-08$ & 236.03 & $\mathrm{~Pa}-234 \mathrm{~m}$ & -> & $U-234$ \\
\hline 3.97E-08 & 557.7 & $\mathrm{~Pa}-234 \mathrm{~m}$ & $->$ & U-234 \\
\hline $3.42 \mathrm{E}-08$ & 718.94 & $\mathrm{~Pa}-234 \mathrm{~m}$ & $->$ & U-234 \\
\hline $3.20 \mathrm{E}-08$ & 721.33 & $\mathrm{~Pa}-234 \mathrm{~m}$ & -> & U-234 \\
\hline $2.87 E-08$ & 1719.5 & Pa-234 & -> & $U-234$ \\
\hline $1.99 \mathrm{E}-08$ & 750.5 & $\mathrm{~Pa}-234 \mathrm{~m}$ & -> & $U-234$ \\
\hline $1.65 E-08$ & 516.8 & $\mathrm{~Pa}-234 \mathrm{~m}$ & $->$ & $U-234$ \\
\hline $1.58 \mathrm{E}-08$ & 45.28 & Pa-234 & -> & $U-234$ \\
\hline $1.58 \mathrm{E}-08$ & 432.8 & $\mathrm{~Pa}-234$ & -> & $U-234$ \\
\hline $1.56 \mathrm{E}-08$ & 73.9 & $\mathrm{~Pa}-234 \mathrm{~m}$ & -> & $\mathrm{Pa}-234$ \\
\hline $1.29 \mathrm{E}-08$ & 58.31 & Pa-234 & $->$ & $\mathrm{U}-234$ \\
\hline 5.17E-09 & 34.37 & $\mathrm{~Pa}-234$ & $->$ & U-234 \\
\hline 2.87E-09 & 1988 & $\mathrm{~Pa}-234$ & -> & U-234 \\
\hline $1.44 \mathrm{E}-09$ & 1959 & $\mathrm{~Pa}-234$ & -> & $U-234$ \\
\hline $1.44 \mathrm{E}-09$ & 1998 & $\mathrm{~Pa}-234$ & $->$ & $U-234$ \\
\hline $9.10 \mathrm{E}-10$ & 53.23 & U-234 & $->$ & Th-230 \\
\hline $3.14 \mathrm{E}-10$ & 120.912 & $U-234$ & -> & Th-230 \\
\hline $1.99 \mathrm{E}-13$ & 454.97 & $U-234$ & $->$ & Th-230 \\
\hline $1.12 \mathrm{E}-13$ & 508.2 & U-234 & $->$ & Th-230 \\
\hline $9.18 \mathrm{E}-14$ & 581.78 & U-234 & $->$ & Th-230 \\
\hline 4.97E-14 & 67.6758 & Th-230 & -> & $\mathrm{Ra}-226$ \\
\hline $7.65 E-15$ & 503.53 & U-234 & $->$ & Th-230 \\
\hline $7.65 E-15$ & 677.67 & U-234 & $->$ & Th-230 \\
\hline $6.42 \mathrm{E}-15$ & 143.876 & Th-230 & $->$ & $\mathrm{Ra}-226$ \\
\hline $6.12 \mathrm{E}-15$ & 624.44 & U-234 & -> & Th-230 \\
\hline $1.47 \mathrm{E}-15$ & 253.732 & Th-230 & $\rightarrow$ & $\mathrm{Ra}-226$ \\
\hline
\end{tabular}




\begin{tabular}{|c|c|c|c|c|}
\hline $1.16 \mathrm{E}-15$ & 186.057 & Th-230 & $\rightarrow$ & Ra-226 \\
\hline $8.22 \mathrm{E}-16$ & 609.311 & $\mathrm{Bi}-214$ & $->$ & Po-214 \\
\hline $2.83 \mathrm{E}-16$ & 1764.49 & $\mathrm{Bi}-214$ & $->$ & Po-214 \\
\hline $2.67 \mathrm{E}-16$ & 1120.273 & $\mathrm{Bi}-214$ & $->$ & Po-214 \\
\hline $1.12 \mathrm{E}-16$ & 253.84 & Th-230 & $->$ & Ra-226 \\
\hline $1.06 \mathrm{E}-16$ & 1238.107 & $\mathrm{Bi}-214$ & $->$ & Po-214 \\
\hline $8.90 \mathrm{E}-17$ & 2204.09 & $\mathrm{Bi}-214$ & $->$ & Po-214 \\
\hline 8.70E-17 & 768.35 & $\mathrm{Bi}-214$ & $->$ & Po-214 \\
\hline $7.17 \mathrm{E}-17$ & 1377.659 & $\mathrm{Bi}-214$ & $->$ & Po-214 \\
\hline $5.63 \mathrm{E}-17$ & 934.039 & $\mathrm{Bi}-214$ & $->$ & Po-214 \\
\hline $5.44 \mathrm{E}-17$ & 1729.58 & $\mathrm{Bi}-214$ & $->$ & Po-214 \\
\hline $4.42 \mathrm{E}-17$ & 1407.97 & $\mathrm{Bi}-214$ & $->$ & Po-214 \\
\hline $3.90 \mathrm{E}-17$ & 1509.22 & $\mathrm{Bi}-214$ & $->$ & Po-214 \\
\hline $3.78 \mathrm{E}-17$ & 1847.41 & $\mathrm{Bi}-214$ & $->$ & Po-214 \\
\hline $3.01 \mathrm{E}-17$ & 1155.183 & $\mathrm{Bi}-214$ & $->$ & Po-214 \\
\hline $2.78 \mathrm{E}-17$ & 665.442 & $\mathrm{Bi}-214$ & $->$ & Po-214 \\
\hline $2.76 \mathrm{E}-17$ & 2447.68 & $\mathrm{Bi}-214$ & $->$ & Po-214 \\
\hline $2.62 \mathrm{E}-17$ & 1280.952 & $\mathrm{Bi}-214$ & $->$ & Po-214 \\
\hline $2.48 \mathrm{E}-17$ & 1401.48 & $\mathrm{Bi}-214$ & $->$ & Po-214 \\
\hline $2.19 \mathrm{E}-17$ & 806.155 & $\mathrm{Bi}-214$ & $->$ & Po-214 \\
\hline $2.16 \mathrm{E}-17$ & 2118.53 & $\mathrm{Bi}-214$ & $->$ & Po-214 \\
\hline $2.05 \mathrm{E}-17$ & 1661.258 & $\mathrm{Bi}-214$ & $->$ & Po-214 \\
\hline $1.73 \mathrm{E}-17$ & 79.29 & $\mathrm{Bi}-214$ & $->$ & Po-214 \\
\hline $1.39 \mathrm{E}-17$ & 1385.295 & $\mathrm{Bi}-214$ & $->$ & Po-214 \\
\hline $1.28 \mathrm{E}-17$ & 1583.22 & $\mathrm{Bi}-214$ & $->$ & Po-214 \\
\hline $1.03 \mathrm{E}-17$ & 76.838 & $\mathrm{Bi}-214$ & -> & Po-214 \\
\hline $8.41 \mathrm{E}-18$ & 703.07 & $\mathrm{Bi}-214$ & $->$ & Po-214 \\
\hline $8.20 \mathrm{E}-18$ & 1207.764 & $\mathrm{Bi}-214$ & $->$ & Po-214 \\
\hline $7.80 \mathrm{E}-18$ & 109.97 & Th-230 & $->$ & Ra-226 \\
\hline $7.31 \mathrm{E}-18$ & 1538.49 & $\mathrm{Bi}-214$ & -> & Po-214 \\
\hline $7.31 \mathrm{E}-18$ & 388.95 & $\mathrm{Bi}-214$ & $->$ & Po-214 \\
\hline $7.18 \mathrm{E}-18$ & 719.856 & $\mathrm{Bi}-214$ & $->$ & Po-214 \\
\hline $6.83 \mathrm{E}-18$ & 11.119 & $\mathrm{Bi}-214$ & $->$ & Po-214 \\
\hline $6.83 \mathrm{E}-18$ & 1838.37 & $\mathrm{Bi}-214$ & $->$ & Po-214 \\
\hline $6.83 \mathrm{E}-18$ & 964.07 & $\mathrm{Bi}-214$ & $->$ & Po-214 \\
\hline $6.60 \mathrm{E}-18$ & 13.502 & $\mathrm{Bi}-214$ & $->$ & Po-214 \\
\hline $6.42 \mathrm{E}-18$ & 386.834 & $\mathrm{Bi}-214$ & $->$ & Po-214 \\
\hline $6.24 \mathrm{E}-18$ & 1543.347 & $\mathrm{Bi}-214$ & $->$ & Po-214 \\
\hline $6.15 \mathrm{E}-18$ & 89.639 & $\mathrm{Bi}-214$ & $->$ & Po-214 \\
\hline $5.95 \mathrm{E}-18$ & 1599.3 & $\mathrm{Bi}-214$ & $->$ & Po-214 \\
\hline $5.78 \mathrm{E}-18$ & 2293.29 & $\mathrm{Bi}-214$ & $->$ & Po-214 \\
\hline $5.67 \mathrm{E}-18$ & 454.832 & $\mathrm{Bi}-214$ & $->$ & Po-214 \\
\hline $5.62 \mathrm{E}-18$ & 1051.95 & $\mathrm{Bi}-214$ & $->$ & Po-214 \\
\hline $5.61 \mathrm{E}-18$ & 302.25 & $\mathrm{Hg}-206$ & $->$ & TI-206 \\
\hline $5.53 \mathrm{E}-18$ & 786.42 & $\mathrm{Bi}-214$ & $->$ & Po-214 \\
\hline $5.08 \mathrm{E}-18$ & 1070.02 & $\mathrm{Bi}-214$ & $->$ & Po-214 \\
\hline $4.72 \mathrm{E}-18$ & 1594.78 & $\mathrm{Bi}-214$ & $->$ & Po-214 \\
\hline $4.55 \mathrm{E}-18$ & 1133.65 & $\mathrm{Bi}-214$ & $->$ & Po-214 \\
\hline $4.21 \mathrm{E}-18$ & 1683.99 & $\mathrm{Bi}-214$ & $->$ & Po-214 \\
\hline $4.03 E-18$ & 1873.112 & $\mathrm{Bi}-214$ & $->$ & Po-214 \\
\hline
\end{tabular}




\begin{tabular}{|c|c|c|c|c|c|c|c|}
\hline $3.21 \mathrm{E}-18$ & 273.7 & $\mathrm{Bi}-214$ & $\rightarrow$ & Po-214 & & & \\
\hline $3.16 \mathrm{E}-18$ & 1896.28 & $\mathrm{Bi}-214$ & $->$ & Po-214 & & & \\
\hline $2.98 \mathrm{E}-18$ & 405.73 & $\mathrm{Bi}-214$ & $->$ & Po-214 & & & \\
\hline $2.67 \mathrm{E}-18$ & 821.166 & $\mathrm{Bi}-214$ & $->$ & Po-214 & & & \\
\hline $2.37 \mathrm{E}-18$ & 752.843 & $\mathrm{Bi}-214$ & $->$ & Po-214 & & & \\
\hline $2.37 \mathrm{E}-18$ & 469.76 & $\mathrm{Bi}-214$ & $->$ & Po-214 & & & \\
\hline $2.16 \mathrm{E}-18$ & 1303.76 & $\mathrm{Bi}-214$ & $->$ & Po-214 & & & \\
\hline $2.10 \mathrm{E}-18$ & 474.51 & $\mathrm{Bi}-214$ & $->$ & Po-214 & & & \\
\hline $1.96 \mathrm{E}-18$ & 426.5 & $\mathrm{Bi}-214$ & $->$ & Po-214 & & & \\
\hline $1.87 \mathrm{E}-18$ & 92.673 & $\mathrm{Bi}-214$ & $->$ & Po-214 & & & \\
\hline $1.87 \mathrm{E}-18$ & 904.33 & $\mathrm{Bi}-214$ & $->$ & Po-214 & & & \\
\hline $1.78 \mathrm{E}-18$ & 1103.7 & $\mathrm{Bi}-214$ & $->$ & Po-214 & & & \\
\hline $1.71 \mathrm{E}-18$ & 1032.22 & $\mathrm{Bi}-214$ & $->$ & Po-214 & & & \\
\hline $1.69 \mathrm{E}-18$ & 333.6 & $\mathrm{Bi}-214$ & $->$ & Po-214 & & & \\
\hline $1.64 \mathrm{E}-18$ & 826.44 & $\mathrm{Bi}-214$ & $->$ & Po-214 & & & \\
\hline $1.59 \mathrm{E}-18$ & 1890.259 & $\mathrm{Bi}-214$ & $->$ & Po-214 & & & \\
\hline $1.55 \mathrm{E}-18$ & 2109.91 & $\mathrm{Bi}-214$ & $->$ & Po-214 & & & \\
\hline $1.53 \mathrm{E}-18$ & 1317.02 & $\mathrm{Bi}-214$ & $->$ & Po-214 & & & \\
\hline $1.52 \mathrm{E}-18$ & 542.84 & $\mathrm{Bi}-214$ & $->$ & Po-214 & & & \\
\hline $1.46 \mathrm{E}-18$ & 572.67 & $\mathrm{Bi}-214$ & $->$ & Po-214 & & & \\
\hline $1.44 \mathrm{E}-18$ & 280.93 & $\mathrm{Bi}-214$ & $->$ & Po-214 & & & \\
\hline $1.43 \mathrm{E}-18$ & 1104.766 & $\mathrm{Bi}-214$ & $->$ & Po-214 & & & \\
\hline $1.41 \mathrm{E}-18$ & 683.21 & $\mathrm{Bi}-214$ & $->$ & Po-214 & & & \\
\hline $1.33 E-18$ & 710.84 & $\mathrm{Bi}-214$ & $->$ & Po-214 & & & \\
\hline 1.27E-18 & 15.874 & $\mathrm{Bi}-214$ & $->$ & Po-214 & & & \\
\hline 1.27E-18 & 536.93 & $\mathrm{Bi}-214$ & $->$ & Po-214 & & & \\
\hline $1.25 \mathrm{E}-18$ & 615.77 & $\mathrm{Bi}-214$ & $->$ & Po-214 & & & \\
\hline $1.25 \mathrm{E}-18$ & 2052.93 & $\mathrm{Bi}-214$ & $->$ & Po-214 & & & \\
\hline $1.25 \mathrm{E}-18$ & 1637.37 & $\mathrm{Bi}-214$ & $->$ & Po-214 & & & \\
\hline $1.23 E-18$ & 1479.19 & $\mathrm{Bi}-214$ & $->$ & Po-214 & & & \\
\hline $1.12 \mathrm{E}-18$ & 1898.9 & $\mathrm{Bi}-214$ & $->$ & Po-214 & & & \\
\hline $1.09 E-18$ & 2192.52 & $\mathrm{Bi}-214$ & $->$ & Po-214 & & & \\
\hline $1.07 E-18$ & 235.01 & Th-230 & $->$ & Ra-226 & & & \\
\hline $1.07 E-18$ & 347.1 & $\mathrm{Bi}-214$ & $->$ & Po-214 & & & \\
\hline $1.07 E-18$ & 633.14 & $\mathrm{Bi}-214$ & $->$ & Po-214 & & & \\
\hline $1.05 E-18$ & 649.18 & $\mathrm{Bi}-214$ & $->$ & Po-214 & & & \\
\hline $1.03 \mathrm{E}-18$ & 1172.93 & $\mathrm{Bi}-214$ & $->$ & Po-214 & & & \\
\hline $1.03 E-18$ & 1173.04 & $\mathrm{Bi}-214$ & $->$ & Po-214 & & & \\
\hline $1.02 \mathrm{E}-18$ & 797.88 & $\mathrm{TI}-210$ & $->$ & $\mathrm{Pb}-210$ & Po-214 & $->$ & $\mathrm{Pb}-210$ \\
\hline $1.02 \mathrm{E}-18$ & 334.9 & $\mathrm{Bi}-214$ & $->$ & Po-214 & & & \\
\hline $9.98 E-19$ & 2089.55 & $\mathrm{Bi}-214$ & $->$ & Po-214 & & & \\
\hline 9.09E-19 & 1935.8 & $\mathrm{Bi}-214$ & $->$ & Po-214 & & & \\
\hline $8.73 E-19$ & 2010.79 & $\mathrm{Bi}-214$ & $->$ & Po-214 & & & \\
\hline $8.72 E-19$ & 72.873 & $\mathrm{Hg}-206$ & $->$ & TI-206 & & & \\
\hline 8.38E-19 & 733.64 & Bi-214 & $->$ & Po-214 & & & \\
\hline $8.16 \mathrm{E}-19$ & 298.1 & TI-210 & $->$ & $\mathrm{Pb}-210$ & Po-214 & $->$ & $\mathrm{Pb}-210$ \\
\hline 8.02E-19 & 1130.6 & $\mathrm{Bi}-214$ & $->$ & Po-214 & & & \\
\hline $8.02 E-19$ & 723.32 & $\mathrm{Bi}-214$ & $\rightarrow$ & Po-214 & & & \\
\hline 7.67E-19 & 660.75 & $\mathrm{Bi}-214$ & $->$ & Po-214 & & & \\
\hline 7.31E-19 & 799.75 & $\mathrm{Bi}-214$ & $->$ & Po-214 & & & \\
\hline
\end{tabular}




\begin{tabular}{|c|c|c|c|c|}
\hline $7.13 \mathrm{E}-19$ & 814.87 & $\mathrm{Bi}-214$ & $->$ & Po-214 \\
\hline $6.95 \mathrm{E}-19$ & 338.5 & $\mathrm{Bi}-214$ & $->$ & Po-214 \\
\hline 6.95E-19 & 740.87 & $\mathrm{Bi}-214$ & $->$ & Po-214 \\
\hline 6.61E-19 & 205.1 & Th-230 & $->$ & $\mathrm{Ra}-226$ \\
\hline $6.60 \mathrm{E}-19$ & 697.89 & $\mathrm{Bi}-214$ & $->$ & Po-214 \\
\hline 6.06E-19 & 304.42 & $\mathrm{Bi}-214$ & $->$ & Po-214 \\
\hline $6.06 \mathrm{E}-19$ & 617.1 & $\mathrm{Bi}-214$ & -> & Po-214 \\
\hline $5.78 \mathrm{E}-19$ & 2694.67 & $\mathrm{Bi}-214$ & -> & Po-214 \\
\hline $5.70 E-19$ & 286.9 & $\mathrm{Bi}-214$ & $->$ & Po-214 \\
\hline $5.70 \mathrm{E}-19$ & 547.1 & $\mathrm{Bi}-214$ & $->$ & Po-214 \\
\hline $5.53 E-19$ & 639.36 & $\mathrm{Bi}-214$ & -> & Po-214 \\
\hline 5.35E-19 & 396 & $\mathrm{Bi}-214$ & -> & Po-214 \\
\hline 5.19E-19 & 650.21 & $\mathrm{Hg}-206$ & $->$ & TI-206 \\
\hline 5.19E-19 & 70.832 & $\mathrm{Hg}-206$ & $->$ & TI-206 \\
\hline $5.17 \mathrm{E}-19$ & 440.4 & $\mathrm{Bi}-214$ & -> & Po-214 \\
\hline $5.17 \mathrm{E}-19$ & 1067.3 & $\mathrm{Bi}-214$ & -> & Po-214 \\
\hline 5.17E-19 & 1045.4 & $\mathrm{Bi}-214$ & -> & Po-214 \\
\hline $4.81 \mathrm{E}-19$ & 1226.8 & $\mathrm{Bi}-214$ & $->$ & Po-214 \\
\hline $4.56 \mathrm{E}-19$ & 2769.99 & $\mathrm{Bi}-214$ & -> & Po-214 \\
\hline $4.10 \mathrm{E}-19$ & 1341.5 & $\mathrm{Bi}-214$ & -> & Po-214 \\
\hline $4.10 \mathrm{E}-19$ & 976.2 & $\mathrm{Bi}-214$ & $->$ & Po-214 \\
\hline $4.10 \mathrm{E}-19$ & 915.8 & $\mathrm{Bi}-214$ & $->$ & Po-214 \\
\hline $4.10 \mathrm{E}-19$ & 832.34 & $\mathrm{Bi}-214$ & $->$ & Po-214 \\
\hline $4.03 E-19$ & 3053.89 & $\mathrm{Bi}-214$ & -> & Po-214 \\
\hline $3.96 \mathrm{E}-19$ & 570.5 & Th-230 & -> & $\mathrm{Ra}-226$ \\
\hline $3.92 \mathrm{E}-19$ & 2331.2 & $\mathrm{Bi}-214$ & $->$ & Po-214 \\
\hline $3.92 \mathrm{E}-19$ & 1230.84 & $\mathrm{Bi}-214$ & $->$ & Po-214 \\
\hline $3.57 \mathrm{E}-19$ & 9.658 & $\mathrm{Bi}-214$ & -> & Po-214 \\
\hline 3.39E-19 & 2021.7 & $\mathrm{Bi}-214$ & -> & Po-214 \\
\hline 3.39E-19 & 1636.6 & $\mathrm{Bi}-214$ & $->$ & Po-214 \\
\hline 3.39E-19 & 1392.5 & $\mathrm{Bi}-214$ & $->$ & Po-214 \\
\hline $3.21 \mathrm{E}-19$ & 2266.67 & $\mathrm{Bi}-214$ & -> & Po-214 \\
\hline $3.21 \mathrm{E}-19$ & 502.2 & $\mathrm{Bi}-214$ & -> & Po-214 \\
\hline $3.11 \mathrm{E}-19$ & 10.259 & $\mathrm{Hg}-206$ & -> & TI-206 \\
\hline $3.11 \mathrm{E}-19$ & 82.434 & $\mathrm{Hg}-206$ & $->$ & TI-206 \\
\hline 3.03E-19 & 631.2 & $\mathrm{Bi}-214$ & $->$ & Po-214 \\
\hline $3.03 E-19$ & 847.2 & $\mathrm{Bi}-214$ & -> & Po-214 \\
\hline $3.03 E-19$ & 943.3 & $\mathrm{Bi}-214$ & $->$ & Po-214 \\
\hline 3.03E-19 & 1038 & $\mathrm{Bi}-214$ & $->$ & Po-214 \\
\hline $2.85 \mathrm{E}-19$ & 1782.1 & $\mathrm{Bi}-214$ & $->$ & Po-214 \\
\hline $2.85 \mathrm{E}-19$ & 2147.8 & $\mathrm{Bi}-214$ & -> & Po-214 \\
\hline $2.85 \mathrm{E}-19$ & 525 & $\mathrm{Bi}-214$ & -> & Po-214 \\
\hline 2.80E-19 & 2922.09 & $\mathrm{Bi}-214$ & $->$ & Po-214 \\
\hline $2.80 \mathrm{E}-19$ & 727.8 & $\mathrm{Bi}-214$ & $->$ & Po-214 \\
\hline $2.70 \mathrm{E}-19$ & 12.313 & $\mathrm{Hg}-206$ & -> & TI-206 \\
\hline $2.62 \mathrm{E}-19$ & 2978.79 & $\mathrm{Bi}-214$ & $->$ & Po-214 \\
\hline $2.17 \mathrm{E}-19$ & 1314 & $\mathrm{TI}-210$ & $->$ & $\mathrm{Pb}-210$ \\
\hline $2.14 \mathrm{E}-19$ & 1020.5 & $\mathrm{Bi}-214$ & $->$ & Po-214 \\
\hline $2.14 \mathrm{E}-19$ & 989.2 & $\mathrm{Bi}-214$ & $->$ & Po-214 \\
\hline $2.14 \mathrm{E}-19$ & 1814.01 & $\mathrm{Bi}-214$ & $->$ & Po-214 \\
\hline
\end{tabular}




\begin{tabular}{|c|c|c|c|c|c|c|c|}
\hline $2.14 \mathrm{E}-19$ & 596 & $\mathrm{Bi}-214$ & $->$ & Po-214 & & & \\
\hline $2.14 \mathrm{E}-19$ & 1471.1 & $\mathrm{Bi}-214$ & $->$ & Po-214 & & & \\
\hline $2.10 \mathrm{E}-19$ & 2376.99 & $\mathrm{Bi}-214$ & $->$ & Po-214 & & & \\
\hline $1.96 \mathrm{E}-19$ & 1330 & $\mathrm{Bi}-214$ & $->$ & Po-214 & & & \\
\hline $1.78 \mathrm{E}-19$ & 2085 & $\mathrm{Bi}-214$ & $->$ & Po-214 & & & \\
\hline $1.78 \mathrm{E}-19$ & 1013.4 & $\mathrm{Bi}-214$ & $->$ & Po-214 & & & \\
\hline $1.64 \mathrm{E}-19$ & 2880.4 & $\mathrm{Bi}-214$ & $->$ & Po-214 & & & \\
\hline $1.60 \mathrm{E}-19$ & 494.6 & $\mathrm{Bi}-214$ & $->$ & Po-214 & & & \\
\hline $1.60 \mathrm{E}-19$ & 2259.7 & $\mathrm{Bi}-214$ & $->$ & Po-214 & & & \\
\hline $1.60 \mathrm{E}-19$ & 394 & $\mathrm{Bi}-214$ & $->$ & Po-214 & & & \\
\hline $1.57 E-19$ & 2999.99 & $\mathrm{Bi}-214$ & $->$ & Po-214 & & & \\
\hline $1.33 E-19$ & 551.8 & Th-230 & $->$ & $\mathrm{Ra}-226$ & & & \\
\hline $1.33 E-19$ & 620 & Th-230 & $->$ & Ra-226 & & & \\
\hline $1.25 \mathrm{E}-19$ & 2251.2 & $\mathrm{Bi}-214$ & $->$ & Po-214 & & & \\
\hline $1.24 \mathrm{E}-19$ & 1068 & TI-210 & $->$ & $\mathrm{Pb}-210$ & & & \\
\hline $1.14 \mathrm{E}-19$ & 2893.59 & $\mathrm{Bi}-214$ & $->$ & Po-214 & & & \\
\hline $1.14 \mathrm{E}-19$ & 12.678 & TI-210 & $->$ & $\mathrm{Pb}-210$ & & & \\
\hline $1.12 \mathrm{E}-19$ & 364.2 & $\mathrm{Bi}-214$ & $->$ & Po-214 & & & \\
\hline $1.08 \mathrm{E}-19$ & 344.96 & $\mathrm{Hg}-206$ & $->$ & TI-206 & & & \\
\hline $1.07 E-19$ & 693.3 & $\mathrm{Bi}-214$ & $->$ & Po-214 & & & \\
\hline $1.07 E-19$ & 687.7 & $\mathrm{Bi}-214$ & $->$ & Po-214 & & & \\
\hline $1.05 \mathrm{E}-19$ & 2505.58 & $\mathrm{Bi}-214$ & $->$ & Po-214 & & & \\
\hline $1.05 E-19$ & 2423.32 & $\mathrm{Bi}-214$ & $->$ & Po-214 & & & \\
\hline $1.05 \mathrm{E}-19$ & 2786.09 & $\mathrm{Bi}-214$ & $->$ & Po-214 & & & \\
\hline $1.02 \mathrm{E}-19$ & 520.4 & $\mathrm{Bi}-214$ & $->$ & Po-214 & & & \\
\hline $9.80 E-20$ & 12.085 & $\mathrm{Bi}-214$ & $->$ & Po-214 & & & \\
\hline $9.29 E-20$ & 2428 & $\mathrm{TI}-210$ & $->$ & $\mathrm{Pb}-210$ & & & \\
\hline $9.09 E-20$ & 2284.4 & $\mathrm{Bi}-214$ & $->$ & Po-214 & & & \\
\hline $9.09 E-20$ & 1419.7 & $\mathrm{Bi}-214$ & $->$ & Po-214 & & & \\
\hline $8.91 E-20$ & 1994.7 & $\mathrm{Bi}-214$ & $->$ & Po-214 & & & \\
\hline $8.73 E-20$ & 10.541 & TI-206 & $->$ & $\mathrm{Pb}-206$ & TI-210 & $->$ & $\mathrm{Pb}-210$ \\
\hline $8.73 E-20$ & 626.4 & $\mathrm{Bi}-214$ & $->$ & Po-214 & & & \\
\hline $8.73 E-20$ & 376.6 & $\mathrm{Bi}-214$ & $->$ & Po-214 & & & \\
\hline $8.72 E-20$ & 85.185 & $\mathrm{Hg}-206$ & $->$ & TI-206 & & & \\
\hline $8.26 \mathrm{E}-20$ & 2358 & TI-210 & $->$ & $\mathrm{Pb}-210$ & & & \\
\hline $7.67 E-20$ & 3081.7 & $\mathrm{Bi}-214$ & $->$ & Po-214 & & & \\
\hline $7.12 \mathrm{E}-20$ & 2008 & $\mathrm{TI}-210$ & $->$ & $\mathrm{Pb}-210$ & & & \\
\hline $7.12 \mathrm{E}-20$ & 1110 & $\mathrm{TI}-210$ & $->$ & $\mathrm{Pb}-210$ & & & \\
\hline $7.12 \mathrm{E}-20$ & 860 & $\mathrm{TI}-210$ & $->$ & $\mathrm{Pb}-210$ & & & \\
\hline $5.29 E-20$ & 74.969 & TI-206 & $->$ & $\mathrm{Pb}-206$ & TI-210 & $->$ & $\mathrm{Pb}-210$ \\
\hline $5.17 E-20$ & 2270 & $\mathrm{Bi}-214$ & $->$ & Po-214 & & & \\
\hline $5.17 E-20$ & 2369.3 & $\mathrm{Bi}-214$ & $->$ & Po-214 & & & \\
\hline $5.17 E-20$ & 2004.5 & $\mathrm{Bi}-214$ & $->$ & Po-214 & & & \\
\hline $5.06 \mathrm{E}-20$ & 1408 & $\mathrm{TI}-210$ & $->$ & $\mathrm{Pb}-210$ & & & \\
\hline $5.06 \mathrm{E}-20$ & 2088 & $\mathrm{TI}-210$ & $->$ & $\mathrm{Pb}-210$ & & & \\
\hline $4.99 E-20$ & 2698.86 & Bi-214 & $->$ & Po-214 & & & \\
\hline $4.98 E-20$ & 14.407 & $\mathrm{Hg}-206$ & $->$ & TI-206 & & & \\
\hline $4.46 E-20$ & 2827 & $\mathrm{Bi}-214$ & $->$ & Po-214 & & & \\
\hline $4.16 \mathrm{E}-20$ & 13.066 & $\mathrm{~Pb}-210$ & $->$ & $\mathrm{Bi}-210$ & & & \\
\hline $4.13 E-20$ & 354 & TI-210 & $->$ & $\mathrm{Pb}-210$ & & & \\
\hline
\end{tabular}




\begin{tabular}{|c|c|c|c|c|c|c|c|}
\hline $4.13 E-20$ & 95 & TI-210 & $->$ & $\mathrm{Pb}-210$ & & & \\
\hline $3.74 \mathrm{E}-20$ & 2482.417 & $\mathrm{Bi}-214$ & $->$ & Po-214 & & & \\
\hline $3.68 \mathrm{E}-20$ & 10.828 & $\mathrm{~Pb}-210$ & $->$ & $\mathrm{Bi}-210$ & $\mathrm{~Pb}-214$ & $->$ & $\mathrm{Bi}-214$ \\
\hline $3.57 E-20$ & 2390.9 & $\mathrm{Bi}-214$ & $->$ & Po-214 & & & \\
\hline $3.39 E-20$ & 2324.8 & $\mathrm{Bi}-214$ & $->$ & Po-214 & & & \\
\hline $3.39 E-20$ & 2360.9 & $\mathrm{Bi}-214$ & $->$ & Po-214 & & & \\
\hline $3.22 \mathrm{E}-20$ & 72.803 & TI-206 & $->$ & $\mathrm{Pb}-206$ & TI-210 & $->$ & $P b-210$ \\
\hline $3.21 \mathrm{E}-20$ & 2719.21 & $\mathrm{Bi}-214$ & $->$ & Po-214 & & & \\
\hline $3.10 \mathrm{E}-20$ & 2268 & TI-210 & $->$ & $\mathrm{Pb}-210$ & & & \\
\hline $3.10 \mathrm{E}-20$ & 908 & TI-210 & $->$ & $\mathrm{Pb}-210$ & & & \\
\hline $3.10 \mathrm{E}-20$ & 380 & TI-210 & $->$ & $\mathrm{Pb}-210$ & & & \\
\hline $3.03 E-20$ & 2940.5 & $\mathrm{Bi}-214$ & $->$ & Po-214 & & & \\
\hline $2.85 E-20$ & 3142.6 & $\mathrm{Bi}-214$ & $->$ & Рo-214 & & & \\
\hline $2.67 E-20$ & 3183.6 & $\mathrm{Bi}-214$ & $->$ & Po-214 & & & \\
\hline $2.30 \mathrm{E}-20$ & 14.836 & TI-206 & $->$ & Pb-206, & TI-210 & $->$ & $\mathrm{Pb}-210$ \\
\hline $2.14 \mathrm{E}-20$ & 2928.7 & $\mathrm{Bi}-214$ & $->$ & Po-214 & & & \\
\hline $2.07 E-20$ & 478 & TI-210 & $->$ & $\mathrm{Pb}-210$ & & & \\
\hline $2.07 E-20$ & 1538 & TI-210 & $->$ & $\mathrm{Pb}-210$ & & & \\
\hline 2.07E-20 & 1588 & TI-210 & $->$ & $\mathrm{Pb}-210$ & & & \\
\hline 2.07E-20 & 668 & $\mathrm{Tl}-210$ & $->$ & $\mathrm{Pb}-210$ & & & \\
\hline 2.07E-20 & 1648 & TI-210 & $->$ & $\mathrm{Pb}-210$ & & & \\
\hline 2.07E-20 & 81 & TI-210 & $->$ & $\mathrm{Pb}-210$ & & & \\
\hline $1.96 \mathrm{E}-20$ & 2988.7 & $\mathrm{Bi}-214$ & $->$ & Po-214 & & & \\
\hline $1.89 \mathrm{E}-20$ & 84.789 & TI-206 & $->$ & $\mathrm{Pb}-206$ & TI-210 & $->$ & $\mathrm{Pb}-210$ \\
\hline $1.62 \mathrm{E}-20$ & 46.52 & $\mathrm{~Pb}-210$ & $->$ & $\mathrm{Bi}-210$ & & & \\
\hline 1.60E-20 & 2631 & $\mathrm{Bi}-214$ & $->$ & Рo-214 & & & \\
\hline $1.50 \mathrm{E}-20$ & 8.953 & $\mathrm{Hg}-206$ & $->$ & TI-206 & & & \\
\hline $1.02 \mathrm{E}-20$ & 2934.9 & $\mathrm{Bi}-214$ & $->$ & Рo-214 & & & \\
\hline $9.99 E-21$ & 15.537 & $\mathrm{~Pb}-210$ & $->$ & $\mathrm{Bi}-210$ & & & \\
\hline $9.09 E-21$ & 3160.5 & $\mathrm{Bi}-214$ & $->$ & Po-214 & & & \\
\hline $9.09 E-21$ & 3093.9 & $\mathrm{Bi}-214$ & $->$ & Po-214 & & & \\
\hline $8.02 E-21$ & 2604.5 & $\mathrm{Bi}-214$ & $->$ & Рo-214 & & & \\
\hline $6.95 E-21$ & 2551 & $\mathrm{Bi}-214$ & $->$ & Po-214 & & & \\
\hline $6.06 \mathrm{E}-21$ & 3136.3 & $\mathrm{Bi}-214$ & $->$ & Po-214 & & & \\
\hline $6.06 \mathrm{E}-21$ & 2860.9 & $\mathrm{Bi}-214$ & $->$ & Po-214 & & & \\
\hline $5.41 \mathrm{E}-21$ & 87.632 & TI-206 & $->$ & $\mathrm{Pb}-206$ & TI-210 & $->$ & $\mathrm{Pb}-210$ \\
\hline $5.17 E-21$ & 2662.23 & $\mathrm{Bi}-214$ & $->$ & Po-214 & & & \\
\hline $4.32 E-21$ & 9.185 & TI-206 & $->$ & $\mathrm{Pb}-206$ & TI-210 & $->$ & $\mathrm{Pb}-210$ \\
\hline $3.95 E-21$ & 10.994 & $\mathrm{Hg}-206$ & $->$ & TI-206 & & & \\
\hline $3.57 E-21$ & 3233.3 & $\mathrm{Bi}-214$ & $->$ & Po-214 & & & \\
\hline 2.07E-21 & 11.349 & TI-210 & $->$ & $\mathrm{Pb}-210$ & & & \\
\hline $1.88 \mathrm{E}-21$ & 9.419 & $\mathrm{~Pb}-210$ & $->$ & $\mathrm{Bi}-210$ & $\mathrm{~Pb}-214$ & $->$ & $\mathrm{Bi}-214$ \\
\hline $1.78 \mathrm{E}-21$ & 3269.7 & $\mathrm{Bi}-214$ & $->$ & Po-214 & & & \\
\hline $1.45 \mathrm{E}-21$ & 12.71 & TI-206 & $->$ & $\mathrm{Pb}-206$ & & & \\
\hline $9.19 E-22$ & 803.13 & TI-206 & $->$ & $\mathrm{Pb}-206$ & Po-210 & $->$ & Pb-206 \\
\hline $7.89 \mathrm{E}-22$ & 384.06 & $\mathrm{Hg}-206$ & $->$ & TI-206 & & & \\
\hline $3.16 \mathrm{E}-22$ & 11.712 & $\mathrm{~Pb}-210$ & $->$ & $\mathrm{Bi}-210$ & $\mathrm{~Pb}-214$ & $->$ & $\mathrm{Bi}-214$ \\
\hline $2.30 \mathrm{E}-23$ & 11.439 & TI-206 & $->$ & Pb-206 & & & \\
\hline $4.80 E-31$ & 266.15 & $\mathrm{Bi}-210$ & $->$ & TI-206 & & & \\
\hline $0.00 E+00$ & 87.19 & $\mathrm{~Pb}-214$ & $->$ & $\mathrm{Bi}-214$ & & & \\
\hline
\end{tabular}




\begin{tabular}{|c|c|c|c|c|}
\hline $0.00 \mathrm{E}+00$ & 83.787 & $\mathrm{Ra}-226$ & $\rightarrow$ & $\mathrm{Rn}-222$ \\
\hline $0.00 E+00$ & 81.067 & $\mathrm{Ra}-226$ & $->$ & $\mathrm{Rn}-222$ \\
\hline $0.00 E+00$ & 12.855 & $\mathrm{Ra}-226$ & $->$ & $\mathrm{Rn}-222$ \\
\hline $0.00 E+00$ & 10.137 & Ra-226 & $->$ & $\mathrm{Rn}-222$ \\
\hline $0.00 \mathrm{E}+00$ & 77.107 & $\mathrm{~Pb}-214$ & $->$ & $\mathrm{Bi}-214$ \\
\hline $0.00 E+00$ & 74.814 & $\mathrm{~Pb}-214$ & $->$ & $\mathrm{Bi}-214$ \\
\hline $0.00 E+00$ & 53.172 & $\mathrm{~Pb}-214$ & $->$ & $\mathrm{Bi}-214$ \\
\hline $0.00 E+00$ & 16.874 & Ra-226 & $->$ & $\mathrm{Rn}-222$ \\
\hline $0.00 E+00$ & 15.439 & $\mathrm{~Pb}-214$ & $->$ & $\mathrm{Bi}-214$ \\
\hline $0.00 \mathrm{E}+00$ & 14.336 & Ra-226 & $->$ & $\mathrm{Rn}-222$ \\
\hline $0.00 E+00$ & 11.713 & $\mathrm{Ra}-226$ & $->$ & $\mathrm{Rn}-222$ \\
\hline $0.00 E+00$ & 13.088 & $\mathrm{~Pb}-214$ & $->$ & $\mathrm{Bi}-214$ \\
\hline $0.00 E+00$ & 838.999 & $\mathrm{~Pb}-214$ & $->$ & $\mathrm{Bi}-214$ \\
\hline $0.00 E+00$ & 785.827 & $\mathrm{~Pb}-214$ & $->$ & $\mathrm{Bi}-214$ \\
\hline $0.00 E+00$ & 765.9 & $\mathrm{~Pb}-214$ & $->$ & $\mathrm{Bi}-214$ \\
\hline $0.00 \mathrm{E}+00$ & 600.83 & $\mathrm{Ra}-226$ & $->$ & Rn-222 \\
\hline $0.00 E+00$ & 580.06 & $\mathrm{~Pb}-214$ & $->$ & $\mathrm{Bi}-214$ \\
\hline $0.00 \mathrm{E}+00$ & 543.91 & $\mathrm{~Pb}-214$ & $->$ & $\mathrm{Bi}-214$ \\
\hline $0.00 E+00$ & 538.7 & $\mathrm{~Pb}-214$ & $->$ & $\mathrm{Bi}-214$ \\
\hline $0.00 E+00$ & 533.5 & $\mathrm{~Pb}-214$ & $->$ & $\mathrm{Bi}-214$ \\
\hline $0.00 \mathrm{E}+00$ & 511 & $\mathrm{~Pb}-214$ & -> & $\mathrm{Bi}-214$ \\
\hline $0.00 \mathrm{E}+00$ & 510 & $\mathrm{Rn}-222$ & $->$ & Po-218 \\
\hline $0.00 E+00$ & 487.13 & $\mathrm{~Pb}-214$ & -> & $\mathrm{Bi}-214$ \\
\hline $0.00 \mathrm{E}+00$ & 480.32 & $\mathrm{~Pb}-214$ & $->$ & $\mathrm{Bi}-214$ \\
\hline $0.00 \mathrm{E}+00$ & 470.8 & $\mathrm{~Pb}-214$ & $->$ & $\mathrm{Bi}-214$ \\
\hline $0.00 \mathrm{E}+00$ & 462.05 & $\mathrm{~Pb}-214$ & $->$ & $\mathrm{Bi}-214$ \\
\hline $0.00 E+00$ & 449.5 & $\mathrm{Ra}-226$ & $->$ & $\mathrm{Rn}-222$ \\
\hline $0.00 \mathrm{E}+00$ & 414.72 & $\mathrm{Ra}-226$ & $->$ & $\mathrm{Rn}-222$ \\
\hline $0.00 \mathrm{E}+00$ & 351.87 & $\mathrm{~Pb}-214$ & $->$ & $\mathrm{Bi}-214$ \\
\hline $0.00 E+00$ & 324.3 & $\mathrm{~Pb}-214$ & $->$ & $\mathrm{Bi}-214$ \\
\hline $0.00 \mathrm{E}+00$ & 314.2 & $\mathrm{~Pb}-214$ & $->$ & $\mathrm{Bi}-214$ \\
\hline $0.00 \mathrm{E}+00$ & 305.5 & $\mathrm{~Pb}-214$ & $->$ & $\mathrm{Bi}-214$ \\
\hline $0.00 \mathrm{E}+00$ & 295.091 & $\mathrm{~Pb}-214$ & $->$ & $\mathrm{Bi}-214$ \\
\hline $0.00 \mathrm{E}+00$ & 274.56 & $\mathrm{~Pb}-214$ & -> & $\mathrm{Bi}-214$ \\
\hline $0.00 \mathrm{E}+00$ & 262.41 & Ra-226 & $->$ & $\mathrm{Rn}-222$ \\
\hline $0.00 \mathrm{E}+00$ & 258.94 & $\mathrm{~Pb}-214$ & -> & $\mathrm{Bi}-214$ \\
\hline $0.00 \mathrm{E}+00$ & 241.92 & $\mathrm{~Pb}-214$ & $->$ & $\mathrm{Bi}-214$ \\
\hline $0.00 \mathrm{E}+00$ & 196.3 & $\mathrm{~Pb}-214$ & $->$ & $\mathrm{Bi}-214$ \\
\hline $0.00 E+00$ & 186.11 & Ra-226 & $->$ & $\mathrm{Rn}-222$ \\
\hline $0.00 \mathrm{E}+00$ & 141.3 & $\mathrm{~Pb}-214$ & $->$ & $\mathrm{Bi}-214$ \\
\hline $0.00 \mathrm{E}+00$ & 137.4 & $\mathrm{~Pb}-214$ & $->$ & $\mathrm{Bi}-214$ \\
\hline $0.00 \mathrm{E}+00$ & 97.907 & $\mathrm{Ra}-226$ & $->$ & $\mathrm{Rn}-222$ \\
\hline $0.00 \mathrm{E}+00$ & 94.677 & $\mathrm{Ra}-226$ & $->$ & $\mathrm{Rn}-222$ \\
\hline $0.00 \mathrm{E}+00$ & 90.128 & $\mathrm{~Pb}-214$ & $->$ & $\mathrm{Bi}-214$ \\
\hline
\end{tabular}

Am-241 $100 \% \quad$ Aged 5 years

507 lines computed 
Sorted by Energy

\begin{tabular}{|c|c|c|c|c|}
\hline \multirow{2}{*}{$\begin{array}{c}\text { Energy } \\
\text { kev }\end{array}$} & \multirow{2}{*}{$\frac{\text { Intensity }}{\mathrm{ph} / \mathrm{s} / \mathrm{gm}}$} & \multicolumn{3}{|c|}{ Initial Isotopes and Decays } \\
\hline & & & & \\
\hline 8.953 & $1.54 \mathrm{E}-11$ & $\mathrm{Bi}-213$ & $->$ & TI-209 \\
\hline 9.185 & $1.27 \mathrm{E}-08$ & TI-209 & $->$ & $\mathrm{Pb}-209$ \\
\hline 9.658 & 7.82E-08 & $\mathrm{Bi}-213$ & $->$ & Po-213 \\
\hline 9.897 & $1.30 \mathrm{E}-07$ & Fr-221 & $->$ & At-217 \\
\hline 10.259 & $3.34 \mathrm{E}-10$ & $\mathrm{Bi}-213$ & $->$ & Tl-209 \\
\hline 10.381 & $1.33 \mathrm{E}-06$ & Ac-225 & $->$ & Fr-221 \\
\hline 10.541 & $2.47 \mathrm{E}-07$ & Tl-209 & $->$ & $\mathrm{Pb}-209$ \\
\hline 10.871 & $1.02 E-06$ & $\mathrm{Ra}-225$ & $->$ & Ac- 225 \\
\hline 10.994 & $4.01 \mathrm{E}-12$ & $\mathrm{Bi}-213$ & $->$ & TI-209 \\
\hline 11.118 & $3.20 \mathrm{E}-03$ & $U-233$ & $->$ & Th-229 \\
\hline 11.119 & $1.50 \mathrm{E}-06$ & $\mathrm{Bi}-213$ & $->$ & Po-213 \\
\hline 11.349 & $3.41 E-09$ & TI-209 & $->$ & $\mathrm{Pb}-209$ \\
\hline 11.372 & $2.35 E+03$ & $\mathrm{~Np}-237$ & $->$ & $\mathrm{Pa}-233$ \\
\hline 11.414 & $2.45 \mathrm{E}-06$ & Fr-221 & $->$ & At-217 \\
\hline 11.62 & $2.14 \mathrm{E}+03$ & $\mathrm{~Pa}-233$ & $->$ & U-233 \\
\hline 11.871 & $1.02 \mathrm{E}+09$ & Am-241 & $->$ & $\mathrm{Np}-237$ \\
\hline 12.017 & $2.42 \mathrm{E}-05$ & Ac- 225 & $->$ & $\mathrm{Fr}-221$ \\
\hline 12.085 & $2.03 \mathrm{E}-08$ & $\mathrm{Bi}-213$ & $->$ & Po-213 \\
\hline 12.311 & $2.68 \mathrm{E}-10$ & $\mathrm{Bi}-213$ & $->$ & TI-209 \\
\hline 12.466 & $5.17 \mathrm{E}-08$ & Fr-221 & $->$ & At-217 \\
\hline 12.636 & $1.75 \mathrm{E}-05$ & $\mathrm{Ra}-225$ & $->$ & $A c-225$ \\
\hline 12.703 & $2.34 \mathrm{E}-07$ & TI-209 & $->$ & $\mathrm{Pb}-209$ \\
\hline 12.952 & $5.55 \mathrm{E}-02$ & U-233 & $->$ & Th-229 \\
\hline 13.255 & 4.96E-07 & Ac- 225 & $->$ & Fr-221 \\
\hline 13.274 & $3.92 E+04$ & $\mathrm{~Np}-237$ & $->$ & $\mathrm{Pa}-233$ \\
\hline 13.504 & $1.39 \mathrm{E}-06$ & $\mathrm{Bi}-213$ & $->$ & Po-213 \\
\hline 13.6 & $3.60 \mathrm{E}+04$ & Pa-233 & $->$ & U-233 \\
\hline 13.911 & $2.97 \mathrm{E}-06$ & Fr-221 & $->$ & At-217 \\
\hline 13.927 & $1.64 \mathrm{E}+10$ & Am-241 & $->$ & Np-237 \\
\hline 14.082 & $2.89 E-07$ & $\mathrm{Ra}-225$ & $->$ & $A c-225$ \\
\hline 14.402 & $5.35 \mathrm{E}-11$ & $\mathrm{Bi}-213$ & $->$ & TI-209 \\
\hline 14.511 & $1.22 \mathrm{E}-03$ & U-233 & $->$ & Th-229 \\
\hline 14.772 & 2.97E-05 & $A c-225$ & $->$ & Fr-221 \\
\hline 14.871 & $4.21 \mathrm{E}-08$ & TI-209 & $->$ & $\mathrm{Pb}-209$ \\
\hline 14.953 & $9.61 \mathrm{E}+02$ & $\mathrm{~Np}-237$ & $->$ & $\mathrm{Pa}-233$ \\
\hline 15.4 & $4.60 \mathrm{E}+02$ & Pa-233 & $->$ & $U-233$ \\
\hline 15.662 & $1.94 \mathrm{E}-05$ & $\mathrm{Ra}-225$ & $->$ & Ac- 225 \\
\hline 15.861 & $4.15 E+08$ & Am-241 & $->$ & $\mathrm{Np}-237$ \\
\hline 15.878 & 2.67E-07 & $\mathrm{Bi}-213$ & $->$ & Po-213 \\
\hline 16.141 & 7.04E-02 & $U-233$ & $->$ & Th-229 \\
\hline 16.361 & $6.16 \mathrm{E}-07$ & Fr-221 & $->$ & At-217 \\
\hline 16.632 & $5.11 \mathrm{E}+04$ & $\mathrm{~Np}-237$ & $->$ & Pa-233 \\
\hline 17.058 & $3.80 E+04$ & $\mathrm{~Pa}-233$ & $->$ & $U-233$ \\
\hline
\end{tabular}




\begin{tabular}{|c|c|c|c|c|}
\hline 17.269 & $7.20 E+00$ & $\mathrm{~Pa}-233$ & $->$ & $U-233$ \\
\hline 17.349 & $5.59 E-07$ & Th-229 & $->$ & $\mathrm{Ra}-225$ \\
\hline 17.449 & $6.50 \mathrm{E}-06$ & Ac- 225 & $->$ & Fr-221 \\
\hline 17.611 & $2.54 \mathrm{E}+10$ & Am-241 & $->$ & $\mathrm{Np}-237$ \\
\hline 18.593 & $4.44 \mathrm{E}-06$ & $\mathrm{Ra}-225$ & $->$ & Ac- 225 \\
\hline 19.148 & $1.71 \mathrm{E}-02$ & U-233 & $->$ & Th-229 \\
\hline 19.718 & $1.19 \mathrm{E}+04$ & $\mathrm{~Np}-237$ & $->$ & Pa-233 \\
\hline 20.42 & $9.00 E+03$ & Pa-233 & $->$ & U-233 \\
\hline 20.997 & $6.54 \mathrm{E}+09$ & Am-241 & $->$ & $\mathrm{Np}-237$ \\
\hline 25.304 & $2.58 \mathrm{E}-05$ & U-233 & $->$ & Th-229 \\
\hline 25.373 & $1.18 \mathrm{E}-07$ & Th-229 & $->$ & $\mathrm{Ra}-225$ \\
\hline 25.98 & $4.65 E-09$ & AC- 225 & $->$ & Fr-221 \\
\hline 26.3445 & $3.02 E+09$ & Am-241 & $->$ & $\mathrm{Np}-237$ \\
\hline 28.578 & $1.30 \mathrm{E}+02$ & $\mathrm{~Pa}-233$ & $->$ & U-233 \\
\hline 29.191 & $1.45 \mathrm{E}-04$ & U-233 & $->$ & Th-229 \\
\hline 29.378 & $2.64 \mathrm{E}+04$ & $\mathrm{~Np}-237$ & $->$ & $\mathrm{Pa}-233$ \\
\hline 31.24 & $5.76 \mathrm{E}-06$ & U-233 & $->$ & Th-229 \\
\hline 31.37 & $1.35 \mathrm{E}-05$ & Th-229 & $->$ & Ra-225 \\
\hline 32.2 & $2.19 E+07$ & Am-241 & $->$ & $\mathrm{Np}-237$ \\
\hline 32.27 & $2.11 \mathrm{E}-05$ & U-233 & $->$ & Th-229 \\
\hline 33.192 & $1.51 \mathrm{E}+08$ & Am-241 & $->$ & $\mathrm{Np}-237$ \\
\hline 36.65 & $4.65 \mathrm{E}-08$ & Ac-225 & $->$ & Fr-221 \\
\hline 37.85 & 7.68E-06 & U-233 & $->$ & Th-229 \\
\hline 38.51 & $2.79 E-08$ & Ac-225 & $->$ & Fr-221 \\
\hline 40.34 & $9.20 \mathrm{E}-05$ & Ra-225 & $->$ & $A c-225$ \\
\hline 40.415 & $7.20 \mathrm{E}+01$ & $\mathrm{~Pa}-233$ & $->$ & U-233 \\
\hline 41.718 & $2.60 \mathrm{E}+01$ & $\mathrm{~Pa}-233$ & $->$ & $U-233$ \\
\hline 42.441 & $1.28 \mathrm{E}-03$ & $\mathrm{U}-233$ & $->$ & Th-229 \\
\hline 42.64 & $1.26 \mathrm{E}+06$ & Am-241 & $->$ & $\mathrm{Np}-237$ \\
\hline 42.722 & $5.26 \mathrm{E}-07$ & Th-229 & $->$ & Ra-225 \\
\hline 43.415 & $9.18 \mathrm{E}+07$ & Am-241 & $->$ & $\mathrm{Np}-237$ \\
\hline 46.57 & $2.72 E+02$ & $\mathrm{~Np}-237$ & $->$ & $\mathrm{Pa}-233$ \\
\hline 49.2 & $3.35 \mathrm{E}-08$ & Ac- 225 & $->$ & Fr-221 \\
\hline 51.013 & $3.14 \mathrm{E}+04$ & Am-241 & $->$ & $\mathrm{Np}-237$ \\
\hline 52.613 & $5.33 \mathrm{E}-06$ & U-233 & $->$ & Th-229 \\
\hline 53.559 & $9.17 \mathrm{E}-05$ & $U-233$ & $->$ & Th-229 \\
\hline 53.82 & $5.88 \mathrm{E}-08$ & Ac- 225 & $->$ & Fr-221 \\
\hline 54.04 & $7.55 \mathrm{E}+05$ & Am-241 & $->$ & $\mathrm{Np}-237$ \\
\hline 54.702 & 2.99E-04 & U-233 & $->$ & Th-229 \\
\hline 55.528 & $2.52 E+07$ & $A m-241$ & $->$ & $\mathrm{Np}-237$ \\
\hline 56.58 & $1.09 \mathrm{E}-06$ & Th-229 & $->$ & $\mathrm{Ra}-225$ \\
\hline 57.149 & 7.97E+02 & $\mathrm{Np}-237$ & $->$ & $P a-233$ \\
\hline 57.77 & $1.30 \mathrm{E}-08$ & Ac- 225 & $->$ & Fr-221 \\
\hline 59.536 & $4.52 E+10$ & Am-241 & $->$ & $\mathrm{Np}-237$ \\
\hline 62.66 & $2.45 \mathrm{E}+01$ & $\mathrm{~Np}-237$ & $->$ & $\mathrm{Pa}-233$ \\
\hline 62.93 & $1.70 \mathrm{E}-06$ & $A c-225$ & $->$ & Fr-221 \\
\hline 63.69 & 7.04E-07 & U-233 & $->$ & Th-229 \\
\hline 63.95 & $3.27 E+01$ & $\mathrm{~Np}-237$ & $->$ & Pa-233 \\
\hline 64.23 & $1.73 \mathrm{E}-07$ & Ac-225 & $->$ & Fr-221 \\
\hline 64.817 & $1.76 \mathrm{E}+05$ & Am-241 & $->$ & $\mathrm{Np}-237$ \\
\hline
\end{tabular}




\begin{tabular}{|c|c|c|c|c|}
\hline 66.11 & $1.79 E-05$ & $U-233$ & $->$ & Th-229 \\
\hline 67.46 & $5.53 E+05$ & Am-241 & $->$ & $\mathrm{Np}-237$ \\
\hline 67.96 & $6.83 \mathrm{E}-06$ & $\mathrm{U}-233$ & $->$ & Th-229 \\
\hline 68.14 & 3.29E-07 & Th-229 & $->$ & Ra-225 \\
\hline 68.88 & $3.62 \mathrm{E}-07$ & Th-229 & $->$ & Ra-225 \\
\hline 68.97 & $2.30 \mathrm{E}-06$ & U-233 & $->$ & Th-229 \\
\hline 69.76 & $3.02 E+07$ & Am-241 & $->$ & $\mathrm{Np}-237$ \\
\hline 69.85 & $1.58 \mathrm{E}-08$ & Ac-225 & $->$ & Fr-221 \\
\hline 70.35 & $1.28 \mathrm{E}-05$ & U-233 & $->$ & Th-229 \\
\hline 70.62 & $3.27 \mathrm{E}+01$ & Np-237 & $->$ & Pa-233 \\
\hline 70.832 & $5.35 \mathrm{E}-10$ & $\mathrm{Bi}-213$ & $->$ & TI-209 \\
\hline 70.92 & $3.10 \mathrm{E}-08$ & Ac-225 & $->$ & Fr-221 \\
\hline 71.71 & 4.55E-08 & Ac-225 & $->$ & Fr-221 \\
\hline 71.847 & $5.76 \mathrm{E}-05$ & U-233 & $->$ & Th-229 \\
\hline 72.803 & $4.01 \mathrm{E}-07$ & TI-209 & $->$ & $\mathrm{Pb}-209$ \\
\hline 72.821 & $1.26 \mathrm{E}-05$ & U-233 & $->$ & Th-229 \\
\hline 72.873 & 8.70E-10 & $\mathrm{Bi}-213$ & $->$ & TI-209 \\
\hline 73.6 & 5.27E-08 & Ac- 225 & $->$ & Fr-221 \\
\hline 73.84 & $1.00 \mathrm{E}-06$ & Ac- 225 & $->$ & Fr-221 \\
\hline 74.46 & $2.27 E+01$ & Np-237 & $->$ & $\mathrm{Pa}-233$ \\
\hline 74.5 & $3.20 \mathrm{E}-05$ & U-233 & $->$ & Th-229 \\
\hline 74.9 & $1.12 \mathrm{E}-07$ & Ac-225 & $->$ & Fr-221 \\
\hline 74.969 & $6.76 \mathrm{E}-07$ & TI-209 & $->$ & Pb-209 \\
\hline 75.17 & $1.64 \mathrm{E}-06$ & Th-229 & $->$ & $\mathrm{Ra}-225$ \\
\hline 75.343 & $2.34 \mathrm{E}+03$ & $\mathrm{~Pa}-233$ & $->$ & $U-233$ \\
\hline 75.83 & $1.38 \mathrm{E}+05$ & Am-241 & $->$ & $\mathrm{Np}-237$ \\
\hline 76.32 & $8.32 \mathrm{E}-06$ & U-233 & $->$ & Th-229 \\
\hline 76.858 & $2.36 \mathrm{E}-06$ & $\mathrm{Bi}-213$ & $->$ & Po-213 \\
\hline 77.1 & $1.54 \mathrm{E}-05$ & $U-233$ & $->$ & Th-229 \\
\hline 78.41 & $1.28 \mathrm{E}-06$ & $U-233$ & $->$ & Th-229 \\
\hline 78.947 & $2.63 E-06$ & Fr-221 & $->$ & At-217 \\
\hline 79.29 & $3.91 \mathrm{E}-06$ & $\mathrm{Bi}-213$ & $->$ & Po-213 \\
\hline 81.517 & 4.37E-06 & Fr-221 & $->$ & At-217 \\
\hline 82.434 & $3.34 \mathrm{E}-10$ & $\mathrm{Bi}-213$ & $->$ & TI-209 \\
\hline 82.9 & $4.65 E-07$ & Ac-225 & $->$ & Fr-221 \\
\hline 82.957 & 3.84E-06 & U-233 & $->$ & Th-229 \\
\hline 83.229 & $3.90 \mathrm{E}-06$ & $A c-225$ & $->$ & Fr-221 \\
\hline 84.27 & $1.60 \mathrm{E}-06$ & $U-233$ & $->$ & Th-229 \\
\hline 84.789 & $2.41 \mathrm{E}-07$ & TI-209 & $->$ & Pb-209 \\
\hline 85.185 & $8.70 \mathrm{E}-11$ & $\mathrm{Bi}-213$ & $->$ & TI-209 \\
\hline 85.402 & $4.05 E-06$ & U-233 & $->$ & Th-229 \\
\hline 86.105 & 6.44E-06 & Ac- 225 & $->$ & Fr-221 \\
\hline 86.23 & $1.25 \mathrm{E}-06$ & Th-229 & $->$ & Ra-225 \\
\hline 86.44 & 9.87E-06 & Th-229 & $->$ & $\mathrm{Ra}-225$ \\
\hline 86.5 & $2.86 \mathrm{E}+04$ & $\mathrm{~Np}-237$ & $->$ & $\mathrm{Pa}-233$ \\
\hline 86.652 & $3.52 E+03$ & $\mathrm{~Pa}-233$ & $->$ & $U-233$ \\
\hline 86.74 & 2.77E-06 & U-233 & $->$ & Th-229 \\
\hline 87.24 & 4.05E-06 & U-233 & $->$ & Th-229 \\
\hline 87.39 & 8.98E-07 & Ac-225 & $->$ & Fr-221 \\
\hline 87.632 & 6.89E-08 & TI-209 & $->$ & $\mathrm{Pb}-209$ \\
\hline
\end{tabular}




\begin{tabular}{|c|c|c|c|c|}
\hline 88.05 & $3.68 \mathrm{E}+02$ & $\mathrm{~Np}-237$ & $->$ & $\mathrm{Pa}-233$ \\
\hline 88.43 & $9.39 \mathrm{E}-06$ & U-233 & $->$ & Th-229 \\
\hline 89.639 & $1.39 \mathrm{E}-06$ & $\mathrm{Bi}-213$ & $->$ & Po-213 \\
\hline 89.957 & $2.22 \mathrm{E}-04$ & $U-233$ & $->$ & Th-229 \\
\hline 90.994 & $7.04 \mathrm{E}-06$ & $U-233$ & $->$ & Th-229 \\
\hline 92.136 & $1.55 \mathrm{E}-06$ & Fr-221 & $->$ & At-217 \\
\hline 92.282 & $3.25 E+03$ & $\mathrm{~Np}-237$ & $->$ & $\mathrm{Pa}-233$ \\
\hline 92.673 & $4.24 \mathrm{E}-07$ & $\mathrm{Bi}-213$ & $->$ & Po-213 \\
\hline 93.36 & $3.61 \mathrm{E}-04$ & $U-233$ & $->$ & Th-229 \\
\hline 94.66 & $2.04 \mathrm{E}+04$ & $\mathrm{~Pa}-233$ & $->$ & $U-233$ \\
\hline 94.723 & $1.55 E+03$ & $\mathrm{~Np}-237$ & $->$ & $\mathrm{Pa}-233$ \\
\hline 94.87 & $4.96 \mathrm{E}-07$ & Ac- 225 & $->$ & Fr-221 \\
\hline 95.265 & $4.80 E-07$ & Fr-221 & $->$ & At-217 \\
\hline 95.858 & $5.93 E+03$ & $\mathrm{~Np}-237$ & $->$ & $\mathrm{Pa}-233$ \\
\hline 96.17 & $9.29 \mathrm{E}-08$ & Ac- 225 & $->$ & Fr-221 \\
\hline 96.215 & $2.99 \mathrm{E}-05$ & U-233 & $->$ & Th-229 \\
\hline 96.67 & $5.91 E+04$ & Am-241 & $->$ & $\mathrm{Np}-237$ \\
\hline 97.071 & $1.48 \mathrm{E}+06$ & Am-241 & $->$ & $\mathrm{Np}-237$ \\
\hline 97.143 & 4.69E-04 & U-233 & $->$ & Th-229 \\
\hline 97.2 & $5.88 \mathrm{E}-08$ & Fr-221 & $->$ & At-217 \\
\hline 97.272 & $2.32 \mathrm{E}-06$ & Ac- 225 & $->$ & Fr-221 \\
\hline 98.443 & $3.20 E+04$ & $\mathrm{~Pa}-233$ & $->$ & $U-233$ \\
\hline 98.951 & $2.55 E+07$ & Am-241 & $->$ & $\mathrm{Np}-237$ \\
\hline 99.58 & $1.95 \mathrm{E}-06$ & Ac-225 & $->$ & Fr-221 \\
\hline 99.82 & $5.20 \mathrm{E}-06$ & $A c-225$ & $->$ & Fr-221 \\
\hline 100.008 & 1.17E-06 & U-233 & $->$ & Th-229 \\
\hline 100.599 & $7.43 E-07$ & Ac- 225 & $->$ & Fr-221 \\
\hline 100.88 & $1.86 \mathrm{E}-07$ & Ac-225 & $->$ & Fr-221 \\
\hline 101.066 & $2.48 \mathrm{E}+06$ & Am-241 & $->$ & Np-237 \\
\hline 101.8 & $1.92 \mathrm{E}-06$ & U-233 & $->$ & Th-229 \\
\hline 102.966 & $2.45 E+07$ & $A m-241$ & $->$ & Np-237 \\
\hline 103.375 & $2.13 \mathrm{E}-06$ & $\mathrm{U}-233$ & $->$ & Th-229 \\
\hline 103.44 & $2.79 E-08$ & Ac- 225 & $->$ & Fr-221 \\
\hline 103.921 & $1.38 \mathrm{E}+03$ & $\mathrm{~Pa}-233$ & $->$ & $U-233$ \\
\hline 105.362 & $1.30 \mathrm{E}-04$ & U-233 & $->$ & Th-229 \\
\hline 106.13 & $1.14 \mathrm{E}+02$ & $\mathrm{~Np}-237$ & $->$ & $\mathrm{Pa}-233$ \\
\hline 107.16 & $2.63 \mathrm{E}-06$ & Th-229 & $->$ & Ra-225 \\
\hline 108.166 & $1.92 \mathrm{E}+03$ & $\mathrm{~Np}-237$ & $->$ & $\mathrm{Pa}-233$ \\
\hline 108.36 & $8.36 \mathrm{E}-07$ & $A c-225$ & $->$ & Fr-221 \\
\hline 108.69 & $1.49 \mathrm{E}+02$ & $\mathrm{~Np}-237$ & $->$ & $\mathrm{Pa}-233$ \\
\hline 108.99 & $4.33 \mathrm{E}-05$ & U-233 & $->$ & Th-229 \\
\hline 109.47 & $6.61 \mathrm{E}-06$ & $U-233$ & $->$ & Th-229 \\
\hline 111.298 & $1.20 \mathrm{E}+04$ & $\mathrm{~Pa}-233$ & $->$ & $U-233$ \\
\hline 111.56 & $1.01 \mathrm{E}-06$ & Ac- 225 & $->$ & Fr-221 \\
\hline 111.897 & $6.34 \mathrm{E}+02$ & $\mathrm{~Np}-237$ & $->$ & $\mathrm{Pa}-233$ \\
\hline 111.935 & $1.00 \mathrm{E}-05$ & U-233 & $->$ & Th-229 \\
\hline 113.3 & $2.98 \mathrm{E}+05$ & Am-241 & $->$ & $\mathrm{Np}-237$ \\
\hline 114.23 & $5.94 \mathrm{E}+05$ & Am-241 & $->$ & $\mathrm{Np}-237$ \\
\hline 114.28 & $5.33 \mathrm{E}-06$ & U-233 & $->$ & Th-229 \\
\hline 114.445 & $4.00 E+03$ & $\mathrm{~Pa}-233$ & $->$ & $U-233$ \\
\hline
\end{tabular}




\begin{tabular}{|c|c|c|c|c|}
\hline 115.19 & $5.11 \mathrm{E}+00$ & Np-237 & $->$ & $\mathrm{Pa}-233$ \\
\hline 116.26 & $4.48 \mathrm{E}-06$ & U-233 & $->$ & Th-229 \\
\hline 117 & $5.42 \mathrm{E}-06$ & TI-209 & $->$ & $\mathrm{Pb}-209$ \\
\hline 117.155 & 5.97E-05 & $U-233$ & $->$ & Th-229 \\
\hline 117.58 & $2.89 \mathrm{E}+05$ & Am-241 & $->$ & $\mathrm{Np}-237$ \\
\hline 117.689 & $3.29 E+02$ & $\mathrm{~Np}-237$ & $->$ & $\mathrm{Pa}-233$ \\
\hline 118.47 & $1.08 \mathrm{E}-07$ & Fr-221 & $->$ & At-217 \\
\hline 118.97 & $6.83 \mathrm{E}-05$ & $U-233$ & $->$ & Th-229 \\
\hline 119.92 & $1.86 \mathrm{E}-07$ & Ac- 225 & $->$ & Fr-221 \\
\hline 120.81 & $4.48 \mathrm{E}-05$ & U-233 & $->$ & Th-229 \\
\hline 122.994 & $1.26 \mathrm{E}+06$ & Am-241 & $->$ & $\mathrm{Np}-237$ \\
\hline 123.76 & $5.88 \mathrm{E}-07$ & Ac-225 & $->$ & Fr-221 \\
\hline 123.91 & $1.39 \mathrm{E}-05$ & U-233 & $->$ & Th-229 \\
\hline 124.51 & 3.29E-06 & Th-229 & $->$ & $\mathrm{Ra}-225$ \\
\hline 124.72 & 1.97E-06 & Th-229 & $->$ & $\mathrm{Ra}-225$ \\
\hline 124.81 & $1.55 \mathrm{E}-07$ & Ac-225 & $->$ & Fr-221 \\
\hline 125.292 & $5.13 E+06$ & Am-241 & $->$ & $\mathrm{Np}-237$ \\
\hline 125.398 & $1.39 \mathrm{E}-06$ & $U-233$ & $->$ & Th-229 \\
\hline 126.23 & 4.34E-08 & Ac- 225 & $->$ & Fr-221 \\
\hline 129.19 & $1.33 \mathrm{E}-08$ & Ac-225 & $->$ & Fr-221 \\
\hline 129.2 & $1.49 E-06$ & U-233 & $->$ & Th-229 \\
\hline 131.09 & $1.74 \mathrm{E}+02$ & $\mathrm{~Np}-237$ & $->$ & $\mathrm{Pa}-233$ \\
\hline 131.2 & 7.04E-07 & U-233 & $->$ & Th-229 \\
\hline 131.92 & $1.09 \mathrm{E}-06$ & Th-229 & $->$ & $\mathrm{Ra}-225$ \\
\hline 134.23 & $1.37 \mathrm{E}+02$ & Np-237 & $->$ & $\mathrm{Pa}-233$ \\
\hline 135.01 & $1.24 \mathrm{E}-07$ & $A c-225$ & $->$ & Fr-221 \\
\hline 135.328 & $4.69 E-05$ & U-233 & $->$ & Th-229 \\
\hline 137.02 & $5.26 \mathrm{E}-06$ & Th-229 & $->$ & $\mathrm{Ra}-225$ \\
\hline 138.2 & $6.19 \mathrm{E}-08$ & $A C-225$ & $->$ & Fr-221 \\
\hline 138.5 & 4.27E-07 & U-233 & $->$ & Th-229 \\
\hline 139.53 & $6.72 E+03$ & Am-241 & $->$ & $\mathrm{Np}-237$ \\
\hline 139.76 & $2.24 \mathrm{E}-06$ & U-233 & $->$ & Th-229 \\
\hline 140.61 & $3.68 \mathrm{E}+01$ & $\mathrm{~Np}-237$ & $->$ & $\mathrm{Pa}-233$ \\
\hline 142.98 & $1.41 \mathrm{E}-06$ & Th-229 & $->$ & $\mathrm{Ra}-225$ \\
\hline 143.227 & $7.97 E+02$ & $\mathrm{~Np}-237$ & $->$ & $\mathrm{Pa}-233$ \\
\hline 144.52 & $6.40 \mathrm{E}-06$ & U-233 & $->$ & Th-229 \\
\hline 145.15 & $3.90 E-07$ & $A c-225$ & $->$ & Fr-221 \\
\hline 145.286 & $3.41 \mathrm{E}-05$ & U-233 & $->$ & Th-229 \\
\hline 146.347 & $1.34 \mathrm{E}-04$ & $U-233$ & $->$ & Th-229 \\
\hline 146.557 & $5.79 \mathrm{E}+05$ & Am-241 & $->$ & Np-237 \\
\hline 148.15 & 7.68E-06 & U-233 & $->$ & Th-229 \\
\hline 148.37 & $3.29 E-06$ & Th-229 & $->$ & $\mathrm{Ra}-225$ \\
\hline 149.8 & $2.71 \mathrm{E}-06$ & U-233 & $->$ & Th-229 \\
\hline 149.99 & 2.17E-07 & Fr-221 & $->$ & At-217 \\
\hline 150.08 & $2.08 \mathrm{E}-06$ & Ac- 225 & $->$ & Fr-221 \\
\hline 150.11 & $1.01 \mathrm{E}+05$ & Am-241 & $->$ & $\mathrm{Np}-237$ \\
\hline 151.423 & $4.91 E+02$ & $\mathrm{~Np}-237$ & $->$ & $\mathrm{Pa}-233$ \\
\hline 152.64 & $1.15 \mathrm{E}-07$ & $A c-225$ & $->$ & Fr-221 \\
\hline 153.31 & $1.17 \mathrm{E}-06$ & U-233 & $->$ & Th-22 \\
\hline 153.72 & $1.41 \mathrm{E}+01$ & $\mathrm{~Np}-237$ & $->$ & $\mathrm{Pa}-23$ \\
\hline
\end{tabular}




$\begin{array}{ccrll}153.93 & 4.74 \mathrm{E}-07 & \mathrm{Ac}-225 & -> & \mathrm{Fr}-221 \\ 154.37 & 2.30 \mathrm{E}-06 & \mathrm{Th}-229 & -> & \mathrm{Ra}-225 \\ 154.4 & 1.01 \mathrm{E}+04 & \mathrm{Am}-241 & -> & \mathrm{Np}-237 \\ 154.69 & 3.20 \mathrm{E}-06 & \mathrm{U}-233 & -> & \mathrm{Th}-229 \\ 155.263 & 1.88 \mathrm{E}+02 & \mathrm{~Np}-237 & -> & \mathrm{Pa}-233 \\ 155.989 & 1.24 \mathrm{E}-06 & \mathrm{U}-233 & -> & \mathrm{Th}-229 \\ 156.4 & 1.51 \mathrm{E}+04 & \mathrm{Am}-241 & -> & \mathrm{Np}-237 \\ 156.46 & 3.29 \mathrm{E}-06 & \mathrm{Th}-229 & -> & \mathrm{Ra}-225 \\ 157.24 & 9.60 \mathrm{E}-07 & \mathrm{Ac}-225 & -> & \mathrm{Fr}-221 \\ 158.48 & 1.76 \mathrm{E}+03 & \mathrm{Am}-241 & -> & \mathrm{Np}-237 \\ 161.7 & 1.13 \mathrm{E}+04 & \mathrm{Am}-241 & -> & \mathrm{Np}-237 \\ 162.51 & 7.56 \mathrm{E}+01 & \mathrm{~Np}-237 & -> & \mathrm{Pa}-233 \\ 162.52 & 1.60 \mathrm{E}-06 & \mathrm{U}-233 & -> & \mathrm{Th}-229 \\ 164.512 & 1.41 \mathrm{E}-04 & \mathrm{U}-233 & -> & \mathrm{Th}-229 \\ 164.58 & 8.39 \mathrm{E}+04 & \mathrm{Am}-241 & -> & \mathrm{Np}-237 \\ 165.493 & 8.32 \mathrm{E}-06 & \mathrm{U}-233 & -> & \mathrm{Th}-229 \\ 165.92 & 3.02 \mathrm{E}+04 & \mathrm{Am}-241 & -> & \mathrm{Np}-237 \\ 168.98 & 1.43 \mathrm{E}-06 & \mathrm{U}-233 & -> & \mathrm{Th}-229 \\ 169.18 & 1.47 \mathrm{E}+02 & \mathrm{~Np}-237 & -> & \mathrm{Pa}-233 \\ 169.56 & 2.11 \mathrm{E}+05 & \mathrm{Am}-241 & -> & \mathrm{Np}-237 \\ 170.67 & 3.88 \mathrm{E}+01 & \mathrm{~Np}-237 & -> & \mathrm{Pa}-233 \\ 170.794 & 2.99 \mathrm{E}-06 & \mathrm{U}-233 & -> & \mathrm{Th}-229 \\ 170.91 & 2.79 \mathrm{E}-08 & \mathrm{Ac}-225 & -> & \mathrm{Fr}-221 \\ 171.29 & 2.17 \mathrm{E}-07 & \mathrm{Fr}-221 & -> & \mathrm{At}-217 \\ 172.35 & 7.47 \mathrm{E}-07 & \mathrm{U}-233 & -> & \mathrm{Th}-229 \\ 172.56 & 1.39 \mathrm{E}+01 & \mathrm{~Np}-237 & -> & \mathrm{Pa}-233 \\ 172.9 & 7.24 \mathrm{E}-07 & \mathrm{Th}-229 & -> & \mathrm{Ra}-225 \\ 174.162 & 4.91 \mathrm{E}-06 & \mathrm{U}-233 & -> & \mathrm{Th}-229 \\ 175.09 & 2.26 \mathrm{E}+04 & \mathrm{Am}-241 & -> & \mathrm{Np}-237 \\ 176.09 & 4.09 \mathrm{E}+01 & \mathrm{~Np}-237 & -> & \mathrm{Pa}-233 \\ 176.1 & 9.17 \mathrm{E}-07 & \mathrm{U}-233 & -> & \mathrm{Th}-229 \\ 177.78 & 4.27 \mathrm{E}-07 & \mathrm{U}-233 & -> & \mathrm{Th}-229 \\ 178.39 & 4.96 \mathrm{E}-08 & \mathrm{Ac}-225 & -> & \mathrm{Fr}-221 \\ 193.29 & 1.68 \mathrm{E}-06 & \mathrm{Th}-229 & -> & \mathrm{Ra}-225 \\ 193.59 & 1.51 \mathrm{E}-05 & \mathrm{Th}-229 & -> & \mathrm{Ra}-225 \\ 194.74 & 1.00 \mathrm{E}+02 & \mathrm{~Np}-237 & -> & \mathrm{Pa}-233 \\ 195.04 & 4.11 \mathrm{E}+02 & \mathrm{~Np}-237 & -> & \mathrm{Pa}-233\end{array}$




\begin{tabular}{|c|c|c|c|c|}
\hline 195.75 & $4.49 E-07$ & $A c-225$ & $->$ & Fr-221 \\
\hline 196.89 & $5.11 \mathrm{E}+01$ & $\mathrm{~Np}-237$ & $->$ & $\mathrm{Pa}-233$ \\
\hline 197 & $1.64 \mathrm{E}+04$ & Am-241 & $->$ & $N p-237$ \\
\hline 198.72 & $6.19 \mathrm{E}-08$ & $A C-225$ & $->$ & Fr-221 \\
\hline 200.17 & $7.15 E+00$ & $\mathrm{~Np}-237$ & $->$ & Pa-233 \\
\hline 200.67 & $2.13 \mathrm{E}-08$ & U-233 & $->$ & Th-229 \\
\hline 201.72 & $8.58 \mathrm{E}+01$ & Np-237 & $->$ & $\mathrm{Pa}-233$ \\
\hline 202.85 & $7.36 \mathrm{E}+00$ & $\mathrm{~Np}-237$ & $->$ & $\mathrm{Pa}-233$ \\
\hline 203.9 & $3.65 E+03$ & Am-241 & $->$ & $\mathrm{Np}-237$ \\
\hline 205.99 & $1.39 \mathrm{E}-06$ & U-233 & $->$ & Th-229 \\
\hline 208 & $9.95 \mathrm{E}+05$ & Am-241 & $->$ & $\mathrm{Np}-237$ \\
\hline 208.149 & 5.33E-05 & U-233 & $->$ & Th-229 \\
\hline 209.19 & $3.17 \mathrm{E}+01$ & $\mathrm{~Np}-237$ & $->$ & Pa-233 \\
\hline 210.94 & $1.09 \mathrm{E}-05$ & Th-229 & $->$ & Ra-225 \\
\hline 212.29 & $2.99 \mathrm{E}-06$ & U-233 & $->$ & Th-229 \\
\hline 212.412 & $3.11 \mathrm{E}+02$ & $\mathrm{~Np}-237$ & $->$ & $\mathrm{Pa}-233$ \\
\hline 214.08 & $8.58 \mathrm{E}+01$ & $\mathrm{~Np}-237$ & $->$ & $\mathrm{Pa}-233$ \\
\hline 216.08 & $1.41 \mathrm{E}-05$ & U-233 & $->$ & Th-229 \\
\hline 216.2 & 1.05E-06 & $A c-225$ & $->$ & Fr-221 \\
\hline 216.86 & $1.30 \mathrm{E}-06$ & Ac- 225 & $->$ & Fr-221 \\
\hline 217.133 & 7.47E-05 & U-233 & $->$ & Th-229 \\
\hline 217.61 & 1.07E-06 & U-233 & $->$ & Th-229 \\
\hline 217.98 & $3.38 \mathrm{E}-05$ & Fr-221 & $->$ & At-217 \\
\hline 218.15 & $4.60 \mathrm{E}-07$ & Th-229 & $->$ & Ra-225 \\
\hline 219.424 & $3.20 \mathrm{E}-06$ & U-233 & $->$ & Th-229 \\
\hline 221.45 & $5.28 \mathrm{E}+04$ & Am-241 & $->$ & $\mathrm{Np}-237$ \\
\hline 223.357 & 7.04E-07 & U-233 & $->$ & Th-229 \\
\hline 224.64 & $2.48 \mathrm{E}-07$ & Ac- 225 & $->$ & Fr-221 \\
\hline 225 & $2.13 \mathrm{E}-07$ & U-233 & $->$ & Th-229 \\
\hline 226.74 & $2.13 \mathrm{E}-07$ & $U-233$ & $->$ & Th-229 \\
\hline 228.07 & $4.27 \mathrm{E}-07$ & U-233 & $->$ & Th-229 \\
\hline 229.98 & $2.68 \mathrm{E}+01$ & $\mathrm{~Np}-237$ & $->$ & $\mathrm{Pa}-233$ \\
\hline 230.086 & $1.43 E-06$ & $U-233$ & $->$ & Th-229 \\
\hline 232.86 & $5.79 E+03$ & Am-241 & $->$ & $\mathrm{Np}-237$ \\
\hline 234.352 & $8.80 E+02$ & Am-241 & $->$ & $\mathrm{Np}-237$ \\
\hline 236.32 & $1.18 \mathrm{E}-07$ & Th-229 & $->$ & Ra-225 \\
\hline 236.39 & $1.07 E-06$ & U-233 & $->$ & Th-229 \\
\hline 237.95 & $1.31 \mathrm{E}+02$ & $\mathrm{~Np}-237$ & $->$ & $\mathrm{Pa}-233$ \\
\hline 240.364 & $8.11 \mathrm{E}-06$ & U-233 & $->$ & Th-229 \\
\hline 240.76 & $4.03 E-08$ & Ac- 225 & $->$ & Fr-221 \\
\hline 242.4 & $3.77 E+04$ & Am-241 & $->$ & $\mathrm{Np}-237$ \\
\hline 245 & $3.77 E+04$ & Am-241 & $->$ & $N p-237$ \\
\hline 245.294 & 7.47E-05 & U-233 & $->$ & Th-229 \\
\hline 246.66 & $3.02 E+03$ & Am-241 & $->$ & Np-237 \\
\hline 248.25 & $1.08 \mathrm{E}+02$ & $\mathrm{~Pa}-233$ & $->$ & U-233 \\
\hline 248.661 & $9.60 \mathrm{E}-05$ & $U-233$ & $->$ & Th-229 \\
\hline 248.7 & $6.19 E-08$ & Ac- 225 & $->$ & Fr-221 \\
\hline 248.91 & $9.81 \mathrm{E}+00$ & $\mathrm{~Np}-237$ & $->$ & Pa-233 \\
\hline 249.1 & $5.03 E+03$ & Am-241 & $->$ & $\mathrm{Np}-237$ \\
\hline 252.5 & $8.11 \mathrm{E}-07$ & U-233 & $->$ & Th-229 \\
\hline
\end{tabular}




\begin{tabular}{|c|c|c|c|c|}
\hline 253.51 & $3.10 \mathrm{E}-07$ & $A c-225$ & $->$ & Fr-221 \\
\hline 255.92 & $9.17 \mathrm{E}-07$ & U-233 & $->$ & Th-229 \\
\hline 256.98 & $1.37 \mathrm{E}+01$ & $\mathrm{~Np}-237$ & $->$ & Pa-233 \\
\hline 258.46 & $1.08 \mathrm{E}+01$ & Рa-233 & $->$ & U-233 \\
\hline 259.3 & $3.84 \mathrm{E}-06$ & U-233 & $->$ & Th-229 \\
\hline 260.4 & $2.30 \mathrm{E}-06$ & $U-233$ & $->$ & Th-229 \\
\hline 260.9 & $1.26 \mathrm{E}+03$ & Am-241 & $->$ & Np-237 \\
\hline 261.88 & $6.61 \mathrm{E}-06$ & $U-233$ & $->$ & Th-229 \\
\hline 262.41 & $1.37 \mathrm{E}+01$ & $\mathrm{~Np}-237$ & $->$ & Pa-233 \\
\hline 264.87 & $1.13 E+04$ & Am-241 & $->$ & $\mathrm{Np}-237$ \\
\hline 267.54 & $3.31 E+04$ & Am-241 & $->$ & $\mathrm{Np}-237$ \\
\hline 268.619 & $5.33 \mathrm{E}-06$ & U-233 & $->$ & Th-229 \\
\hline 271.58 & $8.07 E+02$ & Am-241 & $->$ & $N p-237$ \\
\hline 271.597 & $5.60 \mathrm{E}+02$ & $\mathrm{~Pa}-233$ & $->$ & U-233 \\
\hline 272.27 & $1.34 \mathrm{E}-06$ & U-233 & $->$ & Th-229 \\
\hline 274.692 & $1.00 \mathrm{E}-05$ & $U-233$ & $->$ & Th-229 \\
\hline 275.68 & $8.30 E+03$ & Am-241 & $->$ & $\mathrm{Np}-237$ \\
\hline 278.059 & $2.60 \mathrm{E}-05$ & $U-233$ & $->$ & Th-229 \\
\hline 279.27 & $1.08 \mathrm{E}-07$ & $A c-225$ & $->$ & Fr-221 \\
\hline 282.54 & 2.79E-08 & Fr-221 & $->$ & At-217 \\
\hline 284.22 & $2.13 \mathrm{E}-07$ & $U-233$ & $->$ & Th-229 \\
\hline 285.5 & $3.10 \mathrm{E}-08$ & Ac- 225 & $->$ & Fr-221 \\
\hline 287.949 & $2.30 \mathrm{E}-05$ & U-233 & $->$ & Th-229 \\
\hline 291.21 & $3.90 E+03$ & Am-241 & $->$ & $\mathrm{Np}-237$ \\
\hline 291.317 & $1.11 \mathrm{E}-04$ & U-233 & $->$ & Th-229 \\
\hline 292.76 & $1.74 \mathrm{E}+04$ & Am-241 & $->$ & $\mathrm{Np}-237$ \\
\hline 292.79 & $1.33 \mathrm{E}-06$ & $\mathrm{Bi}-213$ & $->$ & Po-213 \\
\hline 293.923 & 2.99E-06 & $U-233$ & $->$ & Th-229 \\
\hline 294.9 & $6.92 E+04$ & Am-241 & $->$ & $\mathrm{Np}-237$ \\
\hline 295.2 & $4.91 \mathrm{E}-07$ & U-233 & $->$ & Th-229 \\
\hline 298.87 & $5.06 \mathrm{E}+01$ & $\mathrm{~Pa}-233$ & $->$ & U-233 \\
\hline 300.175 & $1.24 \mathrm{E}+04$ & $\mathrm{~Pa}-233$ & $->$ & $U-233$ \\
\hline 302.84 & 1.49E-06 & $U-233$ & $->$ & Th-229 \\
\hline 304.2 & $1.26 \mathrm{E}+03$ & Am-241 & $->$ & $\mathrm{Np}-237$ \\
\hline 309.37 & $1.56 \mathrm{E}-06$ & U-233 & $->$ & Th-229 \\
\hline 310.3 & $1.89 E+04$ & Am-241 & $->$ & Np-237 \\
\hline 311.38 & 5.97E-07 & U-233 & $->$ & Th-229 \\
\hline 311.9 & $7.60 E+04$ & $\mathrm{~Pa}-233$ & $->$ & $U-233$ \\
\hline 317.133 & $1.88 \mathrm{E}-04$ & U-233 & $->$ & Th-229 \\
\hline 320.508 & $6.61 \mathrm{E}-05$ & $\mathrm{U}-233$ & $->$ & Th-229 \\
\hline 322.54 & $1.91 \mathrm{E}+05$ & Am-241 & $->$ & $\mathrm{Np}-237$ \\
\hline 323.321 & $1.79 \mathrm{E}-05$ & U-233 & $->$ & Th-229 \\
\hline 323.81 & $1.24 \mathrm{E}-08$ & $\mathrm{Bi}-213$ & $->$ & TI-209 \\
\hline 324.1 & $5.27 \mathrm{E}-08$ & Fr-221 & $->$ & At-217 \\
\hline 328.69 & $1.39 E-06$ & $U-233$ & $->$ & Th-229 \\
\hline 332.39 & $1.86 \mathrm{E}+05$ & Am-241 & $->$ & $\mathrm{Np}-237$ \\
\hline 335.43 & $6.21 \mathrm{E}+05$ & Am-241 & $->$ & $\mathrm{Np}-237$ \\
\hline 336.579 & $1.26 \mathrm{E}-05$ & U-233 & $->$ & Th-229 \\
\hline 337.727 & $5.28 \mathrm{E}+03$ & Am-241 & $->$ & $\mathrm{Np}-237$ \\
\hline 339.64 & $1.60 \mathrm{E}-07$ & U-233 & $->$ & Th-229 \\
\hline
\end{tabular}




\begin{tabular}{|c|c|c|c|c|}
\hline 340.59 & $8.40 \mathrm{E}+03$ & $\mathrm{~Pa}-233$ & $->$ & $U-233$ \\
\hline 349.46 & $1.51 \mathrm{E}+03$ & Am-241 & $->$ & $\mathrm{Np}-237$ \\
\hline 351.782 & $9.60 \mathrm{E}-07$ & U-233 & $->$ & Th-229 \\
\hline 354 & $1.24 \mathrm{E}-06$ & U-233 & $->$ & Th-229 \\
\hline 358.36 & $1.51 \mathrm{E}+03$ & Am-241 & $->$ & $\mathrm{Np}-237$ \\
\hline 359.09 & $1.08 \mathrm{E}-07$ & Fr-221 & $->$ & At-217 \\
\hline 365.762 & $1.75 \mathrm{E}-05$ & U-233 & $->$ & Th-229 \\
\hline 368.61 & $2.73 E+05$ & Am-241 & $->$ & $\mathrm{Np}-237$ \\
\hline 370.933 & $6.60 \mathrm{E}+04$ & Am-241 & $->$ & $\mathrm{Np}-237$ \\
\hline 375.518 & $1.16 \mathrm{E}+03$ & $\mathrm{~Pa}-233$ & $->$ & U-233 \\
\hline 376.6 & 1.77E+05 & Am-241 & $->$ & $\mathrm{Np}-237$ \\
\hline 382.05 & $1.08 \mathrm{E}-07$ & Fr-221 & $->$ & At-217 \\
\hline 383.4 & $2.03 \mathrm{E}-06$ & $U-233$ & $->$ & Th-229 \\
\hline 383.744 & $3.55 \mathrm{E}+04$ & Am-241 & $->$ & $\mathrm{Np}-237$ \\
\hline 384 & 4.27E-07 & $U-233$ & $->$ & Th-229 \\
\hline 390.6 & $7.42 E+03$ & Am-241 & $->$ & $\mathrm{Np}-237$ \\
\hline 394.34 & $1.60 \mathrm{E}-07$ & $\mathrm{U}-233$ & $->$ & Th-229 \\
\hline 396.65 & 1.77E-07 & $\mathrm{U}-233$ & $->$ & Th-229 \\
\hline 398.663 & $2.38 \mathrm{E}+03$ & $\mathrm{~Pa}-233$ & $->$ & U-233 \\
\hline 402.37 & 1.77E-07 & U-233 & $->$ & Th-229 \\
\hline 406.4 & $1.76 \mathrm{E}+03$ & Am-241 & $->$ & $\mathrm{Np}-237$ \\
\hline 406.7 & $1.13 \mathrm{E}-07$ & U-233 & $->$ & Th-229 \\
\hline 409.1 & $4.03 E-07$ & Fr-221 & $->$ & At-217 \\
\hline 415.932 & $3.02 E+03$ & $\mathrm{~Pa}-233$ & $->$ & $U-233$ \\
\hline 416.4 & $2.13 \mathrm{E}-07$ & U-233 & $->$ & Th-229 \\
\hline 419.22 & $3.52 E+04$ & Am-241 & $->$ & $\mathrm{Np}-237$ \\
\hline 426.39 & $3.09 E+04$ & Am-241 & $->$ & $\mathrm{Np}-237$ \\
\hline 429.8 & $1.26 \mathrm{E}+03$ & Am-241 & $->$ & $\mathrm{Np}-237$ \\
\hline 435.3 & $2.52 E+03$ & Am-241 & $->$ & $\mathrm{Np}-237$ \\
\hline 436.78 & 1.07E-07 & $U-233$ & $->$ & Th-229 \\
\hline 440.34 & $5.00 \mathrm{E}-05$ & $\mathrm{Bi}-213$ & $->$ & Po-213 \\
\hline 442.75 & $4.40 E+03$ & Am-241 & $->$ & $\mathrm{Np}-237$ \\
\hline 449.6 & $2.13 \mathrm{E}-07$ & U-233 & $->$ & Th-229 \\
\hline 452.41 & $3.02 E+03$ & Am-241 & $->$ & $\mathrm{Np}-237$ \\
\hline 452.43 & $3.41 \mathrm{E}-07$ & Ac- 225 & $->$ & Fr-221 \\
\hline 454.67 & $1.22 \mathrm{E}+04$ & Am-241 & $->$ & $\mathrm{Np}-237$ \\
\hline 459.58 & $4.53 E+03$ & Am-241 & $->$ & $\mathrm{Np}-237$ \\
\hline 459.77 & $1.77 \mathrm{E}-07$ & U-233 & $->$ & Th-229 \\
\hline 467 & $5.42 \mathrm{E}-06$ & TI-209 & $->$ & Pb-209 \\
\hline 468 & $3.65 E+03$ & Am-241 & $->$ & $\mathrm{Np}-237$ \\
\hline 471.3 & $3.20 \mathrm{E}-07$ & U-233 & $->$ & Th-229 \\
\hline 479 & $3.20 \mathrm{E}-07$ & U-233 & $->$ & Th-229 \\
\hline 480.5 & $9.29 E-08$ & Ac- 225 & $->$ & Fr-221 \\
\hline 484.05 & $9.17 \mathrm{E}-08$ & $\mathrm{U}-233$ & $->$ & Th-229 \\
\hline 486.3 & $1.26 \mathrm{E}+03$ & Am-241 & $->$ & $\mathrm{Np}-237$ \\
\hline 512 & $1.45 \mathrm{E}+03$ & Am-241 & $->$ & $\mathrm{Np}-237$ \\
\hline 513.5 & $3.10 \mathrm{E}-08$ & Ac-225 & $->$ & Fr-221 \\
\hline 514.2 & $3.27 E+03$ & Am-241 & $->$ & $\mathrm{Np}-237$ \\
\hline 522 & $1.13 \mathrm{E}+03$ & Am-241 & $->$ & $\mathrm{Np}-237$ \\
\hline 526.03 & $3.10 \mathrm{E}-08$ & Ac- 225 & $->$ & Fr-221 \\
\hline
\end{tabular}




\begin{tabular}{|c|c|c|c|c|}
\hline 540.27 & $1.13 \mathrm{E}-07$ & $U-233$ & $->$ & Th-229 \\
\hline 545.1 & $5.12 \mathrm{E}-08$ & $U-233$ & $->$ & Th-229 \\
\hline 569.37 & $8.11 \mathrm{E}-08$ & U-233 & $->$ & Th-229 \\
\hline 574.05 & $1.26 \mathrm{E}+03$ & Am-241 & $->$ & $\mathrm{Np}-237$ \\
\hline 578.47 & $1.07 \mathrm{E}-07$ & $\mathrm{U}-233$ & $->$ & Th-229 \\
\hline 582.6 & $2.52 E+02$ & Am-241 & $->$ & $\mathrm{Np}-237$ \\
\hline 586.5 & $1.64 \mathrm{E}+03$ & Am-241 & $->$ & $\mathrm{Np}-237$ \\
\hline 590.3 & $3.52 E+03$ & Am-241 & $->$ & $\mathrm{Np}-237$ \\
\hline 597.41 & $1.18 \mathrm{E}+04$ & Am-241 & $->$ & $\mathrm{Np}-237$ \\
\hline 619.011 & $7.42 E+04$ & Am-241 & $->$ & $\mathrm{Np}-237$ \\
\hline 620.87 & 4.91E-08 & U-233 & $->$ & Th-229 \\
\hline 627.2 & $7.04 \mathrm{E}+02$ & Am-241 & $->$ & $\mathrm{Np}-237$ \\
\hline 633 & $1.58 \mathrm{E}+03$ & Am-241 & $->$ & $\mathrm{Np}-237$ \\
\hline 641.436 & $8.93 E+03$ & Am-241 & $->$ & $\mathrm{Np}-237$ \\
\hline 652.99 & $4.74 \mathrm{E}+04$ & Am-241 & $->$ & $\mathrm{Np}-237$ \\
\hline 656.97 & $6.40 \mathrm{E}-08$ & U-233 & $->$ & Th-229 \\
\hline 659.72 & $2.64 \mathrm{E}-07$ & $\mathrm{Bi}-213$ & $->$ & Po-213 \\
\hline 662.456 & $4.62 E+05$ & Am-241 & $->$ & $\mathrm{Np}-237$ \\
\hline 669.9 & $4.78 \mathrm{E}+02$ & Am-241 & $->$ & $\mathrm{Np}-237$ \\
\hline 675.8 & $8.05 E+02$ & Am-241 & $->$ & $\mathrm{Np}-237$ \\
\hline 680.06 & $3.90 E+03$ & Am-241 & $->$ & $\mathrm{Np}-237$ \\
\hline 688.77 & $3.77 E+04$ & Am-241 & $->$ & $\mathrm{Np}-237$ \\
\hline 693.5 & $4.65 E+03$ & Am-241 & $->$ & $\mathrm{Np}-237$ \\
\hline 696.36 & $6.29 E+03$ & Am-241 & $\rightarrow$ & $\mathrm{Np}-237$ \\
\hline 707.47 & $5.76 \mathrm{E}-08$ & U-233 & $->$ & Th-229 \\
\hline 710.95 & $8.05 E+03$ & Am-241 & $->$ & $\mathrm{Np}-237$ \\
\hline 721.962 & $7.55 \mathrm{E}+04$ & Am-241 & $->$ & $\mathrm{Np}-237$ \\
\hline 722.7 & $1.64 \mathrm{E}+05$ & Am-241 & $->$ & $\mathrm{Np}-237$ \\
\hline 729.5 & $1.64 \mathrm{E}+03$ & Am-241 & $->$ & $\mathrm{Np}-237$ \\
\hline 731.5 & $5.91 E+02$ & Am-241 & $->$ & $\mathrm{Np}-237$ \\
\hline 737.3 & $1.01 \mathrm{E}+04$ & Am-241 & $->$ & $\mathrm{Np}-237$ \\
\hline 755.89 & $9.56 \mathrm{E}+03$ & Am-241 & $->$ & $\mathrm{Np}-237$ \\
\hline 759.5 & $2.14 \mathrm{E}+03$ & Am-241 & $->$ & $\mathrm{Np}-237$ \\
\hline 763.4 & $2.49 E+02$ & Am-241 & $->$ & $\mathrm{Np}-237$ \\
\hline 766.79 & $6.29 E+03$ & Am-241 & $->$ & $\mathrm{Np}-237$ \\
\hline 770.49 & $6.29 E+03$ & Am-241 & $->$ & $\mathrm{Np}-237$ \\
\hline 772.1 & $3.35 E+03$ & Am-241 & $->$ & $\mathrm{Np}-237$ \\
\hline 777.2 & $7.55 \mathrm{E}+01$ & Am-241 & $->$ & $\mathrm{Np}-237$ \\
\hline 780.5 & $3.14 \mathrm{E}+02$ & Am-241 & $->$ & $\mathrm{Np}-237$ \\
\hline 788.8 & $4.91 \mathrm{E}+02$ & Am-241 & $->$ & $\mathrm{Np}-237$ \\
\hline 801.9 & $1.64 \mathrm{E}+03$ & Am-241 & $->$ & $\mathrm{Np}-237$ \\
\hline 807.27 & 7.91E-07 & Bi-213 & $->$ & Po-213 \\
\hline 811.8 & $7.55 \mathrm{E}+02$ & Am-241 & $->$ & $\mathrm{Np}-237$ \\
\hline 819.3 & $5.03 E+02$ & Am-241 & $->$ & $\mathrm{Np}-237$ \\
\hline 822.6 & $2.77 E+02$ & Am-241 & $->$ & $\mathrm{Np}-237$ \\
\hline 828.5 & $3.02 E+02$ & Am-241 & $->$ & $\mathrm{Np}-237$ \\
\hline 851.5 & $4.78 \mathrm{E}+02$ & Am-241 & $->$ & $\mathrm{Np}-237$ \\
\hline 854.7 & $2.52 E+02$ & Am-241 & $->$ & $\mathrm{Np}-237$ \\
\hline 859.2 & $1.06 \mathrm{E}+02$ & Am-241 & $->$ & $\mathrm{Np}-237$ \\
\hline 862.6 & $6.67 E+02$ & Am-241 & $->$ & $\mathrm{Np}-23$ \\
\hline
\end{tabular}




$\begin{array}{ccrcc}867.9 & 4.48 \mathrm{E}-08 & \mathrm{U}-233 & \rightarrow & \mathrm{Th}-229 \\ 872.7 & 8.80 \mathrm{E}+02 & \mathrm{Am}-241 & \rightarrow & \mathrm{Np}-237 \\ 887.5 & 2.77 \mathrm{E}+02 & \mathrm{Am}-241 & \rightarrow & \mathrm{Np}-237 \\ 898.4 & 8.80 \mathrm{E}+01 & \mathrm{Am}-241 & \rightarrow & \mathrm{Np}-237 \\ 902.5 & 3.77 \mathrm{E}+02 & \mathrm{Am}-241 & \rightarrow & \mathrm{Np}-237 \\ 912.4 & 3.14 \mathrm{E}+02 & \mathrm{Am}-241 & \rightarrow & \mathrm{Np}-237 \\ 922.2 & 2.39 \mathrm{E}+02 & \mathrm{Am}-241 & \rightarrow & \mathrm{Np}-237 \\ 928.8 & 6.29 \mathrm{E}+01 & \mathrm{Am}-241 & \rightarrow & \mathrm{Np}-237 \\ 945.7 & 6.29 \mathrm{E}+01 & \mathrm{Am}-241 & \rightarrow & \mathrm{Np}-237 \\ 955.7 & 7.29 \mathrm{E}+02 & \mathrm{Am}-241 & \rightarrow & \mathrm{Np}-237 \\ 1003 & 1.77 \mathrm{E}-07 & \mathrm{U}-233 & \rightarrow & \mathrm{Th}-229 \\ 1100.06 & 8.54 \mathrm{E}-07 & \mathrm{Bi}-213 & \rightarrow & \mathrm{Po}-213 \\ 1119 & 1.77 \mathrm{E}-07 & \mathrm{U}-233 & \rightarrow & \mathrm{Th}-229 \\ 1566 & 6.56 \mathrm{E}-06 & \mathrm{Tl}-209 & \rightarrow & \mathrm{Pb}-209\end{array}$

\begin{tabular}{|c|c|c|c|c|}
\hline Am241 & $\begin{array}{c}\text { Aged } 5 \\
\text { years }\end{array}$ & \multicolumn{3}{|c|}{ Sorted by Intensity } \\
\hline Intensity & Energy & \multicolumn{3}{|c|}{ Initial Isotopes and Decays } \\
\hline $\mathrm{ph} / \mathrm{s} / \mathrm{gm}$ & $\mathrm{keV}$ & & & \\
\hline $4.52 E+10$ & 59.536 & Am-241 & $->$ & $\mathrm{Np}-237$ \\
\hline $2.54 \mathrm{E}+10$ & 17.611 & Am-241 & $->$ & $\mathrm{Np}-237$ \\
\hline $1.64 \mathrm{E}+10$ & 13.927 & Am-241 & $->$ & $\mathrm{Np}-237$ \\
\hline $6.54 \mathrm{E}+09$ & 20.997 & Am-241 & $->$ & $\mathrm{Np}-237$ \\
\hline $3.02 E+09$ & 26.3445 & Am-241 & $->$ & $\mathrm{Np}-237$ \\
\hline $1.02 \mathrm{E}+09$ & 11.871 & Am-241 & $->$ & Np-237 \\
\hline $4.15 E+08$ & 15.861 & Am-241 & $->$ & $\mathrm{Np}-237$ \\
\hline $1.51 \mathrm{E}+08$ & 33.192 & Am-241 & $->$ & $\mathrm{Np}-237$ \\
\hline $9.18 \mathrm{E}+07$ & 43.415 & Am-241 & $->$ & $\mathrm{Np}-237$ \\
\hline $3.02 E+07$ & 69.76 & Am-241 & $->$ & $\mathrm{Np}-237$ \\
\hline $2.55 E+07$ & 98.951 & Am-241 & $->$ & $\mathrm{Np}-237$ \\
\hline $2.52 E+07$ & 55.528 & Am-241 & $->$ & $\mathrm{Np}-237$ \\
\hline $2.45 E+07$ & 102.966 & Am-241 & $->$ & $\mathrm{Np}-237$ \\
\hline $2.19 E+07$ & 32.2 & Am-241 & $->$ & $\mathrm{Np}-237$ \\
\hline $5.13 E+06$ & 125.292 & Am-241 & $->$ & $\mathrm{Np}-237$ \\
\hline $2.48 \mathrm{E}+06$ & 101.066 & Am-241 & $->$ & $\mathrm{Np}-237$ \\
\hline $1.48 \mathrm{E}+06$ & 97.071 & Am-241 & $->$ & $N p-237$ \\
\hline $1.26 \mathrm{E}+06$ & 42.64 & Am-241 & $->$ & Np-237 \\
\hline $1.26 \mathrm{E}+06$ & 122.994 & Am-241 & $->$ & Np-237 \\
\hline $9.95 \mathrm{E}+05$ & 208 & Am-241 & $->$ & $\mathrm{Np}-237$ \\
\hline $7.55 \mathrm{E}+05$ & 54.04 & Am-241 & $->$ & $\mathrm{Np}-237$ \\
\hline $6.21 E+05$ & 335.43 & Am-241 & $->$ & $\mathrm{Np}-237$ \\
\hline $5.94 \mathrm{E}+05$ & 114.23 & Am-241 & $->$ & $\mathrm{Np}-237$ \\
\hline $5.79 E+05$ & 146.557 & Am-241 & $->$ & $\mathrm{Np}-237$ \\
\hline $5.53 E+05$ & 67.46 & Am-241 & $->$ & Np-237 \\
\hline $4.62 E+05$ & 662.456 & Am-241 & $->$ & Np-237 \\
\hline $2.98 \mathrm{E}+05$ & 113.3 & Am-241 & $->$ & $\mathrm{Np}-237$ \\
\hline $2.89 E+05$ & 117.58 & Am-241 & $->$ & Np-237 \\
\hline $2.73 E+05$ & 368.61 & Am-241 & $->$ & $\mathrm{Np}-237$ \\
\hline
\end{tabular}




\begin{tabular}{|c|c|c|c|c|}
\hline $2.11 E+05$ & 169.56 & Am-241 & $\rightarrow$ & $\mathrm{Np}-237$ \\
\hline $1.91 \mathrm{E}+05$ & 322.54 & Am-241 & $->$ & $\mathrm{Np}-237$ \\
\hline $1.86 \mathrm{E}+05$ & 332.39 & Am-241 & $->$ & $\mathrm{Np}-237$ \\
\hline $1.77 \mathrm{E}+05$ & 376.6 & Am-241 & $->$ & $\mathrm{Np}-237$ \\
\hline $1.76 \mathrm{E}+05$ & 64.817 & Am-241 & $->$ & $\mathrm{Np}-237$ \\
\hline $1.64 \mathrm{E}+05$ & 722.7 & Am-241 & $->$ & $\mathrm{Np}-237$ \\
\hline $1.38 \mathrm{E}+05$ & 75.83 & Am-241 & $->$ & $\mathrm{Np}-237$ \\
\hline $1.01 \mathrm{E}+05$ & 150.11 & Am-241 & $->$ & $\mathrm{Np}-237$ \\
\hline $8.39 E+04$ & 164.58 & Am-241 & $->$ & $\mathrm{Np}-237$ \\
\hline $7.60 E+04$ & 311.9 & $\mathrm{~Pa}-233$ & $->$ & $U-233$ \\
\hline $7.55 E+04$ & 721.962 & Am-241 & $->$ & $\mathrm{Np}-237$ \\
\hline $7.42 E+04$ & 619.011 & Am-241 & $->$ & $\mathrm{Np}-237$ \\
\hline $6.92 E+04$ & 294.9 & Am-241 & $->$ & $\mathrm{Np}-237$ \\
\hline $6.60 E+04$ & 370.933 & Am-241 & $->$ & $\mathrm{Np}-237$ \\
\hline $5.91 E+04$ & 96.67 & Am-241 & $->$ & $\mathrm{Np}-237$ \\
\hline $5.28 E+04$ & 221.45 & Am-241 & $->$ & $\mathrm{Np}-237$ \\
\hline $5.11 \mathrm{E}+04$ & 16.632 & $\mathrm{~Np}-237$ & $->$ & Pa-233 \\
\hline $4.74 E+04$ & 652.99 & Am-241 & $->$ & $\mathrm{Np}-237$ \\
\hline $3.92 E+04$ & 13.274 & $\mathrm{~Np}-237$ & $->$ & $\mathrm{Pa}-233$ \\
\hline $3.80 E+04$ & 17.058 & $\mathrm{~Pa}-233$ & $->$ & U-233 \\
\hline $3.77 E+04$ & 688.77 & Am-241 & $->$ & $\mathrm{Np}-237$ \\
\hline $3.77 E+04$ & 245 & Am-241 & $->$ & $\mathrm{Np}-237$ \\
\hline $3.77 E+04$ & 242.4 & $A m-241$ & $->$ & $\mathrm{Np}-237$ \\
\hline $3.60 E+04$ & 13.6 & $\mathrm{~Pa}-233$ & $->$ & U-233 \\
\hline $3.55 E+04$ & 383.744 & $A m-241$ & $->$ & $\mathrm{Np}-237$ \\
\hline $3.52 E+04$ & 419.22 & Am-241 & $->$ & $\mathrm{Np}-237$ \\
\hline $3.31 E+04$ & 267.54 & $A m-241$ & $->$ & $\mathrm{Np}-237$ \\
\hline $3.20 E+04$ & 98.443 & $\mathrm{~Pa}-233$ & $->$ & U-233 \\
\hline $3.14 \mathrm{E}+04$ & 51.013 & Am-241 & $->$ & $\mathrm{Np}-237$ \\
\hline $3.09 E+04$ & 426.39 & Am-241 & $->$ & $\mathrm{Np}-237$ \\
\hline $3.02 E+04$ & 165.92 & Am-241 & $->$ & $\mathrm{Np}-237$ \\
\hline $2.86 E+04$ & 86.5 & $\mathrm{~Np}-237$ & $->$ & $\mathrm{Pa}-233$ \\
\hline $2.64 \mathrm{E}+04$ & 29.378 & $\mathrm{~Np}-237$ & $->$ & $\mathrm{Pa}-233$ \\
\hline $2.26 \mathrm{E}+04$ & 175.09 & Am-241 & $->$ & $\mathrm{Np}-237$ \\
\hline $2.04 \mathrm{E}+04$ & 94.66 & $\mathrm{~Pa}-233$ & $->$ & U-233 \\
\hline $1.89 \mathrm{E}+04$ & 310.3 & Am-241 & $->$ & $\mathrm{Np}-237$ \\
\hline $1.74 \mathrm{E}+04$ & 292.76 & Am-241 & $->$ & $\mathrm{Np}-237$ \\
\hline $1.64 \mathrm{E}+04$ & 197 & Am-241 & $->$ & $\mathrm{Np}-237$ \\
\hline $1.51 \mathrm{E}+04$ & 156.4 & Am-241 & $->$ & $\mathrm{Np}-237$ \\
\hline $1.24 \mathrm{E}+04$ & 300.175 & Pa-233 & $->$ & U-233 \\
\hline $1.22 \mathrm{E}+04$ & 454.67 & Am-241 & $->$ & $\mathrm{Np}-237$ \\
\hline $1.20 \mathrm{E}+04$ & 111.298 & $\mathrm{~Pa}-233$ & $->$ & U-233 \\
\hline $1.19 \mathrm{E}+04$ & 19.718 & $\mathrm{~Np}-237$ & $->$ & $\mathrm{Pa}-233$ \\
\hline $1.18 \mathrm{E}+04$ & 597.41 & Am-241 & $->$ & $\mathrm{Np}-237$ \\
\hline $1.13 E+04$ & 161.7 & Am-241 & $->$ & $\mathrm{Np}-237$ \\
\hline $1.13 E+04$ & 264.87 & Am-241 & $->$ & $\mathrm{Np}-237$ \\
\hline $1.01 \mathrm{E}+04$ & 154.4 & Am-241 & $->$ & $\mathrm{Np}-237$ \\
\hline $1.01 \mathrm{E}+04$ & 737.3 & Am-241 & $->$ & $\mathrm{Np}-237$ \\
\hline $9.56 \mathrm{E}+03$ & 755.89 & $A m-241$ & $->$ & $\mathrm{Np}-237$ \\
\hline $9.00 E+03$ & 20.42 & Pa-233 & $->$ & U-233 \\
\hline
\end{tabular}




\begin{tabular}{|c|c|c|c|c|}
\hline $8.93 E+03$ & 641.436 & Am-241 & $->$ & $\mathrm{Np}-237$ \\
\hline $8.40 E+03$ & 340.59 & Pa-233 & $->$ & U-233 \\
\hline $8.30 E+03$ & 275.68 & Am-241 & $->$ & $\mathrm{Np}-237$ \\
\hline $8.05 E+03$ & 710.95 & Am-241 & $->$ & Np-237 \\
\hline $7.42 E+03$ & 390.6 & $A m-241$ & $->$ & $\mathrm{Np}-237$ \\
\hline $6.72 E+03$ & 139.53 & Am-241 & $->$ & $\mathrm{Np}-237$ \\
\hline $6.29 E+03$ & 770.49 & Am-241 & $->$ & $\mathrm{Np}-237$ \\
\hline $6.29 E+03$ & 766.79 & Am-241 & $->$ & $\mathrm{Np}-237$ \\
\hline $6.29 E+03$ & 696.36 & Am-241 & $->$ & $\mathrm{Np}-237$ \\
\hline $5.93 E+03$ & 95.858 & $\mathrm{~Np}-237$ & $->$ & Pa-233 \\
\hline $5.79 E+03$ & 232.86 & Am-241 & $->$ & $\mathrm{Np}-237$ \\
\hline $5.28 \mathrm{E}+03$ & 337.727 & Am-241 & $->$ & $\mathrm{Np}-237$ \\
\hline $5.03 E+03$ & 249.1 & Am-241 & $->$ & $\mathrm{Np}-237$ \\
\hline $4.65 E+03$ & 693.5 & Am-241 & $->$ & $N p-237$ \\
\hline $4.53 E+03$ & 459.58 & Am-241 & $->$ & $\mathrm{Np}-237$ \\
\hline $4.40 E+03$ & 442.75 & Am-241 & $->$ & $\mathrm{Np}-237$ \\
\hline $4.00 E+03$ & 114.445 & Pa-233 & $->$ & U-233 \\
\hline $3.90 E+03$ & 680.06 & Am-241 & $->$ & $\mathrm{Np}-237$ \\
\hline $3.90 E+03$ & 291.21 & Am-241 & $->$ & $\mathrm{Np}-237$ \\
\hline $3.65 E+03$ & 468 & Am-241 & $->$ & $\mathrm{Np}-237$ \\
\hline $3.65 E+03$ & 203.9 & Am-241 & $->$ & $\mathrm{Np}-237$ \\
\hline $3.52 E+03$ & 590.3 & Am-241 & $->$ & $\mathrm{Np}-237$ \\
\hline $3.52 E+03$ & 86.652 & Рa-233 & $->$ & U-233 \\
\hline $3.35 E+03$ & 772.1 & Am-241 & $->$ & $\mathrm{Np}-237$ \\
\hline $3.27 E+03$ & 514.2 & Am-241 & $->$ & $\mathrm{Np}-237$ \\
\hline $3.25 E+03$ & 92.282 & Np-237 & $->$ & Pa-233 \\
\hline $3.02 E+03$ & 415.932 & Pa-233 & $->$ & U-233 \\
\hline $3.02 E+03$ & 452.41 & Am-241 & $->$ & $N p-237$ \\
\hline $3.02 E+03$ & 246.66 & Am-241 & $->$ & $\mathrm{Np}-237$ \\
\hline $2.77 E+03$ & 190.4 & Am-241 & $->$ & $\mathrm{Np}-237$ \\
\hline $2.52 E+03$ & 435.3 & Am-241 & $->$ & $\mathrm{Np}-237$ \\
\hline $2.38 \mathrm{E}+03$ & 398.663 & Pa-233 & $->$ & U-233 \\
\hline $2.35 E+03$ & 11.372 & $\mathrm{~Np}-237$ & $->$ & $\mathrm{Pa}-233$ \\
\hline $2.34 \mathrm{E}+03$ & 75.343 & Pa-233 & $->$ & U-233 \\
\hline $2.14 \mathrm{E}+03$ & 11.62 & $\mathrm{~Pa}-233$ & $->$ & U-233 \\
\hline $2.14 \mathrm{E}+03$ & 759.5 & Am-241 & $->$ & $\mathrm{Np}-237$ \\
\hline $1.92 \mathrm{E}+03$ & 108.166 & $\mathrm{~Np}-237$ & $->$ & Pa-233 \\
\hline $1.76 \mathrm{E}+03$ & 158.48 & Am-241 & $->$ & $\mathrm{Np}-237$ \\
\hline $1.76 \mathrm{E}+03$ & 406.4 & Am-241 & $->$ & $\mathrm{Np}-237$ \\
\hline $1.64 \mathrm{E}+03$ & 801.9 & Am-241 & $->$ & $\mathrm{Np}-237$ \\
\hline $1.64 \mathrm{E}+03$ & 729.5 & Am-241 & $->$ & $\mathrm{Np}-237$ \\
\hline $1.64 \mathrm{E}+03$ & 586.5 & Am-241 & $->$ & $\mathrm{Np}-237$ \\
\hline $1.58 E+03$ & 633 & Am-241 & $->$ & $\mathrm{Np}-237$ \\
\hline $1.55 E+03$ & 94.723 & $\mathrm{~Np}-237$ & $->$ & $\mathrm{Pa}-233$ \\
\hline $1.51 E+03$ & 349.46 & Am-241 & $->$ & $\mathrm{Np}-237$ \\
\hline $1.51 E+03$ & 358.36 & Am-241 & $->$ & $\mathrm{Np}-237$ \\
\hline $1.45 E+03$ & 512 & Am-241 & $->$ & $\mathrm{Np}-237$ \\
\hline $1.38 \mathrm{E}+03$ & 103.921 & Pa-233 & $->$ & U-233 \\
\hline $1.26 \mathrm{E}+03$ & 429.8 & Am-241 & $->$ & $\mathrm{Np}-237$ \\
\hline $1.26 \mathrm{E}+03$ & 486.3 & Am-241 & $->$ & $\mathrm{Np}-237$ \\
\hline
\end{tabular}




\begin{tabular}{|c|c|c|c|c|}
\hline $1.26 \mathrm{E}+03$ & 260.9 & Am-241 & $->$ & $\mathrm{Np}-237$ \\
\hline $1.26 \mathrm{E}+03$ & 304.2 & Am-241 & $->$ & $\mathrm{Np}-237$ \\
\hline $1.26 \mathrm{E}+03$ & 574.05 & Am-241 & $->$ & $\mathrm{Np}-237$ \\
\hline $1.16 \mathrm{E}+03$ & 375.518 & $\mathrm{~Pa}-233$ & $->$ & U-233 \\
\hline $1.13 E+03$ & 522 & Am-241 & $->$ & $\mathrm{Np}-237$ \\
\hline $9.61 \mathrm{E}+02$ & 14.953 & $\mathrm{~Np}-237$ & $->$ & $\mathrm{Pa}-233$ \\
\hline $8.80 E+02$ & 234.352 & Am-241 & $->$ & $\mathrm{Np}-237$ \\
\hline $8.80 E+02$ & 872.7 & Am-241 & $->$ & $\mathrm{Np}-237$ \\
\hline $8.07 E+02$ & 271.58 & Am-241 & $->$ & $\mathrm{Np}-237$ \\
\hline $8.05 E+02$ & 675.8 & Am-241 & $->$ & $\mathrm{Np}-237$ \\
\hline $7.97 E+02$ & 57.149 & $\mathrm{~Np}-237$ & $->$ & $\mathrm{Pa}-233$ \\
\hline $7.97 E+02$ & 143.227 & $\mathrm{~Np}-237$ & $->$ & $\mathrm{Pa}-233$ \\
\hline $7.55 E+02$ & 811.8 & Am-241 & $->$ & $\mathrm{Np}-237$ \\
\hline $7.29 E+02$ & 955.7 & Am-241 & $->$ & $\mathrm{Np}-237$ \\
\hline $7.04 \mathrm{E}+02$ & 627.2 & Am-241 & $->$ & $\mathrm{Np}-237$ \\
\hline $6.67 E+02$ & 862.6 & Am-241 & $->$ & $\mathrm{Np}-237$ \\
\hline $6.34 \mathrm{E}+02$ & 111.897 & $\mathrm{~Np}-237$ & $->$ & Pa-233 \\
\hline $5.91 E+02$ & 731.5 & Am-241 & $->$ & $\mathrm{Np}-237$ \\
\hline $5.60 \mathrm{E}+02$ & 271.597 & Pa-233 & $->$ & U-233 \\
\hline $5.03 E+02$ & 819.3 & Am-241 & $->$ & $\mathrm{Np}-237$ \\
\hline $4.91 \mathrm{E}+02$ & 788.8 & Am-241 & $->$ & $\mathrm{Np}-237$ \\
\hline $4.91 E+02$ & 151.423 & $\mathrm{~Np}-237$ & $->$ & $\mathrm{Pa}-233$ \\
\hline $4.78 E+02$ & 851.5 & Am-241 & $->$ & $\mathrm{Np}-237$ \\
\hline $4.78 E+02$ & 669.9 & Am-241 & $->$ & $\mathrm{Np}-237$ \\
\hline $4.60 E+02$ & 15.4 & $\mathrm{~Pa}-233$ & $->$ & $U-233$ \\
\hline $4.11 \mathrm{E}+02$ & 195.04 & $\mathrm{~Np}-237$ & $->$ & $\mathrm{Pa}-233$ \\
\hline $3.77 E+02$ & 902.5 & Am-241 & $->$ & $\mathrm{Np}-237$ \\
\hline $3.68 E+02$ & 88.05 & $\mathrm{~Np}-237$ & $->$ & $\mathrm{Pa}-233$ \\
\hline $3.29 E+02$ & 117.689 & $\mathrm{~Np}-237$ & $->$ & $\mathrm{Pa}-233$ \\
\hline $3.14 \mathrm{E}+02$ & 912.4 & Am-241 & $->$ & $\mathrm{Np}-237$ \\
\hline $3.14 \mathrm{E}+02$ & 780.5 & Am-241 & $->$ & $\mathrm{Np}-237$ \\
\hline $3.11 E+02$ & 212.412 & $\mathrm{~Np}-237$ & $->$ & $\mathrm{Pa}-233$ \\
\hline $3.02 E+02$ & 828.5 & Am-241 & $->$ & $\mathrm{Np}-237$ \\
\hline $2.77 E+02$ & 822.6 & Am-241 & $->$ & $\mathrm{Np}-237$ \\
\hline $2.77 E+02$ & 887.5 & Am-241 & $->$ & $\mathrm{Np}-237$ \\
\hline $2.72 E+02$ & 46.57 & $\mathrm{~Np}-237$ & $->$ & $\mathrm{Pa}-233$ \\
\hline $2.72 E+02$ & 191.9 & Am-241 & $->$ & $\mathrm{Np}-237$ \\
\hline $2.52 E+02$ & 582.6 & Am-241 & $->$ & $\mathrm{Np}-237$ \\
\hline $2.52 E+02$ & 854.7 & Am-241 & $->$ & $\mathrm{Np}-237$ \\
\hline $2.49 E+02$ & 763.4 & $A m-241$ & $->$ & $\mathrm{Np}-237$ \\
\hline $2.39 E+02$ & 922.2 & Am-241 & $->$ & $\mathrm{Np}-237$ \\
\hline $1.88 \mathrm{E}+02$ & 155.263 & $\mathrm{~Np}-237$ & $->$ & $\mathrm{Pa}-233$ \\
\hline $1.74 \mathrm{E}+02$ & 131.09 & $\mathrm{~Np}-237$ & $->$ & $\mathrm{Pa}-233$ \\
\hline $1.49 E+02$ & 108.69 & $N p-237$ & $->$ & $\mathrm{Pa}-233$ \\
\hline $1.47 \mathrm{E}+02$ & 169.18 & $\mathrm{~Np}-237$ & $->$ & $\mathrm{Pa}-233$ \\
\hline $1.37 \mathrm{E}+02$ & 134.23 & $\mathrm{~Np}-237$ & $->$ & $\mathrm{Pa}-233$ \\
\hline $1.31 \mathrm{E}+02$ & 237.95 & $\mathrm{~Np}-237$ & $->$ & $\mathrm{Pa}-233$ \\
\hline $1.30 \mathrm{E}+02$ & 28.578 & $\mathrm{~Pa}-233$ & $->$ & U-233 \\
\hline $1.14 \mathrm{E}+02$ & 106.13 & $\mathrm{~Np}-237$ & $->$ & $\mathrm{Pa}-233$ \\
\hline $1.08 \mathrm{E}+02$ & 248.25 & $\mathrm{~Pa}-233$ & $->$ & U-233 \\
\hline
\end{tabular}




\begin{tabular}{|c|c|c|c|c|}
\hline $1.06 \mathrm{E}+02$ & 859.2 & Am-241 & $->$ & $\mathrm{Np}-237$ \\
\hline $1.04 \mathrm{E}+02$ & 193.29 & $\mathrm{~Np}-237$ & $->$ & $\mathrm{Pa}-233$ \\
\hline $1.00 \mathrm{E}+02$ & 194.74 & $\mathrm{~Np}-237$ & $->$ & $\mathrm{Pa}-233$ \\
\hline $8.80 E+01$ & 898.4 & Am-241 & $->$ & $\mathrm{Np}-237$ \\
\hline $8.58 E+01$ & 214.08 & $\mathrm{~Np}-237$ & $->$ & $\mathrm{Pa}-233$ \\
\hline $8.58 E+01$ & 201.72 & $\mathrm{~Np}-237$ & $->$ & $\mathrm{Pa}-233$ \\
\hline $7.56 \mathrm{E}+01$ & 162.51 & $\mathrm{~Np}-237$ & $->$ & $\mathrm{Pa}-233$ \\
\hline $7.55 E+01$ & 777.2 & Am-241 & $->$ & $\mathrm{Np}-237$ \\
\hline $7.20 \mathrm{E}+01$ & 40.415 & Pa-233 & $->$ & U-233 \\
\hline $6.29 E+01$ & 945.7 & Am-241 & $->$ & $\mathrm{Np}-237$ \\
\hline $6.29 E+01$ & 928.8 & Am-241 & $->$ & $\mathrm{Np}-237$ \\
\hline $5.52 \mathrm{E}+01$ & 191.46 & $\mathrm{~Np}-237$ & $->$ & $\mathrm{Pa}-233$ \\
\hline $5.11 \mathrm{E}+01$ & 196.89 & $\mathrm{~Np}-237$ & $->$ & $\mathrm{Pa}-233$ \\
\hline $5.06 \mathrm{E}+01$ & 298.87 & $\mathrm{~Pa}-233$ & $->$ & U-233 \\
\hline $4.70 E+01$ & 180.801 & $\mathrm{~Np}-237$ & $->$ & $\mathrm{Pa}-233$ \\
\hline $4.09 E+01$ & 176.09 & $\mathrm{~Np}-237$ & $->$ & $\mathrm{Pa}-233$ \\
\hline $3.88 E+01$ & 170.67 & $\mathrm{~Np}-237$ & $->$ & $\mathrm{Pa}-233$ \\
\hline $3.68 \mathrm{E}+01$ & 140.61 & $\mathrm{~Np}-237$ & $->$ & $\mathrm{Pa}-233$ \\
\hline $3.27 E+01$ & 70.62 & $\mathrm{~Np}-237$ & $->$ & $\mathrm{Pa}-233$ \\
\hline $3.27 E+01$ & 63.95 & $\mathrm{~Np}-237$ & $->$ & $\mathrm{Pa}-233$ \\
\hline $3.17 \mathrm{E}+01$ & 209.19 & $\mathrm{~Np}-237$ & $->$ & $\mathrm{Pa}-233$ \\
\hline $2.68 \mathrm{E}+01$ & 229.98 & $\mathrm{~Np}-237$ & $->$ & $\mathrm{Pa}-233$ \\
\hline $2.60 E+01$ & 41.718 & $\mathrm{~Pa}-233$ & $->$ & U-233 \\
\hline $2.45 E+01$ & 62.66 & $\mathrm{~Np}-237$ & $->$ & $\mathrm{Pa}-233$ \\
\hline $2.27 E+01$ & 74.46 & $\mathrm{~Np}-237$ & $->$ & Pa-233 \\
\hline $1.41 \mathrm{E}+01$ & 153.72 & $\mathrm{~Np}-237$ & $->$ & $\mathrm{Pa}-233$ \\
\hline $1.39 \mathrm{E}+01$ & 172.56 & $\mathrm{~Np}-237$ & $->$ & $\mathrm{Pa}-233$ \\
\hline $1.37 \mathrm{E}+01$ & 256.98 & $\mathrm{~Np}-237$ & $->$ & $\mathrm{Pa}-233$ \\
\hline $1.37 \mathrm{E}+01$ & 186.7 & $\mathrm{~Np}-237$ & $->$ & $\mathrm{Pa}-233$ \\
\hline $1.37 \mathrm{E}+01$ & 262.41 & $\mathrm{~Np}-237$ & $->$ & $\mathrm{Pa}-233$ \\
\hline $1.08 \mathrm{E}+01$ & 258.46 & $\mathrm{~Pa}-233$ & $->$ & U-233 \\
\hline $9.81 E+00$ & 248.91 & $\mathrm{~Np}-237$ & $->$ & $\mathrm{Pa}-233$ \\
\hline $7.36 \mathrm{E}+00$ & 202.85 & $\mathrm{~Np}-237$ & $->$ & $\mathrm{Pa}-233$ \\
\hline $7.20 \mathrm{E}+00$ & 17.269 & $\mathrm{~Pa}-233$ & $->$ & U-233 \\
\hline $7.15 E+00$ & 200.17 & $\mathrm{~Np}-237$ & $->$ & $\mathrm{Pa}-233$ \\
\hline $5.11 \mathrm{E}+00$ & 115.19 & $\mathrm{~Np}-237$ & $->$ & $\mathrm{Pa}-233$ \\
\hline 7.04E-02 & 16.141 & U-233 & $->$ & Th-229 \\
\hline $5.55 \mathrm{E}-02$ & 12.952 & $U-233$ & $->$ & Th-229 \\
\hline $1.71 \mathrm{E}-02$ & 19.148 & $U-233$ & $->$ & Th-229 \\
\hline $3.20 \mathrm{E}-03$ & 11.118 & $U-233$ & $->$ & Th-229 \\
\hline $1.28 \mathrm{E}-03$ & 42.441 & $U-233$ & $->$ & Th-229 \\
\hline $1.22 \mathrm{E}-03$ & 14.511 & $U-233$ & $->$ & Th-229 \\
\hline 4.69E-04 & 97.143 & $U-233$ & $->$ & Th-229 \\
\hline $3.61 \mathrm{E}-04$ & 93.36 & $U-233$ & $->$ & Th-229 \\
\hline 2.99E-04 & 54.702 & $U-233$ & $->$ & Th-229 \\
\hline $2.22 \mathrm{E}-04$ & 89.957 & $U-233$ & $->$ & Th-229 \\
\hline $1.88 \mathrm{E}-04$ & 317.133 & $U-233$ & $->$ & Th-229 \\
\hline $1.45 \mathrm{E}-04$ & 29.191 & $U-233$ & $->$ & Th-229 \\
\hline $1.41 \mathrm{E}-04$ & 164.512 & $U-233$ & $->$ & Th-229 \\
\hline $1.34 \mathrm{E}-04$ & 146.347 & $U-233$ & $->$ & Th-229 \\
\hline
\end{tabular}




\begin{tabular}{|c|c|c|c|c|}
\hline $1.30 \mathrm{E}-04$ & 105.362 & $U-233$ & $->$ & Th-229 \\
\hline $1.11 \mathrm{E}-04$ & 291.317 & $U-233$ & $->$ & Th-229 \\
\hline $9.60 \mathrm{E}-05$ & 248.661 & $U-233$ & $->$ & Th-229 \\
\hline $9.20 \mathrm{E}-05$ & 40.34 & $\mathrm{Ra}-225$ & $->$ & Ac- 225 \\
\hline $9.17 \mathrm{E}-05$ & 53.559 & U-233 & $->$ & Th-229 \\
\hline 7.47E-05 & 217.133 & $U-233$ & $->$ & Th-229 \\
\hline 7.47E-05 & 245.294 & $U-233$ & $->$ & Th-229 \\
\hline $6.83 E-05$ & 118.97 & $U-233$ & $->$ & Th-229 \\
\hline $6.61 E-05$ & 320.508 & $U-233$ & $->$ & Th-229 \\
\hline 5.97E-05 & 117.155 & $U-233$ & $->$ & Th-229 \\
\hline $5.76 \mathrm{E}-05$ & 71.847 & $U-233$ & $->$ & Th-229 \\
\hline 5.33E-05 & 208.149 & U-233 & $->$ & Th-229 \\
\hline 5.00E-05 & 440.34 & $\mathrm{Bi}-213$ & $->$ & Po-213 \\
\hline 4.69E-05 & 135.328 & $U-233$ & $->$ & Th-229 \\
\hline 4.48E-05 & 120.81 & $U-233$ & $->$ & Th-229 \\
\hline 4.33E-05 & 108.99 & $U-233$ & $->$ & Th-229 \\
\hline 4.27E-05 & 187.942 & $U-233$ & $->$ & Th-229 \\
\hline $3.41 \mathrm{E}-05$ & 145.286 & $U-233$ & $->$ & Th-229 \\
\hline $3.38 \mathrm{E}-05$ & 217.98 & Fr-221 & $->$ & At-217 \\
\hline $3.20 \mathrm{E}-05$ & 74.5 & U-233 & $->$ & Th-229 \\
\hline 2.99E-05 & 96.215 & U-233 & $->$ & Th-229 \\
\hline 2.97E-05 & 14.772 & Ac- 225 & $->$ & Fr-221 \\
\hline $2.60 \mathrm{E}-05$ & 278.059 & U-233 & $->$ & Th-229 \\
\hline $2.58 \mathrm{E}-05$ & 25.304 & $U-233$ & $->$ & Th-229 \\
\hline $2.42 \mathrm{E}-05$ & 12.017 & $A c-225$ & $->$ & Fr-221 \\
\hline $2.30 \mathrm{E}-05$ & 287.949 & U-233 & $->$ & Th-229 \\
\hline $2.11 \mathrm{E}-05$ & 32.27 & U-233 & $->$ & Th-229 \\
\hline $1.94 \mathrm{E}-05$ & 15.662 & $\mathrm{Ra}-225$ & $->$ & Ac-225 \\
\hline $1.79 E-05$ & 66.11 & U-233 & $->$ & Th-229 \\
\hline $1.79 E-05$ & 323.321 & $U-233$ & $->$ & Th-229 \\
\hline $1.75 \mathrm{E}-05$ & 365.762 & $U-233$ & $->$ & Th-229 \\
\hline $1.75 \mathrm{E}-05$ & 12.636 & $\mathrm{Ra}-225$ & $->$ & Ac- 225 \\
\hline $1.54 \mathrm{E}-05$ & 77.1 & U-233 & $->$ & Th-229 \\
\hline $1.51 \mathrm{E}-05$ & 193.59 & Th-229 & $->$ & $\mathrm{Ra}-225$ \\
\hline $1.41 \mathrm{E}-05$ & 216.08 & U-233 & $->$ & Th-229 \\
\hline $1.39 E-05$ & 123.91 & $U-233$ & $->$ & Th-229 \\
\hline $1.35 \mathrm{E}-05$ & 31.37 & Th-229 & $->$ & $\mathrm{Ra}-225$ \\
\hline $1.28 \mathrm{E}-05$ & 70.35 & $\mathrm{U}-233$ & $->$ & Th-229 \\
\hline $1.26 \mathrm{E}-05$ & 336.579 & $U-233$ & $->$ & Th-229 \\
\hline $1.26 \mathrm{E}-05$ & 72.821 & $U-233$ & $->$ & Th-229 \\
\hline $1.09 E-05$ & 210.94 & Th-229 & $->$ & $\mathrm{Ra}-225$ \\
\hline $1.00 \mathrm{E}-05$ & 111.935 & U-233 & $->$ & Th-229 \\
\hline $1.00 \mathrm{E}-05$ & 274.692 & U-233 & $->$ & Th-229 \\
\hline 9.87E-06 & 86.44 & Th-229 & $->$ & $\mathrm{Ra}-225$ \\
\hline $9.39 \mathrm{E}-06$ & 88.43 & U-233 & $->$ & Th-229 \\
\hline $8.32 \mathrm{E}-06$ & 76.32 & $U-233$ & $->$ & Th-229 \\
\hline 8.32E-06 & 165.493 & $U-233$ & $->$ & Th-229 \\
\hline $8.11 \mathrm{E}-06$ & 240.364 & $U-233$ & $->$ & Th-229 \\
\hline 7.68E-06 & 37.85 & $U-233$ & $->$ & Th-229 \\
\hline $7.68 \mathrm{E}-06$ & 148.15 & $U-233$ & $->$ & Th-229 \\
\hline
\end{tabular}




\begin{tabular}{|c|c|c|c|c|}
\hline 7.04E-06 & 90.994 & $U-233$ & $->$ & Th-229 \\
\hline $6.83 E-06$ & 67.96 & $U-233$ & $->$ & Th-229 \\
\hline $6.61 \mathrm{E}-06$ & 109.47 & $U-233$ & $->$ & Th-229 \\
\hline 6.61E-06 & 261.88 & $U-233$ & $->$ & Th-229 \\
\hline $6.56 \mathrm{E}-06$ & 1566 & TI-209 & $->$ & Pb-209 \\
\hline $6.50 \mathrm{E}-06$ & 17.449 & Ac- 225 & $->$ & Fr-221 \\
\hline $6.44 \mathrm{E}-06$ & 86.105 & Ac- 225 & $->$ & Fr-221 \\
\hline $6.40 \mathrm{E}-06$ & 144.52 & $U-233$ & $->$ & Th-229 \\
\hline $5.76 \mathrm{E}-06$ & 31.24 & $U-233$ & $->$ & Th-229 \\
\hline 5.42E-06 & 117 & Tl-209 & $->$ & Pb-209 \\
\hline 5.42E-06 & 467 & Tl-209 & $->$ & Pb-209 \\
\hline 5.33E-06 & 114.28 & $U-233$ & $->$ & Th-229 \\
\hline 5.33E-06 & 268.619 & $U-233$ & $->$ & Th-229 \\
\hline 5.33E-06 & 52.613 & $U-233$ & $->$ & Th-229 \\
\hline $5.26 \mathrm{E}-06$ & 137.02 & Th-229 & $->$ & Ra-225 \\
\hline $5.20 \mathrm{E}-06$ & 99.82 & Ac- 225 & $->$ & Fr-221 \\
\hline 4.91E-06 & 174.162 & U-233 & $->$ & Th-229 \\
\hline $4.48 \mathrm{E}-06$ & 116.26 & $U-233$ & $->$ & Th-229 \\
\hline 4.44E-06 & 18.593 & $\mathrm{Ra}-225$ & $->$ & Ac- 225 \\
\hline $4.37 \mathrm{E}-06$ & 81.517 & Fr-221 & $->$ & At-217 \\
\hline $4.05 E-06$ & 87.24 & $U-233$ & $->$ & Th-229 \\
\hline $4.05 E-06$ & 85.402 & $U-233$ & $->$ & Th-229 \\
\hline $3.91 \mathrm{E}-06$ & 79.29 & $\mathrm{Bi}-213$ & $->$ & Po-213 \\
\hline $3.90 E-06$ & 83.229 & Ac- 225 & $->$ & Fr-221 \\
\hline $3.84 \mathrm{E}-06$ & 259.3 & U-233 & $->$ & Th-229 \\
\hline $3.84 \mathrm{E}-06$ & 82.957 & $U-233$ & $->$ & Th-229 \\
\hline $3.29 E-06$ & 148.37 & Th-229 & $->$ & Ra-225 \\
\hline $3.29 E-06$ & 156.46 & Th-229 & $->$ & Ra-225 \\
\hline $3.29 E-06$ & 124.51 & Th-229 & $->$ & $\mathrm{Ra}-225$ \\
\hline $3.20 E-06$ & 219.424 & U-233 & $->$ & Th-229 \\
\hline $3.20 \mathrm{E}-06$ & 154.69 & $U-233$ & $->$ & Th-229 \\
\hline $2.99 \mathrm{E}-06$ & 293.923 & $U-233$ & $->$ & Th-229 \\
\hline $2.99 \mathrm{E}-06$ & 212.29 & $U-233$ & $->$ & Th-229 \\
\hline 2.99E-06 & 170.794 & $U-233$ & $->$ & Th-229 \\
\hline 2.97E-06 & 13.911 & Fr-221 & $->$ & At-217 \\
\hline $2.77 \mathrm{E}-06$ & 86.74 & U-233 & $->$ & Th-229 \\
\hline $2.71 \mathrm{E}-06$ & 149.8 & $U-233$ & $->$ & Th-229 \\
\hline $2.63 E-06$ & 78.947 & Fr-221 & $->$ & At-217 \\
\hline $2.63 \mathrm{E}-06$ & 107.16 & Th-229 & $->$ & $\mathrm{Ra}-225$ \\
\hline $2.45 \mathrm{E}-06$ & 11.414 & Fr-221 & $->$ & At-217 \\
\hline $2.36 \mathrm{E}-06$ & 76.858 & $\mathrm{Bi}-213$ & $->$ & Po-213 \\
\hline $2.32 \mathrm{E}-06$ & 97.272 & Ac- 225 & $->$ & Fr-221 \\
\hline $2.30 \mathrm{E}-06$ & 260.4 & U-233 & $->$ & Th-229 \\
\hline $2.30 \mathrm{E}-06$ & 68.97 & $U-233$ & $->$ & Th-229 \\
\hline $2.30 \mathrm{E}-06$ & 154.37 & Th-229 & $->$ & Ra-225 \\
\hline $2.24 \mathrm{E}-06$ & 139.76 & U-233 & $->$ & Th-229 \\
\hline $2.13 \mathrm{E}-06$ & 103.375 & U-233 & $->$ & Th-229 \\
\hline $2.08 \mathrm{E}-06$ & 150.08 & $A c-225$ & $->$ & Fr-221 \\
\hline $2.03 E-06$ & 383.4 & U-233 & $->$ & Th-229 \\
\hline 1.97E-06 & 124.72 & Th-229 & $->$ & $\mathrm{Ra}-225$ \\
\hline
\end{tabular}




\begin{tabular}{|c|c|c|c|c|}
\hline $1.95 \mathrm{E}-06$ & 99.58 & $A c-225$ & $->$ & Fr-221 \\
\hline $1.92 \mathrm{E}-06$ & 101.8 & $\mathrm{U}-233$ & $->$ & Th-229 \\
\hline $1.70 \mathrm{E}-06$ & 62.93 & Ac- 225 & $->$ & Fr-221 \\
\hline $1.68 \mathrm{E}-06$ & 179.74 & Th-229 & $->$ & Ra-225 \\
\hline $1.64 \mathrm{E}-06$ & 75.17 & Th-229 & $->$ & Ra-225 \\
\hline $1.60 \mathrm{E}-06$ & 84.27 & U-233 & $->$ & Th-229 \\
\hline $1.60 \mathrm{E}-06$ & 162.52 & $U-233$ & $->$ & Th-229 \\
\hline $1.56 \mathrm{E}-06$ & 309.37 & U-233 & $->$ & Th-229 \\
\hline $1.55 \mathrm{E}-06$ & 92.136 & Fr-221 & $->$ & At-217 \\
\hline $1.50 \mathrm{E}-06$ & 11.119 & $\mathrm{Bi}-213$ & $->$ & Po-213 \\
\hline $1.49 \mathrm{E}-06$ & 302.84 & $U-233$ & $->$ & Th-229 \\
\hline $1.49 \mathrm{E}-06$ & 129.2 & $U-233$ & $->$ & Th-229 \\
\hline $1.43 \mathrm{E}-06$ & 168.98 & $U-233$ & $->$ & Th-229 \\
\hline $1.43 \mathrm{E}-06$ & 230.086 & U-233 & $->$ & Th-229 \\
\hline $1.42 \mathrm{E}-06$ & 187.99 & Ac- 225 & $->$ & Fr-221 \\
\hline $1.41 \mathrm{E}-06$ & 142.98 & Th-229 & $->$ & Ra-225 \\
\hline $1.39 E-06$ & 13.504 & $\mathrm{Bi}-213$ & $->$ & Po-213 \\
\hline $1.39 \mathrm{E}-06$ & 89.639 & $\mathrm{Bi}-213$ & $->$ & Po-213 \\
\hline $1.39 \mathrm{E}-06$ & 328.69 & $U-233$ & $->$ & Th-229 \\
\hline $1.39 \mathrm{E}-06$ & 125.398 & $U-233$ & $->$ & Th-229 \\
\hline $1.39 \mathrm{E}-06$ & 205.99 & $U-233$ & $->$ & Th-229 \\
\hline $1.34 \mathrm{E}-06$ & 272.27 & $U-233$ & $->$ & Th-229 \\
\hline $1.33 \mathrm{E}-06$ & 292.79 & $\mathrm{Bi}-213$ & $->$ & Po-213 \\
\hline $1.33 \mathrm{E}-06$ & 10.381 & Ac- 225 & $->$ & Fr-221 \\
\hline $1.30 \mathrm{E}-06$ & 216.86 & Ac- 225 & $->$ & Fr-221 \\
\hline $1.28 \mathrm{E}-06$ & 78.41 & U-233 & $->$ & Th-229 \\
\hline $1.25 \mathrm{E}-06$ & 86.23 & Th-229 & $->$ & Ra-225 \\
\hline $1.24 \mathrm{E}-06$ & 155.989 & U-233 & $->$ & Th-229 \\
\hline $1.24 \mathrm{E}-06$ & 354 & $U-233$ & $->$ & Th-229 \\
\hline $1.17 \mathrm{E}-06$ & 100.008 & $U-233$ & $->$ & Th-229 \\
\hline $1.17 \mathrm{E}-06$ & 153.31 & $U-233$ & $->$ & Th-229 \\
\hline $1.09 \mathrm{E}-06$ & 56.58 & Th-229 & $->$ & Ra-225 \\
\hline $1.09 \mathrm{E}-06$ & 131.92 & Th-229 & $->$ & Ra-225 \\
\hline $1.07 \mathrm{E}-06$ & 217.61 & U-233 & $->$ & Th-229 \\
\hline $1.07 E-06$ & 236.39 & $U-233$ & $->$ & Th-229 \\
\hline $1.05 \mathrm{E}-06$ & 216.2 & Ac- 225 & $->$ & Fr-221 \\
\hline $1.02 \mathrm{E}-06$ & 10.871 & $\mathrm{Ra}-225$ & $->$ & Ac- 225 \\
\hline $1.01 \mathrm{E}-06$ & 111.56 & Ac- 225 & $->$ & Fr-221 \\
\hline $1.00 \mathrm{E}-06$ & 73.84 & Ac- 225 & $->$ & Fr-221 \\
\hline $9.60 \mathrm{E}-07$ & 157.24 & Ac- 225 & $->$ & Fr-221 \\
\hline $9.60 \mathrm{E}-07$ & 351.782 & U-233 & $->$ & Th-229 \\
\hline 9.17E-07 & 255.92 & $U-233$ & $->$ & Th-229 \\
\hline $9.17 \mathrm{E}-07$ & 176.1 & U-233 & $->$ & Th-229 \\
\hline $8.98 \mathrm{E}-07$ & 87.39 & AC- 225 & $->$ & Fr-221 \\
\hline $8.54 \mathrm{E}-07$ & 1100.06 & $\mathrm{Bi}-213$ & $->$ & Po-213 \\
\hline $8.53 E-07$ & 185.782 & $U-233$ & $->$ & Th-229 \\
\hline $8.53 \mathrm{E}-07$ & 192.12 & U-233 & $->$ & Th-229 \\
\hline $8.36 \mathrm{E}-07$ & 108.36 & $A C-225$ & $->$ & Fr-221 \\
\hline $8.11 \mathrm{E}-07$ & 252.5 & U-233 & $->$ & Th-229 \\
\hline 7.91E-07 & 807.27 & $\mathrm{Bi}-213$ & $->$ & Po-213 \\
\hline
\end{tabular}




\begin{tabular}{|c|c|c|c|c|}
\hline $7.56 \mathrm{E}-07$ & 183.96 & Th-229 & $->$ & Ra-225 \\
\hline 7.47E-07 & 172.35 & $\mathrm{U}-233$ & $->$ & Th-229 \\
\hline 7.43E-07 & 100.599 & Ac- 225 & $->$ & Fr-221 \\
\hline $7.24 \mathrm{E}-07$ & 172.9 & Th-229 & $->$ & Ra-225 \\
\hline 7.04E-07 & 223.357 & U-233 & $->$ & Th-229 \\
\hline 7.04E-07 & 131.2 & $U-233$ & $->$ & Th-229 \\
\hline 7.04E-07 & 63.69 & $U-233$ & $->$ & Th-229 \\
\hline $6.76 \mathrm{E}-07$ & 74.969 & TI-209 & $->$ & $\mathrm{Pb}-209$ \\
\hline $6.16 \mathrm{E}-07$ & 16.361 & Fr-221 & $->$ & At-217 \\
\hline 5.97E-07 & 311.38 & $U-233$ & $->$ & Th-229 \\
\hline $5.88 \mathrm{E}-07$ & 123.76 & Ac-225 & $->$ & Fr-221 \\
\hline 5.59E-07 & 17.349 & Th-229 & $->$ & Ra-225 \\
\hline 5.33E-07 & 184.17 & U-233 & $->$ & Th-229 \\
\hline $5.26 \mathrm{E}-07$ & 42.722 & Th-229 & $->$ & Ra-225 \\
\hline $4.96 \mathrm{E}-07$ & 94.87 & Ac- 225 & $->$ & Fr-221 \\
\hline 4.96E-07 & 13.255 & Ac- 225 & $->$ & Fr-221 \\
\hline 4.91E-07 & 295.2 & U-233 & $->$ & Th-229 \\
\hline 4.80E-07 & 95.265 & Fr-221 & $->$ & At-217 \\
\hline 4.74E-07 & 153.93 & $A c-225$ & $->$ & Fr-221 \\
\hline 4.65E-07 & 82.9 & Ac- 225 & $->$ & Fr-221 \\
\hline 4.60E-07 & 218.15 & Th-229 & $->$ & Ra-225 \\
\hline 4.49E-07 & 195.75 & Ac-225 & $->$ & Fr-221 \\
\hline 4.27E-07 & 228.07 & U-233 & $->$ & Th-229 \\
\hline $4.27 E-07$ & 177.78 & $U-233$ & $->$ & Th-229 \\
\hline 4.27E-07 & 138.5 & $U-233$ & $->$ & Th-229 \\
\hline $4.27 E-07$ & 384 & $U-233$ & $->$ & Th-229 \\
\hline $4.24 \mathrm{E}-07$ & 92.673 & $\mathrm{Bi}-213$ & $->$ & Po-213 \\
\hline 4.03E-07 & 409.1 & Fr-221 & $->$ & At-217 \\
\hline $4.01 \mathrm{E}-07$ & 72.803 & TI-209 & $->$ & $P b-209$ \\
\hline $3.90 E-07$ & 145.15 & Ac- 225 & $->$ & Fr-221 \\
\hline $3.62 \mathrm{E}-07$ & 68.88 & Th-229 & $->$ & $\mathrm{Ra}-225$ \\
\hline $3.41 \mathrm{E}-07$ & 452.43 & Ac- 225 & $->$ & Fr-221 \\
\hline $3.29 E-07$ & 68.14 & Th-229 & $->$ & Ra-225 \\
\hline $3.20 \mathrm{E}-07$ & 479 & U-233 & $->$ & Th-229 \\
\hline $3.20 \mathrm{E}-07$ & 471.3 & $U-233$ & $->$ & Th-229 \\
\hline $3.10 \mathrm{E}-07$ & 253.51 & Ac-225 & $->$ & Fr-221 \\
\hline 2.89E-07 & 14.082 & Ra-225 & $->$ & Ac- 225 \\
\hline 2.67E-07 & 15.878 & $\mathrm{Bi}-213$ & $->$ & Po-213 \\
\hline $2.64 \mathrm{E}-07$ & 659.72 & $\mathrm{Bi}-213$ & $->$ & Po-213 \\
\hline $2.48 \mathrm{E}-07$ & 224.64 & Ac- 225 & $->$ & Fr-221 \\
\hline $2.47 \mathrm{E}-07$ & 10.541 & Tl-209 & $->$ & $\mathrm{Pb}-20 \mathrm{~S}$ \\
\hline $2.41 \mathrm{E}-07$ & 84.789 & TI-209 & $->$ & $P b-209$ \\
\hline $2.34 \mathrm{E}-07$ & 12.703 & TI-209 & $->$ & Pb-209 \\
\hline $2.17 \mathrm{E}-07$ & 149.99 & Fr-221 & $->$ & At-217 \\
\hline $2.17 \mathrm{E}-07$ & 171.29 & Fr-221 & $->$ & At-217 \\
\hline $2.13 \mathrm{E}-07$ & 416.4 & $U-233$ & $->$ & Th-229 \\
\hline $2.13 \mathrm{E}-07$ & 226.74 & $U-233$ & $->$ & Th-229 \\
\hline $2.13 \mathrm{E}-07$ & 449.6 & $U-233$ & $->$ & Th-229 \\
\hline $2.13 \mathrm{E}-07$ & 225 & U-233 & $->$ & Th-229 \\
\hline $2.13 \mathrm{E}-07$ & 284.22 & $U-233$ & $->$ & Th-22 \\
\hline
\end{tabular}




\begin{tabular}{|c|c|c|c|c|}
\hline $1.86 \mathrm{E}-07$ & 100.88 & $A c-225$ & $->$ & Fr-221 \\
\hline $1.86 \mathrm{E}-07$ & 119.92 & Ac- 225 & $->$ & Fr-221 \\
\hline $1.77 \mathrm{E}-07$ & 1003 & U-233 & $->$ & Th-229 \\
\hline $1.77 \mathrm{E}-07$ & 396.65 & $U-233$ & $->$ & Th-229 \\
\hline $1.77 \mathrm{E}-07$ & 402.37 & $U-233$ & $->$ & Th-229 \\
\hline $1.77 \mathrm{E}-07$ & 1119 & U-233 & $->$ & Th-229 \\
\hline $1.77 \mathrm{E}-07$ & 459.77 & $\mathrm{U}-233$ & $->$ & Th-229 \\
\hline $1.73 \mathrm{E}-07$ & 64.23 & Ac- 225 & $->$ & Fr-221 \\
\hline $1.60 \mathrm{E}-07$ & 339.64 & U-233 & $->$ & Th-229 \\
\hline $1.60 \mathrm{E}-07$ & 394.34 & $U-233$ & $->$ & Th-229 \\
\hline $1.55 \mathrm{E}-07$ & 124.81 & $A C-225$ & $->$ & Fr-221 \\
\hline $1.30 \mathrm{E}-07$ & 9.897 & Fr-221 & $->$ & At-217 \\
\hline $1.24 \mathrm{E}-07$ & 135.01 & $A c-225$ & $->$ & Fr-221 \\
\hline $1.18 \mathrm{E}-07$ & 236.32 & Th-229 & $->$ & Ra-225 \\
\hline $1.18 \mathrm{E}-07$ & 25.373 & Th-229 & $->$ & Ra-225 \\
\hline $1.15 \mathrm{E}-07$ & 152.64 & Ac- 225 & $->$ & Fr-221 \\
\hline $1.13 \mathrm{E}-07$ & 406.7 & U-233 & $->$ & Th-229 \\
\hline $1.13 \mathrm{E}-07$ & 540.27 & $U-233$ & $->$ & Th-229 \\
\hline $1.12 \mathrm{E}-07$ & 74.9 & Ac- 225 & $->$ & Fr-221 \\
\hline $1.08 \mathrm{E}-07$ & 279.27 & Ac- 225 & $->$ & Fr-221 \\
\hline $1.08 \mathrm{E}-07$ & 359.09 & Fr-221 & $->$ & At-217 \\
\hline $1.08 \mathrm{E}-07$ & 382.05 & Fr-221 & $->$ & At-217 \\
\hline $1.08 \mathrm{E}-07$ & 118.47 & Fr-221 & $->$ & At-217 \\
\hline $1.07 \mathrm{E}-07$ & 578.47 & $U-233$ & $->$ & Th-229 \\
\hline $1.07 E-07$ & 436.78 & $U-233$ & $->$ & Th-229 \\
\hline $9.29 E-08$ & 96.17 & $A c-225$ & $->$ & Fr-221 \\
\hline $9.29 \mathrm{E}-08$ & 480.5 & $A C-225$ & $->$ & Fr-221 \\
\hline $9.17 \mathrm{E}-08$ & 484.05 & U-233 & $->$ & Th-229 \\
\hline $8.11 \mathrm{E}-08$ & 569.37 & $U-233$ & $->$ & Th-229 \\
\hline 7.82E-08 & 9.658 & $\mathrm{Bi}-213$ & $->$ & Po-213 \\
\hline $6.89 E-08$ & 87.632 & TI-209 & $->$ & Pb-209 \\
\hline $6.40 \mathrm{E}-08$ & 656.97 & U-233 & $->$ & Th-229 \\
\hline $6.19 \mathrm{E}-08$ & 248.7 & Ac- 225 & $->$ & Fr-221 \\
\hline $6.19 E-08$ & 138.2 & Ac- 225 & $->$ & Fr-221 \\
\hline $6.19 \mathrm{E}-08$ & 186.12 & Ac- 225 & $->$ & Fr-221 \\
\hline $6.19 \mathrm{E}-08$ & 198.72 & Ac- 225 & $->$ & Fr-221 \\
\hline $5.88 \mathrm{E}-08$ & 53.82 & $A c-225$ & $->$ & Fr-221 \\
\hline $5.88 E-08$ & 97.2 & Fr-221 & $->$ & At-217 \\
\hline $5.76 \mathrm{E}-08$ & 707.47 & U-233 & $->$ & Th-229 \\
\hline $5.27 E-08$ & 73.6 & $A c-225$ & $->$ & Fr-221 \\
\hline $5.27 \mathrm{E}-08$ & 324.1 & Fr-221 & $->$ & At-217 \\
\hline $5.17 \mathrm{E}-08$ & 12.466 & Fr-221 & $->$ & At-217 \\
\hline $5.12 \mathrm{E}-08$ & 545.1 & U-233 & $->$ & Th-229 \\
\hline $4.96 \mathrm{E}-08$ & 178.39 & Ac- 225 & $->$ & Fr-221 \\
\hline 4.91E-08 & 620.87 & U-233 & $->$ & Th-229 \\
\hline $4.65 E-08$ & 36.65 & Ac- 225 & $->$ & Fr-221 \\
\hline $4.55 E-08$ & 71.71 & Ac- 225 & $->$ & Fr-221 \\
\hline $4.48 E-08$ & 867.9 & U-233 & $->$ & Th-229 \\
\hline $4.34 \mathrm{E}-08$ & 126.23 & $A c-225$ & $->$ & Fr-221 \\
\hline $4.21 \mathrm{E}-08$ & 14.871 & TI-209 & $->$ & Pb-209 \\
\hline
\end{tabular}




\begin{tabular}{|c|c|c|c|c|}
\hline $4.03 E-08$ & 240.76 & Ac-225 & $\rightarrow$ & Fr-221 \\
\hline $3.35 E-08$ & 49.2 & $A c-225$ & $->$ & Fr-221 \\
\hline $3.10 \mathrm{E}-08$ & 285.5 & Ac- 225 & $->$ & Fr-221 \\
\hline $3.10 \mathrm{E}-08$ & 513.5 & Ac- 225 & $->$ & Fr-221 \\
\hline $3.10 \mathrm{E}-08$ & 526.03 & Ac- 225 & $->$ & Fr-221 \\
\hline $3.10 \mathrm{E}-08$ & 70.92 & Ac- 225 & $->$ & Fr-221 \\
\hline $2.79 E-08$ & 38.51 & Ac- 225 & $->$ & Fr-221 \\
\hline $2.79 \mathrm{E}-08$ & 170.91 & $A c-225$ & $->$ & Fr-221 \\
\hline $2.79 E-08$ & 103.44 & Ac- 225 & $->$ & Fr-221 \\
\hline $2.79 E-08$ & 282.54 & Fr-221 & $->$ & At-217 \\
\hline $2.13 E-08$ & 200.67 & $U-233$ & $->$ & Th-229 \\
\hline $2.03 E-08$ & 12.085 & $\mathrm{Bi}-213$ & $->$ & Po-213 \\
\hline $1.58 \mathrm{E}-08$ & 69.85 & Ac- 225 & $->$ & Fr-221 \\
\hline $1.33 \mathrm{E}-08$ & 129.19 & $A c-225$ & $->$ & $\mathrm{Fr}-221$ \\
\hline $1.30 \mathrm{E}-08$ & 57.77 & Ac- 225 & $->$ & Fr-221 \\
\hline $1.27 \mathrm{E}-08$ & 9.185 & Tl-209 & $->$ & Pb-209 \\
\hline $1.24 \mathrm{E}-08$ & 323.81 & $\mathrm{Bi}-213$ & $->$ & TI-209 \\
\hline 4.65E-09 & 25.98 & Ac- 225 & $->$ & Fr-221 \\
\hline $3.41 \mathrm{E}-09$ & 11.349 & TI-209 & $->$ & $\mathrm{Pb}-209$ \\
\hline 8.70E-10 & 72.873 & $\mathrm{Bi}-213$ & $->$ & TI-209 \\
\hline $5.35 E-10$ & 70.832 & $\mathrm{Bi}-213$ & $->$ & TI-209 \\
\hline $3.34 \mathrm{E}-10$ & 82.434 & $\mathrm{Bi}-213$ & $->$ & TI-209 \\
\hline $3.34 \mathrm{E}-10$ & 10.259 & $\mathrm{Bi}-213$ & $->$ & TI-209 \\
\hline $2.68 \mathrm{E}-10$ & 12.311 & $\mathrm{Bi}-213$ & $->$ & TI-209 \\
\hline 8.70E-11 & 85.185 & $\mathrm{Bi}-213$ & $->$ & TI-209 \\
\hline $5.35 E-11$ & 14.402 & $\mathrm{Bi}-213$ & $->$ & TI-209 \\
\hline $1.54 \mathrm{E}-11$ & 8.953 & $\mathrm{Bi}-213$ & $->$ & TI-209 \\
\hline $4.01 \mathrm{E}-12$ & 10.994 & $\mathrm{Bi}-213$ & $->$ & TI-209 \\
\hline Am-243 & $100 \%$ & & $\mathrm{Ag}$ & 5 years \\
\hline
\end{tabular}

$811 \quad$ lines computed.

Sorted by Energy

\begin{tabular}{|c|c|c|c|c|c|c|c|}
\hline Energy & Intensity & \multicolumn{6}{|c|}{$\begin{array}{l}\text { Initial Isotopes and } \\
\text { Decays }\end{array}$} \\
\hline $\mathrm{keV}$ & $\mathrm{ph} / \mathrm{s} / \mathrm{gm}$ & & & & & & \\
\hline 8.953 & $6.65 E-13$ & $\mathrm{Bi}-211$ & $->$ & TI-207 & & & \\
\hline 9.185 & $1.36 \mathrm{E}-15$ & TI-207 & $->$ & $\mathrm{Pb}-207$ & & & \\
\hline 9.3 & $4.48 \mathrm{E}-15$ & Ac- 227 & $->$ & Th-227 & & & \\
\hline 9.419 & $5.89 E-13$ & $\mathrm{~Pb}-211$ & $->$ & $\mathrm{Bi}-211$ & & & \\
\hline 9.658 & $6.70 \mathrm{E}-13$ & $\mathrm{Rn}-219$ & $->$ & Рo-215 & & & \\
\hline 10.137 & $1.73 \mathrm{E}-11$ & Ra-223 & $->$ & $\mathrm{Rn}-219$ & & & \\
\hline 10.259 & $1.35 \mathrm{E}-11$ & $\mathrm{Bi}-211$ & $->$ & TI-207 & & & \\
\hline 10.541 & $2.73 \mathrm{E}-14$ & TI-207 & $->$ & Pb-207 & & & \\
\hline 10.622 & $3.27 \mathrm{E}-11$ & Fr-223 & $->$ & Ra-223, & Th-227 & $->$ & $\mathrm{Ra}-223$ \\
\hline 10.828 & $1.15 \mathrm{E}-11$ & $\mathrm{~Pb}-211$ & $->$ & $\mathrm{Bi}-211$ & & & \\
\hline
\end{tabular}




\begin{tabular}{|c|c|c|c|c|c|c|c|}
\hline 10.871 & $1.09 \mathrm{E}-09$ & $\mathrm{~Pa}-231$ & $->$ & $A C-227$ & & & \\
\hline 10.994 & $1.75 \mathrm{E}-13$ & $\mathrm{Bi}-211$ & $->$ & TI-207 & & & \\
\hline 11.118 & $2.60 \mathrm{E}-05$ & Ac- -227 & $->$ & Th-227, & $U-235$ & $->$ & Th-231 \\
\hline 11.119 & $1.30 \mathrm{E}-11$ & Rn-219 & $->$ & Po-215 & & & \\
\hline 11.349 & $3.54 \mathrm{E}-16$ & TI-207 & $->$ & $\mathrm{Pb}-207$ & & & \\
\hline 11.372 & 7.78E-05 & Th-231 & $->$ & $\mathrm{Pa}-231$ & & & \\
\hline 11.712 & $1.86 \mathrm{E}-13$ & $\mathrm{~Pb}-211$ & $->$ & $\mathrm{Bi}-211$ & & & \\
\hline 11.713 & $3.20 \mathrm{E}-10$ & $\mathrm{Ra}-223$ & $->$ & $\mathrm{Rn}-219$ & & & \\
\hline 12.085 & $2.38 \mathrm{E}-13$ & $R n-219$ & $->$ & Po-215 & & & \\
\hline 12.124 & $1.09 E+08$ & $N p-239$ & $->$ & Pu-239 & & & \\
\hline 12.313 & $1.23 \mathrm{E}-11$ & $\mathrm{Bi}-211$ & $->$ & TI-207 & & & \\
\hline 12.325 & $5.68 \mathrm{E}-10$ & Fr-223 & $->$ & Ra-223, & Th-227 & $->$ & $\mathrm{Ra}-223$ \\
\hline 12.636 & $2.00 \mathrm{E}-08$ & Pa-231 & $->$ & $A c-227$ & & & \\
\hline 12.703 & $2.48 \mathrm{E}-14$ & TI-207 & $->$ & $\mathrm{Pb}-207$ & & & \\
\hline 12.855 & $4.55 \mathrm{E}-12$ & $\mathrm{Ra}-223$ & $->$ & $\mathrm{Rn}-219$ & & & \\
\hline 12.952 & 5.71E-04 & Ac- -227 & $->$ & Th-227, & $U-235$ & $->$ & Th-231 \\
\hline 13.082 & $1.27 \mathrm{E}-11$ & $\mathrm{~Pb}-211$ & $->$ & $\mathrm{Bi}-211$ & & & \\
\hline 13.495 & $1.43 \mathrm{E}-11$ & $\mathrm{Rn}-219$ & $->$ & Po-215 & & & \\
\hline 13.662 & $1.20 \mathrm{E}-11$ & Fr-223 & $->$ & Ra-223, & Th-227 & $->$ & $\mathrm{Ra}-223$ \\
\hline 13.724 & 1.27E-03 & Th-231 & $->$ & $\mathrm{Pa}-231$ & & & \\
\hline 14.082 & $3.37 \mathrm{E}-10$ & $\mathrm{~Pa}-231$ & $->$ & $A c-227$ & & & \\
\hline 14.262 & $1.74 \mathrm{E}+09$ & $\mathrm{~Np}-239$ & $->$ & Pu-239 & & & \\
\hline 14.341 & $3.08 \mathrm{E}-10$ & Ra-223 & $->$ & $\mathrm{Rn}-219$ & & & \\
\hline 14.407 & $2.25 \mathrm{E}-12$ & $\mathrm{Bi}-211$ & $->$ & TI-207 & & & \\
\hline 14.511 & 5.71E-06 & Ac- -227 & $->$ & Th-227, & U-235 & $->$ & Th-231 \\
\hline 14.886 & $4.54 \mathrm{E}-15$ & TI-207 & $->$ & $\mathrm{Pb}-207$ & & & \\
\hline 14.953 & $1.04 \mathrm{E}-05$ & Th-231 & $->$ & $\mathrm{Pa}-231$ & & & \\
\hline 15.2 & $1.21 \mathrm{E}-12$ & $A C-227$ & $->$ & Th-227 & & & \\
\hline 15.213 & $8.80 \mathrm{E}-12$ & Fr-223 & $->$ & Ra-223 & & & \\
\hline 15.218 & $6.92 \mathrm{E}-10$ & Th-227 & $->$ & Ra-223 & & & \\
\hline 15.397 & $2.79 \mathrm{E}-12$ & $\mathrm{~Pb}-211$ & $->$ & $\mathrm{Bi}-211$ & & & \\
\hline 15.69 & $2.28 \mathrm{E}-08$ & $\mathrm{~Pa}-231$ & $->$ & $A c-227$ & & & \\
\hline 15.856 & $2.89 \mathrm{E}-12$ & $R n-219$ & $->$ & Po-215 & & & \\
\hline 16.074 & 5.17E-12 & Ac- 227 & $->$ & Th-227 & & & \\
\hline 16.119 & 3.90E-04 & U-235 & $->$ & Th-231 & & & \\
\hline 16.333 & $2.80 E+07$ & $N p-239$ & $->$ & Pu-239 & & & \\
\hline 16.397 & $3.46 \mathrm{E}-10$ & $\mathrm{~Pa}-231$ & $->$ & Ac-227 & & & \\
\hline 16.559 & $9.59 E-04$ & Th-231 & $->$ & $\mathrm{Pa}-231$ & & & \\
\hline 16.918 & $6.09 \mathrm{E}-11$ & $\mathrm{Ra}-223$ & $->$ & $\mathrm{Rn}-219$ & & & \\
\hline 17.187 & $5.70 \mathrm{E}-06$ & Th-231 & $->$ & Pa-231 & & & \\
\hline 17.997 & $1.58 \mathrm{E}-10$ & Th-227 & $->$ & Ra-223 & & & \\
\hline 18.016 & $1.99 \mathrm{E}-12$ & Fr-223 & $->$ & Ra-223 & & & \\
\hline 18.08 & $1.84 \mathrm{E}+09$ & $\mathrm{~Np}-239$ & $->$ & Pu-239 & & & \\
\hline 18.607 & 5.38E-09 & $\mathrm{Pa}-231$ & $->$ & $A c-227$ & & & \\
\hline 18.998 & $3.01 \mathrm{E}-10$ & $\mathrm{~Pa}-231$ & $->$ & Ac- 227 & & & \\
\hline 19.118 & $6.75 \mathrm{E}-05$ & U-235 & $->$ & Th-231 & & & \\
\hline 19.308 & $1.38 \mathrm{E}-12$ & Ac- 227 & $->$ & Th-227 & & & \\
\hline 19.811 & $1.92 \mathrm{E}-04$ & Th-231 & $->$ & $\mathrm{Pa}-231$ & & & \\
\hline 20.27 & $6.96 \mathrm{E}-12$ & Fr-223 & $->$ & Ra-223, & Th-227 & $\rightarrow$ & Ra-223 \\
\hline 21.622 & $4.13 E+08$ & $\mathrm{~Np}-239$ & $->$ & Pu-239 & & & \\
\hline
\end{tabular}




$\begin{array}{cc}23.55 & 1.55 \mathrm{E}-13 \\ 24.5 & 1.21 \mathrm{E}-13 \\ 24.56 & 2.73 \mathrm{E}-11 \\ 25.464 & 8.20 \mathrm{E}-11 \\ 25.642 & 3.79 \mathrm{E}-04 \\ 27.396 & 8.48 \mathrm{E}-09 \\ 29.58 & 2.09 \mathrm{E}-13 \\ 29.869 & 3.31 \mathrm{E}-12 \\ 29.996 & 8.38 \mathrm{E}-11 \\ 30.08 & 1.11 \mathrm{E}+00 \\ 31.04 & 8.20 \mathrm{E}-12 \\ 31.136 & 4.87 \mathrm{E}+06 \\ 31.566 & 2.64 \mathrm{E}-12 \\ 31.58 & 6.38 \mathrm{E}-12 \\ 31.585 & 4.16 \mathrm{E}-07 \\ 31.87 & 3.14 \mathrm{E}-15 \\ 33.52 & 4.61 \mathrm{E}-13 \\ 35.884 & 1.46 \mathrm{E}-11 \\ 38.235 & 1.36 \mathrm{E}-10 \\ 38.66 & 1.11 \mathrm{E}+02 \\ 39.61 & 1.28 \mathrm{E}-12 \\ 40.006 & 1.09 \mathrm{E}-11 \\ 40.16 & 6.59 \mathrm{E}-13 \\ 40.41 & 1.71 \mathrm{E}+00 \\ 41.13 & 7.79 \mathrm{E}-07 \\ 41.88 & 1.32 \mathrm{E}-12 \\ 41.96 & 1.56 \mathrm{E}-06 \\ 42.06 & 1.74 \mathrm{E}+00 \\ 42.52 & 5.47 \mathrm{E}-12 \\ 42.828 & 1.50 \mathrm{E}-06 \\ 43.03 & 4.28 \mathrm{E}+06 \\ 43.09 & 6.38 \mathrm{E}-12 \\ 43.53 & 1.65 \mathrm{E}-12 \\ 43.537 & 3.76 \mathrm{E}+08 \\ 43.73 & 7.58 \mathrm{E}-12 \\ 44.06 & 1.82 \mathrm{E}-08 \\ 44.08 & 2.31 \mathrm{E}-13 \\ 44.213 & 5.47 \mathrm{E}-11 \\ 44.37 & 4.29 \mathrm{E}-13 \\ 44.665 & 7.67 \mathrm{E}+06 \\ 46.13 & 6.75 \mathrm{E}-16 \\ 46.218 & 7.79 \mathrm{E}+00 \\ 46.393 & 1.90 \mathrm{E}-10 \\ 46.69 & 6.13 \mathrm{E}-01 \\ 47.52 & 3.07 \mathrm{E}-01 \\ 49.87 & 3.30 \mathrm{E}-13 \\ 50.14 & 7.74 \mathrm{E}+06 \\ 50.8 & 2.96 \mathrm{E}-12 \\ & 1.92 \mathrm{E}+05\end{array}$

\begin{tabular}{|c|c|c|c|c|c|}
\hline $\mathrm{Pa}-231$ & $->$ & Ac- 227 & & & \\
\hline Ac- 227 & $->$ & Th-227 & & & \\
\hline $\mathrm{Pa}-231$ & $->$ & $A c-227$ & & & \\
\hline Pa-231 & $->$ & Ac- 227 & & & \\
\hline Th-231 & $->$ & $\mathrm{Pa}-231$ & & & \\
\hline $\mathrm{Pa}-231$ & $->$ & Ac- 227 & & & \\
\hline Fr-223 & $->$ & Ra-223, & Th-227 & $->$ & $\mathrm{Ra}-223$ \\
\hline Fr-223 & $->$ & Ra-223, & Th-227 & $->$ & Ra-223 \\
\hline Pa-231 & $->$ & $A c-227$ & & & \\
\hline Pu-239 & $->$ & U-235 & & & \\
\hline $\mathrm{Pa}-231$ & $->$ & Ac- 227 & & & \\
\hline Am-243 & $->$ & $\mathrm{Np}-239$ & & & \\
\hline Th-227 & $->$ & Ra-223 & & & \\
\hline $\mathrm{Pa}-231$ & $->$ & Ac- 227 & & & \\
\hline U-235 & $->$ & Th-231 & & & \\
\hline Ra-223 & $->$ & $\mathrm{Rn}-219$ & & & \\
\hline Th-227 & $->$ & Ra-223 & & & \\
\hline $\mathrm{Pa}-231$ & $->$ & Ac- 227 & & & \\
\hline Pa-231 & $->$ & $A c-227$ & & & \\
\hline Pu-239 & $->$ & U-235 & & & \\
\hline Рa-231 & $->$ & Ac-227 & & & \\
\hline Pa-231 & $->$ & $A c-227$ & & & \\
\hline Th-227 & $->$ & Ra-223 & & & \\
\hline Pu-239 & $->$ & U-235 & & & \\
\hline U-235 & $->$ & Th-231 & & & \\
\hline Th-227 & $->$ & Ra-223 & & & \\
\hline U-235 & $->$ & Th-231 & & & \\
\hline Pu-239 & $->$ & U-235 & & & \\
\hline $\mathrm{Pa}-231$ & $->$ & $A c-227$ & & & \\
\hline Th-231 & $->$ & Pa-231 & & & \\
\hline Am-243 & $->$ & Np-239 & & & \\
\hline Pa-231 & $->$ & $A c-227$ & & & \\
\hline Th-227 & $->$ & Ra-223 & & & \\
\hline Am-243 & $->$ & Np-239 & & & \\
\hline Fr-223 & $->$ & Ra-223, & Th-227 & $->$ & $\mathrm{Ra}-223$ \\
\hline Th-231 & $->$ & $\mathrm{Pa}-231$ & & & \\
\hline Th-227 & $->$ & Ra-223 & & & \\
\hline $\mathrm{Pa}-231$ & $->$ & Ac- 227 & & & \\
\hline Th-227 & $->$ & Ra-223 & & & \\
\hline Np-239 & $->$ & Pu-239 & & & \\
\hline Ac-227 & $->$ & Fr-223 & & & \\
\hline Pu-239 & $->$ & U-235 & & & \\
\hline Рa-231 & $->$ & Ac-227 & & & \\
\hline Pu-239 & $->$ & U-235 & & & \\
\hline Pu-239 & $->$ & U-235 & & & \\
\hline Fr-223 & $->$ & Ra-223, & Th-227 & $->$ & Ra-223 \\
\hline Np-239 & $->$ & Pu-239 & & & \\
\hline Fr-223 & $->$ & Ra-223, & Th-227 & $->$ & Ra-223 \\
\hline Fr-223 & $->$ & Ra-223, & Th-227 & $->$ & Ra-223 \\
\hline $\mathrm{Am}-243$ & $->$ & Np-239 & & & \\
\hline
\end{tabular}




\begin{tabular}{|c|c|c|c|c|c|c|c|}
\hline 50.82 & $5.27 \mathrm{E}-13$ & Th-227 & $->$ & Ra-223 & & & \\
\hline 50.97 & $1.28 \mathrm{E}-12$ & $\mathrm{~Pa}-231$ & $->$ & Ac-227 & & & \\
\hline 51.179 & $5.19 \mathrm{E}-07$ & $U-235$ & $->$ & Th-231 & & & \\
\hline 51.29 & $9.89 \mathrm{E}-14$ & Fr-223 & $->$ & Ra-223, & Th-227 & $->$ & Ra-223 \\
\hline 51.624 & $2.87 E+02$ & Pu-239 & $->$ & $U-235$ & & & \\
\hline 52.763 & $7.75 \mathrm{E}-11$ & $\mathrm{~Pa}-231$ & $->$ & Ac- 227 & & & \\
\hline 54.02 & $1.79 \mathrm{E}+00$ & Pu-239 & $->$ & $U-235$ & & & \\
\hline 54.18 & $2.64 \mathrm{E}-13$ & Fr-223 & $->$ & Ra-223, & Th-227 & $->$ & Ra-223 \\
\hline 54.632 & $7.38 \mathrm{E}-11$ & $\mathrm{~Pa}-231$ & $->$ & $A c-227$ & & & \\
\hline 55.4 & $6.78 \mathrm{E}+05$ & Am-243 & $->$ & Np-239 & & & \\
\hline 56.03 & $1.65 \mathrm{E}-13$ & Th-227 & $->$ & Ra-223 & & & \\
\hline 56.63 & $2.31 \mathrm{E}-13$ & Th-227 & $->$ & Ra-223 & & & \\
\hline 56.8 & $5.47 \mathrm{E}-12$ & $\mathrm{~Pa}-231$ & $->$ & Ac- 227 & & & \\
\hline 56.825 & $1.19 \mathrm{E}+01$ & Pu-239 & $->$ & U-235 & & & \\
\hline 56.9 & $1.00 \mathrm{E}-11$ & $\mathrm{~Pa}-231$ & $->$ & Ac- 227 & & & \\
\hline 57.2 & $1.37 \mathrm{E}-11$ & $\mathrm{~Pa}-231$ & $->$ & Ac- 227 & & & \\
\hline 57.233 & $2.28 \mathrm{E}-11$ & $\mathrm{~Pa}-231$ & $->$ & Ac- 227 & & & \\
\hline 57.2759 & $1.09 \mathrm{E}+07$ & Np-239 & $->$ & Pu-239 & & & \\
\hline 57.278 & $4.42 \mathrm{E}+05$ & $N p-239$ & $->$ & Pu-239 & & & \\
\hline 58.562 & $1.24 \mathrm{E}-05$ & Th-231 & $->$ & $\mathrm{Pa}-231$ & & & \\
\hline 59.5 & $2.97 \mathrm{E}-13$ & Th-227 & $->$ & Ra-223 & & & \\
\hline 60.526 & $6.38 \mathrm{E}-12$ & $\mathrm{~Pa}-231$ & $->$ & Ac- 227 & & & \\
\hline 61.435 & $2.64 \mathrm{E}-12$ & Th-227 & $->$ & Ra-223 & & & \\
\hline 61.462 & $7.08 \mathrm{E}+07$ & Np-239 & $->$ & Pu-239 & & & \\
\hline 62.05 & $6.59 \mathrm{E}-14$ & Th-227 & $->$ & Ra-223 & & & \\
\hline 62.36 & 7.91E-12 & Th-227 & $->$ & Ra-223 & & & \\
\hline 62.54 & $2.97 \mathrm{E}-13$ & Th-227 & $->$ & Ra-223 & & & \\
\hline 63.7 & $4.56 \mathrm{E}-11$ & $\mathrm{~Pa}-231$ & $->$ & Ac- 227 & & & \\
\hline 63.834 & $5.96 \mathrm{E}-07$ & Th-231 & $->$ & $\mathrm{Pa}-231$ & & & \\
\hline 64.348 & 5.19E-07 & $U-235$ & $->$ & Th-231 & & & \\
\hline 64.37 & $9.23 \mathrm{E}-13$ & Th-227 & $->$ & $\mathrm{Ra}-223$ & & & \\
\hline 65.516 & $2.39 \mathrm{E}-12$ & $\mathrm{~Pb}-211$ & $->$ & $\mathrm{Bi}-211$ & & & \\
\hline 65.7 & $3.49 \mathrm{E}-01$ & Pu-239 & $->$ & $U-235$ & & & \\
\hline 66.1 & $1.98 \mathrm{E}-13$ & Th-227 & $->$ & Ra-223 & & & \\
\hline 66.3 & $2.31 \mathrm{E}-13$ & Th-227 & $->$ & Ra-223 & & & \\
\hline 67.69 & $1.50 \mathrm{E}+00$ & Pu-239 & $->$ & U-235 & & & \\
\hline 67.8462 & $6.63 E+06$ & $\mathrm{~Np}-239$ & $->$ & Pu-239 & & & \\
\hline 68.5 & $1.48 \mathrm{E}-07$ & Th-231 & $->$ & $\mathrm{Pa}-231$ & & & \\
\hline 68.7 & $1.88 \mathrm{E}-13$ & Th-227 & $->$ & Ra-223 & & & \\
\hline 68.73 & $1.27 \mathrm{E}+00$ & Pu-239 & $->$ & U-235 & & & \\
\hline 68.75 & $1.32 \mathrm{E}-12$ & Th-227 & $->$ & $\mathrm{Ra}-223$ & & & \\
\hline 69.13 & $2.65 \mathrm{E}-15$ & Ac- 227 & $->$ & Fr-223 & & & \\
\hline 69.65 & 2.97E-13 & Th-227 & $->$ & Ra-223 & & & \\
\hline 69.83 & $8.20 \mathrm{E}-15$ & Ac- 227 & $->$ & Fr-223 & & & \\
\hline 70.54 & $6.38 \mathrm{E}-12$ & $\mathrm{~Pa}-231$ & $->$ & Ac- 227 & & & \\
\hline 70.832 & $2.31 \mathrm{E}-11$ & $\mathrm{Bi}-211$ & $->$ & TI-207 & & & \\
\hline 71.92 & $1.82 \mathrm{E}-12$ & $\mathrm{~Pa}-231$ & $->$ & Ac- 227 & & & \\
\hline 72.57 & 3.65E-12 & $\mathrm{Pa}-231$ & $->$ & $A c-227$ & & & \\
\hline 72.71 & $2.86 \mathrm{E}-06$ & U-235 & $->$ & Th-231 & & & \\
\hline 72.767 & $6.51 \mathrm{E}-06$ & Th-231 & $->$ & $\mathrm{Pa}-231$ & & & \\
\hline
\end{tabular}




\begin{tabular}{|c|c|c|c|c|c|c|c|}
\hline 72.8 & $9.23 \mathrm{E}-13$ & Th-227 & $->$ & Ra-223 & & & \\
\hline 72.803 & $4.54 \mathrm{E}-14$ & TI-207 & $->$ & Pb-207 & & & \\
\hline 72.873 & $3.91 \mathrm{E}-11$ & $\mathrm{Bi}-211$ & $->$ & TI-207 & & & \\
\hline 73.66 & $6.26 \mathrm{E}-13$ & Th-227 & $->$ & Ra-223 & & & \\
\hline 74.209 & $2.28 \mathrm{E}-11$ & $\mathrm{~Pa}-231$ & $->$ & $A c-227$ & & & \\
\hline 74.672 & $4.42 E+09$ & Am-243 & $->$ & Np-239 & & & \\
\hline 74.8 & $1.56 \mathrm{E}-06$ & U-235 & $->$ & Th-231 & & & \\
\hline 74.814 & 7.94E-12 & $\mathrm{Pb}-211$ & $->$ & $\mathrm{Bi}-211$ & & & \\
\hline 74.89 & 4.02E-01 & Pu-239 & $->$ & U-235 & & & \\
\hline 74.969 & $7.57 E-14$ & TI-207 & $->$ & Pb-207 & & & \\
\hline 75.1 & $2.97 \mathrm{E}-13$ & Th-227 & $->$ & Ra-223 & & & \\
\hline 76.858 & $1.65 \mathrm{E}-11$ & $\mathrm{Rn}-219$ & $->$ & Po-215 & & & \\
\hline 77.107 & $1.33 \mathrm{E}-11$ & $\mathrm{~Pb}-211$ & $->$ & $\mathrm{Bi}-211$ & & & \\
\hline 77.405 & $6.20 \mathrm{E}-11$ & Pa-231 & $->$ & $A c-227$ & & & \\
\hline 77.593 & $4.52 E+00$ & Pu-239 & $->$ & U-235 & & & \\
\hline 78.37 & $1.79 E+00$ & Pu-239 & $->$ & $U-235$ & & & \\
\hline 79.29 & $2.76 \mathrm{E}-11$ & $\mathrm{Rn}-219$ & $->$ & Po-215 & & & \\
\hline 79.72 & 7.33E-11 & Fr-223 & $->$ & Ra-223, & Th-227 & $->$ & $\mathrm{Ra}-223$ \\
\hline 81 & $1.40 \mathrm{E}-12$ & $\mathrm{~Pb}-211$ & $->$ & $\mathrm{Bi}-211$ & & & \\
\hline 81.067 & 4.77E-10 & Ra-223 & $->$ & $\mathrm{Rn}-219$ & & & \\
\hline 81.229 & 2.33E-05 & Th-231 & $->$ & Pa-231 & & & \\
\hline 82.434 & $1.38 \mathrm{E}-11$ & $\mathrm{Bi}-211$ & $->$ & TI-207 & & & \\
\hline 83.787 & $7.91 \mathrm{E}-10$ & Ra-223 & $->$ & $\mathrm{Rn}-219$ & & & \\
\hline 83.81 & $1.77 \mathrm{E}-12$ & $\mathrm{~Pb}-211$ & $->$ & $\mathrm{Bi}-211$ & & & \\
\hline 84.203 & $1.71 \mathrm{E}-04$ & Th-231 & $->$ & $\mathrm{Pa}-231$ & & & \\
\hline 84.979 & $2.70 \mathrm{E}-14$ & Tl-207 & $->$ & $\mathrm{Pb}-207$ & & & \\
\hline 85.185 & $3.88 \mathrm{E}-12$ & $\mathrm{Bi}-211$ & $->$ & TI-207 & & & \\
\hline 85.429 & $6.28 \mathrm{E}-11$ & Fr-223 & $->$ & Ra-223, & Th-227 & $->$ & $\mathrm{Ra}-223$ \\
\hline 86.57 & $2.21 E+07$ & Am-243 & $->$ & $\mathrm{Np}-239$ & & & \\
\hline 87.19 & $4.72 \mathrm{E}-12$ & $\mathrm{~Pb}-211$ & $->$ & $\mathrm{Bi}-211$ & & & \\
\hline 87.632 & $7.88 \mathrm{E}-15$ & TI-207 & $->$ & Pb-207 & & & \\
\hline 87.673 & $4.34 \mathrm{E}-10$ & $\mathrm{~Pa}-231$ & $->$ & $A C-227$ & & & \\
\hline 88.057 & $4.35 E+05$ & $\mathrm{~Np}-239$ & $->$ & Pu-239 & & & \\
\hline 88.2 & $5.27 \mathrm{E}-13$ & $\mathrm{~Pb}-211$ & $->$ & $\mathrm{Bi}-211$ & & & \\
\hline 88.471 & $1.03 \mathrm{E}-10$ & Fr-223 & $->$ & Ra-223, & Th-227 & $->$ & $\mathrm{Ra}-223$ \\
\hline 89.639 & $9.84 \mathrm{E}-12$ & $\mathrm{Rn}-219$ & $->$ & Po-215 & & & \\
\hline 89.85 & $1.12 \mathrm{E}-13$ & Th-227 & $->$ & Ra-223 & & & \\
\hline 89.954 & $2.44 \mathrm{E}-05$ & Th-231 & $->$ & Pa-231 & & & \\
\hline 89.955 & $8.73 \mathrm{E}-05$ & U-235 & $->$ & Th-231 & & & \\
\hline 90.128 & $1.40 \mathrm{E}-12$ & $\mathrm{~Pb}-211$ & $->$ & $\mathrm{Bi}-211$ & & & \\
\hline 90.886 & $7.11 \mathrm{E}-10$ & $\mathrm{~Pa}-231$ & $->$ & Ac- 227 & & & \\
\hline 92.279 & $1.01 \mathrm{E}-05$ & Th-231 & $->$ & $\mathrm{Pa}-231$ & & & \\
\hline 92.673 & $2.98 \mathrm{E}-12$ & $\mathrm{Rn}-219$ & $->$ & Po-215 & & & \\
\hline 93.07 & $1.27 \mathrm{E}-06$ & Th-231 & $->$ & $\mathrm{Pa}-231$ & & & \\
\hline 93.36 & $1.43 E-04$ & U-235 & $->$ & Th-231 & & & \\
\hline 93.93 & $4.61 \mathrm{E}-11$ & Th-227 & $->$ & $\mathrm{Ra}-223$ & & & \\
\hline 94.3 & $3.41 \mathrm{E}-13$ & $\mathrm{~Pb}-211$ & $->$ & $\mathrm{Bi}-211$ & & & \\
\hline 94.66 & $4.44 \mathrm{E}+01$ & Pu-239 & $->$ & U-235 & & & \\
\hline 94.677 & $2.79 E-10$ & Ra-223 & $->$ & Rn-219 & & & \\
\hline 94.89 & $5.58 \mathrm{E}-13$ & $\mathrm{~Pb}-211$ & $->$ & $\mathrm{Bi}-211$ & & & \\
\hline
\end{tabular}




\begin{tabular}{|c|c|c|c|c|c|c|c|}
\hline 94.9 & $3.96 \mathrm{E}-13$ & Th-227 & $->$ & Ra-223 & & & \\
\hline 95.863 & $1.63 \mathrm{E}-05$ & Th-231 & $->$ & $\mathrm{Pa}-231$ & & & \\
\hline 96.06 & $1.98 \mathrm{E}-12$ & Th-227 & $->$ & Ra-223 & & & \\
\hline 96.154 & $2.23 \mathrm{E}-06$ & U-235 & $->$ & Th-231 & & & \\
\hline 96.16 & $3.89 \mathrm{E}-01$ & Pu-239 & $->$ & $U-235$ & & & \\
\hline 96.979 & $8.02 E-11$ & $\mathrm{~Pa}-231$ & $->$ & Ac- 227 & & & \\
\hline 97.3 & $3.57 E-13$ & $\mathrm{~Pb}-211$ & $->$ & $\mathrm{Bi}-211$ & & & \\
\hline 97.907 & $8.92 \mathrm{E}-11$ & Ra-223 & $->$ & $\mathrm{Rn}-219$ & & & \\
\hline 98.43 & $5.90 E+05$ & Am-243 & $->$ & Np-239 & & & \\
\hline 98.441 & $7.21 \mathrm{E}+01$ & Pu-239 & $->$ & U-235 & & & \\
\hline 98.79 & $1.29 E+01$ & Pu-239 & $->$ & $U-235$ & & & \\
\hline 99.28 & $3.11 \mathrm{E}-06$ & Th-231 & $->$ & Pa-231 & & & \\
\hline 99.522 & $1.11 \mathrm{E}+09$ & Np-239 & $->$ & Pu-239 & & & \\
\hline 99.56 & $4.29 \mathrm{E}-13$ & $\mathrm{Fr}-223$ & $->$ & Ra-223, & Th-227 & $->$ & Ra-223 \\
\hline 99.64 & $6.59 \mathrm{E}-14$ & Th-227 & $->$ & $\mathrm{Ra}-223$ & & & \\
\hline 99.7 & $1.54 \mathrm{E}-14$ & Ac- 227 & $->$ & Fr-223 & & & \\
\hline 99.915 & 3.69E-11 & $\mathrm{Fr}-223$ & $->$ & Ra-223, & Th-227 & $->$ & Ra-223 \\
\hline 100.31 & 3.30E-12 & Fr-223 & $->$ & Ra-223, & Th-227 & $->$ & Ra-223 \\
\hline 100.96 & $2.92 \mathrm{E}-11$ & $\mathrm{~Pa}-231$ & $->$ & $A C-227$ & & & \\
\hline 101.943 & $5.16 \mathrm{E}+04$ & $\mathrm{~Np}-239$ & $->$ & Pu-239 & & & \\
\hline 102.255 & $1.06 \mathrm{E}-05$ & Th-231 & $->$ & $\mathrm{Pa}-231$ & & & \\
\hline 102.45 & $3.96 \mathrm{E}-14$ & Th-227 & $->$ & Ra-223 & & & \\
\hline 102.613 & $2.55 \mathrm{E}-10$ & $\mathrm{~Pa}-231$ & $->$ & $A c-227$ & & & \\
\hline 103.035 & $2.24 \mathrm{E}+00$ & Pu-239 & $->$ & U-235 & & & \\
\hline 103.341 & $1.21 \mathrm{E}-11$ & Fr-223 & $->$ & Ra-223, & Th-227 & $->$ & Ra-223 \\
\hline 103.734 & $1.76 \mathrm{E}+09$ & Np-239 & $->$ & Pu-239 & & & \\
\hline 103.85 & $5.34 \mathrm{E}-13$ & Ra-223 & $->$ & $\mathrm{Rn}-219$ & & & \\
\hline 104.9 & $1.32 \mathrm{E}-12$ & Th-227 & $->$ & Ra-223 & & & \\
\hline 105.362 & $5.14 \mathrm{E}-05$ & U-235 & $->$ & Th-231 & & & \\
\hline 105.803 & $1.84 \mathrm{E}-07$ & Th-231 & $->$ & $\mathrm{Pa}-231$ & & & \\
\hline 106.1 & $6.75 \mathrm{E}-16$ & Ac- 227 & $->$ & Fr-223 & & & \\
\hline 106.1272 & 1.67E+09 & $\mathrm{Np}-239$ & $->$ & Pu-239 & & & \\
\hline 106.137 & $8.38 \mathrm{E}-11$ & $\mathrm{~Pa}-231$ & $->$ & Ac- 227 & & & \\
\hline 106.488 & $3.54 \mathrm{E}+06$ & Np-239 & $->$ & Pu-239 & & & \\
\hline 106.581 & $4.41 \mathrm{E}-07$ & Th-231 & $->$ & $\mathrm{Pa}-231$ & & & \\
\hline 106.66 & $6.91 \mathrm{E}-13$ & Ra-223 & $->$ & $\mathrm{Rn}-219$ & & & \\
\hline 107.92 & $2.31 \mathrm{E}-13$ & Th-227 & $->$ & $\mathrm{Ra}-223$ & & & \\
\hline 108.166 & 5.91E-06 & Th-231 & $->$ & $\mathrm{Pa}-231$ & & & \\
\hline 108.99 & $1.71 \mathrm{E}-05$ & $U-235$ & $->$ & Th-231 & & & \\
\hline 109.1 & $1.98 \mathrm{E}-13$ & Th-227 & $->$ & $\mathrm{Ra}-223$ & & & \\
\hline 109.16 & $4.00 \mathrm{E}-05$ & $U-235$ & $->$ & Th-231 & & & \\
\hline 110.421 & $8.41 E+00$ & Pu-239 & $->$ & $U-235$ & & & \\
\hline 110.58 & $1.65 \mathrm{E}-13$ & Th-227 & $->$ & Ra-223 & & & \\
\hline 110.8 & $1.51 \mathrm{E}-12$ & $\mathrm{Ra}-223$ & $->$ & $\mathrm{Rn}-219$ & & & \\
\hline 111.3 & $1.64 \mathrm{E}+01$ & Pu-239 & $->$ & U-235 & & & \\
\hline 111.89 & $3.25 \mathrm{E}-01$ & Pu-239 & $->$ & $U-235$ & & & \\
\hline 111.897 & 1.97E-06 & Th-231 & $->$ & $\mathrm{Pa}-231$ & & & \\
\hline 112.54 & $2.64 \mathrm{E}-13$ & Th-227 & $->$ & Ra-223 & & & \\
\hline 113.12 & $4.94 \mathrm{E}-12$ & Th-227 & $->$ & Ra-223 & & & \\
\hline 113.19 & $1.85 \mathrm{E}-11$ & Th-227 & $->$ & Ra-223 & & & \\
\hline
\end{tabular}




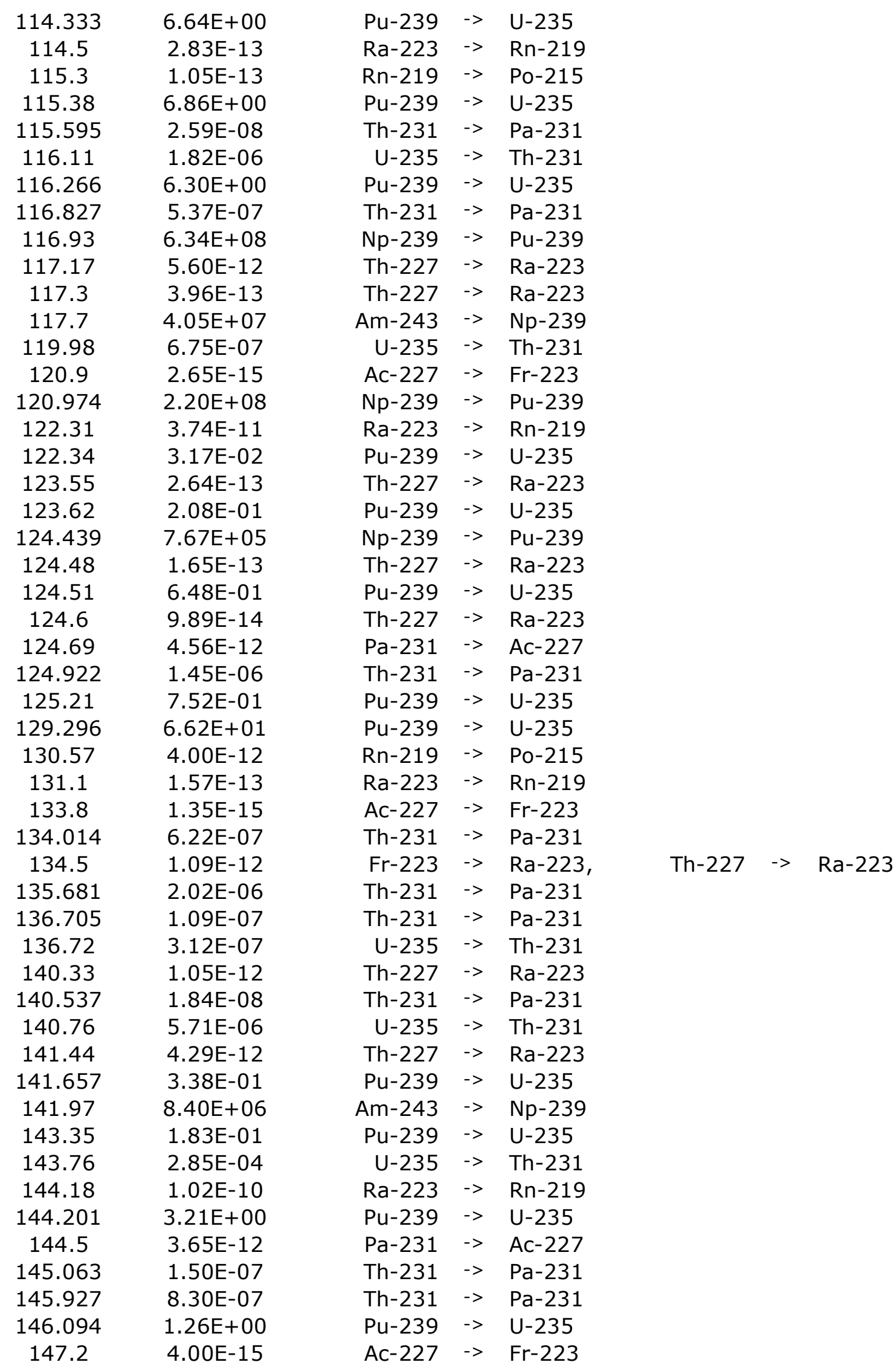




\begin{tabular}{|c|c|c|c|c|c|c|c|}
\hline 150.02 & $3.30 \mathrm{E}-13$ & Th-227 & $->$ & Ra-223 & & & \\
\hline 150.93 & $2.08 \mathrm{E}-06$ & U-235 & $->$ & Th-231 & & & \\
\hline 154.18 & $1.76 \mathrm{E}-10$ & $\mathrm{Ra}-223$ & $->$ & $\mathrm{Rn}-219$ & & & \\
\hline 158.59 & $2.16 \mathrm{E}-11$ & $\mathrm{Ra}-223$ & $->$ & $\mathrm{Rn}-219$ & & & \\
\hline 160.19 & $6.56 \mathrm{E}-02$ & Pu-239 & $->$ & U-235 & & & \\
\hline 161.482 & $1.30 \mathrm{E}+00$ & Pu-239 & $->$ & $U-235$ & & & \\
\hline 162.18 & $2.31 \mathrm{E}-13$ & Th-227 & $->$ & Ra-223 & & & \\
\hline 163.114 & $4.02 E-06$ & Th-231 & $->$ & $\mathrm{Pa}-231$ & & & \\
\hline 163.33 & $1.32 \mathrm{E}-04$ & U-235 & $->$ & Th-231 & & & \\
\hline 164.97 & $1.01 \mathrm{E}-07$ & Th-231 & $->$ & $\mathrm{Pa}-231$ & & & \\
\hline 165.5 & $1.57 \mathrm{E}-13$ & Ra-223 & $->$ & $\mathrm{Rn}-219$ & & & \\
\hline 166.364 & $1.25 E+06$ & Np-239 & $->$ & Pu-239 & & & \\
\hline 167.81 & $3.10 \mathrm{E}-02$ & Pu-239 & $->$ & U-235 & & & \\
\hline 168.19 & $4.61 \mathrm{E}-13$ & Th-227 & $->$ & Ra-223 & & & \\
\hline 169.637 & $3.11 \mathrm{E}-08$ & Th-231 & $->$ & $\mathrm{Pa}-231$ & & & \\
\hline 169.98 & $9.89 \mathrm{E}-14$ & Th-227 & $->$ & Ra-223 & & & \\
\hline 170.14 & $8.85 E+04$ & Am-243 & $->$ & Np-239 & & & \\
\hline 171.21 & $3.30 \mathrm{E}-14$ & Th-227 & $->$ & $\mathrm{Ra}-223$ & & & \\
\hline 171.393 & $1.16 \mathrm{E}+00$ & Pu-239 & $->$ & $U-235$ & & & \\
\hline 171.4 & $1.98 \mathrm{E}-15$ & $A c-227$ & $->$ & $\mathrm{Fr}-223$ & & & \\
\hline 172.27 & $2.60 \mathrm{E}-07$ & U-235 & $->$ & Th-231 & & & \\
\hline 173.38 & $5.64 \mathrm{E}-13$ & Fr-223 & $->$ & Ra-223, & Th-227 & $->$ & $\mathrm{Ra}-223$ \\
\hline 173.7 & $4.23 E-02$ & Pu-239 & $->$ & U-235 & & & \\
\hline 174.157 & $4.75 E-07$ & Th-231 & $->$ & $\mathrm{Pa}-231$ & & & \\
\hline 175.23 & $5.93 E-13$ & Th-227 & $\rightarrow$ & Ra-223 & & & \\
\hline 175.55 & $5.34 \mathrm{E}-13$ & Ra-223 & $->$ & $\mathrm{Rn}-219$ & & & \\
\hline 176.8 & $1.26 \mathrm{E}-13$ & Ra-223 & $->$ & $\mathrm{Rn}-219$ & & & \\
\hline 177.35 & $1.48 \mathrm{E}-12$ & Ra-223 & $->$ & $\mathrm{Rn}-219$ & & & \\
\hline 179.22 & $6.98 \mathrm{E}-01$ & Pu-239 & $->$ & U-235 & & & \\
\hline 179.28 & $6.59 E-14$ & Th-227 & $->$ & Ra-223 & & & \\
\hline 179.69 & $4.80 \mathrm{E}-12$ & $\mathrm{Ra}-223$ & $->$ & $\mathrm{Rn}-219$ & & & \\
\hline 181.716 & $8.18 E+06$ & $\mathrm{~Np}-239$ & $->$ & Pu-239 & & & \\
\hline 182.61 & 8.83E-06 & U-235 & $->$ & Th-231 & & & \\
\hline 183.483 & 8.53E-07 & Th-231 & $->$ & $\mathrm{Pa}-231$ & & & \\
\hline 184.55 & $2.24 \mathrm{E}-02$ & Pu-239 & $->$ & $U-235$ & & & \\
\hline 184.68 & $1.29 \mathrm{E}-12$ & Fr-223 & $->$ & Ra-223, & Th-227 & $->$ & Ra-223 \\
\hline 185.72 & $1.49 \mathrm{E}-03$ & U-235 & $->$ & Th-231 & & & \\
\hline 188.23 & $1.15 \mathrm{E}-01$ & Pu-239 & $->$ & $U-235$ & & & \\
\hline 188.756 & 8.30E-08 & Th-231 & $->$ & $\mathrm{Pa}-231$ & & & \\
\hline 189.36 & $8.78 \mathrm{E}-01$ & Pu-239 & $->$ & $U-235$ & & & \\
\hline 194.94 & $1.64 \mathrm{E}-05$ & U-235 & $->$ & Th-231 & & & \\
\hline 195.3 & $6.19 E+04$ & Am-243 & $->$ & Np-239 & & & \\
\hline 195.679 & $1.13 E+00$ & Pu-239 & $->$ & U-235 & & & \\
\hline 196.87 & $3.91 \mathrm{E}-02$ & Pu-239 & $->$ & U-235 & & & \\
\hline 197.61 & $3.96 \mathrm{E}-13$ & Th-227 & $->$ & Ra-223 & & & \\
\hline 198.9 & $1.09 \mathrm{E}-06$ & U-235 & $->$ & Th-231 & & & \\
\hline 198.96 & $5.10 \mathrm{E}-12$ & Pa-231 & $->$ & Ac- 227 & & & \\
\hline 199.43 & $9.42 \mathrm{E}-14$ & Ra-223 & $->$ & $\mathrm{Rn}-219$ & & & \\
\hline 200.48 & $6.59 E-13$ & Th-227 & $->$ & Ra-223 & & & \\
\hline 201.64 & $6.59 E-13$ & Th-227 & $->$ & Ra-223 & & & \\
\hline
\end{tabular}




\begin{tabular}{|c|c|c|c|c|c|c|c|}
\hline 202.11 & $2.81 \mathrm{E}-05$ & $U-235$ & $->$ & Th-231 & & & \\
\hline 202.4 & $1.98 \mathrm{E}-13$ & Th-227 & $->$ & Ra-223 & & & \\
\hline 203.54 & $5.92 E+00$ & Pu-239 & $->$ & $U-235$ & & & \\
\hline 204.2 & $7.58 \mathrm{E}-12$ & Fr-223 & $->$ & Ra-223, & Th-227 & $->$ & $\mathrm{Ra}-223$ \\
\hline 204.95 & $4.94 \mathrm{E}-12$ & Fr-223 & $->$ & Ra-223, & Th-227 & $->$ & $\mathrm{Ra}-223$ \\
\hline 205.31 & $1.30 \mathrm{E}-04$ & U-235 & $->$ & Th-231 & & & \\
\hline 206.04 & $7.58 \mathrm{E}-12$ & Fr-223 & $->$ & $\mathrm{Ra}-223$, & Th-227 & $->$ & $\mathrm{Ra}-223$ \\
\hline 209.7554 & $2.45 E+08$ & $\mathrm{~Np}-239$ & $->$ & Pu-239 & & & \\
\hline 210.58 & $3.73 \mathrm{E}-11$ & Fr-223 & $->$ & Ra-223, & Th-227 & $->$ & Ra-223 \\
\hline 212.56 & $2.31 \mathrm{E}-12$ & Th-227 & $->$ & $\mathrm{Ra}-223$ & & & \\
\hline 212.69 & $5.60 \mathrm{E}-13$ & Th-227 & $->$ & Ra-223 & & & \\
\hline 215.305 & 7.01E-07 & U-235 & $->$ & Th-231 & & & \\
\hline 217.934 & $1.04 \mathrm{E}-06$ & Th-231 & $->$ & $\mathrm{Pa}-231$ & & & \\
\hline 218.45 & $1.06 \mathrm{E}-02$ & Pu-239 & $->$ & U-235 & & & \\
\hline 218.77 & $1.98 \mathrm{E}-12$ & Th-227 & $->$ & Ra-223 & & & \\
\hline 218.94 & $1.32 \mathrm{E}-12$ & Th-227 & $->$ & Ra-223 & & & \\
\hline 220.2 & $2.19 \mathrm{E}-13$ & $\mathrm{~Pa}-231$ & $->$ & Ac- 227 & & & \\
\hline 220.38 & $1.04 \mathrm{E}-12$ & $\mathrm{Ra}-223$ & $->$ & $\mathrm{Rn}-219$ & & & \\
\hline 221.38 & $3.12 \mathrm{E}-06$ & U-235 & $->$ & Th-231 & & & \\
\hline 221.9 & $9.52 \mathrm{E}-13$ & $\mathrm{Rn}-219$ & $->$ & Po-215 & & & \\
\hline 224.68 & $4.94 \mathrm{E}-13$ & Fr-223 & $->$ & Ra-223, & Th-227 & $->$ & $\mathrm{Ra}-223$ \\
\hline 225.39 & $1.72 \mathrm{E}-01$ & Pu-239 & $->$ & U-235 & & & \\
\hline 226.382 & $2.51 E+07$ & Np-239 & $->$ & Pu-239 & & & \\
\hline 226.77 & $2.37 \mathrm{E}-12$ & $\mathrm{~Pa}-231$ & $->$ & $A c-227$ & & & \\
\hline 228.16 & $2.19 \mathrm{E}-13$ & $\mathrm{~Pa}-231$ & $->$ & Ac- 227 & & & \\
\hline 228.1865 & $7.89 E+08$ & Np-239 & $->$ & Pu-239 & & & \\
\hline 228.77 & $2.08 \mathrm{E}-07$ & U-235 & $->$ & Th-231 & & & \\
\hline 230.17 & $2.31 \mathrm{E}-14$ & Th-227 & $->$ & Ra-223 & & & \\
\hline 233.5 & 7.53E-07 & U-235 & $->$ & Th-231 & & & \\
\hline 234.82 & $1.62 \mathrm{E}-11$ & Fr-223 & $->$ & Ra-223, & Th-227 & $->$ & Ra-223 \\
\hline 235.97 & $3.69 \mathrm{E}-10$ & Fr-223 & $->$ & $\mathrm{Ra}-223$, & Th-227 & $->$ & $\mathrm{Ra}-223$ \\
\hline 235.985 & $2.39 \mathrm{E}-07$ & Th-231 & $->$ & $\mathrm{Pa}-231$ & & & \\
\hline 237.75 & $1.59 \mathrm{E}-01$ & Pu-239 & $->$ & U-235 & & & \\
\hline 240.25 & 7.26E-09 & Th-231 & $->$ & $\mathrm{Pa}-231$ & & & \\
\hline 240.85 & $1.40 \mathrm{E}-06$ & U-235 & $->$ & Th-231 & & & \\
\hline 242 & 7.29E-12 & Pa-231 & $->$ & Ac- 227 & & & \\
\hline 242.12 & $8.78 \mathrm{E}-02$ & Pu-239 & $->$ & U-235 & & & \\
\hline 242.508 & $2.18 \mathrm{E}-08$ & Th-231 & $->$ & $\mathrm{Pa}-231$ & & & \\
\hline 243.17 & $4.28 \mathrm{E}-11$ & $\mathrm{~Pa}-231$ & $->$ & Ac- 227 & & & \\
\hline 243.36 & $2.45 \mathrm{E}-01$ & Pu-239 & $->$ & U-235 & & & \\
\hline 244.86 & 5.50E-02 & Pu-239 & $->$ & U-235 & & & \\
\hline 245.19 & $2.83 \mathrm{E}-13$ & $\mathrm{Ra}-223$ & $->$ & $\mathrm{Rn}-219$ & & & \\
\hline 245.438 & $6.38 \mathrm{E}-12$ & $\mathrm{~Pa}-231$ & $->$ & Ac- 227 & & & \\
\hline 245.77 & $6.29 E-12$ & $\mathrm{~Pa}-231$ & $->$ & Ac- 227 & & & \\
\hline 246.07 & $3.63 \mathrm{E}-13$ & Th-227 & $->$ & Ra-223 & & & \\
\hline 246.84 & $1.38 \mathrm{E}-06$ & U-235 & $->$ & Th-231 & & & \\
\hline 248.88 & 7.93E-02 & Pu-239 & $->$ & U-235 & & & \\
\hline 249.36 & $2.31 \mathrm{E}-13$ & Th-227 & $->$ & $\mathrm{Ra}-223$ & & & \\
\hline 249.49 & $1.16 \mathrm{E}-12$ & Ra-223 & $->$ & $\mathrm{Rn}-219$ & & & \\
\hline 249.56 & $2.02 E-08$ & Th-231 & $->$ & $\mathrm{Pa}-231$ & & & \\
\hline
\end{tabular}




\begin{tabular}{|c|c|c|c|c|c|c|c|}
\hline 250.12 & $1.22 \mathrm{E}-11$ & Fr-223 & $->$ & Ra-223, & Th-227 & $->$ & $\mathrm{Ra}-223$ \\
\hline 250.33 & $4.29 E-12$ & Th-227 & $->$ & $\mathrm{Ra}-223$ & & & \\
\hline 250.43 & $1.69 \mathrm{E}-08$ & Th-231 & $->$ & $\mathrm{Pa}-231$ & & & \\
\hline 251.12 & $1.10 \mathrm{E}-12$ & Ra-223 & $->$ & $\mathrm{Rn}-219$ & & & \\
\hline 251.58 & $9.73 E-13$ & Ra-223 & $->$ & $\mathrm{Rn}-219$ & & & \\
\hline 252.46 & $3.63 \mathrm{E}-12$ & Th-227 & $->$ & Ra-223 & & & \\
\hline 254.421 & $7.30 \mathrm{E}+06$ & Np-239 & $->$ & Pu-239 & & & \\
\hline 254.66 & $2.64 \mathrm{E}-11$ & Fr-223 & $->$ & Ra-223, & Th-227 & $->$ & Ra-223 \\
\hline 254.98 & $1.00 \mathrm{E}-12$ & Ra-223 & $->$ & $\mathrm{Rn}-219$ & & & \\
\hline 255.384 & $8.46 \mathrm{E}-01$ & Pu-239 & $->$ & $U-235$ & & & \\
\hline 255.74 & $1.57 \mathrm{E}-13$ & $\mathrm{Ra}-223$ & $->$ & $\mathrm{Rn}-219$ & & & \\
\hline 255.858 & $9.11 \mathrm{E}-11$ & Pa-231 & $->$ & Ac- 227 & & & \\
\hline 256.24 & $2.21 \mathrm{E}-10$ & Fr-223 & $->$ & Ra-223, & Th-227 & $->$ & Ra-223 \\
\hline 258.43 & $2.10 \mathrm{E}-12$ & Pa-231 & $->$ & $A c-227$ & & & \\
\hline 260.29 & $1.66 \mathrm{E}-10$ & Pa-231 & $->$ & $A c-227$ & & & \\
\hline 260.4 & $1.88 \mathrm{E}-13$ & Ra-223 & $->$ & $\mathrm{Rn}-219$ & & & \\
\hline 262.72 & $3.30 \mathrm{E}-12$ & Fr-223 & $->$ & Ra-223, & Th-227 & $->$ & Ra-223 \\
\hline 263.9 & $2.70 \mathrm{E}-01$ & Pu-239 & $->$ & $U-235$ & & & \\
\hline 265.81 & 3.17E-02 & Pu-239 & $->$ & U-235 & & & \\
\hline 266.3 & $8.24 \mathrm{E}-14$ & Th-227 & $->$ & Ra-223 & & & \\
\hline 266.483 & $1.56 \mathrm{E}-07$ & U-235 & $->$ & Th-231 & & & \\
\hline 267.62 & $3.01 \mathrm{E}-08$ & Th-231 & $->$ & Pa-231 & & & \\
\hline 267.82 & $3.30 \mathrm{E}-13$ & Th-227 & $->$ & Ra-223 & & & \\
\hline 269.39 & $4.27 \mathrm{E}-10$ & Ra-223 & $->$ & $\mathrm{Rn}-219$ & & & \\
\hline 270.34 & $2.64 \mathrm{E}-13$ & Th-227 & $->$ & Ra-223 & & & \\
\hline 270.76 & $1.05 \mathrm{E}-12$ & Th-227 & $->$ & Ra-223 & & & \\
\hline 271.13 & $3.14 \mathrm{E}-10$ & $\mathrm{Rn}-219$ & $->$ & Po-215 & & & \\
\hline 272.852 & $5.53 E+06$ & $\mathrm{~Np}-239$ & $->$ & Pu-239 & & & \\
\hline 272.95 & $1.62 \mathrm{E}-11$ & $\mathrm{Fr}-223$ & $->$ & Ra-223, & Th-227 & $->$ & $\mathrm{Ra}-223$ \\
\hline 273.17 & $5.19 \mathrm{E}-11$ & $\mathrm{~Pa}-231$ & $->$ & Ac- 227 & & & \\
\hline 274.14 & $7.78 \mathrm{E}-10$ & Th-231 & $->$ & $\mathrm{Pa}-231$ & & & \\
\hline 275.2 & $4.94 \mathrm{E}-14$ & Th-227 & $->$ & Ra-223 & & & \\
\hline 275.47 & $1.27 \mathrm{E}-07$ & U-235 & $->$ & Th-231 & & & \\
\hline 277.17 & $5.65 E-11$ & $\mathrm{~Pa}-231$ & $->$ & Ac- 227 & & & \\
\hline 277.43 & $3.01 \mathrm{E}-12$ & Pa-231 & $->$ & Ac-227 & & & \\
\hline 277.6016 & $1.05 \mathrm{E}+09$ & Np-239 & $->$ & Pu-239 & & & \\
\hline 279.7 & $2.31 \mathrm{E}-12$ & Fr-223 & $->$ & Ra-223, & Th-227 & $->$ & $\mathrm{Ra}-223$ \\
\hline 281 & $2.31 \mathrm{E}-13$ & Th-227 & $->$ & Ra-223 & & & \\
\hline 281.12 & $2.22 \mathrm{E}-02$ & Pu-239 & $->$ & U-235 & & & \\
\hline 281.31 & 5.27E-12 & Th-227 & $->$ & Ra-223 & & & \\
\hline 281.45 & $1.56 \mathrm{E}-07$ & U-235 & $->$ & Th-231 & & & \\
\hline 282.98 & $1.30 \mathrm{E}-07$ & $U-235$ & $->$ & Th-231 & & & \\
\hline 283.673 & $1.45 \mathrm{E}-09$ & $\mathrm{~Pa}-231$ & $->$ & $A c-227$ & & & \\
\hline 284.24 & $1.65 \mathrm{E}-12$ & Fr-223 & $->$ & Ra-223, & Th-227 & $->$ & $\mathrm{Ra}-223$ \\
\hline 285.27 & $1.59 \mathrm{E}-02$ & Pu-239 & $->$ & U-235 & & & \\
\hline 285.4624 & $5.45 \mathrm{E}+07$ & $N p-239$ & $->$ & Pu-239 & & & \\
\hline 285.48 & $1.81 \mathrm{E}-12$ & Th-227 & $->$ & $\mathrm{Ra}-223$ & & & \\
\hline 286.11 & $5.24 \mathrm{E}-11$ & Fr-223 & $->$ & Ra-223, & Th-227 & $->$ & Ra-223 \\
\hline 288.15 & $4.83 \mathrm{E}-12$ & Ra-223 & $->$ & Rn-219 & & & \\
\hline 289.5 & $2.97 E-13$ & Th-227 & $->$ & Ra-223 & & & \\
\hline
\end{tabular}




\begin{tabular}{|c|c|c|c|c|c|c|c|}
\hline 289.57 & $1.82 \mathrm{E}-07$ & U-235 & $->$ & Th-231 & & & \\
\hline 289.6 & $4.78 \mathrm{E}-13$ & Fr-223 & $->$ & Ra-223, & Th-227 & $->$ & Ra-223 \\
\hline 291.72 & $3.64 \mathrm{E}-07$ & $U-235$ & $->$ & Th-231 & & & \\
\hline 292.3 & $1.98 \mathrm{E}-12$ & Fr-223 & $->$ & Ra-223, & Th-227 & $->$ & Ra-223 \\
\hline 293.8 & $2.06 \mathrm{E}-12$ & $\mathrm{Rn}-219$ & $->$ & Po-215 & & & \\
\hline 296.54 & $1.42 \mathrm{E}-11$ & Th-227 & $->$ & Ra-223 & & & \\
\hline 297.46 & $5.27 E-01$ & Pu-239 & $->$ & U-235 & & & \\
\hline 299.97 & $6.92 \mathrm{E}-11$ & Fr-223 & $->$ & $\mathrm{Ra}-223$, & Th-227 & $->$ & Ra-223 \\
\hline 300.07 & 2.17E-09 & Pa-231 & $->$ & $A c-227$ & & & \\
\hline 300.34 & $6.59 \mathrm{E}-12$ & Th-227 & $->$ & Ra-223 & & & \\
\hline 301.72 & $1.30 \mathrm{E}-07$ & $U-235$ & $->$ & Th-231 & & & \\
\hline 302.638 & $5.83 \mathrm{E}-10$ & $\mathrm{~Pa}-231$ & $->$ & $A c-227$ & & & \\
\hline 302.671 & $1.56 \mathrm{E}-09$ & Pa-231 & $->$ & $A C-227$ & & & \\
\hline 302.89 & $6.03 E-02$ & Pu-239 & $->$ & U-235 & & & \\
\hline 304.51 & $3.59 \mathrm{E}-11$ & Fr-223 & $->$ & Ra-223, & Th-227 & $->$ & Ra-223 \\
\hline 307.82 & $6.56 \mathrm{E}-02$ & Pu-239 & $->$ & U-235 & & & \\
\hline 307.89 & $5.67 \mathrm{E}-14$ & Fr-223 & $->$ & Ra-223, & Th-227 & $->$ & Ra-223 \\
\hline 308.49 & $4.94 \mathrm{E}-13$ & Th-227 & $->$ & Ra-223 & & & \\
\hline 308.75 & $1.01 \mathrm{E}-08$ & Th-231 & $->$ & Pa-231 & & & \\
\hline 310.19 & $1.28 \mathrm{E}-12$ & Pa-231 & $->$ & Ac-227 & & & \\
\hline 310.71 & $1.04 \mathrm{E}-07$ & U-235 & $->$ & Th-231 & & & \\
\hline 311 & $7.52 \mathrm{E}-08$ & Th-231 & $->$ & Pa-231 & & & \\
\hline 311.698 & $1.03 E+05$ & Np-239 & $->$ & Pu-239 & & & \\
\hline 311.78 & $2.73 \mathrm{E}-01$ & Pu-239 & $->$ & U-235 & & & \\
\hline 312.57 & $1.55 \mathrm{E}-11$ & Fr-223 & $->$ & $\mathrm{Ra}-223$, & Th-227 & $->$ & $\mathrm{Ra}-223$ \\
\hline 313.058 & $1.03 \mathrm{E}-10$ & Pa-231 & $->$ & Ac-227 & & & \\
\hline 313.39 & $2.11 \mathrm{E}-02$ & Pu-239 & $->$ & U-235 & & & \\
\hline 313.85 & $9.62 \mathrm{E}-13$ & $P b-211$ & $->$ & $\mathrm{Bi}-211$ & & & \\
\hline 314.78 & $9.89 \mathrm{E}-13$ & Th-227 & $->$ & Ra-223 & & & \\
\hline 314.82 & $1.52 \mathrm{E}-11$ & Th-227 & $->$ & Ra-223 & & & \\
\hline 315.8825 & $1.17 \mathrm{E}+08$ & Np-239 & $->$ & Pu-239 & & & \\
\hline 316.4 & $1.49 \mathrm{E}-01$ & Pu-239 & $->$ & U-235 & & & \\
\hline 317.12 & $2.60 \mathrm{E}-08$ & U-235 & $->$ & Th-231 & & & \\
\hline 317.93 & 2.07E-09 & Th-231 & $->$ & Pa-231 & & & \\
\hline 318.1 & $1.82 \mathrm{E}-12$ & Pa-231 & $->$ & Ac-227 & & & \\
\hline 318.58 & $1.98 \mathrm{E}-13$ & Th-227 & $->$ & Ra-223 & & & \\
\hline 319.18 & $1.04 \mathrm{E}-12$ & Fr-223 & $->$ & Ra-223, & Th-227 & $->$ & Ra-223 \\
\hline 320.188 & 2.85E-09 & Th-231 & $->$ & Pa-231 & & & \\
\hline 322.267 & $4.20 E+05$ & Np-239 & $->$ & Pu-239 & & & \\
\hline 323.77 & $5.70 \mathrm{E}-01$ & Pu-239 & $->$ & U-235 & & & \\
\hline 323.88 & $1.22 \mathrm{E}-10$ & Ra-223 & $->$ & Rn-219 & & & \\
\hline 325.08 & $1.98 \mathrm{E}-13$ & Th-227 & $->$ & $\mathrm{Ra}-223$ & & & \\
\hline 326.12 & $1.65 \mathrm{E}-13$ & Th-227 & $->$ & Ra-223 & & & \\
\hline 327.23 & $2.74 \mathrm{E}-11$ & Pa-231 & $->$ & $A c-227$ & & & \\
\hline 328.08 & $4.57 \mathrm{E}-14$ & TI-207 & $->$ & Pb-207, & Po-211 & $->$ & Pb-207 \\
\hline 328.49 & $6.22 \mathrm{E}-12$ & Ra-223 & $->$ & Rn-219 & & & \\
\hline 329.84 & $9.00 \mathrm{E}-11$ & Fr-223 & $->$ & Ra-223, & Th-227 & $->$ & Ra-223 \\
\hline 330.066 & $1.20 \mathrm{E}-09$ & Pa-231 & $->$ & Ac-227 & & & \\
\hline 332.853 & $5.33 E+00$ & Pu-239 & $->$ & U-235 & & & \\
\hline 333.87 & $3.11 \mathrm{E}-12$ & Ra-223 & $->$ & $\mathrm{Rn}-219$ & & & \\
\hline
\end{tabular}




\begin{tabular}{|c|c|c|c|c|c|c|c|}
\hline 334.3136 & $1.51 \mathrm{E}+08$ & Np-239 & $->$ & Pu-239 & & & \\
\hline 334.38 & $3.26 \mathrm{E}-11$ & Fr-223 & $->$ & Ra-223, & Th-227 & $->$ & Ra-223 \\
\hline 336.116 & $1.20 \mathrm{E}+00$ & Pu-239 & $->$ & U-235 & & & \\
\hline 338.28 & $8.73 \mathrm{E}-11$ & Ra-223 & $->$ & $R n-219$ & & & \\
\hline 339.1 & $4.61 \mathrm{E}-14$ & Th-227 & $->$ & Ra-223 & & & \\
\hline 339.45 & $1.29 \mathrm{E}-13$ & $\mathrm{Fr}-223$ & $->$ & Ra-223, & Th-227 & $->$ & Ra-223 \\
\hline 340.873 & $1.59 \mathrm{E}-10$ & $\mathrm{~Pa}-231$ & $->$ & Ac-227 & & & \\
\hline 341.17 & $2.00 \mathrm{E}-11$ & Pa-231 & $->$ & $A c-227$ & & & \\
\hline 341.526 & 7.00E-01 & Pu-239 & $->$ & U-235 & & & \\
\hline 342.44 & $1.25 \mathrm{E}-11$ & $\mathrm{Fr}-223$ & $->$ & Ra-223, & Th-227 & $->$ & Ra-223 \\
\hline 342.9 & $6.28 \mathrm{E}-12$ & Ra-223 & $->$ & Rn-219 & & & \\
\hline 343.02 & $1.05 \mathrm{E}-12$ & $\mathrm{~Pb}-211$ & $->$ & $\mathrm{Bi}-211$ & & & \\
\hline 343.78 & 7.79E-08 & U-235 & $->$ & Th-231 & & & \\
\hline 345.014 & $5.91 \mathrm{E}+00$ & Pu-239 & $->$ & U-235 & & & \\
\hline 345.921 & 7.53E-07 & $U-235$ & $->$ & Th-231 & & & \\
\hline 346.39 & $2.47 E-13$ & Th-227 & $->$ & Ra-223 & & & \\
\hline 348.4 & $1.98 \mathrm{E}-13$ & Th-227 & $->$ & Ra-223 & & & \\
\hline 350.1 & $3.94 \mathrm{E}-10$ & $\mathrm{Bi}-211$ & $->$ & TI-207 & & & \\
\hline 350.48 & $3.63 \mathrm{E}-12$ & Th-227 & $->$ & Ra-223 & & & \\
\hline 351.82 & $1.82 \mathrm{E}-09$ & Th-231 & $->$ & Pa-231 & & & \\
\hline 352.57 & $3.30 \mathrm{E}-13$ & Th-227 & $->$ & Ra-223 & & & \\
\hline 354.02 & $8.46 \mathrm{E}-03$ & Pu-239 & $->$ & U-235 & & & \\
\hline 354.63 & 7.29E-11 & $\mathrm{Pa}-231$ & $->$ & Ac- 227 & & & \\
\hline 356.068 & $1.30 \mathrm{E}-07$ & $U-235$ & $->$ & Th-231 & & & \\
\hline 357.2 & $1.40 \mathrm{E}-10$ & $\mathrm{~Pa}-231$ & $->$ & Ac- 227 & & & \\
\hline 359.51 & $8.20 \mathrm{E}-12$ & Pa-231 & $->$ & $A C-227$ & & & \\
\hline 361.8 & $1.35 \mathrm{E}-12$ & Ra-223 & $->$ & Rn-219 & & & \\
\hline 361.84 & $1.24 \mathrm{E}-01$ & Pu-239 & $->$ & U-235 & & & \\
\hline 362.55 & $1.65 \mathrm{E}-13$ & Th-227 & $->$ & Ra-223 & & & \\
\hline 364.03 & $6.74 \mathrm{E}-12$ & Pa-231 & $->$ & Ac- 227 & & & \\
\hline 367.048 & $9.17 \mathrm{E}-01$ & Pu-239 & $->$ & U-235 & & & \\
\hline 368.543 & $9.52 \mathrm{E}-01$ & Pu-239 & $->$ & U-235 & & & \\
\hline 368.78 & $2.51 \mathrm{E}-13$ & Ra-223 & $->$ & $R n-219$ & & & \\
\hline 369.32 & $2.08 \mathrm{E}-13$ & Fr-223 & $->$ & Ra-223, & Th-227 & $->$ & Ra-223 \\
\hline 369.48 & $6.28 \mathrm{E}-13$ & Ra-223 & $->$ & $\mathrm{Rn}-219$ & & & \\
\hline 370.85 & $2.31 \mathrm{E}-13$ & Th-227 & $->$ & Ra-223 & & & \\
\hline 371.8 & $1.54 \mathrm{E}-11$ & Ra-223 & $->$ & $\mathrm{Rn}-219$ & & & \\
\hline 372.47 & $2.22 \mathrm{E}-02$ & Pu-239 & $->$ & U-235 & & & \\
\hline 374.93 & $4.61 \mathrm{E}-14$ & Th-227 & $->$ & Ra-223 & & & \\
\hline 375.039 & $1.66 \mathrm{E}+01$ & Pu-239 & $->$ & U-235 & & & \\
\hline 376.26 & $1.98 \mathrm{E}-13$ & Th-227 & $->$ & Ra-223 & & & \\
\hline 376.9 & $2.22 \mathrm{E}-13$ & $R n-219$ & $->$ & Po-215 & & & \\
\hline 379.05 & $1.11 \mathrm{E}-02$ & Pu-239 & $->$ & U-235 & & & \\
\hline 379.4 & $4.19 \mathrm{E}-11$ & Rn-219 & $->$ & Рo-215, & Pa-231 & $->$ & AC-227 \\
\hline 380.16 & $3.23 \mathrm{E}+00$ & Pu-239 & $->$ & U-235 & & & \\
\hline 382.15 & $1.98 \mathrm{E}-13$ & Th-227 & $->$ & Ra-223 & & & \\
\hline 382.732 & $2.74 \mathrm{E}+00$ & Pu-239 & $->$ & U-235 & & & \\
\hline 383.12 & $1.26 \mathrm{E}-13$ & Ra-223 & $->$ & $\mathrm{Rn}-219$ & & & \\
\hline 383.47 & $1.58 \mathrm{E}-12$ & Th-227 & $->$ & Ra-223 & & & \\
\hline 384.97 & 3.74E-12 & Pa-231 & $->$ & $A C-227$ & & & \\
\hline
\end{tabular}




\begin{tabular}{|c|c|c|c|c|c|c|c|}
\hline 387.267 & $4.56 \mathrm{E}-13$ & $\mathrm{~Pa}-231$ & $->$ & $A c-227$ & & & \\
\hline 387.874 & $9.87 \mathrm{E}-08$ & U-235 & $->$ & Th-231 & & & \\
\hline 387.9 & $1.27 \mathrm{E}-14$ & $\mathrm{Rn}-219$ & $->$ & Po-215 & & & \\
\hline 388.3 & $4.40 \mathrm{E}-13$ & Ra-223 & $->$ & $\mathrm{Rn}-219$ & & & \\
\hline 388.98 & $9.75 \mathrm{E}-13$ & $\mathrm{~Pa}-231$ & $->$ & Ac- 227 & & & \\
\hline 390.32 & $2.60 E-08$ & U-235 & $->$ & Th-231 & & & \\
\hline 391 & $9.42 \mathrm{E}-14$ & Ra-223 & $->$ & Rn-219 & & & \\
\hline 391.85 & $6.20 \mathrm{E}-12$ & $\mathrm{~Pa}-231$ & $->$ & Ac-227 & & & \\
\hline 392.24 & $2.97 E-13$ & Th-227 & $->$ & Ra-223 & & & \\
\hline 392.3 & $1.18 \mathrm{E}+05$ & Np-239 & $->$ & Pu-239 & & & \\
\hline 392.914 & $5.85 \mathrm{E}+00$ & Pu-239 & $->$ & U-235 & & & \\
\hline 395.8 & $2.37 \mathrm{E}-12$ & $\mathrm{~Pa}-231$ & $->$ & Ac-227 & & & \\
\hline 398.4 & $8.48 \mathrm{E}-12$ & $\mathrm{~Pa}-231$ & $->$ & Ac- 227 & & & \\
\hline 398.6 & 2.97E-13 & Th-227 & $->$ & Ra-223 & & & \\
\hline 399.46 & $6.45 E-02$ & Pu-239 & $->$ & U-235 & & & \\
\hline 401.7 & $2.11 \mathrm{E}-10$ & $R n-219$ & $->$ & Po-215 & & & \\
\hline 402.42 & $3.96 \mathrm{E}-13$ & Th-227 & $->$ & $\mathrm{Ra}-223$ & & & \\
\hline 404.86 & $1.19 \mathrm{E}-10$ & $\mathrm{~Pb}-211$ & $->$ & $\mathrm{Bi}-211$, & At-215 & $->$ & $\mathrm{Bi}-211$ \\
\hline 407.98 & $3.10 \mathrm{E}-11$ & $\mathrm{~Pa}-231$ & $->$ & Ac- 227 & & & \\
\hline 410.269 & 7.79E-08 & U-235 & $->$ & Th-231 & & & \\
\hline 410.84 & $1.73 \mathrm{E}-12$ & $\mathrm{~Pa}-231$ & $->$ & Ac- 227 & & & \\
\hline 410.94 & 8.46E-02 & Pu-239 & $->$ & U-235 & & & \\
\hline 412.83 & $2.11 \mathrm{E}-04$ & Pu-239 & $->$ & $U-235$ & & & \\
\hline 413.713 & $1.55 \mathrm{E}+01$ & Pu-239 & $->$ & $U-235$ & & & \\
\hline 415.09 & $5.60 \mathrm{E}-14$ & Th-227 & $->$ & Ra-223 & & & \\
\hline 422.598 & $1.29 E+00$ & Pu-239 & $->$ & U-235 & & & \\
\hline 426.64 & $2.42 \mathrm{E}-01$ & Pu-239 & $->$ & U-235 & & & \\
\hline 427 & $5.34 \mathrm{E}-11$ & $\mathrm{~Pb}-211$ & $->$ & $\mathrm{Bi}-211$ & & & \\
\hline 429.87 & $2.73 E+05$ & Np-239 & $->$ & Pu-239 & & & \\
\hline 430.16 & $5.18 \mathrm{E}-02$ & Pu-239 & $->$ & U-235 & & & \\
\hline 430.26 & $1.99 \mathrm{E}-13$ & $\mathrm{~Pb}-211$ & $->$ & $\mathrm{Bi}-211$ & & & \\
\hline 430.53 & 5.97E-13 & $\mathrm{Ra}-223$ & $->$ & $\mathrm{Rn}-219$ & & & \\
\hline 432.29 & $1.65 E-13$ & Th-227 & $->$ & Ra-223 & & & \\
\hline 432.34 & $1.04 \mathrm{E}-12$ & Ra-223 & $->$ & $\mathrm{Rn}-219$ & & & \\
\hline 434.9 & $9.07 E+05$ & $\mathrm{~Np}-239$ & $->$ & Pu-239 & & & \\
\hline 435.38 & $3.01 \mathrm{E}-12$ & $\mathrm{~Pa}-231$ & $->$ & Ac-227 & & & \\
\hline 436.137 & $6.12 \mathrm{E}+04$ & Np-239 & $->$ & Pu-239 & & & \\
\hline 438.24 & $3.74 \mathrm{E}-12$ & Pa-231 & $->$ & Ac- 227 & & & \\
\hline 438.8 & $1.26 \mathrm{E}-12$ & Po-215 & $->$ & $\mathrm{Pb}-211$ & & & \\
\hline 444.94 & $3.99 \mathrm{E}-11$ & Ra-223 & $->$ & $\mathrm{Rn}-219$ & & & \\
\hline 445.81 & $9.31 \mathrm{E}-02$ & Pu-239 & $->$ & $U-235$ & & & \\
\hline 448 & $5.36 \mathrm{E}-15$ & Fr-223 & $->$ & Ra-223, & Th-227 & $->$ & $\mathrm{Ra}-223$ \\
\hline 448.3 & $1.84 \mathrm{E}+04$ & $\mathrm{~Np}-239$ & $->$ & Pu-239 & & & \\
\hline 448.42 & $2.60 \mathrm{E}-08$ & U-235 & $->$ & Th-231 & & & \\
\hline 451.481 & $2.00 \mathrm{E}+00$ & Pu-239 & $->$ & U-235 & & & \\
\hline 452.52 & $4.22 \mathrm{E}-15$ & Fr-223 & $->$ & Ra-223, & Th-227 & $->$ & Ra-223 \\
\hline 454.568 & $9.14 \mathrm{E}+04$ & Np-239 & $->$ & Pu-239 & & & \\
\hline 455.12 & $2.08 \mathrm{E}-08$ & U-235 & $->$ & Th-231 & & & \\
\hline 457.07 & $2.96 \mathrm{E}-15$ & Fr-223 & $->$ & Ra-223, & Th-227 & $->$ & Ra-223 \\
\hline 457.57 & $1.63 \mathrm{E}-02$ & Pu-239 & $->$ & $U-235$ & & & \\
\hline
\end{tabular}




\begin{tabular}{|c|c|c|c|c|c|c|c|}
\hline 461.25 & $2.11 \mathrm{E}-02$ & Pu-239 & $->$ & $U-235$ & & & \\
\hline 461.9 & $1.18 \mathrm{E}+05$ & Th-227 & $->$ & Ra-223, & Np-239 & $->$ & Pu-239 \\
\hline 463.8 & $1.69 \mathrm{E}-03$ & Pu-239 & $->$ & $U-235$ & & & \\
\hline 466.2 & $1.65 \mathrm{E}-15$ & Th-227 & $->$ & Ra-223 & & & \\
\hline 469.7 & $7.81 \mathrm{E}+04$ & $N p-239$ & $->$ & Pu-239 & & & \\
\hline 471.33 & $1.37 \mathrm{E}-13$ & $\mathrm{~Pa}-231$ & $->$ & $A c-227$ & & & \\
\hline 474.26 & $9.52 \mathrm{E}-04$ & Pu-239 & $->$ & $U-235$ & & & \\
\hline 478 & $4.03 E-13$ & $\mathrm{~Pb}-211$ & $->$ & $\mathrm{Bi}-211$ & & & \\
\hline 478.22 & $7.02 E-14$ & $\mathrm{~Pa}-231$ & $->$ & Ac-227 & & & \\
\hline 480.25 & $1.04 \mathrm{E}-14$ & Fr-223 & $->$ & Ra-223, & Th-227 & $->$ & Ra-223 \\
\hline 481.1 & 8.07E-13 & $\mathrm{Pb}-211$ & $->$ & $\mathrm{Bi}-211$ & & & \\
\hline 481.78 & $4.86 \mathrm{E}-02$ & Pu-239 & $->$ & U-235 & & & \\
\hline 482.14 & $5.45 \mathrm{E}-15$ & Fr-223 & $->$ & Ra-223, & Th-227 & $->$ & $\mathrm{Ra}-223$ \\
\hline 484.3 & $7.59 E+04$ & $\mathrm{~Np}-239$ & $->$ & Pu-239 & & & \\
\hline 486.99 & 2.64E-03 & Pu-239 & $->$ & U-235 & & & \\
\hline 487.59 & $3.14 \mathrm{E}-13$ & Ra-223 & $->$ & $\mathrm{Rn}-219$ & & & \\
\hline 492 & 4.34E-13 & $\mathrm{Pb}-211$ & $->$ & $\mathrm{Bi}-211$ & & & \\
\hline 492.2 & $4.35 E+05$ & $N p-239$ & $->$ & Pu-239 & & & \\
\hline 493.15 & $1.91 \mathrm{E}-14$ & Fr-223 & $->$ & Ra-223, & Th-227 & $->$ & $\mathrm{Ra}-223$ \\
\hline 493.25 & $9.31 \mathrm{E}-03$ & Pu-239 & $->$ & U-235 & & & \\
\hline 497.71 & $2.36 \mathrm{E}+05$ & $\mathrm{~Np}-239$ & $->$ & Pu-239 & & & \\
\hline 498.8 & $2.29 E+05$ & $N p-239$ & $->$ & Pu-239 & & & \\
\hline 500.4 & $3.41 \mathrm{E}-13$ & $\mathrm{~Pb}-211$ & $->$ & $\mathrm{Bi}-211$ & & & \\
\hline 501.33 & $5.47 \mathrm{E}-13$ & $\mathrm{~Pa}-231$ & $->$ & $A c-227$ & & & \\
\hline 503.983 & $1.03 E+05$ & $\mathrm{~Np}-239$ & $->$ & Pu-239 & & & \\
\hline 504.33 & $4.65 E-13$ & $\mathrm{~Pb}-211$ & $->$ & $\mathrm{Bi}-211$ & & & \\
\hline 507.64 & $1.46 \mathrm{E}-14$ & Fr-223 & $->$ & Ra-223, & Th-227 & $->$ & $\mathrm{Ra}-223$ \\
\hline 515.7 & $1.27 \mathrm{E}-12$ & $\mathrm{Rn}-219$ & $->$ & Po-215 & & & \\
\hline 515.96 & $7.00 \mathrm{E}-15$ & Fr-223 & $->$ & Ra-223, & Th-227 & $->$ & $\mathrm{Ra}-223$ \\
\hline 516.45 & $1.18 \mathrm{E}-12$ & $\mathrm{~Pa}-231$ & $->$ & $A c-227$ & & & \\
\hline 517.2 & $1.04 \mathrm{E}-08$ & U-235 & $->$ & Th-231 & & & \\
\hline 524.02 & $5.82 \mathrm{E}-15$ & Fr-223 & $->$ & Ra-223, & Th-227 & $->$ & $\mathrm{Ra}-223$ \\
\hline 527.3 & $2.23 \mathrm{E}-12$ & Ra-223 & $->$ & Rn-219 & & & \\
\hline 534.9 & $3.30 \mathrm{E}-15$ & Th-227 & $->$ & Ra-223 & & & \\
\hline 535.45 & $5.10 \mathrm{E}-13$ & $\mathrm{~Pa}-231$ & $->$ & Ac- 227 & & & \\
\hline 536.88 & $3.83 E-14$ & Fr-223 & $->$ & Ra-223, & Th-227 & $->$ & $\mathrm{Ra}-223$ \\
\hline 538.9 & $3.17 E-03$ & Pu-239 & $->$ & $U-235$ & & & \\
\hline 540.1 & $1.90 \mathrm{E}-13$ & $\mathrm{Rn}-219$ & $->$ & Po-215 & & & \\
\hline 544.55 & $1.25 E+03$ & Am-243 & $->$ & Np-239 & & & \\
\hline 546.9 & $4.56 \mathrm{E}-13$ & Pa-231 & $->$ & Ac- 227 & & & \\
\hline 550.6 & 4.23E-03 & Pu-239 & $->$ & $U-235$ & & & \\
\hline 552.09 & $9.71 \mathrm{E}-15$ & Fr-223 & $->$ & Ra-223, & Th-227 & $->$ & Ra-223 \\
\hline 555.91 & 8.04E-15 & Fr-223 & $->$ & Ra-223, & Th-227 & $->$ & $\mathrm{Ra}-223$ \\
\hline 557.7 & $6.34 \mathrm{E}-04$ & Pu-239 & $->$ & $U-235$ & & & \\
\hline 568.76 & $3.69 E-14$ & Fr-223 & $->$ & Ra-223, & Th-227 & $->$ & $\mathrm{Ra}-223$ \\
\hline 569.15 & $4.49 \mathrm{E}-14$ & Po- 211 & $->$ & $\mathrm{Pb}-207$ & & & \\
\hline 572.3 & $4.56 \mathrm{E}-13$ & $\mathrm{~Pa}-231$ & $->$ & Ac- 227 & & & \\
\hline 575.8 & $1.77 \mathrm{E}-14$ & Fr-223 & $->$ & Ra-223, & Th-227 & $->$ & $\mathrm{Ra}-223$ \\
\hline 578.2 & $5.07 \mathrm{E}-15$ & Fr-223 & $->$ & Ra-223, & Th-227 & $->$ & $\mathrm{Ra}-223$ \\
\hline 586.27 & $1.48 \mathrm{E}-03$ & Pu-239 & $->$ & $U-235$ & & & \\
\hline
\end{tabular}




\begin{tabular}{|c|c|c|c|c|c|c|c|}
\hline 588.17 & $2.09 \mathrm{E}-15$ & Fr-223 & $->$ & Ra-223, & Th-227 & $->$ & Ra-223 \\
\hline 596.44 & $1.35 \mathrm{E}-15$ & Fr-223 & $->$ & Ra-223, & Th-227 & $->$ & Ra-223 \\
\hline 598.69 & $2.83 \mathrm{E}-12$ & Ra-223 & $->$ & Rn-219 & & & \\
\hline 606.9 & $1.59 \mathrm{E}-03$ & Pu-239 & $->$ & U-235 & & & \\
\hline 607.2 & $6.58 \mathrm{E}-15$ & Fr-223 & $->$ & Ra-223, & Th-227 & $->$ & Ra-223 \\
\hline 608.5 & $1.27 \mathrm{E}-13$ & $\mathrm{Rn}-219$ & $->$ & Po-215 & & & \\
\hline 609.08 & $1.98 \mathrm{E}-12$ & Ra-223 & $->$ & $\mathrm{Rn}-219$ & & & \\
\hline 609.62 & $7.14 \mathrm{E}-13$ & $\mathrm{~Pb}-211$ & $->$ & $\mathrm{Bi}-211$ & & & \\
\hline 612.93 & 8.57E-03 & Pu-239 & $->$ & U-235 & & & \\
\hline 617.43 & $2.16 \mathrm{E}-02$ & Pu-239 & $->$ & $U-235$ & & & \\
\hline 618.94 & $2.58 \mathrm{E}-02$ & Pu-239 & $->$ & U-235 & & & \\
\hline 621.92 & $2.15 \mathrm{E}-15$ & Fr-223 & $->$ & Ra-223, & Th-227 & $->$ & Ra-223 \\
\hline 623.38 & $2.51 \mathrm{E}-13$ & Ra-223 & $->$ & $\mathrm{Rn}-219$ & & & \\
\hline 623.52 & $5.27 \mathrm{E}-15$ & Th-227 & $->$ & Ra-223 & & & \\
\hline 624.8 & $4.23 E-03$ & Pu-239 & $->$ & U-235 & & & \\
\hline 631.116 & $2.43 E+04$ & Am-243 & $->$ & Np-239 & & & \\
\hline 633.15 & $2.68 \mathrm{E}-02$ & Pu-239 & $->$ & U-235 & & & \\
\hline 633.2 & $4.29 E-15$ & Th-227 & $->$ & Ra-223 & & & \\
\hline 637.862 & $2.71 \mathrm{E}-02$ & Pu-239 & $->$ & U-235 & & & \\
\hline 640.096 & 8.67E-02 & Pu-239 & $->$ & U-235 & & & \\
\hline 641.45 & $6.59 E-16$ & Th-227 & $->$ & Ra-223 & & & \\
\hline 643.93 & $2.90 \mathrm{E}-15$ & Fr-223 & $->$ & Ra-223, & Th-227 & $->$ & Ra-223 \\
\hline 645.969 & $1.57 \mathrm{E}-01$ & Pu-239 & $->$ & U-235 & & & \\
\hline 647.296 & $2.01 \mathrm{E}-03$ & Pu-239 & $->$ & U-235 & & & \\
\hline 648.43 & $6.96 \mathrm{E}-16$ & Fr-223 & $->$ & Ra-223, & Th-227 & $->$ & Ra-223 \\
\hline 649.377 & 7.53E-03 & Pu-239 & $->$ & U-235 & & & \\
\hline 650.529 & $2.75 \mathrm{E}-03$ & Pu-239 & $->$ & $U-235$ & & & \\
\hline 652.052 & 6.93E-02 & Pu-239 & $->$ & U-235 & & & \\
\hline 654.89 & $2.38 \mathrm{E}-02$ & Pu-239 & $->$ & $U-235$ & & & \\
\hline 658.919 & $1.05 \mathrm{E}-01$ & Pu-239 & $->$ & U-235 & & & \\
\hline 662.251 & $7.00 \mathrm{E}+04$ & Am-243 & $->$ & Np-239 & & & \\
\hline 662.8 & $1.98 \mathrm{E}-15$ & Th-227 & $->$ & Ra-223 & & & \\
\hline 664.587 & $1.75 \mathrm{E}-02$ & Pu-239 & $->$ & U-235 & & & \\
\hline 675.33 & $1.99 \mathrm{E}-13$ & $\mathrm{~Pb}-211$ & $->$ & $\mathrm{Bi}-211$ & & & \\
\hline 676.4 & $1.90 \mathrm{E}-13$ & Rn-219 & $->$ & Po-215 & & & \\
\hline 686.16 & $9.41 \mathrm{E}-03$ & Pu-239 & $->$ & U-235 & & & \\
\hline 690.85 & $5.82 \mathrm{E}-03$ & Pu-239 & $->$ & $U-235$ & & & \\
\hline 691.46 & $5.49 \mathrm{E}-15$ & Fr-223 & $->$ & Ra-223, & Th-227 & $->$ & Ra-223 \\
\hline 703.8 & $4.10 \mathrm{E}-02$ & Pu-239 & $->$ & U-235 & & & \\
\hline 704.19 & $4.68 \mathrm{E}-15$ & Fr-223 & $->$ & Ra-223, & Th-227 & $->$ & Ra-223 \\
\hline 704.51 & $1.49 \mathrm{E}-11$ & $\mathrm{~Pb}-211$ & $->$ & $\mathrm{Bi}-211$ & & & \\
\hline 707.18 & $1.69 \mathrm{E}-15$ & Fr-223 & $->$ & Ra-223, & Th-227 & $->$ & Ra-223 \\
\hline 711.2 & $1.10 \mathrm{E}-13$ & $\mathrm{Ra}-223$ & $->$ & $\mathrm{Rn}-219$ & & & \\
\hline 714.58 & $9.52 \mathrm{E}-04$ & Pu-239 & $->$ & $U-235$ & & & \\
\hline 718 & $2.96 \mathrm{E}-02$ & Pu-239 & $->$ & U-235 & & & \\
\hline 718.22 & $1.10 \mathrm{E}-15$ & Fr-223 & $->$ & Ra-223, & Th-227 & $->$ & Ra-223 \\
\hline 722.6 & $2.41 \mathrm{E}-14$ & Fr-223 & $->$ & Ra-223, & Th-227 & $->$ & Ra-223 \\
\hline 723.86 & $4.91 \mathrm{E}-14$ & Fr-223 & $->$ & Ra-223, & Th-227 & $->$ & Ra-223 \\
\hline 727.84 & $1.15 \mathrm{E}-02$ & Pu-239 & $->$ & U-235 & & & \\
\hline 733.9 & $6.54 \mathrm{E}-15$ & Fr-223 & $->$ & Ra-223, & Th-227 & $->$ & $\mathrm{Ra}-223$ \\
\hline
\end{tabular}




\begin{tabular}{|c|c|c|c|c|c|c|c|}
\hline 734.6 & $6.20 \mathrm{E}-15$ & Fr-223 & $->$ & Ra-223, & Th-227 & $->$ & Ra-223 \\
\hline 736.76 & $2.96 \mathrm{E}-15$ & Fr-223 & $->$ & Ra-223, & Th-227 & $->$ & Ra-223 \\
\hline 742.52 & $1.04 \mathrm{E}-08$ & $U-235$ & $->$ & Th-231 & & & \\
\hline 746.24 & $1.41 \mathrm{E}-14$ & Fr-223 & $->$ & Ra-223, & Th-227 & $->$ & $\mathrm{Ra}-223$ \\
\hline 748.56 & $1.73 \mathrm{E}-14$ & Fr-223 & $->$ & $\mathrm{Ra}-223$, & Th-227 & $->$ & Ra-223 \\
\hline 753.44 & $6.17 \mathrm{E}-15$ & Fr-223 & $->$ & Ra-223, & Th-227 & $->$ & $\mathrm{Ra}-223$ \\
\hline 754.17 & $6.35 \mathrm{E}-15$ & Fr-223 & $->$ & $\mathrm{Ra}-223$, & Th-227 & $->$ & Ra-223 \\
\hline 756.42 & $3.56 \mathrm{E}-02$ & Pu-239 & $->$ & U-235 & & & \\
\hline 756.79 & $1.26 \mathrm{E}-14$ & Fr-223 & $->$ & Ra-223, & Th-227 & $->$ & Ra-223 \\
\hline 757.03 & $3.31 \mathrm{E}-14$ & Fr-223 & $->$ & Ra-223, & Th-227 & $->$ & Ra-223 \\
\hline 762.3 & $9.50 \mathrm{E}-15$ & Fr-223 & $->$ & Ra-223, & Th-227 & $->$ & $\mathrm{Ra}-223$ \\
\hline 764.52 & $4.65 E-14$ & Fr-223 & $->$ & Ra-223, & Th-227 & $->$ & $\mathrm{Ra}-223$ \\
\hline 766.35 & $2.20 \mathrm{E}-11$ & $\mathrm{~Pb}-211$ & $->$ & $\mathrm{Bi}-211$ & & & \\
\hline 766.6 & 2.91E-05 & Pu-239 & $->$ & $U-235$ & & & \\
\hline 769.19 & $1.26 \mathrm{E}-01$ & Pu-239 & $->$ & $U-235$ & & & \\
\hline 772.85 & $4.52 \mathrm{E}-15$ & Fr-223 & $->$ & $\mathrm{Ra}-223$, & Th-227 & $->$ & $\mathrm{Ra}-223$ \\
\hline 775.82 & $2.36 \mathrm{E}-13$ & Fr-223 & $->$ & Ra-223, & Th-227 & $->$ & $\mathrm{Ra}-223$ \\
\hline 779.46 & $1.41 \mathrm{E}-03$ & Pu-239 & $->$ & $U-235$ & & & \\
\hline 780.59 & $1.17 \mathrm{E}-14$ & Fr-223 & $->$ & $\mathrm{Ra}-223$, & Th-227 & $->$ & Ra-223 \\
\hline 784.04 & $6.35 E-15$ & Fr-223 & $->$ & Ra-223, & Th-227 & $->$ & Ra-223 \\
\hline 786.9 & $4.43 E-15$ & Fr-223 & $->$ & Ra-223, & Th-227 & $->$ & $\mathrm{Ra}-223$ \\
\hline 787.3 & 8.88E-04 & Pu-239 & $->$ & U-235 & & & \\
\hline 788.36 & $1.32 \mathrm{E}-15$ & Th-227 & $->$ & Ra-223 & & & \\
\hline 792.6 & $1.32 \mathrm{E}-15$ & Th-227 & $->$ & Ra-223 & & & \\
\hline 793 & $2.64 \mathrm{E}-04$ & Pu-239 & $->$ & U-235 & & & \\
\hline 793.11 & $5.56 \mathrm{E}-17$ & Fr-223 & $->$ & Ra-223 & & & \\
\hline 793.12 & $9.89 \mathrm{E}-16$ & Th-227 & $->$ & Ra-223 & & & \\
\hline 794.72 & $1.56 \mathrm{E}-08$ & U-235 & $->$ & Th-231 & & & \\
\hline 796.5 & $3.38 \mathrm{E}-04$ & Pu-239 & $->$ & $U-235$ & & & \\
\hline 796.96 & $3.07 E-14$ & Fr-223 & $->$ & Ra-223, & Th-227 & $->$ & $\mathrm{Ra}-223$ \\
\hline 803.3 & $4.65 E-04$ & Pu-239 & $->$ & $U-235$ & & & \\
\hline 803.58 & $5.82 \mathrm{E}-14$ & Fr-223 & $->$ & $\mathrm{Ra}-223$, & Th-227 & $->$ & Ra-223 \\
\hline 808.2 & $1.55 \mathrm{E}-03$ & Pu-239 & $->$ & $U-235$ & & & \\
\hline 808.26 & $1.93 \mathrm{E}-15$ & Fr-223 & $->$ & Ra-223, & Th-227 & $->$ & $\mathrm{Ra}-223$ \\
\hline 812.15 & $9.53 E-14$ & Fr-223 & $->$ & Ra-223, & Th-227 & $->$ & $\mathrm{Ra}-223$ \\
\hline 813.9 & $6.56 \mathrm{E}-04$ & Pu-239 & $->$ & U-235 & & & \\
\hline 817.31 & $5.61 \mathrm{E}-15$ & Fr-223 & $->$ & Ra-223, & Th-227 & $->$ & $\mathrm{Ra}-223$ \\
\hline 818.23 & $9.89 \mathrm{E}-16$ & Th-227 & $->$ & $\mathrm{Ra}-223$ & & & \\
\hline 821.1 & 5.39E-04 & Pu-239 & $->$ & U-235 & & & \\
\hline 822.99 & $8.70 \mathrm{E}-14$ & $\mathrm{Fr}-223$ & $->$ & $\mathrm{Ra}-223$, & Th-227 & $->$ & $\mathrm{Ra}-223$ \\
\hline 825.96 & $2.94 \mathrm{E}-14$ & Fr-223 & $->$ & Ra-223, & Th-227 & $->$ & $\mathrm{Ra}-223$ \\
\hline 825.97 & $6.02 \mathrm{E}-16$ & Fr-223 & $->$ & $\mathrm{Ra}-223$ & & & \\
\hline 828.31 & $7.15 \mathrm{E}-15$ & Fr-223 & $->$ & Ra-223, & Th-227 & $->$ & $\mathrm{Ra}-223$ \\
\hline 828.53 & $3.90 \mathrm{E}-16$ & Fr-223 & $->$ & Ra-223, & Th-227 & $->$ & Ra-223 \\
\hline 828.8 & $1.46 \mathrm{E}-03$ & Pu-239 & $->$ & $U-235$ & & & \\
\hline 831.86 & $1.18 \mathrm{E}-10$ & $\mathrm{~Pb}-211$ & $->$ & $\mathrm{Bi}-211$ & & & \\
\hline 831.93 & $2.64 \mathrm{E}-04$ & Pu-239 & $->$ & $U-235$ & & & \\
\hline 834.2 & $3.17 \mathrm{E}-14$ & $\mathrm{Rn}-219$ & $->$ & Po-215 & & & \\
\hline 837.36 & $1.63 \mathrm{E}-14$ & Fr-223 & $->$ & Ra-223, & Th-227 & $->$ & Ra-223 \\
\hline 839 & $4.23 E-04$ & Pu-239 & $->$ & U-235 & & & \\
\hline
\end{tabular}




\begin{tabular}{|c|c|c|c|c|c|c|c|}
\hline 842.02 & $2.27 \mathrm{E}-14$ & Fr-223 & $->$ & Ra-223, & Th-227 & $->$ & Ra-223 \\
\hline 843.8 & $1.69 \mathrm{E}-03$ & Pu-239 & $->$ & $U-235$ & & & \\
\hline 846.56 & $2.15 \mathrm{E}-14$ & Fr-223 & $->$ & $\mathrm{Ra}-223$, & Th-227 & $->$ & $\mathrm{Ra}-223$ \\
\hline 848.88 & $3.51 \mathrm{E}-15$ & Fr-223 & $->$ & Ra-223, & Th-227 & $->$ & Ra-223 \\
\hline 854.2 & $2.31 \mathrm{E}-15$ & Th-227 & $->$ & Ra-223 & & & \\
\hline 857.89 & $2.15 \mathrm{E}-15$ & Fr-223 & $->$ & Ra-223, & Th-227 & $->$ & Ra-223 \\
\hline 858.4 & $9.35 E-15$ & Fr-223 & $->$ & $\mathrm{Ra}-223$, & Th-227 & $->$ & Ra-223 \\
\hline 863.2 & $1.59 \mathrm{E}-15$ & Fr-223 & $->$ & Ra-223, & Th-227 & $->$ & Ra-223 \\
\hline 865.81 & $1.99 \mathrm{E}-13$ & $\mathrm{~Pb}-211$ & $->$ & $\mathrm{Bi}-211$ & & & \\
\hline 867.23 & $2.90 \mathrm{E}-15$ & Fr-223 & $->$ & Ra-223, & Th-227 & $->$ & Ra-223 \\
\hline 876.14 & $2.49 \mathrm{E}-14$ & Fr-223 & $->$ & Ra-223, & Th-227 & $->$ & Ra-223 \\
\hline 878.16 & $3.97 E-15$ & Fr-223 & $->$ & Ra-223, & Th-227 & $->$ & Ra-223 \\
\hline 879 & $5.29 E-04$ & Pu-239 & $->$ & $U-235$ & & & \\
\hline 887.7 & $4.76 \mathrm{E}-14$ & $\mathrm{Rn}-219$ & $->$ & Po-215 & & & \\
\hline 891 & $1.59 \mathrm{E}-15$ & Fr-223 & $->$ & Ra-223, & Th-227 & $->$ & Ra-223 \\
\hline 891.1 & $8.14 \mathrm{E}-04$ & Pu-239 & $->$ & U-235 & & & \\
\hline 892.8 & $1.03 \mathrm{E}-15$ & Fr-223 & $->$ & Ra-223, & Th-227 & $->$ & Ra-223 \\
\hline 896.41 & $1.15 \mathrm{E}-14$ & Fr-223 & $->$ & Ra-223, & Th-227 & $->$ & Ra-223 \\
\hline 897.23 & $7.31 \mathrm{E}-12$ & TI-207 & $->$ & Pb-207, & Po-211 & $->$ & $\mathrm{Pb}-207$ \\
\hline 908.03 & $7.52 E-14$ & Fr-223 & $->$ & Ra-223, & Th-227 & $->$ & $\mathrm{Ra}-223$ \\
\hline 909.85 & $4.94 \mathrm{E}-16$ & Th-227 & $->$ & $\mathrm{Ra}-223$ & & & \\
\hline 920.06 & $3.96 \mathrm{E}-16$ & Th-227 & $->$ & Ra-223 & & & \\
\hline 926.28 & $9.72 \mathrm{E}-16$ & Fr-223 & $->$ & Ra-223, & Th-227 & $->$ & Ra-223 \\
\hline 938.34 & $3.30 \mathrm{E}-16$ & Th-227 & $->$ & $\mathrm{Ra}-223$ & & & \\
\hline 940.1 & 4.44E-04 & Pu-239 & $->$ & U-235 & & & \\
\hline 941.41 & $2.37 \mathrm{E}-15$ & Th-227 & $->$ & Ra-223 & & & \\
\hline 951 & $6.83 \mathrm{E}-13$ & $\mathrm{~Pb}-211$ & $->$ & $\mathrm{Bi}-211$ & & & \\
\hline 956.4 & $5.92 \mathrm{E}-04$ & Pu-239 & $->$ & $U-235$ & & & \\
\hline 958.6 & $2.04 \mathrm{E}-15$ & Th-227 & $->$ & $\mathrm{Ra}-223$ & & & \\
\hline 969.91 & $9.89 E-16$ & Th-227 & $->$ & Ra-223 & & & \\
\hline 971.28 & 3.30E-16 & Th-227 & $->$ & Ra-223 & & & \\
\hline 979.52 & $2.43 E-04$ & Pu-239 & $->$ & $U-235$ & & & \\
\hline 986.7 & $1.27 \mathrm{E}-04$ & Pu-239 & $->$ & U-235 & & & \\
\hline 990.2 & $1.15 \mathrm{E}-15$ & Th-227 & $->$ & $\mathrm{Ra}-223$ & & & \\
\hline 992.47 & $2.43 E-04$ & Pu-239 & $->$ & U-235 & & & \\
\hline 994.9 & $2.31 \mathrm{E}-16$ & Th-227 & $->$ & Ra-223 & & & \\
\hline 999.78 & $9.89 E-16$ & Th-227 & $->$ & Ra-223 & & & \\
\hline 1005.63 & $1.27 \mathrm{E}-04$ & Pu-239 & $->$ & $U-235$ & & & \\
\hline 1014.48 & $5.55 E-13$ & $\mathrm{~Pb}-211$ & $->$ & $\mathrm{Bi}-211$ & & & \\
\hline 1015.1 & $4.94 \mathrm{E}-16$ & Th-227 & $->$ & Ra-223 & & & \\
\hline 1020 & $6.59 \mathrm{E}-16$ & Th-227 & $->$ & $\mathrm{Ra}-223$ & & & \\
\hline 1024.8 & $4.94 \mathrm{E}-16$ & Th-227 & $->$ & Ra-223 & & & \\
\hline 1055.8 & $1.90 \mathrm{E}-14$ & $\mathrm{Rn}-219$ & $->$ & Po-215 & & & \\
\hline 1057.32 & $4.76 \mathrm{E}-04$ & Pu-239 & $->$ & U-235 & & & \\
\hline 1080.19 & 4.75E-13 & $\mathrm{Pb}-211$ & $->$ & $\mathrm{Bi}-211$ & & & \\
\hline 1090.5 & 8.07E-14 & $\mathrm{Pb}-211$ & $->$ & $\mathrm{Bi}-211$ & & & \\
\hline 1103.4 & $1.58 \mathrm{E}-13$ & $\mathrm{~Pb}-211$ & $->$ & $\mathrm{Bi}-211$ & & & \\
\hline 1109.37 & $4.56 \mathrm{E}-12$ & $\mathrm{~Pb}-211$ & $->$ & $\mathrm{Bi}-211$ & & & \\
\hline 1196.61 & $3.97 \mathrm{E}-13$ & $\mathrm{~Pb}-211$ & $->$ & $\mathrm{Bi}-211$ & & & \\
\hline 1234.3 & $4.03 E-14$ & $\mathrm{~Pb}-211$ & $->$ & $\mathrm{Bi}-211$ & & & \\
\hline
\end{tabular}


$\begin{array}{lllll}1270.67 & 2.76 \mathrm{E}-13 & \mathrm{~Pb}-211 & \rightarrow & \mathrm{Bi}-211\end{array}$

\begin{tabular}{|c|c|c|c|c|}
\hline \multirow{2}{*}{$\begin{array}{c}\text { Am243 } \\
\text { Intensity }\end{array}$} & $\begin{array}{l}\text { Aged } 5 \\
\text { years }\end{array}$ & \multicolumn{3}{|c|}{ Sorted by Intensity } \\
\hline & Energy & \multicolumn{3}{|c|}{$\begin{array}{l}\text { Initial Isotopes and } \\
\text { Decays }\end{array}$} \\
\hline $\mathrm{ph} / \mathrm{s} / \mathrm{gm}$ & keV & & & \\
\hline $4.42 E+09$ & 74.672 & Am-243 & $->$ & $\mathrm{Np}-239$ \\
\hline $1.84 \mathrm{E}+09$ & 18.08 & $\mathrm{~Np}-239$ & $->$ & Pu-239 \\
\hline $1.76 \mathrm{E}+09$ & 103.734 & Np-239 & $->$ & Pu-239 \\
\hline $1.74 \mathrm{E}+09$ & 14.262 & $\mathrm{~Np}-239$ & $->$ & Pu-239 \\
\hline $1.67 \mathrm{E}+09$ & 106.1272 & $\mathrm{~Np}-239$ & $->$ & Pu-239 \\
\hline $1.11 \mathrm{E}+09$ & 99.522 & $\mathrm{~Np}-239$ & $->$ & Pu-239 \\
\hline $1.05 \mathrm{E}+09$ & 277.6016 & $\mathrm{~Np}-239$ & $->$ & Pu-239 \\
\hline $7.89 E+08$ & 228.1865 & $\mathrm{~Np}-239$ & $->$ & Pu-239 \\
\hline $6.34 \mathrm{E}+08$ & 116.93 & Np-239 & $->$ & Pu-239 \\
\hline $4.13 E+08$ & 21.622 & $\mathrm{~Np}-239$ & $->$ & Pu-239 \\
\hline $3.76 \mathrm{E}+08$ & 43.537 & Am-243 & $->$ & $\mathrm{Np}-239$ \\
\hline $2.45 E+08$ & 209.7554 & $\mathrm{~Np}-239$ & $->$ & Pu-239 \\
\hline $2.20 \mathrm{E}+08$ & 120.974 & $\mathrm{~Np}-239$ & $->$ & Pu-239 \\
\hline $1.51 \mathrm{E}+08$ & 334.3136 & $\mathrm{~Np}-239$ & $->$ & Pu-239 \\
\hline 1.17E+08 & 315.8825 & Np-239 & $->$ & Pu-239 \\
\hline $1.09 \mathrm{E}+08$ & 12.124 & Np-239 & $->$ & Pu-239 \\
\hline $7.08 \mathrm{E}+07$ & 61.462 & $\mathrm{~Np}-239$ & $->$ & Pu-239 \\
\hline $5.45 \mathrm{E}+07$ & 285.4624 & Np-239 & $->$ & Pu-239 \\
\hline $4.05 E+07$ & 117.7 & Am-243 & $->$ & $\mathrm{Np}-239$ \\
\hline $2.80 E+07$ & 16.333 & $\mathrm{~Np}-239$ & $->$ & Pu-239 \\
\hline $2.51 \mathrm{E}+07$ & 226.382 & $\mathrm{~Np}-239$ & $->$ & Pu-239 \\
\hline $2.21 E+07$ & 86.57 & Am-243 & $->$ & $\mathrm{Np}-239$ \\
\hline $1.09 E+07$ & 57.2759 & $\mathrm{~Np}-239$ & $->$ & Pu-239 \\
\hline $8.40 E+06$ & 141.97 & Am-243 & $->$ & $\mathrm{Np}-239$ \\
\hline $8.18 \mathrm{E}+06$ & 181.716 & Np-239 & $->$ & Pu-239 \\
\hline $7.74 \mathrm{E}+06$ & 49.4152 & Np-239 & $->$ & Pu-239 \\
\hline $7.67 E+06$ & 44.665 & Np-239 & $->$ & Pu-239 \\
\hline $7.30 \mathrm{E}+06$ & 254.421 & Np-239 & $->$ & Pu-239 \\
\hline $6.63 E+06$ & 67.8462 & Np-239 & $->$ & Pu-239 \\
\hline $5.53 E+06$ & 272.852 & Np-239 & $->$ & Pu-239 \\
\hline $4.87 E+06$ & 31.136 & $\mathrm{Am}-243$ & $->$ & $\mathrm{Np}-239$ \\
\hline $4.28 \mathrm{E}+06$ & 43.03 & Am-243 & $->$ & $N p-239$ \\
\hline $3.54 \mathrm{E}+06$ & 106.488 & Np-239 & $->$ & Pu-239 \\
\hline $1.25 \mathrm{E}+06$ & 166.364 & $N p-239$ & $->$ & Pu-239 \\
\hline $9.07 \mathrm{E}+05$ & 434.9 & $\mathrm{~Np}-239$ & $->$ & Pu-239 \\
\hline $7.67 E+05$ & 124.439 & Np-239 & $->$ & Pu-239 \\
\hline $6.78 \mathrm{E}+05$ & 55.4 & Am-243 & $->$ & $\mathrm{Np}-239$ \\
\hline $5.90 \mathrm{E}+05$ & 98.43 & Am-243 & $->$ & Np-239 \\
\hline $4.42 E+05$ & 57.278 & $\mathrm{~Np}-239$ & $->$ & Pu-239 \\
\hline $4.35 \mathrm{E}+05$ & 492.2 & Np-239 & $->$ & Pu-239 \\
\hline $4.35 E+05$ & 88.057 & $\mathrm{~Np}-239$ & $->$ & Pu-239 \\
\hline
\end{tabular}




\begin{tabular}{|c|c|c|c|c|c|c|c|}
\hline $4.20 E+05$ & 322.267 & $\mathrm{~Np}-239$ & $->$ & Pu-239 & & & \\
\hline $2.73 E+05$ & 429.87 & Np-239 & $->$ & Pu-239 & & & \\
\hline $2.36 \mathrm{E}+05$ & 497.71 & $\mathrm{~Np}-239$ & $->$ & Pu-239 & & & \\
\hline $2.29 \mathrm{E}+05$ & 498.8 & Np-239 & $->$ & Pu-239 & & & \\
\hline $1.92 \mathrm{E}+05$ & 50.8 & Am-243 & $->$ & Np-239 & & & \\
\hline $1.18 \mathrm{E}+05$ & 461.9 & Th-227 & $->$ & $\mathrm{Ra}-223$, & Np-239 & $->$ & Pu-239 \\
\hline $1.18 \mathrm{E}+05$ & 392.3 & Np-239 & $->$ & Pu-239 & & & \\
\hline $1.03 E+05$ & 503.983 & $\mathrm{~Np}-239$ & $->$ & Pu-239 & & & \\
\hline $1.03 E+05$ & 311.698 & Np-239 & $->$ & Pu-239 & & & \\
\hline $9.14 \mathrm{E}+04$ & 454.568 & $\mathrm{~Np}-239$ & $->$ & Pu-239 & & & \\
\hline $8.85 E+04$ & 170.14 & $\mathrm{Am}-243$ & $->$ & Np-239 & & & \\
\hline $7.81 E+04$ & 469.7 & $N p-239$ & $->$ & Pu-239 & & & \\
\hline $7.59 E+04$ & 484.3 & $\mathrm{~Np}-239$ & $->$ & Pu-239 & & & \\
\hline $7.00 E+04$ & 662.251 & Am-243 & $->$ & Np-239 & & & \\
\hline $6.19 E+04$ & 195.3 & Am-243 & $->$ & Np-239 & & & \\
\hline $6.12 \mathrm{E}+04$ & 436.137 & $\mathrm{~Np}-239$ & $->$ & Pu-239 & & & \\
\hline $5.16 \mathrm{E}+04$ & 101.943 & Np-239 & $->$ & Pu-239 & & & \\
\hline $2.43 E+04$ & 631.116 & Am-243 & $->$ & Np-239 & & & \\
\hline $1.84 \mathrm{E}+04$ & 448.3 & $N p-239$ & $->$ & Pu-239 & & & \\
\hline $1.25 \mathrm{E}+03$ & 544.55 & Am-243 & $->$ & $\mathrm{Np}-239$ & & & \\
\hline $2.87 E+02$ & 51.624 & Pu-239 & $->$ & $U-235$ & & & \\
\hline $1.11 \mathrm{E}+02$ & 38.66 & Pu-239 & $->$ & $U-235$ & & & \\
\hline $7.21 \mathrm{E}+01$ & 98.441 & Pu-239 & $->$ & $U-235$ & & & \\
\hline $6.62 \mathrm{E}+01$ & 129.296 & Pu-239 & $->$ & $U-235$ & & & \\
\hline $4.44 \mathrm{E}+01$ & 94.66 & Pu-239 & $->$ & $U-235$ & & & \\
\hline $1.66 \mathrm{E}+01$ & 375.039 & Pu-239 & $->$ & $U-235$ & & & \\
\hline $1.64 \mathrm{E}+01$ & 111.3 & Pu-239 & $->$ & $U-235$ & & & \\
\hline $1.55 \mathrm{E}+01$ & 413.713 & Pu-239 & $->$ & $U-235$ & & & \\
\hline $1.29 \mathrm{E}+01$ & 98.79 & Pu-239 & $->$ & $U-235$ & & & \\
\hline $1.19 \mathrm{E}+01$ & 56.825 & Pu-239 & $->$ & $U-235$ & & & \\
\hline $8.41 E+00$ & 110.421 & Pu-239 & $->$ & $U-235$ & & & \\
\hline $7.79 E+00$ & 46.218 & Pu-239 & $->$ & $U-235$ & & & \\
\hline $6.86 \mathrm{E}+00$ & 115.38 & Pu-239 & $->$ & U-235 & & & \\
\hline $6.64 \mathrm{E}+00$ & 114.333 & Pu-239 & $->$ & $U-235$ & & & \\
\hline $6.30 \mathrm{E}+00$ & 116.266 & Pu-239 & $->$ & U-235 & & & \\
\hline $5.92 E+00$ & 203.54 & Pu-239 & $->$ & $U-235$ & & & \\
\hline $5.91 \mathrm{E}+00$ & 345.014 & Pu-239 & $->$ & $U-235$ & & & \\
\hline $5.85 E+00$ & 392.914 & Pu-239 & $->$ & $U-235$ & & & \\
\hline $5.33 \mathrm{E}+00$ & 332.853 & Pu-239 & $->$ & $U-235$ & & & \\
\hline $4.52 E+00$ & 77.593 & Pu-239 & $->$ & $U-235$ & & & \\
\hline $3.23 E+00$ & 380.16 & Pu-239 & $->$ & $U-235$ & & & \\
\hline $3.21 E+00$ & 144.201 & Pu-239 & $->$ & $U-235$ & & & \\
\hline $2.74 \mathrm{E}+00$ & 382.732 & Pu-239 & $->$ & $U-235$ & & & \\
\hline $2.24 \mathrm{E}+00$ & 103.035 & Pu-239 & $->$ & $U-235$ & & & \\
\hline $2.00 E+00$ & 451.481 & Pu-239 & $->$ & $U-235$ & & & \\
\hline $1.79 \mathrm{E}+00$ & 78.37 & Pu-239 & $->$ & $U-235$ & & & \\
\hline $1.79 \mathrm{E}+00$ & 54.02 & Pu-239 & $->$ & $U-235$ & & & \\
\hline $1.74 \mathrm{E}+00$ & 42.06 & Pu-239 & $->$ & $U-235$ & & & \\
\hline $1.71 \mathrm{E}+00$ & 40.41 & Pu-239 & $->$ & $U-235$ & & & \\
\hline $1.50 \mathrm{E}+00$ & 67.69 & Pu-239 & $->$ & $U-235$ & & & \\
\hline
\end{tabular}




\begin{tabular}{|c|c|c|c|c|}
\hline $1.30 \mathrm{E}+00$ & 161.482 & Pu-239 & $->$ & U-235 \\
\hline $1.29 \mathrm{E}+00$ & 422.598 & Pu-239 & $->$ & $U-235$ \\
\hline $1.27 \mathrm{E}+00$ & 68.73 & Pu-239 & $->$ & $U-235$ \\
\hline $1.26 \mathrm{E}+00$ & 146.094 & Pu-239 & $->$ & $U-235$ \\
\hline $1.20 \mathrm{E}+00$ & 336.116 & Pu-239 & $->$ & $U-235$ \\
\hline $1.16 \mathrm{E}+00$ & 171.393 & Pu-239 & $->$ & U-235 \\
\hline $1.13 E+00$ & 195.679 & Pu-239 & $->$ & $U-235$ \\
\hline $1.11 \mathrm{E}+00$ & 30.08 & Pu-239 & $->$ & U-235 \\
\hline $9.52 \mathrm{E}-01$ & 368.543 & Pu-239 & $->$ & U-235 \\
\hline $9.17 \mathrm{E}-01$ & 367.048 & Pu-239 & $->$ & U-235 \\
\hline $8.78 \mathrm{E}-01$ & 189.36 & Pu-239 & $->$ & $U-235$ \\
\hline 8.46E-01 & 255.384 & Pu-239 & $->$ & U-235 \\
\hline 7.52E-01 & 125.21 & Pu-239 & $->$ & $U-235$ \\
\hline 7.00E-01 & 341.526 & Pu-239 & $->$ & $U-235$ \\
\hline $6.98 \mathrm{E}-01$ & 179.22 & Pu-239 & $->$ & $U-235$ \\
\hline $6.48 \mathrm{E}-01$ & 124.51 & Pu-239 & $->$ & $U-235$ \\
\hline $6.13 \mathrm{E}-01$ & 46.69 & Pu-239 & $->$ & $U-235$ \\
\hline $5.70 \mathrm{E}-01$ & 323.77 & Pu-239 & $->$ & $U-235$ \\
\hline $5.27 \mathrm{E}-01$ & 297.46 & Pu-239 & $->$ & U-235 \\
\hline $4.02 \mathrm{E}-01$ & 74.89 & Pu-239 & $->$ & $U-235$ \\
\hline 3.89E-01 & 96.16 & Pu-239 & $->$ & U-235 \\
\hline $3.49 \mathrm{E}-01$ & 65.7 & Pu-239 & $->$ & U-235 \\
\hline $3.38 \mathrm{E}-01$ & 141.657 & Pu-239 & $->$ & U-235 \\
\hline $3.25 \mathrm{E}-01$ & 111.89 & Pu-239 & $->$ & U-235 \\
\hline 3.07E-01 & 47.52 & Pu-239 & $->$ & U-235 \\
\hline $2.73 \mathrm{E}-01$ & 311.78 & Pu-239 & $->$ & $U-235$ \\
\hline $2.70 \mathrm{E}-01$ & 263.9 & Pu-239 & $->$ & U-235 \\
\hline $2.45 \mathrm{E}-01$ & 243.36 & Pu-239 & $->$ & $U-235$ \\
\hline $2.42 \mathrm{E}-01$ & 426.64 & Pu-239 & $->$ & $U-235$ \\
\hline $2.08 \mathrm{E}-01$ & 123.62 & Pu-239 & $->$ & $U-235$ \\
\hline $1.83 \mathrm{E}-01$ & 143.35 & Pu-239 & $->$ & $U-235$ \\
\hline $1.72 \mathrm{E}-01$ & 225.39 & Pu-239 & $->$ & $U-235$ \\
\hline $1.59 \mathrm{E}-01$ & 237.75 & Pu-239 & $->$ & U-235 \\
\hline $1.57 \mathrm{E}-01$ & 645.969 & Pu-239 & $->$ & U-235 \\
\hline $1.49 \mathrm{E}-01$ & 316.4 & Pu-239 & $->$ & $U-235$ \\
\hline $1.26 \mathrm{E}-01$ & 769.19 & Pu-239 & $->$ & U-235 \\
\hline $1.24 \mathrm{E}-01$ & 361.84 & Pu-239 & $->$ & $U-235$ \\
\hline $1.15 \mathrm{E}-01$ & 188.23 & Pu-239 & $->$ & $U-235$ \\
\hline $1.05 \mathrm{E}-01$ & 658.919 & Pu-239 & $->$ & $U-235$ \\
\hline $9.31 \mathrm{E}-02$ & 445.81 & Pu-239 & $->$ & $U-235$ \\
\hline 8.78E-02 & 242.12 & Pu-239 & $->$ & $U-235$ \\
\hline 8.67E-02 & 640.096 & Pu-239 & $->$ & $U-235$ \\
\hline 8.46E-02 & 410.94 & Pu-239 & $->$ & U-235 \\
\hline 7.93E-02 & 248.88 & Pu-239 & $->$ & U-235 \\
\hline 6.93E-02 & 652.052 & Pu-239 & $->$ & U-235 \\
\hline $6.56 \mathrm{E}-02$ & 160.19 & Pu-239 & $->$ & $U-235$ \\
\hline $6.56 \mathrm{E}-02$ & 307.82 & Pu-239 & $->$ & U-235 \\
\hline $6.45 \mathrm{E}-02$ & 399.46 & Pu-239 & $->$ & U-235 \\
\hline $6.03 E-02$ & 302.89 & Pu-239 & $->$ & U-235 \\
\hline $5.50 \mathrm{E}-02$ & 244.86 & Pu-239 & $->$ & U-235 \\
\hline
\end{tabular}




$\begin{array}{lcccc}\text { 5.18E-02 } & 430.16 & \mathrm{Pu}-239 & -> & \mathrm{U}-235 \\ 4.86 \mathrm{E}-02 & 481.78 & \mathrm{Pu}-239 & -> & \mathrm{U}-235 \\ 4.23 \mathrm{E}-02 & 173.7 & \mathrm{Pu}-239 & -> & \mathrm{U}-235 \\ 4.10 \mathrm{E}-02 & 703.8 & \mathrm{Pu}-239 & -> & \mathrm{U}-235 \\ 3.91 \mathrm{E}-02 & 196.87 & \mathrm{Pu}-239 & -> & \mathrm{U}-235 \\ 3.56 \mathrm{E}-02 & 756.42 & \mathrm{Pu}-239 & -> & \mathrm{U}-235 \\ 3.17 \mathrm{E}-02 & 265.81 & \mathrm{Pu}-239 & -> & \mathrm{U}-235 \\ 3.17 \mathrm{E}-02 & 122.34 & \mathrm{Pu}-239 & -> & \mathrm{U}-235 \\ 3.10 \mathrm{E}-02 & 167.81 & \mathrm{Pu}-239 & -> & \mathrm{U}-235 \\ 2.96 \mathrm{E}-02 & 718 & \mathrm{Pu}-239 & -> & \mathrm{U}-235 \\ 2.71 \mathrm{E}-02 & 637.862 & \mathrm{Pu}-239 & -> & \mathrm{U}-235 \\ 2.68 \mathrm{E}-02 & 633.15 & \mathrm{Pu}-239 & -> & \mathrm{U}-235 \\ 2.58 \mathrm{E}-02 & 618.94 & \mathrm{Pu}-239 & -> & \mathrm{U}-235 \\ 2.38 \mathrm{E}-02 & 654.89 & \mathrm{Pu}-239 & -> & \mathrm{U}-235 \\ 2.24 \mathrm{E}-02 & 184.55 & \mathrm{Pu}-239 & -> & \mathrm{U}-235 \\ 2.22 \mathrm{E}-02 & 372.47 & \mathrm{Pu}-239 & -> & \mathrm{U}-235 \\ 2.22 \mathrm{E}-02 & 281.12 & \mathrm{Pu}-239 & -> & \mathrm{U}-235 \\ 2.16 \mathrm{E}-02 & 617.43 & \mathrm{Pu}-239 & -> & \mathrm{U}-235 \\ 2.11 \mathrm{E}-02 & 313.39 & \mathrm{Pu}-239 & -> & \mathrm{U}-235 \\ 2.11 \mathrm{E}-02 & 461.25 & \mathrm{Pu}-239 & -> & \mathrm{U}-235 \\ 1.75 \mathrm{E}-02 & 664.587 & \mathrm{Pu}-239 & -> & \mathrm{U}-235 \\ 1.63 \mathrm{E}-02 & 457.57 & \mathrm{Pu}-239 & -> & \mathrm{U}-235 \\ 1.59 \mathrm{E}-02 & 285.27 & \mathrm{Pu}-239 & -> & \mathrm{U}-235 \\ 1.15 \mathrm{E}-02 & 727.84 & \mathrm{Pu}-239 & -> & \mathrm{U}-235 \\ 1.11 \mathrm{E}-02 & 379.05 & \mathrm{Pu}-239 & -> & \mathrm{U}-235 \\ 1.06 \mathrm{E}-02 & 218.45 & \mathrm{Pu}-239 & -> & \mathrm{U}-235 \\ 9.41 \mathrm{E}-03 & 686.16 & \mathrm{Pu}-239 & -> & \mathrm{U}-235 \\ 9.31 \mathrm{E}-03 & 493.25 & \mathrm{Pu}-239 & -> & \mathrm{U}-235 \\ \text { 8.57E-03 } & 612.93 & \mathrm{Pu}-239 & -> & \mathrm{U}-235 \\ \text { 8.46E-03 } & 354.02 & \mathrm{Pu}-239 & -> & \mathrm{U}-235 \\ \text { 7.53E-03 } & 649.377 & \mathrm{Pu}-239 & -> & \mathrm{U}-235 \\ \text { 9.59E-04 } & 16.559 & \mathrm{Th}-231 & -> & \mathrm{Pa}-231 \\ \text { 9.52E-04 } & 474.26 & \mathrm{Pu}-239 & -> & \mathrm{U}-235 \\ 9.52 \mathrm{E}-04 & 714.58 & \mathrm{Pu}-239 & -> & \mathrm{U}-235\end{array}$




\begin{tabular}{|c|c|c|c|c|c|c|c|}
\hline 8.88E-04 & 787.3 & Pu-239 & $->$ & $U-235$ & & & \\
\hline $8.14 \mathrm{E}-04$ & 891.1 & Pu-239 & $->$ & $U-235$ & & & \\
\hline $6.56 \mathrm{E}-04$ & 813.9 & Pu-239 & $->$ & $U-235$ & & & \\
\hline $6.34 \mathrm{E}-04$ & 557.7 & Pu-239 & $->$ & $U-235$ & & & \\
\hline $5.92 \mathrm{E}-04$ & 956.4 & Pu-239 & $->$ & $U-235$ & & & \\
\hline $5.71 \mathrm{E}-04$ & 12.952 & $A c-227$ & $->$ & Th-227, & $U-235$ & $->$ & Th-231 \\
\hline $5.39 \mathrm{E}-04$ & 821.1 & Pu-239 & $->$ & U-235 & & & \\
\hline $5.29 E-04$ & 879 & Pu-239 & $->$ & $U-235$ & & & \\
\hline $4.76 \mathrm{E}-04$ & 1057.32 & Pu-239 & $->$ & U-235 & & & \\
\hline 4.65E-04 & 803.3 & Pu-239 & $->$ & $U-235$ & & & \\
\hline $4.44 \mathrm{E}-04$ & 940.1 & Pu-239 & $->$ & $U-235$ & & & \\
\hline $4.23 E-04$ & 839 & Pu-239 & $->$ & $U-235$ & & & \\
\hline 3.90E-04 & 16.119 & U-235 & $->$ & Th-231 & & & \\
\hline $3.79 \mathrm{E}-04$ & 25.642 & Th-231 & $->$ & $\mathrm{Pa}-231$ & & & \\
\hline $3.38 \mathrm{E}-04$ & 796.5 & Pu-239 & $->$ & U-235 & & & \\
\hline $2.85 \mathrm{E}-04$ & 143.76 & $U-235$ & $->$ & Th-231 & & & \\
\hline $2.64 \mathrm{E}-04$ & 793 & Pu-239 & $->$ & U-235 & & & \\
\hline $2.64 \mathrm{E}-04$ & 831.93 & Pu-239 & $->$ & $U-235$ & & & \\
\hline $2.43 \mathrm{E}-04$ & 992.47 & Pu-239 & $->$ & U-235 & & & \\
\hline $2.43 \mathrm{E}-04$ & 979.52 & Pu-239 & $->$ & $U-235$ & & & \\
\hline $2.11 \mathrm{E}-04$ & 412.83 & Pu-239 & $->$ & $U-235$ & & & \\
\hline $1.92 \mathrm{E}-04$ & 19.811 & Th-231 & $->$ & $\mathrm{Pa}-231$ & & & \\
\hline $1.71 \mathrm{E}-04$ & 84.203 & Th-231 & $->$ & $\mathrm{Pa}-231$ & & & \\
\hline $1.43 \mathrm{E}-04$ & 93.36 & $U-235$ & $->$ & Th-231 & & & \\
\hline $1.32 \mathrm{E}-04$ & 163.33 & U-235 & $->$ & Th-231 & & & \\
\hline $1.30 \mathrm{E}-04$ & 205.31 & $U-235$ & $->$ & Th-231 & & & \\
\hline $1.27 \mathrm{E}-04$ & 1005.63 & Pu-239 & $->$ & U-235 & & & \\
\hline $1.27 \mathrm{E}-04$ & 986.7 & Pu-239 & $->$ & $U-235$ & & & \\
\hline $8.73 \mathrm{E}-05$ & 89.955 & $U-235$ & $->$ & Th-231 & & & \\
\hline $7.78 \mathrm{E}-05$ & 11.372 & Th-231 & $->$ & $\mathrm{Pa}-231$ & & & \\
\hline $6.75 E-05$ & 19.118 & U-235 & $->$ & Th-231 & & & \\
\hline $5.14 \mathrm{E}-05$ & 105.362 & U-235 & $->$ & Th-231 & & & \\
\hline 4.00E-05 & 109.16 & $U-235$ & $->$ & Th-231 & & & \\
\hline $2.91 \mathrm{E}-05$ & 766.6 & Pu-239 & $->$ & $U-235$ & & & \\
\hline $2.81 \mathrm{E}-05$ & 202.11 & U-235 & $->$ & Th-231 & & & \\
\hline $2.60 \mathrm{E}-05$ & 11.118 & $A c-227$ & $->$ & Th-227, & U-235 & $->$ & Th-231 \\
\hline $2.44 \mathrm{E}-05$ & 89.954 & Th-231 & $->$ & $\mathrm{Pa}-231$ & & & \\
\hline $2.33 \mathrm{E}-05$ & 81.229 & Th-231 & $->$ & $\mathrm{Pa}-231$ & & & \\
\hline $1.71 \mathrm{E}-05$ & 108.99 & U-235 & $->$ & Th-231 & & & \\
\hline $1.64 \mathrm{E}-05$ & 194.94 & $U-235$ & $->$ & Th-231 & & & \\
\hline $1.63 \mathrm{E}-05$ & 95.863 & Th-231 & $->$ & $\mathrm{Pa}-231$ & & & \\
\hline $1.24 \mathrm{E}-05$ & 58.562 & Th-231 & $->$ & $\mathrm{Pa}-231$ & & & \\
\hline $1.06 \mathrm{E}-05$ & 102.255 & Th-231 & $->$ & $\mathrm{Pa}-231$ & & & \\
\hline $1.04 \mathrm{E}-05$ & 14.953 & Th-231 & $->$ & $\mathrm{Pa}-231$ & & & \\
\hline $1.01 \mathrm{E}-05$ & 92.279 & Th-231 & $->$ & $\mathrm{Pa}-231$ & & & \\
\hline $8.83 \mathrm{E}-06$ & 182.61 & U-235 & $->$ & Th-231 & & & \\
\hline $6.51 \mathrm{E}-06$ & 72.767 & Th-231 & $->$ & $\mathrm{Pa}-231$ & & & \\
\hline 5.91E-06 & 108.166 & Th-231 & $->$ & $\mathrm{Pa}-231$ & & & \\
\hline $5.71 \mathrm{E}-06$ & 14.511 & $A c-227$ & $->$ & Th-227, & U-235 & $->$ & Th-231 \\
\hline $5.71 \mathrm{E}-06$ & 140.76 & U-235 & $->$ & Th-231 & & & \\
\hline
\end{tabular}




\begin{tabular}{|c|c|c|c|c|}
\hline $5.70 \mathrm{E}-06$ & 17.187 & Th-231 & $->$ & $\mathrm{Pa}-231$ \\
\hline $4.02 E-06$ & 163.114 & Th-231 & $->$ & Pa-231 \\
\hline $3.12 \mathrm{E}-06$ & 221.38 & U-235 & $->$ & Th-231 \\
\hline $3.11 \mathrm{E}-06$ & 99.28 & Th-231 & $->$ & $\mathrm{Pa}-231$ \\
\hline $2.86 \mathrm{E}-06$ & 72.71 & U-235 & $->$ & Th-231 \\
\hline $2.23 E-06$ & 96.154 & U-235 & $->$ & Th-231 \\
\hline $2.08 \mathrm{E}-06$ & 150.93 & U-235 & $->$ & Th-231 \\
\hline $2.02 E-06$ & 135.681 & Th-231 & $->$ & $\mathrm{Pa}-231$ \\
\hline 1.97E-06 & 111.897 & Th-231 & $->$ & $\mathrm{Pa}-231$ \\
\hline $1.82 \mathrm{E}-06$ & 116.11 & U-235 & $->$ & Th-231 \\
\hline $1.56 \mathrm{E}-06$ & 41.96 & $U-235$ & $->$ & Th-231 \\
\hline $1.56 \mathrm{E}-06$ & 74.8 & $U-235$ & $->$ & Th-231 \\
\hline $1.50 \mathrm{E}-06$ & 42.828 & Th-231 & $->$ & $\mathrm{Pa}-231$ \\
\hline $1.45 \mathrm{E}-06$ & 124.922 & Th-231 & $->$ & Pa-231 \\
\hline $1.40 \mathrm{E}-06$ & 240.85 & U-235 & $->$ & Th-231 \\
\hline $1.38 \mathrm{E}-06$ & 246.84 & U-235 & $->$ & Th-231 \\
\hline $1.27 \mathrm{E}-06$ & 93.07 & Th-231 & $->$ & $\mathrm{Pa}-231$ \\
\hline 1.09E-06 & 198.9 & U-235 & $->$ & Th-231 \\
\hline $1.04 \mathrm{E}-06$ & 217.934 & Th-231 & $->$ & Pa-231 \\
\hline 8.53E-07 & 183.483 & Th-231 & $->$ & $\mathrm{Pa}-231$ \\
\hline $8.30 \mathrm{E}-07$ & 145.927 & Th-231 & $->$ & $\mathrm{Pa}-231$ \\
\hline 7.79E-07 & 41.13 & $U-235$ & $->$ & Th-231 \\
\hline $7.53 \mathrm{E}-07$ & 345.921 & U-235 & $->$ & Th-231 \\
\hline $7.53 E-07$ & 233.5 & U-235 & $->$ & Th-231 \\
\hline 7.01E-07 & 215.305 & U-235 & $->$ & Th-231 \\
\hline $6.75 \mathrm{E}-07$ & 119.98 & U-235 & $->$ & Th-231 \\
\hline $6.22 \mathrm{E}-07$ & 134.014 & Th-231 & $->$ & $\mathrm{Pa}-231$ \\
\hline $5.96 \mathrm{E}-07$ & 63.834 & Th-231 & $->$ & Pa-231 \\
\hline $5.37 \mathrm{E}-07$ & 116.827 & Th-231 & $->$ & $\mathrm{Pa}-231$ \\
\hline $5.19 \mathrm{E}-07$ & 51.179 & U-235 & $->$ & Th-231 \\
\hline 5.19E-07 & 64.348 & $U-235$ & $->$ & Th-231 \\
\hline 4.75E-07 & 174.157 & Th-231 & $->$ & $\mathrm{Pa}-231$ \\
\hline 4.41E-07 & 106.581 & Th-231 & $->$ & Pa-231 \\
\hline $4.16 \mathrm{E}-07$ & 31.585 & $U-235$ & $->$ & Th-231 \\
\hline $3.64 \mathrm{E}-07$ & 291.72 & $U-235$ & $->$ & Th-231 \\
\hline $3.12 \mathrm{E}-07$ & 136.72 & U-235 & $->$ & Th-231 \\
\hline $2.60 E-07$ & 172.27 & U-235 & $->$ & Th-231 \\
\hline $2.39 \mathrm{E}-07$ & 235.985 & Th-231 & $->$ & Pa-231 \\
\hline $2.08 \mathrm{E}-07$ & 228.77 & $U-235$ & $->$ & Th-231 \\
\hline $1.84 \mathrm{E}-07$ & 105.803 & Th-231 & $->$ & $\mathrm{Pa}-231$ \\
\hline $1.82 \mathrm{E}-07$ & 289.57 & U-235 & $->$ & Th-231 \\
\hline $1.56 \mathrm{E}-07$ & 281.45 & U-235 & $->$ & Th-231 \\
\hline $1.56 \mathrm{E}-07$ & 266.483 & $U-235$ & $->$ & Th-231 \\
\hline $1.50 \mathrm{E}-07$ & 145.063 & Th-231 & $->$ & Pa-231 \\
\hline $1.48 \mathrm{E}-07$ & 68.5 & Th-231 & $->$ & $\mathrm{Pa}-231$ \\
\hline $1.30 \mathrm{E}-07$ & 301.72 & U-235 & $->$ & Th-231 \\
\hline $1.30 \mathrm{E}-07$ & 356.068 & U-235 & $->$ & Th-231 \\
\hline $1.30 \mathrm{E}-07$ & 282.98 & $U-235$ & $->$ & Th-231 \\
\hline $1.27 \mathrm{E}-07$ & 275.47 & U-235 & $->$ & Th-231 \\
\hline $1.09 \mathrm{E}-07$ & 136.705 & Th-231 & $->$ & Pa-231 \\
\hline
\end{tabular}




\begin{tabular}{|c|c|c|c|c|c|c|c|}
\hline $1.04 \mathrm{E}-07$ & 310.71 & U-235 & $->$ & Th-231 & & & \\
\hline $1.01 \mathrm{E}-07$ & 164.97 & Th-231 & $->$ & $\mathrm{Pa}-231$ & & & \\
\hline $9.87 \mathrm{E}-08$ & 387.874 & U-235 & $->$ & Th-231 & & & \\
\hline 8.30E-08 & 188.756 & Th-231 & $->$ & $\mathrm{Pa}-231$ & & & \\
\hline 7.79E-08 & 410.269 & U-235 & $->$ & Th-231 & & & \\
\hline 7.79E-08 & 343.78 & $U-235$ & $->$ & Th-231 & & & \\
\hline $7.52 \mathrm{E}-08$ & 311 & Th-231 & $->$ & Pa-231 & & & \\
\hline $3.11 \mathrm{E}-08$ & 169.637 & Th-231 & $->$ & $\mathrm{Pa}-231$ & & & \\
\hline $3.01 \mathrm{E}-08$ & 267.62 & Th-231 & $->$ & $\mathrm{Pa}-231$ & & & \\
\hline $2.60 \mathrm{E}-08$ & 448.42 & U-235 & $->$ & Th-231 & & & \\
\hline $2.60 \mathrm{E}-08$ & 317.12 & $U-235$ & $->$ & Th-231 & & & \\
\hline $2.60 \mathrm{E}-08$ & 390.32 & $U-235$ & $->$ & Th-231 & & & \\
\hline 2.59E-08 & 115.595 & Th-231 & $->$ & $\mathrm{Pa}-231$ & & & \\
\hline $2.28 \mathrm{E}-08$ & 15.69 & $\mathrm{~Pa}-231$ & $->$ & Ac- 227 & & & \\
\hline $2.18 \mathrm{E}-08$ & 242.508 & Th-231 & $->$ & $\mathrm{Pa}-231$ & & & \\
\hline $2.08 \mathrm{E}-08$ & 455.12 & U-235 & $->$ & Th-231 & & & \\
\hline $2.02 \mathrm{E}-08$ & 249.56 & Th-231 & $->$ & Pa-231 & & & \\
\hline $2.00 \mathrm{E}-08$ & 12.636 & $\mathrm{~Pa}-231$ & $->$ & Ac- 227 & & & \\
\hline $1.84 \mathrm{E}-08$ & 140.537 & Th-231 & $->$ & $\mathrm{Pa}-231$ & & & \\
\hline $1.82 \mathrm{E}-08$ & 44.06 & Th-231 & $->$ & Pa-231 & & & \\
\hline $1.69 \mathrm{E}-08$ & 250.43 & Th-231 & $->$ & $\mathrm{Pa}-231$ & & & \\
\hline $1.56 \mathrm{E}-08$ & 794.72 & U-235 & $->$ & Th-231 & & & \\
\hline $1.04 \mathrm{E}-08$ & 517.2 & $U-235$ & $->$ & Th-231 & & & \\
\hline $1.04 \mathrm{E}-08$ & 742.52 & U-235 & $->$ & Th-231 & & & \\
\hline $1.01 \mathrm{E}-08$ & 308.75 & Th-231 & $->$ & Pa-231 & & & \\
\hline 8.48E-09 & 27.396 & Pa-231 & $->$ & Ac- 227 & & & \\
\hline $7.26 \mathrm{E}-09$ & 240.25 & Th-231 & $->$ & $\mathrm{Pa}-231$ & & & \\
\hline $5.38 \mathrm{E}-09$ & 18.607 & $\mathrm{~Pa}-231$ & $->$ & Ac- 227 & & & \\
\hline $2.85 \mathrm{E}-09$ & 320.188 & Th-231 & $->$ & $\mathrm{Pa}-231$ & & & \\
\hline 2.17E-09 & 300.07 & $\mathrm{~Pa}-231$ & $->$ & Ac- 227 & & & \\
\hline 2.07E-09 & 317.93 & Th-231 & $->$ & Pa-231 & & & \\
\hline $1.82 \mathrm{E}-09$ & 351.82 & Th-231 & $->$ & $\mathrm{Pa}-231$ & & & \\
\hline $1.56 \mathrm{E}-09$ & 302.671 & $\mathrm{~Pa}-231$ & $->$ & Ac- 227 & & & \\
\hline $1.45 \mathrm{E}-09$ & 283.673 & $\mathrm{~Pa}-231$ & $->$ & Ac- 227 & & & \\
\hline $1.20 \mathrm{E}-09$ & 330.066 & $\mathrm{~Pa}-231$ & $->$ & Ac- 227 & & & \\
\hline $1.09 \mathrm{E}-09$ & 10.871 & $\mathrm{~Pa}-231$ & $->$ & Ac- 227 & & & \\
\hline $7.91 \mathrm{E}-10$ & 83.787 & Ra-223 & $->$ & Rn-219 & & & \\
\hline $7.78 \mathrm{E}-10$ & 274.14 & Th-231 & $->$ & $\mathrm{Pa}-231$ & & & \\
\hline $7.11 \mathrm{E}-10$ & 90.886 & $\mathrm{~Pa}-231$ & $->$ & Ac- 227 & & & \\
\hline $6.92 \mathrm{E}-10$ & 15.218 & Th-227 & $->$ & Ra-223 & & & \\
\hline $5.83 \mathrm{E}-10$ & 302.638 & Pa-231 & $->$ & Ac-227 & & & \\
\hline $5.68 \mathrm{E}-10$ & 12.325 & Fr-223 & $->$ & Ra-223, & Th-227 & $->$ & Ra-223 \\
\hline 4.77E-10 & 81.067 & $\mathrm{Ra}-223$ & $->$ & $\mathrm{Rn}-219$ & & & \\
\hline $4.34 \mathrm{E}-10$ & 87.673 & $\mathrm{~Pa}-231$ & $->$ & Ac- 227 & & & \\
\hline $4.27 \mathrm{E}-10$ & 269.39 & $\mathrm{Ra}-223$ & $->$ & $\mathrm{Rn}-219$ & & & \\
\hline $3.94 \mathrm{E}-10$ & 350.1 & $\mathrm{Bi}-211$ & $->$ & TI-207 & & & \\
\hline $3.69 \mathrm{E}-10$ & 235.97 & Fr-223 & $->$ & Ra-223, & Th-227 & $->$ & $\mathrm{Ra}-223$ \\
\hline $3.46 \mathrm{E}-10$ & 16.397 & Pa-231 & $->$ & Ac- 227 & & & \\
\hline $3.37 \mathrm{E}-10$ & 14.082 & $\mathrm{~Pa}-231$ & $->$ & Ac- 227 & & & \\
\hline $3.20 \mathrm{E}-10$ & 11.713 & Ra-223 & $->$ & $\mathrm{Rn}-219$ & & & \\
\hline
\end{tabular}




$\begin{array}{lc}3.14 \mathrm{E}-10 & 271.13 \\ 3.08 \mathrm{E}-10 & 14.341 \\ 3.01 \mathrm{E}-10 & 18.998 \\ 2.95 \mathrm{E}-10 & 50.14 \\ 2.79 \mathrm{E}-10 & 94.677 \\ 2.55 \mathrm{E}-10 & 102.613 \\ 2.21 \mathrm{E}-10 & 256.24 \\ 2.11 \mathrm{E}-10 & 401.7 \\ 1.90 \mathrm{E}-10 & 46.393 \\ 1.76 \mathrm{E}-10 & 154.18 \\ 1.66 \mathrm{E}-10 & 260.29 \\ 1.59 \mathrm{E}-10 & 340.873 \\ 1.58 \mathrm{E}-10 & 17.997 \\ 1.40 \mathrm{E}-10 & 357.2 \\ 1.36 \mathrm{E}-10 & 38.235 \\ 1.22 \mathrm{E}-10 & 323.88 \\ 1.19 \mathrm{E}-10 & 404.86 \\ 1.18 \mathrm{E}-10 & 831.86 \\ 1.03 \mathrm{E}-10 & 88.471 \\ 1.03 \mathrm{E}-10 & 313.058 \\ 1.02 \mathrm{E}-10 & 144.18 \\ 9.11 \mathrm{E}-11 & 255.858 \\ 9.00 \mathrm{E}-11 & 329.84 \\ 8.92 \mathrm{E}-11 & 97.907 \\ 8.73 \mathrm{E}-11 & 338.28 \\ 8.38 \mathrm{E}-11 & 106.137 \\ 8.38 \mathrm{E}-11 & 29.996 \\ 8.20 \mathrm{E}-11 & 25.464 \\ 8.02 \mathrm{E}-11 & 96.979 \\ 7.75 \mathrm{E}-11 & 52.763 \\ 7.38 \mathrm{E}-11 & 54.632 \\ 7.33 \mathrm{E}-11 & 79.72 \\ 7.29 \mathrm{E}-11 & 354.63 \\ 6.92 \mathrm{E}-11 & 299.97 \\ 6.28 \mathrm{E}-11 & 85.429 \\ 6.20 \mathrm{E}-11 & 77.405 \\ 6.09 \mathrm{E}-11 & 16.918 \\ 5.65 \mathrm{E}-11 & 277.17 \\ 5.47 \mathrm{E}-11 & 44.213 \\ 5.34 \mathrm{E}-11 & 427 \\ 5.24 \mathrm{E}-11 & 286.11 \\ 5.19 \mathrm{E}-11 & 273.17 \\ 4.61 \mathrm{E}-11 & 93.93 \\ 4.56 \mathrm{E}-11 & 63.7 \\ 4.28 \mathrm{E}-11 & 243.17 \\ 4.19 \mathrm{E}-11 & 379.4 \\ 3.99 \mathrm{E}-11 & 444.94 \\ 3.91 \mathrm{E}-11 & 72.873 \\ 3.74 \mathrm{E}-11 & 122.31 \\ 3.73 \mathrm{E}-11 & 210.58\end{array}$

\begin{tabular}{|c|c|c|c|c|c|}
\hline $\mathrm{Rn}-219$ & $->$ & Po-215 & & & \\
\hline Ra-223 & $->$ & $\mathrm{Rn}-219$ & & & \\
\hline $\mathrm{Pa}-231$ & $->$ & $A c-227$ & & & \\
\hline Fr-223 & $->$ & Ra-223, & Th-227 & $->$ & Ra-223 \\
\hline $\mathrm{Ra}-223$ & $->$ & $\mathrm{Rn}-219$ & & & \\
\hline $\mathrm{Pa}-231$ & $->$ & $A c-227$ & & & \\
\hline $\mathrm{Fr}-223$ & $->$ & Ra-223, & Th-227 & $->$ & Ra-223 \\
\hline Rn-219 & $->$ & Po-215 & & & \\
\hline $\mathrm{Pa}-231$ & $->$ & Ac- 227 & & & \\
\hline Ra-223 & $->$ & Rn-219 & & & \\
\hline $\mathrm{Pa}-231$ & $->$ & Ac- 227 & & & \\
\hline $\mathrm{Pa}-231$ & $->$ & Ac- 227 & & & \\
\hline Th-227 & $->$ & Ra-223 & & & \\
\hline $\mathrm{Pa}-231$ & $->$ & $A c-227$ & & & \\
\hline $\mathrm{Pa}-231$ & $->$ & Ac- 227 & & & \\
\hline Ra-223 & $->$ & $\mathrm{Rn}-219$ & & & \\
\hline $\mathrm{Pb}-211$ & $->$ & $\mathrm{Bi}-211$, & At-215 & $->$ & $\mathrm{Bi}-211$ \\
\hline $\mathrm{Pb}-211$ & $->$ & $\mathrm{Bi}-211$ & & & \\
\hline Fr-223 & $->$ & Ra-223, & Th-227 & $->$ & Ra-223 \\
\hline $\mathrm{Pa}-231$ & $->$ & Ac- 227 & & & \\
\hline $\mathrm{Ra}-223$ & $->$ & $\mathrm{Rn}-219$ & & & \\
\hline $\mathrm{Pa}-231$ & $->$ & Ac-227 & & & \\
\hline $\mathrm{Fr}-223$ & $->$ & Ra-223, & Th-227 & $->$ & Ra-223 \\
\hline Ra-223 & $->$ & $\mathrm{Rn}-219$ & & & \\
\hline Ra-223 & $->$ & $\mathrm{Rn}-219$ & & & \\
\hline $\mathrm{Pa}-231$ & $->$ & Ac-227 & & & \\
\hline $\mathrm{Pa}-231$ & $->$ & Ac- 227 & & & \\
\hline $\mathrm{Pa}-231$ & $->$ & Ac- 227 & & & \\
\hline $\mathrm{Pa}-231$ & $->$ & Ac- 227 & & & \\
\hline Pa-231 & $->$ & Ac- 227 & & & \\
\hline $\mathrm{Pa}-231$ & $->$ & $A c-227$ & & & \\
\hline Fr-223 & $->$ & Ra-223, & Th-227 & $->$ & Ra-223 \\
\hline $\mathrm{Pa}-231$ & $->$ & $A c-227$ & & & \\
\hline Fr-223 & $->$ & Ra-223, & Th-227 & $->$ & Ra-223 \\
\hline $\mathrm{Fr}-223$ & $->$ & Ra-223, & Th-227 & $->$ & Ra-223 \\
\hline $\mathrm{Pa}-231$ & $->$ & Ac-227 & & & \\
\hline $\mathrm{Ra}-223$ & $->$ & $\mathrm{Rn}-219$ & & & \\
\hline Pa-231 & $->$ & $A C-227$ & & & \\
\hline Pa-231 & $->$ & Ac- 227 & & & \\
\hline $\mathrm{Pb}-211$ & $->$ & $\mathrm{Bi}-211$ & & & \\
\hline Fr-223 & $->$ & Ra-223, & Th-227 & $->$ & Ra-223 \\
\hline $\mathrm{Pa}-231$ & $->$ & Ac- 227 & & & \\
\hline Th-227 & $->$ & $\mathrm{Ra}-223$ & & & \\
\hline $\mathrm{Pa}-231$ & $->$ & Ac-227 & & & \\
\hline $\mathrm{Pa}-231$ & $->$ & Ac-227 & & & \\
\hline $\mathrm{Rn}-219$ & $->$ & Po-215, & Pa-231 & $->$ & Ac-227 \\
\hline Ra-223 & $->$ & $\mathrm{Rn}-219$ & & & \\
\hline $\mathrm{Bi}-211$ & $->$ & TI-207 & & & \\
\hline $\mathrm{Ra}-223$ & $->$ & $\mathrm{Rn}-219$ & & & \\
\hline $\mathrm{Fr}-223$ & $->$ & Ra-223, & Th-227 & $->$ & Ra-223 \\
\hline
\end{tabular}




$\begin{array}{lc}3.69 \mathrm{E}-11 & 99.915 \\ 3.59 \mathrm{E}-11 & 304.51 \\ 3.27 \mathrm{E}-11 & 10.622 \\ 3.26 \mathrm{E}-11 & 334.38 \\ 3.10 \mathrm{E}-11 & 407.98 \\ 2.92 \mathrm{E}-11 & 100.96 \\ 2.76 \mathrm{E}-11 & 79.29 \\ 2.74 \mathrm{E}-11 & 327.23 \\ 2.73 \mathrm{E}-11 & 24.56 \\ 2.64 \mathrm{E}-11 & 254.66 \\ 2.31 \mathrm{E}-11 & 70.832 \\ 2.28 \mathrm{E}-11 & 74.209 \\ 2.28 \mathrm{E}-11 & 57.233 \\ 2.20 \mathrm{E}-11 & 766.35 \\ 2.16 \mathrm{E}-11 & 158.59 \\ 2.00 \mathrm{E}-11 & 341.17 \\ 1.85 \mathrm{E}-11 & 113.19 \\ 1.73 \mathrm{E}-11 & 10.137 \\ 1.65 \mathrm{E}-11 & 76.858 \\ 1.62 \mathrm{E}-11 & 234.82 \\ 1.62 \mathrm{E}-11 & 272.95 \\ 1.55 \mathrm{E}-11 & 312.57 \\ 1.54 \mathrm{E}-11 & 371.8 \\ 1.52 \mathrm{E}-11 & 314.82 \\ 1.49 \mathrm{E}-11 & 704.51 \\ 1.46 \mathrm{E}-11 & 35.884 \\ 1.43 \mathrm{E}-11 & 13.495 \\ 1.42 \mathrm{E}-11 & 296.54 \\ 1.38 \mathrm{E}-11 & 82.434 \\ 1.37 \mathrm{E}-11 & 57.2 \\ 1.35 \mathrm{E}-11 & 10.259 \\ 1.33 \mathrm{E}-11 & 77.107 \\ 1.30 \mathrm{E}-11 & 11.119 \\ 1.27 \mathrm{E}-11 & 13.082 \\ 1.25 \mathrm{E}-11 & 342.44 \\ 1.23 \mathrm{E}-11 & 12.313 \\ 1.22 \mathrm{E}-11 & 250.12 \\ 1.21 \mathrm{E}-11 & 103.341 \\ 1.20 \mathrm{E}-11 & 13.662 \\ 1.15 \mathrm{E}-11 & 10.828 \\ 1.09 \mathrm{E}-11 & 40.006 \\ 1.00 \mathrm{E}-11 & 56.9 \\ 9.84 \mathrm{E}-12 & 89.639 \\ 8.80 \mathrm{E}-12 & 15.213 \\ 8.48 \mathrm{E}-12 & 398.4 \\ 8.20 \mathrm{E}-12 & 31.04 \\ 8.20 \mathrm{E}-12 & 359.51 \\ 7.94 \mathrm{E}-12 & 74.814 \\ 7.91 \mathrm{E}-12 & 62.36 \\ 7.58 \mathrm{E}-12 & 204.2\end{array}$

\begin{tabular}{|c|c|c|c|c|c|}
\hline Fr-223 & $->$ & Ra-223, & Th-227 & $->$ & Ra-223 \\
\hline Fr-223 & $->$ & Ra-223, & Th-227 & $->$ & Ra-223 \\
\hline Fr-223 & $->$ & Ra-223, & Th-227 & $->$ & Ra-223 \\
\hline Fr-223 & $->$ & Ra-223, & Th-227 & $->$ & Ra-223 \\
\hline $\mathrm{Pa}-231$ & $->$ & $A c-227$ & & & \\
\hline Pa-231 & $->$ & Ac- 227 & & & \\
\hline$R n-219$ & $->$ & Po-215 & & & \\
\hline $\mathrm{Pa}-231$ & $->$ & Ac- 227 & & & \\
\hline Pa-231 & $->$ & Ac-227 & & & \\
\hline Fr-223 & $->$ & Ra-223, & Th-227 & $->$ & $\mathrm{Ra}-223$ \\
\hline $\mathrm{Bi}-211$ & $->$ & TI-207 & & & \\
\hline $\mathrm{Pa}-231$ & $->$ & Ac- 227 & & & \\
\hline Pa-231 & $->$ & Ac- 227 & & & \\
\hline $\mathrm{Pb}-211$ & $->$ & $\mathrm{Bi}-211$ & & & \\
\hline Ra-223 & $->$ & Rn-219 & & & \\
\hline Pa-231 & $->$ & Ac- 227 & & & \\
\hline Th-227 & $->$ & Ra-223 & & & \\
\hline $\mathrm{Ra}-223$ & $->$ & Rn-219 & & & \\
\hline$R n-219$ & $->$ & Po-215 & & & \\
\hline Fr-223 & $->$ & Ra-223, & Th-227 & $->$ & Ra-223 \\
\hline Fr-223 & $->$ & Ra-223, & Th-227 & $->$ & Ra-223 \\
\hline Fr-223 & $->$ & Ra-223, & Th-227 & $->$ & Ra-223 \\
\hline Ra-223 & $->$ & Rn-219 & & & \\
\hline Th-227 & $->$ & Ra-223 & & & \\
\hline $\mathrm{Pb}-211$ & $->$ & $\mathrm{Bi}-211$ & & & \\
\hline Pa-231 & $->$ & Ac- 227 & & & \\
\hline Rn-219 & $->$ & Po-215 & & & \\
\hline Th-227 & $->$ & Ra-223 & & & \\
\hline $\mathrm{Bi}-211$ & $->$ & TI-207 & & & \\
\hline Pa-231 & $->$ & Ac- 227 & & & \\
\hline $\mathrm{Bi}-211$ & $->$ & TI-207 & & & \\
\hline$P b-211$ & $->$ & $\mathrm{Bi}-211$ & & & \\
\hline$R n-219$ & $->$ & Po-215 & & & \\
\hline $\mathrm{Pb}-211$ & $->$ & $\mathrm{Bi}-211$ & & & \\
\hline Fr-223 & $->$ & Ra-223, & Th-227 & $->$ & Ra-223 \\
\hline $\mathrm{Bi}-211$ & $->$ & TI-207 & & & \\
\hline Fr-223 & $->$ & Ra-223, & Th-227 & $->$ & $\mathrm{Ra}-223$ \\
\hline Fr-223 & $->$ & Ra-223, & Th-227 & $->$ & Ra-223 \\
\hline Fr-223 & $->$ & Ra-223, & Th-227 & $->$ & Ra-223 \\
\hline $\mathrm{Pb}-211$ & $->$ & $\mathrm{Bi}-211$ & & & \\
\hline $\mathrm{Pa}-231$ & $->$ & Ac- 227 & & & \\
\hline Pa-231 & $->$ & Ac- 227 & & & \\
\hline$R n-219$ & $->$ & Po-215 & & & \\
\hline Fr-223 & $->$ & Ra-223 & & & \\
\hline Pa-231 & $->$ & Ac- 227 & & & \\
\hline Pa-231 & $->$ & Ac- 227 & & & \\
\hline Pa-231 & $->$ & Ac-227 & & & \\
\hline $\mathrm{Pb}-211$ & $->$ & $\mathrm{Bi}-211$ & & & \\
\hline Th-227 & $->$ & Ra-223 & & & \\
\hline Fr-223 & $->$ & Ra-223, & Th-227 & $->$ & Ra-223 \\
\hline
\end{tabular}




\begin{tabular}{|c|c|c|c|c|c|c|c|}
\hline $7.58 \mathrm{E}-12$ & 206.04 & Fr-223 & $->$ & Ra-223, & Th-227 & $->$ & Ra-223 \\
\hline $7.58 \mathrm{E}-12$ & 43.73 & Fr-223 & $->$ & Ra-223, & Th-227 & $->$ & Ra-223 \\
\hline $7.31 \mathrm{E}-12$ & 897.23 & TI-207 & $->$ & Pb-207, & Po-211 & $->$ & Pb-207 \\
\hline $7.29 \mathrm{E}-12$ & 242 & $\mathrm{~Pa}-231$ & $->$ & $A c-227$ & & & \\
\hline $6.96 \mathrm{E}-12$ & 20.27 & Fr-223 & $->$ & Ra-223, & Th-227 & $->$ & Ra-223 \\
\hline $6.96 \mathrm{E}-12$ & 49.85 & Fr-223 & $->$ & Ra-223, & Th-227 & $->$ & Ra-223 \\
\hline $6.74 \mathrm{E}-12$ & 364.03 & $\mathrm{~Pa}-231$ & $->$ & Ac- 227 & & & \\
\hline $6.59 \mathrm{E}-12$ & 300.34 & Th-227 & $->$ & Ra-223 & & & \\
\hline $6.38 \mathrm{E}-12$ & 245.438 & $\mathrm{~Pa}-231$ & $->$ & Ac- 227 & & & \\
\hline $6.38 \mathrm{E}-12$ & 43.09 & $\mathrm{~Pa}-231$ & $->$ & Ac- 227 & & & \\
\hline $6.38 \mathrm{E}-12$ & 60.526 & Pa-231 & $->$ & $A c-227$ & & & \\
\hline $6.38 \mathrm{E}-12$ & 31.58 & $\mathrm{~Pa}-231$ & $->$ & Ac- 227 & & & \\
\hline $6.38 \mathrm{E}-12$ & 70.54 & Pa-231 & $->$ & Ac- 227 & & & \\
\hline $6.29 \mathrm{E}-12$ & 245.77 & $\mathrm{~Pa}-231$ & $->$ & $A c-227$ & & & \\
\hline $6.28 \mathrm{E}-12$ & 342.9 & Ra-223 & $->$ & $\mathrm{Rn}-219$ & & & \\
\hline $6.22 \mathrm{E}-12$ & 328.49 & Ra-223 & $->$ & $\mathrm{Rn}-219$ & & & \\
\hline $6.20 \mathrm{E}-12$ & 391.85 & $\mathrm{~Pa}-231$ & $->$ & Ac- 227 & & & \\
\hline $5.60 \mathrm{E}-12$ & 117.17 & Th-227 & $->$ & Ra-223 & & & \\
\hline $5.47 \mathrm{E}-12$ & 42.52 & $\mathrm{~Pa}-231$ & $->$ & $A c-227$ & & & \\
\hline $5.47 \mathrm{E}-12$ & 56.8 & Pa-231 & $->$ & Ac- 227 & & & \\
\hline $5.27 \mathrm{E}-12$ & 281.31 & Th-227 & $->$ & Ra-223 & & & \\
\hline $5.17 \mathrm{E}-12$ & 16.074 & $A c-227$ & $->$ & Th-227 & & & \\
\hline $5.10 \mathrm{E}-12$ & 198.96 & $\mathrm{~Pa}-231$ & $->$ & $A c-227$ & & & \\
\hline $4.94 \mathrm{E}-12$ & 113.12 & Th-227 & $->$ & Ra-223 & & & \\
\hline 4.94E-12 & 204.95 & Fr-223 & $->$ & Ra-223, & Th-227 & $->$ & Ra-223 \\
\hline $4.83 \mathrm{E}-12$ & 288.15 & Ra-223 & $->$ & $\mathrm{Rn}-219$ & & & \\
\hline $4.80 \mathrm{E}-12$ & 179.69 & Ra-223 & $->$ & $\mathrm{Rn}-219$ & & & \\
\hline $4.72 \mathrm{E}-12$ & 87.19 & $\mathrm{~Pb}-211$ & $->$ & $\mathrm{Bi}-211$ & & & \\
\hline $4.56 \mathrm{E}-12$ & 1109.37 & $\mathrm{~Pb}-211$ & $->$ & $\mathrm{Bi}-211$ & & & \\
\hline $4.56 \mathrm{E}-12$ & 124.69 & $\mathrm{~Pa}-231$ & $->$ & $A c-227$ & & & \\
\hline $4.55 \mathrm{E}-12$ & 12.855 & Ra-223 & $->$ & $\mathrm{Rn}-219$ & & & \\
\hline $4.29 E-12$ & 250.33 & Th-227 & $->$ & $\mathrm{Ra}-223$ & & & \\
\hline $4.29 \mathrm{E}-12$ & 141.44 & Th-227 & $->$ & Ra-223 & & & \\
\hline $4.00 \mathrm{E}-12$ & 130.57 & $\mathrm{Rn}-219$ & $->$ & Po-215 & & & \\
\hline $3.88 \mathrm{E}-12$ & 85.185 & $\mathrm{Bi}-211$ & $->$ & Tl-207 & & & \\
\hline $3.74 \mathrm{E}-12$ & 438.24 & $\mathrm{~Pa}-231$ & $->$ & $A c-227$ & & & \\
\hline $3.74 \mathrm{E}-12$ & 384.97 & $\mathrm{~Pa}-231$ & $->$ & Ac- 227 & & & \\
\hline $3.65 E-12$ & 144.5 & $\mathrm{~Pa}-231$ & $->$ & $A c-227$ & & & \\
\hline $3.65 E-12$ & 72.57 & $\mathrm{~Pa}-231$ & $->$ & $A c-227$ & & & \\
\hline $3.63 \mathrm{E}-12$ & 252.46 & Th-227 & $->$ & Ra-223 & & & \\
\hline $3.63 E-12$ & 350.48 & Th-227 & $->$ & $\mathrm{Ra}-223$ & & & \\
\hline $3.31 \mathrm{E}-12$ & 29.869 & Fr-223 & $->$ & Ra-223, & Th-227 & $->$ & $\mathrm{Ra}-223$ \\
\hline 3.30E-12 & 262.72 & Fr-223 & $->$ & Ra-223, & Th-227 & $->$ & $\mathrm{Ra}-223$ \\
\hline 3.30E-12 & 100.31 & Fr-223 & $->$ & Ra-223, & Th-227 & $->$ & Ra-223 \\
\hline $3.11 \mathrm{E}-12$ & 333.87 & Ra-223 & $->$ & $\mathrm{Rn}-219$ & & & \\
\hline $3.01 \mathrm{E}-12$ & 435.38 & $\mathrm{~Pa}-231$ & $->$ & Ac-227 & & & \\
\hline $3.01 \mathrm{E}-12$ & 277.43 & Pa-231 & $->$ & Ac- 227 & & & \\
\hline $2.98 \mathrm{E}-12$ & 92.673 & $\mathrm{Rn}-219$ & $->$ & Po-215 & & & \\
\hline $2.89 \mathrm{E}-12$ & 15.856 & $\mathrm{Rn}-219$ & $->$ & Po-215 & & & \\
\hline $2.83 \mathrm{E}-12$ & 598.69 & Ra-223 & $->$ & $\mathrm{Rn}-219$ & & & \\
\hline
\end{tabular}




$\begin{array}{lc}2.79 \mathrm{E}-12 & 15.397 \\ 2.64 \mathrm{E}-12 & 31.566 \\ 2.64 \mathrm{E}-12 & 61.435 \\ 2.39 \mathrm{E}-12 & 65.516 \\ 2.37 \mathrm{E}-12 & 226.77 \\ 2.37 \mathrm{E}-12 & 395.8 \\ 2.31 \mathrm{E}-12 & 279.7 \\ 2.31 \mathrm{E}-12 & 212.56 \\ 2.25 \mathrm{E}-12 & 14.407 \\ 2.23 \mathrm{E}-12 & 527.3 \\ 2.10 \mathrm{E}-12 & 258.43 \\ 2.06 \mathrm{E}-12 & 293.8 \\ 1.99 \mathrm{E}-12 & 18.016 \\ 1.98 \mathrm{E}-12 & 292.3 \\ 1.98 \mathrm{E}-12 & 609.08 \\ 1.98 \mathrm{E}-12 & 218.77 \\ 1.98 \mathrm{E}-12 & 96.06 \\ 1.82 \mathrm{E}-12 & 318.1 \\ 1.82 \mathrm{E}-12 & 71.92 \\ 1.81 \mathrm{E}-12 & 285.48 \\ 1.77 \mathrm{E}-12 & 83.81 \\ 1.73 \mathrm{E}-12 & 410.84 \\ 1.65 \mathrm{E}-12 & 284.24 \\ 1.65 \mathrm{E}-12 & 43.53 \\ 1.58 \mathrm{E}-12 & 383.47 \\ 1.51 \mathrm{E}-12 & 110.8 \\ 1.48 \mathrm{E}-12 & 177.35 \\ 1.40 \mathrm{E}-12 & 90.128 \\ 1.40 \mathrm{E}-12 & 81 \\ 1.38 \mathrm{E}-12 & 19.308 \\ 1.35 \mathrm{E}-12 & 361.8 \\ 1.32 \mathrm{E}-12 & 41.88 \\ 1.32 \mathrm{E}-12 & 104.9 \\ 1.32 \mathrm{E}-12 & 218.94 \\ 1.32 \mathrm{E}-12 & 68.75 \\ 1.29 \mathrm{E}-12 & 184.68 \\ 1.28 \mathrm{E}-12 & 50.97 \\ 1.28 \mathrm{E}-12 & 39.61 \\ 1.28 \mathrm{E}-12 & 310.19 \\ 1.27 \mathrm{E}-12 & 515.7 \\ 1.26 \mathrm{E}-12 & 438.8 \\ 1.21 \mathrm{E}-12 & 15.2 \\ 1.18 \mathrm{E}-12 & 516.45 \\ 1.16 \mathrm{E}-12 & 249.49 \\ 1.10 \mathrm{E}-12 & 251.12 \\ 1.09 \mathrm{E}-12 & 134.5 \\ 1.05 \mathrm{E}-12 & 343.02 \\ 1.05 \mathrm{E}-12 & 140.33 \\ 1.05 \mathrm{E}-12 & 270.76 \\ 1.04 \mathrm{E}-12 & 319.18\end{array}$

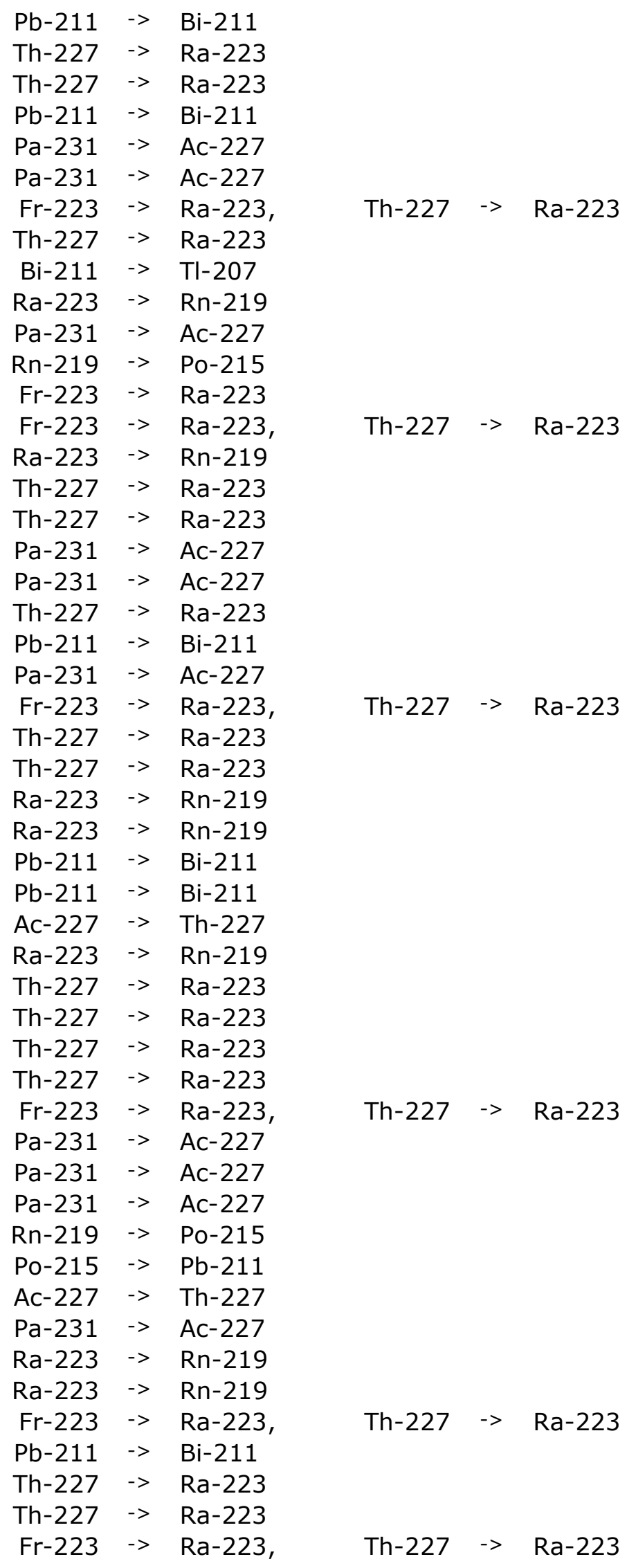




\begin{tabular}{|c|c|c|c|c|c|c|c|}
\hline $1.04 \mathrm{E}-12$ & 220.38 & $\mathrm{Ra}-223$ & $->$ & $\mathrm{Rn}-219$ & & & \\
\hline $1.04 \mathrm{E}-12$ & 432.34 & $\mathrm{Ra}-223$ & $->$ & $\mathrm{Rn}-219$ & & & \\
\hline $1.00 \mathrm{E}-12$ & 254.98 & $\mathrm{Ra}-223$ & $->$ & $\mathrm{Rn}-219$ & & & \\
\hline $9.89 \mathrm{E}-13$ & 314.78 & Th-227 & $->$ & Ra-223 & & & \\
\hline $9.75 \mathrm{E}-13$ & 388.98 & $\mathrm{~Pa}-231$ & $->$ & Ac- 227 & & & \\
\hline $9.73 E-13$ & 251.58 & Ra-223 & $->$ & $\mathrm{Rn}-219$ & & & \\
\hline $9.62 \mathrm{E}-13$ & 313.85 & $\mathrm{~Pb}-211$ & $->$ & $\mathrm{Bi}-211$ & & & \\
\hline $9.52 \mathrm{E}-13$ & 221.9 & $R n-219$ & $->$ & Po-215 & & & \\
\hline $9.23 E-13$ & 72.8 & Th-227 & $->$ & Ra-223 & & & \\
\hline $9.23 E-13$ & 64.37 & Th-227 & $->$ & Ra-223 & & & \\
\hline $8.07 \mathrm{E}-13$ & 481.1 & $\mathrm{~Pb}-211$ & $->$ & $\mathrm{Bi}-211$ & & & \\
\hline $7.14 \mathrm{E}-13$ & 609.62 & $\mathrm{~Pb}-211$ & $->$ & $\mathrm{Bi}-211$ & & & \\
\hline $6.91 \mathrm{E}-13$ & 106.66 & $\mathrm{Ra}-223$ & $->$ & $\mathrm{Rn}-219$ & & & \\
\hline $6.83 \mathrm{E}-13$ & 951 & $\mathrm{~Pb}-211$ & $->$ & $\mathrm{Bi}-211$ & & & \\
\hline $6.70 \mathrm{E}-13$ & 9.658 & $\mathrm{Rn}-219$ & $->$ & Po-215 & & & \\
\hline $6.65 \mathrm{E}-13$ & 8.953 & $\mathrm{Bi}-211$ & $->$ & TI-207 & & & \\
\hline $6.59 \mathrm{E}-13$ & 201.64 & Th-227 & $->$ & Ra-223 & & & \\
\hline $6.59 \mathrm{E}-13$ & 200.48 & Th-227 & $->$ & Ra-223 & & & \\
\hline $6.59 \mathrm{E}-13$ & 40.16 & Th-227 & $->$ & $\mathrm{Ra}-223$ & & & \\
\hline $6.28 \mathrm{E}-13$ & 369.48 & Ra-223 & $->$ & $\mathrm{Rn}-219$ & & & \\
\hline $6.26 \mathrm{E}-13$ & 73.66 & Th-227 & $->$ & Ra-223 & & & \\
\hline $5.97 E-13$ & 430.53 & Ra-223 & $->$ & $\mathrm{Rn}-219$ & & & \\
\hline $5.93 E-13$ & 175.23 & Th-227 & $->$ & Ra-223 & & & \\
\hline $5.89 \mathrm{E}-13$ & 9.419 & $\mathrm{~Pb}-211$ & $->$ & $\mathrm{Bi}-211$ & & & \\
\hline $5.64 \mathrm{E}-13$ & 173.38 & Fr-223 & $->$ & Ra-223, & Th-227 & $->$ & $\mathrm{Ra}-223$ \\
\hline $5.60 \mathrm{E}-13$ & 212.69 & Th-227 & $->$ & $\mathrm{Ra}-223$ & & & \\
\hline $5.58 \mathrm{E}-13$ & 94.89 & $\mathrm{~Pb}-211$ & $->$ & $\mathrm{Bi}-211$ & & & \\
\hline $5.55 \mathrm{E}-13$ & 1014.48 & $\mathrm{~Pb}-211$ & $->$ & $\mathrm{Bi}-211$ & & & \\
\hline $5.47 \mathrm{E}-13$ & 501.33 & $\mathrm{~Pa}-231$ & $->$ & Ac- 227 & & & \\
\hline $5.34 \mathrm{E}-13$ & 175.55 & $\mathrm{Ra}-223$ & $->$ & $\mathrm{Rn}-219$ & & & \\
\hline $5.34 \mathrm{E}-13$ & 103.85 & Ra-223 & $->$ & $\mathrm{Rn}-219$ & & & \\
\hline $5.27 \mathrm{E}-13$ & 88.2 & $\mathrm{~Pb}-211$ & $->$ & $\mathrm{Bi}-211$ & & & \\
\hline $5.27 E-13$ & 50.82 & Th-227 & $->$ & Ra-223 & & & \\
\hline $5.10 \mathrm{E}-13$ & 535.45 & $\mathrm{~Pa}-231$ & $->$ & Ac- 227 & & & \\
\hline $4.94 \mathrm{E}-13$ & 224.68 & Fr-223 & $->$ & Ra-223, & Th-227 & $->$ & $\mathrm{Ra}-223$ \\
\hline $4.94 \mathrm{E}-13$ & 308.49 & Th-227 & $->$ & $\mathrm{Ra}-223$ & & & \\
\hline $4.78 \mathrm{E}-13$ & 289.6 & Fr-223 & $->$ & Ra-223, & Th-227 & $->$ & $\mathrm{Ra}-223$ \\
\hline $4.75 E-13$ & 1080.19 & $\mathrm{~Pb}-211$ & $->$ & $\mathrm{Bi}-211$ & & & \\
\hline $4.65 E-13$ & 504.33 & $\mathrm{~Pb}-211$ & $->$ & $\mathrm{Bi}-211$ & & & \\
\hline $4.61 \mathrm{E}-13$ & 168.19 & Th-227 & $->$ & Ra-223 & & & \\
\hline $4.61 \mathrm{E}-13$ & 33.52 & Th-227 & $->$ & Ra-223 & & & \\
\hline $4.56 \mathrm{E}-13$ & 387.267 & $\mathrm{~Pa}-231$ & $->$ & Ac- 227 & & & \\
\hline $4.56 \mathrm{E}-13$ & 546.9 & $\mathrm{~Pa}-231$ & $->$ & $A c-227$ & & & \\
\hline $4.56 \mathrm{E}-13$ & 572.3 & $\mathrm{~Pa}-231$ & $->$ & Ac- 227 & & & \\
\hline $4.40 \mathrm{E}-13$ & 388.3 & Ra-223 & $->$ & $\mathrm{Rn}-219$ & & & \\
\hline $4.34 \mathrm{E}-13$ & 492 & $\mathrm{~Pb}-211$ & $->$ & $\mathrm{Bi}-211$ & & & \\
\hline $4.29 E-13$ & 99.56 & Fr-223 & $->$ & Ra-223, & Th-227 & $->$ & $\mathrm{Ra}-223$ \\
\hline $4.29 E-13$ & 44.37 & Th-227 & $->$ & $\mathrm{Ra}-223$ & & & \\
\hline $4.03 \mathrm{E}-13$ & 478 & $\mathrm{~Pb}-211$ & $->$ & $\mathrm{Bi}-211$ & & & \\
\hline $3.97 E-13$ & 1196.61 & $\mathrm{~Pb}-211$ & $->$ & $\mathrm{Bi}-211$ & & & \\
\hline
\end{tabular}




\begin{tabular}{|c|c|c|c|c|c|c|c|}
\hline $3.96 \mathrm{E}-13$ & 197.61 & Th-227 & $->$ & Ra-223 & & & \\
\hline $3.96 \mathrm{E}-13$ & 402.42 & Th-227 & $->$ & Ra-223 & & & \\
\hline $3.96 \mathrm{E}-13$ & 94.9 & Th-227 & $->$ & Ra-223 & & & \\
\hline $3.96 \mathrm{E}-13$ & 117.3 & Th-227 & $->$ & Ra-223 & & & \\
\hline $3.63 E-13$ & 246.07 & Th-227 & $->$ & Ra-223 & & & \\
\hline $3.57 E-13$ & 97.3 & $\mathrm{~Pb}-211$ & $->$ & $\mathrm{Bi}-211$ & & & \\
\hline $3.41 \mathrm{E}-13$ & 94.3 & $\mathrm{~Pb}-211$ & $->$ & $\mathrm{Bi}-211$ & & & \\
\hline $3.41 \mathrm{E}-13$ & 500.4 & $\mathrm{~Pb}-211$ & $->$ & $\mathrm{Bi}-211$ & & & \\
\hline $3.30 \mathrm{E}-13$ & 48.27 & Fr-223 & $->$ & Ra-223, & Th-227 & $->$ & Ra-223 \\
\hline $3.30 \mathrm{E}-13$ & 267.82 & Th-227 & $->$ & $\mathrm{Ra}-223$ & & & \\
\hline $3.30 \mathrm{E}-13$ & 150.02 & Th-227 & $->$ & Ra-223 & & & \\
\hline $3.30 \mathrm{E}-13$ & 352.57 & Th-227 & $->$ & Ra-223 & & & \\
\hline $3.14 \mathrm{E}-13$ & 487.59 & $\mathrm{Ra}-223$ & $->$ & $\mathrm{Rn}-219$ & & & \\
\hline $2.97 E-13$ & 392.24 & Th-227 & $->$ & Ra-223 & & & \\
\hline $2.97 E-13$ & 59.5 & Th-227 & $->$ & Ra-223 & & & \\
\hline $2.97 E-13$ & 398.6 & Th-227 & $->$ & Ra-223 & & & \\
\hline $2.97 E-13$ & 62.54 & Th-227 & $->$ & Ra-223 & & & \\
\hline $2.97 \mathrm{E}-13$ & 69.65 & Th-227 & $->$ & Ra-223 & & & \\
\hline $2.97 \mathrm{E}-13$ & 289.5 & Th-227 & $->$ & Ra-223 & & & \\
\hline $2.97 \mathrm{E}-13$ & 75.1 & Th-227 & $->$ & Ra-223 & & & \\
\hline $2.83 \mathrm{E}-13$ & 114.5 & $\mathrm{Ra}-223$ & $->$ & $\mathrm{Rn}-219$ & & & \\
\hline $2.83 \mathrm{E}-13$ & 245.19 & Ra-223 & $->$ & $\mathrm{Rn}-219$ & & & \\
\hline $2.76 \mathrm{E}-13$ & 1270.67 & $\mathrm{~Pb}-211$ & $->$ & $\mathrm{Bi}-211$ & & & \\
\hline $2.64 \mathrm{E}-13$ & 54.18 & Fr-223 & $->$ & Ra-223, & Th-227 & $->$ & Ra-223 \\
\hline $2.64 \mathrm{E}-13$ & 123.55 & Th-227 & $->$ & $\mathrm{Ra}-223$ & & & \\
\hline $2.64 \mathrm{E}-13$ & 270.34 & Th-227 & $->$ & Ra-223 & & & \\
\hline $2.64 \mathrm{E}-13$ & 112.54 & Th-227 & $->$ & $\mathrm{Ra}-223$ & & & \\
\hline $2.51 \mathrm{E}-13$ & 368.78 & $\mathrm{Ra}-223$ & $->$ & $\mathrm{Rn}-219$ & & & \\
\hline $2.51 \mathrm{E}-13$ & 623.38 & Ra-223 & $->$ & Rn-219 & & & \\
\hline $2.47 \mathrm{E}-13$ & 346.39 & Th-227 & $->$ & Ra-223 & & & \\
\hline $2.38 \mathrm{E}-13$ & 12.085 & Rn-219 & $->$ & Po-215 & & & \\
\hline $2.36 \mathrm{E}-13$ & 775.82 & Fr-223 & $->$ & Ra-223, & Th-227 & $->$ & $\mathrm{Ra}-223$ \\
\hline $2.31 \mathrm{E}-13$ & 370.85 & Th-227 & $->$ & $\mathrm{Ra}-223$ & & & \\
\hline $2.31 \mathrm{E}-13$ & 44.08 & Th-227 & $->$ & Ra-223 & & & \\
\hline $2.31 \mathrm{E}-13$ & 107.92 & Th-227 & $->$ & Ra-223 & & & \\
\hline $2.31 \mathrm{E}-13$ & 56.63 & Th-227 & $->$ & Ra-223 & & & \\
\hline $2.31 \mathrm{E}-13$ & 281 & Th-227 & $->$ & $\mathrm{Ra}-223$ & & & \\
\hline $2.31 \mathrm{E}-13$ & 249.36 & Th-227 & $->$ & Ra-223 & & & \\
\hline $2.31 \mathrm{E}-13$ & 162.18 & Th-227 & $->$ & Ra-223 & & & \\
\hline $2.31 \mathrm{E}-13$ & 66.3 & Th-227 & $->$ & Ra-223 & & & \\
\hline $2.22 \mathrm{E}-13$ & 376.9 & $\mathrm{Rn}-219$ & $->$ & Po-215 & & & \\
\hline $2.19 \mathrm{E}-13$ & 228.16 & Рa-231 & $->$ & Ac- 227 & & & \\
\hline $2.19 \mathrm{E}-13$ & 220.2 & Pa-231 & $->$ & Ac- 227 & & & \\
\hline $2.09 E-13$ & 29.58 & Fr-223 & $->$ & Ra-223, & Th-227 & $->$ & $\mathrm{Ra}-223$ \\
\hline $2.08 \mathrm{E}-13$ & 369.32 & Fr-223 & $->$ & Ra-223, & Th-227 & $->$ & Ra-223 \\
\hline $1.99 \mathrm{E}-13$ & 675.33 & $\mathrm{~Pb}-211$ & $->$ & $\mathrm{Bi}-211$ & & & \\
\hline $1.99 \mathrm{E}-13$ & 865.81 & $\mathrm{~Pb}-211$ & $->$ & $\mathrm{Bi}-211$ & & & \\
\hline $1.99 \mathrm{E}-13$ & 430.26 & $\mathrm{~Pb}-211$ & $->$ & $\mathrm{Bi}-211$ & & & \\
\hline $1.98 \mathrm{E}-13$ & 66.1 & Th-227 & $->$ & Ra-223 & & & \\
\hline $1.98 \mathrm{E}-13$ & 348.4 & Th-227 & $->$ & Ra-223 & & & \\
\hline
\end{tabular}




\begin{tabular}{|c|c|c|c|c|c|c|c|}
\hline $1.98 \mathrm{E}-13$ & 325.08 & Th-227 & $->$ & Ra-223 & & & \\
\hline $1.98 \mathrm{E}-13$ & 318.58 & Th-227 & $->$ & Ra-223 & & & \\
\hline $1.98 \mathrm{E}-13$ & 109.1 & Th-227 & $->$ & Ra-223 & & & \\
\hline $1.98 \mathrm{E}-13$ & 376.26 & Th-227 & $->$ & Ra-223 & & & \\
\hline $1.98 \mathrm{E}-13$ & 382.15 & Th-227 & $->$ & $\mathrm{Ra}-223$ & & & \\
\hline $1.98 \mathrm{E}-13$ & 202.4 & Th-227 & $->$ & Ra-223 & & & \\
\hline $1.90 \mathrm{E}-13$ & 540.1 & $R n-219$ & $->$ & Po-215 & & & \\
\hline $1.90 \mathrm{E}-13$ & 676.4 & $\mathrm{Rn}-219$ & $->$ & Po-215 & & & \\
\hline $1.88 \mathrm{E}-13$ & 260.4 & Ra-223 & $->$ & $\mathrm{Rn}-219$ & & & \\
\hline $1.88 \mathrm{E}-13$ & 68.7 & Th-227 & $->$ & Ra-223 & & & \\
\hline $1.86 \mathrm{E}-13$ & 11.712 & $\mathrm{~Pb}-211$ & $->$ & $\mathrm{Bi}-211$ & & & \\
\hline $1.75 \mathrm{E}-13$ & 10.994 & $\mathrm{Bi}-211$ & $->$ & TI-207 & & & \\
\hline $1.65 \mathrm{E}-13$ & 362.55 & Th-227 & $->$ & Ra-223 & & & \\
\hline $1.65 \mathrm{E}-13$ & 56.03 & Th-227 & $->$ & Ra-223 & & & \\
\hline $1.65 \mathrm{E}-13$ & 110.58 & Th-227 & $->$ & Ra-223 & & & \\
\hline $1.65 \mathrm{E}-13$ & 432.29 & Th-227 & $->$ & $\mathrm{Ra}-223$ & & & \\
\hline $1.65 \mathrm{E}-13$ & 326.12 & Th-227 & $->$ & Ra-223 & & & \\
\hline $1.65 \mathrm{E}-13$ & 124.48 & Th-227 & $->$ & Ra-223 & & & \\
\hline $1.58 \mathrm{E}-13$ & 1103.4 & $\mathrm{~Pb}-211$ & $->$ & $\mathrm{Bi}-211$ & & & \\
\hline $1.57 \mathrm{E}-13$ & 255.74 & Ra-223 & $->$ & $\mathrm{Rn}-219$ & & & \\
\hline $1.57 \mathrm{E}-13$ & 131.1 & $\mathrm{Ra}-223$ & $->$ & $\mathrm{Rn}-219$ & & & \\
\hline $1.57 \mathrm{E}-13$ & 165.5 & Ra-223 & $->$ & $\mathrm{Rn}-219$ & & & \\
\hline $1.55 \mathrm{E}-13$ & 23.55 & $\mathrm{~Pa}-231$ & $->$ & Ac- 227 & & & \\
\hline $1.37 \mathrm{E}-13$ & 471.33 & $\mathrm{~Pa}-231$ & $->$ & Ac-227 & & & \\
\hline $1.29 \mathrm{E}-13$ & 339.45 & Fr-223 & $->$ & Ra-223, & Th-227 & $->$ & $\mathrm{Ra}-223$ \\
\hline $1.27 \mathrm{E}-13$ & 608.5 & $\mathrm{Rn}-219$ & $->$ & Po-215 & & & \\
\hline $1.26 \mathrm{E}-13$ & 176.8 & $\mathrm{Ra}-223$ & $->$ & $\mathrm{Rn}-219$ & & & \\
\hline $1.26 \mathrm{E}-13$ & 383.12 & Ra-223 & $->$ & $\mathrm{Rn}-219$ & & & \\
\hline $1.21 \mathrm{E}-13$ & 24.5 & Ac- 227 & $->$ & Th-227 & & & \\
\hline $1.12 \mathrm{E}-13$ & 89.85 & Th-227 & $->$ & Ra-223 & & & \\
\hline $1.10 \mathrm{E}-13$ & 711.2 & Ra-223 & $->$ & $\mathrm{Rn}-219$ & & & \\
\hline $1.05 \mathrm{E}-13$ & 115.3 & $\mathrm{Rn}-219$ & $->$ & Po-215 & & & \\
\hline $9.89 \mathrm{E}-14$ & 51.29 & Fr-223 & $->$ & Ra-223, & Th-227 & $->$ & $\mathrm{Ra}-223$ \\
\hline $9.89 \mathrm{E}-14$ & 169.98 & Th-227 & $->$ & $\mathrm{Ra}-223$ & & & \\
\hline $9.89 \mathrm{E}-14$ & 124.6 & Th-227 & $->$ & $\mathrm{Ra}-223$ & & & \\
\hline $9.53 \mathrm{E}-14$ & 812.15 & Fr-223 & $->$ & Ra-223, & Th-227 & $->$ & Ra-223 \\
\hline $9.42 \mathrm{E}-14$ & 391 & $\mathrm{Ra}-223$ & $->$ & $\mathrm{Rn}-219$ & & & \\
\hline $9.42 \mathrm{E}-14$ & 199.43 & Ra-223 & $->$ & $\mathrm{Rn}-219$ & & & \\
\hline 8.70E-14 & 822.99 & Fr-223 & $->$ & Ra-223, & Th-227 & $->$ & $\mathrm{Ra}-223$ \\
\hline $8.24 \mathrm{E}-14$ & 266.3 & Th-227 & $->$ & $\mathrm{Ra}-223$ & & & \\
\hline 8.07E-14 & 1090.5 & $\mathrm{~Pb}-211$ & $->$ & $\mathrm{Bi}-211$ & & & \\
\hline 7.57E-14 & 74.969 & TI-207 & $->$ & $P b-207$ & & & \\
\hline $7.52 \mathrm{E}-14$ & 908.03 & Fr-223 & $->$ & Ra-223, & Th-227 & $->$ & $\mathrm{Ra}-223$ \\
\hline $7.02 \mathrm{E}-14$ & 478.22 & $\mathrm{~Pa}-231$ & $->$ & Ac- 227 & & & \\
\hline $6.59 \mathrm{E}-14$ & 62.05 & Th-227 & $->$ & Ra-223 & & & \\
\hline $6.59 \mathrm{E}-14$ & 179.28 & Th-227 & $->$ & Ra-223 & & & \\
\hline $6.59 \mathrm{E}-14$ & 99.64 & Th-227 & $->$ & Ra-223 & & & \\
\hline $5.82 \mathrm{E}-14$ & 803.58 & Fr-223 & $->$ & Ra-223, & Th-227 & $->$ & $\mathrm{Ra}-223$ \\
\hline $5.67 \mathrm{E}-14$ & 307.89 & Fr-223 & $->$ & Ra-223, & Th-227 & $->$ & Ra-223 \\
\hline $5.60 \mathrm{E}-14$ & 415.09 & Th-227 & $->$ & $\mathrm{Ra}-223$ & & & \\
\hline
\end{tabular}




$\begin{array}{lc}4.94 \mathrm{E}-14 & 275.2 \\ 4.91 \mathrm{E}-14 & 723.86 \\ 4.76 \mathrm{E}-14 & 887.7 \\ 4.65 \mathrm{E}-14 & 764.52 \\ 4.61 \mathrm{E}-14 & 339.1 \\ 4.61 \mathrm{E}-14 & 374.93 \\ 4.57 \mathrm{E}-14 & 328.08 \\ 4.54 \mathrm{E}-14 & 72.803 \\ 4.49 \mathrm{E}-14 & 569.15 \\ 4.03 \mathrm{E}-14 & 1234.3 \\ 3.96 \mathrm{E}-14 & 102.45 \\ 3.83 \mathrm{E}-14 & 536.88 \\ 3.69 \mathrm{E}-14 & 568.76 \\ 3.31 \mathrm{E}-14 & 757.03 \\ 3.30 \mathrm{E}-14 & 171.21 \\ 3.17 \mathrm{E}-14 & 834.2 \\ 3.07 \mathrm{E}-14 & 796.96 \\ 2.94 \mathrm{E}-14 & 825.96 \\ 2.73 \mathrm{E}-14 & 10.541 \\ 2.70 \mathrm{E}-14 & 84.979 \\ 2.49 \mathrm{E}-14 & 876.14 \\ 2.48 \mathrm{E}-14 & 12.703 \\ 2.41 \mathrm{E}-14 & 722.6 \\ 2.31 \mathrm{E}-14 & 230.17 \\ 2.27 \mathrm{E}-14 & 842.02 \\ 2.15 \mathrm{E}-14 & 846.56 \\ 1.91 \mathrm{E}-14 & 493.15 \\ 1.90 \mathrm{E}-14 & 1055.8 \\ 1.77 \mathrm{E}-14 & 575.8 \\ 1.73 \mathrm{E}-14 & 748.56 \\ 1.63 \mathrm{E}-14 & 837.36 \\ 1.54 \mathrm{E}-14 & 99.7 \\ 1.46 \mathrm{E}-14 & 507.64 \\ 1.41 \mathrm{E}-14 & 746.24 \\ 1.27 \mathrm{E}-14 & 387.9 \\ 1.26 \mathrm{E}-14 & 756.79 \\ 1.17 \mathrm{E}-14 & 780.59 \\ 1.15 \mathrm{E}-14 & 896.41 \\ 1.04 \mathrm{E}-14 & 480.25 \\ 9.71 \mathrm{E}-15 & 552.09 \\ 9.50 \mathrm{E}-15 & 762.3 \\ 9.35 \mathrm{E}-15 & 858.4 \\ 8.20 \mathrm{E}-15 & 69.83 \\ 8.04 \mathrm{E}-15 & 555.91 \\ 7.88 \mathrm{E}-15 & 87.632 \\ 7.15 \mathrm{E}-15 & 828.31 \\ 7.00 \mathrm{E}-15 & 515.96 \\ 6.58 \mathrm{E}-15 & 607.2 \\ 6.54 \mathrm{E}-15 & 733.9 \\ 6.35 \mathrm{E}-15 & 754.17\end{array}$

\begin{tabular}{|c|c|c|c|c|c|}
\hline Th-227 & $->$ & Ra-223 & & & \\
\hline Fr-223 & $->$ & Ra-223, & Th-227 & $->$ & Ra-223 \\
\hline $\mathrm{Rn}-219$ & $->$ & Po-215 & & & \\
\hline Fr-223 & $->$ & Ra-223, & Th-227 & $->$ & $\mathrm{Ra}-223$ \\
\hline Th-227 & $->$ & Ra-223 & & & \\
\hline Th-227 & $->$ & Ra-223 & & & \\
\hline TI-207 & $->$ & Pb-207, & Po-211 & $->$ & $\mathrm{Pb}-207$ \\
\hline TI-207 & $->$ & $\mathrm{Pb}-207$ & & & \\
\hline Po-211 & $->$ & Pb-207 & & & \\
\hline $\mathrm{Pb}-211$ & $->$ & $\mathrm{Bi}-211$ & & & \\
\hline Th-227 & $->$ & $\mathrm{Ra}-223$ & & & \\
\hline Fr-223 & $->$ & Ra-223, & Th-227 & $->$ & $\mathrm{Ra}-223$ \\
\hline Fr-223 & $->$ & Ra-223, & Th-227 & $->$ & $\mathrm{Ra}-223$ \\
\hline Fr-223 & $->$ & Ra-223, & Th-227 & $->$ & $\mathrm{Ra}-223$ \\
\hline Th-227 & $->$ & $\mathrm{Ra}-223$ & & & \\
\hline $\mathrm{Rn}-219$ & $->$ & Po-215 & & & \\
\hline $\mathrm{Fr}-223$ & $->$ & Ra-223, & Th-227 & $->$ & $\mathrm{Ra}-223$ \\
\hline Fr-223 & $->$ & Ra-223, & Th-227 & $->$ & $\mathrm{Ra}-223$ \\
\hline TI-207 & $->$ & $\mathrm{Pb}-207$ & & & \\
\hline TI-207 & $->$ & $\mathrm{Pb}-207$ & & & \\
\hline Fr-223 & $->$ & Ra-223, & Th-227 & $->$ & $\mathrm{Ra}-223$ \\
\hline TI-207 & $->$ & $\mathrm{Pb}-207$ & & & \\
\hline Fr-223 & $->$ & Ra-223, & Th-227 & $->$ & Ra-223 \\
\hline Th-227 & $->$ & $\mathrm{Ra}-223$ & & & \\
\hline Fr-223 & $->$ & Ra-223, & Th-227 & $->$ & Ra-223 \\
\hline Fr-223 & $->$ & Ra-223, & Th-227 & $->$ & $\mathrm{Ra}-223$ \\
\hline Fr-223 & $->$ & Ra-223, & Th-227 & $->$ & $\mathrm{Ra}-223$ \\
\hline $\mathrm{Rn}-219$ & $->$ & Po-215 & & & \\
\hline Fr-223 & $->$ & Ra-223, & Th-227 & $->$ & $\mathrm{Ra}-223$ \\
\hline Fr-223 & $->$ & Ra-223, & Th-227 & $->$ & $\mathrm{Ra}-223$ \\
\hline $\mathrm{Fr}-223$ & $->$ & Ra-223, & Th-227 & $->$ & $\mathrm{Ra}-223$ \\
\hline$A c-227$ & $->$ & Fr-223 & & & \\
\hline Fr-223 & $->$ & Ra-223, & Th-227 & $->$ & Ra-223 \\
\hline $\mathrm{Fr}-223$ & $->$ & Ra-223, & Th-227 & $->$ & $\mathrm{Ra}-223$ \\
\hline $\mathrm{Rn}-219$ & $->$ & Po-215 & & & \\
\hline Fr-223 & $->$ & Ra-223, & Th-227 & $->$ & Ra-223 \\
\hline Fr-223 & $->$ & Ra-223, & Th-227 & $->$ & Ra-223 \\
\hline Fr-223 & $->$ & Ra-223, & Th-227 & $->$ & Ra-223 \\
\hline Fr-223 & $->$ & Ra-223, & Th-227 & $->$ & $\mathrm{Ra}-223$ \\
\hline Fr-223 & $->$ & Ra-223, & Th-227 & $->$ & Ra-223 \\
\hline Fr-223 & $->$ & Ra-223, & Th-227 & $->$ & $\mathrm{Ra}-223$ \\
\hline Fr-223 & $->$ & Ra-223, & Th-227 & $->$ & $\mathrm{Ra}-223$ \\
\hline Ac- 227 & $->$ & $\mathrm{Fr}-223$ & & & \\
\hline Fr-223 & $->$ & Ra-223, & Th-227 & $->$ & $\mathrm{Ra}-223$ \\
\hline TI-207 & $->$ & $\mathrm{Pb}-207$ & & & \\
\hline Fr-223 & $->$ & Ra-223, & Th-227 & $->$ & $\mathrm{Ra}-223$ \\
\hline Fr-223 & $->$ & Ra-223, & Th-227 & $->$ & Ra-223 \\
\hline Fr-223 & $->$ & Ra-223, & Th-227 & $->$ & Ra-223 \\
\hline Fr-223 & $->$ & Ra-223, & Th-227 & $->$ & Ra-223 \\
\hline Fr-223 & $->$ & Ra-223, & Th-227 & $->$ & Ra-223 \\
\hline
\end{tabular}




$\begin{array}{lc}6.35 \mathrm{E}-15 & 784.04 \\ 6.20 \mathrm{E}-15 & 734.6 \\ 6.17 \mathrm{E}-15 & 753.44 \\ 5.82 \mathrm{E}-15 & 524.02 \\ 5.61 \mathrm{E}-15 & 817.31 \\ 5.49 \mathrm{E}-15 & 691.46 \\ 5.45 \mathrm{E}-15 & 482.14 \\ 5.36 \mathrm{E}-15 & 448 \\ 5.27 \mathrm{E}-15 & 623.52 \\ 5.07 \mathrm{E}-15 & 578.2 \\ 4.68 \mathrm{E}-15 & 704.19 \\ 4.54 \mathrm{E}-15 & 14.886 \\ 4.52 \mathrm{E}-15 & 772.85 \\ 4.48 \mathrm{E}-15 & 9.3 \\ 4.43 \mathrm{E}-15 & 786.9 \\ 4.29 \mathrm{E}-15 & 633.2 \\ 4.22 \mathrm{E}-15 & 452.52 \\ 4.00 \mathrm{E}-15 & 147.2 \\ 3.97 \mathrm{E}-15 & 878.16 \\ 3.51 \mathrm{E}-15 & 848.88 \\ 3.30 \mathrm{E}-15 & 534.9 \\ 3.14 \mathrm{E}-15 & 31.87 \\ 2.96 \mathrm{E}-15 & 457.07 \\ 2.96 \mathrm{E}-15 & 736.76 \\ 2.90 \mathrm{E}-15 & 643.93 \\ 2.90 \mathrm{E}-15 & 867.23 \\ 2.65 \mathrm{E}-15 & 120.9 \\ 2.65 \mathrm{E}-15 & 69.13 \\ 2.37 \mathrm{E}-15 & 941.41 \\ 2.31 \mathrm{E}-15 & 854.2 \\ 2.15 \mathrm{E}-15 & 857.89 \\ 2.15 \mathrm{E}-15 & 621.92 \\ 2.09 \mathrm{E}-15 & 588.17 \\ 2.04 \mathrm{E}-15 & 958.6 \\ 1.98 \mathrm{E}-15 & 171.4 \\ 1.98 \mathrm{E}-15 & 662.8 \\ 1.93 \mathrm{E}-15 & 808.26 \\ 1.69 \mathrm{E}-15 & 707.18 \\ 1.65 \mathrm{E}-15 & 466.2 \\ 1.59 \mathrm{E}-15 & 863.2 \\ 1.59 \mathrm{E}-15 & 891 \\ 1.36 \mathrm{E}-15 & 9.185 \\ 1.35 \mathrm{E}-15 & 133.8 \\ 1.35 \mathrm{E}-15 & 596.44 \\ 1.32 \mathrm{E}-15 & 788.36 \\ 1.32 \mathrm{E}-15 & 792.6 \\ 1.15 \mathrm{E}-15 & 990.2 \\ 1.10 \mathrm{E}-15 & 718.22 \\ 1.03 \mathrm{E}-15 & 892.8 \\ 9.89 \mathrm{E}-16 & 818.23\end{array}$

\begin{tabular}{|c|c|c|c|c|c|}
\hline Fr-223 & $->$ & Ra-223, & Th-227 & $\rightarrow$ & $\mathrm{Ra}-223$ \\
\hline Fr-223 & $->$ & Ra-223, & Th-227 & $->$ & Ra-223 \\
\hline Fr-223 & $->$ & $\mathrm{Ra}-223$, & Th-227 & $->$ & $\mathrm{Ra}-223$ \\
\hline Fr-223 & $->$ & Ra-223, & Th-227 & $->$ & $\mathrm{Ra}-223$ \\
\hline Fr-223 & $->$ & Ra-223, & Th-227 & $->$ & Ra-223 \\
\hline Fr-223 & $->$ & Ra-223, & Th-227 & $->$ & $\mathrm{Ra}-223$ \\
\hline Fr-223 & $->$ & Ra-223, & Th-227 & $->$ & Ra-223 \\
\hline Fr-223 & $->$ & Ra-223, & Th-227 & $->$ & $\mathrm{Ra}-223$ \\
\hline Th-227 & $->$ & $\mathrm{Ra}-223$ & & & \\
\hline Fr-223 & $->$ & Ra-223, & Th-227 & $->$ & Ra-223 \\
\hline Fr-223 & $->$ & Ra-223, & Th-227 & $->$ & Ra-223 \\
\hline TI-207 & $->$ & Pb-207 & & & \\
\hline Fr-223 & $->$ & Ra-223, & Th-227 & $->$ & $\mathrm{Ra}-223$ \\
\hline$A c-227$ & $->$ & Th-227 & & & \\
\hline Fr-223 & $->$ & Ra-223, & Th-227 & $->$ & Ra-223 \\
\hline Th-227 & $->$ & $\mathrm{Ra}-223$ & & & \\
\hline $\mathrm{Fr}-223$ & $->$ & Ra-223, & Th-227 & $->$ & $\mathrm{Ra}-223$ \\
\hline Ac- 227 & $->$ & $\mathrm{Fr}-223$ & & & \\
\hline Fr-223 & $->$ & Ra-223, & Th-227 & $->$ & Ra-223 \\
\hline $\mathrm{Fr}-223$ & $->$ & Ra-223, & Th-227 & $->$ & $\mathrm{Ra}-223$ \\
\hline Th-227 & $->$ & $\mathrm{Ra}-223$ & & & \\
\hline $\mathrm{Ra}-223$ & $->$ & $\mathrm{Rn}-219$ & & & \\
\hline Fr-223 & $->$ & Ra-223, & Th-227 & $->$ & Ra-223 \\
\hline Fr-223 & $->$ & Ra-223, & Th-227 & $->$ & $\mathrm{Ra}-223$ \\
\hline Fr-223 & $->$ & Ra-223, & Th-227 & $->$ & $\mathrm{Ra}-223$ \\
\hline Fr-223 & $->$ & Ra-223, & Th-227 & $->$ & $\mathrm{Ra}-223$ \\
\hline$A c-227$ & $->$ & Fr-223 & & & \\
\hline$A C-227$ & $->$ & Fr-223 & & & \\
\hline Th-227 & $->$ & $\mathrm{Ra}-223$ & & & \\
\hline Th-227 & $->$ & $\mathrm{Ra}-223$ & & & \\
\hline Fr-223 & $->$ & Ra-223, & Th-227 & $->$ & $\mathrm{Ra}-223$ \\
\hline Fr-223 & $->$ & Ra-223, & Th-227 & $->$ & Ra-223 \\
\hline Fr-223 & $->$ & Ra-223, & Th-227 & $->$ & Ra-223 \\
\hline Th-227 & $->$ & $\mathrm{Ra}-223$ & & & \\
\hline Ac- 227 & $->$ & Fr-223 & & & \\
\hline Th-227 & $->$ & $\mathrm{Ra}-223$ & & & \\
\hline Fr-223 & $->$ & Ra-223, & Th-227 & $->$ & $\mathrm{Ra}-223$ \\
\hline Fr-223 & $->$ & Ra-223, & Th-227 & $->$ & Ra-223 \\
\hline Th-227 & $->$ & $\mathrm{Ra}-223$ & & & \\
\hline Fr-223 & $->$ & Ra-223, & Th-227 & $->$ & Ra-223 \\
\hline Fr-223 & $->$ & Ra-223, & Th-227 & $->$ & Ra-223 \\
\hline TI-207 & $->$ & $\mathrm{Pb}-207$ & & & \\
\hline Ac- 227 & $->$ & Fr-223 & & & \\
\hline $\mathrm{Fr}-223$ & $->$ & Ra-223, & Th-227 & $->$ & $\mathrm{Ra}-223$ \\
\hline Th-227 & $->$ & Ra-223 & & & \\
\hline Th-227 & $->$ & Ra-223 & & & \\
\hline Th-227 & $->$ & Ra-223 & & & \\
\hline Fr-223 & $->$ & Ra-223, & Th-227 & $->$ & Ra-223 \\
\hline Fr-223 & $->$ & Ra-223, & Th-227 & $->$ & Ra-223 \\
\hline Th-227 & $->$ & Ra-223 & & & \\
\hline
\end{tabular}




\begin{tabular}{|c|c|c|c|c|c|c|c|}
\hline $9.89 \mathrm{E}-16$ & 793.12 & Th-227 & $->$ & Ra-223 & & & \\
\hline $9.89 E-16$ & 999.78 & Th-227 & $->$ & Ra-223 & & & \\
\hline $9.89 \mathrm{E}-16$ & 969.91 & Th-227 & $->$ & Ra-223 & & & \\
\hline $9.72 \mathrm{E}-16$ & 926.28 & Fr-223 & $->$ & Ra-223, & Th-227 & $->$ & $\mathrm{Ra}-223$ \\
\hline $6.96 \mathrm{E}-16$ & 648.43 & Fr-223 & $->$ & Ra-223, & Th-227 & $->$ & $\mathrm{Ra}-223$ \\
\hline $6.75 \mathrm{E}-16$ & 106.1 & Ac- 227 & $->$ & Fr-223 & & & \\
\hline $6.75 \mathrm{E}-16$ & 46.13 & $A c-227$ & $->$ & Fr-223 & & & \\
\hline $6.59 E-16$ & 1020 & Th-227 & $->$ & Ra-223 & & & \\
\hline $6.59 E-16$ & 641.45 & Th-227 & $->$ & Ra-223 & & & \\
\hline $6.02 \mathrm{E}-16$ & 825.97 & Fr-223 & $->$ & Ra-223 & & & \\
\hline $4.94 \mathrm{E}-16$ & 1015.1 & Th-227 & $->$ & Ra-223 & & & \\
\hline $4.94 \mathrm{E}-16$ & 1024.8 & Th-227 & $->$ & Ra-223 & & & \\
\hline $4.94 \mathrm{E}-16$ & 909.85 & Th-227 & $->$ & Ra-223 & & & \\
\hline $3.96 \mathrm{E}-16$ & 920.06 & Th-227 & $->$ & Ra-223 & & & \\
\hline $3.90 \mathrm{E}-16$ & 828.53 & Fr-223 & $->$ & Ra-223, & Th-227 & $->$ & Ra-223 \\
\hline $3.54 \mathrm{E}-16$ & 11.349 & TI-207 & $->$ & $\mathrm{Pb}-207$ & & & \\
\hline $3.30 \mathrm{E}-16$ & 938.34 & Th-227 & $->$ & Ra-223 & & & \\
\hline $3.30 \mathrm{E}-16$ & 971.28 & Th-227 & $->$ & $\mathrm{Ra}-223$ & & & \\
\hline $2.31 \mathrm{E}-16$ & 994.9 & Th-227 & $->$ & $\mathrm{Ra}-223$ & & & \\
\hline $5.56 \mathrm{E}-17$ & 793.11 & Fr-223 & $->$ & Ra-223 & & & \\
\hline
\end{tabular}

\section{Cm-244}

$100 \%$
Aged: 5 years

344 lines computed.

Sorted by Energy:

\begin{tabular}{|c|c|c|c|c|c|c|c|}
\hline \multirow{2}{*}{$\begin{array}{c}\text { Energy } \\
\text { keV }\end{array}$} & \multirow{2}{*}{$\begin{array}{c}\text { Intensity } \\
\mathrm{ph} / \mathrm{s} / \mathrm{gm}\end{array}$} & \multicolumn{3}{|c|}{ Initial Isotopes and Decays } & & & \\
\hline & & & & & & & \\
\hline 8.953 & $1.86 \mathrm{E}-13$ & $\mathrm{Bi}-212$ & $->$ & TI-208 & & & \\
\hline 9.185 & $8.02 E-14$ & TI-208 & $->$ & $\mathrm{Pb}-208$ & & & \\
\hline 9.419 & $1.17 \mathrm{E}-12$ & $\mathrm{~Pb}-212$ & $->$ & $\mathrm{Bi}-212$ & & & \\
\hline 9.658 & $4.63 E-15$ & $\mathrm{Bi}-212$ & $->$ & Po-212 & & & \\
\hline 10.137 & $2.51 \mathrm{E}-14$ & Ra-224 & $->$ & $\mathrm{Rn}-220$ & & & \\
\hline 10.259 & $3.76 \mathrm{E}-12$ & $\mathrm{Bi}-212$ & $->$ & TI-208 & & & \\
\hline 10.541 & $1.60 \mathrm{E}-12$ & TI-208 & $->$ & $\mathrm{Pb}-208$ & & & \\
\hline 10.622 & $6.28 \mathrm{E}-13$ & Th-228 & $->$ & $\mathrm{Ra}-224$ & & & \\
\hline 10.828 & $2.27 \mathrm{E}-11$ & $\mathrm{~Pb}-212$ & $->$ & $\mathrm{Bi}-212$ & & & \\
\hline 10.994 & $3.50 \mathrm{E}-14$ & $\mathrm{Bi}-212$ & $->$ & TI-208 & & & \\
\hline 11.118 & $2.19 \mathrm{E}-01$ & Ac- 228 & $->$ & Th-228, & $U-236$ & $->$ & Th-232 \\
\hline 11.119 & $9.05 \mathrm{E}-14$ & $\mathrm{Bi}-212$ & $->$ & Po-212 & & & \\
\hline 11.349 & $2.15 \mathrm{E}-14$ & TI-208 & $->$ & $\mathrm{Pb}-208$ & & & \\
\hline 11.712 & $2.99 \mathrm{E}-13$ & $\mathrm{~Pb}-212$ & $->$ & $\mathrm{Bi}-212$ & & & \\
\hline 11.713 & $4.48 \mathrm{E}-13$ & Ra-224 & $->$ & $\mathrm{Rn}-220$ & & & \\
\hline 12.085 & $1.37 \mathrm{E}-15$ & $\mathrm{Bi}-212$ & $->$ & Po-212 & & & \\
\hline 12.124 & $5.69 E+09$ & $\mathrm{Cm}-244$ & $->$ & Pu-240 & & & \\
\hline
\end{tabular}




\begin{tabular}{cc}
12.282 & $4.33 \mathrm{E}-12$ \\
12.325 & $1.08 \mathrm{E}-11$ \\
12.701 & $1.50 \mathrm{E}-12$ \\
12.76 & $2.35 \mathrm{E}-10$ \\
12.855 & $1.17 \mathrm{E}-14$ \\
12.952 & $3.73 \mathrm{E}+00$ \\
13.099 & $2.06 \mathrm{E}-11$ \\
13.5 & $1.24 \mathrm{E}-09$ \\
13.501 & $9.05 \mathrm{E}-14$ \\
13.662 & $3.28 \mathrm{E}-13$ \\
14.262 & $9.89 \mathrm{E}+10$ \\
14.336 & $6.20 \mathrm{E}-13$ \\
14.511 & $1.04 \mathrm{E}-01$ \\
14.563 & $1.01 \mathrm{E}-12$ \\
14.884 & $2.76 \mathrm{E}-13$ \\
15.15 & $1.24 \mathrm{E}-10$ \\
15.23 & $1.64 \mathrm{E}-11$ \\
15.376 & $3.89 \mathrm{E}-12$ \\
15.869 & $1.76 \mathrm{E}-14$ \\
16.154 & $2.48 \mathrm{E}-10$ \\
16.161 & $5.15 \mathrm{E}+00$ \\
16.18 & $5.57 \mathrm{E}-10$ \\
16.333 & $2.22 \mathrm{E}+09$ \\
16.881 & $1.34 \mathrm{E}-13$ \\
17.952 & $3.70 \mathrm{E}-12$ \\
18.135 & $9.89 \mathrm{E}+10$ \\
18.8 & $1.61 \mathrm{E}-10$ \\
19.094 & $1.15 \mathrm{E}+00$ \\
19.113 & $5.69 \mathrm{E}-11$ \\
19.4 & $1.11 \mathrm{E}-11$ \\
21.554 & $2.47 \mathrm{E}+10$ \\
39.846 & $1.36 \mathrm{E}-12$ \\
42.817 & $6.18 \mathrm{E}+08$ \\
45.244 & $6.47 \mathrm{E}+05$ \\
49.369 & $8.55 \mathrm{E}-02$ \\
57.81 & $6.50 \mathrm{E}-12$ \\
59 & $1.74 \mathrm{E}-11$ \\
70.832 & $9.28 \mathrm{E}-14$ \\
72.803 & $2.64 \mathrm{E}-12$ \\
72.873 & $1.57 \mathrm{E}-13$ \\
74.814 & $3.61 \mathrm{E}-11$ \\
74.969 & $4.44 \mathrm{E}-12$ \\
76.858 & $1.32 \mathrm{E}-13$ \\
77.107 & $6.09 \mathrm{E}-11$ \\
79.29 & $2.21 \mathrm{E}-13$ \\
81.067 & $4.13 \mathrm{E}-13$ \\
82.434 & $5.57 \mathrm{E}-14$ \\
83.787 & $6.89 \mathrm{E}-13$ \\
84.26 & $4.22 \mathrm{E}-12$ \\
84.789 & $1.57 \mathrm{E}-12$ \\
& \\
\hline
\end{tabular}

\begin{tabular}{|c|c|c|c|c|c|}
\hline $\mathrm{Bi}-212$ & $->$ & TI-208 & & & \\
\hline Th-228 & $->$ & Ra-224 & & & \\
\hline TI-208 & $->$ & $\mathrm{Pb}-208$ & & & \\
\hline Ra-228 & $->$ & Ac- 228 & & & \\
\hline Ra-224 & $->$ & $\mathrm{Rn}-220$ & & & \\
\hline Ac- 228 & $->$ & Th-228, & $U-236$ & $->$ & Th-232 \\
\hline $\mathrm{Pb}-212$ & $->$ & $\mathrm{Bi}-212$ & & & \\
\hline $\mathrm{Ra}-228$ & $->$ & Ac- 228 & & & \\
\hline $\mathrm{Bi}-212$ & $->$ & Po-212 & & & \\
\hline Th-228 & $->$ & Ra-224 & & & \\
\hline $\mathrm{Cm}-244$ & $->$ & Pu-240 & & & \\
\hline Ra-224 & $->$ & $\mathrm{Rn}-220$ & & & \\
\hline Ac- 228 & $->$ & Th-228, & $U-236$ & $->$ & Th-232 \\
\hline $\mathrm{Bi}-212$ & $->$ & TI-208 & & & \\
\hline TI-208 & $->$ & $\mathrm{Pb}-208$ & & & \\
\hline $\mathrm{Ra}-228$ & $->$ & $A c-228$ & & & \\
\hline Th-228 & $->$ & Ra-224 & & & \\
\hline $\mathrm{Pb}-212$ & $->$ & $\mathrm{Bi}-212$ & & & \\
\hline $\mathrm{Bi}-212$ & $->$ & Po-212 & & & \\
\hline Ac- 228 & $->$ & Th-228 & & & \\
\hline U-236 & $->$ & Th-232 & & & \\
\hline $\mathrm{Ra}-228$ & $->$ & Ac- 228 & & & \\
\hline Cm-244 & $->$ & Pu-240 & & & \\
\hline $\mathrm{Ra}-224$ & $->$ & $\mathrm{Rn}-220$ & & & \\
\hline Th-228 & $->$ & Ra-224 & & & \\
\hline $\mathrm{Cm}-244$ & $->$ & Pu-240 & & & \\
\hline Ra-228 & $->$ & $A c-228$ & & & \\
\hline U-236 & $->$ & Th-232 & & & \\
\hline Ac- 228 & $->$ & Th-228 & & & \\
\hline Ra-228 & $->$ & Ac- 228 & & & \\
\hline $\mathrm{Cm}-244$ & $->$ & Pu-240 & & & \\
\hline $\mathrm{Bi}-212$ & $->$ & Tl-208 & & & \\
\hline $\mathrm{Cm}-244$ & $->$ & Pu-240 & & & \\
\hline Pu-240 & $->$ & $U-236$ & & & \\
\hline U-236 & $->$ & Th-232 & & & \\
\hline Ac- 228 & $->$ & Th-228 & & & \\
\hline Th-232 & $->$ & $\mathrm{Ra}-228$ & & & \\
\hline $\mathrm{Bi}-212$ & $->$ & TI-208 & & & \\
\hline TI-208 & $->$ & $\mathrm{Pb}-208$ & & & \\
\hline $\mathrm{Bi}-212$ & $->$ & TI-208 & & & \\
\hline $\mathrm{Pb}-212$ & $->$ & $\mathrm{Bi}-212$ & & & \\
\hline TI-208 & $->$ & $P b-208$ & & & \\
\hline $\mathrm{Bi}-212$ & $->$ & Po-212 & & & \\
\hline $\mathrm{Pb}-212$ & $->$ & $\mathrm{Bi}-212$ & & & \\
\hline $\mathrm{Bi}-212$ & $->$ & Po-212 & & & \\
\hline Ra-224 & $->$ & $\mathrm{Rn}-220$ & & & \\
\hline $\mathrm{Bi}-212$ & $->$ & TI-208 & & & \\
\hline Ra-224 & $->$ & $R n-220$ & & & \\
\hline Th-228 & $->$ & Ra-224 & & & \\
\hline TI-208 & $->$ & $\mathrm{Pb}-208$ & & & \\
\hline
\end{tabular}




\begin{tabular}{|c|c|c|c|c|}
\hline 85.185 & $1.55 \mathrm{E}-14$ & $\mathrm{Bi}-212$ & $->$ & TI-208 \\
\hline 85.429 & $6.11 \mathrm{E}-14$ & Th-228 & $->$ & $\mathrm{Ra}-224$ \\
\hline 87.19 & $2.16 \mathrm{E}-11$ & $\mathrm{~Pb}-212$ & $->$ & $\mathrm{Bi}-212$ \\
\hline 87.632 & $4.53 E-13$ & TI-208 & $->$ & $\mathrm{Pb}-208$ \\
\hline 88.471 & $1.01 \mathrm{E}-13$ & Th-228 & $->$ & Ra-224 \\
\hline 89.639 & 7.72E-14 & $\mathrm{Bi}-212$ & $->$ & Po-212 \\
\hline 89.955 & $4.21 \mathrm{E}-11$ & Ac- 228 & $->$ & Th-228 \\
\hline 90.128 & $6.40 \mathrm{E}-12$ & $\mathrm{~Pb}-212$ & $->$ & $\mathrm{Bi}-212$ \\
\hline 92.673 & $2.43 \mathrm{E}-14$ & $\mathrm{Bi}-212$ & $->$ & Po-212 \\
\hline 93.35 & $6.93 \mathrm{E}-11$ & Ac- 228 & $->$ & Th-228 \\
\hline 94.66 & $9.77 \mathrm{E}+02$ & Pu-240 & $->$ & U-236 \\
\hline 94.677 & $2.48 \mathrm{E}-13$ & $\mathrm{Ra}-224$ & $->$ & $R n-220$ \\
\hline 97.907 & $7.92 \mathrm{E}-14$ & Ra-224 & $->$ & $\mathrm{Rn}-220$ \\
\hline 98.441 & $1.44 \mathrm{E}+03$ & Pu-240 & $->$ & $U-236$ \\
\hline 98.855 & $3.71 \mathrm{E}+07$ & $\mathrm{Cm}-244$ & $->$ & $\mathrm{Pu}-240$ \\
\hline 99.55 & $1.61 \mathrm{E}-11$ & Ac- 228 & $->$ & Th-228 \\
\hline 99.915 & $3.60 \mathrm{E}-14$ & Th-228 & $->$ & Ra-224 \\
\hline 103.341 & $1.17 \mathrm{E}-14$ & Th-228 & $->$ & $\mathrm{Ra}-224$ \\
\hline 104.244 & $1.00 \mathrm{E}+05$ & Pu-240 & $->$ & $U-236$ \\
\hline 105.362 & $2.48 \mathrm{E}-11$ & Ac- 228 & $->$ & Th-228 \\
\hline 108.99 & $8.29 \mathrm{E}-12$ & AC- 228 & $->$ & Th-228 \\
\hline 110.421 & $1.70 \mathrm{E}+02$ & Pu-240 & $->$ & U-236 \\
\hline 111.3 & $5.17 \mathrm{E}+02$ & Pu-240 & $->$ & $U-236$ \\
\hline 112.75 & $2.08 \mathrm{E}-02$ & U-236 & $->$ & Th-232 \\
\hline 114.866 & $1.44 \mathrm{E}+02$ & $\mathrm{Pu}-240$ & $->$ & $U-236$ \\
\hline 115.122 & $2.03 \mathrm{E}-12$ & $\mathrm{~Pb}-212$ & $->$ & $\mathrm{Bi}-212$ \\
\hline 124 & $3.94 \mathrm{E}-12$ & Th-232 & $->$ & $\mathrm{Ra}-228$ \\
\hline 129.03 & $3.59 \mathrm{E}-11$ & AC- 228 & $->$ & Th-228 \\
\hline 131.5 & 4.47E-13 & Th-228 & $->$ & $\mathrm{Ra}-224$ \\
\hline 135.68 & $2.10 \mathrm{E}-13$ & Ac- 228 & $->$ & Th-228 \\
\hline 141.19 & $6.06 \mathrm{E}-13$ & Ac- 228 & $->$ & Th-228 \\
\hline 143.99 & $1.24 \mathrm{E}-14$ & $\mathrm{Bi}-212$ & $->$ & TI-208 \\
\hline 146.06 & $3.59 \mathrm{E}-12$ & Ac- 228 & $->$ & Th-228 \\
\hline 152.622 & $2.42 \mathrm{E}+07$ & $\mathrm{Cm}-244$ & $->$ & Pu-240 \\
\hline 153.89 & $1.04 \mathrm{E}-11$ & Ac- 228 & $->$ & Th-228 \\
\hline 160.28 & $6.04 \mathrm{E}+03$ & Pu-240 & $->$ & U-236 \\
\hline 164 & $6.19 \mathrm{E}-15$ & $\mathrm{Bi}-212$ & $->$ & TI-208 \\
\hline 164.15 & $1.72 \mathrm{E}-14$ & $\mathrm{~Pb}-212$ & $->$ & $\mathrm{Bi}-212$ \\
\hline 166.43 & $2.86 \mathrm{E}-13$ & Th-228 & $->$ & Ra-224 \\
\hline 174.18 & $3.96 \mathrm{E}-13$ & Ac- 228 & $->$ & Th-228 \\
\hline 176.577 & $1.75 \mathrm{E}-13$ & $\mathrm{~Pb}-212$ & $->$ & $\mathrm{Bi}-212$ \\
\hline 184.72 & $1.73 \mathrm{E}-12$ & Ac- 228 & $->$ & Th-228 \\
\hline 191.29 & $1.49 \mathrm{E}-12$ & AC- 228 & $->$ & Th-228 \\
\hline 199.54 & $3.47 \mathrm{E}-12$ & Ac- 228 & $->$ & Th-228 \\
\hline 204.37 & $1.94 \mathrm{E}-12$ & Ac- 228 & $->$ & Th-228 \\
\hline 205.75 & $9.77 \mathrm{E}-14$ & Th-228 & $->$ & Ra-224 \\
\hline 209.39 & $5.07 \mathrm{E}-11$ & Ac- 228 & $->$ & Th-228 \\
\hline 210.76 & $2.85 \mathrm{E}-12$ & Ac- 228 & $->$ & Th-228 \\
\hline 211.31 & $2.10 \mathrm{E}-13$ & Tl-208 & $->$ & Pb-208 \\
\hline 212.46 & $4.31 E+02$ & Pu-240 & $->$ & U-236 \\
\hline
\end{tabular}




\begin{tabular}{|c|c|c|c|c|}
\hline 215.75 & $9.67 \mathrm{E}-13$ & Th-228 & $->$ & $\mathrm{Ra}-224$ \\
\hline 216.24 & $9.90 \mathrm{E}-12$ & $A c-228$ & $->$ & Th-228 \\
\hline 220.49 & $1.73 \mathrm{E}-13$ & Ac- 228 & $->$ & Th-228 \\
\hline 223.72 & 8.17E-13 & Ac- 228 & $->$ & Th-228 \\
\hline 232.3 & $7.43 E-13$ & $A c-228$ & $->$ & Th-228 \\
\hline 233.32 & $3.82 \mathrm{E}-13$ & TI-208 & $->$ & $\mathrm{Pb}-208$ \\
\hline 238.578 & $1.50 \mathrm{E}-10$ & $\mathrm{~Pb}-212$ & $->$ & $\mathrm{Bi}-212$ \\
\hline 240.76 & $1.34 \mathrm{E}-11$ & $\mathrm{Ra}-224$ & $->$ & $R n-220$ \\
\hline 251.43 & $2.99 E+05$ & $\mathrm{Cm}-244$ & $->$ & Pu-240 \\
\hline 252.45 & $9.87 \mathrm{E}-13$ & TI-208 & $->$ & $\mathrm{Pb}-208$ \\
\hline 257.29 & $3.96 \mathrm{E}-13$ & $A c-228$ & $->$ & Th-228 \\
\hline 263.33 & $1.41 \mathrm{E}+06$ & $\mathrm{Cm}-244$ & $->$ & Pu-240 \\
\hline 263.57 & $7.18 \mathrm{E}-13$ & Ac- 228 & $->$ & Th-228 \\
\hline 270.26 & $4.70 \mathrm{E}-11$ & Ac- 228 & $->$ & Th-228 \\
\hline 277.28 & 8.39E-12 & Tl-208 & $->$ & $\mathrm{Pb}-208$ \\
\hline 279.3 & $2.85 \mathrm{E}-12$ & Ac- 228 & $->$ & Th-228 \\
\hline 282.02 & $1.10 \mathrm{E}-12$ & Ac- 228 & $->$ & Th-228 \\
\hline 288.08 & $4.22 \mathrm{E}-13$ & $\mathrm{Bi}-212$ & $->$ & TI-208 \\
\hline 290 & $3.10 \mathrm{E}-14$ & Ra-224 & $->$ & $\mathrm{Rn}-220$ \\
\hline 300.034 & $1.15 \mathrm{E}-11$ & $\mathrm{~Pb}-212$ & $->$ & $\mathrm{Bi}-212$ \\
\hline 302.96 & $3.81 E+05$ & $\mathrm{Cm}-244$ & $->$ & Pu-240 \\
\hline 321.9 & $3.09 E-12$ & Ac- 228 & $->$ & Th-228 \\
\hline 327.67 & $1.61 \mathrm{E}-12$ & Ac- 228 & $->$ & Th-228 \\
\hline 327.93 & $1.70 \mathrm{E}-13$ & $\mathrm{Bi}-212$ & $->$ & TI-208 \\
\hline 328.07 & $4.33 \mathrm{E}-11$ & Ac- 228 & $->$ & Th-228 \\
\hline 332.48 & $5.82 \mathrm{E}-12$ & Ac- 228 & $->$ & Th-228 \\
\hline 338.42 & $1.53 \mathrm{E}-10$ & Ac- 228 & $->$ & Th-228 \\
\hline 340.94 & $6.44 \mathrm{E}-12$ & $A c-228$ & $->$ & Th-228 \\
\hline 356.83 & $2.48 \mathrm{E}-13$ & Ac- 228 & $->$ & Th-228 \\
\hline 372.3 & $1.11 \mathrm{E}-13$ & Ac- 228 & $->$ & Th-228 \\
\hline 388.9 & $1.86 \mathrm{E}-13$ & Ac- 228 & $->$ & Th-228 \\
\hline 396.9 & $3.96 \mathrm{E}-13$ & Ac- 228 & $->$ & Th-228 \\
\hline 399.2 & $4.58 \mathrm{E}-13$ & Ac- 228 & $->$ & Th-228 \\
\hline 409.3 & $1.38 \mathrm{E}-14$ & Ra-224 & $->$ & $\mathrm{Rn}-220$ \\
\hline 409.62 & $2.72 \mathrm{E}-11$ & Ac- 228 & $->$ & Th-228 \\
\hline 415.156 & $9.63 \mathrm{E}-14$ & $\mathrm{~Pb}-212$ & $->$ & $\mathrm{Bi}-212$ \\
\hline 416.2 & $2.10 \mathrm{E}-13$ & Ac-228 & $->$ & Th-228 \\
\hline 419.23 & $3.22 \mathrm{E}-13$ & Ac- 228 & $->$ & Th-228 \\
\hline 433.51 & $1.73 \mathrm{E}-14$ & $\mathrm{Bi}-212$ & $->$ & TI-208 \\
\hline 440.49 & $1.86 \mathrm{E}-12$ & $A c-228$ & $->$ & Th-228 \\
\hline 449.57 & $8.05 E-13$ & Ac- 228 & $->$ & Th-228 \\
\hline 452.77 & $4.49 E-13$ & $\mathrm{Bi}-212$ & $->$ & TI-208 \\
\hline 460.84 & $6.19 E-13$ & Ac- 228 & $->$ & Th-228 \\
\hline 463.1 & $5.69 \mathrm{E}-11$ & $A c-228$ & $->$ & Th-228 \\
\hline 471.4 & $4.33 \mathrm{E}-13$ & $A c-228$ & $->$ & Th-228 \\
\hline 473.36 & $6.19 \mathrm{E}-14$ & $\mathrm{Bi}-212$ & $->$ & TI-208 \\
\hline 474.32 & $3.59 \mathrm{E}-13$ & Ac- 228 & $->$ & Th-228 \\
\hline 478.2 & 2.97E-12 & $A c-228$ & $->$ & Th-228 \\
\hline 481.8 & $1.11 \mathrm{E}-13$ & Ac-228 & $->$ & Th-228 \\
\hline 485.78 & $6.17 \mathrm{E}-14$ & TI-208 & $->$ & $\mathrm{Pb}-208$ \\
\hline
\end{tabular}




\begin{tabular}{|c|c|c|c|c|}
\hline 492.36 & $2.48 \mathrm{E}-13$ & Ac- 228 & $->$ & Th-228 \\
\hline 492.62 & $7.55 E-15$ & $\mathrm{Bi}-212$ & $->$ & TI-208 \\
\hline 498.26 & $5.32 \mathrm{E}-13$ & Ac- 228 & $->$ & Th-228 \\
\hline 503.7 & $2.60 \mathrm{E}-12$ & Ac- 228 & $->$ & Th-228 \\
\hline 507.22 & $1.88 \mathrm{E}+05$ & $\mathrm{Cm}-244$ & $->$ & Pu-240 \\
\hline 509.17 & $6.06 \mathrm{E}-12$ & Ac- 228 & $->$ & Th-228 \\
\hline 510.606 & $2.66 \mathrm{E}-11$ & Tl-208 & $->$ & $\mathrm{Pb}-208$ \\
\hline 515.2 & $5.32 \mathrm{E}-13$ & Ac- 228 & $->$ & Th-228 \\
\hline 519.97 & $9.65 E-13$ & Ac- 228 & $->$ & Th-228 \\
\hline 523.18 & $1.49 \mathrm{E}-12$ & Ac- 228 & $->$ & Th-228 \\
\hline 538.11 & $1.72 \mathrm{E}+00$ & Pu-240 & $->$ & $U-236$ \\
\hline 540.5 & $3.59 E-13$ & Ac- 228 & $->$ & Th-228 \\
\hline 546.36 & $2.72 \mathrm{E}-12$ & Ac- 228 & $->$ & Th-228 \\
\hline 549.7 & $2.41 \mathrm{E}-13$ & $\mathrm{Rn}-220$ & $->$ & Po-216 \\
\hline 554.54 & $1.95 \mathrm{E}+06$ & $\mathrm{Cm}-244$ & $->$ & $\mathrm{Pu}-240$ \\
\hline 555.3 & $6.06 \mathrm{E}-13$ & Ac- 228 & $->$ & Th-228 \\
\hline 562.65 & $1.25 \mathrm{E}-11$ & Ac- 228 & $->$ & Th-228 \\
\hline 570.2 & $2.35 \mathrm{E}-12$ & Ac- 228 & $->$ & Th-228 \\
\hline 572.5 & $2.35 \mathrm{E}-12$ & Ac- 228 & $->$ & Th-228 \\
\hline 580.4 & $9.78 \mathrm{E}-16$ & $\mathrm{Bi}-212$ & $->$ & TI-208 \\
\hline 583.022 & $1.10 \mathrm{E}-10$ & TI-208 & $->$ & $\mathrm{Pb}-208$, \\
\hline 583.28 & $1.86 \mathrm{E}-12$ & Ac- 228 & $->$ & Th-228 \\
\hline 587.82 & $4.93 \mathrm{E}-14$ & TI-208 & $->$ & $\mathrm{Pb}-208$ \\
\hline 597.35 & $1.11 \mathrm{E}+06$ & $\mathrm{Cm}-244$ & $->$ & Pu-240 \\
\hline 606.07 & $1.78 \mathrm{E}+05$ & $\mathrm{Cm}-244$ & $->$ & Pu-240 \\
\hline 615.9 & $1.11 \mathrm{E}-12$ & Ac- 228 & $->$ & Th-228 \\
\hline 619.88 & $1.36 \mathrm{E}-12$ & AC- 228 & $->$ & Th-228 \\
\hline 620.3 & $4.46 \mathrm{E}-15$ & $\mathrm{Bi}-212$ & $->$ & TI-208 \\
\hline 629.93 & $6.19 \mathrm{E}-13$ & Ac- 228 & $->$ & Th-228 \\
\hline 640.7 & $7.55 \mathrm{E}-13$ & Ac- 228 & $->$ & Th-228 \\
\hline 642.5 & $1.81 \mathrm{E}+02$ & Pu-240 & $->$ & $U-236$ \\
\hline 649.19 & $5.32 \mathrm{E}-13$ & Ac- 228 & $->$ & Th-228 \\
\hline 650.1 & $2.41 \mathrm{E}-14$ & Ra-224 & $->$ & $R n-220$ \\
\hline 650.14 & $4.44 \mathrm{E}-14$ & TI-208 & $->$ & $\mathrm{Pb}-208$ \\
\hline 651.44 & $1.25 \mathrm{E}-12$ & Ac- 228 & $->$ & Th-228 \\
\hline 666.4 & $5.69 \mathrm{E}-13$ & Ac- 228 & $->$ & Th-228 \\
\hline 673.86 & $1.24 \mathrm{E}-12$ & Ac- 228 & $->$ & Th-228 \\
\hline 677.07 & $1.11 \mathrm{E}-11$ & Ac- 228 & $->$ & Th-228 \\
\hline 687.59 & $3.88 \mathrm{E}+01$ & Pu-240 & $->$ & $U-236$ \\
\hline 692.5 & $4.21 \mathrm{E}-14$ & Ac- 228 & $->$ & Th-228 \\
\hline 701.8 & $2.35 \mathrm{E}-12$ & Ac- 228 & $->$ & Th-228 \\
\hline 705.24 & $2.71 \mathrm{E}-14$ & TI-208 & $->$ & $\mathrm{Pb}-208$ \\
\hline 707.49 & $1.86 \mathrm{E}-12$ & Ac- 228 & $->$ & Th-228 \\
\hline 721.91 & $2.50 \mathrm{E}-13$ & Tl-208 & $->$ & $\mathrm{Pb}-208$ \\
\hline 726.63 & $1.08 \mathrm{E}-11$ & Ac- 228 & $->$ & Th-228 \\
\hline 727.25 & $1.47 \mathrm{E}-11$ & $\mathrm{Bi}-212$ & $->$ & Po-212 \\
\hline 737.7 & $5.07 E-13$ & Ac- 228 & $->$ & Th-228 \\
\hline 748.58 & $5.30 \mathrm{E}-14$ & TI-208 & $->$ & $\mathrm{Pb}-208$ \\
\hline 755.28 & $1.63 \mathrm{E}-11$ & Ac- 228 & $->$ & Th-228 \\
\hline 758.64 & $3.78 \mathrm{E}+05$ & $\mathrm{Cm}-244$ & $->$ & $\mathrm{Pu}-240$ \\
\hline
\end{tabular}




\begin{tabular}{|c|c|c|c|c|}
\hline 763.06 & $2.02 \mathrm{E}-12$ & TI-208 & $->$ & $\mathrm{Pb}-208$ \\
\hline 772.28 & $1.35 \mathrm{E}-11$ & $A c-228$ & $->$ & Th-228 \\
\hline 774 & $1.11 \mathrm{E}-12$ & Ac- 228 & $->$ & Th-228 \\
\hline 782.12 & $7.30 \mathrm{E}-12$ & Ac- 228 & $->$ & Th-228 \\
\hline 785.51 & $2.44 \mathrm{E}-12$ & $\mathrm{Bi}-212$ & $->$ & Po-212 \\
\hline 791.05 & $2.60 \mathrm{E}-13$ & $A c-228$ & $->$ & Th-228 \\
\hline 791.2 & $3.59 E-13$ & Ac- 228 & $->$ & Th-228 \\
\hline 794.79 & $5.69 \mathrm{E}-11$ & Ac- 228 & $->$ & Th-228 \\
\hline 804.9 & $6.21 \mathrm{E}-15$ & Po-216 & $->$ & $\mathrm{Pb}-212$ \\
\hline 816.62 & $4.33 \mathrm{E}-13$ & $A c-228$ & $->$ & Th-228 \\
\hline 817.87 & $1.66 \mathrm{E}+06$ & $\mathrm{Cm}-244$ & $->$ & Pu-240 \\
\hline 821.14 & $4.93 \mathrm{E}-14$ & TI-208 & $->$ & Pb-208 \\
\hline 825.3 & $7.18 \mathrm{E}-13$ & Ac- 228 & $->$ & Th-228 \\
\hline 830.59 & $7.80 \mathrm{E}-12$ & Ac- 228 & $->$ & Th-228 \\
\hline 835.6 & $2.12 \mathrm{E}-11$ & Ac- 228 & $->$ & Th-228 \\
\hline 840.44 & $1.16 \mathrm{E}-11$ & $A c-228$ & $->$ & Th-228 \\
\hline 853.6 & $1.73 \mathrm{E}-13$ & Ac- 228 & $->$ & Th-228 \\
\hline 857.5 & $1.51 \mathrm{E}+05$ & $\mathrm{Cm}-244$ & $->$ & Pu-240 \\
\hline 860.3 & $1.48 \mathrm{E}-11$ & TI-208 & $->$ & $\mathrm{Pb}-208$ \\
\hline 870.47 & 8.17E-13 & $A c-228$ & $->$ & Th-228 \\
\hline 873.94 & $8.34 \mathrm{E}+00$ & Pu-240 & $->$ & U-236 \\
\hline 874.43 & $1.04 \mathrm{E}-12$ & $A c-228$ & $->$ & Th-228 \\
\hline 877.65 & $2.48 \mathrm{E}-13$ & Ac- 228 & $->$ & Th-228 \\
\hline 883.27 & $3.82 \mathrm{E}-14$ & TI-208 & $->$ & $\mathrm{Pb}-208$ \\
\hline 884 & $1.24 \mathrm{E}-12$ & Ac- 228 & $->$ & Th-228 \\
\hline 887.46 & $2.48 \mathrm{E}-13$ & $A c-228$ & $->$ & Th-228 \\
\hline 893.42 & $8.10 \mathrm{E}-13$ & $\mathrm{Bi}-212$ & $->$ & Po-212 \\
\hline 900.31 & $4.20 E+04$ & $\mathrm{Cm}-244$ & $->$ & Pu-240 \\
\hline 904.29 & $1.10 \mathrm{E}-11$ & Ac-228 & $->$ & Th-228 \\
\hline 911.16 & $3.59 E-10$ & Ac- 228 & $->$ & Th-228 \\
\hline 919.19 & $3.47 E-13$ & Ac- 228 & $->$ & Th-228 \\
\hline 922.28 & $2.60 \mathrm{E}-13$ & $A c-228$ & $->$ & Th-228 \\
\hline 927.42 & $1.54 \mathrm{E}-13$ & TI-208 & $->$ & $\mathrm{Pb}-208$ \\
\hline 931.1 & $1.86 \mathrm{E}-13$ & Ac- 228 & $->$ & Th-228 \\
\hline 943.9 & $1.32 \mathrm{E}-12$ & Ac- 228 & $->$ & Th-228 \\
\hline 948.4 & $1.51 \mathrm{E}-12$ & $A c-228$ & $->$ & Th-228 \\
\hline 952.17 & $3.88 \mathrm{E}-13$ & $\mathrm{Bi}-212$ & $->$ & Po-212 \\
\hline 958.3 & $3.84 \mathrm{E}-12$ & Ac- 228 & $->$ & Th-228 \\
\hline 964.64 & 7.18E-11 & $A c-228$ & $->$ & Th-228 \\
\hline 968.97 & $2.15 \mathrm{E}-10$ & Ac- 228 & $->$ & Th-228 \\
\hline 979.7 & $2.85 \mathrm{E}-13$ & $A c-228$ & $->$ & Th-228 \\
\hline 982.52 & $2.43 \mathrm{E}-13$ & TI-208 & $->$ & $\mathrm{Pb}-208$ \\
\hline 987.87 & $4.21 \mathrm{E}-12$ & Ac- 228 & $->$ & Th-228 \\
\hline 987.94 & $2.35 \mathrm{E}-12$ & Ac- 228 & $->$ & Th-228 \\
\hline 1016.12 & 3.09E-13 & Ac- 228 & $->$ & Th-228 \\
\hline 1019.7 & 3.09E-13 & Ac- 228 & $->$ & Th-228 \\
\hline 1033.32 & $1.92 \mathrm{E}-12$ & Ac- 228 & $->$ & Th-228 \\
\hline 1039.97 & $6.81 \mathrm{E}-13$ & $A c-228$ & $->$ & Th-228 \\
\hline 1041.35 & $3.96 \mathrm{E}-13$ & $A c-228$ & $->$ & Th-228 \\
\hline 1054.3 & $3.96 \mathrm{E}-13$ & $A c-228$ & $->$ & Th-228 \\
\hline
\end{tabular}




\begin{tabular}{|c|c|c|c|c|}
\hline 1065.05 & $2.12 \mathrm{E}-12$ & Ac- 228 & $->$ & Th-228 \\
\hline 1074.1 & $3.53 E-14$ & $\mathrm{Bi}-212$ & $->$ & Po-212 \\
\hline 1078.69 & $1.18 \mathrm{E}-12$ & $\mathrm{Bi}-212$ & $->$ & Po-212 \\
\hline 1093.63 & $4.56 \mathrm{E}-13$ & TI-208 & $->$ & $\mathrm{Pb}-208$ \\
\hline 1095.87 & $1.63 \mathrm{E}-12$ & Ac- 228 & $->$ & Th-228 \\
\hline 1104.01 & $2.10 \mathrm{E}-13$ & Ac- 228 & $->$ & Th-228 \\
\hline 1110.7 & $4.79 E-12$ & Ac- 228 & $->$ & Th-228 \\
\hline 1116.97 & $7.55 \mathrm{E}-13$ & Ac- 228 & $->$ & Th-228 \\
\hline 1125.6 & $6.17 \mathrm{E}-15$ & Tl-208 & $->$ & Pb-208 \\
\hline 1135.39 & $1.49 \mathrm{E}-13$ & Ac- 228 & $->$ & Th-228 \\
\hline 1142.7 & $1.24 \mathrm{E}-13$ & Ac- 228 & $->$ & Th-228 \\
\hline 1153.69 & 1.97E-12 & Ac- 228 & $->$ & Th-228 \\
\hline 1160.55 & $1.36 \mathrm{E}-14$ & Tl-208 & $->$ & Pb-208 \\
\hline 1163.6 & $9.28 \mathrm{E}-13$ & Ac- 228 & $->$ & Th-228 \\
\hline 1174.79 & $3.59 \mathrm{E}-13$ & Ac- 228 & $->$ & Th-228 \\
\hline 1185.1 & $2.10 \mathrm{E}-14$ & TI-208 & $->$ & $\mathrm{Pb}-208$ \\
\hline 1216.4 & $3.22 \mathrm{E}-13$ & Ac- 228 & $->$ & Th-228 \\
\hline 1245.23 & $1.11 \mathrm{E}-12$ & Ac- 228 & $->$ & Th-228 \\
\hline 1246.6 & $7.05 \mathrm{E}-12$ & Ac- 228 & $->$ & Th-228 \\
\hline 1249.81 & $7.43 E-13$ & Ac- 228 & $->$ & Th-228 \\
\hline 1277.5 & $2.48 \mathrm{E}-13$ & Ac- 228 & $->$ & Th-228 \\
\hline 1282.7 & $6.41 \mathrm{E}-14$ & Tl-208 & $->$ & $\mathrm{Pb}-208$ \\
\hline 1286.6 & $1.47 \mathrm{E}-12$ & Ac- 228 & $->$ & Th-228 \\
\hline 1309.6 & $2.60 \mathrm{E}-13$ & Ac- 228 & $->$ & Th-228 \\
\hline 1314.76 & $2.60 \mathrm{E}-13$ & Ac- 228 & $->$ & Th-228 \\
\hline 1347.6 & $1.61 \mathrm{E}-13$ & Ac- 228 & $->$ & Th-228 \\
\hline 1357.6 & $3.59 E-13$ & Ac- 228 & $->$ & Th-228 \\
\hline 1374.26 & $2.48 \mathrm{E}-13$ & Ac- 228 & $->$ & Th-228 \\
\hline 1381 & $8.64 \mathrm{E}-15$ & TI-208 & $->$ & $\mathrm{Pb}-208$ \\
\hline 1415.7 & $3.22 \mathrm{E}-13$ & Ac- 228 & $->$ & Th-228 \\
\hline 1431 & $3.96 \mathrm{E}-13$ & Ac- 228 & $->$ & Th-228 \\
\hline 1451.2 & $2.23 E-13$ & Ac- 228 & $->$ & Th-228 \\
\hline 1459.19 & $1.31 \mathrm{E}-11$ & Ac- 228 & $->$ & Th-228 \\
\hline 1468.8 & $2.48 \mathrm{E}-13$ & Ac- 228 & $->$ & Th-228 \\
\hline 1481.2 & $2.48 \mathrm{E}-13$ & Ac- 228 & $->$ & Th-228 \\
\hline 1496 & $1.30 \mathrm{E}-11$ & Ac- 228 & $->$ & Th-228 \\
\hline 1501.44 & $7.18 \mathrm{E}-12$ & Ac- 228 & $->$ & Th-228 \\
\hline 1503.87 & $3.59 E-13$ & Ac- 228 & $->$ & Th-228 \\
\hline 1512.75 & $6.91 \mathrm{E}-13$ & $\mathrm{Bi}-212$ & $->$ & Po-212 \\
\hline 1528.9 & $8.29 E-13$ & Ac- 228 & $->$ & Th-228 \\
\hline 1537.4 & $6.06 \mathrm{E}-13$ & Ac- 228 & $->$ & Th-228 \\
\hline 1548.5 & $5.32 \mathrm{E}-13$ & Ac- 228 & $->$ & Th-228 \\
\hline 1557 & $2.48 \mathrm{E}-12$ & Ac- 228 & $->$ & Th-228 \\
\hline 1572.03 & $6.19 \mathrm{E}-13$ & Ac- 228 & $->$ & Th-228 \\
\hline 1573.3 & $6.81 \mathrm{E}-13$ & Ac- 228 & $->$ & Th-228 \\
\hline 1580.3 & 8.79E-12 & Ac- 228 & $->$ & Th-228 \\
\hline 1588.23 & $4.46 \mathrm{E}-11$ & Ac- 228 & $->$ & Th-228 \\
\hline 1620.66 & $3.33 \mathrm{E}-12$ & $\mathrm{Bi}-212$ & $->$ & Po-212 \\
\hline 1625.1 & $3.96 \mathrm{E}-12$ & AC- 228 & $->$ & Th-228 \\
\hline 1630.47 & $2.41 \mathrm{E}-11$ & Ac- 228 & $->$ & Th-228 \\
\hline
\end{tabular}




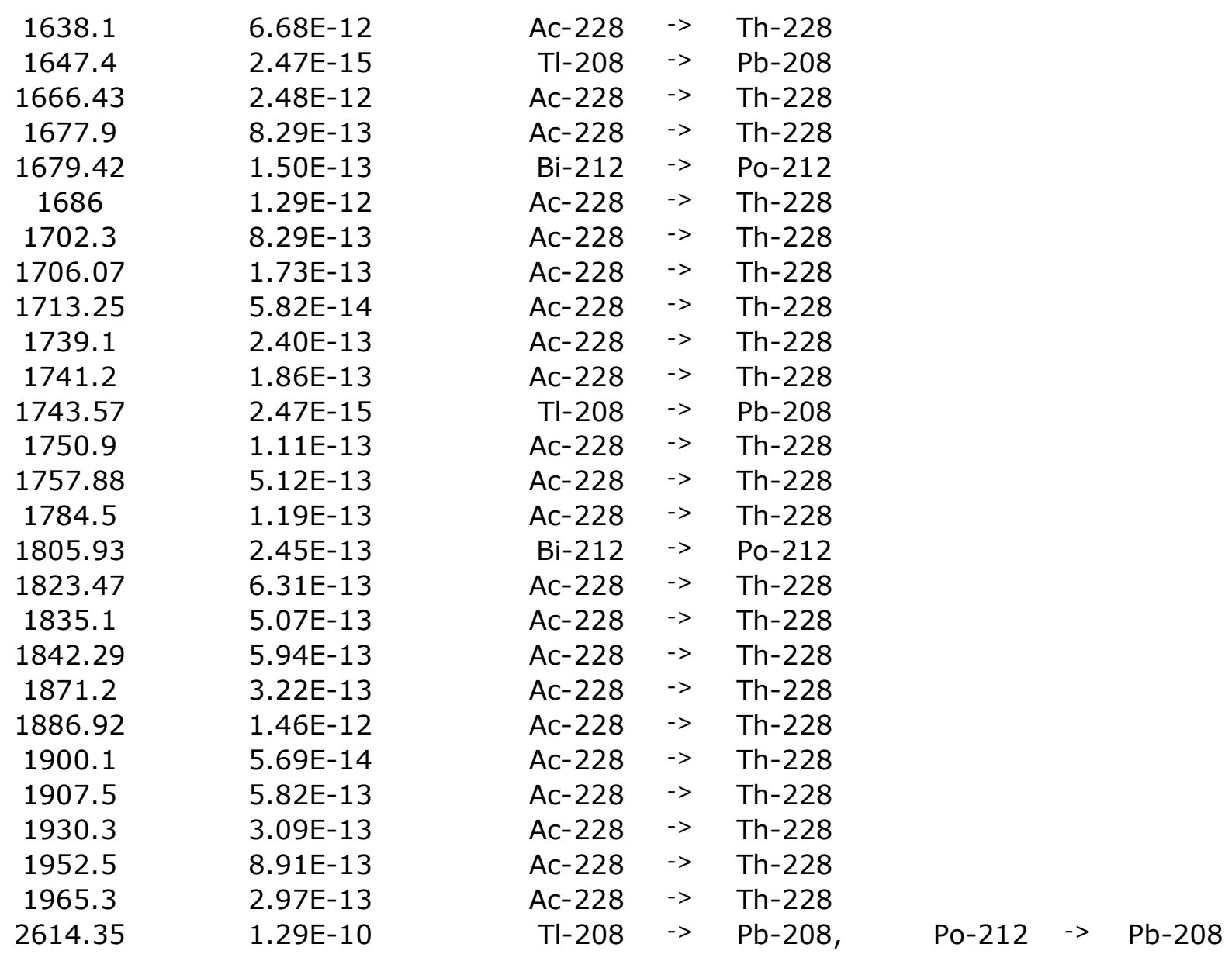

Cm244 Aged 5 years Sorted by Intensity

\begin{tabular}{|c|c|c|c|c|}
\hline Intensity & Energy & \multicolumn{3}{|c|}{ Initial Isotopes and Decays } \\
\hline $\mathrm{ph} / \mathrm{s} / \mathrm{gm}$ & keV & & & \\
\hline $9.89 E+10$ & 14.262 & $\mathrm{Cm}-244$ & $->$ & Pu-240 \\
\hline $9.89 E+10$ & 18.135 & $\mathrm{Cm}-244$ & $->$ & Pu-240 \\
\hline $2.47 E+10$ & 21.554 & $\mathrm{Cm}-244$ & $->$ & Pu-240 \\
\hline $5.69 E+09$ & 12.124 & $\mathrm{Cm}-244$ & $->$ & Pu-240 \\
\hline $2.22 E+09$ & 16.333 & $\mathrm{Cm}-244$ & $->$ & Pu-240 \\
\hline $6.18 \mathrm{E}+08$ & 42.817 & $\mathrm{Cm}-244$ & $->$ & Pu-240 \\
\hline $3.71 E+07$ & 98.855 & $\mathrm{Cm}-244$ & $->$ & Pu-240 \\
\hline $2.42 E+07$ & 152.622 & $\mathrm{Cm}-244$ & $->$ & Pu-240 \\
\hline $1.95 \mathrm{E}+06$ & 554.54 & $\mathrm{Cm}-244$ & $->$ & Pu-240 \\
\hline $1.66 \mathrm{E}+06$ & 817.87 & $\mathrm{Cm}-244$ & $->$ & Pu-240 \\
\hline $1.41 \mathrm{E}+06$ & 263.33 & $\mathrm{Cm}-244$ & $->$ & Pu-240 \\
\hline $1.11 \mathrm{E}+06$ & 597.35 & $\mathrm{Cm}-244$ & $->$ & Pu-240 \\
\hline $6.47 E+05$ & 45.244 & Pu-240 & $->$ & $U-236$ \\
\hline $3.81 \mathrm{E}+05$ & 302.96 & $\mathrm{Cm}-244$ & $->$ & Pu-240 \\
\hline $3.78 \mathrm{E}+05$ & 758.64 & $\mathrm{Cm}-244$ & $->$ & Pu-240 \\
\hline $2.99 E+05$ & 251.43 & $\mathrm{Cm}-244$ & $->$ & Pu-240 \\
\hline
\end{tabular}




\begin{tabular}{|c|c|c|c|c|c|c|c|}
\hline $1.88 \mathrm{E}+05$ & 507.22 & $\mathrm{Cm}-244$ & $->$ & Pu-240 & & & \\
\hline $1.78 \mathrm{E}+05$ & 606.07 & $\mathrm{Cm}-244$ & $->$ & $\mathrm{Pu}-240$ & & & \\
\hline $1.51 \mathrm{E}+05$ & 857.5 & $\mathrm{Cm}-244$ & $->$ & Pu-240 & & & \\
\hline $1.00 \mathrm{E}+05$ & 104.244 & Pu-240 & $->$ & $U-236$ & & & \\
\hline $4.20 \mathrm{E}+04$ & 900.31 & $\mathrm{Cm}-244$ & $->$ & Pu-240 & & & \\
\hline $6.04 \mathrm{E}+03$ & 160.28 & Pu-240 & $->$ & $U-236$ & & & \\
\hline $1.44 \mathrm{E}+03$ & 98.441 & Pu-240 & $->$ & $U-236$ & & & \\
\hline $9.77 E+02$ & 94.66 & Pu-240 & $->$ & $U-236$ & & & \\
\hline $5.17 E+02$ & 111.3 & Pu-240 & $->$ & $U-236$ & & & \\
\hline $4.31 E+02$ & 212.46 & Pu-240 & $->$ & $U-236$ & & & \\
\hline $1.81 \mathrm{E}+02$ & 642.5 & Pu-240 & $->$ & $U-236$ & & & \\
\hline $1.70 \mathrm{E}+02$ & 110.421 & Pu-240 & $->$ & $U-236$ & & & \\
\hline $1.44 \mathrm{E}+02$ & 114.866 & Pu-240 & $->$ & $U-236$ & & & \\
\hline $3.88 \mathrm{E}+01$ & 687.59 & Pu-240 & $->$ & $U-236$ & & & \\
\hline $8.34 \mathrm{E}+00$ & 873.94 & Pu-240 & $->$ & $U-236$ & & & \\
\hline $5.15 E+00$ & 16.161 & U-236 & $->$ & Th-232 & & & \\
\hline $3.73 E+00$ & 12.952 & Ac- 228 & $->$ & Th-228, & $U-236$ & $->$ & Th-232 \\
\hline $1.72 \mathrm{E}+00$ & 538.11 & Pu-240 & $->$ & $U-236$ & & & \\
\hline $1.15 \mathrm{E}+00$ & 19.094 & U-236 & $->$ & Th-232 & & & \\
\hline 2.19E-01 & 11.118 & AC- 228 & $->$ & Th-228, & $U-236$ & $->$ & Th-232 \\
\hline $1.04 \mathrm{E}-01$ & 14.511 & Ac- 228 & $->$ & Th-228, & U-236 & $->$ & Th-232 \\
\hline $8.55 E-02$ & 49.369 & U-236 & $->$ & Th-232 & & & \\
\hline $2.08 \mathrm{E}-02$ & 112.75 & U-236 & $->$ & Th-232 & & & \\
\hline $1.24 \mathrm{E}-09$ & 13.5 & $\mathrm{Ra}-228$ & $->$ & $A C-228$ & & & \\
\hline $5.57 \mathrm{E}-10$ & 16.18 & Ra-228 & $->$ & $A c-228$ & & & \\
\hline $3.59 \mathrm{E}-10$ & 911.16 & Ac- 228 & $->$ & Th-228 & & & \\
\hline $2.48 \mathrm{E}-10$ & 16.154 & Ac- 228 & $->$ & Th-228 & & & \\
\hline $2.35 \mathrm{E}-10$ & 12.76 & $\mathrm{Ra}-228$ & $->$ & Ac- 228 & & & \\
\hline $2.15 \mathrm{E}-10$ & 968.97 & Ac- 228 & $->$ & Th-228 & & & \\
\hline $1.61 \mathrm{E}-10$ & 18.8 & $\mathrm{Ra}-228$ & $->$ & $A c-228$ & & & \\
\hline $1.53 \mathrm{E}-10$ & 338.42 & Ac- 228 & $->$ & Th-228 & & & \\
\hline $1.50 \mathrm{E}-10$ & 238.578 & $\mathrm{~Pb}-212$ & $->$ & $\mathrm{Bi}-212$ & & & \\
\hline $1.29 \mathrm{E}-10$ & 2614.35 & TI-208 & $->$ & $\mathrm{Pb}-208$, & Po-212 & $->$ & $\mathrm{Pb}-208$ \\
\hline $1.24 \mathrm{E}-10$ & 15.15 & $\mathrm{Ra}-228$ & $->$ & $A C-228$ & & & \\
\hline $1.10 \mathrm{E}-10$ & 583.022 & TI-208 & $->$ & $\mathrm{Pb}-208$ & Po-212 & $->$ & $\mathrm{Pb}-208$ \\
\hline 7.18E-11 & 964.64 & Ac- 228 & $->$ & Th-228 & & & \\
\hline $6.93 \mathrm{E}-11$ & 93.35 & Ac- 228 & $->$ & Th-228 & & & \\
\hline $6.09 \mathrm{E}-11$ & 77.107 & $\mathrm{~Pb}-212$ & $->$ & $\mathrm{Bi}-212$ & & & \\
\hline $5.69 E-11$ & 19.113 & Ac- 228 & $->$ & Th-228 & & & \\
\hline $5.69 \mathrm{E}-11$ & 794.79 & Ac- 228 & $->$ & Th-228 & & & \\
\hline $5.69 \mathrm{E}-11$ & 463.1 & Ac- 228 & $->$ & Th-228 & & & \\
\hline $5.07 \mathrm{E}-11$ & 209.39 & Ac- 228 & $->$ & Th-228 & & & \\
\hline 4.70E-11 & 270.26 & Ac- 228 & $->$ & Th-228 & & & \\
\hline $4.46 \mathrm{E}-11$ & 1588.23 & Ac- 228 & $->$ & Th-228 & & & \\
\hline 4.33E-11 & 328.07 & Ac- 228 & $->$ & Th-228 & & & \\
\hline $4.21 \mathrm{E}-11$ & 89.955 & Ac- 228 & $->$ & Th-228 & & & \\
\hline $3.61 \mathrm{E}-11$ & 74.814 & $\mathrm{~Pb}-212$ & $->$ & $\mathrm{Bi}-212$ & & & \\
\hline $3.59 \mathrm{E}-11$ & 129.03 & Ac- 228 & $->$ & Th-228 & & & \\
\hline $2.72 \mathrm{E}-11$ & 409.62 & $A c-228$ & $->$ & Th-228 & & & \\
\hline $2.66 \mathrm{E}-11$ & 510.606 & TI-208 & $->$ & $\mathrm{Pb}-208$ & & & \\
\hline
\end{tabular}




\begin{tabular}{|c|c|c|c|c|}
\hline $2.48 \mathrm{E}-11$ & 105.362 & Ac- 228 & $->$ & Th-228 \\
\hline $2.41 \mathrm{E}-11$ & 1630.47 & Ac- 228 & $->$ & Th-228 \\
\hline 2.27E-11 & 10.828 & $\mathrm{~Pb}-212$ & $->$ & $\mathrm{Bi}-212$ \\
\hline $2.16 \mathrm{E}-11$ & 87.19 & $\mathrm{~Pb}-212$ & $->$ & $\mathrm{Bi}-212$ \\
\hline $2.12 \mathrm{E}-11$ & 835.6 & $A C-228$ & $->$ & Th-228 \\
\hline $2.06 \mathrm{E}-11$ & 13.099 & $\mathrm{~Pb}-212$ & $->$ & $\mathrm{Bi}-212$ \\
\hline $1.74 \mathrm{E}-11$ & 59 & Th-232 & $->$ & $\mathrm{Ra}-228$ \\
\hline $1.64 \mathrm{E}-11$ & 15.23 & Th-228 & $->$ & Ra-224 \\
\hline $1.63 \mathrm{E}-11$ & 755.28 & Ac- 228 & $->$ & Th-228 \\
\hline $1.61 \mathrm{E}-11$ & 99.55 & $A c-228$ & $->$ & Th-228 \\
\hline $1.48 \mathrm{E}-11$ & 860.3 & TI-208 & $->$ & $\mathrm{Pb}-208$ \\
\hline $1.47 \mathrm{E}-11$ & 727.25 & $\mathrm{Bi}-212$ & $->$ & Po-212 \\
\hline $1.35 \mathrm{E}-11$ & 772.28 & Ac- 228 & $->$ & Th-228 \\
\hline $1.34 \mathrm{E}-11$ & 240.76 & $\mathrm{Ra}-224$ & $->$ & $\mathrm{Rn}-220$ \\
\hline $1.31 \mathrm{E}-11$ & 1459.19 & Ac- 228 & $->$ & Th-228 \\
\hline $1.30 \mathrm{E}-11$ & 1496 & Ac- 228 & $->$ & Th-228 \\
\hline $1.25 \mathrm{E}-11$ & 562.65 & Ac- 228 & $->$ & Th-228 \\
\hline $1.16 \mathrm{E}-11$ & 840.44 & Ac- 228 & $->$ & Th-228 \\
\hline $1.15 \mathrm{E}-11$ & 300.034 & $\mathrm{~Pb}-212$ & $->$ & $\mathrm{Bi}-212$ \\
\hline $1.11 \mathrm{E}-11$ & 677.07 & Ac- 228 & $->$ & Th-228 \\
\hline $1.11 \mathrm{E}-11$ & 19.4 & $\mathrm{Ra}-228$ & $->$ & Ac- 228 \\
\hline $1.10 \mathrm{E}-11$ & 904.29 & Ac- 228 & $->$ & Th-228 \\
\hline $1.08 \mathrm{E}-11$ & 12.325 & Th-228 & $->$ & Ra-224 \\
\hline $1.08 \mathrm{E}-11$ & 726.63 & Ac- 228 & $->$ & Th-228 \\
\hline $1.04 \mathrm{E}-11$ & 153.89 & Ac- 228 & $->$ & Th-228 \\
\hline $9.90 \mathrm{E}-12$ & 216.24 & Ac- 228 & $->$ & Th-228 \\
\hline 8.79E-12 & 1580.3 & Ac- 228 & $->$ & Th-228 \\
\hline 8.39E-12 & 277.28 & TI-208 & $->$ & $\mathrm{Pb}-208$ \\
\hline 8.29E-12 & 108.99 & Ac- 228 & $->$ & Th-228 \\
\hline 7.80E-12 & 830.59 & Ac- 228 & $->$ & Th-228 \\
\hline 7.30E-12 & 782.12 & Ac- 228 & $->$ & Th-228 \\
\hline $7.18 \mathrm{E}-12$ & 1501.44 & Ac- 228 & $->$ & Th-228 \\
\hline 7.05E-12 & 1246.6 & Ac- 228 & $->$ & Th-228 \\
\hline $6.68 \mathrm{E}-12$ & 1638.1 & Ac- 228 & $->$ & Th-228 \\
\hline $6.50 \mathrm{E}-12$ & 57.81 & Ac- 228 & $->$ & Th-228 \\
\hline $6.44 \mathrm{E}-12$ & 340.94 & Ac- 228 & $->$ & Th-228 \\
\hline $6.40 \mathrm{E}-12$ & 90.128 & $\mathrm{~Pb}-212$ & $->$ & $\mathrm{Bi}-212$ \\
\hline $6.06 \mathrm{E}-12$ & 509.17 & $A c-228$ & $->$ & Th-228 \\
\hline $5.82 \mathrm{E}-12$ & 332.48 & Ac- 228 & $->$ & Th-228 \\
\hline 4.79E-12 & 1110.7 & Ac- 228 & $->$ & Th-228 \\
\hline $4.44 \mathrm{E}-12$ & 74.969 & TI-208 & $->$ & $\mathrm{Pb}-208$ \\
\hline $4.33 \mathrm{E}-12$ & 12.282 & $\mathrm{Bi}-212$ & $->$ & TI-208 \\
\hline $4.22 \mathrm{E}-12$ & 84.26 & Th-228 & $->$ & $\mathrm{Ra}-224$ \\
\hline $4.21 \mathrm{E}-12$ & 987.87 & Ac- 228 & $->$ & Th-228 \\
\hline $3.96 \mathrm{E}-12$ & 1625.1 & Ac- 228 & $->$ & Th-228 \\
\hline $3.94 \mathrm{E}-12$ & 124 & Th-232 & $->$ & $\mathrm{Ra}-228$ \\
\hline $3.89 \mathrm{E}-12$ & 15.376 & $\mathrm{~Pb}-212$ & $->$ & $\mathrm{Bi}-212$ \\
\hline $3.84 \mathrm{E}-12$ & 958.3 & $A c-228$ & $->$ & Th-228 \\
\hline $3.76 \mathrm{E}-12$ & 10.259 & $\mathrm{Bi}-212$ & $->$ & TI-208 \\
\hline $3.70 \mathrm{E}-12$ & 17.952 & Th-228 & $->$ & $\mathrm{Ra}-224$ \\
\hline
\end{tabular}




\begin{tabular}{|c|c|c|c|c|}
\hline $3.59 \mathrm{E}-12$ & 146.06 & Ac- 228 & $->$ & Th-228 \\
\hline $3.47 \mathrm{E}-12$ & 199.54 & Ac- 228 & $->$ & Th-228 \\
\hline $3.33 \mathrm{E}-12$ & 1620.66 & $\mathrm{Bi}-212$ & $->$ & Po-212 \\
\hline $3.09 \mathrm{E}-12$ & 321.9 & $A c-228$ & $->$ & Th-228 \\
\hline $2.97 \mathrm{E}-12$ & 478.2 & Ac- 228 & $->$ & Th-228 \\
\hline $2.85 \mathrm{E}-12$ & 279.3 & Ac- 228 & $->$ & Th-228 \\
\hline $2.85 \mathrm{E}-12$ & 210.76 & Ac- 228 & $->$ & Th-228 \\
\hline $2.72 \mathrm{E}-12$ & 546.36 & Ac- 228 & $->$ & Th-228 \\
\hline $2.64 \mathrm{E}-12$ & 72.803 & TI-208 & $->$ & $\mathrm{Pb}-208$ \\
\hline $2.60 \mathrm{E}-12$ & 503.7 & $A c-228$ & $->$ & Th-228 \\
\hline $2.48 \mathrm{E}-12$ & 1557 & Ac- 228 & $->$ & Th-228 \\
\hline $2.48 \mathrm{E}-12$ & 1666.43 & Ac- 228 & $->$ & Th-228 \\
\hline $2.44 \mathrm{E}-12$ & 785.51 & $\mathrm{Bi}-212$ & $->$ & Po-212 \\
\hline $2.35 \mathrm{E}-12$ & 701.8 & $A C-228$ & $->$ & Th-228 \\
\hline $2.35 \mathrm{E}-12$ & 570.2 & Ac- 228 & $->$ & Th-228 \\
\hline $2.35 \mathrm{E}-12$ & 572.5 & Ac- 228 & $->$ & Th-228 \\
\hline $2.35 \mathrm{E}-12$ & 987.94 & Ac- 228 & $->$ & Th-228 \\
\hline $2.12 \mathrm{E}-12$ & 1065.05 & Ac- 228 & $->$ & Th-228 \\
\hline $2.03 E-12$ & 115.122 & $\mathrm{~Pb}-212$ & $->$ & $\mathrm{Bi}-212$ \\
\hline $2.02 \mathrm{E}-12$ & 763.06 & TI-208 & $->$ & $\mathrm{Pb}-208$ \\
\hline $1.97 \mathrm{E}-12$ & 1153.69 & Ac- 228 & $->$ & Th-228 \\
\hline $1.94 \mathrm{E}-12$ & 204.37 & Ac- 228 & $->$ & Th-228 \\
\hline $1.92 \mathrm{E}-12$ & 1033.32 & Ac- 228 & $->$ & Th-228 \\
\hline $1.86 \mathrm{E}-12$ & 440.49 & Ac- 228 & $->$ & Th-228 \\
\hline $1.86 \mathrm{E}-12$ & 583.28 & Ac- 228 & $->$ & Th-228 \\
\hline $1.86 \mathrm{E}-12$ & 707.49 & Ac- 228 & $->$ & Th-228 \\
\hline $1.73 \mathrm{E}-12$ & 184.72 & Ac- 228 & $->$ & Th-228 \\
\hline $1.63 \mathrm{E}-12$ & 1095.87 & Ac- 228 & $->$ & Th-228 \\
\hline $1.61 \mathrm{E}-12$ & 327.67 & Ac- 228 & $->$ & Th-228 \\
\hline $1.60 \mathrm{E}-12$ & 10.541 & TI-208 & $->$ & $\mathrm{Pb}-208$ \\
\hline $1.57 \mathrm{E}-12$ & 84.789 & TI-208 & $->$ & $\mathrm{Pb}-208$ \\
\hline $1.51 \mathrm{E}-12$ & 948.4 & Ac- 228 & $->$ & Th-228 \\
\hline $1.50 \mathrm{E}-12$ & 12.701 & TI-208 & $->$ & $\mathrm{Pb}-208$ \\
\hline $1.49 \mathrm{E}-12$ & 191.29 & Ac- 228 & $->$ & Th-228 \\
\hline $1.49 \mathrm{E}-12$ & 523.18 & Ac- 228 & $->$ & Th-228 \\
\hline $1.47 \mathrm{E}-12$ & 1286.6 & Ac- 228 & $->$ & Th-228 \\
\hline $1.46 \mathrm{E}-12$ & 1886.92 & Ac- 228 & $->$ & Th-228 \\
\hline $1.36 \mathrm{E}-12$ & 619.88 & $A c-228$ & $->$ & Th-228 \\
\hline $1.36 \mathrm{E}-12$ & 39.846 & $\mathrm{Bi}-212$ & $->$ & TI-208 \\
\hline $1.32 \mathrm{E}-12$ & 943.9 & Ac- 228 & $->$ & Th-228 \\
\hline $1.29 \mathrm{E}-12$ & 1686 & Ac- 228 & $->$ & Th-228 \\
\hline $1.25 \mathrm{E}-12$ & 651.44 & Ac- 228 & $->$ & Th-228 \\
\hline $1.24 \mathrm{E}-12$ & 673.86 & Ac- 228 & $->$ & Th-228 \\
\hline $1.24 \mathrm{E}-12$ & 884 & Ac- 228 & $->$ & Th-228 \\
\hline $1.18 \mathrm{E}-12$ & 1078.69 & $\mathrm{Bi}-212$ & $->$ & Po-212 \\
\hline $1.17 \mathrm{E}-12$ & 9.419 & $\mathrm{~Pb}-212$ & $->$ & $\mathrm{Bi}-212$ \\
\hline $1.11 \mathrm{E}-12$ & 1245.23 & Ac- 228 & $->$ & Th-228 \\
\hline $1.11 \mathrm{E}-12$ & 774 & $A c-228$ & $->$ & Th-228 \\
\hline $1.11 \mathrm{E}-12$ & 615.9 & Ac- 228 & $->$ & Th-228 \\
\hline $1.10 \mathrm{E}-12$ & 282.02 & Ac- 228 & $->$ & Th-228 \\
\hline
\end{tabular}




\begin{tabular}{|c|c|}
\hline $1.04 \mathrm{E}-12$ & 874.43 \\
\hline $1.01 \mathrm{E}-12$ & 14.563 \\
\hline $9.87 \mathrm{E}-13$ & 252.45 \\
\hline $9.67 \mathrm{E}-13$ & 215.75 \\
\hline $9.65 E-13$ & 519.97 \\
\hline $9.28 \mathrm{E}-13$ & 1163.6 \\
\hline 8.91E-13 & 1952.5 \\
\hline 8.29E-13 & 1677.9 \\
\hline 8.29E-13 & 1702.3 \\
\hline $8.29 E-13$ & 1528.9 \\
\hline $8.17 \mathrm{E}-13$ & 870.47 \\
\hline $8.17 E-13$ & 223.72 \\
\hline $8.10 \mathrm{E}-13$ & 893.42 \\
\hline $8.05 E-13$ & 449.57 \\
\hline $7.55 \mathrm{E}-13$ & 640.7 \\
\hline $7.55 E-13$ & 1116.97 \\
\hline $7.43 \mathrm{E}-13$ & 232.3 \\
\hline $7.43 E-13$ & 1249.81 \\
\hline $7.18 \mathrm{E}-13$ & 825.3 \\
\hline $7.18 \mathrm{E}-13$ & 263.57 \\
\hline $6.91 \mathrm{E}-13$ & 1512.75 \\
\hline $6.89 E-13$ & 83.787 \\
\hline $6.81 \mathrm{E}-13$ & 1039.97 \\
\hline $6.81 \mathrm{E}-13$ & 1573.3 \\
\hline $6.31 \mathrm{E}-13$ & 1823.47 \\
\hline $6.28 \mathrm{E}-13$ & 10.622 \\
\hline $6.20 \mathrm{E}-13$ & 14.336 \\
\hline $6.19 \mathrm{E}-13$ & 1572.03 \\
\hline $6.19 \mathrm{E}-13$ & 460.84 \\
\hline $6.19 \mathrm{E}-13$ & 629.93 \\
\hline $6.06 \mathrm{E}-13$ & 141.19 \\
\hline $6.06 \mathrm{E}-13$ & 555.3 \\
\hline $6.06 \mathrm{E}-13$ & 1537.4 \\
\hline $5.94 \mathrm{E}-13$ & 1842.29 \\
\hline $5.82 \mathrm{E}-13$ & 1907.5 \\
\hline $5.69 E-13$ & 666.4 \\
\hline $5.32 \mathrm{E}-13$ & 1548.5 \\
\hline $5.32 \mathrm{E}-13$ & 515.2 \\
\hline $5.32 \mathrm{E}-13$ & 498.26 \\
\hline $5.32 \mathrm{E}-13$ & 649.19 \\
\hline $5.12 \mathrm{E}-13$ & 1757.88 \\
\hline $5.07 E-13$ & 1835.1 \\
\hline $5.07 \mathrm{E}-13$ & 737.7 \\
\hline $4.58 \mathrm{E}-13$ & 399.2 \\
\hline $4.56 \mathrm{E}-13$ & 1093.63 \\
\hline $4.53 E-13$ & 87.632 \\
\hline $4.49 E-13$ & 452.77 \\
\hline $4.48 \mathrm{E}-13$ & 11.713 \\
\hline $4.47 E-13$ & 131.5 \\
\hline $4.33 E-13$ & 471.4 \\
\hline
\end{tabular}

\begin{tabular}{|c|c|c|}
\hline Ас- 228 & -> & Th-228 \\
\hline $\mathrm{Bi}-212$ & $->$ & TI-208 \\
\hline TI-208 & -> & b-208 \\
\hline Th-228 & $->$ & Ra-224 \\
\hline Ac-228 & $->$ & Th-228 \\
\hline$c-228$ & -> & Th-228 \\
\hline$c-228$ & $->$ & Th-228 \\
\hline Ac- 228 & -> & Th-228 \\
\hline$c-228$ & $->$ & Th-228 \\
\hline Ac- 228 & $->$ & Th-228 \\
\hline Ac- 228 & $->$ & Th-228 \\
\hline Ac- 228 & $->$ & h-228 \\
\hline $\mathrm{Bi}-212$ & $->$ & Po-212 \\
\hline$A c-228$ & $->$ & Th-228 \\
\hline Ac- 228 & $->$ & Th-228 \\
\hline Ac- 228 & $->$ & Th-228 \\
\hline Ac- 228 & $->$ & Th-228 \\
\hline$A C-228$ & $->$ & Th-228 \\
\hline$A c-228$ & $->$ & Th-228 \\
\hline Ac- 228 & $->$ & Th-228 \\
\hline $\mathrm{Bi}-212$ & $->$ & Po-212 \\
\hline Ra-224 & $->$ & $\mathrm{Rn}-220$ \\
\hline Ac- 228 & $->$ & Th-228 \\
\hline Ac- 228 & $->$ & Th-228 \\
\hline Ac- 228 & $->$ & Th-228 \\
\hline Th-228 & $->$ & Ra-224 \\
\hline$a-224$ & $->$ & $\mathrm{Rn}-22 \mathrm{C}$ \\
\hline Ac- 228 & $->$ & Th-228 \\
\hline Ac- 228 & $->$ & Th-228 \\
\hline Ac- 228 & $->$ & Th-228 \\
\hline Ac- 228 & $->$ & Th-228 \\
\hline Ac- 228 & $->$ & Th-228 \\
\hline Ac- 228 & $->$ & Th-228 \\
\hline Ac- 228 & $->$ & Th-228 \\
\hline Ac- 228 & $->$ & Th-228 \\
\hline AC-228 & $->$ & Th-228 \\
\hline Ac- 228 & $->$ & Th-228 \\
\hline Ac- 228 & $->$ & Th-228 \\
\hline Ac- 228 & $->$ & Th-228 \\
\hline Ac- 228 & $->$ & Th-228 \\
\hline Ac- 228 & $->$ & Th-228 \\
\hline Ac- 228 & $->$ & Th-228 \\
\hline Ac- 228 & $->$ & Th-228 \\
\hline Ac- 228 & $->$ & Th-228 \\
\hline TI-208 & $->$ & $\mathrm{Pb}-208$ \\
\hline TI-208 & $->$ & $\mathrm{Pb}-208$ \\
\hline $\mathrm{Bi}-212$ & $->$ & TI-208 \\
\hline Ra-224 & $->$ & $\mathrm{Rn}-220$ \\
\hline Th-228 & $->$ & $\mathrm{Ra}-224$ \\
\hline Ac- 228 & $->$ & Th-228 \\
\hline
\end{tabular}




\begin{tabular}{|c|c|c|c|c|}
\hline $4.33 E-13$ & 816.62 & $A c-228$ & $->$ & Th-228 \\
\hline $4.22 \mathrm{E}-13$ & 288.08 & $\mathrm{Bi}-212$ & $->$ & TI-208 \\
\hline $4.13 E-13$ & 81.067 & Ra-224 & $->$ & $\mathrm{Rn}-220$ \\
\hline $3.96 \mathrm{E}-13$ & 257.29 & Ac- 228 & $->$ & Th-228 \\
\hline $3.96 \mathrm{E}-13$ & 174.18 & Ac- 228 & $->$ & Th-228 \\
\hline $3.96 \mathrm{E}-13$ & 396.9 & Ac- 228 & $->$ & Th-228 \\
\hline $3.96 \mathrm{E}-13$ & 1431 & $A c-228$ & $->$ & Th-228 \\
\hline $3.96 \mathrm{E}-13$ & 1041.35 & Ac- 228 & $->$ & Th-228 \\
\hline $3.96 \mathrm{E}-13$ & 1054.3 & $A c-228$ & $->$ & Th-228 \\
\hline $3.88 \mathrm{E}-13$ & 952.17 & $\mathrm{Bi}-212$ & $->$ & Po-212 \\
\hline $3.82 \mathrm{E}-13$ & 233.32 & TI-208 & $->$ & $\mathrm{Pb}-208$ \\
\hline $3.59 \mathrm{E}-13$ & 540.5 & Ac- 228 & $->$ & Th-228 \\
\hline $3.59 \mathrm{E}-13$ & 791.2 & Ac- 228 & $->$ & Th-228 \\
\hline $3.59 E-13$ & 1357.6 & $A c-228$ & $->$ & Th-228 \\
\hline $3.59 \mathrm{E}-13$ & 1503.87 & Ac- 228 & $->$ & Th-228 \\
\hline $3.59 \mathrm{E}-13$ & 474.32 & Ac- 228 & $->$ & Th-228 \\
\hline $3.59 \mathrm{E}-13$ & 1174.79 & $A c-228$ & $->$ & Th-228 \\
\hline $3.47 \mathrm{E}-13$ & 919.19 & $A c-228$ & $->$ & Th-228 \\
\hline $3.28 \mathrm{E}-13$ & 13.662 & Th-228 & $->$ & $\mathrm{Ra}-224$ \\
\hline $3.22 \mathrm{E}-13$ & 419.23 & Ac- 228 & $->$ & Th-228 \\
\hline $3.22 \mathrm{E}-13$ & 1415.7 & Ac- 228 & $->$ & Th-228 \\
\hline $3.22 \mathrm{E}-13$ & 1216.4 & $A c-228$ & $->$ & Th-228 \\
\hline $3.22 \mathrm{E}-13$ & 1871.2 & Ac- 228 & $->$ & Th-228 \\
\hline $3.09 \mathrm{E}-13$ & 1930.3 & Ac- 228 & $->$ & Th-228 \\
\hline $3.09 \mathrm{E}-13$ & 1019.7 & $A c-228$ & $->$ & Th-228 \\
\hline $3.09 E-13$ & 1016.12 & Ac- 228 & $->$ & Th-228 \\
\hline $2.99 \mathrm{E}-13$ & 11.712 & $\mathrm{~Pb}-212$ & $->$ & Bi-212 \\
\hline $2.97 E-13$ & 1965.3 & $A c-228$ & $->$ & Th-228 \\
\hline $2.86 \mathrm{E}-13$ & 166.43 & Th-228 & $->$ & $\mathrm{Ra}-224$ \\
\hline $2.85 E-13$ & 979.7 & $A c-228$ & $->$ & Th-228 \\
\hline $2.76 \mathrm{E}-13$ & 14.884 & TI-208 & $->$ & $\mathrm{Pb}-208$ \\
\hline $2.60 \mathrm{E}-13$ & 922.28 & Ac- 228 & $->$ & Th-228 \\
\hline $2.60 \mathrm{E}-13$ & 1309.6 & Ac- 228 & $->$ & Th-228 \\
\hline $2.60 \mathrm{E}-13$ & 1314.76 & Ac- 228 & $->$ & Th-228 \\
\hline $2.60 \mathrm{E}-13$ & 791.05 & $A c-228$ & $->$ & Th-228 \\
\hline $2.50 \mathrm{E}-13$ & 721.91 & Tl-208 & $->$ & $\mathrm{Pb}-208$ \\
\hline $2.48 \mathrm{E}-13$ & 94.677 & Ra-224 & $->$ & $\mathrm{Rn}-220$ \\
\hline $2.48 \mathrm{E}-13$ & 887.46 & $A c-228$ & $->$ & Th-228 \\
\hline $2.48 \mathrm{E}-13$ & 877.65 & $A c-228$ & $->$ & Th-228 \\
\hline $2.48 \mathrm{E}-13$ & 1277.5 & Ac- 228 & $->$ & Th-228 \\
\hline $2.48 \mathrm{E}-13$ & 492.36 & Ac- 228 & $->$ & Th-228 \\
\hline $2.48 \mathrm{E}-13$ & 1481.2 & $A c-228$ & $->$ & Th-228 \\
\hline $2.48 \mathrm{E}-13$ & 356.83 & Ac- 228 & $->$ & Th-228 \\
\hline $2.48 \mathrm{E}-13$ & 1468.8 & Ac- 228 & $->$ & Th-228 \\
\hline $2.48 \mathrm{E}-13$ & 1374.26 & Ac- 228 & $->$ & Th-228 \\
\hline $2.45 E-13$ & 1805.93 & $\mathrm{Bi}-212$ & $->$ & Po-212 \\
\hline $2.43 E-13$ & 982.52 & Tl-208 & $->$ & $\mathrm{Pb}-208$ \\
\hline $2.41 \mathrm{E}-13$ & 549.7 & $\mathrm{Rn}-220$ & $->$ & Po-216 \\
\hline $2.40 \mathrm{E}-13$ & 1739.1 & $A c-228$ & $->$ & Th-228 \\
\hline $2.23 E-13$ & 1451.2 & $A c-228$ & $->$ & Th-228 \\
\hline
\end{tabular}




\begin{tabular}{|c|c|c|c|c|}
\hline $2.21 \mathrm{E}-13$ & 79.29 & $\mathrm{Bi}-212$ & $\rightarrow$ & Po-212 \\
\hline $2.10 \mathrm{E}-13$ & 416.2 & $A c-228$ & $->$ & Th-228 \\
\hline $2.10 \mathrm{E}-13$ & 135.68 & Ac- 228 & $->$ & Th-228 \\
\hline $2.10 \mathrm{E}-13$ & 1104.01 & Ac- 228 & $->$ & Th-228 \\
\hline $2.10 \mathrm{E}-13$ & 211.31 & TI-208 & $->$ & $\mathrm{Pb}-208$ \\
\hline $1.86 \mathrm{E}-13$ & 388.9 & $A c-228$ & $->$ & Th-228 \\
\hline $1.86 \mathrm{E}-13$ & 1741.2 & $A c-228$ & $->$ & Th-228 \\
\hline $1.86 \mathrm{E}-13$ & 931.1 & Ac- 228 & $->$ & Th-228 \\
\hline $1.86 \mathrm{E}-13$ & 8.953 & $\mathrm{Bi}-212$ & $->$ & TI-208 \\
\hline $1.75 \mathrm{E}-13$ & 176.577 & $\mathrm{~Pb}-212$ & $->$ & $\mathrm{Bi}-212$ \\
\hline $1.73 \mathrm{E}-13$ & 220.49 & Ac- 228 & $->$ & Th-228 \\
\hline $1.73 \mathrm{E}-13$ & 853.6 & Ac- 228 & $->$ & Th-228 \\
\hline $1.73 \mathrm{E}-13$ & 1706.07 & Ac- 228 & $->$ & Th-228 \\
\hline $1.70 \mathrm{E}-13$ & 327.93 & $\mathrm{Bi}-212$ & $->$ & TI-208 \\
\hline $1.61 \mathrm{E}-13$ & 1347.6 & $A c-228$ & $->$ & Th-228 \\
\hline $1.57 \mathrm{E}-13$ & 72.873 & $\mathrm{Bi}-212$ & $->$ & TI-208 \\
\hline $1.54 \mathrm{E}-13$ & 927.42 & TI-208 & $->$ & $\mathrm{Pb}-208$ \\
\hline $1.50 \mathrm{E}-13$ & 1679.42 & $\mathrm{Bi}-212$ & $->$ & Po-212 \\
\hline $1.49 \mathrm{E}-13$ & 1135.39 & $A c-228$ & $->$ & Th-228 \\
\hline $1.34 \mathrm{E}-13$ & 16.881 & Ra-224 & $->$ & $\mathrm{Rn}-220$ \\
\hline $1.32 \mathrm{E}-13$ & 76.858 & $\mathrm{Bi}-212$ & $->$ & Po-212 \\
\hline $1.24 \mathrm{E}-13$ & 1142.7 & Ac- 228 & $->$ & Th-228 \\
\hline $1.19 \mathrm{E}-13$ & 1784.5 & Ac- 228 & $->$ & Th-228 \\
\hline $1.11 \mathrm{E}-13$ & 481.8 & Ac- 228 & $->$ & Th-228 \\
\hline $1.11 \mathrm{E}-13$ & 1750.9 & $A c-228$ & $->$ & Th-228 \\
\hline $1.11 \mathrm{E}-13$ & 372.3 & Ac- 228 & $->$ & Th-228 \\
\hline $1.01 \mathrm{E}-13$ & 88.471 & Th-228 & $->$ & $\mathrm{Ra}-224$ \\
\hline $9.77 \mathrm{E}-14$ & 205.75 & Th-228 & $->$ & Ra-224 \\
\hline $9.63 \mathrm{E}-14$ & 415.156 & $\mathrm{~Pb}-212$ & $->$ & $\mathrm{Bi}-212$ \\
\hline $9.28 \mathrm{E}-14$ & 70.832 & $\mathrm{Bi}-212$ & $->$ & TI-208 \\
\hline $9.05 E-14$ & 13.501 & Bi-212 & $->$ & Po-212 \\
\hline $9.05 \mathrm{E}-14$ & 11.119 & $\mathrm{Bi}-212$ & $->$ & Po-212 \\
\hline $8.02 \mathrm{E}-14$ & 9.185 & TI-208 & $->$ & $\mathrm{Pb}-208$ \\
\hline $7.92 \mathrm{E}-14$ & 97.907 & Ra-224 & $->$ & $\mathrm{Rn}-220$ \\
\hline $7.72 \mathrm{E}-14$ & 89.639 & $\mathrm{Bi}-212$ & $->$ & Po-212 \\
\hline $6.41 \mathrm{E}-14$ & 1282.7 & TI-208 & $->$ & $\mathrm{Pb}-208$ \\
\hline $6.19 \mathrm{E}-14$ & 473.36 & $\mathrm{Bi}-212$ & $->$ & TI-208 \\
\hline $6.17 \mathrm{E}-14$ & 485.78 & TI-208 & $->$ & $\mathrm{Pb}-208$ \\
\hline $6.11 \mathrm{E}-14$ & 85.429 & Th-228 & $->$ & $\mathrm{Ra}-224$ \\
\hline $5.82 \mathrm{E}-14$ & 1713.25 & $A c-228$ & $->$ & Th-228 \\
\hline $5.69 \mathrm{E}-14$ & 1900.1 & $A c-228$ & $->$ & Th-228 \\
\hline $5.57 \mathrm{E}-14$ & 82.434 & $\mathrm{Bi}-212$ & $->$ & TI-208 \\
\hline $5.30 \mathrm{E}-14$ & 748.58 & TI-208 & $->$ & $\mathrm{Pb}-208$ \\
\hline $4.93 \mathrm{E}-14$ & 587.82 & TI-208 & $->$ & $\mathrm{Pb}-208$ \\
\hline $4.93 \mathrm{E}-14$ & 821.14 & TI-208 & $->$ & $\mathrm{Pb}-208$ \\
\hline $4.44 \mathrm{E}-14$ & 650.14 & TI-208 & $->$ & $\mathrm{Pb}-208$ \\
\hline $4.21 \mathrm{E}-14$ & 692.5 & Ac- 228 & $->$ & Th-228 \\
\hline $3.82 \mathrm{E}-14$ & 883.27 & TI-208 & $->$ & $\mathrm{Pb}-208$ \\
\hline $3.60 \mathrm{E}-14$ & 99.915 & Th-228 & $->$ & $\mathrm{Ra}-224$ \\
\hline $3.53 E-14$ & 1074.1 & $\mathrm{Bi}-212$ & $->$ & Po-212 \\
\hline
\end{tabular}




$\begin{array}{lcrll}3.50 \mathrm{E}-14 & 10.994 & \mathrm{Bi}-212 & \rightarrow> & \mathrm{Tl}-208 \\ 3.10 \mathrm{E}-14 & 290 & \mathrm{Ra}-224 & \rightarrow & \mathrm{Rn}-220 \\ 2.71 \mathrm{E}-14 & 705.24 & \mathrm{Tl}-208 & \rightarrow & \mathrm{Pb}-208 \\ 2.51 \mathrm{E}-14 & 10.137 & \mathrm{Ra}-224 & -> & \mathrm{Rn}-220 \\ 2.43 \mathrm{E}-14 & 92.673 & \mathrm{Bi}-212 & -> & \mathrm{Po}-212 \\ 2.41 \mathrm{E}-14 & 650.1 & \mathrm{Ra}-224 & -> & \mathrm{Rn}-220 \\ 2.15 \mathrm{E}-14 & 11.349 & \mathrm{Tl}-208 & -> & \mathrm{Pb}-208 \\ 2.10 \mathrm{E}-14 & 1185.1 & \mathrm{Tl}-208 & -> & \mathrm{Pb}-208 \\ 1.76 \mathrm{E}-14 & 15.869 & \mathrm{Bi}-212 & -> & \mathrm{Po}-212 \\ 1.73 \mathrm{E}-14 & 433.51 & \mathrm{Bi}-212 & -> & \mathrm{Tl}-208 \\ 1.72 \mathrm{E}-14 & 164.15 & \mathrm{~Pb}-212 & -> & \mathrm{Bi}-212 \\ 1.55 \mathrm{E}-14 & 85.185 & \mathrm{Bi}-212 & -> & \mathrm{Tl}-208 \\ 1.38 \mathrm{E}-14 & 409.3 & \mathrm{Ra}-224 & -> & \mathrm{Rn}-220 \\ 1.36 \mathrm{E}-14 & 1160.55 & \mathrm{Tl}-208 & -> & \mathrm{Pb}-208 \\ 1.24 \mathrm{E}-14 & 143.99 & \mathrm{Bi}-212 & -> & \mathrm{Tl}-208 \\ 1.17 \mathrm{E}-14 & 103.341 & \mathrm{Th}-228 & -> & \mathrm{Ra}-224 \\ 1.17 \mathrm{E}-14 & 12.855 & \mathrm{Ra}-224 & -> & \mathrm{Rn}-220 \\ 8.64 \mathrm{E}-15 & 1381 & \mathrm{Tl}-208 & -> & \mathrm{Pb}-208 \\ 7.55 \mathrm{E}-15 & 492.62 & \mathrm{Bi}-212 & -> & \mathrm{Tl}-208 \\ 6.21 \mathrm{E}-15 & 804.9 & \mathrm{Po}-216 & -> & \mathrm{Pb}-212 \\ 6.19 \mathrm{E}-15 & 164 & \mathrm{Bi}-212 & -> & \mathrm{Tl}-208 \\ 6.17 \mathrm{E}-15 & 1125.6 & \mathrm{Tl}-208 & -> & \mathrm{Pb}-208 \\ 4.63 \mathrm{E}-15 & 9.658 & \mathrm{Bi}-212 & -> & \mathrm{Po}-212 \\ 4.46 \mathrm{E}-15 & 620.3 & \mathrm{Bi}-212 & -> & \mathrm{Tl}-208 \\ 2.47 \mathrm{E}-15 & 1647.4 & \mathrm{Tl}-208 & -> & \mathrm{Pb}-208 \\ 2.47 \mathrm{E}-15 & 1743.57 & \mathrm{Tl}-208 & -> & \mathrm{Pb}-208 \\ 1.37 \mathrm{E}-15 & 12.085 & \mathrm{Bi}-212 & -> & \mathrm{Po}-212 \\ 9.78 \mathrm{E}-16 & 580.4 & \mathrm{Bi}-212 & -> & \mathrm{Tl}-208\end{array}$

